

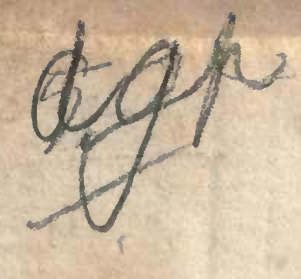




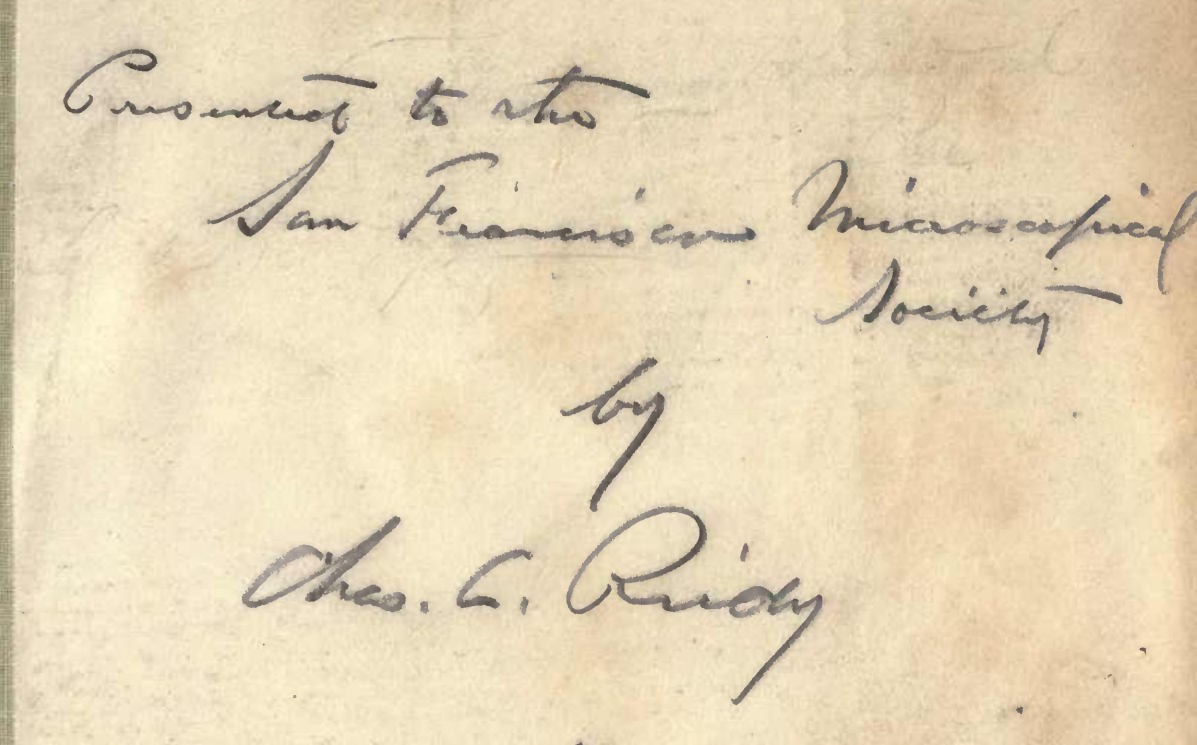


x 




\section{A N U A L}

or

HUMAN MICROSCOPICAL ANATOMY. 



\section{MANUAL}

OF

\section{HUMAN MICROSCOPICAL ANATOMY.}

PROFESSOR OF ANATOMX AND PHYSIOLOGY IN WÜRZBCRG.

TRANSLATED BY

GEORGE BUSK, F.R.S., aNd THOMAS HUXLEY, F.R.S.

EDITED, WITH NOTES AND ADDITIONS,

BY

J. DA COSTA, M.D.

ILLUSTRATED BY THREE HUNDRED AND THIRTEEN ENGRAVINGS ON WOOD,

PHILADELPHIA:

LIPPINCOTT, GRAMBO \& CO.,

$185 \%$ 



\section{C.ONTENTS.}

\section{INTRODUCTION.}

BECT.

1. Historical Introduction

2. Present position of the Science, $\quad$ - $\quad$. $\quad$. $\quad 34$

3. Aids to the Study (Literature, Microscope, Preparations), - 36

\section{GENERAL HISTOLOGY.}

\section{I.-OF THE ELEMENTARY PARTS, pp. 39, 40.}

4. Simple and compound elementary parts, $\quad \ldots \quad \ldots \quad \ldots \quad \ldots \quad$. $\quad . \quad 39$

5. Plastic fluid, fundamental substance, . $\quad$. . . . 40

A. Simple elementary parts, pp. 41-70.

1. Elementary Granules,-vesicles, nuclei-

6.

\section{Of the Cells:-}

7. Composition,

8. Form, chemical relations, nucleus, nucleolus, $\quad . \quad \ldots \quad . \quad 44$

9. Cell-formation,

10. Free cell-formation,

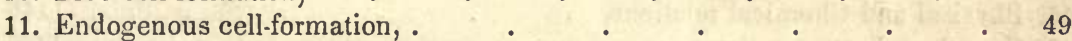

12. Multiplication of cells by division, . . . . . . 54

13. Theory of cell-formation, . . . . . . . . $\quad$. 56

14. Vital phenomena of cells, growth, . $\quad . \quad \ldots \quad$. $\quad . \quad 59$

15. Processes in the interior of the cells-Absorption, ${ }^{\text {1 }} 61$

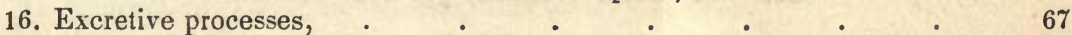

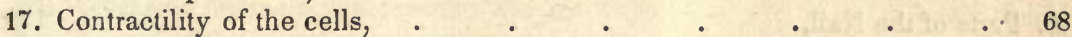

18. Metamorphoses of the cells, kinds of cells, . $\quad . \quad \ldots \quad$. 69

B. HIGHER ELEMENTARY PARTs, pp. 70-72.

19.

II.-OF THE TISSUES, ORGANS, AND SYSTEMS, pp. 72-119.

20. Enumeration of them, 
22. Cartilaginous tissue, .

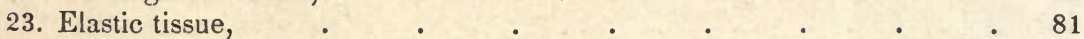

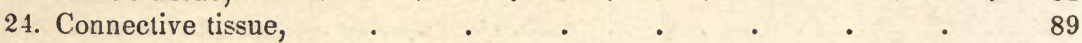

25. Osseous tissue, . . . . . . . $\quad . \quad 99$

26. Tissue of the smooth muscles, . . . . 102

27. Tissue of the striped muscles, . . . . _ . . . 106

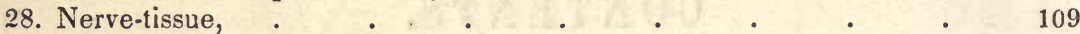

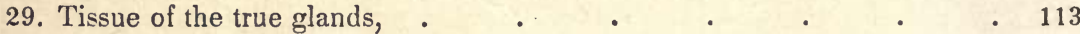

30. Tissue of the blood-vascular glands, $\quad$. $\quad . \quad \ldots \quad$. $\quad$. 116

\section{SPECIAL HISTOLOGY.}

\section{OF THE EXTERNAL INTEGUMENT, pp. 119--222.}

I.-OF THE SKIN IN THE MORE RESTRICTED SENSE, pp. 119-159.

A. CUTis Derma, pp. 119-139.

31. Parts of the common integument, . $\quad$ - $\quad$. $\quad$. $\quad$. $\quad 119$

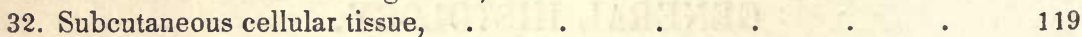

33. Parts of the corium, tactile papillæ, . . . . . . $\quad$. 120

34. Connective tissue, elastic fibres, and muscles of the corium, . . . 123

35. Fat-cells, • • . . . . . . . . . 125

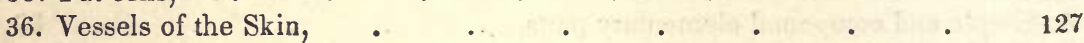

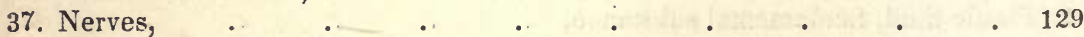

38. Development of the Cutis, . $\quad$. $\quad$. $\quad . \quad . \quad$. 134

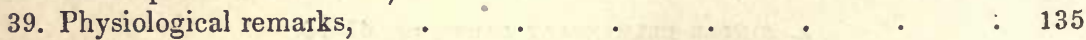

B. CUTICLE OR EPIDERMIS, pp. 139-159.

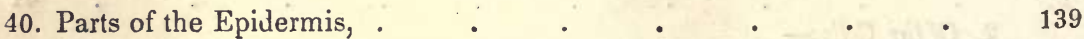

41. Mucous layer, . . . . . . . $\quad$. 140

42. Horny layer, . $\quad$. $\quad$. . . . . . 142

43. Colour of the Epidermis, . . . . . . . $\quad$. 143

44. Thickness of the Epidermis, . . . . . 145

45. Physical and Chemical relations, . . . . . . . 146

46. Growth and regeneration, . . . . . . . $\quad .150$

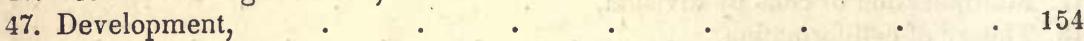

II.-OF THE NAILS, pp. 159-171.

48. Parts of the Nail,

49. Structure of the Nail,

50. Relation of the Nail to the epidermis, . . . . . 164

51. Growth of the Nails, . . . . . . . . 165

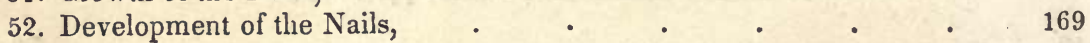

III.-OF THE HAIRS, pp. 171-199.

OF THE HAIRS IN THE MORE RESTRICTED SENSE.

53. Parts of the Hair, 
54. Disposition and size of the Hairs,

55. External peculiarities, and Chemical composition of the Hairs, . $\quad$. 172

56. Structure of the Hairs, cortical substance, . $\quad$. $\quad$. $\quad 173$

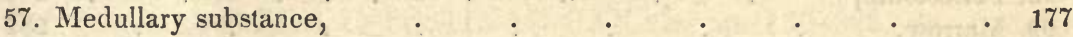

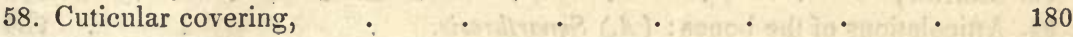

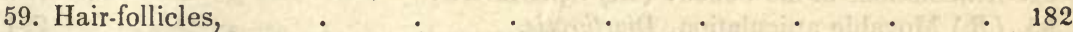

60. Hair-follicle in the more restricted sense, _ $\quad$. $\quad . \quad$. $\quad 182$

61. Root-sheaths, . . . . . . . . 184

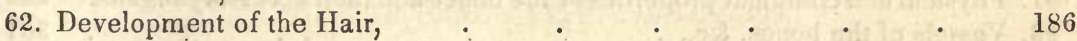

63. Shedding of the Hair, . . . . . . . . . . 191

64. Physiological remarks, _ . . . . . . . 193

\section{IV.-OF THE CUTANEOUS GLANDS, pp. 199, 222.}

A. OF THE SUDORIPAROUS GLANDS, pp. 199-209.

65. Disposition,

66. Structure, ․ . . . . . . . 199

67. More intimate structure of the glandular coil, . . . . 200

68. Secretion of the sudoriparous glands, . $\quad . \quad \ldots \quad$. $\quad 202$

69. Sweat-Ducts, . . . . . . . . 204

70. Development of the sudoriparous glands, . . . . 205

B. of the Ceruminots gland, pp. 209-212.

71. Structure,

72. Secretion and development,

c. of the sebaceous glands, pp. 212-222.

73. Strueture, form, and disposition, . . . . . $\quad 212$

74. More intimate structure, . . . . . . . . 216

75. Development, . $\quad$. $\quad$. $\quad$. $\quad$. . 218

\section{OF THE MUSCULAR SYSTEM, pp. 222-266.}

76. Definition of it, . . . . . . . . . . 222

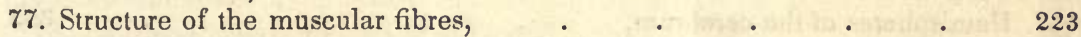

78. The mode in which they are associated, . . . . . 229

79. Connection with other parts, . . . . . . 231

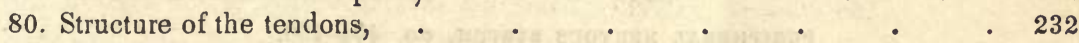

81. Connection of the tendons with other parts, . . . . $\quad 235$

82. Accessory organs of the muscles and tendons, . . . . 238

83. Vessels of the muscles and accessory organs, . . . . . 243

84. Nerves of the muscles, . . . . . . . . . . 245

85. Chemical and physical relations of the muscles, . . . $\quad 250$

86. Development of the muscles and tendons, . $\quad$. $\quad . \quad 253$

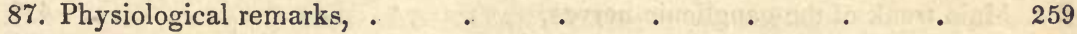

OF THE OSSEOUS SYSTEM, pp. 266-345.

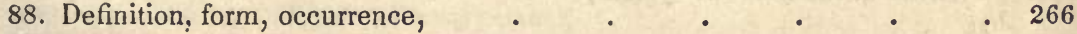

89. Intimate structure of the osseous tissue, _ $\quad . \quad \ldots \quad$. 267 
SECr.

90. Matrix of bone, • +270

91. Lacunæ and canaliculi, . . . . . . . 275

92. Periosteum, .

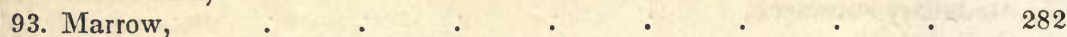

94. Articulations of the bones: (A.) Synarthrosis, . . . . 284

95. (B.) Movable articulation, Diarthrosis, . . . . . 291

96. Articular capsules, . . . . . . . . . . 295

97. Physical and chemical properties of the bones and their accessory organs, 299

98. Vessels of the bories, \&c., . . . . . . . 301

99. Nerves of the osseous system, . $\quad . \quad \ldots \quad \ldots \quad$. $\quad . \quad 303$

100. Development of the bones, . . . . . . . 306

101. Primordial cartilaginous skeleton, . $\quad$. $\quad . \quad 306$

102. Metamorphoses of the primordial cartilaginous skeleton, . $\quad 310$

103. Changes in the ossifying cartilage, . . . . . 314

104. Ossification of the cartilage, . . . . . . . 316

105. Elementary processes in the layers formed from the periosteum, . $\quad 324$

106. Bones, not primarily cartilaginous, . . . . . 330

107. Growth of the secondary cranial bones, . . . . 330

108. Vital phenomena in the mature bones, . . . . 336

\section{OF THE NERVOUS SYSTEM, pp. 345-436.}

109. Definition, division,

ELEMENTS OF THE NEIROUS SYSTEM, pp. 345-359.

110. Nerve-tubes or fibres,

111. Nerve-cells,

Centrad nervous system, pp. 359-404.

112. Spinal cord,

113. Conjectural course of the fibres in the spinal cord,

• $\quad \cdot \quad 369$

114. Medulla oblongata and Pons Varolii,

115. Cerebellum, . . . . . . . . . 379

117. Ganglia of the cerebrum, . . . . . . . . 382

117. Hemispheres of the cerebrum, . . . . . . 385

118. Membranes and vessels of the central nervous system, . . . 392

PERIPHERAL NERVOUS system, pp. 404-436.

119. Spinal nerves, .

120. Structure of the spinal ganglia, . . . . . . . 405

121. Further course of the spinal nerves, . . . . . . 411

122. Cerebral nerves, . . . . . . . . . . . 416

123. Ganglionic nerves, . . . . . . . . 418

124. Main trunk of the ganglionic nerves, . . . . . . 418

125. Peripheral distribution of the ganglionic nerves, . . . . 423

126. Development of the elements of the nervous system, . . . 426

127. Functions of the nervous system, . . . . . . 431 


\section{OF THE DIGESTIVE ORGANS, pp. 436-568.}

\section{OF THE INTESTINAL CANAL, pp. 436-528.}

SECT.

128. General structure, .

OF THE ORAL CAVITY, pp. 436-499.

A. of the mucous membrane of the oral cavity, pp. 436-441.

129. Mucous membrane and submucous tissue,

B. OF THE TONGUE, pp. 441-455.

131. Muscular structure of the tongue, .

132. Mucous membrane of the tongue,

c. of the glands of the oral cavitr, pp. 455-467.

1. Mucous Glands:-

133. Different kinds of glands, .

134. Their more intimate structure,

2. Follicular Glands:-

135. Simple follicles and Tonsils,

3. Salivary Glands:-

D. OF THE TEETH, pp. $46 \pi-499$.

137. Constituent parts, .

138. Dentine (substantia eburnea), . . . . . . . 468

139. Enamel (substantia vitrea), . . . . . . . . . 476

140. Cement (substantia osteoidea), . . . . . . . 480

141. Soft parts of the teeth, . . . . . . . 483

142. Development of the teeth, . . . . . . 484

143. Physiological conditions of the teeth, . . . . . . 494

OF THE ORGANS OF DEGLUTITION, pp. 499-502.

1. THE PHARYNX.

144.

1. THE GSOPHAGUS.

145.

OF THE ALIMENTARY CANAL, pp. 502-528.

146. General conformation,

147. Peritoneum,

148. Muscular coat of the alimentary canal,

149. Mucous membrane of the stomach, 
SECT.

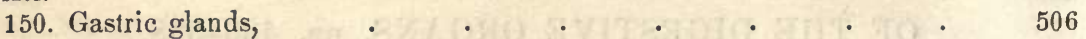

151. Other particulars of the mucous membrane, . $\quad$. $\quad$. 509

152. Mucous membrane of the small intestine, . . . . 511

153. Villi of the small intestine, $\quad$ - . $\quad$. $\quad$. $\quad 511$

154. Glands of the small intestine, . $\quad$. $\quad$. $\quad$. $\quad$. $\quad 518$

155. Closed follicles of the small intestine, . . . . . . . . . 520

156. Mucous Membrane of the large intestine, . $\quad$. . . 524

157. Development of the intestinal canal, _ . $\quad . \quad \ldots \quad$. 526

\section{OF THE LIVER, pp. 528-549.}

158. General structure,

159. More intimate structure,

160. Hepatic cells and cell networks, . $\quad$. $\quad$. 532

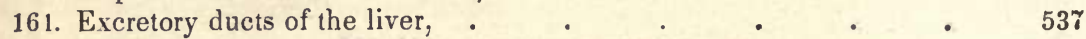

162. Vessels and nerves of the liver, . $\quad$. $\quad . \quad$. $\quad 541$

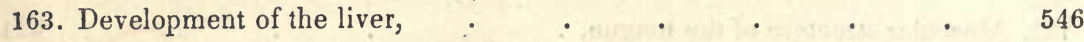

OF THE PANCREAS, pp. 549-551.

164.

\section{OF THE SPLEEN, pp. 551-568.}

165. General structure, .

166. Coats and trabecular structure of the spleen, . _ _ _ _ _ $\quad 551$

167. Malpighian structure of the spleen,

168. Red substance of the spleen,

169. Vessels and nerves of the spleen, .

170. Physiological remarks;

\section{OF THE RESPIRATORY ORGANS, pp. 568-595.}

171. Enumeration of the respiratory organs,

OF THE LUNGS, pp. 568-585.

172. General structure, .

173. Larynx,

174. Trachea,

175. Lungs, .

176. Air-vessels and cells,

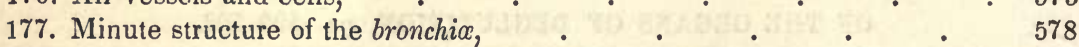

178. Vessels and nerves of the lungs, . $\quad . \quad \ldots \quad$. $\quad . \quad 580$

179. Development of the lungs, . $\quad$. $\quad$. $\quad$. 583

OF THE THYROID GLAND, pp. 585-589.

180. General structure of the thyroid gland,

\section{OF THE THYMUS, pp. 589-595.}

182. General structure of the thymus,

183. Minute structure,

184. Development of the thymus, 
OF THE URINARY ORGANS, pp. 595-613.

SECT.

185. Enumeration of the urinary organs,

186. Kidneys-general structure of, .

187. Composition of the renal substance,

188. Tubuli uriniferi,

189. Vessels and nerves of the kidneys,

190. Urinary passages,

191. Physiological remarks,

\section{OF THE SUPRARENAL GLANDS, pp. 613-618.}

192. General description of the suprarenal glands, . $\quad . \quad \ldots \quad$. 613

193. Minute structure, . . . . . . . 613

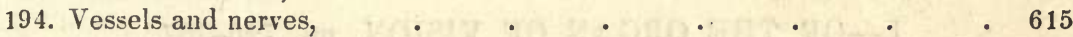

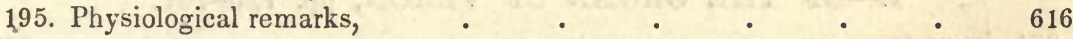

\section{OF THE SEXUAL ORGANS, pp. 618-667.}

A. Male sexual organs, pp. 618-640.

196. Enumeration of the male sexual organs,

197. Testes,

198. Tubuli seminiferi,

199. Membranes, vessels and nerves of the testes,

200. Vasa deferentia, vesicula seminales, and accessory glands, . $\quad 627$

201. Organ of copulation,

630

202. Physiological remarks,

634

B. Female sexual organs, pp. 640-660.

203. Enumeration of the female sexual organs,

204. Ovary, parovarium,

205. Detachment and re-formation of the ova,.

206. Uterus and oviducts,

643

207. Changes in the uterus at the menstrual period and in pregnancy, . 646

208.

209. Physiological remarks,

c. OF THE LACteal GLaNds, 660-667.

210. Their structure,

211. Physiological remarks,

\section{OF THE VASCULAR SYSTEM, pp. 667-715.}

212. Its elements,

I.-OF THE HEART, pp. 667-674.

213.

\section{II.-OF THE BLOODVESSELS, pp. 674-693.}

214. General structure of the bloodvessels,

215. Arteries,

216. Veins,

217. Capillaries, 
III.-OF THE LYMPHATIC SYSTEM, pp. 693-699.

SECT.

218. Lymphatic vessels,

219. Lymphatic glands,

IV.-OF THE BLOOD AND LYMPH, pp. 699-725.

220. Different kinds of fluids included in those terms, and their mode of

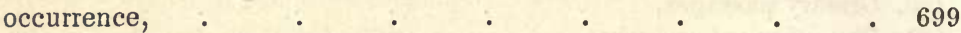

221. General structure of the morphological elements, . . . 700

222. Of the blood, . . . . . . . . 703

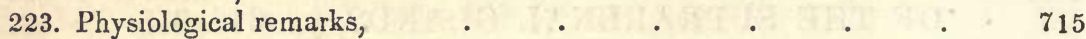

OF THE HIGHER ORGANS OF SENSE, pp. 725-785.

I.-OF THE ORGAN OF VISION, pp. 725-767.

224. Its parts, .

A. OF THE EXEBALL, pp. 725-757.

225. Fibrous tunic of the eye,

226. Vascular tunic,

227. Nervous tunic, .

228. The lens,

229. The vitreous humor,

B. ACCESSORT ORGANs, pp. 757-760.

230. Eyelids, conjunctiva, lachrymal apparatus,

231. Physiological remarks,

II.-OF THE ORGAN OF HEARING, pp. 767-778.

232.

$\circ \quad \cdot \quad 767$

233. External and middle ear, $\quad$. $\quad . \quad$. $\quad . \quad 467$

234. The vestibule and semicireular canals, $\quad . \quad$. $\quad . \quad 4 \quad 469$

235. Cochlea,

III.-OF THE OLFACTORY ORGAN, pp. 778-785.

236. Its parts,

\section{APPENDIX.}

1. Corpuscula tactûs and Pacinian bodies, . . . . . $\quad 785$

2. Malpighian bodies of the spleen, . . . . . . 786

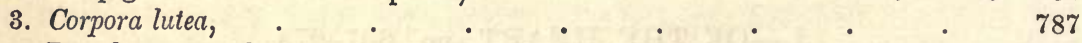

4. Development of the teeth, . $\quad$. $\quad$. $\quad$. $\quad$. $\quad 789$ 


\section{LIST OF ILLUSTRATIONS.}

FIG.

1. Nerve-cells of the Thalamus Opticus of Man,

2. Contents of a Malpighian Corpuscle of the $\mathrm{Ox}, \quad$. $\quad$. $\quad$. 48

3. Cells from the Cephalic Cartilage of a Tadpole, $\quad$. $\quad$. $\quad$. 50

4. Nuclei from the Ovum of an Ascaris dentata, . $\quad$. $\quad$. 50

5. Ova of Ascarus nigrovenosa, . $\quad . \quad$. $\quad . \quad$. $\quad . \quad 51$

6. Cartilage-cells from Articular Cartilage of the Condyle of the Femur of Man, 53

7. Cells from the Medullary Cavities of the Flat Bones of the Skull in Man, 53

8. Dividing Blood-corpuscles of the Chick, . . . . . 54

9. Dentine Cells, from the Dog, . . . . . . . $\quad$. 55

10. Cartilage Cells of Man, . . . . . . . . . 60

11. Bone-cells from a Rachitic Bone, • . . . . . 60

12. Plates of the Horny Layer of the Epidermis in Man, . . . $\quad 75$

13. Epidermis of a two months' Human Embryo, . • . • 76

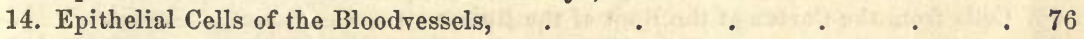

15. Epithelium of the Intestinal Villi of the Rabbit, . . . . 76

16. Ciliated cells from the finer Bronchiæ, . $\quad$. $\quad$. $\quad$. $\quad$. 76

17. A simple Papilla with manifold Vessels and Epithelium from the Gums of a Child, 77

18. Ciliated Epithelium from the Trachea of Man, $\quad$ - $\quad . \quad$. $\quad$. 77

19. Portion of the Chorda Dorsalis of an Embryo Sheep, . . . 80

20. Cartilage Cells from the White Layer of the Cricoid Cartilage, . $\quad$. 80

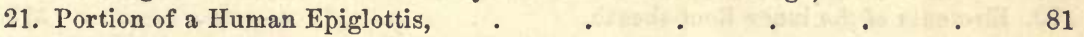

22. Elastic Network from the Tunica Media of the Pulmonary Artery of a Horse, 81

23. Bundles of Connective Tissue from the Arachnoid of Man, - $\quad 82$

24. Network of Fine Elastic Fibres from the Peritoneum of a Child, - . 82

25. Elastic Membrane from the Tunica Media of the Carotid of a Horse, $\quad 82$

26. Formative Cells of Elastic Fibres, from the Tendo-Achillis, . . . 83

27. Stellate Formative Cells of the Nucleus Fibres of Tendo-Achillis of a new-

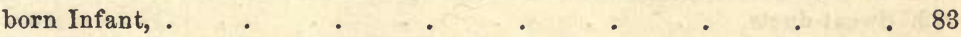

28. Lax Connective Tissue with Fat-cells, from Man, . $\quad$ _ $\quad$ • $\quad$ - $\quad 89$

29. Formative Cells of the Connective Tissue from the Skin of a Sheep's Embryo, 90

30. Formative Cells of the Areolated Connective Tissue from the Allantois of a Sheep's Embryo, . $\quad$. $\quad . \quad$. $\quad . \quad 4 \quad .90$

31. Perpendicular section of a Parietal Bone, . $\quad$. $\quad$. $\quad$. $\quad 99$

32. Developing Bone-cells from a Rachitic Bone, . • . . 100

33. Muscular Fibre-cell from the Small Intestine of Man, . . 103

34. Muscular Fibre-cell from the Investment of the Spleen of a Dog, . . 103

35. Muscular Fibres from Man, . . . . . 106

36. Primitive Fibrils from a Primitive Bundle of the Axolotl, . . . 106 
FIG.

37. Tubular Nerve-fibres of Man,

38. Nerve-cell of the Pike, .

39. Nerve-cells from the floor of the Fourth Ventricle in Man,

40. Network of Hepatic Cells,

41. Two of the Smallest Lobes of the Lung from a new-born Child, . 113

42. Gastric Gland from the Pylorus of the Dog, . . . . . . 114

43. Glandular Vesicles from the Thyroid Gland of a Child, • . 117

44. Malpighian Corpuscle from the Spleen of the Ox, . $\quad$ • . 117

45. Perpendicular section of the Skin of the Ball of the Thumb, . $\quad 119$

46. Compound Papillæ of the Surface of the Hand, . $\quad$. $\quad$. 121

47. Horizontal Section of the Skin of the Heel, . . . . 121

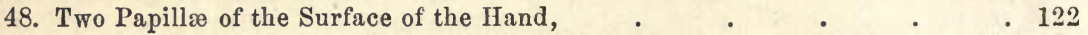

49. Elastic Fibres from the Fascia Lata of Man, . . . . 123

50. Normal Fat-cells from the Breast, . $\quad$. $\quad$. $\quad$. 125

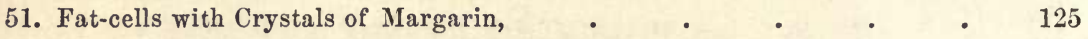

52. Vessels of the Fat-cells ; after Todd and Bowman, . $\quad$ • $\quad$. $\quad 128$

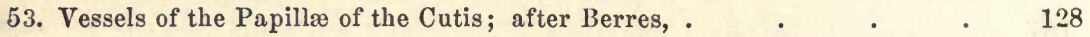

54. Two Papillæ from the extremities of the Fingers, with Axile-corpuscles, $\quad 129$

55. A. Surface of the Palm from within, . . . . $\quad 139$

55. B. Perpendicular section of the Skin of the Negro, . . . . 140

56. Horny Epidermic Plates of Man, . . . . . . $\quad 142$

57. Horny Plates boiled with Caustic Potassa, . $\quad$. $\quad$. 148

58. Transverse section through the Body and Bed of the Nail, _ $\quad$ - 159

59. Capillaries of the Bed of the Nail; after Berres, . . . . 160

60. Longitudinal section through the Matrix of the Nail, . . . 161

61. Transverse section through the Body of the Nail, . . . . 162

62. Nail Plates boiled with Caustic Soda, . . . . . . 163

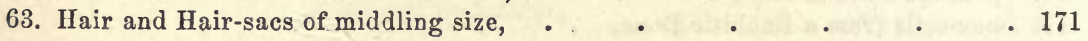

64. Plates of the Cortical Substance of a Hair, _ . _ . . . 174

65. White Hair after treatment with Caustic Soda, . . . . . 175

66. Cells from the Cortex of the Root of the Hair, . . . . 176

67. Cells from deepest part of Hair Bulb, . $\quad$. . . . 176

68. Root of a dark Hair acted upon by Caustic Soda, . _ . . . 177

69. Medullary Cells of Hair, . . . . . . . . . .

70. Surface of the Shaft of a White Hair, . . . . . 180

71. Portion of the transverse fibrous layer and structureless membrane of a Human

Hair-sac, . . . . . . . . . 183

72. Elements of the inner Root-sheath, $\quad . \quad \ldots \quad . \quad . \quad . \quad 185$

73. Rudiment of the Hair from the Brow of a Human Embryo, . . . . 187

74. Rudiment of the Hair from the Eyebrow, . $\quad$. $\quad$. $\quad$. $\quad 189$

75. Rudimental Hair from the Eyebrow, . $\quad$. $\quad . \quad$. $\quad$. 189

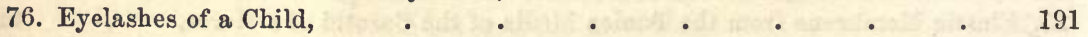

77. Eyelashes with the Root-sheaths from a Child, _ . . . 191

78. A Sudoriparous Coil and its Vessels, _ . . . . . 199

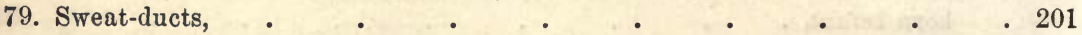

80. Perpendicular section through the Epidermis and outer surface of the Corium, . $\quad . \quad . \quad . \quad . \quad . \quad . \quad 204$

81. Rudiment of a Sudoriparous Gland of a Human Embryo, . • . 206

82. Rudiment of a Sudoriparous Gland from a seven months' Fœtus, . 206

83. Perpendicular section through the Skin of the External Auditory Meatus, 210

84. Sebaceous Glands from the Nose, . . . . . . . 213

85. A Gland from the Nose, with Hair-sac opening into it, . . . 213

86. Two Sebaceous Glands, . . . . . . . . . 215

87. A Glandular Vesicle of a common Sebaceous Gland, . $\quad$ • 216 
88. The development of the Sebaceous Glands in a six months' Fotus, . $\quad 219$

89. Primitive Fibrils from a primitive fasciculus of the Axolotl, . . 223

90. Transverse sections of Muscular Fibre, . . . . . . 224

91. Human Muscular Fibre treated with Acetic Acid, .. . . 224

92. A Primitive Fasciculus, separating transversely into dises; after Bowman, 225

93. Primitive Fibres from a transversely striated Muscle of a Bug, . . 226

94. Transverse section of Rectus Capitis Anticus Major of Man, . . . 230

95. Transverse section of a Tendon of a Calf, . . . . . . 233

96. Tendon of the Tibialis Posticus, . . . . . . . . $\quad$. 234

97. A Primitive Fasciculus from Intercostal Muscle of Man, . . . 235

98. Disposition of Muscular Fibres at their insertion into the Tendon, . . 235

99. Insertion of the Tendo-Achillis into the Calcaneum, . . . 237

100. Cartilage-cells from the Vaginal Ligaments surrounding the Tendons of the Poplitæus, . . . . . . . . . . . 241

101. Capillary Vessels in Muscle, . . . . . . . . . 243

102. Expansion of the Nerves in the Omo-hyoid muscle of Nan, . . $\quad 246$

103. Divisions of the Primitive Nerve-fibres in Muscle, . . . . 246

104. Divisions of Nerve-fibres in Thoracic Muscle of the Frog, . . 250

105. Primitive Fasciculi of a Human Embryo, . . . . . . . 254

106. Primitive Fibres of a Human Embryo, . . . . . 254

-107. From the Tendo-Achillis of a New-born Child, . . . . . 256

108. Primitive Fibres from the Alar Muscles of the Dung-fly, . _ . 259

109. Primitive Fasciculæ of a Frog's Muscle in different degrees of Extension, . 264

110. Transverse section of Human Femur, . . . . 268

111. Haversian Canals from Superficial Layer of Human Femur, • 268

112. Transverse section of Human Metacarpal Bone, . . . . 271

113. Transverse section of Shaft of Humerus, . $\quad$. $\quad . \quad$. . 271

114. Perpendicular section of Parietal Bone, . . . . . . . 273

115. Transverse section of Shaft of Humerus, . $\quad$. . . . $\quad$. 276

116. Section parallel with Surface of Human Femur, . . . . $\quad 276$

117. Lacunæ and Canaliculi of Parietal Bone, . . . . . . 277

118. Surface of a Tibia of Calf, . . . . . . 279

119. Bony Spiculæ from Apophysis, _ . . . . . . . 280

120. Fat-cells from Marrow of Human Femur, . . . . . 283

121. Transverse section of Ligamentum Nuchæ of $\mathrm{Ox}, \quad$. $\quad . \quad$. $\quad$ - 285

122. Cells from Gelatinous Nucleus of the Lig. Intervertebralia, . . 286

123. Cartilaginous border of Human Symphysis, $\quad . \quad 0 \quad$. $\quad . \quad$. 289

124. Human Cartilage Cells, . . . . . . 290

125. Perpendicular section of Articular Cartilage of Human Metacarpal Bone, . 292

126. Diagram of Transverse Section of Phalangeal Articulation, . . 295

127. Synovial Membrane of Phalangeal Articulation, . . . . . 297

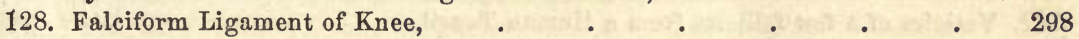

129. Cartilage Cells from Humerus of Embryo of Sheep, . _ . . . 308

130 Perpendicular section of Ossifying border of Shaft of Femur, . . $\quad 314$

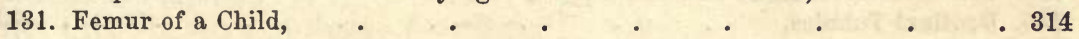

132. Femur of a Rachitic Child, . . . . . . 317

133. Transverse section of Metatarsus of Calf, . $\quad . \quad$. $\quad . \quad$. 324

134. Diagram of Growth of Cylindrical Bone, . $\quad$. $\quad . \quad$. $\quad 327$

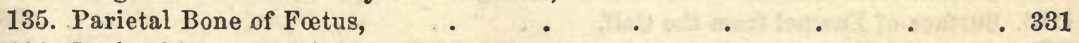

136. Parietal Bone of new-born Child, . _ . _ . . . . 331

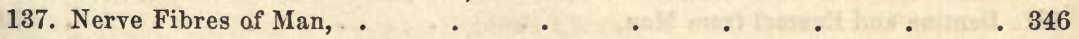

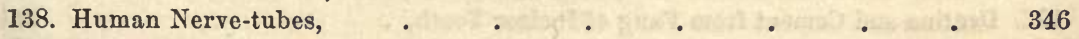

139. Nerve Fibres, .

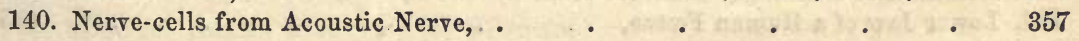


141. Transverse section of Spinal cord,

142. Cells from Gray Central Nucleus of Cord,

143. Nerve-cells from Anterior Cornua of Cord in Man, . . . . . 364

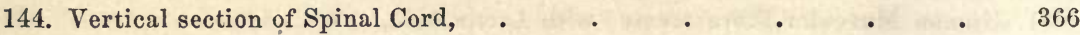

145. Five transverse sections of Human Spinal Cord, . . . . . 370

146. Transverse section through Human Medulla Oblongata, . . ' . 373

147. Nerve-cells of the Substantia Ferraginea, . . . . . . . 376

148. Large Cells of Cortical Substance of Human Cerebellum, • • 380

149. Internal portions of Gray Layer of Human Cerebral Convolutions, . $\quad 387$

150. Finest Nerve-tubes of Superficial White Substance of Human Cerebrum, 388

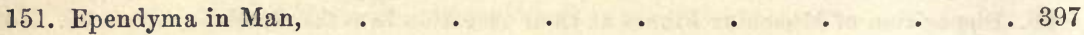

152. Vessels of Cerebral Substance of Sheep, . $\quad . \quad$. $\quad . \quad 400$

153. Brain-sand from Pineal Gland, . . . . . . . 401

154. Lumbar Ganglion of Young Dog, . $\quad$ • . . . 405

155. Ganglion Globules from Gasserian Ganglion of Cat, . 407

156. Cells from Sheath of Nerve-cells of Spinal Ganglia in Man, . $\quad 407$

157. Coccygeal Nerve, with an adherent Nerve-cell, . . . . . 408

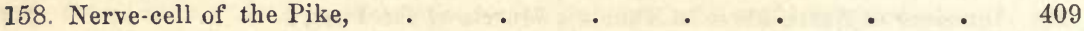

159. A Pacinian Body in Man, . . . . . . . . . $\quad$. 413

160. Transverse section of Ischiatic Nerve, . $\quad$ • $\quad$. $\quad$. 414

161. Ganglion of Sympathetic Nerve of Rabbit, . $\quad$. $\quad$ • 419

162. From the Sympathetic in Man, . . . . . . . 419

163. Nerve-cells from the Cardiac Ganglion of the Frog, . • • . 424

164. Nerve-cells from Spinal Ganglion of Human Embryo, . • . 427

165. Nerve-fibres from Ischiatic Nerve of Human Embryo, . . . 427

166. Nerve from the Tail of a Tadpole, . . . . . . 428

167. Simple Papilla, with Vessels and Epithelium, . . . . 438

165. Epithelial cells in Oral Cavity of Man, . . . . . . 439

169. Longitudinal section of Human Tongue, $\quad$ • $\quad$ • $\quad 442$

170. Transverse section of Human Tongue, . . . . . 443

171. Portion of longitudinal section of Human Tongue, . . . . 445

172. Branched Primitive Muscular Bundle from Tongue of Frog, . . 446

173. Two Papillæ Filiformes of Man; after Todd and Bowman, . . . 448

174. Papilla Fungiformis; after Todd and Bowman, . . . . 448

175. Papilla Circumvallata of Man, • • • • • . $\quad 450$

176. Epithelial Cells covered with Granular Matrix of Fungus, . . 451

177. Papilla Filiformis invested by a Fungus, . . . . . 452

178. Racemose Mucous Glands from the Floor of the Oral Cavity, . . 457

179. Diagram of two Ducts of a Lobe of a Mucous Gland, . $\quad$ • 457

180. Two. Glandular Vesicles of a racemose Mucous Gland of Man, . . 458

181. Follicular Gland from the root of the Tongue in Man, . $\quad$. 460

182. Vesicles of a few follicles from a Human Tonsil, . . . . . 461

183. Human Molar Tooth, . . . . . . . . . . 468

184. Transverse section of Dentinal Canals, . $\quad$ • $\quad$. $4 \quad$. 469

185. Dentinal Tubules, . $\quad . \quad$. $\quad . \quad$. . . . $\quad .470$

186. Transverse section through the Dentinal Canal of a Human Tooth, . 470

187. Perpendicular section of the Apex of an Incisor Tooth, . . 473

188. Dentine with Dentinal Globular and Interglobular Spaces, . . 473

189. Surface of Enamel from the Calf, . . . . . . . . 478

190. Enamel Prisms from Man, . . . . . . . . $\quad$. 478

191. Dentine and Enamel from Man, . . . . . . 479

192. Dentine and Cement from Fang of Incisor Tooth, . . . . 481

193. Cement and Dentine of the Root of an old Tooth, . . . . . 482

194. Lower Jaw of a Human Fœtus, . . . . . . 485 
195. Diagram of the Development of a Milk-tooth; after Goodsir,

196. Tooth-sac of Incisor of a Fœtus, . • • • • . 488

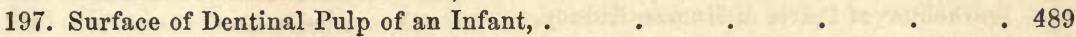

198. Transverse section of Human Esophagus, _ . . . . 501

199. Muscular-fibre Cells from Esophageal Mucous Membrane, . • . 501

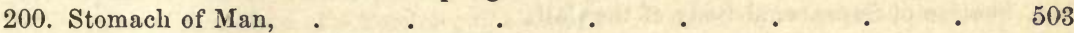

201. Muscular-fibre Cells from Small Intestine, . . . . . . 504

202. Bloodvessels of Smooth Muscles of Intestine, . . . . 505

203. Perpendicular section through Tunics of Pig's Stomach, . . . 506

204. Mucous Gastric Gland of a Dog, . . . _ . $\quad$ • 507

205. Vessels of Large Intestine of a Dog, . . _ _ . 510

206. Section through the Walls of a Calf's Ileum, _ _ . $\quad$ - 512

207. Intestinal Villus of a Young Kitten, . $\quad . \quad \ldots \quad$. $\quad . \quad$. 512

208. Vessels of Villi of the Mouse; after Gerlach, . . . . 513

209. Villi without Epithelium and with a Lacteal, . _ _ . . . 513

210. Intestinal Villi of the Cat,. . . . . . 514

211. Villi with the Epithelium, from the Rabbit, . . . . . 515

212. Lieberkühnian Glands of the Pig, . $\quad . \quad$. $\quad . \quad$. 519

213. Peyer's Patch, in Man, . . . . . . . . 521

214. Portion of a Peyer's Patch of an Old Man, . . . . 521

215. Horizontal section from the middle of Peyer's Follicle of the Rabbit, . 522

216. Solitary Follicle from the Small Intestine; after Böhm, . . . 523

217. Solitary Follicle from the Colon of a Child, . . . . . . . 525

218. Segment of a Pig's Liver, . $\quad . \quad$. $\quad . \quad$. $\quad . \quad 5530$

219. Portal Vessel of the Pig; after Kiernan, . . . . . . . 530

220. Hepatic Cells of Man, . . . . . . . . 532

221. Hepatic Cell-network, . . . . . . . . . 533

222. Hepatic Cell-network and its capillaries, . $\quad$. $\quad . \quad$. $\quad 542$

223. Hepatic Veins of the Rabbit, . . . . . . . . 542

224. Arterial Network upon surface of a Child's Liver, . . . $\quad 545$

225. Vessels of the Pancreas of a Rabbit, . . . . . . 550

226. Section through the middle of the Spleen of an Ox, . . . 551

227. Fibres from the Pulp of Human Spleen, . . . . . . 552

228. Artery covered with Malpighian Corpuscles from Spleen of Dog, . 553

229. Malpighian Corpuscles from Spleen of an $0 x$, . $\quad . \quad . \quad . \quad$. 554

230. Contents of a Malpighian Corpuscle, . $\quad . \quad$. $\quad . \quad$. 554

231. Blood-corpuscle-holding Cells and their Metamorphoses, . . . . 559

232. Artery from the Spleen of a Pig, . . . . . . 563

233. Ciliated Epithelium from the Human Trachea, . . . . 571

234. Vertical Section through Anterior Wall of Human Trachea, . $\quad 573$

235. Lymphatics in the Tracheal Mucous Membrane of Man, . . . 574

236. Pulmonary Lobules from a Child, . . . . . . . 575

237. External Surface of the Lung of a Cow, . . . . . . 577

238. Human Air-cell, . $\quad . \quad$. . . . . $\quad 579$

239. Capillary plexus of Human Air-cell, . . . . . . 581

240. Gland-vesicles from the Thyroid Gland of a Child, . . . 586

241. Gland-vesicles of Thyroid filled with Colored Matter, . • . 587

242. Portion of Thymus of a Calf,

243. Half of Human Thymus,

244. Section of an injected Lobule of Thymus of a Child, . . . 591

245. Vertical section of an injected Rabbit's Kidney, . . . . . 597

246. Tubuli Uriniferi of Man, . . . . . . . . . . $\quad . \quad 599$

247. A Malpighian Corpuscle, . . . . . . . . . 599

248. From the Human Kidney; after Bowman, . . . . 603 
FIG.

249. Malpighian Corpuscle from Kidney of a Horse,

250. Transverse section through Cortical Tubules, .

251. Epithelium of Pelvis of Human Kidney,

252. Vertical section through Suprarenal Capsule in IIan,

253. From the Suprarenal Capsule of Man, . . . . . . 614

254. Section of Suprarenal Body of the Calf, . . . . . 616

255. Section through Testis and its Tunics in Man, . . . . 619

256. Diagram of the Course of a Spermatic Tubule, . . . . . 619

257. Human Testis and Epididymis; after Arnold, . . . . $\quad$ • 619

258. Portion of a Spermatic Tube in Man, . . . . . . . . . 621

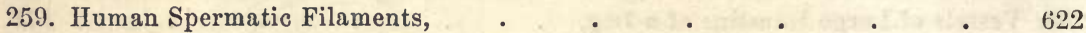

260. Development of the Spermatic Filaments in a Rabbit, _ . . 622

261. Glands of a Littre, .

262. Arteries from Corpora Cavernosa Penis,

263. Section through the Ovary of a Woman,

264. Graafian Follicle of the Sow,

265. Human Ovulum,

266. Two Corpora Lutea,

267. Muscular Elements from the Uterus in Pregnancy,

268. Muscular Fibre-cell from a Gravid Uterus, .

269. Uterine Gland,

270. Muscular Fibre-cell of the Uterus,

271. Graafian Follicles from Ovary of a Newly-born Child,

272. Lobules of the Lacteal Gland of a Puerperal Female,

273. Development of Lacteal Gland,

274. Elementary Forms in Milk,

275. Anastomosing Primitive Fasciculug from the Human Heart,

281. Transverse section of Art. Prof. Femoris of Man, . . . . 681

282. Transverse section of Aorta, . . . . . . . 682

283. Muscular Fibre-cell from innermost layer of Axillary Artery, . . 683

284. Transverse section of the Saphena Vein, . . . . . 685

285. Muscular Fibre-cell from Renal Vein of Man, . . . . 686

286. Longitudinal section of Inferior Vena Cava, . . . . . . . 688

287. Finest Vessels on the arterial side of the Capillaries, . . . 691

288. Capillary Lymphatic from the tail of a Tadpole, . . . . 693

289. Transverse section of Human Thoracic Duct, . . . . 695

290. Elements of the Chyle, . . . . . . . 700

291. Human Blood-globules, . . . . . . . 703

292. Colorless Blood-globules, . . . . . . . 707

293. Blood-cells of the Frog and of the Pigeon, . . . $\quad 713$

294. Capillaries from the Tail of a Tadpole, . . . . . . 717

295. Blood-corpuscles of a Fotal Lamb, . . . . . 718

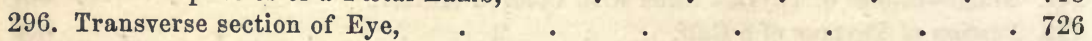

297. Capillaries and Lymphatics of Cornea of a Kitten, . . . 731

298. Nerves of the Cornea of a Rabbit, . . . . . . . 732

299. Cells from the Stroma of the Choroid, . . . . . 734

300. Cells of the Pigmentum Nigrum of Man, . . . . . 735

301. Vessels of the Choroid and Iris of a Child ; after Arnold, . $\quad 737$

302. Vertical Transverse section of Human Retina, . . . . . 739 
FI .

PAGI

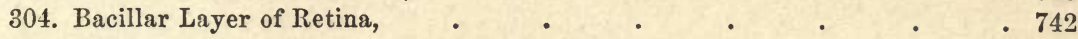

305. Nerve-cells from Retina of the $\mathrm{Ox}, \quad \ldots \quad \ldots \quad \ldots \quad$. $\quad . \quad$. 743

306. Fibres of the Lens, from the Ox, . $\quad$. $\quad$. $\quad$. $\quad$. 751

307. Human lens; after Arnold, * . . . . . . . $\quad 753$

308. Transverse section of a Semicircular Canal, . $\quad$. $\quad$ • $\quad$ • 769

309. Vertical section of the Lamina Spiralis ; after Corti, . . $\quad 771$

310. Vestibular surface of the Lamina Spiralis Membranacea, . . • 771

311. Bipolar Ganglion-globule from Lamina Spiralis of a Pig; after Corti, . 774

312. From Nasal Mucous Membrane of the Sheep, • • . . 780

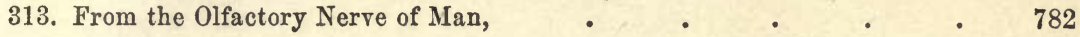





\section{INTRODUCTION.}

$\S 1$. THe doctrine of the elementary structure of Plants and Animals, belongs to the last two centuries, originating with Marcellus Malpighi (1628-94), and Anton van Leeuwenhoek (1632-1723), at the period when the assistance of magnifying glasses, powerful, though of very simple form, was first offered to observers. The ultimate constituents in respect of form, of organisms, were unknown to antiquity and to the middle ages, for although Aristotle and Galen speak of the homogeneous and heterogeneous parts of the body (partes similares et dissimilares), and Fallopius (1523-62) defined still more exactly the idea of "Tissues," and even attempted to classify them ("Tractatus quinque de partibus similaribus,' opera, tom. ii. Francof. 1600), yet the minuter structures were completely hidden from these investigators. Brilliant as were the first efforts of the young science under the guidance of these men and afterwards of a Ruysch, Swammerdam, and others, yet they were not adequate to acquire a safe footing for it, since, on the one hand, the philosophers were far too little masters of microscopic investigation to strive at once, with a clear insight, towards the true goal; while, on the other, the development of other branches of study, as of the grosser Anatomy, of Physio$\log y$, of Embryology, and of Comparative Anatomy, claimed too large a share of their attention. It thus happened that, with the exception of a few to some extent important works (Fontana, Muys, Lieberkühn, Hewson, Prochaska), Histology made no considerable progress during the whole of the 18 th century, and acquired no importance greater than that due to a disjointed collection of isolated observations. It was in the year 1801 that it first acquired a rank co-equal with that of its sister anatomical sciences by the genius of a man to whom indeed, Histology owes no great discoveries, but who understood, as no one before him had done, so to arrange existing materials and so to connect them with Physiology and Medicine, that for the future its independence was assured. In fact, Bichat's 'Anatomie Générale' (Paris, 1801), was the first attempt to treat Histology scientifically, and on this account merely, it constitutes an epoch; but besides this, its importance 
was still greater, inasmuch as the tissues were not merely clearly defined and fully and logically treated of, but full account was taken of their physiological functions and morbid alterations. To this great internal progress, the present century has added an ever-increasing perfection of the external aids of the microscope, and a steadily increasing zeal in the investigation of nature, so that it is not to be wondered at, that in its five decades, it has left far behind all that was done in the century and $a$ half of its earlier existence. In the last thirty years particularly, discoveries have so trodden upon one another's heels, that it must be considered truly fortunate that a bond of connection has arisen, and that Microscopical Anatomy has thus escaped the danger of becoming, as in earlier days, lost in minutir. In the year 1838, in fact, the demonstration by Dr. Th. Schwann, of the originally perfectly identical cellular composition of all animal organisms, and of the origin of their higher structures from these elements, afforded the appropriate conception which united all previous observations, and afforded a clue for further investigations. If Bichat founded histology more theoretically by constructing a system and carrying it out logically, Schwann has, by his investigations, afforded a basis of fact, and has thus won the second laurels in this field. What has been done in this science since Schwann, has been indeed of great importance to physiology and medicine, and in part of great value in a purely scientific point of view, inasmuch as a great deal which Schwann only indicated, or shortly adverted to, as the genesis of the cell, the import of the nucleus, the development of the higher tissues, their chemical relations, \&c., has received a further development; but all this has not amounted to a step so greatly in advance as to constitute a new epoch. If, without pretensions to prescience, it be permitted to speak of the future, this condition of Histology will last as long as no essential advance is made towards penetrating more deeply into organic structure, and becoming acquainted with those elements, of which that which we at present hold to be simple, is composed. If it be possible that the molecules which constitute cell-membranes, muscular fibrils, axile fibre of nerves, \&c., should be discovered, and the laws of their apposition, and of the alterations which they undergo in the course of the origin, the growth, and the activity of the present so-called elementary parts, should be made out, then a new era will commence for Histology, and the discoverer of the law of cell genesis, or of a molecular theory, will be as much or more celebrated than the originator of the doctrine of the composition of all animal tissues out of cells.

$\S 2$. In characterizing the present position of Histology and of its objects, we must by no means forget that, properly speaking, it considers only one of the three aspects which the elementary parts present 
to observation, namely, their form. Microscopical anatomy is concerned with the understanding of the microscopic forms, and with the laws of their structure and development, not with any general doctrine of the elementary parts. Composition and function are only involved, so far as they relate to the origin of forms and to their variety. Whatever else respecting the activity of the perfect elements and their chemical relations is to be found in Histology, is there either on practical grounds in order to give some useful application of the morphological conditions, or to complete them; or from its intimate alliance with the subject, it is added only because physiology proper does not afford a due place for the functions of the elementary parts.

If Histology is to attain the rank of a science, its first need is to have as broad and certain an objective basis as possible. To this end the minuter structural characters of animal organisms are to be examined on all sides, and not only in fully formed structures, but in all the earlier periods from their first development. When the morphological elements have been perfectly made out, the next object is to discover the laws according to which they arise, wherein one must not fail to have regard also to their relations of composition and function. In discovering these laws, here as in the experimental sciences generally, continual observation separates more and more, among the collective mass of scattered facts and observations, the occasional from the constant, the accidental from the essential, till at last a series of more and more general expressions of the facts arises, - from which, in the end, mathematical expressions or formulæ proceed, and thus the laws are enunciated.

If we inquire how far Histology has satisfied these requirements, and what are its prospects in the immediate future, the answer must be a modest one. Not only does it not possess a single law, but the materials at hand from which such should be deduced, are as yet relatively so scanty, that not even any considerable number of general propositions appear well founded. Not to speak of a complete knowledge of the minuter structure of animals in general, we are not acquainted with the structure of a single creature throughout, not even of man, although he has been so frequently the object of investigation, -and therefore it has hitherto been impossible to bring the science essentially any nearer its goal. It would, however, be unjust to overlook and depreciate what we do possess; and it may at any rate be said that we have acquired a rich store of facts and a few more trustworthy general propositions. To indicate only the more important of the former, it may be mentioned, that we have a very sufficient acquaintance with the perfect elementary parts of the higher animals, and that we also understand their development, with the exception of the elastic tissue, and of the elements of the teeth and bones. The mode in which 
these are united into organs has been less examined, yet on this head also, much has been added of late, especially in man, whose individual organs with the exception of the nervous system, the higher organs of sense, and a few glands (the liver, blood-vascular glands), have been almost exhaustively investigated. If the like progress continue to be made, the structure of the human body will in a few years be so clearly made out, that, except perhaps in the nervous system, nothing more of importance will remain to be done with our present modes of investigation. With comparative Histology it is otherwise; hardly commenced, not years but decades will be needed to carry out the necessary investigations. Whoever will do good work in this field must, by monographs of typical forms embracing their whole structure from the earliest periods of development, ${ }^{*}$ obtain a general view of all the divisions of the animal kingdom, and then, by the methods above described, strive to develop their laws.

As regards the general propositions of Histology, the science has made no important progress since Schwann, however much has been attained by the confirmation of the broad outlines of his doctrines. The position that all the higher animals at one time consist wholly of cells and develop from these their higher elementary parts, stands firm, though it must not be understood as if cells, or their derivatives, were the sole possible or existing elements of animals. In the same way, Schwann's conception of the genesis of cells, though considerably modified and extended, has not been essentially changed, since the cell nucleus still remains as the principal factor of cell-development and of cell-multiplication. Least advance has been made in the laws which regulate the origin of cells and of the higher elements, and our acquaintance with the elementary processes which take place during the formation of organs must be regarded as very slight. Yet the right track in clearing up these points has been entered upon; and a logical investigation of the chemical relations of the elementary parts and of their molecular forces, after the manner of Donders, Ludwig, and others, combined with a more profound microscopical examination of them, such as has already taken place with regard to the muscles and nerves,further, a histological treatment of embryology, such as has been attempted by Reichert, Vogt, and myself, will assuredly raise the veil, and bring us, step by step, nearer to the desired though perhaps never to be reached, end.

§ 3. The aids in studying Histology may here be best shortly adverted to. With respect to the literature of the subject, the more impor-

* [See a very praiseworthy monograph of this kind by Leydig, Beiträge zur Mikroskopischen Anatomie und Entwickelungs-geschichte der Rochen u. Haie, 1852. (Microscopic Anatomy and Development of the Rays and Sharks.)-Trs.] 
tant monographic works are cited under their appropriate section, and here only those large independent works will be noticed, in which further instruction is to be found. It is right to head the list with Schwann's 'Mikroskopische Untersuchungen über die Uebereinstimmung in der Struktur und dem Wachsthum der Thiere und Pflanzen' (Berlin, 1839),* abstracted in Froriep's 'Neue Notizen' (1838), as the most fitting introduction to Histology. Beside this, we may name X. Bichat, 'Anatomie Générale,' Tom. iv. (Paris, 1801); E. H. Weber, 'Handbuch der Anatomie des Menschen von Hildebrandt,' Bd. 1, 'Algemeine Anatomie' (Braunschweig, 1830), a work distinguished in its day, and even now indispensably necessary, as a store of old literature [or Ed. 4 (Stuttgart, 1833)]; Brun's 'Lehrbuch der Allgemeinen Anatomie des Menschen' (Braunschweig, 1841), very clear, concise, and good; Henle, 'Allgemeine Anatomie' (Leipzig, 1841), containing a classical account of Histology in the year 1840, many original statements, and physiological, pathological, and historical remarks; G. Valentin, article 'Gewebe,' in R. Wagner's 'Handwörterbuch d. Physiologie,' Bd. i. (1842); R. B. Todd and W. Bowman, 'The Physiological Anatomy and Physiology of Man,' Parts i. ii. (London 1845-47), mostly based upon original observations, very comprehensive and good [also Parts iii. iv. (1847-52)]; Bendz, 'Haandbog i den almindelige Anatomie' (Kiöbenhavn, 1846-47), with industriously collected historical data; A. Kölliker, 'Mikroskopische Anatomie oder Gewebelehre des Menschen, Band II. Specielle Gewebelehre, 1, Hälfte. u. 2 ; Hälfte. 1 Abtheilung' (Leipzig, 1850-52), containing an exposition, as complete as possible, of the minute structure of the organs and systems of man. With these are to be compared the yearly Reports of Henle, in Cannstatt's 'Jahresbericht,' and those of Reichert, in Müller's 'Archiv,' in the latter of which, more objective views and an earlier appearance would be desirable.

Useful figures are found in all the works above cited, with the exception of those of Bichat, Weber, and Bruns; furthermore, the figures of injections in Berres' 'Anatomie der Mikroskopischen Gebilde des menschlichen Körpers,' Heft 1-12 (Wien, 1836-42), are for the most part excellent, as are the representations of tissues in R. Wagner's 'Icones Physiologicæ,' second edition, by A. Ecker. Those of Langenbeck, 'Mikroskopisch-anatomische Abbildungen.' Lief. 1-4 (Grottingen, 1846-51); of A. H. Hassall, 'The Microscopic Anatomy of the Human Body' (London, 1846-49); and Mandl, 'Anatomie Microscopique' (Paris 1838-48), are middling; while on the other hand, those given by Quekett, 'Catalogue of the Histological Series in the Museum of the Royal College of Surgeons of England' (London, 1850), are admirable.

As regards Microscopes, I may express my opinion that of the more easily accessible, those of Plössl, Oberhäuser, and Schiek, take the first

* Translated for the Sydenham Society, 1847. 
rank. In Italy, Amici ; in England, Ross, Powell, and others, produce instruments quite equal to the above, but out of the question for Germany; among small, cheap, but not particularly useful instruments for students and physicians, for 115 to 150 francs, George Oberhäuser (Rue Dauphine, 19, Paris), furnishes the best. The much-famed instruments of Nachet are good, but inferior to those of Oberhäuser; on the other hand, the small ones of Schiek for 40 thalers [ 30 dollars], and those of Plössl for 70 to $100 \mathrm{Fl}$. [30 to 45 dollars], would be very serviceable if these artists were as productive as Oberhäuser.* For the use of the microscope Irefer to J. Vogel, "Anleitung zum Gebrauche des Mikroskops" (Leipzig, 1841); H. von Mohl, "Mikrographie" (Tübingen, 1846); Harting, "Het Mikroskoop deszelfs gebruik, geschiedenis en tegenwoordige toestand" (Utrecht, 1848-50), 3 Theile; Purkinje, article "Mikroskop," in Wagner's "Handwörterbuch der Physiologie," Bd. 2, 1844; in which works, as well as in that of Quekett, "A Practical Treatise on the Use of the Microscope" (London, 1848, translated, by Hartmann, Weimar, 1850, [also Ed. 2, London, 1852)]; and Robin, "Du Microscope et des Injections dans leurs applications à l'Anatomie et à la Pathologie" (Paris, 1848), the preparation of microscopical objects is in part very elaborately treated of.

A collection of microscopical preparations is indispensably necessary for a more exact study of Histology, especially sections of bones and teeth and injections. Every one may with a little trouble, form a moderate collection for himself, hints towards which he will find in the paragraphs standing at the end of each section of the special part, as well as in the works just cited. Microscopical preparations may also be exchanged with or purchased of Hyrtl, in Vienna; Dr. Oschatz, in Berlin; Topping, Smith, and Beck, Hett and others, in London; and also in Paris. The largest private and public collections of microscopical preparations exist in Vienna, with Hyrtl (injections); in Utrecht, with Harting and Schröder van der Kolk (injections, sections, muscles, nerves); in London, in the College of Surgeons (animal and vegetable tissues of all kinds); with Tornes (sections of bones and teeth); and with Carpenter (hard tissues of the lower animals).

* [The opinion expressed in the above lines with regard to microscopes seems entirely too national. For clearness of definition of the object glasses and neatness of the stand, the instruments furnished by Ross, and Powell, and Lealand, are not only "quite equal," but far superior to those of Plössl, Oberhanuser, or Schiek, the only objection to them being their high price. Of the cheaper microscopes, those most used at present in this country and in England are the small instruments of Nachet (Rue Serpente, Paris), the glasses of which are superior to those of Plosssl and Schiek, whilst the great convenience of the present stand, modelled according to the English style, renders them preferable to those of Oberhauser. In this country microscopes have been made by Mr. Spencer, of New York, the lenses of which are not inferior to the best glasses either of Ross, or of Powell and Lealand, but which are as yet too expensive to be introduced into general use. $-\mathrm{DAC}_{\mathrm{A}}$.] 


\section{GENERAL ANATOMY OF THE TISSUES.}

\section{OF THE ELEMENTARY PARTS.}

$\S 4$. IF the solid and fluid constituents of the human body be examined with the aid of strong magnifying powers, it appears at once that the smallest parts which they exhibit to the naked eye, as granules, fibres, tubes, membranes, \&c., are not the ultimate elements in respect of form, but on the contrary, that all, in conjunction with a universally distributed, fluid, semi-fluid, or even solid, homogeneous, uniting substance, contain minute particles which differ in different organs but in the same organs have always a similar appearance. There are various kinds of these so-called elementary parts, simple and compound. The simplest are quite homogeneous, without any trace of their being composed of heterogeneous portions and are nearly allied to the inorganic forms, the crystalline granules and crystals, which also occur in the animal organism. Others already show that they have suffered a differentiation into an investment and determinate, though homogeneous contents : in others again, the contents present differences. The most important among all these forms, which may be comprehended under the general title of "simple elementary parts," are the cells, which not only form the starting-point of every animal and vegetable organism, but also, either as cells or after having undergone manifold metamorphoses, make up the body of the perfect animal, and in the simplest animal and vegetable formations (unicellular animals and plants), even enjoy an independent existence. Compared with cells, all other simple elementary parts have quite a subordinate importance, so far as their direct participation in the formation of the tissues and organs is concerned; while, from their being almost all contained in the interior of cells and from their being concerned in many and most important ways in the vital processes of these cells, their importance in other respects is very great.

The simple elementary parts, which at first wholly comprise the commencing animal (or plant), often unite in the course of development in 
such a manner that they lose their independence and cease to exist as isolated elements. In this manner compound forms arise, each of which answers genetically to a whole series of simple ones, and which may most fittingly be called the "higher elementary parts." Such a coalescence has been observed with certainty only in cells, and from these most of the tubular and fibrous elements of the body are produced.

\section{§5. Formative and Nutritive Fluid-Interstitial substance or matrix.} -While in plants the elementary parts in by far the majority of cases, unite directly with one another, in animals there is a very wide difference; a peculiar interstitial substance which combines them, and is ultimately derived from the blood, is always in a lower or more distant relation therewith. If this take a direct share in the formation of the elementary parts it is called "formative fluid," Cytoblastema (Schleiden), from

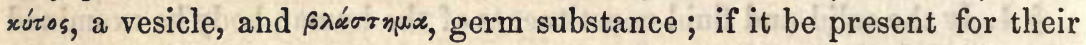
maintenance, it is called "nutritive fluid;" if it have nothing to do with either the one or the other of these functions, it is called the "matrix" or connecting substance. The cytoblastema is usually quite fluid, as in the blood, in the chyle, in many glandular secretions, in the contents of the glandular follicles, and in many embryonic organs; more rarely, viscid and like mucus, as in the gelatinous cellular tissue of embryos (vide infra), still more rarely solid, as the blastema from which the villi of the chorion arise and grow. The "nutritive fluid" takes the place of the formative fluid in all perfect organs; and except when it is contained in special canals and cavities, as in bones, teeth, and perhaps in some cellular organs, is present in so small a quantity, that it cannot be directly observed. A matrix, lastly, is found in cartilages and bones as a solid, homogeneous, granular, or even fibrous substance connecting the cellular elements and for the most part arising from the blood, independently of them.

The occurrence of a solid blastema, growing independently, in the villi of the chorion and of a solid matrix deposited directly out of the blood demonstrates that all parts of the body are not, as Schwann was disposed to believe, without exception developed from cells or in dependence upon cells. A few more recent authors, as Reichert, Donders, and Virchow, also consider that the connective tissue, excepting its elastic element, is to be reckoned among those tissues which are not at all, or not wholly, derived from cells; but, as we shall see below, incorrectly. On the other hand, it is certain that in pathological formations such masses very frequently occur, fibrinous exudations becoming changed in great measure, without previous organization, $i$. $e$. cell formation into permanent tissues.*

[* The Enamel and the Dentine of the teeth, and the so-called Cuticle of the hair (see \$\$ 0.1 Hair and Teeth, and 'Quarterly Journal of Microscopical Science' for April, 1853), must 


\section{A. SIMPLE ELEMENTARY PARTS.}

\section{ELEMENTARY GRANULES, ELEMENTARY VESICLES, NUCLEI.}

$\S 6$. In almost all animal fluids, whether contained in canals, or enclosed in cells, as well as in many more solid tissues, there are found and often in immense quantities, roundish corpuscles of very small, hardly meaurable dimensions. Henle has called them "elementary granules," and has expressed the opinion that they are vesicular. This, however, is not always true, since it is demonstrable that many of these corpuscles possess no investment. Such is the case with the fatty particles which occur in many cells and glandular secretions, with the granules of the black pigment of the eye and of other colored cells, the granular precipitates of biliary coloring matter, of different salts in the kidneys, and in the urine; lastly, the protein granules (albuminous granules) which are found free in certain portions of the gray substance of the central nervous system and of the retina. Among the pathological but very common formations, we must enumerate here amorphous deposits, the colloid granules in the thyroid and elsewhere, and the corpuscula amylacea of the central nervous system, although these sometimes attain a very considerable size. All these granules want the properties observed in the higher elementary parts, such as endogenous growth, multiplication, assimilation, and excretion; and so far incline towards the purely inorganic forms-crystals; which are also found, though less commonly, in the organism, as for example in the spleen, in the lungs (black columns), in the ear, in the cells of the præputial glands of the rat, in the blood-corpuscles of the dog and of fishes, in the fat-cells of man, and in the cells of the chorion of the embryo of sheep.

Elementary vesicles also occur very frequently, and are for the most part allied, physiologically, with the elementary granules, since, once formed they do not increase, and neither multiply by division nor by endogenous development. The milk-globules may with tolerable certainty be arranged among these; at first included within the cells of the nascent milk, they are subsequently found free, in enormous numbers, in the perfect secretion, and, as Henle first stated, consist of the fatty matter of the milk, with an investment of casein. The immeasurably small molecules of the chyle and of the blood, are also, according to H. Müller's investigation, fat globules with a protein envelop, and similar vesicles may be found in most other fluids containing fat and albumen in abundance. In fact, since the discovery of Ascherson (Müller's 'Archiv,' 1840, p. 49), that whenever fluid fat and fluid albumen are shaken together, the fat globules which are formed always

certainly be regarded as structures which are not derived directly from the metamorphosis of cells. We are inclined also to believe, that the opinion of Reichert, Donders, and Virchow, as to the nature of the connective tissue deserves much more attention than Professor Kölliker seems disposed to bestow on it. See $\$$ on Connective and Elastic Tissues.-Trs.] 
become surrounded by an albuminous coat, it is more than probable that whenever, in the body, fat and albumen in the fluid condition come into contact, similar vesicles are produced.

A peculiar class of elementary vesicles is formed by the elements which occur in the yelk of certain animals. We are best acquainted with them in the yelk of the hen's egg, ${ }^{*}$ in whose proper yelk-substance and yelk-cavity the globules which have been so long known are all vesicular, but have not the nature of cells. The membranes of these yelkvesicles are excessively delicate and consist of a protein compound; the contents are fluid albumen, and, in the globules of the yelk-cavity, there is usually a large parietal fat globule, while in the others there are many smaller and larger ones. The development of these vesicles proceeds, in all probability, from the fat globules as in other elementary vesicles, from which, however, they are distinguished by the fact that they distinctly possess the power of growth, during which their contents undergo metamorphosis, since in many the number of fat globules increases with age. Similar vesicles exist, also, in the yelk of fishes, crustacea, and spiders, and here, as in birds, they have only a temporary importance, since they are not directly applied to the formation of the embryo, but only serve to nourish it.

Lastly, free nuclei occur in many localities, either temporarily, where cells are formed immediately round nuclei, as in the chyle, the bloodvascular glands, the Peyerian patches; or permanently, as proper elements of the tissue, in the wall of the thymus vesicles, in the rust-colored layer of the cerebellum, and in the granular layer of the retina. $f$

Von Wittich ('De Hymenogonia albuminis,' Regimontanii. 1850), has lately given some information upon the formation of the so-called Aschersonian vesicles. According to Wittich, whenever oil and albumen come in contact, a portion of the oil is saponified by uniting with the alkali of the layer of albumen in contact with it, and this layer being thus rendered insoluble by the deprivation of its alkali becomes precipitated and thus forms the Aschersonian so-called haptogen membrane. According to this explanation the process would be purely chemical and not physical, and still less vital. In opposition to this view, however, Harting ('Ned. Lanc.' Sept. 1851), observed, not long/ago, the formation of pseudo-cells by the agitation of albumen with mercury, in which case the albumen must be solidified, in the same way as by the mere shaking with water or otherwise (Melsens, in 'Bull. de l'Acad. de Belgique,' 1850. Harting, \&c.). Again, if by the bringing together of

* [It is, however, by no means eertain that the yelk-corpuscles of the hen's egg are elementary granules. According to Dr. H. Meckel (Die Bildıng der für partielle Furchung bestimmten Eier der Vogel, \&c., Siebold and Kölliker's 'Zeitschrift'' 1852,) they are altered cells.Trs.]

$\dagger[$ The blood corpuscles of man and the mammalia should be added to this list. See Wharton Jones, 'Phil. Transactions,' 1846.-TRs.] 
albumen and chloroform, serum-casein and fat, chondrin and chloroform,-albuminous, casein, and chondrin membranes are formed, as Panum observed (see in part, 'Archiv f. Path. Anatomie,' iv. 2), it can hardly be permissible to assume any chemical action.

\section{OF THE CELLS.}

7. The cells, celluloe, called also elementary cells, or nucleated cells, are perfectly closed vesicles of $0.005-0.01$ of a Paris line,* in mean

* [The measurements employed by Prof. Kölliker are, according to the old French standard, of inches and lines. In the original work a line is expressed by '", an inch by ". As these signs are not used in this country, I have, in every instance, substituted the words for the signs. Most English and American authors express the size of objects in fractions of an inch, but rarely in lines. The English inch and line differ but little from the Paris inch and line. The old Paris inch (") was divided into 12 lines ("'); one line being equal to $\frac{8}{90}$ ths, or rather more than the 11th of an English inch: hence the difference between a Paris line and an English line is very slight; $\frac{1}{2} \frac{1}{0}$ th of an English line being equal to $\frac{1}{2} \mathrm{~d} d$ of a Paris line; an English inch is thus exactly 11.26 Paris lines. All the French and many of the continental microscopists employ the recent French measurements : the centimetre and millimetre. The proportion they bear to the English and French inch is as follows: one millimetre $\left({ }^{\mathrm{mm}}\right)$ is equal to 0.03937 , or about $\frac{1}{9} \frac{1}{0}$ th of an English inch, or to $\frac{1}{2} \mathrm{~d}$ of a Paris line. The subjoined table of the main measurements, noted throughout the work in lines, reduced to fractions of an English inch, and parts of millimetres, will aid the student in forming a comparative estimate of the size of objects, as measured by observers in different countries:-

TABLE OF MEASUREMENTS.

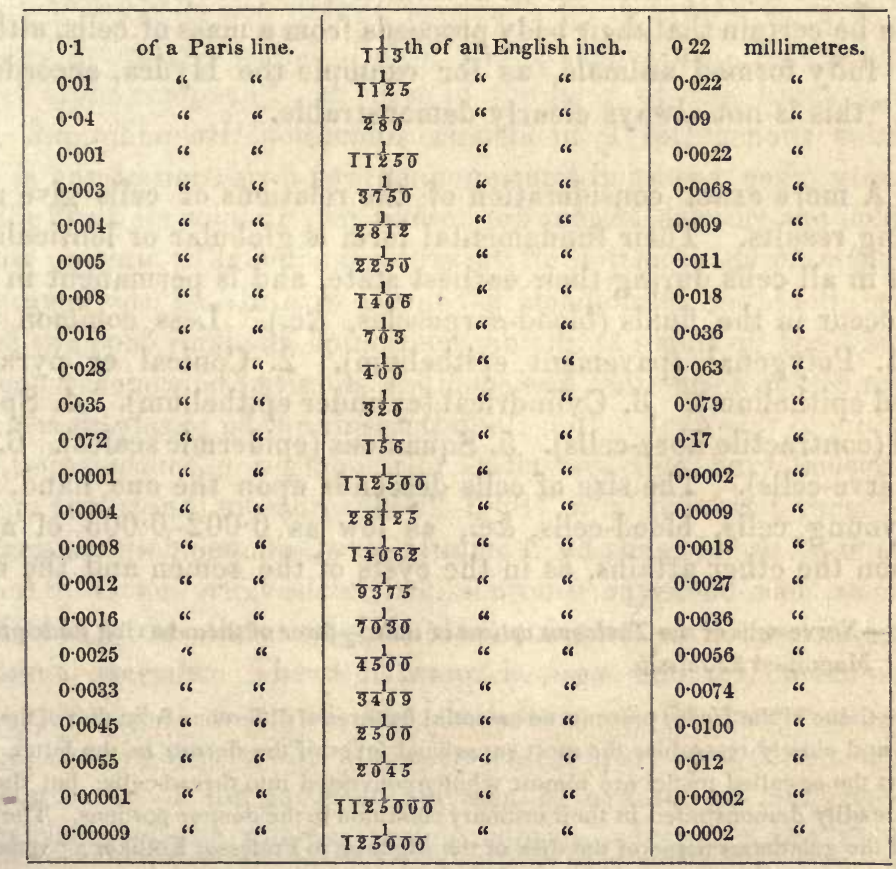


diameter, in which we may distinguish a special investment, the cell membrane and contents. The latter are

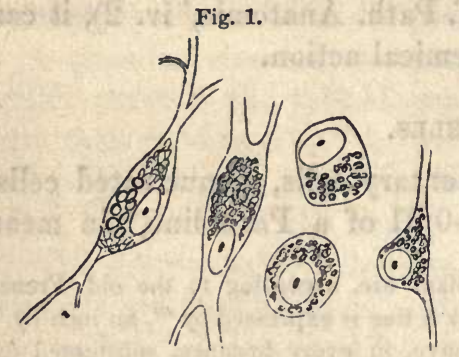
always composed of a fluid, containing formed particles of various kinds, and a peculiar rounded body, the cell-nucleus, which again contains in its interior a fluid and a still smaller corpuscle, the nucleolus. These cells, which must be considered to be endowed with peculiar vital powers, and to be capable of absorption and assimilation, of growth and of multiplication, not only at the earliest period entirely compose the body of the higher and that of most of the lower animals, but almost wholly generate the higher elementary parts of the fully developed body. In fact, even in adult animals, we find in very many places that the elements are simply in the condition of cells, and that as such they take a more or less marked share in the performance of the organic functions.

It is not yet quite decided what part cells play in the composition of the simplest animals. Siebold and I have expressed the opinion that the Protozoa, like the simplest plants, are unicellular organisms, but it is granted that no demonstration of this has been given in many, especially the Rhizopods. In all creatures above the Protozoa, it would seem to be certain that their body proceeds from a mass of cells, although in the fully formed animals, as for example the Hydra, according to Ecker, ${ }^{*}$ this is not always clearly demonstrable.

$\S 8$. A more exact consideration of the relations of cells give us the following results. Their fundamental form is globular or lenticular; it is such in all cells during their earliest state, and is permanent in those which occur in the fluids (blood-corpuscles, \&c.). Less common forms are: 1. Polygonal (pavement epithelium). 2. Conical or pyramidal (ciliated epithelium). 3. Cylindrical (cylinder epithelium). 4. Spindleshaped (contractile fibre-cells). 5. Squamous (epidermic scales). 6. Stellate (nerve-cells). The size of cells descends upon the one hand, as in many young cells, blood-cells, \&c., as low as 0.002-0.003 of a line, and upon the other attains, as in the cysts of the semen and the nerve-

Fia. 1.-Nerve-cells of the Thalamus opticus of man,-three of them having their processes torn off. Magnified 350 diam.

* [The tissue of the Hydra presents no essential features of difference from that of the higher animals, and closely resembles the most superficial layer of the dermis in the latter. In its outer part the so-called nuclei are almost wholly converted into thread-cells; but they may be very readily demonstrated in their ordinary condition in the deeper portions. The resemblance of the gelatinous tissue of the disk of the Medusæ to Professor Kolliker's " reticulative connective tissue" is still more striking.-Trs.] 
cells, that of $0.02-0.04$ of a line. The largest animal cells are the yeik-cells or ova, especially those of birds and amphibia, and a few of those animals which consist of single cells, these, in certain Gregarinæ, attaining 0.7 of a line.

The membrane of the cells is generally very delicate, smooth, hardly separable, and marked by a single contour, rarely of any considerable density or measurable thickness; with our present optical instruments it exhibits no structure of any kind. In the interior of the cells there are invariably found, at a certain time, one or many nuclei, besides fluid and granules of various proportions and of different natures. Cells which contain only fluid are rare (fat-cells, blood-cells, cells of the chorda dorsalis), and it is colorless or reddish; in general they contain, in addition, corpuscles in greater or less number (elementary granules, elementary vesicles, perhaps crystals), and in fact, as a rule, young cells possess few, while older ones contain many, which are very often more densely grouped round the nucleus, or occupy only a single spot (colored nerve-cells).

The chemical composition of the cells is as yet very obscure. The contents in most cells present certain generally disseminated substances, which occur dissolved in the nutritive fluid or cytoblastema, as water, albumen, fat, extractive matter, salts; a nitrogenous substance, which is precipitated by water and by dilute acids, thus resembling mucus, is very extensively distributed, and considerably impedes the microscopical analysis of the cells and tissues, inasmuch as it causes them to be obscure and granular, instead of clear and transparent. Many cells contain yet other compounds, as those of the liver, of the kidneys, of the blood, \&c. The cell-membrane consists of a nitrogenous substance, which is unquestionably a protein compound in young cells, as we may conclude from its solubility in acetic acid (partly even in the cold) and in dilute caustic alkalies. Subsequently the membrane in many cells, yet by no means in all (e.g. not in the blood-corpuscles, in the deepest cells of the epidermis and epithelium, nor in the cells of the glandular follicles), becomes less soluble, and here and there more or less approximates the substance of the elastic tissue.

The cell-nucleus is a globular or lenticular, clear, or yellowish body, which in the mean measures $0.002-0.004$ of a line, and rarely, as in the ganglion-globules and ova, attains a diameter of $0.01-0.04$ of a line. All nuclei are vesicles, as Schwann supposed and as I have recognized to be their original and universal structure in embryos and adult animals. The membrane is very delicate in the smaller ones, appearing as a simple, fine, dark line; in the larger it is more marked, even of measurable thickness and lirited by a double contour, as in the nuclei of the ganglion-globules, of ova and of many embryos. The contents of the nuclear vesicle consist, excepting the nucleolus, 
almost invariably of a pellucid or slightly yellowish-never more darkly tinged-fluid, in which water and acetic acid precipitate the same dark granules, as in the cells, for which reason, the nuclei never preserve their natural homogeneous clear appearance, when examined according to the ordinary methods. More rarely the nuclei have formed contents, as the spermatic filament in the semen; in ova peculiar granules, the socalled germinal spots; also in the fat of $P$ iscicola (Leydig). In respect of their chemical composition, only this much can be said of the nuclei, that their membranes are nitrogenous, and in general but little different from the substance forming the younger cell-membranes; they are, however, dissolved more slowly in alkalies, and are but slightly attacked by dilute acetic and mineral acids. In the latter circumstance they approximate the elastic tissue, from which, however, they are most essentially distinguished by their easy solubility in alkalies.

The nuclei are found, so far as I have observed, in all cells of embryos without exception, and in those of adults, so long as the cells are still young. In general only a single nucleus exists in each cell, except when it is multiplying; in the latter case, however, two or more nuclei arise, according to the number of the developing cells. In certain cells we meet with more numerous nuclei; thus, in those of the semen, 4 , 10,20 , and more; also in the substantia grisea centralis of the spinal cord, of the supra-renal capsules, of the pituitary body, in the hepatic cells of embryos, in the foetal medullary cells of bone, and elsewhere. That nuclei also occur free, and take part in the formation of certain tissues, has already been stated.

The nucleoli are round, sharply defined, generally dark, fat-like granules, which, on the average, measure $0.001-0.0015$ of a line, are often almost immeasurably small, and in embryos, in the germinal vesicles of ova as the germinal spots, and in the ganglion-globules, attain the size of $0.003-0.01$ of a line. In all probability they are always vesicular, as may be surmised from their sharply circumscribed form, their similarity to elementary vesicles, and also from the circumstance that in certain cells, especially in ova and ganglion-globules, a larger or smaller cavity filled with fluid frequently becomes developed in them. The chemical composition of the nucleoli is unknown: their. external appearance, their similarity to the elementary vesicles, their disappearance in caustic alkalies, and their insolubility in acetic acid, would lead us to suppose them to be fat; the membranes may, as in the elementary vesicles, be a protein compound. Nucleoli are found in the great majority of nuclei, so long as these are still young, and in many during their whole existence; but nuclei also exist, in which nucleoli cannot be recognized with certainty, or at least become obvious only at a later period; and therefore, at present, the nucleolus cannot be so unconditionally recognized to be an essential constituent 
of the cell, as the nucleus. Generally, a nucleus contains only one nucleolus, frequently there are two, rarely three, and, in solitary cases, four or five may be present, which are then either eccentric or lie free in the nucleus.

A short time since, Donders, in a very remarkable work (vide infra), expressed the opinion that all cell-membranes consist of one and the same, or at least of very nearly allied substances, which agree in their characters with the elastic tissue. For my own part I believe that all animal cell-membranes consist originally of the same substance-of a protein compound, in fact ; that, however, in consequence of its subsequent metamorphoses, it may acquire differences of composition and of reaction. Many membranes in this manner become more resistant with time and, as Donders justly states, approach elastic tissue; others change into collagenous tissue, as those of the formative cells of the connective tissue, ${ }^{*}$ and of the cartilage cells during ossification; others into syntonin, as in the smooth muscles; into the so-called horn, and so on. If we assume the primitive cell-membrane to be a protein compound, and from the reaction of young cells and of embryonic parenchyma it can hardly be otherwise, we obtain a correspondence with the regetable cell, since in this case the primordial utricle, consisting of a protein compound, can be considered as the analogue of the animal cellmembrane, whilst the cellulose membrane appears as a secondary product, as an excretion. Such may be the true condition also in those animal structures of the Tunicata which are formed of cellulose, in which case my assertion that here the cell-membranes are composed of woody fibre, and that of Schacht (Müller's "Archiv," 1851), that they are nitrogenous, would coincide. If future investigation justify this comparison of the animal cell with the primordial utricle of plants, the further question would arise in animals, whether perhaps all the so-called metamorphoses of the cell-membrane are not to be laid to the account of deposits which are thrown down upon the outer side of it, similarly to the cellulose in plants, so that, perhaps, together with the original protein membrane, other secondary collagenous or elastic membranes, \&c., might be distinguished, and even the most considerable thickenings of the animal cell be produced, in a manner analogous to that which occurs in the ligneous tissues of plants on the outer side of the protein membrane; so that, for example, within ossified cartilage-cells the original cell-membrane might perhaps still exist.

In all normal cells of the higher animals, the nuclei can be readily shown by the vesicles, and most beautifully so in embryos; only in those cells which arise directly around nuclei, are the nuclei at first more

" [The term "connective tissue" (Bindegewebe) has been used by the translators to designate the tissue more generally known as " white fibrous tissue."- $\mathrm{DAC}_{\mathrm{A}}$.] 
homogeneous, and subsequently exhibit a distinct membrane. In pathological formations, this character of the nucleus, which may be called an undeveloped form, is very frequent, and the nucleus-like structures in the Protozoa are also for the most part homogeneous bodies.

§ 9. Development of Cells. - With regard to the development of cells, we have to distinguish between their free origin and their production by the intermediation of other cells. In the former case the cells are developed, independently of others, in a plastic fluid, the cytoblastema of Schleiden, containing chiefly fat, protein, and salts in solution; in the other, or in cell-multiplication, the existent cells either produce the so-called daughter or secondary cells within themselves, or multiply by division; endogenous cell-formation and fissiparous cell-formation. Both kinds of cell-formation agree in this, that the cell nuclei play a very important part, and appear to be the proper centres of development of the young cells.

§ 10. Free cell-development is, in man and the higher animals, far less common than has been hitherto assumed, and under this category we can enumerate, so far as is at present known, only the development of the chyle and lymph corpuscles, of the cells of certain glandular secretions (spermatic cells, ova), and gland-like organs (closed follicles of the intestine, lymph glands, splenic corpuscles and pulp, thymus); lastly, of the cellular elements in the pregnant uterus, in the corpus Fig. 2. luteum, in the medulla of foetal bones, and in the soft

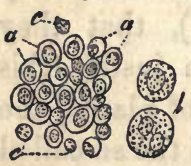
ossifying blastemata. The separate steps of the process in this mode of cell-development have as yet been traced principally in the first-named cells, but much is yet wanting to complete our knowledge of it. This much is certain, that the origin of the cells is always preceded by the development of cell-nuclei, while it is doubtful, on the other hand, how these are formed. In the chyle and in the spleen we see as the first indication of cell-formation rounded homogeneous-looking corpuscles of $0.001-.002$ of a line, which, increasing somewhat in size, soon clearly appear to be vesicles, and often, upon the addition of water, exhibit in their interior, together with small granules, a large granule, like a nucleolus. Whether this last, as is certainly the case in the dependent mode of cell-development, arises before the nucleus and is the condition of the development of the latter, or whether it is formed subsequently therein; how, again, the nuclei themselves are developed, whether as originally homogeneous corpuscles, which subsequently exhibit a differentiation

Frg. 2.-Contents of a Malpighian corpuscle of the ox: $a$, small; $b$, larger cells; $c$, free nuclei; magnified 350 diameters. 
into inner and outer parts, - envelop, and contents, or whether they are not, from the first, vesicular, cannot at present be decided.

The nuclei being once formed, the cell-membranes are developed around them, though not always in the same way. In the first place they may be applied directly around the nucleus, so that the nascent cell is but little larger than its nucleus; or, in the second place, the latter may become surrounded by a greater or smaller quantity of solidifying cytoblastema, and it is only around this enveloping mass, which I have called an investing globule, that a membrane forms. This last occurrence in the free cell-formation has hitherto been observed only in the ovum, in which the germinal vesicle, i. e. the nucleus of the eggcell, being first formed, surrounds itself with some yelk before the vitellary membrane appears. On the other hand, cell-development directly round the nucleus takes place in all the other localities which have been mentioned above, and is demonstrated by the occurrence, among free nuclei and large cells, of very small cells, which closely invest the nucleus or are but little separated from it. It may, however, be remarked, that perhaps in these cases also, the cell-membranes at their origin are separated from the nuclei by a very small quantity of cytoblastena, so small as to be incapable of detection.

Free cell-formation is exceedingly frequent in pathological productions, and the cells in pus and in exudations of all kinds arise in this manner; in fact, all pathological cell-formation properly comes under this head. Usually the cell-membranes here arise directly round the nucleus, less commonly as it would seem round investing globules. With regard to physiological processes, as has been already shown, free cell-development has been much too readily taken for granted; and especially as regards the epithelial and horny tissues, as well as in many glandular secretions, it has been assumed without any sufficient grounds. Botany knows no free cell-development.*

$\S 11$. The development of cells within other cells, or their endogenous origin, is of very frequent occurrence, and easy to be observed in embryos. The commonest form of this cell genesis is, that a so-called parent cell produces two secondary cells, which from the first wholly fill

[There cannot be said to be any evidence of the occurrence of free cell-development in animals, so long as in any case cited it is not shown that the first-formed particles which make their appearance cannot have derived their origin from pre-existing formed particles, either by the detachment or fission of the latter. Not only does this condition remain unfulfilled for all the instances cited, but it has not been attempted, and would seem to be impossible. In pathological exudations, for instance, who shall determine that the first structural elements which appear, granules, free "nuclei," exudation corpuscles, \&c., are not. directly derived either from the blood, or from the tissue into which the exudation has taken place?-Trs.] 
it. The first thing to be observed in this case, in the parent cell, is a metamorphosis of its nucleus, which grows, acFig. 3.

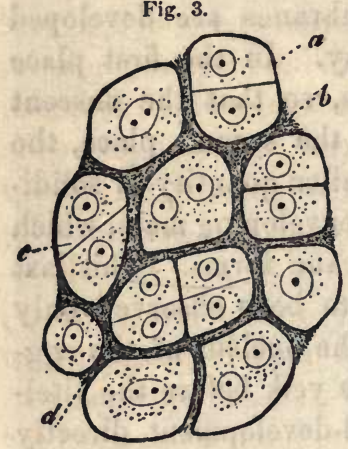
quires two nucleoli, becomes elongated, and divides into two. When this has once taken place, the nuclei become somewhat divaricated and then a wall of separation arises between the cells, which divides tha parent cell into two perfectly distinct spaces, each of which contains a single nucleus and one half of the contents. The mode in which the multiplication of the nucleus takes place, has not yet been made out with exactness. This much, however, is certain, that where clear observation is possible, it is always the nucleoli which first divide into two and then diverge a little. In the nuclei, which have at the same time slightly elongated, there then usually appears, making the first trace of their division, a median partition, which in favorable cases may be recognized as composed of two secondary nuclei applied to one another by their flat sides and completely filling the parent nucleus. Very frequently we see, in the course of this process of multiplication of the nuclei, no-

Fig. 4. thing more than, first an elongated nucleus, with a partition and - two nucleoli, and then two hemispherical nuclei applied by their plane faces to one another, without its being possible to demonstrate with certainty any endogenous development of nuclei ; so much the less, as it is not to be doubted that, together with the latter process, a multiplication of nuclei by division takes place, in which an elongated parent nucleus, with two nucleoli, breaks up into two by the formation of a constriction which gradually deepens in the middle.

The further destiny of the parent cells, with a partition and two nuclei, is not always the same. As a rule, it appears that in each, two perfect secondary cells afterwards become evident, which may serve as a demonstration that the partition is double from the very first. At other times, distinct secondary cells are not recognizable, which however does not imply that there exists a mode of cell-development by the mere formation of partitions, but only that in such cases the secondary cells do not become distinctly separated from the parent cells. Whether the one process or the other take place, it rarely stops at one performance, but is generally repeated a certain, often very considerable number of times; in fact, as long as the organism grows. The parent cells either remain, or they cease earlier or later to be histologically distinct struc-

FIa. 3.-From the cephalic cartilage of an advanced tadpole. Parent cells, with 1 and 2 nuclei, or 2-4 secondary cells, and some interstitial substance; magnified 350 diameters.

Fig. 4.- An elongated nucleus, and one containing two secondary nuclei, from the ovum of an Ascaris dentata; magnified 350 diameters. 
tures, and coalesce with the substance which unites the cells as a matrix. The occurrence of this endogenous cell-development, which may be called cell-development around the collective contents, and which agrees in all essential points with free cell-development around investing masses, has been made out with certainty in the young cartilage of all animals, and probably occurs in embryonic organs in general, in which, from the moment when they consist of actual cells, the total growth essentially depends upon a self-multiplication of the cells without free-cell development. Since, however, it is as yet undecided, whether perhaps cell-development by division, to which attention has been very lately drawn, does not play a part in the one case or in the other, our judgment, so far as regards the latter, must as yet be suspended, until more particular investigations have been undertaken; and the same holds good for many organs of the adult, as the horny tissues and certain glandular secretions. Only, when secondary cells are observer in parent cells, as especially in the pituitary body and in the supra-renal capsules, there can of course be no doubt as to the existence of endogenous cell-development.

Besides these most usual forms of cell-development, there exist yet a few others.

1. In the ova of most animals a peculiar process, the so-called cleavage of the yelk, occurs at the earliest period of development, which is to be regarded as the introduction to the formation of the first cells of the embryo; and since the ovum has the nature of a simple cell, it is a case of endogenous cell-development. This cleavage takes place as follows. After the original nucleus of the egg-cell, the germinal vesicle, has disappeared with the occurrence of fecundation, the granules of the yelk no longer form a compact mass as before, but become dispersed and fill the whole eggcell. Then, as the earliest sign of commencing development, there arises in the midst of the yelk, around a new nucleolus, a new

Fig. 5.

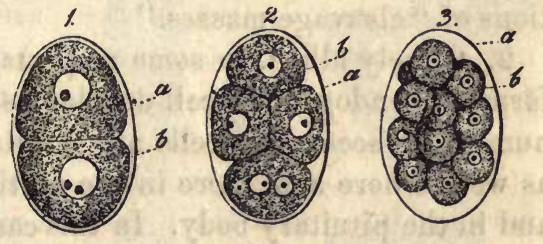
nucleus, the primary nucleus of the embryo, which operates as a centre of attraction upon the yelk and unites it again into a globular mass, the first "cleavage mass" (Furchungs-kugel). In the further course of development two new nuclei are formed by endogenous development from the first nucleus, and these, as soon as they have become freed by the solution of the parent nucleus, separate from one another for a short. distance, act as new centres upon the yelk, and thus break up the first

Fig. 5. - The ova of Ascaris nigovenosa, -1 from the second, 2 from the third, and 3 from the fifth stage of division, with 2,4 , and 16 division-masses: $a$, chorion; $b$, cleavage masses. In 1 the nucleus of the lower mass contains two nucleoli, in 2 the lowest contains two nuclei. 
cleavage mass into two. In this way the multiplication of nuclei and of cleavage masses proceeds, - the former always taking the lead, until a very great number of small globules are produced which fill the whole cavity of the yelk-cell; it is only in exceptional cases that the cleavagemasses break up after the development of three or four nuclei within them; so that then, instead of two, three or four cleavage-masses immediately proceed from one. This process is called total cleavage, because here the whole yelk is disposed around the newly-developed nuclei: partial cleavage is essentially similar, differing only in the circumstance that it is not the whole yelk, but a greater or lesser portion, according to the animal, which invests the nascent nuclei.

When the process has attained a certain stage, the cleavage-masses all together, or in successivelayers, surround themselves with membranes, and become actual cells, whence we are justified in considering this to be a process of endogenous cell-development. In fact, it is nothing else than an introduction to cell-development in the egg-cell, and differs from the ordinary phenomena of that class only in this, that firstly, the nucleus of the parent cell or the germinal vesicle, in most cases (Müller saw a division of it occur in the Molluses which are developed within Synapta digitata*) has nothing to do with it; secondly, that the parent cell itself persists; and thirdly, that the investing globules developed by the successive multiplication of nuclei become cells only in the latest generations. This view is the more justifiable, as the cells which have arisen in consequence of the metamorphosis of the last cleavage-masses long continue to multiply by endogenous development; and the whole process of division may be regarded as a kind of endogenous cell-development, in which, on account of the rapidity with which the nuclei multiply, no formation of cell-membrane takes place in the early generations of "cleavage-masses."

2. Closely allied, in some respects, to the cleavage process, are those forms of endogenous cell-development, in which, a greater or smaller number of secondary cells are developed within persistent parent cells, as we see here and there in the cartilages, in the supra-renal capsules, and in the pituitary body. In this case there either arise in the ordinary manner, in a cell, two secondary cells, which wholly or partially fill it, and from these by continued multiplication, other generations, which sometimes lie quite free, and sometimes are wholly or partially included in the parent cells of the second generation; or a more free development of a secondary cell within a parent cell occurs, from which cell-development then takes place in one mode or in the other.

In connection with the process of endogenous cell-development, we may very properly speak of the formation of a great number of nuclei within cells, a process which is frequently the precursor of cell-development,

* [Dr. Nelson (Phil. Trans., 1852, p. 580), has observed the same thing in Ascaris mystax. -Trs.] 
but which may also continue alone. Even in the common endogenous cell-development (and also in the cleavage process) we not unfrequently observe three and four nuclei in a parent cell, so that then, instead of two secondary cells, many arise at once, e. $g$. in the hepatic cells of embryos. In certain animals (Cucullanus, Ascaris dentata, Distoma, Cestoidea), instead of cleavage masses, nuclei alone are developed in the first stages of development, and it is only later, when by successive endogenous multiplication these have increased to a great bulk, that they become surrounded by cell membranes. Something similar appears to take place in the cells of the

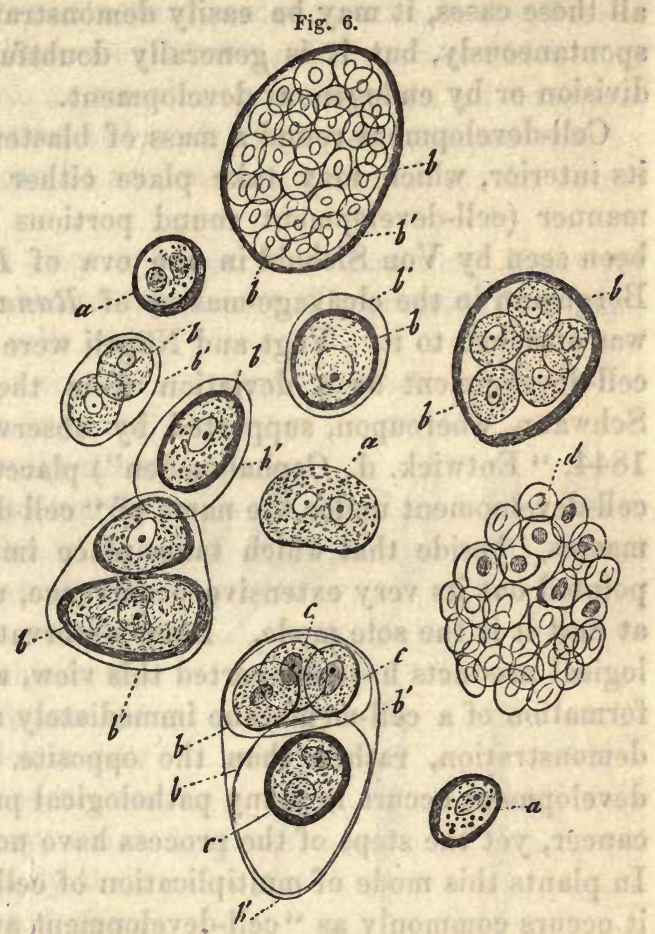
germ in the Crustacea, in which 10-20 nuclei are often found (Rathke, 'De Anim. Crustac. gen.', Regim. 1844.) On the other hand, the numerous nuclei in the spermatic cells of most animals are, in general, in no way connected with cell-development, since the spermatic filaments are developed in them; and the like holds good of those cells of the lower animals, whose multitudinous nuclei are changed into thread-cells. The import of the number of nuclei in certain nerve-cells, and in the large

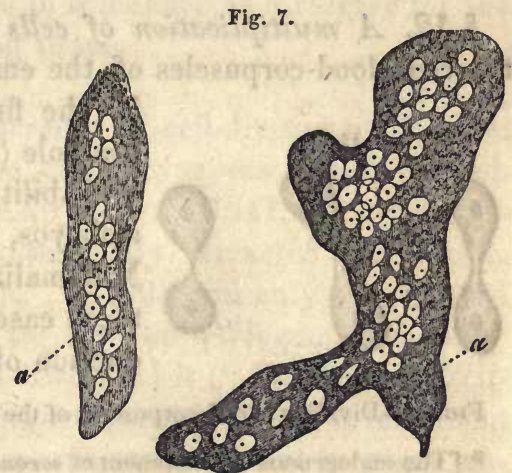
cells of the bone-medulla, which Robin and I have observed, is doubtful; in the latter, I think it is not improbable that the multiplication

Fig. 6.-Cartilage cells from a fibrous, velvety, articular cartilage of the condyle of the femur of man, magnified 350 diameters; all lying in a fibrous matrix, and readily isolated: $a$, simple cells, with or without a thickened wall, with 1 or 2 nuclei; $b$, secondary cells, or cells of the first generation, with 1 or 2 nuclei; $1,2-5$ or more, in parent cells; $b, c$, cells of the second generation, 1-3 in number, in cells of the first; $d$, freed groups of secondary cells.

Fig. 7.- a. Peculiar granulated cells with many nuclei from the youngest medullary cavities of the flat bones of the skull in man. Magnified 350 diameters. 
of the nuclei is preliminary to their breaking up into smaller cells. In all these cases, it may be easily demonstrated that the nuclei multiply spontaneously, but it is generally doubtful whether this happens by division or by endogenous development.

Cell-development round a mass of blastema containing a nucleus in its interior, which may take place either freely or in an endogenous manner (cell-development round portions of contents), had long ago been seen by Von Siebold in the ova of Distoma globiporum, and by Bergmann in the cleavage-masses of Rana, but no further importance was attached to it. Vogt and Nägeli were the first who regarded this cell-development as a deviation from the theory of Schleiden and Schwann, whereupon, supported by observations upon embryos, I (in 1844, "Entwick. d. Cephalopoden") placed this as a second kind of cell-development under the name of "cell-development round investing masses," beside that which takes place immediately round nuclei, and pointed out its very extensive occurrence, especially in embryos, where at first it is the sole mode. Later observations upon normal and pathological products have supported this view, and at the present time the formation of a cell-membrane immediately around the nucleus requires demonstration, rather than the opposite method. Endogenous celldevelopment occurs in many pathological products,-most frequently in cancer, yet the steps of the process have not yet been exactly made out. In plants this mode of multiplication of cells is the most extensive, and it occurs commonly as "cell-development around portions of contents," more rarely (in the embryo sac) by free development within parent cells.*

§12. A multiplication of cells by division certainly takes place in the red blood-corpuscles of the embryos of Birds and Mammalia, and

Fig. 8.

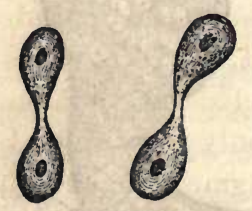
in the first colorless blood-corpuscles of the Tadpole (Remak). It takes place also, in all probability, in the colorless blood-corpuscles of embryos, and in the chyle-corpuscles of adult Mammalia under certain circumstances. In all these cases, we see, in elongating cells, the production of two nuclei from the originally simple

Fig. 8.-Dividing blood corpuscles of the chick; magnified 350 diam.

* [The endogenous development of secondary "nuclei" seems to us to be extremely doubtful, even upon the evidence adduced, and we have been unable to observe anything indicating that regularity of occurrence and importance of function attributed to the nucleolus by Professor Kölliker. In cartilage, in the tooth-pulp, in the homogeneous layers of the cutis, and in other localities, in which unaltered "nuclei" occur, the presence and number of the granules which might be called nucleoli is in the highest degree variable and uncertain. The same irregularity as to the presence of nucleoli occurs in the plant (vide Von Mohl, l. c., and Schacht, "Die Pflanzenzelle," p. 30).

Upon this subject consult the valuable memoir of Remak (Ueber extra-cellulare Fnstehung Thierischen Zellen," \&c., Müll. Archiv, 1852), which by no means deserves the epithet of " no longer available ;" in fact, Remak's views seem to be essentially correct.-TRs.] 
nucleus, apparently by division; the cells then suffer constriction in the middle, and contract more and more around the nuclei, as they recede from each other, and at last separate into two cells, each of which contains a nucleus. In the chick we see blood-corpuscles in all conceivable stages of separation, so that at length they are connected only by a delicate thread, and there can be no doubt whatever as to the actual occurrence of this process.

Whether division ever take place in other cells than these is not yet determined. If it be allowable to explain, by this process of division, the occurrence of constricted cells with two nuclei, we may suppose it to take place in the nerve-cells, which, in young mammalia, are not unfrequently more or less divided or even united merely by a narrow isthmus, and also in the ciliated epithelium cells, which, although rarely, present two or three enlargements lying one behind the other, each with a nucleus. A peculiar kind of cell-development, which is very closely related to division, occurs in the formative cells of the ivory, which as they go on growing, multiply their nuclei and become constricted from time to time, so that whilst the portion

Fig. 9.

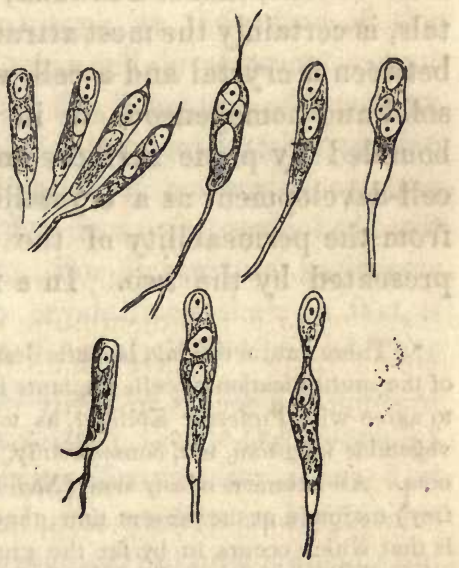
next the ivory ossifies, the other serves in a manner as a reserve for the subsequent formation of fresh ossifying tissue.

Schwann knew nothing of the occurrence of cell-division. The first who observed it in the blood-corpuscles of embryos was Remak ("Med. Verein." 1841, No. 27; Schmidt, "Jahrbücher," 1841, p. 145; Canstatt, "Jahresb.," 1841), yet he subsequently retracted his opinion ("Diagn. und Pathol. Untersuchungen," p. 100), and only now, since I have confirmed it and declared it to be true (Wiegm. "Archiv," Jahrg. 13, Bd. 1, p. 19), has he again advocated it ("Entwick. d. Wirbelthiere,' I.) It is extremely probable that this mode of cell-development occurs very extensively, and it may perhaps turn out that in many embryos and adult tissues, in which a self-multiplication of the cell is certain, and yet in which no parent cells with secondary cells can be demonstrated, cell-development by division may occur instead of endogenous cell-development. It is certain that the transverse and longitudinal division of the Protozoa is to be placed here, since these animals have 
the structure of simple cells, and the nucleus-like body they contain takes a share in the process of cell division, like that of the cell-nucleus in common cells. In pathological formations cell division has not yet been observed. In the vegetable kingdom it is rare, and has been seen only in the lower organisms; unless, indeed, we are to reckon here the constriction of the primordial utricle observed by Von Mohl in the course of endogenous cell-development.*

\section{§ 13. Theory of Cell-development.-Among the few hypotheses which} have, up to the present time, been proposed in explanation of the development of cells, that of Schwann, who compares it with the formation of crystals, is certainly the most attractive. Without overlooking the differences between a crystal and a cell, which chiefly consist in the former being solid and homogeneous, in its growing by apposition, and in its being bounded by plane surfaces and angles, Schwann endeavors to explain cell-development as a crystallization of organic matter, and to deduce from the permeability of the latter the differences in the phenomena presented by the two. In a fluid containing organic matters dissolved

* [There can, we think, be little doubt that Von Mohl is quite correct in the view he takes of the multiplication of cells in plants by division, and therefore we are by no means inclined to agree with Professor Kolliker, as to the rarity of this form of cell-multiplication in the vegetable kingdom, nor, consequently, in what he says at the conclusion of the preceding note. All botanists of any note (Nageli, Von Mohl, Hoffmeister, Alex. Braun, Schacht, Henfrey) maintain at the present time, that the process of cell-division so far from being "rare," is that which occurs in by far the great majority of cases in plants. "That the formation of cells, in all organs of plants (except the cells originating in the embryo sac), depends upon the division of older cells, is an opinion, which could not for a long time past be opposed by any careful cbserver, unless he were misled by preconceived notions." (Von Mohl, "Anatomy and Physiology of the Vegetable Cell," 1851, Henfrey 's translation). Nor can we agree with Professor Kollikers estimate of the relative frequency of occurrence and importance of endogenous cell-development and cell-division in the animal world. In young cartilage, which is cited by our author as a locality in which endogenous cell-clevelopment takes place, we must affirm, on the contrary, that the process is as much one of cell-division as it is in any plant. At this period the so-called "nuclei" of the cartilage completely fill their cavities (e. g. nasal cartilage of four montlıs' fœtus), and may be seen in all stages of division. The walls of the cavities grow in, pari passu, and eventually form a partition between the two nuclei, or rather primordial utricles, which have been thus developed from one.

Remak, who, in a very valuable paper (Ueber die Entstehung der Bindegewebes und Knorpels, Mull. Archiv, 1852), has advocated this view, so far as cartilage and connective: tisue are concerned, does not appear to have seen the necessity of extending it to the other tissues. As Reichert, however, long since pointed out (see note, $\S$ on Connective Tissue) whatever determines the nature of the cartilage corpuscle, and of its matrix, determines that of all the other tissues whose anatomical continuity with cartilage can be traced directly or indirectly. Thus a direct anatomical continuity may be slown to exist between the matrix of cartilage, the apparent fibrillæ of connective tissue, the fibrillæ of muscle, the homogeneous matrix of the cutis and of its papillæ, and the so-called walls of the epithelial cells; wbile a perfect identity in size, structure, and relation, may be traced between the corpuscles of cartilage, the "nuclei" of connective tissue, those of muscle, of the papillæ and of the epithelial cells.-Trs.] 
in considerable proportions, a granule, the nucleolus, is precipitated. Once formed, this attracts nutriment from the cytoblastema, and thus becomes the nucleus, which Schwann considers to be solid. This still goes on attracting to itself a substance, which, becoming more and more condensed, at last forms a membrane; which, allowing the passage of fluid cytoblastema through its pores, becomes detached from the nucleus, and we have a cell. In this exposition, we must admire not only the skilful, acute working out of the fundamental idea which the original treatise manifests, but also the assumption of a molecular attraction in cell-development, analogous to that which occurs in the formation of crystals, for the existence of which there is, in fact, decisive evidence (only in part, however, known to Schwann), such as the action of the nuclei in the cleavage process, in cell-division, in cell-development, round portions of contents, in the cyclosis, and in the formation of granular precipitates in cells.* On the other hand, it is evidently going too far to call cell-development simply a crystallization of permeable organic substances, since in this case important differences are overlooked, and non-essentials are made unduly prominent. For it must not be forgotten that organic permeable substances also crystallize; that, in fact, if Reichert have observed correctly (Müller's "Archiv," 1849), and I see no reason for doubt, histogenetic substances capable of forming tissues, as albumen for instance, assume a crystalline form. Hence the molecular attraction concerned in the formation of cells is so far peculiar, that-1, it never produces geometrical solids, but even in the nucleus and nucleolus determines the globular form ; 2 , that it aggregates, not homogeneous but chemically different substances, as those which constitute the nucleus and the cell-membrane; 3 , lastly, that without exception, in the development of the cell-membrane it limits itself, and does not, like the crystallizing force, repeatedly apply layer upon layer. Of these differences, the two latter might perhaps be set aside, if with regard to the second point, it were assumed that the nuclei at first consist of the same substance as the cell-membranes, or are almost identical with them in chemical composition; or if we referred to the fact that in crystallization also, different substances may unite into one crystal, or that a substance, $b$, may crystallize round a substance, $a$.

In order to diminish the force of the third fact adduced (this objection, indeed, does not hold with regard to endogenous development, and therefore in almost all plants, since it is impossible here that the cells should produce any more layers around themselves), it might be urged that the permeability of the organic membranes, the exchange of constituents which take place between the juices of the cell and the cytoblastema, and the application of the molecules attracted from the cytoblastema to the growth of the membrane, and to precipitates within the

$$
\text { * [See note, p. 52.-TRs. }]
$$


interior, are perhaps the reasons why the cells develop no new circumferential layers. It is not necessary to carry out this last possibility any further, nor to bring forward the difficulties which are opposed to this view also,-among which not the smallest is, that the organic development of vesicles does not stop at the formation of nuclei, but is only finished with the completion of the cell membrane; since in any case the facts brought forward are more than enough to demonstrate the insufficiency of ISchwann's hypothesis. I do not see, however, anything better or more positive to substitute in its place, and I therefore think it will be most expedient simply to group together the ascertained facts into a few general propositions, which may, perhaps, be done as follows.

1. The nucleus of the cell arises in the first place, as a precipitate in an organizable fluid, and afterwards becomes consolidated in such a manner, that a special investment and contents with a nucleolus appear. Its development may in this case be compared to that of inorganic precipitates, yet the constantly globular figure, and the size of nuclei which are just formed, indicate some essential though not yet recognized condition peculiar to them. Secondly, cell-nuclei are produced endogenously in nuclei, or by their division under the influence of a nucleolus, which also divides. Here is one condition which is never presented by crystals, - the division from an internal cause; while the other, the influence of the nucleoli upon the nucleus, can hardly be comprehended in any but a physical way, as a molecular attraction proceeding from the nucleoli, of an indefinable nature, which at last draws the entire half of the parent nucleus within its influence.

2. In the development of cells by division, the cell-nucleus plays exactly the same part which was previously ascribed to the nucleolus, and the occurrence of the formation of cells in this manner demonstrates that chemical conditions are not necessarily concerned therein.

3. In cell development around portions of contents, and in the cleavage process, the nuclei also opcrate as simple centres of attraction (einfach attrahirend) upon a certain mass of blastema, and then follows the formation of a membrane upon the surface of this mass; which is most simply understood as a condensation of the blastema.

4. In cell-development directly around the nucleus, the investment with blastema is wanting, and the nucleus develops the membrane immediately around itself. This process admits of both a physical and a chemical explanation. In the first place, we may with Schwann assume that the nucleus attracts molecules, which, when they have reached a certain amount, condense into a membrane, and, by growing, become detached from the nucleus. Or secondly, it is conceivable, that the nucleus in some manner initiates chemical processes, which terminate with the formation of a membrane around it. In this way a coagulable sub- 
stance might be produced within and excreted from it; or, like rennet upon casein, it might act upon the protein combinations in the cytoblastema, in such a manner that they should coagulate where in contact with it; or lastly, by the extraction of the alkali it might render an albuminous substance insoluble, as is the case in the development of Ascherson's vesicles. Which of these various possibilities really obtains, must at present remain undetermined, yet, for my own part, I should prefer the first view, in order to retain one and the same condition, a physical one, for all the different modes of cell-development.

I do not think it necessary to enter at greater length, in the present place, into this very obscure subject, and I will therefore only once more express my opinion, that I hold the physical processes of celldevelopment, which may pass under the general name of molecular attraction, to be something quite different from those which attend crystallization. 'Although in both, solids arise out of fluids, and grow by the further agglomeration of molecules, yet in cell-development different substances are as a rule superimposed, plane geometrical solids are never formed, and the process is always limited in the same way, after the formation of the cell-membrane. Since organic and even histogenetic substances are crystallizable, the reason of cell-development is not to be found in permeability nor in any of the other properties of organic compounds, which in fact, even if the substances did not crystallize, would not suffice to explain all the peculiarities of cells in question, nor their power of self-division and multiplication; but in those peculiar, as yet unknown combinations of the powers of nature which are concerned in organic development. To discover these is the further and difficult task of Histology, which to this end must be wholly directed to the socalled molecular forces of organic forms, especially to those electrical phenomena which must indubitably occur in the cells as well as in their derivative structures, the nerve-tubes and muscular fibres.*

§14. Vital Phenomena of the Perfect Cells. Growth.-The cells, when once completed, perform a considerable number of functions, which relate as well to the form of the whole cell and of its contents, as to their chemical composition, and are called growth and change of substance.

- EThe essential distinction between living organized matter (the cell) and mere inorganic formed matter (the crystal) appears to us to be here overlooked. If some inorganic substance should be discovered crystallizing in the form of nucleated cells, it would not the more approximate to an animal or vegetable cell; for the essential character of the latter, which is its passage through a definite succession of states and not its form merely, would still be absent. It is this characteristic peculiarity of organized living beings, which has been exhibited with so much force by Alex. Braun, in the plant, under the somewhat fanciful title of 'Verjünung' (rejuvenescence), but which equally obtains in the animal. The crystal tends to attain a permanent condition, the cell towards its own disappearance, either by death or division. The crystal tends towards an equilibrium with the forces around it, the cell incessantly disturbs that equilibrium,--life and change being one,-Trs.] 
As to growth, it occurs perhaps in all cells, though not in all to the same extent. It is clearly manifested in all those cells which are formed directly round a nucleus, since in this case the membranes which at first closely invest the nucleus, in time become more and more separated, whilst the cells which arise around portions of contents or investing masses, and are from the first provided with contents, often increase in size but very slightly. Growth is either in surface or in thickness. The former appears very usually to be general, when cells increase without altering their form, e. $g$. the ova, many nerve-cells, \&c.; frequently however it is partial, as in all cells which depart from the primitive globular form, in such a manner that the cell-membranes cnly add new substance and extend at two or more points. Growth in

Fig. 10.

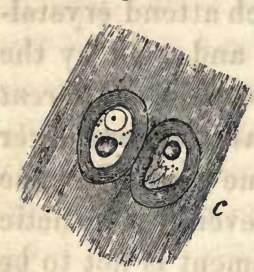

thickness also occurs, to a certain extent, in all cells, since all cellmembranes become somewhat thicker with age; and it produces in some localities a very considerable thickening of the membrane, occasionally with evident lamination (as in the cartilage cells), and
Fig.11.

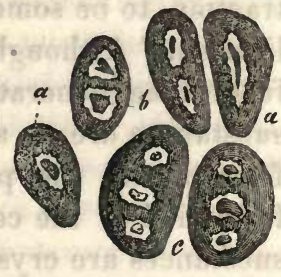

even gives rise to certain structures (Fig. 11), which have the greatest similarity to the sclerogenous cells of plants (bone-cells).

The nuclei and nucleoli also take part, to a certain extent, in the growth of the cells. In the former, a general growth is easily demonstrable in all growing cells; in many, as in those of the smooth muscles, of the epithelium of the vessels, of the formative cells of elastic tissue, and others, there is also a partial growth, in consequence of which they often assume the form of long slender rods. The nucleoli also not unfrequently grow with their cells (nerve-cells, ova); but except when dividing they never assume any but the globular form.

Schwann has given an explanation of the growth, as well of the cell as of the nucleus. He considers that the molecules of the cell-membrane exert an attractive influence upon the fluid which surrounds them, and deposits its newly-formed particles among themselves; if the deposition take place between molecules already present in the substance of the membrane, the cell becomes distended; if it take place only in the direction of the radius of the cell, the membrane becomes thickened.

FIG. 10.-Cartilage cells of man. Two cells with thickened walls, from the cartilage of the great cornu of the hyoid bone, containing a clear clrop of fat beside their nucleus. Magnified 350 diameters.

Fra. 11.-Six developing bone-cells from a rickety bone as yet sharply defined from the interstitial substance:- $a$, simple bone-cells; $b$, a compound one, answering to a parent cell with two secondary cells; $c$, similar ones with three cells. Magnified 350 diameters.

* [This increased thickness of the cell-wall with age is well seen in all epithelial tissues normal as well as pathological.-DAC.] 
The nucleus grows less than the cell, because as soon as the latter is formed it no longer comes into direct contact with the concentrated cytoblastema. General growth takes place when the molecules of the membranes all attract equally; partial growth, when this happens only or especially at particular spots, where the apposition of new matter takes place to a greater extent. With reference to the mode of formation of precipitates and of crystals, this theory appears to me to explain very well the phenomena of general growth, supposing that we ascribe to the cell-membrane the faculty of readily taking up molecules and applying them to its increase. Such, however, must be the case, for the relations of the nuclei, which even when free, never grow very considerably, and particularly never in one direction,* show that the power of growth is not simply innate in every organic membrane, manifesting itself when sufficient formative material is offered, but requires peculiar conditions which are realized only in the cell-membrane. To account for partial growth, Schwann's view must be somewhat extended; for only those modes of growth in which the cells, during their increase in certain directions, lose nothing of their original dimensions in others, can be interpreted in Schwann's way, but not those in which the cells become narrower as they elongate; here we must assume that whilst new substance is deposited in the one direction, in the other an absorption takes place, for we can in nowise consider the process to be a mechanical one. For the rest, it may be remarked, that partial growth may depend upon the occurrence of assimilation, in particular cells, only in certain directions, as in the thickened vegetable cells with pore-canals, which is possibly connected with a one-sided direction of the currents in the cell-contents.

$\$ 15$. Processes in the Interior of the Cells.-In order to obtain a clear conception of the processes which go on in the interior of cells, it would before all things be necessary to have a more exact acquaintance with the chemical composition of the cell-contents than we at present possess. Only two kinds of cells, the ovum and the blood-globule, have been investigated with care (see Remarks); but these have such peculiar relations that they can hardly be regarded as types of cells in general. However, we may from these analyses draw certain inferences with regard to other cells, and bearing in mind what micro-chemical investigation teaches us, it may be permissible to regard the cell-contents in general, as a moderately concentrated solution of protein with alkaline and earthy salts, and dissolved or suspended fatty particles. From these common characters, presented without doubt by all cells, at least in their young condition, many cells differ very widely, insomuch as either some of these constituents greatly predominate or altogether new

* [This is surely incorrect. The " nuclei" in the hair-pulp, in the tooth pulp, in connective tissue, in organic muscle, grow in one direction to a very considerable extent.-TRs]. 
substances occur. Thus, there exist cells with much protein, as the nerve-cells; and with much fat, as the fat-cells, the cells of the sebaceous follicles, of the milk-glands, \&c. ;-then such as contain hæmatin, pigment, biliary, and urinary constituents, mucus (epithelium-cells), milk, sugar, \&c. \&c.

The phenomena manifested by these, so variously constituted cellcontents, during life, may be best enumerated as-absorption, assimilation, and excretion. These depend principally upon chemical and physical conditions, and are to a great extent capable of microscopic investigation, since very frequently the changes of form in the cell and the changes of its contents go hand in hand. Absorption is manifested in all cells, but to far the greatest extent in those which at first have little or no contents save the nucleus. In these, the primary cause of the absorption is not to be sought in endosmose, but, as Schwann has indicated, in this-that while the membranes grow by the attraction of material from the surrounding fluid, by virtue of their porosity they allow substances to penetrate into the interior. This filling, however, does not take place by the cells admitting every kind of matter indiscriminately, but they exhibit peculiar relations to the cytoblastema, varying with the period and with the locality; so that they take up one constituent and reject another; and the like occurs with the absorptive powers of those cells which possess contents from their earliest existence.

That this is actually the case, is demonstrated, for instance, by the fact, that in embryos, notwithstanding the identity of the formative material in all cells, $i$. $e$. the plasma of the blood, some take up more of one substance, some more of another; and it is still more clearly evidenced by the fact, that the cell-contents of probably all cells are chemically different from the cytoblastema out of which they are formed and by which they are nourished, as has been clearly shown lately, in the ova and blood-corpuscles, which for example contain far more potassa than the blood. The reason of this phenomenon may be generally stated to be, that the cell-membranes do not act as mere filters, but allow one substance or another to permeate them, according to their chemical composition, the constitution of the fluid which imbues them, their condition of aggregation, and their thickness.

Endosmose must also be taken into account as a conditicn in the absorptive actions of cells, though hitherto it has been too freely appealed to, and cells have been too often considered as vesicles provided with merely indifferent porous membranes. That endosmose operates is not to be denied, when it is observed how the addition of concentrated or diluted solutions, causes cells to dilate or to collapse, yet it is not easy to determine what influence such conditions have during life, nor what results are produced by the combined operation of the cell-membranes 
and their contents. From a few facts in vegetable physiology (growth of plants in arsenical and cupreous solutions, without the admission of these substances), it might be believed that the membranes exercised the more important influence in determining absorption.

It is an important question whether the substances received by the cells and composing them become modified by their vital processes. Schwann has answered it in the affirmative, and has denominated metabolic processes of cells, all those chemical metamorphoses which go on in them and in their separate parts; and justly so, for the occurrence of such chemical changes is not only very probable $a$ priori, since in plants all such metamorphoses (and these of the most various kinds) take place in the cells, but may very easily be demonstrated by observation. These changes affect, firstly, the cell-membrane, and secondly, the cell-contents. As regards the former, this much is certain, that the membranes of most cells not only become denser and more solid with age, but also that they take on a different chemical constitution, though it is impossible in particular cases to say on what the change depends. In the horny tissues, the membranes of the young cells are easily soluble in alkalies and acids, whilst subsequently they sometimes offer extreme resistance to their action: the same takes place in a few of the higher elementary parts, as the nervous tubules, the animal muscles, and the capillaries, in which the sarcolemma, the sheath of the nerve fibre, the capillary membranes, which are metamorphosed cell-membranes, react in a very different manner from the original formative cells. In the cartilage cells also, the membrane becomes more resistant with age, and in the course of ossification not only thickens, but is for the most part changed into collagenous tissue, which is subsequently impregnated with calcareous salts. These examples, which might be multiplied, may suffice to demonstrate the occurrence of a metamorphosis of the cell-membranes; further investigations will be needed to show upon what it depends, whether, as it would seem, the original animal cell-membrane actually alters in composition in course of time, or whether the change in the reaction depends upon the addition of foreign substances, on the incrustation of the membranes with salts, and so forth, such as botanists are inclined to assume for the vegetable cell-membranes, or whether it depends upon secondary deposits on the exterior of the original membranes.

The changes of the cell-contents are of two kinds, formative and resolvent. Both processes are easily followed in the embryos of different animals, in which, in the first place, the primary formative cells, which at the beginning are distended with the elements of the yelk, especially with oil, acquire by degrees more fluid and homogeneous contents, the yelk granules dissolving, sometimes from the cell-membrane towards the nucleus, sometimes from within outwards; and secondly, in cells thus formed, the most various new formations take place, among which that 
of hæmatin, of different kinds of pigment, and of fat, are the most obvious. But metamorphoses of the cell-contents are very common in adult animals also, and are at the same time very important, since in many places, on account of the great number of cells which are affected in the same way at the same time, unexpectedly great results are produced, as one of the most important of which we may name the biliary secretion, which is brought about, so to say, only by the activity of the many millions of cells which form the liver. A pretty series of changes may also be traced in the fat-cells, which, according to the deficiency or superfluity of nutritive fluid, in the one case lose their proper contents, and may even become cells containing mere serum, in others are filled to bursting with drops of oil; again, in the cells of the fat-secreting glands, which at first contain but little fat, and finally become crammed with it; and also in the lymph-corpuscles, which develop the coloring matter of the blood within themselves, and thus become blood-corpuscles.* The formation of mucus, again, must probably be assigned to the epithelial cells of the mucous glands and mucous membranes; that of the so-called pepsin to the cells of the gastric glands; and that of semen to the spermatic cells. A multiplicity of confirmatory evidence is afforded by comparative anatomy, and I will here only advert to the development of the concretions of uric acid in the renal cells of the mollusca, that of sepia in the cells of the ink bag of the Cephalopod, of crystals and concretions of different kinds in the cells of the invertebrata, and of certain pigments in those of the mollusca. Pathological anatomy affords us the pigment formations, the metamorphoses of cells containing blood-corpuscles, and the fatty deposits in cells of all kinds.

Manifold morphological phenomena go hand in hand with these changes, such as the thickenings of the cell-membrane, which have been already adverted to, with laminated depositions upon their inner surface, and even with the formation of pore-canals; as the precipitation in the cell-contents of granules of different sorts, as of pigment, albumen, casein (in the yelk, perhaps in the hepatic cells); and as the formation of fat drops, of elementary vesicles, of concretions, crystals, and nuclei. Even movements resembling the cyclosis of plants appear to occur in the cells of the lower animals (seen by me in the cells of the arms of a minute Medusa, a new Aginopsis from the Mediterranean, and of Polyclinum stellatum), and in Protozoa (currents in Loxodes bursaria, contracted vesicles in different genera); while on the other hand, the Brownian molecular movements, $i$. e. a more or less active tremulous motion of granules without further change of place, which may be observed in many cells under the microscope, most beautifully in the pigment cells of the eye, are also, perhaps, hardly to be reckoned among vital phenomena.

* [In the oviparous vertebrata.-TRs.] 
The nuclei also occasionally, though upon the whole rarely, take part in the changes of the cells. The commonest of these appearances is their becoming clear, as a consequence of the liquefaction of the at first more viscid contents, upon which circumstance it depends that in young cells they are homogeneous, while in the larger they evidently appear to be vesicles. A formation of granules in the nuclei is very rare (see above); concretions, coloring matters, and crystals, are also not found here in animals; on the other hand, the development of the urticating threads in certain animals and that of the spermatozoa, takes place in nuclei.

In endeavoring to explain the metabolic processes of cells, we must in all cases especially regard the cell-nucleus; for just as it excites the development of the cell, so is it the centre of the currents of the contents, and of the deposits and solutions; but it is not to be regarded as the sole agent, for, firstly, it does not appear why the cell-contents should not, like the cytoblastema, become changed of themselves; and, secondly, the changes of the cell-membrane are, at all events, more independent, and probably also have a certain influence upon the cell-contents, as the depositions which take place upon it, and the solution of the solid contents which often occurs in its neighborhood, demonstrate. To assume with Schwann a special metabolic force is incorrect, for, in the first place, the causes of the metabolic phenomena are certainly very various; and, secondly, there is every reason to reduce them to known molecular forces. Thus, for instance, even the action of the nucleus* may not unfittingly be compared with the so-called catalytic, or contact action, inasmuch as it is hardly at all altered during the cbanges of the cells, and consists of a nitrogenous substance, which like pepsin (which is also nothing but cell-contents), very readily produces a chemical alteration in other substances. The relation of the cell-membrane to absorption also, may even now be referred to the general laws of imbibition and diffusion.

I here give two analyses as examples of the chemical composition of the cell-contents. The yelk of the hen's egg contains : water, 48.55 ; casein, 13.93 ; albumen, mixed with casein, 0.892 ; albumen, 2.841 ; membranes of the yelk vesicles, 0.459 ; fats, $31 \cdot 146(30.46$ accurding to Gobley), consisting of olein and margarin; 21.304 ; cholesterine, 0.438 ; lecithin (containing phosphoric acid), 8.426 ; and cerebrin; salts, 1.523 ; a hundred parts of the ash yielded, potass, $8.60-8.93$; chloride of sodium, $9 \cdot 12$; phosphatic salts, $66 \cdot 7-67 \cdot 8$; lime, $12 \cdot 21$; magnesia, 2.07 ; oxide of iron, $1 \cdot 45$; silica, 0.055 . The blood-corpuscles contain :

* [This "action of nucleus" is a wholly hypothetical though a very general assumption It is important to bear in mind, on the contrary, that there is every reason to believe that the molecular and chemical changes of the cell-membrane and the nucleus are independent of one another.-Trs.] 
water, 68.88 ; hæmatin, 1.67 ; globulin and membranes, 28.22 ; fat, 0.23 ; extractive matters, 0.26 ; mineral substances (without iron), 0.81 ; of which chlorine, 0.16 ; sulphuric acid, 0.006 ; phosphoric acid, 0.4 ; potassium, 0.33 ; sodium, 0.10 ; oxygen, 0.06 ; phosphate of lime, 0.01 ; phosphate of magnesia, $0 \cdot 007$. To these must also be added, free oxygen and carbonic acid, which likewise occur in the yelk.

We have here instances of cells containing much protein, and especially fat, and may consider them to be fair examples of this kind of cell. The comparison of the contents of these cells with the plasma of the blood, out of which those of the one kind are formed, while the others live in it, is very interesting. In the blood-globules there is a considerable preponderance of solid constituents, since the blood-plasma only contains about 10 per cent. of solids, which is evidence that there are cells whose contents do not attain an equilibrium with the cytoblastema by which they are supported. With regard to particular substances, the blood-corpuscles contain more fat; hæmatin, which is not found in the plasma; more potassa and phosphoric acid; less chlorine, extractive matters, soda, and earths. The yelk of the hen's egg contains also considerably more solid constituents than the blood, which however is here less surprising than in the blood-corpuscles which swim in the bloodplasma. It is interesting, that the relative proportions of the different substances are quite different in this case from the other. We have, namely, an exceedingly large quantity of fat, more protein and salts; and among the latter, again, more potassa, and also more earthy salts.

Even these facts indicate a considerable independence of action in the cells; but those which have been lately made known by Ludwig, tend still more forcibly in the same direction; for the influence of the nerves upon the salivary glands discovered by this observer must, I believe, be so interpreted, that it is not only the membrance proprix of the vesicles of the salivary glands, which are so altered in their molecular relations by the nervous influence that they directly exercise an energetic attraction upon the blood-plasma which surrounds them, but the epithelial cells which line them also. If this really be the case, we have an insight into an altogether new condition regulating the absorptive powers of cells, and at the same time cell-life is brought into such connection with the activity of the nervous system, that it no longer appears out of reason to speak of the positive functions of the latter. Such relations are in nowise opposed to analogy, since in the contractile elements we have already a connection between nervous activity and the modification of cell-contents, which perhaps upon further investigation may come under the same general category as the foregoing. In any case, these considerations lead anew to an exact investigation of the molecular forces of cells, especially of those electrical phenomena which will certainly be found in them. 
Very recently Donders (Nederlandsch. Lancet.) has justly brought forward a character which until now had received no attention, viz., the elasticity of the cell-membranes and the pressure consequently exercised upon the cell-contents. It is an ascertained fact that the cell-membranes are elastic; and it thence naturally follows that, according to the greater or less amount of the contents of the cells, so will these suffer a greater or less pressure. This, however, reacts again upon the absorptive and excretive processes, so that under a more considerable pressure the latter, under a less, the former prevails, and in certain circumstances it may conduce to the maintaining of a regular interchange of substances. Donders believes, that the greater density of the cell-contents may be derived from their always being under greater pressure than the cytoblastema.

$\S 16$. Excretive processes. - The vegetative functions of animal cells are not limited to mere absorption and metamorphosis, but substances are excreted as a result of their operation. This may take place in two ways.

1. The cells give out unaltered, the substances which they have received from without.-This occurs in the epithelium cells of those glands which, like the kidneys, lachrymal glands, lungs, \&c., simply permit of the discharge of substances from the blood, also in those cells which line the serous membranes, and probably many others.

2. The cells excrete substances which they have prepared within themselve8. - Thus the blood-cells give up their hromatin in dilute bloodplasma; the fat-cells their fat in emaciated persons; the hepatic cells, bile; those of the gastric glands, gastric juice; those of the mucous glands and membranes, mucus.

The occurrence of these excretions, of which, in fact, there are assuredly very many with which we are still unacquainted, may in some cases be explained by exosmose; in others, however, as in the secretions of the glands, this cannot take place. Here the exit of the contents is a consequence of the pressure to which they are exposed, which pressure is to be referred upon the one hand to the force of the blood, on the other to an attractive force exercised by the cells themselves in absorbing the substances, and to the elasticity of the cell-membranes.

The excreted matters in general no longer continue in the organism, but are completely removed as in the glands; in a few localities they remain, taking a solid form, as extra-cellular substance, outside the cells, and form the genuine membrance proprioe of the glands $(e . g$. of the renal canals), the proper envelop of the chorda dorsalis, and probably also the so-called vitreous membranes (capsule of the lens, membrana Demoursii). An intercellular substance is rare in animals, for the matrix of the cartilages and bones, which for the most part is not excreted by the 
cells, but is deposited from the blood-plasma or is even formed out of cells, does not come under this head. It may be said to be present in a smaller quantity and more fluid, however, not only in the cellular tissues, but also in the higher structures, among which there everywhere exists a small quantity of connecting substance. Intercellular spaces developed by the excretions of cells between one another, have not been demonstrated with certainty in animals, yet it is probable that most glandular cavities and those of the heart and of the great ressels are of this nature, since they appear to rise by the excretion of fluid in the interior of originally compact masses of cells.

My view, that the genuine, membrance proprice and the vitreous membranes are formed as excretions, is founded particularly upon the examination of the chorda dorsalis and of the renal canals, in which it may be readily shown that the structureless membranes are secondary formations, arise in intimate union with the cells of this part, and from the very first appear perfectly homogeneous. The supposition of many authors, especially of Reichert, that these membranes belong to the homogeneous connective tissue, is readily refuted by chemical examination, since they yield no gelatine, but consist of a substance which most closely approximates to the sarcolemma and elastic tissue (comp. Mensonides in 'Nederl. Lancet.' d. iv. 694, and Donders, ibid. August, 1851, p. 73). To what extent homogeneous membranes formed by excretions from cells, occur among animals is not yet determined, but the homogeneous chitin-investments of the intestine, and of the external surface in the Articulata, appear to be of this nature.

$\S 17$. Contractility of the Cells.-Among the vital phenomena of cells must be enumerated those contractions which are manifested by cellmembranes and also by cell-contents. Contractile cell-membranes are possessed by many if not all Protozoa; and among subordinate cells, by the yelk-cells of the Pladariæ, the heart-cells of many embryos (Alytes, Sepia, Limax), the cells of the tail of embryo Botrylli. The cilia also, as processes of the cell-membrane, may be mentioned here. Contractile cell-contents are found in the fibre-cells of the smooth muscles, in the stellate cells of the skin of the embryo of Limax, and in the animal muscular fibres; which last, as they consist of a number of united cells, may be here enumerated. Here also I place the contractile phenomena exhibited by the contents of the Protozoa (contractile vesicles) and by the Rhizopoda.*

* [To this list of contractile cells must be added the colorless corpuscle of the blood of man, the Frog, and the Skate, and probably that of other Vertebrata (Wharton Jones, l. c.), the cells which lie in the meshes of the areolar tissue of the disc of the Medusæ (Cyanaa) and the young epithelium cells (mucus-corpuscles) of the mucous membranes, in which most distinct protean movements, like those of the colorless corpuscle, may be observed. 
Donders has recently promulgated the view that it is only the cellcontents which are contractile, not the cell-membranes. Although it must be granted, that it is difficult, in the cases in question, to decide what part of the cell contracts, yet it seems very hazardous to endeavor to refer the movements of the cilia in plants and animals, in free and combined cells, to indemonstrable contents in these cilia communicating with the cell. In the cells of the Planariæ and in the Protozoa, any one who has actually seen the movements will hardly refer them to anything but the cell-membranes. In the transversely striated muscular fibres on the other hand, it is evidently the fibrilla or the contents which are contractile, and the sarcolemma, as an elastic yielding body, only moves with them: the same appears to hold good with the muscular fibre-cells, in which a special membrane cannot be demonstrated.

\section{§ 18. Metamorphoses of Cells-Kinds of Cells. - The destination of} the cells which are found at an early period in the organism is very various. A very considerable portion of them remain but for a short time in their primitive condition, and subsequently coalesce with others to form the higher elementary parts. Another portion, while they enter into no such combinations, change more or less their previous nature; as the horny plates of the epidermis and nails. Many cells, lastly, never become metamorphosed at all, but remain as cells, until sooner or later, often not before the decay of the organism, they disappear accidentally or typically, as the epithelia, glandular parenchymata, \&c.

The permanent cells may be most conveniently arranged under the following heads :-

1. True cells, which have in no essential respect altered their cellular nature. These occur in the epidermis (stratum Malpighii) and the epithelia; in the blood, chyle, lymph; in the glandular secretions, in the fatty tissue, in the gray nervous substance, the red bone-medulla; in the glands (liver, spleen, suprarenal capsules, closed glandular follicles), and the cartilages. According to their form, these cells may be divided into round, discoid, cylindrical, conical with cilia, and stellate; according to their contents, they may be distinguished as containing fat, protein or serum, hrmatin, bilin, pepsin, mucus, or pigment; and as to their modes of occurrence, some are either isolated in fluids or in solid tissues, others are united into a simple cellular parenchyma, while others are conjoined by an intercellular substance of one kind or another.

It is certainly the membrane which contracts in these cases, for it pushes out processes which are only subsequently filled by the granular contents.

In the lower plants $(A \lg \propto)$ the occurrence of contractile processes in the shape of cilia is universal, and the contractility of the cell substance in the zoospores of Volvox is evinced by the occurrence of a rhythmically contracting space in them. (See Busk on Volvox globator, "Quarterly Journ. of Micr. Sc.," No. 2, 1853.)-TRs.] 
2. Metamorphosed cells, which have more or less altered their original structure. To these belong:-

a. The horny scales : flattened, polygonal, or fusiform: their membrane being fused into one mass with the contents. In the epidermis, the laminated pavement epithelium, the hairs and nails.

b. The contractile fibre cells: fusiform, slightly flattened, considerably elongated cells, whose membrane, together with its soft solid contents is changed into a contractile substance. In the smooth muscles.

c. The tubules of the lens: very much elongated cells, with viscid, albuminous contents.

d. The prisms of the enamel: greatly elongated, prismatic, and strongly calcified cells.

e. The bone cells: thickened cells with pore canals which have coalesced with the homogeneous matrix of the bones and anastomose by means of excavations in it.

f. The transversely striated muscular cells: large polygonal cells, whose contents have become metamorphosed into a transversely striated tissue, such as is found in the transversely striated muscular fibre. In the endocardium of ruminants.

\section{B. HIGHER ELEMENTARY PARTS.}

$\S 19$. The higher elementary parts correspond, genetically, to a whole series of the simple ones, and it is the cells only, so far as we know, which possess the faculty of producing them. The manner in which this takes place varies. Either the cells while they coalesce retain their cellular nature, and to a certain extent their independence, in which case we have, according as they are fusiform or stellate cells, cell-fibres or cellreticulations; or the cells in uniting totally surrender their independence, in which case they form, if they are arranged in lines, elongated elementary parts; or are united by many offsets,-networks; or are fused together upon all sides,-membranes. The two former of these again, according to the kind of modification undergone by the contents of the united cells, appear either as fibres, bundles of fibrilloe and tubes, or as fibre-networks and tubular plexuses. Since all these elementary parts will be spoken of at length afterwards, among the tissues, we may here simply enumerate them as follows. They are :-

a. The cell-fibres and cell-networks. - To these belong a part of the nuclear fibres of authors, the cartilage cells of certain Plagiostome fishes (see Leydig, "Beiträge zur mikr. Anat. u. Entwickel. d. Rochen u. Haie.," Leipzig, 1852), the pigment cells of the lamina fusca, pia mater, and of Batrachian larvæ, the networks of the nerve cells in the brain of the Torpedo (R. Wagner), the fatty substance of the Lepidoptera (H. Meyer, "Zeitschrift für wiss. Zool.," Bd. i. st. 178). 
b. The elastic fibres, fibrous networks, and fibres.

c. The fibres of connective [areolar] tissue, the networks of connective tissue (reticulated connective tissue), and the membranes composed of connective tissue (homogeneous connective tissue).

d. The transversely striated muscular fibres and muscular fibre networks.

e. The nerve-fibres and nerve-fibre networks.

$f$. The capillary plexuses of the blood-vessels and lymphatics.

$g$. The tracher and tracheal plexuses of the invertebrata.

All these higher elementary parts possess essentially the same properties as cells, especially growth in length and thickness, absorption, metamorphoses, and excretion, and to some extent contractility; together with other functions which may perhaps also be demonstrated in cells. Their growth is manifested by the fact, that all, without exception, are much shorter and narrower immediately after their formation than subsequently; their absorptive powers, by the dependence of their functions upon the circulation, by the phenomena of resorption in the lymphatics and blood-vascular capillaries, and by the above-mentioned growth, which can only take place by the reception of substances into their interior. A metamorphic and an excretive power must be assumed to exist in them; it is testified by the well-known peculiar products of decomposition of the muscles, and also by the continual transmission of blood-plasma through the walls of the capillaries. The muscular fibrils possess contractility, and the processes in the nerve-fibres, though very peculiar, and at present not to be defined more nearly, may nevertheless in some respects be compared to the functions of the nervecells.

With regard to the trachex, which are placed here only for completeness' sake, I long since found that their terminations are formed by the coalescence of stellate cells into tubes, in which the original cell-contents either remain or become developed into a spiral fibre; and I published a concise notice of the fact in the year 1849 ("Zeitschrift für wiss. Zool.," Bd. i. p. 215, Anmerkung), a view which has since been confirmed by H. Meyer (Ibid. Bd. i.), and more recently by Leydig (Ibid. Bd. iii. Heft 4.)

Literature of the Elementary Parts. - In addition to Schwann's work quoted above, may be named: Kölliker, "die Lehre von der thierischen Zelle," in Schleiden u. Nägeli's " Zeitschrift für wiss. Botanik.," Heft ii. 1845; Remak, "Ueber extracellulare Entstehung thierischen Zellen und die Vermehrung derselben durch Theilung u. über Entstehung des Bindegewebes u. d. Knorpel," in Müller's "Archiv," 1852, i. (No longer available. Remak assumes quite confidently, what I only indicated, that animal cells have a primordial utricle, without giving any demonstration of 
the fact; he ascribes the multiplication of cells by division to be widely extended through embryonic tissues; finds (what others will not easily succeed in doing) two membranes in the later cleavage-masses, and wrongly, denies altogether the occurrence of free cell-development); also the treatise of Donders, cited below under the head of elastic tissue; and the embryological monographs of Reichert, Bischoff, Vogt, Remak, and myself. Inasmuch also, as the doctrine of the vegetable cell is important for zoologists, I call attention to Schleiden's first treatise ("Abhandlung über die Bildung d. Pflanzenzelle," Müll. "Arch.," 1837); to his "Elements of Botany;" to Nägeli's Essay "Ueber die Pflanzenzelle," in the "Zeitschrift für wissenschaftlich. Botanik," Heft ii.; and to Mohl's monograph upon this subject, in Wagner's "Handwörterbuch" ("Mohl on the Vegetable Cell," translated by A. Henfrey, London, 1852). [To these should be added the important monographs of Schleiden, "Beiträge zur Phytogenesis," in Müller's Archiv, p. 137; of Rosenthal, "De formatione granulosa in nervis aliisque partibus organismi animalis," Vratislaviæ, 1837; of Kramer "Bemerkungen ïber das Zellenleben in der Entwickelung des Froscheies" in Müller's Archiv, 1848 , and the more recent works of Dr. Schacht, on the "Vegetable Cell" (Die "Pflanzenzelle") Berlin, 1852; and of Alex. Brown on "Rejuvenescence" (Verjüngung), Leipzig, 1851.-DAC.]

\section{OF THE TISSUES, ORGANS, AND SYSTEMS.}

$\S 20$. The elementary parts of both the simpler and the higher kinds, are not dispersed irregularly in the body, but are united according to determinate laws, into the so-called tissues and organs. Under the first denomination comes every constant arrangement of the elementary parts always recurring in similar modes in the same parts; under that of an organ, on the other hand, a certain sum of elementary parts of a definite form and function. When several or many organs of a similar or different kind are united into a higher unity, this is called a system.

The tissues are of different kinds, according as structural elements of one kind only occur in them, or as various elements and even organs take part in their formation. We can thence distinguish simple and complex tissues, which, however, cannot be sharply separated from one another, and which may be most fittingly arranged in the following series:-

(a.) Simple tissues.

1. Epidermic tissue.

2. Cartilaginous tissue.

3. Elastic tissue.

4. Connective tissue.*

* [Fibrous tissue, see note, p. 47.-Ev.] 
(b.) Complex tissues.

5. Osseous tissue.

6. Smooth muscular tissue.

7. Transversely striated muscular tissue.

8. Nervous tissue.

9. The tissue of the blood-vascular glands.

10. The tissue of the true glands.

The organs may be divided like the tissues, into simple and complex. To the simple belong:-

(a.) Horny tissue, as the epidermis, the epithelia, hairs, nails, and the lens, which consist solely and wholly of epithelial cells of one kind or another.

(b.) The true cartilages and the elastic cartilages, which in their interior, with few exceptions, consist only of cartilaginous tissue, though externally they possess a vascular and nervous coat, the perichondrium.

(c.) The elastic ligaments, consisting of elastic fibres, with some connective tissue, and containing only at the surface a few vessels and nerves.

(d.) The tendons, ligaments, true fibrous membranes, and fibro-cartilages, containing a preponderance of connective tissue, intermixed with fine elastic fibres, and sometimes cartilage cells in small quantities, and almost entirely devoid of vessels and nerves.

Complex organs are :-

(e.) The smooth muscles and muscular membranes; and-

(f.) The transversely striated muscles and muscular membranes, both of which, besides their contractile elements, are abundantly intermingled with connective tissue, nerves, and blood-vessels.

(g.) The nerves, ganglia, and higher central organs of the nervous system, contain besides gray and white nervous substance, many bloodvessels, and special fibrous investments.

(h.) The vessels are composed of connective and elastic tissue, muscles and epithelium in various proportions, and are provided with vessels and nerves, only in their outermost layers.

(i.) The bones and teeth, which, together with their characteristic tissues, have peculiar soft structures, containing many vessels and nerves, and the former medulla also.

(k.) The blood-vascular glands, composed of a peculiar glandular element, in the form of closed follicles of different kinds, and many bloodressels; with nerves also, and with abundant but generally non-contractile fibrous tissue.

(l.) The true glands; glandular follicles, vesicles or tubes with many vessels, nerves, and investing fibrous tissue.

(m.) The vascular membranes, as the skin, the mucous, serous, and proper vascular membranes, which in a matrix composed of connective 
and elastic tissue, generally contain very numerous blood-vessels and lymphatics, in part also simple glands and nerves, and are invested by special epithelial layers.

(n.) The separate organs of the tractus intestinalis, as the tongue, the oral cavity, the pharynx, the œesophagus, the stomach, and so forth, into the constitution of which, mucous, muscular, and serous membranes, grouped in various ways, enter.

'(o.) The higher organs of sense, into which almost all the tissues and many more simple organs enter.

Lastly, the organs enter into the formation of peculiar systems, of which we may distinguish the following:-

1. The external cutaneous system, consisting of the corium, the epidermis, the horny tissues, and the larger (lacteal gland) and smaller glands of the skin.

2. The osseous system, consisting of the bones, cartilages, ligaments, and articular capsules.

3. The muscular system, consisting of the muscles of the trunk and of the extremities, the tendons, fasciæ, tendinous ligaments, and bursce mucosce.

4. The nervous system, composed of the larger and smaller central organs, the nerves and the higher organs of sense.

5. The vascular system, consisting of the heart, the blood and lymphvessels, and the lymphatic glands.

6. The intestinal system, composed of the intestinal canal, the organs of respiration, with the thymus and thyroid, the salivary glands, the liver, and the spleen.

7. The urinal and sexual systems.

As the separate organs and systems are particularly considered in the special part of this work, it is not necessary to speak at greater length of them here, and it is only requisite to define the tissues somewhat more closely-taking occasion at the same time to refer to some generalities concerning the organs.

§21. Epidermic Tissue.-The morphological character of the epidermis is, that it is wholly constituted by independent cells intimately united together without any visible matrix, which are generally nucleated and in part are true vesicles, while in part they are metamorphosed into solid scales. In its chemical characters this tissue is but little known, though this much has been made out, that its cells contain chiefly an albuminous substance, in part also mucous; and at first all possess easily soluble protein membranes, which however, subsequently, become partially changed into a substance which more or less resists acids and alkalies,-the so-called horn. The physiological import of the epidermic tissue is to serve as a defensive covering to those parts of the organism 
which abound in vessels and nerves, and by the activity of its elements to take part in secretion and absorption. All epidermic tissues are nonyascular, and support themselves from a plasma which is yielded by the deeper-seated vessels. They are very easily regenerated when their superficial portions are removed, and in this case they grow chiefly by the development of new elements in the deeper layers; even when wholly lost they are readily reproduced.

The epidermic tissue takes the following forms:-

1. Corneous tissue. - This always consists of compact masses of cells, which are soft in the neighborhood of their vascular basis, but at a greater distance become more or less solid and hard (corneous), and frequently lose their originally vesicular constitution and nucleus, and become the so-called horny scales. The following organs are formed by this tissue :-

a. The Epidermis; which invests the exterior of the body, and is continuous at the great apertures of the internal cavities with the epithelium. It consists of two tolerably distinct layers; the mucous layer (rete mucosum), with soft, rounded polygonal cells, which, under certain circumstances, contain coloring matter. This layer applies itself accurately to all the inequalities of the corium (which nourishes the epidermis), and externally passes into the polygonal scales of the horny Fig. 12.

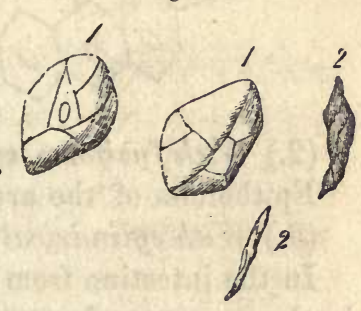
layer.

b. The Nails. - These may be regarded as a modification of the epidermis, whose horny layer has attained a still greater density; and, with its rete mucosum, lies upon a special depressed surface of the cutis, - the bed of the nail; and is partly sunk in a peculiar cleft,- the fold of the nail.

c. The Hairs.-Filiform epidermic* structures, seated upon a vascular papilla, in a peculiar sac, the hair sac, which is a process of the corium, and is lined by a continuation of the epidermis. The structural elements in the region of the papilla are soft and vesicular; the more distant are metamorphosed into three kinds of cells-plates, flat fibres, and more or less rounded irregular cells.

2. Epithelium.-Soft nucleated cells, nowhere densely corneous; rounded, polygonal, fusiform, cylindrical or conical in shape; sometimes possessing cilia, sometimes not, and occurring in one or many layers. Hence we have the following forms :-

FIG. 12.-Plates of the horny layer in man, magnified 350 diameters. 1, without addition, viewed from the surface, one, with a nucleus: 2 , from the side.

* [It is to be questioned if the hairs are truly epidermic structures, vide infra, § Hair.-TRs.] 
a. Epithelium in a single stratum.

(1.) With rounded, polygonal cells (pavement-epithelium in a single layer).

This exists as an investment of the true serous membranes, of most synovial membranes, of the cerebral ventricles (ependyma) of the membrane of Demours, of the back of the iris, and of the inner surface of the choroid (pigment layer), of the capsule of the lens and of the retina, of the internal ear, of the endocardium, of the veins, of many glandular vesicles and canals (racemose glands, kidneys, sudoriparous and ceruminous glands, lungs), and of the ductus interlobulares of the liver.

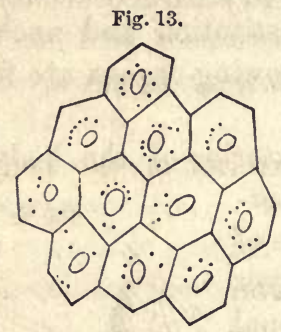

Fig. 14.
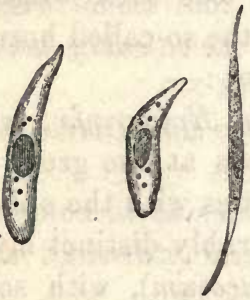

(2.) With fusiform, superficially-united cells (fusiform epithelium). Epithelium of the arteries, and of many veins.

(3.) With cylindrical cells (cylinder-epithelium).

In the intestine from the cardia to the anus, in Lieberkühn's glands, in the excretory ducts of the gastric glands, as well as of all the other glands which open into the intestine; also of the lacteal and lachrymal glands; in the male urethra, the vas deferens, the vesiculoe seminales, the excretory ducts of the prostate, of Cowper's, Bartholini's, and the uterine glands.

Fig. 15.

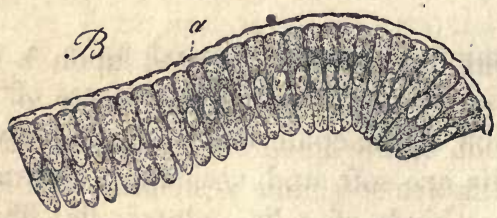

Fig. 16.

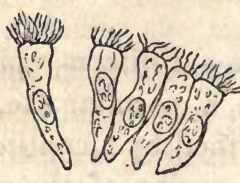

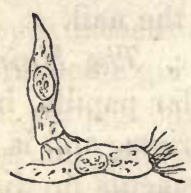

(4.) With cylindrical or conical ciliated cells (simple, ciliated cylinderepithelium).

Epithelium of the finest bronchiæ, of the nasal cavities, of the inner surface of the membrana tympani, of the Eustachian tube, of the uterus,

FIG. 13.-Epidermis of a two months' human embryo, still soft like epithelium; magnified 350 diam.

Fra. 14.-Epithelial cells of the vessels, the longer ones from the arteries, the shorter from the veins.

Frg. 15.-Epithelium of the intestinal villi of the rabbit; magnified 300 diam.

Frg. 16.-Ciliated cells from the finer bronchiæ; magnified 350 diam. 
from the middle of the cervix, up the Fallopian tubes, as far as the outer surface of the fimbrice.

(5.) With rounded ciliated cells (simple ciliated pavement-epithelium). Epithelium of the cerebral cavities of embryos.

b. Epithelium in many layers:-

(1.) With cylindrical or rounded cells below, rounded, polygonal, more or less flattened cells above (laminated pavement-epithelium).

Epithelium of the oral cavity, of the lower half of the pharynx, of the osophagus, of the lachrymal canals, of the conjunctiva, of the tympanic cavity, of the vagina and female urethra, of the urinary bladder, of the ureters and pelves of the kidneys, and of certain synovial membranes.

(2.) With rounded cells below, more elongated ones in the middle, and ciliated conical ones above (laminated ciliary epithelium).

Epithelium of the larynx, trachea, and larger bronchir; of the nasal

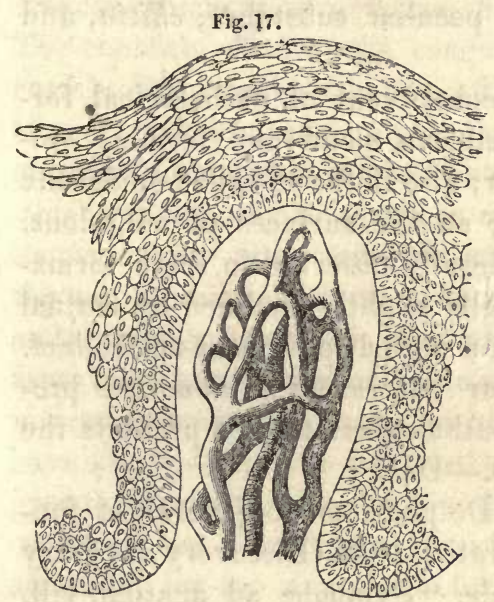

Fig. 18.

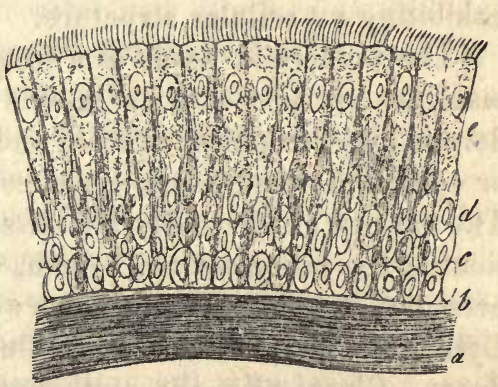

cavity, with the exception of the regio olfactoria; of the lachrymal sac and duct; of the upper half of the pharynx.

Among the epidermic tissues, we may also enumerate the crystalline lens and the enamel.* The former consists of long tubular cells filled with albumen, which, as the study of development teaches, are formed by the metamorphosis of a part of the epidermis. The latter contains prismatic, densely-ossified long fibres, which, in all probability, are also nothing more than excessively elongated epithelial cells of the enamel organ of the embryonic tooth sac.

The epidermic tissue is found through almost the whole animal kingdom, and as regards its elements, it exhibits no very considerable devia-

FrG. 17.-A simple papilla with manifold vessels and epithelium, from the gums of a child; magnified 250 diam.

Fig. 18. - Ciliated epithelium from the trachea of a man; magnified 300 diam.; a. outermost part of the elastic longitudinal fibres; $b$, homogeneous outermost layer of the mucous membranes; $c$, deepest round cells; $d$, median long cells; e, outernost ciliated cells.

* [Vide infra, note $\$$ on the Teeth.] 
tions in animals. One of its kinds, the horny tissue, appears to occur more generally, and to some extent in peculiar forms. To it belong (a), among structures which appertain to the skin: claws, hoofs, horns, spines, plates, and discs, bristles, feathers, and penis-spines. (b), among appendages of the mucous membranes: the horny sheaths of the beaks of birds, of Chelonia, of the Siren and Ornithorhynchus; the horny teeth of the cyclostome fishes; of the Ornithorhynchus, of the gill rays of fishes, and of Batrachian larvæ; the whalebone, the spines and plates of the tongue of Birds, Mammalia, and some Amphibia; the spines of the cesophagus of Chelonia; the jaws of Cephalopoda and other invertebrata; the gastric teeth of many mollusks; the horny plates of the bird's stomach. In all these structures, but often only by the aid of caustic alkalies, horny plates of one kind or another, as in the corneous structures of man, are discoverable. On the other hand, the hard tissues of the Articulata differ not only morphologically, but also chemically from them, consisting as they do of a peculiar substance, chitin, and exhibiting no cellular structure.

[The epidermic tissue is a frequent constituent of pathological, formations: It is met with in skin and mucous membrane, forming epidermic hypertrophies, as corns and warts; also in the so-called epidermic or epithelial tumors, and as a covering on the surfaces of adhesions. The variety of the epidermic tissue generally observed in these formations is the pavement-epithelium, with cells slightly modified by mutual pressure, but not differing otherwise from normal pavement-epithelium. Cylindrical and ciliated epithelium occur but rarely as abnormal products. Chemically, the epithelium of pathological tissues presents the same reactions as that of normal tissues.-DAC.]

Literature.-Purkinje et Valentin, "De phænomeno generali et fundamentali motus vibratorii continui," Vratisl. 1835 (Discovery of ciliary movement in the higher animals); Henle, "Symbolæ ad anatom. vill. int.," Berol. 1837; "On the distribution of the epithelium in the human body," Berlin, 1838; and upon the development of mucus and pus, and their relation to the epidermis (first exact description of the different epidermic cells); Valentin, art. Ciliary Motion, in Wagner's "Handwörterbuch ;" Jäsche, "De telis epithelialibus in specie et de iis vasorum in genere," Dorp. 1847. [Pathologically, the epidermic tissue is considered by Mayer; "Thèse sur les tumeurs epidermiques," Paris, 1846 ; by Bennett, "On Cancerous and Cancroid Growths," Edinb., 1849; by Lebert, in his Monograph, "Du Cancer et du Cancroide de la Peau," Paris, 1851; by Virchow, Wurzb. Verhandl., 1850, and in the recent works of Paget, "Surgical Pathology," London, 1853, and of Wedl, "Grundzüge der Pathologischen Histologie," Wien, 1854.-DAC.]

§ 22. Cartilage.-Cartilage consists of a solid, but elastic, bluish, milkwhite or yellowish substance, which presents two morphological condi- 
tions; appearing, firstly, as a simple parenchyma composed of cells; and secondly, as a cellular tissue, with an intermediate substance or matrix between the elements. The cartilage-cells present little peculiarity in respect of form; they are generally round or elongated, frequently flattened or fusiform, very rarely stellate (in Cuttle-fishes and Sharks, and in enchondroma). Their membrane is ordinarily thick, frequently invested by concentric laminæ; the contents are clear and more fluid, with a single nucleus, and, though not constantly, with one or many fat globules. The interstitial substance is either homogeneous or finely granulated or fibrous, even with clear separable fibres. The chemical characters of cartilage are in some respects but little known. It is ascertained, however, that the cells and the intermediate substance are composed of different substances. The membranes of the cartilage cells, in fact, are not dissolved by boiling, and offer a lengthened resistance to alkalies and acids, peculiarities which distinguish them from the substances which yield gelatine, but approximate them to elastic tissue. The contents of the cells coagulate in water and dilute acids, and are readily dissolved by alkalies. The interstitial substance is, in most cartilages, chondrin, and only in the reticulated cartilages is it a substance closely allied to that of the elastic tissue. Consequently the cartilages, which consist only of cartilage cells, yield no gelatine upon boiling, and its occurrence is no essential character of cartilage. Physiologically, the solidity and elasticity of the cartilages are particularly to be noted, as by these properties it is fitted for its various uses. In growing cartilages the change of material is very energetic; they constantly contain, in certain localities, numerous bloodvessels in peculiar canals; and, as I have demonstrated in the nasal cartilage of the calf, even nerves. Their growth takes place, firstly, by endogenous multiplication of cells, traces of which are always clearly to be observed in perfect cartilages; and secondly, by the deposition between the cells, which originally exist alone in all cartilages, of an interstitial substance from the blood-plasma, which, according to Schwann, at first yields no chondrin even in the true cartilages, and subsequently gradually increases in quantity. In perfect cartilages the nutrition is by no means energetic; and it has, apart from the vessels of the perichondrium which invests many cartilages, and those of the neighboring bone, no particular agent, except in the cartilages (septum of the nose) of a few mammalia, and in the plagiostome fishes, in some of which last, according to Leydig, even in old individuals, vascular canals exist (Raja), in others anastomosing, fusiform, or stellate corpuscles (Sharks). With age, the intermediate substance of certain true cartilages readily becomes fibrous, and very similar in its chemical characters to that of the reticulated cartilage, which demonstrates that these two kinds of cartilage are not widely separated; the true cartilages also not uncommonly ossify, vessels, cartilage, and 
medulla being formed in them at the same time. Cartilage possesses no power of regeneration, nor do wounds in cartilage unite by cartilage; on the other hand, an adventitious development of cartilage is not uncommon.

The different cartilages are:-

a. Cartilage without interstitial substance, or parenchymatous cartilage. To this belong the chorda dorsalis of the embryo and of many adult fish; many fotal cartilages; the cartilages of the gill laminæ of fishes in part; and those of the external ear of many mammalia.

b. Cartilage with interstitial substance.

1. With a more homogeneous, chondro-yielding substance : true cartilage, hyaline cartilage: it is found in the larger cartilages of the respiratory organs, those of the articulations, of the ribs, and of the nose; also in all symphyses and synchondroses immediately in contact with the bones; in the talus ossis cuboidei, and in the so-called ossifying cartilages of the fœtus.

2. With a fibrous interstitial substance, yielding no chondrin, or but very little: reticulated cartilage, yellow cartilage, fibro-cartilage in

Fig. 19.

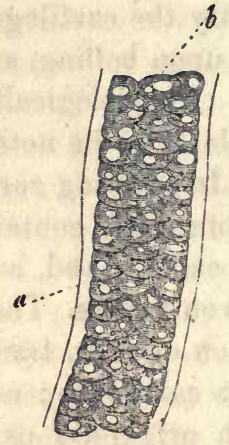

Fig. 20.

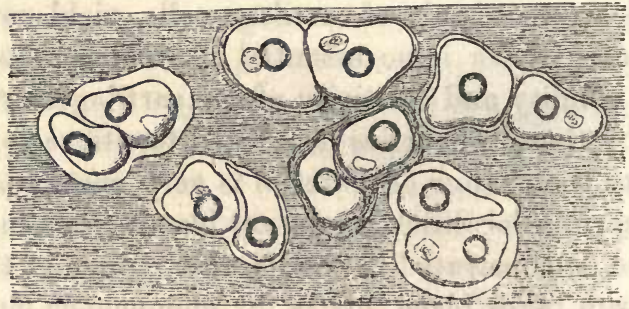

part; epiglottis, cartilagines Santoriniance, Wrisbergiance, cartilage of the ear and of the Eustachian tube; ligamenta intervertebralia in part.

In the Invertebrata many tissues of a similar consistence to cartilage are found, but true cartilage has hitherto been discovered only in the Cuttle-fishes.

[Cartilage is frequently observed in situations where it does not occur normally. This pathological cartilage resembles morphologically and chemically, the ordinary cartilage, and more especially the variety with an interstitial substance.- $\mathrm{D}_{\mathrm{A}} \mathrm{C}$.]

Fia. 19.-Portion of the chorda dorsalis of an embryo sheep, 6 lines long: $a$, sheath ; $b$, cells, with clear vascular spaces.

Fic. 20.-Cartilage cells from the white layer of the cricoid cartilage: from man-magnified 350 diameters. 
Literature.-Meckauer, "De penitiori cartilaginum structurâ Diss.," Vratisl. 1836; J. Müller, in "Poggendorf's Annalen," 1836, p. 293; Rathke, in Froriep's "Notizen," 1847, p. 306; A. Bergmann, "De cartilaginibus Disq. micr.," Mitaviæ, 1850. [Leidy, "On the structure of articular cartilage," American Journal of Med. Sci. April, 1849.]

§ 23. Elastic Tissue.*-The elements of elastic tissue are cylindrical or band-like fibres, with dark contours, which vary in their diameter from immeasurable fineness up to a thickness of 0.003 , and even 0.005 of a line (in animals even to as much as 0.008 of a line), and when they are present in quantity, exhibit a yellowish color. These so-called elastic fibres are, when perfectly formed, quite solid, but may subsequently acquire little cavities in particular spots; and these, in one animal, the Giraffe (Quekett, "Histological Catalogue," i.), are so regular, that the fibres present a pretty transversely striated appearance. The margins of the elastic fibres are in general quite rectilinear, but in some rare cases appear to be notched and even, as Virchow saw them, in newly-developed tissues, beset with a great number of shorter and longer pointed processes. Hitherto the elastic fibres have been separated from the nucleus-fibres: since, however, the latter are dis-

Fig. 21.

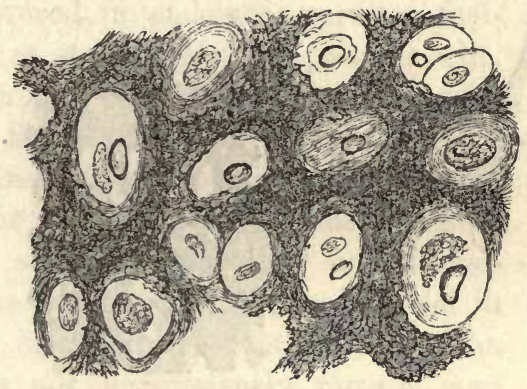

Fig. 22.

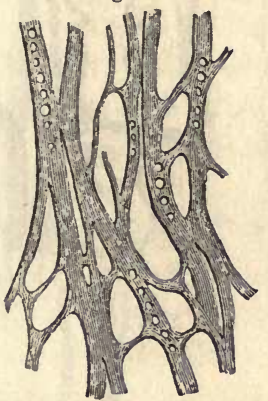

tinguished from the former in nothing but their diameter; furthermore, as all elastic fibres are originally as fine as nucleus-fibres; and since, finally, the latter are not formed of nuclei alone, it will be better wholly to suppress the name of nucleus-fibres, and to divide the elastic fibres simply into finer and coarser. The elastic fibres are found either isolated as longer or shorter fibres, which may be straight or wind spirally round other parts (bundles of connective tissue, nerves), and in this case they are commonly of the finer kind; or by the anastomosis of fibres of different sizes, a so-called fibrous elastic network is formed, which is some-

Fig. 21.-Portion of a human epiglottis; magnified 3 - 0 diameters.

Fia. 22.-Elastic network from the tunica media of the pulmonary artery of a horse, with lacunæ in the fibre; magnified 350 diameters.

* [Yellow fibrous tissues - DaC.] 
times expanded in a membranous form, and sometimes penetrates other tissues to various depths. A modification of this fibrous elastic network is formed by the elastic membranes, in which the fibres are so closely interwoven, that a connected membrane arises, which in the most extreme cases no longer exhibits any indication of its previous nature, and appears as a perfectly homogeneous membrane with smaller gaps (the fenestrated membrane of Henle).

Fig. 23.

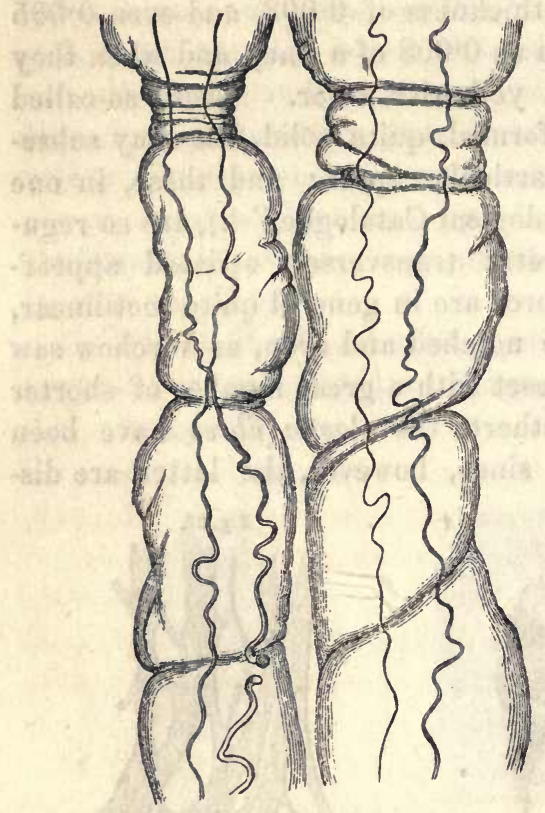

Fig. 24.

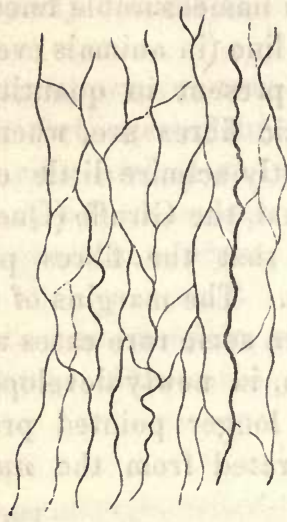

Fig. 25.

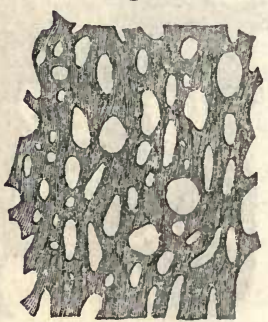

Chemically, elastic tissue presents very decided reactions, but the composition of its substance is not yet exactly known. In cold concentrated acetic acid, the elastic fibres, except that they swell a little, are not affected;* if boiled for a whole day, however, they are gradually dis-

FIG. 23.-Two secondary bundles of connective tissue from the arachnoid of man with coiled and straight (intestinal) fine elastic fibres, and acetic acid added; magnified 350 diameters.

FIG. 24.-Network of fine elastic fibre from the peritoneum of a child; magnified 350 diameters.

FIG. 25.-Elastic membrane from the tunica media of the carotid of the horse; magnified 350 diameters.

* [The difficulty with which acetic acid acts on elastic (yellow fibrous) tissue, presents an important means of distinction between it and the connective (white fibrous) tissue, the fibres of which are readily rendered transparent by its action. In tissues in which the elastic fibres are mixed up, or concealed by the fibres of connective tissue, acetic acid 
solved: nitric acid colors them yellow; Millon's test* for protein tinges them red; whilst sulphuric acid $†$ and sugar have no action (red coloration) upon them. In a moderately diluted solution of potassa, elastic tissue remains, for a long time, unaltered in the cold, except that it swells up and becomes somewhat paler. Heated for a day with it, it becomes converted into a gelatinous mass. In water this tissue does not alter, even after sixty hours' boiling, but changes by boiling for thirty hours in Papin's digester into a brownish substance, smelling like glue, but not gelatinizing, which is precipitated by tannic acid, tincture of iodine, and corrosive sublimate, but not by the other tests for chondrin.

Physiologically, the prominent characteristic of this tissue is its elasticity, in consequence of which it forms a most essential support to the motor organs, and also plays an important part in other situations, e. $g$. in the vocal ligaments. With respect to its development, the supposition of Schwann, that this tissue proceeds from cells, receives increasing support from modern investigations; in fact, in all those organs which subsequently contain elastic tissue, there may be discovered in embryos, peculiar fusiform or stellate, sharply-pointed cells, which by their coalescence produce long fibres or reticulations, in which at first, those localities where the bodies of the cells previously existed may still be recognized as enlargements containing elongated nuclei in their interior. In this condition the fibres not unfrequently remain, and they then form a modification of what were formerly called nucleus-fibres, or every trace of their previous composi-

Fig. 26.

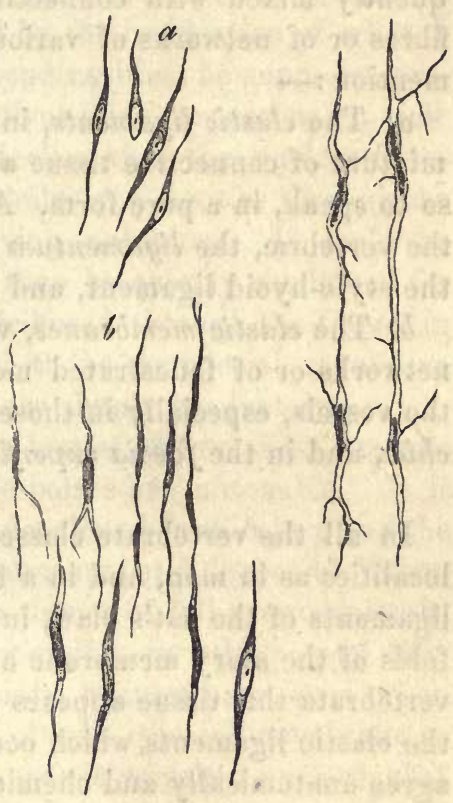

FIG. 26.-Formative cells of the elastic fibres, from the tendo-Achillis: $a$, of a four months' embryo; $b$, from a seven months' fœetus, - a few cells free, with one and two processes, others united by twos and threes.-Magnified 350 diameters.

Fig. 27.-Stellate formative cells of the nucleus-fibres out of the tendo-Achillis of a newborn infant.-Magnified 350 diameters.

by rendering the latter transparent, permits us to investigate the former. In preparations thus treated the subsequent addition of ammonia, by neutralizing the acid, causes the fibres. of the connective tissue to reappear, and those of the elastic tissue to recede.-DaC.]

* [Millon's test (see Compt. Rend. t. 27, p. 42-44), is the most delicate test for protein known. It consists in heating the suspected fluid or tissue from $60^{\circ}$ to $100^{\circ}$ in a solution of one part of mercury, in two parts of nitric acid. If protein be present an intense red: color results, which does not disappear on prolonged boiling.-DaC.]

$\dagger$ [The immediate effect of sulphuric acid is to render the fibres more distinct.-DaC.] 
tion disappears, so that quite homogeneous fibres or fibrous reticulations are produced. These may then either remain through life as fine elastic fibres and networks, or by increasing in thickness they may pass into the coarser form of the tissue. The more homogeneous elastic membranes are nothing but close elastic networks, whose fibres have so much increased in diameter, that only narrow spaces remain between them. The perfect elastic tissue appears to undergo very little change of substance-at least it is, so to speak, non-vascular, even when it occurs in large masses; on the other hand, in those forms which still present indications of the original cells, a certain movement of the juices may still take place. Elastic tissue is not known to be regenerated, but new formations of it are not rare.

The elastic tissue is rarely found in large masses ; but it is very frequently mixed with connective tissue, either in the form of isolated fibres or of networks of various kinds. As true elastic organs we may mention:-

a. The elastic ligaments, in which the tissue, with only a slight admixture of connective tissue and hardly any vessels and nerves, exists, so to speak, in a pure form. As such we have the ligamenta subflava of the vertebræ, the ligamentum nucho, certain ligaments of the larynx, the stylo-hyoid ligament, and the ligamentum suspensorium penis.

b. The elastic membranes, which appear in the form either of fibrous networks or of fenestrated membranes, and are found in the walls of the vessels, especially in those of the arteries, in the trachea and bronchioe, and in the fascia superficialis.

In all the vertebrate classes the elastic tissue is found in the same localities as in man, and in a few particular situations besides, as in the ligaments of the cat's claw, in the alary membrane of mammals, in the folds of the alary membrane and in the lung sacs of birds. In the Invertebrata this tissue appears to be rare, and it is not even certain that the elastic ligaments which occur in them (as, for example, in bivalves), agree anatomically and chemically with the elastic tissues of the higher animals.

Of the different parts belonging to the elastic tissues, the so-called nucleus-fibres of Gerber are almost alone those whose development has been examined. With regard to these, Henle's view, * that they arise by the coalescence of elongated nuclei, was almost universally received, until lately Virchow and Donders nearly contemporaneously brought forward another conception. Both these authors proceed from the investigation of the connective tissue, and show that what in it have been held to be elongated, isolated, or more or less coalesced nuclei, are nothing more than fusiform or stellate cells, with fine processes, which

* [See article "Kernfasern," Maller's Archiv. 1838.-DaC.] 
closely surround a generally elongated nucleus, and are partly united into fibres or networks. With regard to the development of these cells, Virchow deduces from Schwann's observations, and Donders from his own investigations, the result that the well-known fusiform cells in the rudimentary areolar tissue of embryos are nothing more than the formative cells of the so-called nucleus-fibres; to which, then, as a consequence, it is added that the proper connective tissue does not proceed from cells, but is nothing else than fibrillated cytoblastema. Hence both these authors agree in placing connective tissue and cartilage side by side and, in comparing the formative cells of the so-called nucleusfibres, which Virchow calls "connective-tissue corpuscles," (Bindegewebskörperchen), with the cartilage-cells; the interstitial substance of the cartilage, with the fibrous part of the connective tissue. Virchow goes still further, and compares even the bone substance to connective tissue; especially that which is developed by the ossification of what I have called soft blastema, in which the bone cavities, he supposes, proceed from stellate anastomosing "connective-tissue corpuscles," a view which seems chiefly to have led him to declare that the nucleus-fibres are hollow, and form a great system of tubules and cavities through the connective tissue, thus probably subserving nutrition. It could be imagined that the nutritious fluid might thus be quickly conducted for considerable distances, and uniformly distributed through the tissue, in which case the nuclei must be considered to be the special regulative portions of the apparatus, while the cells are simply conductors.

If we submit these different views to the test of observation, it results that much is quite correct, but that some points are untenable. It is true, that the so-called nucleus-fibres are developed from cells; and the fact is, indeed, noted in certain earlier statements and figures of Valentin, Hassall, Quekett, and others. In the tissues of full-grown animals, it is in many localities unquestionably impossible, in others very difficult, to attain to certainty upon these points, because here, even when the nucleus-fibres still present indications of cells, the cell-membrane so closely embraces the elongated and by no means vanished nucleus, that it is often quite out of the question to decide whether we have a cell with two or more slender processes, or a fusiform or stellate nucleus; on the other hand in young animals, in which also Virchow made his first observations, and especially in embryos, it is easy to come to a clear decision upon the matter. In man, I find the tendons, ligaments, and the aponeurosis palmaris and plantaris, to be especially serviceable objects; but in all the places in which elastic tissue is mixed with connective tissue, I was able to follow their development. The observation is most successful in the foetus of three to four months. Here, in all the more solid organs composed of connective tissue,-tendons, ligaments, fascia, corium, - the proper connective-tissue fibrils are already 
quite well developed, while of nuclear fibres, so to speak, there are no traces. Instead of these, however, we find between the often very distinct bundles of connective tissue, a great number of fusiform cells of $0.01-.015$ of a line in length, which in their middle (of $0.002-0.003$ of a line in breadth) enclose an elongated roundish clear nucleus with a nucleolus, which completely fills them, and are prolonged at their ends into fine dark threads. If we trace these cells, among which are always scattered many round and elongated cells out of which they are formed, and in older embryos a few stellate ones, with from 3 to 5 processes, it is found that they gradually become longer and narrower, and from the sixth month begin to coalesce with one another into elongated fibres or networks; up to a late period, however (even 7 to 8 months), these formative cells of the elastic tissue may be easily isolated in abundance from all forms of connective tissue, either singly or combined by twos and threes. In the foetus at birth this can no longer be done; but here the complete nucleus-fibres, at least in the more solid forms of connective tissue, still clearly exhibit their composition out of fusiform and stellate cells with nuclei; which, as we have already seen, is occasionally in some localities even the case in the adult.

What holds good of the nucleus-fibres may be asserted also of the elastic fibres, which are not further treated of by Donders and Virchow. Valentin (Wagner's "Handw. d. Phys.," I. p. 668) found that the elastic fibres of the ligamentum nuchoe of the calf are considerably finer than those of the ox, and I stated ("Zeitschrift für Wiss. Zool.," I. p. 77, Anm.) that all the thick elastic fibres of the adult have at one time been common nucleus-fibres. In fact, we find in the new-born child not a single true elastic fibre, since even those of the ligt. nuchoe, of the ligt. flava, and of the aorta, when largest, do not measure more than $0.0008-\cdot 001$ of a line. This circumstance alone, might, if we take into account the close resemblance of the elastic and so-called nucleus-fibres in other respects, be considered as a demonstration that the former are also developed out of cells, but we have in addition direct evidence that this is their mode of development

In the aorta, in the ligt. nucho, and in the fascia superficialis abdominis of human embryos of the fourth and fifth months, we find the same short fusiform cells as in the common connective tissue; and their coalescence into originally finer fibres, though perhaps not quite so readily demonstrable as in the former localities, may yet be made out with certainty, so that the agreement in their genesis of the finer with the coarser elastic fibres, may be considered to be established.

Not, however, to the same extent, as with regard to the genesis of the finer elastic fibres, can I agree with the authors mentioned in other points. In the first place, concerning the physiological import of the so-called nucleus-fibres, I grant to Virchow, that even in the adult, 
in some places, they appear to retain more their original character of a system of canals; yet I can by no means allow, that these nucleusfibres are to be regarded as a system of tubules subserving nutrition. In my opinion, all fine elastic fibres, which no longer present any trace of the original cell, $i$.e. those of the areolar connective tissue of the corium, fascix, of the perimysium, of the periosteum, of the dura mater, of the serous membranes, of the walls of the vessels, and of mucous membranes, are solid fibres, and only of service to the organism so far as they are elastic.

A relation to nutrition can only be supposed of those elastic elements of the tendons, ligaments, and of the cornea, which present conditions more nearly embryonic; but even with respect to these it does not appear so evident that it can be decidedly affirmed. In the tendons and ligaments, for instance, it is plain that a part only of the elastic elements are not quite fully developed, so that possibly cavities may still exist in them; while the rest, much more considerable, are as completely developed as elsewhere, and offer no trace of a cavity. If, now, it were assumed that the former have a determinate relation to the conduction of the nutritive fluid, it would yet remain unexplained why, in certain regions, these organs were more favored than in others. If it be further considered, that in the tendons and ligaments, as in the connective tissue in general, vegetative molecular changes and nutrition are assuredly at their lowest stage,-furthermore, that the arrangement of the nucleus-fibres (their more longitudinal course, the want of anastomosis of the nucleus-fibres of the different secondary bundles in tendons) appears very little fitted to conduct nutritious fluids from the surfaces of these organs, where only the vessels are found, into their interior,-there will appear no great necessity for entering further into this hypothesis. For the cornea alone, where the elastic tissue remains in a quite embryonic stage, should I be inclined to adopt Virchow's hypothesis; and, as respects the other tissues, I can only grant that, when the elastic elements are in such an imperfectly developed condition that they still contain canals for greater or less distances, they may have a share in the distribution of the nutritive fluid which natu-. rally interpenetrates these organs, and, therefore, in their nutrition, but that this must rather be regarded as a secondary function, and will not justify their approximation to the fine canals of the teeth and bones, which exist specially for the purpose of nutrition.

Such a function might much rather be ascribed to the undeveloped nucleus-fibres and their formative cells in the immature (pathological or normal) connective tissue; for here, at least, the anatomical and physiological relations of the tissue are not opposed to such an assumption; it would amount to little more, however, than what may be asserted of every undeveloped fibrous tissue. 
A second and much more important point in which I differ from Donders and Virchow is the general mode of looking at the connective tissue. Both these writers hold that it is not composed of cells, but is developed by the fibrillation of a homogeneous cytoblastema; and they believe that all the fusiform embryonic cells, which since Schwann's time have been regarded as its formative cells, belong not to it, but to the elastic tissue. I can by no means admit such a view, and it seems to me to be comprehensible, only when we recollect that these authors arrived at it more upon theoretical grounds, than by direct observation. With Virchow, a passage in Schwann appears to have been conclusive, where he describes the embryonic connective tissue as a gelatinous homogeneous mass, which dissolves upon boiling, and contains cells distributed through it which are not affected by the boiling. Virchow does not hesitate to extend this to all connective tissue, and to assume that the substance soluble in water answers to the subsequently fibrous connective tissue, while the insoluble cells are the formative cells of the socalled nucleus-fibres. Here, however, he has omitted to notice that Schwann speaks only of a determinate form of tissue, the lax or areolated, and describes in a totally different manner the formation of the more solid connective tissue, e. $g$. of a tendon. In this case we find, in direct contrast to the former, no trace of cytoblastema, which can in no way be directly observed, the tendon consisting throughout of fibre-cells, either isolated or united into bundles of connective tissue.

To observe this, however, the examination must be made at a very early period, since, as Schwann has justly remarked, the elements of the fibrous tissue are very early developed; a circumstance from neglecting to observe which, it seems that Donders has been led to adopt the same view as Virchow. For my own part I have found Schwann's statements confirmed in all essential points, with the single exception that he was unacquainted with the formative cells of the elastic fibres, and confounds them with those of the connective tissue. My observations upon these points are to be found in the following section. Hence I cannot admit that cartilage and connective tissue are nearly allied, inasmuch as the fundamental substances of both, even if chemically agreeing, yet, genetically, are very different.

Literature.-A. Eulenberg, "de tela elastica," 1836; Virchow, "die Identität von Knochen, Krorpel und Bindegewebskörperchen, sowie ueber Schleimgewebe," in the "Verhandlungen der Phys. Med. Gesellschaft in Würzburg," Bd. II. 1851, p. 150; and "Weitere Beiträge z. Kenntniss d. Structur der Gewebe der Bindesubstanz." Ebend. II. p. 314 ; Donders in the "Nederlansch Lancet.," 1851, July and August; and in the "Zeitschrift für wissen. Zool.," Bd. III. p. 348; Kölliker, "Ueber die Entwicklung der sogenannten Kernfasern, der elastichen Fasern und des Bindegewebes;" in "Verh. d. Phys. Med. Ges. in Würzburg," Bd. III. H. 1. 
§24. Connective Tissue [fibrous and areolar tissue].-The elementary parts which are found in connective tissue may be divided into the essential, never-failing components, and those which are met with only in certain localities. To the former belongs the fasciculated as well as the more homogeneous connective tissue; to the latter, elastic fibres in their different forms and conditions of development, fat cells, cartilage cells, and pigment cells of different kinds. Besides these, connective tissue contains also no inconsiderable quantity of a gelatinous intermediate substance. The bundles of the connective tissue are, among the essential elements, those which occur most frequently; each of them consists of a certain number of very fine fibrils, the connective fibrils, which are distinguished from their nearest allies, the finest elastic fibres and muscular fibrils, by their smaller diameter $(0.0003-0.0005$ of a line), their pale color, their homogeneous appearance, and the complete absence of striation. They are united by means of a small quantity of a clear connecting substance, and thus form the bundles in question, which in many respects re-

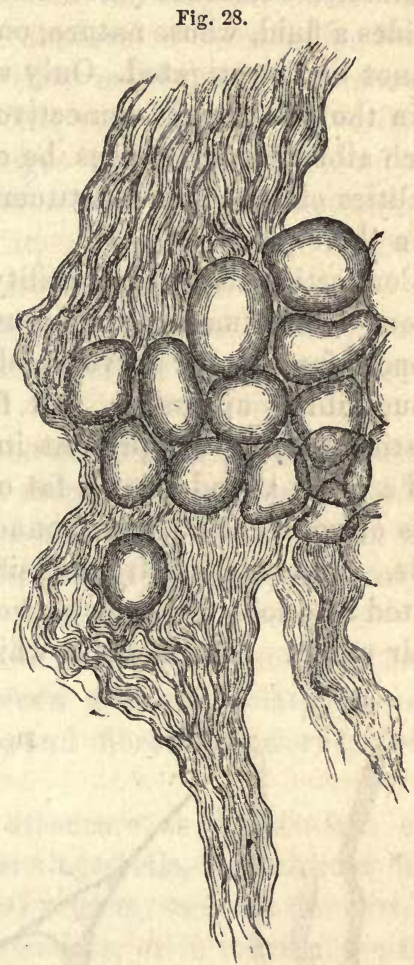
semble those of the transversely striated muscles, but differ from them in the absence of any special investment comparable to the sarcolemma, and in their smaller mean diameter $(0.004-0.005$ of a line.) They are either long, slightly wavy cords, of uniform thickness throughout, which are not directly connected together, but arranged in different ways near and above one another, forming great lamellæ and bundles; or they coalesce like the elastic networks into meshes, and thus form what I have called the reticulative connective tissue. In rare cases the bundles appear not to be composed of fibrils, but are more homogeneous, as in the neurilemma, where they are known as Remak's fibres. Besides this form of connective tissue, there exists a second, rarer kind, in which neither bundles nor fibrils can be clearly distinguished, but only a membranous or more or less solid, finely granulated, or slightly striated, even perfectly homogeneous, clear tissue; homogeneous (or Reichert's) connective tissue. The other elements which occur in connective tissue pre-

Fig. 28.-Lax connective tissue with fat-cells from man; magnified 350 diam. 
sent nothing remarkable, and will be more particularly treated of in their proper places in the special part.

The chemical relations of connective tissues are well known: proper connective substance when boiled yields common gelatine, and contains besides a fluid, whose nature, on account of its generally minute quantity, cannot be investigated. Only where it exists in considerable proportion, as in the gelatinous connective tissue of embryos, can the presence of much albumen and mucus be easily demonstrated in it. The chemical qualities of the other constituents of the connective tissue will be spoken of in their place.

Connective tissue is of utility to the organism according to its composition,--sometimes as a solid unyielding substance; sometimes as a soft support for vessels, nerves, and glands; sometimes, finally, as a yielding tissue, filling up spaces, and facilitating changes of position. Where elastic elements are present in it in great quantities, its nature alters; and a great abundance of fat or cartilage cells gives it an unusual softness or resistance. The connective tissue is invariably developed from cells, and, in fact, from fusiform or stellate vesicles, which become united into long fibres or networks, and often break up into fibrils before their union. The mode in which this takes place is not yet quite made

Fig. 29.

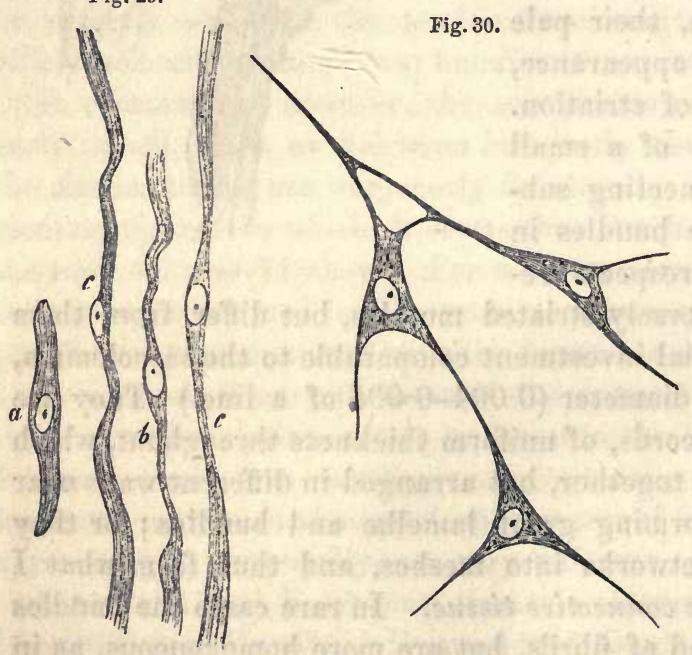
out, but it is most probable that the cells, as they elongate, change with their membrane and contents, into a homogeneous softish mass, which subsequently breaks up into a bundle of fine fibrils and some intermediate substance. The development of the homogeneous connective tissue has as yet been little investigated, but it would seem, like the other, to proceed from a fusion of rounded or elongated cells, which

are perhaps united by an intermediate substance, in which the metamorphic process has only gone so far as the development of a homogeneous mass, but has not attained the stage of fibrillation. The bundles

Frg. 29.-Formative cells of the connective tissues from the skin of the trunk in a sheep's embryo, 7 lines long: $a$, cell without any indication of fibrils; $b$, with commencing; $c$, with distinct fibrils.-Magnified 350 diameters.

FIG. 30.-Three formative cells of the areolated connective tissue from the allantois of a sheep's embryo, 7 lines long.-Magnified 350 diameters. 
of the connective tissue, when once formed, grow in length and thickness like the elastic fibres, until they have attained the size which they possess in the adult; however, there arise subsequently, in many places, additional elements, which are combined with the original ones. The perfect connective tissue, when unmixed, is almost non-vascular, and with regard to nutrition, it is certainly very low in the scale, whence it undergoes hardly any morbid changes. The vascular connective tissue is an exception to this rule, but the changes in this case depend not upon any.peculiarity in the connective tissue itself, but are determined by the vessels, fat-cells, \&c., contained in it. The bundles of fibrils of the connective tissue and the elastic fibres stand at the bottom of the series of the higher elementary parts, and thence most readily adapt themselves to the regeneration of lost substance, or to the increase of parts which already exist.

The union of the different elements of the connective tissue is effected in many ways. but the following forms are most worthy of distinction:-

1. Solid connective tissue (formed connective tissue, Henle). In this the elements are intimately united, and in such a manner, that simple organs of well-marked form proceed from them. To this belong:-

a. The tendons and ligaments, with parallel bundles, united by loose connective tissue into larger cords, between which a relatively very small number only of fine elastic fibres, and fibrous networks, penetrate.

b. The fibro-cartilages have the same structure as the tendons and ligaments, but with numerous scattered cartilage cells, and without finer elastic fibres. They exist either as special organs, such as the cartilagines interarticulares and the cotyloid ligaments, or in particular parts of other organs composed of connective tissue, especially in the tendons, the tendinous sheaths, and the ligaments.

$c$. The fibrous membranes are distinguished from $a$, only by the frequent interweaving of the bundles, and generally by the more considerable number of the elastic fibres. Here may be enumerated:-

1. The muscular fascioe, which have more the structure of tendons.

2. The periosteal membranes and the perichondrial membranes, containing sometimes a great number of elastic elements.

3. The white dense tunics of many soft organs, as the dura mater, the neurilemma, the sclerotic and cornea, the fibrous coat of the spleen and kidneys, the tunica albuginea of the ovaries and testes, penis, and clitoris. In the last-mentioned parts, and in the spleen, these coats, which consist of a solid connective tissue and numerous fine elastic fibres, are continued into the interior, where, mixed to some extent with smooth muscles, they constitute a more or less complete framework, which appears in the form of partitions, or of a stroma, or of a trabecular network. In the cornea we find a modification, inasmuch as the connective 
tissue is transparent, contains fine elastic tissue in a more embryonic state, and when boiled in water yields chondrin and not gelatine.

d. The serous membranes consist of a connective tissue, rich in fine elastic fibres, whose bundles anastomose, or are interwoven in different modes; and sometimes also, especially at the surface of these membranes, appear more homogeneous. The serous membranes, which never possess glands, and upon the whole but few vessels and nerves, line the cavities which contain the viscera, and present an inner surface, which is smooth and shining from the presence of an epithelial investment. 'They do not necessarily form closed sacs, as was formerly believed, but may have apertures in certain localities (abdominal apertures of the Fallopian tubes), or may be wholly wanting, as upon the articular cartilages; or the areolar foundation may be absent, as in the so-called external lamina of the arachnoidea cerebri. To these membranes belong I., the true serous membranes, as the arachnoidea, the pleura, the pericardium, the peritoneum, and the tunica vaginalis propria, which all, normally, secrete only a minute quantity of serous fluid; and 2 , the synovial membranes or capsules of the joints, bursce mucosoe, and the tendinous sheaths, which afford a viscid yellow secretion,-the synovia-containing albumen and mucus.

$e$. The corium consists of a dense network of bundles of connective tissue, which at the surface, and in the papilla, gives place to an indistinctly fibrillated, in part even more homogeneous tissue, and contains a great quantity of finer and coarser elastic networks, as well as very numerous vessels and nerves.

The corium supports the papillæ upon its outer surface, and is here covered by the epidermis, in connection with which it forms the external skin; from the deeper parts it is separated by a soft tissue, generally very rich in fat, the subcutaneous connective tissue, adipose membrane, or panniculus adiposus.

$f$. The mucous membranes essentially consist of a very vascular basis of connective tissue, well supplied with nerves,- the proper mucous membrane-of an epithelial layer covering it, and of a submucous areolar tissue, which in the intestine is also called the tunica nervea. The former is of the same structure as the corium, only softer, and not unfrequently poor in elastic tissue. The mucous membranes are distinguished from the serous, in general by their greater vascularity, their more considerable thickness, their numerous glands, and the mucous secretion, which may be especially ascribed to their soft epithelium; though there are mucous membranes which are as delicate and glandless as serous membranes; and, on the other hand, the synovial capsules may approximate the mucous membranes in their vascularity and the nature of their secretion. The mucous membranes and the external skin are analogous in all their principal components, whence the transitions 
between the two, such as exist upon the lips, eyelids, and elsewhere, are not surprising. To the mucous membranes belong the innermost coat of the tractus intestinalis; the lining of the nasal passages, and of their secondary cavities; the Eustachian tube, the tympanum and mastoid cells, and the conjunctiva. Among the glands, all the larger, present in their excretory ducts, a distinct mucous membrane, as the lungs from the glottis to the finest bronchrce; the liver in the larger gall-ducts and in the gall-bladder; the pancreas in the ductus pancreaticus; the urinary and sexual organs; in the urethra, bladder, ureters, pelvis of the kidneys, vagina, uterus, and oviducts ; and in the ducts and follicles of the mammary gland; in the seminal vesicles, and in the vas deferens. In all these glands the coats of the mucous membrane pass immediately into the walls of the glandular tubes and vesicles, which might thus be regarded as composed of a more delicate mucous membrane. The same might be said of the smaller glands, as those of the intestine, which are directly connected with the larger mucous expansions, only in that case the smaller glands of the skin must be regarded as attenuated processes of it. Inasmuch as both physiology and development support this view, it would seem to be at any rate justifiable; yet every one is free, notwithstanding, to look more to the differences which certainly do exist between the parts in question, and to consider them as distinct structures.

g. The membranes of the veins, lymphatics, the adventitious coat of the arteries, and the endocardium, consist of a loose connective tissue not altogether dissimilar to that of the fibrous membranes, and of finer or coarser elastic fibrous networks, with which in the veins smooth muscles are also partly mixed.

h. The so-called vascular membranes (tunicce vasculosce), to which belong the pia mater, with the plexus choroidei, the choroid coat and the iris, all contain very numerous vessels, which, however, appear to have less reference to the membranes themselves than to the nutrition of other organs. Supporting these vessels we have either a common connective tissue, in which there are no elastic fibres (iris, pia mater), with parallel, matted, and anastomosing bundles, or a homogeneous connective tissue (plexus choroidei, choroidea), to which, as in the choroid, peculiar elements, namely, anastomosing cells, generally filled with more or less pigment, may be added.

$i$. The homogeneous connective tissue.-In many organs we find membranes whose chemical nature agrees with that of connective tissue, but which neither contain distinct bundles nor fibres, and appear to be more homogeneous. Such is the homogeneous tissue which often invests the bundles of the arachnoid singly, or in a number together; the coats of the Malpighian corpuscles of the spleen, and of the glandular follicles of the intestine (tonsils, lingual-follicles, the solitary and Peyerian 
glands), certain of the so-called membrance proprice of the glands appear to come under this head also; yet, since some of them do not belong here, and consist of a very different substance from connective tissue, as, for example, that of the kidneys, and since we have no thorough investigation of these structures, for the present nothing decided can be said upon the subject.

k. Loose or areolated connective tissue* ("amorphous connective tissue" of Henle), consists of a soft meshwork of reticulated, or variously interwoven bundles of connective tissue, which in larger or smaller quantity constitute a filling up and uniting mass between the organs and their parts, and appear under two forms :-

1. As adipose tissue, when numerous fat-cells are contained in the meshes of an areolated tissue which is usually very poor in elastic fibres.

2. As common lax connective tissue, when the latter are few or wanting. The adipose tissue occurs principally in the skin forming the panniculus adiposus; in the larger cylindrical bones, as yellow bonemedulla; in the orbit; around the kidneys; in the mesentery and the omentum; around the spinal marrow; in the nerves and vessels, and in muscles. The areolated connective tissue is widely distributed between the separate organs and the viscera of the neck, thorax, abdomen, and pelvis, and everywhere along the course of the vessels and nerves, and in the interior of the muscles, nerves, and glands.

The connective tissue is found in all the four classes of the vertebrata in about the same condition as in man; while, on the other hand, in the invertebrata it is very rare, and when present is more homogeneous, or consists of isolated cells and intermediate substance, rarely more fibrous, as in Cephalopoda, in the mantle of bivalves, in the peduncle of the Lingulæ, and of the Cirripeds. Fat-cells also do not occur among the lower animals to the same extent as among the higher. The firm connective tissue is here replaced by a chitinous substance, or by one consisting of cellulose, and by calcareous or horny tissues.

Opinions are still divided as to the structure and development of the connective tissue. Whilst the majority ascribe a distinctly fibrous structure to it, and suppose it to consist of bundles, and these again of fibrils, Reichert considers this tissue to be more homogeneous, and regards the fibrillation partly as artificial, partly as the expression of a folding, a view to which Bidder and Virchow are also inclined. For my own part, I find a certain amount of truth in Reichert's conception, insomuch as it is not to be denied that there also exists a non-fibrillated, more homogeneous connective tissue, which had previously been little investigated; but I am nevertheless of opinion, that, as applied to the

* [This second variety, "loose or areolated connective tissue," is generally described as areolar tissue, or under the faulty name of cellular tissue.-DaC.] 
great mass of the organs composed of connective tissue, it is incorrect. The possibility of making out fibrils in delicate membranes, even without preparation, the ease in which these may be isolated in tendons and ligaments, and lastly, the circumstance that the fibrils may be demonstrated upon transverse sections of the tendons, and of the more solid connective tissue in general, are for me sufficient reasons for retaining the old view.

With respect to the development of the connective tissue, I distinguish two types which correspond with its two principal forms, the solid and the areolated. The former is developed out of masses of cells without any demonstrable matrix, by the elongation of the cells, their breaking up into fibrils, and their coalescence. This is most obvious in the tendons and ligaments, which, as observations upon Batrachian larve and upon mammalian embryos show, at first consist entirely of common, rounded, formative cells, which about the same time as the transversely striated muscles are formed (in mammalia in the second month), become fusiform. The further development demonstrates (what had escaped Schwann) that only one portion of these fusiform cells, and in fact cells which are remarkable for their size and paler contours, become bundles of connective tissue, while the others, which Schwann in part depicts rightly (Tab. III. fig. 11 ; the smallest cell, fig. 6, from connective tissue, the cell $b$, and the lowest cell upon the right side), remain for a time as fusiform elements, and only subsequently become fused into elastic fibres. There arises, at last, out of the cells alone, with no distinguishable matrix, a compact tissue composed of two chemically quite distinct fibres. The areolated connective tissue differs from the former in the circumstance that, if not from the beginning yet from the time at which the cells become elongated, an abundant gelatinous intermediate substance is developed between them, which does not yield gelatine, and never becomes converted into it, but contains albumen and a substance similar to mucus; Schwann, indeed, found a substance resembling pyin, in this tissue. Although all embryologists know that the areolated connective tissue is at first of a gelatinous consistence, as for example, under the skin, in the neck, in the omentum, behind the peritoneum, in the orbit, and in the bones, no one has yet drawn attention to the general occurrence of that intermediate substance which was observed by Schwann in a single locality. I originally became acquainted with this tissue between the chorion and amnion, and at first paid more attention to its reticulated anastomosing cells. Subsequently when I examined it more closely in the enamel organ of the embryonic tooth sac, I paid attention to the peculiar intermediate substance, and at the same time Virchow described this tissue from the umbilical cord, where the gelatinous tissue of Wharton entirely consists of it. Virchow believed that it ought to be distinguished from connective tissue, and 
proposed the denomination of mucous tissue (tissu muqueux) for it. I considered it from the first to be connective tissue, and I now feel the more inclined to remain of this opinion, because I find that every description of the areolated connective tissue of embryos originally commences under this form, and therefore the circumstance that the tissue in the umbilical cord never arrives at perfection, cannot determine its nature.

The mode in which the gelatiniform connective tissue is developed is this: one portion of the cells contained in the gelatinous basis changes into connective tissue by becoming fusiform, and breaking up into common or reticulated anastomosing connective tissue, which, however, as Schwann has already stated, at first yields no gelatine. In this manner a closer or denser network arises, in the interspaces of which the intermediate substance or matrix, and a remainder of the previous formative cells, are contained. In the further course of development, new cells proceed from the matrix, which hereby diminishes by degrees in quantity, and at the same time the original network consolidates, fresh cells being added to it, a part of which also become elastic fibres and vessels. If subsequently the areolated connective tissue includes no adipose cells, the gelatinous tissue ends by completely disappearing, and nothing remains but a loose fibrous tissue, containing at most somewhat less fluid, and loose cells in its meshes; if, on the other hand, it becomes converted into an adipose tissue, the spaces remain, and a great part of the cells which have arisen at the expense of the gelatinous substance, subsequently pass, by the development of fat in their interior, into fatcells.

In the gelatinous tissue of Wharton, between the chorion and amnion, and in part of the enamel organ, the areolated connective tissue remains more in its fotal condition of a gelatinous tissue, yet there exists no natural line of demarcation from ordinary connective tissue, so much the less, since in the gelatinous substance of Wharton, in older embryos even fibrils are quite evident, and in the enamel organ the passage of a part of the gelatinous tissue into common connective tissue is demonstrable.

So much for the two types of development of the connective tissue. We have yet to state how the bundles become chemically and morphologically what they are. In the first place, I may observe that the formative cells of the connective tissue are not originally distinguishable from the other formative cells of the embryo, do not dissolve by boiling in water, and therefore contain no gelatine. Even when the cells have evidently become fusiform, and have already coalesced into bundles and networks, they still, as Schwann has already stated, yield no gelatine. Therefore, in this case, the change of the cells into a collagenous substance, goes on as slowly as in the matrix of the cartilages, which, 
according to Schwann, also, at first, yields no gelatine, and therefore it is no objection to the above view of the nature of Wharton's gelatinous tissue, that it yields no gelatine on boiling, as Scherer has found. How the collagenous matter is formed out of cells, whether the contents only, or the membrane also, takes part therein, it is very difficult to say; in any case, from what we know of the contents of embryonic cells, it can hardly be any but a protein substance which yields the gelatine, and, from what takes place in the ossification of the cartilage cells, it seems very probable that the cell-membranes and contents together become metamorphosed into a collagenous substance.

The morphological change which the formative cells of the connective tissue undergo, in the course of their passage into bundles of fibrils, is very probably this, that after their membranes and contents are fused into a homogeneous semi-solid mass, they then secondarily break up into fibrils; the latter process taking place in the same manner as we see it occur in the contents of the animal muscular fibres. Herewith, as a rule, the nuclei of the cells eventually disappear, or if they remain, as we see occasionally in connective tissue, still they never become changed into the so-called nucleus fibres.

Though in physiological connective tissue, development from cells must be most decidedly affirmed, it does not therefore follow that a substance which chemically and morphologically closely resembles connective tissue, may not arise in a different manner. We know, in fact, that the collagenous basis of cartilage, when it breaks up into fibres, becomes deceptively similar to connective tissue, and furthermore, that fibrous exudations may become changed into a fibrous substance which is scarcely, perhaps not at all, to be distinguished from genuine connective tissue. There also exists, however, a pathological true connective tissue in cicatrices of all kinds, and perhaps elsewhere, which is developed from cells; and for my own part, therefore, I am opposed to the classing together of all connective tissues. We must in our classifications not only distinguish similarity or identity in structure and chemical composition, but embrace all the conditions, and especially the genesis; and thence we must distinguish both the collagenous fibrous cartilage and the collagenous organized fibrine, from true connective tissue,-just as we separate the true elastic fibre, from the chemically and morphologically, very similar fibres of the reticulated cartilages and from certain forms of metamorphosed fibrine. On the other hand, the connective tissue which has not been developed from cells, may justly and properly be arranged with cartilage.*

* [The arguments brought forward by Professor Kolliker in support of his views with regard to the nature and mode of development of connective tissue, appear to us not to preponderate against those of Reichert, Virchow, and Remak, and to be opposed to 


\section{Literature.-C. B. Reichert, "Vergleichende Beobachtungen über das Bindegewebe und die verwandten Gebilde," Dorpat, 1845; Luschka,}

our own observations, which agree in all essential points with those of the last-named authors.

There are two questions in dispute. The first, the structure of the connective tissue; the second, the homology of its various constituents with those of other tissues, and of cells in general.

With respect to the first question, it is admitted on all hands that ordinary connective tissue (e. g. of the tendons) is composed of two elements: $a$, a network of elastic tissue, which is not acted upon by cold acetic acid; $b$, a substance which is swollen up by acetic acid, and has a more or less fibrillated appearance, contained in the meshes of the elastic tissue. Now it has been demonstrated by Virchow, and the fact is admitted by both Kölliker (supra) and Reichert (Zur Strêitfrage über die Gebilde der Binde-substanz, über die Spiralfaser, \&c., Muller's "Archiv," 1852), that the elastic fibres are originally cells, and therefore that they are homologous with the cartilage-cell, $i$. e. the cartilage-cavity with its wall plus the cartilage-corpuscle or nucleus. That this is the case is very evident upon examining in a young animal (e.g. kitten) the insertion of the tendo-Achillis into the cartilaginous extremity of the os calcis. It is here easy enough to see that the oval or rounded cells of the true cartilage pass in the rnost gradual manner into the elongated elastic fibres of the true tendon. The cells retain their cavities for a considerable time, but eventually the nuclei and the thin layer of substance which immediately forms the wall of the cavity, become fused into one mass, and altered in chemical composition. A like alteration affects the matrix in various irregular directions, so that the delicate elastic connecting fibres are formed, and constitute a network through the whole tendon. These connecting fibres are often branched, and even appear fibrillated at the ends, especially if torn out from their connection with one another, and in this condition they exactly resemble the bodies figured by Professor Kolliker as the "fusiform formative cells" (Fig. 29.) That they have nothing to do with the development of the "fibrillated" collagenous substance is, however, obvious, from this very simple circumstance,- that the latter lies between them, and in part replaces the rest of the matrix of the cartilage, into which it can be directly traced. It will not be said in this case, that the "fibrillated" tissue of the tendo-Achillis is only "deceptively similar" to true connective tissue-and yet the transition of true cartilage into true connective tissue, is not less certainly demonstrable in the intervertebral cartilages, \&c.

As Reichert, then, long since indicated, in illustrating his "law of continuity" (a law whose full importance, it may be observed, has yet to be developed), and as he and Virchow have since demonstrated, the elastic element of fully-formed connective tissue represents the cartilage-cells, while the collagenous element represents the matrix of the cartilage, and is not developed from distinct cells.

With regard to the structure of the latter element, Reichert, in his last communication, after considering Kolliker's arguments, denies the truth of his statement, that the ends of the fibrils may be seen in transverse sections of the tendons ( $\$$ Tendon, infra,) and retains his opinion that it is not truly fibrillated in the uninjured state, but that it is simply plaited. Some remarkable observations upon the behavior of the "connective fibril bundles" with acids and alkalies, to which Reichert first drew attention in 1846, and which have been since extended by Dr. Paulsen (Bericht., Müller's "Archiv," 1849), are, as the former points out, of the greatest importance in determining the nature of this tissue, and remind one somewhat of the equally puzzling structure of the starch-corpuscle. Dr. Paulsen states, that if a piece of tendon be kept for twenty-four hours in a solution of caustic potash of ten per cent. strength, it changes into a viscid hyaline mass, so transparent that it can hardly be distinguished from the surrounding fluid. This substance can be torn with equal ease in any direction, and no fibrous structure can in any way be detected in it. Under the microscope the mass is quite transparent, and shows no trace of the well-known striation. However, the connective tissue is at this time by no means dissolved, nor is its texture destroyed. If the potass be removed by acetic acid, and this, if it be in excess, by washing, the original 


\section{"Die Structur der serösen Häute." Besides which, consult the works of Virchow, Donders, Remak, and myself, cited above.}

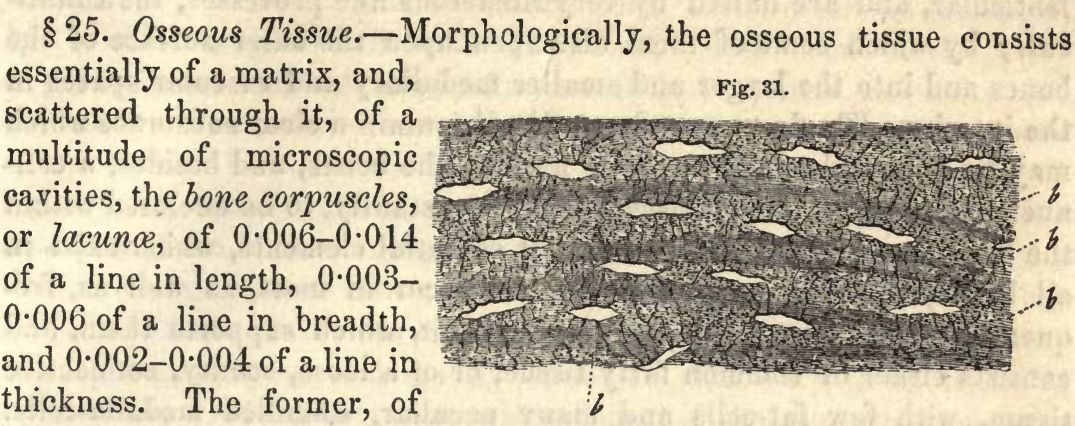
a white color, is sometimes more homogeneous, sometimes finely granular,

Fra. 31.-A portion of a perpendicular section of a parietal bone ; magnified 350 diameters: $a$, lacunæ with pale only partially visible canaliculi, filled as in the natural condition with fluid; $b$, granulated matrix. The striated parts indicate the boundaries of the lamellæ.

texture returns. The author justly remarks, that if the connective tissue consisted of separate fibrils the impossibility of isolating them in the distended condition would be quite inexplicable. It is however intelligible, that in consequence of such an alteration in the connective tissue its cleavability may be diminished or destroyed, which does away with the necessity of supposing a fibrous structure. On the other hand, if a piece of tendon be hardened by a strong solution of caustic potash, or by nitric or hydrochloric acids, no fibres can be demonstrated in it (Bericht., pp. 40,41). It is easy enough to verify the truth of these statements, by treating a piece of tendinous tissue with acetic acid, when, as is well known, the fibrillated appearance disappears; then keeping in view one of the distended and transparent "bundles," slowly add a solution of caustic ammonia, the transparent mass will be seen gradually to shrink, and eventually to resume what appears to be a most distinctly fibrous appearance.

The gelatinous or rather gelatiniform areolated connective tissue of Professor Kolliker is simply ordinary connective tissue, in which the collagenous element is not yet or but little formed. Its development may be readily traced to the most superficial layer of the skin and mucous membranes, or in the tooth-pulp, or the so called actinenchyma of the enamel organ in the calf, \&c. The epiglottis of the kitten is particularly to be recommended, as this tissue can be observed passing on the one side into the homogeneous layer of the corium next to the epithelium, and on the other into the so-called fibro-cartilage of the epiglottis.

In all these cases, the mode of development of the areolated connective tissue is essentially similar to that observed by Remak (Ueber die Entstehung des Bindegewebes, \&c., Mall. "Archiv," 1852, I.) in the frog. The layer of the tissue next the epidermis or epithelium, is composed of a nearly homogeneous substance (matrix,) in which lie corpuscles (so-called nucler), the whole in fact corresponding exactly with embryonic cartilage. Internal to this, vacuolar cavities have been formed in the rhatrix between the corpuscles, the substance of the matrix appearing as bands or fibres between these vacuola. The latter enlarging, the substance of the matrix is more and more broken up into bands, in which dilatations remain where the "nuclei" are situated, so that the bands often resemble fusiform or stellate cells. A structure of this kind which undergoes no further chemical or morphological alteration, constitutes the gelatiniform connective tissue; and it is unquestionable, that its subsequent conversion into perfect areolated connective tissue is effected, as Professor Kolliker states, by the direct passage of these fusiform bodies into the pseudo-fibrillated 
very frequently lamelluted, and hard and brittle from its being intimately combined with calcareous salts; the lacunce are for the most part lenticular, and are united by very numerous fine processes, the canaliculi; by which some of them also open upon the outer surface of the bones and into the larger and smaller medullary and vascular spaces in the interior. The lacunce and canaliculi contain a clear substance which may be regarded as the nutritive fluid of the bones, and besides, a cellnucleus appears in many cases, perhaps constantly, to be enclosed within the lacunæ. Besides these two most essential elements, which exist in all bones, numerous vessels and nerves occur in most, as well as, frequently, a peculiar substance, the medulla, which supports them, and consists either of common fatty tissue, or of a loose, scanty, connective tissue, with few fat-cells and many peculiar, so-called medulla-cells. These soft parts fill up the larger cavities in the interior of the bones and in the spongy substance; but are to be found also, at least partially, in narrow canals which penetrate the compact substance, the vascular or Haversian canals, which open in all directions upon the outer and inner surfaces of the bones.

The matrix of the osseous tissue is composed of an intimate combination of an organic substance, which perfectly agrees with that of the connective tissue, and of inorganic compounds, among which the phosphate and carbonate of lime are the principal constituents. : The fluid contained in the cavities and canals is not thoroughly understood, but it probably presents a preponderance of albumen, fat, and salts, like the serum. The bones, from their solidity and inflexibility, serve as supFig. 32.

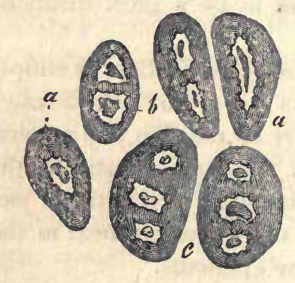
ports to the softer organs or for their more secure enclosure; and also perform special functions; as, for example, the auditory ossicles and the parts of the labyrinth which conduct the sonorous vibrations. The development of the bones takes place in two modes: firstly, by the metamorphosis of genuine cartilage, and secondly, by that of a soft blastema composed of indifferent

FIG. 32.-Six developing bone-cells from a rickety bone, as yet sharply defined from the interstitial substance : $a$, simple bone-cells; $b$, compound ones running to a parent cell, with two secondary cells; $c$, such arising from three cells.-Magnified 350 diameters.

bundles of the collagenous substance. But it is their outer portion only, that therefore which corresponds with the matrix of cartilage, which becomes thus changed-the elastic element being developed as before, not from separate cells, but by the chemical metamorphosis of the matrix immediately around the cavity which contains the "nucleus," and in various other directions.

That the pseudo-fibrillated portion of the connective tissue corresponds with the matrix of the cartilages is then, we think, certain. Whether with Remak we are to regard both these as cell-walls, or with Reichert as intercellular substances, must be discussed hereafter. (See General Appendix.) - Trs.] 
cells and of a fibrous substance similar to connective tissue. In both cases it is the cells-in the one the cartilage cells, in the other, cells without any defined character-which form the lacunoe and canaliculi by the thickening of their walls, with a contemporaneous development of pore canals, which subsequently grow into the matrix and unite with one another; whilst the matrix of the cartilage and the fibrous substance harden into the matrix of the bone by the deposition of calcareous salts, which likewise infiltrate the thickened cell-walls. The nutrition of the bones is very energetic, and is effected by the vessels of the investing periosteum, and, if they be present, by those of the medulla and the Haversian canals also. The bones have a great capacity of regeneration, and readily unite; in fact, very great losses of substance are repaired, or even whole bones, if the periosteum be left: adventitious development of bone is also very common.

The osseous tissue is found, firstly, in the bones of the skeleton, to which also the auditory ossicles and the hyoid bone belong; secondly, in the bones of the muscular system, as the sesamoid bones and the ossifications of tendons; thirdly, in the substantia osteoidea, or tooth cement. Many cartilages ossify with tolerable regularity as they grow older; as the costal-cartilages, and those of the larynx.

Dentine may be regarded as a modification of osseous substance, which, instead of solitary lacunce, presents long canals, - the dental canals; besides which, it exhibits some chemical modifications. The development of the dentine leads to the conclusion that it is an osseous structure, whose cells, in the course of their ossification and thickening, become united into tubes, and have very little or no intermediate substance; a view which gains additional support from the numerous transitional forms, to be observed in animals, between typical dentine and osseous tissue.

In the Vertebrata, bone is found more extensively distributed than in man. It exists in the skin (Armadillo, Tortoises, Lizards, Fishes), in the heart (the cardiac bone of the Ruminants and Pachydermata), in the muscular system (diaphragmatic bone of the Camel, Lama, and Porcupine, ossified tendons of birds), in the eye (sclerotic ring of Birds, Chelonians, and Saurians, bony scales of the sclerotic of many Fishes), in the external portion of the nose (proboscis of the Pig and Mole, os proenasale of the Sloth), in the tongue (os entoglossum of Fishes and Birds), in the respiratory organs (laryngeal, tracheal, and bronchial bones of many Birds), in the sexual organs (penis-bone of Mammalia), in the osseous system (ossa sterno-costalia of birds and some mammals). In the Invertebrata true bones are never found, being, in them, replaced by the so-called calcareous skeletons, which principally consist of car- 
bonate of lime, and arise in different structures as incrustations of homogeneous tissues and of cellular parenchymata, as solidifying excretions of calcareous matter, or as deposits of calcareous concretions. The teeth are limited to the three well-known classes of vertebrata. In the Plagiostomata, structures precisely similar to the teeth occur as cutaneous spines.

- Literature.-Deutsch, "De penitiori ossium structurâ Observationes," Diss. Vrat., 1834; Miescher, "De inflammatione ossium eorumque anatome generali." Accedunt observat. auct. J. Müller, Berol., 1836 ; Schwann, article "Knochengewebe," in "Berl. encyclop. Wörterbuch der med. Wiss.," Bd. xx. p. 102 ; Tomes, article Osseous Tissue, in "Cyclop. of Anatomy," vol. iii.*

\$26. Structure of the Smooth Muscles. - The smooth muscles consist essentially of microscopic, usually fusiform, more rarely shorter and broader fibres, to which I have given the name of "contractile or muscular fibre-cells." Each of these elements, in the mean from $0.02-$ 0.04 of a line long, $0.002-0.003$ of a line broad, is an elongated cell, wherein, however, no difference between contents and membrane can be distinguished; but which consists of an apparently homogeneous, often finely granulated or slightly striated, soft substance, in which without exception in the middle of the fibre a generally columnar elongated nucleus exists. These fibre-cells are united by means of a substance which cannot be directly demonstrated, into flattened or rounded cords, the bundles of the smooth muscles; which are then united, by delicate investments of connective tissue with fine elastic fibres (a kind of perimysium), into more considerable masses, in which numerous vessels and a relatively small number of nerves are distributed. Chemically, the principal constituent of smooth muscle is a nitrogenous substance similar to fibrin, the so-called muscular fibrin or syntonin (Lehmann), which, from the observations that have hitherto been made, is distinguished from blood fibrin only in this, that it is not dissolved by solution of nitre, nor by carbonate of potass, but very easily by dilute hydrochloric acid.

* [While perfectly agreeing with Professor Kölliker's general view of the relations between dentine and bone, namely, that the canals in the former represent the cavities and canaliculi which exist in the latter structure, we do not think that his statement of the mode in which the process of calcification of the dentine takes place is correct. So far as we have seen, the dentine is never developed by the immediate ossification of cells, nor do the latter take any direct share in its formation. (See Quarterly Journal of Micros. Sc., April, 1852.) It may be said that dentine is bone, in which, in consequence of the early disappearance of the "nuclei" from the ossifying blastema, the lacunce are not formed, the dentinal tubes presenting only the canaliculi.-Trs.] 
The physiological importance of the smooth muscles lies in their contractile power; in consequence of which they afford considerable assistance to the functions of the different viscera. The development of their elements takes place simply by the elongation of rounded cells, the membranes and contents uniting into a homogeneous soft substance. The nutrition of the smooth muscles would seem to go on very actively, according to the later investigations upon the fluid which bathes them, which, according to Lehmann, has most generally a distinctly acid reaction, and together with lactic, acetic, and butyric acid, contains creatin and inosit; and the same conclusion may be deduced from the frequent occurrence of physiological (in the uterus) and pathological hypertrophies and atrophies of them. Whether smooth muscles are regenerated, or whether loss of their substance is replaced by a similar tissue, is unknown; on the other hand, new formations of them appear to occur in uterine tumors.

The smooth muscular fibres never form large isolated muscles in the human body; as, for example, is the case in the genito-rectal muscles of mammalia, but exist either scattered in the connective tissue, or in the form of muscular membranes. In both cases the bundles are either parallel or interwoven into networks. Their distribution is as follows :-

1. In the Intestinal canal the smooth muscle forms: first, the tunica musculosa from the lower half of the csophagus, where smoth bundles are still mingled with transversely striated fibres, as far as the sphincter ani internus: secondly, the muscular layers of the mucous membrane, from the œsophagus to the anus: and thirdly, scattered muscular bundles in the villi.

2. In the Respiratory organs, a layer of smooth muscles appears in the posterior wall of the trachea, and accompanies the bronchioe, even to their finest ramifications, as a complete circularly fibrous membrane.

3. In the Salivary glands, this tissue is found solely in Wharton's duct; and here only scantily, and forming an incomplete coat.

4. The Liver has a perfect muscular layer in the gall-bladder, and scattered smooth muscles also in the ductus choledochus.

5. The Spleen has this kind of muscle in many animals in its outer

FIG. 33.-Muscular fibre-cell from the small intestine of man.

Fig. 34.-Muscular fibre-cell from the fibrous investment of the spleen of the dog; magnified 350 diam. 
coat, and in the trabeculce, mixed with connective tissue and elastic fibres.

6. In the Urinary organs the smooth muscles are found in the calices and pelves of the kidneys, form a complete muscular layer in the ureters and urinary bladder, but are only sparingly to be found in the urethra.*

7. The Female sexual organs possess smooth muscles in the oviducts, the uterus, where during pregnancy their elements become excessively developed, and attain a length of $\frac{1}{4}$ of a line, the vagina, the corpora cavernosa, and in the broad ligaments of the uterus in different places.

8. In the Male sexual organs they are found in the dartos, between the $t$. vaginalis communis and propria, in the vas deferens, vesiculae seminales, the prostate, around Cowper's glands, and in the corpora cavernosa penis.

9. In the Vascular system smooth muscles exist in the tunica media of all, especially of the smaller arteries; also in that of most veins, and of the lymphatics, with the exception of the finest; furthermore, in the lymphatic glands (Heyfelder); and lastly in the tunica adventitia of many veins. The elements, in vessels of middle dimensions, are everywhere fusiform fibre-cells; in the large arteries, on the other hand, shorter plates, which often resemble certain forms of pavement epithelium; and in the smallest arteries they are more elongated, or even round cells, forms which must be considered as less developed.

10. In the Eye, smooth muscles form the sphincter and dilator pupilloe and the tensor choroidece.

11. In the Skin, lastly, this tissue appears besides in the dartos, in the form of minute muscles upon the hair sacs, in the areola, and in the nipple, and in many of the sudoriparous and sebaceous follicles. $\dagger$

The elements of the smooth muscles were formerly universally regarded as elongated bands containing many nuclei, which were supposed to be developed by the coalescence of numerous mutually applied cells. In $1847 \mathrm{I}$ showed that this is not the case; that, on the other hand, the elements of these muscles are only modified simple cells; and at the same time I demonstrated, that these contractile fibre-cells occur wherever contractile connective tissue had previously been assumed to

* [Mr. Hancock (On the Anatomy and Physiology of the Male Urethra, London, 1852), who had made out the existence of the organic muscular layer in the urethra independently, attributes to it much more anatomical and physiological importance. (See below, § Urinary Organs.)-TRs.]

$\uparrow[$ These muscles have also been seen by Mr. Lister (Quart. Journal of Microscop. Science, vol.i. p. 203), in the scalp. Mr. Lister found the muscles in this situation smaller ( $\frac{1}{2} \frac{1}{0}$ th of an inch) than those measured by Kolliker. They possessed extremely distinct nuclei, but instead of uniting in flat bundles were often circular, sometimes elliptical or polygonal.-DaC.] 
exist, and also, that they are to be found in many localities in which their presence had not been suspected. These views, notwithstanding contradiction at first from certain quarters, are now universally confirmed; a result to which Reichert, by the discovery of a reagent, which readily enables even those who are less practised, easily to isolate the contractile fibre-cells, viz. : nitric and hydrochloric acids of 20 per cent. (Müller, "Archiv," 1849, and Paulsen, "Obs. Microchem.," 1849); and Lehmann, by his chemical investigations upon this tissue, have contributed their share. Contractile fibre-cells occur in all four classes of the Vertebrata, but appear to be wholly wanting in the Invertebrata, since the smooth fibres of these creatures, which have been thought to be such, are allied genetically to the transversely striated muscles of the higher animals.

Their occurrence in the Vertebrata is in some respects peculiar, and I will here mention the following localities in which they are found: In the skin of Birds, as the muscles of the quill-feathers-in this case with tendons of elastic tissue; in that of the Orang-outang, in the hairsacs, as in man; in the iris of the Amphibia; in the campanula Halleri of the osseous Fishes (Leydig); in the swimming bladder of Fishes; in the lungs of the Frog (in Triton they are here wanting); in the mesentery of the Plagiostomata, or Psammosaurus and Leposternon (Leydig u. Brücke); in the genito-rectal muscle of Mammals. In the gizzard of birds these muscles are of a bright red color, and are united with a tendinous membrane.

Literature.-Kölliker, "Ueber den Bau und die Verbreitung der glatten Muskeln.," in the "Mittheilungen der Naturf. Gesellschaft in Zürich," 1847, p. 18, and "Zeitschrift für wiss. Zool.," Bd. I. 1849; C. R. Walther, "Nonnulla de musculis lævibus.," Diss. Lips. 1851.* [Jos. Lister, "Observations on the Contractile Tissue of the Iris," Quart. Journ. Mic. Sc., vol. I. p. 8, Pl. i., and "Observations on the Muscular Tissue of the Skin," Quart. Journ. Mic. Sc. vol. I. p. 263.$\mathrm{DaC}$.

* [Reichert (Bericht, 1849, Müller, "Archiv.,") states that, according to Paulsen, the action of a solution of caustic potass of 50 per cent. causes the smooth muscles to become wavy, and thus to assume a transversely striated appearance under the microscope. Macerated in such a solution for three days, they break up into small globules; striated muscle behaves in a similar manner, and the globules correspond in size to the interval between two striæ.

Eylandt (Obs. Microscop. de musculis organicis in hominis cute obviis. Diss. inaug., Dorp. 1850 , c. Tab. lithog.), denies the existence of free smooth muscles in the papilla and areola mamma, in the scrotum, in the skin of the penis or of the prepuce, and in the perinæum. Nor does he find them in the outer layers of the hair-sacs (apart from the arrectores pill), in the glandula sudorifere of the axilla, of the anus, \&c., nor in the glandula ceruminosa. The smooth muscles observed in the papilla and areola mamma, in the skin of the penis, and the perinæum, he considers to belong to a greatly developed vascular layer. (See, however, the remarks of Prof. Kolliker upon Eylandt's statements, at the end of \$ 34.)-Trs.] 
\$27. Transversely Striated Muscular Tissue.-The elements of this tissue consist essentially of the so-called muscular fibres or primitive muscular bundles, each of which, 0.004-0.03 of a line thick, consists of fine fibrils surrounded by a special homogeneous, delicate, elastic investment, the sarcolemma: the fibrils are generally enlarged at regular intervals, so that they appear to consist of a series of many portions, and give a
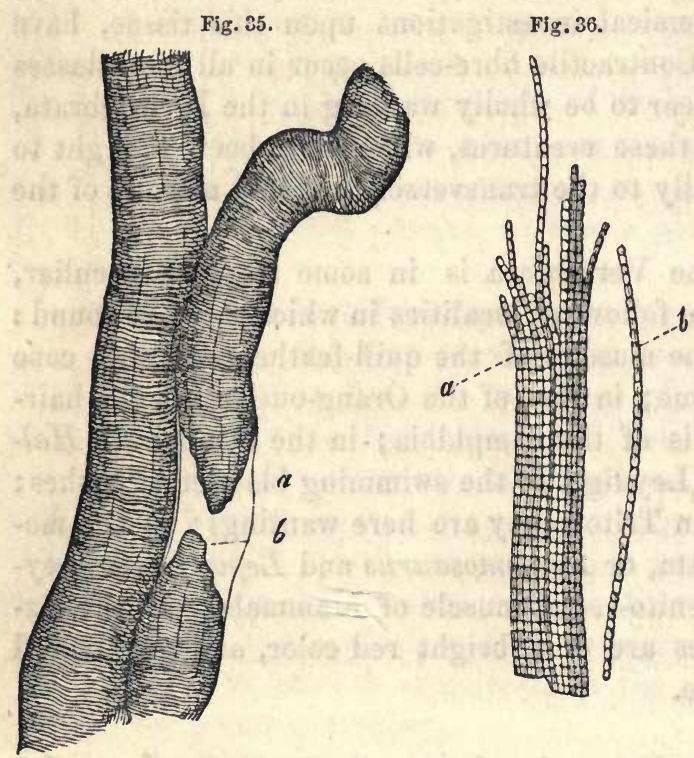
transversely striated aspect to the muscular fibres, or they appear more even, and then the primitive bundles present a longitudinal striation. Besides these fibrils, the muscular fibres contain nothing but a small quantity of viscid substance uniting them, and a certain number of rounded or elongated cell-nuclei, which generally lie against the inner surface of the sarcolemma. The association of the muscular fibres into muscles and muscular membranes occurs in such a manner that they either apply themselves parallel to one another, or are united into true networks of transversely striated muscles. They then receive an investment of more delicate or firmer connective tissue, the so-called perimysium, with which finer elastic fibres and also fat-cells are frequently mingled; and are, besides, surrounded by numerous blood-vessels and nerves.

In chemical characters the principal substance of the transversely striated muscular fibres agrees perfectly with the syntonin referred to in the previous section. The sarcolemma is very resistant to acids and alkalies, whilst the nuclei present the common characters of those organs. A fluid with an acid reaction may be expressed from the muscles, in which Liebig and Scherer have discovered an interesting series of nonnitrogenous and nitrogenous products of the decomposition of the muscular tissue.

The transversely striated muscles are in a high degree contractile, and

Fig. 35.-Two muscular fibres of man; magnified 350 diam. In one the bandle of fibrils, $b$, is torn, and the sarcolemma, $a$, is to be seen as a mere empty tube.

FIG. 36.-Primitive fibrils from a primitive bundle of the Axolotl (Siredon pisciformis); $a$, a small bundle of them; $b$, an isolated fibril, magnified 600 diam. 
are the chief instruments of the animal motions. Their elements are developed by the coalescence of round or stellate cells, whose contents change into a homogeneous, semi-fluid substance, and then break up into fibrils. Once formed, the muscular fibres grow by the elongation and thickening of their elements, and in their complete condition they enjoy a very energetic nutrition, which is especially manifested by the multiform products of their decomposition, as well as by the circumstance that their powers are exhausted in a short time when the circulation is suspended. Wounds of the muscles never heal by transversely striated muscular substance; but an adventitious formation of this tissue appears to occur sometimes, though rarely.

Transversely striated muscular tissue is found in the following parts:

1. In the muscles of the trunk and extremities; of the globe of the eye, and all those of the ear.

2. In the muscles of many organs; as the larynx, pharynx, tongue, and osophagus (upper half), the end of the rectum (sphincter externus, levator ani), the genital organs (bulbo-ischio-cavernosus, urethralis transversus, transversi perincei, cremaster, muscular fibres of the round ligaments of the uterus, in part).

3. In certain parts of the vascular system, e. $g$. in the heart and in the walls of the great veins which open into it.

The muscular fibres of animals are not all composed of bundles of transversely striated fibrils, but present a series of other forms, which may best be grouped in the following manner:-

1. Muscular tubes, with homogeneous, semi-solid, not transversely striated contents (most Mollusks, Worms, and Radiata).

2. Muscular tubes with a membrane, a semi-fluid, homogeneous, cortical layer in contact with it, and a fluid or granular, frequently transversely striated or nucleated central substance. (Muscles of Petromyzon in part, certain muscles [of the lateral line and of the spiracles] of the plagiostome and osseous Fishes. Muscles of the Hirudinida, Lumbricidoe, of Paludina in part, and of Carinaria.)

3. Similar muscular tubes with a transversely striated cortical layer without distinct fibrils. (Many muscular fibrils of the Hirundinidoe, and of the muscles of Fishes enumerated under 2.)

4. Muscular fibres without any internal cavity, with a sarcolemma and transversely striated contents, which do not break up into fibrils, but frequently into discs (Bowman), Salpoe, some Radiata, many Articulata.

5. Similar muscular fibres, which readily break up into fibrils. (Most Vertebrata, certain muscles of Insects.)

6. Simple isolated cells, whose contents are changed into a transversely striated substance, which either fills the whole cell or forms only a thin layer upon its membrane. Here my observations lead me to place 
the peculiar cartilaginous striæ, which Purkinje (Mikr. neurol. Beobachtungen, in Müll. "Arch.," 1845) found in the endocardium of Ruminants. They consist of large polygonal cells with beautiful nuclei, which internally, but as it seems only upon their wall, contain a transversely striated substance, which is not distinguishable from that in the muscular fibres.

All these forms are readily comprehended, if the genesis of the true transversely striated muscular fibres in the higher vertebrata be properly understood (see the special part, Muscles); and I cannot agree with the supposition of Stannius (Gött. Nachr., 1851, p. 17), that the transversely striated muscular fibrils are developed according to many essentially different types. Even the gap which has hitherto separated the smooth from the transversely striated muscles becomes less, when we remember that the so-called transversely striated muscular fibrils may also have homogeneous non-striated contents, and also that even when transversely striated they may appear as isolated cells.*

Muscular fibres of the same description as the transversely striated muscles, and in part actually striated, are very widely distributed. In the Vertebrata such muscles are found in the osophagus of some Mammalia and of the plagiostome fishes, in the intestine of Tinca chrysitis, in the stomach of Cobitis fossilis, around the poison gland of Snakes, and in the contractile organ of the pharynx of the Carp; in the skin of Mammalia, Birds, Snakes, and tailless Batrachians (so called cutaneous muscles), in the tactile hairs of mammals, in the lymph hearts of many Birds and Amphibia ; in the auriculo-ventricular valve of the right side in Birds, and the Ornithorhynchus; upon the vena cava inferior of the Seal, close above the diaphragm : in the interior of the eye of Birds; and round Cowper's and the anal glands of mammals. In the Invertebrata, as we have mentioned, all the muscles belong to this category, whether they be transversely striated or not; and they are found, therefore, in the heart, the intestine, the genitalia, and often clearly striated.

The anastomosis of the primitive bundles of the muscles, with which Leeuwenhoek was already acquainted $\dagger$ and which I rediscovered in the heart of the frog, has now been seen in many places, and appears to be constant in the hearts of the lymph and blood-vascular systems of all animals, and in the muscles of the Invertebrata, especially those of the vegetative and generative organs. (Hessling, Leydig.) Simple arbores-

* [The muscles of the Medusæ consist of flat, fusiform bands, whose ends are interlaced like those of smooth muscle, but which present the most distinct transverse striæ.-TRs.]

$\dagger[$ It has been pointed out to us by Professor Sharpey, that Leeuwenhoek was not acquainted with the anastomosis of the primary bundles of the cardiac muscles, but has described and figured only that of the secondary bundles, which is indeed obvious upon reference to Leeuwenhoek's Plate ("Experimenta et Contemplationes," Op. On. Lugd. Bat. tom. i. p. 409, 1722); the ascription in the text is therefore an error. For other remarks upon the muscular tissue, vide infra, \$ Muscle.-Trs.] 
cent branchings of muscular fibres, which Corti and I noticed in the tongue of the frog, are on the other hand rare, and have been seen elsewhere only in Artemia salina and in the oral and anal disc of Piscicola (Leydig).*

Literature.-W. Bowman, article Muscle and Muscular Motion, in Todd's "Cyclopædia of Anatomy," and "On the Minute Structure of Voluntary Muscle," in "Phil. Trans.," 1840 II. 1841, I. ; J. Holst, "De Structurâ Musculorum in genere et annulatorum Musculis in Specie," Dorp. 1846; M. Barry, "Neue Unters. über die schraubenförmige Beschaffenheit der Elementarfasern d. Muskeln, nebst Beobachtungen über die musculös. Natur d. Flimmerhärchen" (Müll. "Arch.," 1850, p. 529).

§28. Nervous tissue.-The essential elements of this tissue are of two kinds, the nerve-fibres and nerve-cells (ganglion-globules). The primitive fibres or tubules of the nerves have either a distinct medulla or they have none. The former consist of three parts: of a structureless delicate membrane, the sheath of the primitive tubules; of a central, soft, but elastic fibre, the central or axis band (axis cylinder, Purkinje; primitive band of Remak); and of a viscid white layer placed between them, the medullary sheath. In the tubules without medulla, which in man occur only in certain peripheral expansions (retina, olfactory organ, cornea, Pacinian corpuscles), the structureless coat contains nothing but a homogeneous or finely-granular, clear substance, which appears to be identical with the central band of the other tubules, and at any rate may be considered analogous to it, so that the medullary layer may be supposed to be absent in these. The primitive nerve-tubules of both kinds, especially of the former, occur of very different dimensions, and may thence be divided into fine ones of 0.0005-0.002 of a line, those of a medium size of $0.002-0.004$, and thick ones of $0.004-0.01$ of a line. Their course is either isolated, so that one tubule runs from the centre to the periphery; or they divide, especially in their terminal expansions, into a greater or smaller number of branches; or, lastly, they form actual anastomoses and networks. Besides this, many nerve-tubules are connected with nerve-cells, so that they either arise from them or are interrupted in their course by interposed nerve-cells. These nerve-cells, or as they are called in the ganglia, ganglion-cells, or ganglion-globules, are endowed with the common attributes of cells. Their membrane presents no peculiarity, except that, frequently, it is very delicate, and even, as in the great central masses, eventually perhaps wholly disappears. The contents are finely-granulated, semi-solid, often contain

* [Suclı branched muscular fibres may be found beautifully marked in the upper lip of the Rat, and in the tongue of Man and Animals. See article "Tongue," by Dr. Hyde Salter, in Todd's "Clycopædia."-Trs.] 
pigment, and without exception enclose a distinct vesicular nucleus with a large nucleolus. In size, the nerve-cells vary from $0.003-0.04$ of a line, and as regards their form, they may be distinguished principally

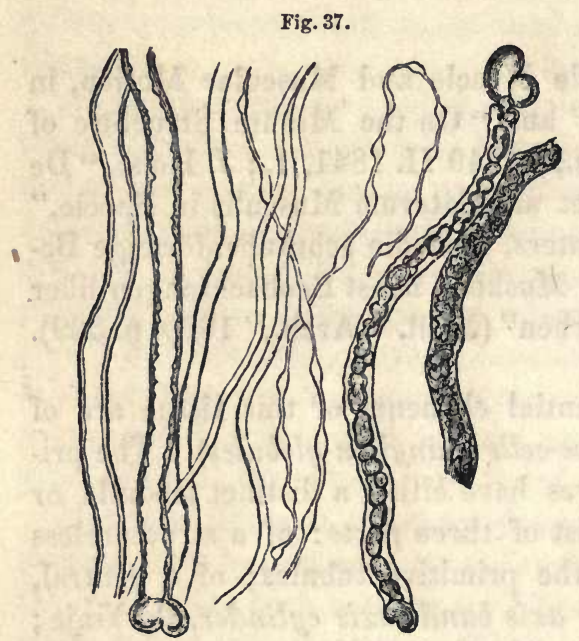

into round, fusiform, and stellate. The two latter kinds are produced by the prolongation of many nerve-cells into two, three, to eight and more, pro-

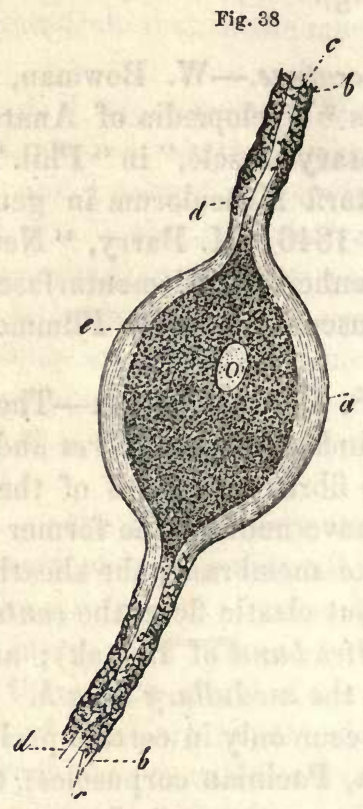
cesses, which in some cases, after a short course, pass into medullated nerve-fibres, in others, present a more marked independence, since, preserving a complete resemblance to non-medullated nerves, they often run for a considerable distance, and branch out in manifold ways. In what manner finally these processes end, whether free or in connection with nerve-tubules or by anastomosis with similar processes, is not yet made out; though, upon the whole, it would seem to be not improbable that all three possibilities may occur in different localities.

Nerve-fibres and nerve-cells are combined into two substances, which in extreme cases present very wide differences, the gray and the white substances. The former constitutes the so-called white medulla or medullary substance of the spinal cord and brain, and the nerves; it consists essentially of nerve tubules, united into bundles or interwoven into plexuses, with bloodvessels; added to which, in the peripheral nerves, we have

Fig. 37.-Tubular nerve fibres of man. Four of them fine, two of them being varicose, one of a medium thickness with a simple contour, and four thick ones; two having double contours, and two with granular contents.-Magnified 350 diameters.

Fig. 38.-Nerve-cell of the Pike (so-called bipolar), passing at its two ends into dark-bordered nervous tubules, treated with arsenious acid: $a$, membrane of the cell; $b$, nervesheaths; $c$, medulla of the nerve; $d$, axis-fibres connected with the contents of the nervecell; $e$, retracted from the membrane.-Magnified 350 diameters. 
special investments of connective tissue, the so-called neurilemma. The gray substance contains a great preponderance of nerve-cells, besides which, in certain localities, there is a finely-granular matrix and free nuclei; but it is rarely found quite unmixed, being usually mingled more

Fig. 39.

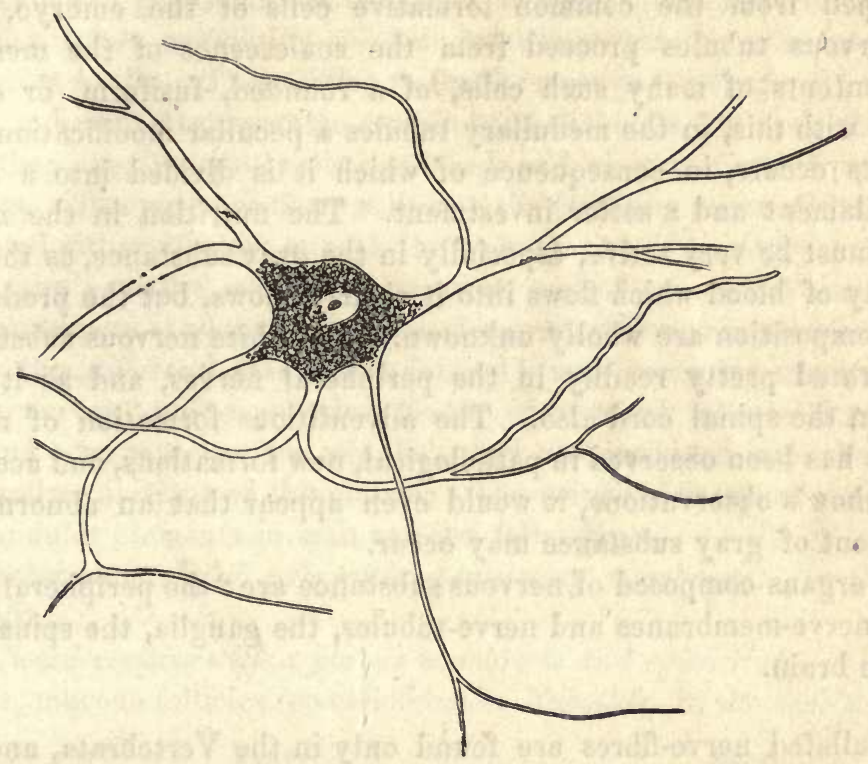

or less with nerve-fibres. This is more especially the case in most ganglia, in the gray substance of the spinal cord, and in the so-called ganglia of the cerebrum; while, on the other hand, in the gray cortex of the cerebrum and cerebellum, it is found in some localities almost without nervous fibres. This substance possesses vessels even in much greater abundance than the white; and in the peripheral ganglia there are also different forms of connective tissue, which serve to invest their separate parts.

The chemical composition of the nervous substance has hitherto, by no means, been sufficiently investigated. In the white substance, the central bands of the nerve-tubules consist of a protein compound very similar to the fibrin of the muscles; the medullary sheath, chiefly of fats of different kinds, and the membrane, of a substance similar to the sarcolemma. The gray substance contains a preponderance of albuminous matter, besides a considerable quantity of fat.

The physiological importance of the nervous tissue consists, in the first place, in its subserving movement and sensation; secondly, in its

FIG. 39.-Nerve-cells of the substantia ferruginea from the floor of the fourth ventricle in man; magnified 350 diameters. 
exerting a certain influence upon the vegetative functions ; and thirdly, in its serving as a substratum to the psychical activities; in all which capacities, according to what we know at present, the gray substance performs the more important part, the white acting rather as a connecting conductor between, it and the organs. The nerve-cells are developed from the common formative cells of the embryo, whilst the nervous tubules proceed from the coalescence of the membrane and contents of many such cells, of a rounded, fusiform, or stellate shape; with this, in the medullary tubules a peculiar modification of the contents occurs, in consequence of which it is divided into a central solid filament and a softer investment. The nutrition in the nervous tissue must be very active, especially in the gray substance, as the great quantity of blood which flows into it clearly shows, but the products of its decomposition are wholly unknown. The white nervous substance is regenerated pretty readily in the peripheral nerves, and as it would seem, in the spinal cord also. The adventitious formation of nervous tubules has been observed in pathological, new formations, and according to Virchow's observations, it would even appear that an abnormal development of gray substance may occur.

The organs composed of nervous substance are : the peripheral nervecords, nerve-membranes and nerve-tubules, the ganglia, the spinal cord, and the brain.

Medullated nerve-fibres are found only in the Vertebrata, and even in that class not in every division, as for example, in Petromyzon (Stannius). Fibres without medulla always occur together with the former, and in general in the same localities as in man; but in other situations also, as in the skin of the Mammalia, in the electric organs of Fishes, and in the sympathetic nerve of the Plagiostomata (Leydig). Where nerves are found in the Invertebrata, they contain only pale fibres without medulla, whose structure often completely resembles that of the embryonic fibres of higher animals, especially as regards the occurrence of great nucleated enlargements in the terminal expansions, which remains of the original formative cells, have, recently, less properly been considered to be ganglion-globules.

Literature.-G. Valentin, "On the course and termination of the nerves," in the "Nov. Act. Natur. Curios.," vol. xviii. t. i.; R. Remak, "Observations anatomicæ et microscop. de syst. nerv. struct.," Berol., 1838; A. Hannover, "Recherches microscopiques sur le système nerveux," Copenhague, 1844 ; R. Wagner, "Neue Unters. über den Bau und die Endigungen der Nerven und die Structur der Ganglien," Leipzig, 1847; and "Neurologische Untersuchungen," in Göttingen "Anzeige," 1850 ; Bidder and Reichert, "Zur Lehre vom Verhältniss der Ganglienkörper zu den Nervenfasern," Leipzig, 1847 ; Ch. Robin, in "l'Institut.," 
1846, Nos. 687-699, and 1848, No. 733 ; Kölliker, "Neurologische Bemerkungen," in "Zeitsch. für wiss. Zool.," i. p. 135.

§29. True Glandular Tissue.-The most essential constituents of the true glands are the secreting elements, which appear as aggregations of cells, as closed glandular vesicles, and as open glandular vesicles and glandular tubes, containing as their most important constituent the socalled gland-cells. These cells are for the most part polygonal or cylindrical, and perfectly resemble certain epithelial cells, but upon the other hand, they are frequently distinguished and characterized by peculiar contents. The union of these cells into the secreting parts of the glands is effected either directly or with the co-operation of homogeneous membranes, the so-called membrance proprice, and of connective tissue. In this manner the secreting glandular elements, different in nature according to the different glands, are formed; and becoming invested with vessels, nerves, and connective tissue, with which elastic fibres, fatcells, and even muscles, are mingled, they are combined into the larger and smaller divisions of the glands. The principal forms of the secreting glandular elements in man are the following:-

1. Solid networks of cells without investing membrane. In the liver (Fig. 40).

2. Closed vesicles with a fibrous membrane and epithelium. Graafian vesicles, mucous follicles (so-called ovula Nabothi), in the cervix uteri.

Fig. 40.

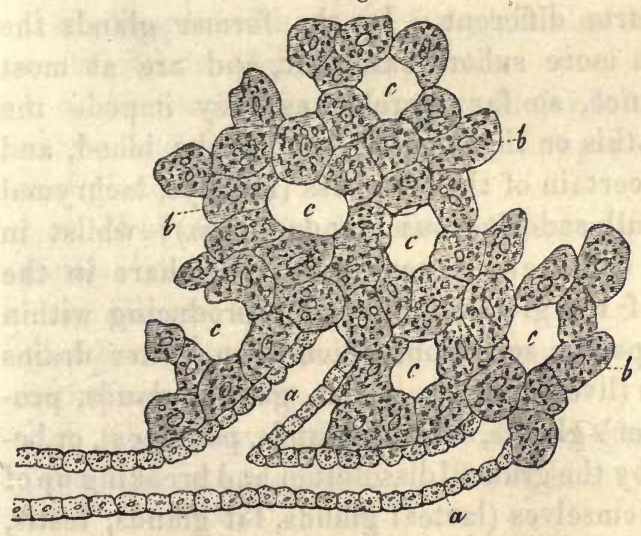

Fig. 41.

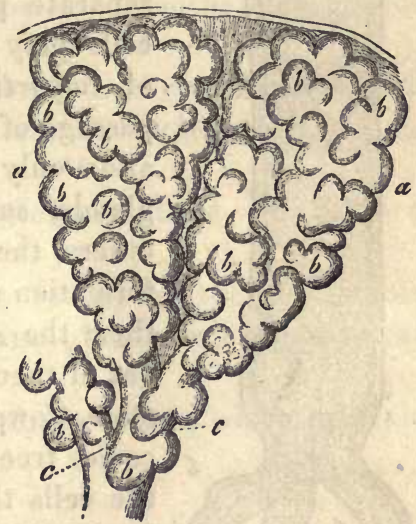

3. Rounded or elongated glandular vesicles, with a membrana propria and an epithelium. In the racemose glands (Fig. 41).

Fra. 40.-Network of hepatic cells, $b$; and finest ductus interlobulares, $a$; of man after nature; the union of both diagrammatic; $c$, vascular spaces.-Magnified 350 diameters.

Fig. 41.-Two of the smallest lobes of the lung, $a a$; with air-cells, $b b$; and the finest bronchial ramifications, $c c$; upon which also air-cells are seated. From a new-born child; semi-diagrammatic figure.-Magnified 25 diameters. 


\section{Glandular tubes, with a membrana propria, or a fibrous membrane} and an epithelium. Tubular glands (Fig. 42).

To these elements are also added (except in those glands enumerated under 2, which become emptied of their contents by the occasional bursting of their follicles, and the simplest tubular glands) special excretory ducts, which, after manifold ramifications either pass directly into the glandular vesicles and glandular tubes, or, as in the liver, are simply applied to the secreting networks of cells. These ducts are at first similar in their structure to the secreting parts, but they always possess epithelial cells, which have not the specific contents of the proper gland cells, and mostly also exhibit a different form. The wider excretory ducts consist of a fibrous investment and of an epithelium, and often also, possess a muscular layer, and in their ultimate divisions, a fibrous, a muscular, and a mucous layer very frequently exist as special structures.

Chemically, the glands are, as yet, little known. The glandular cells, the most important structures, are allied in this respect also to the epithelial structures, only that frequently, they contain in their interior peculiar substances, - as fat, the constituents of the bile, of the urine, of the gastric juice, mucus, \&c., and thence assume a specific character.

Fig. 42.

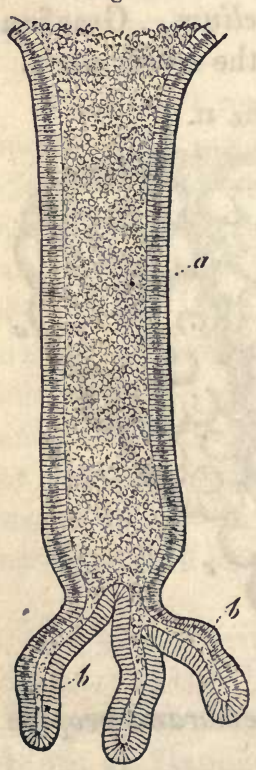

The true glands either separate certain constituents from the blood, or by means of it, elaborate peculiar substances of structural elements, and according as they do the one or the other, is the import of their separate parts different. In the former glands the cells play a more subordinate part, and are at most of importance, so far merely, as they impede the passage of this or that constituent of the blood, and allow only certain of them to pass (kidneys, lachrymal glands, small sudoriparous glands, lungs); whilst in others, the cells take a very important share in the formation of the glandular fluid, by producing within them the specific secretion, which then either drains out of them (liver, mucous glands, gastric glands, prostate, Cowper's glands, salivary glands, pancreas), or becomes free by the gradual dissolution and breaking up of the cells themselves (lacteal glands, fat glands, testis, larger sudoriparous and ceruminous glands). In the former case, as in the Graafian follicles, a peculiar celldevelopment may take place in the secretion which is formed, whilst in the latter, new elements continually arise in place of those gland-cells which are removed as they attain their full develop-

FIG. 42.-Gastric gland from the pylorus of the dog, with cylinder-epithelium: $a$, larger glandular cavity $b$, tubular appendages of it. 
ment, in consequence of which the character of these cells as a coating of the glandular canals is frequently lost, and they appear simply as a part of the secretion (testis, lacteal gland during lactation). All the glands here mentioned, with the exception of the sexual, are developed from the internal and external epithelial structures of the body, conjoined with the vascular membranes which support these epithelia. Some of them originate as involutions of these membranes, and retain the cavities throughout the course of their development (lungs, small intestinal glands), others are at first hollow, but afterwards increase by the addition of solid out-growths (liver); others, again, are solid from the very first, continue to grow in this condition, and only secondarily come to possess cavities (cutaneous glands, racemose glands). The nutrition of the glands goes on with great energy, and they belong to the most vascular organs of the body. Except in the uterine glands no regeneration of the glandular substance takes place, but hypertrophy occurs in them, and even the accidental formation of minute glands.

The true glands of the human body may, according to the form of their ultimate elements, above described, be divided as follows :-

1. Glands with closed glandular vesicles, which dehisce periodically. Ovary, follicles of the uterus.

2. Glands whose parenchyma consists of cells united into a network. Liver.

3. Racemose glands, in which rounded and elongated glandular vesicles are seated upon the ultimate ends of the excretory ducts.

a. Simple, with one or few glandular lobules. Mucous glands, sebaceous glands, Meibomian glands.

b. Composite, with many glandular lobules. Lachrymal glands, salivary glands, pancreas, prostate, Cowper's and Bartholini's glands, lacteal glands, lungs.

4. Tubular glands, whose secreting elements have the form of canals.

a. Simple, consisting of only one or a few creal tubes. Tubular glands of the stomach and intestine, uterine glands, sudoriparous and ceruminous glands.

b. Composite, with many branched glandular canals, which may also be united into a network. Testis, kidney.

The forms of the glands of animals, notwithstanding their variety, may, with few exceptions, be brought under one of the four categories here established. The following are worthy of particular notice: 1 . The glandular cells, with peculiar excretory ducts, to be found in some Articulata, which either, singly, form glands, or are united together in numbers by a membrana propria. 2. The occurrence of a structureless, chitinous membrana intima in many glands of the Articulata. 3. 
The formation of certain secretions [Uric acid and bilin in Mollusks, bilin in Crustacea] within special spontaneously enlarging "secreting vesicles," (Nägeli, H. Meckel), which may be compared to the yelk vesicles $(\S 6)$. 4. The colossal size (up to 0.1 of a line) of many glandular cells of Insects, and the peculiar ramifications of their nuclei.

Literature.-J. Müller, "De Glandularum secernentium structurâ penitiori," Lips. 1830 ; H. Meckel, "Micrographie einiger Drüsenapparate niederer Thiere," in Müll. "Arch.," 1846; Fr. Leydig's "Vergleichend-anatomische Abhandlungen," in "Zeitschrift für wiss. Zool."

$\S$ 30. Tissue of the Blood-vascular Glands.-Under this denomination are most appropriately comprised, a series of organs, which agree in this, that in a peculiar glandular structure, they elaborate from the blood or other juices certain substances which are not excreted by special, permanent, or periodically-formed excretory ducts, but simply by infiltration from the tissue, and are afterwards applied in one way or another to the general purposes of the organism.

It may be, that this wide definition includes organs, which it will be necessary to separate in future; but with our present slight knowledge of these structures, it is the only one which is possible without entering more fully into the subject.

The essential glandular tissue of the organs in question appears under the following forms:-

1. As a parenchyma of larger and smaller cells, imbedded in a stroma of connective tissue. Supra-renal bodies, anterior lobes of the hypophysis cerebri. Some of the cells here attain the great size of 0.04 of a line; and then contain, together with a granular substance, many nuclei, and perhaps secondary cells.

2. As closed follicles, each of which consists of a membrana propria with an epithelium upon its inner side, and has clear contents: thyroidea. The follicles, which are not enlarged cells, are surrounded by a large quantity of connective tissue, and are united by it into smaller and larger lobules.

3. As closed follicles, with a membrane of connective tissue, and contents consisting of nuclei, cells and some fluid. Among these I enumerate-

a. The solitary follicles of the stomach and intestine; and

b. The aggregated follicles of the small intestine, or Peyer's patches (in animals those of the stomach and large intestine also), both of which contain numerous bloodvessels in the interior of the follicles.

c. The follicular glands in the root of the tongue, the tonsils, and the pharyngeal follicles, which in the walls of their sacs, contain many 
closed follicles like those above mentioned, but, so far as we as yet know, without vessels in their interior.
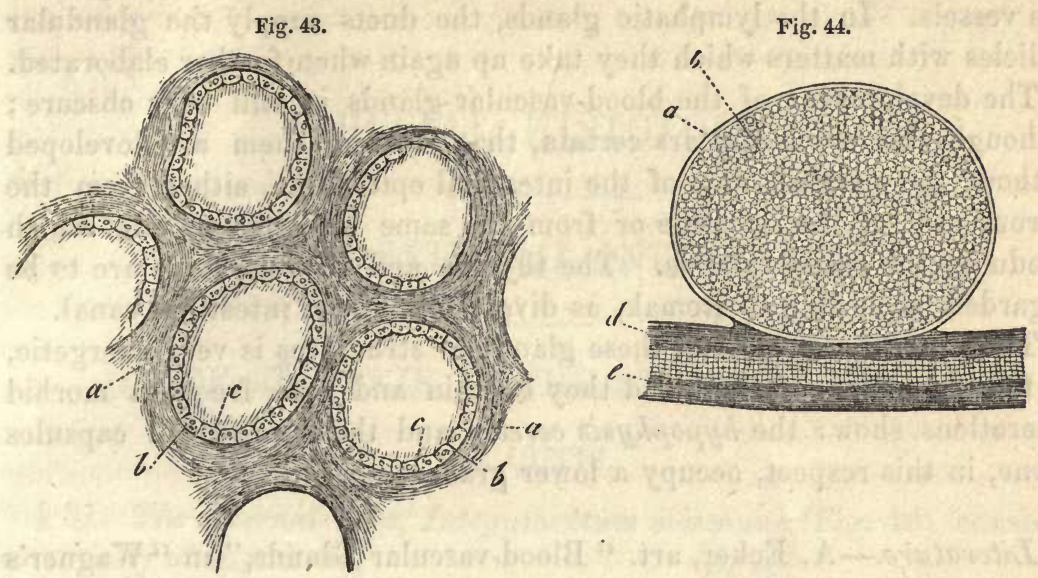

d. The lymphatic glands, which appear to consist of follicles like those of the Peyerian patches.

4. As a cellular parenchyma, which contains numerous closed follicles like those just described: Spleen.

5. As racemose, aggregated, glandular vesicles opening into a common ciosed canal or broad space, whose thick walls are formed of a delicate investment of connective tissue, and of a soft substance consisting of many nuclei and of vessels: Thymus.

We know little of the chemical nature of these organs, which are all more or less richly supplied with bloodvessels. Those enumerated under 1,2,3, and 5, contain much protein and fat in their tissue, as also do the follicles of those included under the fourth form, while the remaining parenchyma of the spleen possesses peculiar corpuscles, not yet completely investigated, which seem to indicate an energetic, retrogressive metamorphosis. We know little of the physiological functions of these glands; and here it need merely be remarked, that in the spleen, the thyroid, the thymus, the supra-renal capsules, and the pituitary body, it can only be the blood which yields material to them, and only the blood-and lymph-vessels which again receive the substances given off externally or internally (thymus) by them. In the follicular glands of the mouth and pharynx, the secretions are poured into the wider cavities of the glands, and ultimately into those organs, whilst in

Fra. 43.-A few of the glandular vesicles from the thyroid gland of a child: $a$, connective tissue between them; $b$, membrane of the glandular vesicles; $c$, their epithelium-Magnified 250 diameters.

FIG. 44.-A Malpighian corpuscle from the spleen of the ox: $a$, wall of the corpuscle; $b$, contents; $c$, wall of the artery upon which it is seated; $d$, sheath of the latter.-Magnified 150 diameters. 
the intestinal follicles, it is doubtful whether they excrete substances into the intestine, or receive them from thence to give them up again to the vessels. In the lymphatic glands, the ducts supply the glandular follicles with matters which they take up again when further elaborated.

The development of the blood-vascular glands is still very obscure; although this much appears certain, that most of them are developed without the participation of the intestinal epithelium, either from the fibrous wall of the intestine or from the same blastema as that which produces the sexual glands. The thymus and thyroid alone are to be regarded, according to Remak, as diverticula of the intestinal canal.

The nutrition of most of these glandular structures is very energetic, as the abundance of the blood they contain and their frequent morbid alterations show: the hypophysis cerebri and the supra-renal capsules alone, in this respect, occupy a lower grade.

Literature.-A. Ecker, art. "Blood-vascular Glands," in "Wagner's Handw. d. Phys.," Bd. IV. 1849. [H. Gray, "On the Development of the Ductless Glands in the Chick," Philosoph. Trans., 1852.-Trs.] 


\section{SPECIAL HISTOLOGY.}

\section{OF THE EXTERNAL INTEGUMENT.}

\section{I.-OF THE SKIN IN THE STRICTER SENSE.}

\section{A. CUTIS.}

§ 31. The external skin, Integumentum commune (Fig. 45), consists essentially of an internal layer formed principally of connective tissue, and rich in vessels and nerves, the true skin, cutis, derma (Fig. 45, $c, d)$; and of an external layer composed of cells only, the epidermis (Fig. $45, a, b)$; and it contains in addition many peculiar, glandular and horny organs.

The cutis may be again subdivided into two layers, the subcutaneous cellular tissue, tela cellulosa subcutanea (Fig. $45, d)$; and the proper corium (Fig. 45, Fig. 45.

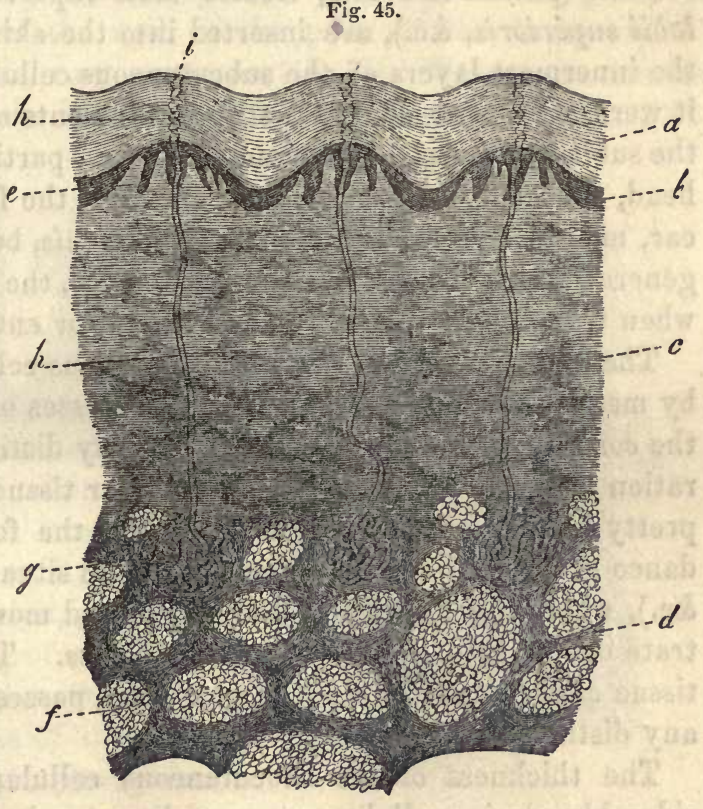

c); the latter of which, from its rich nervous and vascular supply, forms the most important part of the skin.

$\S 32$. The subcutaneous cellular tissue is a tolerably firm membrane, constituted chiefly of connective tissue, which in by far the most parts

Frg. 45.-Perpendicular section through the whole skin of the ball of the thumb, transversely through three ridges of the cutis: $a$, horny layer of the epidermis; $b$, its mucous layer; e, corium ; d, panniculus adiposus (upper part) ; $e$, papillæ of the cutis ; $f$, fat masses ; $g$, sudoriparous glands; $h$, their canals; $i$, sweat-pores.-Magnified 20 diameters. 
of the body encloses within its meshes a considerable quantity of fat-cells (Fig. $45, f$ ), thus forming the panniculus adiposus; in some situations, however, as for example in the scrotum, the penis, and the nymphoe, \&c., it contains but little or even no fat. The innermost layer of the subcutaneous cellular tissue, which upon the trunk and thighs forms a tolerably firm fatless texture, the fascia superficialis, rests upon different organs, as muscular fascice, periosteum, and perichondrium, muscles, and the deeper accumulations of fat, and is more or less closely united with them. The union is looser upon the trunk, the two distal divisions of the limbs, the back of the hand and foot, the neck, and especially on the eyelids; the penis, scrotum, and on the extensor side of the articulations, where the subcutaneous mucous bursc, as they are called, are frequently situated, as, for instance, in the knee, elbow, and phalangeal joints. A more close connection sometimes exists-as where tendinous fibres or processes (aponeurosis palmaris and plantaris, linea alba), or muscles (palmaris brevis, levator labii superioris alceque nasi, levator labii superioris, \&c.), are inserted into the skin; sometimes, -as where the innermost layers of the subcutaneous cellular tissue are blended, as it were, by means of short, strong, filaments of connective tissue with the subjacent muscle, fascio, tendons, \&c., particularly, therefore, on the head, especially on the alce nasi and lips, the forehead and temples, the ear, mouth, and occiput; on the glans penis, beneath the nails, \&c. In general, where the fat forms a thick layer, the skin is less movable than when from any cause it is less abundant or entirely absent.

The external surface of the subcutaneous cellular tissue, is connected by means of numerous filamentary processes of connective tissue, with the corium, and is not everywhere clearly distinct from it; but a separation between the subcutaneous cellular tissue and the corium may be pretty readily effected, especially when the former contains an abundance of fat, with the exception of certain situations (head, cheeks, chin, \&c.), where the follicles of the larger and more closely set hairs penetrate deeply into the panniculus adiposus. The subcutaneous cellular tissue of the penis, scrotum (dartos), \&c., passes into the corium without any distinct limitation.

The thickness of the subcutaneous cellular tissue varies very considerably, as is well known, according to situation, age, sex, and the individual. The fatless subcutaneous cellular tissue of the eyelids, and of the upper and outer part of the ear, measures, according to Krause $\frac{1}{4}$, on the penis $\frac{1}{3}$, on the scrotum $\frac{2}{3}$ of a line. The panniculus adiposus is 1 line thick on the cranium, brow, nose, lobe of the ear, neck, dorsum of the hand and foot, the knee and elbow; in most other situations it is 2 to 6 lines, though in fat persons it may exceed 1 inch in thickness, and in thin ones may sink below 1 line.

$\S 33$. The proper corium is a tough, slightly-elastic membrane, and is 
composed principally of connective tissue, which in the thicker parts presents two, though not very well-defined layers, which may be designated the "reticular" and the "papillary" portions ( $p$. reticularis and $p$. papillaris). The former constitutes the inner layer of the corium, and consists of a white, reticulated membrane, frequently distinctly laminated in its deeper portions, and containing in special, narrow or wide, scanty or numerous meshes, the hair follicles and cutaneous glands, together with much fat. The papillary part of the corium is the reddishgray external superficial layer (Fig. 45 ), which in its dense, firm tissue, contains the upper portion of the hair-follicles, and cutaneous glands, and the terminal expansions of the vessels and nerves of the skin. Its most important element consists in the cutaneous or tactile papillæ, papillce tactûs (Fig. 46); small, semi-transparent, flexible, but tolerably solid elevations of the external surface of the corium, which are ordinarily conical or clavate in form, but in certain places present numerous points (compound papilloe). With regard to their number and position, the papilloe of the bed of the nail, of the palm of the hand, and of the sole of the foot are very numerous (E. H. Weber enumerates upon 1 square line of the vola manus, 81 compound or 150 to 200

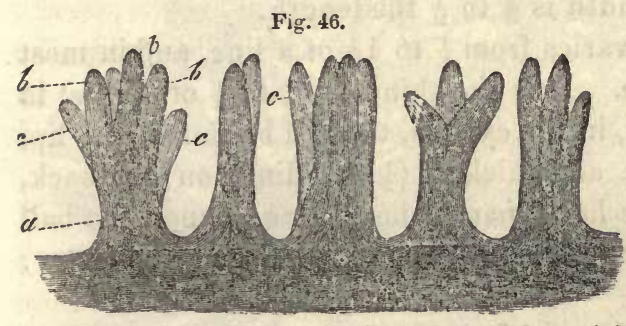

smaller papilloe), and disposed with tolerable regularity in two principal series, each of which has 2 to 5 papillæ in the transverse direction, placed upon linear elevations, $\frac{1}{10}$ to $\frac{1}{3}$ of a line broad, by $\frac{1}{20}$ to $\frac{1}{6}$ of a line high, - the ridges of the corium. The course of these ridges is visible, even externally in the epidermis,

Fig. 47.

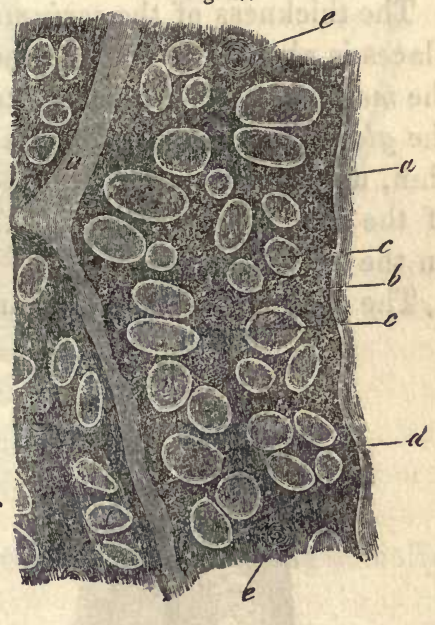

FrG. 46.-Compound papillæ of the surface of the hand, with two, three, and four points : $a$, base of a papilla ; $b b$, their separate processes; $c c$, processes of papillæ whose base is not visible.-Magnified 60 diameters.

Fra. 47.-Horizontal section of the skin of the heel through the apices of the papillæ of one entire and two half ridges. The serial arrangement of the papillæ corresponding with the ridges of the cutis, is obvious. a, Horny layer of the epidermis between the ridges, which from their undulating course are cut through in making a section through the points of the papillæ. $b$, Stratum Malpighii of the epidermis. $c$, Papillæ which are placed in more than two rows; since, however, many of them are always seated upon a common base, there are, so to say, only two rows of compound papillæ present. d, Stratum Malpighii between the papillæ belonging to a common base, which, because it has a less thickness here, appears somewhat clearer. e, Sweat canals.-Magnified 60 diameters. 
and therefore needs no further description. Elsewhere the papilloe are more irregularly scattered, either very close together, as in the labia minora, the clitoris, the penis, and the nipple, or somewhat more widely apart, as upon the extremities, with the exception of the places named, on the scrotum, the neck, chest, abdomen, and back.

The size of the papilloe varies considerably; the shortest $\left(\frac{1}{66}\right.$ to $\frac{1}{40}$, of a line) occur in the face, especially upon the eyelids, brow, nose, cheeks, and chin, where they are even wholly wanting, or are replaced by a network of depressed ridges; next upon the female breast $\left(\frac{1}{80}\right.$ to $\left.\frac{1}{60}\right)$, upon the scrotum, and at the base of the penis ( $\left(\frac{1}{66}\right.$ to $\frac{1}{4} 6$ of a line). In most other situations their length is from $\frac{1}{22}$ to $\frac{1}{33}$ of a line. The longest, $\left(\frac{1}{20}\right.$ to $\frac{1}{30}$ of a line) exist on the surface of the palm of the hand, sole of the foot, and the nipple, where they are generally of the compound kind; further, the anterior and posterior extremities of the bed of the nail $\left(\frac{1}{14}\right.$ to $\left.\frac{1}{10}\right)$, and the labia minora $\left(\frac{1}{20}\right.$ to $\frac{1}{10}$ of a line). The breadth of the base in most of the papillæ about equals or is somewhat less than the length; in a few, as in those of the scrotum, prepuce, and root of the penis, it even exceeds the length by $\frac{1}{3}$ or more, whence these papillæ exactly resemble warts, or even short ridges; in the longest papillre, lastly, the breadth is $\frac{1}{3}$ to $\frac{1}{2}$ the length.

The thickness of the corium varies from $\frac{1}{8}$ to $1 \frac{1}{2}$ of a line, and in most places is about $\frac{1}{4}$ to $\frac{3}{4}$ of a line. It is the thinnest ( $\left(\frac{1}{5}\right.$ to $\frac{1}{8}$ of a line) in the meatus auditorius externus, in the eyelids, the red border of the lip, the glans penis, and clitoridis; and thickest ( $\frac{1}{2}$ to 1 line) on the back, chin, upper and lower lip, (the hairy part), the aloe nasi, upon the ball of the sole, the extremity of the great toe, the scapula, and the nates; on the heel, 1 to $1 \frac{1}{2}$ lines.

The principal chemical characters of the corium agree with those of the connective tissues, of which it is

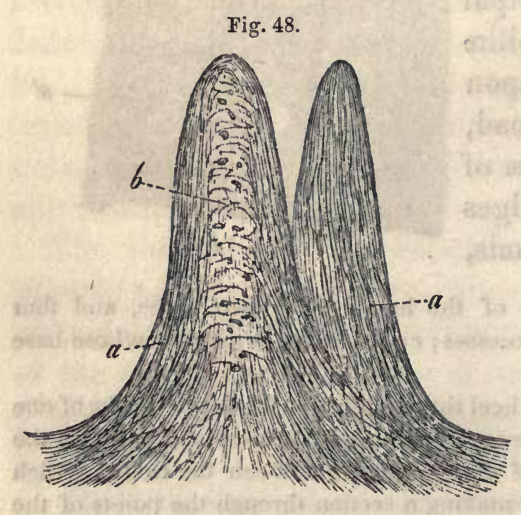
principally constituted. It putrefies with difficulty, and not at all when tanned; it may be easily dried, and then becomes yellowish, transparent, and hard, but flexible and no longer subject to putrefaction. In boiling water, it at first shrinks, eventually however dissolving, but not with equal facility in all animals, and in the young, more quickly than in the old, into gelatine, colla; and the same change is effected at the

FIG. 48.-Two papillæ of the surface of the hand, from a slightly macerated skin; magnified 350 diameters. $a$, Wavy, remarkably distinct fibrils of connective tissue; $b$, Transverse elastic fibrils lying in the axis of a papilla and transverse nuclei, axile corpuscles, the corpuscula tactûs of R. Wagner (see \$ 37); of nerves no trace is to be seen without reagents. 
ordinary temperatures, when it is treated with dilute acids and alkalies.

$\S 34$. The corium is principally composed of connective and elastic tissue, containing in addition, smooth muscles, fat-cells, bloodvessels, nerves, and lymphatics, in great abundance.

The connective tissue consists of the ordinary bundles, which are in part united into a network, as in the subcutaneous cellular tissue; in part into large secondary bundles, trabeculoe and laminoe, which, in the panniculus adiposus, circumscribe larger and smaller spaces filled with fat; whilst in the fascia superficialis, and in the corium, their connection is very intimate, and they form, especially in the latter, a very dense tissue, with indications of lamination. In the papilloe the fibrous structure is not everywhere distinct, and instead of it there often exists a more homogeneous tissue,* which frequently appears to be bounded by a

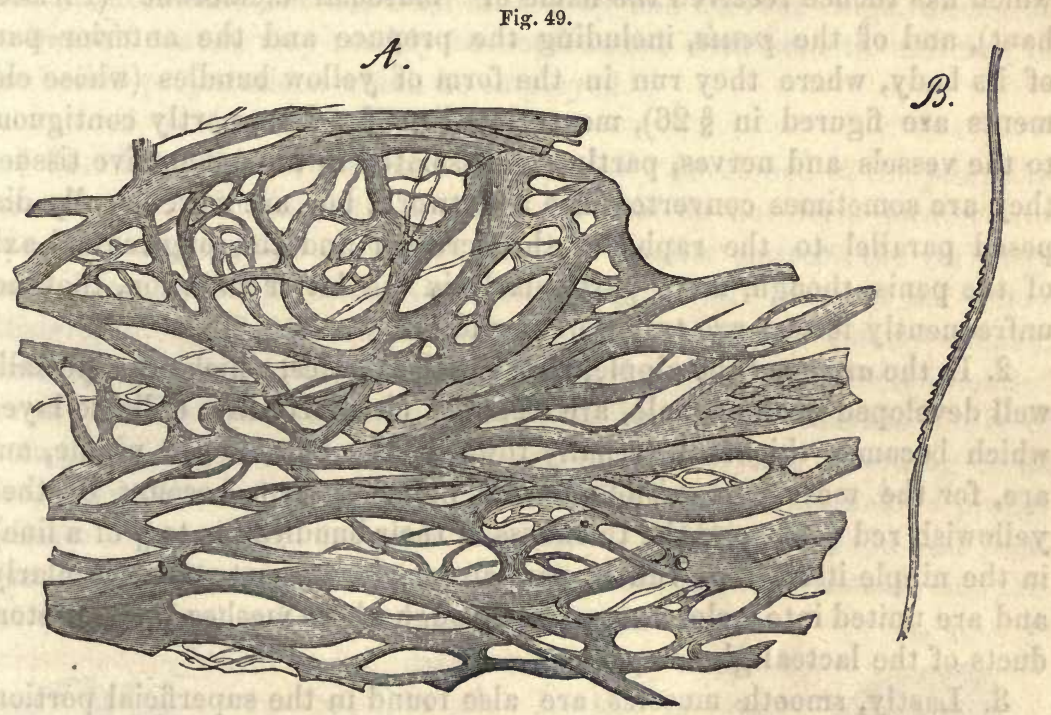

structureless membrane, which, however, does not admit of being actually isolated.

The bursce mucosce subcutanea are nothing but larger, simple, or partially subdivided reticular spaces in the subcutaneous cellular tissue, in

Fra. 49.- $A$, Elastic fibres from the inner part of the fascia lata of man, closely interwoven, and appearing like an elastic membrane; magnified 450 diameters. $B, A n$ elastic fibre with a serrated edge, such as may also be seen occasionally in the cutis.

* [The most superficial layer of the cutis is invariably composed of a transparent matrix, homogeneous or nearly so, in which nuclei are imbedded. The "indications of lamination" are simply the commencement of the breaking up of this tissue into areolated connective tissue, such as we have already described; see note, p. 83.-Trs.] 
the fascia superficialis (bursa olecranii), or between the laminæ of the fascia muscularis (bursa patelloe). The internal walls, smooth but uneven, are formed of common connective tissue, possess no epithelium, and include a somewhat viscid, clear fluid.

The elastic tissue exists abundantly in almost all parts of the cutis ; but, in general, far more sparingly than the connective tissue. More rarely it occurs in the form of true elastic membranes, which may even resemble the densest elastic networks of the arteries, as in the fascia superficialis of the abdomen and thigh; while more commonly it represents a loose reticulation of coarser or finer fibres, as in the corium. The papilloe (but not all), and the pannicuilus adiposus, in which they are sometimes wholly wanting, contain only fine elastic (nucleus) fibre.

1. Smooth muscles, according to my observations, occur far more extensively in the skin than has hitherto been supposed, and particularly in the subcutaneous cellular tissue of the scrotum, or the tunica dartos, which has thence received the name of "muscular membrane" (Fleischhaut), and of the penis, including the prepuce and the anterior part of its body, where they run in the form of yellow bundles (whose elements are figured in $\S 26$ ), measuring $\frac{1}{3}$ to $\frac{1}{2}$ a line, partly contiguous to the vessels and nerves, partly more isolated in the connective tissue; they are sometimes converted into a network, but are more usually disposed parallel to the raphe of the scrotum and the longitudinal axis of the penis, though, more particularly in the latter situation, they not unfrequently form large transverse bundles.

2. In the areola of the nipple, the smooth muscles, which are especially well developed in the female, are disposed circularly in a delicate layer, which becomes thicker internally towards the base of the nipple, and are, for the most part, visible to the naked eye, on account of their yellowish red color, and the thickness of their bundles (up to $\frac{1}{3}$ of a line); in the nipple itself they run in part circularly, in part perpendicularly, and are united into a close network, through whose meshes the excretory ducts of the lacteal glands pass.

3. Lastly, smooth muscles are also found in the superficial portions of the corium, and in fact in all situations where hairs occur, in the form of flat bundles, $0 \cdot 1-0 \cdot 16$ of a line broad, which, singly or in pairs, are invariably placed near the upper part of the hair-follicles and sebaceous glands.* They arise, probably, from the superficial part of the corium, and running obliquely from without inwards, towards the hairfollicles, surround the sebaceous glands, and are inserted close behind and near the base of those glands into the hair-follicles.

Quite recently Eylandt and Henle have added to our knowledge of the smooth muscles of the skin. The existence of the little muscles of

* [Smooth muscular fibres have also been found in the scalp. See note p. 104.-DaC.] 
the hair-follicles, termed by Eylandt, arrectores pili, has been confirmed by both writers, only that they find them to be more delicate (Eylandt 0.02 , Henle 0.04 of a line). Eylandt never noticed more than one bundle passing to a hair-follicle, and Henle states that they subdivide upwards into many bundles of 0.004 of a line, and may be traced im. mediately under the epidermis as far as the papillæ. In the scrotum, in the skin of the penis, the perinceum, the areola mammoe, and in the nipple, Eylandt could not find smooth muscles; and he imagines that I have confounded the circular muscles of the vessels with them, a supposition which I should not have allowed myself to entertain even against a beginner. Henle has seen the smooth muscles in all these situations, which it is, in fact, very easy to do, though I think that he goes to the other extreme in assuming the existence of smooth muscular fasciculi in the hairless portions of the skin also, in the sudoriparous glands, and in the vascular ramuscules (on their exterior), and, I believe, that in these cases, he has been misled by fine nervous twigs, which, as he himself states, may readily be confounded with smooth muscles, in the boiled preparations which he employed.

\$35. Fat-cells.-These cells are especially developed in the panniculus adiposus. In this situation the fat-cells do not form large continuous expansions, but occupy, in larger or smaller clusters, the variously formed meshes of the connective tissue (Fig. $45 f$ ). Each of the yellow clusters, or fat-lobules, which appear to the naked eye clearly defined, has a special coating of connective tissue, in which the ressels intended for the nutrition of the fat-cells are distributed, and consists either of a simple aggregation of cells, or of a number, varying according to its size, of smaller and smallest lobules, each of which again has its proper delicate investment of connective tissue. According to Todd and Bowman, every cell even, has its own special covering and vessels; but this

Fig. 50. though true in many cases, is certainly not so
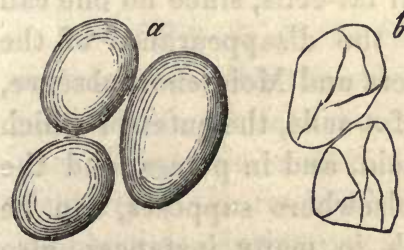
in all. In the corium the fat-cells are found more in the deeper part. 6 round the hair-follicles and sebaceous glands, while they are wholly wanting in the pars papillaris. In persons in tole-

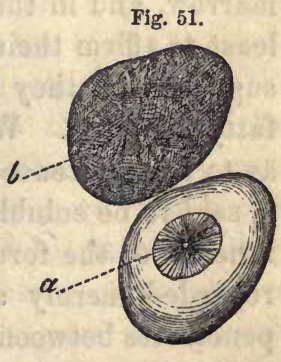

FIG. 50.-Normal fat-cells from the breast; magnified 350 diameters: $a$, without reagents; $b$, after being treated with ether, whereby the fat is exhausted, and the folded delicate membrane remains.

FIG. 51.-Fat-cells with erystals of margarin; magnified 350 diameters : $a$, cell with a star of crystalline needles, as they may be found not uncommonly in normal fat; $b$, cell quite filled with crystals, from the white fat-lobules of an emaciated subject. 
rably good condition, the fat-cells are always rounded or oval, $0.01-0.06$ of a line in diameter, with a dark border, filled with fluid, pale yellow fat, which forms a single drop-and with a parietal nucleus which is not readily rendered visible (Fig. 50). In emaciated subjects, on the other hand, hardly any cells of this kind are met with, but instead, more or less abnormal forms: 1. Granular cells, with numerous small fatdrops, forming whitish-yellow clustered lobules; 2. Fat-cells containing serum, in yellow or reddish-brown minute lobular masses, which, together with the fat (which has become more or less diminished in quantity, and usually appears as a single dark-colored globule), contain a clear fluid and a distinct nucleus, and are considerably smaller than the normal cells, $0.01-0.015$ of a line; 3 . Cells which contain no fat, but only serum, with a distinct nucleus, and having a delicate or thickened membrane; they occur in more gelatinous fatty tissue, or mingled with the others; they are also met with in oedema; 4. Lastly, Fat-cells containing crystals, either presenting 1 to 4 stars of acicular crystals (margarin), together with a drop of fat, or being completely filled with crystalline needles. The former occur among our normal cells, the latter only in the white, more isolated, fat-lobules.

The nuclei in the fat-cells of the adult have not, as far as I am aware, yet been observed, excepting by Bendz (Almind. "Anat." p. 122, tab. I. fig. 4), who rarely, very rarely, noticed even two pale nuclei with nucleoli. It is true that Mulder (p. 601), states that they are furnished with one, rarely with two, nuclei, but Donders and Moleschott (ib., p. 602 , et seq.), upon whom Mulder appears principally to rely, expressly say that they did not detect the nuclei; nor does Donders (in the "Holländ. Beitr.," I. pp. 57,61), say anything about nuclei. I invariably find them when the fat has partially disappeared from the cell. In cells completely filled, I first distinctly noticed them, in some cases in the marrow, and in the fat-cells in the muscles; but I do not hesitate in the least to affirm their constant occurrence in all fat-cells, since no one can suppose that they are not formed until after the disappearance of the fatty contents. With respect to what Donders and Moleschott observe, as to the existence of two membranes in the fat-cells, the outer of which is said to be soluble in concentrated acetic acid, and in potass, and the inner not; the former, as Donders himself elsewhere supposes, can be regarded merely as connective tissue, which, in many instances, also penetrates between the separate cells and connects them together, or, probably, is occasionally replaced by a homogeneous connective substance (modified cytoblastema). The crystals in the fat-cells are considered by Vögel to be margarin. As the forms of margarin and margaric acid are very similar, the question can be decided only on chemical grounds, and these appear to favor the latter. 
The pathological conditions of the fat-cells, although as yet but little investigated, corroborate my assertion of the constant occurrence of the nucleus. Without relying upon Schwann's observation, that the fatcells of the subcutaneous cellular tissue of a rachitic child a year old, all contained a nucleus, I would more particularly adduce the condition of the fat-cells in cutaneous dropsy. In this affection, as long as the fat in the panniculus adiposus has not entirely disappeared, cells containing serum, and but a small quantity of fat, are extremely abundant, and exactly of the same form as those which are found in emaciated subjects, all with distinct nuclei; and, besides these, there are numerous cells containing nothing but serum and also nucleated. In cases where the fat may be said to have altogether disappeared, and the colorless subcutaneous cellular tissue is infiltrated throughout with water, I find the lastmentioned cells in greatly preponderating quantity, and associated with them, others of peculiar form. In the first place, fusiform or stellate cells, with from three to five irregular, often tolerably long processes, with a distinct nucleus, and mostly only scanty and minute dark fatgranules; these, as the very numerous and various transitionary forms indicate, being developed froin diminished cells containing serum, and from which the fat has been partially or wholly removed; secondly, roundish or elongated minute cells (0.003-0.006 of a line) closely filled with dark granules, and without a visible nucleus, which, as is also easy to be perceived, owe their origin to a diminution of the fat-cells coincident with a change in their contents, and, on the other hand, are metamorphosed into the cellules with little or no fat, and containing serum, with which they are found associated. I may also mention that, in the inflamed medulla in the articular ends of the bones, as, according to Hasse, appears to be the case in rheumatism, I have seen the common fat cells transformed into round and even fusiform cells, containing serum and little fat, and occasionally furnished with nuclei. (From Kölliker, "Mikrosk. Anat.," Vol. II. p. 18.)

$\S 36$. Vessels of the Skin.-In the subcutaneous cellular tissue the arteries entering the skin give off many branches to the hair-follicles (see below), the fat-lobules and the smooth muscles, which, for the most part, form wide-rneshed networks of fine capillaries; more rarely, particularly in the fat-lobules, the network is closer. More externally they supply the sudoriparous and sebaceous glands (see below), and also form terminal expansions in the inner part of the corium (pars reticularis), but not many: finally, they penetrate into the outermost part of the papillary layer, and into the papilloe themselves, where they terminate in a close network of capillaries with narrow meshes. This consists, wherever there are papillae, of two portions; firstly, of a horizontal 
plexus lying immediately under the surface covered by the epidermis, and which is composed of larger vessels (of $0.01-0.005$ of a line) with
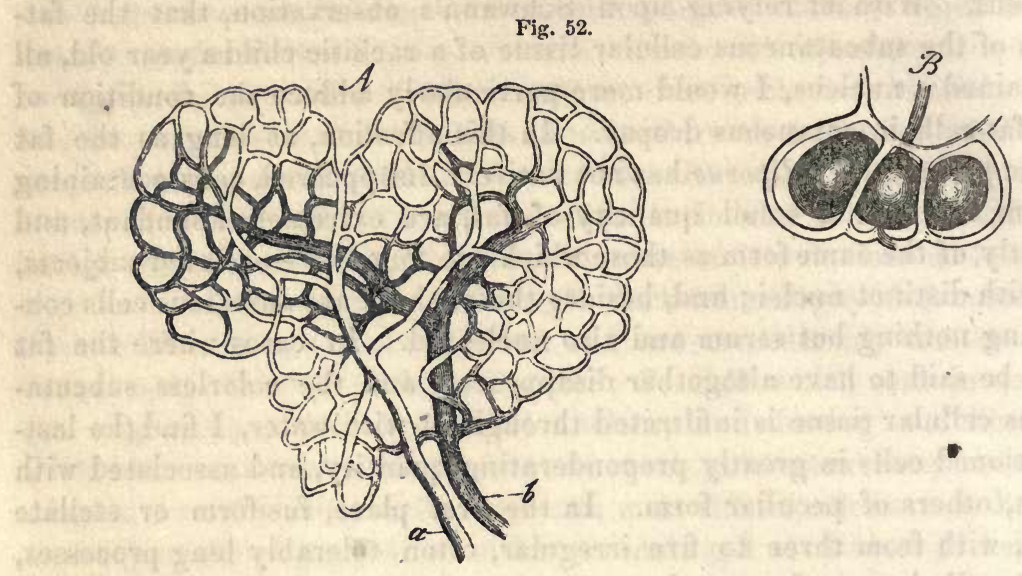

wide, and of capillaries (of $0.003-0.005$ of a line) with narrow meshes; and Fig. 53.

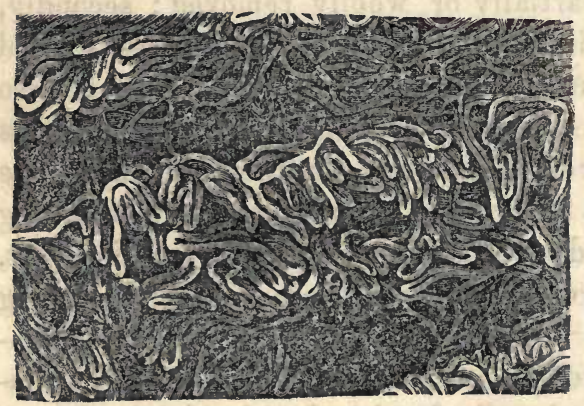
secondly, of very many separate loops of the finest vessels $\{0.003$ -0.004 of a line) which are given off to the papilloe. With certain exceptions ( $v$. $\S 37)$ every papilla possesses its own capillary loop (Fig. 53), (the branched papillo have many), which runs either in the axis of the papilla or near the surface, almost as far as its apex.

The larger trunks of the lymphatic vessels are very easily recognizable in the subcutaneous cellular tissue, and are very numerous. In the corium itself different anatomists, Hasse, Lauth, Fohmann, \&c., have demonstrated the lymphatics by injecting them with quicksilver. All agree in this, that they form an excessively close network of fine vessels in its outermost part, - according to Krause (l. c., p. 111) of $\frac{1}{15}-\frac{1}{20}$ of a line in diameter; the meshes of which become wider internally, and finally open by single trunks into the vessels of the subcutaneous cellular tissue. However, it is not by any means known, whether the ressels composing these plexuses are really the true commencement of the cutaneous lymphatics.

Fra. 52.-Vessels of the fat-cells. $A$. Vessels of a small fat-lobule; $a$, artery; $b$, vein. $B$. Three fat-cells with their capillaries more magnified; after Todd and Bowman. Magnified 100 diameters.

FIa. 53.-Vessels of the papilla of one entire and two half ridges of the cutis; after Berres. 
$\S 37$. Nerves.-The skin, in those parts of it which border upon the epidermis, particularly in some localities, is one of the structures most richly provided with nerves in the human organism, whilst in its deeper parts it is remarkable for their scantiness. In the panniculus adiposus, and in the fascia superficialis, as yet, no nerves are known besides those, which, giving off a succession of branches, traverse those parts to reach the corium, or to supply the hair-glands, smooth muscles, and Pacinian corpuscles, of which we shall speak further on. In the corium itself, the trunks which enter through the meshes of the deeper layers ascend by degrees, continually ramifying, but without actually forming, terminal expansions, towards the pars papillaris. Here they anastomose frequently, and form rich terminal plexuses, in which deeper and more superficial portions may be clearly distinguished, the former consisting of fine branches still containing many primitive tubules, with wide meshes; the second of fibres single or united in pairs,

Fig. 54.

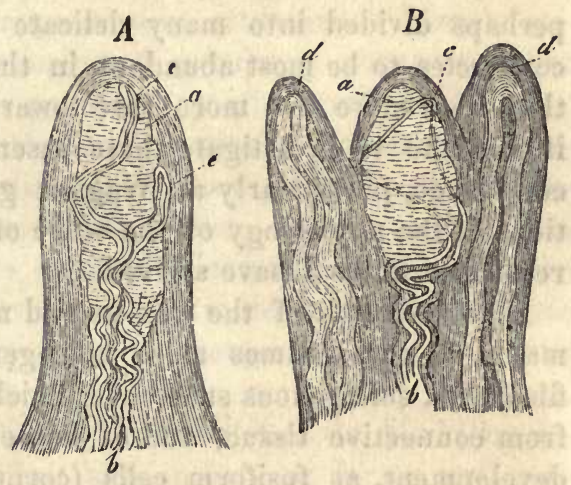
with narrow meshes. In this last or the fine terminal plexus, there also occur (whether in all the fibres is as yet undecided) in man and in animals actual divisions of the primitive tubules, so that they divide, generally at an acute angle, into two; and from the plexus itself, the tubules finally enter the base of the papilloe in pairs, in order to run to their extremitics, and there unite in a loop.

The elements of the nerves of the skin exhibit no striking peculiarities; the diameter of some, in the trunks in the subcutaneous cellular tissue, is still as much as $0.005-0.006$ of a line, and also in the deepest part of the corium, whilst they become finer and finer outwards. In the terminal plexus I find they vary according to the locality, from 0.003 to 0.0016 , in the papilloe from $0.0008-0.002$ of a line. In the hand and foot the finest tubules vary between $0.0012-0.002$ of a line; in the glans penis, in the lips and nose, on the other hand, only from $0.0008-0.0012$ of a line.

R. Wagner has recently published some statements ("Allg. Zeitung," Jan., Feb., 1852; "Götting. Nachricht," Feb., 1852), according to.

Fra. 54.-Two papilla from the extremities of the fingers, without epithelium and with axile corpuscles, $a$, and nerves, $b$. A. Simple papilla, with four nerve-fibres and two terminal loops, c. B. Compound papilla, with two vascular points with capillary loops, $d$; and one nervous point with a terminal loop, $e$. 
which the relations of the nerves of the skin have hitherto been entirely misconceived. From the investigations of G. Meissner and himself, which were instituted upon the nerves of the palm of the hand, Wagner divides the papillce into nervous and vascular. The former are said to contain a peculiar oval corpuscle in their axis, which consists of superimposed saccular or band-like laminæ, resembling a fir-cone, and this structure is regarded by Wagner, as a peculiar sensory apparatus, and named by him "tactile corpuscle" (corpusculum tactûs). Into these the nerves-1 to 3 fine dark-bordered tubules-are said to enter from below, or from the side, and to terminate within them, either free, or perhaps divided into many delicate branches. Wagner found these corpuscles to be most abundant in the points of the fingers, "and that they were more and more rare towards the wrist. I have considered it requisite to investigate these assertions, which are made with much confidence, particularly as Wagner grounds upon them great expectations of the physiology of the sense of touch, and the following are the results at which $I$ have arrived.

Independently of the vessels and nerves, the papillæ consist, in the main, of a sometimes more homogeneous, sometimes more distinctly fibrillated collagenous substance, which there is no reason to distinguish from connective tissue, and of fine elastic fibres in different stages of development, as fusiform cells (corpuscles of the connective tissue, of Virchow), cell networks, isolated fine elastic fibres and fibrous networks. These elements are so distributed, that in most papilloe a cortical layer and an axile tract can be distinguished. In the former the fibrous elements are disposed longitudinally, and the connective tissue is often distinctly fibrillated, with the exception of the most superficial layer, which forms a clear, homogeneous but not separable margin. In the latter, on the other hand, the substance is more uniform and clear, and in many places is separated from the outer layer by transverse elastic fibres. When these latter, true elastic fibres, are not very closely disposed, no one would be led to consider that there is anything peculiar in this arrangement; but undeveloped and very close together, as they are in Wagner's corpuscle, it is otherwise. These are, in fact nothing but the clear axis marked by transverse nuclei and nucleated fibres, which I have already described; and, if no reagents be added, they present no other appearance than that which I have figured in my "Microscopic Anatomy," Fig. 4, or in Fig. 48 of this work.

Dilute solution of soda, of which almost solely I have availed myself in investigating the course of the nerves in the papillæ, often does not render their contour at all more distinct, and I therefore paid no further attention to their structure; while, on the other hand, acetic acid, which was also employed by Wagner and Meissner, brings out the axes of the papillæ generally, though not always, as oval, or cylindrical, 
more sharply-defined bodies, to which the numerous transverse strix give a certain vague similarity to a fir-cone (Fig. 54). In its more intimate structure, such an "axile corpuscle," as I call it, does not consist of superimposed discs, as Wagner supposes, but of an internal mass of homogeneous connective tissue, which is most distinct in transverse sections, and when viewed from above; and of an external generally single layer of undeveloped elastic tissue, which, in the form of fusiform cells probably connected together and, more or less, drawn out into fine fibres, with shorter or longer nuclei (these last were also seen by Wagner), closely surrounds the internal substance in which here and there similar corpuscles also appear to be contained. Morphologically, then, such a corpuscle is by no means peculiarly constructed, but resembles the axis of certain other papillæ (e.g. of the sole of the foot), which are surrounded by true elastic fibres, particularly their often lessdeveloped summits; it is very similar, again, to the bundles of connective tissue, with elastic fibres wound round them, such as are found in the corium; indeed, the difference consists principally in this, that the axis-corpuscles contain more undeveloped elastic tissue; a circumstance easily comprehensible, since the papilloe, as compared with the cutis itself, consist altogether of a tissue which is in a more embryonic state.

With regard to their occurrence, axile corpuscles of the kind here described occur only in certain papillæ; and, in fact, so far as my investigations hitherto extend, only in those of the palm and surface of the hand, the red edges of the lips, and the tip of the tongue, not in those of the toes, thorax, back, glans penis, nymphre, and only rarely in those of the back of the hand and of the sole of the foot.* In the hand they

* [I have recently succeeded in detecting these tactile corpuscles in the toes. They correspond exactly to the tactile corpuscles, as described by Wagner and Kolliker in other parts, only they were far less numerous than on the palm of the hand, or on the edges of the lip. I first discovered them in the papillæ of the toe of an infant, and have twice since verified my observation. Their minute structure apparently consisted of superimposed discs, such as described by Wagner. They may be most conveniently studied in fine transverse or perpendicular sections, which have been treated with acetic acid, or a dilute solution of soda.

The nature of these so-called "tactile" or "axile" corpuscles is as yet a matter of dispute. Most recent observers seem to entertain regarding them the same views as Kolliker (see Mr. Dalzell's, in the Edinburgh "Monthly Journal of Medical Science," March, 1853; also Mr. Huxley, "Quart. Journal of Micr. Sc.," Oct. 1853, and in Appendix to this work); yet Wagner, (in his reply to Kolliker, in "Müler's Archiv. 1852,") and more recently Meissner, ("Beitrăge Zur Anatomie und Physiologie der Haut," Leipsig,1853) add additional observations in confirmation of the nature of these "corpuscula tactus" as first described by them. Meissner has presented us with some very interesting researches on the relative frequency of their occurrence in the papillæ in different parts of the body. Thus he found on a surface of 7 lines in length on the lips and apex of the tongue, only 4 or 5 ; on one square line comprising 400 papillæ of the last joint of the index finger, 108, but only 40 on the second joint, 18 on the first. In the papillæ at the root of the tongue, he was not able to discover any of these bodies.

The tactile corpuscles are probably surrounded by a special membrane, since they can be isolated. They have as yet only been observed in Man, and in the Ape.-DaC.] 
appear especially in the compound papilloe, in particular cusps, one or two together, which may project more or less, and are sometimes shorter, frequently longer; they occur more rarely in the simple papilla, as oval or cylindrical bodies, occupying $\frac{1}{3}$ to $\frac{2}{3}$ the width of the summits of the papilla, and $\frac{1}{4}$ to $\frac{1}{2}$, or as much as $\frac{3}{4}$ the length. In the points of the fingers, they occur in the proportion of 1 to every 2-4 papilloe; in the first phalanx, on the other hand, in the length of 1 line, only 2-6 are to be found, and in the palm they are still more rare. Frequently the axile corpuscles exhibit local constrictions, especially after the addition of acetic acid, are even spirally contorted, so that they often have a certain similarity to a bundle of connective tissue treated in the same way, or with a spiral sudoriparous duct. Upon the back of the fingers and in the heel there appeared, in many individuals to be no axile corpuscles in the papillæ; in a small number, however, they were to be found even here, but scattered and small, in a few papillæ. In the lips, I saw in two individuals axile corpuscles similar to those in the hand; in one they were wanting. They existed only in that part of the red margin of the lip, which is visible when the mouth is closed; they were very minute, and were placed partly in small projecting points of the larger papillæ, partly in depressions between two of their processes. In the tongue, in which, according to Wagner, something similar to his corpuscles appears to exist, I met, in two cases, with no axile corpuscles; whilst, in a third, I found them tolerably well developed in the papillo fungiformes of the point of the tongue (whether they are to be found in the posterior ones I know not), whilst they were wanting in the $p$. filiformes and $p$. circumvallate. In the $p$. fungiformes, one or many were situated in the point of the principal papilla, without extending into its simple processes, and therefore lay, as it were, at the bottom of a terminal pit, surrounded by the simple papillæ.

With regard to the course of the nerves of the skin, Wagner confirms the fact discovered by me, that even in man, the primitive tubules divide in the terminal plexuses (which I have recently also observed in the hand, the lips, and the tongue); and he further states that, in the palm at least, only those papillæ contain nerves which possess the axile corpuscles, while they have no vessels. As regards the latter important circumstance, all those who have occupied themselves more particularly with the investigation of the skin, must be aware that nerves are not to be found in all the papillæ; but seeing the difficulty of discovering the nerves in a dense organ like the skin, no one has thought it requisite on this account to depart from the old notion that every papilla contains a nerve, and is therefore a tactile process. Wagner having observed the sharply-defined axile corpuscles of the hand, appears to have been surprised that they occurred only in certain papillæ, and that these had nerves; and struck with this circumstance, adopted 
the view referred to. As for myself, having again made long-continued investigations into the skin of the palm of the hand, I find that those points of the papillæ, or those independent papillæ, which contain axile corpuscles, do generally exhibit dark-bordered nerve-tubules very distinctly; but from this I should, for the present at any rate, by no means be led to conclude that the other papillæ contain no nerves, but only vessels.

If it be considered that dark-bordered nerve-tubules, though indeed rarely in proportion, are contained in vascular papillæ without axile corpuscles, in the hand; furthermore that in other places, as in the sole of the foot and the lips, such papillæe are found ; and finally, that the investigation of the cutaneous nerves is very difficult, it seems more judicious to suspend one's judgment upon this question, especially as it is possible, that pale, non-medullated, nerve-tubules, similar to those which I discovered in the skin of the Mouse, exist in man also. However, I am by no means disinclined to agree with Wagner this far, that in the palm it is almost exclusively the papillæ with axile corpuscles which contain dark-bordered nerves, for to say the least, it is very remarkable that in these papillæ the nerves are so readily and satisfactorily displayed. As to the possible existence of non-medullated fibres in the papillæ without axile corpuscles, it is certainly too soon to express any definite opinion. With regard to the vessels, it is incorrect, unconditionally to deny their existence in those papillæ which contain nerves. In the compound papillæ it is unquestionably true, that the cusps with axile corpuscles and nerves frequently contain no vessels; at other times, however, even these contain a capillary loop, and this is still more frequently the case in the simple papilla with nerves. In the lip, the papillæ containing nerves, whether they possess axile corpuscles or not contain vessels for the most part, if not always, and there are relatively very few papillæ in which no nerves are visible. The tongue possesses vessels and nerves in all the larger papillæ; on the other hand, I have as yet been unable to discover nerves in the simple papillae buried in the epithelium. It is yet to be ascertained how the nerves are disposed in other parts of the skin. It is surprising to me, that even in the sole of the foot, dark-bordered nerve-tubules can so rarely be perceived in the papillæ, while in many situations they cannot be found at all.

Further investigations are required to determine to what extent darkbordered nerves are distributed in the papillæ of the skin; whether, perhaps, non-medullated fibres occur instead of them; or whether, in certain situations, the nerves do not enter the papillæ at all, but end in the well-known superficial plexus at their base.

With respect to the dark-bordered nerves in the papillæ of the hand, Wagner is wrong in asserting that the nervous loops which $I$ have figured are bloodvessels. He has only imperfectly seen the nerves of the papillæ in question, perhaps on account of his having preferred the use of caustic soda, which more easily destroys them. Latterly, in making 
very delicate investigations, I have used only acetic acid, and have arrived at the following results:-Each point of a papillæ, or each papilla with an axile corpuscle, generally contains two, or as frequently happens at the points of the fingers four, dark-bordered tubules, which, surrounded by a neurilemma* which has escaped previous observers, pass upwards through the axis of the papilla to reach the base of the axile corpuscle, as a fine, convoluted nervous twig of $0.006-.012$ of a line in thickness. Here the nerve frequently becomes invisible, so that, as has happened to Wagner, one may be led to believe that it enters the corpuscle, which is seated upon it, as upon a stalk, and there ends. However, if a number of fresh preparations be treated with acetic acid and examined, the conviction is soon arrived at that this is merely apparent, the nervous tubules in reality proceeding along the outer surface of the corpuscle, either as far as its point, or very nearly so. In the meanwhile they either remain together or take an isolated course. In both cases their neurilemma becomes excessively delicate, appearing finally to vanish entirely, while the nerves themselves surround the axile corpuscle, passing round it either more directly, though in a slightly undulating course, or forming one or several spiral coils (Fig. 54, B). As regards the actual termination of the nervous tubules, I retain the opinion I formerly expressed, inasmuch as, in at least six cases, I have again most distinctly seen loops (Fig. 54). It is, however, always difficult to observe them, and very frequently impossible, in spite of every exertion; and therefore, as we are all liable to error, I will blame no one for considering the termination of the nerves of the papillæ to be unknown, or for believing in the existence of free ends, which perhaps also exist, and, at any rate, very frequently appear to exist. I only state what, according to my best belief, I have seen; and while I have no prejudice in favor of loops, neither can I see anything alarming in their existence. This much, however, is certain, that Wagner has not traced the nerves in the papillæ so far as they may be traced, and therefore, at present at all events, can lay no claim to a decisive voice in the matter. How the nerves in the papillæe of the lips, tongue, and elsewhere, are disposed, I have not yet ascertained with certainty; but with regard to the first of these, I believe I can also affirm, that they do not terminate in the axile corpuscles, but either merely pass by them or wind round them. In the lips, in a single instance, I found well-marked nerve-coils in small papillæ, or at the base of the large ones.

$\S 38$. Development of the Cutis.-The following may be taken as a

* [It is by no means easy to recognize this neurilemma. Indeed, even its existence is questioned by Meissner, who states that after the most careful observation, he has not been able to detect a neurilemma in one single instance. The nerve itself he thinks does not terminate in loops, as supposed by Kolliker, but penetrates into the tactile corpuscles. The cross-strix considered by Kölliker as elastic tissue, he justly describes as the termination of the darkbordered nerve-tubules, for the action of soda on them proves them to be such.-DaC.] 
general sketch of the development in the foetus, of the cutis in the broadest sense of the term. It consists at first of cells, which though not in man, yet in animals (the Frog, for instance) may be easily traced back to the earliest formative cells of the embryo. A considerable proportion of these cells are changed into connective tissue, becoming fusiform, coalescing, and eventually being converted into bundles of fibrils; a process which appears to occur first in the fascia superficialis, the subcutaneous connective tissue, then in the pars reticularis of the corium, and finally in the papillary layer. Another portion of the cells are converted into vessels and nerves, as ean be seen even in man, and very beautifully in the Batrachia (see my Memoir in the "Annales des Sciences Naturelles," 1846); while a third, growing and developing fat in its interior, becomes elastic fibres and fat-cells (vide supra, § 23). The first foundations of all these parts having been laid, they continue to increase in a manner which is not yet exactly made out. The cutis obviously grows from within outwards (so that the papillæ arise and are developed last of all), partly by the growth of its primitive elements, partly at the expense of cells, which are perhaps mostly of new formation, and do not proceed from the original formative cells. The panniculus adiposus also increases, partly by the increase of the cells of which it at first consists, partly by the subsequent development of others, as well as of connective tissue and vessels. In this manner, the skin grows for a long time after birth. In children below seven years of age, for example, the cutis is, according to Krause, only half as thick as in the adult, until at last, though at a time which is yet undetermined, the new development of cells ceases, as at a later period, perhaps, does the extension of those elements, cells, fibres, \&c., which are already formed. The fat-cells of adults, in which the process of growth is especially obvious, according to Harting, are in the orbit twice, in the palm three times as large as in the new-born infant; whence it results that they increase in size in proportion to the parts of the body to which they belong.

In embryos of two months the skin is $0.006-0.01$ of a line thick, and wholly composed of cells. At the third month it is about 0.06 of a line, and already presents tolerably distinct connective tissue. In the fourth month the first lobules of fat appear, and the ridges of the hand and sole of the foot. From the seventh month onwards the panniculus adiposus is rapidly developed, and at birth it is relatively thicker than in the adult.

§39. Physiological Remarks.-If we attempt to harmonise the anatomical data here brought together, with the phenomena of sensation exhibited by the skin, we meet with considerable difficulties. The more intimate anatomy of the skin, as it is here detailed, fails to demonstrate nerves in all the papillæ, or even in the majority of them; and yet ex- 
periment teaches that though all points of the skin may not feel with the same delicacy, they are all nevertheless sensitive. I hoped to be able to submit Wagner's doctrine of the absence of nerves in many papillæ to experimental proof, by examining the sensitiveness of various parts of the body with the finest possible English sewing needle. At first I really thought that I had found some places which were quite insensible, whilst in others the slightest touch produced sensation; but on carrying the investigation further, it appeared that the very same place was often sometimes sensible, sometimes not; so that, finally, I came to the conclusion that the very smallest portions of the skin are sensitive. But since even in the palm of the hand the papillæ containing nerves are widely dispersed, and in other places occur but rarely, or even not at all, it only remains either to assume the existence of non-medullated nerve-fibres in all the papillæ, or to have recourse to the nervous plexus at the base of the papillæ. I should unhesitatingly prefer the latter explanation, were it not: (1) that these plexuses are in many places so very scanty, and (2) that the slightest touch of the epidermis produces sensation; for the present, therefore, I believe this must remain an open question.

If we are not in a condition to understand how it is that every point of the skin is sensitive, still less are we competent to explain the different kinds of sensations. In this respect the following very general statement may be made.

The excitement of the terminations of the nerves in the outermost parts of the cutis and the papillæ is either direct or indirect. The former as it is produced, for example, when the cutis is laid bare, by penetrating instruments and by fluids, is much more intense than that which takes place through the mediation of the epidermis, one of the functions of the latter being to act as a defence against too violent impressions, and to blunt them according to its greater or less thickness. It can now be partly explained on anatomical grounds, why the delicacy and vivacity of the sense of touch are not everywhere equal, why they are less upon the hairy scalp, the back, the two upper divisions of the extremities, than on the face, on the genitalia, the hand and foot, the chest, and abdomen. In the first place, where the tactile sense is delicate, the epidermis is in itself thin, as upon the eyelids and face, or has, at least a thin horny layer, as upon the penis and clitoris, whilst upon the back and extremities it is considerably thicker. Yet this circumstance is not a sufficient explanation, for parts with a thicker epidermis, as the palm of the hand and the sole of the foot are delicately sensitive, more so, in fact than others with a thinner covering, as the back of the hand and foot. Another condition must here obviously come into play, and it is, I think, that the skin is not equally well provided with nerves in all its parts. Simple inspection teaches that the nerves upon the palm of the hand and 
the sole of the foot are more numerous than upon the back of the hand and foot; upon the glans penis and clitoridis, the nipple, the face, they are more abundant than upon the abdomen, back, and thigh, \&c. \&c.; and this is to some extent confirmed by my measurement of the sensitive roots of the spinal nerves (vide "Mic. Anat.," p. 433). With the number of the nerres, is connected that of the actually demonstrable dark-contoured nerves in the papillæ and the superficial nervous plexus, for nowhere is this more considerable than in the points of the fingers, the lips, the tip of the tongue, and the glans penis.

As to the local sensibility of the skin, it is the province of anatomy, especially, to afford information, with regard to these two points : 1 , how it is that we do not distinguish with the same clearness and exactness, in all parts of the body, the point at which a single irritation is applied: and 2, why two stimuli operating at the same time, under certain circumstances, appear double, under others single (Weber's experiment). I think that Weber's experiment cannot be explained by the mode of distribution of the peripheral nerves, but depends very probably upon their central relations. It seems to me to be simplest to assume, that every peripheral end of a nerve is capable, when irritated, of producing a conscious sensation, but that, on account of the small number of nervous fibres (in the cerebrum) which unite these with the seat of consciousness, if many contiguous, or even more distant, cutaneous nerves are excited, only a single conscious sensation results. In this case, the nerves of acutely sensitive parts must be connected with the seat of consciousness by more numerous intermediate fibres than those of other localities, and at the ends of these fibres, also, we must suppose a sort of interlacement to take place. Upon this hypothesis, the former of the two points might be explained. A local irritation is, indeed, felt locally; but, according as the nerves implicated are united with the brain by more or fewer conductors in the spinal cord, are we able to assign, more or less exactly, the precise spot; so that, in some cases the limits of error will not exceed $\frac{1}{2}-1$ line, while in others, they may extend to 1 or $1 \frac{1}{2}$ inch and more.

E. H. Weber has endeavored to demonstrate, in his last able Treatise upon the sense of touch, that it is only the termination of the nerves in the skin, not the fibres in the nervous trunks, which are the mediators of the sensations of pressure, warmth, and cold ; and he has expressed a supposition, that tactile organs as yet unknown may exist in the skin. R. Wagner believes that he has, in fact, found these organs in his so-called corpuscula tactûs, and he supposes that, composed as they are, according to him, of superimposed membranes, in the interspaces of which a very minute quantity of fluid is lodged, like elastic cushions, or a bladder filled with water, they are specially fitted to receive impressions from the epidermis at the extremity which is directed towards it, and to transmit them to the ends of the nerves which lie in and upon them.

In my opinion, Weber's view of the greater sensibility of the termi- 
nations of the nerves in the skin, can hardly be doubted; but, on the other hand, there is no obvious reason, à priori, why peculiar hitherto unknown organs should exist to that end; nor why the condition to which I have already referred, the more isolated course of the fibres of the nervous tubules in the papillæ and terminal plexuses, their fineness, superficial position, and the delicacy or absence of the neurilemma, may not abundantly suffice as an explanation. That Wagner's so-called corpuscula tactûs, my axile corpuscles, are not tactile organs in the sense intended by Weber, is easily demonstrable. Independently of the erroneousness of his views of their structure, and of the fact that the nerves are not distributed in them, but only proceed along them, outside, in order, in many cases, to terminate even beyond them-we find that all the essential functions of the skin are also performed without such corpuscles. The feeling of warmth and cold, of orgasm, of tickling, of pressure, of pricking, of burning, of pain, are found partly over the whole surface of skin, partly in places where these corpuscles are certainly absent, which sufficiently shows that they have not in the remotest degree the signification ascribed to them by Wagner. However, it is not likely that they exist for nothing in those particular localities, in which the sensibility to pressure is the greatest, and which we use especially as tactile organs, as the ends of the fingers, the point of the tongue, and the border of the lips; and I consider them as parts, which in consequence of their being composed principally of dense, imperfectly-formed elastic tissue, confer a certain solidity upon the points of the papillæ, and serve as a firm support for the nerves, in consequence of which, a pressure, which in other situations is not sufficient to affect the nerve, here takes effect. They would, in fact, be organs, like the nails and phalangeal bones, not essential and indispensable to the sense of touch, but only conferring upon it a greater acuteness than elsewhere. If, in this sense, they are to be called tactile corpuscles, I have nothing to say against the term, but then the phalanges and the nails, the "whiskers" of animals, \&c., equally deserve the name of tactile organs.

The contractility of the skin is exhibited in the wrinkling of the scrotum and of the skin of the penis, the erection of the nipple, and the occurrence of the so-called cutis anserina. It depends upon the smooth muscles of the skin already described, which, as Froriep and subsequently Brown-Séquard and I have found, contract by electricity, inasmuch as by this means, even in the living subject, the cutis anserina, the erection of the nipple, and, in recently-executed persons, a wrinkling of the scrotum can be produced. In the erection of the nipple by gentle mechanical irritation, the whole areola becomes diminished by the contraction of its circular fibres, and thus protrudes the nipple whose muscular fibres, in this case, seem to be relaxed. Cold causes the areola and the nipple to contract, both becoming small and firm. The cutis anserina, which consists in wholly local contractions of the portions of 
the skin around the hair sacs by which their apertures are protruded conically, is explained simply by the existence of the muscles which I discovered, and which pass obliquely from the superficial part of the cutis down to the hair sacs, and when they act, extrude the sacs, and retract those portions of the skin whence they arise. The assumption of a contractile connective tissue in the skin, as well as in other parts, I must repudiate here, as I have already done (Mittheil. der Züricher Gesellschaft, 1847, p. 27), because the smooth muscles, which can be microscopically demonstrated in the skin, and whose contraction by galvanism may be experimentally shown, sufficiently account for all the contractile phenomena which it exhibits.

\section{B. EPIDERMIS.}

$\S 40$. The corium is everywhere covered by a semitransparent membrane formed wholly of cells, and containing neither vessels nor nerves, - the epidermis, which applies itself exactly to all the elevations and

Fig. $55 \mathrm{~A}$.

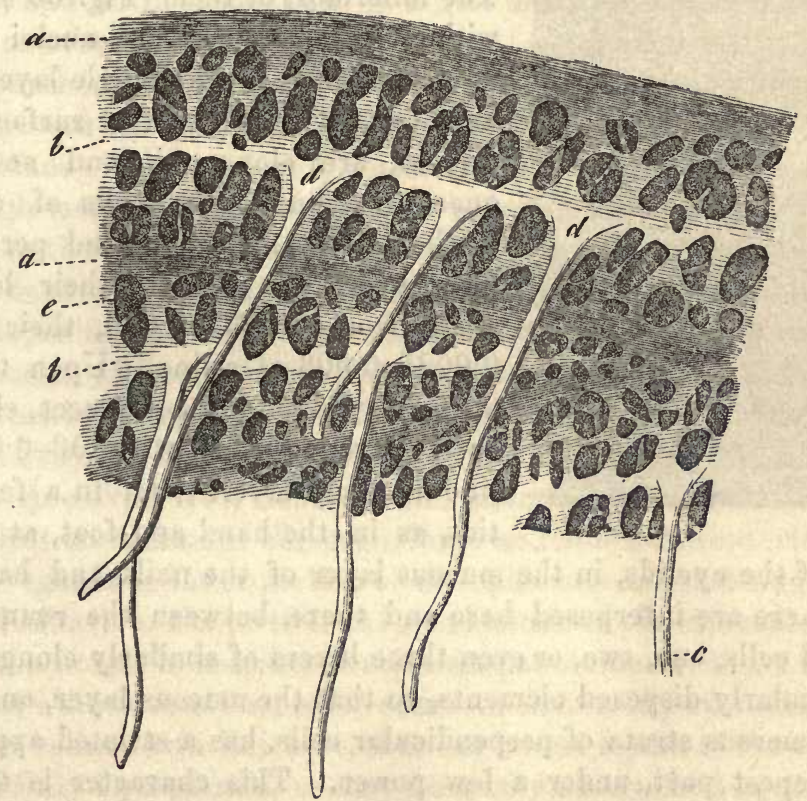

depressions, and which accordingly upon its inner surface presents an exact cast of the outer surface of the corium, the convexities and concavities of course being reversed. Upon its outer surface, also, the epidermis, to some extent, represents the form of the corium since the

Fig. 55 A.-Surface of the palm, from within: $a$, ridge answering to the groove between the ridges of the cutis; $b$, a similar one corresponding with the cleft between the rows of papillæ; $c$, sweat ducts; $d$, broader points of insertion of these into the epidermis; $e$, depressions for the simple and compound papillæ. 
more marked elevations and depressions, such as the ridges of the palm of the hand and of the sole of the foot, the folds at the joints, muscular insertions, \&c. are expressed in it,-the latter even more strungly; on the other hand, the papillæ produce either no perceptible projection, or hardly any.

The epidermis consists of two layers, chemically and morphologically distinct, and which are separated by a tolerably sharp line of demarcation, viz., the mucous layer and the horny layer.

\section{$\S 41$. The mucous layer, stratum Malpighii, rete or mucus Malpighii} (rete mucosum), of many authors, is that part of the epidermis which lies immediately upon the corium, and almost everywhere appears undulated; in many places it is distinguishable even to the naked eye by its color, which is whitish or variously tinged with brown, and it is further characterized by its small, soft, easily destroyed, peculiarly disposed cells.

Fig. 55 B.

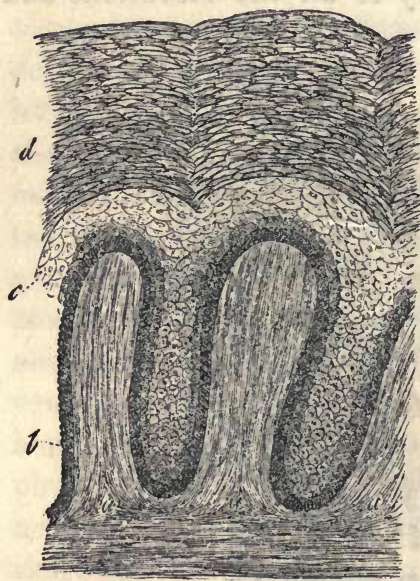

The form of these cells and their disposition are not the same in all localities. The innermost of them (Fig. 55 ), which, without interspersed free nuclei or semifluid substance, form a single layer resting immediately upon the free surface of the corium, are elongated, and not unfrequently resemble the cells of cylinderepithelium; they are placed perpendicularly upon the corium; their length is about from $0.0033-0.006$, their breadth $0.0025-0.003$ of a line. Upon these immediately follow in most places, elongated or even round cells of $0.003-0.004$ of a line in many layers; but in a few localities, as in the hand and foot, at the free margin of the eyelids, in the mucous layer of the nails and hairs (vide infra), there are interposed here and there, between the rounded and elongated cells, one, two, or even three layers of similarly elongated and perpendicularly disposed elements, so that the mucous layer, on account of the numerous strata of perpendicular cells, has a striated appearance in its deepest part, under a low power. This character is the more striking, since the other elements of the mucous layer, the further they are followed from the first, round cells outwards, become thinner in another direction, $i$. e. become horizontally flattened (Fig. $55 \mathrm{c}$ ), and finally in the uppermost layers are transformed into thick vesicles, $0.006-0.016$ of a line broad, and $0.002-0.008$ of a line thick. At the

FIG. 55 B.-Perpendicular section of the skin of the Negro (from the leg). $a$ a, cutispapillæ; $b$, deepest intensely-colored layer of perpendicularly elongated cells of the stratum mucosum; $c$, upper layer of the stratum mucosum : d, horny layer.-Magnified 250 diameters. 
same time, from their mutual pressure, they acquire a polygonal form which may even be recognized in isolated cells.

All the cells of the mucous layer agree, in essential points, in their structure, and are nucleated vesicles distended with fluid. Their membrane is pale, often difficult of demonstration in the smallest, frequently quite distinct, always delicate, thicker in the larger ones, yet by no means to be compared to that of the cells in the horny layer. The contents are never quite fluid; but also, excepting in the colored epidermis (vide infra), never normally contain larger particles, granules or fat-drops for example; but are finely granulated, with more or less clearly-defined granules, which invariably diminish in number in the more external cells. The nucleus, lastly, is small in the smallest cells, $(0.0015-0.0025$ of a line), in the large, of greater size (0.003-0.005 of a line); globular or lenticular in the round and flattened cells; elongated in the elongated cells. In the larger cells it appears obviously vesicular, often with a nucleolus, and lies centrally in the midst of the contents; in the smaller it is apparently more homogeneous, without any visible nucleolus, and so disposed that it is not unfrequently in contact with one part or other of the cell-walls.

[Most authors admit the existence of a special membrane between the cuticle and the corium. Krause (loc. cit. p. 112) describes on the upper surface of the corium a perfectly transparent, textureless, semifluid, tough mass of $\frac{1}{2} \frac{1}{0}$ th to $\frac{1}{3} \frac{1}{0}$ th of a line in thickness, which he believes to be the cytoblastema of the epidermic cells, covered by a layer of free nuclei and real cells. Henle (loc. cit. p. 1010) considers the inferior layer of the cuticle as a continuous cytoblastema with interspersed nuclei, and calls it the "intermediate skin." Such free nuclei are admitted by Bruns (loc. cit. p. 358), by Gunther (loc. cit. p. 257), by Hyrtl (p. 379), by Hassall (loc. cit. p. 242), and others, whilst Reichert (Müller's Archiv, 1845), denies their occurrence, and also that of the structureless membrane of Krause. Todd and Bowman (loc. cit. p.413, Fig. 84), regard the lower portion of the epidermis as consisting of fullydeveloped cells (loc. cit. p. 404-411), and admit as the external border of the corium a simple homogeneous and transparent membrane, "basement membrane," which view Carpenter seems to share. My own observations lead me to believe with Reichert, that the lowest layers of the epidermis consist of fully-formed cells, as may be easily ascertained in most cases by the addition of acetic acid, or diluted alkalies to fine vertical sections. We are, therefore, not justified in drawing conclusions from those cases in which the cells are not distinct, which, it is true, does not unfrequently occur; for even then the regularity of distance separating the nuclei, the traces of cell-membranes observable as delicate striæ between them, and the distinct limitation of the Malpighian stratum, as it approaches the corium, afford certain evidences of the existence of fully-formed cells. The homogeneous membrane of Krause, and the basement membrane of 
the English authors, I believe to be identical. They are but the external portion of the corium, which appears, especially around and between the papillæ, as a structureless and homogeneous membrane. A separation from the corium of this narrow, and on its under surface, by no means distinctly limited membrane, ought not to be attempted; for although a distinct membrane in the embryo, it cannot be obtained as a special layer in the adult. (From Kölliker's Microsc. Anat. vol. ii. p. 47.-DaC.)]

$\S 42$. The horny layer (stratum corneum), forms the external semitransparent part of the epidermis, which in white people is colorless, and is composed almost wholly of uniform cells metamorphosed into plates. The deepest plates are still very similar to the uppermost cells of the stratum mucosum; but even in the second or third layer we find the widely different epidermic or horny plates. They (Fig. 56, 1, 2, 3)

Fig. 56.
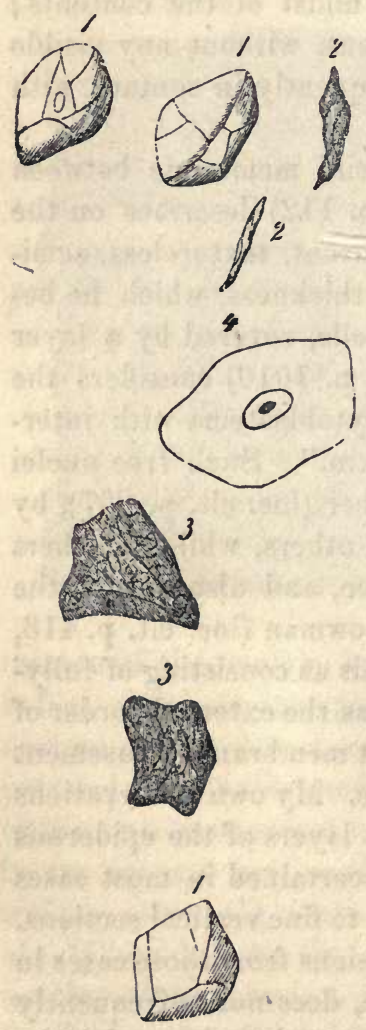
are, in fact, plates of moderate thickness, which in the lower and middle parts of the horny layer retain tolerably regular polygonal (4-5-6-sided) and smooth surfaces; in the upper layers, on the other hand, they present more irregular outlines, are variously crooked and curved, and thence often appear to be wrinkled and folded. These plates must be regarded as cells, completely flattened, and containing a very minute quantity of viscid fluid; and not, as might at first be imagined, as homogeneous lamellæ, composed throughout of the same substance; for by the addition of various reagents, especially of acetic acid and potassa, they swell up and assume the form of resicles (Fig. 57); at the same time, a rudimentary nucleus becomes obvious in a few, particularly in those from the middle and inner layers, but by no means in the majority of them, in the form of a flat, homogeneous, rounded or elongated corpuscle, $0.003-0.004$ of a line in length, and 0.0020.003 of a line in breadth, which, especially when seen from the side, is easily recognized by the dark contours which it then presents. The size of the plates of the ordinary horny layer varies from $0.008-0.016$ of a line, and in the outer layer it is commonly somewhat

FrG. 56.-Horny plates of man: 1, without addition, viewed from the surface one with a nucleus; 2, from the side; 3 , treated with water, granular and dark; 4, nucleated plate, such as occur on the outer surface of the labia minora and on the glans penis.-Magnified 350 diameters. 
greater than in the inner. Upon the body of the penis the cells measure $0.008-0.012$ of a line; upon the glans, the largest are $0.016-0.02$ of a line; upon the outer side of the labia minora $0.012-0.02$; on the labia majora $0.01-0.016$ of a line. These last, larger cells, all possess distinct nuclei, and are almost identical with the epithelial plates, e. $g$. of the cavity of the mouth and of the vagina (Fig. 56, 4).

Whilst the stratum Malpighii, except in its upper layer, is but indistinctly laminated, a clear lamination is obvious in the horny layer, inasmuch as its plates applied together horizontally, form strata in number proportionate to the thickness of the horny layer (Fig. 55). It must not be imagined that these strata are distinctly defined from one another; they are connected by their surfaces, and can only be detached and demonstrated adhering together in numbers, by dissection, which is much facilitated by boiling or macerating the epidermis. The innermost, like the stratum Malpighii, taken together, exhibit a wavy course wherever papillæ exist, projecting outwards over the points of the papillæ, and following the depressions between them. This takes place in the most striking manner where very much developed papillæ coexist with a moderately thick rete Malpighii, especially in the palm and in the sole of the foot, in which (see the figure in the section on the sudoriparous glands), the horny layer penetrates so deeply between the papillæ, that its deepest cells are on a level with half their height: where the papillæ are smaller, the horny layer sinks less deeply between them, or even lies quite flat upon the stratum Malpighii, as is the case where the papillo are absent. From this cause the boundary-line between the horny layer and the stratum mucosum in perpendicular sections, is sometimes straight, sometimes wavy, with smaller or greater elevations and depressions. The other parts of the horny layer take a more even course the further they are from the mucous layer; yet not merely in the hand and foot, where, as is well known, the ridges of the corium are marked externally upon the epidermis, but in many other localities, a slightly wavy course of the uppermost layers may be perceived in perpendicular sections, and slight elevations indicate externally the points beneath which the papillæ are seated. In the separate lamellæ the plates are sometimes irregular, sometimes arranged circularly, as around the excretory ducts of the glands and hair-follicles, and also round the papillæ on the palm of the hand and sole of the foot, as may be seen most readily at the apertures of the sudoriparous glands.

$\S 43$. As regards the color of the epidermis, in the white races, as has been said, the horny layer is colorless and transparent or slightly yellowish, the mucous layer yellowish white or brownish. The color is deepest in the areola and in the nipple, passing eren into blackishbrown, especially in women during pregnancy and after they have borne 
children; it is less intense in the labia majora, the scrotum, and the penis, where for the rest it varies greatly, being sometimes almost entirely absent, sometimes very distinct, and is least considerable in the axilla and round the anus. Besides these situations, which in most individuals are more or less tinged, in the dark-complexioned more than in the fair, a lighter or more deeply colored, frequently very dark pigment is deposited in various other localities in the stratum Malpighii; in pregnant women in the linea alba, and in the face (rhubarb-colored spots); in persons who are exposed to the sun, in the face, especially the brow, chin, and cheeks; in the neck, the thorax, the back of the hands, the forearm; and in dark persons over almost the whole body. These tints are not produced by special pigment cells, but are seated in the common cells of the mucous layer, round whose nuclei a finely granular or more homogeneous coloring matter or actual pigment granules are deposited. When the skin is only slightly colored, it is mostly only the neighborhood of the nuclei and in fact only of the lowermost layers of cells which is implicated, so that in perpendicular sections the papillæe are seen to be surrounded by a yellowish fringe; darker shades are produced by the extension of the color to two, three, four, and more layers of cells, and over the whole cell contents; sometimes by a darker coloration of the deepest layer of cells; the two conditions commonly coexisting. The horny layer also of the colored places of the skin, has according to Krause, its cell walls slightly tinged; this appears, however, only upon comparing them with those of uncolored parts of the skin, and only in the more deeply-tinged parts. In the negro, and the other colored human races, it is also only the epidermis which is colored, whilst the corium completely resembles that of Europeans. The pigment, however, is far darker and more abundant. In the Negro (Fig. $55)$, in whom, as regards the arrangement and size of the cells, the epidermis is precisely like that of the European, it is the perpendicular cells of the deepest part of the mucous layer which are darkest (dark brown or blackish-brown), and they form a sharply marked fringe contrasted against the clear corium. To these succeed clearer but still brown cells, which are accumulated particularly in the depressions between the papillæ, but are also found on their points and lateral portions in many layers; finally at the boundary close to the horny layer there follow brownish-yellow or yellow, often rather pale, more transparent layers. All these cells are colored throughout, with the exception of their membranes, and especially the parts round the nuclei, which, in the internal layers, are by far the darkest portions of the cells.* The horny layer of the negro also inclines to yellow or brownish.

* [The pigment in these cells seems of two kinds: a less intense yellowish pigment, and a dark granular pigment. The latter is irregularly dispersed throughout the contents of the cell, and in some parts it is conglomerated into small masses. It is generally especially 
In the yellowish skin of a Malay head in the anatomical collection at Würzburg, I find the same appearance as in a dark-colored European scrotum. It follows, then, that the epidermis of the colored races is, in no essential point, distinguishable from the colored regions in the white man, and it even agrees in nearly all respects with that of certain localities (the areola of the nipple, for instance).

Pathological coloration of the epidermis (freckles, mothers' marks, \&c.), according to Simon, Krause, Bärensprung, and my own observations, is produced exactly as the more intensely colored spots in the white man, and as the color of the negro's skin. Pigment deposits in the corium and in the papillæ, such as may be seen in cicatrices, after chronic inflammation of the skin, and frequently as in ichthyosis and many novi, associated with a colored epidermis, in which the pigment is developed directly from the blood-corpuscles and their coloring matter, must be carefully distinguished from the foregoing.* Numerous instances of partially or entirely white negroes and of black Europeans, not as a consequence of change of climate, but as a congenital or subsequently arising abnormal condition of the skin, have been noticed (see Hildebrandt.-Weber, II. fig. 526 ; Flourens, Compt. rendus XVII.) But, for the future, it will have to be remembered, so far as the dark coloration of Europeans is concerned, that it may also arise from a deposition of the coloring matter of the bile.

$\S 44$. The thickness of the epidermis as a whole, varies exceedingly, depending especially upon that of the horny layer. It measures :-

1-75th to 1-50th of a line, upon the chin, the cheeks, and brow, in the external auditory passages, and upon the eyelids:

1-50th to 1-25th of a line, upon the bridge of the nose, on the breast and nipple of a female, on the back of the toes and fingers, upon the neck and back, on the inner and outer side of the thigh, on the scrotum and the labia minora:

1-25th to 1-16th of a line, on the edge of the eyelids, on the male chest and nipple, the hairy scalp, the chin, penis, prepuce, and glans penis :

1-16th to 1-10th of a line, on the red external parts of the lips, on the back of the hand:

1-10th to 1-7th of a line, on the flexor side of the fingers and toes:

dense around the nucleus, which is itself but rarely recognizable, except by the aid of reagents, and does not seem to contain any of these darker granules.- $\mathrm{DaC}$.]

* [A colored epidermis may also be produced by a parasitic vegetable growth, as in the disease called pityriasis versicolor, in which the yellowish color of the epidermis, is owing to layers of filaments and spores deposited under the epidermic cells.-DaC.] 
1-3 to 1-2 of a line, on the palm: and-

$3-4$ ths to $1 \frac{1}{3} d$ of a line, on the sole of the foot, in which two latter localities the variations are greatest, independently of the circumstance, that in the furrows and at the joints the skin is thinner than elsewhere.

With regard to the proportionate thickness of the horny and mucous layers I find, in some localities, that the latter is constantly thicker than the former; i.e., in all parts of the face, in the hairy scalp, in the penis, the glans, the scrotum, the nipple, and the skin of the thorax in man; in the labia majora and minora, on the back and neck. Here the mucous layer is 3-6 times, or 2-3 times as thick as the horny layer, according as its thickness is measured from the bases or from the points of the papillae; in a few of the localities mentioned, however, the stratum Malpighii is, in its thinnest parts, of the same thickness as the epidermis, as in the glans. In the rest of the body both layers are either equal in thickness, as in the external auditory passage, and here and there upon the flexor side of the first two sections of the extremities, or the horny layer is 2 to 5 times thicker than the mucous, and in the thickest places even 10 to 12 times as thick.

The absolute thickness of the stratum Malpighii varies (at the base of the papillos) between 0.007 and 0.16 of a line; where it is thicker than the horny layer, it measures in the mean 0.04 of a line, where it is thinner, $0.01-0.02$ of a line. The horny layer by itself measures in many places only 0.005 of a line, in others 1 line or more; when its thickness exceeds that of the stratum Malpighii, it is generally about $0 \cdot 1-04$ of a line, when it is less, 0.01 of a line.

$\S$ 45. Physical and Vital Properties.-The epidermis is but little elastic, flexible in the living condition and not easily frangible, softer in the deeper than in the superficial layers. The cells contain, neither in their membranes nor between them, any demonstrable pores (apart from the sudoriparous ducts and hair-sacs, which, in a manner, have their outermost portions hollowed out in the epidermis), and form a very solid, hardly a permeable substance. Many experiments, especially those of Krause, show, that the horny layer of the epidermis permits no fluids, except those which act chemically upon it, as the mineral acids and the caustic alkalies, to pass through it, either by pores or by imbibition, or by endosmose and exosmose, while it readily takes up gaseous matters, or easily vaporizable substances (alcohol, ether, acetic acid, ammonia, solutions of chloride of iron in ether, of acetate of lead in alcohol), and gives them off (cutaneous evaporation). This conclusion is not invalidated by the undeniable passage of water, liquid substances, ointments, and even solid matter (sulphur, cinnabar), through the unin- 
jured epidermis, since in these cases a mechanical intrusion of the substances, in and through the sudoriparous ducts and hair-sacs, or their penetration into the sweat-ducts, and mingling with the sweat, explains their absorption. The mucous layer, at any rate, is easily penetrated by liquids, as is sufficiently shown by pathological anatomy (exudations which penetrate the mucous and raise up the horny layer into a vesicle the ready occurrence of absorption after the separation of the horny and the superficial portion of the mucous layer, by the action of vesicants).

In their chemical relations, it is indeed well known how the cells and plates of the epidermis behave with regard to certain reagents, but there exists, at present, no perfectly satisfactory total analysis of the epidermis, with regard to its two layers which differ so widely; and the organic combinations, also, which occur in it, are not sufficiently known.

The so-called horn, which forms the membranes of the horny plates, is insoluble in water, easily soluble in concentrated alkalies and concentrated sulphuric acid, whence the skin, if wetted with these substances, feels slippery and greasy ; there remains, however, a small residue insoluble in alkalies; concentrated acetic acid, also, dissolves it, first rendering it gelatinous, by which it is distinguished from the protein compound of the hair. It contains less sulphur than the hair and nails, which is perhaps the reason why salts of lead, mercury, and bismuth, color the hair but not the epidermis. Besides these, Mulder finds in the horny layer a gelatinous matter, which is obtained by long boiling in water, and which would appear to be of a collagenous nature. The epidermis does not putrefy -it melts in the fire without bending or swelling up, and burns with a clear flame.

The behavior of the epidermis towards reagents is particularly of importance for the microscopist, on whose behoof I add the following account.

After long maceration in water, the epidermis becomes detached in portions, and under moderate pressure is resolved into a white powder, consisting of the isolated horny plates, and the uppermost cell of the rete Malpighii. Boiled in water, pieces of the horny layer break up into their elements much more readily. Boiled in concentrated acetic acid from 15 to 25 minutes, all the horny plates become perfectly isolated, forming a cloudy, whitish deposit in the test tube; they are exceedingly pale, so that they are often hardly visible under a full illumination; and are completely swollen up and changed into globular or elongated, distended, but always more or less flattened vesicles of 0.02 0.032 of a line in breadth, and $0.006-0.01$ of a line in thickness, the nuclei when they are present being also pale and hardly to be perceived. The 
Malpighian layer becomes pale under the action of cold, concentrated acetic acid, the cells and nuclei being rendered more distinct. The cell-contents are partially dissolved: by longer action the contours of the deepest layers of cells become invisible. The same thing occurs after boiling for four minutes.

Caustic potass and soda act differently, according as the solutions used are concentrated or diluted. In the latter case, immediately after the addition of the reagent, the horny layer is rendered more clear, it swells up and changes in a short time into a beautiful tissue of pellucid, globular vesicles, without nucleus or granules, and with sharp, moderatelythick contours, $0.02-\cdot 032$ of a line in breadth, and $0.016-0.02$ of a line in thickness. If concentrated, solutions of the caustic alkalies render the plates at first smaller, so that they measure only $0.012-0.016$ of a line, and are at the same time more wrinkled and pale, but with defined, dark contours; in the course of an hour they swell up so as to appear as cells, but it takes two or three hours to give them the aspect of the plates which have been heated with dilute solutions. Boiled with these fluids, even the dry horny layer swells up, in an instant, into the most beautiful tissue of cells, without either granules or nuclei (Fig. 57), and at the same time the dissolving cell-contents mixed with the alkali, are

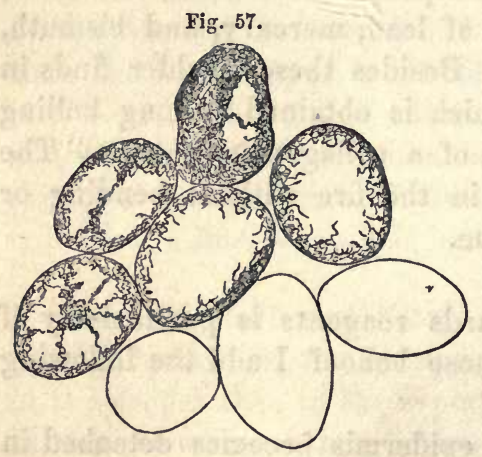
collected in the cells, into greater and smaller granular masses; after the action of the heat for five hours, all the cells disappear without leaving a trace, and yellowish and pale fat-drops in no great number, swim in the liquid. The cells of the Malpighian layer are still more acted upon by alkalies than those of the horny stratum: they swell up at once, and appear distinctly as delicate vesicles; these then dissolve, all but the uppermost two or three layers, which require a longer time, like those of the horny stratum, though less than the latter. The nuclei of all the cells withstand the operation of this reagent even less than the cells; whilst, when the latter are dissolved, a granular or striated substance remains behind, which is, probably, partly fat. Concentrated sulphuric acid, in five minutes, causes the horny layer to swell up so much, that its elements, although still remaining flattened and irregular, appear quite distinctly to be vesicles; after half an hour they are somewhat more distended, and easily separable from one another. By boiling with this acid the plates swell up

FrG. 57.-Horny plates boiled with caustic potassa and distended; the contents partially and wholly distended.-Magnified 350 diameters. 
even in a minute, without exhibiting nuclei, and in two minutes they disappear without leaving any trace. Boiling in dilute sulphuric acid renders the horny layer hard and transparent, and dissolves it wholly in 4-5 hours. The cells of the stratum Malpighii* are little altered by cold sulphuric acid: on boiling, their contours and nuclei at first become more distinct, but in about two minutes the whole is dissolved. Nitric acid colors the epidermis yellow, softens and changes it into xantho-proteic acid. The cells of the horny layers swell up somewhat, after a time, in the cold, and become granular; the stratum Malpighii is rendered granular and indistinct, and sharply defined from the horny layer. Upon boiling, the whole epidermis is entirely dissolved in half a minute. Hydrochloric acid does not tinge the epidermis, and in the cold renders the cells of

* [I have recently investigated the reaction of the pigmental cells in the Malpighian layer of the Negro, and with the following results. Macerated in water they remain unchanged. Caustic potassa renders the outline of the cell more distinct, but produces no effect on the enclosed pigment. After acting for three or four hours it dissolves the cell, and allows the dark pigment granules to escape. Boiled for ten minutes in a solution of caustic potassa, the cells become larger and more distinct; in many, nuclei and dark granules are visible; and their color becomes changed to a yellowish brown. Prolonged boiling dissolves the cells, but does not act on the granular pigment; caustic soda brings the pigment cells distinctly into view and causes them to swell up. Continued action for one or two hours, dissolves the cell and permits the unchanged black pigment to escape. Upon boiling for about four minutes in caustic soda, rupture of the cell-wall with escape of the contents ensues.

Concentrated sulphuric acid has no effect on the Malpighian layer. When first added the cells are rendered more distinct; after twenty-four hours no further effect is observable. Boiling in sulphuric acid produces no change on the cells; after a few minutes the whole epidermis is dissolved. Nitric acid when first added had no decided action; but after a few hours it rendered the cells more indistinct, dissolving some, and staining the intermediate tissue yellow. Soaked for twenty-four hours in nitric acid, the whole pigmentary layer assumes a yellow color; the separate cells are scarcely recognizable. The rapidity with which the whole epidermis when boiled in nitric acid is dissolved, makes it difficult to determine its effect upon the Malpighian layer.

Hydrochloric acid does not act as powerfully as nitric acid. At first its addition produces no effect on the cells; in the course of a few hours, they become more indistinct, but are not dissolved. The same result is obtained by allowing this acid to act for twenty-four hours. The cells are then indistinct, but of a black color; boiled for two minutes they swell up, enlarge, but are not rendered more distinct. More prolonged boiling (four or five minutes) dissolves the whole epidermis. Acetic acid causes the pigment-cells to be more easily distinguished. In a preparation that had been placed for five weeks in concentrated acetic acid, the cells were very distinct; but not otherwise altered. No effect was produced upon the pigment.

The action of Nitrate of silver, as it stains the horny tissue, cannot be well ascertained. In a preparation, that had been placed in a concentrated solution for twenty-four hours, the pigment cells, as far as they could be studied, were of a more intense color, but not otherwise altered. In alcohol and ether the epidermis is hardened and the pigment-cells of the Malpighian layer are well and distinctly seen, but these reagents exert no action on them. Boiled in ether the cells become distinct and of a lighter color. A solution of Iodine, colors the whole epidermis immediately, and thus prevents the study of its special action upon the pigmental cells of the Malpighian layer.-DaC.] 
the horny layer somewhat more distinct than nitric acid. After boiling for a minute the horny layer becomes a beautiful cellular tissue, exactly as after the addition of dilute solution of potass. In carbonate of potass the epidermis is hardly changed at all. After seventeen weeks it is hardened and easily cut with a knife. Nitrate of silver colors it violet or brownish-black, by the formation of oxide of silver, of chloride of silver, and of black sulphuret of silver, in consequence of the chloride of sodium and sulphur which it contains. Investigated microscopically with the help of acetic acid, the tissue of the epidermis is seen to remain quite unchanged, and minute dark granules are visible between its elements. Nitrate of mercury gives the epidermis a reddish-brown hue, sulphurets of the alkalies render it brown and black: many' vegetable colors unite with it. In alcohol and ether it is insoluble, with the exception of a small quantity of fat which it contains.

From all this, it results, with regard to the elementary parts of the epidermis, that they are cells, which, however, as the alkalies show, do not everywhere present the same characters. In the stratum mucosum they are actual vesicles and easily soluble-in the horny layer, scarcely so; and here, in fact, a distinction must be drawn between the resisting cell-membrane and the cell-contents, which swell up and disappear more readily; these, in the natural condition, form an apparently homogeneous simple plate, but the difference between them may be readily exhibited by reagents. In what parts the small quantity of collagenous substance, which has been noticed, has its seat, is not clear; perhaps it forms a portion of the contents, especially of the cells of the mucous layer, or belongs, it may be, to an intermediate substance between the cells, which, however, is not microscopically demonstrable. If the fat of the epidermis is not merely accidental, arising from the cutaneous secretions, it is most probably contained within the Malpighian cells.

Cruns, Todd and Bowman, Valentin and Bruch, recommend the use of alkalies for the investigation of the epidermic tissues, but their full importance was first shown by Donders (Mulder's "Phys. Chemie," p. 257, et seq., and "Holländische Beiträge," I. u. 11). They are now generally recognized as quite indispensable reagents for the investigation of the horny tissues ; but, as Paulsen ("Obs. Microchem.," \&c. Dorpat, 1848) and Reichert (Müll. "Arch," 1847, Jahresbericht.) advise, it is well always to use only definite solutions. I may add, that a great saving of time is effected by the heating of the tissues to be investigated in test tubes, with these and other reagents, as I have already done in examining those tissues of animals which contain cellulose ("Annales d. Sc. Nat.," 1846).

$\S 46$. Growth and Regeneration.-The epidermis possesses no power of continually active growth depending upon intrinsic causes and founded 
upon the vital relations of its cells, or those which it has with the corium; it is essentially a stable tissue, which does not change in its elementary parts, but, somewhat like a cartilage, has all its vital energies directed to its unchanged self-maintenance as a whole (thickness of the whole epidermis, proportion of the rete Malpighii to the horny layer), and in its separate parts. However, since a throwing-off of the external layers, if not necessarily, yet accidentally, takes place almost continually over the whole body to a greater or less extent, the epidermis is, so to speak, continually occupied in replacing what is lost, or in growing, and thus exhibits its vegetative life in a more remarkable manner. Whichever takes place, it is the corium and its vessels from which the fluids required by the epidermis are derived. In every locality, we may suppose, that a certain determinate quantity of plasma, corresponding with the anatomical and physiological relations of the vessels of the corium and the thickness of the epidermis, permeates the latter, and, when it is not growing, simply fills its cells and plates (independently of that more watery fluid which subserves the cutaneous evaporation), maintaining their vital activity, and at the most causing temporary deposits of pigment in the rete Malpighii. If, on the other hand, its outer layers be removed, a certain amount of plasma becomes free and disposable, and then regeneration takes place, which, if it proceed continuously, may even be called growth. It is in this process that the vegetative life of the epidermis-cells is most distinctly evidenced, particularly in the rete Malpighii, where it is unquestionably most intense, exhibiting itself especially in the multiplication and growth of the cells, and in their chemical changes. In the horny stratum the phenomena are less striking, though it must not be considered to be inactive even in the uppermost layer; being by no means dead matter, as we evidently see, when under certain conditions, especially under abnormal states of the coriumthe source of its nutrition-it sometimes becomes hypertrophied, sometimes completely dies away. We have not as yet, however, attained to an exact insight into the vital manifestations of the epidermic cells, and are therefore not in a condition to decide which of the phenomena presented by them are to be ascribed to their own activity, and which to the nature of the plasma which nourishes them. The latter is certainly of the greatest importance for the epidermis, and it is more than probable that most of its peculiarities, as, for instance, its typically different thickness in different parts of the body, the different relations of the stratum Malpighii to the horny layer, and its pathological states, depend upon qualitative and quantitative differences in the plasma. Upon what condition, furthermore, it depends, that in the Malpighian layer, the changes of the cells are far more considerable than in the horny layer, whose elements all closely resemble one another, is as little obvious as 
the cause of the somewhat defined line of demarcation between the two layers, a condition which appears still more strikingly in the nails, and would lead one to suppose that, at the first formation, and in the course of the development of the epidermis and nails, a very considerable alteration suddenly takes place at one point in their cells, thus determining their separation into two layers.

In the deep fold of the skin which surrounds the glans penis and clitoridis, a continual desquamation and reproduction of the epidermic scales, which are here soft and nucleated, takes place, in consequence of which a peculiar secretion, the smegma preputii, is produced. Hitherto this secretion has been erroneously, but almost universally, supposed to be a sebaceous matter secreted by the preputial glands. The microscope shows: 1, that in the female, where the presence of smegma preputii is constant, neither sebaceous nor any other glands exist upon the prepuce or glans clitoridis; 2 , that in the male, in whom such glands are indeed found, they are commonly but insignificant in relation to the quantity of smegma, and are often very few and scattered; 3, finally, that the smegma, in both sexes, consists principally of cells of the same form as those of the prepuce and glans penis and clitoridis; whence, taking also into account the fact, that in the male it is generally distinctly composed of superimposed layers covering the whole prepuce continuously, whilst the sebaceous glands occur only isolated, it naturally follows that the smegma is principally constituted of desquamated epidermis. However, this does not exclude the preputial sebaceous matter in the male from also taking a share in proportion to the number and size of Tyson's glands, in the formation of what goes under the common name of smegma. There would in this locality then, really be a constant desquamation of the external, and a new development of the internal layers of the epidermis, but here there are special purposes in view which elsewhere do not enter into consideration. The preputial fold, in fact, is to be compared to a gland; and as the secretions of these are very often formed only by the continual casting off of the cells which line them (e. $g$. sebaceous glands), so is that of the prepuce. We must recollect that in many animals, e.g., the Weasel, the Beaver (E. H. Weber), without essentially changing the character which it possesses in man, the prepuce takes on a highly glandular nature, and that even in man it yields a secretion which differs considerably from common epidermis. According to Lehmann, the yellow, fatty, strongly-odorous preputial smegma of man contains, when dried, in 100 parts : Ethereal extract, $52 \cdot 8$; alcoholic extract, $7 \cdot 4$; aqueous extract, $6 \cdot 1$; earthy salts, $9 \cdot 7$; albuminous substances soluble in dilute acetic acid, $5 \cdot 6$; insoluble residuum, 18.5. The ethereal extract contained saponifiable fat, choles- 
terin, a non-saponifiable and uncrystallizable fat and bilin (Gallenstoff). The smegma of the horse possessed nearly the same constituents; and among the salts, oxalate of lime; while in man, ammonio-phosphate of magnesia occurred. The watery extract contained neither albumen nor casein.

An extensive desquamation of the entire horny layer of the epidermis, such as takes place in the embryo and in many animals, does not occur in man except in certain morbid states. On the other hand, its power of regeneration is exhibited in other modes than those which have been mentioned. Excised portions of the epidermis, for instance, are very readily replaced, and with tolerable rapidity, so long as the corium be not injured; and, in fact, not by the immediate deposition of epidermis in the wound, but only by the growing up of the whole epidermis from below. If the corium be injured as well, an epidermis is, indeed, formed upon the substance of the cicatrix, but without any of the previous elevations and depressions of the internal and external surface, because the new corium has neither papillæ nor ridges. If the epidermis be raised up into a vesicle by acrid substances, e.g., Tartar emetic, a slight burn, \&c., the wall of the vesicle, which consists of the horny layer and some few layers of cells of the mucous layer, never again becomes adherent; but from the main substance of the mucous layer, which mostly remains lying upon the papillæ, a new horny layer is by degrees developed.

If we inquire more minutely into the mode of regeneration of the epidermis, there can, in the first place, be no doubt that it takes place in the Malpighian layer, inasmuch as losses of substance of the horny layer, $e . g$. a piece cut out, are restored not by the formation of a new portion in the gap, but by the growth outwards of a horny layer from below (the wound remaining wholly unchanged), which gradually raises the bottom of the wound, and brings it to a level with the surrounding epidermis, the latter, in consequence of the pressure that it suffers from the growing portion, becoming everted and exfoliating. The reason of this phenomenon is to be sought, simply in this, that the non-vascular epidermis draws the materials which it requires for its nutrition and regeneration from the superficial vessels of the corium. It is more difficult to ascertain from what portion of the Malpighian layer the regeneration proceeds. If a layer of cytoblastema and of free nuclei existed upon the surface of the corium, as many authors suppose, we might acquiesce in the view, that the epidermis grows by free cell-development in those innermost layers which rest immediately upon it; but such a cytoblastema, as we have seen, does not exist, the stratum Malpighii being invariably formed by perfect cells; and thence nothing remains, but to suppose an endogenous cell-development around portions of contents in 
the deepest round cells, or a multiplication by division, for which latter view the occasional occurrence of two nuclei in some of the softer epidermic cells, seems to speak. It can be more easily made out, how, in the course of the growth of the epidermis, the youngest epidermic cells become changed into horny plates. The small and round vesicles of the deeper layers of the stratum Malpighii become larger and flatter the more they approach the surface, until at last they are completely converted into flattened plates. In the meanwhile their nuclei at first grow a little, and then, as a general rule, disappear wholly in the horny layers; whilst the cell-contents, which are granular in the mucous layer, clearly distinct from the cell-mernbrane, and probably semi-fluid, become more solid and homogeneous in the horny layer, and finally coalesce with the cell-membranes. At the same time the latter are chemically altered, becoming less and less soluble in caustic alkalies.

§47. Development of the Epidermis.-The first layers of the epidermis are developed, in the Mammalia, by the metamorphosis of the most superficial of the original formative cells which compose the young embryo. When the rudiments of the stratum Malpighii and horny layer are once indicated, the former continues to increase in thickness, in consequence of the multiplication of its elements, whilst the horny layer, for the increase of its proper substance and to replace what it loses by desquamation, recruits itself from it exactly as in the adult. How the multiplication of the cells goes on in the rete Malpighii has not been directly observed; but it is certainly not by free cell-development, since in embryos of all ages the mucous layer consists wholly of cells, and free nuclei are altogether absent. As regards the horizontal extension of the epidermis, it appears, as Harting ("Rech. micrométr," p. 47) justly observes, from the circumstance that the epidermic scales of the fotus and of the adult differ very little in superficial size, that it can only in a very slight degree be ascribed to the growth of its elements. In fact the horny plates of the embryo of fifteen weeks already measure 0.009 -0.012 , in the sixth month $0.01-0.012$, in the seventh month 0.01 0.014 , in the new-born infant $0.012-0.016$, in the adult $0.008-0.016$ of a line. Since, however, keeping in mind the structure of the horny layer, it cannot well be supposed that new scales are continually intercalated from below between its elements, and since a superficial multiplication of the cells of the rete, which also do not increase in size, must certainly be granted, it seems impossible to admit any other conclusion than that, in agreement with the great superficial growth of the cutis and of the rete, and the small extensibility of the horny layers, a series of desquamations of the latter take place, which, if this view be correct, must likewise obtain after birth. 
In an embryo of five weeks I found, in the place of the epidermis, nothing but an external layer of polygonal cells of $0.012-0.02$ of a line in diameter, and an internal layer of small cells of $0.003-0.004$ of a line; in embryos of fifteen weeks the epidermis is $0.01-0.012$, of a line thick, and composed as before, only that the deep layer of cells answering to the stratum mucosum is often already double, and the external cells measure only $0.009-0.012$ of a line.

In the fifth month the epidermis in one instance measured on the heel and ball of the hand $0.02-0.024$ of a line, over the ridges of the cutis $0.036-0.04$, in the furrows between them on the back $0.02-0.024$ of a line, of which thickness one-third may be regarded as belonging to the horny layer and two-thirds to the rete Malpighii. In a somewhat older embryo it was, on the heel $0 \cdot 06-064$ of a line (mucous layer $0 \cdot 05$, horny layer $0.01-0.014$ ), on the surface of the hand 0.05 of a line (mucous layer 0.04 , horny layer 0.01 ), on the back $0.02-0.024$ of a line (mucous and horny layers of equal thickness). The mucous layers consisted of many layers of smaller cells, the lowest of which were already elongated,and stood perpendicularly; the horny layer, of at least two layers of polygonal flattened cells, with round nuclei.

In the sixth month the epidermis upon the thorax measures $0.02-$ 0.022 , on the palm of the hand 0.06 , on the sole of the foot 0.07 of a line, and everywhere it consists of many layers of cells. The outermost one or two are composed of horny plates without nuclei $0.01-0.14$ of a line, perfectly similar to those of the external horny layer in adults; then follow 3-4 layers of polygonal cells, the largest $0.01-0.012$ of a line, with nuclei 0.004 of a line; finally a mucous layer, whose thickness equals about one-half or two-fifths that of the whole epidermis, with at least 3 or 4 layers of rounded cells of $0.003-0.004$ of a line, the lowest of which are somewhat elongated, and are seated perpendicularly upon the cutis.

In the seventh month, I found in one embryo, that the epidermis on the heel measured 0.12 of a line (mucous layer 0.072 , horny layer 0.048 ), upon the back 0.07 of a line, (mucous layer 0.04 horny layer 0.03 ): in another it measured on the heel $0.12-0.14$ of a line (mucous layer $0.05-0.06$, horny layer $0.07-0.08)$, on the knee $0.046-0.064$ of a line (mucous layer $0 \cdot 016-0 \cdot 024$, horny layer $0 \cdot 03-0 \cdot 04$ ). Both layers of the epidermis are as sharply separated from one another as in adults, and their elements similar to those of the perfect epidermis, especially the lowest parts of the stratum Malpighii and the plates of the horny layer, which have no nuclei, and measure $0.01-0.014$ of a line in the uppermost layers:

In the new-born infant, apart from the thickness of the epidermis, which in one case measured on the heel $0 \cdot 1-0 \cdot 11$ of a line (mucous layer $0.04-0.05$, horny layer 0.06 ), nothing particular is to be observed, 
except that by maceration, \&c., it is much more easily separated from the corium than in the adult. The non-nucleated horny plates measured $0.012-\cdot 016$, on the labia minora, where they possess nuclei $0.016-0.02$ of a line.

During embryonic life a desquamation of the epidermis occurs, which is perhaps repeated several times. Such is the fate, probably, of the layer of polygonal cells which arises first of all, and which in the second to the fourth months, becomes metamorphosed into an almost structureless membrane, and is then no longer to be found; perhaps also of the layer of epidermis, which covers the points of the hairs which have not yet appeared externally (vide infra § Hairs); and in the second half of the foetal period it may be easily demonstrated as an actively occurring process. From the fifth month onwards, in fact, continually increasing desquamation of the external epidermic cells takes place, and these becoming in most parts mixed up with the sebaceous secretion of the skin, form the so-called vernix caseosa or smegna embryonum. This is a whitish or yellowish, viscid, inodorous material, which, especially from the sixth month onwards, covers the whole surface of the foetus with an often considerably thick and even laminated substance, which is most abundant upon the genitalia, on the flexor side of the joints (axilla, knee, nates), on the sole, the palm, the back, the ear, and on the head in large quantity, and when microscopically examined consists mainly of epidermic cells, but also contains sebaceous cells and fat globules. According to Davy ("Lond. Med. Gaz.," March, 1844) the vernix caseos $\alpha$ contains in 100 parts, 5.75 elain, 3.13 margarin ( 8.88 fat); the rest, 91.12 per cent., must be reckoned as epidermic scales, for since the vernix caseosa contains no free fluid, the 77.87 per cent. water and 13.25 solid substance found by Davy must be laid to the account of epidermic cells. This also holds good of Buek's analysis ("De Vernice caseosa," Halis, 1844) who found in 100 parts, 10.15 fat, 5.40 epithelium, and 84.45 water (so that there was 89.85 of epithelium); and also in two other cases, in which the water was not exactly determined, he found 14.80 and 9.31 per cent. of fat, and therefore 86.20 to 89.69 of moist epithelium. According to Buek, the fat of the vernix caseosa contains no cholesterin, as had been stated by Fromherz and Gugert, but oleic acid, and either stearic or margaric acid, which are probably not free, but combined with glycerine,-a circumstance which also evidences its origin from the sebaceous glands, in which, normally, no cholesterin is formed. Lehmann found (l. c.) in the dry vernix caseosa of a nearly full-grown fotus, $47 \cdot 5$ per cent, of ethereal extract, $15 \cdot 0$ of alcoholic extract, $3 \cdot 3$ of watery extract, $4 \cdot 0$ of acetic acid extract (earthy phosphates and albuminous substances), epidermis and lanugo 23.7. In the ethereal extract the reaction of bilin was absent and the fresh vernix contained a large quantity of water, which in all probability had entered 
its cells from the liquor amnii. The smegma generally appears about the sixth month, varies greatly in quantity, and is, especially in newlyborn infants, sometimes very greatly developed (as much as $3 \frac{1}{2}$ drachms, Buek), sometimes wholly wanting; in which latter case it either becomes mixed with the liquor amnii, which in fact often contains epidermic cells as well as fat (Mark, in Heller's "Archiv," 1845, p. 218), or may have been from the first, less dereloped. After birth the smegma is thrown off in the course of from two to three days, and the permanent epidermis appears, of whose further changes up to the adult state there is little to be said. In a child four months old the epidermis measured:-

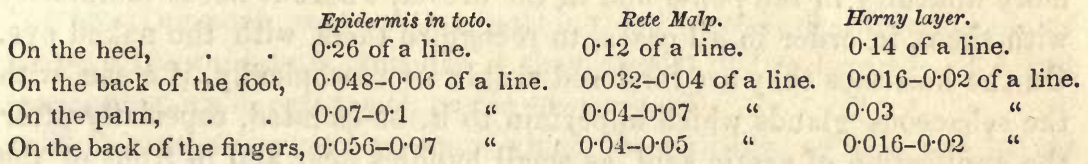

On comparing this with the adult, it is to be observed that the epidermis of the young child is disproportionately thick, and that this thickness depends especially upon the rete Malpighii, whilst the horny layer exhibits only a slight development. The pigment of the rete Malpighii arises, in the colored races, only after birth. P. Camper ("Kleinere Schriften," 1782, Bd. I. p. 24) saw a negro child, which at its birth was reddish, and hardly differed from that of a European, rapidly become tinged black at the edges of the nails and round the nipple. On the third day the genitalia became colored, and on the fifth and sixth the blackness spread over the whole body. In Europeans also, at birth, the pigment of the areola and of the other places which have been mentioned, is not yet present: it is gradually developed in the course of the first year, so that in children of two or three months old it is only indicated.

In investigating the skin, perpendicular and horizontal sections of fresh, dried, and boiled preparations are serviceable: they may be moistened with an indifferent fluid or with various reagents, the most important points in regard to whose effects have been noticed in the foregoing paragraphs. The epidermis is separated from the corium by maceration, by boiling, and where it is not thick, as on the genitalia, by acetic acid and soda, easily and in large flakes, so that its lower surface and the papillæ of the corium become visible in the most beautiful manner, and the latter may be examined singly or in groups. In the fresh skin their position and number are quickly and easily to be recognized in horizontal sections, passing through the papilla and the deep layers of the epidermis. Its vessels are to be studied in thin parts of the skin (genitalia, lips), in the fresh condition, or in injected preparations with those of the rest of the skin; its nerves in perpendicular sections, in isolated 
papillæ, or in thin portions of the skin (prepuce, glans, eyelids, conjunctiva bulbi) after the addition of acetic acid and dilute solution of caustic soda, or according to Gerber's and Krause's method. Gerber boils the skin until it is transparent, lays it a few hours in oil of turpentine until the nerves are white and glistening, and then examines them in fine perpendicular sections made with the double knife. According to Krause, the nerves are seen very well after treating the skin with nitric acid, if the right amount of action is hit upon. The elastic tissue of the skin comes out well under the action of acetic acid, soda, and potassa. The smooth muscles may be readily isolated in the tunica dartos-with more difficulty in the penis and in the areola, where it needs familiarity with them, in order in all cases, to recognize them with the naked eye. On the hair-sacs they are rendered visible microscopically, if a sac, with the sebaceous glands which appertain to it, be isolated, especially after the application of acetic acid, as small bundles near and in front of the sebaceous glands, but best and very easily in perpendicular sections of boiled skin (Henle). The examination of the fat-cells is especially instructive in thin persons, in whom their membranes and nuclei are readily visible: in other cases their membranes are readily demonstrable by the aid of ether, which extracts the fat; but the nuclei are seen with difficulty, though they may occasionally be discovered here and there even in full cells. The epidermis must, for its Malpighian layer especially, be examined fresh, in fine perpendicular sections, to which acetic acid and dilute solution of soda may be added; the horny layer, particularly by the addition of alkalies, in perpendicular and horizontal sections; however, mere maceration in water separates its elements from one another, and those who are practised can discover them in fresh preparations, when viewed both laterally and from the surface.

Literature.-Gurlt, "Vergleichende Unters. über die Haut des Menschen u. d. Haus-säugethiere," \&c., in Müll. "Archiv," 1835, p. 399 (good figures for the period); Raschkow, "Meletemata circa Mammal. dentium evolutionem," Vratisl, 1835 (first more complete description of the elements of the epidermis under Purkinje's guidance); Simon, "Ueber die Structur der Warzen u. über Pigmentbildung in der Haut," Miill. "Arch.", 1840,p. 167 (pigment-cells in the rete of white persons); Krause, article "Haut," in Wagner's "Handwörterbuch," II. 1844, p. 127 (a detailed and excellent treatise); Kölliker, "Zur Entwicklungsgeschichte der äussern Haut," in "Zeitschrift für wiss. Zool.," Bd. II. p. 67 ; "Histological Observations," ibid. II. p. 118; Eylandt, "De musculis organicis in cute humanâ obviis," Dorp. Liv., 1850. Besides these refer particularly to the works of Simon ("Die Hautkrankheiten durch anatomische Untersuchungen erläutert," 2 Aufl., Berl., 1851); Von Bärensprung ("Beiträge zur Anat. u. Pathol. der menschlichen 
Haut," 1848); and Krämer ("Ueber Condylome u. Warzen," Götting. 1847). [Meissner, "Beitrage zur Anatomie und Physiologie der Haut;" Leipzig, 1853.] Figures are given by R. Wagner, "Icon. phys.;" Berres, tab. vi. vii. xxiv. (middling, with the exception of what regards the vessels); Arnold, "Icones org. sens.," tab. xi. (very pretty, but drawn with too low magnifying powers); Hassall, tab. xxiv. xxvi. xxvii. (among others, the skin of the negro also, and the areola of the white from within, colored); and myself, "Mikr. Anatomie," Taf. i.

\section{II.-OF THE NAILS.}

$\S 48$. The nails, ungues, are nothing but peculiarly metamorphosed parts of the epidermis, and like it they consist of two layers, - of a soft mucous, and a horny layer, or the proper nail.

That part of the corium upon which the nail lies, or the bed of the nail, corresponds exactly in form with it, is elongated, quadrangular, arched in the middle, shelving off anteriorly and posteriorly, and especially on the sides. When the nail is removed by maceration in connection with the epidermis, its anterior and middle parts are uncovered whilst its lateral edges and its posterior segment, on the other hand, are invested by a process of the cutis, the wall of the nail, which is anteriorly depressed and rounded off, posteriorly more acute and longer, and, taken together with the bed, forms a fold, the fold of the nail, which receives the lateral edges and the posterior (2-3 lines) portion of its root (Figs. 58, 60).

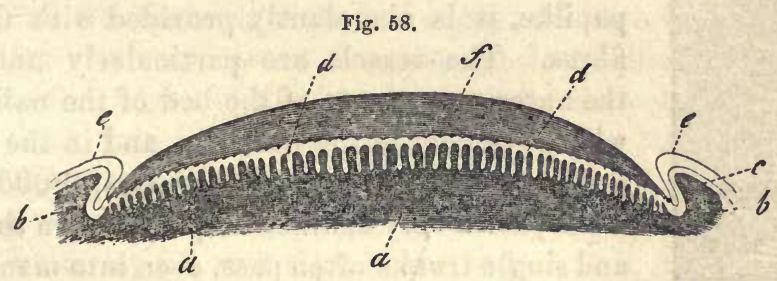

The bed of the nail presents upon its surface peculiar ridges, similar to those of the palm and of the sole of the foot (Fig. 58 a). They begin at the bottom of the fold of the nail, at the posterior border of its bed, and, as Henle (p. 270) justly remarks, they run from the middle of it almost as from a pole. The middle ones pass directly forwards; the lateral at first describe an arc, which is the more convex

Fia. 58.-Transverse section through the body and bed of the nail, $a$, bed of the nail, with its ridges (black); $b$, corium of the lateral parts of the wall of the nail; $c$, stratum Malpighii of the nail with its ridges (white); $e$, horny layer on the wall of the nail; $f$, horny layer of the nail, or proper nail substance, with short notches upon its under surface.-Magnified 8 diameters. 
the further out the ridges lie, and eventually are directed forwards like the others. At a distance of $2 \frac{1}{2}$ to $3 \frac{1}{2}$ lines from their origin, they all at once become more prominent, and take on the form of true lamince of 0.024 to 0.1 of a line in depth, which run directly almost to the anterior edge of the bed of the nail, and then end suddenly, as if truncated. The line of transition of the ridges into the lamince is convex anteriorly, and divides the bed of the nail into two sections, differing both in their extent and in their color: the posterior smaller one is nearly covered by the wall of the nail and underlies its root, the anterior and reddishcolored division underlying its body. The ridges and laminæ of the bed of the nail, the number of which varies between 50 and 90 , are, at their edges, beset with a series of short papillæ of $0.008-0.016$ of a line. In addition, I can confirm Henle's statement that the bottom of the fold of the nail exhibits a few transverse ridges with larger papillæ directed forwards ; further forwards where the lamince cease, there are also long isolated papillæ. On the nail of the little toe, the papillæ are frequently not seated upon ridges, but are more dispersed.

The wall of the nail has no ridges upon its lower surface, and rarely a papilla here and there. These commence again upon its margin, where they are of some length, and are continued thence upon its upper surface, which is in no respect distinguishable from the cutis of the back of the fingers and toes.

The corium of the wall and of the bed of the nail

Fig. 59.

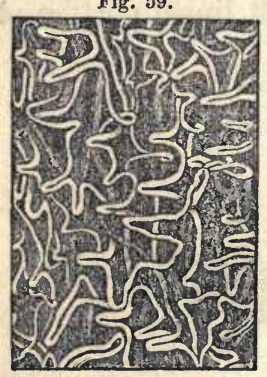
is dense, and for a considerable distance contains but little fat; in the ridges and laminæ with their papillæ, it is abundantly provided with fine elastic fibres. The vessels are particularly numerous in the anterior segment of the bed of the nail ; behind, where the root of the nail lies, and in the wall they are more scanty; their capillaries, 0.005-0.008 of a line, form very distinct simple loops in the papillæ, and single trunks often pass, even into many papillæ. The nerves have the same relations below as in the skin, but I have hitherto been unable to detect their terminal loops or divisions in them.

In the nail itself we may distinguish, the root, the body, and the free edge (Fig. 60). The soft root (Fig. $60 l$ ) corresponds in its extent to the posterior ridged segment of the bed of the nail, and is either wholly hidden in the fold, or exposes a small semilunar surface, the lunula. The posterior edge is attenuated, slightly bent upwards, and is the thinnest and most flexible part of the nail. The hard body, which increases in thickness and breadth from behind forwards $(k)$, lies for the most part with its upper surface uncovered; its somewhat sharp thin edges are hidden in the lateral

FrG. 59.-Capillaries of the bed of the nail, after Berres. 
parts of the fold, and its under surface reposes upon the anterior segment of the bed of the nail : lastly, the free edge $(m)$ is, in cut nails, directed

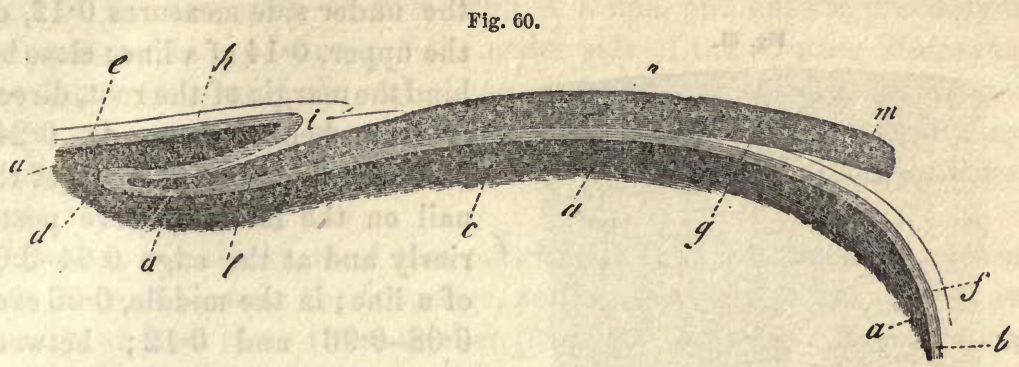

straight forwards, but if uncut, it curves downwards round the ball of the finger and with the rest of the nail, attains to a length of as much as two inches.

The lower surface of the body and of the root answer in their form exactly to the bed of the nail, and we therefore find lamina and ridges upon them, as well as furrows, in the same order as on the latter, only here the edges of the laminæ are straight and not papillated, whilst the furrows, instead of having an even bottom, as in the nail-bed, are provided with shallow pits for the reception of the papillæ. By the mutual interlocking of the elevations and depressions, the intimate union of the nail with the corium is effected, which becomes still more close by the application of the under surface of the wall of the nail upon its base and edges.

The color of the nail, so long as it remains in its natural condition, is whitish and transparent, at its free edges; reddish in the body, with the exception of a very narrow clear margin close behind the commencement of the free edge ; in the lunula, whitish; the color of the last two portions arising principally from the corium and its blood-vessels which shine through them. Separated from the corium and epidermis, the nail is of a tolerably uniform whitish color, and transparent, though somewhat whiter at the root than in the body.

$\S 49$. Structure of the Nail.-The nail consists in its deeper parts of a soft, somewhat pale stratum mucosum, which is distinguished from the hard external horny layer, or proper nail, still more sharply than in the common epidermis. It covers the whole of the lower surface of the root and body of the nail, frequently also a small part of the upper surface

Fra. 60.-Longitudinal section through the middle of the nail and bed of the nail : $a$, bed of the nail, and cutis of the back and points of the fingers; $b$, mucous layer of the points of the fingers; $c$, of the nail ; $d$, of the bottom of the fold of the nail ; $e$, of the back of the finger; $f$, horny layer of the points of the fingers; $g$, beginning of them under the edge of the nail; $h$, horny layer of the back of the fingers; $i$, ends of it upon the upper surface of the root of the nail ; $k$, body; $l$, root $; m$, free edge of the proper substance of the nail.-Magnified 8 diameters. 
of the root, and forms by itself the above-mentioned lamina on the under surface of the nail. Its thickness at the posterior part of the root upon

Fig. 61.

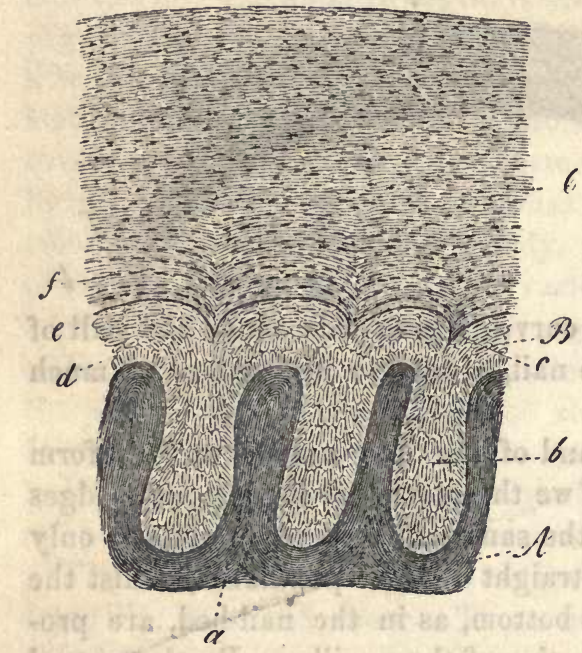
the under side measures $0 \cdot 12$, on the upper, 0.14 of a line; close behind the margin of the root, directly from behind forwards, $0 \cdot 24$ 0.26 of a line; on the body of the nail on the laminæ, more posteriorly and at the edge 0.04-0.05 of a line; in the middle, 0.06 even $0.08-0.96$ and 0.12 ; between these, $0.032-0.04$ of a line.

The Malpighian layer of the nail, like that of the epidermis, consists wholly of nucleated cells, and agrees in all essential points with it, except that the deep portion contains many layers of elongated (of $0.004-0.007$ of a line) perpendicular cells, in consequence of which a striated appearance is produced, which has misled Günther, and probably Rainey also, ${ }^{*}$ into supposing the existence of peculiar glands under the nail. In the Negro, according to Béclard (Anat. Générale, p. 309), the stratum Malpighii of the nail is black; and according to Krause (l. c. p. 124), its cells would in this case appear to contain dark-brown nuclei, as well as yellowish-brown ones, in dark Europeans. According to Hassall (p. 252), the younger cells of the nail (i.e., those of the stratum mucosum), generally contain pigment, which I can confirm, at least in some cases.

The horny layer of the nails, or its proper substance (Figs. $58 f ; 60$ $k, l, m ; 61 c)$, is that hard brittle portion which forms its upper part and its free edge. The under surface of this layer is quite smooth, posteriorly at the root; further forward it exhibits sharp ridges separated by broad furrows, which are inserted into the furrows of the mucous layer of the nail. These ridges of the proper nail substance appear in

Fig. 61.-Transverse section through the body of the nail; magnified 250 diameters. $A$, cutis of the bed of the nail; $B$, mucous layer of the nail; $C$, horny layer of it, or proper nail substance; $a$, layers of the bed of the nail; $b$, layers of the stratum Malpighii of the nail; $c$, ridges of the proper substance of the nail ; $d$, deepest perpendicular cells of the mucous layer of the nail; $e$, upper flat cells of it; $f$, nuclei of the proper substance of the nail.

* [With respect to Rainey's observations, Reichert, in his Report for 1849-50 ("Mall. Arch.," $1850-51$ ) says, that the observation as to the follicles is quite correct, and that with Dr. Ammons, who had studied the growth and regeneration of the nails for some years, he had seen such capsules containing horny cells, with especial distinctness upon the bed of the nail of the great toe.-Trs.] 
transverse sections (Figs. 58, 61), as pointed processes of $0.01-0.02$ of a line in length, which, as a rule, are most strongly developed at the edges of the nail, even to $0.04-0.06$ of a line, and answer precisely in their number to the laminxe of the under side of the stratum Malpighii. The upper surface of the substance of the nail is smooth, taken as a whole, yet sometimes even here, very distinct, parallel, longitudinal streaks appear as the last, almost effaced indications, of the inequalities of its bed.

Usually, the thickness of this part of the nail continually increases from the root to near the free edge, so that the body of the nail is, anteriorly, at least three times thicker (from 0.3 to 0.4 of a line) than the former; at the free edge again, it becomes somewhat less. In its transverse diameter also, with the exception of the posterior edge of the root, the substance of the nail is not everywhere equally thick; it thins considerably towards the lateral edges, so that at last the nails, where they lie in the fold, measure not more than $0.06-0.12$ of a line, and finally terminate quite sharply.

With regard to the structure of the proper substance of the nail, it can hardly be made out without the action of reagents. In perpendicular sections we see, particularly in the body, nothing but horizontal, fine, straight, or curved, closely-approximated lines, which one would be inclined to consider as the optical expression of delicate, superimposed lamellæ, and between these, a multitude of elongated, horizontal, opaque or peculiar reddish-transparent striæ, evidently nuclei. Only upon the most posterior part of the root, and on the under surface, where it meets the stratum Malpighii, do more or less distinctly flattened cells with nuclei appear disposed in layers. Horizontal sections show even less than the perpendicular ones; exhibiting a pale transparent substance, granular here and there, and mostly without indication of any structure whatsoever, occasionally with very indistinct contours of plates similar to those of the horny layer of the epidermis. Very different are " the appearances presented after treating the nail with alkalies and certain acids.

If the substance of the nail be boiled in dilute caustic soda, it becomes changed upon the first bubbling of the fluid into a beautiful cellular tissue (Fig. $62, A, B$ ), whose

Fig. 62 .

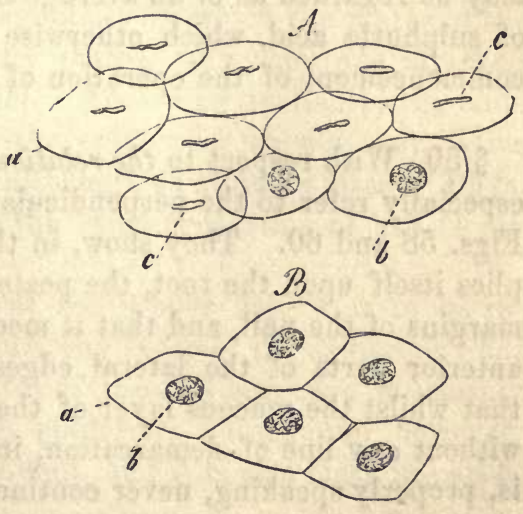

Fia. 62.-Nail plates boiled with caustic soda. $A$, from the side; $B$, from the surface: $a$, membranes of the distended elements of the nail; $b$, their nuclei, from the surface; $c$. from the side.-Magnified 350 diameters. 
polygonal elements all, without exception, the deep as well as the superficial, possess nuclei of $0.0030-0.0046$ of a line in length and breadth, and 0.002 of a line in thickness, which, according as they turn their surfaces or their edges to the observer, appear as rounded, very pale, and finely-granulated discs, or as long, narrow, dark-contoured rods; it deserves further to be noted, that together with these, large and very pale nuclei of $0.006-0.01$ of a line, and more, occur in considerable numbers, probably owing their existence to the excessive action of the reagent which has swollen them up. Caustic soda and potass also (which has a similar action upon the whole, though it acts more upon the nuclei) demonstrate the important fact, that the cells of the nail are flatter in the superficial than in the deeper layers. If, in fact, a fine perpendicular section be moistened with cold, or better, with hot solution of soda, we see the cellular structure of the nail appear almost at the very instant it becomes moistened, without any obvious enlargement of its elements; and it is observable, at the same time, that its deepest cells are at least as thick again as the most superficial.

If the soda solution acts longer, the section gradually swells up, in the under cells first, on account of their greater softness, and only subsequently in the flat and hard upper elements. By treating the nail with cold sulphuric and nitric acids, and also by boiling with hydrochloric acid, its elements are isolated.

Taking these facts, together with what we see in the unaltered nail, it results that its horny layer consists of closely united but not sharply defined lamellæ; each lamella being composed of one or many layers of nucleated, polygonal, flat scales or plates, which, excepting their nuclei, are very similar to those of the horny layer of the epidermis, and in their deepest layers are thicker and somewhat less in circumference than in the upper and uppermost layers: Those of $0.012-0.016$ of a line, may be regarded as of an average size, as may be seen upon the addition of sulphuric acid, which otherwise exerts but little action, and at the commencement of the operation of soda and potass.

$\S 50$. With respect to the relation of the nail to the epidermis, I must especially refer to the perpendicular and transverse sections figured in Figs. 58 and 60 . They show, in the first place, that the epidermis applies itself upon the root, the posterior part of the body, and upon the margins of the nail, and that it meets it under the free edge and on the anterior parts of the lateral edges. This happens in such a manner, that whilst the mucous layer of the epidermis passes continuously, and without any line of demarcation, into that of the nail, the horny layer is, properly speaking, never continued directly into the actual substance of the nail, but partly applies itself with its lamellæ parallel upon the nail, partly abuts upon it at various oblique angles. At the root, the horny layer passes more or less deeply into the fold of the nail, and at 
the same time runs, in a thin layer which becomes very fine anteriorly, upon the upper free part of the nail, as far as the end of the lunula or the beginning of the body. Anteriorly and posteriorly, in which latter region this layer not uncommonly reaches the posterior margin of the root, its cells lie parallel to the upper surface of the nail; while in the middle, where it is thickest (Fig. $60 i$ ), they are oblique or perpendicular to it. At the free edge of the nail the relations of the parts are similar, where the horny layer meeting the end of the under surface of the body of the nail, partly with more horizontal, partly with oblique lamellæ, is perhaps also continued upon the commencement of the free edge. On the lateral edges, again, the horny layer passes anteriorly, in horizontal strata, under the nail ; more posteriorly it is arranged as upon the root, or simply rests against the edge of the nail. The horny layer thus forms a kind of sheath for the nail, which bears some resemblance to the sheath of the hair, though it is much more imperfect. If we compare the nail with the epidermis, we find, in the structure of its mucous layer, not the slightest peculiarity of any importance, while the horny layer is distinguished from that of the epidermis by its cells being nucleated, harder, and chemically different; by their flattening and intimate union. For the rest, the agreement of the two structures is so close, that the proper nail may justly be considered, as it has long been, a modified portion of the horny layer of the last joints of the fingers and toes.

According to the chemical investigations of Scherer and Mulder, the nails agree very closely with the epidermis; and, according to Mulder, they are distinguished from it, only by their somewhat greater proportion of sulphur and carbon. In his last essay, he considers them to be composed of protein + sulphamid (6.8 per cent. of the latter). This agrees with the observed action of reagents, with which the plates of the nail behave almost exactly like horny plates, only they are attacked with more difficulty and possess nuclei. According to Lauth, the nails contain more phosplate of lime than the epidermis, whence they derive their hardness : this may be correct, although, as Mulders tates ("Phys. Chemie," p. 536), both yield about the same proportion of ash (1 per cent.).

As regards the lamellar structure of the proper nail, it is to be regarded in the same light as that of the horny layer of the epidermis; but it is not so distinct, because the plates of the nail are more intimately connected than the elements of the epidermis. Reagents, however, render the lamellar structure very evident, and it is also clear in pathologically thickened and curved nails.

§ 51. Growth of the Nails. - The nails grow continually, as long as they are cut; on the other hand, if uncut, their growth is limited. In 
this case, as may be observed in those who are long confined to their beds by sickness, and in the Eastern Asiatics, the nails become $1 \frac{1}{2}-2$ inches long (in the Chinese, according to Hamilton, 2 inches), and to curve round the points of the fingers and toes.

During the growth of the nail, the mucous layer does not change its position at all, but its horny layer is constantly being thrust forward. The formation of the latter goes on continually wherever it is in contact with the stratum Malpighii, in other words upon its whole under surface, with the exception of the free anterior edge ; further, in many nails, upon a very small portion of the upper surface of the root, finally, at the posterior edge of the root itself. It is, however, the root portion which grows fastest, whilst the body of the nail is more slowly developed, which is demonstrated especially by the fact that it is not much thinner at the boundary between the root and the body than it is anteriorly upon the body itself, and that the transition of the cells of the stratum Malpighii into nail-cells is easily shown at the root, but with difficulty in the body. By the constant addition of new cells at the edge of the root, the nail grows forward; by their addition to its under surface, it is thickened. The longitudinal growth exceeds that in thickness, because the first rounded cells, as they move from behind and below, forwards and upwards, become more and more flattened and elongated.

The mode in which the plates of the nail arise from the cells of the mucous layer, is easily demonstrable at the root of the nail. Here, in fact, the uppermost cells of the mucous layer are constructed very differently from the deeper ones; they are more or less flattened, and closely resemble the cells of the epidermis, but they possess a nucleus, which, however, is only to be discovered by adding caustic soda, and then with difficulty. If we follow these cells, which form a layer of $0.06-0.12$ of a line in thickness, toward the proper substance of the nail, we find that they become more and more flattened, and at last pass without any defined boundary into the latter, uniting together more closely, and taking on a more transparent appearance.

In the body of the nail, the formation of nail substance is demonstrated with more difficulty, yet here, in opposition to Reichert, we must assume that it does take place, because the nail almost invariably increases in thickness even in the body, from behind forwards. However, there is unquestionably, in this part, a sharper demarcation between the two layers of the nail, than in the root; but in fine sections it appears by no means so sharp as in those which are commonly examined, and I find, in fact, that the transition of the cells of the mucous layer into the plates upon the body of the nail, is demonstrable with tolerable readiness, particularly on the addition of alkalies, where the ridges of the under surface of the proper nail are well developed. Between the ridges also, though no direct transition is recognizable, yet it may be observed that 
the plates of the proper nail which border upon the mucous layer are much less flattened than in the interior and on the surface, which also indicates that they are developed upon the spot. In conclusion I must add, in support of my view, that it is only in this way, that it becomes explicable why the under surface of the proper nail substance upon the root of the nail is almost smooth, while on the body of the nail it presents more or less prominent ridges. The origin or increase of these ridges demonstrates clearly that nail-substance is also formed here. Corresponding with these ridges and with the grooves between them, we also find that the lowest layers of the nail plates, which are quite horizontal upon the root, run with an undulating course upon the body (Fig. 61). The general result then is, that whilst the formation of the nail goes on especially at its root, yet that plates are added to the body of the nail from below, though more slowly and scantily, thus producing the anterior thickening, or at least preventing the necessary thinning of the nail anteriorly. It is to be remarked further, that the development of nail-substance takes place in all parts of the middle line of the nail more rapidly than in the lateral portions, which, anteriorly, are almost as thin as in the root, though they possess longer processes below. But even there, substance must be added to the body of the nail, because it becomes broader anteriorly.

The plates of the substance of the nail once formed, alter in certain respects, as they are pushed forwards and upwards by those which come after them. In the first place, their nature becomes altered in a manner which is little understood, the change consisting partly in the deposition of more phosphate of lime, partly in a solidification (conversion into horn) of their organic elements, particularly of the cell-membranes, in consequence of which, from being soft, as at the root and under surface of the nail, they become gradually harder and harder. In the second place, like the horny cells of the epidermis, they are very considerably flattened, and at the same time increase somewhat in their longitudinal and transverse diameters; finally, they coalesce more completely, so that they cannot be separately recognized, without the action of reagents, in the upper and anterior parts of the nail, which appear to be composed of nothing but a homogeneous substance which tears in all directions; whilst in the lower parts the separate nailplates are, at least, indicated, and are occasionally tolerably distinct. On the other hand, the nuclei of the nail-plates do not disappear, and in this lies a characteristic distinction between the horny layer of the nail and that of the epidermis. They are to be seen in perpendicular sections, of fresh nails, and after treatment with caustic soda, and even in the most superficial layers, though somewhat smaller and flatter than in the deep layer. It follows then, that certain metamorphoses go on in the proper substance of the nail, which as in the epidermis, are to be ascribed to a peculiar growth and vital process in the nail-cells them- 
selves. These seem to occur, however, almost solely in the lower and posterior parts of the nail, for if, as Schwann states (Fig. 91), two points be marked upon the posterior portion of the free surface of the nail, one behind the other, by piercing it with a needle and coloring with nitrate of silver, they in no wise alter their relative position in the course of the two or three months, during which they are moving towards the point of the nail.

As to the pathological conditions of the nails, they are readily regenerated when they have been detached, in consequence of crushing, burning, freezing, cutaneous disorders (scarlet fever, \&c.), inflammations, exudations, suppurations, and effusions of blood in the bed of the nail; in fact, as Pechlin ("Obs. Phys. Med.," p. 315) narrates, this regeneration may take place periodically; in a boy, the nails, every autumn became bluish-black and desquamated, together with the epidermis (the horny layer?), and were subsequently regenerated. In such a case, according to Lauth ("Mém. sur divers points d'Anatomie," in the "Annales de la Société d'Histoire Naturelle de Strasbourg," t. i. 1834), and Hyrtl ("Anatomie," p. 382), the whole bed of the nail becomes covered by soft horny plates, which harden by degrees, grow into a regular nail, and eventually project with their free edges beyond the end of the finger. When the last joint of the finger has been lost, rudimentary nails frequently appear upon the back of the second and even of the first phalanx. The older cases are quoted in Pauli ("De vulneribus sanandis," Gottingæ, 1825, p. 98), more recently Hyrtl (l. c.) saw such a nail 2 lines long and 3 lines broad on the first phalanx of the thumb. As the formation of nail-substance depends upon the vessels of the bed of the nail, we may, with Henle, assume that varying conditions of the latter may frequently produce local thickening, thinning, or even detachment of the nail, and that their deformities in cyanosis and phthisis depend on these causes. The thickening and abnormal development of the nails, however, arise very frequently, as I have observed, from a partial obstruction of the capillaries of their bed. Thus in the lamellated nails of old people, greatly thickened and curved downwards in front, I find all the capillaries of the anterior segment of the bed of the nail closely filled with fat granules of different sizes, and wholly impermeable to the blood; in such a case the development of nail-substance can take place only in small lamellæ in the fold, which then, as may be readily understood, are raised up by those which are growing behind into a continually more and more oblique position ; their posterior extremities forming, on the surface of the nail, transverse streaks one behind another at short intervals. After dividing the nervus ischiadicus, Steinrück ("De nervorum regeneratione," pp. 45-49) observed, in the Rabbit, that the nails and hair fell off, which is a result of the influence of the nerves upon the vessels. Finally, the shape of the bed of the nail also influences its formation. It is thus explained, how (see Henle, l. c.), after 
inflammation and closure of the fold of the nail, the formation of new nail at the posterior edge ceases, the nail no longer growing forwards, but at all its edges exactly covering its bed.

$\S 52$. The development of the nail begins in the third month with the formation of the bed and fold, which are marked off from the surrounding parts by the gradual growth of the skin into the wall of the nail. At first the bed of the nail is lined by the same cells as those which form the other parts of the epidermis (see $\$ 47$ ), only that even in the third month the cells of the stratum Malpighii are distinguished by their elongated and polygonal form (length 0.004 , breadth $0.001-0.0016$ of a line). In the fourth month there arises between the stratum Malpighii and the horny layer of the bed of the nail, which latter is formed by a simple layer of polygonal clearly nucleated cells, a simple lamina of pale, flat, but also quadrangular and nucleated cells, 0.009 of a line in diameter, which are closely united together, and must be regarded as the first indication of the proper substance of the nail ; at the same time also, the stratum Malpighii under these cells become thickened so that it is certainly composed of, at least, two layers. The nail is therefore at first wholly included within the epidermis; it is formed over the whole surface of the bed of the nail as a quadrangular plate, and arises between the embryonic mucous layer and the horny layer, without doubt by a metamorphosis of the cells of the mucous layer, as is probable, especially from the minute size of the original cells of the nails. In the course of its further development, the nail is thickened by the addition of new cells from below (in the fifth month its thickness is about 0.024 , in the sixth 0.04 of a line, of which in the latter 0.025 must be reckoned as proper nail-substance); it increases by the extension of its elements, and by the addition of new ones at its edges; but it remains, even to the end of the fifth month, hidden under the horny layer of the epidermis, until finally it becomes free, and in the seventh month, even begins to grow longitudinally, so that at this period, except in its greater softness and its smaller dimensions, it presents no essential difference from the perfect nail. With regard to the bed of the nail, its ridges are already indicated at the end of the fourth month, and in the fifth they are well-marked, $0.02-0.024$ of a line deep, $0.004-0.005$ of a line broad, and $0.008-0.014$ of a line distant; these measurements also indicate the breadth of the laminæ of the stratum Malpighii. At the sixth month they are somewhat larger and further apart.

In the new-born infant, the whole nail is $0 \cdot 3-0.34$ of a line thick; 0.16 of a line being proper nail-substance, $0.14-0.18$ of a line stratum Malpighii. Its elements are still almost identical with those at the sixth month, and they appear with tolerable distinctness in the nail proper without any reagents, as elongated polygonal nucleated plates $0.002-0.28$ of a line, as Schwann has already partly remarked. The free edge, 
projecting far forwards, which is presented in all nails, is worthy of remark. It is considerably thinner and narrower than the body, and is separated from it by a semilunar line; it is rounded anteriorly, as much as 2 lines long, and is plainly nothing but the nail of an earlier period which has been thrust forward by the longitudinal growth of the nail in the course of its development. In fact it nearly corresponds in size with a nail of the sixth month.

Soon after birth the long free edge of the nail of the new-born infant is cast off, at least once (according to Weber many times), in all probability in consequence of external mechanical violence, which it is unable, owing to its delicacy, to resist. In the sixth and seventh months after birth, I find that the nails which the child brought into the world, are completely replaced by new ones, and in the second and third years the nail-plates are not distinguishable from those of the adult, whence it follows that the nail increases in thickness, less in consequence of any enlargement of its elements, than by the addition of new ones to its edges and under-surface.

The investigation of the nail-cells and plates is best made in fine sections of recent nails, with and without the addition of reagents, especially caustic soda and sulphuric acid, concerning whose operation the most important points have already been noted. To examine the relations of the parts of the nail to one another, and to the epidermis, the nails must be separated from the cutis by maceration, or by boiling in water. It is then seen, that the nail is detached, with the cuticle, from the finger; and in transverse and longitudinal sections, its mode of connection with the former is perceived. The bed of the nail also, its lamince and ridges, the fold and the lamince in the stratum Malpighii of the nail, are easily seen, in this way. Since fine sections, in such a nail, are not readily made, precisely in the most important parts-the margins and roots, - it is necessary, for this purpose, to employ fresh nails separated from the bone with the cutis, and dried. These afford all the information required, portions of them swelling up readily in water, and exhibiting the structure of the different layers, with acetic acid and caustic soda, in the most distinct manner.

Literature.-A. Lauth, "Sur la disposition des ongles et des poils," Mem. de la Société d'hist. nat. de Strasbourg, 1830-4; Gurlt, "Ueber die hornigen Gebilde des Menschen u. der Haussäugethiere," Müll. "Arch.," 1836, p. 262 ; Reichert, in Müll. "Arch.," 1841, Jahresbericht; O. Kohlrausch, "Recension von Henle's allgem. Anat.," in Götting. "Anzeigen," 1843, p. 24 ; Rainey, "On the structure and formation of the nails of the fingers and toes," in "Trans. of Microsc. Society," March, 1849; Berthold, "Beobachtungen über das quantitative Verhältniss der Nägel- u. Haarbildung beim Menschen," in Müll. “Arch.," 1850. 


\section{III.-OF THE HAIRS.}

§53. In every hair we distinguish the free part or shaft, scapus, with its tapering point, from the portion enclosed within the sac, the root, radix. In straight hairs the former is generally straight and rounded; in the wavy, undulated and somewhat flattened; in the curly and woolly hairs, it is twisted spirally and quite flat or slightly ribbed. The root is always straight, tolerably cylindrical, and softer and thicker than the shaft, at least in its lower part; in living hairs it ends in a still softer knoblike enlargement, $1 \frac{1}{2}$ to 3 times thicker than the shaft, - the "bulb of the hair" (c), which is placed, cap-like, upon a papillary process of the sac, the "hair-papilla" (less properly termed pulpa or blastema pili, hair-germ), or in other words receives the papilla in an excavation in its base.

§54. Disposition and Size of the Hairs. -The hairs are distributed over almost the whole surface of the body, ${ }^{*}$ but exhibit very considerable differences in size and number, according to their situation, to individual peculiarity, age, sex, and race. As regards the former, three varieties may be admitted, besides many transitional forms: (1) long soft hairs, of 1-3 feet and more in length, $0.02-0.05$ of a line in thickness; (2) short stiff thick hairs, of $\frac{1}{4}-\frac{1}{2}$ of an inch in length, and $0.03-0.07$ of a line in thickness; (3) short, excessively-fine hairs, down (lanugo),

Fig. 63.

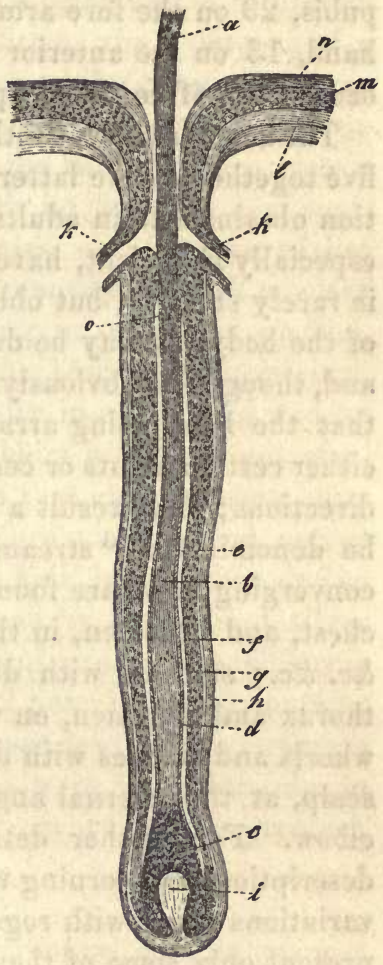
of 1-6 lines in length, and $0.006-0.01$ of a line in thickness. The distribution of the first form is well known; to the second belong the hairs at the entrance of the nostrils (vibrissce), in the external auditory passage, the eyelashes (cilia), and eyebrows; to the third, finally, must be referred the hairs on the face, trunk, and extremities, also those of

FIG. 63.-Hair and hair sacs of middling size ; magnified 50 diameters: $a$, hair shaft; $b$, root of the hair; $c$, bulb of the hair; $d$, epidermis of the hair; $e$, inner root-sheath; $f$, outer root-sheath; $g$, structureless membrane of the hair sac; $h$, transversely and longitudinal fibrous layer thereof; $i$, papilla of the hair; $k$, excretory ducts of the sebaceous glands, with epithelium and layers of fibres; $l$, cutis at the aperture of the hair sac; $m$, stratum mucosum; $n$, horny layer of the epidermis, the latter somewhat retracted into the sac; 0 , end of the inner root-sheath.

* [No hairs exist upon the upper eyelids, the lips, the palm of the hand, and sole of the foot; nor on the dorsum of the last joints of the fingers and toes, the inner surface of the prepuce, and the glans penis.-Trs.] 
the caruncula lachrymalis, and those (frequently absent) of the labia minora (Henle).

The number of hairs upon a given extent of surface varies very much, depending especially upon age, sex, and the color of the hairs. According to Withof, on a surface of $\frac{1}{4}$ of a square inch there were found 147 black, 162 brown, 182 fair hairs. In a moderately hairy man, he found on $\frac{1}{4}$ of a square inch, 293 upon the scalp, 39 on the chin, 34 on the pubis, 23 on the fore arm, 19 upon the outer margin of the back of the hand, 13 on the anterior surface of the leg. In men, closely set hairs occur not unfrequently upon the chests, shoulders, and extremities.

The hairs are placed either singly, or in twos and threes, even four and five together. The latter is the rule in the foetus, but the same disposition obtains also in adults, especially in the lanugo. As Osiander and especially Eschricht, have shown, the direction of the hairs and hair-sacs is rarely straight, but oblique, and in different degrees in different parts of the body, as may be demonstrated with ease in the hairs of embryos, and, though less obviously, in adults also. The regularity depends on this, that the hairs being arranged in curved lines, which converge towards either certain points or certain lines, or diverge from them in two or more directions; there result a multitude of figures, which may with Eschricht be denominated "streams," "whorls," and "crosses." Streams with converging hairs are found, for example, in the median line of the back, chest, and abdomen, in the line which answers to the ridge of the tibia, \&c. \&c.; streams with diverging hairs occur on the line between the thorax and abdomen, on the onc hand, and the back on the other, \&c.; whorls and crosses with diverging hairs are found in the axilla, on the scalp, at the internal angle of the eye; with converging hairs, on the elbow. For further details I must refer to Eschricht's figures and descriptions, concerning which, however, it is to be remarked, that many variations occur with regard to these points, and Eschricht's figures represent only some of them.

§55. External peculiarities and chemical composition of the Hairs.In embryos, the hairs are generally quite colorless and clear; they very slowly become colored, so that in youth they are, in general, paler than in middle age. In the adult the downy hairs, which have remained in a foetal condition as it were, are invariably the palest; the longer ones are always darker, and the darkest are those of the head, beard, and pubis.

The hairs are very elastic; according to Weber, they stretch without breaking to nearly a third more than their length, and if they be stretched only a fifth, they contract again so perfectly, that they remain permanently only $\frac{1}{17}$ th longer. They readily imbibe water, and as readily give it out again; they are therefore sometimes dry and brittle, sometimes moist and soft, according as the skin or the atmosphere con- 
tains much or little moisture. They become longer or shorter, accord- ing to the amount of moisture which they contain, whence their use in Hygrometry. In spite of their extensibility, their strength is considerable, and hairs of the head will bear at least 6 ounces without breaking.

The chemical composition of the hairs is not yet sufficiently understood, but they are chiefly composed of a nitrogenous substance, soluble in alkalies with the evolution of ammonia, and insoluble in boiling acetic acid. Scherer and Von Laer consider it to be a combination of protein with sulphur, and the latter supposes, in addition, the existence of a small quantity of a substance similar to gelatine, whilst Scherer regards a second nitrogenous matter which he found, to be a product of decomposition. Mulder considers the substance of the hairs to be a protein compound combined with sulphamid, of which he finds 10 per cent. Besides their nitrogenous constituents, the hairs, as even the earlier investigations showed, contain a considerable quantity of dark or clear fatty matter, which may be extracted by boiling in ether and alcohol. From horn and epidermis, the substance of the hair is distinguished, according to Mulder, especially by its insolubility in acetic acid and by the same test, from albumen and fibrin. The hairs withstand putrefaction better than any other part of the body, so that even mummy hairs are found to be quite unchanged; in water they are not dissolved, except in Papin's digester. Metallic oxides color the hair just as they do the epidermis; thus, for example, they are blackened by the salts of silver and manganese, sulphurets of these metals being produced. Chlorine bleaches them. The ash amounts to about 1-2 per cent., and contains oxide of iron (more in dark hair); oxide of manganese ; silica (traces); phosphate of magnesia and sulphate of alumina were found by Jahn in white hairs; and according to Laugin, copper occurs in the greenish hairs of those who work in copper and brass.

$\S 56$. With regard to their more intimate structure, two substances may be distinguished in all hairs without exception, and in many there are three: 1, the cortical substance, or better, fibrous substance, which constitutes by far the most considerable portion of the hair and determines its form; 2 , the cuticle, a delicate external investment of the fibrous substance; 3 , lastly, the central medullary substance, which is often absent.

The cortical or fibrous substance is longitudinally striated, very often presents dark dots, is streaked or spotted and except in white hairs, in which it is transparent, is more or less deeply colored; the color is sometimes distributed through the whole substance with tolerable regularity, sometimes more concentrated in certain elongated, granular spots. The more intimate structure of the cortex of the hair, and the signification of its spots and strix, cannot be properly understood without the use of acids and alkalies (which afford important aid in the investigation 
of the hairs in general) and other manipulations. If a hair be treated with concentrated sulphuric acid at a warm temperature, its fibrous substance is much more readily broken up than before, into flat elongated fibres of various breadths (commonly 0.002-0.005 of a line), which are characterized particularly by their rigidity and brittleness, and by their irregular, even notched, margins and ends : in pale hairs they are clear, and in dark ones have a dark tinge. These so-called hair-fibres

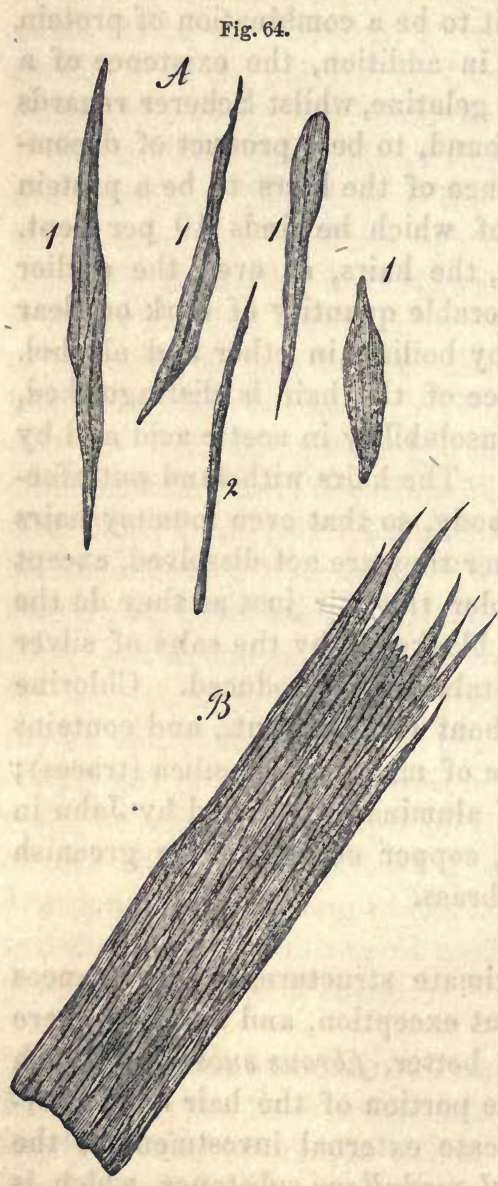
are not, however, the ultimate elements of the fibrous substance; each of them, in fact, consisting of an aggregation of flat, moderately-long fibre-cells or plates, which may be found isolated among the fibres after the thorough action of sulphuric acid. These (Fig. 64), which may best be named the plates of the fibrous substance, or the fibre-cells of the cortex, are flat and generally fusiform, 0.024 -0.033 of a line long, 0.002-0.004, or even 0.005 of a line broad, 0.0012 -0.0016 of a line thick, with uneven surfaces and irregular edges; they do not swell up into vesicles on the addition of caustic alkalies, and they very frequently exhibit a darker streak in their interior, of which we shall speak immediately; under certain circumstances they also contain granular pigment; for the rest they are homogeneous, and present no minuter elements, such as fibrillæe or the like. They appear to be more strongly united longitudinally, than in the direction of their breadth, whence it arises that the cortical substance easily breaks up into the long fibres above mentioned. The fibres themselves (which I should not be inclined to consider as compound elements of the cortical substance, since their constituents are separable, and they themselves are far too irregular), without constituting distinct lamellæ, like the plates of the nail and of the epidermis, form a compact fibrous bundle, and in this

FrG. 64.-Plates or fibre-cells of the cortical substance of a hair treated with acetic acid; magnified 350 diameters: $A$, isolated plates, 1 , from the surface ( 3 single, 2 united); 2 , from the side. $B$, a lamella composed of many such plates. 
manner the cortical substance, which constitutes the principal bulk of the hair, is produced.

The dark spots, dots, and streaks of the cortex, are very various in their nature, and are principally: 1, granular pigment; 2, cavities filled with air or fluid; and 3, nuclei. The action of caustic potass and soda, which soften and swell up the cortical' substance without attacking the spots (Fig. 67), shows that they are in great measure nothing but aggregations of pigment granules, which are deposited in the plates of the hair, are especially frequent in dark hairs, and vary very much in respect to their size and form. Dark spots of a second kind are very similar to the pigment deposits, but turn out on examination to be little cavities filled with air. They are best studied in white hairs, where they cannot possibly be confounded with pigment. Here we see dispersed through the whole cortical substance round dots of $0.0004-.0008$ of a line, or longish streaks of 0.004 of a line in length, $0.0004-0.0008$ of a line in breadth, which, sometimes more scattered, sometimes more numerous, and arranged in irregular lines, run parallel with the axis of the hair. The dark contours and somewhat clear centre of these, attract attention at once, and call to mind fat granules, which, in fact, for a long time I held them to be; but they are nothing but excessively minute cavities filled with air, which occur very frequently also in fair, brightbrown, and bright-red hairs, often in very great numbers, while they are wanting in very dark hairs, and in the lower half of the root of all hairs. Thirdly, there occur in the cortex, other tolerably dark striæ or lines, which in dark hairs are commonly connected with the pigment-spots in such a manner that the striæ form the ends of the spots, or pass through them axially; in white and pale hairs they appear not unfrequently as prolongations of the air cavities, but in both kinds of hairs they often occur independently, in various numbers and degrees of distinctness. I hold these streaks, which are commonly most distinct in pale or bright-brown hairs without any medulla, to be sometimes the expression of the composition of the hairs by the above described fibre-

Fra. $65 .-A$, a piece of a white hair after treatment with caustic soda; magnified 350 diameters : $a$, nucleated cells of the medulla without air; $b$, cortical substance with a fine fibrillation and prominent linear nuclei; $c$, epidermis with its plates projecting more than usual; $B$, three isolated linear nuclei from the cortex. 
cells; in other words, to be the boundary lines of the separate elements of the cortex, and sometimes I consider them to be their nuclei. For, even in the shaft of the hair, the cortical plates all contain fusiform nuclei $0.01-0.016$ of a line long, $0.0005-0.0012$ of a line broad, which may, in fact, be isolated by rubbing down white hairs which have been boiled in caustic soda. Besides these, there appear in the cortical substance, and with especial distinctness in a whitish place immediately above the bulb, certain fine strix, which are produced by inequalities in the surface of the cortical plates, and which do not readily disappear, even after the continued action of alkalies, but eventually give place to a finely fibrous appearance; they cannot be isolated, but are visible in those portions of the cortex which have been separated by sulphuric acid, and sometimes even are very distinct (Fig. 66).

The description of the cortex, which has just been given, holds good especially for the hair-shaft. In the root of the hair, so long as it is still solid and brittle, we find essentially the same conditions; and it is only in its lower half, where it becomes gradually softer, at first finely fibrous and then granular, that the structure of the cortex undergoes a progressive change. Here, in fact, the above described plates are less rigid, and take on more and more distinctly the form of elongated cells (Fig. 66 ) of $0.020-0.024$ of a line in length, and $0.009-0.011$ of a line in breadth, whose cylindrical, straight, or serpentine nuclei of $0.008-0.01$ of a line, are very easily rendered visible by the action of acetic acid, and may also be readily isolated. The soft and shortened plates then pass Fig.66. into elongated, rounded cells, with short nuclei, the Fig.67.

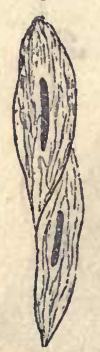
fibrous structure becoming more and more obliterated, and these are finally continued without interruption into the elements of the lowest and thickest part of the hair, the bulb. They (Fig. 67) are nothing more than round cells of $0.003-0.006$ of a line, which lie closely pressed

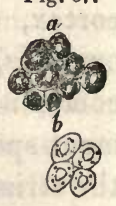
together; and like the cells of the mucous layer of the epidermis sometimes contain only colorless granules, sometimes are so full of dark pigment-granules, that they become true pigmentcells. It must be added, that the chemical relations of the cortex are altered in the lower half of the root, its elements becoming more sensitive to the action of acetic acid, which does not affect the plates of the shaft at all; they swell up and dissolve in alkalies also, much more quickly than those of the shaft.*

Fia. 66.-Two cells from the cortex of the root of the hair (the finely-striated part of it immediately above the root), with distinct nuclei and a striated appearance; magnified 350 diameters.

Fia. 67.-Cells from the deepest part of the bulb of the hair; magnified 350 diameters: $a$, from a colored bulb, with pigment-granules and somewhat hidden nucleus; $b$, from a white hair with a distinct nucleus and few granules.

* [Reichert ("Bericht" for 1850, Mull. "Archiv," 1851) asserts that the cortical substance 
The color of the cortical substance arises partly from spots of pigment, to some extent from air cavities, and partly from a coloring matter diffused through and combined with the substance of the cortical plates. The first or the granular pigment, exhibits all shades from clear yellow, through red and brown, to black; the diffused pigment is quite absent in white hairs, and is scanty in clear, fair hairs; it is most abundant in the more opaque fair hairs, and in red as well as in dark hairs, in which it may by itself give rise to an intense red or brown color. The color of the cortex depends especially upon that of these two pigments, but sometimes the one, sometimes the other predominates, and it is only in the very light and in the very dark hairs that they are developed in about equal proportions.

$\S 57$. The medullary substance is a streak or cord which extends in the axis of the hair, from the neighborhood of the bulb nearly to the point (Figs. 65, 68). It is generally absent in the lanugo and colored hairs of the head, but is usually present in the thick, short hairs, and in the stronger long ones, as well as in the white hairs of the head. If white hairs be boiled with caustic soda until they swell and coil up, we can often, by the use of simple pressure, demonstrate without further trouble, the cellular structure of the medullary cylinder, which is then transparent for trans-

Fig. 68.

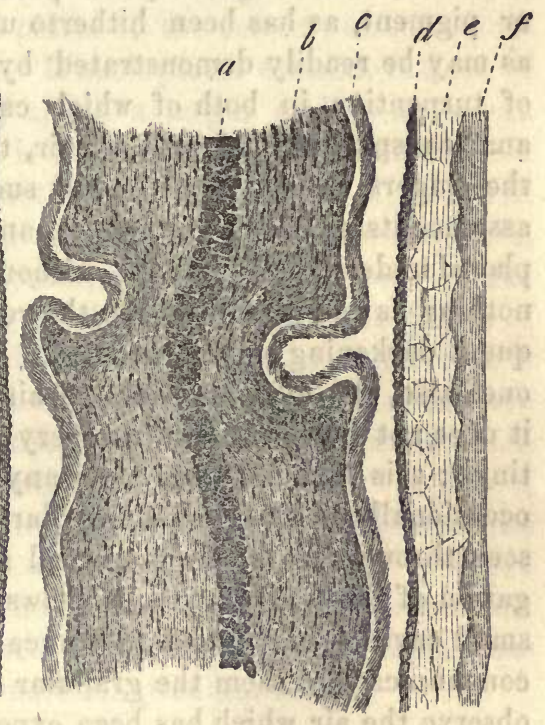

Frg. 68.-A portion of the root of a dark hair slightly acted upon by caustic soda; $a$, medulla, still containing air, and with cells, which appear pretty distinct; $b$, cortex with pigment spots; $c$, inner layer of the epidermis; $d$, outer layer of it $i e$, inner layer of the inner root-sheath (Huxley's layer); $f$, outer fenestrated layer (Henle s layer). Magnified 250 diameters.

of the hair is composed of superimposed laminx, and recommends, in order to demonstrate the fact, that a hair should be treated with a solution of caustic potass of 10 per cent, and then submitted to pressure. Under these circumstances; "beautiful lamellæ appear. The separate layers exhibit no trace of being composed of fusiform cells; they appear finely striated, and in places, hyaline; sometimes elongated spots appear, of which it cannot be determined with certainty whether they are nuclei or perforations in the membrane." In some, there was no trace of these to be seen. Reichert consilers the fibres of the cortex to be artificial products, and was unable to convince himself of the existence of nuclei in this part of the hair.-Trs.] 
mitted light (Fig. 65 a). If a hair thus treated be carefully teased out, it is easy to isolate the medullary cells, either in aggregate masses, Fig. 69. or even completely separate (Fig. 69). They are rectangular or quadrangular, rarely rounded or fusiform of $0.007-0.01$ $6.0 \%$ of a line in diameter, occasionally containing dark, fat-like granules, and often when the alkali has not acted too strongly, a rounded clear spot of $0.0016-0.002$ of a line, which is plainly the rudiment of a nucleus, and which also seems to swell up somewhat in soda.

In fresh hairs, the medulla in the shaft is silvery white or dark, an appearance which, as many more favorable objects show, arises from rounded-angular, granular corpuscles, black (opaque) or of a brilliant white, according to the illumination, tolerably uniform in size, but varying according to the hairs, from $0.0002-0.002$ of a line and occupying the medullary cells in great quantity (Fig. 68). These granules are not fat or pigment, as has been hitherto universally supposed, but air-vesicles, as may be readily demonstrated by boiling a white hair in ether or oil of turpentine, in both of which cases the medulla becomes quite clear and transparent. If such a hair, treated with water, be dried between the fingers, it soon, often quite suddenly and visibly to the naked eye, assumes its previous white color, and if immediately after drying, it be placed under the microscope, without fluid, or with fluid at one end only, nothing is easier than to see the re-entrance of the air and the consequent darkening of the medulla. Not only in white hairs, but in dark ones also, the medulla contains air in the fresh state, only in this case it does not appear of a pure silvery white, but with a blonde, red or brown tinge; this does not arise from any special pigment, which is only found occasionally in the medulla of dark hairs, but proceeds from its being seen through the colored cortical substance. A more careful investigation of the medullary cells shows, that while fresh they contain many small cavities in a viscid substance; in these lie the air vesicles, which communicate to them the granular appearance above described. If we observe the air which has been expelled refilling the medulla of a dried hair, it seems as if all the cavities of one and the same cell communicated with one another, at least the air frequently passes in continuous winding streams from one cavity into the other; indeed from the sudden manner in which the air sometimes fills the medulla, it might almost be believed that the cavities of contiguous cells were connected together. However this may be in certain cases, it is conceivable, that even if the cavities of the different cells are quite closed, and only separated from one another by delicate partitions, the air still may quickly fill the medulla under the appearances we have noted. For the rest, the vacuities of

Fia. 69.-Eight medullary cells, with pale nuclei, and fatty granules, from a hair treated with soda; magnified 350 diameters. 
the medullary cells, whether they are quite closed or not, are of different sizes, the aeriferous medulla appearing sometimes coarsely, sometimes finely granular. I have also seen cases in which the medullary cells obviously contained only a single large air-vesicle, and appeared almost like small fat-cells. Very frequently single larger or smaller spots may be observed in the medulla, which contain no air, and are thence pale, and this is constantly the case in the lowermost part of the medulla, close above the bulb.

The medulla and the cortex are widely different if we compare the extreme forms of their elements; in the one case we have rigid homogeneous elongated plates, almost without contents, in the other rounded vesicles filled with fluid or air. If, however, we take into account all their conditions, we shall find that the limits are not so marked, and in fact are often hardly distinguishable. On the one hand, for instance, the medullary cells are not unfrequently of an elongated or short fusiform figure, whilst on the other the plates of the cortex present a considerable cavity containing pigment. If such plates contain, instead of pigment or the smaller air-vesicles, air in a larger cavity, as occurs sometimes though not frequently, it is still more difficult to distinguish the two kinds of elements from one another, and the more so if, as in red hairs, the medulla and cortex are in places, or for considerable distances, not distinctly defined from one another, the superficial cells of the medulla being scattered and passing quite gradually into the plates of the cortex, which lie very close together and contain much air. It is not intended to imply, by this, that the medulla and the cortex are identical, but only that transitions exist, and that the differences which occur are less marked than is commonly supposed.

The diameter of the medulla is generally, in proportion to that of the hair itself, as 1: $3-5$; relatively and absolutely, it is thickest in short thick hairs, thickest in the down and hairs of the head. In a transverse section it presents a round or flattened figure, and the cells which comprise it are disposed in 1-5 or even more longitudinal series.

The medullary substance, the cells in which were first accurately described by G. H. Meyer, varies most of all the constituents of the hair. In the down of the hairs of the head, it has been stated, by some, that it is wholly absent, which is to be corrected thus far, that it is certainly generally absent in the former, and frequently in the latter, perhaps more frequently, in certain individuals. In white hairs, even those of the head, of a tolerable length and thickness, I have never failed to find it always beautifully distinct. In rare cases the medullary tract is double throughout(Bruns, figure in Hassall), more frequently divided in places into two tracts, which soon unite again. In the lower part of the root, the medulla, which is here clear, is often thicker, and exhibits 
the nuclei of its cells very distinctly, especially after the addition of acetic acid. Steinlin and Eylandt assert of the medullary substance, that it does not belong to the proper hair, but to its papilla, and originally represents a prolongation of this into the free part of the hair, which then dries up. This is incorrect. The papilla or germ of the hair is a part of the cutis, and has the same structure as the papilloe of the latter, whilst the medulla of the hair is composed of isolated cells, which by their resistance to alkalies, are in all respects allied to those of the epidermis. On the other hand, in animals, as has long been known, and as lately Bröcker has especially shown, the papilla often projects far, even to the point of the hairs, bristles or spines, subsequently drying up; but in these instances, according to Bröcker, it never, even after the action of potass, exhibits a cellular texture, whilst this is always obvious in the medullary substance, which is often present at the same time. Such an elongation of the papillæ may occasionally be noticed even in man, to a certain extent; thus Henle found it a few times prolonged into a short point. But any prolongation of this kind must be distinguished as decidedly from the cellular medullary substance, as in animals.

$\S 58$. The cuticle of the Hair (cuticula), is a very thin, transparent pellicle, which completely invests the hair, and is very closely united with the cortical substance. In its normal position, and observed in an unaltered hair, it is evidenced by hardly anything more than by numerous dark, reticulated, irregular or even jagged lines, which surround the hair at intervals $0.002-0.006$ of a line from one another, and occasionally also by small serrations at its apparent edge (Fig. $70 \mathrm{~A}$ ); if, on the other hand, a hair be treated with alkalies, the cuticle is raised in smaller or larger lamella from the fibrous substance, and is even separated into its elements. These are quadrangular or rectangular flat plates without nuclei, generally pale and transparent (Fig. $70 \mathrm{~B}$ ),

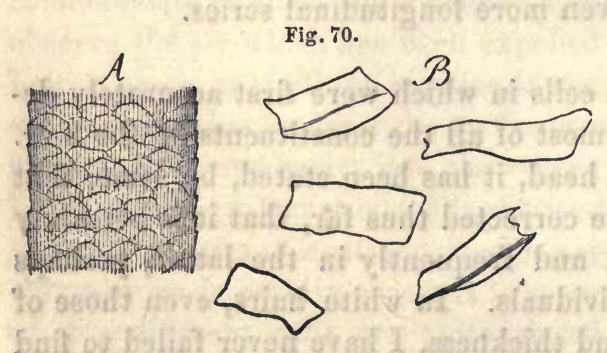
which do not swell up into vesicles by the action of any reagent, and disposed in an imbricated manner, form a simple membrane which completely surrounds the cortex of the hair in such a way, that the deeper or lower cells cover the upper ones. By sulphuric acid also the structure of the epidermis is readily made out; the hair is, as it were,

Fra. 70.- $A$, surface of the shaft of a white hair; magnified 160 diameters. The curved lines mark the free edges of the epidermic plates: $B$, epidermic plates from the surface, isolated by the action of caustic soda ; magnified 350 diameters. One or both of their longer edges are bent round, and so appear dark. 
bristled at the edges with the erected plates and by scraping or rubbing, the cuticle is less easily obtained in large lamellæe, but is readily enough reduced to its elementary parts.

On the shaft of the hair the cuticle consists only of a single layer of plates $0.002-0.003$ of a line thick, which measure $0.024-0.028$ of a line in the transverse direction of the hair; $0.016-0.02$ in that of its length; and are hardly more than 0.0005 of a line in thickness. The same structure exists also in the upper part of the root of the hair; of its lower part, on the other hand, so far as the inner root-sheath extends, two layers of the epidermis constantly occur. The outer (Fig. $(68 d$ is rendered especially obvious by the action of soda or potassa, and with a little pressure frequently comes away from the hair with the inner root-sheath, whilst the inner layer becoming undulated, remains lying upon the cortical substance, and may be easily studied, as well in the side view as upon its surface. In hairs that are torn out, this layer is found only where they are covered by the inner root-sheath, otherwise it remains behind in the hair-sac. Its elements also, are broad cells without nuclei, covering one another like tiles, which do not swell up in alkalies, and are soluble with great difficulty; they are thicker than those of the other layer, and measure only 0.002-0.004 of a line in the direction of the length of the hair. The whole outer layer measures $0.0016-0.002$ of a line, whilst the inner layer upon the root has a thick. ness of $0.0025-0.0035$ of a line. Upon the bulb of the hair, the two layers of cuticular plates pass with a tolerably defined margin into soft nucleated cells, which are broad in the transverse direction of the bulb, very short longitudinally, and somewhat longer in their third diameter, which stands perpendicularly or obliquely to the longitudinal axis of the hair. They are readily attacked by alkalies, or even by acetic acid, possess without exception transverse and longish nuclei, and finally pass, on the bulb, into the already described, round cells of which it is formed.*

* [We cannot agree with Professor Kolliker that the cuticle of the hair passes into the outer cells of the bulb. It may be worth devoting a little space to this matter, as the whole question of the homology of the hair essentially turns upon it. So far from being able to trace the two layers of the cuticle into the round cells of the bulb, we find that they cease somewhat suddenly when the shaft begins to expand, while its substance is fibrous-looking and contains only much elongated nuclei. Below this point, as Henle has correctly, figured in his "Allgemeine Anatomie," Pl. I. Fig. 14, the transverse striations of the cuticle are absent; and if the cuticular layer be viewed in section, it will be seen to be composed of a more transparent substance, which gradually becomes thinner until it is hardly distinguishable as a distinct layer, and at the same time loses the oblique lamination, which it has above, where it is continuous with the two layers of the cuticle proper. The careful addition of caustic ammonia is particularly fitted to demonstrate the structure of this part. In the first place, it raises up the outer layer of the cuticle from the inner, and shows that the former, at any rate, is not continuous with any cells; and secondly, it dissolves and forces out the substance of the lower soft portion of the bulb, so that the lower part of the cuticle may be obtained as a transparent, colorless, and independent sheath, even from 
$\S 59$. The hair-sacs, folliculi pilorum, are flask-like follicles 1-3 lines long, which embrace the roots of the hair tolerably closely, and, in the lanugo, are lodged in the substance of the upper layers of the corium, while in the stronger or long hairs, they generally project into its deeper portion, and even extend for a greater or less distance into the subcutaneous cellular tissue. These follicles are simply to be regarded as involutions of the skin, with its two constituents, the corium and the epidermis, and there may be distinguished, therefore, in each of them, an external fibrous, vascular part, the proper hair-sac, and a non-vascular cellular investment lining this, - the epidermis of the hair-sac; or, since it immediately surrounds the root of the hair, - the "root-sheath" (vagina pili).

$\S 60$. The proper hair-sac consists of two fibrous investments, an external and an internal, and of a structureless membrane; it is on an average $0.015-0.022$ of a line thick, and contains in its lower part a peculiar structure, the papilla of the hair.

The external fibrous membrane (Fig. 63 h), the thickest of the three layers of the hair-sac, determines its external form, and by its innermost layer is very closely connected with the corium. It consists of common connective tissue with longitudinal fibres, without any intermixture of elastic fibres, but with a considerable number of long fusiform nuclei; it contains a tolerably close plexus of capillaries, and exhibits also a few nervous fibrils with occasional divisions.

The internal fibrous membrane (Fig. $71 a$ ) is much more delicate than the external; bounded by smooth surfaces, and everywhere of equal thickness, it extends from the bottom of the hair-sac as far only as the entrance of the sebaceous glands. To all appearance, it contains neither vessels nor nerves, and is composed solely of a simple layer of transverse fibres, with a long narrow nuclei, which may be seen particularly well in the empty hair-sacs of both coarse and fine hairs, with or without the

the very darkest hairs; lastly, under favorable circumstances, this reagent raises up a definite basement membrane from the outer surface of the lowest part of the bulb, in immediate contact with the rounded "nuclei" of this part, and this basement membrane may be traced upwards into direct continuity with the homogeneous portion of the cuticle above-described. (In the "Edinburgh Monthly Journal of Medical Science," for March, 1853, Mr. Dalzell states that the papilla of the hair has a basement membrane. Is it this structure to which he refers?) In all cases in which, in man or in animals, we have isolated the hair-bulb from its sac, it seemed to have a definite limiting outer line down to the narrow neck by which it passes into the hair-sac, though it was not often easy to obtain evidence that this limiting line was the expression of a distinct basement membrane. However, the same difficulty would occur with any dermic papilla; and it seems to us that there is sufficient evidence to show that the cuticle of the hair is not the product of any direct metamorphosis of cells, but represents a modified basement membrane with a subjacent layer of peculiarly altered blastema, corresponding precisely with the "Nasmyth's membrane" and the enamel of the teeth. Vide infra, $\$$ on Teeth.-Trs.] 
addition of acetic acid. They resemble sinooth muscular fibres, although they cannot be completely isolated and actually recognized as true fusiform fibres with a single nucleus; on which account, and especially as no contractions of the hair-sacs have in general been observed, I must for the present refrain from positively deciding upon their nature.

The third layer, lastly (Fig. 71 b), is a transparent structureless membrane, which, when the hairs are torn out, invariably remains behind in the hair-sac, and extends from its base, though, as it would seem, without covering the papilla, as far as the inner root-sheath, and perhaps higher. In the uninjured hair-sac it appears only as a pale streak $0.001-0.0015$, rarely 0.002 of a line thick, between the outer root-sheath and the transversely fibrous layer of the hair-sac; by preparing an empty hair-sac, however, it can readily be obtained in large shreds, and then appears smooth externally; internally it is covered

Fig. 71.

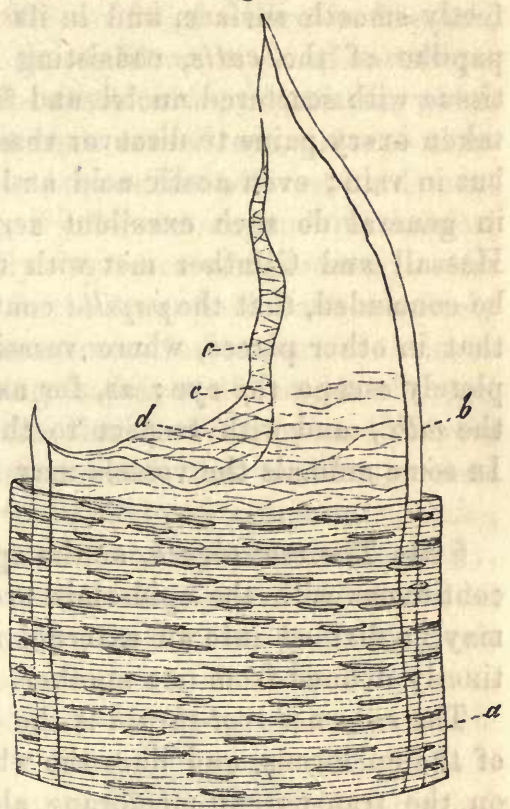
with very delicate, transverse, often anastomosing lines, which, like the membrane itself, remain unchanged in acids and alkalies. Neither acids nor alkalies bring out cells or nuclei in this membrane, and it therefore probably belongs to the category of true structureless membranes.

The papilla of the hair (Fig. $63 i$ ) also, less properly termed the hairgerm, pulpa pili, belongs to the sac, and corresponds with a papilla of the cutis. It is generally seen but indistinctly, especially in dark hairs with a colored bulb, either appearing, only as a clear, indistinctly defined spot, or after the tearing out of the hair, remaining so covered by the cells of the bulb that nothing can be made out of it. It is only in the hair-sacs of white hairs, that its outlines can be more frequently distinguished without wholly isolating it, especially by the help of a little pressure. Reagents, on the other hand, avail nothing, for they attack the papilla to about the same extent as the bulb, with the sole exception of a weak solution of caustic soda, in which it retains its outlines, for a

Fia.71.-A piece of the transverse fibrous layer, and of the structureless membrane (vitreous membrane) of a human hair-sac, treated with acetic acid; magnified 300 diameters: $a$, transversely fibrous layer with elongated transverse nuclei; $b$, vitreous membrane in apparent section; $c$, its edges, where the sheath which it forms is torn; $d$, fine transverse partly anastomosing lines (fibres) on their inner surface. 
time at any rate, whilst the cells of the bulb are freed, and may be pressed out of the sac. The papilla is ovate or fungiform, $\frac{1}{8}-\frac{1}{4} 0$ of a line long, $\frac{1}{11}-\frac{1}{20}$ of a line broad, and is connected with the layer of connective tissue of the sac, by a pedicle; it has sharp contours and a perfectly smooth surface, and in its structure completely agrees with the papillæ of the cutis, consisting of an indistinctly fibrous connective tissue with scattered nuclei and fat granules, but not of cells. I have taken every pains to discover vessels and nerves in the isolated papillce, but in vain; even acetic acid and dilute solution of caustic soda, which in general do such excellent service in these cases, have failed, and Hassall and Günther met with the same results. It must not hence be concluded, that the papilla contains no vessels or nerves, for we know that in other places, where vessels do certainly exist, they often completely escape the eye; as, for example, in the dermal papilloe and in the villi; and with respect to the nerves, in the papillæ of the cutis. In some animals the vessels may very readily be seen.

$\S 61$. The root-sheath, or the epidermic investment of the hair-sac, is continuous with the epidermis around the aperture of the follicle, and may be divided into an external and an internal layer, which are distinctly defined from one another.

The external root-sheath is the continuation of the stratum Malpighii of the epidermis, and lines the whole hair-sac, resting for its lower half on the transparent membrane above described; higher up, when this and the transverse fibres are absent, it lies directly upon the longitudinally fibrous layer. Its structure corresponds exactly with that of the stratum Malpighii, even in the having the outermost cells, which in the negro, according to Krause, are always brown, and in whites are so, at least in the hairs of the latia majora, towards the upper part, arranged perpendicularly. At the bottom of the hair-sac, the outer root-sheath, its cells becoming gradually rounded, passes continuously, and without any sharp line of demarcation, into the round cells of the hair-bulb which cover the papilla. The outer root-sheath is generally about 3-5 times as thick as the inner; but not unfrequently it becomes thinned towards its upper part, and below invariably passes into a very thin lamella. In the coarse hairs it measures in the middle of the root $0.018-0.03$ of a line, and presents 5-12 layers of cells.

The inner root-sheath (Fig. 68-e. g.) is a transparent membrane which extends from almost the very bottom of the hair-sac, over more than two-thirds of it, and then suddenly ceases. It is closely connected externally with the outer root-sheath, internally with the cuticle of the hair (its outer layer), so that normally there is no space between it and the hair; further it is distinguished by its great density and elasticity, and it consists in all but its lowermost part, of two or even three layers 
of polygonal, elongated, transparent, and somewhat yellowish cells, all of which have their longitudinal axes parallel to that of the hair (Fig. 68). The outermost layer (Fig. 72, A), which alone was formerly known, the inner root-sheath of Henle is formed of elongated cells without nuclei, $0.016-0.02$ of a line in length, and $0.004-0.006$ of a line in breadth, which are intimately connected, and in the ordinary mode of investigation, after the addition of acetic acid, caustic soda, or potassa, which swell up the hair, or after the hair has been teased out, present elongated fissures between them, whence they appear like a fenestrated membrane. In quite recent hairs, however, if all reagents and mechanical injury have been avoided, we see hardly any trace of apertures in the upper half of the layer in question, and in the lower half (from the finely fibrous part of the cortex upwards), at most mere indications of them, in the form of strix, clear or dark, according as they are in or out of focus, and similar to those of the cortex of the shaft. We can hardly avoid supposing, therefore, that the openings as they are commonly seen (0.005-0.008 of a line in length, and $0.001-0.03$ of a line in breadth), are produced artificially by the teasing out of the membrane. Secondly, cells also occur in the root-sheath, between which gaps are never visible. These (Fig. 72, B), which form a simple or a double layer (Huxley's

Fig. 72.

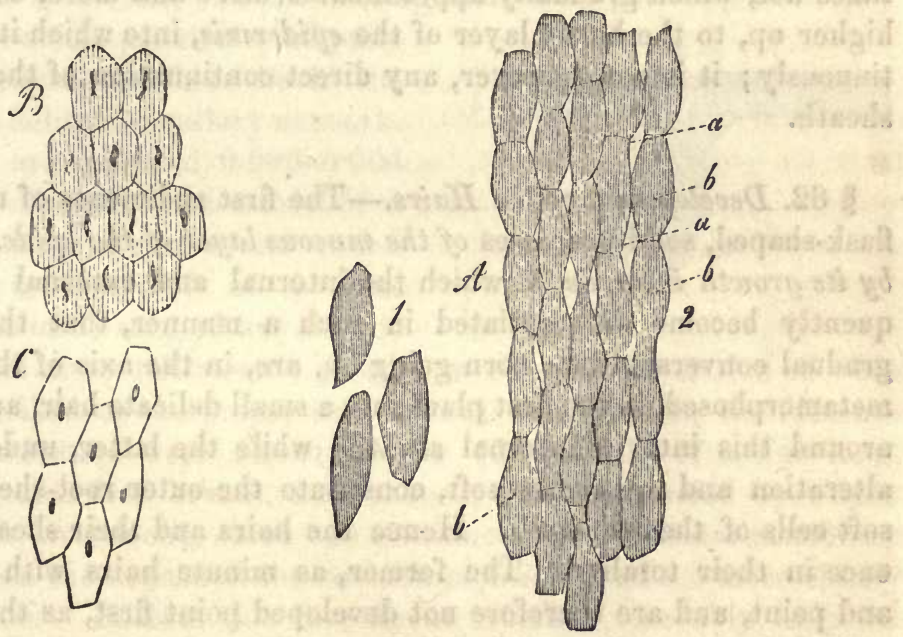

layer), are constantly situated internal to the common, and as far as I have seen, always single, fenestrated layer of cells; they are shorter and

Fig. 72.-Elements of the inner root-sheath; magnified 350 diameters. $A$, from the outer layer, 1 , its isolated plates; 2 , the same in connection, from the uppermost parts of the layer in question, after treatment with caustic soda: $a$, apertures between the cells, $b ; B$, cells of the inner not-perforated layer, with elongated and slightly notched nuclei; $C$, nucleated cells of the lowest part (single layer) of the inner sheath. 
broader than the cells which have already been described $(0.014$ to 0.018 of a line long, 0.006 to 0.009 of a line broad), but are also polygonal, and always possess, at least in the lower half of the root-sheath, distinct elongated nuclei, often prolonged into points of $0.004-0.006$ of line. The dianneter of the whole inner root-sheath is, upon the average, $0.006-0.015$ of a line, whence it follows that its cells, of which there are never more than three layers, are at least $0.002-0.005$ of a line thick. They are recognizable at once in their natural position, and by the teasing out of the root-sheath, and are readily isolated by the use of soda and potassa (Fig. 72), but without swelling up, a character which no less than their great resistance to alkalies altogether, distinguishes their cells, in common with the epidermic scales of the hairs, from all others.

At the bottom of the hair-sac, the inner root-sheath consists only of a single layer of beautiful, large, polygonal, nucleated cells, without any intermediate openings (Fig. 72,C); which becoming at last soft, delicate, and rounded, pass without defined limits into the outer layers of the round cells of the hair-bulb. Superiorly, this membrane not unfrequently becomes somewhat separated from the hair, and ends, not far from the apertures of the sebaceous glands in a sharp, notched edge, formed by its separate more or less projecting cells. Thence upwards, it is replaced by a layer of cells, in some cases at first nucleated, but at other times not, which gradually approximates more and more, as it is traced higher up, to the horny layer of the epidermis, into which it passes continuously; it is not, however, any direct continuation of the inner rootsheath.

$\S 62$. Development of the Hairs. - The first rudiments of the hairs are flask-shaped, solid processes of the mucous layer of the epidermis formed by its growth inwards, in which the internal and external cells subsequently become differentiated in such a manner, that the former, a gradual conversion into horn going on, are, in the axis of the rudiment, metamorphosed, in the first place into a small delicate hair, and secondly, around this into its internal sheath; while the latter, undergoing less alteration and remaining soft, constitute the outer root-sheath and the soft cells of the hair-bulb. Hence the hairs and their sheaths arise at once in their totality. The former, as minute hairs with root, shaft, and point, and are therefore not developed point first, as the teeth are, with their crown first, and still less as Simon has supposed, from their root first. The elements of the youngest hairs are nothing but elongated cells similar to those of the cortex of the later hairs, which are developed by the lengthening and chemical alteration of the innermost cells of the rudiments of the hairs. Medullary cells are entirely wanting, but the cuticle is clearly visible. The inner sheath is striated, presents no openings, and consists of elongated cells, which have been developed 
from those lying between the hair and the outer sheath. The proper hair-sac is formed, in its fibrous layers, essentially in loco, out of the formative cells which surround the rudiment of the hair; possibly, however, they may be considered as an involution of the cutis, produced by the ingrowing process of the epidermis. Its structureless membrane, which appears very early, is, not improbably, closely related to the external cells of the rudiment of the hair, answering to the outer hairsheath, and formed by an excretion from them like the membrance proprice of the glands; as to the papilla, it is hardly possible to consider it as anything but an outgrowth of the fibrous layer of the hair-sac, analogous to the papillæ of the cutis in general; though the circumstance that it appears at a time when the hair-sac is hardly demonstrable as a whole, and that it may always be pulled out together with the rudiments of the hair and root-sheath, is apparently opposed to this view.

The first rudiments of the downy hairs and of their sheaths, are found in the human embryo at the end of the third or at the beginning of the fourth month, upon the forehead and eyebrows. They consist of papilliform masses of cells 0.02 of a line in diameter (Fig. 73) which are visible, even to the naked eye, as minute whitish spots separated by regular intervals. They are continuously connected with the rete Malpighii of the epidermis, and are nothing more than perfectly solid processes of it, which penetrate obliquely into the corium, and here lie in the meshes of a delicate capillary network. These cells are spherical, $0 \cdot 003-0.004$ of a line in diameter, and consist of a clear granular substance and round nuclei of $0.002-0.003$ of a line. Nothing was to be seen of any dermic

Fig. 73.

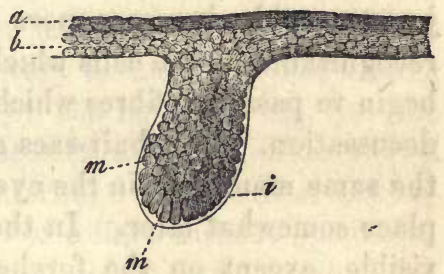
investment of these rudiments ; in other words, the foundation of what I have described as the proper hair-sac was not laid. In the fifteenth week the processes were already larger $(0.025-0.03$ of a line long, $0.013-0.02$ of a line broad), flask-shaped, and surrounded by a thin structureless investment, which was continued into a delicate membrane lying between the cutis and the rete Malpighii, but united more closely with the latter. Besides this investment, which is, probably, only the structureless membrane which I have discovered in the perfect hair (see $\S 60$ ), another external layer of cells occurs on the hair-sacs, which can

Fig. 73.-Rudiment of the hair from the brow of a human embryo, sixteen weeks old; magnified 350 diameters : $a$, horny layer of the epidermis; $b$, its mucous layer; $i$, structureless membrane surrounding the rudiment of the hair and continued between the mucous layer and the corium; $m$, roundish, partly-elongated cells, which especially compose the rudiment of the hair. 
generally be separated only in shreds with it, from the cutis, rarely altogether: this I regard as the first indication of the fibrous layers of the hair-sacs. In the sixteenth and seventeenth weeks, the processes of the mucous layer, which I will henceforward simply call "hair-rudiments," increase in size up to $.004-0.06$ of a line in length, and $0.03-$ 0.04 of a line in breadth, and acquire thicker coverings, but as yet exhibit no trace of a hair. In the eighteenth week these first appear in the eyebrows, as hair-rudiments of $0 \cdot 1-0 \cdot 2$ of a line, their central cells becoming somewhat elongated, and arranging themselves with their longitudinal axes parallel to that of the rudiment, whilst the peripheral cells are disposed with their now longer diameter transversely. A variety of shade in the hitherto homogeneous hair-rudiment arises in this manner, and a central substance, broad below, running above into a sharp point, becomes marked off from an outer portion, which is narrow. below and thick above. When the rudiment has attained a length of 0.22 of a line, this marking off is still more distinct, the rather longer and especially broader, inner cone having a somewhat clearer appearance (Fig. 74). Finally, in rudiments of hair of 0.28 of a line, the inner cone is divided into two structures, a central portion somewhat darker, and an external, perfectly transparent and glassy,-the hair and the inner root-sheath,-whilst the peripheral cells which have remained opaque, constitute unmistakably the outer root-sheath (Fig. 75 A). At the same time the papilla, which was even before (Fig. 74) just traceable, becomes more distinct, and the proper hair-sac also more recognizable, as the cells which lie external to its structureless membrane begin to pass into fibres which may, even at this time, be known by their decussation. The hair-sacs and hairs arise, in other places, exactly in the same manner as in the eyebrows, except that their development takes place somewhat later. In the fifteenth week, no rudiments of hairs are visible, except on the forehead and eyebrows; in the sixteenth and seventeenth week they appear all over the head, back, chest, and abdomen; and not till the twentieth week on the extremities. The hairs themselves never make their appearance earlier than 3-5 weeks after that of the rudiments; in the nineteenth week, for example, the commencement of hairs is nowhere to be seen, except on the forehead and eyebrows; and in the twenty-fourth week they are still absent upon the hand and foot, and partly on the forearm and leg.

Once formed, the hairs and hair-sacs continue to grow. The former sometimes penetrate the epidermis immediately (eyebrows, eyelashes, Fig. 75), sometimes their points are insinuated between the horny layer and the stratum Malpighii, or among the elements of the horny layer itself, and grow for a time covered by the epidermis (chest, abdomen, back, extremities [?]), through which they eventually make their passage. Involutions of the skin growing towards the hairs as they pass 
out, never exist, and the supposition that they do, rests upon a wholly subjective foundation.

Fig. 75.

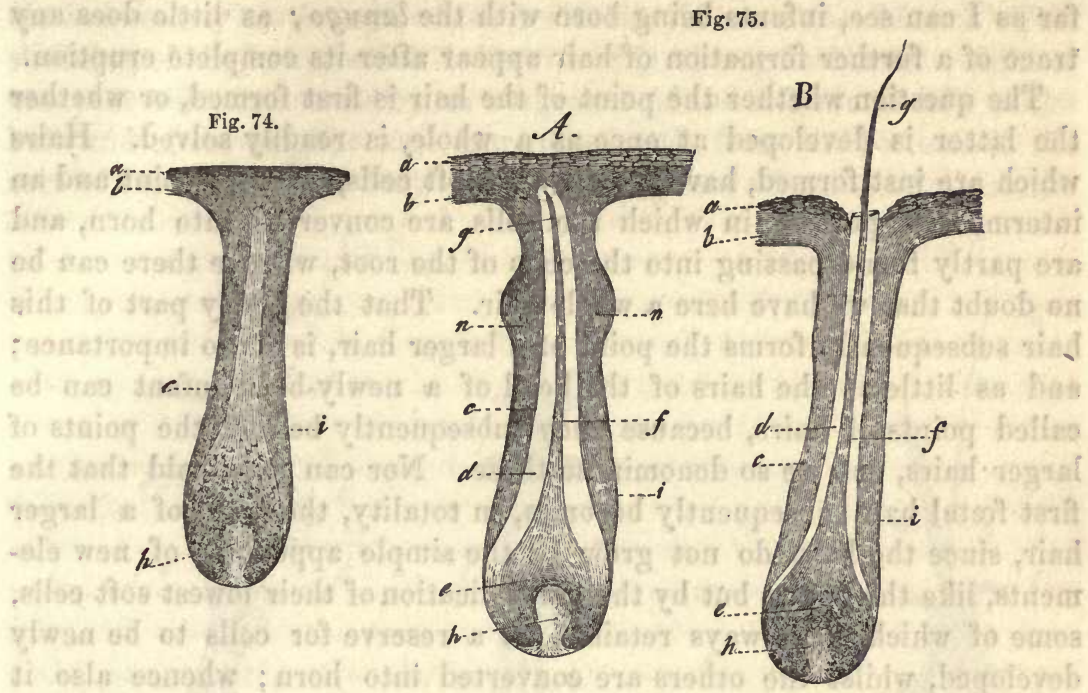

The downy hairs, lanugo, the eruption of which is completed in the 23-25th week, are short fine hairs, whose peculiar arrangement has been noted above. They measure on the bulb 0.01 , on the shaft 0.006 , at the point $0.0012-0.002$ of a line; are pale, or almost colorless, and consist only of cortical substance and a cuticle. In man, the bulb is usually colorless, and often rests upon a very distinct papilla, arising in the ordinary manner from the bottom of the hair-sac. It has the same three layers as in the adult, and possesses a very well-developed epidermic investment, consisting of an external root-sheath of $0.004-$ 0.012 , and an inner sheath of $0.006-0.008$ of a line, without openings.

After their eruption, the downy hairs grow slowly to a length of $\frac{1}{4}-\frac{1}{3}$ of a line, and in fact to a greater length in the head than elsewhere. Generally they remain to the end of foetal life, gradually acquiring a darker color, becoming in many cases, as on the head, even blackish; another small portion falls off into the liquor amnii, is swallowed with

Fra. 74.- Rudiment of a hair from the eyebrow ( 0.22 of a line in length), its inner cells forming a distinct cone, as yet without any hair, but with the papilla indicated: $a$, horny layer of the epidermis; $b$, mucous layer; $c$, outer root-sheath of the subsequent sac; $i$, structureless membrane upon its outer side; $h$, papilla of the hair--Magnified 50 diameters.

Fig. 75.- $A$, rudimental hair from the eyebrows, with just developed but not yet erupted hair, of 0.28 of a line in length. The inner root-sheath projects beyond the point of the hair somewhat at the upper part, and laterally at the neck of the sac; the first rudiments of the sebaceous glands appear in the form of two papillary outgrowths from the outer root-sheath. $B$, hair-sac from the same, with its hair just erupted; the inner root-sheath projects through the aperture of the hair-sac; the rudiments of sebaceous glands are as yet not developed; $a, b, c, h, i$, have the same signification as in Fig. $74: e$, hair-bulb; $f$, hair-shaft; g, hair-point; $n$, rudiments of the sebaceous glands. 
this by the foetus, and may afterwards be found in the meconium. A proper shedding of the hair does not take place at all in the foetus, so far as I can see, infants being born with the lanugo; as little does any trace of a further formation of hair appear after its complete eruption.

The question whether the point of the hair is first formed, or whether the latter is developed at once as a whole, is readily solved. Hairs which are just formed, have a bulb with soft cells, a horny point and an intermediate portion, in which the cells are converted into horn, and are partly found passing into the cells of the root, whence there can be no doubt that we have here a whole hair. That the horny part of this hair subsequently forms the point of a larger hair, is of no importance; and as little as the hairs of the head of a newly-born infant can be called points of hairs, because they subsequently become the points of larger hairs, can we so denominate these. Nor can it be said that the first foetal hair subsequently becomes, in totality, the point of a larger hair, since the hairs do not grow by the simple apposition of new elements, like the bones, but by the multiplication of their lowest soft cells, some of which are always retained as a reserve for cells to be newly developed, whilst the others are converted into horn; whence also it happens, that the cells even of a complete hair-bulb are to be regarded as the successors of those of the fotal hair.*

* [From what has been said above (see note on the Cuticle) it is clear we do not share Professor Kölliker's view tliat the hair is an epidermic production. Reichert's view, on the other hand, that the hair results from the cornification of a dermic papilla or natrix, which drying up and becoming filled with air, remains as the medullary portion, seems to us to be nearer the truth. There can be no doubt of these two facts: 1 , that no line of demarcation can be traced between the papilla of the hair and its shaft; and 2, that in many animals the papilla is vascular and nervous for a considerable distance into the shaft, and, therefore, is certainly a dermic structure.

Whether Reichert's somewhat mechanical notion of the "drying up" of the matrix to form the medulla is correct, is not of much importance, so long as we keep in view the unquestionable continuity of tissue and homological identity, of the medulla and cortex with the dermic papilla.

For us, in fact, the Hair is homologous in all its parts with the Tooth. The substance of the shaft corresponds with the dentine, offering even rudimentary tubes in its aeriferous cavities; the inner layer of the cuticle answers to the enamel, the outer to Nasmyth's membrane; and whoever will compare these structures will be struck by the similarity even in their appearance. The sac answers to the dental capsule; the outer root-sheath to the layer of epithelium (enamel organ) next the capsule; the fenestrated membrane to the stellate tissue; and what Professor Kölliker calls "Huxley's layer," to the columnar epithelial layer of the organon adamantince. The comparison may seem startling at first, but the examination of the development of the teeth of an osseous fish, for example, will suffice, we believe, to afford full justification of it.

With respect to the not very important question, as to the nature of the first rudiment of the hair-shaft, i. e. whether it is the point of a hair or a whole hair, we must confess that we should be tempted to arrive at the opposite conclusion to our author. Inasmuch as the portion of the hair which first appears becomes the point of the fully-grown hair, we should say that the hairs are formed like the teeth, point first.

A hair, like a tooth, has a definite form to attain. As the latter has a peculiarly con- 
\$63. Shedding of the Hair.-After birth, a total shedding of the hairs takes place in consequence of the development of new hairs within the hair-sacs of the lanugo, which gradually force out the old ones. This shedding of the hairs, which I discovered in the eyelashes of a child of one year old, commences by an outgrowth of the soft round cells of the bulb and of the neighboring outer root-sheath, from the bottoms of the sacs of the lanugo, into long processes composed of cells, by which the

Fig. 76.

Fig. 77.
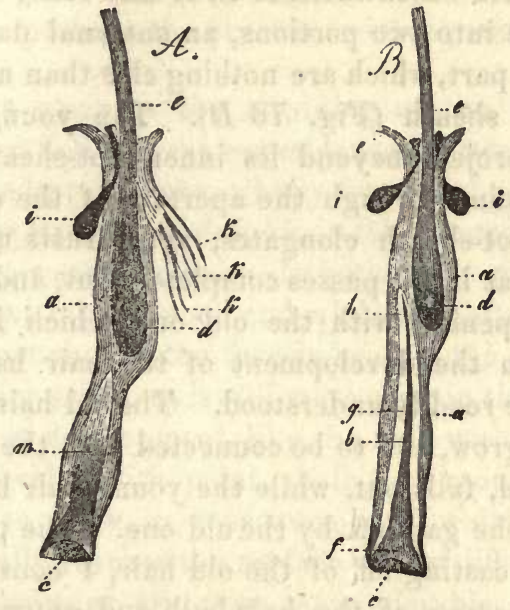

hair is raised from its papilla, whilst at the same time it becomes converted into horn even in its lowermost portion. When these processes have attained a length of 0.25 of a line, a differentiation of their outer and inner cells takes place, similar to that which has been already

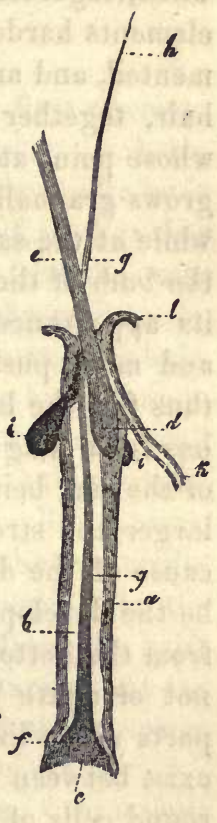

FrG. 76.-The eyelashes of a child of one year old pulled out; magnified 20 diameters: $A$, one with a process of the bulb or of the outer root-sheath, of 0.25 of a line, in which the central cells are elongated (their pigment is not represented), and are clearly defined as a cone from the external ones; $B$, eyelash in whose process, of 0.3 of a line, the inner cone is metamorphosed into a hair and an inner root-sheath; the old hair is pushed up, and like $A$ and Fig. 75, possesses no inner root-sheath : $a$, outer; $b$, inner root-sheath of the young hair; $c$, pit for the papilla of the hair; $d$, bulb; $e$, the shaft of the old hair; $f$, bulb; g, shaft; $h$, point of the young hair; $i$, sebaceous glands; $k$, three sudoriparous canals, which in $A$ open into the upper part of the hair-sac; $l$, transition of the outer root-sheath into the rete mucosum of the epidermis.

Fia. 77.-An eyelash with the root-sheaths from a child one year old, with an old and a growing young hair, magnified 20 diameters : the young hair is wholly extruded, and now two hairs appear at one aperture. A sudoriparous canal opens into the hair-sac. The letters have the same signification as in Fig. 76.

structed and narrowed root when complete, so has the hair when it has attained its full growth a peculiarly constructed bulb; and it is not a perfect hair until this peculiar bulb is developed. Until it has attained this form it goes on growing; but once having reached it, it grows no more, but falls out and is replaced by a new hair (see following \$).-TRs.] 
described as occurring in those processes of the stratum Malpighii, in which the hairs of the lanugo are developed. The outer cells, in fact, remaining round and colorless, as they were before, the inner ones begin to develop pigment in their interior and to elongate, becoming distinguished at the same time from the former, as a conical substance with its point directed upwards. At first (Fig. $76 \mathrm{~A}$ ), this central substance is quite soft, and like the layers of cells which surround it externally, dissolves readily in solution of caustic soda; subsequently, however, when, together with the process which incloses it, it has elongated, its elements harden, and separate into two portions, an enternal dark pigmented, and an external clear part, which are nothing else than a young hair, together with its inner sheath (Fig. $76 \mathrm{~B}$ ). The young hair, whose point at first does not project beyond its inner root-sheath, now grows gradually, forcing its point through the aperture of the old sac, while at the same time its root-sheath elongates, and thrusts upwards the bulb of the old hair, until at last it passes completely out, and makes its appearance at the same opening with the old one, which is more and more pushed up. When the development of the hair has gone thus far, the last stage may be readily understood. The old hair, which has for a long time ceased to grow, and to be connected with the bottom of the sac, being thus extruded, falls out, while the young hair becomes larger and stronger, and fills the gap left by the old one. The primary cause of the dying away and casting off of the old hair, I consider to be the development of the processes of the hair-bulb and outer sheath from the bottom of the sac, which has been described. As the sacs do not elongate to a corresponding extent, they push upwards all those parts which lie above them, and cause a continually increasing space to exist between the papilla and the proper hair, or the point at which the round cells of the bulb begin to elongate and undergo conversion into horny matter.

The hair thus becomes in a manner detached from the source of its nourishment; it receives less and less blastema, at last ceasing to grow, and becoming converted into horn in its lowest part. The cells of the processes, on the other hand, which are connected with the papilla, are incessantly supplied from it with new formative material, which for the time they apply not to the formation of horny matter, but to their own growth. In this manner the processes continue to grow, and mechanically elevate the cornified root of the old hair with its sheaths, to the aperture of the sebaceous glands, where to all appearance a partial solution of the old sheaths takes place : this may be observed with certainty in the inner sheath, and must be assumed to occur in the outer.

All that has been said, holds good only with respect to the eyelashes. The hairs of the head, and the other hairs of the body of the child (almost a year old) in question, never contained more than one hair, though 
their bulbs presented processes without hairs like those which precede the shedding of the eyelashes; such processes, in fact, being of very common occurrence in the hairs of children within the first year. I believe I am not wrong, if from the presence of these processes I deduce the universal occurrence of a shedding of the hairs, particularly as it is certain that in many children within the first 2-6 months after birth, the hairs of the head fall out and are replaced by new ones. However, further observation is necessary to determine what period is occupied by this first shedding of the hair, in what hairs it occurs, and whether perhaps the process is subsequently repeated.

If we compare the shedding of the hairs with their first development, we find a great resemblance between the two processes. In both, elongated projections, wholly formed of round soft cells, shoot like buds from the stratum Malpighii, in the one case of the skin itself, in the other of the hair-sacs and hairs. In both, a separation of the inner from the outer cells next takes place; and while the latter are metamorphosed into the outer root-sheath, the former become the inner root-sheath and the hair. The latter arises, as is still more clear in the shedding of the hairs than in their first development, like the nail, with all its parts at once, as a small hair provided with point, shaft, and root, and which only subsequently begins to grow, in consequence of which it enlarges in all its parts, and finally reaches the surface. The differences between the two modes of development are very inconsiderable, and chiefly depend upon the rudimentary hair-processes, in the one case proceeding from the hairs themselves, but not in the other; and upon the circumstance that the young hairs, although in both cases they lie at first in a closed space, reach the surface more readily in the one case, than in the other.

In the periodical shedding of the hair of animals, the observations of Heusinger and Kohlrausch, and lately those of Langer, Gegenbaur, and Steinlin, show that the new hairs are also developed in the sacs of the old ones; although, according to the last author, with whom however Langer is not quite in accord, the process does not appear to be exactly the same as in man.

$\S 64$. Physiological Observations. - The hairs have a definite length, dependent upon locality and sex, but if they are cut they grow again, and consequently exhibit the same conditions as the other horny textures. The place from whence the growth of the hair proceeds is unquestionably the bottom of the hair-sac. Here there arise around the papillæ with the co-operation of a blastema formed out of its vessels or those of the hair-sac, new elements, by the continual multiplication of the existing cells, while those which are already present, somewhat higher up pass uninterruptedly, the middle ones into medullary cells, the next into cor- 
tical plates, the outermost into epidermic scales, and thus the horny part of the hair is continually forced from below upwards, and elongates. In the latter no formation of elementary parts takes place, but at most a certain metamorphosis of those which are already existent, which produces a gradual thinning of the root from the bulb upwards, until it acquires the thickness of the shaft. Higher up still, these changes of the elementary parts cease, whence cut hairs, for example, do not produce new points. The root-sheaths and the outer layer of the epidermis take no part in the growth of the cut hairs.

The complete hair, though non-vascular, is not a dead substance. Although the processes which go on in it are not at all understood, we may suppose that fluids are diffused through it which subserve its nutrition and maintenance. These fluids are furnished from the vessels of the papilla and sac of the hair, in all probability ascend (particularly from the bulb); without any special canals through the cortex upwards, and thus reach all parts of the hair. Having served for the nutrition of the hair, they evaporate from its outer surface and are replaced by a fresh supply. Perhaps the hairs also absorb fluids from without, though of course only in the condition of vapor, like a hair used as a hygrometer; on the other hand I cannot believe that, as many authors would seem to suppose, the secretion of the sebaceous glands passes from without into the hairs, since the perfectly closed cuticle is probably impervious to it. In the same way it seems to be in nowise proved that the hairs are pervaded by a peculiar oleaginous fluid (Laer), which might proceed from the medullary substance (Reichert), and which keeps it greasy, for such a fluid has not been demonstrated, and the greasiness of the hairs may be more simply explained by the externally adherent sebaceous matter, which is readily visible. The existence of hair in the medullary axis and in the cortex can only arise from a disproportion between the supply of fluid from the hair-sac and the amount eraporated; it is owing as it were, to a drying-up of the hair, which, however, must not be supposed to go so far that the hair contains no fluid in its aeriferous portion. In any case, however, these portions are the most inactive, or relatively dead parts of the hair; the cortex, on the other hand, which is also most readily altered by alkalies and acids, notwithstanding the apparent hardness and density of its elements, is the most rich in juices, and is that in which the nutritive process is most actively going on. Hence it follows, that the hair lives, and is to a certain extent dependent upon the collective organism, particularly on the skin, from whose vessels (i. e. those of the hair-sac) it derives the materials necessary for its maintenance. Therefore, as Henle well says, the condition of the hair is a sort of index of that of the activity of the skin; if they are soft and shining, the skin is turgescent and transpires; if they are dry, brittle, and rough, then it may be concluded that the surface of the body is in a collapsed condition. 
The falling out of the hairs certainly depends, in many cases, - when, for example, it takes place in the course of normal development, - on nothing else than a want of the necessary nutritive material, which in the instance already explained, in speaking of the shedding of the hairs, depends on the detachment of the hair from its matrix by the abundant production of cells at the bottom of the hair-sac. In age, perhaps, it arises simply from the obliteration of the vessels of the hair-sacs.

The whitening of the hairs, which chiefly depends upon a decoloration of the cortex, and less upon that of the almost colorless medulla, should probably be here considered, for its normal occurrence in old age gives it the significance of a retrogressive development.

The frequent occurrence of cases, in which the hair grows gray first at its point or in the middle, and the well-established instances of its rapidly becoming white, are interesting, and strongly testify to the vitality of the hair; but it has not yet been shown, what peculiar processes in the elements of the hair produce the decoloration of its different pigments.

As in youth hairs which are shed are replaced by others, so at a later age something similar appears to occur. It is quite certain that during the period of full health and activity, a continual replacement of the numerous hairs which fall out goes on; furthermore that new hairs in great numbers spring up at the time of puberty in certain localities, but the manner in which this takes place is unknown. Inasmuch as even in adults we find hair-sacs with little processes downwards, whose proper hair has an abrupt clavate end, as in the child; since further, in this case it not unfrequently happens that two hairs come out of one aperture, and even exist together in one sac; and, finally, since in hairs which have fallen out spontaneously, we invariably find roots like those* which exist in the extruded hairs of the first shedding, it may be assumed that an actual shedding of the hairs occurs, even at a later period, in such a manner that the old hair-sacs produce new hairs while they throw off the old ones. I do not, however, intend to affirm by this, that an actual new formation of hairs does not occur after birth, but only this much, that in adults they are certainly regenerated from the already existing hair-sacs, especially if it be recollected that, accord-

* [Henle ("Allg. Anat.," p. 303) gives a very excellent description of this state of the hair-bulb: "Instead of the soft cellular hair-bulb, we find an inconsiderable clavate enlargement, which is solid and fibrous, like the substance of the shaft, only more clear. From its outer surface, short and irregular processes project downwards, which are probably the notched lower edges of the outermost layers of the cortical substance; they look like fibres connecting the hair with the inner wall of the sac. This kind of root is found in hairs which have fallen out spontaneously, and it is, therefore, probable that it belongs to a later stage of development of the hair, or rather marks the conclusion of its development. When the connection with the sac has ceased, which is the case in these clavate roots, the hair grows no longer; probably it is no longer nourished, but falls out."-Trs.] 
ing to Heusinger's observations, the whiskers of dogs, when pulled out, are produced from the same sacs in a few days, and also that during the shedding of the hair in adult animals, according to Kohlrausch, the young hairs are produced from the old sacs. Also, when the hairs which have fallen out after a severe illness, are replaced, it is more probable, since, according to $\mathrm{E}$. $\mathrm{H}$. Weber, the sacs of lost hairs remain for a long time, that they arise in the old sacs, than that new ones are developed.*

The multiplication of the cells of the bulb of the hair during its growth takes place unquestionably, not by free cell-development, since no trace of anything of the kind is to be seen in any bulb, but either by endogenous cell-development round portions of contents, or by division. I do not think that all those hairs which possess a sharply-defined clavate bulb are on that account dead and ready to fall out. It is certainly thus in many cases; but in others this condition indicates nothing more than the normal termination of growth, whence of course, it does not follow that the nutrition also has ceased. In proof of the occutrence of a continual development of the hairs independently of the old hairsacs, the hairs which lie spirally curled up under the epidermis and subsequently break through it, upon the forearm, leg, \&c., are frequently cited. But I do not know that it would not be more correct to consider this, with many pathologists, rather as an abnormal process. In the first place this formation of the hairs by no means occurs in all persons; and secondly, where it does, there are found together with those coiled-up hairs, which are apparently normally developed, others which are evidently abnormal, in great quantities. These, often in considerable number (up to 9), with thick sheaths, lie in one sac and have rounded points, with irregular bulbs. With respect to their relations, it might for the present be wiser, so long as an actual, normal new development of hairs has not been demonstrated, not to assume it, and to consider that, even at a later period, the development of new hairs within the old sacs is the normal mode, especially since Dr. Langer has actually observed it to take place in many instances in the very same manner as that which $I$ have described in children. The reason why the hairs grow continually, if they are cut, but not otherwise, is the same as I have already adduced, to account for the same occurrence in the

* [Berthold (Müll "Archiv," 1850) has communicated some curious statistics relative to the growth of Hairs. The hairs of the head of a female of from 16 to 24 years of age, grow at the rate of 7 lines a month. The growth of the hairs of the beard is quicker the oftener they are cut; shaved every 12 hours they would attain a length of from $5 \frac{1}{2}-12$ inches per

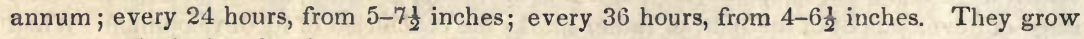
faster by ${ }_{15}^{1}$ during the day than during the night; and in 18 days of summer, 0026 more than in 18 days of winter.-Trs.] 
nails. The vessels of the papilla excrete a certain quantity of nutritive fluid, just so much as is sufficient to keep the whole hair continually moist and in a state of vitality. If the hair be cut, more nutritive fluid is supplied than the hair can use, and therefore it grows by the aid of the superfluity until it has attained its typical length again, or if it be continually cut, it as continually grows.

Dzondi, Tieffenbach ("Nonnulla de regeneratione et transplantatione," Herbip, 1822) and Wiesemann (De coalitu partium, Lips. 1824) have succeeded in transplanting the hairs with their sacs. Hairs are developed also in abnormal places, e. $g$. on mucous membranes, in encysted tumors, ovarian cysts, and in all these cases, even in the lungs (Mohr's case), possess sacs, root-sheaths, and an otherwise normal structure. No hairs are developed upon cicatrices of the skin. No satisfactory reason can be given for the excessive growth of the hairs, nor for their morbid universal falling out, together with their frequent reproduction in the same way; probably the principal causes are to be found in increased or diminished exudations from the vessels of the papilla and of the hair-sac, and more remotely in the state of the skin and the organism in general. In other cases vegetable productions (fungi) in the interior of the hair itself (in Herpes tonsurans, the "Teigne tondante," Mahon), according to Gruby ["Gaz. Méd.," 1844, No. 14], and Malmsten, (Müll. "Arch.," 1848, 1), or under the epidermis of the hair and around it (in the Porrigo decalvans of Willan according to Gruby), are concerned in the production of baldness, which then is limited (Alopecia circumscripta). The process of becoming gray is also obscure, although grief, excessive intellectual activity, and nervous influences are sometimes evidently concerned in it. It is not until physiology and chemistry have approached these latter processes, that we can hope for a scientific pathology and treatment of the hair. Plica polonica, which, according to Bidder (l. c.), is a disease of the shaft of the hair, is said by Guensburg and Walther (Müller's "Archiv," 1844, p. 411, and 1845, p. 34), to arise from a fungus which is developed in the hairs (bulb, shaft), and partly destroys them; whilst Münter (ibid., 1845, p. 42) could find no such fungus. This disease, as well as peculiar yellowish-white rings upon the human hairs, consisting of epithelial cells without nuclei (Svitzer, in "Fror. Notizen," 1848, No. 101), which appear to consist of an altered secretion of the sebaceous glands, are less interesting from a histological point of view, and therefore are but shortly adverted to here.

For microscopic investigation, a white hair with its sac should be chosen in the first instance, subsequently colored ones. Transverse sections may be obtained, either by shaving twice at short intervals (Henle) or by cutting hair on a glass (H. Meyer), or in a bundle between two cards (Bowman), or fixed in a cork (Hartin); longitudinal 
sections, by splicing a finer or splitting a coarser hair. The hair-sacs may be examined, both isolated and with the hair ; their different layers may be separated by preparations, and the nuclei of the external ones may be demonstrated by acetic acid. Concerning the papilloe, all that is necessary has been said above; the whole upper part of the root-sheath generally follows the hair when it is torn out, and in the macerated skin it comes out very readily with the hair; its cells may be made out without addition, or by a little acetic acid or caustic soda. The inner rootsheath is often to be found entire in torn out hairs, and may without further preparation, or by stripping off the outer sheath, be readily recognized in all its parts. Caustic soda and potassa acting for a short time, make it still more distinct. The cuticle must particularly be examined with alkalies and sulphuric acid, like the hair itself. The most important details upon this point have already been given, and more may be found in Donders (l. c.). I will only add that in this case also, the application of a high temperature (see above, in the section on the nails) saves much time. In investigating foetal hairs, in the very young state it is sufficient to tear off the epidermis, attached to which the rudiments of the hairs will be found. In older embryos, fine sections of the skin must be made; or the epidermis and the corium may be stripped off together, in which case caustic soda is of assistance.

Literature.-Eble, "Die Lehre von den Haaren in der gesammten organischen Natur.," 2 Bde., Wein, 1831; Eschricht, "Ueber die - Richtung der Haare am menschlichen Körper," in Müll. "Arch.," 1837, p. 37 ; V. Laer, "De structurâ capill. hum. observationibus microscopicis illustr.," "Dissert. inaug.," Traject. ad Rhenum, 1841, und "Annelin der Chemie u. Pharmacie," Bd. 45, No. 147; G. Simon, "Zur Entwicklungsgeschchite der Haare," Müll. "Arch.,"1841, p. 361; Krause, article "Haut.," in Wagner's "Handwörterbuch d. Phys." 1844, Bd. ii. p. 124 ; Kohlrausch, "Ueber innere Wurzelscheide und Epithelium des Haares," Müll. "Arch.," 1846, p. 300; Jasche, "De telis epithelialibus in genere et de iis vasorum in specie," Dorpat, 1847; Kölliker, "Ueber den Bau der Haarbälge und Haare," in the "Mitthiel d. zürich., naturf.," Ges., 1847, p. 177; Hessling, "Vom Haare und seinen Scheiden in Froriep neue Notizen," 1848, No. 113; Langer, "Ueber den Haarwechsel, bei Thieren und beim Menschen," in den "Denkschr. d. Wien," Akad., $1850, \mathrm{Bd}$. i. The comparative anatomy of the hairs is treated of by Heusinger in Meckel's "Arch," 1822, 1823, und "System der Histiologie ;" Erdl, in "Abh. d. Münch.," Akad., Bd. III. ii.; Gegenbaur, in "Verhund d.phys. med. Gesellschaft zu Würzburg," 1850; Steinlin, in "Zeitschrift, für rationellen Medizin," Bd. IX. The allied horny tissues are described in the "Dorpat. dissertations," by Bröcker, "De texturâ et formatione spinarum," 1849; Hehn, "De text. et form. barbæ Balænæ," 1849 ; Schrenk, "De formatione pennæ," 1849. 
IV. OF THE GLANDS OF THE SKIN.

\section{A. OF THE SUDORIPAROUS GLANDS.}

$\S 65$. The Sudoriparous Glands consist of a single delicate, more or less convoluted tube, which secretes the sweat. They are formed over the whole surface of the skin, with the exception of the concave side of the concha of the ear, of the external auditory meatus, the glans penis, one lamella of the prepuce, and a few other localities; and open upon it by numerous fine apertures.

$\S 66$. In every sudoriparous gland (Fig. 45, Fig. 78), we may distinguish, the glandular coil (Fig. 78 a, Fig. $75 \mathrm{~g}$ ), or the proper gland, from the excretory duct or sudoriparous canal (Fig. 45 $h$, Fig. $78 b$ ). The former is a rounded or elongated corpuscle of a yellowish or transparent yellowish-red color, which in general measures $\frac{1}{7}-\frac{1}{5}$ of a line; but on the eyelids, the integument of the penis, scrotum, nose, convex side of the concha of the ear, on the other hand, not more than $\frac{1}{10}-\frac{1}{12}$ of a line; whilst on the areola of the nipple and in its neighborhood, at the root of the penis, and between

Fig. 78.

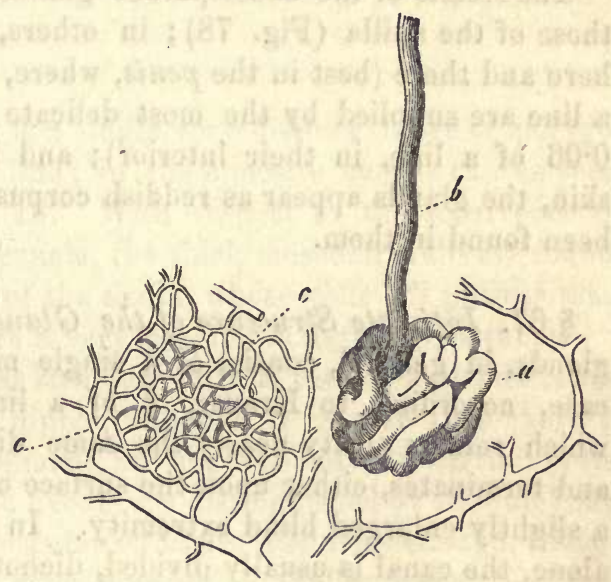
the scrotum and perinæum, it attains as much as $\frac{1}{2}$ a line, and in the hairy parts of the axilla reaches as much as $\frac{1}{2}-1-1 \frac{1}{2}$ line in thickness, and 1-3 lines in breadth.

The sudoriparous glands, in most cases, are lodged in the meshes of the pars reticularis of the corium, sometimes more superficially, sometimes deeper, surrounded by fat and loose connective tissue, together with or among hair-sacs. They occur more rarely in the subcutaneous connective tissue, or at its boundaries, as for example in the axilla, to some extent in the areola mammo, in the eyelids, penis, and scrotum, the palm of the hand and sole of the foot. In the two lastnamed localities, they are disposed in rows under the ridges of the cutis,

FrG. 78.-A sudoriparous coil and its vessels; magnified 35 diameters : $a$, glandular coil; $b$, excretory duct or sweat duct; $c$, vessels of a glandular coil, according to Todd and Bowman. 
and at tolerably equal distances apart; in other places they are met with, usually in a regular manner, singly or in pairs, in each mesh of the corium, although, according to Krause, spaces of $\frac{1}{4}-\frac{1}{2}$ a line exist, where they are totally absent, or occur in groups of three or four close together. In the axilla, the glands form a connective layer under the corium.

According to Krause, there occur on a square inch of the skin be- tween 400 and 600 glands on the back of the trunk, the cheeks, and the two superior segments of the lower extremities; $924-1090$ on the anterior part of the trunk, on the neck; brow, the forearm, back of the hand and foot; 2685 on the sole of the foot; and 2736 on the paln of the hand. The total number of the sudoriparous glands, without reckoning those of the axilla, is estimated (somewhat too highly) by Krause at $2,381,248$, and their collective volume (with those of the axilla) at 39,653 cubic inches.

The vessels of the sudoriparous glands are particularly well seen in those of the axilla (Fig. 78); in others, the vessels may also be seen here and there (best in the penis, where, for example, glands of 0.36 of a line are supplied by the most delicate ramifications of an artery of 0.06 of a line, in their interior); and in successful injections of the skin, the glands appear as reddish corpuscles. Nerves have not hitherto been found in them.

§67. Intimate Structure of the Glandular Coil.-The sudoriparous glands, in general, consist of a single much convoluted canal (in one case, according to Krause, $\frac{3}{4}$ of a line long), twined into a coil, which retains pretty nearly the same diameter throughout its length, and terminates, either upon the surface of the coil, or in its interior, in a slightly enlarged blind extremity. In the large glands of the axilla alone, the canal is usually divided, dichotomously, into branches, which subdivide, and sometimes, though rarely, anastomose; and after giving off small crcal processes, each separate branch finally terminates in a blind extremity. The glandular canals have either thin or thick walls (Fig. 79). The former (Fig. $79 \mathrm{~A}$ ) possess an external fibrous investment, consisting of indistinctly fibrous connective tissue, with scattered elongated nuclei; internally this is sharply limited, perhaps by a membrana propria, and is covered by a single, double, or multiple layer of polygonal cells of $0.005-0.007$ of a line, which in their chemical relations, and otherwise, correspond perfectly with the deep cells of pavement-epithelium, except that they almost invariably contain a few fatty granules, and still more frequently a small quantity of yellowish or brownish pigment-granules.

The thick-coated sudoriparous glandular canals (Fig. 79 B) possess, besides the two layers just described, a middle layer of smooth muscles 
running longitudinally, whose elements are easily separable, as muscular fibre-cells of $0.015-0.04$ of a line long, $0.002-0.005$, or even 0.008 of a line broad, occasionally with a few pigment-granules, and each containing a roundish elongated nucleus. Whenever the glandular tubes con-

Fig. 79.
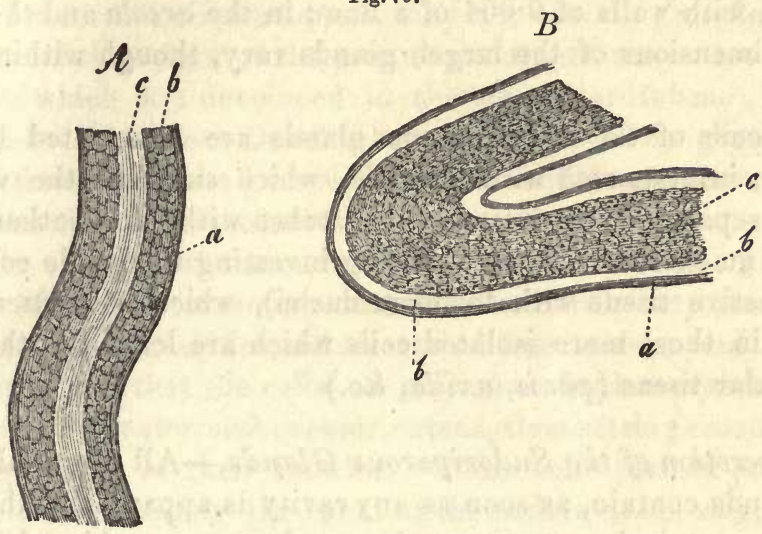

tain only fluid, the epithelium is a single very distinct layer of polygonal cells of $0.006-0.015$ of a line ; in the opposite case it can be seen only with difficulty or not at all. With respect to the occurrence of these two forms of glandular canals, the thick muscular walls are found, especially in the large glands of the axilla, whose cells all possess muscular walls, and thence acquire a very peculiar striated appearance. I have noticed a precisely similar structure only in the large glands of the root of the penis and of the nipple, although it is true that there is occasionally a muscular development, but slighter and only partial, in the glands of the palm, whose wide canals are distinguished by the thickness of their walls, and exhibit a muscular structure distinctly enough, though thinner than elsewhere. The same description applies to certain glands of the scrotum, and even of the back, of the labia majora, of the mons veneris, and of the neighborhood of the anus; yet with this limitation, that often only a small part of the glandular tube, perhaps merely its cæcal extremity, is provided with a muscular coat. The glands of the leg, of the penis, of the thorax (the areola excepted), of the eyelids, and the majority of those of the back and thigh, of the chest and abdomen, as well as of the two prominent segments of the upper extremity, are delicate and without muscles.

The diameter of the glandular canals varies, in the smaller glands from $0.022-0.04$ of a line, and is about 0.03 of a line on the average; the

FiG.79.-Sweat ducts; magnified 350 diameters. $A$, one with thin walls and a central cavity without a muscular coat, from the hand : $a$, connective investment; $b$, epithelium; $c$, cavity. $B$, a portion of a canal without a cavity, and with a delicate muscular layer, from the scrotum: $a$, connective tissue; $b$, muscular layer; $c$, cells which fill the glandular canal with yellow granules among their contents. 
thickness of the walls, $0.002-0.003$; of the epithelium, 0.006 ; of the cavity, $0.004-0.01$ of a line. Among the axillary glands some have canals of $0.07-0.1$, even 0.15 of a line, with walls 0.006 of a line in thickness, without the epithelium, and half of which is formed by the muscular layer; others and in fact the largest glands, possess canals of $0.03-0.06$, with walls of 0.004 of a line; in the areola and the genitalia also, the dimensions of the larger glands vary, though within narrower limits.

All the coils of the sudoriparous glands are penetrated by connective tissue, interspersed with fat-cells, which supports the vessels and unites the separate convolutions of the tubes with one another; some of them have an external fibrous covering investing the whole coil (of common connective tissue with fusiform nuclei), which is particularly well developed in those more isolated coils which are lodged in the subcutaneous cellular tissue (penis, axilla, \&c.)

$\S 68$. Secretion of the Sudoriparous Glands.-All the smaller sudoriparous glands contain, as soon as any cavity is apparent in their canals, which, however, is by no means always the case, nothing but a clear, bright fluid, without any formed contents. In the axillary glands, on the other hand, the contents abound in formed particles, and appear either as a grayish, transparent, semi-fluid substance, with innumerable fine, pale granules, and often with solitary nuclei; or as a whitish-yellow tolerably viscid matter, with a varying quantity of larger, opaque, colorless, or jellow granules, nưclei and cells, similar to the epithelial cells above described. That these cell contents, which, as I have found, contain much protein and fat, differ considerably from the common sweat, which is fluid and presents no formed elements, and probably rather approximate to the sebaceous secretion of the skin, is evident, on which account we might be induced to remove the glands of the axilla from the class of sudoriparous glands, and to regard their secretion as of a peculiar kind. These glands, however, sometimes afford a secretion containing but few granules, or even nothing but fluid; and among the larger axillary glands smaller ones occur, which, so far as regards their contents, exhibit many transitions, on the one hand into the large, and on the other into common small glands.* If we further consider

\footnotetext{
* [However true it may be that this secretion is sometimes fluid, and similar to that of sudoriparous glands in other situations, this is the exception, and by no means the rule. But it is not on account of their secretion, but mainly of their different structure, that these glands have been separated from the common sudoriparous glands. They differ from them by being united into groups, and by their yellowish color. In size, too, they vary. Many are 5 or 6 times larger: some attain the size of 2 lines in diameter. The groups can be readily seen with the unassisted eye, if the adipose tissue adhering to a flap of skin from the axilla be removed. They then appear as small granulations of a reddish or rosy tint, and are soft and pulpy. The excretory ducts are not spirally wound as in the ordinary sudoriparous glands.
}

In the Negro these axillary glands as first pointed out by Prof. Horner (American Journal 
that, occasionally, the sudoriparous glands in other situations, as, for instance, in the areola of the nipple, contain a fluid abounding in granules, it is clear that it is unadvisable to distinguish the large axillary glands from the common kind, on account of the difference in their secretion; and the more so, indeed, because we by no means know whether the latter, under certain circumstances, may not contain granules.

As respects the origin of the granular contents, they must be referred to the cells which are developed in the glandular tubes. For we frequently meet in these with cells containing the same granules, which also occur free within the glandular canals; and frequently may be said to constitute their whole contents. It sometimes happens, also, that in one and the same gland the ends of the glandular tubes contain nothing but cells, while the excretory duct exhibits hardly any trace of them, presenting merely granules and scattered free nuclei; and in this case we can easily see that the cells, as they pass further upwards, become broken up to a greater and greater extent, thus setting free their nuclei and the granules in their interior. These cells plainly proceed from the epithelial cells lining the canal of the sudoriparous coil ; for, in the first place, the cells of the contents of the epithelium resemble one another in all respects ; and secondly, where cellular or granular contents are found in the glands themselves, the epithelium is for the most part completely absent, so that the former rests immediately upon the muscular membrane. Now, since on the other hand, in those glands which contain only a clear fluid, the epithelium is always easily seen, and often presents many dark (even golden yellow) pigment granules in its cells, it may perhaps be assumed, that the cells in the contents are nothing but detached epithelium, and that the secretion mainly depends upon a growth and continual casting off of the epithelial cells.

The examination of the secretion of the sudoriparous glands is neither chemically nor microscopically complete. As regards the former, the fact that the axillary glands secrete fat and a nitrogenous substance in large quantities, appear to me interesting, since from the obvious similarity in structure between these and the other sudoriparous glands, we may perhaps draw some conclusions as to the secretion of the latter. We already know that the ordinary perspiration contains nitrogenous matters (extractive); and as Krause (1. c., p. 146) has clearly shown, fat, also; and it may be asked whether these substances do not perhaps in certain situations (e. g. hand, foot) occur more abundantly, or under certain conditions (local, adhesive, peculiarly odorous perspiration) increase in quantity. The so-called sweat-corpuscles of Henle (l. c., pp. 915 and 939), that is, structures similar to the mucus-corpuscles, I

of Med. Science, 1846), are much larger than in the White. To the secretion of these large glands indeed, the peculiar smell of Negroes is attributed.

In the groin sudoriparous glands very similar in their structure to these axillary glands are met with.-DaC.] 
have hitherto found neither in the sweat of man nor in the smaller glands; but I may remark that almost constantly, even in the smaller sudoriparous glands, certain canals exist which present no cavity, but are wholly filled with epithelial cells. These appeared to me always to be near the blind end (Fig. 79, B), whilst those which are nearer the excretory duct, almost invariably exhibit a cavity $0.004-0.1$ of a line in diameter. I consider it therefore to be not impossible, that in the common sudoriparous glands, a cellular secretion is at times formed and - excreted in the same manner as in the axillary glands; for from what we see in the canals of the latter, it can hardly be doubted that granules, nuclei, and perhaps also remains of cells, occur in the sweat of the axilla. Whether the sweat in different individuals and races of men present notable differences is unknown, for it is not ascertained that the different odor of the cutaneous exhalation in the European and the Negro, for instance, depends on the sweat or the material of the perspiration; nor have its pathological relations been investigated, at all events not microscopically.

\$ 69. Swcat-Ducts. - The excretory ducts of the sudoriparous glands, the sweat-ducts, or spiral canals (Figs. 45, 80), commence at the upper

Fig. 80.

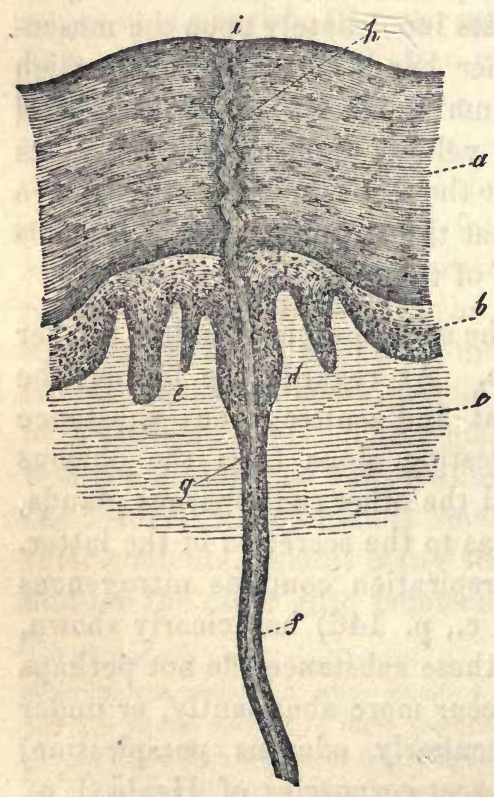
end of the glandular coil as simple canals, ascend with slight undulations vertically through the corium, and then penetrate between the papilloe (never through their points), into the epidermis. Here they begin to twist, and according to the thickness of the cuticle they perform from 2-16 eloser, or more distant spiral turns, until eventually they terminate by small, round, often funnel-shaped apertures, the socalled sweat-pores on the free surface of the epidermis.

The length of the sweat-ducts depends on the situation of the glands and the thickness of the skin. The commencement of the duct is invariably narrower than the canal in the coil itself, measuring $0.009-0.012$ of a line; it continues narrow up to its entrance into the stratum Malpighii

FIG. 80.-Perpendicular section through the epidermis and outer surface of the corium of the bulb of the thumb, transversely through two ridges, treated with acetic acid; $a$, horny layer of the epidermis; $b$, mucous layer; $c$, cutis $; d$, simple papilla ; $e$, compound papilla $; f$, epithelium of a sweat-duct passing into the mucous layer; $g$, cavity of it in the cutis; $h$, in the horny layer; $i$, sweat-pore.-Magnified 50 diameters. 
where it dilates to about double the size, $i$. e., to $0.024-0.28$ of a line (Fig. 80); retaining this breadth, it traverses the epidermis, and terminates in an aperture of $\frac{1}{50}-\frac{1}{20}$ of a line. In the axillary glands, the excretory duct measured in one case at the level of the sebaceous glands $0.06-0.09$ of a line, immediately under the epidermis 0.03 , in the epidermis itself 0.06 of a line. In the corium the sweat-ducts have always a distinct cavity, an external investment of connective tissue, with elongated nuclei (in the glands of the axilla, muscles also), at all events, inferiorly, and an epithelium composed of at least two layers of polygonal, nucleated cells without pigment granules. Where the ducts enter the epidermis, they lose their investment of connective tissue, which coalesces with the outermost layer of the corium, and henceforward they are bounded by nothing but layers of cells, which in the stratum Malpighii are nucleated, but in the horny layer are without nuclei. Chemically and morphologically they completely resemble the epidermic cells, with the sole exception that they are disposed more perpendicularly, particularly in the horny layer. The duct has often a distinct cavity in the epidermis, at other times there is a granular streak in the place of it, which is probably either a secretion or a deposit from the secretion. The sweat-pores, whose disposition, corresponding with that of the glands, is sometimes very regular, at others more irregular, are distinguishable, even with the naked eye, in the palm of the hand and sole of the foot. In other localities they are visible only with the aid of the microscope; occasionally the excretory ducts of two glands unite into a single canal (Krause).

\$70. Development of the Sudoriparous Glands.-The sudoriparous glands first appear in the fifth month of embryonic life, and are originally perfectly solid, slightly flask-shaped, processes of the stratum Malpighii of the epidermis, and are very similar to the first rudiments of the hairsacs. In the earliest condition which I have observed, the processes measured in the sole of the foot $0.03-0.09$ of a line in length, and 0.01 of a line in breadth at the neck, at the bottom $0.018-0.02$ of a line, and even the very longest did not penetrate more than half through the cutis, which was 0.25 of a line thick. They were entirely composed of round cells, perfectly similar to those of the stratum Malpighii of the epidermis; besides which, each process had a delicate investment, which was continuous with the boundary of the inner surface of the epidermis. No trace of sweat-pores or ducts was visible. At the beginning of the sixth month, the glands in the sole of the foot and palm of the hand extend as far as the middle and inner fourth of the cutis, measure at the clavate extremity $0.028-0.04$ of a line, and $0.016-0.02$ of a line in the duct which arises from them, are already slightly serpentine, and present a cavity, at all events partially in their narrow portion; they do not, however, penetrate the cuticle, or in any way open on the 
surface. It was not before the seventh month that I perceived, in the same situations, the first indications of the sweat-pores and ducts in the

Fig. 81.

Fig. 82.

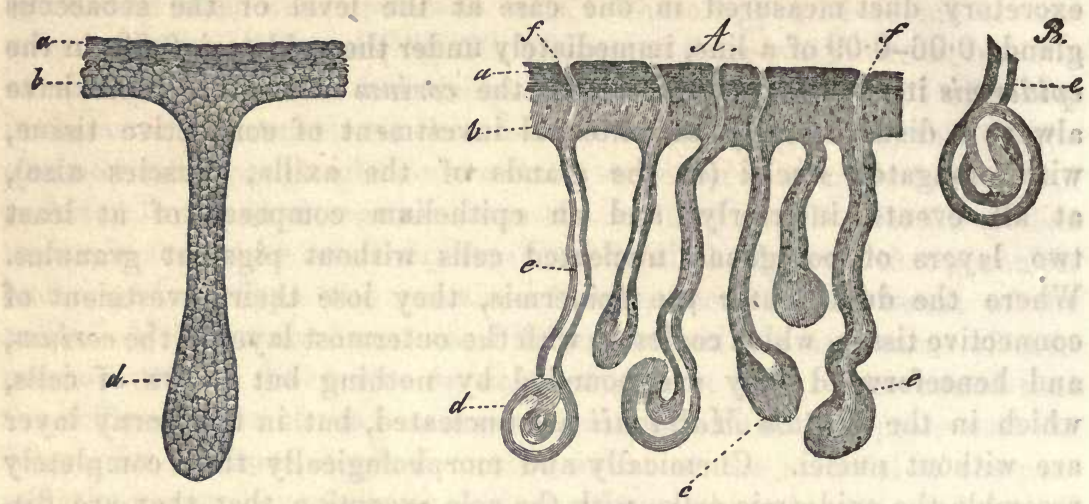

epidermis, though as yet very indistinct, and the latter forming only half a spiral turn (Fig. 82, A); at the same time the part of the gland which projected into the corium was more considerably developed, reached as far as the innermost portion of that structure, and at its cæcal extremity was bent into a hook or even slightly convoluted, so as to afford the first indication of a glandular coil of about 0.04 to 0.06 of a line. The canal arising from it usually presented several marked undulations, and measured in total thickness $0.015-0.022$ of a line, with a cavity of $0.003-0.004$ of a line, which frequently extended even to the terminal coil: like the latter it was composed of the original though thickened membrane continuous with the surface of the corium, and of an epithelium consisting of many layers of pale, polygonal, or rounded cells. The glands of the rest of the body about this period, appeared to me to be similarly constituted. I can say nothing as to their earlier condition, but even those of the axilla were in no wise distinguished from the rest. From this time the development goes on very rapidly; the end of the gland elongates more and more, and coils itself up (Fig. $82 B$ ), so that it assumes an appearance hardly different from that which it presents in the adult. In the new-born infant, the glandular coils in the heel measure $0.06-0.07$ of a line (in a child of four months $0 \cdot 06-0 \cdot 1$ of a line on the heel, in the hand 0.12 of a line), present

FIG. 81.-Rudiment of a sudoriparous gland of a human embryo at five months; magnified 2,50 diameters : $a$, horny layer of the epidermis; $b$, mucous layer; $c$, corium; $d$, rudimentary glands, as yet without any cavity, and consisting of small round cells.

FrG. 82.- $\mathcal{A}$, rudiment of a sudoriparous gland from a seven months' fœtus; magnified 50 diameters. The letters $a, b, d$, as in Fig. 81 . The cavity $e$ is present throughout, only it does not extend quite so far as the end of the thicker part of the rudiment of the gland, which becomes converted into the glandular coils. The continuation of the canals into the epidermis and the sweat-pores, $f$, are present. $B$, a coil of a sudoriparous gland, from a fœtus at the eighth month. 
much convoluted canals of $0.015-0.022$ of a line, and traverse the epidermis with their already twisted ducts (in the corium of 0.008 , in the rete Malpighii of 0.022 of a line).

It results from these facts that the sudoriparous glands are nothing else than involutions of the skin, and do not begin as hollow structures, but are at first a simple development of the stratum mucosum. By a continual process of cell-multiplication, the original rudiments grow deeper and deeper into the skin, acquire their peculiar spiral windings, and divide into the glandular coil and the sweat-duct; while at the same time, either by liquefaction of their central part, which would thus, as it were, represent a first secretion, or by the excretion of a fluid between their cells, a cavity is produced. How the sweat-duct in the epidermis and the pore are formed is doubtful; probably by a formative process in the epidermis itself. According to a few measurements which $I$ have instituted ("Mikroscop. Anat.," II. i. 171), a development of sudoriparous glands appears to take place even after the fifth month, whilst the whole number appears to exist at birth.

Little is known as to the pathological conditions of the sweat-glands. Kohlrausch (Müller's "Archiv," 1843, p. 366), has found them of considerable size ( $\frac{1}{2}$ a line) in an ovarian cyst, together with hairs and sebaceous follicles. In Elephantiasis graecorum, G. Simon and Brücke (Simon, "Hautkrank.," p. 268), noticed an increase in size of the sudoriparous glands, and V. Bärensprung observed the same thing in a kind of wart (1. c., p. 81); the latter also found that these glands were atrophied in corns, and that the duct in the outer layers of the epidermis had disappeared. The condition of the several glands in old age, in cases where the secretion of sweat is altogether wanting, and in abnormal perspirations, is not known. In a remarkable case of Ichthyosis congenita (very similar to that mentioned by Steinhausen, only more marked) in a new-born infant, which was examined by Dr. H. Müller and myself, the sudoriparous glands were present; their excretory ducts, so far as regards their course through the epidermis, which was thickened to 2 lines, were partly disposed as usual, partly they were placed, as in the sole of the foot, with their outer portions almost completely horizontal, and ran in some places for as much as $1 \frac{1}{2}$ line in this manner, so that in superficial sections of the epidermis they appeared as parallel, at first sight altogether abnormal canals, with a cavity of $0.0025-0.003$ of a line. The contents of the ducts were very peculiar, consisting invariably of a multitude of white oil drops. I observed sudoriparous glands also in the case described by Mohr, of a great cavity containing hairs in the lung ("Berlin Med., Central-zeitung," 1839, No. 13), they were about 0.24 of a line in diameter, and were contained in a panniculus adiposus, with common fat-cells; and it may be remarked that the 
wall of the cavity besides the panniculus also presented a corium with papilla, and an epidermis like the external integument.

Method of Investigation.- To examine the position of the sudoriparous glands and their excretory ducts, fine sections of fresh or slightly-dried skin of the palm or sole should be prepared, and made transparent by acetic acid or caustic soda. Gurlt used for this purpose skin hardened and rendered transparent in a solution of carbonate of potassa (liquor kali carbonici). Giraldès macerates the skin for twenty-four hours in dilute nitric acid ( 1 part acid, 2 parts water), and for twenty-four hours in water,-a process which, according to Krause, is very useful, as the glands become yellow, and are readily distinguished. In macerated pieces of the skin, the cellular lining of the sweat-ducts may be drawn out of the corium, in the form of long tubes, with the epidermis; in delicate parts of the skin I have, not unfrequently, succeeded in doing this after treatment with concentrated acetic acid. The investigation of the glandular coils themselves is very easy in the axillary glands; in the others the skin must be prepared from within, and the glands sought for partly upon the inner surface of the cuts, partly in its meshes, - a method which readily succeeds, with a little attention, particularly in the hand, foot, and nipple. The large glands of the ball of the foot of the Dog, described by Gurlt, are particularly well-fitted for demonstration, and still more those of the prepuce and of the integuments of the udder of the Horse, which lie quite loose in the subcutaneous tissue. If it be desired to count the glands, their apertures may be sought for, or a piece of skin of determinate size may be treated according to Giraldès' method, and examined portion by portion (Krause). For the study of the development of the glands, sections of the fresh and dried skin of the heel and palm of embryos, may be made with the double knife or razor. In embryos preserved in spirit, if the sections be fine, the glands may also be very well seen, especially in the first moments of the action of caustic soda.

Literature.-Breschet et Roussel de Vauzème, "Recherches anatomiques et physiologiques sur les appareils tégumentaires des animaux," in the "Annales des Sciences Nat.," 1834, pp. 167 and 321 (discovery of the sudoriparous glands); Gurlt, "Vergleichende Untersuchungen über die Haut des Menschen und der Haussäugethiere, besonders in Bezug auf die Absonderungsorgane des Hauttalges und des Schweisses," in Müller's "Archiv," 1835, p. 399 (first good figures of the glands themselves). [Robin, "Note sur une espèce particulière des glandes de la peau de l'homme," in the "Annales des Sciences Nat.," 1845; Horner, "On the Odoriferous Glands of the Negro," in American Journal of Med. Sciences, 1846.] Besides these, compare especially the general works of Todd and Bowman, Henle, Valentin, Hassall, and myself; the above-cited treatises of Krause, myself, Simon, Von Bärensprung, and Wilson; further the figures of Berres, tab. XXIV.; R. Wagner, "Icon. 
Phys.," tab. XVI., fig. 9 ; F. Arnold, "Icon. Org. Sens.," tab. XI., and my own "Mikr. Anat.," tab. I.

\section{B. OF THE CERUMINOUS GLANDS.}

$\S 71$. The ceruminous glands of the Ear are brownish simple glands, in external appearance precisely similar to the sudoriparous glands, which do not exist in the whole external auditory meatus, but only in its cartilaginous portion, where they are situated between the lining membrane of the passage and the cartilage, or the fibrous substance which supplies its place, in a tough subcutaneous tissue, containing little fat. They form a connected yellowish-brown layer, visible enough to the naked eye, which is thickest in the inner half of the cartilaginous meatus, and becomes gradually thinner and more lax externally, extending, however, quite as far as the cartilaginous meatus itself. Each ceruminous gland consists of a glandular coil and an excretory duct. The former (Fig. $83 d), \frac{1}{10}-\frac{1}{4}-\frac{3}{4}$ of a line in size, is formed by the multitudinous convolutions of a single canal of $0.03-0.06$ on the average $0.04-0.05$ of a line in thickness, which occasionally, although not constantly, throws out little diverticula, and terminates in a blind slightly enlarged end. From the coil a short straight excretory duct, $0.017-0.024$ of a line thick, passes perpendicularly upwards, penetrates the corium and epidermis of the auditory meatus, and usually opens independently in a circular pore of 0.044 of a line, or else into the upper part of a hair-sac.

The following is the intimate structure of the ceruminous glands. The canals of the coil present a fibrous coat, and an epithelium, the former being $0.004-0.005$, the latter 0.004 of a line in thickness. The fibrous covering presents exactly the same conditions as in the larger sudoriparous glands, that is, it consists of an internal longitudinal layer of smooth muscles, $0.0023-0.0026$ of a line in diameter, and an external layer of connective tissue, with scattered nuclei, and occasionally very fine transverse nucleus-fibres. The epithelium rests immediately upon the muscular layer, and consists of polygonal cells of $0.006-0.01$ of a line in a single layer, which contain a greater or smaller number of yellowishbrown pigment-granules, of immeasurable minuteness, insoluble in acids and alkalies in the cold, or whitish fat-globules up to 0.001 of a line in size, and which are so disposed that the whole lengths of a gland contain generally only one and the same kind of granules; whence it arises that they appear either uniformly brownish or opaque (by reflected light whitish). The contents of the glandular canals are sometimes a clear fluid, sometimes a granular substance composed principally of cells analogous to those of the epithelium, whence it would seem that the same kind and mode of secretion occurs in them as in the sudoriparous glands. The excretory ducts possess a coat of connective tissue, and an epithelium consisting of several layers, and constituted of small nucleated 
cells, without fat or pigment-granules. In their cavity, which is, however, not always distinct, they sometimes contain a clear fluid, sometimes a small quantity of finely-granulated substance.

Fig. 83.

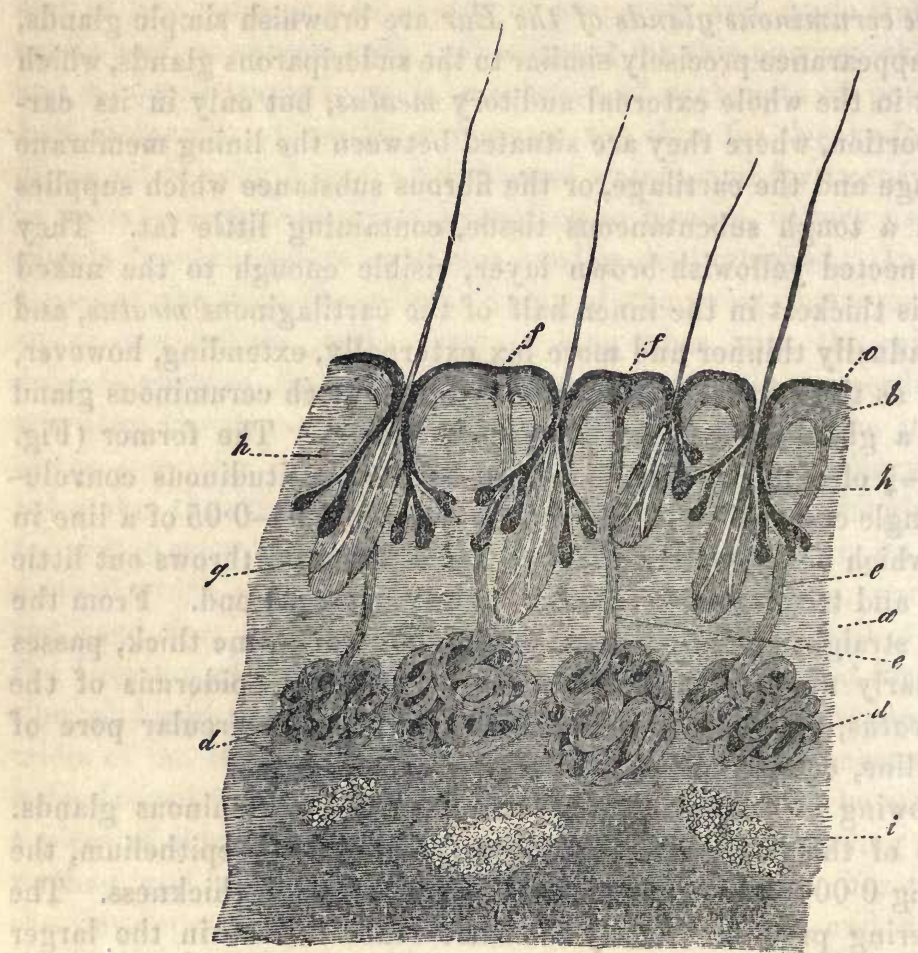

$\S 72$. The Cerumen of the ear is commonly considered to be the secretion of these glands, though this is only partially correct. If we examine the yellow or brownish, soft or more solid, viscid substance which is formed within the cartilaginous meatus, it is found to contain various constituents: independently of a few hairs, occasionally an Acarus folliculorum, and epidermic cells in various numbers, there occur,-1. Very many cells completely filled with pale fatty matter of $0.009-0.02$ of a line, usually of an oval, flattened, irregular shape; in which, on the addition of water, or still better of caustic soda, the fat is separated in isolated, round, or irregular dark drops. 2. Much free fatty matter in the form of pale, small yellowish round drops, which, on the addition of water, appear as dark spherical granules, from an immeasurable minuteness up to 0.002 of a line and more; and it is only upon this addition that they become quite distinct, but at the same time are decolorized.

Frg. 83.-Perpendicular section through the skin of the external auditory meatus; $a$, corium; $b$, stratum Malpighii; $c$, horny layer of the epidermis; $d$, coil of the ceruminons glands; $e$, their excretory ducts; $f$, their apertures; $g$, hair-sacs; $h$, sebaceous glands of the meatus; $i$, masses of fat.-Magnified 20 diameters. 
3. Yellow or brownish granules, and masses of granules, free or rarely in cells, few upon the whole. 4. Lastly, when the secretion is more fluid, also a small quantity of a clear liquid. I consider that the first-named cells belong to the sebaceous secretion of the external meatus; but that the remainder is the secretion of the ceruminous glands, which would, therefore, eliminate oily fluid with scattered brown granules. This being the case, the analysis by Berzelius of the common ear-wax, a mixture of the sebaceous and proper ceruminous secretion, must only be admitted with caution. In my opinion, the brownish-yellow bitter substance, soluble in alcohol and water, found by him, and the pale yellow strong-tasted extractive matter, hardly soluble in water, and not at all in alcohol, must be attributed to the ceruminous glands; the remaining fat, the horny matter, and probably also most of the albumen, to the sebaceous glands; whilst the relations of the salts must, of course, be left undetermined.

The vessels of the ceruminous glands are disposed like those of the sudoriparous; in one case I noticed, in addition, a fine nervous fibre of 0.003 of a line in the midst of a gland. As to the development of these glands I can only say, that in a foetus of five months they had the form of straight, pale processes of the stratum Malpighii of the epidermis of the external auditory meatus, were entirely composed of nucleated cells, and ended by a slightly enlarged termination somewhat twisted upon its axis, in which the first indication of a glandular coil was presented. In other words, these rudimentary glands exactly resembled the sudoriparous glands at the same period; and considering the great anatomical resemblance between the two structures, I do not doubt for a moment that the ceruminous glands, both in their first commencement and subsequently, go through the same phases as the former.

According to all that I have seen of the ceruminous glands, I must consider them to be mere modifications of the sudoriparous. In speaking of these it has already been remarked, that their secretions are certainly not everywhere identical, being in one locality more aqueous, in another fatty and albuminous, with peculiar odorous ingredients. Even although the cerumen may, to some extent, contain peculiar substances, e. g., the yellow bitter substance, which, however, according to Lehmann, is not bilin, nevertheless, taking into account the other correspondences (consider the almost constant and often very abundant yellow granules in the sudoriparous glands, which are also insoluble in acids and alkalies), we may associate the ceruminous glands with the sudoriparous, especially with the larger among the latter, which are both anatomically and physiologically most closely allied to them; in fact, I am inclined, for my own part, to think, that the smallest pale ceruminous glands at the commencement of the meatus are hardly distinguishable from common sudoriparous glands. Nothing is known of the pathological conditions of the ceruminous glands-of the cerumen 
itself we know that it is often quite solid, at other times fluid, puriform, and pale colored. In the latter case, which is seen in congested conditions of the external meatus, it contains far more fluid and free fat than usual, and very beautiful cells containing fat.* With regard to the mode of examining the ceruminous glands, I must refer to the sudoriparous glands, with which they wholly agree in position, chemical relation to acids, alkalies, \&c. \&c.

Literature.-R. Wagner, "Icones Phys.," tab. xvi. fig. 11, $A, B$; Krause and Kohlrausch, in Müller's "Archiv," 1839, p. cxvi. ; Pappenheim, "Beiträge zur Kentniss der Structur des gesunden Ohres," in Froriep's "Neue Notizen," 1838, No.141, p. 131, and Specielle Gebelehre d. Gehörorgans (Breslau, 1840); Henle, "Allg. Anat." pp. 915, 916, 934, 941 ; Huschke "Eingeweidelehre," p. 819; Hassall, "Microsc. Anatomy," \&c., p. 427, pl. lvii.; Valentin, article "Gewebe," in Wagner's "Handw. d. Phys.," i. p. 755.

\section{OF THE SEBACEOUS GLANDS.}

$\S 73$. The Sebaceous Glands are small whitish glands, which exist in almost every part of the skin, and which afford the cutaneous sebaceous or fatty secretion.

In form they vary very considerably; the simplest (Fig. $84, A$ ) are short follicles of an elongated or pyriform shape; in others-the simple racemose glands-two, three, or even more follicles or vesicles are united with a shorter or longer peduncle; whilst in others, lastly (Figs. $84 B, 85)$, two, three, or more simple clusters of follicles communicate with a common duct, constituting an elegant compound racemose gland. Besides these three forms, which represent only the chief varieties, there are a good many intermediate ones, which do not require any detailed description.

The sebaceous glands occur principally in the hairy parts of the body, opening, in common with the hair-sacs, upon the surface, whence they have also been termed the glands of the hair-sacs.

In all the coarser hairs, the glands appear to be lateral appendages of the hair-sacs, and open by narrow excretory ducts into them (Figs.

* [There is an occasional ingredient in the so-called cerumen which is worthy of notice, viz. a mucedinous fungus. Attention has been recently called to its occurrence by Dr. Inman ("Quarterly Journal of Micros. Science," January, 1853), who states that a pellet of ear-wax which he examined was composed of nothing but this fungus, with a minute portion of epithelium. However, Professor Mayer, of Bonn, so long ago as 1844 ("Beobachtung Von Cysten mit Fadenpilzen aus dem aussern Gehörgange," \&c., Müller's "Archiv," 1844 , p. 404), described at length the structure of certain sacs containing fungi, which were extracted from the external auditory meatus of a girl eight years old,-in whom they appear to have been at first accompanied by considerable deafness and irritation. The sacs were as large as a pea, and open at one end; externally they were composed of layers of epithelium scales, from which mucedinous threads, terminated by globular sporangia, projected into the cavity of the sac. These sacs appear to have been repeatedly formed and discharged, to a very considerable number.-TRs.] 
$75,76,77,83)$, whilst in the lanugo the ducts and the hair-sacs are often of about the same diameter (Fig. $84 \mathrm{~B}$ ), and open into a common canal, which may be regarded as a continuation of the one as much as of the other; or the ducts may even be the larger (Fig. 85), the hairs bearing a subordinate relation to them, so that their sacs open into the glands, and the hairs come out through the glandular opening itself. In the hairless parts of the surface, sebaceous glands occur only in the labia minora (vide § 54), and in the glans penis and prepuce, whilst they do not exist in the glans and prepuce of the clitoris. In general, the glands are situated close to the hair-sacs in the superficial layer of the corium, and are larger in the finer hairs than in the coarser; in

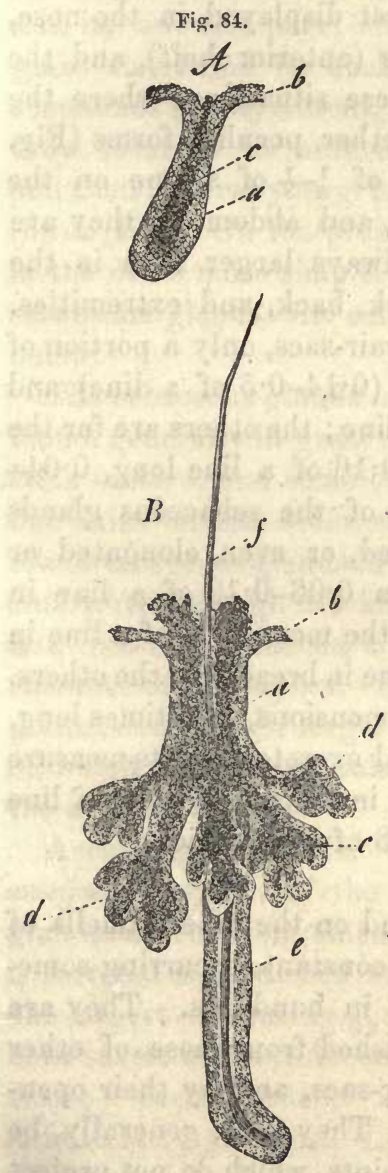

Fig. 85 .

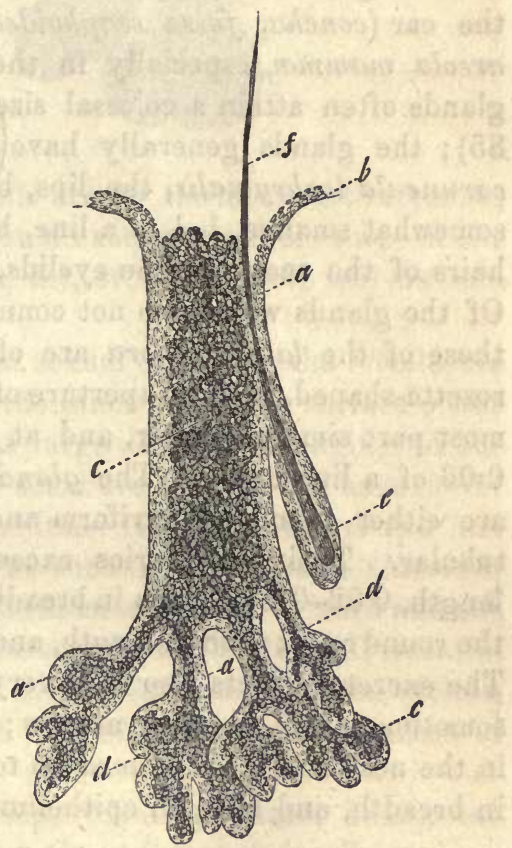

particular cases, however, they present many differences. With respect to the glands of the larger hair-sacs, they are usually of the simple racemose kind, having an average size of $\frac{1}{10}-$ $\frac{3}{10}$ of a line, and are disposed around the sac to the number of from 2 to 5 . The smallest, of $0.1-0.16$ of a line, occur in pairs, attached to

FIG. 84.-Sebaceous glands from the nose; magnified 50 diameters. $A$, simple tubular gland without any hair; $B$, compound gland, which has a common opening, with a hairsac: $a$, glandular epithelium, connected with $b$, the stratum Malpighii of the epidermis; $c$, contents of the glands, sebaceous cells, and free fat; $d$, the separate racemes of the compound gland; $e$, hair-sacs (root-sheath), with the hair, $f$.

Fra. 85.-A large gland from the nose, with a little hair-sac opening into it; magnified 50 diameters. The letters $a-f$ as in Fig. 84 . 
each hair of the scalp; they are somewhat larger, $0.16-0.24$ of a line in the hairs of the beard, and the longer hairs of the chest and axilla, in which situations several glands are usually disposed around the hair follicle; the largest of all exist on the mons veneris, the labia majora, and the scrotum, where, at all events in the last-mentioned locality, they are found at the deepest boundary of the corium, and the glands, from four to eight being connected together, represent bea utiful rosettes $\frac{1}{4}-\frac{1}{2}$ -1 line broad. Attached to the sacs of the smaller coarse hairs, I find smaller sebaceous glands of $0.06-0.24$ of a line, mostly in pairs; and also in the eyebrows, eyelids, and the hairs at the entrance of the nostrils. The lanuginous hairs have generally larger glands, or aggregations of glands of $\frac{1}{4}-1$ of a line; these are best displayed in the nose, the ear (concha, fossa scaphoidea), the penis (anterior half), and the areola mammo, especially in the first of these situations, where the glands often attain a colossal size, and altogether peculiar forms (Fig. 85); the glands generally have a diameter of $\frac{1}{5}-\frac{1}{3}$ of a line on the caruncula lachrymalis, the lips, brow, thorax, and abdomen; they are somewhat smaller, $\frac{1}{5}-\frac{1}{6}$ of a line, but almost always larger than in the hairs of the scalp, in the eyelids, cheeks, neck, back, and extremities. Of the glands which are not connected with hair-sacs, only a portion of those of the labia minora are of large size $(0.14-0.5$ of a line) and rosette-shaped, with an aperture of 0.033 of a line; the others are for the most part simply tubular, and at most $0.12-0.16$ of a line long, $0.04-$ 0.06 of a line broad. The glandular vesicles of the sebaceous glands are either round, or pyriform and flask-shaped, or even elongated or tubular. Their size varies exceedingly, from $0 \cdot 06-0 \cdot 16$ of a line in length, $0.02-0.1$ of a line in breadth, and is in the mean 0.04 of a line in the round ones; 0.08 in length, and 0.03 of a line in breadth in the others. The excretory ducts also have very different dimensions, sometimes long, sometimes short, broad or narrow; the principal excretory ducts measure in the nose and labia minora up to $\frac{1}{3}$ of a line in length, $\frac{1}{15}-\frac{1}{6}$ of a line in breadth, and have an epithelium $0.015-0.03$ of a line thick.

The sebaceous glands on the glans penis, and on the inner lamella of the prepuce or "Tyson's glands," are very inconstant, occurring sometimes only in a very small number, sometimes in hundreds. They are ordinary sebaceous glands, which are distinguished from those of other regions by their not being connected with hair-sacs, and by their opening independently on the surface of the skin. They may, generally, be perceived by the naked eye as small whitish points, which do not project above the skin; and in sections of the skin treated with caustic soda or acetic acid, their peculiarities may be readily studied with the microscope. They appear to be sometimes tubular, at others, simply racemose; the former present a round or pyriform follicle of $0.048-0.12$ of a line in diameter, and a straight excretory duct of $\frac{1}{10}$ of a line in length, and 
$0.024-0.035$ of a line in breadth; the latter have two to three, or at most five, terminal vesicles, and measure $0.08-0.18$ of a line altogether. The apertures of both kinds of glands, which have a diameter of $0.02-0.06$ of a line, are easily seen. With regard to the position of the glands, I would remark that I have never failed in finding them, 10-50 and more, in number, on the inner lamella of the prepuce, especially in the neighborhood of the frcenulum, and its anterior part; while on the glans itself and its neck, they are sometimes totally absent, sometimes they occur on its anterior surface, and then generally in great numbers

Fig. 86.

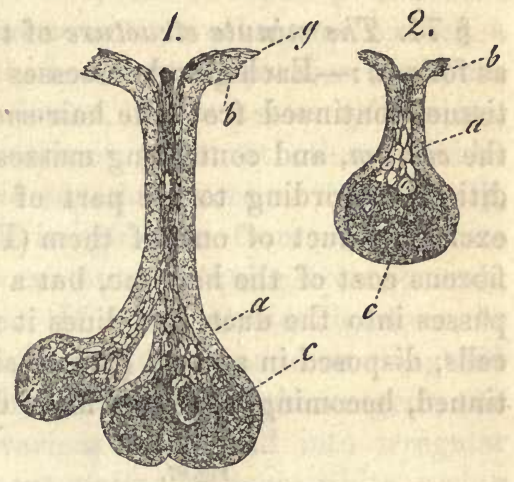
(up to 100). On the prepuce, the glands are for the most part racemose, in the penis more simple. Their contents exactly resemble those of the sebaceous glands, viz. cells containing fat, of which more will be said below.

The sebaceous glands of the external sexual organs in the female, are found, generally in great numbers, on the inner and outer surface of the labia minora, and some of them are as large as those belonging to the fine hairs on the labia majora, while some are smaller. I have never found sebaceous glands in the glans and inner.lamella of the proputium clitoridis, although Burkhardt speaks of such in the corona clitoridis, kut, in a few instances, I have met with them about the urethra and the entrance of the vagina. Resembling the sebaceous glands in all essential points, except their larger size, are the Meibomian glands in the eyelids, of which a more particular description will be given when we treat of the eye.

According to E. H. Weber (Froriep, "Notiz.," Marz, 1849), the smegma proputii of the Beaver, the "Castor" is not, in the main, a glandular secretion, since only a small portion of the pouch in which it is secreted is furnished with very simple, rounded, lenticular glandules, the largest measuring $\frac{1}{33} \mathrm{~d}$ of a line. The secretion, in individuals of both sexes, may rather be described as a laminated substance lining the entire "castor pouch," and consisting merely of epidermic cells and minute fatty globules. Leydig ("Zeitsch. f. w. Zool.," Bd. II., pp. 22, 31, et seq.), finds no glands at all in the "castor-pouch;" and according

FIG. 86.-Two sebaceous glands; the larger, 1, from the inner lamella of the prepuce; the smaller, 2 , from the glans penis: $a$, glandular epithelium continued into the stratum Malpighii of the skin; $b, c$, contents of the gland, with scattered larger fat drops; g, horny layer of the epiclermis, projecting somewhat into the duct.-Magnified 50 diameters. 
to him, the same is the case in the præputial sac of the Weasel, whilst in the Rat and Mouse, the prepuce contains true sebaceous glands of a complicated structure.

$\S 74$. The minute structure of the sebaceous glands may be described as follows:-Each gland possesses an external delicate coat of connective tissue, continued from the hair-sac, or, in the case of free glands, from the corium, and containing masses of cells, which exhibit different conditions, according to the part of the gland. If we proceed from the excretory duct of one of them (Fig. $88 \mathrm{~B}$ ), we see, that not only the fibrous coat of the hair-sac, but a portion of its inner root-sheath, also, passes into the duct, and lines it with nucleated, rounded, or polygonal cells, disposed in several (two to six) layers. This cellular layer is continued, becoming more and more delicate, into the remoter parts of the

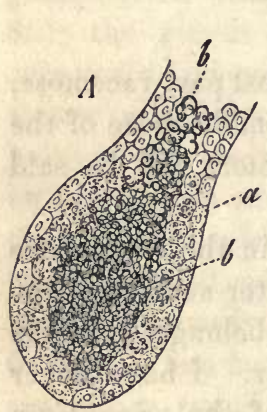

Fig. 87 .

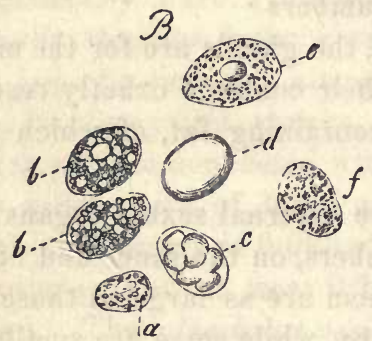
gland, and ultimately penetrates into the proper glandular vesicles, clothing them with a single, rarely a double, layer. Internally to these cells, which are distinguished by a greater or smaller number of fat granules from the epithelial cells above them, there immediately succeed, in the glandular vesicles themselves, others (Fig. $87 \mathrm{~B} b$ ) containing more fat; and these finally pass into the innermost cells of the glandular vesicles, which are invariably larger (of $0.016-0.028$ of a line) than the middle and outermost cells, are rounded or elongated in their form, and so filled with colorless fat that they might, not improperly, be termed sebaceous cells (Fig. $87 \mathrm{~B}$ ). The fat contained in them appears either still to retain the form of discrete drops (bb), as in the outer cells, or, as is indeed more frequently the case, under that of larger drops; and in many cells there are but a few of them, or even only a single one, which quite fills the cell $(d)$; in consequence of which these cells greatly resemble the fat-cells of the panniculus adiposus. If these innermost cells, which rarely exhibit any nucleus, are traced onward towards the excretory duct, nothing is

Fig. 87.- $A$, a glandular vesicle of a common sebaceous gland; magnified 250 diameters: $a$, epithelium sharply defined, but without any investing membrana propria, and passing continuously into the fat-cells, $b$ (their contours are too indistinctly drawn), in the interior of the glandular tube. $-B$, sebaceous cells from the glandular tube, and the cutaneous sebaceous matter; magnified 350 diameters : $a$, smaller nucleated cells, still more of an epithelial character, and containing but little fat; $b$, cells abounding in fat, without visible nucleus; $c$, cell in which the fat is beginning to flow into one mass; $d$, cell with one fat-drop; $e, f$, cells from which the fat has partially escaped. 
more easy to observe than that similar cells, applied uninterruptedly one to the other, are continued into this also, i.e. into the canal lined by its epithelium; then, entering the hair-sac, they occupy the space between the hair and the epidermis of the hair-sac, and are finally extruded. These cells are the sole sources of the cutaneous sebaceous matter, a substance which, when fresh and at the common temperature, is semifluid, but in the dead subject more consistent, like butter or soft cheese, whitish or whitish-yellow in color, sometimes viscid, at others friable. Its cells, in the fresh secretion, adhere together more or less closely, and are thence generally flattened and irregular in form; their membrane is not recognizable, and their contents are quite homogeneous, and transparent, with a yellowish hue. If dilute alkalies, however, be added, they swell up after a short time into beautiful round or elongated vesicles, in which, in consequence of the penetration of the reagent, the fat divides into separate drops of various sizes, and into irregular masses; at the same time the sebaceous matter becomes white, owing to the numerous minute fatty particles which are produced, and larger fat-drops are formed, probably in consequence of the solution of many cells. Besides that in the cells, the sebaceous matter also contains free $f a t$, in larger or smaller quantity, and in some cases, perhaps, an excessively minute amount of a clear fluid.

It appears, then, that the cutaneous sebaceous matter is a secretion, consisting, so to speak, only of formed elements, either cells containing fat alone, or cells together with drops of fat. These constituents are formed in the vesicular ends of the glands, in consequence of a production of cells, which, as in the epidermic tissues in general, proceeds entirely from the pre-existing cells, unaided by free cell-development, of which there is in this case no indication. By endogenous development round portions of contents, or by division, cells are continually produced at the bottom of the glandular vesicles. These are at first pale, and contain but few granules, like the epithelial cells from which they arise; but as they are forced towards the interior by cells developed after them, they are very soon completely filled with moderately large, round, dark, fat-granules. They thus proceed towards the excretory ducts; and the fat drops contained in them running more and more together, and the membranes themselves becoming rather more resistant, they eventually assume the form of the sebaceous cells above described. The free fatty matter in the sebaceous secretion is formed, in certain cases, by the solution of the cells whilst still in the interior of the glandular vesicles, for, in fact, in many glands, free fat, in smaller or larger, often very considerable drops (Fig. $86 \mathrm{~B}$ ), is met with, even in the terminal vesicles; however, it is also, perhaps, produced in consequence of its draining from closed cells, a supposition which is not a little strengthened by the circumstance, that the fat-containing cells in the excreted seba- 
ceous matter are seldom filled to distension, but appear for the most part variously flattened, or even corrugated, and contain only a small quantity of fat. Understood in this way, the formation of the cutaneous sebaceous matter resembles in many respects that of the cuticle. The young, easily soluble cells at the bottom of the glandular follicles may be compared to the Malpighian cells of the epidermis, and the less soluble ones of the secretion filled with fat, to the horny plates, which seems the more appropriate, if we consider, 1 , that the deep layer of the epidermis of the hair-sac is continued into the ducts of the glands, and even the outermost cells of the terminal vesicles; and 2 , that the epidermis, in some situations being constantly detached, form secretions (I refer to the smegma proputii of the penis and clitoris), substances which are, moreover, to all appearance chemically allied to the sebaceous secretion; for the latter, it may be remarked, according to an analysis of the contents of a distended gland by Esenbeck (Gmelin's "Handbuch der Chemie," Bd. ii.), contains principally, fat, 24.2 ; albumen and casein, $24 \cdot 2$; extractive matters, 24 ; and phosphate of lime, 20 per cent.; substances which are found, at all events in part, in the smegma.

Of nerves, I have seen no indication in the sebaceous glands, nor of vessels distributed upon and between their lobules; whilst numerous minute véssels and even capillaries, undoubtedly exist around the larger glands, most distinctly in the penis and scrotum, as well as in the ear. I would, moreover, refer to the smooth muscles described above, when speaking of the cutis, which are found in the neighborhood of the sebaceous glands, and whose contraction is, perhaps, not inoperative towards the emptying of their contents.

§ 75. Development of the Sebaceous Glands. - The first formation of the sebaceous glands takes place at the end of the fourth and in the fifth month, and is intimately connected with that of the hair-sacs, since they make their appearance simultaneously with the hairs, or shortly after, as outgrowths of the hair-sacs; whence they are not all formed at once, but those of the eyebrows, forehead, \&c., first, those of the extremities last. The mode of their development, more precisely described, is as follows: When the rudiments of the hair-sacs have attained a considerable development, and the first indication of the hair is visible in them (Fig. 75, A, B), there are perceptible, on their outer surface, small, indistinctly-bounded papillary processes $(u, v)$, which consist of a cellular substance, solid throughout and continuous with the outer rootsheath, and of a delicate investment, which is continuous with that of the hair-sac. These processes of the external root-sheaths of the hair-sacs, as they may properly be called, at first of $0.02-0.03$ of a line in length, and $0.01-0.016$ of a line in thickness, now begin to grow in proportion to the hair-sacs, become globular, and finally, while they extend them- 
selves and incline obliquely towards the bottom of the sac, pyriform and flask-shaped. A formation of fat in the internal cells now commences (Fig. 88, $A$ ), which, beginning at the bottom of the pyriform processes, is continued, also, into their pedicles, and finally includes the cells of the outer root-sheath, until at last the fat cells reach as far as the canal of the hair-sac (Fig. $88, B$ ). The gland and its contents are

Fig. 88.

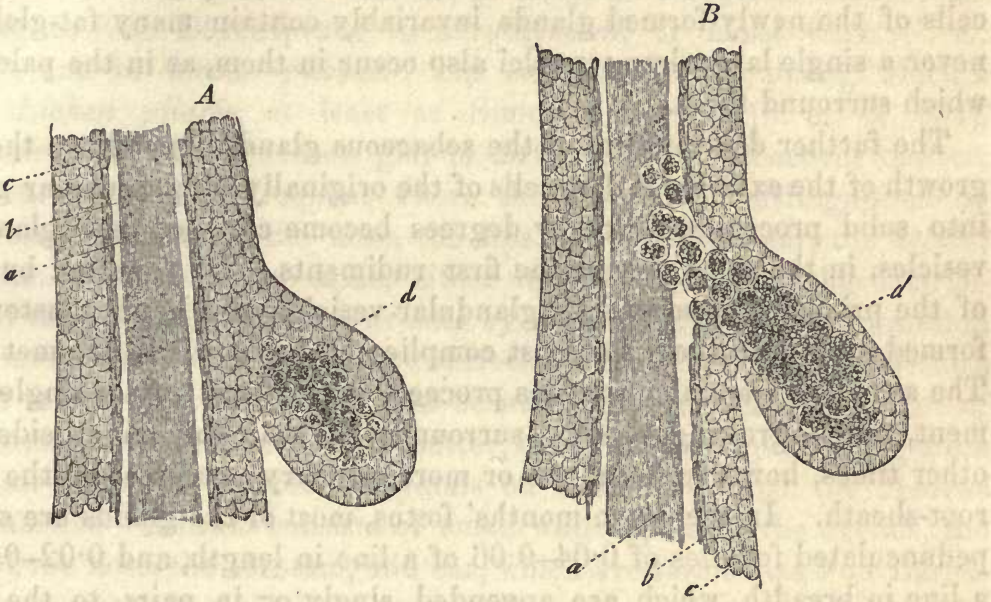

now complete, and it needs only that the cells at the bottom of the gland, or the glandular vesicles, should multiply, to force the sebaceous cells in the duct into the hair-sac, and fully to establish the secretion. The sebaceous glands, therefore, like the sudoriparous, are, at first, solid outgrowths of the Malpighian layer of the skin, for which an external opening is not developed till afterwards, and the first cutaneous sebaceous matter is formed by a metamorphosis of the inner cells of the rudiment of the gland, while the space which these cells occupied becomes the cavity of the gland, which, however, never appears empty, but is continually filled by successive generations of cells.

The development of the glands, up to this point, proceeds pretty quickly. It may be stated generally, that so long as the hairs have not appeared externally, the rudiments of the glands are papillary, measure scarcely more than 0.03 of a line, and for the most part contain cells which are still quite pale; after the hairs have made their appearance externally, we find larger pyriform rudiments with a thicker end, of $0.024-0.05$ of a line, the cells of which are partly still pale, partly

Fig. 88.-To elucidate the development of the sebaceous glands in a six-months' fœtus: $a$, hair; $b$, inner root-sheath, here more closely resembling the horny layer of the epidermis; $c$, outer root-sheath; $d$, rudiments of the sebaceous glands. $A$, flask-shaped rudiment of the gland, with fat developed in the central cells; $B$, larger rudiments; the development of fat has taken place also in their neck, and fatty cells have been excreted into the hair-sac, giving rise to the glandular cavity and the secretion.-Magnified about 250 diameters. 
contain fat, and which now soon open into the hair-sac. In the fifth month, therefore, the secretion has already begun in many places, and in the sixth it is everywhere established. At the same time, however, it is to be observed that, together with the original glands, which occur, one or two together to each sac, in the sixth month, new rudiments are produced, which generally lie deeper, and taking on the same course as that which has been described, soon become secreting glands. The fatty cells of the newly-formed glands invariably contain many fat-globules, never a single large drop; nuclei also occur in them, as in the pale cells which surround them.

The further devclopment of the sebaceous glands depends on the outgrowth of the external fatless cells of the originally simple tubular gland, into solid processes, which by degrees become changed into glandular vesicles, in the same way as the first rudiments. By repeated budding of the primitive or secondary glandular vesicles, the larger clusters are formed, and from them the most complicated forms which are met with. The so-called glandular rosettes proceed, very often, from a single rudiment, which, growing rapidly, surrounds the hair-sac on all sides; at other times, however, from two or more primary processes of the outer root-sheath. In the seven months' fotus, most of the glands are simple pedunculated follicles of $0.04-0.06$ of a line in length, and $0.02-0.03$ of a line in breadth, which are appended, singly or in pairs, to the hairsacs; in the ear alone, do four or five glands of the simplest kind surround a sac, and form rosettes of not more than 0.06 of a line in diameter; in the nose, simple clusters of at most $0 \cdot 1$ of a line are presented. In the new-born infant, instead of the simple follicles, simple racemoseglands are found in all the above-mentioned situations, one, or more rarely two, to a sac $0 \cdot 1-0 \cdot 12$ of a line in length, and only $0.04-0.06$ of a line in breadth; on the chest, the glands are rosette-like, also on the ear, temple, nose, nipple, labia majora, and scrotum, where they measure $0 \cdot 1$, in the last four places even 0.4 of a line and more. From these data, it results, that after birth an increase in size takes place in most of the glands, and assuredly in the same manner as during the foetal period, a view which is favored by the occasional occurrence of pale, solid, glandular lobules, even in the adult; certain glands arise only after birth, viz. those of the labia minora.

Sebaceous glands also occur in abnormal localities; thus Kohlrausch (Mull. "Arch.," 1843, p. 365), observed them in an ovarian cyst, and Von Bärensprung (l. c., p. 104) in a subcutaneous cystic tumor of the brow; in both places they were connected with hair-sacs, whence it may, perhaps, be concluded, that they are very frequently to be found in cysts which contain hair. In fact I met with very beautiful sebaceous glands, with a considerable amount of sebaceous matter, in the walls of 
the cyst containing hair, mentioned above, from the lung (Mohr's case); Von Bärensprung has, he believes, though rarely, observed a new development of sebaceous glands in cicatrices of some years' standing. When the hairs fall out, the sebaceous glands seem to disappear, at least I have repeatedly failed in finding them in bald places. Hypertrophy* of the sebaceous glands takes place, according to E. H. Weber (Meckel's "Archiv," 1827, p. 207), in cutaneous cancer; according to Von Bärensprung in akrothymion, or moist warts (l. c., p. 81), and in noevus pilosus. The comedones also, among which I place Lichen pilaris, at least as Simon defines it (1. c., p. 334), are hair-sacs and sebaceous glands distended with sebaceous matter, which are especially frequent where the glands are distinguished by their large size, as on the nose, the lips, the chin, the ear, the areola, and the scrotum. They arise, either in consequence of the obstruction of the apertures of the hair-sacs by impurities, or of the formation of a more viscid and consistent secretion; and they contain, besides one or many hairs, which may also be wanting, fatty cells, like those of the normal cutaneous sebaceous matter, epidermic cells proceeding from the hair-sacs, free fat, often crystals of cholesterin and the Acarus folliculorum. Milium consists of small white spots on the eyelids, the root of the nose, the scrotum, and ear, which are formed, as Von Bärensprung is certainly right in supposing, from the sebaceous glands also, by their distension alone, without the hair-sacs; in consequence of which, rounded prominences without any aperture are formed and raise up the skin : their secretion, similar to that of the comedones, may still frequently be pressed out through the hair-sacs. Finally, there can no longer be any doubt that the sebaceous cysts which lie in the corium itself (atheroma, steatoma, meliceris, and molluscum), must also be regarded as colossal hair-sacs with sebaceous glands. Further details may be found in the works cited.

With respect to a little parasite, the Acarus folliculorum, which resides in healthy and distended hair-sacs and sebaceous glands, I must refer to G. Simon (l. c., p. 287). In the case of Ichthyosis congenita above referred to, Dr. H. Müller and I found the excretory ducts of the sebaceous glands in the epidermis everywhere dilated to $0.02-0.06$ of a line, with saccular diverticula, often lying many together one behind another, of $0.04-0.12$ of a line, and quite full of sebaceous matter. Here and there a hair was found in one of these ducts, so that it appeared at the same time to be a hair-sac.

In investigating the sebaceous glands, they should either be prepared from within, by cutting them with the hair-sacs which belong to them from the cutis, or perpendicular sections, not too fine, may be made.

* [These glands, when hypertrophied, frequently lose their glandular structure, and are changed into yellowish granular masses.-DaC.] 
The minuter structure may be best studied, at first in the glands of the scrotum and penis, or labia minora, as these can be isolated without any trouble; to which end acetic acid, which renders the surrounding parts transparent, is very serviceable. With the others, so far as form, position, and size, are concerned, the use of alkalies, especially of caustic soda, is most advisable, inasmuch as they clear all the other parts, while they act but little on the glands on account of the quantity of fat they contain. If it be desired to study, not so much the investments, as the cells of the glands, obtaining at the same time a view of their whole figure, there is no plan better than maceration; the hairs with their root-sheaths, and the cellular masses of the sebaceous glands, epithelium and contents, may then be drawn out altogether. Where the epidermis is thin (on the scrotum, labia majora, glans penis), the same end may be attained in a short time by the dropping on it concentrated acetic acid, and also by using caustic soda in the same manner, though with greater destruction of the glandular cells. To study development, the maceration of foetal skin, and the rendering it transparent by acetic acid, are of great use. The fat-cells in the interior of the glands are isolated with great ease by teasing out a large gland, and the secretion may be examined without addition, and also with water and caustic soda.

Literature.-Compare the works cited above under the head of "Skin," by Gurlt, p. 409; Krause, p. 126; G. Simon, p. 9; Valentin, p. 758; the "Essay on the Hairs," by Eschricht, which has been mentioned; then the general works by Henle, p. 899; Todd and Bowman, p. 424, fig. 92; Hassall (pl. liv. should be liii.), p. 401; Bruns, p. 349; Gerber, p. 75 , figs. $40,42,43,44,45,239$; Arnold, part II., the figures of Wagner, "Icon. Phys.," tab. xvi., fig. 11, c ; Arnold, "Icon. Anatom.," Fasc. II., tab. xi., fig. 10, and Berres, tab. xxiv., besides that G. Simon, "Ueber die sogenannten Tyson'schen Drüsen an der Eichel des männlichen Gliedes," in Müller's "Archiv," 1844, p. 1.

\section{OF THE MUSCULAR SYSTEM.}

§ 76. To this system belong all the transversely striped muscles, which, together with their accessory appendages, the tendons. and fascix, serve for the movements of the skeleton, of the proper organs of sense, and of the integuments. These muscles constitute a system situated between the integuments and the bones, and between the bones themselves, the individual parts of which are so associated and united by common membranes, that they may conveniently be regarded as a whole.

$\S 77$. The proper elements of the muscles in question, visible even to 
the naked eye,-the transversely striated (animal or voluntary) muscular fibres, or primitive fasciculi, - are distinguished, especially by their size and the distinctness of their individual parts, from those of most of the striped muscles occurring in other situations (heart, large venous trunks, pharynx, oesophagus, larynx, urethra). With respect to this latter characteristic, it is to be remarked that the sheath of the primitive fasciculus, or the sarcolemma,* in every fasciculus without exception, especially on the addition of water, acetic acid, and alkalies, may at once be recognized as a perfectly structureless, transparent, elastic, smooth membrane, which in man, as in the mammalia, is distinguished by its delicacy from the same tissue in the lower Vertebrata, and particularly in the naked Amphibia. The muscular or primitive fibrils may, though not without difficulty, be isolated, especially in muscles that have undergone slight maceration, or have been boiled, or immersed in alcohol or chromic acid. In general they are varicose, that is to say, present more or less distinct enlargements, at intervals of $0.0004-0.001$ of a line ; in consequence of which arrangement, and owing to the circumstance that the thicker and thinner spaces, throughout the entire thickness of the fasciculus, are placed regularly on the same level, the latter for the most part appears to be marked with delicate transverse bands. Occasionally, moreover, in addition, a fine parallel striation is evident, or, more rarely, where the enlargements on the fibrils are less appaFig. 89.

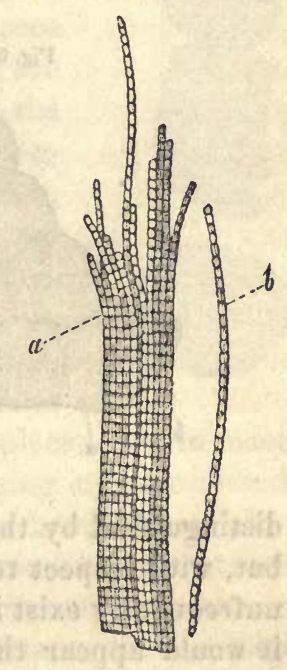
rent or quite imperceptible, simply a longitudinal

striation. In adults, the fibrils do not enclose any central space or canal (Jacquemin, Skey, Valentin), but, with the addition of a small quantity of a connecting interstitial substance, constitute perfectly compact fasciculi (Fig. 90). On the inner side of the sarcolemma,

FIG. 89.-Primitive fibrils from a primitive fasciculus of the Axolotl (Siredon pisciformis); magnified 600 diameters: $a$, a small fascicular composed of them; $b$, an isolated fibril.

* [It is greatly to be doubted whether the universality of the occurrence of this structure should be so strongly affirmed. We have been unable to detect it either in the muscular bundles of the heart, or in the great majority of those of the tongue, or in any of the muscles of a seven-months' fœtus. In fact, the question of the existence of a sarcolemma as an independent structure very much resembles that of the existence of "fibrils." The sarcolemma must be considered merely as the outer portion of the transparent, homogeneous matrix, in which the "sarcous elements" are imbedded (vide infra); and the possibility of raising it up by artificial means, or of observing its optical expression, as a distinct structure, will depend upon the amount to which it is developed relatively to the various elements, and the extent of chemical differentiation which has gone on it as compared with the rest of the matrix.-Trs.] 
numerous nuclei always exist, of a lenticular or fusiform shape, frequently with nucleoli, and from $0.003-0.005$ of a line long. These nucle $i$ are not placed with any regularity; sometimes two or more at the same level, or in rows, or alternately one behind the other. Fatty, or yellowish pigment-granules, also, frequently occur around the nuclei and between the fibrils, chiefly, however, in muscular fibres which are not in a perfectly normal condition.

The form of the muscular fasciculi is rounded-polygonal. In thickness they vary from $0.005-0.03$ of a line, or more. In the trunk and extremities they are invariably thicker $(0.016-0.03$ of a line) than on the head, in which situation, especially in the facial muscles, they are

Fig. 90.

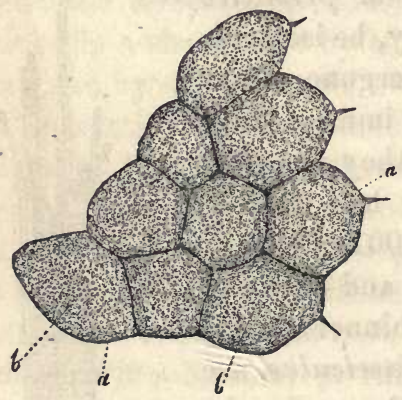

Fig. 91.

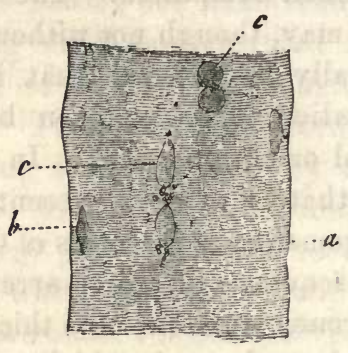

distinguished by the smallness of their fibres $(0.005-0.016$ of a line); but, with respect to this, it is to be remarked that great differences not unfrequently exist in one and the same muscle. From all that is known, it would appear that there is no absolute difference in the size of the muscular fibre in man and in woman, or between weak and robust individuals. On the other hand, it is not improbable that in one case one extreme, and in a second the other, may prevail. The thickness of the primitive fibrils, in man, amounts on the average to 0.0005 of a line; their number, in one of the larger fasciculi, must reach several hundreds, but is not accurately known. The distance between the transverse striæ varies usually from 0.0004 to 0.001 of a line.

Various controversial opinions still prevail with respect to the constitution of the muscular fibres. Several authors assert, or at all events consider it probable, that the primitive fibrils are produced artificially.

Fig. 90.-Transverse section of some muscular fibres or primitive fasciculi from the gastrocnemius of man; magnified 300 diameters: $a$, sarcolemma and interstitial connective tissue; $b$, transverse section of the muscular fibrils, with the interstitial substance.

Fic. 91.-Portion of a muscular fibre of man, treated with acetic acid, magnified 450 diameters: $a$, sarcolemma; $b$, a simple nucleus; $c$, double nucleus, surrounded with fatty molecules. 
This is the opinion entertained by Remak, who thinks a pre-existing division of the muscular cylinder still problematical; of Brücke, who appears to regard the contents of the muscle-tubes during life as fluid; of Du Bois-Reymond, and, above all, of Bowman. According to the latter, a division of the muscular fibres into "dises" (Fig. 92) is quite as natural, although not so frequent, as that into fibrils, and that they may be considered as columns composed of such discs, quite as correctly as bundles of fibrils. Were a muscular fibre completely divided in the direction of both the transverse and longitudinal striæ, rounded, angular, minute particles would be produced, which may be termed primitive particles, or "sarcous elements." In the fibre, these elementary particles are connected in both directions, the same particles in the one case constituting a "disc," and in the other a segment or joint of the fibrils. The division into dises, upon which Bowman lays especial stress, would in my opinion have been of importance had it occurred as frequently as that into fibrils, and also, occasionally, in recent muscle; but it is not so,-for, in the first place, nothing of the sort can ever be seen in recent muscles

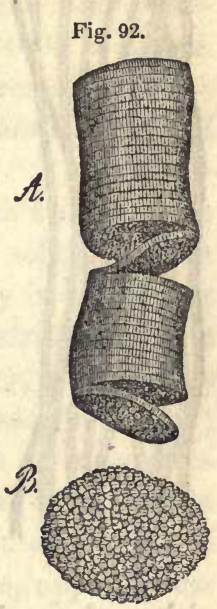
of man and the higher animals; and in the second place, even in macerated or otherwise manipulated fasciculi, the breaking up into discs is an extremely rare phenomenon; whilst, on the other hand, the isolation and exhibition of the fibrils may be obtained, in almost every instance, by any one at all conversant with the subject. Moreover, in transverse sections of perfectly fresh living muscles, as, for instance, of the thigh of a Frog, made by means of the double-bladed knife, the transverse section of the fibrils is just as evident and distinct as in dried muscles, whilst, in precisely similar longitudinal sections, not a trace of the "discs" can be detected. This fact at once sets aside all those views, according to which the muscular fibres, during life, consist of a homogeneous, solid, or fluid substance, or of minute particles, connected in two directions. To Bowman's opinion, moreover, is opposed the fact that his assumed "elementary particles," except in macerated muscles, where such a thing readily occurs, can only with difficulty be obtained in an isolated form, whilst, according to his view, such a disintegration, in cases where these particles do not cohere firmly, either in a longitudinal or transverse direction, would necessarily take place with equal

Fra. 92.- $A$, a primitive fasciculus, separating transversely into discs; magnified 350 diameters. It exhibits distinct transverse and fainter longitudinal striæ. The discs, of which one more highly magnified is seen at $B$, are granular, and consist of the primitive particles (sarcous elernents) of Bowman, or segments of the fibrils according to other authors (after Bowman). 
facility in either; and in the second place, that, in the thoracic muscles of insects, the individual fibrils may be very distinctly and beautifully seen (Fig. 93) in the muscles, when quite fresh. When we consider the

Fig. 93.

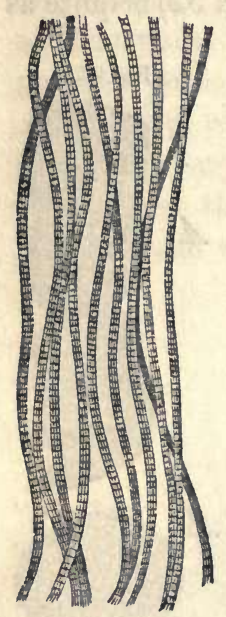
great similarity between the muscles of insects and of the higher animals, in every essential particular, this fact appears to me to be of a striking nature. I am, therefore, from this and the other reasons assigned, thoroughly convinced of the existence of fibrils during life, and believe that, where they do not so readily admit of being isolated, as in man and many animals, they are connected by a homogeneous, tenacious (albuminous), interstitial substance, which is very evident in a transverse section, and in fact so firmly, that, under certain circumstances, transverse rupture of the fasciculi may take place, that is to say, in the direction of the thinner spaces of the fibrils; as also occurs in other fibres; for instance, in the elastic tissue, smooth muscles, and even in the corneous cells (internal root-sheath and cortex of the hair). With all this, it must not be supposed that fibrils exist in all muscular fibres of animals, either themselves striped or corresponding to the striped fibres here described. The study of development and of comparative anatomy much rather teaches that the muscular fibre occurs in various conditions, and, particularly, that it frequently exhibits more homogeneous contents, with or without transverse striation, and without fibrils. This, however, of course, affords no ground for the assumption of such a condition in man and the mammalia also; and although such muscular fibres, in certain animals, readily break up into transverse segments (Leydig), still, it is not thereby proved that in the higher animals a similar division of the contents is to be regarded as natural, and that into fibrils as artificially produced.

The diameter of the primitive fasciculi varies, not inconsiderably, in different muscles, or in one and the same muscle. Henle (who is followed by Gerlach), at an earlier period, assigned to them a diameter of $0.005-0.006$, and at most of 0.017 of a line; but more lately (Stadelmann, "Sectiones transversæ"), has declared that these measurements are not universally correct. I will here give some particulars upon which the measurements stated above, in the text, are founded. In a female, the fasciculi of the sacro-lumbalis measured $0.016-0.028$, the majority $0.020-0.022$ of a line; in the pectoralis major, $0.01-0.03$, most of them 0.02 of a line; deltoid, $0.016-0.026$, the majority $0.02-$ 0.022 of a line; in the masseter, $0.006-0.02$, the majority $0.01-0.018$

Fia. 93.-Primitive fibres from a quite recent transversely-striated muscle of a Bug; magnified 350 diameters. 
of a line; in the retrahens auriculo, $0.006-0.015$, the greater part 0.008 0.01 of a line. In a male, their diameter in the pectoralis amounted to $0.018-0.28$ of a line, the greater number measuring $0.02-0.022$ of a line; in the deltoid, $0.012-0.024$ for the largest, and for the smaller 0.016-0.02 of a line; in the obliquus abdominis externus, $0.16-0.024$ and $0.016-0.02$ of a line; in the orbicularis oris, $0.008-0.016$ and $0.01-$ 0.012 of a line; in the frontalis, $0.006-0.014$ and $0.008-0.01$ of a line. In a second individual, the pectoralis major contained fibres of 0.0068 0.024 , most of them $0.018-0.02$ of a line; the pyramidalis, some of $0.01-0.028$, the majority 0.02 of a line.

With respect to the nature of the primitive fibrils, much still remains to be cleared up. In general they must be regarded as solid; and, in fact, there is nothing to indicate the existence of a cavity in them. It is fully ascertained that it is to them that the transverse striation of the primitive fasciculus is due. It is still doubtful, however, whence the appearance of transverse striation in the fibrils themselves arises; whether from their being spirally twisted (Arnold), from zigzag curvatures (Will), or from varicosities. All that I have seen leads me to adopt the latter view, which is also that most generally entertained. I do not deny that, in the examination of numerous fibrils, appearances are occasionally met with, favorable to the other two views, and particularly to that of Will; but it is much more usual to find simple nodular enlargements. The large fibrils in the perennibranchiate Amphibia (Siren, Proteus, Menopoma), (Fig. 89), are above all others adapted for this investigation. In these animals, when they have been preserved in spirit, the fibrils become isolated in considerable numbers, and may be examined on all sides. It is the same with the muscles of the thorax in Insects.

Quite lately Dr. Barry has propounded the view that each muscular fibril is constituted of two spirally convoluted filaments running in the same direction. I have seen nothing of the kind, and do not hesitate to describe the whole of Dr. Barry's exposition as nothing but a myth, and his figures as fantastical images.

As regards the notion adopted by Bowman, Dobie, and others, that the fibrils are constituted of still more minute particles (sarcous elements), it may perhaps be stated, as the study of development shows, that the fibrils do, in fact, at first appear to consist of separate particles. But the question is whether in the adult such elementary particles continue to be evident, and this, at present, I am inclined to deny.

The nuclei of the muscular fibres, in man, are situated, as I agree with Schwann in stating, only on the inner surface of the sarcolemma, and not within the fibrils; that they are not placed externally on the fasciculi, as was formerly stated by Henle and Stadelmann, and more lately by Gerlach, is readily perceived, when the muscles are treated 
with alkalies. Under these circumstances the partially-swollen nuclei escape, together with the fibrils, in a state of solution, from the sheaths, which remain behind, and before they are dissolved may be easily examined in an isolated state. In many muscles, even when there are no granules between the fibrils, larger or smaller fatty molecules occur around the nuclei.*

* [With regard to the vexed question of the ultimate structure of striped muscle, we question if any rea! improvements have been made upon the description originally given by $\mathrm{Mr}$. Bowman ("Phil. Trans.," 1840), viz., that it consists of minute, dark, subangular particles, the "sarcous elements," imbedded in a more transparent connecting substance or matrix; that neither discs nor fibrils can be said to exist in the normal state,-the breaking up of the muscular bundle into either of these elements, resulting simply from the manner in which the lines of greatest cohesion are disposed, at the time when mechanical violence is applied to it. The assertion in the text that the fresh muscle of man and the mammalia does not break up into discs, is decidedly erroneous-as we have seen it occur repeatedly.

Nor can we grant the invisibility of the dises in longitudinal sections of muscle: what may be the case in such sections made with the double knife, we do not know,-but in those accidental longitudinal fractures of the muscular bundles of man, mammals, and insects, which constantly occur, the edges of the dises are most distinct. Again, without making any section at all, the discs may, especially in insects, be traced, by altering the focus of the microscope, through the entire thickness of the bundles. The argument in the text, in fact, proves too much; for if the fibrils are visible over the whole transverse section, their dark parts (discs) alternating with the light ones, must be as visible in a section, made in any longitudinal plane, as they are on the surface. However, that the appearance of discs should be absent in any longitudinal section of striped muscle is, to us, simply incomprehensible.

With regard to the thoracic muscles of insects, it is to be observed, in the first place, that they do not represent ultimate fibrils, but non-fibrillated primitive bundles. Dr. Auber, in a valuable paper in "Siebold and Kölliker's Zeitschrift" (H. 3 and 4, 1853), has already shown that there is no defined line of demarcation to be drawn between these and the ordinary muscles of insects, the two forms passing into one another by the peculiar flat bundles of the Libellulidæ, though he still considers the thoracic muscles to represent ultimate fibrils. His sole argument, however, is their resemblance to the ultimate fibrils of the higher animals, which we think loses all force, when we consider a fact that he has overlooked, namely, that the muscles of the legs, \&c., present a very beautiful, though very delicate fibrillationthe fibrils being not more than $\frac{20}{0} \frac{1}{0} 00-3 \frac{1}{30} 00$ th of an inch in diameter; that is, not more than from one-third to one-sixth the diameter of the thoracic muscles. Examined carefully with a high power (600), with a good definition, the edges of the dises, which under a lower power appeared very sharp and even, are seen to be distinctly granular, and to be composed of minute, somewhat fusiform or rounded particles, not more than $20 \frac{1}{1} \sigma^{\text {th }}$ of an inch in diameter, distinct from one another, and imbedded in the general transparent matrix, which is marked by fine longitudinal lines running between the rows of particles. Occasionally, the broad, dark discs appear to be separated by a delicate line, and this line, if carefully examined, is found to be composed of similar, but far more minute and paler particles. However, this appearance, though very common, is not to be met with in all the bundles. Acetic acid swells the muscle up, and renders the sarcous elements still more distinct, thongh the whole becomes very pale. If dilute ammonia be added to such a bundle, so as to neutralize the acid, it resumes its original dimensions, and almost its original appearance, except that the sarcous elements have often a wonderful sharpness of outline.

The thoracic fibres, treated with acetic acid, become exceedingly pale, and the distance between the discs is much increased. The latter often assume a granular appearance, but not so distinctly as in the former case; nor have we been able to detect any fibrillation of the intermediate substance, nor any minute sarcous elements, in them. They share the former character, however, no less than the latter, with multitudes of unquestionable muscular bundles-so that taking into consideration the existence of fibrils very much minuter 


\section{$\S 78$. The muscular fibres in the trunk and extremities are, in general,} so associated, without the existence of any divisions, reticular connection, or termination in the interior of the muscles, as to constitute contiguous prismatic bundles extending the entire length of the muscle.

than the thoracic fibres in the muscles of insects, and the gradual transition of the latter into undoubted bundles, we do not hesitate to regard these thoracic fibres as homologous, not with primitive fibrils, but with primitive bundles; and therefore to neglect any argument which may be drawn from their existence, to that of primitive fibrils during life in the higher animals.

The answer to the question, whether primitive fibrils exist during life or not, in fact, depends very much upon the meaning of the words. If it be meant that the muscular bundle is like a rope, the fibrils being the separate strands, united by "a homogeneous, tenacious substance,"-we should say that nothing of the kind exists during life. If, on the other hand, it be meant only that the molecules of the muscle are so arranged as to break up more readily and more frequently in the longitudinal direction than any other (just as a bar of wrought iron would tear into longitudinal fibres rather than in any other way, though it could by no means be said that it was composed of longitudinal fibres), why, there can be no question that such is the fact. The behavior of a muscular fasciculus, under the alternate action of acetic acid and ammonia, is as instructive with regard to this point, as that of bundles of connective tissue.

The existence of varicosities of the fibrils must depend very much upon their state of extension. Normally, they do not exist, unless, perhaps, the fibril has been split off from the very edge of a bundle, where the sarcous elements often project strongly. When very much stretched again, since the sarcous elements are more solid and resisting than the matrix, they will form knots, and the fibrils will appear more or less varicose. The great majority of instances in which the fibrils appear varicose, however, depend on imperfect definition-and the same may be said of supposed zigzag bendings; while the spiral fancies, on the other hand, are more probably connected with an imperfect judgment.

Recently, Drs. Sharpey ("Quain's Elements") and Carpenter ("Manual of Physiology") have advocated a view, the former, however, with some doubts (to which Professor Kolliker does not refer), founded upon an examination of the preparations of muscular fibrils, made by Mr. Lealand. They distinguish quadrilateral dark spaces in the fibrillæ, each of which is set, as it were, in a transparent frame of the same shape; these joined together constitute the fibril, the lines of junction of the frames, or "cells" being indicated by a dark line. We have repeatedly seen the appearances which are thus described; but so far as we have been able to discover, they invariably arise from that peculiar interposition of rows of minute paler sarcous elements, between the ordinary broad dark ones, to which we have referred above in describing the muscles of insects.

Very often, the finer sarcous elements are completely wanting, as in the thoracic muscles of Insects, in the muscles of the Frog, and in many of the bundles in Mammals; and in these cases there is, of course, no evidence at all of the existence of any such "cells."

In conclusion, we may state the view which we are led to take of the structure of striped muscle, in a few words. In a homogeneous, transparent matrix, definite particles are imbedded-the sarcous elements, - which are arranged side by side, in even transverse rows. In some cases the sarcous elements are all of one size; in others, they are alternately larger and smaller. The reason of this difference does not at present appear, but it is very possibly connected with the nutrition of the muscle. The matrix usually tends to break up into longitudinal bands - the "fibrils," - which have the diameter either of a single sarcous element, or of some multiple thereof; it likewise tends to break up in the transverse direction, giving way between the pairs of rows (discs) of sarcous elements; but these cleavage lines are no indication of the existence of discs or fibrils, as such in the unaltered muscle. The sarcolemma is simply the outer portion of the matrix, and its demonstrability as a separate structure depends upon the extent to which it is developed, and the amount of chemical change which it may have undergone relatively to the inner portion.-TRs.] 
These secondary muscular fasciculi, as they are termed, are, each of them, enclosed by a special envelop of connective tissue, and, several together, united by stronger membranes into tertiary fasciculi, which, lastly, in a greater or less number, unite and constitute the separate bellies of the muscle, or muscles themselves. If the muscular fasciculi are placed in the same plane, they constitute the membranous or flat muscles; and when disposed in a thick bundle, the elongated or columnar muscles. The muscles consequently are aggregations of numerous,

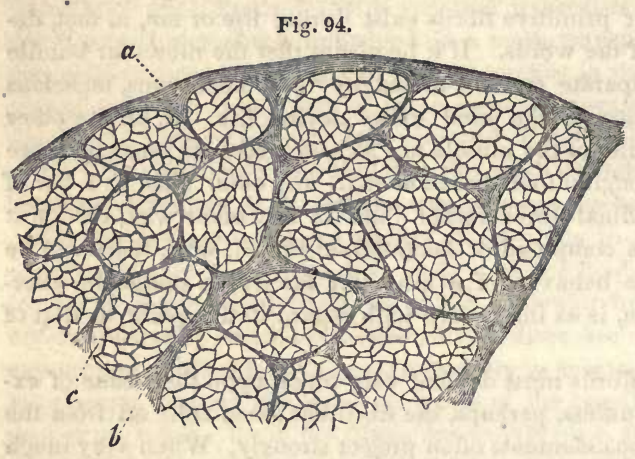

larger and smaller secondary and tertiary fasciculi, the sheaths or perimysium of which constitute a connected system, in which that portion which surrounds the entire muscle, as the perimysium externum or muscular sheath (vagina muscularis), is to be distinguished from the more internal elements immediately surrounding the larger and smaller fasciculi and the muscular fibres-the perimysium internum. The thickness of the secondary fasciculi varies from $\frac{1}{6}$ to $\frac{1}{2}$ a line; that of the tertiary and still larger bundles, which are most evident in muscles with coarse fibres (glutoeus maximus, deltoideus), is so various, and the division of the muscle in these more remote constituents is so arbitrary, that there is nothing specially to be said with respect to them.

The muscular sheaths or envelops, perimysium, composed of connective tissue, which are for the double purpose of conveying the vessels and nerves of the muscles, and of connecting the muscular fibres and supporting them when in action, vary in thickness according to the greater or less size of the groups of fasciculi surrounded by them. They are always, however, delicate, dull-white, non-glistening tunics, consisting of common connective tissue, and minute, isolated or anastomosing elastic fibres, of at most 0.001 of a line in thickness, the latter occurring in greater number, especially in the perimysium externum, which may, consequently, very properly and conveniently, be regarded as a semi-elastic membrane, and its function estimated in accordance with this structure. In all muscles, especially in those of a more lax construction, a certain number of adipose cells of the usual kind (fre-

Fra. 94.-Transverse section from the rectus capitis anticus major of Man, magnified 350 diameters: $a$, external perimysium; $b$, perimysium internum; $c$, primitive fásciculus and secondary muscular fasciculus. 
quently containing beautiful fat-crystals) occur, and in fat persons they are found quite in the interior.

[It was formerly supposed that the primitive muscular fasciculi ran in a perfectly straight direction towards their insertions, without dividing or anastomosing; but this is not correct. Recent investigations, made partly alone, partly with the assistance of Dr. Corti, have demonstrated the anastomosis of the fasciculi of striped muscles in the hearts probably of all Mammalia. We observed anastomosing fasciculi in Man, in the Rabbit, Dog, Cat, Calf, Frog, Heron, and Leydig has seen them in the Ruff. In the Mammalia, and in Man, they are frequent, and extremely delicate, and the anastomosis occurs by means of short transverse or oblique branches extending between parallel fasciculi. In the larynx, œsophagus, pharynx, and the tongue of the Rabbit, nothing similar to this was met with, but we found in the tongue of the Frog, immediately under the mucous membrane (which is readily removed in boiled preparations), the most delicate divisions, although no anastomoses. Fasciculi of 0.03 of a line or more, could be observed to form, by successively dividing at acute angles, large bundles of fine branches (the finest, 0.0012 to 0.0016 of a line), which were inserted into the mucous membrane of the tongue between its glands. I have seen, also, in the lymph-heart of frogs, an anastomosis of the striped muscles similar to that occurring in the heart, and Dr. Leydig (Zeitschrift für Wissenschaftliche Zoologie, Bd. I. Heft 2, 3), has observed anastomoses in the muscles of Paludina virspara, which genetically correspond to the striped muscles. In the muscles of the trunk and extremities in Man and in the Mammalia, I have never been able to discover even the trace of an anastomosis, although it occasionally appeared to me, as if some fasciculi beforo or at their connection with tendons, divided within a short space two or three times. I have certainly seen this division in the tails of batrachian-larvæ, where single fibres at their insertion into tendons separated into from three to five conical branches. (From Kölliker's Micr. Anat., I. 1, p. 210.)-DaC.]

§79. Connection of the Muscles with other parts. - The muscular fibres are connected with the movable parts, the bones, cartilages, articular capsules, the skin, \&c., partly in a direct manner, partly with the intervention of fibrous elements, the tendons, fascice, certain forms of muscular ligaments and membranes (lig. interossece, membrance, obturatorice). Those muscles which are attached either wholly, or at one or, the other end without the intervention of tendons, constitute on the whole the smaller number. Where the muscular fibres arise directly from bone (obliqui, iliacus, psoas, glutxi, \&c.) and cartilage (transversus abdominis, diaphragm), or rest immediately upon those structures (serrati, omohyoideus, sterno-hyoideus, aural muscles), they never extend 
further than to the periosteum or perichondrium, terminating abruptly on those membranes, with the fibres of which they are not, in any way, continuous, nor do they come into immediate contact with the bone or cartilage. Where the muscles extend to the skin, they either expand immediately beneath, and without any connection with it, or radiate in it, in the form of larger or smaller divergent fasciculi (facial muscles); in which case they appear to be inserted, at all events occasionally, at once into the filamentary processes of connective tissue.* But the precise mode of connection of these tissues has not yet been ascertained.

$\S 80$. The sinews, tendons, are brilliant, white or yellowish structures, composed almost entirely of connective tissue. They are subdivided, according to their figure, into the rounded, cord-like, true tendons, and into membranous aponeuroses (centrum tendineum, galea, tendons of the abdominal muscles, latissimus dorsi, cucullaris, \&c.). The two forms, either in their external configuration or internal constitution, do nat admit of definite distinction; they consist of connective tissue, which is characterized by the parallelism of its elementary fibres, its consistence, and its poverty in elastic filaments. The elements of the connective tissue, the fibrilloe, may be readily perceived, in fresh tendon, to be, as they are everywhere, extremely minute. In the cord-like tendons, they are slightly wavy in their course, all perfectly uniform in size, parallel to the long axis of the tendon, and in the recent state so closely approximated, that the demonstration of the existence of primitive fasciculi is not easy. Such fasciculi, however, do exist, having a breadth of $0.006-0.008$ of a line, and a rounded polygonal figure, as may be seen, especially in transverse sections of dried tendons, particularly on the addition of alkalies. But in the natural state they are so firmly united that they cannot be isolated.

On the other hand, in true tendons, in the recent state, secondary and tertiary fasciculi are very evident (Fig. 95). Delicate dissepiments, in fact, of loose connective tissue, penetrate the substance of the tendon,

* [The insertion of muscles without the intermediation of tendons, directly into the connective tissue of the skin and mucous membranes, is seen very beautifully in the tongue and in the facial muscles of Mammals. The former case has been well-described by Dr. Salter (Todd's "Cyclopædia," article "Tongue"); the latter may be examined with great ease in the levator labii superioris of the Rat. Here the muscular bundles run in the subcutaneous connective tissue, keeping a pretty even diameter until they nearly reach their insertions. They then divide into many branches, each of which either tapers off to a conical extremity, or divides into a number of delicate pointed processes. In either case, the ends of the muscular fibre gradually or suddenly lose their striation, and pass directly into the irregular nucleated bands of the connective tissue. No sarcolemma can be demonstrated in the branched ends of the muscles, and the bands of the connective tissue are directly continuous with the matrix of the muscle; the change, from the one to the other, being evidenced merely by the appearance of the sarcous elements. Nothing can afford a more complete proof of the homology between the pseudo-fibrillated tissue of muscle and that of connective tissue, than what we find here.-Trs.] 
and by their mutual connection form a continuous system of parallel tubes, thus dividing the tendinous fibrils or primitive fasciculi into numerous larger or smaller groups. Secondary fasciculi, mostly of a polygonal, or perhaps rounded or elongated figure, and having a diameter of $0.03-0.06$ of a line, may be very readily distinguished; and tertiary fasciculi, with polygonal contours, of $0 \cdot 1-0.05$ of a line, and more in diameter, and bounded by rather stronger dissepiments; there are, also, generally apparent, still larger subdivisions, composed of numerous tertiary fasciculi, and which, being closely united in very various numbers and groups, by a common envelop

Fig. 95.

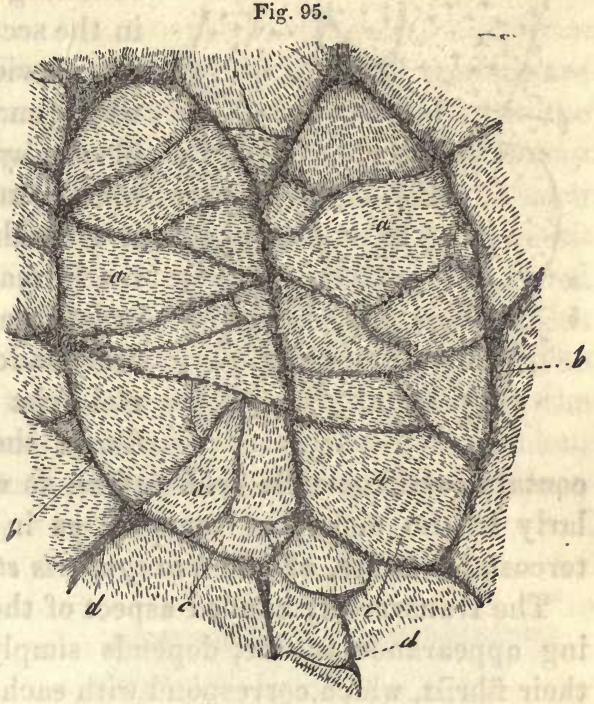
of lax connective tissue, constitute the tendon itself. The aponeuroses are constituted either in the same way as the true tendons, and consist of several layers of parallel, secondary fasciculi, disposed contiguously in the same plane, or more resemble the fibrous membranes, and present primary and secondary fasciculi decussating in two or more directions (abdominal muscles, diaphragm).

Fine elastic fibres (the so-termed nuclear fibres) occur in the secondary fasciculi of all tendons, in various conditions of development : sometimes as a series of slender fusiform cells connected by delicate processes; sometimes as fully-formed fibres of uniform thickness, or as isolated fusiform cells. The arrangement of these elastic elements is uniform throughout, and they run at regular distances, parallel to, and among the fasciculi of connective tissue. Consequently, in the transverse section of a tendon, the dark ends of the elastic fibres are apparent, distributed, at constant distances of $0.007-0.008$ of a line apart, over the whole section. But, besides these stronger elastic filaments, measuring from $0.0005-0.001$ of a line, there exist in most, perhaps in all tendons, extremely delicate fibrils of $0.0002-0.0004$ of a line, connecting the former in various directions, so that in reality there is, in every tendon, an elastic network, penetrating and entwining the fasciculi of con-

FIG. 95.-Transverse section of a tendon of the calf, magnified 20 diameters : $a$, secondary fasciculus; $b$, tertiary; $c$, nuclear fibres not quite in transverse section, but appearing as little streaks in the former; $d$, interstitial connective tissue. 
nective tissue. These fibrils may also be distinguished on a transverse

Fig. 96.

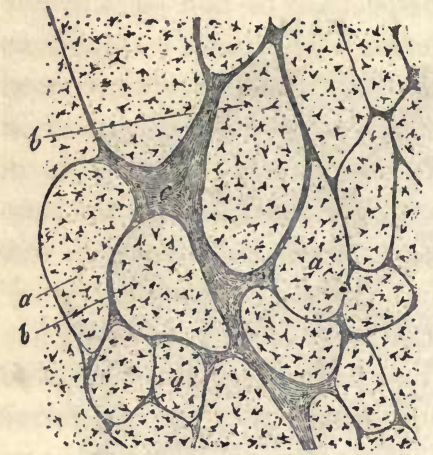
section, as minute dark points, or as lines radiating from the coarser points exhibited in the section (Fig. 96); and they are still more evident in longitudinal sections, in which, more especially, the whole of the fibrous system just described comes very readily into view. In such sections, also, it is evident that, in every case in which the formative cells of which the fibres are constituted still retain a certain degree of independence, very distinct elongated nuclei exist in them. Besides these elastic fibres, the tendons, in certain situations, contain cartilage-cells (vid. infra), as well as common fat-cells, particularly in the more lax tendons, as in the tendinous fibres of the intercostal muscles, of the triangularis sterni, masseter, \&c.

The transversely banded aspect of the tendons, to which their glistening appearance is due, depends simply upon the numerous curves of their fibrils, which correspond with each other throughout the fasciculus; this appearance is destroyed when the tendon is forcibly stretched, and merely indicates its innate elasticity, which comes into play in the relaxed condition.

The primary tendinous fasciculi, according to Donders and Moleschott, are seen in transverse sections treated with potass. This reagent, according to them, separates the secondary fasciculi into smaller ones, each of which consists of from 5 to 10 primitive fasciculi. In moistened transverse sections of dried tendon of man and the mammalia, I can very distinctly recognize the primitive fasciculi, although they have extremely delicate outlines. The appearance thus obtained affords an indistinct image of that presented in a transverse section of muscle. Even the very fibrils are, in this way, rendered distinct, a circumstance which appears to me of the greatest importance. When a transverse, not a longitudinal, section of tendon moistened with water or acetic acid is examined, there will be observed in all the secondary fasciculi, or in the primary when they can be distinguished, if not in all, yet in most cases, an extremely regular and minute punctation, nearly like that of the muscular fasciculi (Fig. 90), only not quite so distinct. The apparent granules are pale, round, of the same diameter as the tendinous fibrils which are obtained in other ways, and can be explained in no other manner than as being the transverse sections of such fibrils.

FiG. 96.-Tendon of the tibialis posticus (Man), magnified 60 diameters: $a$, secondary fasciculi; $b$, thicker nuclear fibres; $c$, interstitial connective tissue. 
These facts, better than any other, contradict Reichert's view, according to which the tendinous tissue is composed of a homogeneous substance. (Vid. § 24, note.)

§ 81. Connections of the Tendons with other parts. -The tendons are connected on the one side with the muscles, and on the other with the various parts moved by the muscles. Even by the naked eye, it may be seen that the former connection is effected in the one case in such a way that the tendon and muscle are continued into each other rectilinearly, and in the other so that the muscular fibres, with rounded extremities, join the borders and surfaces of the tendons and aponeuroses at an acute angle, as in the instance of the penniform muscles. The microscopic conditions in these two cases are widely different. In the former, the muscular fasciculi pass immediately into those of the tendon, in such a way that no sharply-defined limit exists between the two tissues,

Fig. 97.

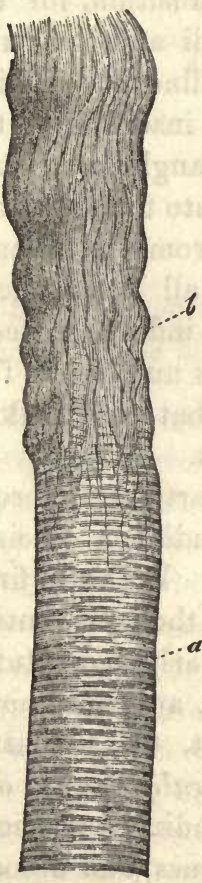

Fig. 98.

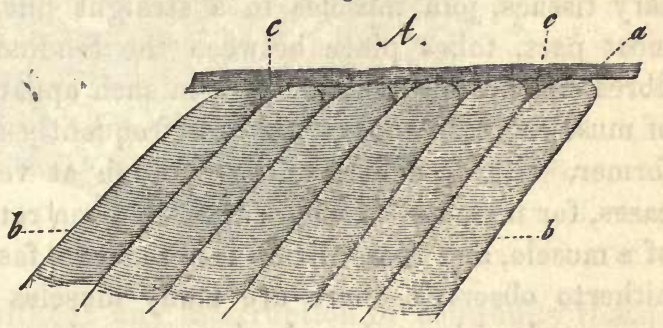

and the entire fasciculus of muscular fibrils is continuous with a nearly equal-sized bundle of tendinous fibrils (Fig. 97). Extraordinary as it may sound, I must say -in order to describe the impression that this sort of conjunction of muscle and tendon gives me-that it is that of a continuous connection of the muscular and tendinous fibrils. Where the muscular fasciculi join the tendons and aponeuroses at an acute angle, we find, in complete contrast with the condition just described, an abrupt limit between the muscle and tendon (Fig. 98). For, in this case, the fibres of the muscle really end, for the most part, obliquely truncated, with a slightly conical projecting terminal

Fra. 97.-A primitive fasciculus: $a$, from one of the internal intercostal muscles of Man, continuous into a tendinous fasciculus, $b$, into which it passes without any defined limit.Magnified 350 diameters.

FIG. 98.-Disposition of the muscular fibres at their oblique insertion into the tendon of the gastrocnemius (Man): $a$, a portion of the tendon cut longitudinally; $b$, muscular fibres witl slightly conical or truncated extremities, affixed in small depressions on the inner aspect of the tendon, to the border of which the perimysium internum, $c$, is connected.-Magnified 350 diameters. 
surface, or, more rarely, perceptibly attenuated, though always rounded, and are attached at a more or less acute angle to the surfaces of the tendons and aponeuroses, and on the borders of the former. Notwithstanding this, however, the connection between the two tissues is of the most intimate kind. The extremities of the primitive fasciculi are inserted into minute pits in the surface of the tendon, whilst, at the same time, the connective tissue between them, the perimysium internum, is continuous with that on the surface of the tendon. These relations are best observed in muscles which have lain a long time in spirit, or been boiled; in which, also, the sacciform blind extremity of the sarcolemma may occasionally be clearly seen. The last-described condition occurs whenever muscular fibres and tendons meet obliquely, consequently in all semipenniform and penniform muscles; in those whose tendons of insertion commence as membranous expansions (soleus, gastrocnemius, \&c.), and which arise from the surfaces of fascir, bones, and cartilages. Where, on the other hand, aponeuroses or tendons, with their elementary tissues, join muscles in a straight line, a real transition, for the most part, takes place between the tendinous fasciculi and muscular fibres, but not always, for, even in such apparently rectilinear transition of muscles into tendons, there is frequently an oblique insertion of the former, with free extremities, though at very acute angles; in such cases, for instance, as where tendons penetrate deeply into the substance of a muscle, and there divide into separate fasciculi. From what I have hitherto observed, there are many muscles in which all the fasciculi connected with tendons begin or terminate free, and indeed scarcely one in which this is not the case, with a greater or less number of fasciculi; whence it may be deduced, as a general rule, that the tendons have for the most part a less diameter than the muscles.

Besides muscles, tendons are connected with bones, cartilages, fibrous membranes (sclerotica, sheath of the optic nerve, tendinous fascix), ligaments, and synovial membranes (subcruralis, \&c.). With the firstnamed textures, the connection is either indirect, with the intervention of the periosteum and perichondrium, into the similarly constituted elements of which the tendinous fibres, for the most part, are continuous, or to the thickness of which they appear to add or direct. In the latter case (tendo Achillis, tendons of the quadriceps, pectoralis major, deltoideus, latissimus dorsi, ilio-psoas, glutoci, \&c.), the tendinous fasciculi rest, at an acute or right angle, on the surface of the bones, and become attached, without the intervention of the periosteum, which is wholly wanting in these situations, to all the elevations and depressions of the surface (Fig. 99). Close to the bones, the tendons frequently contain, throughout a certain extent, delicate, isolated cartilage cells, which are sometimes, however, contiguous and disposed in small rows. In exceptional cases, I have also seen the tendinous fibrils, at their extre- 
mities next the bone, entirely incrusted with calcareous salts, in the form of granules (ossified). In fibrous membranes, the tendons cease

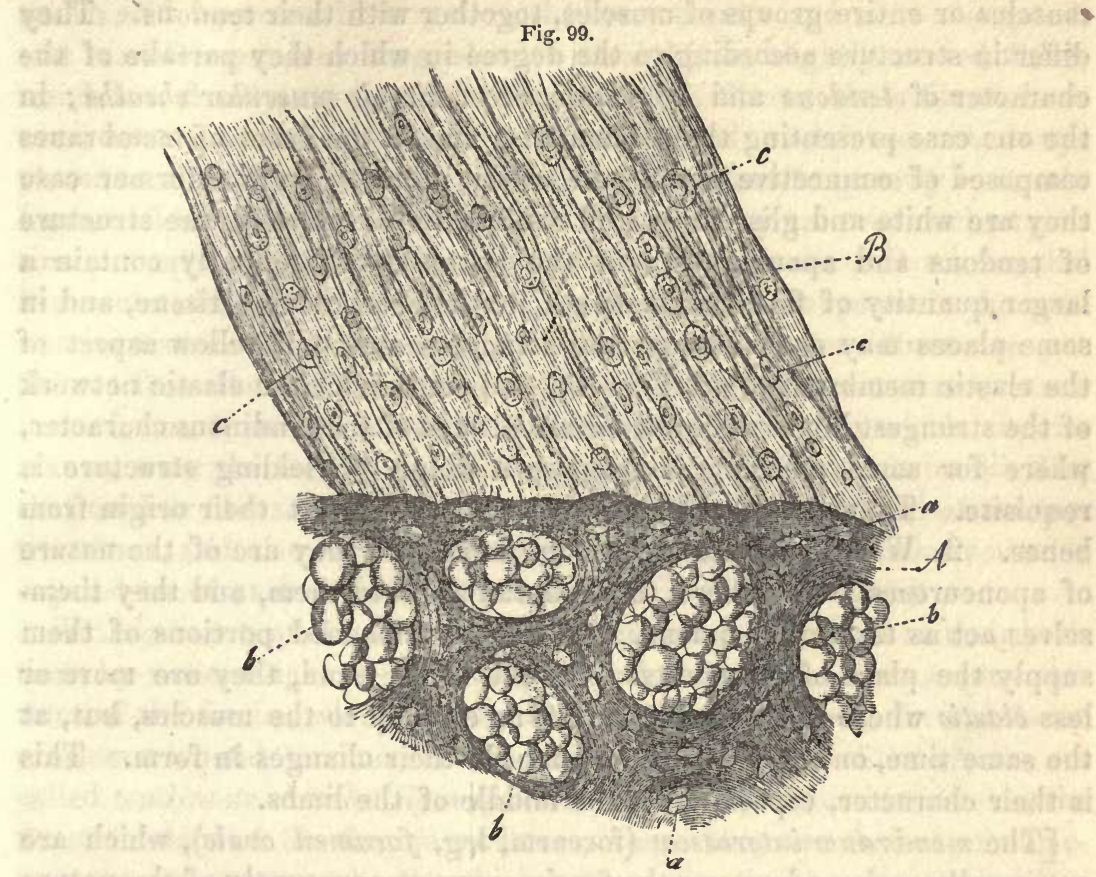

quite imperceptibly, and without any interruption of continuity (tensor fascioe, biceps humeri).

In man, I must positively deny that the tendinous fasciculi are ever connected merely with the sarcolemma (Reichert). Nor could I satisfy myself that this is the case in the River-crab, in which the tendons, it may be remarked, consist of chitine. Whilst other animals have afforded indubitable evidence of the existence of the same conditions as in man, the Frog, in particular, presents evidence of this fact; in the tadpole of which, owing to the sparing development of pigment in the tail, the transition of the extremities of the muscular fibres, which are frequently divided into three and five serrations, into the same number of minute tendons, may be very distinctly seen. In the caudal muscles, also, of the Cod, I noticed, very distinctly, the continuous connection of the tendons and muscles; in this case, owing to the shortness of the muscles, many muscular fibres were even seen in their entire length, together with the tendinous fasciculi at each end.*

Fra. 99.-Insertion of the tendo Achillis into the calcaneum of a Man sixty years old: $A$, bone with lacunæ, $a$; canalli and fat-cells, $b ; B$, tendon with tendinous fibrils and cartilage cells, c.-Magnified 300 diameters.

* [There can be no doubt that both the modes of connection between muscles and their tendons, described above, exist. Is it not possible that the gradual transition or the sharp 


\section{\$ 82. Accessory organs of the Muscles and Tendons.-A. The mus-} cular envelops or fascice are fibrous membranes surrounding single muscles or entire groups of muscles, together with their tendons. They differ in structure according to the degree in which they partake of the character of tendons and ligaments, or of simple muscular sheaths; in the one case presenting that of tendons, and in the other of membranes composed of connective tissue and elastic fibres. In the former case they are white and glistening, and exhibit, in all respects, the structure of tendons and aponeuroses; in the latter they frequently contain a larger quantity of fine elastic fibres in their connective tissue, and in some places may even assume the structure and dull-yellow aspect of the elastic membranes (vid. Fig. 49), and contain a close elastic network of the strongest kind. The fascice are always of the tendinous character, where for some mechanical purpose a tough unyielding structure is requisite. They are of this kind, therefore:-1. At their origin from bones. 2. Where muscles arise from them, and they are of the nature of aponeuroses. 3. Where tendons radiate into them, and they themselves act as terminal tendons. 4. Where thickened portions of them supply the place of ligaments. On the other hand, they are more or less elastic where they constitute a firm envelop to the muscles, but, at the same time, one which does not impede their changes in form. This is their character, especially in the middle of the limbs.

[The membrance interossece (forearm, leg, foramen ovale), which are not usually reckoned among the fascir, are not apparently of the nature of ligaments of the bones, but rather of intermuscular ligaments. The plantar and palmar a poneuroses serve, in part, as tendons for the smaller muscles of the hand and foot, but chiefly as ligaments for the retention of the flexor tendons, in which respect they are analogous to the lig.cruciatum carpi dorsale, \&c. In them, even in the adult, the entire course of development of the nuclear fibres (minute elastic fibres), may be studied. Between the fasciculi of connective tissue, straight series of 10 to 20 and more, thickly placed, elliptical cells of 0.006 to 0.012 of a line, with round

line of demarcation between the muscle and its tendon may have some connection with the age and completeness of the particular bundle examined? In the Frog, we have noticed that, among neighboring bundles, some exhibit transitions between the proper muscular tissue and the tendon, while others have the former very sharply defined; and the examination of the insertion of the triceps extensor cubiti of a seven-months' fotus has afforded us the most evident transitions from tendon into muscle, although the insertion of the bundles is here very oblique. The best way of expressing the mode of connection of muscles with their tendons, perhaps, would be to say that the matrix of the muscle and the matrix of the connective tissue, into which it is inserted, whether in the form of tendon or otherwise, are invariably continuous; the appearance of continuity or of discontinuity of the two tissues, arising solely from the sudden or gradual cessation of the deposit of the sarcous elements at their point of junction.

The nature of the corpuscles which are to be found at the junction of tendons with bones and cartilages-Professor Kölliker's "cartilage-corpuscles"-has been adverted to in the note at p. 97.-Trs.] 
nuclei, and 2 to 6 minute opaque fat-granules, occasionally occur; the cells afterwards disappear, and the nuclei, which, on the addition of acetic acid, appear a little yellowish, become more and more elongated, and transformed into long, slender, straight, or slightly-curved fibres, which are, finally, conjoined into long nuclear fibres; these fibres, however, upon the whole are rare. The elongated nuclei are not always placed in a straight line, one behind the other, but frequently in an oblique, and in various other directions. In this way are produced serpentine nuclear fibres, which, even when fully formed, are still surrounded with isolated fat-granules, and lie as it were in vacant spaces in the connective,tissue. In this case, consequently, the nuclear fibres are not formed from the nuclei of the cells, from which the connective tissue is formed, but from special cells of a temporary nature; which circumstance, were the fact of general application, would make it intelligible, that nuclear fibres may both surround the secondary fasciculi of connective tissue, and also exist without any such tissue (membranous reticular expansions of nuclear fibres, "Micr. Anat.," II. 1, p. 226)].

$B$. Ligaments of the tendons. - The tendons are retained in their positions by various ligaments. Independently of certain ligamentous portions of the fascix, which, being attached to the bones, form tubular processes around tendons, or otherwise confine them, there are the socalled tendinous sheaths (lig. vaginalia tendinum), as for instance on the flexor tendons of the fingers and toes, where they are formed of numerous successive narrow bands, which in these situations serve to strengthen the mucous sheaths. Other ligaments to be referred to this class, are the lig. carpi proprium, the trochlea, and the retinacula tendinum.

C. Mucous bursoe and sheaths,-bursce mucosce et vaginoe synoviales.Where muscles or tendons, in their movements, rub upon hard parts (bones, cartilages), or on other muscles, tendons, and ligaments, there are found, between the parts in question, spaces filled with a slightly viscid fluid, which, according to Virchow (Würzb. "Verh." II. 281), contain not mucus, but a material very similar to colloid matter, and which anatomists have been used to regard as lined with a special membrane, a synovial membrane. These spaces are said to constitute closed sacs of a rounded or elongated form, which either simply invest the opposed surfaces of bones and tendons, bones and muscles, \&c.,-bursce mucosee; or in the form of double, although connected tubes, cover at the same time the surface of the tendons, and of the parts between which the tendons play,-vagince synoviales. The truth of the matter is this, that it is only the smallest of these spaces which are lined with a continuous membrane; most of them are in many situations without such a lining. With respect to the mucous burse, those appertaining to the muscles (psoas, iliacus, deltoid, \&c.), are, eminently, to be considered as continuous sacculi, whilst in those belonging to the tendons, a 
membrane can only be detached in parts, and is found to be almost wholly wanting exactly at the points where the mutually gliding parts are in contact. Precisely the same thing obtains in the synovial sheaths, among which the common sheaths of the flexor tendons of the fingers and toes, only in a certain measure, retain the form of a so-termed serous sac, although, even in this case, many parts of the surface of the tendons are without any such membranous lining. Whence it would appear, that in this case, as in many others, the old doctrine of the existence of continuous serous sacs requires thorough emendation. In most of the synovial sheaths, and in many mucous bursæ, are found occasionally, particularly in the retinacula, smallor or larger, reddish fimbriated processes, exactly resembling those of the joints, and which, in like manner, are nothing but vascular processes of the synovial membrane.

D. Fibro-cartilages and sesamoid bones.-The tendons of some muscles (tibialis posticus, peronous longus), in those portions which run in the tendinous sheaths, contain, imbedded in their substance, dense semi-cartilaginous bodies, which are known under the name of sesamoid cartilages (fibro-cartilagines sesamoideoe), and when, as occasionally happens, they become ossified, of sesamoid bones (ossa sesamoidea); the latter occur normally, imbedded in the flexor tendons of the fingers and toes, presenting one surface towards an articulation.

Respecting the more intimate structure of the last-mentioned parts, the following is to be remarked. The sesamoid bones consist of common, finely cancellated osseous substance, are on one side closely surrounded by tendinous or ligamentous tissue, and on the other, which is invested with a thin layer of cartilaginous substance, project into an articulation. The ligaments of tendons, in correspondence with their function, possess exactly the same firm structure as that of the tendinous portions of the fascice and of the tendons themselves, and exhibit occasionally fine elastic fibres in process of development, or the round formative cells of such fibres disposed in rows. The retinacula tendinum have a more delicate structure; their function being rather to convey vessels to the tendons, they consequently contain chiefly a more lax connective tissue, with fine elastic fibres, and also fat-cells. The mucous bursæ, which are invariably thin-walled, consist, in as far as they possess a distinct membrane, of fasciculi of connective tissue, crossing each other in the most various directions, loosely connected, and in many places anastomosing, together with some fine elastic fibres. The mucous sheaths, on the other hand, in agreement with their double function, which in one respect is that of a mucous bursa, and in another that of ligaments confining the tendon or of tendinous sheaths, present in their thinner parts the structure of bursce mucosoe, and in the thicker, an unmixed, dense connective tissue, frequently with cells disposed in rows, which pass into elastic 
fibres. Both of these kinds of sacs are lined, on the inner surface, together with the parts contained in or otherwise bounding them, but only in places, with an epithelium, consisting for the most part of a simple layer of nucleated polygonal cells $0.004-0.007$ of a line in diameter. The parts which are bare of epithelium, are:-many portions of the mucous sheaths, and the tendons lying in them, and certain spots of the bursce mucosce themselves, which are distinguishable by their dull lustre and yellowish aspect, and which occur especially in those situations where the tendons and parts surrounding them are exposed to a greater degree of pressure. The common flexor sheath of the fingers is lined throughout with epithelium; and the same may be said of the mucous bursæ, in which it is only certain loop-like ligaments, which beyond the limits of the bursæ still surround the tendons, that do not present any cellular covering, as is the case, occasionally, in the subscapularis poplitous, \&c.

All these bare places, which are uncovered by epithelium, invariably exhibit, almost throughout, the nature of fibro-cartilages, the dense connective tissue of which they are composed, and which for the most part is furnished with but few elastic elements, containing a greater or less, often a very considerable number of cartilage-cells (Fig. 99), amongst which, the most frequent are rounded cells, with a dark contour, although by no means with thick walls, measuring $0.006-0.012$ of a line, with a roundish nucleus of 0.003 of a line, and clear fluid, Fig. 100.

with or without some minute, opaque fatty granules. Besides these, there are, moreover, elongated cells, with one or two nuclei; round, thin-walled cells, containing $1,2-20$ secondary cells, with thick walls, and dark contours; the mother-cells measuring as much as $0.02-0.03$ of a line; and lastly, elongated cells, with concentric deposits, inclosing a nucleus, or nucleated secondary cell. In the tendons, the simpler forms of cells almost exclusively occur, and the cells, although frequently extremely numerous, are for the most part isolated, or, at most, disposed in rows or groups of $2-6$, which are contained in the connective tissue, both superficially and more deeply. In most cases, the common connective tissue alternates with one more resembling fibro-cartilage, so that the tendon, on a transverse section, presents a speckled, white and yellowish aspect; or it maybe, that the outer surface only of the tendon contains cartilage, the deeper portions retaining their usual condition. Where the deposition of the cartilage cells is most abundant, the tendons become thickened, or even studded, as it were, with distinct, fibro-cartilaginous masses (peroneus longus, tib. posticus). In the mucous bursæ and the other parts above named, the cartilage cells are placed, not un-

Frg. 100.-Cartilage-cells from the vaginal ligaments surrounding the tendons of the poplitcus, magnified 350 diameters: $a$, cell with one; $b$, with two nuclei; $c$, cell with one; $d$, with two secondary cells, both of which have rather thick contents. 
frequently, in closer aggregation, or in longer rows of 5-10 cells or more, in which rows the terminal cells are invariably the smallest, and the middle ones the largest. On the cuboid bone, where the tendon of the peroneus longus passes over it, there is a layer of true cartilage $\frac{1}{3}-\frac{1}{2}$ a line thick.

The vascular processes of the tendinous sheaths and mucous bursæ, correspond with those of the articulations, only that they are for the most part of smaller size.

The synovial sacs of the muscular system are not mere meshes of connective tissue, like the subcutaneous mucous bursæ, since they have, invariably, an epithelial lining in certain places; they bear just as little resemblance, however, to the proper serous sacs (pleura, peritonoum, \&c.), because, with a few exceptions, their epithelium is never complete, and also because the cellular coat of the serous membrane is almost universally wanting entirely in some spots. The synovial sacs of the muscular system, on the other hand, and the synovial capsules, which also never possess a complete epithelium, and frequently communicate with mucous bursæ (quadriceps femoris, popliteus, subscapularis, \&c.), belong to one and the same category, and differ in some points from the serous sacs; with respect to which, however, it must not be forgotten that transitionary forms between these two kinds of sacs exist.

No one seems to have remarked upon the occurrence of cartilage cells in the various textures which go to the construction of the synovial sacs of the muscular system (except in the fibro-cartilages of the tendons); and the more so, because even Henle refers the fibro-cartilage of the tendinous sheaths to his interarticular cartilages (bandscheiben). It is quite true that the cartilage cells, while often occurring isolated in the connective tissue, or more frequently only in certain spots aggregated together, are not always readily seen; they may, however, be recognized in sufficiently thin sections, and very distinctly on the addition of acetic acid. The cell-membranes are not in this case utterly destroyed, any more than they are in the cartilage, cells of the interarticular ligaments, \&c., and no doubt can be entertained as to their being true cartilage cells, which, almost without exception, exist, not as a tissue, but rather dispersed in the connective tissue. Those spots in which they exist in great quantity may be described as fibro-cartilaginous places; but the distinction between these fibro-cartilages and those of true fibres not of the nature of cellular tissue (epiglottis, ossifying bones), must not be lost sight of. Genuine cartilage, as on the cuboid bone, I have never as jet met with in any other tendinous sheath; not even in the sulcus malleoli externi et interni; in the sulcus of the heel; nor in the sheath of the peronceus on the longus calcaneum; in which situations, cartilage cells are, indeed, everywhere to be seen, but only scattered in the connective tissue. 
With respect to the rows of cells which are met with in ligaments of the tendons, and in the tendinous sheaths, the nuclei of which, after the disappearance of the cell, continue to grow and arrange themselves together in the form of nuclear fibres, I cannot avoid remarking upon their close resemblance to the more simple cartilage cells of the tendinous sheaths and tendons, a resemblance so close, that I should almost be inclined to indicate it as marking their identity, if it did not sound altogether strange, to speak of a transition of the nuclei of cartilage cells into nuclear fibres. If not as identical, still they may be regarded as analogous formations; and the rather so, because in almost every case where cartilage cells occur in the connective tissue, rows of cells of this kind, and their relation to elastic fibres may be shown to exist, as well as in the interarticular cartilages, or ligamentous discs of Henle, as they are termed, afterwards to be described. On the other hand, it is true, similar rows of cells are to be found in the palmar fascia, tendons and ligaments, although those structures possess no indubitable cartilage-cells.

\$ 83. Vessels of the Muscles and their accessory Organs. A. Bloodvessels.-The ramifications of the larger vessels present little that is peculiar. The trunks reach the muscles in an oblique or transverse direction and then subdivide, running in the perimysium internum, in an arborescent manner, and at acute or obtuse angles, so that every part of the muscle is supplied by them. The minutest arteries and veins usually run parallel with the muscular fibres, between which they constitute a vascular plexus, so characteristic that, once seen, it can never be mistaken. The interstices of the plexus are rectangular, with the longer sides parallel to the longitudinal axis of the muscle, and it is of course composed of two sets of vessels, one longitudinal, which, as is shown most conclusively in transverse sections of injected muscle, lie in the fissures between two muscular fasciculi, or in the irregular spaces left between several of them, and the other transverse, which -anastomosing Fig. 101.

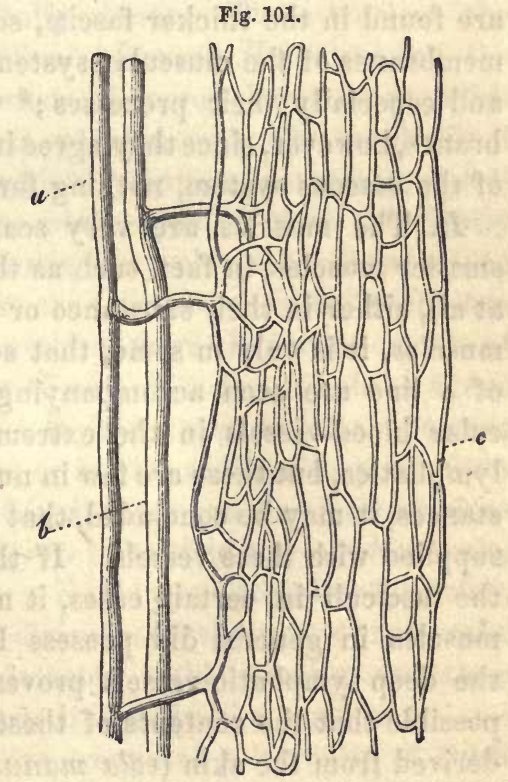
in various ways with the former, surround the muscular fibres. Thus

Fig. 101.-Capillary vessels in muscle, magnified 350 diameters: $a$, artery; $b$, vein; c, capillary plexus. 
each separate primitive fasciculus is lodged, to a certain extent, in a plexus of capillaries, and being surrounded on all sides by them, is very abundantly supplied with blood. The capillaries of muscle are among the most minute in the human body, their diameter being often less than that of the blood-corpuscles themselves. In one of Hyrtl's preparations, they measure $0.0025-0.003$ of a line; in the pectoralis major, when filled with blood, $0.002-0.003$, and when empty $0.0016-$ 0.0020 of a line.

The tendons may be reckoned amongst those parts of the body which are the most scantily supplied with blood-vessels. The smaller tendons, in the interior, present no trace of vessels, whilst externally, in the more lax connective tissue by which they are surrounded, there exists a widemeshed capillary plexus. In the larger tendons, a few vessels occur in the superficial layers, and in the largest, by means of the microscope and injection, a scanty vascular network may also be rendered evident in the deeper layers; but even in this case the innermost portions of the tendon are entirely without vessels. The tendon-ligaments present the same conditions as the tendons, only, that in them even still fewer vessels can be perceived. The thinner fascix, also, are altogether nonvascular; sparing ramifications, exclusive of those in the lax connective tissue, amply supplied with blood-vessels, which covers their surface, are found in the thicker fasciæ, such as the fascia lata. The synovial membranes of the muscular system, on the other hand, are very vascular, and especially their processes ;* with respect to these synovial membranes, however, since they agree in all respects with the synovial capsules of the osseous system, nothing further need be remarked in this place.

$B$. The muscles are very scantily supplied with lymphatics; the smaller muscles, in fact, such as the omohyoid, subcrural, \&c., have none at all, either in their substance or on the surface; and among the largest muscles, it is only in some, that solitary lymphatics, measuring $\frac{1}{4}$ and $\frac{1}{5}$ of a line are seen accompanying the blood-vessels. The deep or muscular blood-vessels in the extremities, it is true, are accompanied by lymphatics, but these are few in number; and from the latter two circumstances, it may be concluded, that even the larger muscles are but poorly supplied with these vessels. If they had not actually been observed in the fasciculi in certain cases, it might have been a question, whether muscles in general did possess lymphatics at all; the occurrence of the deep lymphatic vessels proves nothing towards this, it being quite possible that the contents of these vessels, scanty as they are, might be derived from the skin (vola manus, planta pedis, \&c.), from the joints, or perhaps from the bones. It may also be concluded, that if a few

* [The vessels generally form loops, which communicate with each other by means of delicate branches, and which can readily be traced to the extremity of each process. - DaC.] 
lymphatics really exist in the larger muscles, they do not run among the secondary fasciculi, but only in the more vascular perimysium between the larger and more lax subdivisions, and especially where the latter contains adipose tissue, and is consequently soft, as, for instance, in the glutceus, and in the superficial layers of the muscles.

Lymphatic vessels have never yet been noticed in tendons, fasciæ, and the synovial capsules of the muscular system. At the same time, it cannot be said, at all events in the latter instance, that lymphatics may not, as in other serous membranes, be contained in the sub-serous connective tissue.

$\S 84$. Nerves of Muscles.-The distribution of the muscular nerves, with respect even to their coarser relations, presents considerable peculiarity, it being evident, in most muscles, that the nerves come in contact with their fibres only at a few limited points, and are by no means connected with them throughout their entire length. With respect to the ultimate termination of the nerves, it may be stated that in all muscles there exist anastomoses of the smaller branches, forming the sotermed "plexuses." These anastomoses among the larger branches are seen chiefly, if not altogether, where the entire ramification of the nerves takes place within an extremely limited compass (vid. note); elsewhere they rarely occur, or are wholly absent. Those between the smaller and smallest branches (terminal plexuses, Valentin), on the other hand, are very numerous everywhere, forming elongated roundish meshes, which run for the most part parallel with the longitudinal direction of the fasciculi. These terminal plexuses, composed, sometimes of smaller, sometimes of larger, meshes, and formed principally by the ramules of one small branch, though not altogether isolated one from the other, proceed to form what are termed by Valentin the terminal loops; by which I understand nothing more than anastomoses of the ultimate twigs, effected by means of one. or a few primitive fibres passing from one twig into another. It is consequently unimportant whether they follow a straight course, or are curved in a looplike form (Fig. 102). Whether, besides these loops, there are also free terminations of the nerve-fibres, such as are known to exist in the lower animals, and as I believed I once noticed in a Rabbit, is altogether doubtful; whilst it is certain that, even in man, divisions of the nerve-fibres take place, although they are rare, and detected with difficulty, and their relation to the loops, it must be confessed, is still to be made out.

The trunks entering the muscles are composed principally of thick nerve-fibres, about twelve of the finer ones occurring, on an average, among 100 of the larger (Volkmann). They become smaller in the interior of the muscle, so that the terminal plexus consists only of extremely minute fibrils, measuring $000 \cdot 1-0.0025$ of a line in diameter. 
In some cases, even, the gradual attenuation of the fibres may be directly observed, proving that the diminution in size does not take place, in this case at least, in consequence of division. Thus, in the

Fig. 102.

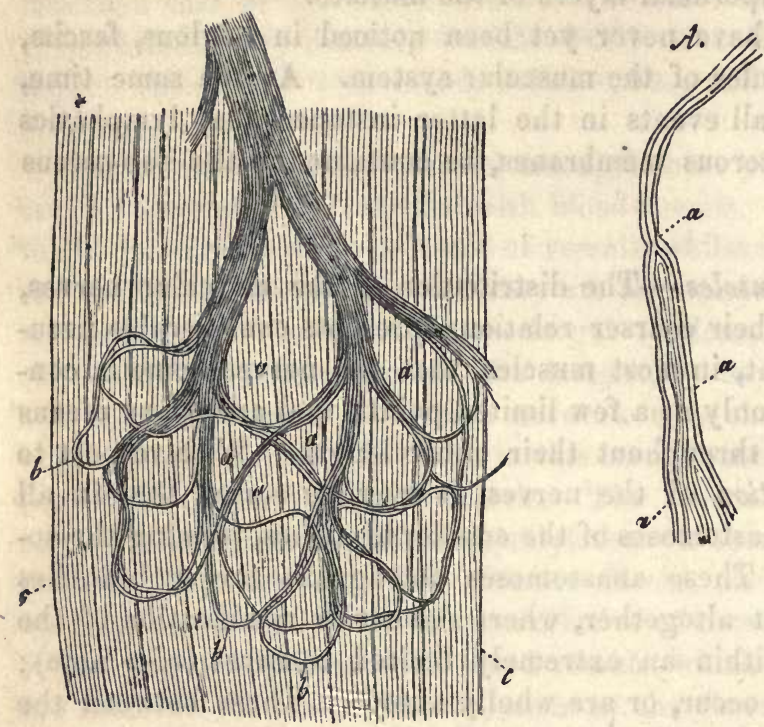

Fig. 103.

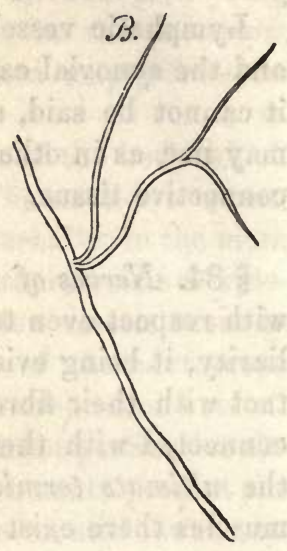

omohyoid, I have noticed several nerve-fibres of $0.004-0.0053$ of a line, derived from trunks measuring $0.05-0.07$ of a line, become attenuated (within a distance of $0.15-0.2$ of a line), to a diameter of $0.002-0.0026$ of a line, and, after a further course of $0: 4-0.5$ of a line, acquire that of the smallest fibrils, or 0.001 of a line. Simultaneously with this change in size, the nerve-fibres assumed in all respects the aspect of the so-termed sympathetic nerves, and ultimately became pale, with a simple contour line, and disposed to form varicosities; at the same time that they appeared to lose every vestige of a coat composed of connective tissue, they still retained dark borders, and consequently were not nonmedullated fibres (or free axis-cylinders), such as are seen in other terminations of nerves.

Nervi vasorum (vascular nerves), accompanying the bundles of vessels, occur in all muscles, and, according to the size of the latter, form larger or smaller branches. They are composed only of the smallest fibres, and always follow the course of the large vessels, which can still

FIG. 102.-Ultimate expansion of the nerves in the omohyoid muscle of Man, magnified 350 diameters, and treated with soda: $a$, interstices of the terminal plexus; $b$, terminal loops; $c$, muscular fibres.

F1G. 103.-Divisions of the primitive nerve-fibres in muscle, magnified 350 diameters: $\mathcal{A}$, double division from the omohyoid muscle in Man; $a$, neurilemma; $B$, division of a nerve from a facial muscle of the Rabbit into three apparently pointed twigs. 
be recognized as arteries and veins. I have not seen how they terminate; and this much only I know-that they are never met with on the capillaries, and very frequently, also, are not to be found on the smallest arteries and veins. Occasionally, one or more fibrils from the terminal plexus of the muscular nerves may be seen to join these vessels; a circumstance quite in accordance with the demonstrable fact that the vascular nerves in many parts, for instance in the extremities, are derived from the spinal nerves.

The smaller tendons contain no nerves, and the larger, such as the tendo Achillis and the tendon of the quadriceps femoris, only vascular nerves. The fascice and sheaths of tendons are also without nerves, as well as the synovial capsules of the muscular system, so far as I am at present aware.

In many of the small muscles, the extent of space included in the distribution of the nerve is extremely limited, as for instance in the superior belly of the omohyoid, in a portion of which, three inches long, the space over which the nerves are distributed, does not exceed from five to eight lines in length. The trunk of the nerve entering in the middle of the transverse axis, divides into two equal primary branches, one passing towards the left, and the other towards the right, border of the muscle, and each giving rise to numerous anastomosing branches of all sizes, and thus supplying the entire thickness of the muscle from the most superficial to the deepest layers. Whilst this distribution of the nerve takes place at one point, - a distribution not unlike that in an organ of sense, - the rest of the muscle presents the utmost poverty, or even a complete deficiency, of nerves. In one case, which I examined closely, I was unable, besides the few vascular nerves in these portions, to detect more than three small nervous twigs of $0.021,0.028,0.042$ of a line, which, though derived from the main nerves, differed from the other branches in their distribution. Two of them ran directly towards the lower, and one towards the upper, end of the belly of the muscle, giving off a few filaments composed of one or two primitive fibrils which passed through the muscle, and terminated, a little before reaching the intermediate and terminal tendons, in the most minute twigs and single nerve-fibrils. I found the same conditions of the nerves in the subcruralis, and in one of the costo-cervical muscles (arising from the first rib in the cervical fascia), as in the omohyoid; in the sternohyoid, sternothyroid, omohyoid (inferior belly), the same condition in some parts was noticed, whilst, in others, one apparently different existed, that is to say, the branches of the nerves frequently did not all divide at the same level, but were more widely spread. It was easily seen, however, that the above-described mode of division essentially obtained, also, in this case,-viz.: that the separate portions of the muscles are in 
connection with the nervous plexuses, only at a point of limited extent. The proof of the existence of similar conditions in other small muscles was more difficult, as in those of the orbit, where the nerves reach the muscles at acute angles, follow a longer course in them, with their primary branches, and form their ultimate ramifications at various, more or less widely separated points; yet even in this case it was tolerably well made out. It is easy to understand that, in the larger muscles, a microscopic examination, in toto, is impossible; but it can be shown in other ways, as by the preparation and examination of minute flat fasciculi taken in their entire length, that conditions exist, at all events in some of them, similar to those which appear to be evidenced in the small muscles. It is thus seen, especially in muscles of lax structure, that each fasciculus presents precisely the same conditions as an entire smaller muscle. How the distribution of the nerves is effected in muscles with long fasciculi (sartorius, latissimus dorsi, \&c.), I have not examined; it is probable that in this case each primitive fasciculus is joined by the nerves at several points, widely apart.

Valentin and Emmert, in the year 1836, simultaneously described the terminations of the primitive nerve-fibres in the muscles, to be in the form of loops, and the former maintained that the nerves of sensation terminated in a similar way. But Physiology having more recently shown that she does not well know what is to be done with these loops, and Microscopic Anatomy having distinctly demonstrated the existence, in many situations, of other modes of termination of the nerves (Pacinian bodies, \&c.), the loops have fallen into such discredit, that the question now is, not as before, whether, besides the loops, there are other modes of termination, but rather, whether loops really exist anywhere? With respect to the muscles especially, anatomists seem inclined to deny their existence altogether, since divisions and terminations of nerve-fibres have been discovered in them; but this conclusion, from what has been remarked above, would be incorrect. Henle also, in Canstatt's Jahresb. f. 1847, p. 63 , says, that in his opinion the loops had been too rashly discarded; while on the other hand, Wagner, with reference to this question, places the analogy with what is seen in the Frog, \&c., above direct observation, and denies the existence of loops. With respect to divisions of the nerves, Wagner ("Gött. Nach.," 1852, p. 27), finds them to be tolerably frequent in the muscles of the Mouse. I would, moreover, remark, that in one case, I think I noticed a minute ganglion with about five cells on a nervous twig in the omohyoid of man; the observation, however, was not satisfactory, the muscle having been previously treated with soda.

In the Invertebrata, many observers have long since described free terminations of the nerve fibrils, and their insertion with expanded ends into the muscular fibres, as Doyère in the Tardigrada, and Quatrefages 
in Eolidina, and some Rotifera ("Ann. d. Sc. N.," 1843, p. 300, and pl. 11, fig. 12). I myself, in a larva of Chironomus (a dipterous insect), noticed a single nerve-fibre, proceeding to the two muscular fasciculi of the simple tarsus, bifurcate into two branches, which were implanted upon the surface of the muscle, with somewhat expanded terminations. In the Vertebrata, Müller and Brücke first described division of the nerves in the orbital muscles of the Pike (J. Müller, "Physiol.," 4th ed. vol. 1, p. 524), and in Amphioxus, Quatrefages noticed conditions precisely like those met with in the Invertebrata above mentioned. The observation is easily confirmed, as respects the orbital muscles of the Pike, in which, upon the teasing out of the fasciculus either of the fresh muscle as well as after it had been treated with corrosive sublimate, and rendered transparent by acetic acid, numerous divisions of the nerves are apparent. They are nevertheless not nearly so frequent in this case, as in the Frog, nor are the divisions more than bifid or trifid. Besides this, I was especially struck with the glaring contrast that was presented, to what is seen in the Mammalia, in the enormous extent of space included in the distribution of the nerve-fibres; a distribution so extensive, that it is by no means easy to find a single primitive fasciculus which has not a nervefibre going to it; in many places even, the latter were seen in apposition with a fasciculus throughout a great extent, and surrounding it with loops, or with a variable number of spiral convolutions. A similar condition was observed by R. Wagner in the orbital muscles of the Torpedo, whilst in other muscles the nerves were very scantily supplied ("Gött. Nach.," Oct., 1851). In the Amphibia, divisions and free terminations of the nerves have been described by Wagner. The former are remarkably beautiful and numerous. They commence in nerve-fibres, measuring 0.004-0.006 of a line, in the smaller trunks and branches, and are several times repeated, with a gradual diminution of the fibres, until extremely minute filaments measuring $0.001-0.0015$ of a line are formed. The divisions are for the most part di- or tri-chotomous, more rarely multiple; in one instance, however, Wagner noticed eight ramusculi. The ultimate filaments are pale, and have a simple contour line. They never penetrate into the muscular fasciculus, but, after running a short distance, are either applied obliquely or transversely to it, or proceed for some distance in close contiguity and parallel with it; in either case, becoming attenuated to a sharp point, and frequently as fine as a fibril of connective tissue. All these conditions are best seen in the mylohyoideus (Wagner), and above all, in a delicate cutaneous muscle of the thorax, as was pointed out to me by Ecker, and in which the distribution of the nerves has recently been very accurately described by Reichert. $\mathrm{He}$ observed in this case, as I had done in man, that only a small portion of the muscle was well supplied with nerves, which were but sparingly distributed to the other portions. The trunk of the nerve supply- 
ing the 160-180 fasciculi of this muscle, contains, according to Reichert, 7-10 fibres, and ultimately, by continual division, forms 290-340

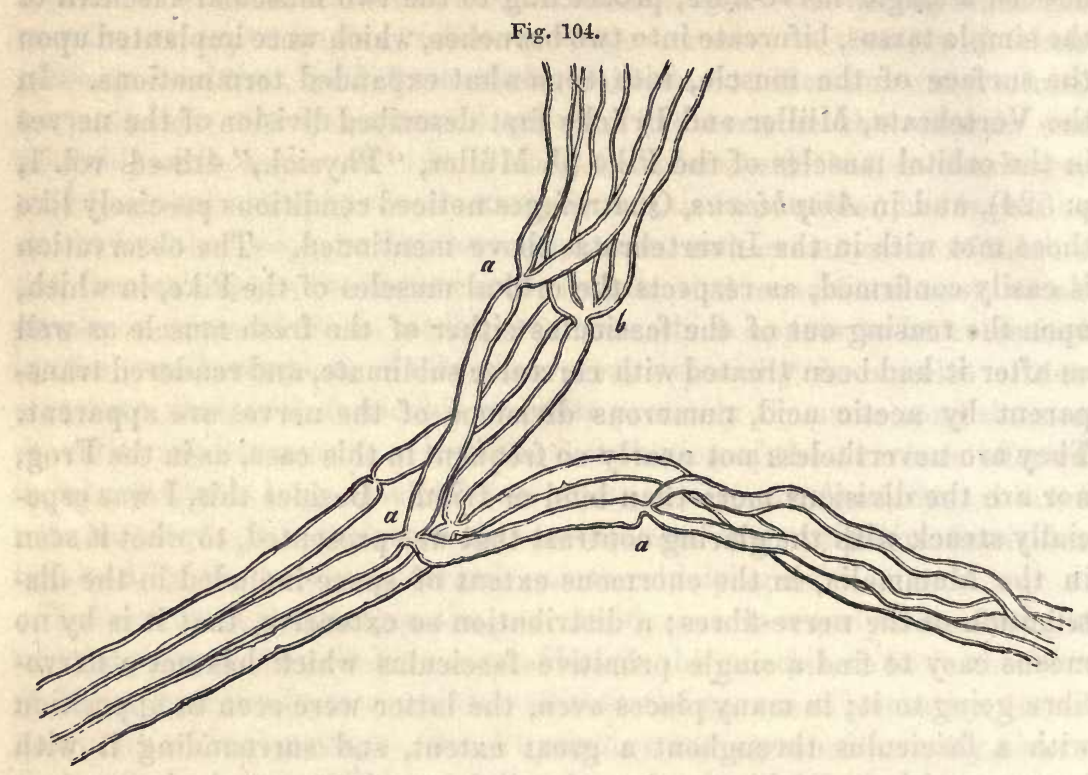

terminal filaments, so that there is more than one for each muscular fasciculus.

\$ 85. Chemical and Physical Relations of the Muscles.-In 100 parts of fresh beef there are contained, according to Bibra, 72.5674.45 parts of water. The solid constituents $(25 \cdot 55-27 \cdot 44)$ in a man 59 years old, were composed of a residue insoluble in boiling water, alcohol, and ether, 16.83; soluble albumen and coloring matter, 1.75; substance affording gelatine, 1.92 ; extractive matter and salts, 2.80 ; fat, $4 \cdot 24$. The fat is derived chiefly from the blood, the fat-cells in the muscles and their nerves, and in part perhaps from the muscular fibres themselves, in which microscopic fat granules are, at all events occasionally, evident. The gelatine is derived from the perimysium, in smaller proportion also from the vessels and neurilemma; none, on the contrary, is afforded by the sarcolemma, which is still apparent in muscles completely exhausted by boiling, whence (in opposition to Reichert) it is evident that the sarcolemma should not be referred to connective tissue. The inorganic salts and the albumen are principally afforded, probably by the muscular fibre itself, as are also and above all the salts described by Liebig and Scherer in the juice of muscles, of the lactic, acetic, butyric, and formic acids, the free lactic acid, the creatin and creatinin,

Fra. 104.-Divisions of nerve-fibres, in a small twig from the cutaneous thoracic muscle of the Frog; magnified 350 diameters: $a$, bifurcation; $b$, threefold division. 
the sugar of muscles or inosit, and the coloring matter, which substances, even the last-named, are lodged partly in the fibrils themselves, partly and chiefly, and this is the case especially with the albumen, in the interstitial substance, by which the fibrils are connected together. The 16.83 parts of insoluble residue belong in part to the elastic tissue in the vessels and perimysium, and to the smooth muscle in the vessels, but principally to the muscular fibrils themselves, which, as we have before seen ( $\$ 27)$, consist of a substance allied to fibrin. The sarcolemma is less affected by alkalies and acids than the fibrils, and approaches in its nature more nearly to the membrana propria of the glands, the walls of the capillaries, and the membrane of many cells. The coloring matter of the muscles (and the muscles themselves), like the blood, becomes bright red in the air, or still more in oxygen gas, and is rendered dark by sulphuretted hydrogen. It is extracted, and indeed readily, by water, but not by salts, in which circumstance, that is to say, in an alteration in the degree of concentration of the plasma with which the muscle is imbued, is perhaps to be sought the principal reason for the readiness with which the color of the muscles is altered in disease.

The muscles, although softer and more easily torn than the tendons, possess, nevertheless, considerable tenacity, particularly during life, and they have a certain degree of elasticity. During life, as has been correctly remarked by $\mathrm{E}$. Weber, even when not under the influence of the nerves, they are not for the most part in their natural form, but stretched, or in a state of tension, and like harp-strings in the same condition, exert an elastic force. This is satisfactorily shown when the tendons of the extensor muscles in an animal's limb which is strongly flexed, are cut through, the nerves having been previously divided, whereupon the tendons are very considerably retracted (E. Weber). This tension of the muscles varies very much, according to the position of the limbs. It is very slight when the body is at rest with the limbs semiflexed, still less or even wholly absent when a muscle falls into a state of repose after it has acted powerfully upon the limb; greater, and manifested in the greatest degree, when the antagonists of a muscle are acting with all their force. According to Weber, the living muscle, when in a state of inactivity, may be compared with caoutchouc, seeing that, like that substance, they possess a very great elastic extensibility; or, in other words, a slight but very perfect elasticity; as may be readily perceived in the muscles even of dead animals, which may be alternately stretched and allowed to retract. Owing to their elasticity, the muscles offer scarcely any hindrance to the movements of the limbs, and in consequence of its perfect nature, they recover their previous form and length even after the greatest possible extension. This is exemplified in the stretching of the abdominal muscles in pregnancy and in certain pathological conditions. When the muscles are in a state of activity, 
their elasticity alters in a very remarkable manner: 1 . During the contrāction they become more extensible or less elastic, on which account they exert a much less force by their contraction than would otherwise be the case, had their elasticity remained unchanged, and the same as in the inactive condition. 2. The elasticity of the active muscle, in one and the same muscle, is extremely variable; it continues to diminish as long as it is in action, whence arise the phenomena of fatigue and loss of power in the muscles (E. Weber).

In the dead muscle, according to the same observer, the elasticity is less perfect; that is to say, the dead muscle, when stretched, does not altogether resume its pristine form, and consequently is more readily torn, although such a muscle as the gracilis may still be capable of supporting a weight of eighty pounds without breaking. But at the same time it is also less extensible, more rigid, less flexible,-or its elasticity is greater. The phenomena of fatigue in the muscles are consequently to be distinguished from those induced by death. In the former state, the diminution of elasticity occurs during the influence of the nerves and the contractions of the muscle itself, probably in consequence of changed conditions in the molecular nutrition of the muscle, and is consequently a vital phenomenon; whilst in the latter case, innervation, nutrition, and contraction have ceased, and the increase of elasticity, which produces what is termed the rigor mortis, is a purely physical phenomenon, and not to be confounded with the increased tension, which, under the influence of life, takes place during the contraction of the muscles, simultaneously with a diminution of the elasticity.

The tendons are very firm, and but slightly elastic; and contain, according to Chevreuil, in 100 parts, only 62.03 of water, considerably less therefore than the muscles. They consist principally of a substance affording gelatin, although they are transformed with more difficulty than other parts into that principle.

In my opinion the muscles are sometimes in a state of tension, sometimes in their natural form, sometimes even compressed, and to all these three conditions vital contraction may be superadded. If a muscle in a state of extension contract, so as not to assume its natural form, it will still be in a state of tension after the remission of the contraction, and if divided will retract. On the other hand, if a muscle in its natural form contract, it will, after the cessation of the nervous influence, immediately become extended; as, for instance, the contracted heart, or an isolated muscle excited by galvanism. Consequently, when we speak of the elasticity of muscles, their tension, not only when they are extended, but also in the compressed condition, must be considered; and this appears to me of some physiological importance, as in this way the extension of contracted muscles (heart), and of muscles whose 
antagonists are paralyzed, becomes intelligible. With respect to the cadaveric rigidity, the important facts have quite recently come to light, that it may be arrested by the injection of blood (Brown-S équard); and also that it takes place even in the living animal, when the supply of blood to a group of muscles is entirely cut off (Stannius). In the latter case, the irritability of the nerves ceases at the same time, and on the restoration of the circulation the normal conditions in both muscles and nerves are also restored. By these facts, all hypotheses respecting the occurrence of the cadaveric rigidity, except that of Weber, are contradicted; even that of Brücke, which asserts that it is caused by the coagulation of the fibrin existing in the muscular fibre. But at the same time the question also arises, as to what is the proximate cause of the change in the elastic conditions of the muscles, whether it be due to the death or cessation of activity of the nerves, or to the deficient supply of blood to the muscles themselves? Stannius decides in favor of the former supposition, and is consequently driven to the conclusion, that during life the motor nerves act upon the muscles, by reducing, during the state of repose, their natural amount of elasticity, whilst in the contraction of the muscle the influence of the nerves is momentarily relaxed. Thus, according to Stannius, the rigidity connected with contraction, and vital contraction, would be identical, and nothing more than the condition of the muscle when freed from all nervous influence, and lasting until the nerve again puts the muscle into a state of rest, or its substance is decomposed. I must own that in this view, which moreover had already been proposed by Engel ("Zeitsch. der Wiener," Aerzte, 1849), I do not at present agree; and in particular would remark, that the circumstance of the contractions which occur during life being much more considerable than those which attend the rigor mortis, appears to be opposed to it.

§ 86. Development of the Muscles and Tendons.-The rudiments of the muscles consist, originally, of the same formative cells as those of which the rest of the body of the embryo is constituted; and it is not till afterwards that the muscles, tendons, \&c., are gradually developed by a histological differentiation. In man, the muscles are not evident before the end of the second month; at first, however, they cannot be detected by the unaided eye; they are soft, pale, gelatinous, and not to be distinguished from their tendons. In the tenth and twelfth week they are more distinct, especially in specimens preserved in alcohol; and at this time the tendons also may be distinguished as somewhat clearer, but at the same time transparent streaks.

In the fourth month, both the muscles and tendons are still more distinct, the former being, on the trunk, of a light reddish color, the latter less transparent, and grayish, both retaining a soft consistence. 
From this period, both textures acquire more and more of the configuration which they afterwards retain, so that at the maturity of the embryo,-excepting that the muscles are still softer and paler, and the tendons more vascular and less white,-they no longer present any difference worth notice.

With respect to their intimate conditions, the primitive fasciculi, in the embryo, at the end of the second month, present the aspect of elongated bands (Fig. 105) 0.001 of a line broad, with nodular enlargements at different points, at which places are situated elongated nuclei; the bands exhibit either a homogeneous or finely granular aspect, and but rarely an extremely faint indication of transverse striation. In their further development, these primitive muscular fasciculi, which, as comparative histology teaches, originate in cells arranged in a linear series, continue to increase in breadth and length, and their contents, the original cell-contents, are developed into the muscular fibrils. In the fourth month (Fig. 106) they measure for the most part $0.0028-0.005$, some even 0.006 of a line, whilst others do not exceed 0.0016 , and 0.002 of a line. The larger ones are, still, always flattened, but of uniform width, and also considerably thicker than before, mostly with evident longitudinal and transverse strix, and even with fibrils, which admit of being isolated. It is partially evident, even in a longitudinal

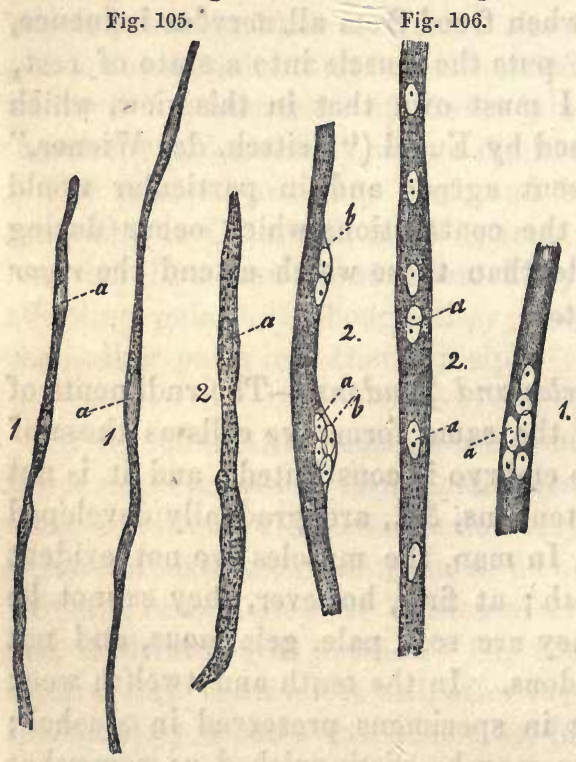
view, but still better in a transverse section, that in many cases, the fibrils do not occupy the entire thickness of the primitive tube, but that they are deposited around its periphery, the interior being as yet filled with a homogeneous substance as at first, and which now appears like a canal within the fibrils. All the primitive tubules possess a sarcolemma (b), which on the application of acetic acid or soda, appears as a very delicate membrane, which by the imbibition of water, may occasionally be raised from the fibrils. The tubes, moreover,

FrG. 105.-Primitive fasciculi of an eight to nine weeks' human embryo; magnified 350 diameters: 1 , two fibres without transverse striæ; 2 , fibres presenting the first indications of transverse striation; $a$, nuclei.

FrG. 106.-Primitive fibres of a four months' human embryo; magnified 350 diameters: 1 , a fasciculus, with a clear, as yet, non-fibrillated substance in the interior: 2 , fasciculus without such contents, with an indication of transverse striation; $a$, nuclei; $b$, sarcolemma. 
as at first, present nuclei lying close upon the sarcolemma, and which frequently cause rounded elevations on the surface of the tube, and may be observed actively engaged in the process of multiplication. They are all vesicular, roundish or elongated, with very distinct, simple or double nucleoli measuring $0.0004-0.0008$ of a line, and frequently with two secondary cells in the interior. They are much more numerous than previously, and most frequently disposed in pairs closely approximated; but often, also, in groups of three or four or even six, either contiguous or arranged serially. From this period to that of birth, no further important change takes place in the muscular fasciculi, except an increase in their size. In the new-born infant they measure 0.0056 -0.0063 of a line, are solid, rounded, polygonal, longitudinally or transversely striated, according to circumstances, as in the adult, with very readily isolated fibrils, and no longer any appearance of nuclei.

From what has been remarked, it is clear that the sarcolemma represents the sum of the membranes of the coalesced cells, and that the nuclei of the youngest fasciculi are the original cell-nuclei, whose descendants are represented in the nuclei of the older fibres, which have multiplied by an endogenous process. The muscular fibrils are the altered contents of the original tubes, become solid; they appear, demonstrably in many instances, to be formed on the inner surface of the sarcolemma, from without to within, but in other cases probably in the whole of the tube at once.

The growth of the entire muscle is chiefly to be referred to the increase, both longitudinal and in thickness, of the primitive fasciculi; and the rudiments of all the future primitive fasciculi appear to be formed, probably even as early as the original rudiments of the muscle itselfin every case at the middle period of foetal life. In the embryo, at the fourth or fifth month, they are perhaps five times as thick as in one at two months; in the new-born infant they measure for the most part twice, occasionally even three and four times as much as in the fourth and fifth month, and in the adult their size is perhaps five times greater than in the new-born child. The number of fibrils must necessarily increase in proportion to the size of the fasciculus, because, according to Harting, they are but little thicker in the adult than in the foetus. The perimysium is developed, as I find in agreement with Valentin and Schwann, after the type of the common connective tissue, from fusiform, coalesced formative cells.

The elementary parts of the tendons are, in no case, formed earlier than those of the muscles; for, in embryos from the eighth to the ninth week, I have never been able to detect a trace of them, although at this time the muscular fibres are quite distinct. It is not till the third or fourth month, when, moreover, they become distinctly visible to the naked eye, that their elementary constituents can be made out, at this time presenting the appearance of long parallel bands with elongated 
nuclei, which, as the observations of Schwann and myself ( $\$ 24)$ on very young animals show, are formed by the coalescence of fusiform cells. As early as the fourth month they may be distinctly recognized as primitive fasciculi, which are wavy, and present, at intervals, elongated nuclei $0.0035-0.006$ of a line long, and 0.0016 of a line broad, but are as yet without distinct fibrils, and not more than $0.0012-0.0016$ of a line wide.

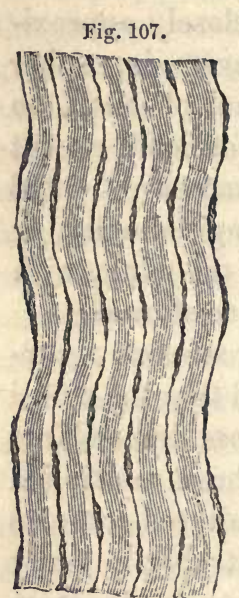
From this period up to the end of foetal life, the fasciculi gradually increase in width, so that in the newborn infant they measure $0.002-0.0025$ of a line; at the same time their fibrils are developed, as are also fine elastic filaments among the fasciculi, from special fusiform formative cells (vide $\S 23$ ). If these fasciculi be compared with those of the adult, measuring 0.006 -0.008 of a line, it is obvious, that the fasciculi of the tendons are continually acquiring an increase in thickness from their first origin, so that their proportional sizes in the four months' foetus, the new-born child, and the adult, are about as $1: 1,8: 6$; and, also, that in every case the growth of the tendons must in a great measure be referred to the increased thickness and elongation of their fasciculi. It would, moreover, appear, that subsequent to the primary rudiments of the tendons, new fasciculi continue to be added during foetal life.

Some controversial opinions are still entertained with respect to the development of the muscular fibres. Reichert and Holst maintain that each fibril is the product of a single cell, and regard it as the equivalent of the smooth muscular fibre, or contractile fibre-cell. This view is erroneous, as is readily shown by the examination of the mammalian and human embryo. Leydig's declaration, quite recently, in its favor (Beitr. p. 78), is explained by his having confounded the peculiar secondary muscular fasciculi in the plagiostomous fishes with the primitive fasciculi of the higher Vertebrata. In the Batrachia, according to Lebert and Remak, in the development of the muscles, elongated simple cells, with self-multiplying nuclei, are found, the contents of which cells undergo a metamorphosis similar to that occurring in the elongated muscular tubules formed of numerous cells, which, according to my observations, also exist in these animals. The contractile part of the muscular fibre, whether it be transversely striped or not, and whether it presents fibrils or not, is generally developed from without to within, in the sarcolemma, forming a sort of tube which does not become solid till afterwards; less frequently it appears as a more solid cord on one side

FIG. 107.-From the tendo Achillis of a new-born child, magnified 250 diameters, and treated with acetic acid, in order to show the formation of fine elastic fibres. 
within the muscular fibre. In the former case, the nuclei and the original contents of the formative cells, which often contain a large quantity of fatty matter, are situated in the interior of the embryonic muscular tubule, or between it and the sarcolemma; in the latter always close upon the sarcolemma.

With respect to the pathological relations of these tissues, the following remarks may be offered: The substance of the striated muscles is not regenerated, and wounds of muscles heal simply with a tendinous callus. A new formation of them has been noticed by Rokitansky ("Zeitsch. der Wiener," Aerzte, 1849, p. 331), in a case of tumor of the testis in an individual 18 years old, and by Virchow ("Verh. der Wuirzb.," Ges., I.) in an ovarian tumor. In the latter case, which carne under my own observation, there were elongated, fusiform, transversely striated cells, each with a nucleus, similar to those described by Remak in the Tadpole. The state of the elementary parts in hypertrophy of the muscles is uncertain.* This condition, however, except in the tongue, heart, and certain respiratory muscles (Bardeleben), does not perhaps occur at all; it is at all events extremely rare in the striped muscles. (Romberg, "Nervenkr.," p. 291, asserts, that such a condition ensues upon long-continued cramps, though it appears to me that this point is not yet sufficiently established.) Equally uncertain, also, is the intimate condition of the muscular elements in the increased development caused by exercise, and whether this depend upon the growth of the pre-existing muscular fasciculi, or on the introduction of new ones-the latter of which supposition may perhaps be affirmed without much chance of error, in the case of the extreme degrees of pathological hypertrophy. Atrophy of the muscles is very frequent, as in old age, paralysis, particularly of the tongue, and in cases of lead-poisoning, and in the development of cancer, fibrous tumors (consequent on inflammation), and of fat, \&c., in the substance of the muscles. The processes, however, which are set up in these cases, have as yet been but little investigated. In extreme old age I find the fasciculi small, presenting occasionally a diameter of not more than $0.004-0.008$ of a line, easily broken up, mostly without transverse stripes, and with the fibrils indistinct, whilst they frequently contain yellowish or brown granules, as much as 0.001 of a line in size, often in large quantity, and very many vesicular nuclei with nucleoli.

* [Wedl has observed longitudinal rows of yellow or brownish granules in hypertrophied as well as in atrophied muscle. These granules are not dissolved by acetic acid or alkalies; and seem deposited around the nuclei of the sarcolemma. In hypertrophied muscle, a peculiar gelatinous substance in the interstitial tissue is sometimes met with. This causes the primitive fasciculi to adhere to each other, and generally results in a complete softening and destruction of the sarcolemma, in the place of which we find a fine granular mass.

The interstitial substance is sometimes found in an hypertrophied state. If this occur to any extent it produces an atrophy of the muscular fibre.-DaC.] 
The nuclei often form continuous rows, or are accumulated on the inner surface of the sarcolemma, exhibiting in a peculiar manner the same distinct indications of an energetic multiplication by endogenous formation, as are presented in the embryo (vide this $\S$, supra). In fatty degeneration, the muscular fasciculi are, by degrees, replaced by connective tissue and fat cells which are developed between them; whilst, at the same time, minute fatty molecules are developed in great number within them, in place of the fibrils, which gradually disappear.

Paralyzed muscles were found by Reid "On the relation between Muscular Contractility and the Nervous System," "Edinburgh Monthly Journal of Med.," 1841) to be thinner, softer, and paler; and Valentin ("Phys.," 2 ed. 2 Th., p. 62) noticed in such cases that the transverse stripes were indistinct, or had disappeared, and could no longer be produced by water, alcohol, \&c.; the longitudinal stripes existed, but did not present their usual aspect, more resembling those of macerated muscle. Subsequently the altered fasciculi disappeared in part, and were to some extent replaced by fat. In a case of atrophy of the pectoralis major caused by cancer, I noticed conditions similar to those I had observed in old age, viz.: destruction of the fibrils, the development of brownish granules, and the presence of numerous nuclei, together with a clear fluid in the persistent sarcolemma; and lastly a diminution of the fasciculi, which did not measure more than 0.002 0.004 of a line in width. I also believe, that I noticed in many fasciculi the development of larger, serially disposed cells, with very large and distinct nuclei, exactly like the so-termed cancer-cells. The condition of the muscles in emaciation is unknown. In an emaciated Frog, which had fasted for eight months, Donders observed that the fasciculi were more slender, which he attributed chiefly to the removal of the interstitial substance between the fibrils. Paleness of the muscles is very common in dropsy, chlorosis, paralysis, lead-poisoning, old age, \&c. ; in which cases, probably the numerous brown or yellow granules are formed from a portion of the coloring matter. This condition is generally associated with softening, in which the fasciculi no longer exhibit any. distinct transverse strix or fibrils, and readily break up into numerous particles, or even into a pultaceous matter. In tetanus, in which rupture of a muscle frequently occurs, Bowman ("Phil. Transact.," 1841, p. 69) observed on the fasciculi numerous nodular enlargements, in which the transverse strix were very closely approximated, and between them either actual rupture of the fibrils, or at all events a considerable stretching and disorganization of them, both of which states are clearly to be referred to a powerful and irregular contraction. The muscles sometimes contain concretions, particularly as the result of the cretification of pus, tubercles, and cysticercus-vesicles; sometimes also true bones, such as are produced after prolonged exercise in the deltoid and other muscles (Exercirknochen). 
Of parasites are to be noticed the not unfrequent Cysticercus cellulosce and Trichina spiralis; and, besides these, in the Eel a nematoid worm, observed by Bowman ("Cyclop. of Anat." II. p. 512) alive, in the almost empty sarcolemma. I met with something analogous to the latter, some years ago, in the abdominal muscles of the Rat (as have V. Siebold and Miescher also in the Mouse); that is to say white streaks 4-7 lines long, and 0.09-0.01 of a line wide, which, on microscopic examination, proved to be hollow primitive fasciculi, entirely filled with elliptical, slightly curved corpuscles, $0.004-0.005$ of a line long, by 0.0019 of a line wide, and manifestly ova. The portions of the fasciculi thus transformed into pouches, had walls $0.009-0.01$ of a line thick, with transverse stripes, and were continuous at either end with the perfectly normal fibre.

§ 87. Physiological Remarks. - The most remarkable peculiarity of the muscles is their contractility. In each contraction, the primitive fasciculi shorten themselves in a rectilinear direction, and at the same time become thicker; they do not, however, undergo any considerable condensation. It is probable, that the contractions generally take place simultaneously in every part of a fasciculus, although at the same time it is not, of course, intended to be said that the contraction does not commence at the points where the terminations of the nerves occur, and that this contraction does not precede, though by a space of time immeasurably short, or at all events inappreciable by the eye, that of the other portions of the fasciculus. Under certain conditions, howerer, successive, progressive, and partial contractions are observed. If during the contraction of a muscle, its longitudinal and transverse striæ are noticed, it is difficult to show that where the former exist, they disappear during the contraction, and give place to transverse markings; and that the latter, where they were already present, become more distinct, and more closely approximated. Moreover, in the easily isolated fibrils of the thoracic muscles of insects, it is easy to perceive that they exhibit very variable conditions in different animals, and vary frequently in one and the same individual. Sometimes they are almost without transverse markings, and very pale; sometimes darker, and with distinct transverse lines; sometimes, again, very distinctly ringed; and together with these varying conditions, does the thickness of the fibrils and the distance between the transverse strix, vary also; so that the

Fig. 108.

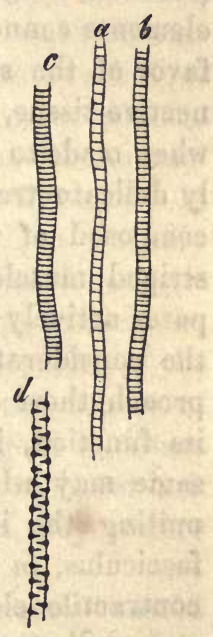

Fig. 108. Primitive fibres from the alar muscles of the "Dung-Fly:" $a$, slender fibril, with very distant delicate transverse striæ; $b$, thicker fibre, with closer, alternately stronger and fainter striæ; $c$, still thicker fibril, with the striæ more closely approximated; $d$, fibril with lateral, alternate elevations (they have come out too dark).-Magnified 350 diameters. 
fibrils which exhibit the most distinct striation, are almost as thick again as the others, and their transverse strix are placed almost twice as closely together. It may thence, perhaps, be allowable to conclude, that in the act of contraction the principal phenomenon consists in the shortening and thickening of the fibrils, and also, that the changes in the fasciculus above noticed depend upon these changes in the fibrils. The further question now arises: how is this shortening of the fibrils effected? and whence does the transverse striation arise? Is the latter connected with the vital conditions of the muscle, or is it produced independently of these? It is unnecessary to answer the latter query in the affirmative, for dead muscles exhibit transverse striæ, and indeed, under the same conditions as the living. This is best shown in muscles successively subjected to various degrees of tension; and consequently, all notion of a merely partial contraction of the fibril, which arises on the first observation of these conditions, must be relinquished. The transverse striation is manifestly merely a physical, not a vital phenomenon. It arises, either because the fibrils are not homogeneous throughout their whole length, but divided into numerous small segments, some of which are possessed of greater elasticity than the others; or, in the opposite case, it may depend upon the circumstance, that the fibrils are soft filaments, which, in shortening, become curved in a zigzag or wavy manner, or varicose. Which of these two views is the correct one, cannot at present be determined; and this much only can be said, that in favor of the former supposition, the fact can be adduced, that fibrils, after maceration, readily break up into minute particles (sarcous elements, Bowman), and possibly consist of a series of such elements connected by a heterogeneous interstitial substance; whilst in favor of the second, are the conditions presented in the fibrils of connective tissue, which are undoubtedly homogeneous throughout, and yet when made to contract by the application of acetic acid, exhibit extremely delicate transverse markings, in consequence of which, the fasciculi composed of them frequently offer a deceptive resemblance to those of striped muscle. It is difficult to say whether the sarcolemma participates actively in the shortening of the fibrils, although, especially from the consideration of its chemical and physical properties, which approach those of elastic tissue, I am rather inclined to the opinion, that its function, in the contraction of the fibre, is merely passive. The same may with greater certainty be affirmed of the albuminous fluid uniting the individual fibrils. Consequently, it is not the muscular fasciculus, in toto, but only the fibrils, which are to be regarded as the contractile elements; a position which is not shaken by the circumstance, that other conditions occur in the smooth muscles, and in many muscles in the Invertebrata (those that exhibit no fibrils).

This is not the place to dilate upon the causes to which the contrac- 
tions of the muscles are due, and by which they are necessarily produced, and I will merely offer the following remarks. There can be no doubt that the contractility of the muscular substance is a proper and inherent attribute, and only called into manifest action to a certain extent through the nerves; whilst it is equally certain, that there are no facts which conclusively demonstrate, that the striped muscles contract independently of a previous nervous influence. What the processes are which take place in the fibrils during the contraction is wholly doubtful; but it is to be hoped that the further investigation of the laws of the electric currents in the muscles, prosecuted in the way so successfully pursued by $\mathrm{Du}$ Bois Reymond ("Untersuchungen über thier. Electricität," Berlin, 1848-49), * will throw some light upon this, as yet, obscure subject. It would be more than bold to hazard an assertion with respect to the nature and mode of action of the nerves upon the muscles, since we are quite as much in the dark as to the processes which take place in the nerves, as we are with regard to those occurring in the muscles themselves. From the anatomical facts, which prove, that in many animals the motor nerve-fibres come in contact with each primitive muscular fasciculus only at a few points, and never penetrate into its interior, it is, however, rendered evident, that in the contraction of a muscle, the nervous influence must act from a certain distance.

The muscles also possess sensibility, though of a rather peculiar kind, because punctures, burns, and incisions into their substance, excite scarcely any sensations worth naming, whilst every muscle, after longcontinued activity, as well as when affected with cramps or spasms, becomes painful and very sensitive to pressure. They are also endowed with an extremely delicate sense of feeling for their own state of contraction, so that they are capable of estimating very minute variations in the force with which they act. The apparent contradiction between these facts is easily accounted for, by the consideration that the muscular nerves contain but very few sensitive fibres, as is readily shown in the nerves of the orbital muscles, \&c. These fibres, to which probably belong the few filaments above described, which are distributed over the whole muscle, though too scanty to render a muscle sensible to local impressions, nevertheless suffice, when implicated in the contraction of the entire muscular substance, to convey to the sensorium the degree of pressure to which they are subjected, and, when the organs are overexerted, to induce pain, in consequence of the frequently-repeated irritation which they have undergone, or of the compression they endure from the rigidity of the muscle.

The mechanical relations of the muscles have been excellently treated of in the article by E. Weber (l. c.), from which the following conclusions may be drawn. The extent of the shortening of the muscles amounts, 
in experiments upon animals, on the average to $\frac{3}{4}$ ths, or in powerful muscles even to $\frac{5}{6}$ ths. The contractile force of a muscle does not depend, coteris paribus, upon its length, but solely on its transverse sectional area; that is to say, on that of all its primitive fasciculi, so that a longer and a shorter muscle exert the same force, when the sum of the transverse sections of all the fasciculi is the same in both. According to the observations of Schwann and Weber, the elasticity of the muscles diminishes at each contraction, and consequently the molecular motions, called into play in them under the nervous influence, must be connected with a change in their substance of an altogether peculiar kind, which, however, can certainly only be regarded as a secondary effect. The degree of contraction differs according to the amount of antagonism with which it meets; if the latter be sufficiently powerful, no true movement of the limb takes place, that is to say, the points of origin and insertion of a flexor muscle (for instance) do not approximate; nevertheless, the fibres themselves contract to a certain extent, in consequence of which the whole muscle becomes tense. This tension must be carefully distinguished from that dependent upon the muscular elasticity, which is generally much less considerable. What has been termed the "tone"-tonus-of muscles, does not in most cases depend upon contraction, but is an elastic tension; I therefore hold, that the posture of the body and the occlusion of the transversely striated sphincters during sleep, has nothing to do with a contraction of the muscles, although such contraction is indubitably requisite to bring the body into this posture. In my opinion, during sleep, all the muscles (of course, with the exception of the respiratory) are at rest, being held in a state of tension, and of antagonism to their opponents merely by their elastic force, and are consequently in the condition of a muscle when supported, in a person in the waking state. As for instance, a biceps, when the arm is bent, may immediately lose its tension if the arm be supported, so in the same way may all other voluntary muscles; only it must not be forgotten, that such a condition of muscular rest may ensue upon all conceivable degrees of contraction. Even the orbicularis oris, when contracted, may be at rest and lose its vital tension. The mouth, nevertheless, will remain closed, for this reason, that although the elastic force, as always after a contraction, will not fail to exert a certain degree of extension upon it, it is unable to open the mouth, owing to its limited amount and inability to overcome the weight of the lips. I do not believe in any muscular "tone," if under that term be understood a long-continued involuntary contraction (though at first excited by the will); but am of opinion, that what has been most generally described under this name, is merely an elastic tension, which has been confounded with the contraction upon which it has ensued. From all we know, the nerves are incapable of exciting a long persistent contraction in the striped muscles, 
but very capable of producing great effects, when the states of contraction and of rest are duly alternated, as for instance in walking, running, \&c., and in the heart and respiratory muscles.

The importance of this view of the nature of the muscular "tone," as regards the physiology of the nerves, is sufficiently obvious; but in pathology also, it may be employed, in explanation of the retraction of divided muscles, and the shortening which takes place in muscles whose antagonists are paralyzed. The former, as correctly pointed out by $\mathrm{E}$. Weber, depends upon the elastic force, and takes place, as far as I know, only in extended, tense muscles, but not in those which are in a state of contraction, which, on the contrary, when cut across, immediately become lengthened, as may be readily observed in the Frog. It is quite true, that contractions also take place in divided muscles, in consequence of nervous influence; but these are never more than local, and cease without the production of any important effect on the form of the wound in the muscle.

The shortening which occurs in the antagonists of paralyzed muscles, is not referable either to the elastic force of the non-paralyzed muscles, which is much too slight to influence the position of a limb, or to their persistent "tone," but depends simply upon the voluntary innervation of the muscles, which are still in an active condition, and which, no longer meeting with any opposition from their antagonists, draw the limb in their own direction. The persistence of the oblique position which now ensues, may be readily explained without our necessarily assuming the existence of a permanent contraction, when it is considered that muscles, the antagonists of which are paralyzed, never again become elastically tense. In lead-palsy, for instance, when the first contraction of the flexors, consequent upon the paralysis of the extensors, ceases, the former, even under the most favorable circumstances, become extended only so far as to assume their natural form, a condition from which necessarily results the semiflexed position of the part affected. In accordance with this view, I regard the permanent condition of the unaffected side of the face, in one-sided paralysis of the facial nerve, and that of the upper eyelids in blepharoptosis, as produced, not by a persistent contraction, but as indicative of a state of perfect rest in the muscles, except when voluntary movements take place. The falling of the upper eyelid is explained by the paralysis of the levator, and the inability of the orbicularis, by its extension after a previous closure, to raise the eyelid beyond a certain point. In the same way the distortion of the face is produced, at first by the voluntary contraction consequent on the paralysis, upon the cessation of which it is impossible that the previous symmetry of the features should be restored, because the antagonist muscles on the opposite side are paralyzed, and their slight elastic force during life is insufficient, simply upon the cessation of the contrac- 
tion, to restore the pristine position of the lips, angle of the mouth, \&c. An actual distortion, therefore, dependent upon persistent muscular contraction, can only take place in consequence of morbid conditions of the central organs.

In the investigation of the muscles it is necessary that they should be studied in the fresh state, and with the aid of various reagents. The primitive fasciculi are most easily isolated in muscles which have been boiled or immersed in spirit, in which also, the transverse striæ are for the most part very well displayed, as is also the case after treatment with corrosive sublimate or chromic acid. In the study of the transverse strix, it is above all indispensable that the muscles should be viewed in various degrees of extension and contraction (Fig. 109). The former Fig. 109.

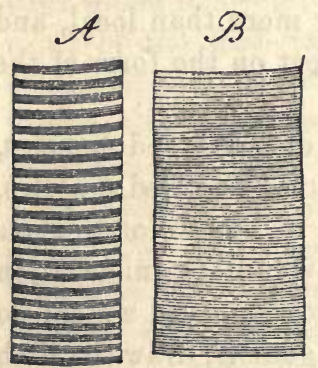
conditions, which are well worth observation, are readily viewed, if long slender muscles, such as the hyoglossi of the Frog, \&c., are examined on a wooden stage having a central opening filled in with glass. It will then be seen, when no extension whatever is employed, that the transverse strix are narrow (about 0.0004 of a line) and very closely approximated, and that the fasciculus itself is broad; whilst, when it is extended to the utmost, the stripes are 0.0008 of a line wide, and placed at the same distance apart, and that the fasciculus is narrower. The contractions must be observed either in fresh muscles still quivering, and kept moist with serum, albumen, or vitreous humor; or in the way proposed by $\mathrm{E}$. Weber,-and which consists in the galvanizing, by means of the rotation apparatus, of the muscle to be examined, such, for instance, as the abdominal muscles and slender muscles of the extremities in the Frog, the diaphragm and cutaneous muscles of the smaller Mammalia, \&c. For this purpose the muscle must be placed upon a piece of looking-glass, from a small space in the middle of which the metallic coating has been removed. One of the conducting wires is brought through an opening in the stage, or else affixed to it so as to be immovably in contact with one of the portions of finfoil. If the muscle now be viewed under a magnifying power of 100 linear, whilst the second conducting. wire is brought in contact with the other portion of tinfoil, the moment the circuit is completed, its fibres will be seen to contract in a rectilinear direction, and at the same time to become thicker, whilst the transverse striæ are more closely approximated (vide Fig. 109, which represents both a contracted and an extended muscle). The muscular fibres remain in this condition so long as the galvanic influence is kept

Frg. 109.-A primitive fasciculus of a Frog's muscle in different degrees of extension: $A$, the fasciculus, stretched and slender, with broad distant transverse striæ; $B$, the same not extended, broader, and with narrower, closely approximated striæ-Magnified 350 diameters. 
up, whilst when the circuit is broken they elongate themselves as rapidly as they contracted, and present zigzag flexures, when the muscle is lying free, but not when it is stretched by small weights attached to it by threads. From this it is evident, that if zigzag flexures take place during life, which is not yet known to occur, they can only arise when muscles in the quiescent condition are not in a state of tension; as, for instance, in the case of a flexor muscle, which has come into a state of rest after it has produced its full effect upon the limb. The sarcolemma is readily seen in the muscles of Amphibia and Fishes, especially in specimens preserved in spirit, in which it frequently, but for the most part in places, appears at a distance from the fibrils. In the higher animals and in man, it is occasionally seen when the fasciculi are teased out; and also in macerated and boiled muscles, and on the addition of acetic acid or alkalies. For this purpose I would especially recommend caustic soda, which in many cases renders the contents of the muscular tubules so fluid, that they escape in a continuous stream together with the nuclei, when the sheaths come very clearly into view. In no case, however, is the sarcolemma, in man, more beautifully exhibited than it is in softened, atrophied muscles which have undergone fatty or other degenerations; and, in fact, the greater the degree of degeneration, the more distinctly is this structure exhibited. The muscular fibrils, in fresh muscles, are constantly visible only in a transverse section, and in the thoracic muscles of insects, elsewhere it is true they are occasionally seen, but more by chance than otherwise. They are easily isolated artificially in preparations preserved in spirit, particularly in the perennibranchiate Reptiles (Siredon, Proteus, \&c.), by treatment with chromic acid (Hannover), by maceration for from 8 to 21 days, at a temperature of $1-8^{\circ} \mathrm{R}$. in water, to which, for the prevention of putrefaction, some corrosive sublimate has been added (Schwann); maceration also in the fluids of the mouth (Henle) allows of their being readily exhibited; whilst, according to Frerichs (Wagner, "Handwörterb.," III. I. p. 814), in the stomach, the fasciculi break up into Bowman's discs. The nuclei of the fasciculi are best studied under the application of acetic acid; by soda (vide supra) they may be isolated, and by potassa be made to swell considerably (Donders). On the subject of the effect of various reagents on the elementary tissues of muscle, the treatises of Donders (Holländ. "Beiträge") and Paulsen ("Observ. michrochem.," Dorpat, 1849) may be consulted. The vessels of muscle are studied in fresh, thin muscles, and in injected preparations; the nerves in the smallest human muscles, in the muscles of the smaller Mammalia, in the cutaneous muscle on the thorax of the Frog, with or without the addition of soda. The perimysium, and the form and position of the muscular fibres, are very well shown in transverse sections of half-dried muscles; and the same observation holds good with respect to the elementary tissues of the tendons. The 
insertions of the latter into the bones, and their cartilage-cells in those situations are readily seen; in the tendo Achillis, for instance, in vertical sections of dried preparations; with respect to their relation to the muscular fasciculi, vide supra, $\$ 81$. In order to examine the cartilagecells in tendons, thin horizontal sections are taken from the surface, which are treated with acetic acid, or a very dilute solution of soda. For the study of the development of muscle, the naked Amphibia must be placed in the first rank, and the Mammalia only in the second.

Literature.-Besides the memoirs, cited in $\$ 27$, there are to be mentioned: G.Valentin, article "Muscles," in the "Encyclopædic Dictionary of the Medical Sciences," vol. xxiv. pp. 203-220, Berlin, 1840; H. R. Ficinus, "De fibræ muscularis formâ et structurâ Diss. inaug.," Lips., 1836 , 4, cum tab. ; F. Will, some remarks upon the origin of the transverse stripes of muscles, in Müller's "Archiv," 1843, p. 358; R. Remak, on the "Development of the Primitive muscular Fasciculi," in Froriep's "N. Notiz.," 1845, Nr. 768 ; Ed. Weber, art. "Muscular Motion," in R. Wagner's "Manual of Physiology," vol. iii. 2d division, 1846; Kölliker, in "Ann. d. Sc. Nat.," 1846; Dobie, "Observations on the Minute Structure and Mode of Contraction of Voluntary muscular Fibre," in "Ann. Nat. Hist.," N. Ser. III. 1849; Lebert, "Recherches sur la Formation des Muscles dans les Animaux vertebrés, in Ann. d. Sc. N.," 1850, p. 205.

\section{OF THE OSSEOUS SYSTEM.}

$\S 88$. The Osseous System consists of a great number of hard organs, the Bones, of a peculiar, uniform structure, which are united either immediately or by means of other tissues, such as cartilage, ligaments, or articular capsules, into a connected whole-the skeleton.

The osseous tissue, in man, presents two principal forms-the compact and spongy. The perfect solidity of the former, however, is only apparent, as, even to the naked eye, it is seen to be penetrated by narrow channels which run in various directions, and by a still greater number of similar but smaller canals, which are brought into view by the microscope. These vascular or Haversian canals (medullary canals of authors), may be said to be almost entirely absent in the spongy substance, in which they are represented by wider, rounded, or elongated spaces, visible to the unassisted eye, which are filled with marrow, in some bones occupied by veins or nerves (cochlea), and termed the medullary spaces or cells (cancelli, celluloe medullares). These spaces all anastomose together, and are formed by the reticular arrangement of the small quantity of osseous tissue, which is disposed in the form of fibres, laminæ, and small rods. When the spaces are of a larger size, the substance is 
termed subst. cellularis, and when smaller, subst. reticularis. The latter, in some situations where the cavities are smaller, and the osseous partitions stronger, approaches in character the compact substance, although it does not actually become such; and in others it passes without any defined limit, into compact tissue. This does not, however, prove that the two substances are identical, but as we learn from observation of their development, depends simply upon the circumstance that the spongy substance very frequently arises in a partial expansion of the compact. The share taken by the two substances in the formation of the different bones, and parts of bones, varies very considerably. It is only in a few situations that the compact substance is met with by itself without vascular canals - as in the lamina papyracea of the ethmoid bone, some portions of the lachrymal and palate bones, \&c. It occurs more frequently, however, with vascular canals, and without spongy substance as in many individuals in the thinnest portion of the scapula, ilium, acetabulum, cranial bones (ala magna, parva of the sphenoid, the orbital process of the frontal bone, \&c.) Spongy substance with a thin compact cortex, without vascular canals, exists in the auditory bones, on the surfaces covered with cartilage of all bones, probably also in the smaller spongy bones. In all other cases, and consequently in most situations, the two substances are conjoined, but in such a way, that sometimes the spongy substance predominates (spongy bones and parts of bones), as in the vertebrce, carpal and tarsal bones; sometimes the compact, as in the diaphyses of the long bones; or the two are in equal proportions, as in the flat bones.

§ 89. Intimate Structure of the Osseous "Tissue.-The osseous tissue consists of a dense, for the most part indistinctly lamellar fundamental substance or matrix, penetrated by vascular canals and numerous minute microscopic spaces-the bone-cells, or lacunce (bone-corpuscles of authors), having very minute hollow processes, the bone-canaliculi.

The vascular canals of the bones, or the Haversian canals (canaliculi medullares), are minute tubules, having an average diameter of $0.01-$ 0.05 of a line, and in the extremes one varying from 0.004 to 0.18 of a line, and which, except in the thinner parts of the facial bones, as above mentioned, exist universally in the compact substance, forming in it a wide network similar to that of the capillaries. In the long bones, and also in the ribs, clavicle, pubis, ischium, and lower jaw, they run chiefly in a direction parallel to the long axis of the bone, and, as shown in longitudinal sections, either parallel to the surface or perpendicular to it, at distances varying from 0.06 to 0.14 of a line apart. They are connected by transverse or oblique branches, which run in the direction both of the radius and of the tangents of a transverse section of the bone. Consequently, under a low magnifying power, in longitudinal 
sections of one of those bones, either parallel to the surface or perpendicular to it, closely approximated canals running parallel to each

Fig. 110.

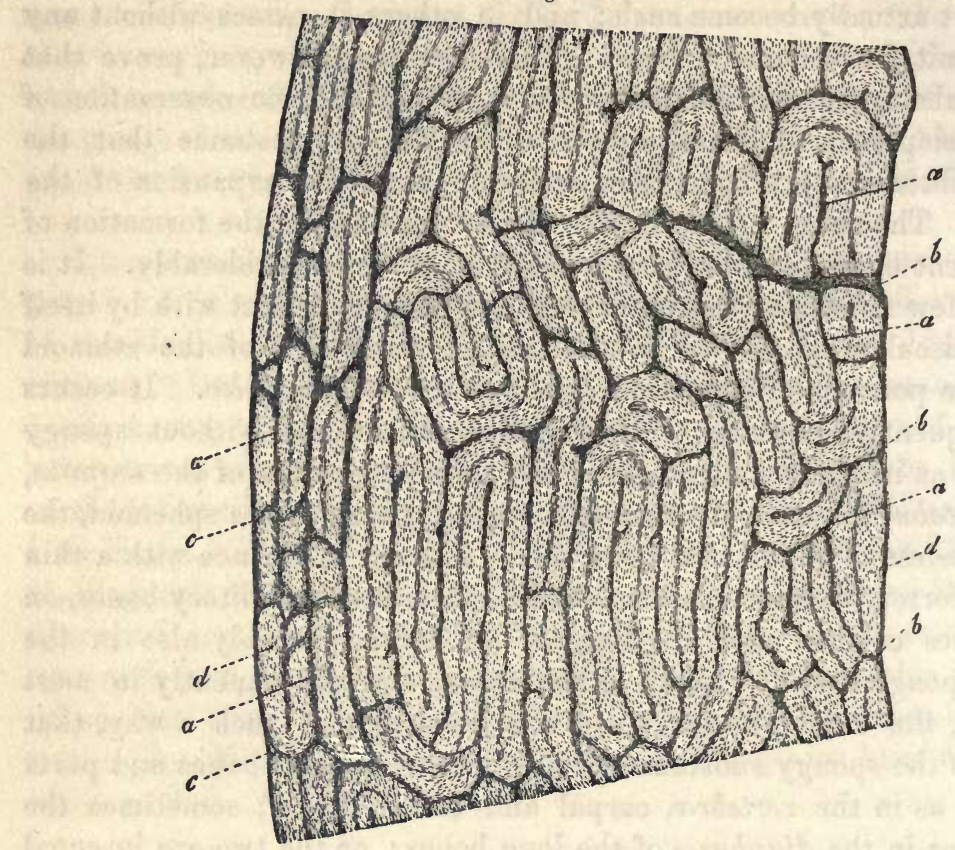

other, chiefly in a longitudinal direction, are seen, here and there with connecting branches, and thus forming a network, consisting of elongated, and most generally rectangular meshes (Fig. 111). And in a

Fig. 111.

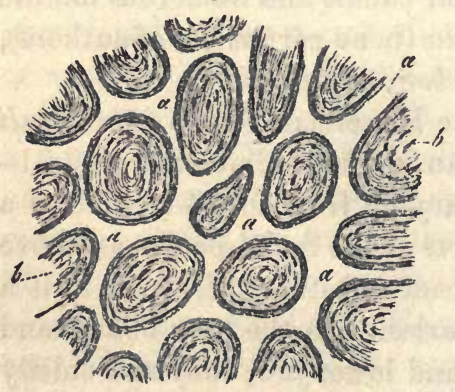

transverse section-transverse sections of the canals, placed at tolerably definite but small distances apart, are principally apparent (Fig. 112); which, more especially in younger bones, are occasionally connected by a tangential branch, and some anastomoses in the direction of the radius. In transverse sections of foetal and undeveloped bone (in man even at the age of eighteen), scarcely any transverse canals occur, but chiefly those running horizontally

Fra. 110.-Segment of a transverse section from the shaft of the femur of an individual 18 years old: $a$, Haversian canals; $b$, their openings internally; $c$, externally; $d$, osseous substance with lacunæ. In this figure transverse sections of vascular canals and fundamental lamellæ are not shown.-Magnified 350 diameters.

Fig. 111.-Haversian canals from the superficial lamellæ of the femur of an individual 18 years old, treated with hydrochloric acid: $a$, canals; $b$, osseous substance with lacunæ.Magnified 60 diameters. 
in the direction of the tangents and radius (Fig. 110), so that the bones appear to consist entirely of short thick lamellæ, each of which, upon closer examination, is seen to belong to two canals, and exhibits a pale central line, indicating the division between the two constituent portions of which it is formed.

In the flat bones, the greater number of the canals do not run in the direction of the thickness of the bone, but almost all, parallel with its surface, and indeed in lines which may be conceived as radiating from one point (tuber parietale, frontale, upper and anterior angle of the scapula, articular portion of the ilium) in a penicillar or stellate manner towards one or several sides; or less frequently, as in the sternum, are all parallel to each other. In the short bones, lastly, there is most usually one predominant direction in which the canals run, as the vertical in the vertebræ,--that of the long axis of the extremity in the carpal and tarsal bones, \&c.; it must be remarked, however, that the larger processes of these bones, as, for instance, the spinous processes of the vertebræ, differ in this respect from the rest of the bone, and, like those of other bones, such as the coracoid and styloid processes, exhibit the same disposition of the canals as that which exists in one of the shorter cylindrical bones. The lamellæ, fibres, and bars of the spongy substance, occasionally present a few vascular canals, but only when they are of some thickness.

As the Haversian canals are vascular channels, they open in certain situations: 1 , externally, on the outer surface of the bone; and, 2, internally, on the walls of the medullary cavities and spaces. In both situations, excessively fine and coarser pores may be everywhere perceived, partly visible to the naked eye, and which are more numerous in proportion to the thickness of the cortex of the bone. But the relation of the vascular canals in the compact substance to these canals thus proceeding from within and without, only partially resembles that between the branches and trunks of vessels, and only in the outermost and innermost lamellæ of the cortical substance. In the interior of the cortical portion of a bone the canals are independent, and morphologically may be most aptly compared to a capillary network, which at its borders is in connection at many points with larger canals. Where the cortical substance rests upon the spongy substance, as in the interior of the ends of the diaphyses, and in the lateral periphery of the apophyses, the vascular canals are continuous, sometimes abruptly, sometimes quite gradually, expanding in an infundibuliform manner, and frequently anastomosing, with smaller or larger medullary spaces, so that, very often, no definite limit is perceptible between them. I have never yet noticed crcal terminations of the vascular canals; it is, however, certain that in many situations on the surface they must constitute, over extensive spaces, closed networks, especially where very few or no vessels 
enter the compact substance, as at the points of insertion of many tendons and ligaments, and beneath several muscles (temporal).*

\section{$\S 90$. The matrix of bone is lamellar, and the lamellæ (Fig. 112) are} apparent in thin sections, but are still better shown in bones from which the earthy matter has been removed, or which have been exposed to the weather or calcined, in which cases the lamellæ exfoliate, and, in the cartilage of decalcified bones, may even be raised with the forceps. In the middle portions of the cylindrical bones they constitute two systems: -one general, in which the lamellæ are parallel with the external and internal surfaces of the bone, and numerous special ones, around the separate Haversian canals. These two systems are in some places in immediate connection, but, in most, merely in apposition, and on that account they may conveniently be regarded as of two kinds; a view with respect to them which is in some degree supported by the phenomena presented in their development.

The lamelloe of the Haversian canals (Fig. 112c,113b) surround those canals concentrically, in greater or less number. They constitute, as it were, the walls of the canal, and are intimately united to each

* [A most valuable contribution to our knowledge of the structure and development of Bone has lately been made by Messrs. Tomes and De Morgan, in their "Observations on the Structure of Bone," read before the Royal Society in June, 1852, but not yet published. We are enabled, however, by the kindness of those gentlemen in allowing us to inspect many of their preparations, and in furnishing us with the proofs of their paper, to make some very important additions and corrections to the text. We may add, that although we do not always agree with Messrs. Tomes and De Morgan in the interpretation of the facts, differences which we shall duly note, our own investigations have led us to believe that their paper is by far the most accurate account of the process'of ossification which has yet appeared.

These writers have pointed out the important fact, that, besides the well-known Haversian canals, other cavities exist in bone, which they denominate Haversian spaces. These have irregular outlines similar to that of the surface of exfoliations, while the boundaries of the Haversian canals are always more smooth and rounded. Again, in the latter, the laminæ are more or less conformable with the canal; while the walls of the spaces are formed by the unconformable edges and surfaces of the laminæ of the adjacent Haversian canals, which have, as it were, been eaten away to form the space. In fact, bone, so far from being a permanent or stationary structure, is continually being deposited, and as con. stantly re-absorbed. The Haversian spaces are the result of the absorption of previouslyexisting osseous tissue; but when this process has gone on to a certain extent, deposition commences in the spaces, and they are converted into Haversian canals. The calibre of these canals now becomes narrowed up to a certain point by the continual laminar deposition of ossific matter, which, after a while, is traversed by new absorptive tunnels, or Haversian spaces, and is removed in its turn.

The spaces are very numerous and large in newly-formed bone situated near ossifying cartilage; while, in older bone, they are far less frequent and generally smaller. They are, however, never absent; being found even in old subjects. They may be observed in various conditions in a series of sections. In one place the space will have attained a large size, while, in another part of the same section, its commencement will be seen extending from one side of an Haversian canal. One side of a space may be becoming the seat of a new system, while the opposite is undergoing further enlargement.-TRs.] 
other, much in the same way that the laminæe of the walls of the larger vessels are continuous with each other. The number of lamellæe belong-

Fig. 112.

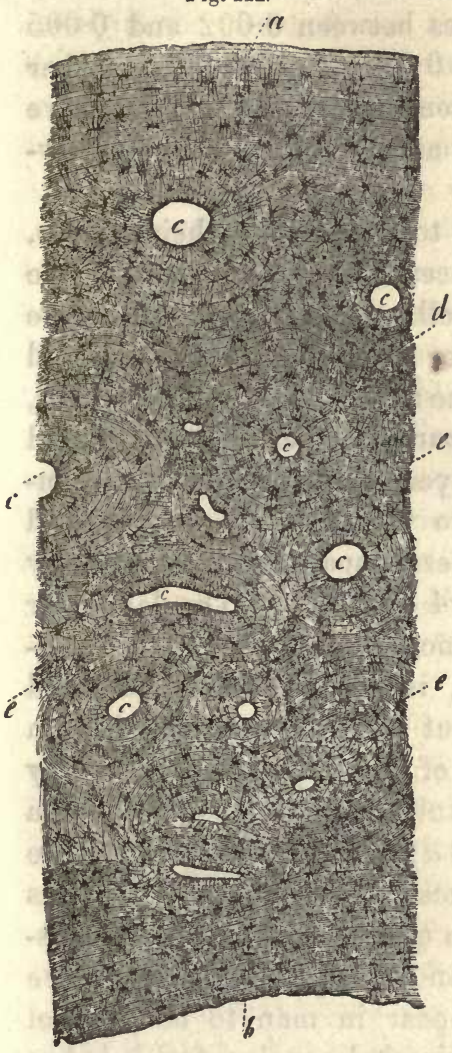

Fig. 113.

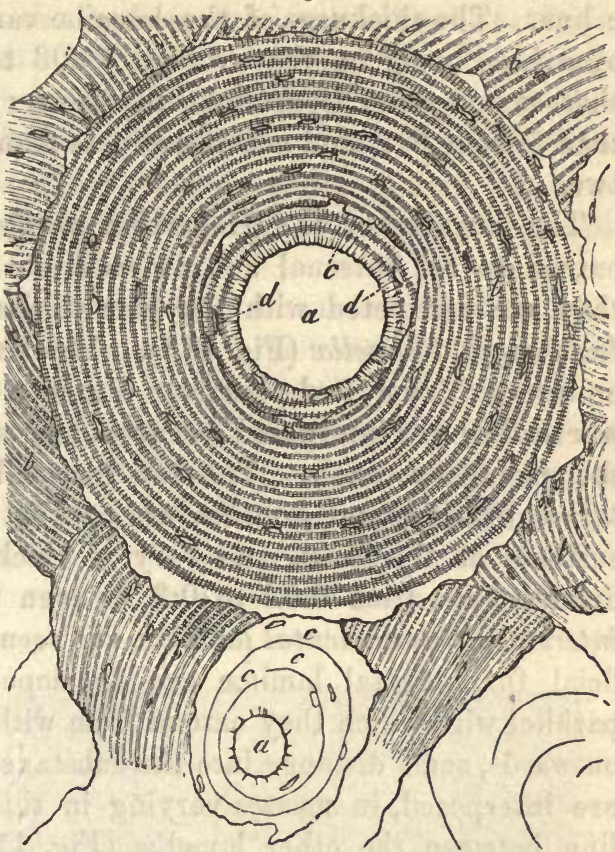

ing to a canal, and the collective thickness of the system formed by them varies not inconsiderably, and bears no constant relation to the size of the canal, as is the case to some extent in the vessels; small canals, therefore, are not unfrequently surrounded by numerous lamellæ, and larger ones by but few.* In general, it may be said that the largest

FiG. 112.-Segment of a transverse section of a human metacarpal bone, treated with oil of turpentine: $a$, external surface of the bone, with the exterior fundamental lamellæ; $b$, internal surface towards the medullary cavity, with the inner lamellæ; $c$, Haversian canals in transverse section with their lamellar systems; $d$, interstitial lamellæ; $e$, lacunæ and processes.-Magnified 90 diameters.

Fig. 113.-Portion of a transverse section of the shaft of the humerus, magnified 350 diameters, treated with oil of turpentine: $a$, Haversian canals; $b$, their lamellar systems, each lamella presenting a more transparent and more opaque portion, with radiating strix in the latter ; $c$, darker lines, which probably indicate greater intermissions in the deposition of the osseous substance; $d$, lacunæ without visible rays. From a preparation by Dr. H. Müller.

* [The "interstitial laminæ" are the remains of Haversian systems, the larger parts of which have been removed by absorption to form new spaces. The irregular outline of the 
canals have thin walls, those of a middle size thick ones, and the most minute, again, walls of little thickness. The thinnest walls $I$ have commonly noticed measure $0.008-0.02$, and the thickest, $0.08-0.1$ of a line. The thickness of the lamellæ varies between 0.002 and 0.005 of a line, being on the average 0.003 to 0.004 of a line; in number there are usually from eight to fifteen; sometimes, however, no more than four or five, and occasionally as many as from eighteen to twentytwo.

The lamellæ of the Haversian canals, together with their canals, extend to the internal and external surfaces of the diaphyses, where they are connected with the general lamellæ above mentioned,-the fundamental lamelloe (Fig. 111). The latter constitute an external and an internal layer, and penetrate also into the substance of the diaphysis, where they are interposed between the separate lamellar systems and the medullary canals. The two former layers, or the external and internal fundamental lamelloe, are parallel to the external and internal surfaces of the bone, and vary in thickness apparently without any definite rule, from 0.02 to 0.3 , or even 0.4 of a line. The latter, or interstitial fundamental lamello, are seen most clearly where the superficial fundamental laminæ are developed, in partial connection and parallel with which they extend from without inwards, and from within outwards, some distance into the substance of the diaphyses, where they are interposed, in masses varying in thickness from 0.02 to 0.12 of a line, between the other lamellæ (Fig. $112 d$ ). In the interior of the compact substance, on the other hand, in man, the Haversian systems are so closely crowded that there can be no question as to the non-existence of lamellar groups between them, and it is evident that those lamellæ, which in a transverse section appear in man to be parallel with the surface, almost all belong to horizontal canals; and it is but rarely that distinct interstitial masses are seen, as is usually the case in other mammalia. The thickness of the separate lamellæ just described is much the same as that of the lamellæ of the Haversian canals, and their number varies from 10 to 100 .

We have hitherto considered only the diaphyses of the long bones. In their apophyses, the thin cortical layer of compact substance naturally presents only a few systems of Haversian canals, which, however, are constituted as elsewhere. The exterior fundamental lamellix are few in number, and internally, owing to the existence there of the spongy substance, they are wholly wanting. In the latter substance, the very few Haversian canals present lamellar systems as usual, except that they are thin, and the remainder, according to the condition of the osseous network, consists of a lamellated and fibrous tissue, which in

outermost of the lamince of an Haversian, canal (see Fig. 113) results from its being the first deposition within the pre-formed irregular Haversian space. (Tomes and De Morgan, l. c., p. 5.)-Trs.] 
general follows the contour of the medullary spaces and cells. The flat and short bones present a similar arrangement internally, whilst the cortical substance of these bones differs from that of the cylindrical, only in the circumstance, that the fundamental lamellæ, in the flat bones, form layers parallel with both surfaces of the bone. The thickness of the fundamental lamellæ in the cranial bones (parietal), is sometimes the same in both aspects, and varies from 0.08 to 0.16 of a line, sometimes they are wanting in vascular situations, and in places, wholly so, on the external aspect of the bone, in which case the Haversian lamellæ reach almost to the surface.

With respect to the intimate structure of the osseous lamellæ, which is best studied in transverse sections, dried, polished, and sufficiently thin, there is usually evident, besides the bone-cells and canaliculi, in the generally not very distinct lamellæ, an extremely fine though very distinct punctuated appearance, so that the whole osseous tissue appears granular, and to be composed as it were of separate, densely crowded, pale granules, measuring 0.0002 of a line (Fig. 114). If water or weak syrup, or albumen, be applied to a slice of bone, it assumes a condition probably similar to that which it possesses during life. The lamellæ, for the most part (both in transverse and perpendicular sections), become clearly visible, and their granular aspect is quite distinct, although not so defined as before the bone was

Fig. 114.

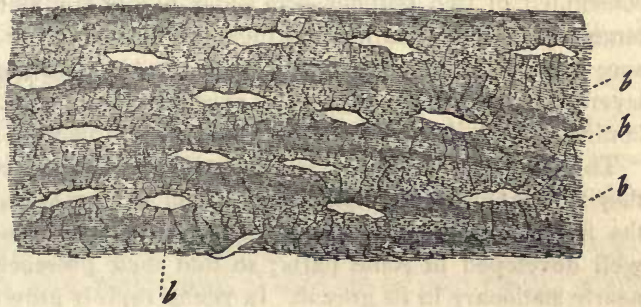
thus treated. For in the first place, together with the granules, there is exhibited a close, pale striation, referable to the canaliculi, which are filled with fluid and which, extending in various directions through the tissue, renders its delineation more complex; there are also apparent in each lamella, as it were, two layers, one pale and more homogeneous, the other darker and granular, which latter chiefly is striated. When this condition is clearly displayed, an extremely delicate marking is produced, resembling that seen in transverse sections of certain urinary calculi (Fig. 113). When once seen in moistened sections, indications of this arrangement will occasionally be observed in dried preparations. In bone treated with hydrochloric acid, the granules and strix (dependent on the canaliculi), in sections both transverse and perpendicular to the

Fia. 114. Portion of a perpendicular section of a parietal bone, magnified 300 diameters: $a$, lacunæ, with pale, only partially-visible prolongations, filled with fluid as in the natural state: $b$, granular matrix. The striated places indicate the boundaries of the lamellæ. 
surface, are less distinctly apparent, whilst the lamellar structure is very manifest, and most generally two layers may be noticed in each lamella, though by no means so clearly as shown in Fig. 113.* In sections parallel to the surface, the bone, in many situations, appears almost homogeneous throughout, presenting no trace of a granular structure, whilst in others a structure of that kind is obscurely visible, together with minute points (Deutsch), and besides these a longitudinal striation; which last gives the whole a fibrous aspect. From this circumstance, many authors appear to have been led to describe the bone as composed of fibres, but quite incorrectly, for although the study of their development shows, that the ossifying parts are, to a certain extent, very distinctly fibrous, it is impossible to demonstrate anything of the sort in perfect bone. On the other hand, there is no doubt that a coarsely fibrous appearance exists, and especially in the bone-cartilage of the compact substance, as has already been remarked by others, and which is probably due to the fibrous fasciculi of the original blastema; care however should be taken not to look upon longitudinal sections of lamellæ as such fibres. $t$ When bone is burnt and the fragments crushed,

* [According to Tomes and De Morgan, the laminæ, when well developed, are always constituted of two portions, - an outer, highly granular, often composed of a single line of large granules, and an inner, which is singularly clear and transparent, and to all appearance without granulation or any recognizable structure. This distinct separation into two layers, however, does not always exist; and in a complete Haversian canal, the innermost lamina of all is frequently clear, glassy, and structureless.

The circumferential laminæ are not so constantly present as is generally supposed, and they rarely entirely surround the shaft of a long bone, still more rarely the flat bones. In the fast-growing bones of young animals they are absent, while in adults they are usually well developed in some parts; so that their presence seems to indicate that the bone is nearly stationary in its growth. In young, rapidly growing bone, the circumferential laminæe are replaced by a series which may be called the undulating lamince. The surface of the bone sends off processes, formed of reduplicated laminæ, which eventually arch over and enclose those vessels of the periosteum which lie nearest them. The spaces thus formed become the seat of Haversian systems. Young growing bone, therefore, may be distinguished from that of adult animals, by its being composed of Haversian systems with intervening undulating laminæ. (Tomes and De Morgan, b. c., pp. 4-6.)-Trs.]

$\dagger[$ Messrs. Tomes and De Morgan (1. c., pp. 13, 14) adduce very good reasons for believing that the fibrous appearance which may often be detected in the laminæ of bone arises from imperfect illumination and definition, and express their belief that bone substance "is composed of granules or granular cells, imbedded in a more or less clear, homogeneous or subgranular matrix." They go on to say, "Thus as regards the basement, homogeneous tissue, it will be found that where lamination is highly developed, the laminæ have a transparent and structureless, and a more opaque and granular part, to which the former appears to be the matrix. The peripheral lamina of the Haversian systems is generally clear and free from granularity, and the internal lamina sometimes presents a similar structureless appearance. The matter which fills up the Haversian systems in the full-grown antlers of the Cervidæ affords another and a very striking example of transparent structureless osseous tissue, which in this instance is the more distinct, from the absence of canaliculi in its sub. stance. Then, again, we have another instance in the clear tissue which is sometimes found between the superficial Haversian systems of ordinary bone. It has already been described as a non-laminated element found on the surface of certain bones. In the instances already cited, and no doubt in many others which may be found in the skeletons of the lower ver- 
it affords, according to Tomes, minute angular granules, from $\frac{1}{5}$ th to $\frac{1}{6}$ th the diameter of the human blood corpuscle, and measuring, according to Todd and Bowman, $\frac{1}{6000}$ th- $-1 \frac{1}{1400}$ th of an inch, and which are also rendered evident when bone is boiled in a Papins' digester. From these particulars, and from the granular aspect of fresh bone, which has also been noticed by Tomes and by Todd and Bowman, and moreover from the pretty nearly equal size of the granules visible in it, with those described by Tomes, and lastly, from the circumstance that bone treated with hydrochloric acid, as well as when calcined, both present a perfectly homogeneous substance without vacuities, it may be assumed that the osseous tissue consists of an intimate mixture of inorganic and organic compounds, in the form of closely connected minute granules.

\section{§ 91. Bone Cavities or Cells, and Canaliculi (lacunce et canaliculi} ossium).-In dried sections of bone, there are visible, scattered throughout the entire osseous substance, in all the lamellæ, microscopic melonseed-shaped corpuscles, with numerous, fine, ramified, and partially anastomosing rays, whose opaque and white color (as viewed by direct light) is due, not to the deposition of calcareous salts, as was formerly supposed, and on which account they were termed "bone," or "calcareous corpuscles," but simply to their being filled with air. In fresh bone, not yet deprived of its watery constituents, nothing can be seen in these bone-cells or lacunæ but clear contents with a nucleus, which may best be described as the nutritive fluid of the bone, and consequently the designation above given to these cavities is the most suitable.

The lacunce are elliptical, flattened cavities, having an average length of 0.01 of a line, 0.004 of a line wide, and 0.003 of a line thick, which give off both from the borders, and particularly from the surfaces, a great number of very fine canals, measuring $0.0005-0.0008$ of a line in diameter-the bone canaliculi above-mentioned (Figs. 115, 116, and 117) The lacunæ are equally numerous in both of the lamellar systems before.

tebrata, we have bone tissue without obvions granularity, and without obvious structure; and although it forms but a small part of the general mass, yet from its constant presence at all ages and in all subjects, it must be regarded as an integral and normal part of mammarial bone. The granular condition of bone tissue is tolerably obvious in all preparations, though it is much more marked in some specimens than in others. The amount of the component granules varies in different parts of the same specimen, and in specimens taken from different parts of the skeleton. Thus, in one situation, we may see laminæ with a highly transparent part gradually merging into a transparent tissue, while in another the laminæ may be granular throughout. Again, in young bone developed in cartilage, the part between the cells becomes highly granular, fragments of which may be found in certain adult bones, as in the petrous portion of the temporal bone. Bone near the articular surface frequently presents a well-marked granularity."

We may remark, in addition to this very just account of the minute structure of bone, that of the lower vertebrata above referred to, the Skate offers one of the best examples of structureless bone, in those polygonal plates which are developed (not on the surface, as is commonly said, but) in the interior of the cartilaginous skeleton.-TRs.] 
described, and are placed so close together, that, according to Harting (1. c., p. 78 ), from 709 to 1120 , or, on the average, 910 of them occur within the space of a square millimeter. They lie for the most part within the lamellæ, but also between them, and are invariably placed with their broad sides parallel with the surfaces of the lamellæ. The canaliculi proceeding from them are much branched, and penetrate the osseous substance in all directions, their course being irregular, and often actually curved. They proceed principally, however, in the first place, from both surfaces of the lacunæe straight through the lamellæ; and secondly, parallel with the Haversian canals, from the two poles of the lacunæ. It is only in certain limited spots that these canaliculi present cacal termina-

Fig. 115.

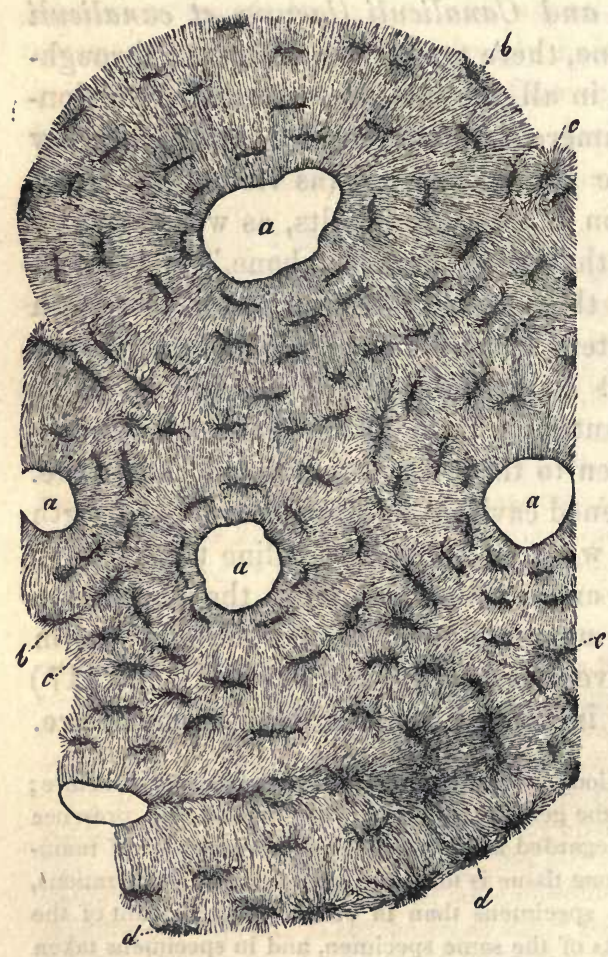

Fig. 116.

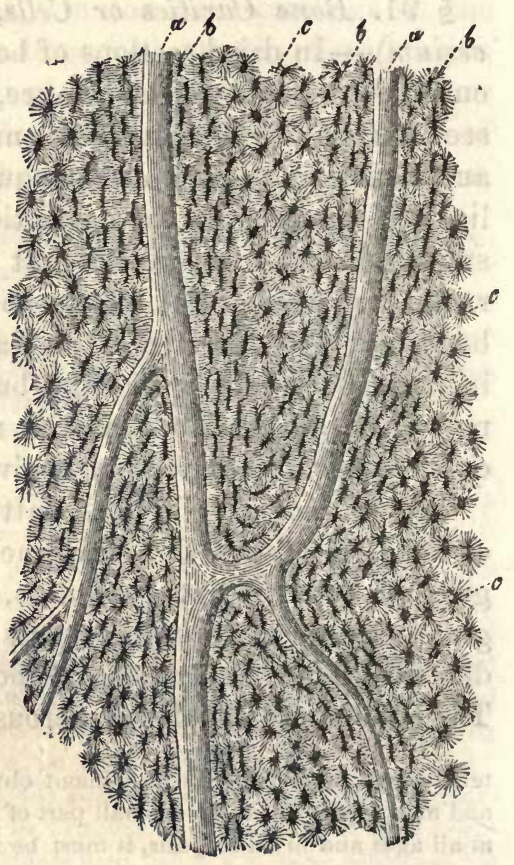

tions; everywhere else some of them anastomose in the most various ways with the canaliculi of the neighboring lacunæ, whilst others

FIG. 115.-From a transverse section of the shaft of the humerus; magnified 300 diam.: $a$, Haversian canals; $b$, lacunæ with their canals, in the Haversian lamellæ; $c$, lacunæ of the interstitial lamellæ; $d$, lacunæ with unilateral canaliculi proceeding to the surface of the Haversian system.

FIG. 116. - Section parallel with the surface from the shaft of a human femur; magnified 100 diam.: $a$, vascular canals : $b$, lacunæ seen from the side, belonging to the lamellæ of these canals; c, lacunæ viewed on the flat side, in lamellæ which are cut horizontally. 
communicate with the vascular canals, the medullary cavities, and the medullary spaces or cancelli of the spongy substance, or open on the surface of the bone. The entire osseous substance, therefore, is penetrated by a connected system of cavities and canaliculi, by means of which the nutritive juice secreted by the vessels is conveyed into its densest tissue.

The lacunæ and canaliculi do not exhibit precisely the same conditions in every part of the bones. In the lamellar systems of the Ha- . versian canals, as seen in a transverse section, the elongated lacunæ, by reason of their curvature, lie as it were concentric to the canal, and their excessively numerous pores or canaliculi necessarily produce a very close striation radiating from the vascular canal (Fig. 115). The lacunæ are sometimes extremely numerous, sometimes more scanty; in the former case they are, for the most part, arranged in tolerably regular alternation, or one behind the other in the direction of the radius of the lamellar system; but they are also frequently disposed very irregularly, either crowded together (vide the lower part of Fig. 115), or separated by wider interspaces. In horizontal and longitudinal sections of Haversian canals (Fig. 116), when the section has passed through the middle of a canal, the lacunæ appear narrow and elongated, and disposed in rows one behind the other, and in numerous layers parallel with the canal; and also furnished with numerous canaliculi, which proceed for the most part

Fig. 117.

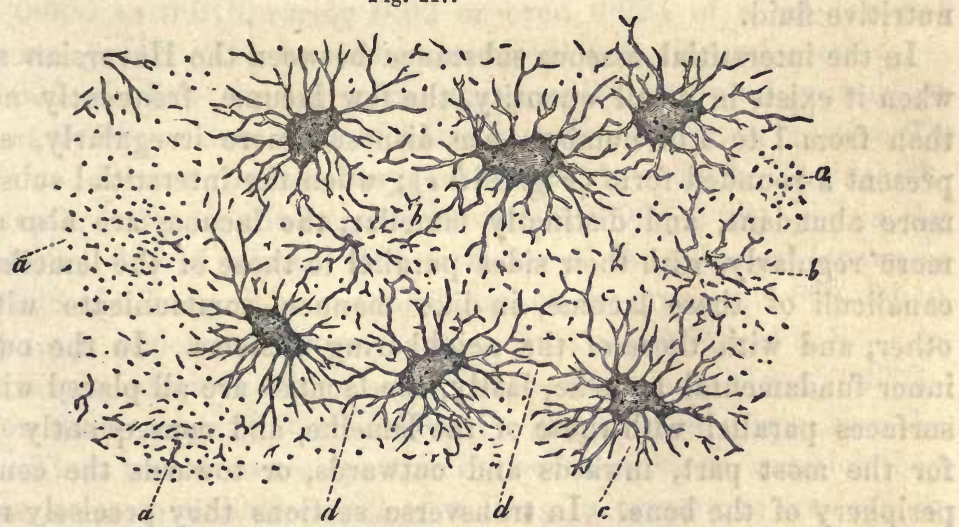

directly inwards and outwards (consequently transversely through the lamellæ), but partly in a direction parallel with the long axis of the

Frg. 117.-Lacunæ viewed on the flat side, with the canaliculi, from the parietal bone; magnified 450 diam.: the spots on the lacunæ or between them belong to eanaliculi, which are cut across, or are the openings of canaliculi into the lacunæ; $a$ a a, groups of transverse sections of canaliculi, each group belonging to a lacuna which has been destroyed in the making of the section. 
canal. If the section strike the surface of a system, the superficial lacunæ come into view, presenting very elegant forms, rounded or oval (Figs. $115 d$, and 117), surrounded in an irregular manner by a complete tuft of canaliculi, which look directly towards the observer, and consequently appear more or less shortened, and by a smaller number of other canaliculi distributed on the surface of the lamellæ. Occasionally, even in the thinnest parts of a section, there occurs a tuft of canaliculi, cut across transversely, and without the lacuna to which they belong, whence these portions of bone exhibit a sievelike aspect. All the canaliculi arising from the inner aspect of the innermost lacunæ of an Haversian system, proceed towards the canal, with which they, by this means, communicate, as may be clearly seen in thin, perpendicular, and transverse sections of bones filled with air, and in the walls of medullary canals laid open longitudinally. From the borders and external aspect of the same lacunæ other canaliculi are given off, which perhaps occasionally terminate in blind extremities, but for the most part communicate with those of the neighboring, and particularly of the outer lacunæ. The succeeding rows of lacunæ are all mutually connected in a similar way, and thus the network of canaliculi and lacunæ extends to the outermost lamellæ of the system, where the lacunæ either commuincate with those of the contiguous systems or of the interstitial lamellæ, or terminate independently, in which latter case (Fig. $115 d$ ) all the canaliculi, or at least most, and the longest of them, proceed inwards, that is to say, towards the vascular canal, from which they derive their nutritive fluid.

In the interstitial osseous substance between the Haversian systems, when it exists in small quantity, the few lacunæ, frequently not more than from 1 to 3 in number, are disposed more irregularly, and also present a rounded form (Fig. $115 \mathrm{e}$ ); when the interstitial substance is more abundant, and distinctly lamellar, the lacunæ are also disposed more regularly, with their sides parallel to those of the lamellæ. The canaliculi of these lacunæ, in like manner, communicate with each other, and with those of the neighboring systems. In the outer and inner fundamental lamellæ, lastly, the lacunæ are all placed with their surfaces parallel with those of the lamellæ, and consequently looking, for the most part, inwards and outwards, or towards the centre and periphery of the bone. In transverse sections they precisely resemble those of the Haversian systems, only that they are but little or not at all curved, except in the smallest cylindrical bones. In longitudinal sections, whether perpendicular or parallel to the surface, they present the conditions above described, with this limitation, however, that a larger number of lacunæ, of course, are seen in the same space in the latter case than in the former, and also that the sievelike aspect described above is more frequently observed, giving the bone considerable resem- 
blance to certain sections of teeth (Fig. 117). The canaliculi of these lamellæ communicate, in part as usual with each other, in part open on the external and internal surfaces of the bone (Fig. 118). At the points of insertion of tendons and ligaments into the bones, the canaliculi of the outermost lacunæ probably terminate in blind extremities; a condition which obtains in every case, in those parts of bones which are covered with cartilage (articular ends, ribs, surfaces of the bodies of the vertebræ, \&c.) In the rods, fibres, and plates of the spongy substance, the lacunæ are disFig. 118.

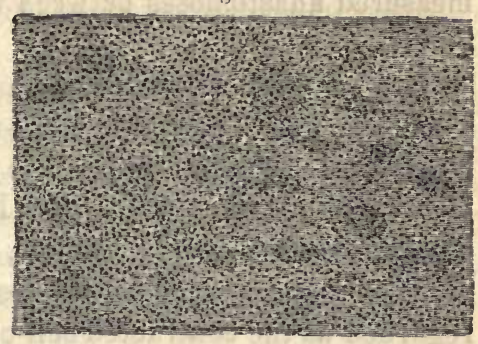
posed in every possible direction, but for the most part, with their long axis parallel to that of the fibres, bars, \&c., and with their flat surfaces directed towards the cancelli. They anastomose also, in these situations, by means of their canaliculi; and the most superficial lacunæ open freely into the cancelli.

The size and shape of the lacunæ, in man, upon the whole, vary but little. By far the greater number are melon-seed-shaped or lenticular, some, more fusiform or spherical. In sections of bone well filled with air, in which alone I have made my measurements, I find their average length to be $0.01-0.014$ of a line, frequently under and above that size, or from 0.006 to 0.016 , rarely 0.02 or even 0.024 of a line (cranial bones, lower jaw). The breadth, measured in horizontal sections, is $0.003-0.006$ of a line; in transverse, it is usually somewhat greater, or as much as 0.008 , or even 0.01 of a line, because the limits between the canaliculi and lacunæ cannot always be accurately defined. Their thickness or depth, lastly, in the smallest lacuna, is $0.003-0.004$, and in the larger $0.002-0.004$ of a line. The diameter of the spherical lacunæ is $0.006-0.008$ of a line. The canaliculi are, on the average, $0.008-0.016$ of a line long, seldom less or more, up to 0.02 and 0.024 of a line; in diameter they measure 0.0004 of a line; at the finest extremities, 0.0005 0.0008 , on the average; $0.0008-0.001$ of a line, at their origin from the lacunæ. Their true distance apart, in horizontal sections, in which they appear as holes, is $0.0008-0.002$ of a line; in transverse sections, in which they produce the radiating strix, in consequence of their being viewed in several planes, they appear to be somewhat closer together, or at distances varying from $0.0008-0.0012$ of a line. The circumference

FIG. 118.-Portion of the surface of the tibia of the calf, viewed on the external aspect, magnified 350 diam.: the numerous points are the openings of the canaliculi; the dark, larger, indistinct spots indicate the lacunæ to which these canaliculi belong, appearing from a greater depth. 
of a lacunæ, together with the radiating canaliculi belonging to it, forms an imperfect sphere, having a diameter of from 0.02 to 0.034 of a line; with reference to which, however, it must not be forgotten, that individual canaliculi transgress the usual length of the others, as I have, in fact, measured anastomoses between two lacunæ of the length of $0.04-0.045$ of a line.

The contents of the lacunoe, according to the later investigations of Donders, Virchow, and myself, appear very closely to resemble those of the cells of cartilage during life; that is to say, they are clear, probably viscid fluid, with a nucleus. If bone-cartilage be boiled in water or caustic soda for 1 or 2 minutes, these nuclei often show themselves very distinctly; or opaque corpuscles make their appearance, which must be regarded as the contracted cell-contents including the nucleus, and analogous to the corpuscles in cartilage. A peculiar phenomenon is seen to occur, when bone is macerated in hydrochloric acid, which was first noticed by Virchow in a diseased, and afterwards in healthy bone, and

Fig. 119.

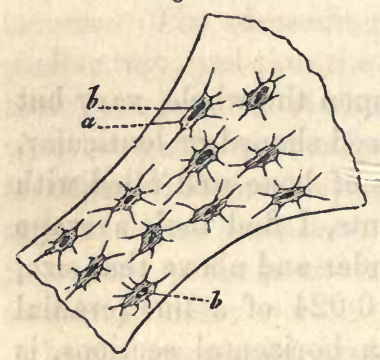
by myself in the cementum of the horse's tooth, - the lacunæ become isolated, having longer or shorter processes, and appear like independent structures, or a sort of stellate cells. This phenomenon seems to depend simply upon the circumstance, that the tissue immediately surrounding the lacunze offers more resistance to the action of the acid than it does elsewhere. In the cementum of the horse's tooth, cells also enclosing the lacunæ, and even Haversian canals, may be isolated,-the best proof, that everything which thus presents itself in an isolated form, is not necessarily a morphological unity.*

FIG. 119.-A bone spicule from an apophysis, with distinct lacunæ and nuclei. Boiled in water, and magnified 350 diameters.

* [It is of very great importance in histology to keep in mind the caution expressed in the last paragraph of the text (see below, note $\$ 101$ ), which applies as well to optical as to chemical distinctness.

Tomes and De Morgan assert that both the lacunce and canaliculi have parietes, which are manifested by appearances similar to those observed in the dentinal tubes. They sometimes found the lacunce and canaliculi filled up to a great extent with solid matter, so as to leave only a small space in the centre.

An important modification of the lacunce is described and figured by these authors (1. c., p. 8 ) in the circumferential laminx. Elongated tubes pass, in bundles or singly, more or less obliquely from the surface towards the interior of the bone. When long, they are sometimes bent once or twice at a sharp angle. They have parietes, and are connected laterally with the canaliculi. They occur irregularly in the circumferential laminæ, and in these only. - [Sinilar tubes exist in the cementum of the Teeth.]

We can confirm Messrs. Tomes and De Morgan's statement that the nuclei may be found without difficulty in recent bone, and they may al ways be brought out with great distinctness by the action of dilute hydrochloric or strong acetic acid. This is especially the case in 
§ 92. The Periosteum.-Among the soft tissues appertaining to bone, the periosteum is one of the most important. It is a more or less transparent, slightly glistening or whitish yellow, vascular, extensible membrane, investing a great part of the surface of bones, and contributing most importantly to their nutrition, by the numerous vessels which it sends into their substance.

The periosteum is not, everywhere, constituted alike. Opaque, thick, and for the most part with the glistening aspect of tendinous structures where it is covered only by the skin, or is connected with fibrous parts, such as ligaments, tendons, fascice, and the dura mater cerebri, it is, on the other hand, thin and transparent in situations where muscular fibres arise directly from it without the intervention of tendon, and also on the -diaphyses, where the muscles nearly rest upon the bone, as on the external surface of the cranium (pericranium), in the vertebral canal, and in the orbit (periorbita). Where mucous membrane rests upon bone, the periosteum is, in most cases, very intimately united to it by the submucous connective tissue, so that the two cannot be separated, and constitute a single membrane, which, as in the palate, alveolar processes, nares, \&c., is of greater, or, as in the maxillary sinus, tympanum, ethmoid cells, \&c., of less thickness.

The connection of the periosteum with the bone itself is either more lax, consisting in simple apposition, and by more delicate vessels which penetrate the bone, or more intimate, taking place by means of larger vessels and nerves, and by numerous tendinous filaments. The former mode of connection is found especially where the periosteum is thin, and the osseous substance more compact, as in the diaphyses, on the inner and outer surfaces, and in the sinuses of the cranium; the latter, where the periosteum is thicker, and the compact substance thinner, as, for instance, in the apophyses, in the short bones, palate, and at the basis of the cranium.

With respect to the intimate structure of the periosteum, it will be

young bone. In old bone we have frequently been unable to discover them. Tomes and De Morgan, however, state that the nuclei are visible in sections of a fossil bone (supposed of a Pterodactyle) in their possession.

Another peculiar condition of the "lacunal cells," described by these authors, is their ossification. They found the light and spongy bones of old people to yield, if broken, a white powder, which was composed of large cells detached or united into masses. They are spherical, and contain a dark granular nucleus, which is surrounded by a thick transparent wall. Similar cells may be found adherent to the walls of the Haversian canals and cancelli; and in this case their nuclei have assumed the form of lacunc, and the canaliculi of adjacent lacunce advance into them. Similar cells may be found in most preparations of adult bone (1. c., p. 12).

We must confess that we doubt the assumption of a lacunal form by the "nucleus" in these cases. We have repeatedly examined these bodies, but if the nucleus was visible at all, we found it unchanged, and often adhering to one side of the lacunc. Again, it is questionable whether they may not rather be compared to the globules of dentine than to cells. -Trs.] 
found to present, almost universally, excepting where muscles arise directly from it, two layers, which, although closely connected, differ, more or less distinctly, in their structure. The outer layer is composed chiefly of connective tissue, with occasional fat-cells, and is the principal seat of the true periosteal vessels and nerves, whilst in the inner layer, elastic fibres, commonly of the finer sort, constitute continuous, and often, very thick networks-true elastic membranes-superimposed one upon another, the connective tissue forming the less important element. Nerves and vessels occur in this layer also, but they do little more than merely pass through it, being destined for the bone itself.

The parts of the surface of bones unprovided with periosteum are: 1. The articular extremities covered with cartilage, and all other places where the bone is covered with cartilage or fibro-cartilage. 2. Where ligaments and tendons are attached to the borders and surfaces of bones at a certain angle, as, for instance, at the insertions of the ligamenta flava, intervertebralia, iliosacra, interossea, teres ossis femoris, patellæ, \&c., of the tendons of the deltoid, coracobrachialis, popliteus, iliopsoas, triceps, surce, quadriceps femoris, glutoei, \&c. In all these situations, the tendons, ligaments, and cartilages, are attached directly to the bone, as has been already in part described, and not a trace of periosteum can be detected.

§ 93. Marrow of the Bones.-Almost all the larger cavities in the bones are occupied by a soft, trasparent, yellowish or reddish, highly vascular substance, the Marrow (medulla ossium). In the cylindrical bones, this substance is found in the medullary canal, and in the cancelli of the apophyses, whilst it is wanting in the compact substance, unless it be in the larger canals; the same is the case in the flat and short bones, the cancelli of which are filled with marrow; but the diploe of the flat cranial bones, besides the marrow, also contains large veins, of which more will be said afterwards. In accordance with what has been remarked, these venous spaces, the canales nutritii, Haversian canals, and the above described nerve-canals and air-cavities of the bones, contain no marrow.

The marrow appears in two forms, one yellow, the other red. The former, as a semifluid substance, occurs principally in the long bones; and according to Berzelius, consists, in the humerus of the $\mathrm{Ox}$, of $96 \cdot 0$ fat, 1.0 connective tissue and vessels, and 3.0 fluid with extractive matter, such as is found in muscle; whilst the latter occurs in the apophyses, flat and short bones, above all in the bodies of the vertetroe, the basis cranii, the sternum, \&c., and is distinguished not only by its reddish or red colour and less consistence, but also by its chemical composition; for, according to Berzelius, this substance, in the diploe, contains $75 \cdot 0$ 
water, $25 \cdot 0$ solid matters, such as albumen, fibrin, extractive matter, and salts, similar to those of muscle, and merely traces of fat. With respect to its structure, it presents, besides vessels and nerves, connective tissue, fat-cells, free fat, a fluid, together with, lastly, peculiar minute cells, marrow-cells. Connective tissue and fat are universally present, though in very various quantities. The former, on the surface of the larger medullary masses of the diaphyses, is of rather firmer consistence, but cannot properly be described as a medullary membrane (endosteum, periosteum internum), because it does not admit of being separated as a continuous structure. In the interior of the marrow in the spongy bones, scarcely any connective tissue can be detected except in the larger masses of it, whilst in the diaphyses, this tissue can be readily demonstrated as a very lax and delicate, areolated structure, containing the fat and supporting the vessels and nerves. Its elements correspond with those of the lax connective tissue (vide $\$ 24$ ); although, as far as I have seen, it does not contain any elastic filaments. Fat-cells of $0.016-0.032$ of a line, not unfrequently with a distinct nucleus, occur in large quantities in the yellow, more dense marrow, quite as abundantly as in the panniculus adiposus, but for the most part not aggregated into distinct lobules. In the reddish marrow, when expressed, they are more rare; and in the red pulp of the bodies of the vertebræ and of the flat cranial bones, they occur only in very minute, scanty accumulations, or altogether isolated, to which circumstance, according to Berzelius, is owing the small quantity of fat in the diploe. In dropsical marrow these cells are frequently only half filled with fat, or with but one or more globules, containing, besides, a large quantity of serum ; and in hyperæmia of the bones, they appear occasionally to be diminished in size, and occasionally elongated and fusiform. Free fat-globules, and a clear or yellowish fluid, are often met with in the softer kinds of marrow, and frequently in considerable quantity. That the former have not been set free from cells, in the preparation of the Fig. 120. specimen, may be satisfactorily shown, but it must remain uncertain whether or not they are to be referred to cells that have ceased to exist. Lastly, there occur, together with some fluid, in all the red, or even only reddish marrow (never in the yellow), minute roundish, nucleated cells, exactly like those of the young medulla (vid. infra, fig. 132). These medulla-cells correspond in every particular with those, which Hasse and I ("Zeitsch. f. ration. Medicin," Bd. V.) found in the hyperæmiated red marrow of the articular extremities of the cylindrical bones, but nevertheless normally exist in the vertebrce, the true cranial bones, in the sternum, and in the ribs, whilst they are wanting in the long and short

FIG. 120.-Two fat-cells from the marrow of the human femur: $a$, nucleus; $b$, cell-membrane; $c$, oil ; magnified 350 diameters. 
bones of the extremities, and in the scapula and os innominatum, occurring apparently in variable number in the bones of the face.*

§ 94. Connections of the Bones.-A. synarthrosis, connection without articulation.

1. By suture. In this mode of connection, the bones are united by an extremely thin, membranous, whitish streak, to which authors have incorrectly given the name of sutural cartilage. It is composed merely of connective tissue, which, like that of the ligaments, extends, in short, parallel fasciculi, from the border of one bone to that of the other, and is characterized solely by the presence of numerous, short, unequal-sized, usually elongated nuclei. This sutural ligament, as it may be termed, is very evident as long as the cranial bones are still growing, at the same time, that it is softer and differently constituted (vide infra). As the growth of the cranium approaches its completion, this tissue gradually disappears, becomes firmer, and, in old age, seems, in many places, especially on the inner part of the sutures, and even before their complete obliteration, to be entirely removed.

2. Connection by ligament, syndesmosis, is effected by means of fibrous and elastic ligaments. The fibrous ligaments, constituting the majority of the ligaments, are white and glistening, corresponding in their structure, partly with the aponeuroses and ligaments of the muscles, and partly with the true tendons.

Elastic ligaments (Fig. 121), are, the ligamenta flava, between the arches of the vertebræ, and the ligamentum nuchx, which, however, is not nearly so well developed in man, as in some others of the Mammalia. The ligamenta flava are yellowish, highly elastic, strong ligaments, the elastic elements of which, in the form of roundish polygonal fibres, 0.0015 -0.004 of a line thick, united into a dense network, run parallel with the long axis of the vertebral column, and give the longitudinal, fibrillar aspect to the ligaments. Between these fibres, which are not collected either into fasciculi or lamelloe, but are continuously connected throughout the entire thickness of each yellow ligament, there is interposed some connective tissue, upon the whole in small quantity, but demonstrable in every preparation, and occurring in the form of lax undulating fasciculi,

* [These nucleated medulla-cells exist in great number in the superior maxillary bone of Man and of many of the Vertebrata. Several of them are sometimes enclosed in a common cell-wall, forming a variety of cell, frequently seen in pathological formations, viz. the parent-cell of the German authors, the plaque a noyaux multiples of Robin.

The occurrence of nucleated cells in the normal medulla of the bones of the face has been mostly overlooked, and has hence given rise to many errors on the part of pathologists. In the description of morbid changes of the bones of the face, especially in those of the superior maxillary bone, representations are frequently given of small cancer-cells or nuclei, taken from the interior of the bone, which are but the normal nucleated cells of the medulla. Many tumors of the upper jaw have thus been pronounced cancerous, which were simply non-malignant hypertrophies of the bone.-DaC.] 
which are arranged parallel with the principal direction of the elastic fibres. According to Todd and Bowman (p. 72), the stylo-hyoid, and internal lateral ligament of the lower jaw, are, also, chiefly composed of strong elastic fibres.

3. By cartilage, synchondrosis. This mode of connection is effected either by cartilage alone, or 'associated with fibro-cartilaginous and fibrous tissue. The former condition is observed in the adult, only between the ribs and sternum, where, however, properly speaking, a true synchondrosis exists only in the case of the first rib, the rest, from the second to the seventh, being connected with the sternum at the anterior extremity by articulation; whilst the false ribs are either free at the extremity, or are incurved one beneath the other. In the symphysis pubis, sacro-iliac synchondrosis, and the junctions of the bodies of the vertebroe, the surfaces of the bones are covered immediately by a layer of true cartilage, which, in the two Fig. 121.

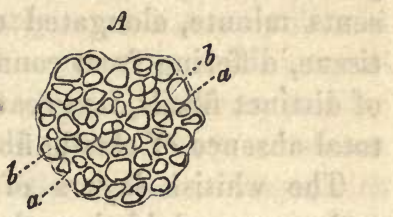

B

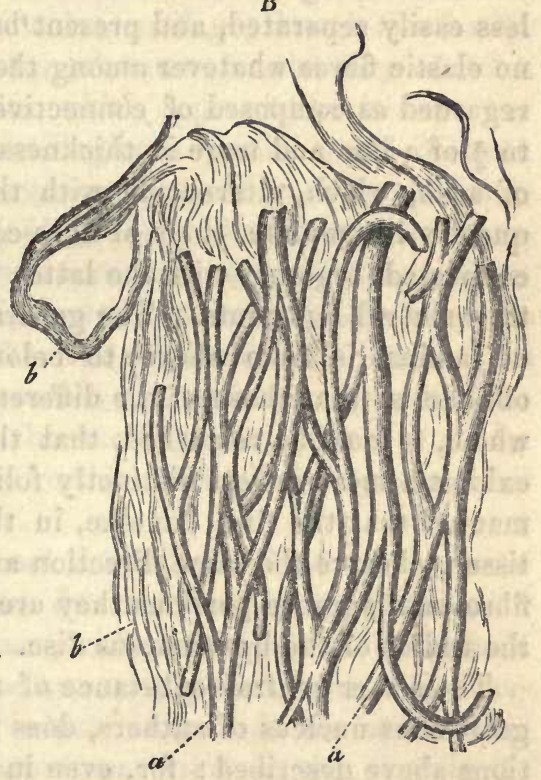
former situations, is directly connected with the opposite layer, and in the latter by means of a fibro-cartilaginous tissue, and is externally encircled by fibro-cartilaginous, and fibrous, concentric layers. In the two former of these instances, there is, not unfrequently, a cavity in the interior of the connecting substance, so that the sacro-iliac sychondrosis, in particular, may also be regarded as a sort of articulation (Zaglas).

The intervertebral ligaments, or ligamentous discs, of the bodies of the vertebræ, consist, 1 , of exterior concentric layers of fibro-cartilage, and whitish connective tissue; 2 , of a central, principally fibro-cartilaginous substance; and 3 , of two cartilaginous layers applied immediately upon the bones.

The concentric lamelloe consist of alternate layers of connective tissue and of fibro-cartilage, which latter, even in fresh transverse sections,

Fia. 121. $-A$, a transverse section through a portion of the ligamentum nuche of the $\mathrm{Ox}$, magnified 350 diameters, and treated with soda: $a$, connective tissue, apparently homogeneous; $b$, transverse section of the elastic fibres $(0.004-001$ of a line in diameter). $B$, elastic fibres; $a$, from a human lig. subflavum, together with some connective tissue, $b$, between them, magnified 450 diameters. 
may be recognized as dull yellow streaks, which become hard and transparent in water. The fibro-cartilage, on microscopic examination, presents minute, elongated cartilage cells, disposed serially in a fibrous tissue, differing from connective tissue in its greater rigidity, the absence of distinct fibrils, its great resistance to alkalies and acetic acid, and the total absence of elastic fibres.

The whitish layers of the outer laminæ, although their fibrils are rather more rigid than those of the common ligaments and tendons, are less easily separated, and present'but few fusiform cells, and frequently no elastic fibres whatever among them, must nevertbeless, at present, be regarded as composed of connective tissue. These laminæ are from $\frac{1}{3}$ to $\frac{5}{4}$ of a line and more in thickness, and form entire circles or segments of such, which, alternating with the somewhat thinner, and also frequently incomplete, rings of fibro-cartilage, with which they are closely connected, together with the latter constitute the larger half of the intervertebral ligaments. The general direction of the fibres of both sets of lamina is from above to below. They are, however, invariably oblique, so that those of the different layers cross each other. Besides which, it must be remarked, that the individual layers themselves also exhibit a more or less distinctly foliated structure, constituted in such a manner that the fine lamellæ, in the portions composed of connective tissue, observe the same direction as the layers themselves, whilst in the fibro-cartilaginous portions they are disposed more in the direction of the radius of the ligamentous disc.

The softer central substance of the intervertebral ligaments, or the gelatinous nucleus of authors, does not differ, essentially, from the portions above described; for, even in this situation, layers of connective tissue occur, although they gradually diminish in proportion to the fibro-cartilage, and are less distinctly defined. The nearer we approach the centre, the less evident is any trace of an alternation of different

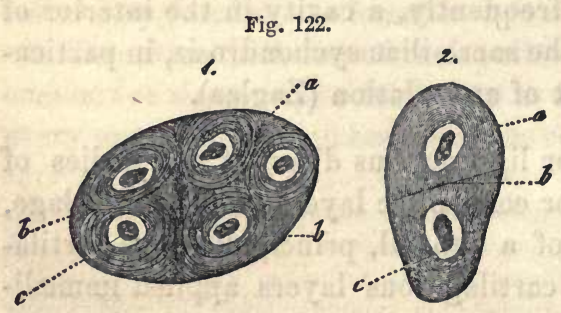
layers, and of a concentric arrangement of them; the whole becomes transparent, soft, and, finally, almost homogeneous. The microscope shows the predominance of fibro-cartilage, with large cells $(0.012-0.024$ of a line), frequently one within the other (Fig. 122); the uniformly thickened walls of which, composed of

Fig. 122.-Cells from the gelatinous nucleus of the lig. intervertebralia: 1, large parent cell, $a$, with a septum derived from two secondary cells of the first generation, and five secondary cells, $b$, of the second generation; with concentrically thickened walls and shrunken nuclei, $c$, in the small cell cavities: 2 , parent cells, $a$, with two secondary cells, separated by a delicate septum, $b$, and which, with uniformly thickened walls, contain a minute cavity and shrunken nucleus, $c$. 
concentric layers, often enclose merely a minute cavity, with a shrunken nucleus; and besides these, smaller cells frequently in process of dissolution, isolated or aggregated together; and, lastly, an indistinctly fibrous or granular matrix, not unfrequently observed in a state of disintegration, and a considerable quantity of fluid contained in larger or smaller areolar spaces in it. The more central portions of this fibrous substance gradually pass into a thin, hard, yellowish lamella of true cartilage, with thickened cells, not unfrequently beset with calcareous particles, which adheres to the bone not unlike an articular cartilage, though less firmly. More externally we find a cartilaginous substance, in the form of isolated, minute, discoid plates or particles, which appear to be in more immediate connection with the fibro-cartilaginous portions, and between these a connective tissue, with scattered cartilage-cells, as in the insertions of the tendons into the bones (vide $\S 81$ ). The more exterior portions of the surfaces of the bodies of the vertebræ, corresponding to these parts of the discoid-ligaments, are, in contradistinction to the more internal portions, as it were porous, after the removal of the ligamentous layer; the medullary cavities or cancelli then being exposed. The pores or cancelli are closed only by the cartilaginous substance of the disc, whilst the fibrous tissue, with its vertical fibres, is firmly connected with the interspaces between them.

Between the sacrum and coccyx, and the individual coccygeal vertebræ, are interposed the so-called false intervertebral ligaments, consisting of a more uniform fibrous substance, without any gelatinous nucleus. The separate bones of the sacrum, at an early period, have true intervertebral ligaments between them, which afterwards become ossified from without to within, but in such a way, nevertheless, that even in the adult, traces of the ligament may still be perceived in the centre. With respect to the nature of the fibres of the intervertebral ligaments, Donders is inclined, especially from the consideration of their chemical relations, to regard almost all of them, not as connective tissue, but as analogous to the matrix of true cartilage, as is also H. Meyer (p. 300, et seq., and p. 310). This opinion may be correct, as regards the central, nuclear portion, and the fibro-cartilaginous laminæe of the outer portions, but hardly so with respect to the purely fibrous parts of the latter. I believe, moreover, that it is not by chemistry, but by the study of the development of these tissues, that the question will be solved, because, although manifest, visible distinctions exist between the fibrils of connective tissue developed from cells, and the fibrous intercellular substance, viewed from a genetic point of view, chemistry probably is not in a condition to distinguish one from the other.* The in-

* [In our note on the connective tissue, we have already expressed the views we entertain of the homologies of the elements of cartilage and connective tissue; and we need merely add that we know of no locality in which the transition of the matrix of cartilage 
tervertebral ligaments are liable to various forms of degeneration; they may become ossified, from their cartilaginous lamellæ outwards, the true fibrous substance probably at the same time disappearing; and in this way anchylosis of two vertebræ frequently takes place. They may become atrophicd, easily broken down, and disintegrated, either in the nuclear portion, or elsewhere in circumscribed spots, into a dirty grumous matter. And lastly, it would appear that although in the normal state, they contain no vessels, vessels may, under certain morbid conditions, be developed in them; at all erents, extravasations of blood are not unfrequently met with, most generally close to the bones or in connection with them.

In the symplysis pubis, the cartilaginous layer, which is thickest in the centre and anteriorly, and connected with the bones by a very uneven surface, consists, at the sides, where it is from $\frac{1}{2}$ to 1 line thick, of true cartilage, with a homogeneous, finely granular matrix and simple cells, measuring $0.01-0.024$ of a line. In the centre, the matrix is softer and fibrous, and in this situation (more particularly, it would appear, in the female sex), there occasionally exists an irregular narrow cavity, with uneven walls, and containing a somewhat slimy fluid, originating evidently in a solution of the innermost cartilaginous layers, and of which manifest traces may be perceived in the cartilaginous substance immediately enclosing it. The outer layers of the symphysis, which, as is well known, are most developed anteriorly and superiorly, do not arise, with the exception of the outermost lamellæ composed of pure connective tissue, directly from the bones, but, properly speaking, unite only the outer portions of the above.described cartilaginous layers, and consist principally of a fibrous substance, to all appearance identical with connective tissue, and occasionally containing cartilage cells.

The formation of the bone-corpuscles, as they are termed, may be traced perhaps more clearly in the symphysis than anywhere else, except in rachitic bone (Fig. 124). For at its osseous borders there are always to be found, either half projecting from, or entirely lodged in, the cartilage, isolated, nucleated bone-corpuscles or cells, with homogeneous, and (from calcareous salts) granular walls, measuring $0.012-0.016$ of a line with respect to which, from their development and from the consideration of the contiguous cartilage-cells, all of which present more or less thickened walls and rudiments of calcareous deposits, not the smallest doubt can be entertained. Well-characterized, half and wholly ossified parent cells of the same kind, with two secondary cells, and measuring $0.015-0.03$ of a line, up to some including ten or twenty secondary cells

into the pseudo-fibrillated collagenous portion of connective tissue is more unmistakably exlibited, than in the intervertebral cartilages of a young animal, e.g., a kitten. We have, in that note, endeavored to show that the notion of the existence of any real difference in the development of the fibrillated element in the different forms of connective tissue is unfounded.-Trs.] 
and having a length of 0.05 of a line, may be distinctly noticed in almost every preparation.

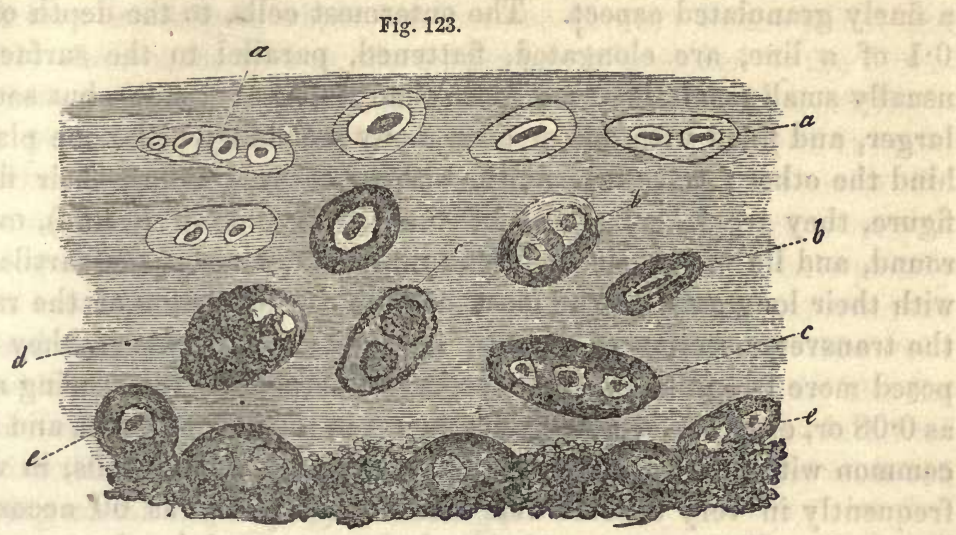

The sacro-iliac synchondrosis is effected by means of a flattened layer of cartilage, $\frac{3}{4}-1 \frac{1}{3}$ of a line in thickness, which is closely attached to the articular surfaces of the corresponding bones, between which it is interposed. The cartilage-cells close to the bone are flattened, with their surfaces directed towards it, and present beautiful transitionary forms into half and wholly isolated bone-cells, which exist on the border of the bone. In the interior of this cartilaginous layer, according to Zaglas, there is always a narrow cavity, which separates the cartilaginous layers of the two bones completely, or almost completely, from each other. It contains a synovia-like fluid, and is bounded by smooth and even walls, which differ from the rest of the cartilaginous substance in their greater hardness, as well as in their structure. The matrix of these cartilaginous layers, in the direction of the surface, is finely fibrous; the cells are all of large size (as much as 0.035 of a line), with numerous secondary cells and uncommonly thick walls, so that the cavities, even of the secondary cells, often appear extremely contracted; but they do not exhibit any distinct indication of pore-canals or calcareous deposit.

The costal cartilages are invested by a strong perichondrium, composed of connective tissue and numerous elastic elements, which commences at the sternal end in connection with the synovial membrane there existing, and at the other is continuous with the periosteum of the ribs. The cartilage, which is in connection with this membrane by a roughened surface, is of considerable firmness although elastic, pale, yellow, or in thin sections, exhibiting a transparent blue tint, internally almost always,

Fig. 123.-Cartilaginous border, towards the cartilage of the symphysis in Man; $a$, cartilage cells with thickened walls; $b$, the same undergoing ossification; $c$, cells nearly ossified, with homogeneous walls free in the matrix of the cartilage; $d$, similar cells with calcareous granules; $e$, ossified cells at the border of the matrix of the bone containing calcareous granules, and half projecting from it.-Magnified 350 diameters. 
in certain spots, of a yellowish-white color, with a silky lustre. Its matrix in the latter situations presents a fibrous structure, and elsewhere a finely granulated aspect. The outermost cells, to the depth of $0.06-$ 0.1 of a line, are elongated, flattened, parallel to the surface, most usually small (sometimes not more than 0.006 of a line), but sometimes larger, and filled with one or even many secondary cells, one placed behind the other; more internally, without entirely losing their flattened figure, they are larger (most of them 0.03-0.05 of a line), oval, and round, and lie with their surfaces towards the ends of the cartilage, and with their long axis for the most part in the direction of the radius of the transverse section of the rib; in many cases, however, they are disposed more irregularly. The largest of these cells (measuring as much as 0.08 or, even 0.1 of a line), are found in the fibrous spots, and they, in common with all the interior cells, contain secondary cells, in varying, frequently in very considerable number (as many as $60^{\circ}$ according to Donders). The most remarkable characteristic of the elementary tissue

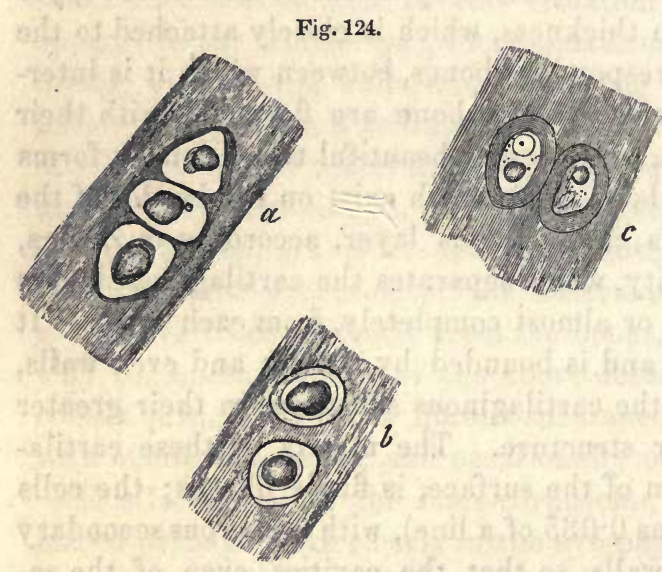
of the costal cartilage is the large quantity of fat contained in it. In the adult, every cell, excepting the most superficial, contains, larger or smaller (from $0.0016-0.008$ of a line), sometimes spherical, sometimes more irregular fatdrops, which frequently so surround the nucleus as entirely to conceal it from view (Fig. $124 a, b$ ), whence it has been assumed, though not quite correctly, that the fat is seated in the latter. The cartilage on the greater cornu of the os hyoides, and between the body and the greater cornu, and the inconstant cartilaginous appendage to the styloid process, differ in no respect from costal cartilage, only that the cartilage cells in those instances do not always contain large fat-globules.

The costal cartilages frequently become ossified in old age; but this ossification, as well as the fibrillation of the matrix, must not be regarded as a normal process, nor be placed in the same category with the usual kind of ossification. The ossification is sometimes more limited,

FrG. 124.-Cartilage cells of Man, magnified 350 diameters : $a$, parent cell with three secondary cells containing oil, from a costal cartilage; $b$, two cells from the same situation in which the globule of oil is surrounded by a pale border; $c$, two cells with thickened walls from the cartilage of the greater cornu of the os hyoides, which together with the globule of oil also contain a distinct nucleus. 
sometimes more extensive. In the former case, it does not proceed further than to the incrustation of the cartilage-cells, and of the matrix in which they are lodged, which has become fibrous; in the latter, and frequently, also, in the former, the ossification is preceded by the formation of hollow spaces in the cartilage, in which is deposited a cartilagemarrow, containing vessels, which are connected, in part with those of the perichondrium, in part with those of the ribs; and the osseous substance is more that of normal bone, though almost always more opaque, less homogeneous, and with imperfectly formed lacunæ, which frequently contain a calcareous deposit. Under the name of cartilage-marrow, are understood the medulla-cells, fat-cells, bundles of connective tissue and vessels which are presented instead of the detritus, afforded by the disintegration of cartilage, and which may be said to correspond in all respects with those of developing foetal bone, and may be readily observed in ossifying costal and laryngeal cartilages.

§ 95. B. Movable Articulation (Diarthrosis). - The articular extremities of the bones, or any other surfaces entering into the formation of a joint, are invariably invested with a thin layer of cartilage, which in the middle of the surfaces covered by it, is of tolerably uniform thickness, gradually thinning as it extends outwardly, and finally terminating with a very abrupt edge. This articular cartilage is closely applied to the bone with a rough, hollowed or raised surface, but is not united to it by any interposed substance; and, on the opposite surface, it is in most joints usually quite bare, and directed towards the cavity of the articulation. Sometimes, however, it is invested with a special fibrous membrane, $a$ perichondrium, which is an immediate prolongation of the periosteum, and extends most generally only over a small portion of the cartilage, gradually ceasing without any defined margin.* In some joints (shoulder, hip) the more secure lodgment of the articular head of the bone is insured by special cartilaginous lips. These are firm, yellowish-white, fibrous rings, attached, at the border of the articular cartilage, by a wider basis, immediately to the bone or partly to the cartilage. They thin off to an acute edge, and for the most part free and uncovered by the synovial membrane, or any epithelium, project into the articulation, being exteriorly in relation with the periosteum and synovial capsule.

* [Reichert, who has paid particular attention to the question of the existence of an epithelium upon the articular cartilages, says, that in the fotal condition of Man and the domestic Mammalia, an epithelium exists over the whole surface of the synovial capsules, and, on the articular cartilage, lies in immediate contact with its substance. It resembles the epithelium of the vessels. In adults, on the other hand, he could discover an epithelium only on those parts of the articular capsules which are not subject to friction; and here it had the same appearance as in the fetal condition. It was wanting upon the articular cartilages and their immediate neighborhood; but it was not uncommon to meet with fine desquarnated flakes of cartilage in the synovia, which fell readily into folds, and thus resembled a fibrocartilaginous tissue (Bericlit, Müller's "Archiv.," 1849, p. 16.)-Trs.] 
As regards the intimate structure of the parts just described, the articular cartilage, on completely formed bones (Fig. 125), and under

Fig. 125.

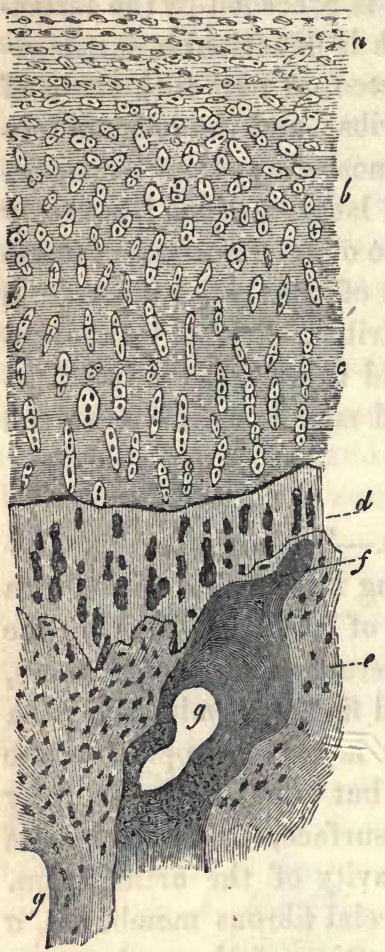
normal conditions, presents throughout, a finely granular, in part almost homogeneous matrix, in which are lodged delicate cartilagecells, which towards the surface of the cartilage are numerous and flattened, and lie parallel to it; more deeply they are oval or rounded, more rare, and disposed in various directions; and lastly, close to the bone they are elongated, and placed vertically with respect to the surface of the bone. These cells all have distinct walls, easily distinguished from the matrix by the use of acetic acid, clear, frequently granular contents, containing, however, but little fat, and a vesicular nucleus. They occur either isolated or in groups, and present very frequently two, four or even more secondary cells, which in the flat cells are placed close together, and in the elongated are disposed in rows. On the condyle of the lower jaw, as on the corresponding surface of the temporal bone, until the bone is completely formed, there is a thick layer of very distinctly marked cartilage-cells, covered, towards the cavity of the articulation; by a layer of connective tissue. This cartilaginous layer disappears by degrees, as the bone approaches its completion, and at last there remains beneath the layer of connective tissue, now become both relatively and absolutely thicker, merely an excessively thin and transparent lamina, the elements of which, although morphologically not true bone-cells, nor as yet ossified, still seem to resemble the latter more closely than cartilagecells.

The cartilaginous lips of the joints consist principally of connective tissue, always containing, however, isolated cartilage-cells of a roundish or elongated form, with a moderately thick membrane, distinct nucleus, and occasionally fat-granules. I have not as yet noticed parent cells in

FrG. 125.-Articular cartilage of a human metacarpal bone, cut perpendicularly: $a$, most superficia!, flattened cartilage cells; $b$, middle round cells; $c$, innermost cells, disposed perpendicularly in small rows; $d$, outermost layer of the bone with ossified fibrous matrix and thick-walled cartilage cells, in this instance appearing dark from their containing air ; $e$, true bone-substance; $f$, ends of the cancelli of the apophyses; $g$, one of the cancelli.-Magnified 90 diameters. 
this situation, whilst cells of the kind already described in the muscular system ( $\$ 82)$, arranged in series, are not unfrequently met with, and might perhaps be regarded as cartilage-cells, although their nuclei exhibit the most evident indications of a transition into nuclear fibres. The articular cartilages, moreover, during their development, which will be entered into more particularly afterwards, have no nerves or vessels, as is the case also with the cartilaginous lips.*

The condition of the bone beneath the articular cartilages, requires special notice. It consists, in almost all joints, in immediate contiguity with the cartilage, of a layer of incompletely formed bone-substance, and, more internally, of that tissue in its usual form (Fig. 125). The layer in question, which is $0.04-0.16$, or on the average 0.12 of a line thick, is composed of a yellowish, mostly fibrous, hard, and truly ossified matrix, containing, however, not a trace of Haversian canals or medullary cavities, nor of any perfectly formed lacunæ; instead of which it presents roundish or elongated corpuscles, aggregated into little masses or rows, the larger of which are $0.016-0.024$ of a line in length, and $0.006-0.008$ of a line in breadth, and the smaller $0.006-0.008$ of a line in length, and $0.004-0.005$ of a line in breadth, which give thin sections of the bone a perfectly opaque aspect, and consequently might be regarded as bone-corpuscles (lacunæ) filled with calcareous particles, as which they have lately been considered by H. Meyer (l. c., p. 325, 326). By the addition of spirit of turpentine, which, however, penetrates with difficulty, this error is dissipated, and it is found, that as in the case of the lacunce of dried bone, the opaque aspect is due only to the air contained in them, and that the bodies in question are nothing more than thick-walled cartilage-cells, retaining their contents (fat, nuclei), presenting occasionally indications of canaliculi, and perhaps also partly calcified; in other words, that they are undeveloped lacunæ. The layer in which these cells are lodged, and which, towards the cartilage, is bounded by a straight line, occasionally dark from calcareous particles, and

* [Besides the elements above mentioned, we find according to Dr. Leidy (see Am. Jour. of Med.Sc., April, 1849), numerous minute lacunæ, as an occasional peculiarity in the structure of articular cartilage. These lacunæ are described by Dr. Leidy as existing in greatest abundance in the deeper part of the cartilage; but decreasing in number, as its free surface is approached. They are lenticular in outline, and measure from 1-1200th-1-3120th of an inch; when well defined, they appear beneath the microscope more translucent than the cartilaginous matrix in which they are situated. When viewed a little within the focus of the instrument, they are of a deep black color, and oppose the transmission of all light. Another peculiarity described by Dr. Leidy is the penetrature of the structure of the cartilage by fibres or columns of bone. These fibres are quite uniform in shape and structure, being compressed and cylindrical; in transverse sections they present an elliptical figure. They are not numerous, and vary from a size not exceeding a cell-group of 5 cells to the size of four or five such groups. They are concentrically laminated, and also present a radiated conformation, resembling somewhat the structure of an Haversian canal, but neither the canal nor the Purkinjean corpuscles are discernible in them.-DaC.] 
towards the true bone by a sinuous contour, in which the limits, as it were, of the individual lacunæ are distinguishable, is not found either exclusively in bones not yet fully formed, as Gerlach believes, nor only at a more advanced age (from 30 upwards, and particularly in old men), as H. Meyer states, but, at all events as far as my observation extends, at all ages, from the complete development of the bone upwards, invariably in every articulation, except that of the lower jaw and those on the os hyoides.*

The articular cartilage on the head of the femur, in a man 25 years old, measured 1-1 $1 \frac{1}{4}$ of a line in thickness; on the condyles in the middle $1 \frac{1}{4}$, on the margin, $\frac{3}{4}-1$ line ; in the fovea patelloe, $1 \frac{1}{2}-1 \frac{3}{4}$ of a line; in the middle of the condyles of the tibia, $1 \frac{1}{2}$ of a line; at the borders, $\frac{1}{2}-\frac{3}{4}$ ths of a line; in the middle of the patella, $1 \frac{1}{2}-1 \frac{3}{4}$ ths of a line; in the glenoid cavity of the tibia, $\frac{1}{2}-\frac{3}{4}$ ths of a line, on the body of the astragalus, on the upper side, ${ }_{5}^{3}$ ths, on the under, $\frac{1}{2}$, on its head, ${ }_{5}^{3}$ ths of a line; at the base of the first metatarsal bone, $\frac{1}{3}-\frac{1}{2}$, on its head $\frac{1}{3} d$ of a line, on the inner cuneiform bone, in front, $\frac{1}{3}-\frac{1}{2}$, behind, $\frac{1}{2}-\frac{3}{4}$ ths of a line. In the foetus, about the middle period of uterine life, the vessels of the synovial membrane, according to Toynbee ("Phil. Transact.," 1841), extend much further upon the articular cartilage; of which fact, however, I have been unable to satisfy myself in the humerus of a five or six month foetus, or in new-born infants. In pathological states endogenous cell-formation is met with in an unusual degree of perfection, and more especially in all kinds of articular cartilages; in which the parent cells, frequently of very considerable size, with one or two generations of secondary cells, and also containing fat, lie tolerably free in the fibrous matrix, and admit of being readily isolated (vide also Ecker in Rosera and Wunderlich's "Archiv," vol. II., 1843, p. 345). In the adult, the articular cartilages are non-vascular, although the vessels of the synovial membrane, at their border, often advance to some distance over them. What Liston ("Med. Chir. Transact.," 1840, pp. 93-4) describes as "vessels in the articular cartilage of several diseased joints, and as running straight in parallel lines from the injected membrane of the bone into the cartilage, and as joining at their further extremities in that tissue, thus forming long loops," were certainly nothing more than the normal vessels of cartilage, which (vide infra) may be very beautifully displayed even in individuals 18 years old. There cannot, therefore, be any question of inflammation of the cartilages in the adult, though they doubtless suffer in morbid conditions of the bones upon which they rest, or in inflammation of the synovial membrane. They frequently assume a fibrous structure, a change which is often attended with a simultaneous

* [This peculiarity of the bone beneath the articular cartilages was first pointed out by Dr. Sharpey (Quain and Sharpey, 5th ed., p. clviii.), and is particularly described by Tomes and De Morgan, l. c., pp. 10, 11.-Trs.] 
increase in thickness, Cruveilhier ("Dict. de Méd. et Chir. prat." III., 514) having noticed fibres of this kind as much as 6 lines in length, thus far exceeding the normal thickness of articular cartilage. They sometimes wear away rapidly, or even disappear altogether (in suppuration in the bone or in the articulation), so that the surface of the bone is left exposed; they also undergo partial loses of substance; when they exhibit ulcerous excavations, which may penetrate to the bone, or commence on the osteal surface of the cartilage.

$\S 96$. The articular capsules (capsulce s. membrance synoviales) are not closed capsules, but short, wide tubular sacs, which are attached by two open ends to the borders of the articular surfaces of the bones, and thus connect them together. They are essentially more or less delicate, transparent membranes, but are in many situations so closely and completely invested externally by fibrous layers - the fibrous capsules as they are termed,-as on cursory inspection to present the aspect of tolerably tough capsules. These fibrous coats are met with especially in situations where the articulation is either wholly unprotected, or but thinly covered by soft parts, or where a very firm connection is required (as in the hipjoint); they are absent for the most part, or are undeveloped, where muscles, tendons, and ligaments rest upon the articulation, or where, for special purposes, the synovial membrane is exposed to more considerable movements (as in the knee and elbow).

The relation of the articular capsules to the bones and articular cartilages, more precisely described, is as follows (Fig. 126):-The articular capsule is attached, either simply to the border of the cartilaginous surface, extending thence directly to the other bone (patella, amphiarthroses); or it may, in the first place, besides the border of the cartilage, also invest a larger or smaller extent of surface of the bone itself, and then pass to the second bone, with which it is connected in the one way or the other. In either of these cases the synovial membrane does not adhere immediately to the hard tissues subjacent to it, but is more or less closely connected with the periosteum and perichondrium, ultimately ceasing without any distinct Fig. 126.

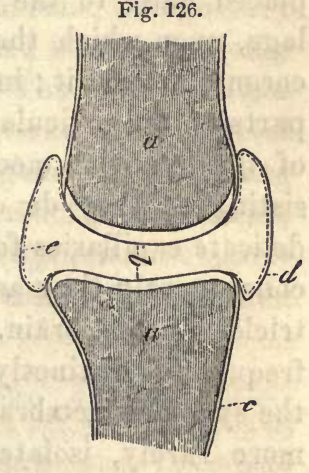
margin, not far from the border of the articular cartilage, with the perichondrium of which it is inseparably united.

With respect to the intimate structure of these tissues, the synovial

Frg. 126.-Diagram of a transverse section of a phalangeal articulation, partly after Arnold : $a$, bones; $b$, articular cartilage; $c$, periosteum continuous with the perichondrium of the articular cartilage; $d$, syrovial membrane at the edge of the cartilage, connected at first with the perichondrium; e, its epithelium. 
membranes, distinct from the fibrous capsules, as they are termed, which possess in all respects the structure of fibrous ligaments, consist: -1 , of a layer of connective tissue, with not very numerous vessels and nerves; and, 2, of an epithelium. The latter is composed of from one to four layers of large tessellated cells, measuring $0.005-0.008$ of a line, with roundish nuclei of $0.002-0.003$ of a line. The former, in its innermost part, is constituted of a layer of parallel fasciculi, with indistinct fibrils and elongated nuclei or fine elastic filaments; more externally of decussating bundles, with a fine elastic network, occasionally also of a network of bundles of connective tissue of very various thickness, with winding elastic fibres, exactly as in the arachnoid. Not unfrequently, common fat-cells occur, dispersed here and there in the meshes of the connective tissue, although upon the whole very rarely; and also a few scattered cartilage-cells, with tolerably thick, opaque walls, and a distinct nucleus. The synovial membranes possess neither glands nor papillæ, whilst they present large adipose masses (plicae adiposce) and vascular processes (plicce vasculoso, plicce synoviales, ligamenta mucosa, of authors). The former, at one time erroneously termed "Haversian glands," are found principally in the hip- and knee-joints, in the form of yellow or yellowish-red soft processes or folds, and consist simply of large collections of fat-cells in vascular portions of the synovial membrane. The latter are met with in almost every joint, constituting, provided that the blood-vessels are filled, red, flattened projections of the synovial membrane, with an indented and plicated margin, and furnished with minute processes. These folds are usually placed close to the junction of the synovial membrane with the cartilage, upon which they lie flat, thus forming, in many cases, a sort of coronal around it; in others they are more isolated, and placed in other parts of the articulation. In their structure, they differ from the rest of the synovial membrane principally in their great vascularity, consisting as they do of little else than minute arteries and veins, and delicate capillaries forming wavy loops at the edge of the processes, and consequently they are very similar to the choroid plexuses in the ventricles of the brain. Besides the vessels, they present a matrix of, frequently, distinctly fibrous, connective tissue, the usual epithelium of the synovial membrane, occasionally solitary or numerous fat-cells, and, more rarely, isolated cartilage-cells. At the edge, they are almost invariably furnished with minute, foliated, conical, membranous processes of the most extraordinary forms (often resembling the stems of a cactus), which also frequently contain vessels, but are for the most part constituted merely of an axis of indistinctly fibrous connective tissue, with occasional cartilage-cells, and an epithelium, very thick in places. The smaller ones frequently consist even of nothing but epithelium, or of little else than connective tissue. 
In many joints there are firm, whitish-yellow, fibrous plates, the so-termed interarticular cartilages or ligaments, which, either Fig. 127. projecting in pairs from the synovial capsule, are interposed between the bones constituting the articulation (knee), or form a single diaphragm transversely across the joint (articulations of the jaw, clavicle, sternum, and wrist). These processes consist of a firm, fibrous tissue, the fibres of which usually cross each other in various directions, and are in all respects closely allied to connective tissue, but presenting less distinct fibrils ; and, besides this, of cartilage-cells and fine elastic fibres.* The cartilage-cells, in the most superficial layers, are more solitary, in the deeper, disposed

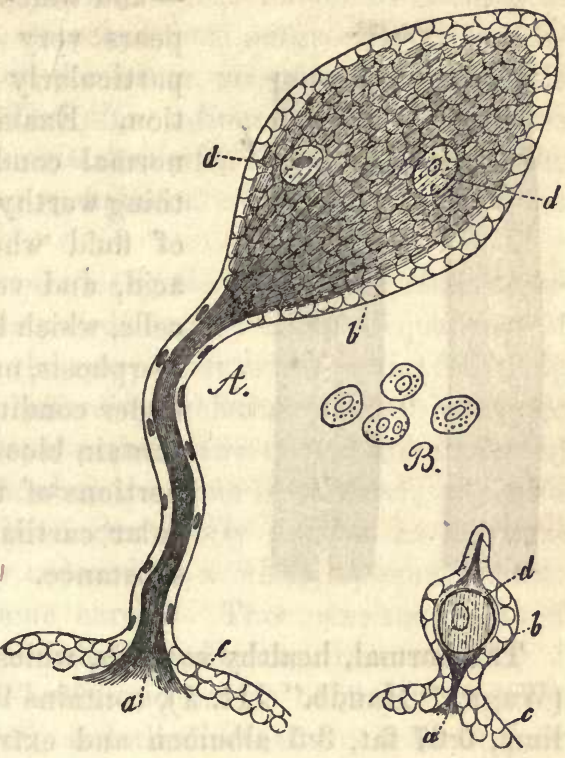
more in rows and smaller, ultimately being replaced by fine elastic fibres, a certain number of which, at all events, appear to originate from cells resembling the cartilage-cells. The interarticular ligaments, which, from what has been said respecting them, must be enumerated among the fibro-cartilages, are not covered by synovial membrane, though they probably have an epithelial investment at the attached border, but only for a small extent, - never over the entire surface. The articular ligaments, with the exception of the softer ligamentum teres, are composed of the same firm connective tissue (in the costal ligaments containing cartilage-cells), as that of which the tendons and the fibrous ligaments, elsewhere, are constituted. The internal ligaments (lig. cruciatoe), however, present softer connective tissue, containing vessels, and covered with epithelium.

Fig. 127.-From the synovial membrane of a phalangeal articulation: $A$, two non-vascular appendages of the synovial processes, magnified 250 diameters: $a$, connective tissue in its axis; $b$, epithelium (in the peduncle of the larger process not distinctly cellular) continuous with that on the free borders of the process; $c, d$, cartilage-cells; $B$, four cells from the epithelium of the synovial membrane of the knee, one with two nuclei; magnified 350 diameters.

* [These interarticular fibro-cartilages lose in old persons their distinct fibrous structure, and assume a yellow or brownisl color. Yet they never, according to Virchow (Archiv. f. Path. Anat.), become entirely homogeneous, since a careful examination will al ways permit two directions of their fibres to be recognized, viz.: one parallel to the free edge, and the other running vertically towards it.-DaC.] 
Within the synovial capsules is contained, in small quantity, a clear yellowish fluid, which may be drawn out into threads, - the synovia,

Fig. 128.

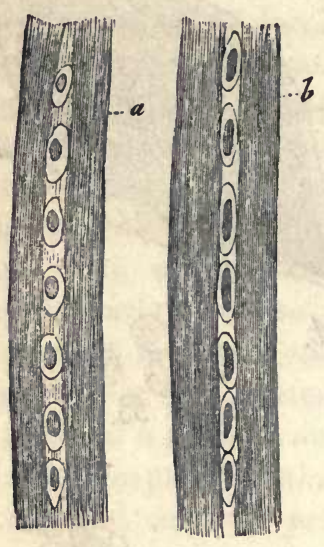

- and which, in its chemical composition, appears very closely to resemble mucus, and particularly in its containing mucin in solution. Examined under the microscope, in its normal condition, this secretion exhibits nothing worthy of much remark, consisting simply of fluid which is rendered turbid by acetic acid, and very frequently contains epithelial cells, which have often undergone a fatty metamorphosis, nuclei of such cells, and fat globules ; under conditions not quite normal, it may also contain blood-and lymph-corpuscles, detached portions of the synovial processes of the articular cartilage, and a structureless gelatinous substance.

The normal, healthy synovia, which in the $0 x$, according to Frerichs (Wagn. "Handb." III. 1), contains 94.8 water, 0.5 mucus and epithelium, 0.07 fat, 3.5 albumen and extractive matter, and 0.9 salts, is a secretion, not having essentially any formed elements in it, which simply exudes from the ressels of the synovial membrane with the intermediation of the epithelium; and, in fact, from all its vascular processes, which are destined as it were for this special function, and always exist at the border of a cartilage requiring a lubricating covering. The nonvascular appendages of these processes give origin to the "loose cartilages," as they are termed; they do this by their increasing in size and solidity, and becoming detached from the vascular folds. These bodies are also met with in mucous bursæ and the sheaths of tendons, which are also furnished with vascular folds (vid. sup. § 82); they consist of connective tissue with elongated nuclei, coated with epithelium, and, though not always, contain a variable number of scattered fat- and true cartilage-cells; and they are not developed externally to the synovial membrane, but from an outgrowth of that membrane itself. Similar solid bodies, moreover, may probably be produced in other ways; Bidder ("Zeitsch. f. rat. Medicin," vol. iii., p. 99, et seq.), at all events, and Virchow ("Med. Zeitung," 1846, Nos. 2 and 3) have observed similar bodies presenting no trace of organization. I am inclined, with Virchow, who has actually demonstrated the presence of fibrin in them, to regard them, in many cases, as fibrinous exudations, and in others as

FIG. 128.-From the falciform ligament of the knee: $a$, a filament of connective tissue with oval cells disposed in a series, and resembling cartilage-cells; $b$, a similar filament with more elongated cells and nuclei. 
solidified deposits from the synovia, which latter supposition is supported by the frequent occurrence of curdy, more or less consistent, structureless masses, evidently inspissated synovia, in the tendinous sheaths of the hand. Portions of bone, also, detached from outgrowths at the circumference of the articular ends of the bones, may find their way into the interior of the articulation. The plicae adiposa have perhaps less to do with the formation of the synovia than with the mechanism of the joint, serving the purpose of filling up hollows.

\$ 97. Physical and chemical properties of the Bones, and their accessory Organs. - The bones are composed, besides a small quantity of water $\left(3-7{ }_{0}^{0}\right.$, according to Stark in the compact substance), and fat (2-3\% Bibra), principally of a substance affording gelatine, and of inorganic elements. The latter, in the adult, constitute two-thirds (68.82 Bibra) of dry bone, and are nearly all left when the bone is calcined; in which case, if due care be taken, the bone completely retains its external aspect, although it may be readily reduced to a white, opaque, friable, heavy powder, the so-termed "bone earth." This consists chiefly of $57-59 \frac{0}{0}$ basic phosphate of lime (according to Heintz, 3 atoms base, 1 atom acid), of carbonate of lime $\left(7-8_{0}^{0}\right)$ and traces of fluate of lime, phosphate of magnesia, silex (traces), and alkaline salts. A small part of the salts of bone is also contained in the walls of the vessels and in the lacunr, and this part is dissolved in water. The collagenous substance is the so-termed bone, formative, or ossifying cartilage. It is obtained when bone is treated at a low temperature with dilute hydrochloric, or nitric acid, in the form of a soft, flexible, elastic, light-yellowish, cartilaginous, transparent substance, retaining accurately the shape of the bone. This bone-cartilage constitutes about $\frac{1}{3}$ of the dry bone, putrefies when moist, and when dried, may be burnt away, leaving a small quantity of ash. It is dissolved by boiling, and from its combination with water is produced the gelatine, usually to the amount of 3 or 4 times its volume, and which may also be obtained directly by long boiling of the bone in a Papin's digester.

With regard to the mode in which the principal constituent elements of the osseous tissue are combined, it is certain that the bone earth does not exist as a distinct deposit in any of the constituent parts of healthy, fully formed bone, but rather, although in a solid form, only in a very intimate union with the tissue. Since both the cartilage and the calcined bone retain the figure of the bone, in all its particulars, independently of each other, there can be no doubt, but that the most intimate union of the two substances exists throughout the entire bone, which, however, cannot be regarded as a chemical combination, principally for the reason, that the proportional relations between the collagenous substance and the phosphate of lime are very variable; and that, by simple 
boiling under an increased pressure, the gelatine is separated from the calcareous salts.

The physical properties of the bones correspond with their composition. Their hardness, density, and rigidity are due to the earthy, whilst their elasticity and flexibility depend upon the organic constituents. In the normal bone of the adult, the two principal constituents are united in such proportions, that the bones, together with considerable hardness and rigidity, have a certain degree of elasticity, though slight, so that they possess a considerable resisting power, and are broken, though not very readily, by the application of greater mechanical force. At an earlier age, when the cartilage is in greater relative proportion, the hardness of the bones is much less, their sustaining power consequently less considerable, and they are more liable to be bent; whilst on the other hand, owing to their greater elasticity, they are much less liable to be broken. This is the case in a much higher degree in rachitis, in which morbid condition, the organic constituents amount to from 70 to 80 per cent. A condition the reverse of this is observed in old age, when the bones, though certainly harder, are more brittle, and therefore more readily fractured, to which liability, however, the rarefaction of the tissue which takes place in consequence of age, partly contributes. The inflammability of bone depends upon its organic basis, and its capability of resisting putrefaction to the inorganic constituents. The latter being so intimately combined with the animal tissues, serve as a protection to them, so that bones from ancient burial-places, and those of fossil animals still retain the full proportion of cartilage.

The true cartilages, even in the foetus, contain, in their organic basis, from 50 to 75 per cent. of water, 3 to 4 per cent. of salts (chiefly of soda and carbonate of lime, and also some phosphate of lime and magnesia). The organic basis has been hitherto supposed to consist entirely of chondrin, a substance allied to gelatin, soluble in boiling water and gelatinising as it cools; but it was noticed by Bruns (p. 216), that the matrix and the cells of cartilage were not equally soluble in water, and Mulder and Donders have rendered it probable that the chondrin, which had hitherto been investigated, is not a simple substance, and that the cartilages consist of several bodies of different natures, the matrix, and the membranes of the parent cells, their contents, and the secondary cells, of which the first is more soluble in water, potass, and sulphuric acid, than the others.

The fibro-cartilages (cartilages containing connective tissue) have been, as yet, but little investigated. J. Müller, in the interarticular cartilages of the knee of the Sheep, found no chondrin ; whilst Donders, on the other hand, met with it in the intervertebral ligaments ("Holl. Beitr.," p. 264); he did not determine whether they also contained gelatin. According to Virchow, the gelatinous, nuclear portion of these 
ligaments, in the new-born child, consists of a substance very nearly allied to that of "colloid" (Wurzb. "Verhandl.," II. 283). The ligaments have the same chemical composition as the tendons.

\$98. Vessels of the Bones and their accessory Organs.-A. Bloodvessels. The periosteum, besides the numerous vessels passing to the bone by which it is traversed, presents in its outer layer, composed of connective tissue, a tolerably close network of minute capillaries $(0.005$ of a line). The blood-vessels of the bone itself are very numerous, as may be seen in injected specimens, and also in recent bone full of blood. In the long bones, the marrow and the spongy substance of the articular ends are supplied by particular vessels, as is also the compact substance of the shaft. The former, or vasa nutritia, enter the bone through large special canals, one or two of which are found in the diaphyses, and many in the apophyses. These vessels, with the exception of a few twigs given off to the innermost Haversian canals of the compact substance, and which possess all the tunics proper to the vessels elsewhere (even to the muscular), ramify in the marrow, where they form a true capillary plexus - the vessels in which vary in size from 0.004 to 0.0052 of a line. The vessels of the compact substance arise, in great part, from those of the periosteum, very soon lose the muscular coat, and form, in the Haversian canals, which they either occupy by themselves, or together with some medullary substance, a network of wide canals, which from their structure, can only in the most trifling extent be referred to the capillary system, most of them possessing a layer of connective tissue and an epithelium, and as it is only in the larger canals that fine capillaries co-exist with the main vessel. The venous blood is returned from all the long bones, in three ways:-1, by a large vein accompanying the nutritious artery, the ramifications of which it follows; 2, by numerous large and small veins at the articular extremities ; and, 3, lastly, by many small veins, which arise independently of each other from the compact substance of the diaphyses, in which their roots, as is correctly stated by Todd and Bowman, occupy the wider spaces and sinuses, or pouch-like excavations, which are very evident even in sections of bone.

All the vessels of bone,- the medullary vessels of the apophyses and of the diaphyses, as well as the vessels of the compact substance,communicate in a multiplicity of ways, so that the vascular system throughout the entire bone constitutes a continuous whole, in which it is possible for the blood from any one part to reach every other part; for it was observed by Bichat ("Anat. Général.," 1812, III. p. 37), in an injected tibia, the nutritious artery of which was obliterated, that the bifurcation of the vessel in the medullary canal was well injected, . and that the nutrition of the marrow was evidently unaffected. 
In the short bones, the bloodvessels present pretty nearly the same conditions as they do in the apophyses of the long bones; the arteries and veins of larger and smaller size entering and quitting the bone at numerous points on the surface, and sometimes, as in the posterior aspect of the bodies of the vertebrce, in very large trunks, - the vence basi-vertebrales of Breschet, furnishing a capillary plexus to the medulla, and also penetrating into the few Haversian canals of these bones.

In the flat bones, such as the scapula and os innominatum, there are distinct nutritious foramina for the larger arteries and veins; the compact substance receiving finer vessels from the periosteum, and the spongy substance being supplied by numerous and even large vessels, as in the neighborhood of the articular cavities. In the flat cranial bones, the arteries, for the most part, enter both the cortical and spongy substance from without, on both surfaces, presenting the usual conditions, whilst the vence diploeticce, as they are termed, have only their extremities free in the medulla, as in other bones, their trunks, and larger and smaller branches running independently and generally unassociated with medullary substance in large, arborescent, special channels, the sotermed "canals of Breschet," which open at determinate points with large apertures (emissaria Santorini), and communicate freely with the veins of the dura mater, with respect to which relations, however, works on special anatomy must be consulted. The size and number of the veins in the cranial bones, is, moreover, extremely variable, and they are constantly becoming obliterated, particularly in old age, concomitantly with the frequent diminution of the diploe, on which account also the venous canals and their openings (emissaria) are of such variable dimensions.

The articular and other cartilages of the osseous system, even the fibro-cartilages, in the adult, normally contain no vessels at all, except those of the perichondrium, which however in this respect is far inferior to the periosteum. But it may happen, that as in the costal cartilages in the middle period of life and afterwards, vessels make their appearance, in which case partial ossification frequently either precedes or follows. The fibrous ligaments are poorly supplied with vessels, and particularly the elastic ligaments, and in this respect may be arranged with the tendons, whilst the synovial membranes are characterized by the considerable number of their bloodvessels. The above described synovial folds are especially rich in this respect, as is also the synovial membrane itself, which, everywhere beneath the epithelium, contains a pretty close plexus of canals, from $0.004-0.01$ of a line in diameter.

B. Lymphatics in bone, have been described by some older and more recent authors (vid. Mikrosk. "Anat.," II. 1, 336), but their existence is still not the less doubtful, and I have in vain endeavored to find such 
vessels. With respect to the other parts of the osseous system, the only question can be, as to whether the periosteum and articular capsules possess lymphatics. In the former, they have not yet been observed, whilst in the latter their existence has been asserted by several authors, Cruveilhier, for example. It must be confessed, however, that it has not been by any means proved that they arise in these structures, at all events it appears to me to be very doubtful, whether the synovial membranes themselves contain vessels of this kind, whilst it is perhaps certain that lymphatics do exist in the loose connective tissue surrounding the articular capsules, and between them and the periosteum of the apophyses, particularly at the knee.

§ 99. Nerves of the Osseous System.-The periosteum is abundantly supplied with nerves, the majority of which, however, do not belong properly to it, but to the bone (vid. infra). With respect to the proper periosteal nerves, it appears that their number, on the whole, is not considerable, so that even in some places they may be entirely absent, as in the neck of the femur, and beneath certain muscles (glutoeus minimus, peroncei, \&c.); but there are perhaps but few bones in which they do not exist in one part or another. These nerves lie in the same layer as the vessels, sometimes along the larger branches, sometimes by themselves, arising, at all events in part, from the larger nerves of the bone itself, and are manifestly distributed over considerable spaces, although their ramifications and anastomoses are scanty. In the larger trunks of these nerves the primitive fibres measure, most generally 0.002 -0.004 of a line, though their size gradually lessens, partly owing to actual divisions, which I have seen with the utmost distinctness in the periosteum of the fossce infra-spinata, and iliaca in man, and J. N. Czermak in that of the frontal bone in the Dog; and in part by a gradual attenuation, to a diameter of $0.0012-0.0016$ of a line, many, and perhaps all, terminating with free extremities. On the articular ends of many bones, such as those of the elbow, knee, and knuckle-joints, I have noticed the nerves to be more abundant than elsewhere, ramifying and anastomosing in the vascular connective tissue covering the periosteum, and following principally the course of the blood-vessels; but in these situations, divisions and terminations of the primitive fibres did not come under my observation.

The nerves of the bone itself, which, with the exception perhaps of the ossicula auditûs and sesamoid bones, are universally present, do not exhibit exactly the same conditions in all bones. In the larger cylindrical bones, they penetrate, in company with the nutrient vessels, in the form of one, or where two nutrient foramina exist, of two, pretty considerable trunks (measuring as much as $0 \cdot 16$ of a line), visible to the naked eye, directly into the medullary cavity, and are there distributed 
into the medulla, following the course of the vessels, though not always in apposition with them, towards the apophyses, and forming multifarious ramifications, but, at least as far as I have seen, only few anastomoses. In the second place, all these bones also present, in the apophyses, numerous finer nerves, accompanying the equally numerous blood-vessels directly into the spongy substance, and ramifying in the medulla; and thirdly, extremely delicate filaments are sent even into the compact substance of the diaphyses, in company with the minute arteries by which it is penetrated. There can be no doubt that these filaments are distributed in the compact substance, although I have never succeeded in finding them within it. The smaller cylindrical bones of the hand and foot present the same conditions with respect to their nerves as the larger ones, except that in them, on account of the undeveloped condition of the medullary cavities, the numerous nerves are not so regularly divided into apophysal and diaphysal.

Of the short bones, I have found the vertebrce to be the most abundantly supplied with nerves, and especially their bodies. The nerves enter posteriorly in company with the arteries and veins (venoe basi-vertebrales), as well as anteriorly and on the sides, together with the vessels, and are distributed in the marrow of the spongy substance. In the astragalus also, calcaneum, os naviculare, cuboideum, and cuneiforme internum, I have noticed, in the larger, several, and in the smaller, at least one nervous filament.

In the scapula and os innominatum, the nerves are very numerous, entering these bones chiefly at the points before indicated, with the larger vessels, sometimes on the expanded portion, sometimes in the neighborhood of the articular cavities. In the sternum also, and in the flat cranial bones, the existence of nerves is demonstrated without difficulty. In the latter, I have observed, even in the new-born infant, in the occipital and parietal bones, nerves entering through the foramina emissaria, which at this period also contain an artery; and in the adult, there are found in the parietal, frontal, and occipital bones, although rarely, yet occasionally, microscopic filaments on the smaller arteries, which enter the compact substance from without, and probably penetrate as far as the diploë.

From these observations, together with those of Kobelt, Beck, Engel, Luschka, \&c., there can be no doubt that the bones are richly supplied with nerves. With respect to the origin of these nerves, they have already been traced by previous observers to the cerebral and spinal nerves, as for instance the nerves of the diaphyses of the femur, tibia and humerus, to the $n n$. cruralis, tibialis, ischiaticus, and perforans Casseri, as well as a nerve of the frontal bone to the $n$. supraorbitalis, which observations, as far as they relate to the tibial nerve, have been confirmed by my own, and by those of Luschka in the case of certain 
of the cranial bones, and of the vertebroe. Nevertheless, the sympathetic participates in their formation, as Luschka, and before him, Kobelt have observed with respect to the vertebral nerves. Microscopical examination confirms this, inasmuch as the nerves of bone, in their trunks and terminations, resemble in every respect the sensitive branches of the spinal nerves, and contain, in the trunks, one-third of fibres, measuring $0.005-0.006$ of a line; two-thirds measuring $0.002-0.004$ of a line; in the larger branches the majority of the fibres measure $0.002-0.003$, but some as much as 0.006 of a line; and lastly, in the finest ramifications, fibres of not more than $0.0012-0.0016$ of a line. The periosteal nerves, also, which may frequently be seen to be connected with the nerves of the bone, and may be traced to the nerves of the extremity, are derived, probably in the greater proportion, from the spinal nerves, although even in their case, perhaps, some participation of the sympathetic cannot be denied.

How the nerves of bone terminate, I have not observed, and can only remark, that from the nerves in the marrow, extremely delicate branches, composed of neurilemma and one or two fibrils, are ultimately developed; but as to what becomes of these I am ignorant. It is also, perhaps, worthy of notice, that in two situations, before their entrance into the bone, I have observed Pacinian bodies on the nerves; viz. on the diaphysal nerve of the titia, two lines before its entrance into the foramen, I noticed a single body, and two others on the largest nerve of the metatarsal bone of the great toe, also just before it entered the bone.

I have never yet detected nerves in the ligaments, in Man (the ligamentum nuchce of the Ox contains some fine nervous twigs, accompanying minute arteries; the twigs measuring 0.004 of a line, with fine fibres of $0.012-0.0015$ of a line), but have no doubt that they, like the tendons, inasmuch as that they contain vessels, are also furnished with a few scattered nerves. On the other hand, the interosseous membrane of the leg contains filaments derived from the interosseal nerve, which, formed of from one to three fibrils, measuring $0.003-0.004$ of a line, present distinct ramifications and free terminations of the primitive fibrils. A nerve of 0.03 of a line, which together with an artery entered the fibrous external part of the symphysis pubis, may here be mentioned. With regard to the cartilages, I have as yet noticed only in the cartilagecanals in the septum narium of the Calf, together with vessels (arteries), very distinct, fine nervous twigs, measuring $0.006-0.01$ of a line, with fibres of $0.0012-0.0016$ of a line thick. In the articular capsules numerous nerves exist, although they belong principally to the so-called fibrous capsules, and to the loose connective tissue external to the synovial membrane. In the knee I have seen nerves, even in the true synovial membrane, although in general they are rare, and are most distinct in the large vascular processes, which besides arteries, contain nerves of 
$0.007-0.008$ of a line, with fine, also dividing filaments of $0.0008-0.002$ of a line. I have also seen in the synovial membrane itself, close to the condyles of the femur, tolerably numerous nerves composed of delicate fibres.

$\S 100$. Development of the Bones.-In respect of their development, the bones fall into two groups, viz., into those which are preformed in $a$ cartilaginous state (primary bones), and into those which from a small beginning are developed in a soft blastema (secondary bones). The former, while yet in the cartilaginous condition, present all their essential parts (diaphyses and apophyses, body, arches, and processes, \&c.), and as far as their cartilaginous basis is concerned, originate like other cartilages, and continue to grow more or less in the same manner. They afterwards become ossified (in man, all of them) from within to without, transforming a portion of the cartilage completely into bone, so that what was the perichondrium becomes the periosteum, and afterwards attaining their ultimate figure, partly by means of the remaining cartilage, which continuing to grow with them is successively ossified, and partly by means of a soft, ossifying blastema, which is deposited layer upon layer on the inner surface of the periosteum. In the second group, the bone is formed from a very limited, soft, non-cartilaginous basis, and continues to grow at the expense of that substance, which is continually developed anew, first at the margins only, but afterwards also on the surfaces. When these bones have attained a certain size, the blastema out of which they have hitherto been developed may become, partially, cartilaginous, in which case the cartilage stands in the same relation to the bone as it does in the former instance. The greatest part of their formative substance, however, always remains in a soft condition, and from it, without its ever becoming cartilaginous, the principal bulk of the bone is produced.

Frequently as the development of the osseous tissue has already been discussed, still, in a general point of view, the mode in which the bones, as organs, originate has hitherto been little considered, and I believe that I was the first, in my "Zootomical Report," Leipzig, 1849, to establish the principal features of the process, and in my "Microscopical Anatomy," II. 1, p. 344, et seq., to trace it in its more particular details. H. Meyer (l. c.) agrees with me in most of the essential points, whilst Robin advances many different views, with which I do not accord, and has to some extent entirely misunderstood my statements.

$\$ 101$. The primary cartilaginous Skeleton of the human body, although less complete than the subsequent osseous framework, is still sufficiently extensive. We find as portions of it: 1. A complete vertebral column, with as many cartilaginous, as there are afterwards osseous 
vertebroe, with cartilaginous processes, and with intervertebral ligaments. 2. Cartilaginous ribs, and a cartilaginous, entire sternum. 3. Wholly cartilaginous extremities, with as many and similarly formed pieces as there are afterwards bones, with the sole exception of the pelvic cartilages, which constitute a single mass. 4. And lastly, an incomplete cartilaginous cranium. This primordial cranium, as it is termed ("Mikrosk. Anat.," tab. iii. figs. 1-3), forms originally a continuous cartilaginous substance, which corresponds, for the greater part, with the occipital bone (except the upper half of the expanded portion), the sphenoid (except the lamina externa of the pterygoid process), the mastoid and petrous portions of the temporal bone, the ethmoid, the inferior turbinated bones, the ossicula. auditus and the hyoid bone; but it also presents some cartilaginous portions, which never become ossified, either remaining in the cartilaginous conditions during life, or afterwards disappearing; as for instance, Meckel's process, two cartilaginous lamellæ below the nasal bones, a narrow cartilaginous band connecting the styloid process with the os hyoides, and two others, one of which extends from the outer part of the ala parva laterally to the lamina cribrosa, whilst the other stretches upwards and forwards from the cartilaginous, mastoid, and petrous portions of the temporal bone. Consequently, in the cartilaginous cranium of man, the vault of the skull is totally wanting, and almost all the lateral portions, as well as nearly all of what afterwards becomes the facial bones; nevertheless, at all events in the true cranium, the parts not formed of cartilage are closed by a fibrous membrane, representing in fact the further development of the soft, primordial, cranial capsule, so that the cranium at this time, though only in part cartilaginous, is yet fully as complete as at an earlier period, and always corresponds to its original soft rudimental form. In other Mammalia, as for instance, in the Pig, the cranium is much more completely cartilaginous ("Microskop. Anatomy," tab. iii. figs. 4, 5).

The complete development of the primordial cartilage, considered histologically, has not yet been accurately traced in all its stages, either in man or in mammalia. If we wish, therefore, to obtain anything like a sufficient idea of it, we must at present have recourse in a great measure to the lower Vertebrata. If the cartilage of the spinal column and of the head be examined in the batrachian larva, it is readily seen, that they are invariably constituted, while still in the soft state, of the same formative cells with vitelline corpuscles, as all the other organs. Before the development of the external branchix, these cartilage-cells present the form of closely approximated spherical cells, 0.007 to 0.009 of a line in size, with nuclei measuring $0.0045-0.006$ of a line, and filled with the well-known vitelline corpuscles; afterwards, when the branchiæ have made their appearance, the granular contents of the cells begin to disappear, from within to without, whilst the nuclei become more distinct, 
lying in a clear fluid within them, and at the same time the cells slowly increase in size. When the branchiæ have disappeared, all the cartilagecells are already quite transparent, with distinct nuclei and walls, and they now gradually increase to the size of $0.018-0.024$ of a line, and the nuclei to that of 0.005 and 0.007 of a line; the cells, from their mutual pressure, become polygonal, and constitute one of the most delicate cellular tissues possible. They now also begin to multiply by endogenous cell-formation around portions of the contents (Nägeli), so that in each cell two secondary cells are formed around the two nuclei produced from the original nucleus, and entirely fill it; at the same time they again increase, though very slowly, particularly in certain cartilages of the head, until they attain a size of $0.013-0.018$. of a line, and in some places of not more than $0.006-0.013$ of a line; whilst between them, a thick interstitial substance is formed out of the coalesced walls of the different generations of cells. With respect to man and the mammalia, it can only be stated as a supposition, that the cartilage-cells originate in a modification of the primordial formative cells. This supposition is favored by the circumstance, that in a human embryo of eight or nine weeks, the outer extremities of which were just developed, they presented scarcely a trace of formed cartilage, the innermost cells of the rudimentary extremities being hardly distinguishable from the outer. They were 0.004-0.006 of a line, in size, spherical, with grayish granular contents, and indistinct nuclei of 0.003 of a line, and formed a tissue of some consistence, without any appre-

Fig. 129.

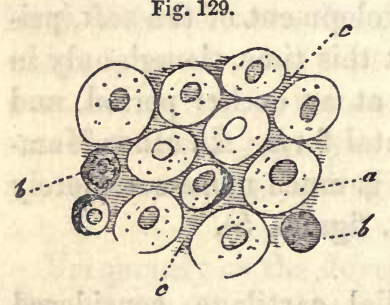
ciable interstitial substance. The corresponding cells in the embryo of a sheep 6-7 lines in length were somewhat larger, although the embryo was smaller than the human foetus above noticed. In this case (Fig. 129) they measured for the most part, $0.006-0.01$ of a line, had distinct walls, nuclei, and clear, aqueous, only slightly granular contents, and were lodged in a scanty homogeneous interstitial substance, so that they were only partially or not at all in contact with each other. The contents of only a very few cells were still in the opaque condition, and these were without any visible nucleus, others exhibited the commencement of transparency from the metamorphosis of their contents. The further development of the cartilage up to the end of foetal life, except in its ossification, presents these characteristics, viz.: (1.) That the cells precisely like those in the batrachian larva, continually increase by endogenous cell-formation, whilst precisely as in the same instance, there is no

FIG. 129.-Cartilage-cells from the humerus of an embryo of the Sheep, 6 lines long: $a$, cells with nucleus and clear contents (two cells retain remains of the earlier thick contents); $b$, cells with consistent contents, without visible nucleus; $c$, intercellular substance. 
indication whatever afforded, of the production of cells independently of those already in existence; and (2.) That the interstitial substance, which in this case is manifestly formed, for the greater part, independently of the cell membranes, is always increasing. With respect to the cells, they are in the second costal cartilage of a four-month foetus, according to Harting, 0.0036 of a line long, 0.0023 of a line wide, and consequently their aggregate bulk pretty nearly corresponds with that of the interstitial substance. In the embryo of the Pig, 3.5 lines long, the space occupied by the nucleated, clear, thin-walled cells, is, according to Schwann, thrice as great as that taken up by the interstitial substance. In a five-months' human embryo, I have myself noticed the cartilage-cells, $0.003-0.008$ of a line in diameter, with and without secondary cells, some with, and some without distinct walls, and separated from each other by a perfectly homogeneous substance, 0.002 0.005 of a line thick. In the new-born child they measure, according to Harting, $0.032-0028^{\mathrm{mm}}$ in length, and $0.0072^{\mathrm{mm}}$ in breadth, and are three or four times as numerous as in the fotus at four months; but on the other hand, they occupy considerably less space, proportionally, than the interstitial substance, the bulk constituted by which is more than double that of the cells. After birth, in the non-ossifying cartilages, the interstitial substance and the cells increase in pretty nearly an equal ratio, so that their relative proportions in the adult are about the same as in the infant at birtb. In the adult the cells are from 8 to 12 times larger than in the new-born child (Harting), but, according to him, their number is diminished, so that they amount to not more than half of what existed in the child, which is explained upon the supposition of a coalescence of the cells. The numbers given by Harting do not appear to me to afford sufficient ground for the establishment of his position; and even should it be established, I cannot agree in the explanation offered, not being aware of a single fact in favor of the notion of a coalescence of cartilage cells.*

* [We have deferred to this place the remarks we have to make with regard to the structure of the cartilage, and its mode of growth; as a just conception upon this subject appears to us to be essentially necessary to a comprehension of the mode of development of Bone,in fact, we might say, to clear notions upon the structure of the tissues generally; for as we shall show more at length below, it was upon the structure of cartilage, and what he supposed to be its similarity to that of vegetable tissue, that Schwann based the whole nomenclature of his cell theory.

Now we may so far anticipate what we shall have to show hereafter, as to premise that Schwann was misled upon two essential points, - the first being the supposition that the histological elements of plants and animals are primarily independent cells; the second, the notion that the "nucleus" of the animal, is homologous with the nucleus of the vegetable tissue. It is, we believe, from the inextricable confusion produced by these fundamental mistakes, which have been adopted by almost all Schwann's successors, that one-half of the controversies with respect to the structure of cartilage and the process of ossification have arisen. And yet to one who is free from them, nothing can be simpler.

We have already (note p. 56) referred to the structure of fœtal cartilage, but it may here 
§102. Metamorphoses of the primordial cartilaginous Skeleton.-Of the primordial cartilages, one portion undergoes further development with the rest of the skeleton, constituting the permanent cartilages of

be described more at length. We found the cartilage of the septum nasi of a four-months' human fœtus to be composed of a homogeneous, soft matrix, without structure of any kind, in which lay imbedded, rounded or irregular vesicular bodies, varying in diameter from $3000^{3}-\frac{3}{500} \sigma^{\text {th }}$ of an inch; the commonest size, however, being $3000-20^{1} \sigma^{\text {th. The }}$ "corpuscles" frequently contained one or more granules, sometimes very small, sometimes larger, and of a distinctly fatty nature; such fatty granules, also, were sometimes to be found in the matrix around the corpuscles.

The cavities in which they lay, were, for the most part, just large enough to contain them, and presented no walls or sharp lines of demarcation of any kind from the surrounding substance.

When the corpuscles were as large as ${ }_{160}^{2}$ th of an inch, they occasionally contained a round body of rather less than $5 \frac{1}{50} 0$ th, as a "nucleus."

The matrix was in some parts pale and indifferent; but where the tissue had taken on its definitely cartilaginous nature, the chondrinous substance into which it was converted refracted the light much more strongly. In this part also, the cavities in which the corpuscles lay, were often of considerably larger dimensions than the latter, and their walls exhibited a sharp, dark line of definition from the surrounding substance, which was often brought out much more strongly by the action of acetic acid. It appeared, in fact, that the conversion into chondrin had not quite reached the inner surface of the cavities, and hence they were chemically and optically distinguished from the surrounding substance.

Now, of course, it matters very little what names are given to these parts, so long as they are used only in one sense. Schwann considered the corpuscles to represent the "nuclei" of plants, and therefore gave them that name. Henle, Reichert, Kolliker, and nearly all their compatriots, Todd and Bowman, Leidy and Sharpey-follow him. As a consequence, they consider the wall of the cavities to represent the cellulose "cell-wall" in plants; and there has been much controversy as to how much of the matrix of the cartilage results from the union of these "cell-walls," how much from the development of an intercellular substance; a controversy which has extended itself to the determination of the hoinologies of the elements of every tissue. We must confess, it seems to us that the disputants have been fighting for a shadow.

If, in fact, the youngest cartilage be composed of cells with distinct walls enclosing the corpuscles, of course these cells may be united by an intermediate, "intercellular" substance; and it will be an important question to determine in the further course of development what arises from the walls and what from the substance which unites them. But if, on the other hand, all this be pure hypothesis; if young cartilage be, as we have said, composed of nothing but a continuous, homogeneous matrix, in which the corpuscles are imbedded, but in which no other structure exists, what becomes of the controversy?

We believe that not merely will the account we have given, be found to be correct, by any one who will without prejudice examine into the subject, but it seems to result from the observations, even of those who have interpreted the facts otherwise.

Schwann ("Mikros. Unters.," pp. 112, 113) describing the development of the cartilage of Pelobates, says: "The new cells arise in the cytoblastema (matrix nobis). . . . . We see at first mere cell-nuclei (corpuscles nobis), which are somewhat smaller than the nuclei of the full-grown cells, $a, b$; partly nuclei, which are closely surrounded by a cell, $c, c$; in short, all transitional forms, from mere cell-nuclei and nuclei surrounded by small eells, to fully formed cells; so that here, development takes place as in small cells, and the nucleus is their actual cytoblast. . . . . The cell-membrane becomes distinct only in the full-grown state."

In the next page, Schwann speaks of the free-swimming nucleated corpuscles which he obtained from the ossifying cartilage of a fœtal pig, and which he considers to be identical with the bodies already described in Pelobates. In reality, however, these bodies are not 


\section{the nose, joints, symphyses, and synchondroses; a second disappears altogether in the course of development (certain cranial cartilages, vide}

cells in the same sense, being merely the nuclei of Schwann and the nucleated form of the corpuscles to which we have referred above.

Whatever Schwann's words may indicate, then, his observations tend to precisely the same conclusion as our own.

Henle ("Allg. Anat." pp. 803-808) follows Schwann, and equally fails to discriminate the cells in Pelobates from the "cells" in the fœtal pig.

Reichert (in his admirable work, "Ueber das Bindegewebe," 1845) recognizes the fact that the cartilage-corpuscles are "nuclei" in Schwann's sense, and refers the appearance of a distinct wall in the cavities, to an optical delusion. He asserts that young cartilage is composed of distinct cells closely united together, without any measurable intercellular substance; as the cartilage grows, the latter increases, and eventually the cell-walls disappear. The only evidence of the existence of these cells and intercellular substance offered by Reichert, however, is the mode in which the tissue may be broken up; a kind of evidence whose value the purport of the rest of his book is to reduce (and most successfully) to nothing.

In effect, therefore, Reichert's observations come to the result already stated, that the foetal cartilage is composed of a homogeneous matrix, in which the corpuscles are dispersed.

Robin ("Observations sur l'Osteogenie") takes nearly the same view of the structure of cartilage as that we have indicated. "Cartilage is composed," he says, " of a homogeneous, amorphous, dense, elastic, hyaline basis (substance fondamentale), in which cavities are hollowed out,- - cartilage-cavities. In each of these cavities we find one or many (sometimes 20 to 30) cells, - cartilage-cells, whose parietes cannot be demonstrated to be distinct from their cavity. These cells are more or less granular, and have a nucleolated nucleus. . . . . In the fœtus, up to the age of four or five months, more or less, the cartilage cavities do not enclose one or more cells, but one or many masses of yellowish granulations, all of nearly the same size. These masses are more or less distinctly defined at their edges, in general indistinctly, and nearly reproduce the form of the cavity without ever filling it. They may be called cartilage-corpuscles. Authors have not generally remarked this fact. By degrees the cells which replace these corpuscles are developed. These cells are formed all at once; but the grades of the process as regards the commencing cell or the pre-existing granulations are as yet but little known. . . . . Some anthors wrongly call the cavities excavated in the fundamental substance, cartilage-cells; and to the true cartilage-cells and masses of yellowish granulations or corpuscles, referred to above as existing in the fœtal state alone, they give the name of contents."

Remak ("Ueber die Entstehung des Bindegewebes und des Knorpels," Müller's "Archiv," 1851-2) appears to have been the first, definitely to recognize the cartilage-corpuscles, as the homologues of the primordial utricles of plants, - a great step, and one which appears to us to lead to most important consequences. Like Schwann, however, led away by the generally assumed anatomical independence of the vegetable cells, Remak interprets the structure of cartilage in the same manner, and speaks of the secretion of the chondrinous wall by the primordial utricles, as "parietal-substance" within the primary cell-membranes. He adduces no evidence, however, that the facts are other than as we have stated them to be.

Virchow. ("Die Identitat von Knochen-, Knorpel-, und Bindegewebe-körperchen so wie uber Schleirngewebe," "Verhandlung d. Phys. Med. Gesellschaft," 1852) is an important witness in this matter. He says, "I have anew convinced myself that the so-called cartilagecorpuscles are actual cells which lie in a cavity of the basis (Grundsubstanz), or in a cellcavity presenting a double contour, and possess a membrane, granular contents, and a frequently nucleolated nucleus. In the neighborhood of the line of ossification, in growing cartilages, as well as in the young callus-cartilage of fractures, these cells are of very large size, clear, and round; in the neighborhood of the articular extremities, excessively small, 


\section{$\S 101)$; the third and greatest part, ultimately becomes ossified, and constitutes all the bones of the trunk and extremities, and a great part of those of the cranium. All these bones are ossified, essentially in the}

compressed, and dark. Under favorable circumstances, the cells, in simple cartilage, may be isolated, and their peculiar relations with regard to acetic acid, which generally renders them darker and collapsed, may be exhibited. Water also causes them to collapse, and they thus occasionally form peculiar, jagged corpuscles, which one might be readily tempted to confound with branched cells. The larger the original cell was, so much the more branched does its collapsed mass appear." ..... (p. 152.) "It might have been expected that in the course of ossification of the cartilage, these cells would be seen to pass into the irregular, anastomosing bone-corpuscles; but nothing of the kind is visible. . . . . A point of difficult determination is, in general, the existence of actual cells in the small flat cavities of cartilage, e. g. towards the surface. Very frequently it would here seem as if the membrane of the cell had coalesced with the intercellular substance, and only the contents with the nucleus had remained behind. But on careful investigation especially under the prolonged operation of acetic acid, frequently after maceration in hydrochloric acid, we clearly see a complete cell with a nucleus and contents in the cavity" (p. 153).

Virchow gives no figures, but the above passages furnish so accurate an account of what we have ourselves seen in young and in fully-formed cartilage, that we have thought we could not do better than cite them. The jagged appearance of the corpuscles to which he refers, is very common, and we have been led to suspect that it may arise from the same cause as the very similar appearance often exhibited by the colorless corpuscles of the blood, viz., a protean throwing out of processes.

When Virchow, however, describes the passage of these "cells" into the branched or stellate corpuscles of fibro-cartilage, and considers the latter to be metamorphosed cartilagecorpuscles, he confounds together things which are essentially different. Careful examination of fotal fibro-cartilage, e. g. the intervertebral fibro-cartilage of the Kitten, shows that the stellate body is the wall of the cartilage cavity, with processes which run out from it, the original corpuscle remaining in the interior of the cavity, either unchanged or becoming gradually lost, or fused into one mass with its walls.

The account of the structure of cartilage given by Tomes and De Morgan (1. c., pp. 15, 16) in all essential points agrees with that of Virchow. They call the corpuscles, granular cartilage cells.

To recapitulate:-the facts contained in other observations, as apart from the interpretation, appear to agree perfectly with our own; the result of which is, that in the fotal state, cartilage is composed of a homogeneous matrix, in which lie the corpuscles, in cavities which they just fill; that their relation to the matrix is exactly that of the primordial utricles to the cellulose wall in plants, and that like this they may or may not develop a nucleus; that with age they enlarge, but not so fast as the cavities, the walls of which become chemically altered into chondrin, a change which often takes place in such a manner as to give rise to a lamination or to a difference in composition of the inner and outer portions. If the cartilage be converted into fibro-cartilage, the outer part becomes changed into collagen, while an alteration into a substance resembling elastic fibre, is effected in the inner portion, and in the direction of certain lines radiating from it, just as we have seen the elastic element to be developed in connective tissue (see $\$$ on Connective Tissue).

So much for the structure of cartilage: with regard to its development and multiplication we must equally demur to the statements in the text. It is, indeed, very true, that no new cartilage-cells arise independently of those which pre-exist: but in opposition to Professor Kölliker we must agree with Leydig, Robin, Remak, and Tomes and De Morgan, that the multiplication of the cartilage-cells invariably takes place by a process of division exactly analogous to that which occurs in plants. So far as we have seen (and in ossifying cartilages, and in that of the Skate, it is easy to trace the process), the corpuscles first become constricted, being found occasionally of an hour-glass shape; and eventually divide. The matrix then grows in, so as to separate the two, and the process of fission is complete.-Trs.] 
same way. At one or more points (puncta ossificationis), in their interior, a deposition of calcareous matter commences, simultaneously with a change in the cartilaginous elements; which transformation proceeds on some or on all sides, continually converting additional portions of the cartilage into bone. Whilst this is going on, the cartilage, in most cases, ceases to grow in one direction, and, consequently, is there soon entirely converted into bone, whilst in others its growth continues, so that a new cartilaginous, plastic material is furnished for the progressive increase of the bone, which material, as in the epiphyses of the cylindrical bones, is sometimes developed into distinct ossific centres or nuclei. When the whole of the cartilage has been converted, and its perichondrium become periosteum, the bone does not cease to enlarge, but a new and peculiar mode of formation is now set up, in all these places, until its growth is completed. This consists in the ossification, from that surface which is in contact with the bone, of an organized, soft, plastic material, which is deposited on the inner surface of the highly vascular periosteum, and in proportion as this conversion into bone takes place on the one side, fresh, fluid materials for it are afforded by the periosteum on the other.

$\S$ 103. Changes in the ossifying Cartilage.-The active vegetative process in the cartilage-cells when ossification is going on, depends upon this, - that the cells which were hitherto of small size, and contained but few secondary cells, begin to grow, and successive generations of cells to be produced in them, as may, also, be seen at the ossifying margins of bones already existing, in which situation larger cells may be noticed close to the bone, and others, which are smaller in proportion to their distance from it. All the cells which are engaged in the incipient formation of the bone, present clearer and, less frequently, granular contents, a distinct, vesicular, round nucleus, with nucleolus and readily distinguishable walls; they are very quickly altered, however, on the addition of water, acetic acid, alcohol, and by drying, \&c., so that the contents contract around the nucleus, and form a roundish, elongated, irregular, even stellate, granular, opaque body (cartilage-corpuscle of authors). Their size and mode of grouping vary, not inconsiderably, according to age and situation. With respect to the former, they exhibit during embryonic life a constant increase, whilst after birth they appear to retain a uniform size; and, as regards the latter, it may be stated as a law, that where the ossification of the cartilage proceeds in one direction only, the cells, at the osseous border, are disposed in rows. This is most distinctly seen, as has been long well known, in the extremities of the diaphyses of the larger cylindrical bones, where the rows of cells are very prettily arranged in parallel lines close together, and are of considerable length; it is also evident in the other long bones, as well as in 
many others where the cartilage ossifies only on one side, as in the connecting surfaces of the vertebroe. Where, however, the ossific nuclei in the centre of a cartilage enlarge on all sides, the cartilage-cells are confusedly grouped in roundish, or oval, irregular little masses, as in the short bones at their first formation, and in the epiphyses. An accurate comparison of the cells which are closer to the ossifying margin, with those more remote from it, and of the groups formed by them, at once

Fig. 130.

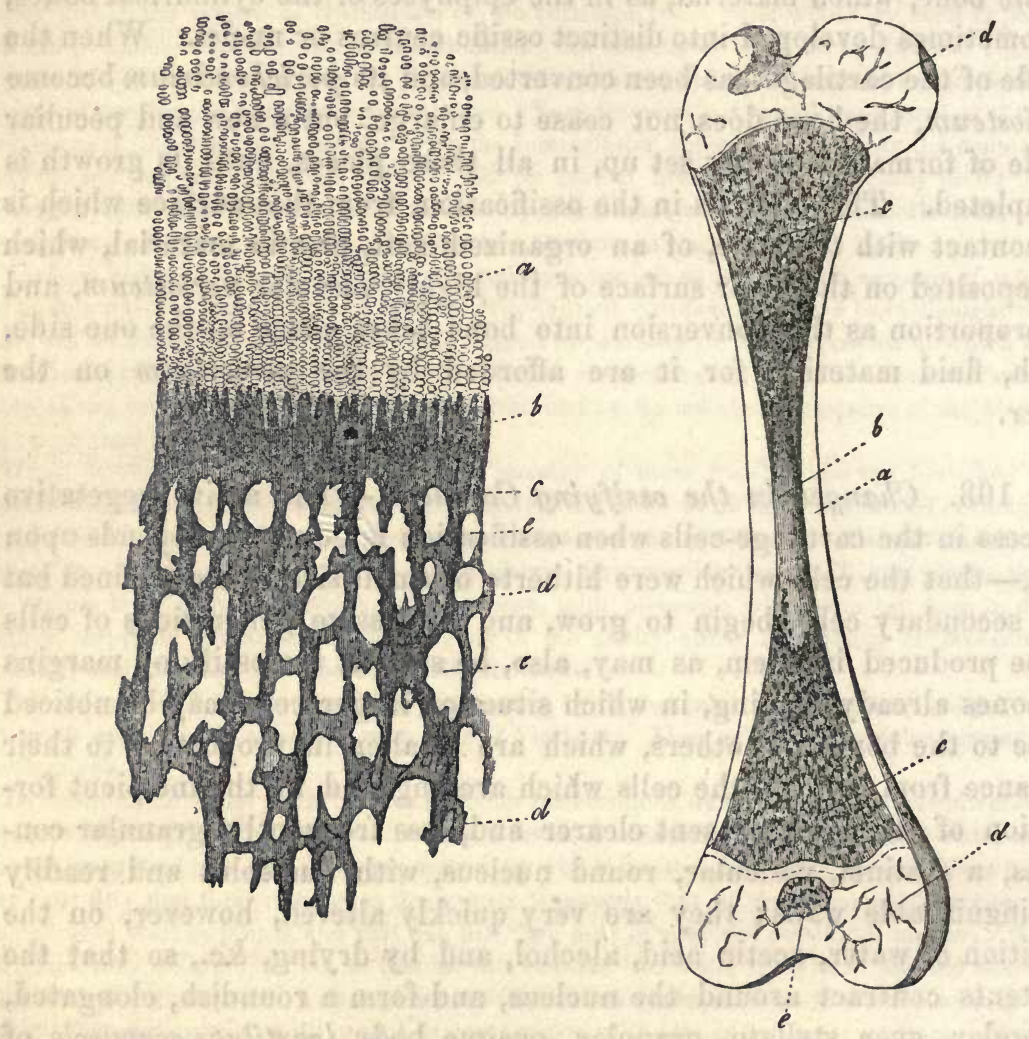

shows that their particular disposition is directly related to their mode of increase. Each individual group (or even two of them) corresponds,

Fia. 130.-Perpendicular section from the ossifying border of the shaft of the femur of a child a fortnight old: $a$, cartilage, in which the cells the nearer they are to the ossifying border are placed together in more extended longitudinal rows; $b$, ossifying border, the dark streaks indicate the progressive ossification in the intercellular substance, the clearer lines the cartilage-cells which ossify subsequently; $c$, compact layer of bone near the ossifying border; $d$, the substantia spongiosa formed in the osseous substance by resorption, with cancelli, the contents of which are not shown.-Magnified 20 diameters.

Fig. 131.-Femur of a child a fortnight old, natural size: a, substantia compacta of the shaft $; b$, medullary cavity; $c$, substantia spongiosa of the shaft $d$, , cartilaginous epiphysis with vascular canals; $e$, osseous nucleus in the inferior epiphysis. 
in a certain measure, with a single primordial cell, and represents all the descendants which in course of development have proceeded from it. In the one case, all these newly-formed cells are disposed, one behind the other, in a single or double linear series; and in this way are produced, by their further increase, the rows of cells above described, whilst in the other they constitute a more globular mass. The primordial cells (first parent-cells) during this procedure, sometimes disappear as distinct organisms, owing to the coalescence or fusion of their walls with the interstitial substance, sometimes not; and the same holds good with those of the subsequent generations. The latter is usually the case in the rounded masses of cells, owing to their smaller size, and around them a contour line may for the most part be recognized, which is nothing more than the distended wall of the first cell ; whilst in the rows of cells, the walls of the original cells are not, usually, so merged in the intercellular substance as to escape recognition. The entire matrix, in which the justdescribed, enlarged, and actively multiplying cells are enclosed, varies very considerably in thickness in the different cartilages; scanty around the osseous nuclei in the epiphyses and short bones, it is $\frac{1}{4}$ to $\frac{1}{2}$ a line thick in the diaphyses. It is universally characterized by its yellowish, transparent color, and its streaky, apparently fibrous fundamental structure, from the other cartilaginous parts, which are, as usual, bluishwhite, with a homogeneous or granular interstitial substance.

The vessels met with in the ossifying cartilages constitute a phenomenon well worth attention; from the middle of foetal life onwards, they occur in many situations, preceding by a shorter or longer time the appearance of the osseous nuclei, and accompanying their increase. I have observed them in the articular cartilage of the epiphyses of the long bones even in a person 18 years old. They entered the cartilage in great number, perpendicularly from the bone, ramifying and terminating a little below its free surface. The cartilage-vessels invariably lie in wide canals (measuring, even in a five months' fotus, 0.02-0.04 of a line), excavated in the cartilage, and bounded by narrow, elongated cartilage-cells, - the vascular canals of cartilage, or cartilage canals,which enter the cartilage from the perichondrium, and, when a vascular ossific nucleus exists (diaphysis), also from the border of the ossifying portion itself (though in less number, at all events at an earlier period), penetrate it in straight lines, in various directions, giving off a few branches, and, to all appearance without any anastomoses, or other kind of interconnection, end, for the most part, in blind, club-shaped dilatations. These canals are produced by a resolution of the elements of the cartilage, in the same way as the medullary cavities of the bone itself, originally contain a plastic material composed of minute rounded cells (cartilage-marrow), corresponding to the foetal cartilage-marrow, and develop in a short time out of this material, true sanguiferous vessels, 
and a wall composed of more or less developed connective tissue, and subsequently also of elastic fibrils. As concerns the vessels themselves, I have sometimes found, in a canal, only one large vessel (frequently very distinctly arterial, with muscular walls), sometimes two such, sometimes capillaries in various numbers, but I am unable to explain how the circulation is carried on in these vessels. There must either be anastomoses between the vessels of different canals, or if the latter be really closed, arteries and veins both probably exist in one and the same canal. The object of these vessels of cartilage appears to be one of a double character; in the first place, to convey the materials requisite for its growth and further development; and secondly, to promote the ossification. The former of these functions is very manifestly carried out in the thick epiphysal cartilages, which grow to such a length before they become ossified, and even afterwards continue to enlarge; and the latter is probably effected principally in the short bones, which do not contain vessels until just before the commencement of ossification. Notwithstanding this, however, it is not intended to imply, that a cartilage cannot grow, nor become ossified without vessels; but although the latter condition does in fact obtain in animals, and probably also in man, normally in certain situations (on the appearance of the first points of ossification of the embryo, those of the ossicula auditus, \&c.), still, this does not prove that the vessels when existing have no concern in the processes adverted to; and consequently it cannot be admitted, as lately supposed by $\mathrm{H}$. Meyer, that they are to be regarded in the light of accidental productions, and as standing in no necessary relation with the development of the bone.

$\S 104 .-$ Ossification of the Cartilage.-The ossification of the matrix generally precedes in some degree that of the cartilage-cells; and, under normal conditions, is primarily effected by a granular deposition of calcareous salts (calcareous granules as they are termed). Where the cells are disposed in rows, at the ossifying border, this deposition of earthy matter always proceeds in the fibrous substance between the rows of cells, forming processes, which, in a longitudinal section, assume the appearance of pointed teeth, and surround the lowest portions of the rows of cells like short tubes. The same disposition, essentially, is also manifested in other situations, where the cartilage cells constitute more rounded groups, only, that in this case the ossifying matrix surrounds them more in a reticular manner. The calcareous granules or particles, the first visible deposit of the earthy salts of bones, are of a rounded angular figure, white by reflected, opaque by transmitted light, easily dissolved with effervescence in acids, and differing in size in different bones, from immeasurable minuteness up to 0.001 , or even 1.002 of a line; their size, however, does not appear to be regulated 
by period or situation, although there is no doubt that they are frequently, in one place, of uniform minuteness, and in others uniformly of coarser character, but rather by some change occurring in the supply of plastic material to the ossifying border. If this earthy deposit be traced in microscopical sections, from the margin of the ossification into the interior of the young bone, it will be apparent, that it is to it, for a certain distance, although with diminishing distinctness, that the granular and opaque aspect of the margin itself is due; the substance gradually becomes more homogeneous, clearer, and more transparent, ultimately acquiring the aspect of perfect bone. According to all appearance, the primordial earthy granules or particles become gradually fused together, and thus impregnate the whole tissue of the matrix of the cartilage, instead of, as before, separate portions, and thenceforth disappear as isolated, distinguishable particles.

With respect to the formation of the bone-cells, I believe, that owing to the discovery of an excellent subject for their observation, viz., rachitic bone, I have put the matter, as regards the most essential particulars, in a clear point of view. The bone-cells are formed, - as Schwann thought possible, and Henle supposed, from analogy with the lignified vegetable cell with pores or dotted canals, - from the cartilage-cells, by the thickening of their wall, with the simultaneous formation of canalicular vacuities in it, and its ossification. In the ossifying shaft of a rickety bone (Fig. 132) the morpho-

Fig. 132.

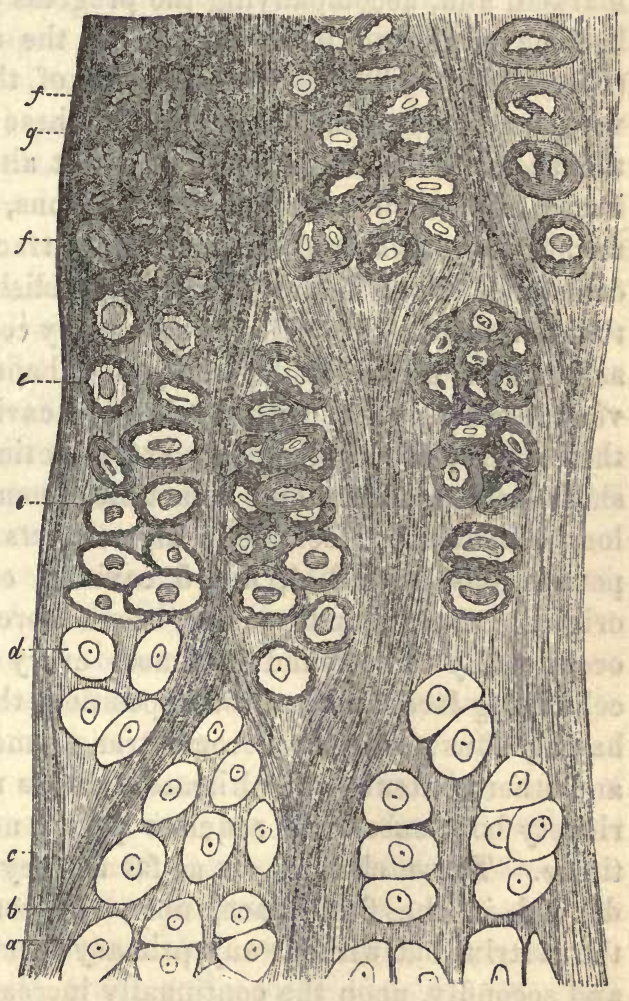
$\operatorname{logy}$ of this process may be most beautifully observed. If the rows of

Frg. 132.-From the ossifying border of the condyle of the femur of a rachitic child, two years old, $a$, cartilage cells, simple and parent cells in series; $b$, more homogeneous; $c$, striated matrix between them; $d$ : cartilage-cells at the commencement of their transformation into bone-cells; $e$, the same further advanced, with very much thickened walls, indication of canaliculi, commencing deposition of calcareous matter in the walls, whence their darker color, though still with distinct nuclei; $f$, bone-cells still more developed and more ossified, in an equally ossified, matrix.-Magnified 300 diameters. 
cartilage-cells of the ossifying border, which in this case are of larger size, be traced from without to within, it will soon be found, that at the point where the deposition of calcareous salts (which takes place for the most part without the formation of the calcareous granules) commences, they exhibit, instead of a membrane indicated by a single, tolerably strong line, a thicker coat, which on the inner side presents delicate indentations. Even when the thickness of this membrane does not exceed 0.001 of a line (Fig. 132, $d$ ), it is obvious that the cartilage-cells are about to be transformed into bone-cells; and this becomes still more evident, when, further on in the bone, the thickness of the membranes in question, together with the simultaneous diminution of the cavity of the cell, is seen to be constantly increasing, the indentations of the interior contour line to become more and more marked, and, accompanying the progress of these changes, the walls to become more and more dark from the addition of calcareous matter (Fig. 132,e). The slow ossification of the matrix between the cells is very favorable to the observation of these changes, allowing not only of the accurate investigation of the first alterations in the cartilage-cells, but also of their subsequent conditions, at a time when they must be termed bone-cells and lacunce, being traced step by step. To this circumstance alone is also due the establishment of the interesting fact, that cartilage-cells, enclosing secondary cells within them, are converted, as a whole, into a single, compound bone-cell. Cells of this kind are very frequently met with, having two cavities, which cells, according to their degree of development, are sometimes wide and furnished with short prolongations, and sometimes from their contracted cavity and long canaliculi, resemble in all respects perfect, bone-lacunæ. Compound cells, with 3,4 , and 5 cavities, each with the remains of the original contents and nucleus, occur more rarely, though even such are occasionally to be found in almost every preparation. The cartilagecells lying free, and in close apposition, though in a non-ossified matrix, having thus evidently become transformed into bone-cells with nuclei and other contents, the ultimate changes now take place from which the rickety bone-substance acquires pretty nearly the nature of the sound tissue. These changes, in as far as they effect the bone-cells, chiefly depend, in the first place, upon the commencement of ossification in the matrix, but without any primary formation of calcareous particles; and secondly, upon the continually increasing deposition of earthy matter in it, and in the thickened cell-walls, owing to which, the new bonesubstance, to the naked eye, becomes more and more white, and under the microscope appears more and more dark and transparent; it now, also, becomes more homogeneous, and the abrupt limits of the bone-cells gradually less and less defined, till at last they appear, not as cellular organisms lodged in the matrix, but to be confused with it, being recog- 
nizable only from their peculiar stellate cavities, - the so-termed bonecorpuscles, or lacunce and canaliculi.

With the knowledge thus obtained of the formation of the lacunx in rachitic bone, the endeavor to arrive at an insight into the same process in normal bone, is no longer attended with as much difficulty as before, when the inquirer was involved in a maze of hypotheses of the most various kinds, and all without any certain foundation. The investigation of the conditions attending the development of bone, both in man and other animals, must nevertheless still be regarded as troublesome, and frequently little worth the pains bestowed upon it. It is, perhaps, certainly manifest (vid. "Mik. Anat.," tab. iii. fig. 6), that the bone-cells, a little beyond the limit of ossification, become thickened, and, still presenting the remains of their cavity and the nucleus, beset with calcareous particles; 'and although such incrusted cells may even be isolated, yet the mode in which the changes are effected further on, is not, beyond a short distance, I must affirm, to be seen with anything like the distinctness that it is in rachitic bone, because, more internally, the newlyformed medulla with its vessels, and the calcareous particles, render almost everything indistinct; and it is not till we get to the homogeneous and more transparent osseous tissue beyond, that distinct, but almost perfectly-formed lacunæ come into view. Nevertheless, from all that we see, there cannot be the least doubt, but that the processes are essentially the same as in rachitis, only, that in the healthy bone the ossification of the thickened walls of the cartilage cells, presents two stages, instead of only one, as in the former case, inasmuch as they first appear granular from the deposition of the calcareous particles, and afterwards homogeneous. Moreover, even in perfectly normal bone, in the adult, I have met with places (some of which, independently of me, have also been lately described by H. Meyer (l. c.)), such as the symphysis pubis, the synchondroses of the vertebroe, and those of the ilium, sacrum, and the points of insertion into the bones of certain tendons containing cartilage-cells. In all of these situations, at the line of junction between the cartilage or tendon and the bone, cartilage-cells of the most characteristic aspect may be seen, lying free in the cartilaginous matrix, and presenting the most various degrees of transformation into bone-cells; some, in particular, having thickened walls, and a more or less copious deposit of calcareous particles; while others are almost perfectly-formed bone-cells, with pores and a more homogeneous wall (Fig. 123); so that I am able to afford a certain support to the statement given above with respect to the mode of origin of the bone-cells, by the conditions presented in normal tissues also. In the last-named situations I have, likewise, very distinctly and very frequently noticed, half- or wholly-ossified parent-cells, containing from two to twelve secondary cells. 
There is another point in the development of the bone-cells still obscure, or at least that has not been directly observed, viz., how their pores or canaliculi become branched cavities, communicate with those of other cells and acquire open orifices in certain situations. All that is apparent in rachitic bone and elsewhere, is merely the circumstance, that the thickening of the ossifying cartilage-cells does not proceed with a straight but with an indented border, which is the case in fact from the beginning up to their completion, and that the bone-cells have, at first, more simple prolongations than afterwards. Observation teaches nothing beyond this. Now, as there can be no doubt that the canaliculi anastomose very freely, and also, that they frequently open on the outer surface of the bone, or into the cavities in its interior, I do not for a moment hesitate to express the opinion, that the canaliculi, arising as simple branches from the lacunce, are continued or further developed by absorption of the already-formed bone-substance. How such an absorption takes place, cannot, it must be confessed, be explained; but that affords no ground of objection to the opinion, because we see a similar process, though on a widely-different scale, take place in the formation of the medullary cavities and cancelli (vid. infra). It would appear to me, that currents of the nutritive fluid in the bone were chiefly concerned in this further development of the canaliculi; and the more so, because the first rudiments of the canaliculi, like the pore-canals of lignifying plant-cells, manifestly indicate nothing more than the points at which the ossifying cartilage-cells continue to admit and emit fluid; on which account, also, their direction is principally towards the internal and external surfaces of the bone, from which the nutritive plasma is derived. It appears to me highly probable, that after the complete ossification of the cartilaginous tissue, the nutritive fluid derived from the blood-vessels of the periosteum and of the medullary cavities (1.) finds new ways for itself towards the lacunce and their prolongations, which, as it may be said, alone are still open to it, and in this way effects their opening on the internal and external surfaces of the bone, and (2.) also burrows passages from the cavities lying nearest to it, and thus ultimately produces a ramification of them, and brings about numerous communications between the different cavities. In accordance with which, a secondary formation of canaliculi must take place, not only in the region of the thickened walls of the original cells, but also in the osseous matrix, and this to a considerable extent, as is at once evident, when the distances between the anastomosing cavities are compared with the diameter of the original cartilage-cells.

The development of the medullary spaces (cancelli) and of the medulla, is to a certain extent the last act in the transformation of cartilage into bone. The medullary spaces do not arise in a coalescence of the cartilagecells, but from a solution of the more or less perfectly formed bone-sub- 
stance, exactly like the large medullary cavities of the cylindrical bones. This is most distinctly and satisfactorily shown by the examination of the diaphyses of a sound or rachitic bone, but especially in the latter. At the limit of the ossification itself, the osseous tissue for a distance of about $\frac{1}{5}$ to $\frac{1}{3}$ of a line, is quite compact, without a trace of larger cavities, and is composed in part of the ossified matrix, and in part of cartilage cells, more or less advanced in their transformation into bonecells ("Mik. Anat.," tab. iii.); beyond this part, however, cavities, at first small, and more internally, larger, come into view, the whole relations of which show most convincingly that they do not originate in any development of the existing elements. They have an extremely irregular contour, are oval, or roundish and angular, and for the most part broader than the cartilage-cells, appearing to be eaten out, as it were, in the substance of the bone, and involving severally the compact tissue, matrix, and bone-cells. When the borders and limitary surfaces of these spaces are closely regarded, it is, in many instances, easy to notice bonecells more or less removed, half projecting from, or buried in the wall, and between them projections of the ossified matrix, so that no doubt can any longer be entertained with respect to the origin of the cavities. It must be confessed that there is as little to be stated, in this case, as in that of the origin of the analogous cartilage-canals, and the further development of the canaliculi of the bone-cells, with respect to the mode in which this absorption takes place; and the process is even still more inexplicable, because, allowing that it really does take place, there would then exist in the ossifying bone, at the same time and almost in immediate contiguity, a formation of bone and a resolution of the tissue, but very little less energetic. The above-described mode of formation of the cancelli, nevertheless, is a morphological fact, and consequently, the explanation of such a curious phenomenon becomes a problem to be solved by chemistry and physiology. As in the diaphyses, so in the ossification of all the other cartilages, medullary spaces are formed by the resorption of the inner portions of that part which is half ossified. But it must be stated, that these spaces do not present the same form, direction, and size in every bone; though with respect to this, it is unnecessary to offer any special remarks, since the relations of this primitive spongy substance are, in the main, the same as they are afterwards. Still, it may be remarked, that in many bones, solitary spaces are apparently developed immediately from cartilage-canals, seeing that some at least of the latter, at the limit of ossification, communicate directly with the spaces in the bone; and, moreover, that not unfrequently, cartilaginous elements not yet wholly converted in to bone-cells, are drawn into the process of resolution.

The medullary cavities, however they arise, are filled with a soft, reddish substance-foetal medulla. This substance at first consists of 
nothing but a small quantity of fluid and many rounded cells, with one or two nuclei and faintly-granular contents, of which I am unable to say how they originate, but only this much, that they are altogether new formations. In process of time these cells, which are in all respects identical with those which occur, in the adult, in certain bones (vid. supra), are developed in the usual way into connective tissue, bloodvessels, fat-cells, and nerves. The formation of bloodvessels proceeds with great rapidity, so that bones, very shortly after the development of the medullary spaces, exhibit bloodvessels in them; that of the fat and nerves takes place more slowly, although the latter, at the period of birth, of course with fewer filaments than subsequently, may be very readily perceived in the large cylindrical bones, even more readily than in the adult, because at this time the medulla may be more easily washed away from them and the great vessels. The fat-cells at this period are but few in number; the medulla, in man at least, being colored entirely red by the blood and the light reddish medulla-cells. After birth they gradually multiply, till at last, the marrow, in consequence of their great increase, and the disappearance of the medulla-cells, which are ultimately all transformed into the elementary tissues of the permanent medulla, acquires its subsequent color and consistence.

In many of the primarily cartilaginous bones of Birds and Amphibia, the ossification of the cartilage commences, according to Rathke and Reichert (1. c.), on the outer aspect of the cartilage, so that at first a cylinder of bone is formed with cartilage internally and at the extremities. The remainder of the internal cartilage then affords space to the medulla, whilst the epiphyses are formed out of that of the extremities.

If the contents of the cartilage-cells, the "cartilage-corpuscles" of authors, be really surrounded by a membrane, as Virchow supposes, it may be assumed that a similar tunic, analogous to the primordial utricle of the plant-cell (vid. sup. $\S 8$ ), exists also around the contents of the bone-cells, and that it takes an essential part, by its throwing out stellate processes, in the first formation of the canaliculi, their further elongation and ultimate anastomoses. In this case, also, the stellate and readily isolated cartilage-cells from an enchondroma described by Virchow (Würz. "Verh.," Bd. 1), around the internal portions of which the contours of rounded cells were visible, would be intelligible, and even the possibility of the isolation of stellate organisms from normal bone (vid. sup.) be explicable. My exposition of the formation of the lacunce in rachitic bone, is confirmed by Rokitansky and Virchow (Würz. "Verh.," II.); whilst Robin declares that it is incorrect, giving a description of their formation which is, to me, unintelligible. I recommend to his notice rachitic bone, the cementum of the horse's tooth, and the symphyses (§95), with which he is manifestly unacquainted, and hope that he 


\section{may then be induced no longer to regard Schwann's and my views as antiquated.*}

* [As we have already said, we must deny the existence of endogenous cell-development in ossifying, or any other cartilage. In fact, the process of multiplication of the corpuscles (nuclei (?) of Kölliker, granular cartilage-cells of Tomes and De Morgan) is so clear, that we are at a loss to comprehend how it can be mistaken. What is meant in the text by "contents," as distinct from the corpuscles, we do not know. Messrs. Tomes and Morgan describe the real changes which precede ossification, very exactly in a few words, thus: "Cartilage previous to its conversion into bone undergoes a rapid growth, which takes place principally in the direction of the long axis of the future bone. Each granular cell becomes divided into two, by segmentation transverse to the line of ossific advance. These are again divided and the process repeated from time to time, until in the place of a single granular cell we have a long line of cells extending from the unchanged cartilage to the point where ossification has taken place" (1. c., p. 16). "If attention be directed to the end of the line furthest from the bone, the cells will be found small in size, granular, and with a perceptible nucleus, but without an outer wall, distinguishable from the hyaline substance, which is abundant between the contiguous lines, but small in quantity between the cells composing the lines. But if the other end of the line be examined, very different conditions will be observed. The granular cells will be seen to have become rounded in form, to have increased to three times their original bulk, and to possess well-marked, circular nuclei. ..." -p. 17.

So far, our own observations are in perfect accordance with those of Tomes and De Morgan. They go on, however, to observe, "in addition to which, each granular cell will have acquired a thick, pellucid, outer wall;" and with this last statement we can by no means agree. Neither in Man, the Calf, the Rabbit, the Skate, nor in enchondroma, have we been able to see anything of the regular development of such an envelop in fact, in the great majority of instances, we have convinced ourselves of the absence of anything of the kindthere being nothing but a clear space between the corpuscle and the ossified wall of the cavity in which it lies. Bodies corresponding with the lacunal cells-cartilage-corpuscles that is,-invested by a thick coat of more or less granular, calcareous matter, may indeed often be obtained free; but they arise, like the corresponding bodies in rickety bone, simply from the deposition of calcareous matter in the cartilage-cavity before it has taken place in the matrix, or from a want of union between the two deposits; and are therefore quite accidental.

The lacunce are developed, according to these authors, by the shooting out of the granular cells into processes, and their direct conversion into the lacunce, the nucleus of the granulecell remaining as the nucleus of the lacuna. On this point also, we must differ from them, and agree with Virchow (1. c., note, \$ 101) and Kolliker (supra, \$ 104), that the development of the canaliculi is, by a process of resolution, quite independent of the corpuscles, which simply diminish in size, and either remain as the so-called "nuclei" of the lacunæ or totally disappear. We can especially recommend the Skate (2) as a subject in which to trace the process of formation of lacunæ, as the bone is homogeneous and transparent, and in consequence of being enclosed in a large mass of firm cartilage, may be cut with ease into very thin sections. We have observed it with great clearness also in enchondroma.

There is one argument which seems to us conclusive on this point. Wherever the canaliculi can be seer at all, however young the tissue, they are perfectly clear and transparent. If, however, they were formed by processes of the granular cells, they ought to be granular, and more or less opaque.

Taking the same view of the structure of cartilage as Messrs. Tomes and De Morgan, then, our view of the nature of the lacunæ, resulting from its ossification, agrees with that of Professor Kölliker. Cartilage becomes bone by the deposit of calcareous salts in the matrix and occasionally in its cavities. The lacunæ are spaces left round the corpuscles, from which, by resorption, processes-the canaliculi,-are subsequently developed. If it be asked how it is that the lacunæ may frequently be demonstrated both optically and chemically as 
§ 105. Elementary processes in the Layers formed from the Periosteum.-The periosteum of the prinarily cartilaginous bones, is proportionally very thick and vascular, consisting, as early as at the fifth month, of common connective tissue and fine elastic filaments, the latter of which in process of time become stronger and stronger, occasionally assuming the nature of elastic fibres. On the inner aspect of this fully formed periosteum, there is now deposited an ossific blastema firmly adherent to the bone (Fig. 133, B); so that when the periosteum is removed, it generally remains upon it as a moderately thick, soft, whitish

Fig. 133.

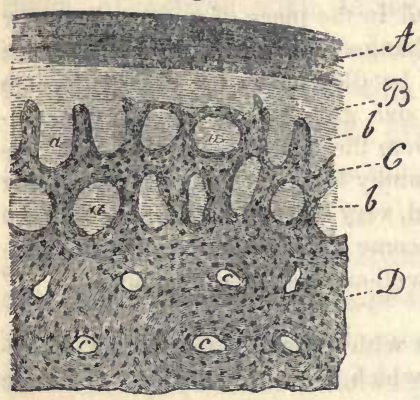
yellow lamella, in which, microscopic examination shows the existence of a fibrous tissue, with a not particularly distinct fibrillar formation, something like immature connective tissue, and granular, oval, or round nucleated cells, measuring 0.006 -0.01 of a line. When this lamella is raised from the bone, it is found to be very intimately connected with the most superficial layers, and on its internal surface a few little detached fragments of bone, and scattered masses of reddish, soft medulla, from the most superficial cancellar spaces, will be observed. The bone thus laid bare, when the removal of the periosteal layer has been carefully conducted, presents a rough, and as it were porous surface, with numerous medullary spaces, and remains, superficially, in spots of greater or less extent, quite soft, pale-yellow, and transparent, whilst more internally it becomes firmer and whiter, ultimately acquiring the usual appearance of perfect osseous tissue. When it is inquired, how the formation of bone, which indubitably takes place in this situation, is effected, we refer to the blastema just described, the cells of which, scattered in the fibrillated connective tissue, have not the least resemblance to those of cartilage, but appear exactly like the foetal medulla-cells, or formative cells of the

FIG. 133.-Transverse section from the surface of the shaft of the metatarsus of the Calf; magnified 45 diam.: $A$, periosteum ; $B$, ossifying blastema ; $C$, young layer of bone, with wide cavities, $a$, in which are lodged remains of the ossifying blastema, and reticular spiculæ, $b$, which towards the blastema present a tolerably abrupt border; $D$, more developed layer of bone, with Haversian canals, $c$, which are surrounded by their lamellæ.

distinct bodies, we must call to mind the fact already referred to, that in cartilage, the wall of the cavities have frequently undergone less change than, or a different change from, the surrounding matrix; and therefore appear both optically and chemically distinct, though they are by no means so, morphologically: and, therefore, that there is no difficulty in supposing the same thing to occur in bone. The chemical differentiation of the wall of the lacuna is, in fact, exactly comparable to that of the wall of the cavity which contains the " nucleus" in connective tissue, and in fibro-cartilage; and which gives rise to the formation of the elastic element in those tissues.-Trs.] 
embryo. In fact, it is now, not difficult to show, that the outermost, still soft bone-lamellæ pass into the blastema in question, with their separate spiculæ and projections, and that (1.) the matrix of the bone arises from its fibrous tissue, by the simple uniform deposition of calcareous salts, although usually, as it seems, without the previous appearance of calcareous granules; and (2.) that the bone-cells are formed out of the formative cells of the blastema. With respect to the latter, however, the transformation cannot be followed step by step, as in rachitic bones. This much, however, is always apparent, that the bonecells at first present larger cavities, less developed rays, and more distinct nuclei (the latter, as we know, remaining), and, as their occasionally visible outlines prove, correspond entirely in size with the cells just mentioned, so that I do not for a moment doubt, that they are formed in this situation exactly as they are elsewhere. With respect to the development of the ossifying blastema itself, it is at least clear, that it is derived from the numerous vessels of the foetal and young periosteum; the origination of its fibres from fusiform cells, I have very frequently observed in man and in animals, but with respect to the cells, can only state that they occur of various sizes, and occasionally intermixed with free nuclci.

The formation of bone in this blastema occurs wherever it is in connection with the bone; it does not, however, take place in connected but in interrupted, reticular lamelloe. The roundish or elongated spaces (Fig. 133, a), which, from the first, remain between the layers of osseous tissue, and in the different layers communicate with each other, are nothing else than the rudiments of the Haversian or vascular canals of the compact substance, and contain a soft, reddish medulla, which at first is obviously nothing more than the unossified portion of the ossific blastema, although it sometimes contains more formative cells than connective tissue. The cells of these spaces are very soon transformed into the usual, light-reddish medulla-cells, and partly into vessels which communicate with those of the interior of the bone, and in part also with those of the periosteum, with which, having once formed a junction, they remain continuous during the entire growth of the bone in thickness, so that the formation of the spaces in the bone is, at least afterwards, preindicated by those, which, in accordance with what has been said, proceed from the periosteum through the ossific blastema to the bone. Besides medulla-cells and vessels as well as some connective tissue, the bone-cavities of the periosteal layers also contain round, elongated, or dentate, flattened, faintly granular cellular corpuscles of $0.01-0.02$ of a line, or more in size, with from 3 to 12 or more vesicular nuclei and nucleoli, which are probably referable to the multiplication of the medulla-cells (vid. § 11). The periosteal layers, which, agreeably to what has been stated, are from the first deposited in the 
form of cribriform lamellæ around the ossific-nuclei formed from cartilage, continue to be produced so long as the general growth of the bone goes on, essentially in the same way, constituting the material by which it increases in thickness; but at the same time, more or less important changes are set up in them; the most considerable of which take place in the large cylindrical bones. In these, we find, more distinctly indeed after birth, that a large cavity is gradually formed in the interior, which at first contains foetal medulla-cells, and afterwards perfectly formed medulla. This medullary cavity is formed, in exact analogy with the medullary spaces described in the preceding paragraphs, by the solution of the osseous tissue of the shaft; at first, only of that which is formed from the primitive cartilaginous rudiment, but soon, also of that deposited from the periosteum upon the former, its development proceeding in a remarkable manner, as long as the general growth of the bone continues. Whence it comes to pass, that, as at the ends of the diaphyses, so also on its surfaces, whilst new bone is continually deposited exteriorly, that which is already formed is as continually absorbed in the interior; and in fact these two processes are so combined, that the bone, during its development is, in a certain measure, several times regenerated, and, for instance in the humerus of the adult, does not contain an atom of the osseous tissue which existed at the time of birth, nor does the bone at that period contain any of the tissue of which it was constituted in the embryo at three months. These conditions will be rendered most distinctly intelligible, and especially with respect to the periosteal and cartilage layers, by means of a diagram (Fig. 134) which I have for a long time employed in my lectures. If, in this figure, we compare the primordial bone $\mathrm{E} \mathrm{E}$ with the almost complete bone $\mathrm{E}^{4} \mathrm{E}^{4}$, it is apparent, that in the longitudinal growth of the diaphysis of the latter on both sides, at the expense of the continually growing epiphysal cartilage, an elongated cone of osseous substance, $1,2,1^{1} 2^{1}$, and 3 , $4,3^{1}, 4^{1}$, is produced, to which, ultimately, the epiphysal nuclei $\mathrm{E}^{4} \mathrm{E}^{4}$, also originating in the cartilage, are joined, whilst, to increase its thickness, the tubular layer $\mathrm{P}, \mathrm{P}^{1}, \mathrm{P}^{2}, \mathrm{P}^{3}$, which are constantly increasing in length and, in the middle, in thickness, are applied to it. In a cylindrical bone of this kind consequently, the entire portion formed from cartilage, presents a figure of a double cone with rounded bases; and that formed from the periosteal layers, $1,{ }^{\prime} 2,3,4, \mathrm{P}^{3}$, and $1^{1}, 2^{1}, 3^{1}, 4^{1}$, $\mathrm{P}^{3}$, the form of an elongated tube thickest in the middle, and resembling an elongated vertebra of a Fish, with conically hollowed, terminal surfaces. The articular cartilage $\mathrm{C}$, is the unossified portion of the epiphysal cartilage, and the medullary cavity which is not shown in the figure (it may be supposed to be indicated pretty nearly by the outlines of the fourth bone $\mathrm{E}^{3} \mathrm{E}^{3}$ ), is formed by the resorption of the entire 
osseous substance derived from the cartilage and periosteum of the younger bones; -in this case the first three, $\mathrm{E} \mathrm{E}, \mathrm{E}^{1} \mathrm{E}^{1}$, and $\mathrm{E}^{2} \mathrm{E}^{2}$.

In the cylindrical bones, without a medullary cavity, and in all other bones containing nothing but spongy substance in the interior, the absoprtion does not proceed to nearly the same extent as it does in the above described cases, that is to say, only to the production of a looser spongy substance in the interior, and, consequently, we find, for instance in the vertebræ, more or less considerable remains even of the earlier bone-substance. In this situation also, the absorption always affects not merely the osseous nucleus, formed from the cartilage, but likewise the periosteal layers, the latest of which only remain more in their original form, as the substantia compacta.

The Haversian canals do not originate, as is sufficiently apparent from what has been said, like the cancelli of the primary bone-substance, from a solution of a preexisting tissue, but are nothing more than open cavities, left from the commencement, in the periosteal layers. They are relatively, of a considerable size at an early period (vid. also "Valentin. Entw." p. 262), measuring in the fotal humerus at five months $0.016-0.024$ of a line in the femur at birth, according to Harting Fig. 134.

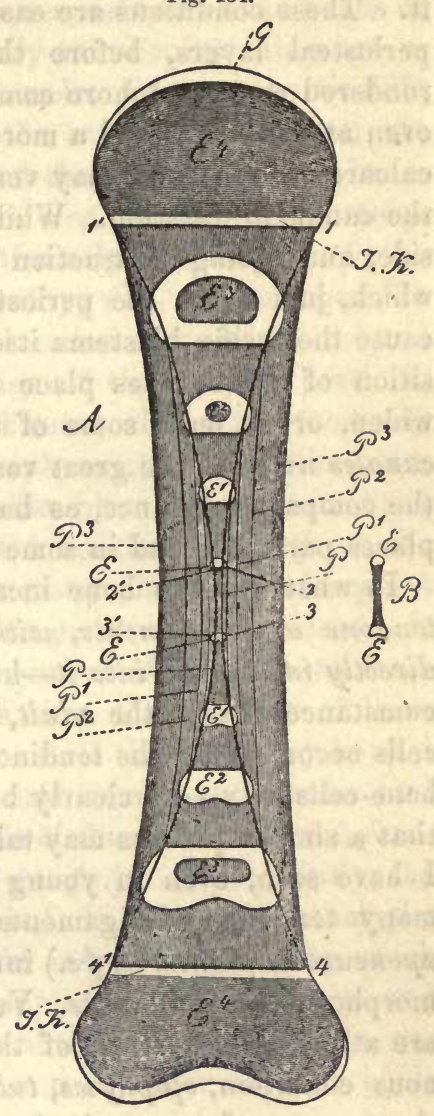
(p. 78), 0.10-0.024 of a line, just as in the most recently formed layers also of a later period. Their contents have been already described. The most important circumstance connected with them remaining to be noticed, is the mode in which their lamellar systems originate. These lamellæ also are formed without the intervention of cartilage, and are nothing more than deposits from the contents of the canals, which sub-

Fig. 134. Diagram of the growth of a cylindrical bone. $B$, primary rudiment, the diaphysis ossified and the epiphyses cartilaginous: $A$, the same bone in four stages of further advance, $\mathrm{E}^{1} \mathrm{PPE} \mathrm{E}^{1}, \mathrm{E}^{2} \mathrm{P}^{1} \mathrm{P}^{1} \mathrm{E}^{2}, \mathrm{E}^{3} \mathrm{P}^{2} \mathrm{P}^{2} \mathrm{E}^{3}, \mathrm{E}^{4} \mathrm{P}^{3} \mathrm{P}^{3} \mathrm{E}^{4} ; \mathrm{P} \mathrm{P}^{1} \mathrm{P}^{2} \mathrm{P}^{3}$, periosteal layers of these four bones; the space contained within $1,2,3,4$, and $1^{1}, 2^{1}, 3^{1}, 4^{1}$, indicates the portion which in the largest bones is formed from cartilage; $E^{\prime} E^{\prime}$, cartilaginous epiphyses of the second bone; $\mathrm{E}^{2} \mathrm{E}^{2}$, epiphyses of the third bone, in one of which is an osseous nucleus ; $\mathrm{E}^{3} \mathrm{E}^{3}, \mathrm{E}^{4}$ $\mathrm{E}^{4}$, epiphyses of the fourth and fifth bones, all with larger epiphysal nuclei : $G$, articular cartilage; $I, K$, interstitial cartilage between the ossified epiphyses and diaphyses. 
stance, as has already been said, in respect of its fibres and cells, entirely corresponds with the ossific blastema beneath the periosteum, and, in a certain degree, is merely an originally unossified remainder of it. These conditions are easily observed in young bones, in which, the periosteal layers, before they have undergone any resolution, are rendered more and more compact by these new, secondary lamellæ; but even at a later period a more or less ossified blastema (always without calcareous granules) may very frequently be perceived on the walls of the canals in question. Whilst the vascular canals are thus, on the one side, undergoing contraction by the deposition of these secondary layers, which, just as in the periosteum itself, appear laminated,-either because the ossific blastema itself is so constructed, or because the deposition of bone takes place with periodical pauses,-they afterwards widen, or at least some of them, by absorption, as for instance, the canales nutritii, the great vascular openings in the apophyses, \&c. ; and the compact substance, as has been already remarked, is also, in many places partially, and in some even entirely, absorbed.

In what way the bone increases in thickness in the situations where tendons and ligaments, without the intervention of periosteum, are directly implanted into it-has not yet been made out. From the circumstance, that in the adult, in many of these situations, true cartilagecells occur among the tendinous fibres, and also, that their passage into bone-cells may very clearly be observed, it might perhaps be concluded that a similar process may take place at an earlier period also. In fact, I have seen, even in young individuals, at the points of insertion of many tendons and ligaments (tendo Achillis, lig. calcaneo-cuboideum, aponeurosis plantaris, \&c.) into the bone, cartilage-cells, and their metamorphosis into bone-cells. Very frequently, also, tendons and ligaments are attached to portions of the bone which remain long in the cartilaginous condition, epiphyses, tuberositas caleanei, fre, and the growth of these parts, of course, is simply to be referred to the cartilage.

The formation of bone on the inner aspect of the periosteum is a fact long well known, although it has, hitherto, generally been thought, that in this situation also, it was preceded by a thin cartilaginous layer, until the contrary was shown by Sharpey and myself. Since the discovery by Duhamel ("Mémoires de l'Academie de Paris," 1742, p. 384, and 1743, p. 138), that the bones of animals fed upon madder are colored red, a great number of experiments have been made with that substance, especially by Flourens, in growing animals ; it being at first believed, that it only colored those parts of the bones which were formed after its administration. This method, however, has lost a good deal of its value since it has been shown by Rutherford (in "Roberti Blake, Hiberni, Dissert. inaugural. med. de dentium formatione et structurâ, in homine 
et in variis animalibus," Edinb. 1780), Gibson ("Memoirs of the Literary and Philosophical Society of Manchester," 2 d series, vol. I. p. 146), Bibra (l. c.), Brullé and Huguény (l. c.), that when animals were fed upon madder, the whole of the growing bones, as well as the bones of adult animals, become colored, and especially so wherever they are in more immediate connection with the bloodvessels; for even the medulla is colored (Bibra). For which reason also, the innermost layers of the Haversian canals, the periosteal surfaces, and the vascular, young bonesubstance, acquire a deeper color. There are, however, still some points worth investigating in this way, particularly with relation to the more recent statements of Brulle and Hugueny, who, relying upon the circumstance, that, as they assert, the decoloration of growing, colored bones, is effected merely by the absorption of the colored portions, believe they have found that the cylindrical bones also deposit osseous substance from within, particularly in the apophyses; whilst on the outer surface, absorption to the same extent takes place; statements upon which I will not, at present, give any decided opinion, although at the same time I hold it as quite certain, that in many places an absorption does take place, on the exterior of the bone to a greater or less extent. It is only by such an absorption that the enlargement of the foramen magnum from the sixth year upwards, at which time the portions of bone surrounding it are united, can be explained. And the same may be said with respect to the arches of the vertebrce, and numerous vascular and nerve-openings (foramen ovale and rotundum of the sphenoid bone, foramina inter transversaria, canalis caroticus, \&c. \&c.). Consequently, the law propounded by Serres (Meck. "Archiv," 1822, p. 455), that the openings in bone enlarge by the growth of the individual pieces by which they are bounded, is wholly incorrect, as applied to the openings and canals in the middle of bones; as had been already, to some extent, declared by E. H. Weber and Henle; and even in other cases it holds good only for the earliest periods.

The periosteal layers present a certain contrast to the osseous tissue developed from cartilage. The former constitute principally the firm cortex of the primarily cartilaginous bone, and are characterized by the occurrence of Haversian canals and their lamellar systems, whilst the latter produces the spongy substance, and contains no vascular canals. It must not, however, be forgotten that even the periosteal layers all have, at first, in a certain degree, a spongy structure, and in all these bones, without exception, contribute, and frequently very essentially, to the formation of the spongy substance; moreover, that in the cellular substance, which originates from the cartilage, in the apophyses for instance, secondary layers, similar to those of the Haversian canals, and of the spongy substance which is formed out of the periosteal layers, only not so much developed, appear to be formed. The morphological 
and chemical relations of the matrix of these two forms of osseous tissue have not as yet been determined. On the other hand the bonelacunæ of both kinds of tissue do not present the least difference.

$\S 106$. Bones not primarily cartilaginous occur, in Man, only in the cranium. They originate outside the primordial cranium, between it and the muscular system, and thus within the structure constituting the vertebral system. They by no means exist as membranous and cartilaginous capsules on the first appearance of the cranium, their formation not commencing till after that of the primordial cranium, from a secondary blastema, whence, in contradistinction to the other primary bones, the formative material of which exists prior to the commencement of ossification, they are termed secondary bones-or, also, because in most places they are in contact with portions of the primordial craniumcovering or overlaying bones (belegknochen). To this class belong, the upper half of the expanded portion of the occipital bone, the parietal, and frontal bones, the squamous portion and tympanic ring of the temporal bone, the nasal, lachrymal, malar, and palate bones, the upper and lower jaw, the vomer, and apparently, the internal lamella of the pterygoid process of the sphenoid, and the cornua sphenoidalia. The blastema of these bones, which differs from that of the primary bones, in its being successively developed in a membranous matrix, simultaneously with the process of ossification, not existing previously in any considerable quantity, presents essentially, exactly the same conditions as that of the periosteal layers, and is also ossified in precisely the same way.

The notion that certain cranial bones, in man and the Mammalia, are not developed from cartilage, is by no means new, although the morphology of the question was first established by Rathke, Reichert, Jacobson, and myself; and its histology by Sharpey and myself. But with respect to the latter subject, a controversy still exists as to the true nature of the ossific blastema (as also, of that of the periosteal layers), -whether it be a kind of connective tissue, as I believe, or a sort of cartilage, as Reichert and A. Bidder assert, with respect to which more will be found in my "Mikroskop. Anat." pp. 374, 375.

$\S 107$. The secondary cranial bones, all, in the first instance commence in the form of a minute, elongated, or rounded, osseous nucleus, consisting of a portion of fundamental substance or matrix, with a few lacunæ, and which is surrounded by a small quantity of soft blastema. How this nucleus originates has not yet been observed, although from the way in which its growth proceeds, it might be assumed with certainty, that shortly previous to its first appearance, a minute lamella of the soft blastema is formed in the situation of the future nucleus, which lamella spreading from a single point, becomes ossified by the addition 
of earthy salts and the metamorphosis of its cells. The primary point of ossification having thus appeared, for instance in the parietal bone, its growth advances simultaneously with the horizontal extension of the membraniform blastema, in such a way that a delicate lamina composed of reticulated osseous spicules is shortly produced, from which, slender rays stretch out into the still unossified blastema (Fig. 135). If this formation be examined more closely, it will be observed, that the individual bone-spicules originate in the membranous blastema, by the ossification of its elements, and, that to a certain extent, the latter is absorbed in the spaces occupied by the spicules, remains of it being left in the interstices; and moreover, that the formation of the osseous elements proceeds exactly in the same way, that it does in the periosteal layers; the

Fig. 135.

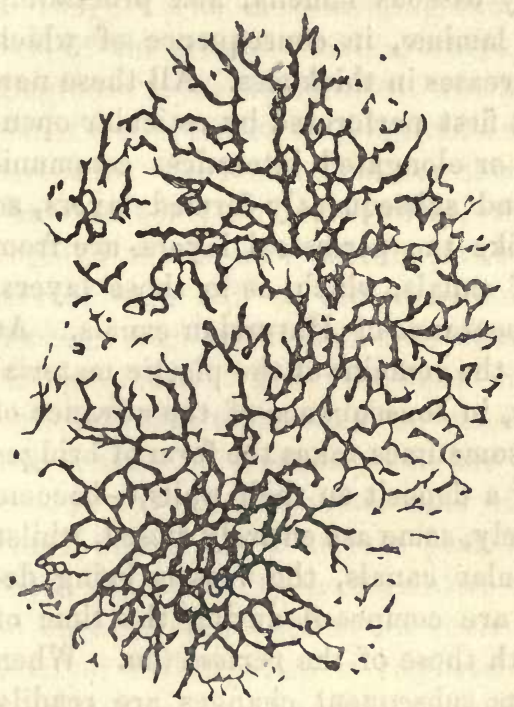

Fig. 136.

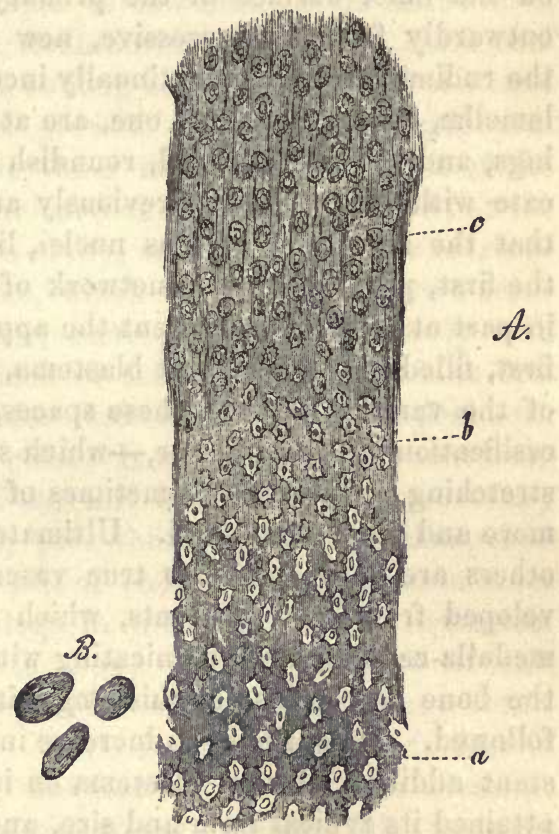

rays of bone as they extend further into the soft blastema becoming softer and paler, and containing less earthy matter, whilst their cells become more and more like the soft formative cells, till, at last, the spicules lose all distinct limitary outline, and are lost in the blastema. At first, the growth of these bones proceeds in a superficial plane only, the rays, as they extend and become connected by transverse branches con-

Fig. 135. Parietal bone of a fourteen-weeks' old fœtus; magnified 18 diameters.

Fig. 136. From the inner surface of the parietal bone of a new-born child; magnified 300 diameters: $a$, bone with lacunæ, still pale-colored and soft; $b$, border of the same; $c$, ossifying blastema with its fibres and cells. $B$, three of these cells, magnified 350 diameters. 
tinuing to add to the size of the original, reticulated lamella, which, however, shortly begins to increase in thickness by the deposition of layers upon both sides of it; the different portions, also, in proportion to their age becoming more and more compact. The formation of the thickening layers is to be referred to the periosteum, which is found on the surfaces of the secondary bones, very soon after their formation has begun, being developed either from their original blastema, or from the contiguous tissues (perichondrium of the primordial cranium, muscular and tendinous coverings), and proceeds exactly in the same way as in the periosteal layers of the primary bones; that is to say, on the inner side of the periosteum, a soft blastema is deposited, which gradually ossifies, from the bone outwards, without its ever being cartilaginous (Fig. 136). In this way are now formed, chiefly on the outer, but also on the inner surface of the primary osseous lamella, and proceeding outwardly from it, successive, new laminæ, in consequence of which the rudimentary bone continually increases in thickness. All these new lamellæ, like the primary one, are at first perforated by reticular openings, and the various sized, roundish or elongated interstices communicate with those of the previously and subsequently formed layers, so that the secondary osseous nuclei, like the periosteal layers, are from the first, penetrated by a network of canals, which, as in those layers, in part at least, soon present the appearance of Haversian canals. At first, filled only with a soft blastema, the remains of the plastic material of the various lamellæ, these spaces, in consequence of the advance of ossification in their interior, - which sometimes takes the form of bridges stretching across them, sometimes of a deposit on their walls,-become more and more contracted. Ultimately, some are entirely closed, whilst others are converted into true vascular canals, the vessels being developed from their contents, which are composed during the time of medulla-cells, and communicating with those of the periosteum. When the bone has arrived at this stage, its subsequent changes are readily followed. It continues to increase in breadth and thickness by the constant addition of new blastema on its edges and surfaces, until it has attained its typical form and size, and at the same time, by the solution of its compact substance, additional spongy tissue (or even large cavities), is formed in its interior, so that eventually, like bone developed from cartilage and periosteal layers, it presents, externally, compact substance with Haversian canals; and internally, medullary spaces (cancelli), although with distinct secondary deposits.

The secondary cranial bones ossify, in part, earlier than the primary, and mostly with only a single nucleus. The soft blastema out of which they are formed, and which, so long as the bones continue to grow, is to be found on their surfaces and edges, does not, like carti- 
lage, grow independently with them, but is developed by degrees, from a plasma successively secreted from the vessels of the periosteum, the two lamellæ of which are conjoined at the margin of the ossifying plate. The cells of this plasma, the metamorphosis of which, as in the periosteal layers, cannot be followed in every particular, are elongated, measuring in man, for the most part, $0.006-0.01$ of a line, and presenting granular contents with oval nuclei of $0.0028-0.0048$ of a line. Such of these cells as are destined for the growth of the bone in thick"ness, with the exception of those of the glenoid cavity of the temporal bone, never present the slightest resemblance to cartilage-cells, and, together with their matrix, invariably ossify without the appearance of any calcareous particles; those on the borders or extremities, on the contrary, may, as it appears, subsequently, take on the nature of true cartilage. The most striking example of this kind occurs in the condyle of the inferior maxilla, where, even during foetal life, a thick cartilaginous layer is deposited, which so long as the growth of the bone continues, precedes its longitudinal growth, exactly like an epiphysal cartilage. I have noticed the same thing in the articular fossa of the temporal bone, where, however, the cartilage is less developed; at the angle of the inferior maxilla (in the Calf), and at the anterior extremities of each half of the same bone, which are connected by a semifibrous, semi-cartilaginous substance, corresponding very nearly with the symphysis. This fact loses much of the singularity which at first sight attaches to it, when we consider that all cartilage is at first soft, and consists of common formative cells. It is, consequently, only necessary that the formative cells of the soft blastema of the secondary bones, should, at a certain period, pass through the same changes as those undergone by the formative cells of embryonic cartilage, in order to effect the production of cartilage in the bones now in question. Further investigation is required to show, whether cartilage of this kind also occurs as a supplementary addition to other secondary bones, and to what extent, in animals. Still, it may be noticed, that in asserting as I have done, that all ossifications from a soft blastema take place without the deposition of calcareous granules, this statement is only in part correct, because it is quite true, in many cases, that this sort of deposition does occur in them, though never at an early period; and, generally speaking, but rarely. The ossifying margin, moreover, in these cases is never abrupt, as it is in ossifying cartilage.

The ultimate changes of the secondary bones have not yet been closely investigated. Their mode of connection with each other, and also with primary bones by suture and coalescence, is tolerably well known. In the vault of the cranium, for instance, as the primary ossific points first appear in the situation of the tuberosities of the parietal and frontal bones, the bones are at first placed widely asunder, 
and are connected merely by a fibrous membrane, the continuation of the periosteal lamella of each, and which is united on the internal aspect with the remains of the membranous cranium of the embryo, and with the dura mater. The bones then continue to grow towards each other, and at last constantly advancing in the above-described continuation of the periosteum, come very nearly into contact at the frontal and sagittal sutures; there remains, however, for a long time one large vacuity, in particular, between them, - the anterior fontanelle,-but which closes in the second year after birth; whilst at the same time, the bones, which, up to this period, adjoined each other with a straight line of juncture, send out interdigitating tooth-like processes, till ultimately, when their blastema is wholly consumed, they continue united only by the remains of the periosteum (the sutural cartilage, as it is termed, or better, the sutural ligament), but which also is capable of becoming ossified sooner or later, and, indeed, invariably first on the inner aspect of the suture, where the tooth-like processes are very little developed. The changes of form in the entire bones during their development are very remarkable, and have hardly been attended to. If a parietal bone, for instance, of a foetus or new-born child, be compared with that of an adult, it will be found that the former is much more curved, and in no way at all represents a piece cut out of the middle of the latter. The adult parietal bone consequently must have undergone a very important alteration in the curvature of its surfaces, and this, as mechanical conditions are out of the question, can only have been effected by an unequal deposition of bone internally and externally, in the middle and at the borders; or by deposition on the one side and absorption on the other. That unequal deposition does actually occur, is seen, for example, in the juga cerebralia and impressiones digitatoe, the sulci meningei, \&c.; but it appears to me, that the whole matter cannot be understood, unless we assume that local absorptions also take place in certain situations. How otherwise can be explained the increase in breadth of the superior orbital ridge, the increase of distance between the frontal eminences, even after the ossific union of the two portions of the frontal bone, the change of form of the lower jaw (the greater distance between the coronoid processes and the mental spine, the alteration in its curvature, the partial removal and renewal of the alveoloe), \&c.? We have already seen, that in the other bones, also, something of the kind must be presumed to take place, and, consequently, cannot hesitate to admit it in the present case, although the particulars of the process be unknown. . That this process occurs in the interior of the secondary bones has been already mentioned. The formation of the diplö̈, which becomes more evident in the tenth year, is to be referred to it, as is also that of the frontal sinuses, and antrum Highmorianum, which however does not take place till a later period. 
I would further remark, that the secondary bones, so long as they are in a growing state, are much more vascular than afterwards, even exceeding, in this respect, the periosteal layers of the other bones; on which account their medulla, containing the multi-nuclear, enigmatical bodies, already referred to, is of a redder color. The vessels enter these bones at innumerable points on the surface, and, in the different bones, run in vertical or horizontal canals. The latter is the case in the flatter bones, in which the vascular channels run principally in the longitudinal direction of the osseous rays proceeding from the primary point of ossification; and the former, in consequence of which the surface of the bone frequently presents an extremely delicate, millepore-like aspect, occurs in the thicker portions. A great many of these canals afterwards become obliterated, or, at all events, very much contracted, whence the surface of the bone is rendered smoother.

In conclusion to these remarks on the development of the bones, I will add a few words regarding their conditions at different periods. Valentin noticed the cartilaginous rudiments of the ribs in a human embryo 6 lines long. That of the cranium is distinctly recognizable in the sixth or seventh week, as well as those of the vertebral zone and that of the extremities; those of the extremities proper do not appear till later (in the eighth or ninth week). Ossification commences as early as the second month, first in the clavicles and lower jaw (fifth to seventh week), then in the vertebroe, the humerus, femur, ribs, and the cartilaginous portions of the lamina of the occipital bone. At the end of the second, and beginning of the third month, ossification is apparent in the frontal bone, scapuloe, bones of the fore-arm and leg, and upper jaw ; in the third month, in the rest of the cranial bones, with few exceptions, the metacarpal and metatarsal bones, and phalanges; in the fourth month, in the ilium and ossicula auditîs; in the fourth or fifth month in the ethmoid, the turbinated bones, the sternum, pubis, and ischium; in the sixth to the seventh month, in the os calcis, and astragalus; in the eighth month, in the os hyoides. At birth, the epiphyses of all the cylindrical bones are still unossified, with the occasional exception of those at the lower extremity of the femur and upper end of the tibia; and besides these all the carpal and the five smaller tarsal bones, the patella, sesamoid bones, and the last segment of the $\operatorname{coccyx}$. After birth, up to the fourth year, the nuclei of these bones also make their appearance; but, in the os pisiforme, not till the twelfth year. The union of most of the epiphyses and processes, with the diaphyses takes place, in part at the time of puberty, in part towards the end of the period of growth.*

* [Dr. Sharpey's discovery that certain bones of the skull are developed in the same manner as those layers which are formed under the periosteum in the long bones, has been a sort of apple of discord among histologists, and has produced a great variety of controversies not only among them, but among comparative anatomists; controversies whose heat has 


\section{$\S 108$. The vital phenomena exhibited in the mature bones are not, during the vigorous period of life, accompanied with any notable or active}

been somewhat increased, as we think, by a want of perception among the combatants, of the fact, that several totally distinct questions are involved. These questions seem to us to be the following, and we shall endeavor to consider them in detail.

1. Whether the tissue from which "secondary" bone proceeds is cartilage, or not?

2. Whether it is morphologically homologous with cartilage, or not?

3. Whether ossification takes place in it in the same manner as in cartilage, or not?

And as the result of the answering these:-4. Whether the differences between the two tissues are sufficient to constitute the basis of a classification of the bones or not?

1. To answer this by saying with Meyer that every tissue which ossifies is cartilage, is simply to beg the whole question. Cartilage we hold to be distinguished from indifferent tissue, by the fact of its matrix containing chondrin. The substance which in the fotus contains no chondrin, but will subsequently become a cartilage-though in common parlance it is very convenient to call it "fœtal cartilage"-is no more cartilage than the cartilaginous basis of a future bone, which might just as properly be called fœtal bone, is osseons tissue. There can be no question then, we think, that Kolliker is in the right, as against Reichert, Meyer, and others, when he says that secondary bone is not developed from cartilage, and that, in this respect, it may be distinguished from primary bone.

2. Though this matrix of secondary bone, however, is assuredly not cartilage, it is another matter whether it is, or is not, morphologically homologous with cartilage. To arrive at any just conclusion on this head, it is necessary to understand the precise structure of this tissue, which Messrs. Tomes and De Morgan have been the first to point out: "If attention be directed to the part furthest removed from the bone, it will be seen that the membrane-like mass is composed of oval cells with slight prolongations from the extremities, which are frequently arranged in the form of bands of fibrous tissue. Dr. Sharpey has observed that the membrane into which the bone extends is like fibrous tissue in an early stage of development; and this observation is strictly true when confined to the part indicated, but the analogy ceases [?] as we extend our examination towards the bone. Here, in the place of cells with elongated processes, or cells arranged in fibre-like lines, we find cells aggregated into a mass, and so closely packed as to leave little room for intermediate tissue. The cells appear to have increased in size at the cost of the processes which existed at an early stage of development and formed a bond of union between them. Everywhere about growing bone, a careful examination will reveal cells attached to its surface, while the surface of the bone itself will present a series of similar bodies ossified. To those we propose to give the name of osteal cells, as distinguished from lacunal and other cells. In microscopic characters, the osteal cells closely resemble the granular cells of temporary cartilage; so closely, indeed, that the latter, when detached from the cartilage, could not well be distinguished from them. They are, for the most part, spherical or oval in form, and lie on the surface of the growing bone in a crowded mass, held together by an intervening and apparently structureless matrix. Here and there we find a cell which has accumulated about itself an outer investment of transparent tissue, and has, in fact, become developed into a lacunal cell destined to become a lacuna" (1. c. p. 23).

The tissue, then, from which secondary bone immediately proceeds, is composed of a homogeneous matrix, in which corpuscles, identical with the cartilage-corpuscles, are imbedded: it is therefore identical, as Dr. Sliarpey described it, with young connective tissue; and as we have seen above (note, $\$ 101$ ), and as the authors state, with fotal cartilage. Though not cartilage, therefore, it is homologous with it (as is, indeed, admitted by Professor Köliiker) ; a fact which is still more strongly evidenced by the transition of cartilage into a similar tissue, at its edges (Tomes and De Morgan, l. c. p. 24), which may readily enough be observed, and which has been particularly shown by Reichert to occur between the primary and secondary bones of the skull ("Zur Streitfrage aber die Gebilde der Bindesubstanz, uber die Spiralfaser und über den Primordial-Schådel," Müller's "Archiv," 1853).

Now it seems to us that a tissue which is identical with the embryonic form of cartilage, 
morphological changes. It is true that, during this period, some of the processes above considered go on-such as the enlargement of the sinuses in the cranial bones, of the points of insertion of muscles

which passes into adult cartilage, and differs from cartilage only in the absence of chondrin (inwhich respect ossified cartilage agrees with it), -is in a morphological point of view homologous with cartilage.

3. With respect to the third question,-Sharpey and Kolliker are of opinion that the deposit of calcareous matter and the formation of lacunæ take place in the same manner as in cartilage, $i$. e. that the calcareous salts are deposited evenly through the matrix, leaving spaces round the corpuscles or "nuclei," from which the canaliculi are subsequently developed by resorption. Messrs. Tomes and De Morgan, on the other hand (see passage cited above), maintain that secondary bone differs from primary, in so far as certain of the corpuscles-"osteal cells,"- " arrange themselves side by side, and together with the transparent blastema in which they lie, become impregnated with ossific matter, and permanently fused with the bone-tissue with which they lie in contact. By the linear arrangement of these osteal cells, lamination is produced. In the case of new laminated bone, the cells are simply ossified without arrangement. Lying amongst the osteal cells will be seen some which have accumulated around them a quantity of tissue which forms a thick investment to them; they then become granular, and take on in every respect the characters of a lacunal cell. These are found deposited at intervals along the line of ossification, and becoming blended with the general mass, the granular cell remaining as a lacuna, and sending out processes in all directions" ("Abstract in Proceedings of Royal Society").

We must confess that all we have seen leads us to believe that the former of these accounts is correct. We have never been able to find evidence of any of the corpuscles becoming converted into "osteal cells," and we believe, for the following reasons, that this process does not take place. In examining the growing Haversian canals in Man, and particularly in the Calf, we have very frequently found the innermost layer transparent, glassy, and structureless-exhibiting nothing but the corpuscles $(d)$ lying in lacunæ without canaliculi. This layer would be as much as $\frac{1}{2} 0_{0} \sigma^{\text {th }}$ of an inch thick; in the layer $(c)$ immediately external to it, however, the "osteal cells" were exceedingly well marked. The inner layer looked like smooth ice, and the outer like ice which bad cracked into innumerable tolerably even portions-but these cracks were by no means produced by the canaliculi, which, as yet, were hardly at all developed. Now it seems clear that if the "osteal cells" were produced by the calcification of certain of the corpuscles, they ought to be more obvious in the young, inner layer, than in the outer; whereas just the reverse occurs. The fact stated by Messrs. Tomes and De Morgan, that lamination is less obvious in young than in old bone, tends to exactly the same conclusion. Again, if the granular substance between the lacunæ were composed of calcified corpuscles-" osteal cells," the action of acids ought to bring them out as strongly as it does those of the lacunæ; whereas neither in young bone nor in old can anything of the kind be seen.

With respect to the lacunæ, again, we have the same remarks to make as when speaking of cartilage. We have never been able to find any trace of the development of the corpuscles (granular cells) into lacunæ. As to the tissue which accumulates round them and forms an investment, we have frequently observed the appearance described; but this investment was nothing but the clear, often homogeneous, calcareous matter, gradually encroaching on the matrix and enclosing the corpuseles.

We consider, then, that the process of ossification in primary and secondary bone is identical; the deposition of the calcareous matter in granules or as a homogeneous infiltration, being of no constancy or importance. In each case the deposit takes place in the matrix, and leaves spaces (lacunæ) round the corpuscles (nuclei, granular cells). Subsequently, the canaliculi are developed in the matrix by a process of resorption; while their walls and those of the lacunæ may or may not become chemically differentiated from it. At the same time, the matrix may or may not break up into laminæ and "osteal cells" or granules. Its 
and ligaments, and of the vascular channels; but a more extensive new formation of bone, whether periosteal or in the Haversian systems, together with a simultaneous and more considerable absorption, never occurs in them. It was formerly believed that the coloration of the bones of adult animals by madder, proved that deposition of bone substance continued to take place even in them, it being assumed that newly forming osseous tissue only became colored; but since it has been shown, that bones already formed were likewise colored by the same agent, and that colored bones in the adult did not lose their color (Brullé and Huguény), this view becomes untenable. Whether in the perfect bone a change, if not of the elementary parts, but still of the atoms, takes place, the same external figure remaining, is another question, for the solution of which microscopy affords no facts. This much is certain, that the organization of bone is such, that notwithstanding the rigidity of their structure, they are in the most general and most intimate relation with the nutritive plasma of the blood. In every situation where the osseous tissue is in connection with vessels, as on the external surface, in the walls of the medullary cavities and cancelli, and those of the Haversian canals, millions of closely crowded minute openings exist. These convey the blood-plasma, by means of the canaliculi, into the lacunæ lying nearest to the surfaces mentioned, from which it is then conducted by wider canaliculi to the more distant lacunæ, as far as the outermost layers of the Haversian lamellæ, and those laminæ of the great lamellar system which are most remote from the vessels. When the enormous number of the canaliculi and their multifarious anastomoses are considered, it must be allowed that no tissue in the human body is better provided for in respect of the distribution of the blood-plasma, whilst in scarcely any other is the direct conveyance of the fluid to the most minute particles more immediately necessary than in it. There can be no doubt that the fluids, which this "plasmatic vascular system" (Lessing) of the bones, obtains from the bloodvessels, probably somewhat modified by the influence of the nucleus which, as I have before endeavored to show, is still retained in every lacunæ, are most indispensably requisite for the maintenance of the bone; for we see, that when the supply of blood to a bone is impeded by the destruction of the

variability in this respect is neither more nor less remarkable than the greater or less fibrillation of the corresponding element of connective tissue, or than the inconstancy of the disposition of the cleavage lines of the saine element in striped muscle.

As little is any line of demarcation , to be drawn between primary and secondary bone as regards the tissues from which they proceed. Indifferent tissue, in which calcareous matter is deposited at once, is the basis of secondary bone; an identical tissue-in which to serve a temporary purpose chondrin is deposited, being subsequently withdrawn and replaced by calcareous salts-is the basis of primary bone. And this paragraph may serve as an answer to the fourth question. If it be correct, we cannot imagine that any distinction of the bones into primary and secondary, upon the ground of their development or non-development from cartilage, can be other than arbitrary.-TRs.] 
periosteum or of the medulla, by ligature of the vessels of the limb, or by obliteration of the periosteal vessels by pressure from without (aneurism, tumors), necrosis of the parts involved certainly ensues, and can scarcely in any case be altogether obviated by the collateral circulation which actually exists also in the bones (vid. supra). On the other hand we are scarcely, at present, in a condition to say, how the circulation of the plasma in the bones is carried on, though its movement to and from vessels (perhaps from the arterial, through several lamellar systems to the venous) must probably be assumed; or what special changes in the course of the nutrition of bone take place; with the latter in particular we are unacquainted, because the chemical investigation of these changes, and especially of the organic products of decomposition, is still altogether imperfect.

That the osseous tissue is in a state of constant, and indeed very energetic molecular change, is evidenced not only in its various morbid conditions, but also by the alterations it undergoes in old age. These alterations consist more espeeially in the disappearance of entire portions of the bones, either externally or internally; of the former, an instance is afforded, in the entire removal of the alveolar processes of the jaws, and the latter is seen in the greater porosity and fragility of every kind of bone, such as the cylindrical bones and those of the cranium, in the enlargement of the vascular openings (vertebroe, apopliyses), and in the greater roughness of the surfaces of the bones. This senile atrophy of the bones may also be attended consecutively with an internal addition of bone-substance, a sclerosis, as it is termed, as in the flat bones of the cranium, in consequence of which, in direct contrast to the phenomena elsewhere presented by senile bone, the diploë disappears, its cancelli becoming filled up by new osseous tissue, whilst the venous spaces and foramina emissaria are obliterated and the entire bone rendered heavier.

With this abundant vascular supply, and certainly not sluggish molecular change, it cannot be surprising that the bones should be so richly furnished with nerves, the principal function of which appears to me to consist in the regulation of the conditions of the vascular system, by their conveying to the central organ (spinal cord) through the sensitive fibres intelligence of the state of the vessels, of the quantity of nutritive fluid in the bone, and probably also of the modus of the molecular change going on in themselves, and by means of the motor elements their bringing a reflex influence from it, to the arteries and veins which are manifestly furnished with contractile fibres. These unconscious and involuntary alternations of influence of sensible and motor filaments, are, as it appears to me, the most important phenomena of the innervation in bones, as well as in all other organs, the nerves of which are not constantly in relation with the external world, and make it intelligible, why it is, that no organ, containing nerves and vessels at all, 
possesses nerves of only one kind. It is not, however, by this, intended to imply that the nerves of bones do not convey conscious perceptions; it is possible that, through them, we obtain a certain degree of knowledge of the processes going on in the bones, of the degree of fulness of the vascular system, the mechanical influences to which they are exposed from without in the movements caused by the action of the muscles, the weight of the body, or of external objects, in lifting weights, mastication, \&c.; but in any case this knowledge would be very indeterminate, and the sensation excited not definitely localized, being confused in the general feelings of fatigue, effort, or relaxation. On the other hand, it is quite certain that the bones, in man, in many diseases, and in consequence of mechanical injury, afford pain, which latter fact has also been frequently noticed in animals, at all events, upon irritation of the larger nervous trunks of the diaphyses. In man the apophyses, in particular, and the vertebral and cranial bones, seem readily to become painful, which is explained by the considerable number of nerves immediately in the spongy substance. The compact substance, on the other hand, might probably be regarded as scarcely obnoxious to pain ; as, for instance, in resections, but not so perhaps the periosteum, which less from its own nerves than as the vehicle of those of the bones before they enter their destination, must naturally be affected in the same way that they are. Whether the nerves of the bones through which, perhaps, the conscious perceptions, but in any case the painful impressions are conveyed, be identical with those through which the reflex actions, above referred to, are carried on, is not determined; but, looking at the origin of most of the bone-nerves from the cerebro-spinal nerves, such an opinion might perhaps be maintained, it being premised that the connections of the nerves with the brain are to be regarded as less intimate than in the case, for instance, of the cutaneous nerves. I would, in addition, call attention to the remarkable occurrence of nerves in the cartilage of the septum narium in the Calf, although I am unable to say anything more with respect to their nature than with regard to that of the nerves of bone.

On the subject of the numerous pathological changes which occur in the bones, only some brief remarks can here be made. Fractures readily unite, under but moderately favorable circumstances, by true bone-substance, which, in the cylindrical bones of animals is preceded by the formation of a true cartilage, a fact of which I and others are satisfied; whilst, according to Paget, this rarely appears to be the case in man. In the spongy bones, in fractures within the articular capsules, and under unfavorable circumstances, the fractured ends frequently unite merely by a fibrous callus, a sort of articulation being formed between them. After loss of substance the osseous tissue is readily regenerated; and it is the periosteum especially, which, in this case, as in 
the growth of a bone in thickness, plays the principal part in the restoration; of course by means of the exudation poured out from its vessels. In aninals, entire bones of the extremities, and ribs are regenerated, pretty nearly in their original figure, not only when the periosteum has been saved, of which many examples are exhibited in Heine's collection in Würzburg, but even after entire excision with the periosteum, a rudiment of the bone is reproduced (Heine). In man also, a good many instances have been afforded of the reproduction of entire bones, such as the lower jaw, the ribs, the scapula (Chopart); and the cases of isolated,-in some instances large, portions of bone being so regenerated are very numerous. It is especially the diaphyses, which are readily replaced, when they have been lost in one way or another, less frequently the spongy bones and spongy parts of bones, and those of the cranium; in the latter, however, openings made by the trephine are in many cases filled up, instead of fibrous membrane, with isolated patches of bone, or even with an entire piece of bone; in fact, trephined portions of bone have united exactly in the same way as has been observed to take place with portions of bone half cut off (Pauli). Hypertrophy of bone assumes the most various forms, all of which may be reduced under two principal types: 1, deposits on the surface, or external hyperostoses, which are formed chiefly from the periosteum; and 2 , internal or scleroses, which consist in the filling up of the medullary cavities and Haversian canals with new bone, and these two forms may occur either separately or combined. The former takes place in inflammations of the periosteum, either idiopathic or in connection with cancer, arthritis, syphilis, \&c., the latter not only consecutively in old age, but also in rachitis, osteomalacia, and syphilis. With respect to the microscopic conditions of these growths, Virchow deserves the credit of having distinctly indicated ("Archiv, f. Pathol. Anat. I.," p. 135), that the bony growths or osteophytes on the cranium are formed by a direct ossification of connective tissue without any preliminary development of cartilage, which is also undoubtedly the case in the filling up of the losses of substance in the cranium, in regeneration proceeding from the periosteum, and in most cases of sclerosis. The newly formed osseous substance is sometimes like the normal (many exostoses), sometimes more dense, with small vascular spaces and large irregular lacunæ: Atrophy of the bones is shown in their disappearance in totality in consequence of chronic diseases, paralysis, anchylosis; or in rarefaction of the osseous tissue analogous to senile atrophy, in syphilis, lepra, mercurial cachexy, paralysis, \&c. Death of bone (necrosis) is observed in cases where the periosteum has been destroyed; in inflammations of that membrane and of the bone, \&c., for the most part attended with an excessive growth of the remaining sound parts.* Peculiar morbid conditions exist in osteomalacia and rachitis, but in neither of these

* [ In necrosed bone, the bone-corpuscles are generally but little changed; the inter-corpuscular structure is granular and of a dark color.-DaC.] 
diseases have microscopical researches afforded anything worth mention here, except what has been made known by H. Meyer and myself (1l. cc.) with respect to ossification in rachitis. In this case $I$ have found: 1 , that in the disproportionately large epiphysal cartilages, the layer of the ossifying cartilage-cells (those disposed in rows), measured, instead of $\frac{1}{2}, 2$ to 5 lines; 2 , that the ossifying border is toothed, owing to the circumstance that the cartilage and bone interlace in various ways; 3 , and lastly, that in decidedly rachitic bones, the deposition of calcareous granular particles is wanting, and the cartilagecells almost invariably, shortly before the matrix, and also without any appearance of calcareous granules, are metamorphosed in to bonecells, on which account the formation of the latter can, in no case, be so well studied as in these bones (vid. supra). Accidental cartilageand bone-formations are very frequent. The former tissue is met with, notwithstanding that it is incapable of regeneration, and that wounds of it heal only with a fibrous tissue, more rarely with bone (ribs), in very many organs (bones, mammary glands, parotid, testicles, lungs, and skin) forming what is termed enchondroma; moreover, as a new covering on the osseous growths, at the border of the worn articular ends of bones (Ecker). The latter is seen in ossifications of the permanent cartilages (ribs, larynx, epiglottis, very rarely) of tendons (exercir-knochen, for example), in the dura mater and arachnoid (Miescher, Rokitansky), in the eye (Valentin), in the ovary, in fibrous membranes (membrana obturatoria), in enchondroma, in fibrous and carcinomatous growths, and in the lungs (Mohr's cysts containing hair). Even in these cases the osseous tissue does not essentially differ from the normal, and it is formed, sometimes from a cartilaginous, sometimes, and in fact mostly, from a soft blastema (Virchow, l. c. p. 137).

In investigations relating to the structure of bone, good sections are, above all things, requisite. With a fine saw, thin slices are made, which are ground with water upon a smooth stone with the finger, or with a second smaller stone, for some minutes $(5-10)$, until they are rendered uniformly transparent. The sections are then cleaned, and (the fat, if they contain any, being removed by ether) may be employed, being wetted with water, for the study of the Haversian canals and disposition of the lacunæ; and with turpentine, for that of the various lamellar systems. The lacunæ and their prolongations, which, in sections, are dark and very distinct, owing to their being filled with air, are completely filled by thin turpentine, so that the latter in great part, and also the former, are very frequently rendered invisible; the same thing happens in water and thicker turpentine, though less rapidly, whence, before these agents have produced their effect throughout, many of the lacunæ and canaliculi are beautifully shown. If it be desired to preserve the lacunæ and canaliculi permanently visible, it is best to polish a thin section, by rubbing it between two glass plates. "It may then be 
examined without the addition of fluid, and presents as perfect figures as those represented in Figs. 115-117. The grinding of the bone with oil is not to be recommended, because the lacunæ, then become filled with the oil, and even after thorough treatment with ether can seldom be rendered distinct. Next to sections of bone, the investigation of the bone-cartilage is the most worth while. This tissue is prepared by the treating of bone in the cold, with diluted hydrochloric acid ( 1 part acid, 10-20 water), until the fluid, which is to be frequently changed, no longer affords any precipitate with ammonia; for which purpose, in small fragments of bone, some hours, in entire bones several days, are required. From the cartilage thus obtained, sections are now to be made with a sharp knife in all directions, suitable chiefly for the study of the Haversian canals and lamellæ, which may even be raised from the surface. The lacun:e, also, are still visible; their prolongations or canaliculi appear as fine streaks, and their nuclei are seen without further trouble, especially also after treatment with potassa, or in cartilage which has been half dissolved by boiling in water. After long maceration in hydrochloric acid, the lacunæe even become isolated, as stellate bodies with delicate walls, or, in the cementum of the horse's tooth, as structures corresponding to the former cartilage-cells. After long softening of bone-cartilage in water, the lamellar systems of the Haversian canals become more or less completely separated, presenting the appearance of short, coarse fibres among the larger lamellæ (Gagliardi's claviculi). If bone be exposed in a platinum capsule to a strong white heat, the organic parts burn away, the bone becoming at first black, and ultimately perfectly white; and, if due care be taken, the earthy constituents are left, completely retaining the original figure of the bone. Preparations of this kind are proper for the study of the laminated structure of the compact substance and of the lamellar systems of the Haversian canals, which, in this case also, sometimes appear isolated, as in macerated bone. For the microscopic examination of the inorganic constituents of bone, sections are subjected to heat on platinum foil, but they must be very thin, as they afterwards become more opaque, and, on account of their fragility, except in minute fragments, do not admit of being ground thinner (Bruns); or sections may be boiled in caustic potassa. In either case, the lacunæ are seen distinct, and empty, with the beginnings of the canaliculi, in a finely granular matrix. The natural condition of the lacunæe is readily seen in perfectly recent bone, in thin sections or laminæ; as, for instance, in many parts of the bones of the face. In recent bone, also, the vessels may be studied, naturally injected, and with the microscope, being thus, far fitter for the purpose than when injections, which often fail, have been practised, and for the closer examination of which, moreover, the bones must afterwards be macerated in hydrochloric acid, and preserved in oil of turpentine. The nerves of the bones may be seen 
by the naked eye, on the nutritious arteries of the larger cylindrical bones, and readily, by the microscope, on the smaller vessels; those of the periosteum must be studied after the membrane has been rendered transparent by caustic soda or acetic acid. The costal and articular cartilages are the most suitable for the study of cartilage, the membranes of the cartilage-cells being evident, sometimes without any addition, sometimes after that of acetic acid or soda, which render the matrix transparent. The development of bone may be investigated in a cylindrical bone, and in the parietal bone; the formation of the lacunæ, in specie, in rachitic bones, and in the osseous surfaces of the symphyses and synchondroses.

Literature.-Besides the works cited in $\$ \S 22$ and 25 , are to be noticed, F. Bidder, "Zur Histogenese der Knochen" ("On the Histogenesis of Bone"), in Müller's "Arch." 1849, p. 292 ; E. v. Bibra, "Chemische Untersuchungen üb. die Knochen und Zähne des Menschen und der Wirbelthiere" ("Chemical Researches on the Bones and Teeth of Man and the Vertebrata"); Schweinfurt, 1844; Vötsch, "Die Heilung der Knochenbrüche per primam intentionem" ("Union of Fractures, \&c."); Heidelberg, 1847 ; Kölliker, "Ueber Verknöcherung bei Rachitis, u. üb. den Bau der Synovialhäute" ("On Ossification in Rachitis, and on the Structure of the Synovial Membranes"); "Mitth. der Zürich. nat. Gesellsch.," 1847, p. 93; Rokitansky, "Beiträge zur Kenntniss des Verknöcherungsprocesses" ("Contributions to a knowledge of the process of Ossification"), in the "Zeitschrift der Wiener Aerzte," 1848, p. 1; A. Krukenberg, "Zur Lehre vom Röhrensysteme der Zähne und Knochen" ("On the Tubular System of the Teeth and Bones"), in Müller's "Archiv.," 1849, p. 403; H. Meyer, "Der Knorpel u. seine Verknöcherung" ("Cartilage and its Ossification"), in Müll. "Archiv.," 1849, p. 292; Virchow, in "Verhandl. der Würzb. phys. med. Ges.," vol. i. No. 13; Robin, "Observations sur le développement de la substance et du tissu des os," in "Mém. de la Société de Biologie," 1850, p. 179 ; Brullé and Hugıény, "Expériences sur la développement des os dans les mammiféres et les oiseaux, Ann. d. Sc. Nat.," 1845, Nov. p. 283 ; Flourens, in "Ann. d. Sc. Nat.," 2 série XIII. 103, ibid. XV.p. 202, ibid. 1845; Août, p. 105, and Déc. p. 358 ; "Compt. rend.," T. XIX. p. 621 ; all his observations collected in "Théorie expérimentale de la formation des os," Paris, 1847-8, avec 7 pl.; Beck, "Abh. üb. ein. in Knochen verlaufende Nerven," Freiburg, 1846; Kölliker, "Ueber die Nerven der Knocken" ("On the Nerves of Bone"), in Wurzb. "Verhandl.," I.; Luschka, "Die Nerven in der harten Hirnhaut" ("The Nerves in the Dura mater"), Tübingen, 1850: and "Die Nerven des Wirbelcanales und der Wirbel" ("Nerves of the vertebral Canal and of the Vertebræ"), Tüb. 1850 . 


\section{OF THE NERVOUS SYSTEM.}

$\S 109$. The nervous system, regarded in the more general anatomical sense, constitutes a connected whole, consisting of two principal massesthe spinal cord and brain, and of numerous cords-nerves-extending from them to almost all the organs of the body. The two former-or the central nervous system, the central organs, are to be regarded not merely from an anatomical point of view, as affording origin to the nerves, but, also, in a physiological sense, as excitors of the movements, and seat of the sensations, as well as of the mental or psychical actions, and consequently as belonging to a higher or governing order of parts, whilst to the latter must be ascribed more of a ministerial office-the communication of the contractions and sensations. This mode of regarding the two divisions of the nervous system, however, is only partially correct, because, in the first place, in the central organs, as in the nerves, very many subordinate parts exist; and, secondly, because in the peripheral nervous system, the so-termed ganglia, physiologically and anatomically, represent central organs. The older division also of the nervous system into animal and vegetative, after the observations of recent times, can no longer be maintained; and the latter,-the sympathetic or ganglionic nervous system, can only be regarded as a portion of the peripheral system, though undoubtedly peculiarly constituted.

\section{ELEMENTS OF THE NERVOUS SYSTEM.}

$\S 110$. The nerve-tubes or fibres (Figs. 137-139), also termed primitive tubes, or primitive fibres of the nerves, are soft, fine, cylindrical filaments having a diameter of $0.0005-0.01$ of a line; they constitute the principal part of the nerves and of the white substance of the central organs, although they are not wanting in the greater part of the gray substance of the latter and in the ganglia. When examined in the recent state and by transmitted light (Fig. 137) they appear as clear as water, transparent, and with simple dark contours; by reflected lightglistening, opaline, like fat, in larger quantities together, white, and for the most part their appearance does not indicate that they are composed of different constituent parts. But it is readily seen upon the application of various methods, that they consist of three, entirely distinct, component structures, viz.: of a delicate coat, and a viscid fluid, in the centre of which is a soft but elastic fibre. The coat, or sheath of the nerve-fibres (limitary membrane, Valentin) (Fig. 139, 1, 2, 3, 4, a) is an excessively delicate, flexible, but elastic, perfectly structureless, and transparent membrane, which, in quite unaltered nerve-fibres, except in certain situations, is altogether invisible. But on the application of 
suitable reagents, at least in the thicker fibres of the nerves and of the central organs, it comes readily into view, corresponding in its chemical characters, in all essential particulars, with the sarcolemma of the muscular fibres. In the finest fibres of the peripheral, as well as of the central nervous system, the existence of this membrane has not yet been

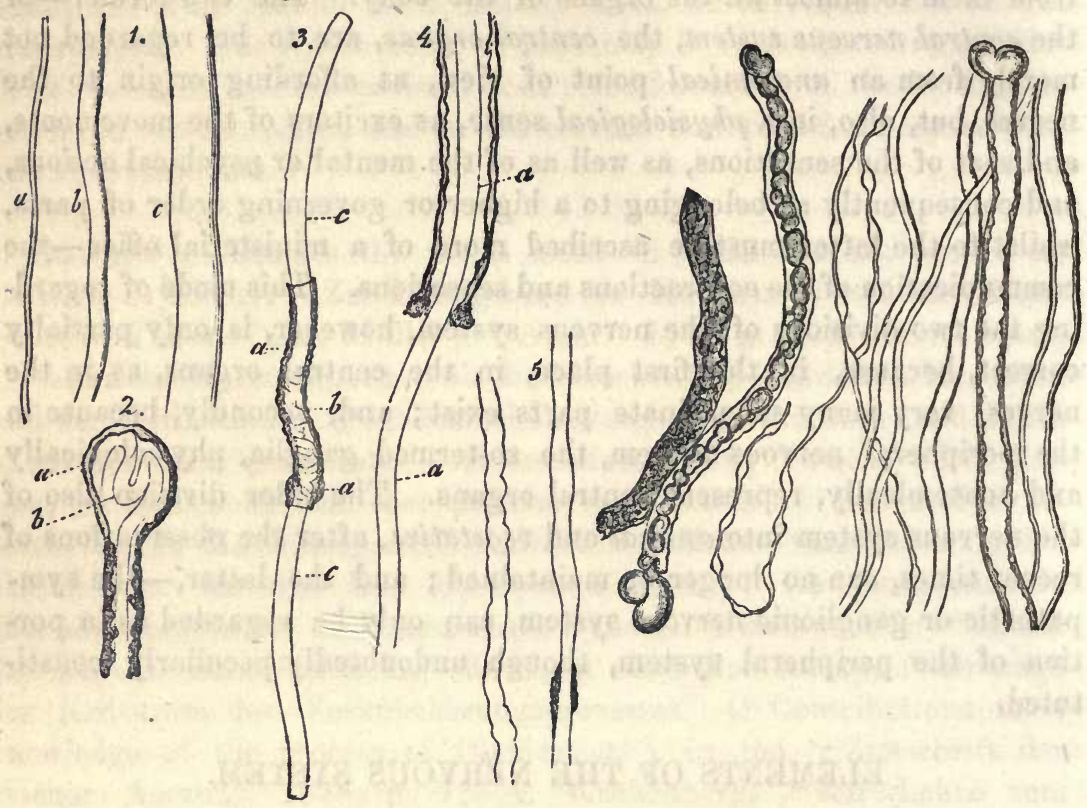

demonstrated, and it must consequently for the present be left undecided whether these fibres possess sheaths or not.

Within the structureless sheath, lies the nerve-medulla, or pulp, ("medullary sheath," Rosenthal and Purkinje, "white substance," Schwann), (Fig. 137, 3, b, Fig. 139, 3, 4, b) in the form of a cylindrical tube, closely and exactly surrounding the central fibre. In the recent nerve-fibre this substance is perfectly homogeneous, fluid, but viscid like a thick oil, and, according to the light by which it may be

Fia. 137.-Nerve-fibres, magnified 350 diameters. 1, from the Dog and Rabbit, in their natural condition; $a$, fine; $b$, of medium thickness; $c$, coarse fibre from the peripheral nerves: 2, from the Frog, with the addition of serum; $a$, drop of the contents expressed ; $b$, axis-cylinder within the drop, continued into the tube: 3 , from the spinal cord of Man, recent, with serum added; $a$, sheath ; $b$, medullary sheath with double contour; $c$, axis-cylinder: 4, double-contoured fibre from the fourth ventricle in Man; the axis-cylinder, a, projecting and visible within the fibre: 5 , two isolated axis-cylinders from the cord, one undulated, the other of unequal thickness, with some medullary substance attached to it.

FIG. 138.-Nerve-tubes of Man, magnified 300 diameters: four fine, two of which are varicose; one of medium size with simple contours; and four thick, two of which have double contours, and two, grumous contents. 
viewed, transparent and clear, or whitish and pearly, and it is obviously to it that the peculiar glistening appearance of the nerves is due. The nerve pulp is rapidly and invariably altered by the application of cold water, of most acids, and of many other reagents, the change consisting principally in a coagulation of it, which takes place gradually from without to within, sometimes involving the entire thickness, sometimes only its outermost layer. In the latter case, are produced the wellknown nerve-fibres with double contour lines (Fig. 137, 2, 3, 4), or in which the medullary sheath is, externally, coagulated to a greater or less extent, remaining fluid internally; in the former case, with the contents apparently wholly grumous and opaque (Fig. 138). The coagulated nervemedulla, in fact, seldom appears homogeneous, but most generally grumous, granular, and as if composed of separate, irregular, larger and smaller masses, and, upon the application of acetic acid, as if formed of minute, separate, or reticularly united rods. The nerve-pulp is also altered very readily by pressure. It sometimes escapes from the ends of the tubes, or from hernial protrusions or ruptures of the sheath, forming larger or smaller drops of every imaginable shape, regularly spherical, clavate, fusiform, cylindrical, filamentary, or of the most bizarre figures, which likewise coagulate either on the surface merely, or throughout, and thence, like the nerves, appear with a double contour, or half or wholly grumous. But, within the fibres also, its structural conditions alter, for, instead of being continued through them as before,-as a cylinder of uniform size,-it accumulates in places into larger masses. In this way are produced the frequently described, varicose nerve-fibres (Fig. 138), in which the medullary sheath presents sometimes, minute moniliform enlargements, sometimes, various sized, irregularly distributed nodosities, or even, in places, complete interruptions. All these forms, in which the sheath frequently participates, but in which the central fibre takes no part, arise artificially, and are developed most readily in the finer fibres with more delicate sheaths, such as are found in the central organs.

The central or axis-fibre of the nerve-tubes ("primitive band," Remak, cylinder axis, Purkinje, Fig. 137, 2, 3, 4, 5; 139, 1), is a cylindrical or slightly flattened filament, which, in entire and unaltered nervefibres, is as little recognizable as the sheath, being surrounded by the pulp, and possessing the same refractive power, whilst it comes readily into view when the fibre is torn or treated with various reagents; and it may thus be recognized as a constant structure, sometimes in the interior of the fibre, and sometimes isolated. Under natural circumstances it is pale, most generally homogeneous, more rarely, finely granular or striated, bordered by straight or occasionally by irregular, pale contour lines, and it is, generally, everywhere of uniform thickness : it is distinguished from the medullary sheath, especially by the circumstance, that 
although soft and flexible, it is still, not fluid and viscous, but elastic and solid, something like coagulated albumen, with which it also ap-

Fig. 139.

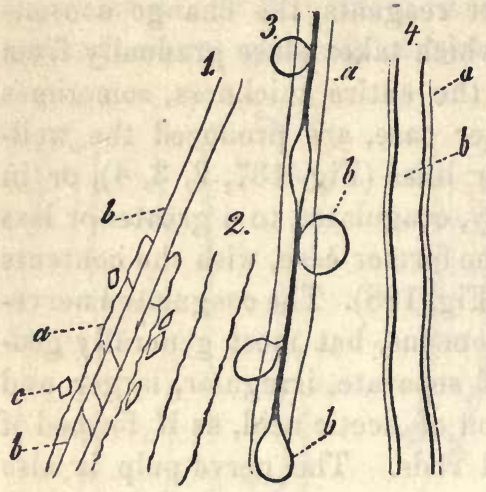

pears to agree in most of its chemical characters. This axis-cylinder exists in all nerve-fibres without exception, even in the finest, and invariably presents the same characters, except only, that it is sometimes thicker, sometimes more slender, according to the size of the fibre itself.

The nerve-fibres, in which the three structures above described can be distinguished, and which we would designate as the medullated or darkbordered, constitute, it is true, the greater proportion of those existing in the body, but besides these there are still some forms requiring more particular description. These are the nerve-fibres in which there exists no trace of a medullary sheath; whilst they have an outer sheath and contents, sometimes identical with the axis-fibre of the other kind of nerves, sometimes similar but more clear. These non-medullated nerve-tubes occur, in the first place, as continuations to the other sort, where the latter are in connection with nerve-cells; and also as more elongated, independent fibres, representing the so-termed processes of the nerve-cells of authors; and lastly, at the terminations of the darkbordered nerves. They may again be arranged in several subdivisions, distinguished respectively by their having or not having nuclei, and more or less transparent, more or less consistent contents. It must also be added, that the dark-bordered fibres differ extremely-partly in respect to the delicacy or firmness of their structure, and partly in their diameter, which varies from 0.0005 to 0.01 of a line, or more, so that they may be distinguished into fine and coarse, delicate or firm fibres; from which it is evident that the nerve-fibres, notwithstanding their general tubular character, still differ pretty widely from each other in various respects.

The tunic or sheath of the nerve-fibres, discovered by Schwann, in most nerves is brought into view with some difficulty. It is only rarely,

Fra. 139.-Nerve-fibres, magnified 350 diameters. 1, from the Frog, boiled with alcohol and acetic acid; $a$, sheath; $b$, axis-cylinder; $c$, crystals (fat ?) $: 2$, isolated sheath of a Frog's nerve, boiled with soda: 3 , from the floor of the fourth ventricle in Man, after treatment with soda ; $a$, sheath $b$, medulla flowing out in drops, the axis-cylinder is wanting (having been drawn out in the preparation), and the pale streak is medulla: 4, from the root of the $n$. abducens of Man, treated with soda; $a$, sheath; $b$, medulla, axis-cylinder not visible. 
as in the roots of certain cerebral nerves (those of the muscles of the eye for instance), and of the spinal nerves that it appears distinct from the contents; its presence however is, with certainty, and readily demonstrated by the aid of chemical reagents. When the nerves are boiled in absolute alcohol, soon after the removal of a considerable part of the fatty matter of the pulp, the sheaths become tolerably distinct, as dark boundary lines; and they are rendered remarkably and beautifully so by a short boiling in acetic acid, during which, the remaining contents of the nerve-sheaths, with the exception of the central fibre, escape from them, whilst at the same time numerous (fat) crystals (Fig. 139, 1) are formed. When boiled in alcohol and treated in the cold with caustic soda, the nerve-fibres also exhibit the sheaths very beautifully, as pale, frequently undulating contours of the colorless, remaining contents ; and when such fibres are boiled for a moment in caustic soda, numerous, elongated fragments of perfectly empty, somewhat swollen nerve-sheaths, are detached, which, from their delicacy, present a striking resemblance to the empty tubules of the membrana propria, of the tubuli uriniferi (Fig. 139, 2). The sheaths, however, are rendered most distinct by means of fuming nitric acid and the subsequent addition of caustic potassa. In this case the fatty matter of the medullary sheath escapes from the tubes in the form of colorless drops, the axis cylinder is dissolved, and the yellow sheaths are left empty, dilated and with swollen walls of $0.0004-0.0008$ of a line in thickness. In nerves treated with corrosive sublimate, according to Czermaàk ("Zeitsch.f. wissensch. Zoologie,"1850), the sheaths are, also, often very prettily shown. It has not yet been determined whether the finest nerve-fibres in the central organs, and in the peripheral nerves (under 0.001 of a line) possess a structureless sheath. Analogy with the coarser fibres is in favor of the existence of such sheaths, but on the other hand there are some facts which would seem to indicate that there are also, sheathless primitive nerve-fibres, both of the medullated and of the non-medullated kinds. I have already (in my "Microscopical Anatomy," II. 1, 396) remarked, that according to my observations in the Tadpole, several dark-bordered fibres are developed in one and the same structureless sheath formed by the coalescence of cell-membranes; and that a similar thing (at least from R. Wagner's figures) occurs in the electric organ of the Torpedo: in which cases special tunics can scarcely be supposed to exist around each separate fibre. And, quite recently, Stannius ("Gött. Nachr.," 1850) has found, in Petromyzon, that the nerve-fibres of the central organs possess neither membranous sheath nor pulp, and are, as it may be expressed, nothing more than free axis-fibres. When to this, it is also added, that the impossibility of demonstrating niembranes, by no means certainly proves their non-existence, still, the facts stated are worthy of all consideration, 
and we must, in this question, for the present, abstain from all conclusions drawn from analogy.

[Of the chemical nature of the sheath of the nerve-fibres but little is known. When boiled for about five minutes in concentrated acetic acid, it is not dissolved, scarcely changed. It resists boiling in caustic soda for a short time without altering, except that it swells up a little; longer boiling dissolves it. It remains unchanged in water, alcohol or ether, even when boiled. Pettenkoffer's test for bilin (sugar and sulphuric acid) which reddens protein and elein, but according to Schulze, neither collagenous substances nor elastic tissue, does not color the nerve-sheath. Yet its action cannot be ascertained with the most desirable accuracy, since the nerve-medulla is simultaneously reddened. Sulphuric acid and potassa render the nerve-sheath yellow (xantho-proteinic acid), producing the same effect as they do, according to Paulsen, on elastic tissue. Hence it seems as if the nervesheath agrees in composition with elastic tissue, except that it is evidently less insoluble in alkalies. From. Kölliker's Micr. Anat. II. 1.-DaC.]

In order to see the medullary sheath or nerve-pulp in its normal condition, a nerve of an animal just killed, without any addition, must be quickly brought under the microscope; in which case some isolated fibres will always be seen quite unchanged, although, as the nerve dries, they are very rapidly altered. Besides this method, I would also recommend the examination of the nerves in the transparent parts of animals, either alive or just killed (nictitating membrane, mucous membrane of the Frog, tail of Tadpole, \&c.), the observing of them on warmed pieces of glass (Stark.), and after treatment with chromic acid, which frequently preserves, particularly the cerebral fibres, quite uninjured. The nervepulp or medulla is obviously a viscid, fluid, extensible, glutinous substance, to be compared in point of consistence with thick oil of turpentine, and which under pressure assumes all possible figures, appearing in the form of globules, filaments, and membranous masses, of very different aspects, with pale or dark borders, and opaque or clear. In chemical composition it consists principally of fatty matter.

The central filament of the nerve-fibres, which was perhaps seen as early as by Fontana, and with which we have become better acquainted under the name of "primitive band" given to it by Remak, or "cylinderaxis" as it has been termed by Rosenthal and Purkinje, is indisputably the most difficult of investigation, and the least known portion of the nerres. There is no microscopist who has not frequently seen this axisfibre, but it may, without fear of contradiction, also be asserted, that there is none, not even excepting Remak himself, its discoverer, who can boast that he has studied and learned its relations in every particular. For this reason, but few, as Hannover and J. Müller, are unconditionally 
agreed with Remak and Purkinje in regarding the axis-cylinder as a constant element in recent nerve, whilst most observers have adopted the views of Valentin ("Repert." 1838, p. 76,1839 , p. 79 ), and Henle ("Allg. Anat."), who regard it as not always present, but rather as a secondary formation, which does not exist during life, and as the uncoagulated central portion of the contents of the nerve-fibre, which, during life, are homogeneous. I have endeavored to the utmost of my power to investigate the relations of this structure, and have arrived at the following results:

1. The axis-cylinder is constantly present in every nerve-fibre, both central and peripheral, in fine and in coarse fibres, and after death is apparent before the nerves are treated with any reagent whatsoever. In the human nerves, in the brain and spinal cord, as they are commonly obtained for examination, the axis-cylinder, when duly sought for, is everywhere and with certainty to be recognized; and in fact by far the most easily in the central organs, where the absence of neurilemma and the delicacy of the nerve-sheaths oppose but little hindrance to the tearing asunder of the fibres. In these situations it may be seen in nearly the finest fibres. It always presents the aspect of a pale filament, which, together with a tolerable degree of consistence, is still very flexible and at the same time highly elastic, as may be readily observed on compression of small portions of the spinal cord (in which case very many axis.cylinders are stretched and torn, retracting considerably, and forming undulating curves). On the average it is about one-third as wide as its nerve-fibre, and consequently varies a good deal in diameter, is obviously quite solid, most generally homogeneous, but not unfrequently also, faintly striated or very finely granular. It most usually follows a straight course, bordered by two parallel, pale contour lines, occasionally, however, it is, in parts, thicker or more slender, though it never presents varicosities like the nerve-fibres; and it may, moreover, be curved or even slightly undulating, and also perhaps with an irregular, even jagged border.

2. When recent nerve-fibres of an animal just killed are treated with proper reagents, the axis-fibre instantaneously appears. If a thin cutaneous nerve of the Frog, whilst under examination with a power magnifying 100 times-be touched with a drop of glacial or concentrated acetic acid, the nerve retracts and there appear instantaneously, at each of the cut ends, large particles of the grumous nerve-pulp, and pale, clear fibres; and the same thing happens if the nerve have been previously teased out, and the fibres brought separately into view. The clear fibres are evidently the axis-fibres, as they may readily be traced into the projecting medullary sheaths and entire nerve-tubes, and in other respects, also, present all the characters of those fibres, only that they are much paler and broader (as much as 0.004 of a line, in the 
peripheral thick fibres) and evidently swollen; they frequently, also, appear convoluted, or even spirally rolled, which is owing, simply to the shortening of the whole nerve caused by the acetic acid. The nervepulp itself is rendered grumous by the same reagent; the grumous particles are sometimes granules, sometimes very short rods, like fatcrystals, which latter may be very often seen on the nerve-fibres, forming stellate, acicular groups (margaric acid); alcohol and ether also render the axis-cylinder very distinct, both, when recent nerves are treated with those reagents in the cold, in which case their action must be rather more prolonged, and when they are boiled in them. I can particularly recommend the boiling in absolute alcohol, by which means excellent preparations of the axis-fibres may be made, and in the shortest time. Under this treatment the nerves become firmer, but still admit of being readily torn into fibres, and always exhibit very numerous isolated central fibres of considerable length, which, contrasted with those brought into view by means of acetic acid, are, as it were, contracted (at most 0.002 of a line wide), yellowish, firmer, and often convoluted or twisted. Ether acts in the same manner. By both reagents the medullary sheaths are rendered paler and grumous, the grumous particles frequently appearing, as it were, to be united into a delicate network. When nerve-fibres are boiled, first in ether and afterwards in alcohol, they become quite pale, but the sheath and axis-cylinder perfectly distinct, the latter presenting precisely the same appearance as after treatment with alcohol alone. Consequently, it would seem, that the axis-fibres contain no trace of fatty matter; at all events, except that they shrink a little, they are not altered by the action of ether and alcohol, and afterwards, also, again enlarge, under acetic acid, into broad pale bands. Besides the reagents above mentioned, the axis-fibres are particularly well displayed by chromic acid (Hannover), corrosive sublimate (Purkinje, Czermàk), and gallic acid, but less readily in recent nerves, in which, it is true that they become instantaneously manifest, although it is never, except by accident, and rarely, that they can be isolated, than, especially after a more prolonged immersion in those. The nerve-fibres under these circumstances appear contracted, the medullary sheath grumous, the axiscylinder more opaque and somewhat diminished, in chromic acid yellowish, but in other respects exactly as above described. In the acoustic nerve of the Sturgeon, Czermàk, by means of corrosive sublimate, bas demonstrated in dividing nerve-fibres, the existence, also, of bifurcating axis-cylinders. Iodine also or iodine combined with aqueous hydriodic acid (Lehmann) act very powerfully. In quite recent nerves it instantaneously renders the medullary sheath wholly grumous, and not only isolates numerous, somewhat shrunken axis-fibres, for a considerable length, but renders them in many nerve-fibres very distinct in situ, and usually appearing convoluted or serpentine. Hydrochloric, sulphuric, 
and fuming nitric acids, in certain cases, also render the axis-cylinders apparent (Lehmann.)*

3. The axis-cylinder consists of a solid protein compound differing from common fitrin, and from the fibrin of the muscles. The chemical nature of the axis-cylinder is difficult of investigation, because it cannot be obtained in an isolated form in large quantity; something, nevertheless, may be learned from microchemical reaction as has been shown by Lehmann and myself. In concentrated acetic acid it swells up considerably, but is dissolved with difficulty, and even after it has been boiled continuously for several minutes, although pale, it always remains unchanged. When boiled for a longer time in acetic acid it dissolves, exactly like coagulated albumen, whilst the sheaths and some of the contents remain undissolved. Alkalies (potassa, soda, ammonia), in the cold, attack the axis-cylinder but slowly, though in soda it instantaneously becomes very pale and swells up to $0.004-0.005$ or even 0.006 of a line. Longer immersion in soda dissolves it, and the same thing takes place upon its being boiled, soon after the commencement of ebullition in the fluid. In fuming nitric acid, it disappears in a short time - less than half a minute,-exactly as is the case with coagulated albumen. Treated with nitric acid and potassa the axis-cylinder is rendered yellow (xanthoproteinic acid) and may be seen spirally contracted, within the nerve-fibres, which are also shortened, but not to the same extent. On the other hand it is not colored by sugar and concentrated sulphuric acid, which redden coagulated albumen, at most acquiring a yellowish or pale-reddish hue. In water the axis-cylinder is unchanged, even when boiled, in which case it is readily isolated and appears somewhat contracted; by ether and alcohol it is undissolved even by boiling, but shrinks to some extent. The latter effect is produced also by corrosive sublimate, chromic acid, iodine, and carbonate of potassa. Viewing all these reactions together, it might perhaps be stated with certainty, that the axis-cylinder is a coagulated protein compound which, however, differs from fibrin, inasmuch as it is insoluble in carbonate of potassa and solution of nitre, and offers much greater resistance to acetic acid and caustic alkalies. On the other hand, it agrees with the substance of which the muscular fibres are composed, in its elasticity and insolubility in carbonate of potassa, differing from it in its insolubility in dilute hydrochloric acid, and difficult solubility in acetic acid.

These are the most important facts connected with the axis-cylinder. The conclusion which may be drawn from them, appears to me, to be simply this, that the axis-cylinder is not an artificial product, but that it must be regarded as an essential constituent of the living nerves.

* [I have always obtained the best preparations by soaking a nerve for six or twelve days in concentrated acetic acid. The axis-fibres can then be readily isolated; but are fragile and devoid of their elasticity. $-\mathrm{DaC}$.] 
The only objection which can be urged against this opinion consists in the circumstance, that the axis-fibre cannot be seen in living fresh nerves, and that it cannot generally be distinguished, as a special structure, in the interior of the nerve-tubes without the aid of reagents. But it must be remarked that it can also be brought into view in nerves that are still warm. Thus I find well-marked projecting axis-fibres at the roots of the cerebral nerves in Frogs just killed, which I have examined as quickly as possible, after the application of a solution of sugar, particularly in those of the optic, trigeminal, and vagus, also in the spinal nerves, for instance in the second. I see them under the same conditions in the peripheral nerves of the Frog that have been teased out, and, in these nerves, have on several occasions, even distinctly noticed the axis-fibres in the form of convoluted filaments, in larger drops of the nerve-pulp expressed from the tubes (Fig. 137, 2). The only fact, therefore, that can be adduced in opposition, is this, that it is quite true that the axis-fibre cannot, with certainty, be perceived in the interior of the recent nerve-tubes themselves, except upon the application of some reagent; but this circumstance obviously proves nothing at all, because neither can it be seen in the interior of tubes of less recent nerve-substance, all of which, as innumerable examples of isolated axis-fibres occurring in them, show, invariably contain such fibres. The axis-cylinder, possessing the same refractive power as the still fluid part of the medullary sheath, is necessarily indistinguishable from it, but from this circumstance we cannot conclude that it is absent, nor, equally, can such a conclusion be drawn from its invisibility in the recent nerve-fibril. Taking all these circumstances together, I am firmly convinced that a special, central structure exists even in recent nerves, which is distinguished from the more external portion,-that is from the medullary sheath, - not only by its chemical composition, as appears to me to have been placed beyond all doubt, but also by its consistence and elasticity, as well as by its possessing a determinate form. The condition in which we obtain the axis-fibre in the human nerves and central organs, by the addition of the serum of the blood, albumen, or vitreous humor, appears to me to represent its natural state; on the other hand alcohol, ether, iodine, corrosive sublimate, gallic, and chromic acid render it more consistent than it is normally; whilst acetic acid, dilute nitric acid, and alkalies exhibit it paler and more swollen. The nerve-pulp forms a semi-fluid cortex around the axis-fibre, and, though intimately connected, is not continuous with it. By pressure, therefore, the pulp may very frequently be expressed, by itself, from the ends of the tubes or from lateral rents of the sheath. The drops of pulp thus formed, usually coagulate on the surface, remaining clear and transparent in the interior, like the central portion of the nerve-tubes. Many authors have described these bodies as por- 
tions of the whole contents of the nerve-tube, and have regarded their formation as a proof against the pre-existence of the axis-fibre, but incorrectly. They belong to the medullary sheath only, which, in the interior of all nerve-fibres with only a double contour, is still for some space perfectly clear and bright. An axis-fibre and a clear space, in fibres having a double contour, are therefore by no means identical, and it is not at all surprising, nor opposed to the existence of an axis-cylinder, that a multitude of drops with a double contour and clear contents should be obtained from such fibres. The medullary sheath may also coagulate entirely, and then the axis-fibre remains evident, sometimes as a transparent streak of uniform breadth throughout; sometimes, when the grumous particles are more numerous, it may be concealed by them, so that the entire contents of the nerve appear to be coagulated. They are so, however, only in appearance, the clear fibre always lying in the interior; and I have never yet seen it coagulated or grumous. Nonmedullated nerve-fibres occur in many situations. I enumerate among them: 1, the pale fibres in the Pacinian bodies; 2 , the nucleated pale fibres in the terminations of the olfactory nerves; 3 , the perfectly transparent, non-nucleated nerve-fibres in the cornea; 4 , the pale, branched, and partially anastomosing terminations of the nerves in the electrical organ of the Torpedo and Ray (R. Wagner, Ecker); 5 , the similarly constituted terminations of the nerves in the skin of the Mouse (vid. "Micr. Anat." $§ 11) ; 6$, the pale processes of the nerve-cells in the central organs and ganglia, even though they may not all pass into dark-bordered fibres. Of these fibres, those which occur at the extremities of nerves were, even by the earliest observers of them, unconditionally regarded as nerve-fibres; and as respects the processes of the nerve-cells, I described this to be their nature as early as the year 1846; but these views could not be considered as fully es tablished, until the relation of the fibres with the elements presenting the dark borders was completely elucidated. But since it has been ascertained by Schwann, Ecker, and myself, that the nerve-fibres of the embryo are in precisely the same condition as the pale fibres now in question, and since, I, Wagner, Robin, and Bidder and Reichert, have shown that the pale processes of the nerve-cells pass into darkbordered fibres, it has become more possible to arrive at positive conclusions on the subject. R. Wagner was the first to broach the supposition, that the pale fibres in the Pacinian bodies, and in the electric organs, were nerve-sheaths, with axis-cylinders, and that the processes which pass into nerve-fibres, were themselves bare axis-cylinders, and, moreover, that the entire granular contents of a nerve-cell are nothing but an axis-cylinder enlarged into a globular form; and after I had demonstrated the constant existence of the axis-cylinder in the living nerve, and that it was a structure distinct from the medullary 
sheath, I considered myself fully justified in asserting that the darkbordered nerve-fibres were in direct connection on the one side through the axis-cylinder, with the pale processes of the nerve-cells and the contents of those cells, and on the other, that they passed into the pale terminal nerves in the situations above mentioned. But this, by itself as I believe, affords no ground for the identification of the pale fibres in question, or the contents of the nerve-cells, with the axis-cylinders. This could only be established, if we knew with certainty that the medullary sheath of the dark-bordered nerve-fibres is superadded from without to the contents of the pale embryonic fibres during the development of the nerves, and is an entirely new formation between those contents and the membranous sheath. This is not the case, however, it being on the contrary more probable that the medullary sheath, which is also albuminous, is developed merely from a metamorphosis of the outermost part of the embryonic nerve-contents, that is to say from the development of fat in it, and that the axis-cylinder is the unaltered innermost part of those contents. In this case all the structures, the nature of which we are now discussing, would represent, not bare axis-cylinders, but an entire embryonic nerve-tube, the contents of which were still homogeneous, or had not undergone differentiation, and would also be in continuous connection with all the parts of the dark-bordered fibres, a mode of explaining them to which, at all events at present, I am disposed to give the preference. In addition I would remark, that the pale nerve-fibres are also met with in different stages of development. The nucleated fibres in the olfactory membrane remain altogether in the stage of embryonic fibres, as also, to all appearance, do the pale ramifications in the electric organ, and the contents of both these kinds of nerve-tubes would appear to have little agreement, in their consistence, with an axis-fibre; in the Pacinian bodies the contents of the pale fibres in all respects, represent an axis-fibre, for it is probable that a sheath also exists in this situation; in the cornea, the contents of the transparent terminal nerve-tubules are apparently more fluid; and, lastly, with respect to the processes of the nerve-cells, they consist, whether they have a delicate sheath or not, of a substance often exactly resembling an axis-cylinder, but which is also frequently of softer consistence, corresponding with the contents of the nerve-cell. The contents of the pale, non-medullated nerve-tubes, therefore, although genetically comprehending more than an axis-fibre, still in all probability are capable pretty nearly of assuming its nature.

$\S$ 111. The nerve-cells (accessory corpuscles (Belegungskörper), nervecorpuscles, Valentin), (Fig. 140), are nucleated cells, occurring in great numbers in the gray or colored substance of the central organs, in the ganglia, and occasionally also in the trunks, and peripheral expansions of the nerves (retina, cochlea, vestibule). The nerve-cells are covered 
externally by a delicate, structureless membrane, which in the cells of the ganglia (ganglion-cells, -globules, -corpuscles), may be demonstrated easily, but with great difficulty in those of the central organs; the

Fig. 140.

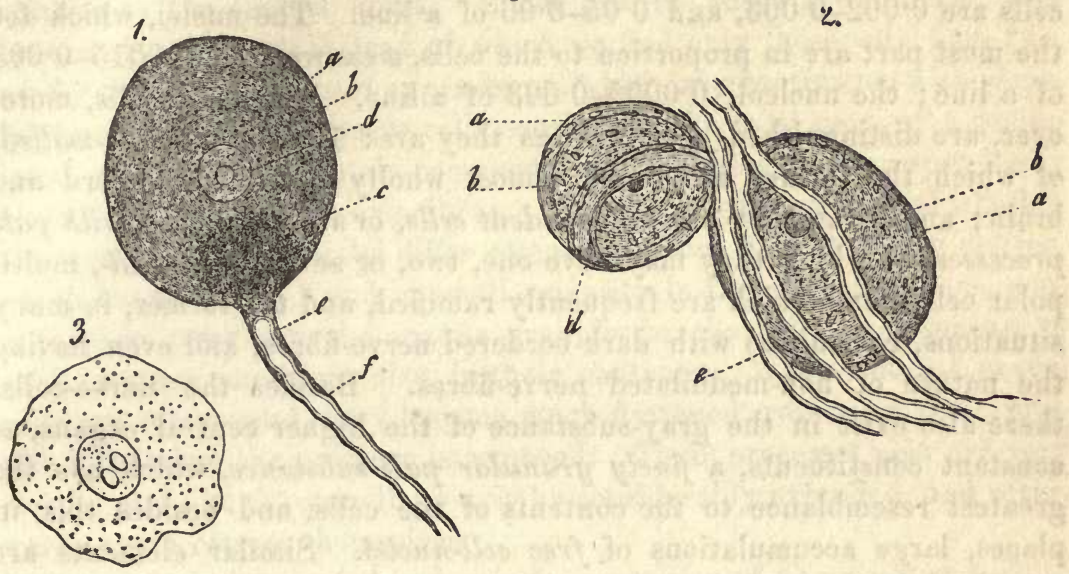

application of reagents, however, will suffice to show, pretty distinctly, that the membrane exists around the larger cells, even in these situations, whilst in the smallest, just as in the finest nerve-fibres, no membrane has yet been observed, although one probably exists. The contents of the nerve-cells are a soft, but tenacious, elastic substance, which besides the nucleus consists of two elements; firstly, of a clear, homogeneous, light-yellowish, or colorless matrix, upon which the physical properties of the contents depend, and which is a protein-compound; and, secondly, of minute granules of different kinds. In the colorless nervecells these present the form of uniform, roundish, for the most part, minute and pale, more rarely, darker and larger corpuscles dispersed throughout the entire contents of the cells, and imbedded in the tenacious matrix; whilst in the colored cells, instead of these granules, more or less yellowish, brown or blackish corpuscles occur. The latter are most usually of a larger size, and are placed, closely aggregated, in a mass near the nucleus; in other instances, they nearly fill the entire cell, giving it the aspect, in all respects, of a brown or blackish pigmentcell. In the midst of these contents lies the nucleus, for the most part as a very clear, spherical vesicle with distinct walls, perfectly transpa-

Fic. 140.-Nerve cells, from the acoustic nerve, magnified 350 diameters: 1 , nerve-cells with the origin of a fibre, from the anastomosis between the facial and auditory nerves, in the meatus audit. int. of the $\mathrm{Ox} ; a$, membrane of the cell ; $b$, contents; $c$, pigment; $d$, nucleus; $e$, continuation of the sheath upon the nerve-fibre; $f$, nerve-fibre : 2 , two nerve-cells with fibres, from the $n$. ampull. infer. of the Ox; $a$, sheath with nuclei; $b$, membrane of the cell; $c$, nucleus; $d$, the origin of a fibre with nucleated sheath : 3 , isolated contents of a nervecell, with nucleus and two nucleoli. For these drawings I am indebted to Dr. Corti. 
rent contents, and one, or more rarely several, large opaque nucleoli, which occasionally exhibit a cavity.

The size of the nerve-cells varies very much; like the fibres, they occur as large, small, and middle-sized. The extreme dimensions of the cells are $0.002-0.003$, and $0.05-0.06$ of a line. The nuclei, which for the most part are in proportion to the cells, measure from $0.0015-0.008$ of a line; the nucleoli $0.0005-0.003$ of a line. The nerve-cells, moreover, are distinguished according as they are: 1, thin or thick-walled, of which the former are found almost wholly in the spinal cord and brain; and 2, as they are independent cells, or are furnished with pale processes, of which they may have one, two, or several (uni-, bi-, multipolar cells), and which are frequently ramified, and the former, in many situations, continuous with dark-bordered nerve-fibres, and even having the nature of non-medullated nerve-fibres. Besides the nerve-cells, there also exist in the gray-substance of the higher central organs, as constant constituents, a finely granular pale substance, which has the greatest resemblance to the contents of the cells, and besides this, in places, large accumulations of free cell-nuclei. Similar elements are contained in the retina, and according to Wagner and Robin in the ganglia of the Plagiostomata.

The nerve-cells are simple cells, as which they were understood even by Schwann; this is clearly and manifestly shown by their form, their chemical composition, and their development. When Bidder, more lately (l. c.), relying upon the fact that the nerve-cells in many situations are in connection at each end with dark-bordered nerve-fibres, propounds the opinion that they are membraneless masses, imbedded in dilatations of the nerve-tubes, he has overlooked those cells from which no fibres are given off, which possess exactly the same membrane as those with processes; and has not considered that there also exist nervecells with a single, and others with numerous processes, as applied to which, his view would be altogether unnatural; and lastly, that the development of these bodies indicates that the nerve-cell is formed in toto, whether it possess processes or not, from a simple cell. It has not yet been determined whether the nerve-cells of the large central organs have membranes or not; Stannius was unable to detect them in the Lamprey, and R. Wagner says the same of the nerve-corpuscles of the electric lobes of the Ray. I think I have seen a membrane in the large, many-rayed corpuscles in the spinal-cord and cerebellum of man, and occasionally, also, in others, but I freely acknowledge that no membrane can be detected in all the smaller cells, nor in the processes of the central cells in general. This does not, however, appear sufficient to justify the denial of the existence of membranes in these instances, and I believe, that in this case, as in that of the finest nerve-tubes, we must 
for the present abstain from any definite opinion. The processes of the nerve-cells, in the brain and spinal cord, which were first noticed by Purkinje, will be more minutely described when we come to speak of the central organs, and the question will there be discussed as to their relation to the central fibres. In the ganglia, there are no cells with branched processes, instead of which we find only those with one, two, rarely three or four, pale appendages, which are continuous with darkbordered tubes. The nerve-cells consist, for the most part, of a coagulated, although soft protein-compound, which appears to correspond very closely with that of the axis-fibres. It has not been ascertained whether the membranes and nuclei differ essentially from it. The fatty matter, which has also been found in small quantity in the gray substance, constitutes in every case the opaque granules in the cells, and appears to exist in other conditions also, in their contents. When isolated nervecells are compressed, they become much flattened, resuming their pristine form when the pressure is removed. Their processes also are very elastic, and like the axis-fibres may be considerably extended, and afterwards again retract themselves.

As our knowledge of the chemical composition of the gray and white substance still leaves much to be desired, I content myself with the following statements. Lassaigne, in the brain of a lunatic, found-

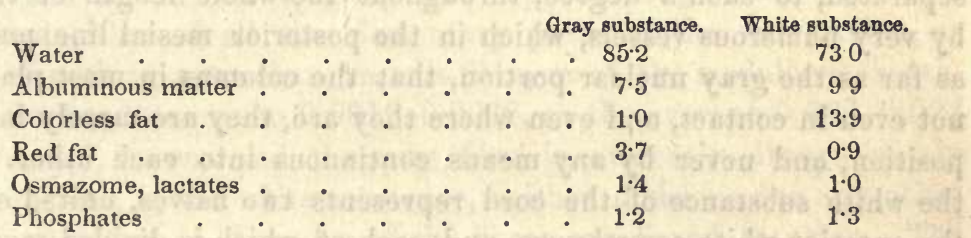

According to Frémy (Comptes rendus, tom. ix. p. 703, "Ann. d. Chem. und Pharm. 1841," vol. xl. p. 69), the brain (both substances together) contains-

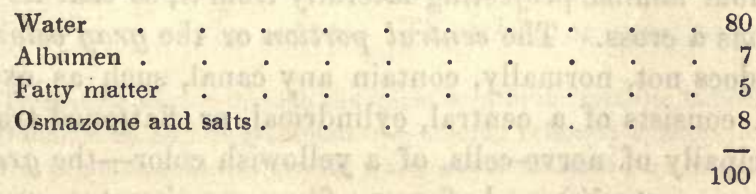

Which almost exactly agrees with Vauquelin's analysis, who moreover estimates the osmazome at $1 \cdot 12$, and the salts at 6.65 ; whilst it differs from that of Denis, who found much more fatty matter $(\mathbf{1 2} 40$ in a man 20 years old, 13.3 in one aged 78$)$, and less water $\left(78\right.$ and $\left.76_{0}^{0}\right)$.

\section{CENTRAL NERVOUS SYSTEM.}

§112. Spinal Cord.-The nervous elements are so disposed in the spinal cord, that its external, white substance is constituted almost ex- 
clusively of nerve-fibres, whilst the gray nuclear portion with its prolongations, the cornua, or horns, is formed, in almost equal proportions, of nerve-fibres and cells.

The white substance of the spinal cord may, for the purpose of description, be most conveniently, and in accordance with usage, divided into two halves, and each of these into three columns. The anterior columns (funiculi anteriores), are, towards the interior, almost completely separated from each other by the anterior fissure (fissura anterior), which extends the whole length of the cord, and into which a vascular process of the pia mater penetrates. At the bottom of the fissure, however, the columns are united by the anterior or white commissure (com. alba); externally, they extend as far as the points of exit of the anterior roots of the nerves, or to the sulcus lateralis anterior, - but are here inseparably connected with the lateral columns (funiculi laterales), which again, at the points of exit of the posterior roots, where the sulcus lateralis posterior is situate, are continuous, without any line of demarcation, with the posterior columns. The latter (funiculi posteriores) appear indeed as if they were in contact in the posterior mesial line, because the posterior longitudinal fissure described by many anatomists, does not exist in man, except in the lumbar enlargement of the cord, and in the superior cervical region; but they are nevertheless separated, to such a degree, throughout the whole length of the cord by very numerous vessels, which in the posterior mesial line penetrate as far as the gray nuclear portion, that the columns in most places are not even in contact, and even where they are, they are merely in juxtaposition, and never by any means continuous into each other. Thus the white substance of the cord represents two halves, united only by the anterior white commissure, and each of which is divided more artificially into three columns, which occupy the depressions left between the projecting processes of the gray substance.

The gray substance presents a central portion, more of a riband-like form, and four laminæ projecting laterally from it, so that its transverse section forms a cross. The central portion or the gray commissure, in the adult, does not, normally, contain any canal, such as exists in the foetus, and consists of a central, cylindrical, or flattened tract, constituted principally of nerve-cells, of a yellowish color-the gray nucleus (subst. grisea. centralis), and of nerve-fibres running transversely, continued beyond the nucleus, before and behind it-the gray or posterior commissures. Of the laminæ, in a transverse section also termed horns, the anterior are thicker, and shorter, of a uniform gray color, composed of larger and smaller nerve-cells, and of delicate nerve-fibres of medium fineness; the posterior, longer and thinner, are constituted at their roots like the former, only most usually of smaller cells; but at the free edge are invested with a more transparent layer, containing 
a preponderance of smaller nerve-cells-the substantia gelatinosa of Rolando. Of the roots of the spinal nerves, the anterior penetrate between the anterior and lateral columns, directly to the anterior horns, and the posterior are lost between the lateral and posterior columns, passing through the substantia gelatinosa into the posterior laminæe or horns.

With respect to the. intimate structure of the spinal cord, we have to distinguish in the white substance:-1, horizontal; and 2 , longitudinal fibres. The latter, in all situations, except in the anterior commissure, are in great part altogether unmixed with horizontal fibres, and everywhere, both superficially and deeply, run parallel with each other, but they are never interlaced, nor do they ever constitute smaller fasciculi. The number diminishes from above downwards, because, as will be afterwards shown, they successively pass inwards towards the gray substance, presenting the general characters of the central nerre-fibres; that is to say, the delicacy of sheath, disposition to the formation of varicosities, and to the breaking up into separate fragments, which are constituted either of all the elementary parts of the nerve-tubes, or consist of nothing more than an axis-fibre, or of the medullary sheath. Their diameter amounts to $0.0012-0.0048$, on the average $0.002-0.003$ of a line, and, in one and the same fibre is, evidently, always nearly the same, since, in the white substance, neither divisions nor any other kind of alteration in diameter of the fibres are found to exist. The transverse fibres occur:-1, in those portions of the lateral and posterior columns which adjoin the horns of the gray substance, and the description of which will be given afterwards with that of the gray substance; 2 , in the white commissure; and, 3, at the points of entrance of the roots of the nerves. The white, or anterior commissure

Frg. 141.-Transverse section through the spinal cord in the superior lumbar region, magnified about 30 diameters, half diagrammatic : $a$, anterior column; $b$, lateral columns, motor portion; $c$, lateral columns, sensitive portion; $d$, posterior columns; $e$, anterior longitudinal fissure; $f$, posterior longitudinal fissure; $g$, motor roots ; $h$, their internal fasciculus; $i$, their external fasciculus; $k$, decussation of the anterior columns in the anterior commissure; $l$, gray fibres of the lateral columns passing into the anterior gray commissure; $m$, gray central nucleus, here internally with two groups of somewhat darker cells; $n$, posterior gray commissure, with a vessel cut across; $o$, fibres of the posterior column passing into the gray commissure; $p$, fibres of the sensitive roots going off to the lateral columns; $q$, similar fibres entering the posterior column; $r$, longitudinal fasciculi of fibres passing into the sensitive roots; $s$, substantia gelatinosa, with traversing bundles of the sensitive roots, $w ; t$, sensitive radical fibres running horizontally forwards to the gray commissure; $u$, large cells of the anterior cornua (the puncta), inner group; $v$, the same, outer group. 
(Fig. 141, $k$ ), is not a commissure in the common sense of the word. It is formed by those nerve-fibres of the anterior columns, which are, in succession, the most deeply placed, and which bending obliquely inwards, cross in front of the gray commissure; the fibres coming from the right anterior column, passing, in a radiating manner and horizontally, to the left anterior horn of the gray substance, and those from the left anterior column passing in like manner to the right anterior horn. The anterior commissure, therefore, represents a decussation, or crossing of the anterior columns, and would be better designated as such. It varies both in thickness and breadth; it is thickest in the region of the two enlargements, and is least so in the middle of the dorsal portion of the cord. Its breadth is regulated pretty nearly by that of the cord, and of the bottom of the anterior fissure; being greatest in the cervical enlargement, and from this point decreasing pretty uniformly in both directions. The decussating fibres measure $0.0012-0.003$ of a line, and, as they diverge in the anterior horns, evidently in some degree decrease in diameter.

The roots of the spinal nerves (Fig. 141, $g, w$ ), without any communication with the longitudinal fibres, are continued, in larger fasciculi, from the sulcus lateralis anterior and posterior, either horizontally or slightly ascending between those fibres, in order, all of them, to enter the anterior and posterior gray laminæ, where we shall again meet them. Their nerve-fibres (in the posterior roots, about $\frac{2}{3} \mathrm{ds}$ measuring $0.004-0.008$, and $\frac{1}{3} d 0.0012-0.003$ of a line, in the anterior about $\frac{3}{4}$ ths measuring from $0.006-0.011$, and $\frac{1}{4}$ th $0.0025-0.003$ of a line) present, as soon as they have entered the cord, all the characters of the central fibres, the largest, at their commencement, measuring about $0.004-0.006$ of a line, in the sensitive, up to 0.008 of a line, in the motor roots. They continue, however, distinctly and constantly decreasing in size, until ultimately, the former, when they enter the gray substance, have a diameter scarcely more than $0.0012-0.0028$ of a line, and the latter in like manner one of not more than 0.004 , or in some of 0.006 of a line.

In the gray substance, the nerve-cells and fibres deserve special consideration. The former present very various forms, all, however, corresponding in one respect, that they are invariably furnished with processes or prolongations, and, for the most part, with many such, which repeatedly branching, ultimately terminate in extremely fine, pale fibrils, like the finest axis-fibres. I distinguish:-1. The cells of the central gray substance. These (Fig. 142) are $0.004-0.008$ of a line, in size, always pale and finely granular, with multiple nuclei and branching pale processes, constituting as it would seem the principal bulk of the central gray substance; but in which, dark, true nervefibres also occur although of nearly the finest kind, which can scarcely be recognized as such, and are indeed very few in number, and quite 
isolated; besides these, there are a good many extremely fine, pale fibrils, like the processes of the cells, only more extended, and running in a transverse and longitudinal direction, of which nothing more can be said, as to whether they are nerve-tubes, or are to be referred to the processes of the cells. The gray central substance, taken altogether, is thickest in the lumbar enlargement, and on a transverse section is of a pyriform, scutate, or cordate figure; next to this stands the cervical enlargement; and lastly, the superior cervical and dorsal portions, in which latter parts its transverse section presents an ellipse, in the cervical region, with the longer diameter much developed. It occasionally exhibits, especially in the lumbar region, indications of its being constituted of two halves, inasmuch as the middle appears somewhat clearer, and the lateral portions, owing to the accumulation of fatty granules in the cells, more opaque. 2. With the above-described cells, those of the sub-

Fig. 142 .

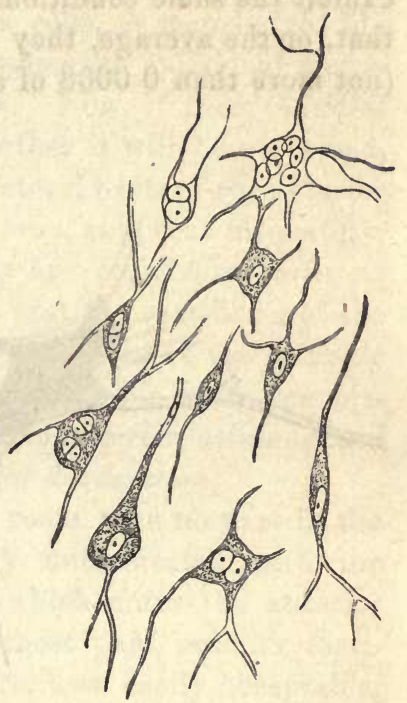
stantia gelatinosa* pretty nearly agree, only that they are of a faint, yellowish color, and have 1-3 processes and simple nuclei. Besides these cells, the substantia gelatinos a also contains the fasciculi of fibres of the posterior roots which pass through it, and numerous other true nerve-fibres (vid. infra). 3. Much-developed, well-marked nerve-cells are seated especially at the apex of the anterior horn, forming an internal and an external group (Fig. 112, u,v), but occurring also in the other portions of the anterior, as well as, though in less number, in the posterior horns, whilst they are never met with in the subst. gelatinosa, nor in the gray commissure. All these cells (Fig. 143) are $0.03-0.06$ of a line, in size, with nuclei measuring $0.005-0.008$ of a line, are fusiform or polygonal, frequently containing brown, pigmentary matter, and furnished with from 2 to 9 , or even more branched processes, which at their origin are often $0.004-0.005$ of a line thick. These processes may be traced to a length of $0 \cdot 1-0.24$ of a line, and ultimately terminate in fine fibrils, all of which, scarcely more than 0.0004 of a line thick, are contained within the gray substance. Besides these large and, for the most part, many-rayed cells, there are also found in the gray substance though more widely scattered among its nerve-fibres, very numerous, smaller cells, which constitute a complete series between the large cells

Fig. 142.-Cells from the gray central nucleus of the cord in Man, magnified 350 diam.

* [The gray matter of the posterior horn.-DaC.] 
and those of the substantia gelatinosa, and consequently require no further description.

The nerve-fibres of the gray substance are excessively numerous, so much so as to constitute in any case the half of its bulk, if not more, and exhibit the same conditions as those of the medullary substance, except that, on the average, they are not more than half as thick, or even less (not more than 0.0008 of a line); fibres, however, also occur of the same

Fig. 143.

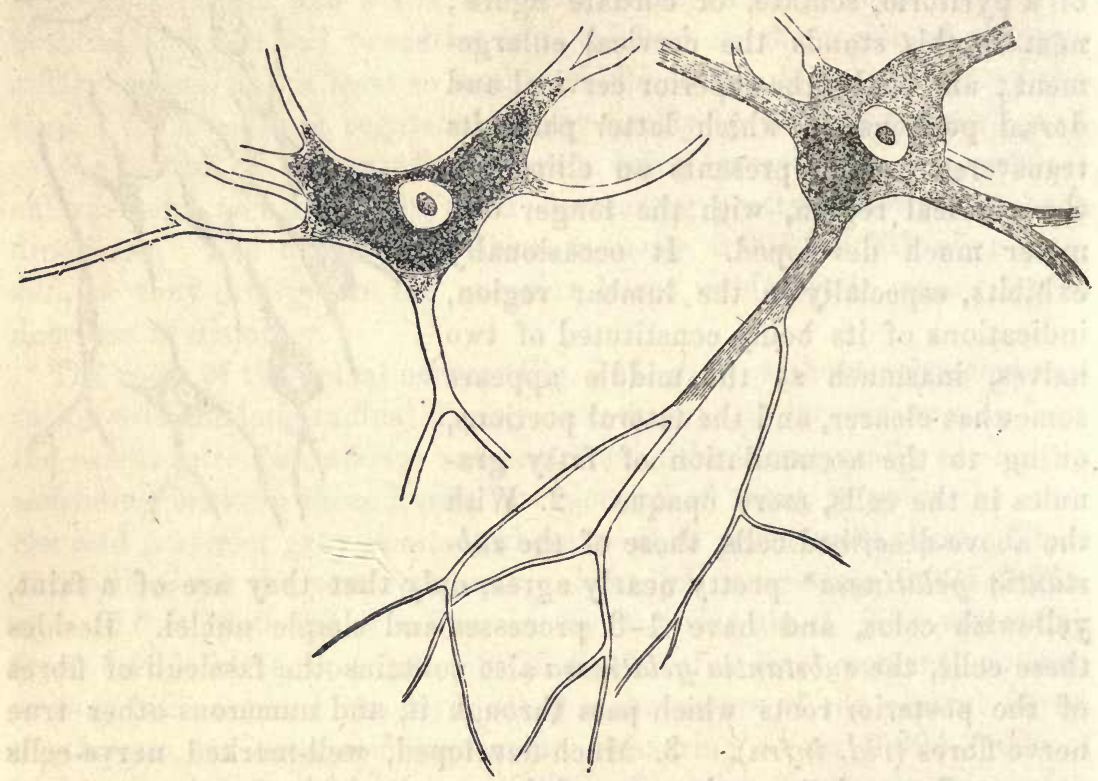

size as those in the white substance and in the entering roots of the nerves, especially in the anterior horns, though more widely scattered, and principally towards the anterior roots. The investigation of the course of these nerve-fibres in the gray substance is one of the most difficult tasks in microscopy. If we observe, above all, the roots of the peripheral nerves (Figs: 141, 144), it is apparent:-1. that the motor filaments in them, after they have entered the sulcus lateralis anterior, and the contiguous portions of the anterior and lateral columns, and penetrated horizontally between the longitudinal fibres of the white substance, are continued further in the gray substance of the anterior horns, principally in two directions. The fibres of one bundle, and indeed of that which enters the most internally (Fig. 141, $h$ ), proceed directly backwards and a little,inwards, without forming any plexus, or

FIG. 143.-Large nerve-cells with processes from the anterior cornua of the spinal cord in Man; magnified 350 diameters. 
subdividing to any considerable extent into subordinate fasciculi, to the innermost portions of the anterior horns, skirting the anterior columns. In this course they pass through the inner group of the many-rayed, large nerve-cells, in perfectly compact bundles, however, and having no connection whatever with the processes of the cells, as may be readily perceived under a strong magnifying power, when the individual nervefibres may be traced quite beyond these cells. Now, if these bundles derived from the anterior roots are traced further it will be perceived, in favorable sections, that they extend to the lateral parts of the anterior commissure, continuing to run in the anterior horns, and, that ultimately, forming more or less well-marked curves, they are continuous with its fibres, and in fact are disposed in such a way, that the root-fibres of the right side pass into the left anterior column, and those of the left side into the right. Consequently there is established in the white commissure $a$ connection of the longitudinal fibres of the anterior columns and of a part of the motor roots, tngether with a total decussation.

A large number of the fibres of the motor roots take no part in the above-described decussation, and are wholly unconnected with the anterior fasciculi,- these are the root-fibres which enter the anterior horns the most externally. Forming, for the most part, smaller fasciculi, or even as separate fibres, and therefore, less easily observable, they run in part directly backwards, and in part arch outwards, but ultimately bend towards the anterior half of the lateral column, where they pass between the outer groups of the large, many-rayed cells of the anterior horns, and then enter the lateral column in a horizontal direction. These transverse fibres now penetrate to various depths (nearly half, or even more) in to the lateral column, then curve upwards, and after running thus a short distance, appear as longitudinal fibres. Consequently, to express the same thing in other words, a second portion of the motor roots arises from the anterior half of the lateral column of the same side, and quits the spinal cord without previously undergoing any decussation.

It is, moreover, worthy of remark, that most of the fibres, perhaps all, which join the motor roots from the anterior and lateral columns, undergo considerable changes in their diameter. Those of the anterior column measure, as has been observed before, at their commencement, on the average, $0.002-0.00 t$ of a line, in the anterior commissure scarcely more than 0.003 , and in the gray substance hardly more than 0.002 of a line; and the same is the case also with those of the lateral columns; which, however, even while still in the interior of the column, where their direction is horizontal, measure scarcely more than 0.002 of a line. This diminution in size, however, is again succeeded by an increase in thickness, which takes place, in part, within the gray substance, in part at the point where the radical bundles quit it, the amount 
of which increase has been already stated in numbers; so that, proceeding from the peripheral nerves, we find them gradually diminishing in

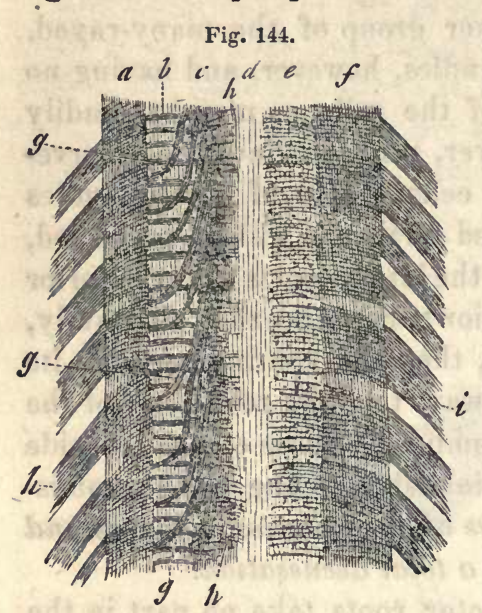
size from their entrance into the cord until they reach the gray substance, and again enlarging from the point where they join the longitudinal elements of the white substance, but not to such an extent as ever to attain nearly their pristine diameter. Of divisions in the fibres of the anterior roots in the anterior horns, I have seen as little indication, as elsewhere in the spinal cord.

The posterior roots of the nerves, as has been already noticed, penetrate, like the anterior, also horizontally, or in a slightly ascending direction, from the sulcus lateralis posterior, through the longitudinal fibres of the white substance as far as the posterior horns. Here they divide into separate, slenderer, or thicker fasciculi (from $0.01-0.02$ of a line), (Figs. 141, s, $144, b)$, and continue, each bundle by itself, in a straight course, and without any direct connection with nerve-cells, quite through the substantia gelatinosa into the substantia grisea. In this course they follow two directions. One portion of them bends upwards, in a uniform curve, or nearly at a right angle, proceeds in the most posterior part of the substantia grisea, close in front of the substantia gelatinosa in a longitudinal direction, and gradually joins chiefly the posterior column, but in part also the posterior portions of the lateral column, being continued further, as its longitudinal fibres (Figs. 141, r, 144, g). A second portion of the sensitive roots (Figs. 141, $t, 144$ ), penetrates, always in a fascicular form, between the above-mentioned longitudinal bundles further forwards, losing itself in the posterior and in the lateral columns, and also entering the gray commissures. In horizontal sections, the former fibres are frequently very distinct, particularly those going off to the posterior columns (Fig. 141, $p, q$ ). I have seen them inost distinctly in the inferior extremity of the spinal cord, below the lumbar enlargement, where they ran towards the conus medullaris, close up to

Frg. 144.-Vertical section through the cord, midway between the gray cornua and the point of entrance of the roots of the nerves, magnified about 25 diameters: a, posterior column with the sensitive roots, $h$, traversing it; $b$, substantia gelatinos $a, c$, prolongations of the posterior roots, which bend round in front of the substantia gelatinosa and run longitudinally, in order there to join more particularly the posterior column; $d$, basis of the posterior cornua, with the ends of the horizontal portion of the sensitive roots apparent (owing to their being cut across); $e$, anterior cornua with the large nerve-cells (the spots), and the also horizontal and divided continuations of the motor roots ; $f$, anterior column traversed by the motor roots, $i$. 
the gray central nucleus, and did not bend backwards until they reached the posterior columns; they were also well displayed in the lumbar enlargement, between the substantia getatinosa and the posterior commissure. The horizontal radical fibres, also, which proceed to the lateral columns, are often exceedingly numerous, although much less so, apparently, than those which enter the posterior columns. The connection of the gray commissures with a portion of the sensitive radical fibres, is, as regards the posterior fibres, not difficult to be seen, these fibres, in part, at least, running backwards along the posterior columns, and being continued directly into the fasciculi of the substantia gelatinosa. In the anterior gray commissure, I have also noticed, though not a direct connection with the sensitive roots, still, fibres which, running horizontally in a direction towards the summits of the posterior horns, entered those processes. The commissural fibres are, besides, connected not only with the sensitive roots, but also, and indeed quite evidently, with the posterior columns, and less distinctly with the lateral; from the anterior portions of which, adjoining the base of the posterior borns, arched fasciculi pass into the commissures and become intermixed with the other commissural fibres (Fig. 141, $o$ and $l$ ). These fibres probably pass over to the opposite side, into the commissures connected with the posterior roots; in which case, like the anterior halves of the cord, a decussation of fibres also takes place in the posterior commissure. In accordance with what has been remarked, the sensitive roots derive their fibres principally from the posterior and lateral columns (the posterior halves) on their own side, and probably also through the gray commissures from the same columns on the other side.

The fibres of the sensitive roots also decrease in size as they traverse the gray substance of the posterior horns. In the roots themselves they still measure about 0.008 of a line; in the substantia gelatinosa, never more than 0.004 , in the substantia grisea, $0.001-0.003$ of a line; in the gray commissures, not more than $0.0008-0.0012$, in the posterior and lateral columns, again, $0.0012-0.004$ of a line; in connection with which, however, it should be remarked, that, in this situation, the increase of size in the fibres which enter these columns horizontally, is not perceptible at their commencement, as is shown more particularly in vertical sections, made in the direction from within to without, through the posterior cornua. The variation in diameter may even be directly observed, in many fibres in this situation; as, for instance, at the entrance of the roots in to the gelatinous substance.

Besides these fibres, belonging to the motory and sensitive roots, there exist, in the gray substance, a considerable number of nerve-fibres not referable to the roots, and which until more is known about them may be termed special spinal-medullary fibres.

The filum terminale contains, so far as it is hollow, as a continuation 
of the gray substance of the cord, a gray, soft substance, consisting chiefly of round, nucleated, pale cells, like nerve-cells, and measuring $0.005-0.006$ of a line. Besides these there occur, in its upper part among the cells, true dark-bordered nerve-fibres, of various, and for the most part small diameter, and also numerous, fine, pale fibres, the nature of which is not clear to me; that is to say, whether they are processes of cells or belong to the finest nerve-fibres. Remak ("Observ.," p. 18) supposes, that in the Mammalia the true nerve-fibres of the filum all go off in lateral branches of it, the existence of which were detected by him.

In the investigation of the course of the fibres in the spinal cord, chromic acid, or instead of it, chromate of potassa, affords the principal aid. It is not easy to hit upon the proper proportion of acid, and so to harden the cord, previously stripped of its dura mater, and cut across with a sharp knife at the points selected, that very thin transverse sections may be taken from it. If the solution be too much diluted, the substance of the cord remains soft in the interior and spoils, if too concentrated it becomes fragile and friable, and larger sections of it cannot be obtained. I have unfortunately neglected to estimate precisely the proper percentage of acid or salt in the solution; and can only say this much, that one of a wine-yellow color acted the best. Objects thus properly hardened may be cut at pleasure with a razor, or other very sharp instrument, if due care be taken, particularly in the avoiding of any sawing motion; and sections suitable even for the highest magnifying powers may be procured and examined, either with or without pressure or reagents, under various degrees of enlargement. The gray substance is scarcely altered by the chromic acid, except that its elements are more easily separated, and I have in hardly any other way seen its nerve-cells, together with their processes, and the nerve-fibres, more beautifully displayed. If it be desired to examine the former, the gray substance is broken up in water, which now no longer produces any change; or, what is best, in the solution of chromic acid itself; but if the examination of the latter be wished, it is by far the best to employ diluted caustic soda or potassa, which renders all the nerve-cells pale. To those who may consider the application of these reagents as too powerful for such delicate organs as the spinal cord, I would remark: 1, that, as stated by Hannover, chromic acid alters the nerve fibres, especially of the gray substance, so little, that most of them do not even become varicose; and $2 \mathrm{~d}$, that soda, added to a preparation made with chromic acid, does not act upon the fibres for a long time, and only so far as to render them more transparent and their medullary contents fluid. I have never, under any circumstances, seen more beautiful nerve-tubes of the gray substance, than in preparations made with 
chromic acid, and I think that of all known means this is the best for their study. In employing pressure, however, great care is requisite. I make use of a compressorium by Nachet, which allows extremely thin covering-glass to be employed, and consequently the highest magnifying powers ; if I wish to apply more considerable pressure for the study of the coarser conditions, the common apparatus suffices, with which, however, with a shorter focal distance of the microscope, only lower powers can be employed. I have had entire transverse sections of the spinal cord before me, in which it might be said, that no part was disturbed from its relative position, and yet, which admitted of the application of a magnifying power of 350 diam. I would, moreover, remark, that the most favorable place in the cord for the first investigation, is the lumbar enlargement. In this situation the cord is not so thick, but that entire sections of it may be obtained, besides which the white substance, which is only an impediment, is thin, and the roots and commissures large and more readily traced.

Whether the nerve-fibres in the cord divide, has not yet been fully ascertained, yet $I$ think that I saw such an appearance, on one occasion, in a dark-bordered fibre, and on another in an isolated axis-cylinder. In any case such divisions cannot be frequent, otherwise I must have noticed them more often, having examined innumerable nerve-fibres and axis-cylinders, expressly with reference to this point. Anastomoses between the processes of branched nerve-fibres, which Schröder van der Kolk thinks he has seen, I must, from my experience so far, doubt, but I am not able to deny their possible occurrence.

\section{$\S$ 113. Probable course of the Fibres in the Cord.-We have found} that the motor and sensitive roots do not terminate at the point where they are implanted into the gray substance of the cord, as at first sight appears to be the case, but that the greater proportion of them are curved upwards, accompanying the longitudinal fibres of the white substance. The important question now arises, viz.: to ascertain what becomes of these fibres, whether, after running a shorter or longer distance, they terminate in the cord, or whether they all ascend to the brain. It is well known, that until recently, most observers have been of the latter opinion, which was founded less upon direct observation than on the ground of probability, until Volkmann, in his deservedly celebrated article, "Physiology of the Nerves," shook it to its foundations, carrying the greater number of physiologists with him. I also was among these, until I had myself investigated the conditions, for there could be no doubt that Volkmann's theory connected, in the most harmonious way, the anatomical facts and the results of physiology as at that time exhibited. When I now, notwithstanding this, abandon Volkmann's theory of the termination of the spinal nerves in the cord, I am induced 
to do so by weighty reasons, and much regretting that $\mathrm{I}$ am unable to maintain a view, which appeared to throw so much light upon many difficult parts of the physiology of the nerves, and to be in accordance with so many other anatomical conditions (ganglia, invertebrate animals).

Volkmann, in his hypothesis of the origination of the fibres in the cord, relies upon the circumstance (l. c., p. 482, et seq.), that the spinal cord is not of a pyramidal form with the base above, as must have been the case had all the fibres of the roots of the nerves ascended towards the cerebrum, but that it rather presents a local increase of the nervous substance at the points of origin of large nerves, which enlargement is not confined to the gray substance, but equally involves the white. That this is the case Volkmann shows from four transverse sections of the spinal cord of the Horse, and from a comparison of the diameter of the cervical cord of Crotalus horridus, with that of all the nerve-roots of the same animal, which was found to be eleven times greater than the former. He also supports his view by the consideration: 1, that the enlargements of the cord are always regulated by the size of the nerves of the extremities, being sometimes wanting and sometimes enormously developed; 2, that the cord, at the points of exit of the largest nerves, instead of becoming suddenly thinner, is in most cases Fig. 145. enlarged; and 3 , that the origin of the spinal accessory nerve in this case loses all that is remarkable in it. Now, if the spinal cord in man be examined with reference to the above points, it will present, in almost all of them, exactly the contrary of what Volkmann noticed in animals. In the first place, the white substance constantly increases in thickness from below upwards, and the enlargements of the cord depend upon an increase of the gray substance more than anything else. That this is the case, is evident at a glance, when sections, such as are represented, after nature, in Fig. 145, are compared with each other, and it also admits of being estimated in numbers (vid. "Mikroskop. Anatomie," II. 1, p. 431).

This fact being established, it remains to determine the proportion borne by the white substance in the superior cervical region to the peripheral nerves. For this purpose I instituted Volkmann's measurements in man, and in a male and female body estimated all the roots of the spinal nerves on the left side; I determined, from the ascertained diameters,

FIG. 145.-Five transverse sections through a human spinal cord, hardened by chromic acid, to show the relative proportions of the gray and white substances, - of the natural size: $A$, from the conus medullaris, the diameter of the cord being 32 lines; $B$, from the lumbar enlargement, transverse diameter $4 \frac{3}{4}$ lines, antero-posterior $4 \frac{1}{2}$ lines; $C$, from the dorsal part of the cord, $4 \frac{1}{2}$ and $3 \frac{3}{4}$ lines; $D$, from the cervical enlargement, $6 \frac{2}{3}$ and $4 \frac{1}{2}$ lines; $E$, from the superior cervical portion, level with the second nerve, 61 and $4 \frac{3}{4}$ lines. 
the transverse sectional surfaces of all the nerves in square lines, and compared with them the transverse sectional area, taken with the utmost possible nicety, of the white substance of the spinal cord, at the level of the second cervical vertebra.

It is quite true that there was now evident a very considerable difference against the spinal cord; but when the very great attenuation of the nerve-fibres of the roots, at their entrance and in their further course in the cord, was brought into account, which was not done by Volkmann, the matter was entirely altered, and it became clear that the cord in the male subject contained more than sufficient fibres to furnish the peripheral ones, and in the female nearly sufficient, particularly when it is considered, moreover, that in the entire enumeration, the numbers were stated rather in favor of the roots of the nerves (vid. the calculation in "Mikroskop. Anatom.," II. 1, § 116).

It appears, therefore, scarcely to admit of doubt, that the notion of a termination of the peripheral nerves in the cord, has no support in measurements such as those which, following Volkmann, I have adduced; - and that the latter, even when all due allowance is made for the uncertainty always incidental to such an inquiry, on the contrary indicate, at all events the probability, that the spinal nerves ascend to the cerebrum. They give no further information, however, and it depends upon other facts, whether such a central origin should be admitted or not, because it is even conceivable, that the peripheral nerves may end in the cord, and that the longitudinal fibres in the cord have a wholly different source. Since it is scarcely probable that the tracing of the nerve-fibres through the entire cord will be effected either at present or perhaps at any time, it is necessary to look round for other facts, which may possibly afford conclusive evidence on the subject; and such facts do exist. In the first place, let us consider the course of the roots of the nerves in the cord, such as it has been described above. We found, that after they had all come, more or less, into contact with the gray substance, the greater number of them could be directly traced into connection with the longitudinal fibres of the anterior, lateral, and posterior columns. From this fact, together with my measurements, the passage of the greater part of the peripheral nerve-fibres into the cerebrum, will appear to many to be proved; but, not to overlook anything, it may be further remarked, that the radical fibres, running longitudinally in the substance of the cord, may terminate in it, or after running in it may again enter the gray substance higher up. The former supposition is now, it must be confessed, but little probable, because in the first place, no one has yet seen the terminations of nerve-fibres in the white substance; and in the second, because anything of the sort, for other reasons, would be very surprising, nerve-fibres being nowhere known to commence in the white substance; and with respect to the latter, any re-entry of the roots of 
the nerves into the substance could not escape notice. Since the junction of the radical fibres with the anterior, posterior, and lateral columns, can be so well and so directly observed, the relation in question would necessarily be evident also ; and yet in the course of my perfectly unprejudiced observations, I have never seen anything of the kind. Nothing, therefore, remains but to assume, that the great majority of the peripheral nerves really have a cerebral origin. Whether they all originate in the brain (where, we shall afterwards see) or in part, though, from my observations, but to a small extent, from the cord, cannot be determined, any more than the question ean be decided, whether the white substance of the cord, besides the fibres derived from the peripheral nerves, also contains others passing from the brain to the cord.

$\S 114$. The medulla oblongata and pons Varolii belong to the most complex parts of the central nervous system, containing white and gray substance, intermixed in very various modes. The white substance is, in part, a continuation of that of the cord, in part distinct from it, and is disposed in the following manner: the anterior columns of the spinal . cord diverge from each other at the commencement of the medulla oblongata, allowing the decussating fibres of the corpora pyramidalia to appear. As they proceed, a smaller division joins the pyramids, forming their outer part, whilst the principal portion surrounding the olivary bodies, internally and externally, whence they are also termed the olivary columns, passes laterally, and then, divided into two bundles, proceeds above the second transverse layer of fibres, through the pons. One of these divisions constitutes the fillet (laqueus), which continued above the crura cerebelli ad cerebrum, enters the posterior corpora quadrigemina, joining, within them, the corresponding division of the opposite side. The second division, or bundle, lies externally and inferiorly to the crura cerebelli, and enters the tegmentum of the cerebral peduncle. Besides this, the olivary columns, corresponding to the anterior columns of the cord, also, as it seems, give off fibres to the pedunculis cerebelli. The lateral columns of the spinal cord divide, on reaching the medulla oblongata, into three bundles; one of which, ascending in a tolerably straight direction, is continuous with the fasciculus lateralis of the restiform body, and with it enters for the most part into the peduncle of the cerebellum, and in small part into the tegmentum; a second division penetrates forwards between the divergent anterior columns, decussates in two or three fasciculi with that of the other side (decussatio pyramidum), constituting the principal bulk of the pyramids; a third division, lastly, appears between the posterior columns at the bottom of the rhomboid fossa, or fourth ventricle, as the eminentia teres. These latter are continued, on the floor of the fourth ventricle and applied to each other, into the tegmentum of the cerebral peduncles, whilst the pyra- 
mids, passing between the first and second transverse layers of fibres of the pons, are continued into the base of the cerebral peduncles. The posterior columns of the cord, lastly, chiefly constitute the fasciculi graciles and cuneati, the latter of which, in great part, enter the pedunculi cerebelli, whilst the remainder, and the fasciculi graciles, situated externally to the eminentice teretes, may be traced into the tegmentum of the crura cerebri. All these fasciculi consist, besides the gray substance, of parallel nerve-fibres of the same dimensions as those of the cord, that is to say, from 0.001-0.004 of a line, seldom more.

Besides this white substance, the pons Varolii and medulla oblongata, omitting the roots of the nerves, present a system of mostly horizontal

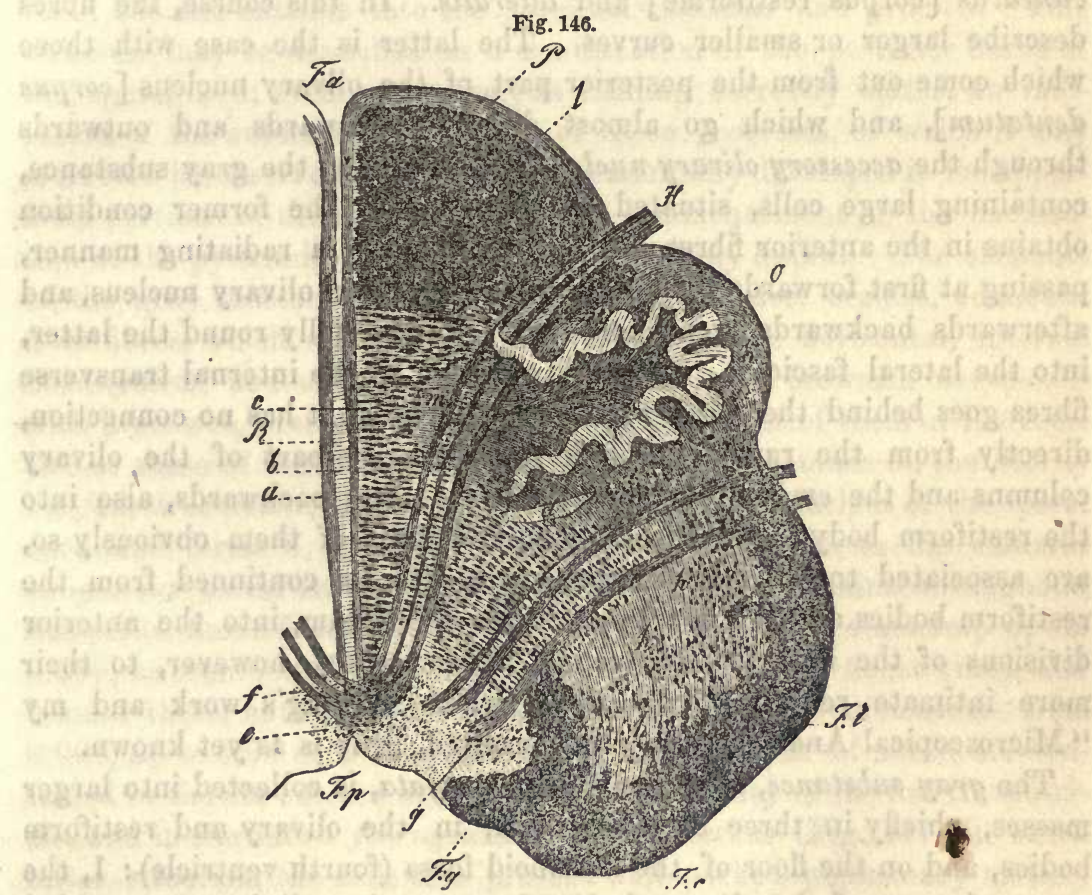

fibres. This system consists: 1 , of the well-known transverse, arcuate fibres, external to the corpora pyramidalia and olivaria; 2 , of straight

FIG. 146.-Transverse section through the medulla oblongata in Man. $P$, pyramids; $O$, olivary bodies; $F . l$, fasciculus lateralis; $F . c$, fasciculi cuneati; $F . g$, fasc. graciles; $H$, root of hypoglossal nerve; $V$, root of $n$. vagus; F.a, fissura anterior; F.p, fissura posterior in the floor of the fourth ventricle, or rhomboid fossa; $R$, raphe: $a$, longitudinal fibres of the raphe; $b$, central gray layer with transverse fibres; $c$, expansion of these fibres in the olivary column and body; $d$, accessory olivary nucleus; $e$, hypoglossal nucleus ; $f$, decussation of the hypoglossal nerve; $g$, nucleus of the vagus; $h h h$, larger nerve-cells in the restiform bodies; $i$, medullary mass in the interior of the olivary body, belonging to the internal transverse fibres; $k$, arcuate fibres external to the olivary body; $l$, transverse fibres external to the pyramids; $m, n, o$, gray nuclei in the pyramids and olivary columns.-Magnified 15 diameters. 
fibres, which extend from before backwards, in the middle, through the medulla oblongata, contributing to the formation of the so-termed raphe (Stilling); 3, and lastly, of very numerous fibres proceeding from this raphe into the lateral halves of the medulla, following a more or less curved direction. These latter, the internal transverse fibres, commence behind the pyramids, and the anterior of them as a large mass, very minutely broken up by fine flattened fasciculi of the pyramidal and olivary columns, penetrate from within into the corpus dentatum olivee, the white substance of which is constituted by them; they then expand in a brush-like form, and are continued through the gray substance of the corpus dentatum, ultimately turning backwards towards the fasciculus cuneatus [corpus restiforme] and lateralis. In this course, the fibres describe larger or smaller curves. The latter is the case with those which come out from the posterior part of the olivary nucleus [corpus dentatum], and which go almost directly backwards and outwards through the accessory olivary nucleus (Stilling), and the gray substance, containing large cells, situated on its exterior; the former condition obtains in the anterior fibres, which spread out in a radiating manner, passing at first forwards between the pyramids and olivary nucleus, and afterwards backwards in a sharp curve, superficially round the latter, into the lateral fasciculi. A second division of the internal transverse fibres goes behind the olivary nucleus with which it has no connection, directly from the raphe, through the posterior part of the olivary columns and the eminentice teretes, outwards and backwards, also into the restiform body. All these fibres, and most of them obviously so, are associated together, and appear to me to be continued from the restiform bodies and the peduncles of the cerebellum, into the anterior divisions of the medulla oblongata. With respect, however, to their more intimate relations, concerning which Stilling's work and my "Microscopical Anatomy" may be consulted, little is as yet known.

The gray substance, in the medulla oblongata, is collected into larger masses, ehiefly in three situations, viz., in the olivary and restiform bodies, and on the floor of the rhomboid fossa (fourth ventricle): 1 , the gray substance of the olivary bodies forms, as is well known, a folded lamella, constituting a capsule closed on all sides except the inner, which, although it occupies the situation of the anterior horns of the spinal cord, which are continued nearly to its inferior border, still has no direct connection with them; appearing, also, to be otherwise isolated from all other gray substance. Within it, besides the very numerous nerve-fibres of the transverse fibre-system, which traverse it for the most part in straight lines, there occur in great numbers smaller nervecells, measuring $0.008-0.012$ of a line in diameter, and of a rounded form, with 3-5 branching processes, and containing in the interior yellowish granules, to which the color of the olivary bodies is due. The 
clnsest observation has failed to afford me any indication of a connection between these cells and the fibres which run among them. On a level with the two upper thirds of the olivary body, is placed, behind the nucleus and wholly isolated from it, the body termed by Stilling the accessory olivary nucleus, in the form of a flattened, yellowish band, of exactly the same structure as the gray substance of the olivary body, and also traversed by horizontal nerve-fibres, and in fact by fibres which have for the most part already passed through the olivary body; 2 , in the restiform bodies, the gray substance (corpus s. nucleus cinereus) assumes the form of an ill-defined, elongated mass intermixed with very numerous nerve-fibres, and which occupies mainly the fasciculus lateralis, but also extends into the fasciculi cuneatus and gracilis. This structure may be described as a continuation of the posterior horns of the spinal cord, even presenting, as Stilling correctly states, an indication of the substantia gelatinosa of those processes, of which it may moreover be observed, that it is very remarkably developed in the uppermost portions of the cord, as far as the commencement of the decussation of the pyramids, and has a position entirely lateral. The elements of the gray substance of the restiform bodies are, besides, numerous finer fibres, which appear to pass chiefly into the horizontal, internal fibre-system, and many, rather pale, but in part brownish nerve-cells with processes, pretty regularly disposed, and most of them of the same size as those of the olivary bodies; 3, the gray substance on the floor of the fourth ventricle, is the continuation of the gray nucleus of the spinal cord, and forms a tolerably thick layer, extending from the calamus scriptorius as far as the aqueductus Sylvii. It contains throughout, numerous nerve-fibres, in part of very considerable diameter, up to 0.006 , or even 0.008 of a line, in part of the finer and finest kinds, and besides these, nothing but caudate nerve-cells of all dimensions from 0.006 , up to 0.03 of a line, and more. The largest of these are contained in the ala cinerea at the posterior extremity of the fourth ventricle, and in the subst. ferruginea s. locus cinereus (Fig. 147), in which latter situation, the cells also present well-marked pigmentary matter, and very numerous, delicately branched processes. The small multinuclear cells, which in the gray nucleus of the cord occur in the form of a compact structure, are here entirely wanting, not being found beyond the decussatio pyramidum. Besides these three masses of gray substance, which can in part be referred to that of the spinal cord, there are found, in the medulla oblongata, some small collections of it, as in the pyramids near the olivary bodies, and in the olivary columns, external to the accessory nucleus, in all of which, as has been already stated by Stilling, are also to be seen in part larger cells, all caudate (in the latter situation measuring as much as 0.025 of a line), and finer nervetubes. One part of the gray substance just described, that namely of 
the anterior half of the fourth ventricle, belongs properly to the pons Varolii. It also contains, in its interior, besides the just described

Fig. 147.

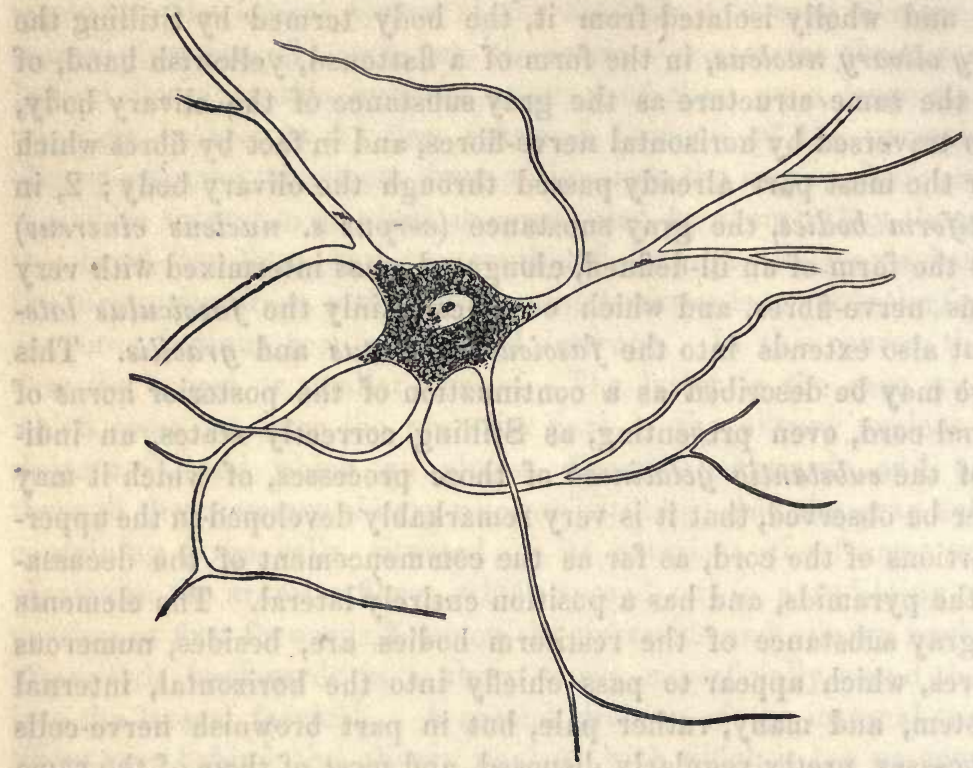

elements, above the transverse fibre-layer, both in the middle as well as more laterally, many accumulations of gray substance, with larger and smaller (as much as 0.02 of a line and more) nerve-cells, all caudate, which are so irregularly imbedded among the longitudinal and transverse fibres, as to require no detailed description, and are connected on the one side with gray nuclei of the medulla oblongata, and on the other with the substantia nigra of the crura cerebri.

The relations of the ten pairs of nerves which arise from the medulla oblongata, the pons, and the crura, constitute a very difficult question. But few inquirers have endeavored to solve it by other means than those usually employed, that is to say, by the tracing of the fibres, with the aid of the scalpel, which here goes no way at all. Among the exceptions are E. Weber (Art. "Muscular Motion," in Wagner's "Handw. d. Phys." III. 2, pp. 20-22), who made his examination in preparations, hardened by carbonate of potassa ; and Stilling, who pursued his by the microscopical examination of sections, similarly hardened by means of alcohol. My own results, obtained from preparations in chromic acid, which had been for the most part made transparent by soda, agree in almost every point with those of Stilling, which, at all events, among all observations on the subject, have gone most deeply into the matter.

Fra. 147.-Nerve-cells of the substantia ferruginea in the floor of the fourth ventricle or rhomboid fossa, of Man; magnified 350 diameters. 
The nerves in question arise, without exception, not from the columns or fibrous substance, out of which they proceed, but all penetrate more or less deeply into the céntral parts, and all probably become connected, some not till they have decussated like the trochleares, with definite parts of the gray substance, which Stilling not inappropriately terms nerve nuclei (accessory nucleus, for instance). It is the floor of the fourth ventricle, and of the aqueduct of Sylvius, which are more particularly concerned in this respect, since all the nerves above named, at least in part, extend to them. The more minute consideration of these relations may be seen in Stilling's Work, and in "Mikroskop. Anatomie," II. 1, pp. $458-462$.

Although a favorable judgment cannot be given upon Stilling and Wallach's work on the spinal cord, I am still very far from disposed to look down upon Stilling's anatomical writings in general, as would seem to have been the fashion for some time past. I am much rather of opinion, in which $\mathrm{R}$. Wagner also coincides, that we have great reason to thank this author for his works on the medulla oblongata and pons Varolii; for although there are some things in them which cannot be maintained, and sufficient attention is not paid to the elementary constituents, still it cannot be denied that they contain a mass of important facts. I have tested, if not all, still the most important of Stilling's statements, and have found them almost all fully confirmed, and am, therefore, glad to take this opportunity of naming him, as the observer to whom we are indebted for the first accurate investigation of the course of the fibres in the central organs. I would also here, add: 1, that in further investigations of this kind, chromic acid, or chromate of potassa, is to be preferred to alcohol, particularly also when caustic soda is cautiously employed for the tracing of the course of the nervefibres in the gray substance thus rendered transparent; and, 2, that in conjunction with lower magnifying powers, the most powerful should be employed, and the relations of the elementary constituents should also be otherwise accurately investigated.

The question as to the origin of the nerves in the medulla oblongata, presents itself as one of the most difficult nature. Most anatomists have hitherto been content to trace the roots of the nerves as far as one or the other column; but this is not sufficient. All the nerves enter at least once, or even several times, into gray substance, in which, and nowhere else, are their origins to be sought for. Now, it must be confessed, that through Stilling's great pains, the fruits of which I can, as it may be said, fully confirm-all the ten pairs of nerves at present under consideration have been traced in their roots, as far as perfectly definite points of the gray substance; but now comes for the first time the further question: do they commence in these situations, or do they 
proceed beyond them? As true origins in the brain have never yet been seen with certainty by any one, there remains nothing but physiological analogies and reasons. As regards the former, we see in all the spinal nerves, that they first penetrate transversely as far as the gray substance, and then, only passing through this, join the white columns, and we may thence suppose that the cerebral nerves, which, in general, so closely resemble them, are in the same condition, and the more so, because these also at first penetrate transversely into the interior of the medulla, and the gray substance, with which they come in contact, corresponds with that of the cord. To this may be added also, that if we make the ten last cerebral nerves terminate in the gray substance, into which they may so readily be traced, the decussated influence of the parts above, upon them, which appears to be established by pathological phenomena, cannot be explained in the case of any one of them except the trochlearis, which decussates before it reaches its gray substance. Now, in the accessorius and hypoglossus it is actually possible to see that the fibres come out from the gray substance, reached by them in the first instance, and afterwards decussate; and the same thing is also at least probable in the oculo-motorius; so that I think it may be, that all the nerves now in question undergo decussation, and do not terminate in the so-termed nuclei of Stilling. "Further investigation will have to show whether this [decussation] takes place in the floor of the fourth ventricle, as would appear to be the case; whether all the fibres of these nerves take part in it ; and where the fibres proceed to after decussation. With respect to the latter, it may be supposed from analogy with the spinal nerves, that the true origin of the cerebral nerves is probably not in the medulla oblongata, but in the corpora striata and optic thalami. Of that portion of the portio major n. trigemini, which is continued into the restiform body, this may especially be remarked, that it certainly does not originate in that part, but winds round it to somewhere above, as is the case also with the $n$. accessorius.

However, in stating, in accordance with what has been said, that I do not consider it directly probable, that the sensitive and motor cerebral' nerves originate in the medulla oblongata and pons, it is by no means intended to imply that these parts may not, as central organs, exert some influence upon them and the more deeply placed nerves. If the medulla oblongata preside over the respiratory movements, if it and the pons be the agents of multiplied reflex motions, this may be the case, without its following that all the nerves called into action should terminate in them, and simply for the reason that the gray substance, so abundantly contained in them, influences the nerves which traverse it, exactly as must be supposed to be the case in the spinal cord. 
$\S 115$. The cerebellum, with respect to the distribution of the elementary tissues, exhibits tolerably simple conditions, gray substance occurring only on the surface of the convolutions, in the nucleusidentatus, and in the roof of the fourth ventricle; all the remainder consists of white substance. The latter is wholly constituted of parallel, probably unbranched, dark-bordered nerve-fibres, possessing all the characters of central fibres (softness, proneness to become varicose, easy isolation of the axis-cylinder, \&c.), are essentially alike in all situations, as far as their condition can be observed, and present a diameter of $0.0012-0.004$ of a line in the extremes, and of 0.002 of a line in the mean. The gray substance occurs, in the first place, very scantily in the roof of the fourth ventricle above the velum medullare inferius, in the form of brown nerve-cells, measuring 0.02-0.03 of a line, scattered in the white substance, and recognizable by a sharp eye without further aid (the substantia ferruginea superior); and, secondly, in the nucleus dentatus, the grayish-red lamella of which contains a considerable number of yellowish pigment nerve-cells of a medium size $(0.008$ 0.016 of a line) with four or five processes, and which have no direct connection with numerous nerve-fibres proceeding from the nucleus dentatus into the medullary substance of the hemispheres, which pass through among them.

The relations of the gray substance on the surface of the convolutions of the cerebellum are more complex (vide "Mikr. Anat.," Pl. IV. fig. 4). It consists everywhere, as is well known, of a layer, internally of a rusty color, externally gray, which, except in the fissures, where the internal layer is most usually thicker, presents pretty nearly the same, but not everywhere an equal thickness.

The internal ferruginous layer contains nerve-fibres and large masses of free nuclei. The former arise, without exception, from the white substance, and run, in general, parallel to each other, although on a transverse section of any convolution slightly diverging in a penicillar manner, directly into the ferrugineous layer. Within this layer they also run from within to without as far as the gray layer, but are broken up into numerous, for the most part, fine fasciculi, which are much interlaced, so that the whole ferrugineous layer is penetrated by a close but delicate network of nerve-fibres, which recalls in appearance the terminal plexuses in peripheral parts, as for instance, in the $n$. acusticus, in the follicles of the vibrisso, \&c. In the meshes formed by these nerve-fibres lie a vast number of opaque, round corpuscles, measuring $0.002-0.004$, in the mean 0.003 of a line, which are nothing else than free nuclei, and which frequently also exhibit a distinct nucleolus, and not unfrequently other granules.

In their passage through the ferrugineous layer, the nerve-fibres of the white substance become gradually attenuated, most of them to a 
diameter of 0.0012 of a line, and in this state enter the external gray layer. This layer, although to outward appearance everywhere perfectly homogeneous, consists of two not well-defined laminæ, the inner of which contains nerve-fibres and very well-marked, large nerve-cells, whilst the outer presents nothing but a finely-granular, pale, light-yellowish substance, which is distributed generally throughout this gray layer, and contains no nerve-cells. The granular substance agrees chemically, morphologically, and physically in all respects with the already-described contents of the nerve-cell; it is tenacious, elastic, rendered more opaque by acetic acid, and more transparent in caustic soda, in which it is, for the most part, dissolved, and exists in the purest form in the outer half of the gray layer, that is to say, next to the pia mater. The small nerve-cells, speaking generally, are very few in number and indistinct. They occur scattered throughout the gray layer, having a diameter of $0.004-0.008$ of a line, more frequent towards the ferrugineous layer than more externally, and when successfully prepared, particularly by means of chromic acid, most of them exhibit delicate processes, which, however, can never be traced to any distance, and are frequently torn off close to the cells. Besides these cells, there also occur here and there, but on the whole rarely, nuclei of $0.002-0.0048$

Fig. 148.

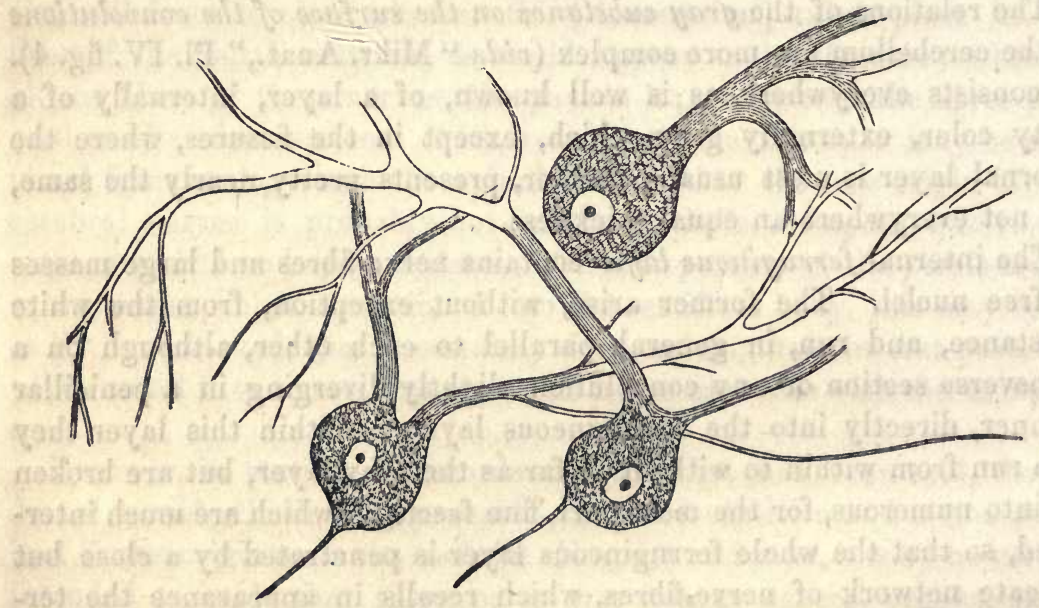

of a line, which, to all appearance, are free, as they are met with even in the most carefully-made preparations. Entirely different from these smaller elements, and very peculiar, are the large cells of the gray layer (Fig. 148) discovered by Purkinje. These cells, measuring 0.016-0.03 of a line, and of a round pyriform or oval figure, with finely-granular, colorless contents, occur only in the innermost portions of the gray,

FIg. 148.-Large cells of the gray layer of the cortical substance of the human cerebellum, magnified 350 diameters. 
close to the ferrugineous layer, and they are, not unfrequently, at least some of them, partly imbedded in its nuclei, in single or multiple layers, and presenting 2-3, rarely 1-4, long and much branched processes, directed particularly towards the outer surface of the convolutions, which are, almost without exception, at all events the strongest of them, given off from the sides of the cells which look from the ferrugineous layer. At their origin these processes are even 0.007 , or as much as 0.008 of a line thick, and extremely finely granular or very delicately striped. As they proceed they become more homogeneous, and at the same time divide into very numerous and extremely slender branches, so that at last, from each process a large bundle of very fine filaments, having a diameter, in the finest, of scarcely 0.0002 of a line, is produced. A portion of these fibrils penetrate more horizontally into the gray layer, although most of them stretch directly outwards, and appear to extend nearly to the outer surface of the gray layer. That they extend very far is certain, for in preparations made with chromic acid, I have isolated some measuring $0 \cdot 15-0 \cdot 2$ of a line, which were still not the finest; and in successful perpendicular sections through the cortical layers of the convolutions, their principal branches appear as parallel, slightly undulating fibres in close contiguity, extending through more than twothirds, or even three-fourths, of the gray layer, to which they give a peculiar striated aspect. Whilst the principal prolongations of the processes are, in this way, continued through the gray layer, they give off their branches at acute or right angles, whence not unfrequently a second striation is produced, crossing the one just described at a greater or less angle.

In the innermost portion of the gray layer, among the large cells, there moreover exists some nerve-fibres; but which, owing to their delicacy and the ease with which they are destroyed, it is very difficult to trace. Quitting the ferrugineous layer, and forming a continuous plexus, they are distributed in the inner third of the gray lamina among the large cells and their processes; there mode of termination has escaped my observation, the result of which amounts only to this: 1 , that they become finer and paler, decreasing from their original thickness of 0.0012 , ultimately to one of 0.0006 and 0.0004 of a line, their dark outlines also being replaced by a paler contour; 2 , that they certainly do not form terminal loops, such as Valentin and Hyrtl, who have probably mistaken a fine plexus for such, think they have noticed; but becoming isolated, and running in a more straight direction, and almost as pale as the processes of the nerve-cells at the border of the inner third of the gray lamina, are lost towards the middle of it.

The crura cerebelli are composed of nothing but parallel nerve-fibres, without any admixture of gray substance, corresponding with those of 
the medullary substance of the cerebellum itself, as a continuation of which they are to be regarded.

\$116. Ganglia of the Cerebrum.-The three pairs of cerebral ganglia, the corpora quadrigemina, optic thalami, and corpora striata, all consist of bulky collections of gray substance, and of nerve-fibres; the former of which are in part quite isolated (corpus striatum), in part mutually connected, and with more deeply lying portions of gray substance (thalami optici, corp. guadrigemina); the latter connect the ganglia, on the one hand with the cerebellum and medulla oblongata, and on the other with the hemispheres of the cerebrum.

The corpus striatum contains two large gray nuclei, the nucleus caudatus anteriorly and superiorly, and the n. lenticularis posteriorly and inferiorly, which are, however, connected in front, constituting a single mass; and besides these, the slender $n$. toeniceformis, with the amygdaloe external to the lenticular nucleus, and is in connection principally with the basis of the cerebral peduncle or continuation of the pyramid which expands in it, forming numerous white fasciculi. The gray substance presents, as almost universally, nerve-cells and fine nervefibres. The former, which measure from $0.006-0.018$ of a line, are, in part, colorless, and in part, contain pigment, as, especially, in the caudate nucleus and third segment of the lenticular nucleus; they are furnished with from two to five processes, and occur in greater numbers according to the depth of color of the gray substance.

The nerve-fibres may be referred for the most part to those of the basis of the crura cerebri. They present the form of dark-bordered tubes from $0.0012-0.005$, most of them from $0.002-0.004$ of a line in size, which, running parallel and close together in a straight direction, enter the first division of the lenticular nucleus, and the most anterior, thickest portion of the caudate nucleus. When traced further in the lenticular nucleus, it will be seen that they form larger and smaller fasciculi, decreasing somewhat in size (most of them measuring from $0.0012-0.003$ of a line) and that, passing straight through the more scanty gray substance of the first divisions of the lenticular nucleus, they are all ultimately lost, spreading out in a penicillar form in its outermost and largest division. That is to say, white fasciculi measuring from $0.04-014$ of a line, with fibres of $0.0012-0.002$ ' of a line, enter this division of the nucleus from the second; and these fasciculi in close contiguity, slightly diverging and subdividing into smaller bundles, are continued further in a direction towards the outer border of the lenticular nucleus, before reaching which they disappear to the naked eye. If traced microscopically in preparations made with chromic acid, it is apparent, that the fasciculi proceed nearly to the - outermost part of the lenticular nucleus, though gradually broken up 
into smaller bundles and separate fibres, and most intricately interlaced with each other. That these fibres terminate here, and do not proceed any further into the medullary substance of the hemispheres, may be considered as made out, not the faintest indication of any further continuation being afforded, which, if it existed, could not escape being seen : on the other hand it is doubtful how they terminate here. All I have to state on the point is this : that the fibres of the nerve-fasciculi, entering the third division of the lenticular nucleus; as may be directly observed in a very great many instances, gradually become so much attenuated, as ultimately to measure not more than $0.0008,0.0006$, or even hardly 0.0004 of a line, and present an almost entirely pale aspect, so that they can scarcely any longer be distinguished from the finer processes of the nerve-cells; with which in fact, unless everything is deceptive, they most probably are actually connected. All the fibres, also, which enter the caudate nucleus, present exactly the same conditions; some of these enter the nucleus directly from the basis of the cerebral peduncle, others, which appear in its thinner portion, are manifestly derived from the lenticular nucleus, the first two divisions of which they traverse in the first instance; in this case, also, there is no transition of the fibres into the medullary substance of the hemispheres, but a separation of the fasciculi into a plexus of the finest, almost nonmedullated fibres takes place, and probably a connection between them and the cells.

Besides the above described, in any case very numerous, nerve-fibres derived from the cerebral peduncles and terminating in the corpora striata, the nuclei of those bodies contain a considerable number of other fibres, whose origin it is, in part, difficult, and, in part, impossible to assign. I think I can trace one set of these fibres to their source. In the most external part of the large nucleus of the corpus striatum, we find, on making various sections, a considerable number of moderately strong fasciculi, though not visible to the naked eye, which in their relative thickness and the diameter of their tubes $(0.0012-0.002$ of a line) differ from the fibres derived from the crus cerebri, which in this situation are reduced to the most extreme attenuation and dispersed in a plexiform manner. It is easily seen that all these fasciculi proceed from the medullary substance of the hemispheres; and, as it appears, after they have run a certain distance parallel with the surface on the border of the nucleus of the corpus striatum, that they enter it. Many of these fibres are continued directly from the medullary substance into the ganglia, and, in this way, decussate, at right angles, with the former fibres. Assembled in fasciculi, these fibres penetrate more or less deeply into the gray substance of the corpus striatum, and of the third division of the lenticular nucleus; and these terminate, as I think I have observed, without any considerable expansion, the formation of 
a plexus, or undergoing any farther decrease in size, their fibres forming loops with closely approximated sides.

Although, speaking relatively, it is not difficult to make out the structure of the corpus striatum, at all events, in its principal features, it is quite otherwise with the optic thalami and corpora quadrigemina, chiefly because the nerve-fibres in these situations are not so much assembled into fasciculi, but are more isolated and most intimately intermixed with the gray substance, on which account they cannot be traced to any great distance. The examination of the gray substance itself, however, is perfectly easy even in these bodies, and its elements - the nerve-cellspresent nothing peculiar, except that, in the optic thalami, they are for the most part more deeply colored, whilst those in the corpora quadrigemina are paler. With respect to the nerve-fibres, it is quite certain that the superior portion of the crura cerebri, that is to say, the crura cerebelli ad corpora quadrigemina, the continuations of the olivary columns, portions of the corpora restiformia, and the eminentice teretes, pass into the ganglia now under consideration, although I have not as yet succeeded in eliciting anything determinate as to the course they take. But I think it may be stated, that the fibrous masses above named, in great part at least, are not continued into the medullary substance of the hemispheres, because, on the one hand, most of their fibres decrease from the original diameter of $0.0012-0.004$ of a line down to the smallest, or less than 0.001 of a line; and on the other, because no such passage of the fibres can be perceived on that side of the optic thalamus, which looks towards the medullary substance of the hemispheres. The superficial white investment, however, of the ganglia in question, must be excepted, which in any case may effect a relation between them and the hemispheres, as its fibres, measuring $0.001-0.003$ of a line, or even more, disposed in fasciculi, and crossing each other - horizontally in various directions, do not appear to terminate in it. Neither is the relation of the optic thalami to the corpora quadrigemina, and that of the fornix to the latter, by any means clear, so that it is pleasing, at all events, to find that another important question admits of a more satisfactory solution. When the external portion of the optic thalamus is examined, it will be found that it adjoins a considerable mass of white substance, which at first sight appears to be a continuation of the basis of the cerebral peduncles passing below and external to the optic thalamus, between the lenticular and caudate nuclei of the corpus striatum, to enter directly into the medullary substance of the hemispheres. Closer observation renders it obvious, that this white substance, as has been said before, in part enters the corpus striatum, particularly the lenticular nucleus, and in part radiates from without inwards, from the hemisphere into the optic thalamus. That is to say, very numerous white fasciculi, visible even to the naked eye, coming from the hemi- 
sphere throughout the entire height of the thalamus, enter the latter, run towards the superior surface to the superior and internal border, and the pulvinar, being ultimately lost exactly in the same way as are the fibres continued from the crus cerebri into the corpus striatum; that is, these fasciculi, the elementary fibres of which originally measure $0.0012-0.0025$ of a line, ultimately subdivide into extremely close plexuses composed of fibres of the most extreme fineness, $0.0004-0.0008$ of a line, the terminations of which cannot be traced.

I will just notice the constitution of some structures connected with the above-described ganglia. The substantia nigra of the crus cerebri presents pigment-cells precisely similar to those of the substantia ferruginea, except that they are for the most part rather smaller, and have ferrer processes, surrounded with nerve-fibres of the finest, and also of the stronger kind. The commissura mollis contains smaller cells, with $1,2,3$, and more processes, and light-colored pigmentary contents; and besides these, very many, plexiform, vertical, and horizontal, fine fibres of $0.0012-0.0016$ of a line, with some still finer, less than 0.001 , and a few stronger measuring as much as 0.004 of a line. The pineal gland exhibits pale, rounded cells, without any processes, and scattered nerve-fibres of $0.001-0.002$ of a line, and also, generally, a considerable quantity of sabulous matter (brain-sand) (vid. § 118). Its peduncles, their anterior prolongations, and the commissura posterior, contain fibres measuring $0.001-0.003$ of a line, and are composed in part also of the finest fibres. The floor of the third ventricle presents, immediately beneath and behind the anterior commissure, extremely large, and smaller, colorless cells, with from one to four, occasionally very thick processes. These are lodged in great number in a close plexus of fine fibres of $0.0004-0.0012$ of a line; and cells, in other respects exactly similar, though not quite of the same size, also exist in the corpus mammillare, likewise intermixed with very numerous fibres of the finest sort; there are other still smaller cells of $0.008-0.012$ of a line, for the most part with only two processes, in the tuber cinereum. The hypophysis cerebri contains, in its anterior, reddish lobe, no nervous elements; but rather, according to Ecker (art. "Blood-vascular Glands," in Wagner, "Handw. d. Physiol."), the elementary tissues of blood-vascular glands; that is to say, a vascular stroma of connective tissue, in the interstices of which, lie vesicles (cells?) measuring $0.030-0.090 \mathrm{~mm}$, containing sometimes only nuclei, and a fine granular substance, sometimes distinct cells, in older persons also colloid-like masses. The posterior, smaller lobe consists of a fine granular substance, with nuclei and bloodvessels, and also contains fine, varicose nerve-fibres, which, like the vessels, descend from the infundibulum.

\section{$\S 117$. Hemispheres of the Cerebrum.-The white substance of the}


hemispheres of the brain consists entirely of nerve-fibres, of 0.00012 0.003 , on the average 0.002 of a line in size, without any admixture of gray substance. These fibres, of whose special course, we, as yet, know extremely little, never form plexiform interlacements or fasciculi, but all run in parallel, and most generally, straight lines, and undoubtedly proceed from the corpus callosum and ganglia of the cerebrum as far as the superficial gray substance, whilst it must remain undetermined whether, in their course, they divide or not. But besides these fibres, omitting also the commissura anterior, the fornix, and the origin of the optic nerves, the hemispheres contain others crossing the former at right angles. I have found these fibres, in the first place, on the outer side of the corpus striatum, in which situation they are to be referred, in part, to the fibres which enter the corpus striatum from the hemispheres and terminate in it ; perhaps, also, in part, to the expansion of the corpus callosum in the inferior lobes; and secondly, in the most superficial layer of the white substance, near the gray cortical substance, where they occur in not inconsiderable numbers, and running, in part, obliquely ; but of their origin nothing satisfactory could be ascertained. Whether there are still other, and what traces of fibres, the future must show.

The more intimate structure of the gray substance of the convolutions is tolerably manifest (vid. "Mikroskop. Anatomie," Pl. IV. fig. 2). It is most conveniently divided into three layers, an external, white; a middle, pure gray; and an internal, yellowish red. The latter, in thickness almost equal to the other two, usually presents, on its outermost border, a clear, frequently white streak, and occasionally, more internally, a second, thinner and less white layer, so that there are in fact four or even six successive laminæ; 1, a yellowish-red layer (inner part); 2 , the first white streak; 3 , yellowish-red layer (outer part); 4 , second white streak; 5 , the gray layer; 6 , superficial white layer. The gray substance contains, in its whole thickness, both nerve-cells and nervefibres; and besides these, much granular matrix-substance, exactly like that of the cerebellum. The nerve-cells are not easily investigated, except in preparations in chromic acid, and in all the three layers they agree in this respect, that by far the greater number of them are furnished with from one to six processes, which give off numerous branches, and ultimately form extremely fine, pale fibrils of about 0.0004 of a line in diameter, differing, however, in respect of size, number, \&c. In the superficial white layer the cells are few, small (0.004-0.008 of a line), with one or two processes, and scattered in an abundant, finely granular matrix. The middle or pure gray layer, most abounds in cells, which in it, are closely aggregated also in a granular matrix. Their size varies very considerably, some being very small $(0.003-0.005$ of a line), frequently appearing as little more than nuclei, whilst there are many 
others of larger dimensions, up to 0.016 and 0.02 of a line (Fig. 149). Their figure is pyriform or fusiform, tri- or multangular, also perhaps more rounded, by far the greater number having from one to six processes, usually three, four, or five; and where this is not the case, they may have been torn off in the preparation, since stumps of them may be very readily noticed in the cells, which are altogether very delicate. In the innermost yellowish-red layer, lastly, the cells are again rather more scanty, though still extremely abundant, otherwise presenting the same characters as those in the gray substance, having sometimes pale, sometimes pigmentary contents; the latter in the inner layers more particularly, and in old persons.

The nerve-fibres of the gray substance of the convolutions, arise, as it is easy to demonstrate, from the medullary substance of the hemispheres,

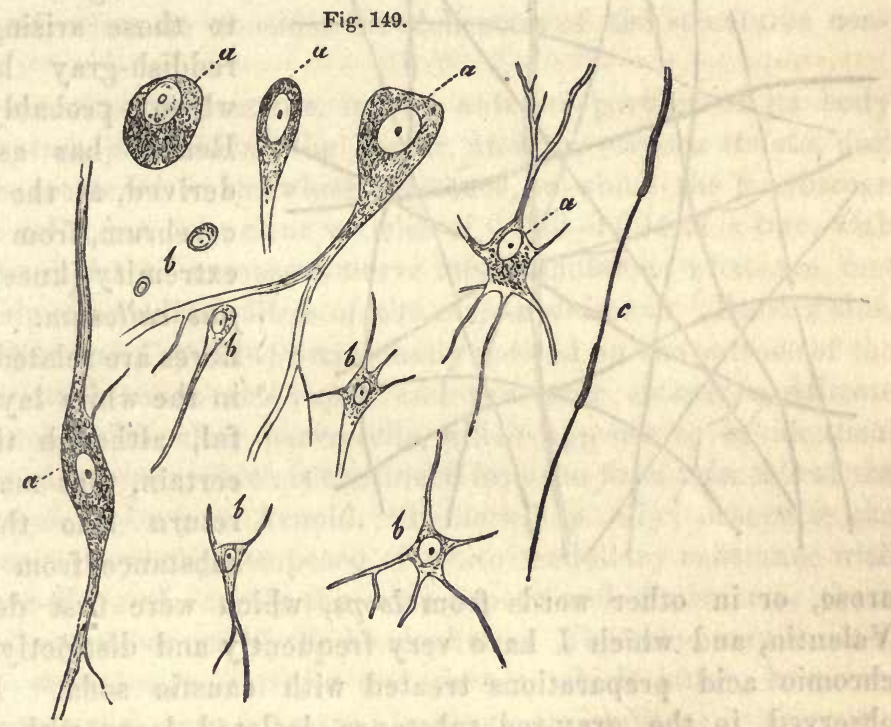

and penetrate, bundle after bundle, directly, and all parallel, into the yellowish-red layer. Arrived here, many fibres separate from the rest, and penetrate the yellowish-red layer in all directions, but more especially parallel to the surface, and consequently crossing the main fasciculi. When these horizontal fibres are more closely aggregated, they produce the above-described whiter or clearer streaks in this layer, the outer of which streaks is situated exactly at the point, where the fasciculi which enter the gray substance, are lost. In fact, as these proceed more outwardly, they constantly decrease in size, owing to their giving off lateral

Fig. 149.-From the internal portions of the gray layer of the convolutions of the human cerebrum, magnified 350 diameters. Nerve-cells : $a$, larger; $b$, smaller; $c$, nerve-fibres with axis-cylinder. . 
fibres, and to the attenuation and separation of their elements, until, when they have reached the gray layer, they become lost to sight, although if more closely traced they may still be perceived as intricately interlaced fibrils of the utmost fineness, and with scarcely any appearance of dark contours, only that there are a certain, though smaller number of fibres, which, upon reaching the gray layer, do not lose their breadth and dark contours, but are continued in a straight or oblique course through it, extending horizontally to a further distance, in the outer white layer. In this layer, consequently, we find a considerable number of finer, and of the very finest fibres (Fig. 150), crossing each other in

Fig. 150.

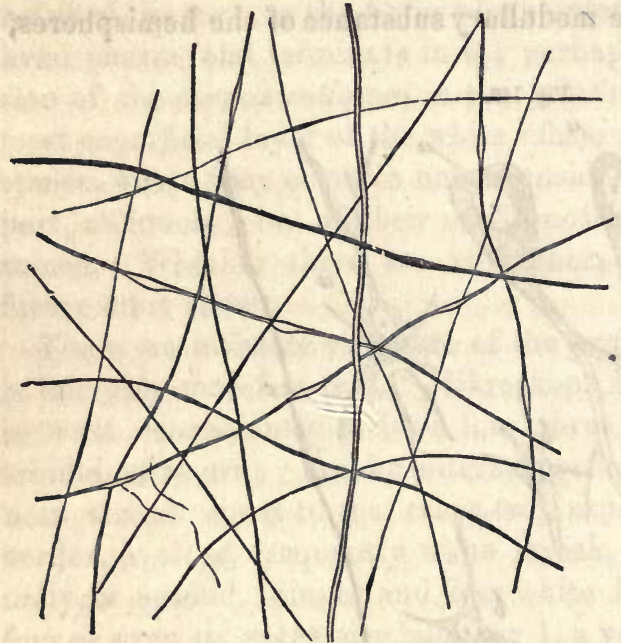

various directions, and in several superimposed layers, which are obviously, as to their origin, to be referred to those arising from the reddish-gray layer; and which probably also, as Remak has assumed, are derived, at the basis of the cerebrum, from the anterior extremity (knee) of the corpus callosum. How these fibres are related to the cells in the white layer is doubtful, although this much is certain, that many of them return into the gray-red substance from which they arose, or in other words from loops, which were first described by Valentin, and which I have very frequently and distinctly noticed in chromic acid preparations treated with caustic soda. I have also observed, in the gray-red substance, isolated loops with closely approximated sides, and also with their convexity looking towards the surface of the brain. The fasciculi of the gray-red substance contain fibres which, at first, measure 0:0012-0.003 of a line, but almost all of which ultimately decrease in size down to 0.001 , and, in the gray substance, acquire the diameter of the smallest nerve-tubes, 0.0004 0.0008 of a line. The fibres given off from these fasciculi, within the gray-red layer are, in part, of the same size as those in the fasciculi, - which is the case particularly with those of the thicker white streak, -in part finer. The fibres which proceed from these fasciculi into the superficial white substance, are also, usually, of greater size, up

Fra. 150.-Finest nerve-tubes of the superficial white substance of the human cerebrum; magnified 350 diameters. 
to 0.003 of a line, many of which form loops; there are, however, in this layer together with these, some of the finest fibrils, measuring 0.0004 of a line. Notwithstanding all my endeavors, I have been unable to discover any connection between the nerve-cells and fibres, in the cortical portion of the cerebrum; but the existence of such a connection would appear to me to be nowhere so probable as here, where the nervefibres, especially in the pure gray layer, assume so much the appearance of processes of the cells, as almost to deceive the observer, and where, in any case, they terminate. There are in this situation an immense number of nerve-fibres, so fine and pale that they could scarcely be regarded as such, were they not straighter than the processes, and did they not, particularly when treated with soda, exhibit minute varicosities. If anywhere in the central organs, an origination of nerve-fibres exists here, although it is quite intelligible that it should not yet have been observed, when we consider the delicacies of the structures concerned.

The corpus callosum presents, in the anterior portions of its body above the septum pellucidum, the fornix, and the corpora striata, dull gray streaks, scattered in the white substance, in which the microscope discovers no cells, but only clear vesicles of $0.003-0.004$ of a line, with nuclei, in the midst of numerous nerve-tubes, similar to what are met with in certain fasciculi of fibres of the corpus striatum. Besides this, Valentin ("Nervenl.," p. 244) occasionally noticed on the surface of the corpus callosum, between the raphe and the strice obtectoe, a delicate gray investment with clear nerve-cells, which appears to be identical with the fasciola cinerea, which is continued into the fascia dentata of the pes hippocampi major (vid. Arnold. "Bemerk," p. 87); otherwise the corpus callosum is wholly composed of white medullary substance with parallel nerve-fibres of exactly the same aspect and diameter as those of the medullary substance of the hemispheres. The same may be said, also, of the commissura anterior and fornix, which latter, however, comes in contact with gray substance in very many ways, as in the optic thalamus, from the tuberculum anterius of which its radix descendens arises; in the corpus mammillare (vid. sup. $\$ 116$ ); at the commencement of the radix ascendens; in the floor of the third ventricle, towards which some delicate fasciculi of the radix ascendens are given off; and at its point of junction with the septum pellucidum, which latter, together with a common thick coat presenting much connective tissue and corpuscula amylacea (vid. § 118), exhibits numerous plexuses of the finest kind of nerve-fibres and nerve-cells, exactly as does the tuber cinereum. The fibres of the fornix measure, in its white portions $0.0008-0.005$, mostly $0.002-0.003$ of a line; in the optic thalamus (upper part), and in the corpus mammillare, the fibres are only of the finest sort, measuring 0.0004-0.001 of a line. The cornu ammonis, and the calcar 
avis (pes hippocampi minor), present nearly the same conditions as those of the cerebral convolutions; in the gray substance of the former, however, there is a peculiar sort of streak, containing chiefly round cells without processes, and closely aggregated.

Lastly, we have to consider the origin of the first two pairs of nerves. The olfactory nerve contains, in the white portion of the tractus olfactorius, fine nerve-fibres, of 0.0004 , or at most 0.002 of a line, the finest, pale-bordered, and apparently non-medullated; and besides these, also some gray substance, with fine granular structure, and cells of 0.007 0.008 of a line. These cells, with some still smaller, down to a diameter of 0.003 of a line, many with branched processes, constitute the bulbus $n$. olfactorii, intermixed with numerous fine fibres, the relation of which to the cells and to the true nerves of smell cannot be made out. The optic nerve arises, with its tractus divided into two crura, from the corpora geniculata, and the corpora quadrigemina and optic thalami; besides which, it is also in connection with the crura cerebri, the substantia perforata antica, the tuber cinereum, and the lamina terminalis. The precise origin of its fibres, dark-bordered tubes of 0.002 of a line, is in Man unknown, but to draw conclusions from experiments in animals, it exists principally in the corpora quadrigemina, whilst we know that they partially decussate in the chiasma (commissure). In this body, however, there are, as stated by Arnold, Todd and Bowman, \&c.: 1, fibres which do not decussate, but are continued from the tractus into the optic nerve of the same side; and 2, commissural fibres, which may indeed be divided into an anterior and posterior set, the latter constituting a commissure between the two points of origin of the optic nerves, whilst the anterior could only unite the two retince. The existence of the first-named fibres is certain, although they are much more scanty than the decussating elements; but that of the others also can hardly be denied. Speaking physiologically, a commissure of the optic thalami and corpora quadrigemina may perhaps be explained, but a commissure also of the retince does not appear to be altogether impossible, because we know that the retina contains gray substance, and in it, nerve-cells with branched processes.

With respect to the origin of the nerve-fibres in the brain and higher central organs, in general, it is several years since I first observed the origin of dark-bordered fine fibres from the processes of the nerve-cells in the spinal cord of the Frog ("Zeitsch. f. wiss. Zool.," vol. I. p. 144, tab. xi. Fig. 7). In man I have not as yet been so fortunate as to perceive anything of the sort with certainty, though I do not myself doubt that similar conditions obtain in this case also. In fact, R. Wagner and Leuckart think they have seen, in man, the processes of the many-rayed cells in the substantia ferruginea, passing into nerve-tubes ("Gott. An- 
zeig.," 1850, No. 43); as has Prof. Domrich, in the cortical substance of the cerebellum, according to a communication to me by letter. R. Wagner again ("Gött. Nachr.," Oct. 1851), has, recently, also found in the electric lobes of the Ray, that from the many-rayed ganglion-globules or nerve-cells, one or more, rarely two, unbranched processes are continued into dark-bordered fibres. He now explains this transition, in the same way as before, saying that the processes were continued as axis-cylinders into the dark-bordered tubes, in which Leydig, who has observed the same transition in the cerebellum of the "Hammer-headed Shark," agrees with him, as does Stannius, in the case of Petromyzon. Nevertheless, it is still not quite evident to me, that any condition should exist in this case, different from that which obtains in the ganglia, where the processes of the nerve-cells are not simply axis-cylinders, but also have a coat, which investing the medullary matter of the nerve, is continuous with the sheath of the dark-bordered tubes; although, seeing that the presence of tunics on the nerve-corpuscles of the central organs, and their processes, in general, is still a disputed point, I am prepared to admit that the fact may be otherwise. These researches have opened the way, and I have no doubt, as I have already said in my Microscopical Anatomy, that in time we shall succeed in demonstrating the origin of dark-bordered tubes in many other situations in the central organs, in man, and other animals. On the other hand, however, supported by repeated investigation of the human brain, I must assert, that it is in the highest degree probable that in many places it will be altogether impossible to demonstrate the origin of fibres from nerve-cells, because very many nerve-tubes, particularly those of the cortical substance of the cerebellum and cerebrum, ultimately become so pale and slender, as not to allow of their being distinguished from the processes of nervecells. Whether the loops which distinctly exist in the convolutions of the cerebrum, and which I have also seen in the corpora striata, are terminations, or whether free prolongations of nerve-tubes exist, we know not, and the less so because it cannot even be asserted that certain fibres really so terminate. It may fairly be assumed that the fibres of the corpus callosum and the commissural fibres in general, commence in the one hemisphere in connection with cells and terminate in the other, and that the fibres which proceed from the surface of the convolutions to the optic thalami and corpora striata terminate in the latter, but to assert, that it is so, is impossible, notwithstanding the visible loops, for it may be that these latter are not terminations at all, and that the fibres in question are all in the one place and the other in connection with nervecells. That nerve-fibres should originate independently of any connection with cells would be contrary to all analogy, but in such an obscure subject we must always be prepared for much that is new, and be careful not wholly to reject any possibility, simply from à priori considerations. 
Several authors have noticed divisions of the nerve-tubes in the central organs, such as, among the older ones, Ehrenberg, Volkmann, E. H. Weber, and more lately also, Hessling ("Fror. N. Notiz.," Ap. 1849, Jenaische, Ann. I. p. 283), E. Harless (ibid., p. 284), and Schaffner ("Zeits. f. rat. Med.," IX.) in the brain of various vertebrate animals, especially at the junction of the white and gray substance. I am not inclined to doubt these statements, especially the latter, but I cannot avoid the remark, that in the human brain, I have, hitherto, in vain sought for divisions of this kind, and have had many hundreds of fibres from the gray substance before me, under the most favorable circumstances, which presented no indications of the sort, whilst I have invariably found such divisions in the spinal cord (vid. supra). The manyrayed nerve-cells with branched processes are not as yet fully known in all their relations. I have described their processes (as will be universally allowed, correctly), as a sort of pale, non-medullated nerve-tubes, and have isolated them occasionally to the extent of $\frac{1}{5}$ and $\frac{1}{4}$ of a line, without being able to notice anything more with regard to their termination, than the fact of their ultimately assuming an extreme degree of fineness. $R$. Wagner states, that those processes, which do not pass into dark-bordered nerve-tubes, serve to connect the separate nervecells together, but in so doing he manifestly says more than actual observation warrants, as he has, hitherto, seen such a connection, only in the electric lobes of the Ray. In the present state of neural Anatomy there is nothing which should be more carefully avoided than the general application of isolated observations, and I am therefore of opinion that this question must as yet be regarded as an open one. It may indeed be very consonant with physiological considerations, to explain the reflex and alternating actions of separate sections of nerves by such connections between the cells, but it is precisely for that reason, that we should be the more careful, and the more so because less obvious theories explain the conditions just as well. I conclude, therefore, from the observations hitherto made, only this much, that nerve-cells may anastomose, leaving it to future inquiries to decide, whether they do so universally and with all their processes, or whether in certain situations the latter do not stretch out without any attachment, exerting a mutual influence and affecting the nerve-fibres simply by juxtaposition, as appears to be the case in the large nerve-cells of the cord and the roots of the spinal nerves.

§ 118. Membranes and Vessels of the central Nervous System.A. Membranes. 1. Spinal cord. The dura mater s. meninx fibrosa is a whitish yellow, occasionally glistening, firm, tolerably elastic membrane, consisting of parallel and mostly longitudinal fasciculi of connective tissue, and of a fine, elastic, fibrous network in almost equal proportions. 
The outer surface of the dura mater is, in front, where the membrane is always at least as thin again as behind, pretty closely united to the fascia longitudinalis posterior of the spinal column, free posteriorly and on the sides, and separated from the arches of the vertebrce and their periosteum by a space, occupied by a lax connective tissue with anastomosing fasciculi scarcely more than $0.004-0.005$ of a line thick (reticular connective tissue), containing a few elastic fibrils (convoluted and longitudinal), and round, fusiform and stellate nucleated cells, similar to the formative cells of the connective tissue, and besides these with larger or smaller aggregations of frequently gelatiniform, transparent fat with cells containing serous fluid. The vessels of this space are in part the well-known plexus venosi, in part finer vessels, and also a network of the finest capillaries in the lax connective tissue itself. The internal surface of the dura mater would appear, from what is generally stated, to be lined with an outer lamella of the arachnoid; nothing, however, is to be seen but an epithelium, composed of polygonal, flattened, nucleated cells, on the innermost layer of the dura mater, and not a trace of any special substratum. The ligamentum denticulatum has no epithelium, and like the thickened processes of the pia mater, to which it is attached, presents, in all respects, a structure similar to that of the dura mater.

The arachnoid membrane is constituted, not of an external and internal lamella, the former of which is united to the dura mater, the latter free, but of a single layer corresponding to the internal lamella of authors. It is an extremely delicate, transparent membrane, exactly corresponding in extent and relations to the dura mater. Its outer surface, in the posterior mesial line of the cervical portion, is connected with the dura mater, above, by tolerably strong processes, lower down, by delicate fibrils, elsewhere it is perfectly smooth and glistening, which appearance depends upon an epithelium precisely like that of the dura mater, and it is merely in apposition with that membrane, as the pulmonary pleura is with the costal. The internal surface of the arachnoid is also smooth, though without epithelium; it is separated from the spinal cord, and cauda equina by a large interspace, the subarachnoid space, affording, however, numerous slender processes to the pia mater and the roots of the nerves, which processes not only accompany the vessels and nerves, but occur, especially in the posterior mesial line, arranged in a consecutive series, and occasionally, particularly in the cervical region, form a perforated or complete septum. As regards its intimate structure, the arachnoid contains, chiefly, reticularly anastomosing bundles of connective tissue of 0.001-0.004 of a line, which are so united as to form lamella, some external with more slender, and some internal with stronger fasciculi, and which are usually so surrounded by fine elastic fibres, as to present a moniliform appearance when swollen by the application of acetic acid (Fig. 23). In many fasciculi, these fibres are very 
fine or wanting, others again, in addition, contain elastic fibres also in the interior.

The vascular membrane, pia mater, very closely invests the spinal cord and the gray substance of the filum terminale, penetrating on the one hand into the anterior and posterior fissures, where it appears within the spinal cord in the form of slender processes, and affording, on the other, delicate sheaths to the roots of the nerves. It contains for the most part common connective tissue with straight fibres, and, more rarely, anastomosing bundles; and besides these a good many nuclei often of a lenticular form, with a few elastic fibrils. Here and there are met with in the pia mater bright yellow or brown pigment-cells, of an irregular, fusiform figure with fine prolonged ends and measuring $0.04-0.05$ of a line in length, which in the cervical region, owing to their greater number, give the membrane, not unfrequently, a brown or even blackish color.

2. Brain.-The membranes of the brain, though corresponding, in general, with those of the spinal cord, yet present some differences. The dura mater, in this situation, consisting of the true fibrous membrane of that name and of the internal periosteum of the cranial bones, which, as the immediate continuations of the corresponding membranes of the spinal canal, become consolidated together at the level of the atlas, is, in general, thicker and also whiter than in the spinal cord. Its external or periosteal lamella, of a whitish-yellow color and rough, is attached more or less firmly to the bones, supports the larger vasa meningea, and is also otherwise more richly supplied with vessels than the internal proper dura mater, with which, at an earlier period, it was more laxly united, and from which, except where the sinuses are contained, it may not unfrequently be separated even in the adult. The internal lamella is less vascular, whiter, presenting in many places a glistening tendinous aspect, and on its surface is quite smooth and for the most part even. The processes of the dura mater, the greater and less falciform processes, and the tentorium, appear as prolongations of this internal lamella; and between the two lamellæ are situated, with few exceptions, the venous canals or sinuses of the dura mater. Both lamellæ contain connective tissue of the same form as that in the tendons and ligaments, with, for the most part, indistinct bundles, and parallel fibrils, which either extend of a uniform size for considerable distances in it, or, especially as in the sinuses, form small, tendinous bands, crossing each other in various directions, and containing among them a good many fine elastic fibres. The internal surface of the dura mater is lined with a single (according to Henle with more than one) layer of tessellated epithelial cells, of $0.005-0.006$ of a line in size, with rounded or elongated nuclei measuring $0.002-0.004$ of a line; possessing no other covering which might be described as a parietal lamella of the arachnoid (vid. Luschka, Seröse Häute, p. 64). 
The arachnoid membrane of the brain differs from that of the spinal cord, not so much in its structure as in its disposition. It is true, that in this situation also, there is but one lamella demonstrable as a membrane composed of connective tissue, which corresponds with the sotermed visceral layer of the arachnoid of authors, and is also very closely applied to the inner surface of the dura mater, but the arachnoid membrane here is in much more intimate relation to the pia mater. That is to say, instead of its being united with the latter, as in the cord, by scattered fibres and lamellæ, it is, in the brain, in many situations, as on all the convolutions, and the projecting parts at the base of the brain, adherent to and coalescent with it, and, elsewhere, where this is not the case, united to it by numerous processes. For this reason, there exists, in the brain, no continuous subarachnoid space, but numerous, larger and smaller spaces, which only partially communicate. The larger of these spaces between the cerebellum and medulla oblongata, and under the pons Varolii, the crura cerebri, the fossa Sylvii, \&c., open directly into the subarachnoid space of the spinal cord, whilst the smaller, corresponding to the sulci, and over which the membrane composed of connective tissue is stretched, are perhaps partially in communication with each other, but, at all events most of them, not with the larger spaces just mentioned. The arachnoid, as has been correctly stated by Henle, is nowhere in connection with the lining membranes of the cerebral ventricles. The structure of the membrane is the same as in the spinal cord, except that the anastomosing fasciculi and spiral elastic fibres are for the most part thicker, measuring as much as 0.01 or even 0.02 of a line; and the former frequently present, as it were, special and more homogeneous sheaths of connective tissue, beneath which, fat- and pigment-granules are often deposited. The outer surface is covered with an epithelium in all respects like that of the dura mater.

The pia mater cerebri is more vascular but more delicate than that of the spinal cord, and covers all the elevations and depressions on the surface of the brain, if not very closely yet quite exactly, with the single exception of the floor of the fourth ventricle, above which it is stretched across from the calamus scriptorius, as far as the nodulus, the free border of the vela medullaria inferiora and the flocculi, forming the tela chorioidea inferior, from which points it proceeds to invest the under surface of the inferior vermiform process and of the tonsilloe. The pia mater penetrates into the interior of the brain only at one point, viz., at the transverse fissure of the cerebrum, where it passes beneath the splenium corporis callosi, investing the vena magna Galeni, as well as the pineal gland, forming the tela chorioidea superior, with the plexus chorioideus ventriculi tertii; and passing beneath the fornix, also constitutes the vascular plexuses of the lateral ventricles, which 
are continuous with the pia mater at the base of the brain, between the crus cerebri and the inferior lobe. With respect to its intimate structure, the cerebral pia mater contains so many vessels, that in parts the connective tissue which forms the matrix appears as a subordinate constituent. It is rarely, as in the spinal cord, distinctly fibrous, for the most part more homogeneous, approaching in character "Reichert's membranes," or immature connective tissue, with a few nuclei and without elastic fibres. Occasionally, however, the pia mater also contains reticular connective tissue, as around the vena Galeni, the pineal gland, the larger vessels, and also on the cerebellum. Fusiform pigment-cells also occur here, as in the spinal cord, particularly on the medulla oblongata, and pons Varolii, but also, more anteriorly, at the base of the brain as far as within the fissure of Sylvius, in which situation I have noticed them even in the $m$. adventitia of smaller arteries.

Those portions of the pia mater which are in relation with the ventricles, the teloe chorioidece and plexus chorioidei, do not differ in their structure from other portions of the membrane, except that, especially in the plexus, they are composed almost wholly of vessels, and are furnished with an epithelium at those points where they are not adherent to the walls of the ventricles. This epithelium consists of a single layer of roundish polygonal cells, $0.008-0.01$ of a line in diameter, and $0.003-0.004$ of a line in thickness, and usually containing together with the rounded nucleus, yellowish granules, often in great numbers, and one or two, dark, round oil-drops of 0.001-0.002 of a line in size. According to Henle, almost all these cells send out, from the angles towards the layer of connective tissue of the plexus, short, slender, acuminate, transparent, and colorless processes, like spines; and according to Valentin ("Physiol.," $2 \mathrm{~d}$ ed., part 2, p. 22), in the Mammalia they also support cilia. The epithelium is succeeded by a thin layer of apparently homogeneous, connective tissue, beneath which is a very close interlacement of larger and smaller vessels, between which no formed connective tissue can be perceived, but only a clear, homogeneous, interstitial substance.

All the portions of the ventricles which are not lined by the continuations of the pia mater, that is to say, the floor of the fourth ventricle, the aqueductus Sylvii, the floor and the sides of the third ventricle, the ventriculus septi lucidi, the roof of the lateral ventricles, the anterior and the posterior cornua, and a considerable part of the descending cornu, in the embryo also the cavity in the olfactory bulb, and the canal in the spinal cord, have a special lining membrane, the so-termed ependyma ventriculorum (Fig. 151). This is a simple tessellated epithelium, which, according to Purkinje and Valentin (Müll. "Arch.," 1836; Val. "Repert.," 1836, p. 156), is said to exhibit ciliary 
motion, a statement, however, which Virchow and I have not been able to confirm in the case of an executed criminal. It lies, normally, immediately upon the nervesubstance, but there is so frequently developed beneath it, especially in the fornix, the stria cornea, and the septum lucidum, a filamentous layer, resembling connective tissue, $0.01-0.05$ of a line thick, that its occurrence at a certain age might almost be described as constant, as in fact it is by Virchow. The epithelium

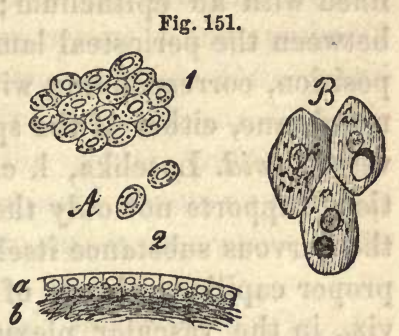
sometimes presents, particularly as in the third ventricle, large cells of $0.008-0.012$ of a line, with pigment-granules and masses, together with nuclei, measuring 0.003 of a line; in other situations, as in the lateral ventricles, the cells are not more than $0.005-0.007$ of a line in size, but almost as thick as wide, with roundish nuclei and a good many yellowish granules, which are generally crowded in the interior.*

The vessels of the membranes just described present very various conditions. In the first place, in the dura mater of the cord, if we except those on the external surface and the numerous arteries and veins of the cord by which it is perforated-the vessels are very few in number, and in this respect the membrane presents more of the conditions of a muscular fascia or tendinous expansion. On the other hand, there exist in this situation, between the dura mater and periosteum of the vertebral canal, the well-known venous plexuses, as well as finer ramifcations in the adipose tissue, which do not demand any further description. In the cranium, on the contrary, the entire dura mater is vascular, but especially in its external periosteal layer, which, partly for its own supply, partly for that of the cranial bones, to which it gives off numerous branches, supports the arterice meningece, and also conveys through its veins a portion of the blood returned from the bones. Besides this, in the cerebral dura mater are lodged the venous sinuses,

Fig. 151.-Ependyma in man. $A$, from the corpus striatum; 1 , from the surface: 2 , from the side; $a$, epithelial cells; $b$, nerve-fibres lying beneath; $B$, epithelium cells from the commissura mollis.-Magnified 350 diameters.

* [According to the recent researches of Virchow, the spinal ependyma differs somewhat from that lining the ventricles of the brain. It is more gelatinous and resistant, and contains cells which are much larger than those of the cerebral ependyma. It lies in the middle of the gray matter, in the exact situation where the spinal cord exists in the fœtus, and forms a continuous filament, extending to the filum terminale.

The ependyma is prolonged without distinct boundaries, between the nervous elements. Virchow has found a continuous layer of a similar substance in the interior of the higher nerves of sense. He has quite recently also discovered in its deeper layers peculiar granules, with the chemical reaction of cellulose. (Vide $\$ 118$, infra.)-DaC.] 
which are simple excavations in it, for the conveyance of blood and lined with an epithelium; and most of which are obviously situated between the periosteal lamella and the proper dura mater, thus, in their position, corresponding with the plexus venosi spinales. The arachnoid membrane, either of the spinal cord or of the brain, contains no proper vessels (vid. Luschka, l. c., p. 71), whilst the pia mater in both situations supports not only the very copious ramifications of the vessels of the nervous substance itself, but is also supplied, with a tolerably rich, proper capillary plexus of its own. In one portion of the pia mater, viz., in the vascular plexuses, the vessels are distributed solely in the membrane itself, the branches entering the nervous substance being of subordinate importance. Lymphatics it is said have recently been injected with air and quicksilver by Fohmann and Arnold, (vid. "Anat." II. p. 618) both in the pia mater on the surface of the cerebrum and cerebellum, as well as in the choroid plexus, but this observation appears to me very much to demand confirmation.

The membranes of the central nervous system, also contain nerves, at all events in part. In the dura mater of the cerebrum they run in the periosteal lamella of the membrane, following pretty nearly the course of the meningeal arteries, and are especially distinct on the $a$. meningea media, which is accompained, not only by twigs of the nervi molles, but also by a special nerve first noticed by Arnold ( $n$. spinosus, Luschka), which, according to Luschka, is derived from the third branch of the n. trigeminus, the former of which are distributed with the vessels, and the latter appears to be destined principally for the bones. Besides these, Purkinje has noticed nerves on the anterior and posterior meningeal arteries, and Arnold long ago described the well-known $n$. tentorii cerebelli, proceeding from the fifth pair, which, as has been lately shown, particularly by Pappenheim and Luschka (l. c.), goes to the larger sinuses of the dura mater. The elements of this white-looking nerve and of the $n$. spinosus of Luschka, are those of the n. trigeminus, those of the others, fine fibres, and in both situations they present divisions. In the dura mater of the spinal cord, I, as well as Purkinje, have been unable to detect any nerves; they occur, however, as has been already mentioned, in the periosteum of the vertebral canal, and on the arteries going to the vertebræ and cord, as well as in the sinuses and lax adipose tissue of the canal (Luschka, l. c.).

In the arachnoid itself I have never noticed any nerves, but on the vessels by which it is penetrated, and in the processes connecting it with the pia mater, they may perhaps be seen, especially at the base of the brain-to which nerves, those seen by Luschka (Seröse Häute, p. 70), notwithstanding the divisions observed in them, appear to me to belong. Bochdalek (1. i.c.) has lately described nerves of the cerebral arachnoid, 
derived from the $n$. accessorius, the portio minor trigemini, and the facial nerve, but fails to show that they terminate in the membrane. When the same author also finds extremely numerous nerves in the arachnoid covering the cauda equina, he falls into the same error, as Rainey had previously encountered in regarding connective tissue, disposed in the more rare reticular form, as nerves. In the cauda equina, I am acquainted with nerves only on the filum terminale, accompanying the vessels, and nowhere else; not even in the dura mater, into which Bochdalek equally supposes he has traced them.

The nerves discovered by Purkinje in the pia mater of the $0 \mathrm{x}$, also exist in man, in whom the pia mater of the cord, including the filum terminale, is richly supplied with plexuses of fine nerves, measuring $0.0015-0.003$ of a line, which throughout do little more than accompany the vessels. At the base of the brain, many similar plexuses occur on the arteries of the circle of Willis, which, in twigs, at most 0.03 of a line in diameter, are distributed through the entire pia mater of the brain, accompanying and always following the course of the various vessels, with the exception of those of the cerebellum; their terminations, however, can nowhere be perceived. It is certain that they do not accompany the arteries into the cerebral substance, and that no nerves exist in the vascular plexuses; whether there are any or not on the vena Galeni, I have not yet inquired. The origin of these nerves has been ascertained by Remak to be in the posterior roots, each of which, as I have satisfied myself, in many situations, and as it appears to me more frequently in the cervical portion of the cord, from the fibres in closest contiguity to each other, sends out fine fibrils across the subarachnoid space into the pia mater. As in this case, so also in the cerebrum, besides the sympathetic nerves (plexus caroticus internus, plexus vertebralis), the cerebral nerves may participate in the supplying of the pia mater, since Bochdalek has noticed numerous fine twigs, given off from the roots of many of the cerebral nerves, of the same structure as the roots themselves, joining the nervous plexuses of the arteries at the base of the brain and of the pia mater of that region, and of the cerebellum; as well as in the plexus chorioideus ventric. quart. (?). He also found that isolated fine filaments entered the pia mater, directly from the medulla oblongata, the pons Varolii, and crura cerebri, which were not previously conjoined with the neighboring nervous trunks.

B. Vessels of the central nervous system. - With respect to the distribution and condition of the bloodvessels-the brain and spinal cord agree almost entirely. After ramifying to a considerable extent in the pia mater, the arteries enter the nervous substance, except in a few situations (substantice perforato, pons), as fine, though still distinct, 
arterial vessels, and ultimately subdivide, by continuous ramification, for

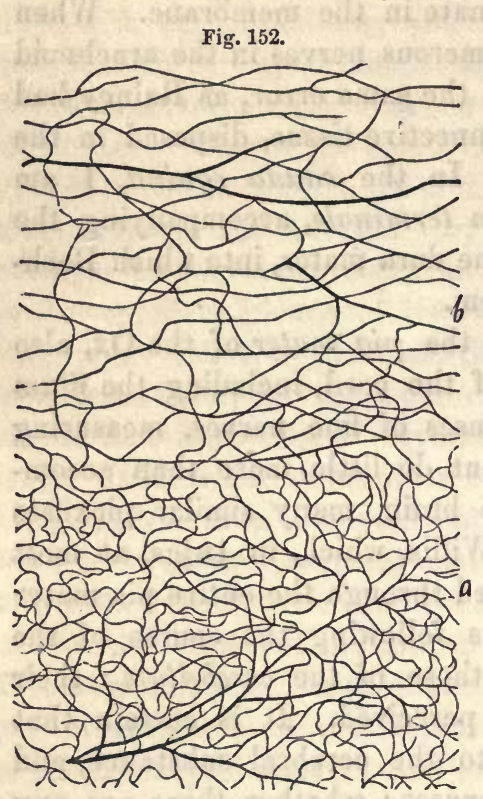
the most part at acute angles into a rather wide network of very fine capillaries, from which again the venous radicles arise, joining so as to form the well-known trunks, both on the surface and in the interior (Fig. 152). The gray substance is invariably much more richly supplied with vessels than the white, the plexus formed by them being closer, and the capillary vessels themselves of less calibre, to which its color is in some respect due. According to E. H. Weber, the interstices of the capillaries in the medullary substance, measure 0.0142 of a line in width and 0.025 of a line in length; in an injected preparation by Gerlach of the sheep's brain, the breadth of the interstices in the gray substance was three or four times less than in the white.

In the spinal cord, the disposition of the entering trunks is sometimes very regularly in series. Two series of vessels of this kind exist in the bottom of the anterior fissure, which, from the processes of the pia mater, penetrate the gray substance on the right and left; whilst a third series corresponds to the posterior fissure, and others not unfrequently also to the roots and the processes of the ligamentum denticulum. All these vessels enter the gray substance without undergoing any direct or considerable decrease in size, and there find their ultimate distribution. In the brain very delicate parallel vessels are met with in the gray substance of the cerebellum, less distinctly in the cerebrum and other parts. The structure of the vessels is, in general, the same as elsewhere. The arteries, upon their entrance into the nervous substance, possess three coats-the tunica adventitia, though resistant, is a thin and apparently quite homogeneous membrane; the $t$. media is purely muscular; and the $t$. intima formed of nothing but a very delicate elastic membrane, with openings, and well-marked fusiform epithelial cells. One after another of these coats is gradually lost, till before the capillaries are reached, we find nothing but the $t$. adventitia, and scattered, transversely placed, elongated cells, with transverse nuclei and an epithelium; with which class of vessels are soon associated capillaries with a structureless membrane and few or more nuclei, sometimes of great minuteness, the finest

Fra. 152.-Vessels of the cerebral substance of the Sheep, from one of Gerlach's injections: $a$, of the gray; $b$, of the white substance. 
measuring in the cord, 0.0022 , in the brain 0.002 of a line. Of the veins, the largest, for the most part, do not present a trace of smooth muscle, exbibiting nothing but connective tissue with nuclei, or fine elastic filaments and epithelium; in the smaller ones I have, occasionally, though very rarely, observed contractile elements.

In the ventricles of the brain there exists, under normal conditions, an extremely small quantity of clear serous fluid, which is manifestly secreted by the arterial plexuses, and which, probably aided by the ciliary movements, assists in the nutrition of the walls of the cavities. A second fluid, the liquor cerebro-spinalis is contained in the subarachnoid spaces above described, which according to Luschka, are lined by an epithelium, and from the largest of which, extending from the base of the brain to the termination of the canal of the dura mater medulloe, the fluid in question may be readily obtained. It is alkaline, contains - of water 98.56 -albumen and extractive matter 0.55 -salts 0.84 per eent., principally chloride of sodium. Its principal function appears to be to conduce to the more free motion of the central nervous system, and to act as a regulator in varying degrees of fulness of the vascular system.

A few pathological points may here be referred to. The ependyma ventriculorum presents not only, as above mentioned, almost constantly in places, a thin fibrous substratum, but is frequently, especially in dropsy of the ventricles, and in old age, very much thickened by a layer of that kind. In either case it invariably contains, as was first mentioned by Purkinje, yellowish bodies, with concentric strix of a round or biscuit shape, and not unlike starch granules. They are scarcely affected by acids, whilst in caustic alkali they become pale and gradually dissolve. I find these corpuscula amylacea (Fig. 153), almost always on the fornix, the stria cornea, and septum pellucidum, and also elsewhere in the walls of the ventricles, as well as in the cortical substance of the brain, in the medullary substance of the cord, and in the filum terminale; in the first-mentioned situations they frequently occur in incredible quantity, close together, in the newly formed connective tissue, or between the nervous elements. That these bodies are a pathological product is certain, but not so of what they consist,

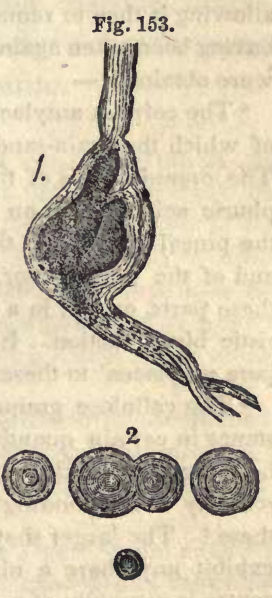
or how they are formed, although everything indicates a nitrogenous

Fia. 153.-1, "Brain-sand" from the pineal gland, in bundles of connective tissue: 2 , corpuscula amylacea from the ependyma of Man; magnified 350 diameters. 
substance, and a formation from successive deposits.* In the plexus chorioidei, in the pineal gland, occasionally in the pia mater and

* [The recent investigations of these corpora amylacea by Virchow, (see Archiv. f. path. Anat. Sept. 1853) have led him to the important discovery of their true nature, viz.: that they are composed of a substance, resembling the cellulose in plants. The fact that in the animal kingdom, starchy substances had only been found in a low class of the invertebrated animals, induced Virchow to examine all starch-like bodies occurring in the higher classes of the animal kingdom, and thus the exact composition of the corpora amylacea was ascertained. As this discovery is of great importance, I shall quote the author's own words.

"In a histological point of view, it has often occurred to me, that the umbilical cord in Man possessed a great similarity in its structure to the cellulose in the Ascidians. (See Würzb. Verhandl. 1851, Vol. II. p. 161, note.) And I was the more confirmed in this idea by Schacht's observations, so that ever since my researches have been more carefully directed to this subject. But in most instances I searched in vain, as in the ova of amphibia and fishes, the peculiar yelk-plates of which I described. (Zeitschrift für Wiss. Zoologie. 1852, Vol. IV. p. 240.) I was more successful, however, recently, when I directed my attention to the so-called "corpora amylacea" of the brain, of the exact nature of which, as compared with the other starch-like bodies in man, I had not been able to form any very definite conclusions. I soon found that on the application of iodine, they assumed a bluish tint, and upon subsequently adding sulphuric acid, the exquisite violet color, which is known to belong to cellulose, and which appeared the more intense, as it formed a distinct contrast to the surrounding yellow or brown nitrogenous substances.

"I have so frequently repeated these investigations, and with so many precautions, that I consider the results as perfectly certain. For I have instituted comparative researches not only in different human bodies, and in the most different situations, but I have allowed the reagents employed to act under all possible conditions."

The best manner, Virchow continues, to obtain this reaction is to allow an hydrated solution of iodine to act on the bodies in question, and then to add a little diluted sulphuric acid. Care should be taken not to add too much iodine at once, and to allow the sulphuric acid to act very slowly. The most beautiful preparations were obtained by adding a drop of sulphuric acid to the edge of the thin glass covering a preparation, and allowing it then to remain undisturbed from twelve to twenty-four hours. Every precaution having been taken against an accidental admixture of starch or cellulose, the following results were obtained :-

"The corpora amylacea are chemically different from the concentric spherical corpuscles of which the brain-sand is composed, and with which they have hitherto been confounded. The organic basis of these brain sand-granules, is evidently nitrogenous; iodine and sulphuric acid color it an intense yellow. The same is true not only of the sandy matter in the pineal gland, and the choroid plexuses, but also of that of the Pacchionian granulations and of the dura mater, as well as of the dentated plates in the spinal arachnoid. In all these parts, except in a few spots in the pineal gland, I have never obtained the characteristic blue reaction. It would, therefore, be henceforth advisable to restrict the term 'corpora amylacea' to these cellulose corpuscles.

"The cellulose granules seem to be connected with the presence of the ependyma substance in certain quantities, and might not improperly be considered as a part of it. But how they are produced from it, it was impossible to recognize. They are usually minute, scarcely corresponding in size to the nuclei of the ependyma. Can they originate from these? The larger they are, the more distinctly laminated they appear. But they do not exhibit anywhere a nitrogenous admixture, distinguishable by its yellow color. Their centre is generally of a darker blue, and hence, perhaps, denser than their border.

"The supposition, of these bodies being introduced from without, is the less probable because a similar substance is nowhere else known. The cellulose in plants exhibits a number of varieties, but this animal cellulose is distinguishable above all by its slight resistance towards reagents; for concentrated acids and alkalies act on it more powerfully than on vegetable cellulose." 
arachnoid (also in the cord), and although rarely, also in the walls of the ventricles, there is furthermore met with, as a constant, though pathological production, the gritty matter of the brain (brainsand). It consists of roundish, simple or mulberry-shaped, opaque, mostly concentrically striated globules of $0.005-0.05$ of a line, and together with them of angular bodies, of a stalactitic, clavate, or other irregular figure, with an uneven, botryoidal, scaly surface; and also in the form of simple, cylindrical, rigid fibres, either branched or reticular, and of fine particles. The brain-sand contains principally carbonate of lime, but also phosphate of lime and magnesia, and an organic substance, which after the salts have been removed, for the most part perfectly retains the figure of the concretion, that is to say, of a concentrically laminated pale body, or as clear fibres. It is quite certain that this brain-sand, when it assumes the form of elongated, branched, reticular bodies, is simply developed in the bundles of connective tissue (Fig. 153), as, not unfrequently, in the pineal gland and in the membranes of the brain; in other cases it appears to be an independent incrustation on fibrinous concretions. Whilst cells impregnated with calcareous matter, which Remak ("Obs.," p. 26) supposed them to be, according to Harless (Müll. "Arch.," 1845, p. 354), do not exist. Lastly, also, may be mentioned the Pacchionian granulations of the pia mater, and ossifications of the membranes. The former, which are situated principally on both sides of the falx major, on the flocculi, in the choroid plexuses, \&c., consist chiefly of a tough fibrous substance, not unlike immature connective tissue, containing also undeveloped elastic tissue, and corpuscula amylacea. The latter, which are true osseous plates, occur sometimes on the inner surface of the cerebral dura mater, sometimes on the arachnoid, particularly of the cauda equina.

The cellulose corpuscles were found only in the substance of the ependyma of the ventricles and its prolongations, including the transparent substance in the spinal marrow described by Kolliker as the " substantia grisea centralis," but not in the cortical layer of the brain, or in the interior of the cerebral substance. Neither was Virchow able to detect them in the brain of a child, or, as Bernard's experiments might lead us to suppose, in the brain of a Rabbit, and he is, therefore, inclined to attribute to them a pathological import. Similar bodies have been found, by Rokitansky in the optic nerve, by Kölliker in the retina, by Luschka in the ganglion of Gasser.

The exact chemical nature of these bodies has not, as yet, been satisfactorily ascertained. They have not the pure reaction of vegetable cellulose, nor, as Donders supposes them to have, that of starch, and they might, perhaps, at present, be more properly called " amyloid" corpuscles. If boiled in water they are dissolved.

This discovery of Virchow's is of great importance, not only with regard to the anatomy of the corpora amylacea, but as establishing as an undoubted fact the existence of vegetable matter as a part of the animal economy. Whether this be a pathological formation or not, it is at present impossible to decide, the former is, however, highly probable, since corpuscles with the same reaction have been found in certain abnormal conditions of the spleen. (Vid. \$ 170, Spleen).-DaC.] 


\section{PERIPHERAL NERVOUS SYSTEM.}

$\S$ 119. Spinal nerves.-The thirty-one pairs of nerves springing from the spinal cord, arise, with few exceptions, by anterior and posterior roots. Receiving a delicate tunic from the pia mater, they converge, and are continued across the subarachnoid space, to perforate, independently of each other, the arachnoid and dura mater, from the latter of which they obtain a firmer coat. Proceeding further, the posterior root forms its ganglion, by the deposition around and among its nerve-fibres, of ganglion-cells, which, to all appearance, give origin to special nerve-tubes, the ganglionic fibres of the spinal-nerves, each for the most part arising from a cell, and which have no further connection with the fibres of the posterior root passing through the ganglion, than that, in their invariably peripheral course, they are in apposition, and intermingled with the latter. The motor root never acquires ganglioncells, merely passing by the ganglion, in more or less close apposition with it. Beyond the ganglion, the two roots are united in such a manner that their elements are very intimately commingled, and a common nervous trunk formed, containing in all its divisions sensitive and motor elements. It is usually connected with the neighboring nerves above and below it, in the formation of the well-known plexuses, afterwards giving off its terminal branches to the muscles, integument, vessels of the trunk and extremities, articular capsules, tendons, and bones. As in the roots, so also in the branches of the common trunk, it is seen that the motor twigs contain principally thick fibres, and those destined for the integument and other organs above named, finer ones; ultimately, however, in the terminal ramifications, all the fibres are of uniform size. The fibres of all the spinal nerves appear to run quite distinct from each other, and to present no divisions in the trunks and branches, whilst, in the terminal ramifications of them, divisions frequently occur, and, at all events in certain animals (Mouse, batrachian larva), also reticular anastomoses. They terminate either in loops, or in free prolongations, the latter being the case, particularly, in the Pacinian bodies, which are structures composed of numerous concentric capsules separated by fluid, of an oval form, and measuring $\frac{1}{2}-2$ lines, found principally in the hand and foot, and which usually contain the termination of a nervefibre.

In the first and last pairs of spinal nerves occasionally only a single root can be perceived, in the former case the motor, and in the latter the sensitive. I have communicated the diameters of all the anterior and posterior roots on the left side in a male and female body, in the "Verh. d. Würzb. phys. med.," Gesellsch. 1850, Heft II. and the transverse sectional areas deduced from these observations are given in 
my "Microscopical Anatomy," § 116. The roots are furnished with a delicate neurilemma, derived from the pia mater, and presenting a similar structure, which forms both an external sheath 0.002 of a line in diameter, as well as internal septa to the individual fasciculi. The contiguous roots frequently anastomose, and this is much more usually the case with the sensitive roots; in the cervical nerves in Man in particular, it is found to take place constantly in one or other of the nerves.

$\S 120$. The structure of the spinal ganglia, in the Mammalia, is a difficult subject of investigation, but I think the following may be stated with certainty respecting them. The sensitive roots, so far as I have hitherto been able to make out, enter into no connection with the nerve-cells in the ganglion, but forming one, or, in the larger ganglia, several, or even numerous fasciculi, which in the latter case anastomose, simply traverse it, to be reunited below the ganglion into a single trunk, which is then immediately blended with the motor root. Most of the nerve-cells themselves appear to be in connection with the nerve-fibres, giving off either one or two, or more rarely, several. These fibres, which I term ganglionfibres, proceed in a preponderating majority perhaps all of them peripherally, joining and strengthening the perforating root-fibres; so that each ganglion is to be regarded as a source of new nervefibres.*

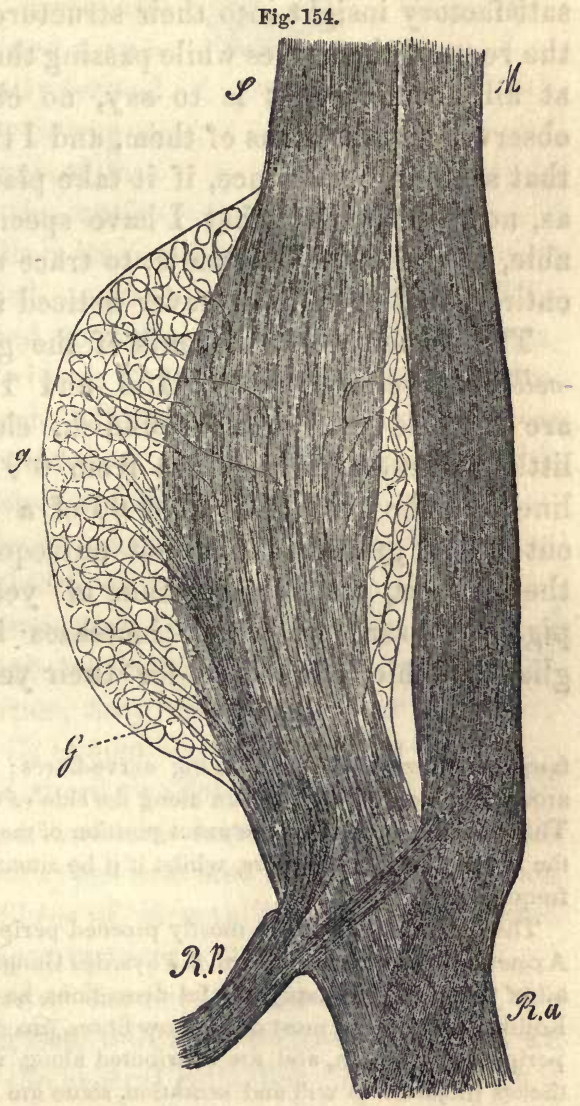

Fia. 154.-A lumbar ganglion of a young Dog, treated with soda, and magnified 45 diameters : $S$, sensitive roots; $M$, motor roots; $R . a$, anterior branch of the spinal nerve; $R . p$, posterior branch; in both their composition from both roots is manifest; $G$, ganglion, with the cells and ganglion-fibres, which assist in the strengthening of the sensitive roots traversing the ganglion.

* [The new "ganglion-fibres" join differently the nerve-fibres on which the ganglion is seated, or by which it is perforated. In the large ganglia the new fibres penetrate in 
The structure of the spinal ganglia (Fig. 154) is a difficult subject for investigation, in Man. No complete results can be obtained from the larger of them, but more may be made out in the smaller or smallest, as in those of the fifth sacral nerve and $n$. coccygeus, which are to be sought within the sac of the dura mater, also perhaps in the fourth sacral and first cervical nerves. If a comparative examination be instituted, of the spinal ganglia of the smaller Mammalia (Rabbit, Puppy, Mole, Mouse, Rat), and if not only the scalpel and needle be employed, but if the entire ganglia be examined after the application of acetic acid, and above all, of a dilute solution of soda, with the aid of the compressorium, a satisfactory insight into their structure may be obtained. The fibres of the roots of the nerves while passing through the ganglia present nothing at all peculiar, that is to say, no change in size; nor have I ever observed any divisions of them, and I think it may be positively asserted, that such an occurrence, if it take place at all, must be extremely rare, as, notwithstanding that I have specially sought for it, and have been able, in the lower Mammalia, to trace numerous nerve-fibres through the entire ganglion, I have never noticed anything of the sort.

The principal constituents of the ganglia-the ganglion-globules or -cells [nerve-cells] (Figs. 155 and 157), have a distinct outer coat, are for the most part roundish, elongated, or pyriform, usually a little flattened, and measure from 0.012 to 0.036 , or even 0.04 of a line; on the average $0.02-003$ of a line. The contents are throughout finely granular, and not unfrequently exhibit, in the vicinity of the nucleus, an accumulation of yellow, or yellowish-brown, larger pigment granules, which increases in age, and to which the ganglia are chiefly indebted for their yellow color. The nuclei measure

fasciculi between the perforating nerve-fibres; in the smaller ganglia they either wind around the nerve-fibres, or run along the side of the nerve on which the ganglion is situated. This depends mainly on the exact position of the ganglion. If it be placed in the middle of the nerve, the former occurs, whilst if it be situated on one side of it, the latter is the more frequent.

The new ganglion-fibres mostly proceed peripherally, but according to a recent observer, Axmann (Beitrage zur Anatom. u. Phys. des Gangl. Syst., Berlin, 1853) this is not the case with all of them. From many careful dissections he satisfied himself that whilst, as stated by Kolliker and others, most of the new fibres, proceeding from every spinal ganglion, run in a peripheral direction, and are distributed along with the cerebro-spinal nerve-fibres, to the tissues subjected to will and sensation, some are connected through the rami communicantes with the sympathetic system. He also found that a small number of the ganglion-fibres penetrate through the roots of the cerebro-spinal nerves into the spinal column and brain, where, he states, they have been frequently mistaken for attenuated cerebro-spinal nervefibres. He adds, further, that he has been able to trace their connection with the spinal ganglia in the Frog, Mouse, and Pike, and also in a human fœus of six montlss. It is, however, difficult to understand how Axmann was able to determine that these fibres proceeded from the ganglia to the spinal marrow, since they might be as readily supposed to have been minute fibres proceeding from the ganglia of the spinal marrow towards the external ganglia.-DaC.] 
$0.004-0.008$, the nucleoti $0.0008-0.002$ of a line. These nerve-cells are situated, in the first place, in larger numbers on the surface of the ganglion, between the neurilemma and the perforating radical fibres; and secondly, at all events in $\mathrm{Man}$, in the interior, where they occupy the interstices of the plexus formed by the nervefibres. The individual cells are retained in their situations by a special tissue, which also separates them from the contiguous cells and from the nerve-fibres. This tissue appears on isolated cells, as if it formed a special coat to them, and is consequently termed their external sheath, but in fact it represents a system of small septa, connected in a complex manner, and pervading the entire

Fig. 155 .
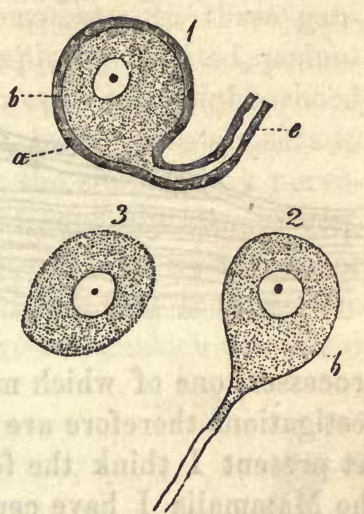
ganglion, receiving the separate cells in its meshes, and only more rarely appearing as a definitely bounded coat on individual cells. This structure is evidently to be referred to connective tissue; it presents, however, several forms, which have been, in part, already, properly distinguished by Valentin (Müll. "Arch." 1839, p. 143), viz. 1, in the form of a sometimes homogeneous, sometimes more fibrous substance, with scattered, flattened, roundish nuclei of $0.002-0.003$ of a line; and, 2, in that of isolated elongated, triangular or fusiform cells, measuring $0.003-0.005$ of a line, with nuclei as above, and which sometimes may be supposed to resemble epithelial cells, although, as is evident from a comparison of their different forms, they rather correspond with the developmental cells of connective, or of elastic tissue (Fig. 156). Besides these two forms, the Fig. 156. former of which occurs everywhere, and the latter principally in the larger ganglia, certain intermediate types are met with in Man, which consist, as it were, of nucleated "fibres of Remak," as they are termed (vid. infra), or, at all events, in the preparation, break up into such.

From by far the greatest number of the nerve-cells, in Man and the Mammalia, are given off pale processes, $0.0015-0.0025$ of a line, in all respects corresponding to those of the central cells, but furnished with a special sheath, and which, as I discovered in the year 1844 ("Selbst. $u$.

FiG. 155.-Ganglion-globules (nerve-cells) from the Gasserian ganglion of the Cat, magnified 350 diam.: 1, cell with a short, pale process, showing the origin of a fibre, $a$; sheath of the cell and nerve-tube, containing nuclei; $b$, cell-membrane of the nerve-cell; 2 , cell with the origin of a fibre, without sheath; $b$, cell-membrane of the nerve-cell; 3 , nerve-cell, deprived, in the preparation of it, of its membrane and external sheath.

Fra. 156.-Cells from the sheath of the nerve-cells of the spinal ganglia in Man, magnified 350 diameters. 
Abh. des Symp. Nerv.," Zürich, 1844, p. 22), are each of them continued into a dark-bordered nerve tube (Figs. 155, 157). The cells

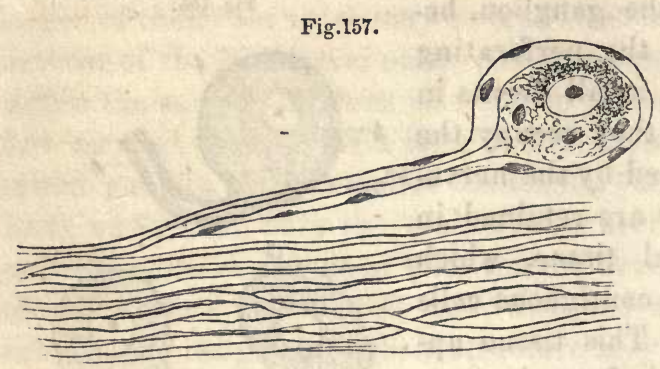

observed by me had but one process, the so-termed unipolar-cells, and I at first thought that such only existed in the spinal ganglia. It now appears, however, from more recent researches, especially from those of Stannius, that they also contain cells with two processes, one of which may again divide; fresh and more extended investigations therefore are required to show how the matter really stands. At present I think the following should be remarked: 1, in Man and the Mammalia I have certainly established the fact of the existence of unipolar-cells, and think it may also be asserted that they are very numerous; 2, quite lately I have myself, although rarely, noticed cells with two, pale processes, and I am willing to admit the possibility that such cells frequently occur, as it is certain that many processes must be torn off in the comparatively rude methods necessarily employed to isolate the cells; 3 , when Stannius very recently noticed in a human foetus, and in a fotal Calf, together with unipolar and apolar cells, in the latter numerous bipolar cells, it should be inquired whether the latter were not cells which afterwards divide?-because divisions of the nerve-cells undoubtedly take place (vid. infra), - and in this way become unipolar; 4, whether the cells give off one or two fibres, one of the latter does not go towards the centre and the other towards the periphery, but both proceed in the latter direction; at all events, in the examination of all small ganglia, only such ganglion-fibres are visible. Stannius, in bipolar cells of this kind from the Calf, also found the two processes closely approximated ; 5 , it is difficult to determine whether cells without processes also occur in the spinal ganglia, seeing that the processes are very readily detached, and that cells thus truncated may very easily be regarded as apolar cells. In small ganglia in the Mammalia a fibre may be traced to each cell, whilst in the smallest spinal ganglia in Man, and in the inconstant ganglia of the posterior roots (vid. seq.), cells are not unfrequently met with, to which no fibre is attached, and, consequently, I would, at present, merely state that, in any case, fibres arise from the majority of the cells. In order to examine these conditions, either the larger ganglia in Man are selected, which

Fra. 157.-Twigs of the coccygeal nerve within the dura mater, with an adherent, pedunculated nerve-cell in its nucleated sheath, from which the derivation of a fibre is very distinctly seen ; magnified 350 diameters. From Man. 
are torn into fibres as carefully as possible under a simple microscope, until the fibres are traced to their origin, which may be done with a little trouble in almost every ganglion, or the small ganglia of the fifth sacral and coccygeal nerves are taken for the purpose. In these ganglia, in almost every individual, solitary and completely isolated, pedunculated, ganglion-globules are met with, close to or in the neighborhood of the ganglia, each in its special sheath, wbich in this case appears to be homogeneous (Fig. 157), and in many cases, the simple, dark nervefibres lying in the peduncle of the globule, and frequently also its connection with the cell, by means of a pale process, may be distinctly perceived. In the ganglia aberrantia also of Hyrtl, that is to say the inconstant, larger or smaller collections of nerve-cells, which are found in every subject upon the posterior roots of the larger nerves, the simple origins of fibres may occasionally be distinctly noticed. The darkcolored fibres, arising from the nerve-cells, simply constitute the continuation of the pale processes of the cells, so that the membranes and contents of each part pass continuously into each other, and thus also the membrane and the contents of the cells are connected with the sheath of the nerve-tubes, the medullary sheath, and the axis-cylinder. In older nervecells, or by the operation of reagents (arsenious acid, chromic acid, iodine), the contents of the cell become detached from the membrane, and the axis-cylinder appears as a direct continuation of the former (Fig. 158), as was first shown by Harting (vid. also Stannius in "Gött. Anzeig.", 1850, and Leydig, l. c. Tab. 1, Fig. 9), which is the best proof that the contents of the

Fig. 158.

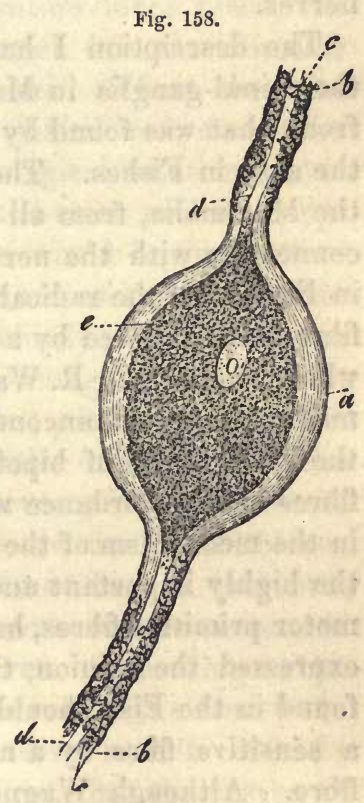
nerve-cells cannot be understood as contained in a dilated nerve-tube.*

Fia. 158.-Nerve-cell of the Pike (bipolar, as they are termed), which is continued at each end into dark-bordered nerve-tubes, treated with arsenious acid: $a$, sheath of the nerve-cell; $b$, sheath of nerve; $c$, nerve-medulla ; $d$, axis-cylinder continuous with the contents of the nerve-cell; $e$, which have shrunk away from the sheath.- Magnified 350 diameters.

* [The axis-cylinder is supposed by some observers not to be, as above stated, a continuation of the contents of the cell, but rather of its nucleus. This continuation of the nucleus into the axis-cylinder is best seen in ganglia, which have been kept for some days in diluted acetic acid. We are thus, according to Axmann (1. c.) not only able to see the connection of the axis-cylinder with the nucleus, but also to isolate the nucleus with a portion of the axiscylinder attached to it. This continuation of the nucleus of the ganglion cell into the axiscylinder has been observed in all classes of animals. It was first described by Harless in the ganglion corpuscles of the electric lobes of the Torpedo Galvani.-DaC.] 
The nerve-tubes or ganglion-fibres thus originating, which frequently arch round or embrace the cells with several circular turns, are at first fine, measuring 0.0015-0.0025 of a line, but (not continuing so as I formerly supposed, when I was acquainted only with their origin), they all very soon increase in size, as may be very readily observed in many fibres, whilst still within the ganglion, up to 0.003 and 0.004 , many even to as much as 0.005 and 0.006 of a line; becoming, consequently, mediumsized, and thick nerve-fibres. The processes of the cells and the nerve-fibres springing from them are also furnished with nucleated sheaths like the cells themselves, the vaginal processes, as they are termed, which they lose, however, at the point where they join the emergent trunk, obtaining instead of it, as a coat, the common neurilemma of the nerves.

The description I have above given of the conditions observable in the spinal-ganglia in Man and the Mammalia, differs very considerably from what was found by Bidder, Reichert, R. Wagner, and Robin, to be the case in Fishes. The chief difference consists in this, that whilst in the Mammalia, from all we know, the roots of the nerves have no direct connection with the nerve-cells, and merely pass through the ganglion, in Fishes, all the radical fibres are connected with the cells, so that each fibre is interrupted by a bipolar cell, and independent ganglion-fibres are wholly wanting. R. Wagner has thought, that what obtains in the Fish might be applied, unconditionally, to all the Vertebrata, and asserts, that the occurrence of bipolar cells in the course of the posterior radical fibres is in accordance with Bell's doctrine, and a necessary contingent in the mechanism of the sensitive fibres ; and moreover, that in this case the highly important and long-sought distinction between sensitive and motor primitive fibres, has been discovered. In opposition to this I have expressed the opinion, that it is not a necessary postulate, that what is found in the Fish should be applied to Man, and that the interruption of a sensitive fibre by a nerve-cell does not distinguish it from a motor fibre. Although Wagner has very recently characterized this opinion of mine as unphysiological, he will not, at the same time, convince any one that the spinal ganglia of the Mammalia are constructed as he thinks, and I shall therefore wait to see whether further observations will confirm my observations or not. In order to complete them, I will moreover mention, that direct measurement of the sensitive roots above and below the ganglia, shows a not inconsiderable difference in favor of the latter situation (vid. "Mikroskop. Anat.," II. p. 509), which as differences in the thickness of the entering and emergent nerve-fibres, and divisions of them within the ganglion do not exist, can only be referred to the fibres which originate in the ganglion and proceed towards the periphery, a view which is also confirmed by direct observation (Fig. 
154). With respect to the interesting observations on the structure of the spinal ganglia of the lower animals, and particularly of Fishes, I would refer especially to the works of R. Wagner, Bidder, Robin, and Stannius, cited below.

$\S 121$. Further course and termination of the Spinal Nerves.-Below the spinal ganglion, the sensitive and motor roots unite to form a common trunk, their fibres being intermixed in diverse ways, as may be very distinctly perceived in small animals. All the subsequent branches, both of the anterior and posterior main divisions, as well as their further continuations, are consequently of a mixed nature, formed of portions derived from both roots; a condition which they retain up to their ultimate distribution. Here, however, an alteration takes place, the motor fibres going off in by far the larger proportion into the muscular branches, and the sensitive chiefly to the cutaneous. Where the ganglion-fibres which arise in the spinal ganglia are distributed, cannot be ascertained anatomically.* When their physiological relations, however, are considered, it would appear as by far the most probable supposition, that they do not, as at first sight one would be inclined to suppose, join the sympathetic in the rami communicantes, but, that accompanying the spinal nerves, they are continued chiefly into the vascular branches, and consequently are distributed in the integuments, muscles, bones, joints, tendons, and membranes (periosteum, pia mater, \&c.), but also, perhaps, to the glands and involuntary muscles of the skin. The nerve-fibres in the main trunks of the spinal nerves present the same diameter as in the roots, that is to say, there are finer and thicker tubes, and a certain number of intermediate forms; but, as they proceed, the fibres separate, the thicker going more to the muscular branches, and the thinner into the cutaneous nerves. According to the statements of Bidder and Volkmann, the proportion of the fine to the thick fibres is, in man, as $1.1: 1$, in the muscular nerves as $0.1-0.33: 1$; statements which I can but confirm, adding to them, that the nerves of the bones contain, in the trunks, one-third of thick and two-thirds of fine, whilst those of the articulations, tendons, and membranes, exhibit a great preponderance of fine fibres. In my opinion, most of the fine fibres contained in the branches of the spinal nerves must be regarded as derived from the spinal cord, and as being, in their function, quite of equal importance with the thick fibres, and at present, the only thing that remains unascertained, is whether they all ascend to the brain, or perhaps in part arise in the spinal cord; upon which point reference may be made to $\S 112$.

The spinal nerves are composed in general of parallel tubes, for the

* [Vid. note, § 120.—DaC.] 
most part undulating, upon which circumstance their transversely banded aspect depends; they exhibit, however, in their course, very frequent anastomoses, in which way the various larger and smaller plexuses with decussating fibres are formed. The formation of these plexuses is due to an interchange of entire fasciculi or fibres, never to a connection between the individual primitive fibres, and in a microscopical point of view affords no point worthy of remark.

Divisions of the nerve-fibres do not occur, according to our present experience, in the trunks and larger branches of the spinal nerves of the Mammalia [in Fishes, Stannius noticed numerous divisions in the trunks of the motor and mixed nerves ("Archiv für phys.," Heilk. 1850, p. $77)]$, nor do they exhibit any considerable change in their diameter; but in the ultimate ramifications, on the other hand, it is certain that such divisions do take place, even in Man, accompanied by a very considerable diminution in the size of the fibres;* with respect to which conditions, and the terminations in the skin, muscles, bones, and membranes in general, reference may be made to the detailed descriptions given in the proper places.

One kind, only, of termination of the spinal nerves, is still to be noticed here,-that in the Pacinian bodies. The small bodies, so named by Henle and myself ("Ueber die Pacin. Körperchen des Menschen und der Thiere," Zürich, 1844), were first accurately described by the Italian, Pacini ("Nuovi organi scoperti nel corpo umano," Pistoja, 1840), especially in the nerves of the palm of the hand and sole of the foot, and, in fact, as Langer of Vienna afterwards showed, had been previously noticed by A. Vater (J.G. Lehmann, "De consensu partium corp. hum.," Vitembergæ, 1741), although their nature had not been recognized. These organs are of an elliptical or pyriform shape, of a whitish transparent color, with whiter streaks internally, and measure $\frac{1}{2}-2$ lines in size; in Man, they are constantly found on the cutaneous nerves of the palm of the hand and sole of the foot, in the subcutaneous connective tissue itself, and most numerously in the fingers and toes, particularly on the third phalanx,-according to Herbst ("Die Pacin. Körperchen und ihre Bedeutung," Gött., 1847), there are about 600 in the hand and not quite so many in the foot; besides which, it must here also be stated, that they are invariably found on the great sympathetic plexus, in front of, and close to the abdominal aorta, behind the peritoneum, particularly near the pancreas, frequently also in the mesentery, close to the intestine; and also occasionally on other nerves, such as the

* [The ultimate ramifications are supposed by Axmann (1.c.), to be merely axis-cylinders surrounded by the sheaths of the nerves, the granular contents of the nerve-tubes having gradually disappeared. $-\mathrm{DaC}$. 
n. pudendus communis, on the glans penis (Fick) and bulb of the urethra, on the intercostal nerves, sacral plexus, cutaneous nerves of the upper- and forearm, on the dorsum of the hand and foot, and the cutaneous nerves of the neck.

The structure of the Pacinian bodies is, upon the whole, simple (Fig. 159). Each of them consists of very numerous (20-60) concentric layers of connective tissue, of which layers the external are separated by wider, and the internal by narrower interspaces, in which is contained a clear serous moisture, which is collected in larger quantity in an elongated central cavity, bounded by the innermost lamella. Each body presents a rounded peduncle, formed from the continuation of its lamellæ, and connected with a nervous twig, and in which a dark nerve-fibre, $0.006-0.068$ of a line (in the Cat, $0.0044-0.0077$ of a line) thick, runs to the Pacinian body. This fibre enters the central cavity from the penduncle, where it becomes 0.006 of a line wide and 0.004 of a line thick, pale, non-medullated, almost like an axis-cylinder, and terminates in the upper part of the cavity, in a free, slightly granular tubercle, the extremity being frequently bifid or trifid. Further observations, and comparative anatomical details with regard to these bodies, which are also found in great number in many Mammalia, as well as in Birds, in the skin, beak, and tongue (Herbst, Will), and with respect to which physiology is still wholly in the dark, will be found in the works above quoted, and also in Reichert ("Bindegewebe," p. 65), Herbst ("Gött. gel. Anz.," 1848, Nos. 162, 163, 1850 ; "Nachr. v. d. Univ.," p. 204, 1851, p. 161), Will ("Sitzungsber. d. Wiener A cad.," Feb., 1850), Osann("Bericht über d. zoot. Anst. in Würzb.," 1849), Strahl (Müller's "Arch.," 1848, p. 163), and Pappenheim ("Comptes rendus," xxiii. p. 68). [Todd and Bowman, "Physiol. Anatomy," Part II., p. 395, figs. 74, 75, 76; and Bowman, art. "Pacinian Bodies," "Cyc. of Anat. and Phys."]

Fig. 159.-A Pacinian body in Man, magnified 350 diam. : $a$, its peduncle; $b$, nervefibre in it; $c$, external; $d$, internal layer of the sheath; $e$, pale nerve-fibre in the central cavity; $f$, divisions and terminations of the same. 
The spinal nerves, from their point of exit through the dura mater, are enclosed by a firm sheath of connective tissue-the nerve-sheath, or neurilemma-which also sends finer prolongations into the interior of the nerves, and, as in the muscles, forms boundaries to larger or smaller fasciculi, as well as extremely delicate septa between the individual tubules (Fig. 160). In the ultimate ramifications, where isolated primi-

Fig. 160.

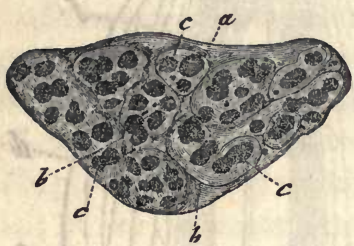
tive fibres, or some few of them, still often retain an external coat, the neurilemma presents the aspect of a homogeneous membrane, with elongated nuclei of 0.003 of a line; and it presents this character also in the smaller twigs of the cutaneous and muscular nerves, only that gradually the substance begins to split, in a longitudinal direction, into fibres, the nuclei become longer $(0.005-0.008$ of a line), frequently almost like those in smooth muscles, and elastic fibres also make their appearance, which are not unfrequently entwined around whole fasciculi. The larger nerves, lastly present common connective tissue, with distinct longitudinal fibrils, as in fibrous membranes, intermixed with numerous reticulated elastic filaments; they still, however, exhibit, especially in the interior, immature forms of connective tissue.

All the larger nerves contain vessels, although not in great number; they run principally in a longitudinal direction, and form a loose plexus of minute capillaries of $0.002-0.004$ of a line, with elongated interstices, which invests the fasciculus, and, in fact, penetrates between its elements; never, however, surrounding individual primitive fibres, but only entire divisions of them. The ganglia contain a delicate capillary plexus, in the form of a network, so that each nerve-cell is surrounded by special vessels. The Pacinian bodies also contain vessels, which even penetrate as far as the central cavity (Todd and Bowman, II. p. 397, Fig. 75, and p. 399, Fig. 76; Herbst, Tab. IV. Figs. 1 and 2).

On the subject of the condition of the cutaneous nerves of animals, I would here add a few remarks. In the skin of the tail of batrachian larvæ (Rana, Bufo, Triton, Bombinator, Alytes), I have described the very delicate ramifications and plexuses of the embryonic pale nervefibres; and moreover, quite evident loops of the fully formed dark nervetubes, and isolated divisions of the latter ("Ann. des S. Nat.," 1846, p. 102, pl. 6, 7). In the full-grown Frog, according to Czermák (Müll. "Archiv," 1849, p. 252), the nerves destined to the skin, form, on its inner aspect, a wide network, already described by Burdach, from which

Fic. 160.-Transverse section of the ischiatic nerve, magnified 15 diam.: $a$, general sheath of the nerve; $b$, neurilemma of the tertiary fasciculi; $c$, secondary nervous fasciculi, in part with special sheaths. From the Calf. 
again numerous fasciculi are given off, penetrate the derma perpendicularly, and, having reached the superficial glandular layer of the skin, form a superficial nervous-plexus between the glands. With respect to the true termination of the nerve-fibres, Czermák arrived at no definite results, but made the interesting discovery that thick and thin nervefibres of the deeper plexus divide dichotomously very frequently and repeatedly, and thus spread themselves over larger surfaces; of which divisions I have most fully satisfied myself from preparations furnished by Czermák. Similar conditions were found by Leydig ("Zeitsch. f. wissen. Zool.," III.) in the skin of Fishes; where also exist superficial and deeper plexuses, with numerous divisions of finer and thicker tubes, all of which on the surface ultimately become quite fine, pale, and finally invisible. In the Invertebrata; as appears from Leydig's researches in Argulus, and especially in Carinaria, conditions are met with perfectly analogous to those described by me in the nerves of the Tadpole; and I cannot agree with Leydig, when he describes the nucleated enlargements as nerve-cells. On the other hand, the conditions observed in Artemia and Corethra are perhaps, peculiar, because in these instances larger branches of the cutaneous nerves are, at their extremities, in connection with numerous roundish vesicles, which might have the function of nerve-cells ("Zeitsch. f. wiss. Zool.," vol. I. iii.).

In the integuments of the Mammalia and of Man, except in the Pacinian bodies, until a short time since, no one had seen anything of divisions in the nerve-tubes; all observers rather agreeing that terminal loops existed there, especially in the papillæ. But it now appears, from the researches of myself, J. N. Czermák, and C. Gegenbaur, that probably loops and divisions, and occasionally even free terminations, all exist in that situation. That in Man, terminal loops occur in the papillæ, and divisions in the terminal plexuses, I have already mentioned; the latter are especially well shown in the conjunctiva scleroticce, where free terminations also appear to exist, and where peculiar convolutions of nerves (nerven-knäuel), similar to those formerly described by Gerber* (vide "Micr. Anat.," II. i. p. 31, Fig. 13 A, 3), present themselves. Czermak, moreover, observed divisions of the cutaneous nerves in the Mouse, and I myself a transition of the dark-bordered nerves into pale anastomosing filaments, of $0.001-0.0005$ of a line, exactly resembling the embryonic fibres in the Tadpole ("Micr. Anat.," II. i. p. 24); lastly, Gegenbaur has noticed numerous divisions in the expansion of the nerves of the tactile hairs in the Mammalia. Further experience will have to show in what relative proportions the loops, divisions, and free terminations, stand with respect to each other, and whether in the different Mammalia, notwithstanding any apparent difference, some correspondence obtains or not.

* ["General Anatomy," translated by G. Gulliver, p. 263, pl. 19, Figs. 99, 100.-Trs.] 
$\S 122$. Cerebral Nerves. - The sensitive and motor nerves arising in the brain, correspond in most particulars so closely with the spinal nerves, that a short description of them will suffice; and with respect to the higher nerves of sense, they will be afterwards described, more fully, in connection with the organs to which they belong.

The motor cerebral nerves, the third, fourth, sixth, seventh, and twelfth pairs, with respect both to their roots and to their course and distribution, present exactly the same conditions as the motor roots and muscular branches of the spinal nerves, with the sole exception, that by all these nerves, from their anastomosing with sensitive nerves, some sensitive fibres are conveyed to the muscles. It deserves remark: 1, that according to Rosenthal and Purkinje, nerve-cells exist in the trunk of the oculo-motorius in the $\mathrm{Ox}$, which, however, Bidder (p. 32) was unable to find; 2 , that the facial nerve, in its gangliform enlargement, presents a number of larger nerve-cells, through which, however, according to Remak, only part of the fibres pass (Müll. "Archiv." 18्41); 3 , that according to Volkmann (in Bidder's "Ganglien-körper," p. 68), the small root of the hypoglossal nerve in the Calf, which is furnished with a ganglion, produces motor effects. What is the significance of this occurrence of nerve-cells in motor nerves has not been ascertained. Probably simple fibres having a peripheral destination arise from them, exactly as in the spinal ganglia. In any case it shows that ganglia are not necessarily placed only on sensitive nerves. The fifth, ninth, and tenth pairs, resemble the spinal nerves, inasmuch as that they all contain motor and sensitive elements. In the trigeminus the small root exhibits a preponderance of thick fibres; the larger, numerous fine fibres. The Gasserian ganglion, as well as the smaller ganglionic body seated upon it, contains many larger and smaller nerve-cells of $0.008-$ 0.030 of a line, with nucleated sheaths, and presents the same conditions, according to my observations, in small Mammalia and in Man, as a spinal ganglion, that is to say, it is simply traversed by the fibres of the greater root, and, from unipolar cells, gives origin to numerous nervefibres of medium size, which go to join the emergent branches. Bipolar cells also occur, but, as it appears, in less quantity, and anything that can be said about apolar cells is as applicable here as in the case of the spinal ganglia. The ultimate distribution of the $n$. trigeminus is for the most part similar to that of the cutaneous nerves, and, in particular, the existence of divisions of the nerve-tubes may be distinctly demonstrated in the mucous membranes, as in the conjunctiva at the edge of the cornea, in the ciliary ligament, in the tooth-pulp, and in the papillæ of the tongue. Terminal loops and free terminations appear to exist in the papillæ of the mucous membrane of the mouth and tongue, and in the conjunctiva, whilst in the cornea, the extremities of the nerves are quite 
transparent and pale, and constitute a wide-meshed plexus without any divisions. With respect to the ganglia, which are placed on the $n$. trigeminus (ganglion ciliare, oticum, sphenopalatinum, linguale, supramaxillare), I find their structure more to resemble that of the sympathetic ganglia, only that they contain a considerable number of larger nerve-cells. The glossopharyngeus, although endowed with motor properties, still, according to Volkmann (Müll. "Arch.," 1840, p. 488), has no fibres which do not pass through one or other of its ganglia. In its roots, which contain numerous fine fibres, there are, according to Bidder (1. c. p. 30), in the Mammalia, not unfrequently, isolated nervecells, often placed free upon it, in which, as in similar cells, on the roots of the n. vagus, the giving off of two middle-sized fibres, it is said, may occasionally be readily perceived. The ganglia of the glossopharyngeus present the same conditions as the spinal ganglia, that is to say, the radical fibres simply traverse them, and, within the ganglion, fibres arise from cells, which are for the most part unipolar; its ultimate ramification in the tympanic cavity and in the tongue, contains small ganglia, and otherwise corresponds with that of the $n$. trigeminus ( $p$. major $)$. In Man, all the roots of the $n$. vagus enter the jugular ganglion, whilst in some of the Mammalia-Dog, Cat, Rabbit, according to Remak (in Froriep's "Not.," 1837, No. 54), in the Dog and Sheep, according to Volkmann (Müller's "Arch.," 1840, p. 491), but not in the Calf, in which nerve-cells occur in the apparently motor root, it has also a primary fasciculus, which has no connection with the ganglion. In the ganglion jugulare and in the intumescentia ganglioformis of the facial nerve, I have not been able to find anything different from the spinal ganglia, only, that the nerve-cells measure occasionally no more than 0.009 of a line, although it is true that there are also a great many as large as 0.03 of a line. The ultimate distribution of the nerve exhibits, as Bidder and Volkmann correctly state, a constant kind of separation of thicker and more slender fibres, so that the branches to the œesophagus, heart, and stomach, are composed almost entirely of fine fibres, whilst in those going to the lungs, and in the laryngeus superior, the fine are to the thick fibres as 2 to 1 ; and in the laryngeus inferior and the rami pharynge $i$, as 1 to $6-10$. All these fine fibres are very far from being derived from the sympathetic, as they occur in preponderating quantity even in the roots of the vagus, and are also numerous in the laryngeus superior. Many of them, moreover, may be nothing more than attenuated or originally finer ganglion-fibres, as they are termed, arising in the ganglia of the vagus itself, and which likewise I should not refer to the sympathetic. With respect to the terminations of the vagus, reference must be made below to the proper places. The $n$. accessorius Willisii, although perhaps also in part sensitive, has no nerve-cells, and in its 
distribution and termination, so far as is known, presents nothing peculiar.

Terminal loops within the trunks of nerves had been already noticed by Gerber, and have lately been described by Valentin in the vagus (pectoral portion) of the Mouse and Shrew-mouse, but without their expressing any opinion with respect to their signification. Still more mysterious are the nervous filaments seen by Remak and Bochdalek, coming out from, and again re-entering the brain.

§ 123. Ganglionic Nerves.-Under this name, perhaps, is most suitably designated the n. sympathicus, as it is termed,-the sympathetic or vegetative nervous system, -as it presupposes no physiological hypothesis, but simply expresses the fact, which, anatomically, is most apparent to the eye. The ganglionic nerves are neither a wholly independent part of the nervous system (Reil, Bichat), nor a mere section of the cerebro-spinal nerves; but on the one hand, from the very numerous fine nerve-fibres originating in their ganglia-ganglion-fibres of the sympathetic,-form an independent system; whilst on the other, they are also connected with the spinal cord and brain, owing to their receiving a smaller number of fibres of the other nerves. Upon comparing the ganglionic nerves with the cerebro-spinal, we find, that the former, as they are constituted from a double source, in a certain respect undoubtedly resemble the latter, which are also formed from ganglionic fibres of the spinal ganglia, and from others proceeding from the cord; but they differ, particularly in this respect, that they possess a much greater number of independent elements, of ganglia and ganglionic fibres, and enter into much more numerous anastomoses with each other. Consequently, although we appear to be justified from an anatomical point of view, in considering the ganglionic nerves by themselves, still they must not be regarded as something altogether peculiar, seeing that, essentially, every nerve exhibits the same principal elements, and some cerebral nerves, vagus, glossopharyngeus, possess even numerous peripheral ganglia; and moreover, because comparative Anatomy shows that they are produced from the spinal nerves, and Physiology the absence of peculiar functions in them.

§124. The principal trunk of the ganglionic nerves (nervus sympathicus). The $n$. sympathicus in man appears as a whitish, or white nerve, the dark-bordered fibres of which usually run parallel with each other, without divisions or anastomoses, some measuring 0.0025-0.006 of a line or even more, and others not more than $0.0012-0.0025$ of a 
line. These finer and coarser fibres are partially intermixed, partly

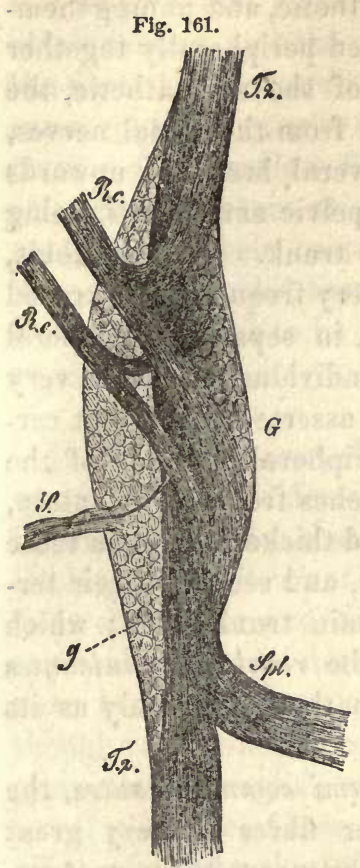

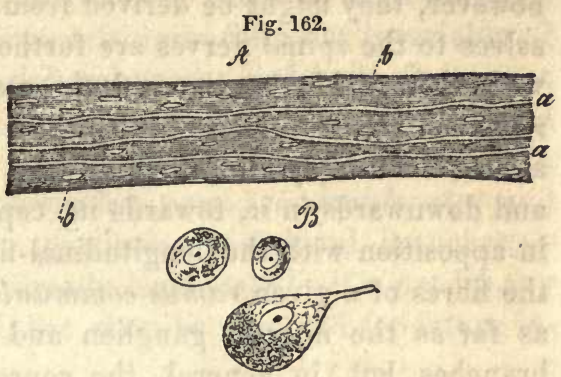

disposed more in a fascicular manner, the latter being the case near the ganglia of the main trunk and in that part itself. The structure of the ganglia is, in the main, similar to that of the spinal ganglia. Each of them consists: 1 , of perforating nerve-fibres, proceeding from one part of the trunk to the other; 2 , of a certain number of finer tubules originating in the ganglion; and 3, of numerous nerve-cells; besides these the rami communicantes also enter the ganglia, and a certain number of peripheral branches are given off from it. The nerve-cells in the sympathetic (Fig. $162 \mathrm{~B}$ ), present, in all essential particulars, precisely the same conditions as those in the spinal ganglia, only that they are, on an average, smaller, measuring $0.006-0.018,0.008-0.01$ of a line in the mean, with less and paler pigment, or even colorless and usually pretty uniformly rounded. As respects the origin of the nerve-fibres of the main trunk, it is, in the first place, evident, that they are in great part derived from the rami communicantes which arise immediately below the spinal ganglia from the trunks of the spinal nerves; that they are in general formed like the sensitive roots of those nerves (that is, contain a preponderance of finer fibres), and, whether simple or compound, that they are manifestly connected with both roots. From all that has hitherto been made out, the fibres of these connecting branches are derived chiefly from the spinal cord and from the spinal ganglia,

FIG. 161.-Sixth thoracic ganglion, on the left side, of the sympathetic nerve of the Rabhit, viewed from behind, treated with soda, and magnified 40 diam.: T. 2 , trunk of the sympathetic; R. c. R. c, rami communicantes, each dividing into two branches; Spl., n. splanclsnicus; $S$, twigs of the ganglion with two stronger fibres and finer filaments, probably going to vessels; $G$, nerve-cells, and ganglion-fibres joining the main trunk.

Fra.162.-From the sympathetic in Man, magnified $350 \mathrm{diam} .: A$, a portion of a gray nerve treated with acetic acid; $a$, fine nerve-tubes; $b$, nuclei of the fibres of Remak. $B$, Three nerve-cells, one with a pale process. 
and are consequently roots of the sympathetic; in a smaller proportion, however, they might be derived from the sympathetic, and joining themselves to the spinal nerves are further distributed peripherally together with them. Having entered the main trunk of the sympathetic, the rami communicantes, so far as they are derived from the spinal nerves, almost invariably run, dividing into two or several branches upwards and downwards in it, towards its cephalic and pelvic extremities, being in apposition with the longitudinal fibres of the trunk. In the Rabbit, the fibres of a given ramus communicans may very frequently be traced as far as the nearest ganglion and beyond it, in separate peripheral branches, but, in general, the course of the individual fasciculi very soon escapes the eye. It may nevertheless be asserted with great certainty, that they all gradually go off in the peripheral branches of the main trunk, for in the first place all these branches frequently contain, in considerable quantity, the same dark-bordered thicker fibres, as those which are contained in the rami communicantes, and secondly their termination or origin is never observed in the main trunk itself; which circumstance is also the principal reason why the rami communicantes can be regarded not as branches of the sympathetic, but only as its roots.

Besides the fine and coarser fibres of the rami communicantes, the main trunk of the sympathetic contains other fibres in very great numbers, which are dark-bordered, but pale, finest nerve-tubes measuring $0.0012-0.002$ of a line, with respect to which I unhesitatingly assert, that they originate in it, and are in no way continuations of the rami communicantes, as has been quite recently supposed, since the discovery of the bipolar ganglion-cells in Fishes. In the Mammalia it is, in fact, extremely easy to prove, by the examination of entire sympathetic ganglia under the careful application of dilute soda and compression, that the great majority of the fibres of the rami communicantes have not the slightest connection with the ganglion-cells, but much rather that they simply pass through the ganglia, and ultimately go off in the peripheral branches. Now, as, besides these fibres in the main trunk, numerous other fibres of the finest kind exist, which can in no way be assigned to the rami communicantes, it is clear, that they must be structures of entirely new formation. This conclusion appears to be the more legitimate, when it is added, that it is not, as I first and many since have shown, by any means so difficult to demonstrate simple origins of fibres in the sympathetic ganglia of the Mammalia and Amphibia, and that, in the ganglia a considerable portion of fine fibres assume the aspect of so-called convoluted fibres, that is to say, of fibres winding about in various directions through the mass of cells. From what I have seen in the Mammalia and man, the sympathetic ganglia correspond so far with those of the spinal nerves, that they contain a 
preponderance of unipolar, rarely of bipolar cells, differing, however, in this respect, that apolar cells certainly exist in them in more considerable quantity, and the ganglion fibres arising in them are invariably of the finest kind, occurring in the peripheral nerves, and probably in most cases, quit the ganglia in various directions. As for a topographical tracing of the various fibres in the main trunk of the sympathetic, with reference to their origin from particular rami communicantes and ganglia, and their continuation into particular peripheral branches, if more be required than what has already been stated-it is not by any means at present to be thought of, but must be reserved for future investigation.

It has been asserted, that the smaller cells in the ganglia of the sympathetic are different from the larger cells in the spinal ganglia, for instance; and also that they are connected only with fine nerve-tubes (Robin), but this is not correct, as is apparent in part from the observations of Wagner and Stannius; for we find: 1, in the ganglia of the cerebral and spinal nerves of the Mammalia and of man, all intermediate sizes between larger and smaller nerve-cells, and also, occasionally, though rarely, larger cells, measuring as much as 0.03 of a line in the sympathetic ganglia; and we may also be convinced, 2 , that the diameter of the nerve-fibres originating in the first-named ganglia, is not at all regulated by that of the cells, all their ganglion-fibres being pretty nearly of the same size, and which is confirmed also by the bipolar cells of Fishes, where the one fibre arising from the cell is often considerably thicker than the other; in Petromyzon, according to Stannius, even six times. Should it be at all supposed that the small cells are peculiar to the sympathetic nerve alone, I must, as above, with respect to the nerve-fibres, remark, that not to mention the ganglia of the roots of the cerebral and spinal nerves, small nerve-cells also occur in situations where there can be no question about the sympathetic, as in the spinal cord and brain, and,-if instances of the same kind in the peripheral nerves be desired-in the retina and cochlea. At all events, this much is certain, that the ganglia of the ganglionic system of nerves constantly present smaller nerve-cells, and that the fibres arising from them are of the fine kind only.

Bidder and Volkmann have shown, in the Frog, that the greater part of the fibres of the rami communicantes are distributed peripherally, with the spinal nerves, and that only a small portion of them, which moreover are derived from the spinal ganglia, should be regarded as roots of the sympathetic. But $I$ think $I$ have noticed in the Rabbit and in Man, that the rami communicantes have chiefly a central destination. Still, in man, fibres also occur very frequently-according to Luschka always, - which must be regarded as branches of the sympa- 
thetic going to the peripheral distribution of the spinal nerves, from which again twigs are given off to nerves of the vertebræ; with respect to which conditions the more detailed observations given in my "Mikr. Anat.," II. p. 525, and particularly those of Luschka ("Nerven des Wirbelcanals," p. 10 et seq.) may be consulted. With regard to the question, whence the fibres are derived which join the main trunk of the sympathetic from the spinal nerves, it is certain that that portion of the rami communicantes, which arises from the motor root, and which, according to Luschka, is always a white filament, takes its origin from the cord (or brain) itself, but as regards the other, proceeding from the sensitive root, it may be formed, in part or wholly, from fibres originating in the ganglion. The latter, however, appears to be improbable, for two reasons: 1 , because in that case, the existence of conscious sensations from parts supplied by the sympathetic would scarcely be conceivable; and 2, because the fibres originating in the spinal ganglia are of medium size, whilst, in the rami communicantes, upon the whole, only a few of that kind occur, and these, moreover, must be referred to the motor root.

We may here offer a few remarks upon the fine fibres of the ganglionic nerves. It has been long known, that the sympathetic contains a larger proportion of finer nerve-fibres than the cerebro-spinal nerves, but it was not till 1842 that Bidder and Volkmann labored to show, that these fibres are not only smaller, but also, in other respects, anatomically different; on which account in contradistinction to the thick fibres of the cerebro-spinal nerves, they termed them sympathetic nervefibres. In opposition to this, Valentin ("Rep.," 1813, p. 103) and I ("Sympath.," p. 10 et seq.) have endeavored to prove, that the fine fibres in the sympathetic do not constitute a special class, and in this I think we were tolerably successful. The principal reasons are as follows : 1. Fine and thick nerve-fibres do not differ intrinsically in any essential respect except in size, and present the most numerous intermediate dimensions. 2. Fine nerve-fibres having exactly the same characters as those of the so-termed sympathetic exist in many other situations, as for instance,-in Man and the Mammalia, -in the posterior roots of the spinal nerves and of the sensitive cerebral nerves, in which situations, as I have already shown, there can be no question whatever as to a derivation of the fibres from the sympathetic, and where we have presented to us, nothing but fine cerebro-spinal fibres; similar fibres are contained by thousands in the spinal cord and brain, as well as in the two higher nerves of sense. 3. All thick nerve-fibres decrease in size in their ultimate ramifications, owing to divisions, or direct diminution, so that ultimately they acquire the diameter and nature of the fine, and finest kinds of fibres. 4. All thick nerve-fibres in the course of their development are, at one time, exactly in the condition of the so- 
termed sympathetic fibres. From these facts it would appear certainly evident, that it is impossible to regard the fine fibres of the sympathetic as altogether of a special nature, and peculiar to it alone, and that it will not do, in the anatomical point of view, to classify the fibres according to their size, very many in fact, in their course, assuming all possible degrees of thickness. Allowing that the great number of very fine pale fibres in the sympathetic is a prominent anatomical fact, as is also indeed the case in the higher nerves of sense and in the gray substance, still, speaking physiologically, I am by no means of opinion that the fineness of the fibres in the sympathetic indicates anything of a special nature in them, and which does not exist elsewhere, but perhaps, that where this condition does exist both in them and in other situations, it is connected with a distinct kind of function.

\section{§ 125. Peripheral distribution of the ganglionic Nerves.-From the} main trunk of the sympathetic arise the branches proceeding to the periphery, which without exception, receive finer and thick fibres from it, but besides these, in part at least, contain other special elements, to which is due their varied aspect. Some of them, for instance, are white, as is the main trunk in most situations, such are the $n$. splanchnici; others grayish white, as the nervi intestinales, the nerves of the unimpregnated uterus (Remak, "Darmnerven System," p. 30); others again gray, and at the same time less firm to the feel, as the $n$. caroticus, internus, the nn. carotici externi s. molles, the nn. cardiaci, the vascular branches in general, the branches connecting the large ganglia and plexuses in the abdomen, those which enter the glands, and the pelvic plexuses. The peculiar condition of the latter nerves depends, in part, upon the paler color of the fine fibres of the sympathetic itself, but in great measure upon the presence of the fibres, named after their discoverer, the fibres of Remak ("gelatinous fibres" of Henle), which were at first regarded as a kind of nerve-tubes, and of which, even now, some cannot be convinced that they are only a sort of connective tissue. They are sometimes more readily isolated, sometimes more united into a compact substance resembling homogeneous connective tissue. In the former case they present the aspect of flat, pale fibres, $0.0015-0.0025$ of a line broad, and 0.0006 of a line thick, of an indistinctly striated, granular, or more homogeneous substance; and which, under the action of dilute organic acids, exhibit precisely the same conditions as connective tissue, and from point to point are furnished with, mostly elongated, or fusiform nuclei, $0.003-0.007$ of a line long, 0.002-0.003 of a line broad. These fibres, again, are found in almost all the gray portions of the ganglionic nerves-I cannot find them in many parts of the pelvic plexuses in Man, where they are replaced by a non-nucleated abundant connective tissue, though they are said by Remak to abound 
in the nerves of the impregnated uterus ("Darmnervensyst.," p. 30) in very great quantity, so that they amount to from three to ten times the number of the dark-bordered true nerve-fibres. They constitute the main part of the proper basis of these trunks, and the dark-bordered tubes extend through them, sometimes more isolated, sometimes assembled in larger or smaller fasciculi; more rarely, and only in the neighborhood of the ganglia themselves, do they appear to form sheaths to individual tubes of the finest kind. Besides these "fibres of Remak," the peripheral ramifications of the sympathetic are, above all, distinguished by a great number of ganglia. These bodies, of a larger or less size, some even microscopic, are placed on the branches or terminations, and, indeed, the microscopic ganglia, so far as is hitherto known, on the nervi carotici, in the pharyngeal plexus, in the heart, at the root of the lungs and in the lungs, on the suprarenal capsules, in the lymphatic glands, in the kidneys of Man occasionally, on the posterior wall of the bladder, in the muscular substance of the neck of the uterus in the Sow, in the plexus cavernosi, and with respect to their distribution, will be further adverted to when we come to speak of the viscera. I will here remark in general, concerning them that with respect to the size and figure of the nerve-cells, and the origination of fine fibres, they present precisely the same conditions as the ganglia of the main trunk. As regards the last point, it may be especially noticed, that in one situation the origin of nerve-fibres from unipolar cells, and the rarity of the double origin of fibres, is particularly well displayed, viz. in the septum of the heart in the Frog (Fig. 163), where R. Wagner has also described

Fig. 163.

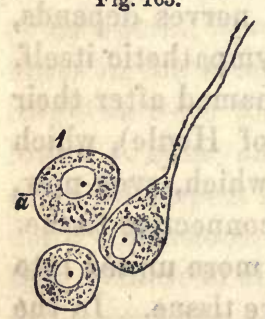

their occurrence. These ganglia, therefore, are also sources of nerve-fibres, and the emergent branches always contain more than the roots, on the supposition that the fibres come out only in one direction, which perhaps in most places may be the case. In the same situation also, it is most readily and satisfactorily seen that many of the cells are apolar and without any processes (Fig. 163); as is also most plainly shown in the cardiac ganglia and small ganglia, on the wall of the urinary bladder in Bombinator, in which ganglia, as well as in the similar ganglia in the Frog, the conditions described are as manifest as possible.

How the fibres arising from these various localities, from the rami communicantes, the ganglia of the main trunk, and the peripheral ganglia, are disposed in their ultimate distribution, is as yet very doubtful. Many peripheral branches anastomose with other nerves, and thus escape all further research, as the $n n$. carotici externi and internus, the latter of which, containing scarcely anything but fine fibres and numerous

Fia. 163.-Nerve-cells from the cardiac ganglia of the Frog, magnified 350 diam.: one with the origin of a nerve-tube. 
"fibres of Remak," I do not look upon in the common sense as a root, but as a branch, arising from the superior cervical ganglion, and probably the other cervical ganglia; as well as the rami communicantes, seeing that individual fibres of them, actually join peripherally the spinal nerves; and the rami cardiaci, pulmonales, \&c. Other branches in the parenchyma of the organs become so fine, that it is impossible to trace them. What has been as yet established respecting their ultimate course, is as follows: 1. Divisions occur in the branches and terminal ramifications of the sympathetic, as in the nerves of the spleen, the Pacinian bodies in the mesentery, in the nerves accompanying the mesenteric vessels in the Frog, in those which exist temporarily in the uterus of the Rodentia, of the lungs and stomach of the Frog and Rabbit, of the dura mater on the meningeal arteries, in branches of the sympathetic of the Sturgeon, in the cardiac nerves of the Amphibia, and in those of the urinary bladder in the Rabbit and Mouse. 2. There are free terminations of the nerves, as in the Pacinian bodies and on the mesenteric vessels in the Frog. 3. The thicker fibres of the sympathetic ultimately so decrease in size as to become of the fine kind; as may be readily seen in the rami intestinales, lineales, and hepatici, which, indeed, even in the interior of the organs in question, contain some coarser nerve-fibres, but ultimately lose them. The actual terminations, however, in the organs themselves, in the heart, lungs, stomach, intestine, kidneys, spleen, liver, uterus, \&c., are as yet quite unknown; although from the impossibility of finding any dark-bordered fibres in the ultimate ramifications of these nerves, it may be supposed that they terminate, almost everywhere, in non-medullated, embryonic fibres. In fact, I have, at all events hitherto, in vain endeavored to find a trace of them. Schaffner says, that in the heart of Bombinator he has seen the passage of the dark-bordered fibres into pale, anastomosing fibrils of the finest kind, whilst Pappenheim (l. c.) describes loops in the nerves of the kidney.

As regards the nature of the "fibres of Remak," most recent observers incline to the opinion first advanced by Valentin ("Repert.," 1838, p. 72 ; Muller's "Archiv," 1839, p. 107), that they are not nervefibres at all, but to be referred to the connective tissue of the nerves; whilst Remak still thinks himself obliged to adhere to his previous opinion, that they are, or may be, in part at least, nerve-fibres ("Darm. nervensyst.," p. 30). As for myself, I freely acknowledge the force of the reasons adduced by the latter observer, which are based chiefly upon the similarity of the fibres in question to the pale embryonic nervefibres, inasmuch as that even in the adult, nucleated nerve-fibres are met with in the olfactory nerve; but I am compelled, nevertheless, as before, fully to concur with Valentin, as do also Bidder and Volkmann, and 
many others. My reasons are the following: 1. The "fibres of Remak," as may be easily shown, arise from the sheath of the nerve-cells of the sympathetic ganglia, and are continued in the nervous trunks, surrounding the nerve-fibres arising from the ganglia. Now as it is certain that these sheaths are a sort of connective tissue, as is apparent also from the spinal ganglia, where they occur in precisely a similar way, only more scantily and without their being continued into the nerves, it follows that the "fibres of Remak" can scarcely be anything else. 2. The finest twigs of the spinal nerves also exhibit nucleated fibres, in all respects like those of Remak, as for instance, those going to the skin, \&c.; with respect to which, as they are wanting in the trunks of the nerves, there can be no question at all of their not being nerve-fibres. 3. The quantity of the "fibres of Remak" always diminishes towards the finest ramifications, which could not be the case were they nerves. It is not, indeed, altogether correct, as stated by Valentin, that they are not to be found in the finer intestinal nerves, for there can be no doubt that they do exist there, though much more rarely than in the trunks of the nerves, and are only to be brought into view by compression. According to Remak (Mull. "Arch.," 1844, p. 464), they also exist in the cardiac nerves of the Mammalia; although as far as I can perceive, only in the immediate neighborhood of the ganglia. Relying upon these reasons, I continue in the firm persuasion that the nucleated fibres in the sympathetic nerve of adult Mammalia are a form of the neurilemma; but I will not omit to remark, that I consider it quite impossible to determine, in undeveloped nerves, what is neurilemma and what young nerve-fibres. Thus in the Rabbit, 2-6 months old, in the n. caroticus internus, not a single developed nerve-fibre is to be met with, and apparently nothing but "fibres of Remak," although it is quite certain that together with them, there must also exist the rudiments of numerous dark-bordered fibres. In the nerves of the spleen, in the Calf, in like manner, numerous nucleated fibres are met with, though in the terminations (vide "Cyclopædia of Anatomy," III. p. 795, figs. 539 and 540), which, probably, afterwards become nerve-fibres. In young animals, consequently, we must not look for a decision of the question; whilst in older ones, it is quite otherwise. In them, a nucleated fibre can only be regarded as a nerve when it can be traced into a dark-bordered fibre, or to a true process of a nerve-cell; and this, as we have seen, is not the case in those of the sympathetic system. It may, however, be remarked, that "fibres of Remak" also occur in the ganglia of the main sympathetic trunk, but that they do not, for the most part, extend to any distance beyond them, so that usually but few are contained in the trunk of the nerve itself.

§ 126. Development of the elements of the Nervous System.-The 
nerve-cells, wherever they may occur, are nothing else than transformations of the so-called embryonic-cells; some of which simply enlarge, whilst others throw out a varying number of processes, and are, at all events in part, connected with nerve-fibres.

Many nerve-cells also appear, at a subsequent period, to increase by division; at all events, I do not know how otherwise to explain the frequent occurrence of two nuclei in the nerve-cells of young animals, especially in the ganglia; and the cells connected by communicating filaments, which have been noticed by various observers.

Fig. 164.

Fig. 165.

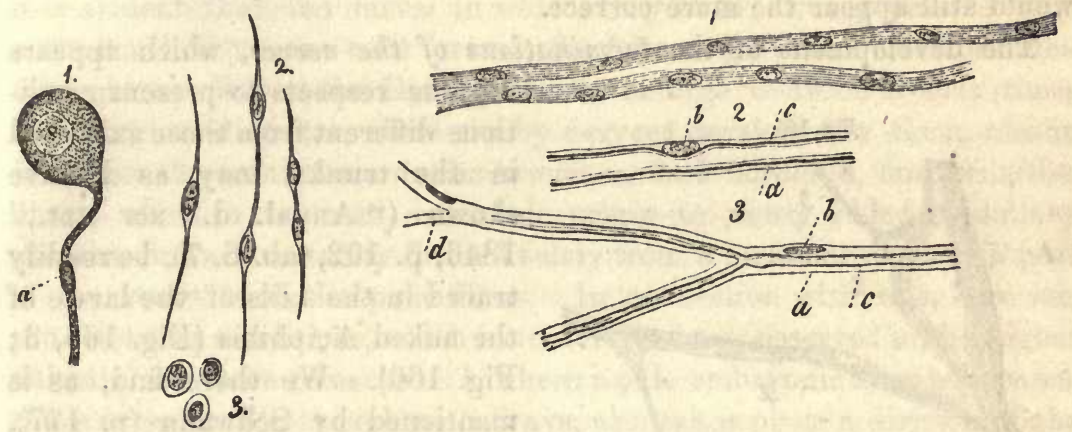

The peripheral nerve-fibres all originate on the spot, but their subsequent development proceeds in such a way that the central extremities always precede the peripheral. With the exception of the extremities of the nerves, they are developed from fusiform nucleated cells, which are nothing else than modifications of the primordial formative cells of the embryo, and are conjoined into pale, flattened, elongated, nucleated tubules or fibres $0.001-0.003$ of a line, broad. Now, at first the nerves consist only of fibres of this kind, and of the rudiment of the neurilemma, being gray or dull white, like the sympathetic filaments; subsequently, in the human embryo at the fourth or fifth month, they always assume a whiter color, and the proper white or medullary substance continues to be more and more developed in them. Of the three possible modes of development of this substance propounded by Schwann, one only, in the present state of things, can come into question, that namely, as to whether the medullary sheath is a structure deposited between the membrane and the contents of the embryonic nucleated fibres; in which case the contents of the latter would become the axisfibre. But besides this, the medullary sheath may originate in what did

FiG. 164.-Nerve-cell from a spinal ganglion of a sixteen weeks' human embryo: $a$, nucleus in the pale process of the cell; 2 , self-developing nerve-tubes from the brain of a two-months' human embryo; 3 , cells from the gray cerebral substance of the same embryo.

Fra. 165.-1, two nerve-fibres from the ischiatic nerve of a sixteen-weeks' embryo; 2 , nerve-tubes from a newly-littered Rabbit; $a$, their sheath; $b$, nucleus; $c$, medullary sheath ; 3 , nerve-fibre from the tail of the Tadpole; $a, b, c$, as before; at $d$, the fibre retains its embryonic character; the dark-bordered fibre shows a division. 
not occur to Schwann, viz., a chemical metamorphosis of the external portion of the contents of the embryonic fibres; and the axis-fibre may be only the remainder of those contents which has not undergone a fatty metamorphosis. It is difficult to determine which of these two views is correct. Direct observation shows only this much, that the contents of the pale embryonic fibres invariably, by degrees, obtain dark contours, and ultimately present the aspect of a true dark-bordered fibre, whilst it teaches nothing with respect to the proper origin of the white substance. Since, however, it can be proved, that the fibres, whilst they undergo this change, do not alter in size, the supposition $I$ have expressed would still appear the more correct.

The development of the terminations of the nerves, which appears

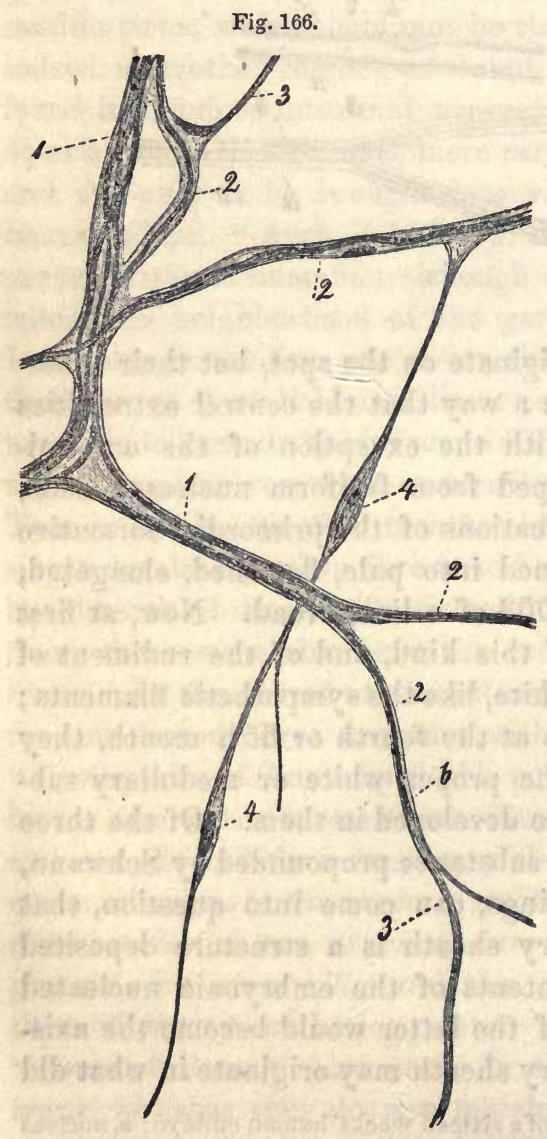
in some respects to present conditions different from those exhibited in the trunks, may, as I have shown ("Annal. d. sc. nat.," 1846 , p. 102 , tab. 6,7 ), be readily traced in the tails of the larvæ of the naked Amphibia (Fig. 165, 3; Fig. 166). We there find, as is mentioned by Schwann (p. 177), the primary rudiments of the nerves to be pale branched fibres, measuring $0.001-0.002$ of a line, which here and there anastomose, all finally terminating in free fibrils of the finest kind, measuring $0.0002-0.0004$ of a line. There is no difficulty in showing that these fibres arise from the coalescence of fusiform or stellate cells, for, in the first place, such cells may be seen, in part, still in close apposition with, but independent of them; in part more or less connected by means of their processes ; and, secondly, cell-nuclei occur at the divisions of the fibres, which are there somewhat dilated; and, at all events, in young larvæ, with them are asso-

Fra. 166.-Nerves from the tail of a Tadpole, magnified 350 diameters: 1, embryonic nerve-fibres, in which more than one dark-bordered tube has become developed; 2 , similar fibres containing but one tube, which in one fibre ceases at $b ; 3$, embryonic pale fibres; 4, fusiform cells connected together, and with a complete nerve-fibre. 
ciated the well-known angular vitelline corpuscles, with which at first, all the cells of the embryo are filled. At first the number of pale embryonic nerves is very small, and limited to a few short trunks closely applied to the muscular structures in the tail; but they are gradually developed, in the direction from the centre towards the periphery, further into the transparent portion of the tail, new cells being continually added in connection with the existing trunks, whilst the latter themselves, almost in the same manner as the capillaries of these larvæ, unite directly by delicate offsets. When these fine ramifications-with respect to the nervous nature of which no doubt can be entertained, as it is evident that the larvæ in which they exist already possess very acute sensibility-are once formed, the following further changes then take place. Whilst the fibres gradually enlarge to twice or four times their orginal diameter, there are by degrees developed in them, and in fact from the trunk towards the branches, dark-bordered, fine primitive fibres, which in no case owe their origin to newly added medullary sheaths, but are certainly formed solely from a metamorphosis of a part of the contents of the pale fibres. In connection with this, however, the following conditions, which have not yet been observed in the higher animals, are to be remarked: 1 , where a pale embryonic fibre bifurcates, there occasionally, though not always, also takes place a division of the dark-bordered tube developed within it; 2 , the dark-bordered tubes scarcely ever completely fill the pale fibres in which they are formed, but a space, frequently of the same diameter as that of the tubes, is most usually left between them and the membranes of the embryonic fibres, in which space occasionally the nuclei of the primordial formative cells may be perceived; 3 , in the trunks and main branches of the embryonic fibres, several (2-4) dark-bordered tubules are undoubtedly developed within one and the same embryonic fibre; a very remarkable condition, which shows that there are even dark-bordered fibres which do not possess a structureless sheath (vid. note to $\$ 110$ ), and resembling what exists in the muscular fasciculus, in which, in like manner, within a single tubule, numerous finer elements are produced. As the tail of the Tadpole is afterwards thrown off, it is to be regretted that its interesting nerves cannot be traced to the same state of completion, as can be done in those of other situations. It is obvious, however, in the oldest Tadpoles, that the nerves are somewhat thicker than they are originally, and that they extend towards the periphery sometimes in loops, sometimes with free ends, but in such a way that the primary pale fibres continue to exist, and proceeding from the dark-bordered fibres, constitute a very fine terminal nervous plexus, with anastomoses and free ends.

I should not have delayed so long on the subject of the nerves in the 
Tadpole, did not similar conditions most probably also obtain in many other terminations of nerves. This is certain as regards those of the electric organ of the Ray, which, even when developed, agree in many respects with those of the more advanced Tadpole, and as Ecker has lately shown ("Zeitsch. für wissensch. Zoologie," 1849, p. 38), are developed in precisely the same manner. The nerves, also, in the skin of the Mouse (vid. note to $\S 121$ ), evidently belong to the same category; and consequently it may hereafter be shown, that wherever peripheral divisions of nerves occur, their development proceeds essentially as it is here described.

With respect to the development of the nerve-fibres in the central organs, we possess but few researches. Of the fibres in the ganglia, I can only observe, that they are developed subsequently to those of the nerves, and probably from smaller fusiform cells, which may be noticed in association with the nerve-cells. On one occasion, in a spinal ganglion of a four months' human embryo, I noticed a cell of this kind in connection with the process of a nerve-cell (Fig. 164). The formation of the fibres in the cord and brain is extremely difficult of investigation, and is best studied with the aid of chromic acid. In the human embryo, I find, as early as the end of the second month, the commencement of the formation of the tubules in question, the white substance being distinctly finely striated, and manifestly containing, in places, very delicate fusiform cells, which are sometimes independent or isolated, sometimes connected, two or three, or several together (Fig. 164). All these cells are at first pale, investing the nucleus, which measures $0.002-0.003$ of a line quite closely, and having processes almost as fine as the fibrils of connective tissue. In the fourth month, when the two kinds of substance are quite distinct, nuclei are still occasionally to be seen in the now wider fibres, but in some they have disappeared, although the fibres are without dark contours; which are not developed before the middle period of fotal life (in the fotal Calf, when more than 12 inches long, according to Valentin), and, indeed, first in the spinal cord.

As regards the subsequent changes in the nerve-fibres, it has already been remarked, that they occasionally increase very considerably in thickness. According to Harting (l. c. p. 75), the fibres of the median nerve which have not yet acquired dark contours, measure in a four months' human embryo, on the average $3 \cdot 4^{\mathrm{mm}}$, in a new-born child $10.4 \mathrm{~mm}$, in the adult $166^{\mathrm{mm}}$. The increased thickness of the nerves themselves appears, according to Harting, from the fourth month onwards, to depend solely upon the enlargement of the already existing elements, the foetus and new-born child already possessing the same number of primitive-fibres as the adult. 
the nervous elements are known. In the nerve-cells of the brain, the deposition of pigmentary matter becomes excessive, particularly in old age; and fatty deposition also takes place (Virchow, "Archiv," I. 1). Valentin thinks that he has observed a regeneration of nerve-cells in the superior cervical ganglion of the Rabbit. Nerve-fibres are readily destroyed, as in consequence of extravasation of blood, tumors, softening, fibroid growths, \&c., in which cases the medullary substance breaks up into larger or smaller, coagulated or fluid masses, of very various configuration, whilst the axis-fibres seem to disappear. In atrophied nerves, the fibres are observed to be thinner, easily broken up, and, instead of the medullary matter, are frequently, in parts, entirely occupied by minute fatty molecules, as was seen on one occasion by Virchow, in a human optic nerve, and by myself in the nerves of a Frog. Nerves that have been cut across, readily unite; portions of peripheral nerves from 8-12 lines even are restored by true nervous tissue (Bidder, 1. c., p. 65 ; Valentin "de funct. nerv.," p. 159, § 323 ; and "Phys.," 2 Aufl. I. 2, 716). Should the union of a divided nerve not take place, the peripheral end undergoes a gradual change in a particular way, with a simultaneous extinction of the nervous activity. The nervefibres generally become yellowish, soft, lacerable, and lose their transversely banded and glistening aspect. They no longer present any trace of a double contour, their medullary substance is wholly coagulated, and their breadth frequently very various (Stannius in Müll. "Arch.," 1847, p. 452). Whether the axis-fibres undergo change, we are, unfortunately, not informed. According to Brown-Sequard, incised wounds, even of the spinal cord, in the Rabbit, united. Hypertrophies of the nerve-substance itself are unknown, although probably such a condition occurs in the neurilemma. Virchow noticed a new formation of fine nerves in pleuritic and peritoneal adhesions, and, according to the same observer, it would appear that gray nerve-substance may be formed on the walls of the cerebral ventricles.

$\S 127$. With respect to the functions of the nervous system, the following remarks, which are immediately pertinent to the anatomical facts, may suffice. As regards the two elementary portions of the nervous system, anatomical investigation shows, that all its divisions, which preside over the higher functions, contain gray substance in greater or less quantity, as in the sympathetic, the ganglia of the spinal and cerebral nerves, and in the spinal cord and brain; whilst the nerves which act only as a conducting apparatus, contain nothing but nerve-fibres. This being admitted to be the attribute of the gray substance, it may further be inquired whether it presents differences in its structure, as it does in its functions. With respect to this I would remark as follows : the largest nerve-cells are met with in situations from which motory effects proceed, 
as in the anterior horns of the spinal cord, amongst the fibres of the anterior roots, in the medulla oblongata, at the points of origin of the motor cerebral nerves, in the cortical substance of the cerebellum, the pons Varolii and crura cerebri; whilst the smallest cells are found in the sensitive regions, as in the posterior horns of the spinal cord, the corpora restiformia, and quadrigemina. There does not, however, appear to be any constant relation between the size of the cells and the existence of sensitive or motor functions, for, in the ganglia of the cerebro-spinal nerves and of the sympathetic, and in the optic thalami, both sorts of fibres arise, in one place, from small, and in another from large cells. It seems, therefore, as in the case of the nerve-fibres, that there are large and small motor cells, as well as sensitive cells of various dimensions, a fact which is confirmed by comparative anatomy, as the large bipolar cells in Fishes are manifestly sensitive. No essential difference can be pointed out between sensitive and motor cells, whether the latter be of uniform or of different size, and in particular the variations existing between such cells are not greater than those between the motor cells in different localities. Even the cells in the cortical substance of the brain, to which physiologists assign the mental manifestations, with our present means of research, exhibit no perceptible peculiarities. The nerve-cells, however, may be divided into those which are in direct connection with nerve-fibres, and those which are not thus connected, but independent. The former, of course, are to be especially regarded as sensitive and motor; with respect to the latter, anatomy to some extent affords no information, inasmuch as, that they present no processes, as in the sympathetic ganglia, and in some situations in the brain; as regards those furnished with processes, particularly the many-rayed cells, which in many situations undoubtedly are not prolonged into nerve-fibres, it might be considered certain that they,-both larger and smaller, by means, of their processes which fulfil the functions of nerves, and whether the latter anastomose or not,-bring different regions of the central organs into mutual connection, and participate in the reflex phenomena, the sympathies, and other modes of association of the functions. Cells of this kind exist in the spinal cord and brain everywhere in very large quantity, but not in the ganglia, although it is not, from this, intended to imply that no reflex actions are performed in those bodies.

Respecting the nerve-fibres, anatomy is not in a condition to point out any difference in them, between the sensitive and motor nerves; a circumstance, however, which, physiologically, can afford no reason to ascribe identical functions to them. As regards the various sizes of the nerve-fibres, the numerous changes in diameter, undergone in their course by all the cerebro-spinal nerves, very obviously indicate that these proportions have no relation to the functions of the fibres in general. Nevertheless, I do not look upon these relations of size as alto- 
gether of little consequence, and in particular does the attenuation of the fibres, where they extend through gray substance (vid. sup., § 112), appear to me to be important, as also their diminution at their origins and terminations. It is, however, difficult to perceive the physiological import of these facts. Were it the case, that in the nerve-fibres the axiscylinder alone was the conducting, and the medullary-sheath, an insulating substance, and could it be proved that the medullary sheaths were wanting in the attenuated portions, the peculiar activity of the nervefibres in these situations (the transverse conduction in the spinal cord, the acuteness of sensibility at the terminations, \&c.) would be satisfactorily explained. It is well known that such a notion has already been entertained by various writers, and its conception has usually proceeded upon the idea that a close alliance or identity exists between electricity and the nervous force, and the medullary sheath abounding in fatty matter, has from this point of view been regarded as an insulator. But (1) it is anything but demonstrated, that the nerves possess no other active force but electricity; and (2) there is nothing to indicate an absence of the medullary sheath, and a free condition of the axis-fibres in many peripheral extremities of the nerves (skin, muscles), and in those portions of the central organs (spinal cord) in which a transverse conduction is evident. The question always remains, whether the medullary sheath, álthough not altogether, yet at all events partially, may not insulate more or less, according to its thickness. Since, however, this membrane is wanting not only in many terminations of nerves, where an insulated conducting faculty might not be required, but also in other situations, as in the Invertebrata and the nerves of Petromyzon generally, as well as in the processes of the nerve-cells which certainly act as nerves, in the central organs of the higher animals, and in the finest nerve-fibres in those situations (brain), the notion that such is its effect in the dark-borderved nerves loses all ground of support. It would seem to me, that the medullary sheath represents nothing more than a protective soft envelope for the tender central fibre. This mode of explanation also, renders it intelligible, why it is, that in dark-bordered nerves, where the medullary sheath is thin or wanting, and the central fibre is in a more free condition, the nerve-fibres are more readily excited and able to communicate their conditions; and as regards the pale nervefibres, in this case they would essentially have the same functions as the others, and the absence of the medullary sheath in them could either be explained on the supposition, that they are less readily excitable, as in the invertebrate animals, and the Cyclostomata, or because they occur in situations where a protective tunic to the nerve-fibres is no longer required, as in the retina, in the nasal mucous membrane, in the gray substance, and in the electric organs, or even where its refractive power upon light would be prejudicial to a certain object, as in the cornea. A 
similar mechanical function appears to me to be performed by the fine granular substance, which in the higher central organs is found in so many situations supporting the most delicate nerve-fibres, cells, and processes.

With respect to the methods to be employed in investigations of the nervous system, the principal have been noticed in the preceding sections. I will, here, once more advert to the importance of preparations made with chromic acid in the investigation of the course of the fibres, and in the examination of the central nerve-cells; and direct attention to the dilute solution of caustic soda for the detecting of nerve-fibres in non-transparent tissues, - wichout which two means very many points would remain in the dark. In this way also the extreme proneness to become changed, of the gray and white substances, and particularly the ready disruption of the processes of the nerve-cells, and the varicosity, coagulation, and destruction of the nerve-fibres, are at once removed or avoided. The brain and spinal cord, as well as the elements of the ganglia, are best studied in the human subject, but the course of the fibres in them, and, above all, the terminations of the nerves, are best investigated in the smaller Mammalia, and only in the second place in Man. In the searching for the minute ganglia in the heart, Ludwig recommends the treatment with phosphoric acid and the solution of iodine in hydriodic acid, the latter so diluted that it has only a tinge of brown. For the development of the nerves, the human and mammalian embryo are quite suitable; but the batrachian larvæ, and if opportunity offer, the electric organs of the embryo Ray, in which the conditions are by far the most clearly displayed, should not be overlooked.

Literature of the Nervous System.-C. G. Ehrenberg, "Beobachtung einer bisher unbekannten Structur des Seelenorgans des Menschen" (Observation of a hitherto unknown structure in the Human Brain), Berlin, 1836; G. Valentin, in Müll. "Archiv," 1839, p. 139, 1840, p. 218, in Valentin's "Repertorium," 1838, p. 77, 1840, p. 79, 1841, p. 96, 1843, p. 96, and: "Hirn- und Nervenlehre" (Treatise on the Brain and Nerves), Leipzic, 1841; J. E. Purkinje, in the "Bericht über die Versammlung deutscher Naturforscher," in Prague, for the year 1837, Prague, 1838, p. 177, and in Müll. "Archiv," 1845, p. 281; R. Remak, in Müll. "Archiv," 1841, p. 506, 1844, p. 461, "Ueb. ein selbständiges Darmnervensystem" (On an independent system of intestinal nerves), Berlin, 1847; J. F. Rosenthal, "De formatione granulosa in nervis aliisque partibus organismi animalis," Vratisl., 1839; A. W. Volkmann, in Müll. "Archiv," 1838, p. 274, and 1840, p. 510; Artic. "Nervenphysiologie," in Wagner's "Handw. der Phys.," II.; F. H. Bidder and A. W. Volkmann, "Die Selbständigkeit des sympathischen Nervensystems durch anatomische Untersuchungen nachgewiesen" (The 
independence of the sympathetic Nervous System, proved by anatomical Researches), Leipzic, 1842; Stilling and Wallach, "Untersuchungen über die Textur des Rückenmarks" (Researches on the Texture of the Spinal Cord), Leipzig, 1842; Stilling, "Ueber die Medulla oblongata," Erlangen, 1843; "Researches on the Structure and Functions of the Brain. I. On the Structure of the pons Varolii," Jena, 1846; A. Kölliker, "Die Selbständigkeit u. Abhängigkeit des sympathischen Nervensystems, durch anatomische Untersuchungen bewiesen" (The independence and dependence of the Sympathetic Nervous System, shown by anatomical researches), Zurich, 1844 ; P. Savi, "Etudes anatomiques sur le système nerveux de la Torpille," Paris, 1843. As an appendix to Matteucci, "Traité des phénoménes électro-physiques des animaux," Paris, 1844 ; R. Wagner, "Ueber d. innern Bau der electrischen Organe im Zitterrochen" (On the intimate Structure of the Electric Organ in the Ray), Göttingen, 1847. With a plate; "Sympathetic Nerve," "Structure of Ganglia," and "Terminations of Nerves," in Wagner's "Handw. d. Physiol.," part III.p. 360 ; "Sympathetic Ganglia of the Heart," ibid., p. 452; "Neurological Researches," in Götting. "Nachricht v. d. Universit., \&c.," Feb. 1851, No. 14; H. Stannius, "Das peripherische Nervensystem der Fische" (Peripheral Nervous System of Fish), Rostock, 1849. Moreover, in the "Archiv dür phys.," Heilk. 1850, and in Gött. "Nachricht, \&c.," 1850, Nos. 6-16, 1851, No. 17; J. N. Czermák, "Ueber die Hautnerven der Frosche" (On the Cutaneous Nerves of the Frog), Müll. "Archiv," 1849, p. 252 ; "Verästelung der Primitivfasern des $N$. acusticus" (Ramifications of the primitive Fibres of the Acoustic Nerve), in "Zeitsch. f. wissen. Zoologie," II. 1850, p. 105. [To these should be added: Robin, "Memoires sur la Structure des Ganglions," in the Journal de l'Institut, 1847, N. 687, and 1848, N. 733, also in the Comptes Rendus, xxiv. p. 1019; Axmann, Beiträge zur Microscopischen Anatomie des Ganglien Nervensystems des Menschen und der Wirbelthiere, Berlin, 1853; Virchow, Uebereine im Gehirn und Rückenmark des Menschen aufgefundene substanz mit der chemischen reaction der cellulose. (On a substance found in the brain and spinal marrow of Man, presenting the chemical reaction of cellulose), in "Archiv" f. Path. Anat. vi. I, 1853.-Ed.] Besides these, should be consulted the general works of Schwann, Henle, Valentin, Todd and Bowman, Bruns, and myself, which also give figures; the Reports of Henle and Reichert; and the memoirs cited under the descriptions of the nerves of the different organs, and in the various sections. 


\section{OF THE DIGESTIVE ORGANS.}

\section{I.-OF THE INTESTINAL CANAL.}

$\S 128$. THE intestinal canal is composed fundamentally of the socalled membranes of the intestine. The innermost of these, the mucous membrane, membrana mucosa, corresponds in its structure with the skin, and like it possesses, (1) a non-vascular investment composed of cellsthe epithelium; (2) the mucous membrane, more strictly speaking, composed of connecting and elastic tissues; containing vessels, nerves, smooth muscular fibres, and different forms of minute glands, and often presenting peculiar processes (papilloe, villi); and (3) an external layer of loose connective tissue, the submucous cellular tissue. The second intestinal tunic, the muscular membrane, tunica muscularis, is provided, for a certain distance at the commencement and at the termination of the intestine, with striated fibres, but in the remainder of its extent the muscles are everywhere of the smooth kind, and form in general two distinct layers; an external, with longitudinal, and an internal, with transverse fibres; more rarely there are three separate layers. The third membrane, the serous, tunica serosa, exists only upon those portions of the intestine which occupy the cavities of the abdomen and pelvis; it is a delicate, transparent membrane, poor in nerves and vessels, and provided with an epithelium; it invests the intestinal canal, and connects it with the walls of the abdominal cavity and with the other viscera.

\section{OF THE ORAL CAVITY.}

A. OF THE MUCOUS MEMBRANE OF THE ORAL CAVITY.

$\S$ 129. The commencement of the intestine may be said to have only one tunic, the mucous membrane, which is applied more or less closely to the bones and muscles bounding the oral cavity; and is distinguished by its not inconsiderable thickness, by its red color, arising from the abundance of its vessels, and by its numerous nerves and papillæ.

The proper mucous membrane, although it is continuous with, and gradually passes into, the cutis upon the lips, is more transparent and softer than the corium; however, it possesses considerable firmness and is still more extensible. Like the thinnest portions of the cutis, it consists of a single layer, $0.1-0.2$ of a line in thickness, and presents a great number of papillæ, like those of the skin, upon its outer surface; they are in general simple but occasionally bifurcated (when hypertrophied they may possess even more processes), are conical or filiform, 
$0.10-0.18$ of a line in length, $0.02-0.04$ of a line in breadth (extremes, $0.024-0.28$ of a line in length, $0.004-0.05$ of a line in breadth), and stand, without any very regular distribution, so close together, that their bases are almost in contact and are rarely more than their own breadth apart.

Besides these papillæ, the mucous membrane presents upon its free surface the orifice of the naso-palatine duct, and a great number of glandular apertures, a few of which are situated at the extremities of large papillary elevations.

The submucous cellular tissue of the mouth varies in its structure. On the floor of the oral cavity, on the anterior surface of the epiglottis, and especially upon the froena of the lips, of the tongue, and of the epiglottis, it is thin and yielding ; and therefore, in these localities, the mucous membrane is very movable upon the subjacent parts. Where glands occur in the submucous tissue, it is more solid, as in the lips and cheeks; at the root of the tongue, and on the soft palate, it may be said to be firmly fixed, and here, especially in the last-named localities, we find large masses of fat in it. The submucous tissue is very dense, firm and of a whitish color, upon the alveolar processes; where, united into one mass with the proper mucous membrane and the periosteum, it forms the gums; upon the hard palate, to which the mucous membrane is attached by an immovable thick fibrous layer, which in some parts contains glands; and finally upon the tongue, where the papillæe are situated. In the latter case, there is a very close union between the mucous membrane and the muscular tissue, the processes of many muscular fibres extending into it and terminating especially in a white, very solid, and dense, tendinous layer, which is in immediate contiguity with the upper longitudinal muscular fibres, and has been described as the fascia linguce (Zaglas).

With respect to the minute structure of the mucous membrane of the mouth, connective tissue is the predominant constituent of the submucous cellular tissue, while throughout the proper mucous membrane, very numerous elastic elements are everywhere found. In both localities, the former usually presents itself in bundles of $0.002-0.005$ of a line in breadth, not united into a network, but while they cross one another in the most various directions, presenting a certain indistinct lamination. The felted mass of connective fibrils becomes densest towards the epithelium, and finally passes into a more structureless layer, which in my opinion is here, as little as in the corium, to be considered a special membrane.* In the interior of the papilloe also, with the exception of those of the tongue, a fibrous structure is usually very indistinct, the whole more resembling a homogeneous substance, slightly granular. The

* [This structureless layer is the "basement membrane" of Todd and Bowman, who describe it as a separate membrane.-DaC.] 
elastic element in the subcutaneous cellular tissue has generally the form of scattered, interstitial, and occasionally, though more rarely, of spirally convoluted, fine fibres; here and there, as in the frenulum epiglottidis, they are not only more abundant, but thicker. The latter is invariably the case in the proper mucous membrane, which, even close to the epithelium, contains in the midst of its connective tissue, very close and intimately connected networks of elastic fibrils, or (and this is the general rule) of moderately thick elastic fibres of $0.001-0.0015$ of a line. Spirally convoluted elastic fibres exist here also, though rarely. In addition, the mucous membrane contains common fat-cells, sometimes in groups, sometimes more isolated, and especially in the submucous layer.

The vessels of the mucous membrane are very numerous, and present essentially the same arrangement as in the skin. The smaller papilloe

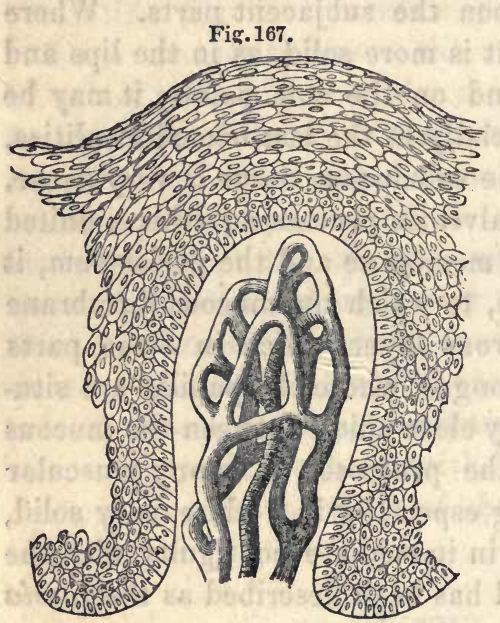
contain only a single capillary loop, whilst in the larger, either simple or branched, a network of capillaries may be observed (Fig. 167); this is especially the case in the gums, the palate, the glandular region of the root of the tongue, the lips, and the lower surface of the tongue. The investigation of the nerves presents many difficulties. If caustic alkalies be added, a wide network of the finer and finest branches is rendered distinct in the outermost layers of the mucous membrane, in which also, divisions of the nervous fibrils may be observed in some localities, particularly upon the anterior surface of the epiglottis; on the other hand, it is often impossible to detect so much as a trace of nerves in the papilloe. Sometimes, however, even in these, especially in the larger, one or two, often twisted, nerve-fibrils of 0.02 of a line in diameter, diminishing to 0.0012 of a line, may be detected, without its being possible to make out their ultimate destination; upon the lip the papilloe possess axile-corpuscles similar to, but smaller than, those of the hand, though not in all individuals. I found here, also, the nerve-coils described by Gerber (see $\S 37$ ). Of the origin and relation, in the t. mucosa itself, of the abundant lymphatic vessels of the oral mucous membrane, nothing is known.

\$130. The epithelium of the cavity of the mouth (Fig. 167), is a so-called pavement epithelium, consisting of many superimposed layers

FrG. 167.-A simple papilla with manifold vessels and epithelium, from the gum of a child; magnified 250 diameters. 
of roundish, polygonal, more or less flattened cells. Taken altogether, this epithelium is a transparent, whitish, pellicle, $0 \cdot 1-0.2$ of a line thick on the average, very flexible, but possessing little elasticity or firmness; it may be detached in considerable flakes by macerating and scraping the mucous membrane, and also by the use of acetic acid. Its elements are, throughout, nucleated cells, whose arrangement and structure, strongly recall those of the epidermis; they are not, as in the latter case, distinguishable into two sharply defined laminæ, but constitute one connected layer, more resembling the mucous layer, but representing the horny layer also. The cells are thus disposed, from within outwards: immediately upon the free surface of the mucous membrane, and upon the papilloe, rest many layers of small vesicles of $0.004-0.005$ of a line (Fig. 167), the deepest of which are, almost without exception, elongated and larger $(0.006-0.009$ of a line), and disposed perpendicularly. To these succeed many layers of roundish, angular, flattened cells, which gradually increase in size, become flatter from within outwards, and assume a more and more distinctly polygonal form (Fig. 168, b).

Fig. 168.

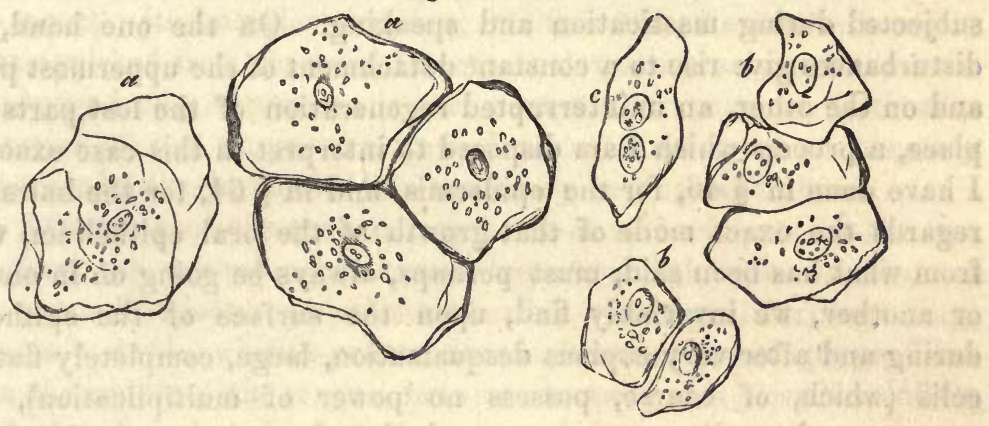

On the outer surface, finally, we meet, gradually proceeding from the deeper cells, with a few layers of the so-called epithelial plates (Fig. $168, a)$, that is, large $(0.02-0.036$ of a line) bodies with rounded corners, in which the flattening has gone so far that they no longer deserve the title of vesicles.

All these cells possess a delicate membrane, easily demonstrable by alkalies and acetic acid; clear contents, present in greater or smaller quantity, according to the amount of flattening, with frequently a few fatty granules, and invariably a nucleus. In the smallest cells the nuclei measure from $0.002-0.003$ of a line, they are elongated or round, and usually without any distinct nucleolus; in the polygonal cells there are invariably one or two beautiful, clearly vesicular, usually spherical nuclei, of $0.004-0.005$ of a line, with clear contents, and 1-2 nucleoli;

Fra. 168.-Epithelial cells in the oral cavity of Man: $a$, large; $b$, middle-sized; $c$, the same with two nuclei; magnified 350 diameters. 
finally, in the plates, the nuclei have begun to retrograde, are smaller, $0.004-0.006$ of a line long, $0.002-0.0015$ of a line broad, generally flattened and more homogeneous, without any distinct cavity or nucleolus, or containing instead several granules. With respect to its chemical relations, the pavement-epithelium of the mouth agrees, so far as we know, in all essential points with the mucous layer of the epidermis, and with the deepest layer of the horny lamina, particularly in the circumstance, that even the plates readily swell up in alkalies; the reader may therefore be referred to $\S 45$.

The most essential physiological characters of the epithelium of the oral cavity are, the continual change to which it is subjected, and its relations as regards absorption and secretion. With respect to the former, it may be said that the epithelium undergoes a continual desquamation, which, however, does not here, any more than in the epidermis, appear to be the effect of special vital energies in the mucous membrane, or in the epithelial cells, but rather to result from the manifold mechanical disturbances to which the surface of the oral mucous membrane is subjected during mastication and speaking. On the one hand, these disturbances give rise to a constant detachment of the uppermost plates ; and on the other, an uninterrupted regeneration of the lost parts takes place, a process which I am disposed to interpret in this case exactly as I have done in $\S 46$, for the epidermis, and in $\S 64$, for the hairs. As regards the exact mode of that growth of the oral epithelium, which, from what has been said, must perhaps, always be going on in one part or another, we invariably find, upon the surface of the epithelium, during and after very copious desquamation, large, completely flattened cells (which, of course, possess no power of multiplication), never younger and smaller structures, and, therefore, the reparation of any loss cannot take place by the formation of new cells at the surface of the epithelium; on the contrary, everything indicates that the renewal occurs in the layers of smallest cells, for although no new development of cells can here be directly observed, yet the analogy with other epidermic structures, and the frequent occurrence of two nuclei in the cells of these layers, nay, even of constricted cells (Bowman, compare § 46), are striking facts in favor of the multiplication of the cells which already exist (by division) and against their actual new formation.

The epithelium of the oral cavity, although thick, is yet readily permeable, differing widely in this respect from the epidermis, which presents similar relations, only in the stratum Malpighii. Fluids of the most different description permeate it from without, and once in contact with the mucous mernbrane, may either be absorbed by its vessels, or perceived by its nerves. Other conditions remaining the same, the activity of the sensitive and absorbent powers will depend upon the 
thinness of the epithelial layer, particularly of the plates, which must always be least permeable, and upon the abundance and superficiality of the vessels and nerves; and these considerations readily explain why the lips, in which the papilloe are very nnmerous, and nearly reach the surface of the epidermis, possess a more delicate sensibility than the gums; and why the point of the tongue, whose papilloe even project with a thinner covering, is still more sensitive (compare, also, on the import of the axile Corpuscles, $\S 39$ ). The epithelium is permeable outwards, as well as inwards, and permits of the passage of plasma from the vessels of the mucous membrane into the cavity of the mouth. In this manner, like the epidermis in relation to the cutaneous perspiration, it participates in the formation of the mucous fluid, which is yielded, not only by the glands which open into the oral cavity, but also by the whole surface of the mucous membrane.

\section{B. OF THE TONGUE.}

$\S 131$. The Tongue is a mass of muscles attached to a particular bone, the os hyoides, and covered by the mucous membrane of the cavity of the mouth; its muscular elements, $0.009-0.023$ of a line in breadth, are distinguished from those of the external transversely striated muscles, only by being interwoven in the most complex manner, so that in the interior of the tongue the lingual muscles cannot be separately demonstrated as such, but only as secondary bundles and fibres.

The framework of the tongue may be said to be formed by the two genio-glossi, the musculus transversus linguo, and the fibro-cartilage of the tongue. The latter, which is also called the lingual cartilage (Fig. $170, e)$, is a dense, whitish-yellow, fibrous lamella, placed perpendicularly in the middle of the tongue, between the two genio-glossi, extending through the whole length of the organ, and is not very appropriately named, inasmuch as it is composed of common tendinous or ligamentous tissue. It commences, low down, upon the body of the hyoid bone, in connection with a broad fibrous lamella, membrana hypoglossa(Blandin), which stretches from the hyoid bone to the root of the tongue, and covers the extremity of the genio-glossus, very soon attains the level of the musculus transversus, and, upon the anterior third of the tongue, gradually diminishes, as far as its point, where it terminates very low down. Superiorly, the septum linguoe, as this fibrous mass, 0.12 of a line thick, might well be termed, ascends to within $1 \frac{1}{2}-2$ lines distance from the dorsum of the tongue; inferiorly, it extends to where the genio-glossi, become lost in the fleshy mass of the tongue, and terminates here, not with a defined border, but by passing into the perimysium, between the two genio-glossi. On each side of this septum, the genio-glossi spread out, fan-like, into the tongue (Fig. 169, $g, 170, g$, $171, f)$, so that they occupy the middle of the organ from its point to 
its root, forming a long, moderately broad, fleshy mass, which, however, is anything but compact. The genio-glossi, in fact, when they have entered the tongue, exchange a few bundles here and there, along the lower edge of the septum, and then break up on each side into a great number of lamellce, which lie one behind the other, separated by small interspaces, in which are the transverse muscular fibres of the tongue ; the lamelloe are, for the most part, perpendicular, but some curve forwards and backwards, towards the dorsum of the tongue.

The fibres of the genio-glossus, thus separated into distinct lamelloe, which have, on the average, a thickness of $0.06-0.14$ of a line, extend as far as the septum, and then gradually take a new arrangement, so as to be directed from behind forwards. For whilst, previously, the genioglossi were broken up into transverse lamello, by the bands of the

Fig. 169.

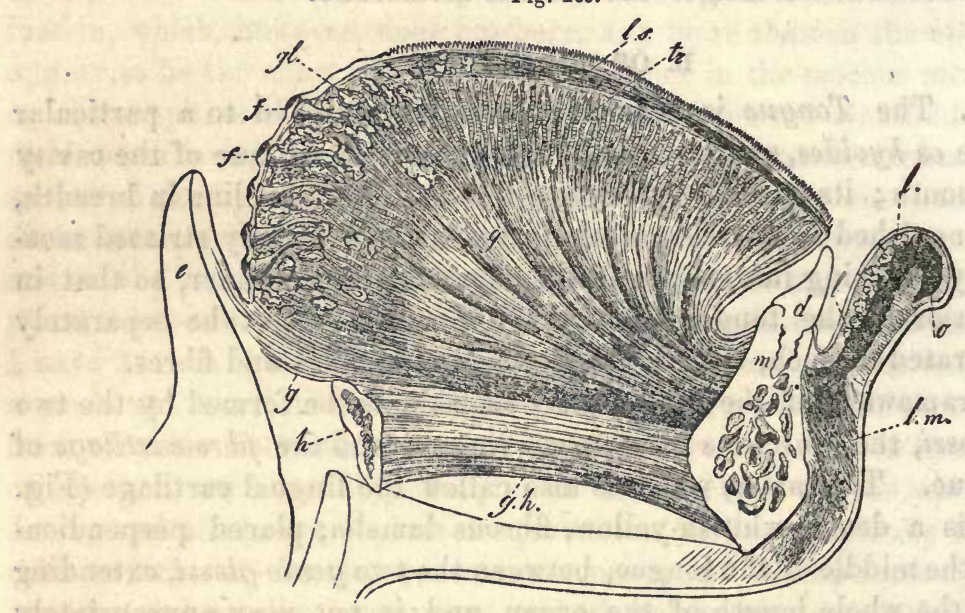

transversus, they are now separated longitudinally by the interposition of the bundles of the superior longitudinal muscle of the tongue, between their fibres. These perpendicular longitudinal lamellae are very distinct in the two anterior thirds of the tongue, less so in the vicinity of the papilloe circumvallatoe, where, especially in the middle of the tongue, the genio-glossus passes, in more isolated bundles to the mucous membrane; in the root of the tongue, finally, they cannot be demonstrated at all. The genio-glossus ends upon the upper surface of the tongue, in such a manner, that its primitive bundles, immediately beneath the mucous membrane, are continuous, in groups, with little tendinous streaks of connective tissue, which then partly become lost in the

- FIG. 169.-Longitudinal section of the human tongue, natural size; the outlines after Arnold Icon. org. (sens.); g.h, genio-hyoideus; h, byoid bone; g, genio-glossus ; g', glosso-epiglotticus; $t r$, transversus lingua ; l.s, longitudinalis superior; e, epiglottis; m, maxilla inferior; $d$, incisor tooth; 0 , orbicularis oris; l.m, levator menti; l. glandula labialis ; folliculi linguales; gl, glandula linguales cum ductibus. 
deeper, very firm layer of the mucous membrane, to be described presently, and partly run as far as the bases of the papilloe. At the root of the tongue, the genio-glossus does not reach so far as the mucous membrane, which may here be easily dissected away, with its mucous sacs, from the more deeply situated racemose glands, but ends upon and between the latter, uniting with them, or with a dense fibrous tissue between them, by means of tendinous strix.

The transverse muscle or the transverse fibres of the tongue (transversus linguce sive fibrce transversales, Fig. 169, tr., 170, tr., 171, g), consists of very numerous lamelloe belonging to each half of the tongue, which penetrate with great regularity between the transverse lamellce of the genio-glossus, and are to be found in all sections of the organ. Each lamella is $0 \cdot 1-0 \cdot 16$ of a line thick, and in the middle of the tongue $\frac{3}{4}$ ths of a line deep; it is usually perpendicular and its muscular fibres extend from the septum linguce to the lateral borFig. 170.

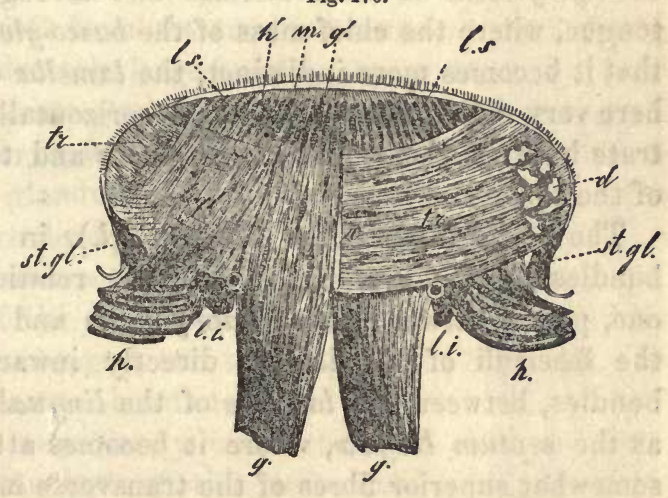
der of the tongue. They arise, so to say, directly from the whole surface of the septum, by the intermediation of a small quantity of a transverse tendinous tissue, distinct from its longitudinal fibres, and pass, united into small flat bundles, at first, directly outwards. In their further course, they curve upwards, and finally, the uppermost shortest fibres reach the sides of the dorsum of the tongue, the inferior longer ones, its proper lateral margin, where they also become attached to the mucous membrane by means of short bands of connective tissue. The other lingual muscles form, in a manner, the sheath of the organ and, in their course, partly follow the above, partly take their own special direction.

The hyo-glossus (baseo- and cerato-glossus of authors), has, at the sides of the tongue, nearly the same relations as the genio-glossus, in the middle. Its coarser bundles, in fact, break up when they have

Frg. 170.-Transverse section of the human tongue, a little in front of the papilla circumvallate: g, genio-glossus; li, longitudinalis inferior (lingualis) with the arteria ranina; tr, transversus, visible in its whole extent on the left side, on the right only at the edge and between the divaricating bundles of the genio-glossus; $; g$, termination of the genio-glossus upon the mucous membrane; $h$, termination of the hyo-glossus; $l . s$, longitudinalis superior, with flat bundles interposed between the perpendicular fibres; $d$, glands of the margin of the tongue; st.gl, stylo-glossus. 
reached the lower surface of the margin of the tongue, into a great number of thin transverse lamello, which, more or less curved, penetrate superiorly between the lamelloe of the transverse muscle and in their further course, present exactly the same relations as those of the genio-glossus, to which they are applied externally, except, that as their fibres ascend towards the dorsum of the tongue, they take a slightly oblique direction inwards. Upon the dorsum of the tongue, the hyoglossus lies between the genio-glossus and the upper edge of the transversus; it presents, like the former, longitudinal plates, with perpendicular fibres, between which the upper longitudinal fibres lie, and it finally, also, terminates in the mucous membrane. The expansion of the hyo-glossus is most distinct and strongest, in the middle of the tongue, where the chief mass of the baseo-glossus lies; it is only behind that it becomes more indistinct, the lamelloe of the cerato-glossus being here very delicate, and lying more horizontally; however, they still penetrate between those of the transversus and terminate upon the dorsum of the tongue.

The stylo-glossus (Fig. 170, st. gl.), in general, divides into two bundles, which have totally different relations; the posterior, smaller one, passes between the cerato-glossus and baseo-glossus, and between the fasciculi of the latter, directly inwards, penetrating, in a few bundles, between the lamelloe of the lingualis and genio-glossus, as far as the septum linguo, where it becomes attached, in common with the somewhat superior fibres of the transverse muscle. The principal mass of the stylo-glossus passes inwards and downwards at the margin of the tongue, unites in front of the hyo-glossus with the lingualis inferior, and terminates in the mucous membrane of the lower surface of the apex of the tongue and of the point itself; the anterior bundles of the two muscles becoming united in an arch.

The lingualis of authors, which I shall call lingualis or longitudinalis inferior (Fig. 170, $l$. $i$ ), is a tolerably strong, longitudinal bundle of muscular fibres placed upon the lower surface of the tongue, between the genio-glossus and hyo-glossus, but whose commencement and termination are not readily discoverable. The posterior portion of the lingualis inferior appears at first to become lost in numerous superimposed flat bundles between the transverse fibres of the genio-glossus (glossopharyngeus), of the stylo-glossus, and of the transversus, at the root of the tongue; more carefully traced, however, it is found that these, like the posterior portions of the genio-glossus, break up into many lamelloe, which ascend, slightly curved, between the transverse fibres, as far as the outer portions of the glandular layer of the root of the tongue; and finally, like the plates of the genio-glossus, which lie internal to them, end in it. Anteriorly the lingualis inferior unites with the larger bundles of the stylo-glossus; ending at the point of the tongue with 
them, and also, applying itself anteriorly to the hyo-glossus, it sends many delicate lamelloe between the transverse muscles as far as the dorsum of the tongue, presenting, in fact, at the border of the anterior third of the tongue, the same relations as the hyo-glossus further backwards.

Finally, there exist in man yet another longitudinalis, or lingualis superior, and isolated perpendicular fibres. The longitudinalis superior (Figs. 169, 170, l.s, 171, e), constitutes a longitudinally fibrous layer placed between the uppermost fibres of the transversus and the mucous membrane, which occupies the whole breadth and length of the tongue and proceeds from the chondro-glossus (overlooked by most anatomists), which arises from the smaller cornu of the hyoid bone as a moderately large bundle, separated from the baseo-and cerato-glossus by the lingual artery and the glosso-pharyngeal nerve. It passes forwards, under the deep glandular layer of the root of the tongue, and in part through the midst of the termination of the genio-glossus and lingualis inferior; occupies, a little in front of the papilloe circumvallatce, almost the entire half of the tongue, and thence passes forwards in the form of narrow bundles, united here and there at acute angles, immediately under the mucous membrane, between the ends of the genio-glossi and hyo-glossi, as far as the point of the tongue, here to become last in the integument on its upper surface.

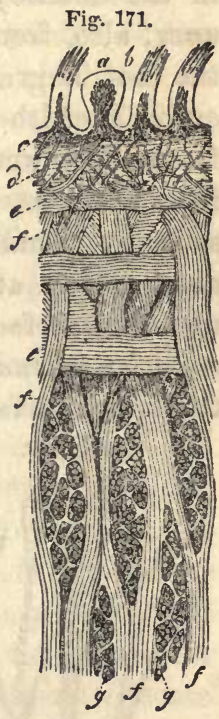
Since these longitudinal fibres become thicker anteriorly, it is probable that independent, superior longitudinal fibres, arising from the mucous membrane of the dorsum of the tongue, and ending upon it, become associated with them. I find perpendicular fibres, which do not arise from without, only in the apex of the tongue, where delicate bundles of them are stretched between the upper and lower layers of mucous membrane.

The lamellæ of the most anterior part of the transversus pass between the inner portions of these bundles, whilst between their extremities the longitudinalis superior and inferior and stylo-glossus penetrate with tolerable regularity, so that transverse sections exhibit an alternation of perpendicular and longitudinal fibres, such as that which appears in the dorsal part of the tongue in Fig. 170.

It remains to be added, that the palato-glossus muscle becomes in part lost, together with the cerato-glossus, in the mucous membrane of

FIG. 171-Portion of a longitudinal section through the side of the human tongue: $a$, papilla fungiformis ; $b$, papilla filiformis; $c$, nucous membrane; $d$, fibrous layer below it ; $e$, longitudinulis superior; $f$, genio-glossus; $g$, transversus, cut across. 
the lateral borders of the tongue, and in part seems to unite with the larger bundles of the stylo-glossus.

If, after thus describing the separate muscles of the tongue, both external and internal, we consider the general structure of the organ, it appears that its proper substance presents essentially only three sets of muscular fibres, which may be denominated perpendicular, transverse, and longitudinal. The perpendicular fibres arise from the genio-glossi in the middle; from the lingualis and hyo-glossus laterally; at the apex, also, from the perpendicularis; and they form from the point to the root, a great number of transverse lamellce, occupying nearly the entire breadth of the halves of the tongue, whose fibres pass in general from the lower surface to the upper. The transverse fibres, derived from the transversus and in part from the stylo-glossus, are inserted in so many, usually somewhat thicker, lamelloe, between the above named, commencing at the septum and ending at the lateral edges and partly upon the surface; the longitudinal fibres, lastly, belong to the lingualis superior (chondro-glossus), the lingualis inferior and stylo-glossus, cover the upper surface, the margin, and in part the lower surface, and lie for

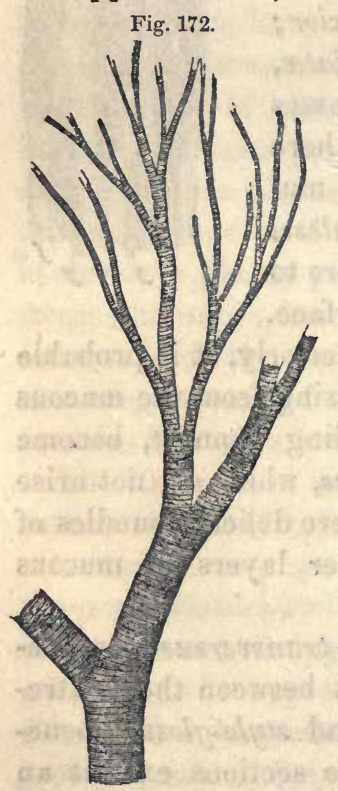
the most part immediately beneath the mucous membrane. The various layers of muscles of the tongue are invariably separated from one another by a thin perimysium, and where larger vessels and nerves run, by thicker masses of connective tissue; besides which, there are in many localities, larger or smaller aggregations of common fatcells, which especially abound between the genioglossi at the septum, at the root of the tongue, and under the mucous membrane.

In the tongue of the Frog very beautiful instances of division of the transversely striated fibres occur (Fig. 172), of which I have not been able to find any certain trace in man. Occasionally, however, it has seemed to me that the fibres of the genio-glossus exhibited divisions shortly before their passage into tendinous bands.

$\S 132$. On the dorsum of the tongue, from the foramen ccecum as far as its point, the mucous membrane differs from that of the rest of the oral cavity, in being very closely united with the subjacent muscular tissue, and in possessing a great number of processes, the well-known lingual or gustatory papilloe. The 6-12 papilloe circumvallatoe consist,

Fra. 172.-A branched primitive muscular bundle, of 0.018 of a line, from the tongue of the Frog; magnified 350 diameters. 
when they are well developed, of a central round papilla, flattened at the end, having a diameter of $\frac{1}{2}-1$ line, and $\frac{1}{4}-\frac{1}{2}$ or even $\frac{3}{4}$ of a line high; and of a lower uniform wall $\frac{1}{5}-\frac{1}{3}$ of a line broad, which closely surrounds the papilla, particularly at its base. These papillo, however, vary much in number, size, and position, and occasionally pass into the fungiform kind; which is especially true of the posterior ones lying in the foramen coecum, or Morgagnii. The papillæ anterior to the circumvallatoe, are arranged in more or less regular rows, which in general run parallel to the latter, and pass, upon the border of the tongue, into laminated, sometimes not even notched folds, which can no longer be considered as papilloe. The papilloe fungiformes or clavatoe are $0.3-0.8$ of a line in length, $0.2-0.5$ of a line in breadth; they have smooth surfaces and, during life, are readily recognised by their red color; they abound particularly upon the anterior half of the tongue, scattered over its surface at tolerably regular intervals of $\frac{1}{4}-1$ line and more; and at the point, indeed, they are often so thickly crowded as to be in contact; they are not absent, however, upon the posterior half, as far back as the papilloe circumvallatce. The papilloe filiformes or conicoe are $\frac{1}{3}-1 \frac{1}{2}$ lines in length, and $0 \cdot 1-0 \cdot 2$ of a line in breadth, and rendered very obvious by their number and whitish color; they occupy, in close contact with one another, the intervals between the fungiform kind, and invariably appear most densely crowded and best developed, with brushlike ends, in the concave side of the $\mathrm{V}$ of the circumvallate papilloe, and in the middle line of the centre of the tongue. Towards the edges and the point, the papilloe themselves, as well as their processes, become shorter, and to some extent more scanty, so that they gradually pass into the lamince to which we have referred, and also in many respects approximate the fungiform papilloe; from which, in fact, so far as the structure of their surface is concerned, they become hardly distinguishable.

Besides these papilloe which project freely, there may also be observed over the whole gustatory region of the tongue, smaller ones completely buried in the epithelium, which are perfectly similar to those of the nongustatory parts of the organ.

With respect to the minuter structure of the mucous membrane of the tongue, that part of it which presents no projecting papilloe, differs in no respect from the mucous membrane of the oral cavity, and possesses, in fact, a laminated pavement epithelium of 0.045 of a line in thickness at the root of the tongue, of $0.06-0.1$ of a line on the lower surface of its apex, with simple small imbedded papillæe of $0.024-0.05$ of a line in length, $0.004-0.02$ of a line in breadth, which are not absent even upon the anterior surface of the epiglottis, and between it and the papilloe circumvallatoe. In the proper gustatory region of the tongue the submucous tissue is wholly absent, the mucous membrane being united with the muscular 
substance by means of a dense layer of connective tissue (see abore $\S 131$ ); it has itself a dense and solid appearance, though in consequence of the presence of a considerable quantity of elastic tissue, of common fat cells of $0.016-0.024$ of a line, and of its abundant vascular supply, it is tolerably elastic.

The papillce filiformes or conicce (Fig. 173), are conical processes of mucous membrane beset either at their extremities only, or over their whole surface, with a certain number $(5-20)$ of smaller secondary papilla of $0 \cdot 1-0 \cdot 14$ of a line in length. The whole is invested with a

Fig. 173.

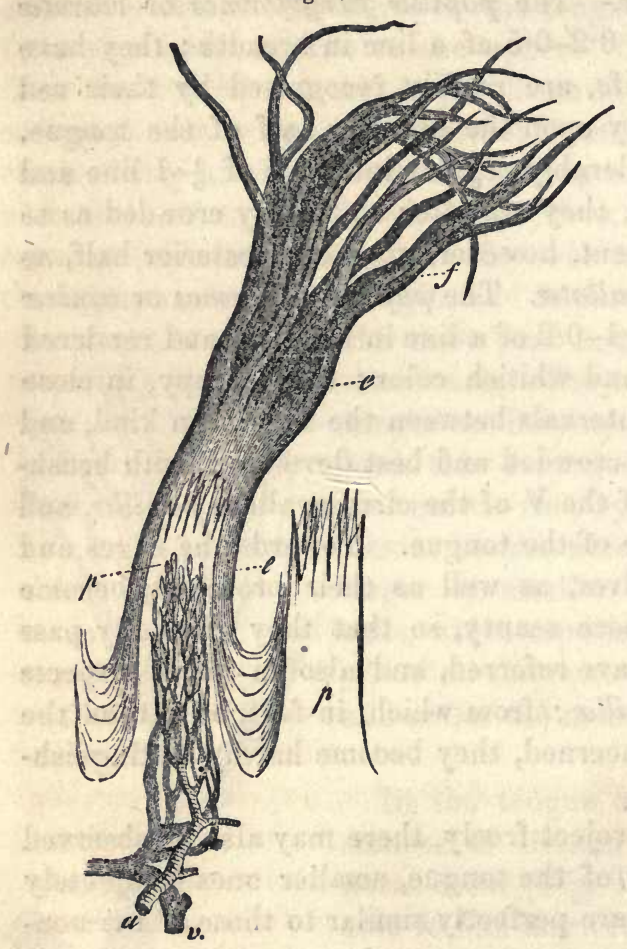

Fig. 174.
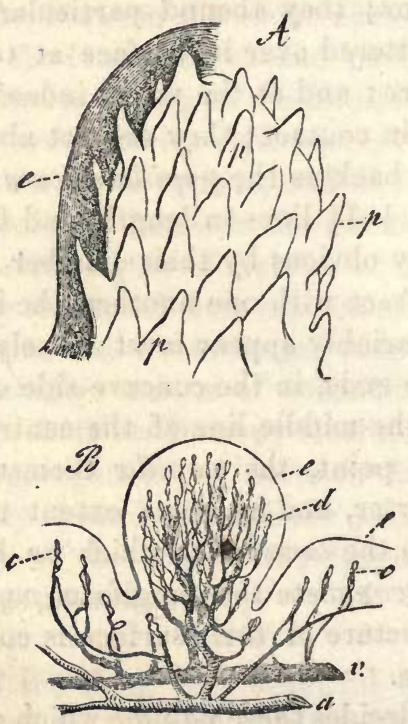

thick epithelial coat drawn out at its extremity into a number of long, thin $(0.01-0.02$ of a line), fine and often subdivided, processes (Fig. 173, $f$ ),

Fig. 173.-Two papilla filiformes of man, one with its epithelium magnified 35 diameters. After Todd and Bowman: $p$, the papilla themselves; $a, v$, arterial and venous vessels of papilla, together with the capillary loops, which, however, ought to enter the secondary papillo.

FIG. 174.-A, papilla fungiformis, with its secondary or simple papillæ, $p$, on one side still covered with epithelium, $e$; magnified 35 diameters. $B$, the same, with only the outlines of the epithelium, $e$, and the vessels; $a$, artery; $v$, vein; $d$, capillary loops of the simple papillæ; $e$, capillaries in the simple papillæ of the mucous membrane at the base of the $p$. fungiformis; magnified 18 diameters. After Todd and Bowman. 
which give the papilla the aspect of a fine brush, and may attain a length of as much as $0.5-0.6-0.7$ of a line, with a breadth of $0.02-0.028$ of a line at their base. The superficial layers of this epithelium resemble the epidermic plates in their long resistance to the action of acids and alkalies, and consist, especially the epithelial processes, only of solid horny scales of 0.022 to 0.028 of a line, which frequently form a more solid axis, and of an external cortex composed of overlapping plates, so that the whole mass may, with some justice, be compared to a hair.

The primary papilla of the $p$. filiformes contains distinct connective tissue, and a very considerable number of elastic fibrils, which as 10-20 wavy threads of $0.0004-0.0008$ of a line, penetrate even to the points of the simple papillæ, and give to the whole cone and its processes a certain solidity and firmness which are not possessed by the simple papillæ of the mucous membrane. A minute artery ramifies in each filiform papilla, in such a manner, that every simple papilla contains a capillary loop of $0.004-0.005$ of a line, from whose reunion a small vein arises. It is difficult to discover the nerves, on account of the abundant elastic tissue; and in many papilla they may be sought in vain. In the majority, however, at least at the base of the papillæ, they are quite distinct, in the form of one or two delicate trunks, with 5-10, dark-edged primitive fibrils of $0.002-0.003$ of a line, which gradually become finer as they run towards the point. I have been unable to make out with certainty how the nerves terminate, yet everything appeared to indicate the existence of loops, not, however, in the simple papillæ, but at their base. In animals these loops are more distinct, as for example, in the calf, where every filiform papilla receives 10-12 primitive fibrils, of $0.002-0.003$ of a line, which diminish to 0.001 of a line, and do not enter the simple papillæ.

The papilloe fungiformes consist of a clavate primary papilla, whose entire surface is beset with closely placed, conical, secondary papillæ, $0.1-0.12$ of a line in length, and invested with a simple epithelium, such as is met with elsewhere in the oral cavity, without filiform processes, or any very horny cells, and which, measured from their points, has a thickness of $0.04-0.05$ of a line. The primary papilla contains far less elastic tissue than the papillo filiformes, and it is almost wholly wanting in the secondary papillæ; on the other hand, a network of bundles of connective tissue of $0.002-0.003$ of a line in breadth, is very distinct. The vessels present the same arrangement as in the $p$. filiformes, only that they are much more numerous; and as regards the nerves, one or two larger trunks of $0.04-0.08$ of a line, enter into. every fungiform papilla, together with many minute filaments, which, spreading out in the form of a brush, and repeatedly anastomosing (see Zeitschrift für Wiss. Zool., B. IV. Tab. IV.), finally diverge in all direc- 
tions towards the secondary papilla, and their axile corpuscles (see $\S 37)$.

The nerves, which in the trunks measured $0.002-0.004$, on the average 0.003 of a line, diminish in size during their course, so that at the base of the papillæ, their diameter is not more than $0.001-0.0015$ of a line, and they also exhibit distinct divisions; I have not yet observed their terminations with certainty, but have thought that in some cases I could detect loops, in others, free ends, without, however, pledging myself either to the one or the other.

In the papillce circumvallatce the central papilla, which may be regarded as a depressed papilla fungiformis, is closely covered upon its

Fig. 175.

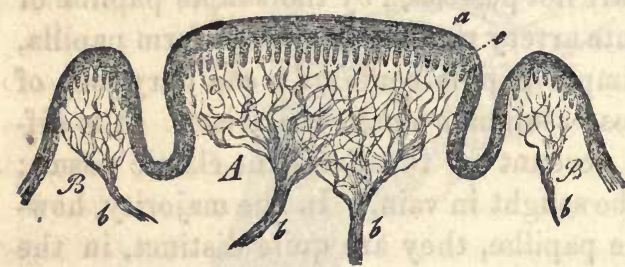

plane terminal surface with simple conical elevations, and is invested externally by an epithelium of uniform thickness, without any special processes and prolongations. The wall is a simple elevation of the mucous membrane, possessing a smooth epithelial investment, beneath which its upper border is produced into many rows of simple conical secondary papillæ. The elastic tissue is usually absent in the papillæ, otherwise, they have the same structure as the fungiform kind, only they are still more abundantly provided with nerves. Every proper papilla circumvallata contains in its lowest portions several nervous trunks of $0.05-0.08$ of a line in diameter, which as they ascend, subdivide into a very elegant plexus, from which the nerves of the secondary papillæ radiate upon all sides. In other respects, they resemble the $p$. fungiformes, except that the nerve-tubules, even in the trunks, have not a greater average diameter than 0.002 , and the largest not more than 0.003 of a line, while at the base of the secondary papillæ it is not more than $0.001-0.0015$ of a line. The walls of these papillæ also contain many nerves, whose ultimate disposition appears to be exactly the same as in the papillæe themselves.

The lingual papillæ present many varieties, the following of which are the most important: 1. The papilloe filiformes are all elongated, and provided with very considerable epithelial processes. The appearance of what is commonly called a gastric furred tongue, depends principally upon the growth of the epithelial processes of the papilloe filiformes, which, all directed backwards and in close apposition, form apparently a peculiar white coating. If the processes become longer, so that the

Fra. 175.-Papilla rircumvallata of Man in section: $A$, proper papilla; $B$, wall; $a$, epithelium; $c$. secondary papilla: $b b$, nerves of the papilla and of the wall.-Magnified about 10 diameters. 
papillæ filiformes measure $1 \frac{1}{2}-2$ lines, we have the lingua hirsuta or villosa, which is not uncommon in various disorders; and at length forms may be produced, in which the tongue looks as if it were covered with hairs, 4-6 lines long. 2. The papillo filiformes possess very small epithelial processes, or none at all, and are hardly distinguishable from the smaller $p$. fungiformes. 3. The papilloe filiformes do not exist as special elevations, but are imbedded in a general epithelial investment of the dorsum of the tongue. Tongues may be observed, particularly in old people, which, without being furred, in some spots or over a large extent, present no papillæ at all, but have either a perfectly smooth surface, or exhibit only a few linear elevations, corresponding with the rows of papillæ which would, otherwise, exist there. In these places we find the epithelium more developed, and beneath it small papillæ, more of the ordinary form. Tongues which, with better developed papillæ present a smooth surface, are again different from these; here the smooth or cracked surface is produced by the papillæ being glued together by superabundant epithelium, mucus, blood, pus-corpuscles, and mucedinous or yeast-like fungi. 4. The epithelial processes of the filiform papilloe are covered with mucedinous fungi. Every microscopist is doubtless acquainted with brownish, elongated bodies $(0 \cdot 12-0.24$ of a line in length, $0.04-0.08$ of a line in breadth), consisting of a dark axis

Fig. 176.

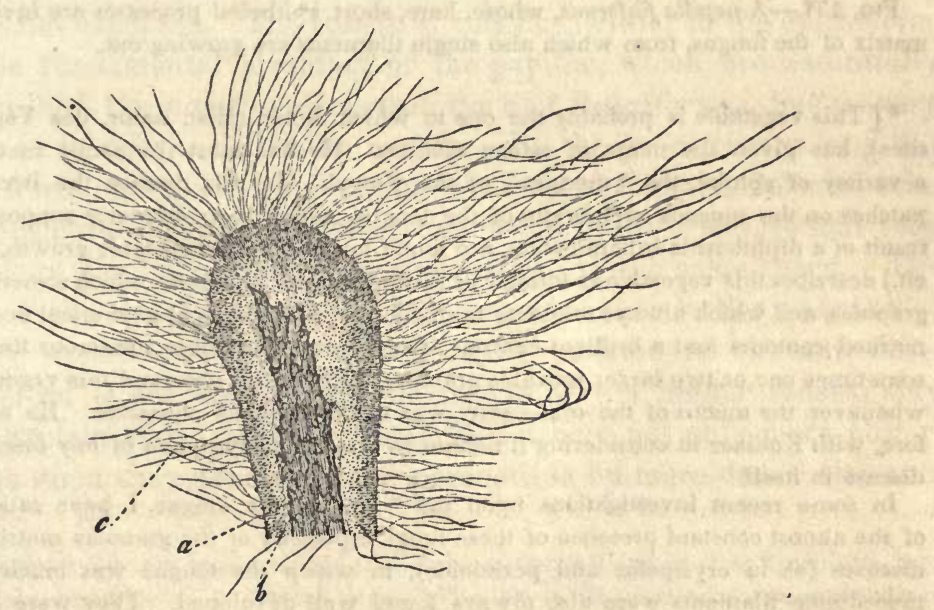

and a finely granular cortex, from the coating of the tongue. The centre of these bodies only is composed of cornified epithelial cells,

Fra. 176.-A mass of epithelial cells covered with the granular matrix of the fungus, $b$, from which a luxuriant growth of mucedinous filaments, $c$, proceeds; magnified 350 diameters. From Man. 
which become isolated and swell up by the action of caustic potassa and

Fig. 177.

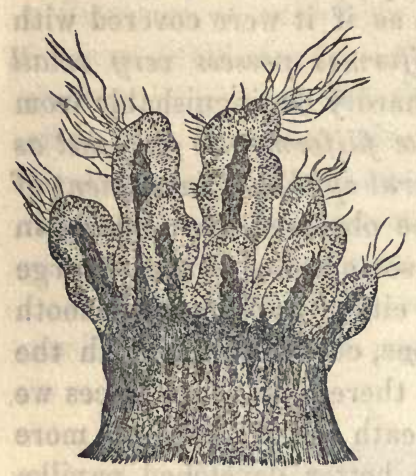
soda, especially with heat, and are derived from the epithelial processes of the papilloe filiformes; the granular cortex again, is nothing but the matrix of a mucedinous fungus of only 0.0006 of a line in diameter, which, agreeing completely with the wellknown filaments upon the teeth, is often rooted in immense quantities in it. In the dead subject we readily recognise the epithelial cells, covered with fungi, either with or without projecting mucedinous filaments, even in situ (Fig. 177); and in living persons, they may be procured in any quantity by scraping the tongue. In twenty or thirty healthy young people, I have hardly once failed to find the granular covering upon the epithelial processes, even in a perfectly clean, red tongue.

The more fur there is, the more abundant is the matrix, and the mucedinous filaments are also apparent, though they are rarely (three or four times in thirty cases) found so clear and distinct as in Fig. 176, and in general are not met with in more than a third of those persons whose papilloe filiformes are not altogether in a normal condition.*

Fra. 177.-A papilla filiformis, whose, here, short epithelial processes are invested by the matrix of the fungus, from which also single filaments are growing out.

* [This vegetable is probably the one to which Robin (Hist. natur. des Végétaux Parasites), has given the name of oidium albicans. He has most frequently met with it in a variety of aphtha, the "muquet" of the French. In this disease the irregular white patches on the mucous membrane of the tongue, which were formerly supposed to be the result of a diphtheritic inflammation, are found to consist of a vegetable growth. Robin (loc. cit.) describes this vegetable as formed by distinct tubular filaments, which sometimes enclose granules, and which always originate from spores. The spores are spherical and have wellmarked contours and a brilliant centre. They generally enclose numerous fine molecules, sometimes one or two larger movable granules. Robin has observed this vegetable growth whenever the mucus of the oral cavity was changed in its character. He agrees, therefore, with Kolliker in considering it neither as a constant symptom of any disease, nor as a disease in itself.

In some recent investigations upon the "fur" of the tongue, I have satisfied myself of the almost constant presence of these fungi, especially of the granular matrix. In acute diseases (as in erysipelas and peritonitis), in which the tongue was much coated, the mucedinous filaments were also always found well developed. They were firmly adherent to isolated epithelial cells, by which they were sometimes concealed, but were easily rendered apparent by the aid of a solution of carbonate of soda. In one case of typhoid fever I discovered large distinct spores. Prof. Clark, of New York, has observed these vegetable growths in cases of extreme debility, especially in infants exhausted by diarrhœa and dysentery.

The main constituents, however, of the fur of the tongue are layers of epithelial cells in different stages of development. If the tongue be much coated, the cells on the 
The physiological results of the anatomical data which have been communicated, may be thus summed up: the papilloe filiformes are neither gustatory nor delicate tactile organs, since their thick, and what is more to the point, greatly cornified epithelium, is very little fitted to allow of the passage of fluids capable of being tasted, or of other influences, to the scattered nerves, which only attain to the base of the simple papillæ. With Todd and Bowman, I consider that the $p$. filiformes have a similar office to the lingual spines of animals, which are nothing but modified filiform papilla, and I therefore ascribe to them a certain importance for the conveyance and retention of the morsels of food, and I consider that their epithelium serves, at the same time, as a protecting investment for the tongue. The two other kinds of papilla subserve the sense of taste and are, besides, the seat of ordinary sensation (for mechanical irritation, temperatures, \&c.), for which functions they are excellently fitted by their thin, soft epithelium, the softness of the tissue of their papillæe, and by the superficial position (in the secondary papillæ), and the great number of their nerves. The sensibility of the tongue is most delicate where the papilloe fungiformes are most closely set, $i$. e., at the apex, which, on that account, and also perhaps by reason of the solid axile corpuscles in many of the papillæe, is especially fitted for a tactile organ; at the root of the tongue it is more obtuse, and is accompanied by peculiar sensations; the sense of taste is much more acute at the root of the tongue than in the other regions, the point not excepted. The reason of this lies neither in the epithelium, nor in the fundamental structure of the papillæ, which are essentially similar in both the papilloe circumvallatoe and fungiformes, but is, very possibly, to be sought for in the nerves. In the $p$. circumvallatoe, the nervous fibres are always finer, and not only absolutely, but also relatively, considerably more numerous than in the fungiformes, so that they possess more papillæ and nervous terminations in the same space. The fineness of the nerves especially, together with the smaller quantity of medullary sheath and the more superficial position of the axis-fibre, which indeed is the case in all the nerves of the higher senses, may perhaps explain why tastable substances act here not only more powerfully, but when they are no longer perceptible by more dense elements

surface are generally very granular, and from being contracted and in close apposition, they bear a certain resemblance to fibrous tissue. The use of reagents, however, especially of alkalies, will always render their cellular nature apparent.

The dark color of the fur, sometimes seen, may be owing to large accumulations of the fungi between the cells, or else to the presence of pigment granules. The latter, if abundant, may cause the tongue to appear as if covered with a thin layer of ink. A case is reported by Dr. Eulenberg (Archiv f. phis. Heilk. 1853), in which the whole tongue was covered by a perfectly black fur, which, when examined microscopically, consisted of distinct, angular pigment corpuscles lying between the epithelial cells. - $\mathrm{DaC}$ ]. 


\section{of the nerves. If this peculiarity be insufficient to account for the} differences in the sense of taste possessed by the two kinds of papillæ, they can only be referred to the central organs, or ascribed to specific actions in the nervous fibres themselves, which, however, is only making a public confession of the hiatus in our knowledge.

\section{Remak* discovered microscopic ganglia upon the expansion of the}

* [Remak, "Ueber die Ganglien der Zunge bei Sängethieren und beim Menschen." The author finds ganglia upon the branches of the glosso-pharyngeal and of the gustatory nerves, and not upon those of the ninth nerve; small ganglia were sometimes observed near branches of the latter, but were never actually connected with them, and probably belonged to neighboring branches of the gustatory. Remak compares these ganglia with those observed by himself in the heart, contractile wall of the bronchiæ, posterior wall of the urinary bladder, and muscular wall of the uterus, and with the ganglia of the cavernous plexus described by Muller; and his reasons against their connection with the nerves of sense appear to us sufficiently important to be given in his own words.

"The terminal branches of both nerves (glosso-pharyngeal and gustatory nerves) form a very dense plexus before entering the papillæ. Neither in this plexus, nor within the papillæ themselves, could ganglion-globules ever be detected. It must be remembered, further, that the ganglia upon the thicker branches of the glosso-pharyngeal and gustatory are always hemiganglia, that is, they do not occupy the whole thickness of the nerve-a bundle of tubules, which takes no share in the formation of the ganglia, passing over them. Those ganglia which lie in the neighborhood of the papillæ have the same structure. Far more numerous are the hologanglia, i. $\epsilon$. those in which all the nervous fibres become lost between the ganglion globules (probably pass into them), but these are found only in the finest lateral branchlets. They are almost always multipolar, i. e. they are connected with more than two nervous trunks, and these are very widely different from the nerves which constitute the papillary plexus. While the latter present very delicate sheaths, and consist of evident dark-edged fibres, the processes of the hologanglia are closely surrounded and enveloped by very dense sheaths, and contain, particularly in the lingual branch of the fifth, both in man and in the sheep and calf, a very large quantity of the well-known nucleated fibres, so that it is at times difficult to find a single dark edged fibre in one of these nerves. In other cases, the processes of the ganglia are delicate nerves (of $\frac{1}{2} \frac{1}{5}$ of a line), which possess a solid sheath, and a single dark-edged nervous fibre enclosed by it. The fine lateral branches of the hemiganglia upon the thicker branches of the nerves present the same appearances. No fibres can ever be traced from a ganglion to the papillæ. Another circumstance which speaks against the relation of the ganglia to those fibres of the gustatory nerve which are distributed to the mucous membrane is, that I could never, in spite of every exertion, find ganglia upon the terminal branches of the gustatory nerve of the apex of the tongue of the sheep. I believe that a certain value may be attributed to this negative result, as I never failed to find ganglia upon the other branches of the gustatory nerve, up to within about an inch of the apex of the tongue."

Remak gives, further, the following reasons for believing that the ganglia are related to the mucous glands. "1. The lingual ganglia always occur in the neighborhood of the mucous glands, or of their excretory ducts. 2. That the smaller number of mucous glands in the anterior region of the tongue (in the sheep or calf), corresponds with the smaller number of ganglia upon the branches of the gustatory nerve. 3. That little ganglia exist upon the branches of the gustatory nerve distributed to the maxillary glands, and to the ductus Whartonianus, whilst in man there is the well-known maxillary ganglion. 4. That in the point of the tongue of the sheep, in which he could find no ganglia on the branches of the fifth, there are no mucous glands. 5. That in the walls of the pharynx and larynx, upon which he also found small ganglia on the branches of the glosso-pharyngeal and superior laryngeal nerves (Med. Zeit., 1840, No. 2), the mucous glands are very numerous. 6 . 
glosso-pharyngeal nerve in the tongue, and these have been recently subjected by myself (Micr. Anat. II, 2, p. 32), and by Remak (Müll. Arch., 1852) to a more exact investigation. Remak found such ganglia also upon the branches of the gustatory division of the fifth nerve in the Sheep and Calf, as far as close to the apex of the tongue, though they were smaller and more scanty than those upon the glosso-pharyngeus; whilst on the other hand, in man, they were wanting upon the thicker branches of the gustatory division of the fifth, and were to be found only as very minute ganglia upon the more delicate internal branches. Remak endeavors to demonstrate some relation between these ganglia and the lingual glands, and draws a functional parallel between them and the ganglion linguale, a view plausible enough, and against which I only have to remark, 1 . That ganglia exist not only upon the branches distributed to the mucous membrane, but also upon those passing to the papille, and in regions of the tongue (the point) where no glands are found; and, 2. That the glandular region of the root of the tongue, also, possesses gustatory sensibility. Upon these grounds, I think it is impossible, for the present, wholly to deny a relation of the ganglia in question, to the sensations.

\section{OF THE GLANDS OF THE ORAL CAVITY.}

\section{I.-MUCOUS GLANDS.}

$\S 133$. The mucous glands of the oral cavity are yellowish or whitish racemose glands, usually of a rounded form, and tuberculated surface of $\frac{1}{3}-2$ lines in diameter, which in general lie immediately external to the mucous membrane and yield a mucous secretion.

According to the localities in which they are found, the mucous glands present somewhat different relations and receive different names.

1. The labial glands, $\frac{1}{2}-1 \frac{1}{2}$ lines in diameter, lie between the muscular layer and the mucous membrane, are very numerous, and form an almost continuous glandular ring round the oral aperture, which commences at 3 lines distance from the red edge of the lips, and possesses a breadth of about $\frac{1}{2}$ a line.

2. The buccal glands, lying further outwards, covered by the buccinator muscle, are tolerably numerous but smaller; a few large glands appear at the aperture of Stenon's duct upon the buccinator muscle, and still further backwards in the neighborhood of the last molar tooth (molar glands).

3. The palatine glands.-Those of the hard palate are small and

That in the sheep and calf he has observed little ganglia upon the surface of the ductus Whartonianus, which are connected with a plexus of delicate nerves investing the duct." Trs.] 
hardly pass beyond its middle anteriorly, while on the other hand, those of the soft palate form a considerable layer of glands upon its under side, which anteriorly measures as much as 3-4 lines, diminishing, however, somewhat towards the free edge and the uvula. On the posterior surface of the soft palate, also, glands exist, but they are much smaller, and do not always form a connected layer.

4. The lingual glands, among which I distinguish:-

a. The mucous glands of the root of the tongue.-These form a stratum, in parts very thick, of glands $\frac{1}{2}-2$ lines in diameter, beneath the simple mucous sacs of the root of the tongue, to which we shall have to refer hereafter, and the papilloe circumvallato; it presents, especially beneath the former, a thickness of as much as 4 lines, and extends almost continuously from one tonsil to the other. In front of the foramen coecum these glands are smaller and more scattered, but a few occur more or less deep in the muscular substance in front of the most anterior papilloe circumvallatoe; they are never found, however, further forwards than the middle of the tongue.

The excretory ducts of the glands, which are interwoven with the extremities of the genio-glossus and partly united with them, are as much as 6 lines long in the posterior glands, and open, as E. H. Weber first showed, by a funnel-shaped expansion into the simple mucous sacs of the root; in the neighborhood of the papilloe circumvallatoe, on the other hand, they open independently between the lingual papillæ, and into the clefts which surround the circumvallate papillæ, a few also on the walls of the foramen coecum.

b. The marginal glands of the root of the tongue.-At the borders of the root of the tongue $\mathrm{we}$ find, at the level of the papilloe circumvallatoe many perpendicular laminated folds, to which reference has already been made, and between them fine apertures, which belong to a special small group of glands lying in the midst of the expansion of the hyo-glossus and transversus. In animals, these glands, as well as the folds (Mayer's organ), are often very greatly developed. (See Brühl, l. c.)

$c$. The glands of the point of the tongue.-On the lower surface of the apex of the tongue, but still in the substance of the lingualis inferior and stylo-glossus, there lie, right and left, two elongated broad glandular masses 6-10 lines long, 2-3 lines thick, 3-4 lines broad, where 5 or 6 excretory ducts open upon peculiar lobed folds of mucous membrane close to the froenulum linguce; these glands were long ago accurately described by Blandin, and have been recently rescued from oblivion by Nuhn.

$\S$ 134. Intimate structure of the mucous glands.-All these glands agree in the essential characters of their intimate organization and invariably consist of a certain number of glandular lobes with a branched 
excretory duct. The lobes, of which in the simplest glands (Fig. 178), only a few (4-8) exist, are in their circumference generally elongated, pyriform, or rounded, not rarely flattened, $0.5-0.72$ of a line long, $0 \cdot 2-0.48$ of a line broad, and are each seated upon a branch $0.03-$ 0.05 of a line thick, of the excretory duct, which measures 0.12 0.3 , or even 0.5 of a line (in the glands of the root of the tongue). They consist of a number of coiled canals, presenting numerous simple or compound, vesicular diverticula (Fig. 179), and appear to be the immediate continuations of the excretory ducts of the lobes, which, as soon as they have entered the latter, usually without diminishing in diameter, break up successively into a certain number of them. What have been called the glandular vesicles or acini, are nothing more than

Fig. 179 .

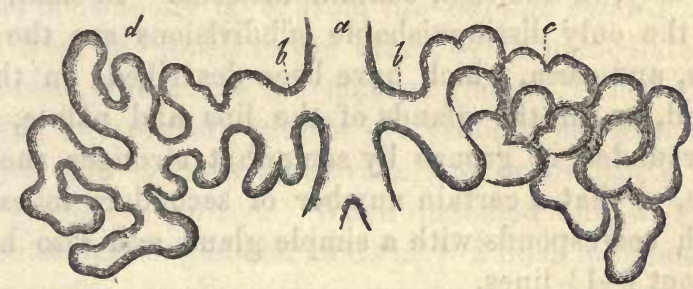

the dilatations and terminations of these canals, or ultimate branches of the excretory ducts. Examined superficially, and under low magnifying powers, they all appear uniformly rounded or pyriform; the exact analysis of a whole lobe, or still better of a dissected and injected gland, shows, however, that their form is very various, rounded, pyriform, or elongated. It is impossible to describe at length all the forms which they assume; I will therefore only remark, that the ends 'of the glandular lobes frequently repeat, on a small scale, the figure and structure of the seminal vesicles, and refer to the subjoined diagrammatic figure.

Fig. 178.-Racemose mucous gland from the floor of the oral cavity: $a$, investment of connective tissue ; $b$, excretory duct ; $c$, glandular vesicles; $d$, ducts of the lobes ; from Man.Magnified 50 diameters.

Fig. 179.-Diagram of two ducts of a lobe of a mucous gland: $a$, excretory duct of the lobe; $b$, secondary branch; $c$, the glandular vesicles upon it in situ; $d$, the same separated and the duct unfolded. 
All the finest glandular ducts and vesicles, whose diameter varies from 0.02 to 0.08 of a line, consist of a peculiar, structureless coat, the membrana propria, of $0.0008-0.0012$ of a line in thickness, and of an epithelium (Fig. 180), which, in fresh preparations, appears as a continuous investment of the glandular extremities, but is

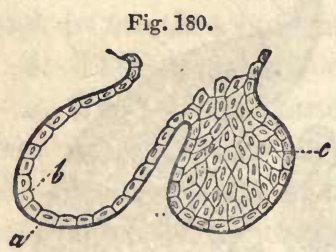
very readily detached, and then fills the glandular vesicles as a granular mass. The epithelial cells constitute a simple layer upon the membrana propria, have $3-6$ sides, are often somewhat elongated, $0.005-0.006$ of a line broad, 0.0030.004 of a line thick, and invariably contain, besides a rounded or elongated nucleus of $0.002-0.003$ of a line, often presenting a distinct nucleolus, a certain number of larger or smaller granules, which sometimes simply resemble white fat, sometimes are colored yellowish and brownish, and contribute to the hue of the glands themselves.

The elements of the glandular lobes which have been just described, though they are all applied very closely together, so, indeed, as not uncommonly to be flattened against one another, yet always present a small quantity of interposed connective tissue, by which the vessels of the lobes are supported. Besides this, the separate lobes and the entire glands are invested by dense coats of a connective tissue, with elastic fibres, which may, in addition, contain fat-cells. In small glands, such as Fig. 178, the only distinguishable subdivisions are the lobes, glandular vesicles, and creca, which have been described; in the larger, on the other hand, as in the glands of the lips and palate, the smallest lobes are surrounded in groups by somewhat stronger sheaths of connective tissue, so that a certain number of secondary lobes are formed, each of which corresponds with a simple gland and also has the same size, $i$. e., about $\frac{1}{2}-1 \frac{1}{2}$ lines.

The excretory ducts of the lobes bave a coat of connective tissue, with networks of fine elastic fibres, and a simple layer of cylindrical cells $0 \cdot 008-0.01$ of a line thick. In the principal excretory ducts, the wall, which is very rich in elastic fibres, measures, even in the smallest glands, 0.02 , in the larger as much as 0.03 and 0.04 of a line, the epithelium $0.01-0.012$ of a line. Of muscular fibres I saw no trace whatsoever, either in the glands themselves or in their excretory ducts; on the other hand, they possess many minute vessels, which penetrate with the excretory duct or otherwise between the lobes, and form, in the interior, a wide network of capillaries of 0.003 of a line, which encircle the separate crea and vesicles, so that each of them is in contact with

FIG. 180.-Two glandular vesicles of a racemose mucous gland of Man, magnified 300 diameters; $a$, membrana propria ; $b$, epithelium, as it appears in the apparent section of a vesicle; $c$, the same seen upon its surface. 
at least 3-4 capillaries. Nerves exist abundantly upon the excretory ducts and, occasionally, moderately fine tubules are found in the glands themselves.

The secretion of the racemose glands is a clear, yellowish mucus, with only accidentally intermingled granules, nuclei, and remains of cells, which coagulates in acetic acid and is insoluble in an excess of it, remaining as a viscid mass, striated, or deceptively similar to a fibrous tissue. It fills the excretory ducts and the other cavities of the gland to their ultimate extremities, being readily rendered obvious in them by acetic acid. I have never found the so-called mucous corpuscles, as they exist in the fluids of the mouth, in a mucous gland, and I believe that, normally, the secretion of mucus goes on without the production of cells.

\section{II.-FOLLICULAR GLANDS.}

$\S 135$. The follicular glands of the cavity of the mouth are: firstly, simple follicles at the root of the tongue and, secondly, compound follicles at the sides of the isthmus faucium,- - the tonsils. These organs a gree so perfectly in structure, that the tonsils may be regarded as an aggregation of simple follicular glands, while, on the other hand, they are so widely different from the mucous glands that they can on no account be classified with them.

The simple follicular glands of the root of the tongue (Fig. 169, $f$ ), form an almost continuous layer from the papilloe vallatce as far as the epiglottis and from one tonsil to the other, lying immediately under the mucous membrane, and above the mucous glands. Their position is so superficial, that the separate glands are visible from without, like little elevations upon the mucous membrane, and allow their number and arrangement to be recognized. When dissected out, we see that each follicle is a lenticular or globular mass of $\frac{1}{2}-2$ lines in diameter, invested upon its outer surface by the mucous membrane, which is here very thin, lying loosely in the submucous tissue and receiving upon its under surface the excretory duct of a more deeply disposed mucous gland. In the midst of the free surface a punctiform aperture, often tolcrably wide $\frac{3}{5}-\frac{1}{2}$ a line, is easily perceived by the naked eye; it leads into a funnelshaped cavity, which is remarkable on the one hand for its narrowness, in relation to the size of the sac, and on the other, for its thick walls, and is usually filled with a grayish mucous material.

Each follicle (Fig. 181) is a thick-coated capsule, externally surrounded by a fibrous investment connected with the deep layers of the mucous membrane; internally, it is lined by a process of the mucous membrane of the oral cavity, with its papillæ and epithelium, and contains between the two, imbedded in a delicate, fibrous, vascular matrix, a certain number of large, completely closed capsules, or follicles (Fig. 181, g), which are about $\frac{1}{10}-\frac{1}{4}$ of a line in diameter, round or elongated in form 
present a whitish color, and closely resemble the capsules of Peyer's patches, the solitary glands, the vesicles of the spleen and of the

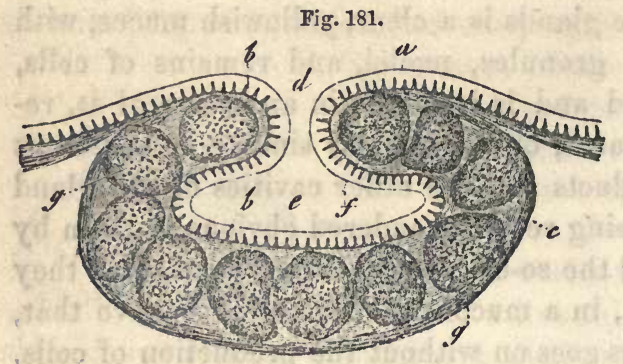
lymphatic glands. They consist of a tolerably solid coat, about $0.002-.003$ of a line thick, composed of more homogeneous connective tissue, without elastic fibres, and of grayish white contents, which, when the follicle is pricked, exude in the form of a drop, which becomes diffused through water, and consists of a fluid with formed particles. The former, alkaline in its reaction, is present in excessively small quantity, so that it appears to be merely the connecting medium of the latter, which consist of cells of $0.003-0.005$ and free nuclei of $0.002-0.0025$ of a line without any determinate character. Acetic acid renders the cells granular, and thence communicates a whitish tinge to the contents; but it precipitates no mucus, the fluid differing decidedly in this respect from the ordinary mucous secretion and agreeing with that of the splenic corpuscles. The position of the follicles is usually such, that they form a connected, almost simple layer, between the external coat and the epithelium of the follicular glands, yet there are localities, at least in animals, where two follicles are found behind one another, or at greater intervals.

The vessels of the follicular glands are very numerous, and may often be traced naturally injected, in man. Small arteries enter from without, passing through the fibrous coat to the interior, ramify between the follicles, as they ascend, in an elegant arborescent manner, and terminate in the papillæ and on the follicles. The vessels of the former present the same relations as those of the other simple papillæ, and are either simple or complex loops; around the follicles they form an exceedingly elegant and abundant network, whose finest vessels, $0.004-0.006$ of a line in diameter, take a wavy course, forming a moderately close network immediately upon the membrane of the follicle. The efferent veins converge from these two localities, and are wide and numerous. Lymphatic vessels also, according to E. H. Weber (Meckel's Archiv, 1827, p. 282), appear to proceed from these glands, and I have myself noticed nerves upon them.

The tonsils are, according to my investigations, nothing but an aggre-

FIG. 181.-Follicular gland from the root of the tongue in Man : $a$, epithelium lining it; $b$, papillæ; $c$, external surface of the follicular gland, with the coat of connective tissue; $e$, cavity of the gland; $f$, epithelium; $g$, follicle in the thick wall of the gland. Magnified 30 diameters. 
gation of a certain number (10 to 20) of compound follicular glands, which, intimately united and held together by a common investrnent, form a large hemispherical organ; the apertures of the follicles frequently unite, so as ultimately to form only a small number. Each section of the tonsil, much as it may vary in the form of its cavity and its external appearance, has exactly the same structure. Proceeding from the oral cavity, we observe that its epithelium enters into the separate cavities of the tonsil and, becoming somewhat thinner, completely lines all the secondary cavities. Beneath it we find a grayish, soft, very vascular membrane $\frac{1}{2}-\frac{1}{3}$ of a line thick; and still more externally, a dense, relatively thick fibrous covering, which, when two lobes or sections of the tonsils are in contact, belongs to them in common, and is in contact at their outer extremities with the common coat of the organ. The soft thick layer between the epithelium and the fibrous investment has the same composition as the corresponding layer of the follicular glands of the root of the tongue. Here also we meet with conical or filiform, even slightly branched papillæ, of $0.06-0.08$ of a line in length, $0.01-0.03$ of a line in breadth, directed towards the epithelium ; internally to these, round, completely closed follicles one close to the other, of the same size and possessing the same contents as those previously described; and finally, a soft fibrous tissue connecting them, and containing numerous vessels. The vessels are still more numerous than in the follicles of the tongue, though their ramifications are essentially similar, except that the papillæ frequently contain multiple loops, and the networks around the capsules are still closer (Fig. 182). The fibrous investment, lastly, consists of connective tissue, with elastic fibres and receives certain muscular fibres from the superior constrictor of the pharynx. Nerves may be detected on the external surface of the tonsil, and in the papillæ, but, as in the case of the follicular glands of the root of the tongue, I have failed to observe them in the proper membrane of the follicles.

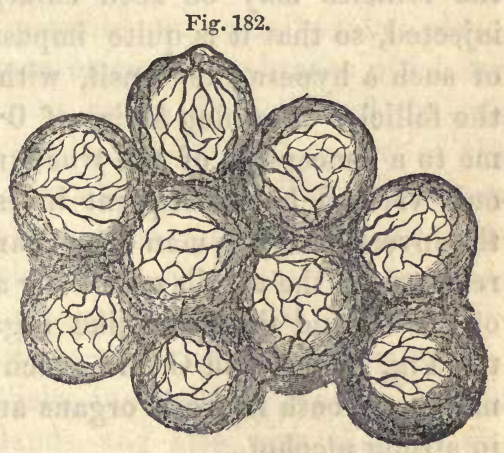

Corresponding with their structural similarity is the resemblance in the secretion of the tonsils and that of the lingual follicles, though the former is not easily obtained pure on account of the tonsils also receiving the ducts of mucous glands. It is a grayish-white mucous substance, which, however, so far as I have been able to observe, contains no mucus, but is composed either of cast-off epithelial plates alone, or of a mixture

Fia. 182.-Vessels of a few follicles from a human tonsil, seen from the cavity of a sac, and magnified 60 diameters. 
of these with cells and nuclei, perfectly identical with those contained in the parietal follicles of the tonsillar cavities. How these cells are formed and whence they arise, I know not. It would seem probable that they proceed from follicles which have burst, a process which may really occur in man, though from what is observed in animals we can hardly assume their dehiscence to be a normal process.*

In man it is quite impossible, in a vast number of cases, to find the follicles which we have described in the walls of the tonsils, a circumstance which appears to me to be explained by the frequent rnorbid changes to which these organs are subject. In fact, in the course of inflammations of the tonsils and their sequeloe, the contents of the follicles appear to alter, the follicles themselves becoming distended, and finally bursting. The closed sacs, filled with purulent or caseous masses, which are described in diseased tonsils, when they do not exceed a certain size, may be nothing else than such follicles; and by their bursting they may yield those masses of secretion which are accumulated in the larger cavities. It thus happens, that the normal structure is frequently no longer recognizable in the walls of the tonsils, and thet we find, at most, recently opened follicles, or more usually nothing but a granular mass interpenetrated by fibres and vessels, with remains of papillæ and of epithelium.

On the other hand, however, the frequent pathological degenerations have this advantage, that if we happen to hit upon the right period, all the follicles may be seen enlarged, but still closed and beautifully injected, so that it is quite impossible to overlook them. An instance of such a hyperæmic tonsil, with distended, lingual follicular glands, the follicles attaining a size of $0.36-0.48$ of a line, was what first led me to a conception of the true structure of these parts, which has been only verified by subsequent investigations. The difficulties attending the investigation in man disappear in many animals, and I can especially recommend the tonsils of the Pig and Sheep, the lingual follicular glands of the $\mathrm{Ox}$ and the tonsil-like organs at the entrance of the larynx in the $\mathrm{Pig}$, Sheep, and $\mathrm{Ox}$, in which the structure may be always readily made out, both in fresh organs and in those which have been hardened in strong alcohol.

With respect to the secretion of the tonsils in man, it is certainly abnormal in many cases, in the dead subjects which are accessible for investigation ; for instance, when the cavities contain considerable quan-

* [In the Mikroskopische Anatomie, B. II. H. II., p. 46, Professor Kölliker adds a few points to the account here given of the structure of the tonsils. In man, racemose glands are not unfrequently met with external to the tonsils, and probably open in them; and in the Calf, a considerable number of such glands may be found between the lobes of the organ. In opposition to Frerichs (Wagn. Handw. III., p. 745), Kolliker states that he has " not yet" found solitary glands like those of the intestine in the mucous membrane of the mouth.-Trs.] 
tities of a grayish, yellowish, or greenish, sometimes softer, sometimes more consistent mucus, if it may be so called, the constituents of these contents being larger and smaller cells, with a single nucleus, some of which have undergone a very obvious fatty metamorphosis, while others have cavities and thickened membranes; further, epithelium (not ciliated cylinders, as Valentin states, having probably confounded with such, the deepest, here very much elongated, cells of the pavement epithelium), occasionally abundant cholesterin crystals and mucedinous fungi. The secretion is more normal if it consist only of epithelium, of small cells without fat and of free nuclei, the two latter elements being perfectly similar to those in the follicles; such great masses of them, however, are often found, that we must suppose they have been developed in excess.

In any case I am disposed to consider such cells and nuclei as the proper secretion of the tonsils, inasmuch as, in animals, as for example, the Sheep, we find similar contents, though their amount is often small. It is difficult to decide whether they are afforded by the follicles or not; certain it is, that they are identical with the contents of the latter, and that in man the follicles burst, but the former might be accidental, and the latter only a morbid process.

In fact, however frequently the tonsils of animals are examined, no ruptured follicles are ever met with; they are always entirely closed, and the epithelium extends them, so that one is led to the belief that the secretion is developed independently, out of a substance excreted into the cavity of the organ. That this is possible and actually takes place, indeed, elsewhere (e.g. suppuration upon mucous membranes which are still covered by their epithelium), is not to be denied; and the sole difficulty about such a hypothesis is, that in this case the import of the tonsillar and lingual follicular glands (for which, also, all that has been said, holds good), becomes highly problematical. If they do not occasionally burst, their function, as regards secretion, can only be to elaborate in their interior a fluid, which when it subsequently enters the cavity of the gland, is especially fitted to form its proper secretion. For the rest, the similarity of the follicles in question, especially with those of the solitary and Peyerian glands, and also with those of the spleen* and lymphatic glands, would indicate another series of possibilities, into which, however, I will not enter, because in all the organs in question the anatomical facts and the physiological relations have hitherto been by no means completely determined.

\section{III.-SALIVARY GLANDS.}

$\S 136$. The salivary glands, $i$. e. the parotid, the submaxillary, the sublingual, and Rivini's glands, agree so closely in their structure with

* [For some additional facts in favor of these resemblances, see notes § Spleen.-Trs.] 
the racemose mucous glands, that it would be quite superfluous to enter into any detailed description of them. They are compound racemose glands, and they might be regarded as aggregations of numerous mucous glandules. In fact, the primary and secondary lobulations which are observed in these glands correspond, the latter to the entire mucous gland, the former to its lobes. The secondary lobulations then become united into still larger groups, and a certain number of these constitute the whole gland. The excretory ducts correspond with the number of the lobulations of the gland; they are more or less branched, and in their final relations resemble those of the mucous glands.

The more intimate structure of the salivary glands presents nothing remarkable. The glandular vesicles have about the same diameter$0.016-0.024-0.03$ of a line, in all three descriptions of glands; they are as variously formed as in the mucous glands, and proceed in a similar manner from the excretory ducts. Their membrana propria frequently presents a double contour, and is, internally, always clothed with a pavement epithelium, whose cells of $0.005-0.008$ of a line have a single nucleus, and may be obtained in beautiful series by coarsely crushing the gland; they are distinguished by their greater proportion of fatty granules and pigment granules from those of most mucous glands, whence the glandular vesicles themselves present a somewhat dark appearance. Here, also, acetic acid makes the contents of the cells turbid, the addition of an excess even not restoring their clearness, and it is not therefore advisable to make use of it in our examinations; a very dilute solution of caustic soda, which allows the epithelial cells to be seen in situ, is more to be recommended.

The excretory ducts of the salivary glands possess a single layer of cylinder epithelium, whose cells measure 0.016 of a line in length. The remaining portion of the wall, which is very thick in Stenon's duct, but much thinner in the others, has a dense, solid structure, and consists of connective tissue, with a very close network of fine, and moderately thick, elastic fibres. It is only in Wharton's duct that we find, external to the epithelium, and to a double layer of elastic membranes whose elements are disposed transversely and longitudinally, a thin stratum of smooth muscles, which, however, can only be demonstrated and isolated with very great difficulty. They are disposed longitudinally, have short nuclei of $0.004-0.006$ of a line, at most 0.008 of a line, and are covered externally by a layer of connective tissue with elastic fibres.

The vessels of the salivary glands are very numerous, and present the ordinary structure. The capillaries have a diameter of $0.003-0.004$ of a line, and form broad networks in which the glandular vesicles are imbedded, so that each vesicle receives its blood from several directions. A considerable number of vessels are distributed also to the excretory ducts. Lymphatics are found in the salivary glands, but their internal 
relations are unknown. Nerves proceed from the plexus caroticus externus, with the ressels, into the interior of the glands; in addition, the ganglion linguale (lingualis and chorda tympani) supplies the two smaller pair of glands, and the facial nerve, probably with the anterior auricular, the parotid. I may remark, with regard to the distribution of these numerous nerves, that here also it is impossible to find any in the smallest lobules of the glands, while on the other hand they are readily discovered upon the larger vessels and the excretory ducts. In animals I found particularly rich nervous networks upon Rivini's ducts, the tubules having a diameter of $0.001-0.002$ of a line.

The secretion of the salivary glands normally contains no formed elements, but may accidentally present cylindrical cells from the excretory ducts, or scattered, half-disorganized cells from the glandular vesicles. Its physical and chemical properties, in the different salivary glands, appear to differ in some respects. The parotid saliva is clear and fluid, and, like the glandular vesicles themselves, contains no mucus. Bernard and Jacubowitsch found the secretion of the sub-maxillary gland in the dog to be viscid, and capable of being drawn out into threads; according to Bernard also, a watery extract of the gland itself contains mucus.

In man, the ductus Whartonianus, if laid open, is usually found to contain a small quantity of a kind of mucous fluid, which, however, consists chiefly of cylinder epithelium and broken-up epithelial cells of the glandular vesicles themselves, containing only a very small amount of a substance which coagulates in acetic acid, and is, perhaps, mucus. The glandular vesicles, on the other hand, if crushed, usually yield a considerable quantity of mucus, which coagulates into threads by the action of acetic acid. The vesicles of the proper sublingual gland contain still more mucus, and the ductus Bartholinianus also commonly presents distinct evidence of it. With respect to Rivini"s ducts, they are filled, in man and animals, with the same yellowish, viscid, amorphous fluid, coagulating into threads by the action of acetic acid, which is met with in the ducts of the small mucous glands, while the glandular vesicles themselves also contain abundant mucus. From all that has been said, it would seem that Rivini's glands, as I will call them, must be excluded from the class of salivary glands, and, as regards the three larger glands, their secretion does not appear to be identical, but sometimes to contain mucus (submaxillary and particularly the sublingual), sometimes to want it (parotid).

We may take this opportunity of making some observations with respect to the salivary, or mucous corpuscles, of authors; rounded cells of 0.005 of a line, with one or many nuclei, which are always to be met 
with in the fluid of the mouth, and are usually supposed to be derived from the mucous or salivary glands, yet wrongly, since the examination of both these kinds of glands, and of their ducts, teaches us that they excrete no formed elements. In my opinion the mucous corpuscles are nothing but products of the mucous membrane of the oral cavity-not normal, although they are almost constant, but a kind of exudation- or pus-corpuscles, with which they have, as is well known, the closest possible resemblarice in structure. Many authors consider them to be abortive epithelial cells of the oral cavity; but in that case the epithelium of the localities in which they are found must want the outermost layer of large, flattened scales, which is by no means the case. In my own person, at any rate, I find mucous corpuscles on the gums, the lips, cheeks, and tongue, in localities in which the epithelium is wholly uninjured; and by scraping with a knife I can often obtain entire lamellæ of epithelial plates, covered with mucous corpuscles. I do not mean to affirm by this, that in little sores, arising from whatever cause, upon the gum, for instance, where the epithelium is wholly or partly wanting, or when it is lost more extensively, in consequence of disease, that mucous- or exudation-corpuscles may not be developed, as upon other sore surfaces, and then might be regarded as abortive epitheliumcells, but only, that this does not take place in the oral cavity under ordinary circumstances. I consider, therefore, that the so-called mucus, or salivary corpuscles, are exuda tion-corpuscles, and consequently totally distinct from epithelial cells; and I regard their formation to be analogous to that of the pus-corpuscles in catarrh, which also very often takes place upon unbroken epithelial surfaces. It is thus readily explained how it is that they are almost entirely absent in many individuals, while in others who are subject to irritation of the mucous membrane of the mouth they are very abundant, and that they have been observed in saliva, obtained from a fistulous aperture (Sebastian, in "Van Setten, Diss. de Saliva." 1837, p. 12).

The best mode of examining the oral mucous membrane is by making perpendicular sections of portions, either fresh, or dried, or hardened in absolute alcohol, in which the papillæ and epithelium are very distinct, and become still more so by the use of very dilute caustic soda ; by the aid of which also the deepest perpendicular epithelial cells are rendered readily visible. The papillæ may be studied in macerated portions, or if it be only required to ascertain their position and form, in perpendicular or horizontal sections, treated with concentrated caustic potassa, on which the epithelium is dissolved. The lingual papillæ may be treated in the same manner; the epithelium upon which, especially on the filiformes, moreover, is often entirely absent. The nerves of all these parts are best seen under the use of dilute caustic soda; acetic 
acid is also frequently of service. The muscular substance of the tongue must be examined by minute dissection, a method which may be carried very far, especially in tongues which are half macerated from having long lain in spirit. Recent tongues are also of use, but are by no means so good, and it is usually necessary to boil them until they are quite soft; and to procure sections for the microscope the tongue may be dried or hardened by boiling, or by strong alcohol. In all three methods caustic soda is of great service in clearing up the tissue, although it undoubtedly attacks the muscular fibres a little. Perpendicular and longitudinal sections in various directions are to be recommended, especially in the glandular region. With respect to the glands, the most important points have been already stated.

Literature.-W. Bowman, Art. "Mucous Membrane," in Todd's "Cyclopædia of Anatomy," April, 1842 ; E. H. Weber, "Ueber die Schleimbälge und zusammengesetzten Drüsen der Zunge und über den Bau der Parotis," in Meckel's "Archiv," 1827, pp. 276-280; A. Sebastian, "Recherches anatomiques, physiologiques et pathologiques sur les glandes labiales," Groningue, 1842; Nuhn, "Ueber eine bis jetzt nicht näher beschriebene Drüse im Innern der Zungenspitze," Mannheim, 1845 ; N. Ward, Art. "Salivary Glands," in Todd's "Cyc. of Anat.," Sept. 1848, part xxxiii. p. 421 ; C. Rahn, "Einiges über die Speichelsecretion," Zürich, 1850; C. Ludwig, "Neue Versuche über die Beihülfe der Nerven zur Speichelsecretion," in "Mitth. der Zürch. nat. Ges.,"-1850, Nos. 53 and 54, and "Zeitschr. f. rat. Med.," 1851 ; C. J. Baur, "Ueber den Bau der Zunge," in Meckel's "Archiv," 1822 , p. 350 ; P. N. Gerdy, "De la Structure de la Langue," in "Recherches d'Anatomie, de Physiologie, et de Pathologie," Paris, 1823; P. F. Blandin, "Sur la structure de la Langue," in the "Archiv. génér. de Médecine," 1823; J. Zaglas on the "Muscular Structure of the Tongue of Man and certain Mammalia," in the "Annals of Anatomy and Physiology," ed. by J. Goodsir, 1850, I. p. 1; H. Hyde Salter, Art. "Tongue," in Todd's "Cycl. of Anat.," iv. June and September, 1850 ; C. B. Brühl, "Ueber den Bau der Zunge der Haussäugethiere," in "Kleine Beiträge zur Anatomie d. Haussäugethiere," Wien, 1850, pp. 1-6; Sappey, "Ueber die Lymphgefässe der Zunge," in "Comptes rendus," 1847, p. 26 ; and "Froriep's Notizen," 1848, vi. p. 88. Besides these, compare the anatomical works of E. H. Weber, Valentin (im Handw. d. Phys.), Todd and Bowman, Henle, Arnold, Huschke, Krause, and myself; the figures of Berres, Arnold, and Langenbeck.

\section{OF THE TEETII.}

$\S 137$. The teeth are hard organs inserted into the alveolar processes of the jaws, which although to some extent identical in structure with 
bone, and in other respects clearly allied to it, must, from their development, be regarded as modifications of the mucous membrane.

In every tooth we must distinguish the tooth proper, and the soft structures; the former consists of a free part, the crown, and of an imbedded portion, the simple or multiple fangs, whose special forms are treated of in anatomical works; they contain internally a small cavity, the pulp cavity, which extends through each fang as an elongated canal opening at its point by a simple, or more rarely double (Havers, Raschkow) fine aperture.

Among the soft parts we may enumerate first, the gum, gingiva, a dense mass formed by the union of the mucous membrane and of the periosteum of the jaw, which surrounds the lower half of the crown or the neck of the tooth. Secondly, the periosteum of the alveolus, which

Fig. 183.

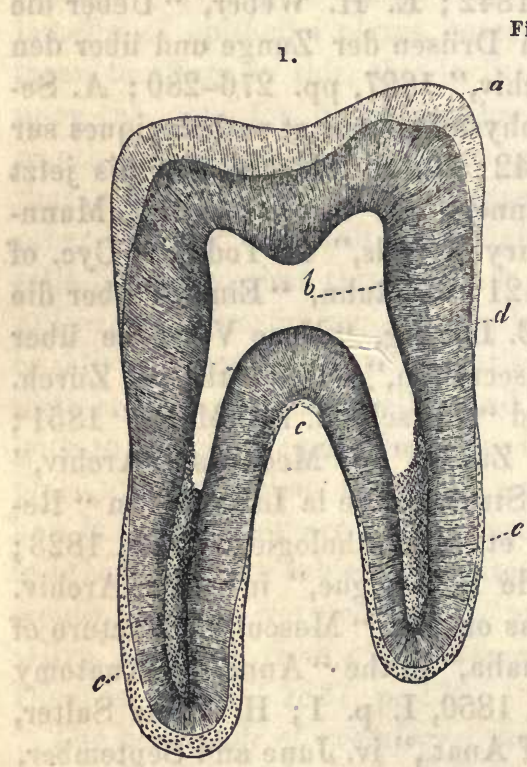

2.

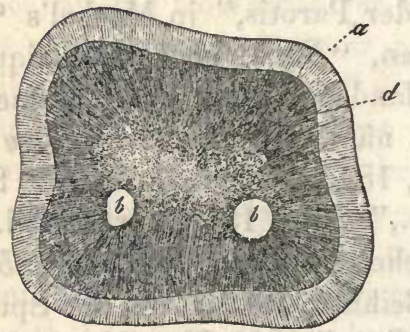

unites the tooth very closely with the alveolus. Finally, the pulp, a soft, vascular and nervous mass, which occupies the cavity of the tooth, and is connected with the periosteum of the alveolus, through the aperture in the fang.

The proper tooth (Fig. 183) consists of three distinct structures. 1. The dentine, which constitutes its principal mass, and determines its general form. 2. The enamel, which forms a tolerably thick investment to the crown; and 3 . The cement, which covers the fang externally.

$\S 138$. The dentine or ivory (Fig. 183, d), is yellowish-white and translucent or transparent, in thin sections of a recent tooth; when dry it has a silky or satiny aspect, in consequence of the reception of air into a special system of canals. It is considerably harder and more

Fig. 183.-Molar tooth (human); magnified about 5 diameters : 1, longitudinal ; 2, transverse section: $a$, enamel ; $b$, pulp cavity; $c$, cement ; $d$, dentine, with its canals. 
brittle than either the cement or bone, but, on the other hand, yields in these qualities to the enamel. With the exception of a very small spot in the root, the dentine forms the sole boundary of the pulp cavity, and in an uninjured tooth it is never exposed, inasmuch as it is covered, even upon the neck of the tooth, by a thin layer of enamel, and when this ceases, by cement.

The dentine consists of a matrix and of a multitude of canals which traverse it, the dentinal tubules or canals. In the recent tooth, the former is, even in the finest sections, quite homogeneous, without the slightest trace of cells, fibres, or other elements. After the extraction of the calcareous salts from the dentine, it exhibits, however, a great tendency to tear up into coarse fibres, parallel to the dentinal canals; from these, finer fibres of $0.002-0.003$ of a line may be detached, their irregular form, however, shows them to be artificial products, and in fact they owe their existence simply to the circumstance, that the dentinal canals run close together and parallel to one another through the dentine. The matrix exists in all parts of the dentine, but not everywhere to the same amount. In general there is less of it in the crown than in the root, and in the neighborhood of the pulp cavity than in that of the enamel and cement.

The dentinal canals (Figs. 184, 187) are microscopic tubules of 0.0006 0.001 of a line (in the root some reach 0.002 of a line), which commence by open mouths upon the wall of the pulp cavity, and traverse the whole thickness of the dentine to the cement and enamel. Each canal has a special wall, rather less in thickness than its diameter, which can only be observed in transverse sections (and then not al-

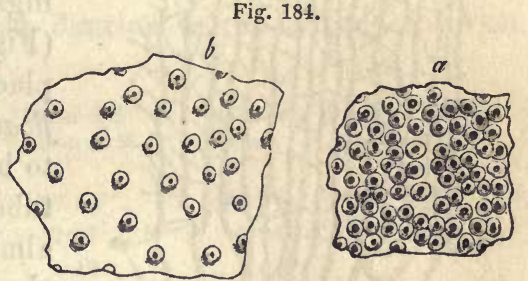
ways), as a narrow, yellowish ring surrounding the cavity; in longitudinal sections, on the other hand, it is almost entirely invisible. During life the canals contain a clear fluid and they cannot therefore readily be detected in recent preparations ; it is different in dry sections, when they become filled with air, and appear separately as black lines by transmitted light, and by reflected, as silvery threads. On account of the immense numbers of the dentinal canals, so great in some situations that their walls are almost in contact, dry sections appear milkwhite, and if not very thin, are quite unfit for microscopic investigation, unless the air has been previously expelled from the canals by any clear and not viscid fluid.

FIG. 184.-Transverse section of dentinal canals as they are commonly seen, magnified 450 diarneters : $a$, canals very close together; $b$, more dispersed. 
The dentinal canals present certain constant peculiarities in their course which may be best gathered from Figs. 185 and 187; it is not straight but wavy, and in addition, they present numerous ramifi-

Fig. 185.

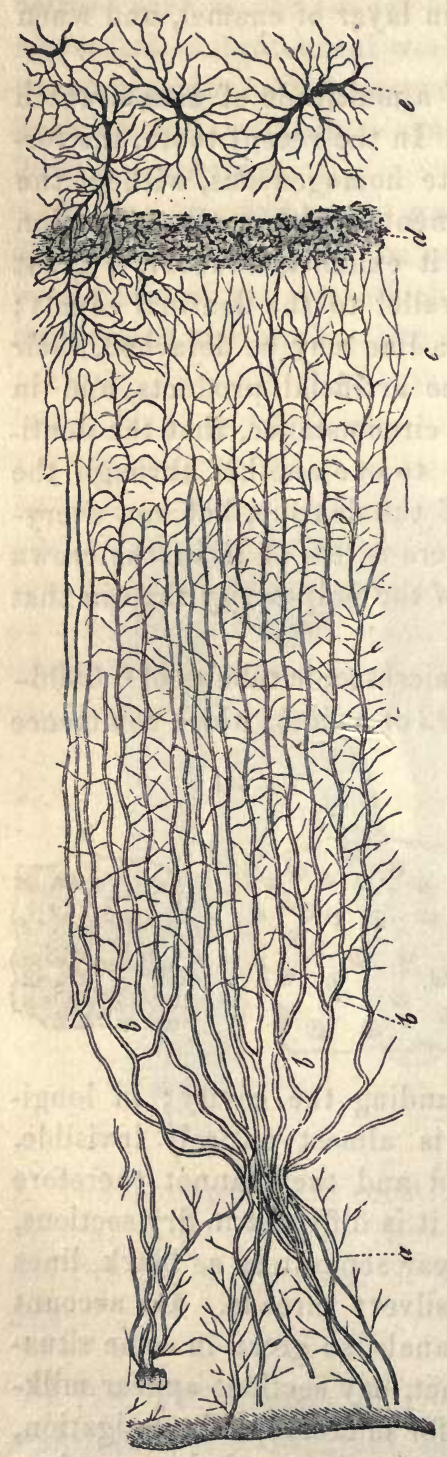

Fig. 186.

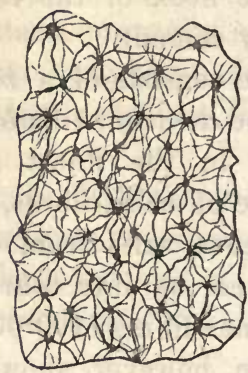

cations and anastomoses. Each canal describes, in general, two or three large curvatures, and a very great number (as many as 200 in 1 line, according to Retzius) of small curvatures, which are sometimes more or less strongly marked and occasionally have even the appearance of actual zigzags, or spiral windings. The ramifications of the canals (Figs. 185 and 186), appear in the first place as divisions, and then as true ramifications; the former are very frequently to be met with close to the origin of the tubules from the pulp cavity, and are almost always bifurcations, of such a kind that one canal divides at an acute angle into two of almost the original diameter. These divisions may be repeated 2-5 times altogether, and even still oftener, so that at last, 4-8-16, and even more canals, proceed from a single one. The canals, already somewhat narrowed after division, then run close together and tolerably parallel, towards the surface of the dentine, and excepting in the root, branch

Fia. 185.-Dentinal tubules from the root, magnified 3\%0 diameters: $a$, internal surface of the dentine, with scattered canals; $b$, their divisions; $c$, terminations with loops; $d$, granu. lar layer, consisting of small dentinal globules at the boundary of the dentine; $e$, bone lacuna, one anastomosing with dentiual canals. From Man.

Fig. 186.-Transverse section throngh the dentinal canals of the root, $a$, in order to exhibit their excessively numerous anastomoses; magnified 350 diameters. From Man. 
again only in its outer half or third; the ramifications appearing, in the root, as fine twigs given off from the principal canal, while in the crown, they more resemble dichotomous divisions. In the latter case, they are for the most part rare, in the former, it is otherwise; the branches, which are usually close together and given off at right or acute angles from the trunk canal, having sometimes the appearance of a feather, sometimes that of a brush, the latter being most common when the twigs are large and undergo further branchings. According to their more or less frequent ramification, are the ends of the dentinal canals more or less fine; frequently appearing merely as excessively fine, pale lines, like fibrils of connective tissue, and finally disappear. When they are distinct, they either become lost upon the surface of the dentine in a granular layer, which we shall have to consider presently, or they enter the innermost portions of the enamel and cement, or finally they are connected in pairs by loops in the dentine (terminal loops of the dentinal canals). The branches of the principal canals are, almost always, very fine and usually simple, though sometimes ramified; they serve, as may be best seen in the root, where they are excessively numerous, to connect neighboring or even distant canals; such anastomoses having either the form of simple, transverse canals or of loops. The ultimate branches present the same relations as the forked or simple terminations of the principal canals, and end either free or by loops, in the dentine, or are continued beyond it.

The chemical composition of dried dentine is, according to Von Bibra:-

\begin{tabular}{|c|c|c|c|c|c|c|c|}
\hline \multirow{2}{*}{\multicolumn{5}{|c|}{ Phosphate of lime, with some fluoride }} & $\begin{array}{c}\text { Molar of a } \\
\text { Woman of } 25 .\end{array}$ & $\begin{array}{l}\text { Molar of a } \\
\text { Man. }\end{array}$ & $\begin{array}{l}\text { Incisor of the } \\
\text { same Man. }\end{array}$ \\
\hline & & & & & & & \\
\hline \multirow{2}{*}{\multicolumn{2}{|c|}{$\begin{array}{l}\text { of calcium, } \\
\text { Carbonate of lime }\end{array}$}} & & & . & 67.54 & 66.72 & . \\
\hline & & & & . & $7 \cdot 97$ & $3 \cdot 36$ & totifing \\
\hline \multicolumn{3}{|c|}{ Phosphate of magnesia, } & • & - & 2.49 & 1.08 & (3) \\
\hline \multirow{3}{*}{$\begin{array}{l}\text { Salts, } \\
\text { Cartilage, } \\
\text { Fat, }\end{array}$} & . & 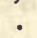 & • & . & $1 \cdot 00$ & 0.83 & \\
\hline & . & & & . & $20 \cdot 42$ & $27 \cdot 61$ & \\
\hline & . & & & . & 058 & 0.40 & \\
\hline 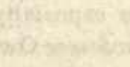 & & & & & $100 \cdot 00$ & $100 \cdot 00$ & \\
\hline \multirow{2}{*}{\multicolumn{2}{|c|}{$\begin{array}{l}\text { Organic substance, } \\
\text { Inorganic substance, }\end{array}$}} & & & & $21 \cdot 00$ & $28 \cdot 01$ & $28 \cdot 70$ \\
\hline & & & . & . & $79 \cdot 00$ & 71.99 & $71 \cdot 30$ \\
\hline
\end{tabular}

In fresh teeth, Pepys found 28 p. c. cartilage, 62 inorganic matter, 10 water and loss; and according to Tomes, teeth, after the pulp is removed, lose in drying $\frac{1}{8}-\frac{1}{17}$ of their weight. The organic basis of the teeth, which may readily be obtained by treating them with hydrochloric acid, is identical in all respects with that of the bones, and is readily changed into gelatin by boiling. This so-called cartilage of 
the tooth retains the exact form of the dentine and its external structure also; the tubules, however, are seen with difficulty. If it be macerated in acids or alkalies until quite soft, the matrix undergoes incipient solution, but the dentinal tubules, with their walls, offer greater resistance, and may be readily and abundantly isolated (see "Mikr. Anat." ii. 2, p. 61, fig. 189). By still longer maceration, all is dissolved. If teeth be heated to redness, or treated with caustic alkalies, the inorganic portions likewise retain the form of the tooth. It follows, then, that the same intimate mixture of inorganic and organic parts occurs in the teeth, as in the bones, with which they so closely agree in their chemical composition.

The apparent walls of the dentinal tubuli, which are commonly visible in transverse sections (Fig. 184), are not the actual walls of the canals, but rings, which result from our invariably viewing a certain length of the canals in the always more or less thick sections, their undulated course giving the walls a greater apparent thickness than they really possess. If in any transverse section the apertures of the canals be exactly brought into focus, we perceive, instead of the dark ring, only a very narrow yellowish border, which is what I consider to be the actual wall. That such is the case, appears from the examination of transverse and oblique sections of canals filled with fluid, in which short, yellow tubules and small rings of almost the same diameter as that of the cavities of the canals, may be clearly recognized.

The dentine occasionally presents indications of lamination in the form of arched lines running more or less parallel and at different distances, often quite close together (Fig. 187); which in transverse sections appear as rings, and are especially distinct in the crown. These, the contour lines of $\mathrm{O}$ wen, $*$ are not the same with the glistening, indistinctly defined striæ observed by Schreger, which run exactly

* [This is not exactly correct. The term "contour lines," as used by Professor Owen ("Report of British Association" for 1838, p. 135, and "Odontography," pp. 460, 464, 611), includes both descriptions of markings mentioned in the text, but is more especially employed for Schreger's. The ordinary contour lines, in fact, are stated by Professor Owen to proceed from "a short bend" of the tubuli "parallel with the outer contour of the crown;" from these, the Professor distinguishes the "strong contour lines," in the ivory of the elephant's tusk, as being produced by "strata of extremely minute opaque cellules." It should be observed, however, that Retzius had long before drawn attention to these peculiar striæ. In his admirable memoir, published in Muller's Archiv, for 1837, he says, p. 507, "In the incisor teeth of the horse, also, many less transparent striæ running parallel with the cavitas pulpa may be seen, like the annual rings in the trunk of a tree. They proceeded in this case, however, not merely from certain parallel flexures of the tubes, but especially from similar calcareous cells, which had accumulated in one zone for the greatest part of the length of the tooth. Tab. xxii. Fig. 3." See also his explanation of the zones in the Elephant's tooth, at pp. 510-11. -Trs.] 
parallel to the pulp cavity, and arise from the primary curvatures of the ciontinal canals, and which are the expression of the laminar mode of deposit of the dentine. In animals they are at times singularly beautiful, especially in the Cetacea and Pachydermata (Zeuglodon, Dugong, Elephant), and also in the Walrus. Here, as well as in fossil teeth, we very frequently observe a breaking up of the dentine into lamellæe (Owen), indications of which may be found also in fresh human teeth and in the dental cartilage.

Upon the crown, the dentinal canals not unfrequently pass for some distance into the enamel, and expand here and there, into larger cavities (Fig. 191), which should perhaps rather be regarded as pathological. Similar not quite normal formations are the interglobular spaces in the dentine itself (Fig. 188). Czermak has conferred this name upon certain very irregular cavities, bounded

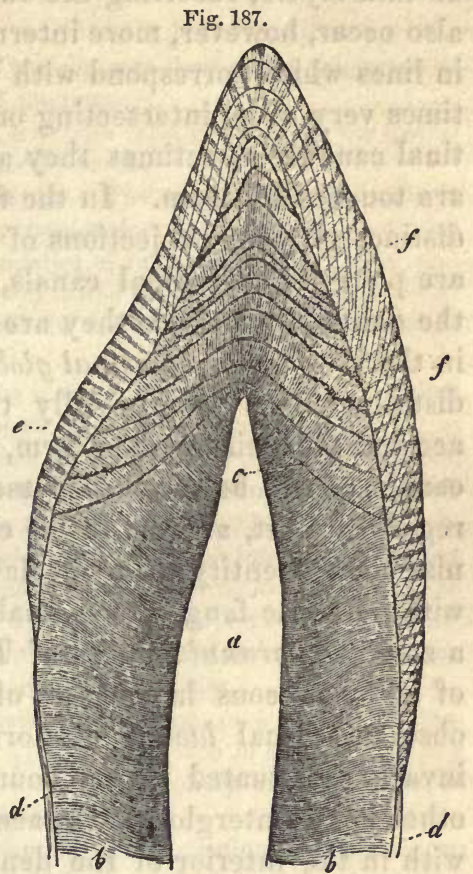
by globular processes of the dentine, which are, it may be said, never entirely absent in the teeth. In the crown they are found most frequently in the neighborhood of the enamel, and

Fig. 188.

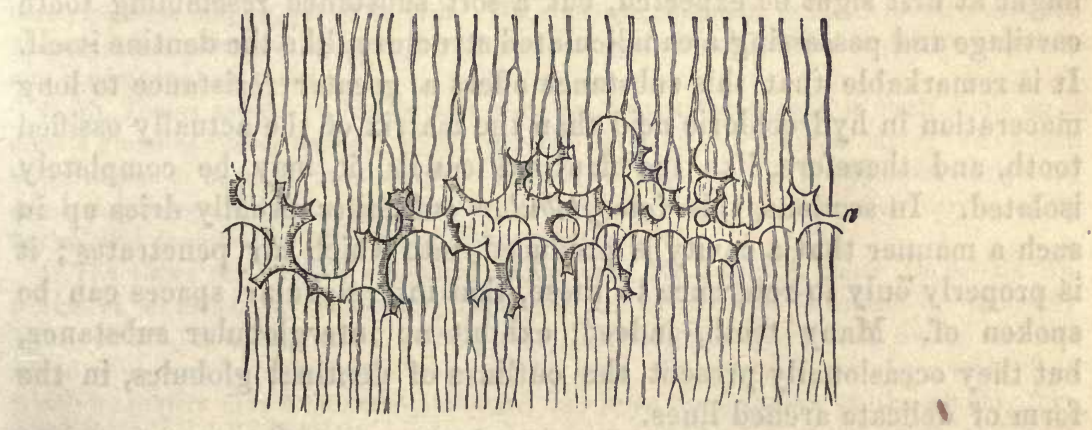

often form a thin curved layer, extending along its whole inner surface,

- FIG. 187.-Perpendicular section of the apex of an incisor tooth (human), magnified 7 diameters : $a$, pulp cavity; $b$, dentine; $c$, arched contour lines, with interglobular spaces; $d$, cement; $e$, enamel, the various directions of the fibres being indicated; $f f$, lines of color of the enamel.

Fig. 188.-A morsel of dentine with dentinal globules and interglobular spaces filled with air between them; magnified 350 diameters. 
which, upon close examination, is seen to be composed of a multitude of thin layers, receiving the ends of the contour lines (Fig. 187). They also occur, however, more internally, but always in longitudinal sections, in lines which correspond with the contour lines. The spaces are sometimes very wide, intersecting or interrupting in their course many dentinal canals; sometimes they are very suall, so that only a few canals are touched by them. In the former case, their limits are formed by distinct globular projections of $0.002-0.012$ of a line, and more, which are pierced by dentinal canals, and have precisely the same aspect as the dentine, of which they are obviously nothing but portions; whilst in the latter, such dentinal globules, as I will term them, are not always distinct. This is especially true of the smallest spaces, which, on account of their notched form, and their communications with dentinal canals, might be taken for osseous lacunce, and indeed have been so regarded; yet, at least in the crown, it is almost always easy to recognize their identity with the larger spaces. Greater difficulty is met with upon the fang, where small interglobular spaces and globules form a zone (the granular layer of 'Tomes), which often appears like a layer of small osseous lacunæ or of simple granules. I have but rarely observed actual lacunce in normal dentine; they were, when present, invariably situated at the boundary of the cement (Fig. 185); on the other hand, interglobular spaces and dentinal globules are to be met with in the interior of the dentine of the root, and with especial distinctness on the walls of the pulp cavity, in which latter locality the globules often give rise to irregularities visible to the naked eye, or even to a botryoidal appearance. The interglobular spaces whose presence is normal in developing teeth, contain during life, not fluid, as might at first sight be expected, but a soft substance resembling tooth cartilage and possessing a canaliculated structure, like the dentine itself. It is remarkable that this substance offers a greater resistance to long maceration in hydrochloric acid than the matrix of the actually ossified tooth, and therefore, like the dentinal canals, it may be completely isolated. In sections, this interglobular substance usually dries up in such a manner that a cavity is produced, into which air penetrates; it is properly only in reference to these, that interglobular spaces can be spoken of. Many teeth, indeed, exhibit no interglobular substance, but they occasionally present the outlines of dentinal globules, in the form of delicate arched lines.

Dentine containing Haversian canals, the so-called "vaso-dentine" of Owen, which exists in many animals, is rarely found in man, and I am only acquainted with one case, observed by Tomes (1. c. p. 225), in which the vascular canals were numerous; on the other hand, in the dentine with irregular tubuli, which is formed in obliteration of the pulp cavity, we occasionally meet with scattered Haversian canals and 


\section{rounded cavities, like osseous lacunæ, constituting the so-called osteo- dentine of Owen.*}

* [Considerable discrepancies will be met with if we compare the various statements of authors who have described the ultimate structure of the dentine.

1. According to Retzius, the dentine contains cells, but these cells, in his view, are cavities analogous to bone lacuna, in which the dentinal canals terminate.

2. Mr. Nasmyth took a totally different view from this. The matrix, or, as he calls it, "interfibrous substance of the dentine," is, he says, composed entirely of cells; but these cells are solid bodies, lie between, and form the boundaries of the canals. His "cells" and those of Retzius had exactly as much, or as little relation to one another, as the "osteal cells" of Tomes and De Morgan (to which we have referred in the note to $\$ 107$, p. 335), have to osseous lacunæ.

3. Professor Owen likewise affirms that the dentine is made up of "cells"-his "dentinal cells,"-which, however, can hardly be identical with Czermák's "dentine globules," as stated in the text. We find it, in fact, somewhat difficult to understand what these "dentinal cells" are, inasmuch as we are unable to reconcile the various definitions of their nature which may be found in the "Odontography." In the first place, at p. 462, it is stated, in a note, that " the true dentinal or calcigerous cells include many tubes and intertubular spaces, and it is much more exact to say, that those cells contain a tubular structure, than that the interstitial space is cellular." In perfect accordance with this, we find, on referring to plate 123, fig. 1, which represents a section of human dentine, that the "dentinal cells" which are marked $d^{\prime} d^{\prime}$, are traversed by from seven to eleven dentinal tubules.

But at p. 463, the passage in which reference is made to this figure, runs thus: "the dentinal cells of the human tooth are subcircular, about $\frac{1}{3000}$ th of an inch in diameter. They seem most numerous from being most conspicuous near the periphery of the dentine, as originally described by me in the dentine of the Crocodile."

And in the Introduction, p. xlvi. we find: "The diameter of the dentinal or calcified primary cells of the pulp, is usually one-fourth or one-half larger than that of the blood-discs manifest in them."

Now, low is it possible, that a body $\frac{1}{30} 0^{\text {th }}$ of an inch in diameter, can have passing through it seven tubules, each of which is $\operatorname{Iot}_{0}^{\frac{1}{0}} \overline{0}_{0}^{\text {th }}$ of an inch in diameter? To say nothing of the circumstance, that these tubules are at the very least $100_{0}^{1} 0^{\text {th }}$ of an inch apart. Halve the actual diameters of the tubules and arrange them close together, and they will barely squeeze into $\frac{1}{30} \sigma_{0}$ th of an inch. We conclude, therefore, as the definitions and the figures of these dentinal cells are at variance, that we are not justified in making any definite statement about them.

4. Mr. Tomes asserts in his lectures, that the "intertubular tissue is itself made up of minute granules closely united," which pass into those of his "granular layer ;" an opinion which seems to us to be most nearly in accordance with fact. We may observe, that the dentine globules and interglobular spaces of Czermák, had been previously very carefully figured and described by Mr. Tomes, in his lectures, p. 45.

5. The views of Czermák are stated in the text. Mr. James A. Salter (On certain Appearances occurring in the Dentine, dependent on its mode of Calcification, "Quarterly Journ. of Mic. Science," vol. I., p. 252, 1853), has confirmed Czermák's results, and has added some very interesting observations of his own. He considers that the contour lines, which he prefers to call "contour-markings," may arise from various causes, not only from those pointed out by Czermák-curvings and lucal enlargements of the canals and interlobular spaces, but also from a difference in density without alteration of structure; and he states as a general law, that the curves of the contour markings are in proportion to the primary curves of the dentinal tubes at any particular spot, and cross them at right angles. No markings are more divergent than the outline of the tooth, and passing from within outward, they abut in succession upon the external surface of the dentine, under the enamel and crusta petrosa, in the form of granular patches. The outer extremities of these patches 


\section{§ 139. The Enamel, substantia vitrea, forms a continuous layer} investing the crown of the tooth; it is thickest upon the masticating surface, and gradually diminishes towards the roots until at last it terminates by a sharply-defined or sometimes slightly-notched border, ceasing sooner upon the contiguous surfaces of the crowns, than upon their inner and outer sides. The external surface of the enamel appears smooth, but is always marked by delicate, close, transverse ridges, among which more marked circular elevations may occur.* A delicate membrane, discovered by Nasmyth, and which I will denominate the cuticle of the enamel [Nasmyth's membrane, Trs.], $\dagger$ entirely covers, but is so closely

look like white rings on the surface of the tooth. They are composed of coarse globular dentine, and gradually thin out internally into mere streaks. When a tooth is macerated in acid, it may be broken up into cones (triangles in section), as Dr. Sharpey first indicated, formed by the normal dentine between the contour markings. In transverse sections, the cones becorne, of course, rings. Finally, Mr. Salter points out that the enamel is almost always imperfect opposite the "patches" at the outer ends of the contour lines.-TRs.]

* [Czermák (1. c. pp. 4,5) states that the fine regular annular ridges and furrows upon the surface of the enamel, characterize the permanent teeth, and are not present upon the enamel of the milk set. The ridges are closest at the margin of the crown, and most distant towards its centre, where they finally disappear. In the space of a line, there were, at the margin of the enamel, 84-72 ridges; more internally, 36-30; and where they began to be indistinct only 18-12.-Trs.]

$\dagger[$ We have ventured to substitute the name "Nasmyth's membrane," for that of the "cuticle of the enamel," used by Professor Kolliker, inasmuch as the latter term gives a false idea of the relations of this important structure, which is much more than a mere "cuticle of the enamel," and is in fact, as one of us has already shown (Huxley, On the Development of the Teeth, "Quarterly Journal of Mic. Science," vol. I. p. 149, 1853), the calcified membrana preformativa of the whole pulp.

This structure was first described, in its true relation to the dental tissues, by Mr. Nasmyth, in a memoir read before the Medical and Chirurgical Society, in January, 1839, and which, illustrated with very good figures, was published in the twenty-second volume of the Society's Transactions (p. 310-328). Mr. Nasmyth states, that his attention had been drawn to fragments of a membrane which he found floating in the acid in which teeth had been macerated; "after a minute and careful examination, however, I was able to demonstrate with the greatest certainty, that they were derived from the external surface of the enamel, and that they were continuous with the structure covering the fang, which latter is itself continued into the chamber of the tooth. I afterwards succeeded in tracing this covering on the whole surface of the enamel and fang of the tooth in one continuous envelop; and eventually, I was enabled to remove it from the crown of the tooth in the form of a distinct coat or capsule; this covering, which I proved to exist externally to the enamel, I have termed 'the persistent dental capsule," " p. 312.

"In all cases where this covering has been removed by means of acid, it has, of course, the appearance of a simple membrane, in consequence of the earthy deposits having been dissolved, and of there being only present the animal tissue. The structure and appearance of the covering detached in this manner from the enamel, are the same in every respect as those observed in the capsule of the unextrucled tooth; consisting, like it, of two layers, fibrous externally, and having on its internal surface the peculiar reticulated appearance common to both, and shown at Plate V. Fig. 6," p. 313.

"On examining carefully fine sections of several teeth under the microscope, I perceived here also, that the structure in question was continuous with the crusta petrosa of the fang of the tooth," p. 313. 
united with, the enamel, that it can be demonstrated only by the use of hydrochloric acid. According to Berzelius and Retzius, * a similar membrane exists between the internal usually irregular surface of the enamel, and the dentine, but $I$ have been unable to find it. The enamel is bluish, transparent in thin sections and much more brittle and harder than the other dental structures, so that it is hardly touched by the knife, and yields sparks with steel (Nasmyth). Chemically, it may be regarded as osseous substance, containing the smallest possible proportion of organic matter; but whether the latter belong to the class of collagenous substances or not is uncertain. According to Von Bibra, the enamel contains:-

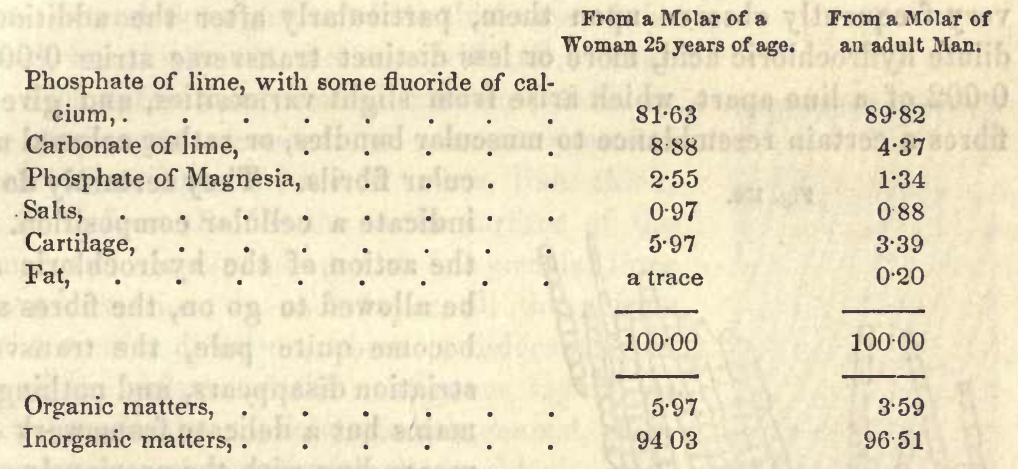

The enamel, as its fibrous structure indicates, consists entirely of the so-called prisms or fibres of the enamel (Fig. 189); long, solid prisms

Mr.. Nasmyth does not distinguish quite clearly in the text, between the proper capsular membrane on the fang and the crusta petrosa itself, though his figures (Pl. 5, Figs, 4, 5) exhibit the two structures as sufficiently distinct, and he hesitates, at p. 316, to decide what relation the outer layer of pale yellowish or brown substance in the cement of the teeth of the Elk, Ox, Bradypus, \&c., may have to the " persistent capsule."

We have not retained Mr. Nasmyth's own term for his discovery, because, as one of us has endeavored to show (Huxley, l. c.) while he accurately described its relations to the other dental tissues, he mistook its true nature. (See Appendix, $\$ 8$, on the Development of the Teeth.) But, on the other hand, as no one has, before or since, distinctly described his "persistent capsule," we have thought it desirable that his name should be associated with the structure.-Trs.]

* [Berzelius and Retzius obviously saw Nasmyth's membrane (Retzius, in Müll. Archiv, 1837, pp. 53, 54). The latter says, that on macerating a large piece of enamel from the fossil tooth of a horse (dug out of a peat-moss) in dilute acid, he found after the enamel was dissolved, a membrane swimming in the fluid, "I examined it immediately under a considerable magnifying power; it appeared to be pierced by a multitude of closely arranged little holes, but exhibited no trace of fibres." But he states expressly, that this membrane was "internal to the enamel fibres, as Berzelius clearly points out," and therefore failed to recognize its true relations with the enamel.-Trs.] 
of $0.0015-0.0022$ of a line in breadth, irregular in shape, but commonly hexagonal or pentagonal, which usually occupy the

Fig. 189.

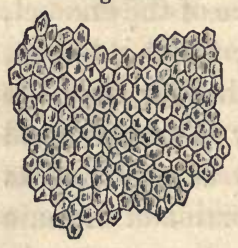
whole thickness of the enamel, resting with one extremity upon the dentine, and with the other upon Nasmyth's membrane. In adult teeth, these elements may be very readily detected in transverse and longitudinal sections, but can hardly be isolated for any great length; it is otherwise in young or developing teeth, where the enamel is much softer and may be cut with a knife. In such isolated prisms, whose broken ends may by accident appear pointed, whence they have been called "enamel needles," the surfaces and edges may be very well seen. We may also very frequently observe upon them, particularly after the addition of dilute hydrochloric acid, more or less distinct transverse striæ $0.0014-$ 0.002 of a line apart, which arise from slight varicosities, and give the fibres a certain resemblance to muscular bundles, or rather colossal mus-

Fig. 190.

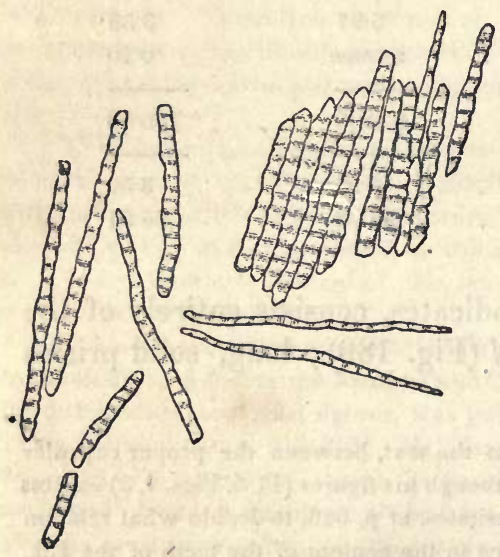
cular fibrils. They certainly do not indicate a cellular composition. If the action of the hydrochloric acid be allowed to go on, the fibres soon become quite pale, the transverse striation disappears, and nothing remains but a delicate framework corresponding with the previously solid fibres, and which often presents certain appearances of tubes. In the end this also becomes almost completely destroyed by the action of the acid, so that in teeth which have been thus macerated hardly anything remains of the enamel, which

does not, like the dentine, retain its form.

The prisms of the enamel are united very intricately without any intermediate substance. I have not been able to convince myself that canals constantly exist between the prisms, * but it is certain that cavi-

FIG. 189.- Surface of the enamel, with the ends of the enamel prisms, magnified 350 diameters. From the Calf.

FiG. 190.-Enamel prisms isolated, after the slight action of hydrochloric acid; magnified 350 diameters. From Man.

* [With respect to this point, opinions differ; Todd and Bowman consider that canals normally exist between the enamel prisms. Tomes finds canals in the enamel prisms of young animals, and sometimes in a part or the whole length of them in old teeth. Kolliker (Mikr. Anat. 77) has not yet observed any such cases. Czermák (1.c. p. 13) believes that, in a few cases, he has observed "very numerous delicate enamel tubules arranged in close series."-Trs.] 
ties of various kinds may be not nufrequently found in the enamel. Such, for instance, are-1. The continuations of the dentinal canals into the enamel, to which reference has been made above, with the elongated cavities at the border of the dentine which arise from their expansion (Fig. 191, c); and 2. The cleft-like gaps in the middle and external portions of the enamel (Fig. 191), which are not in communication with the preceding, are never entirely absent in any enamel, and often occur in very great numbers, as narrower or wider spaces which, however, never contain air.

The general course of the prisms of the enamel resembles that of the dentinal canals of the crown of the tooth, but extensive flexures are only to be met with towards the masticating surface. Most of the prisms extend through the whole thickness of the enamel, but this is not the case with all. They also decussate in a peculiar manner; thus, in some transverse sections, we observe annular layers of prisms, $0.08-0.12$ of a line thick, extending from the dentine to the surface of the enamel, and corresponding with fine circular lines upon its exterior; in each layer, all the prisms take a similar. direction, which is different from that of the prisms of the contiguous layers, so that perpendicular sections of such enamel, especially when moistened with hydrochloric acid, have a very singular striated appearance, arising from the dark transverse, and clear longitudinal sections of the prisms being alternately presented to the eye.

A similar decussation of the prisms occurs constantly at the masticating surface, and here the layers of enamel take a generally annular arrangement, so that they describe circles, in the molars, and ellipses in the incisor teeth; however, towards the centre of the masticating surface, irregularities occur which we are not yet in a condition to explain. Care must be taken not to confound the colorless strice which indicate these peculiar arrangements of the enamel fibres, with certain brownish lines or colored streaks which cross the prisms in various directions, and in perpendicular sections appear like oblique ascending lines or arches (Fig. 187); in transverse sections, like circles in the external layers of

Fig. 191.-Dentine and enamel, magnified 350 diameters; Man : $a$, cuticle of the enamel (Nasmyth's membrane); $b$, enamel prisms with transverse markings and interposed clefts; $c$, larger cavities in the enamel; $d$, dentine. 
enamel, rarely extending through its whole thickness. These I regard as the expression of the lamellated development of the enamel.

Nasmyth's membrane is a calcified, structureless membrane, $0.0004-$ 0.0008 of a line thick, distinguished by the great resistance it offers to chemical reagents, and its consequent appropriateness as a defence for the crown of the tooth. It is not altered by maceration in water, and is not dissolved by boiling in water, concentrated acetic acid, hydrochloric acid, sulphuric acid, and nitric acid; the latter only renders it yellow. In caustic alkalies it remains unchanged. Boiled with caustic potassa and soda, it becomes white and somewhat disintegrated, but still forms a connected mass; the potassa is rendered slightly turbid by the addition of hydrochloric acid, but clears with an excess. The membrane burns with an ammoniacal odor, and yields a calcareous spongy coal.

$\S 140$. The Cement, substantia ostoidea, cementum (Fig. 185), is a cortical layer of true osseous tissue, which covers the fangs, and in the many-fanged teeth, not uncommonly unites them all together. It commences where the enamel ceases, as a very thin layer, either simply abutting upon, or slightly overlapping it, increases in thickness lower down, and finally attains its maximum at the ends of the fangs, and on the alveolar surface of the molar teeth between the fangs. Its internal surface is, in man, very intimately united with the dentine, but without any connecting substance, so that frequently, at least under high powers, the limit of the two structures is not altogether sharply defined. The external surface is very closely surrounded by the periosteum of the alveolar cavity, but is not so firmly united with the gum; after the removal of these soft parts it is usually irregular, and is frequently marked with annular striations. The cement is the least hard of the three dental tissues, and is, chemically, almost identical with bone. Von Bibra found:-

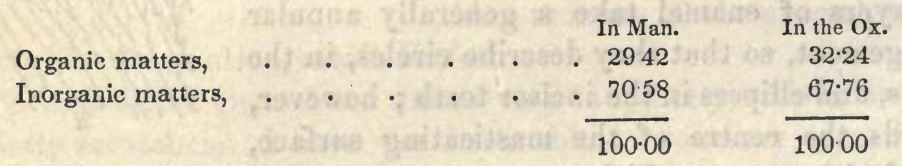

The latter, thus composed:-

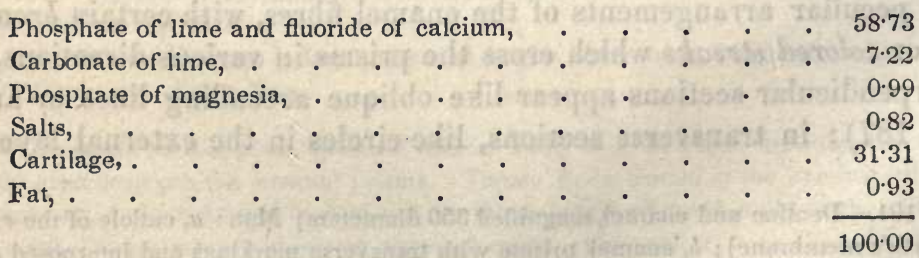

The earthy salts are readily extracted from the cement by acids, a 
white cartilage remaining, which may easily be separated from the dentine, and usually, when boiled, yields gelatin.

Like bone, the cement consists of matrix and of lacunoe, but rarely contains Haversian canals and vessels. Besides these, peculiar canals analogous to those of the dentine are found, and other more abnormal cavities.

The matrix is sometimes granular, sometimes transversely striated, sometimes amorphous, and frequently laminated like bone. The lacunce Fig. 192.

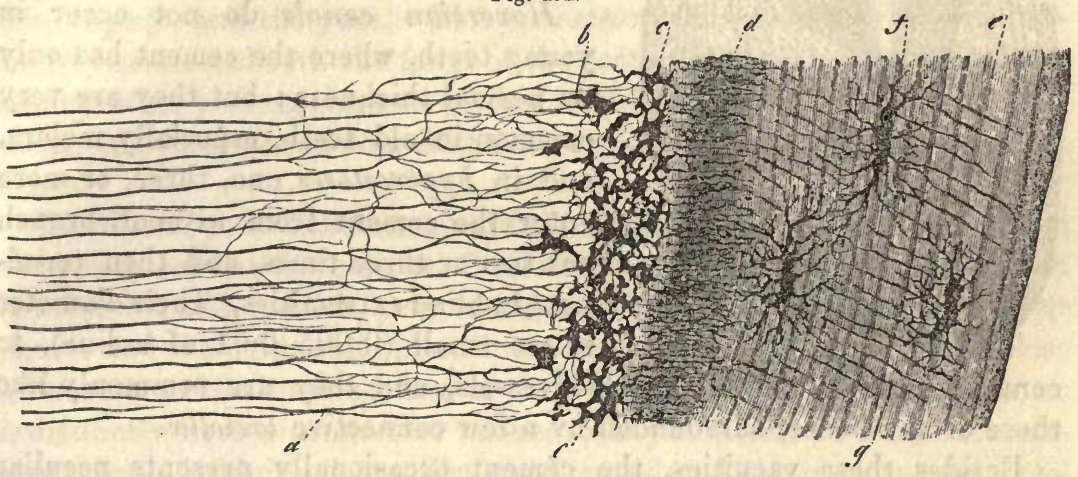

resemble in all essential characters those of the bones, so that any detailed description may be dispensed with. They are distinguished only by the great variety which they present in number, form, and size $(0.005-$ 0.02 , even to 0.03 of a line), and the unusual number and length (as much as 0.03 of a line), of their canaliculi. The majority are oval, and lie parallel to the axis of the tooth, others are rounded and pyriform. Those are most remarkable which have a very elongated form, "together with a narrow, canal-like cavity (Fig. 185), inasmuch as their resemblance to the dentinal canals is unmistakable. The canaliculi often resemble feathers and brushes, and unless the lacunce are altogether isolated, connect them with one anther, and anastomose with the ends of the dentinal canals. In the thinnest part of the cement, towards the crown, the lacunce are invariably absent; they are first met with, as a rule, about the middle of the fang, but are here scattered and solitary; towards the extremity their number gradually increases, and they not unfrequently take on a very regular arrangement, as in the external layer of the long bones, lying in series in the layers of the cement, and sending most of their canaliculi inwards and outwards, so as to give rise to an even, fine, transverse striation of the cement. The thicker layers of

Fra. 192.-Dentine and cement from the middle of the fang of an incisor tooth: $a$, dentinal canals; $b$, interglobular spaces, having the appearance of osscous lacunce; $c$, smaller interglobular spaces; $d$, commencement of the cement, with many canals close together; $e$, its lamella; $f$, lacuna; $g$, canals; from Man. Magnified 350 diameters. 
cement which occur in old teeth, present immense quantities of lacunæ, but these are to a great extent irregular, and have mostly the elongated

Fig. 193.

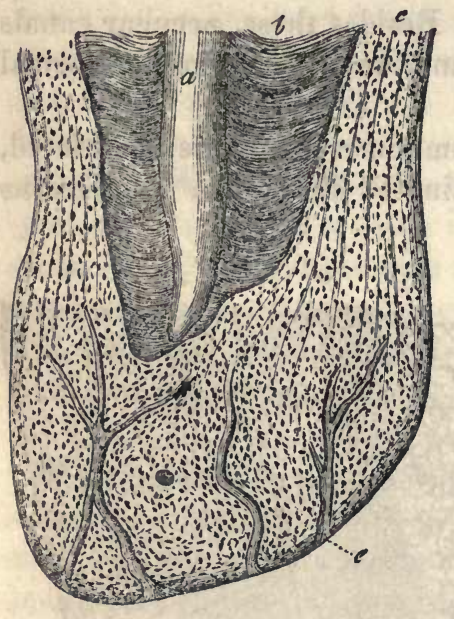

form. Many lacunæ are bordered, singly or in groups, by a very distinct, clear, yellowish, slightly undulated margin, which partially or entirely surrounds them; it has perhaps some relation to the cells from which the cavities are developed.

Haversian canals do not occur in young teeth, where the cement has only its normal thickness; but they are very common in old teeth, especially molars, and in hyperostoses one, three, or more enter the cement from without, branch out two or three times, and then terminate in blind extremities. Their diameter is too small $(0.005-0.01$ of a line $)$, to contain medulla as well as bloodvessels, and they are commonly like those of the bones, surrounded by a few connective lamelloe.

Besides these vacuities, the cement occasionally presents peculiar sinuous cavities, which are certainly pathological products (see "Mikr. Anat." II. 2, p. 82, Fig. 202); and frequently canals, like dentinal canals (Fig. 192), which are sometimes closely set, at others more isolated, occasionally ramified, and very frequently connected with the ends of the dentinal canals, and with the canaliculi of the osseous lacunce.

In the cement of the Solipedia, the osseous lacunce with their canaliculi, of the innermost layers, are frequently enclosed within actual cells, as Gerber first pointed out. If such cement be macerated in hydrochloric acid, these cells may be readily isolated, and present the following characters, which are not unimportant in their bearing upon the nature of the lacunæ. 1. The lacunæ frequently occur in twos and threes in a single cell, exactly as I have seen in rickety bones. 2. The substance which immediately surrounds the cavities and their processes, is not so readily soluble in hydrochloric acid as the other parts of the thickened cell. In fact, while the cells appear generally pale, a dark notched body, which often contains a very distinct cavity, is very obvious in their interior; and as we see by comparing it with these lacunæ of the cement, the contours of whose cells are no longer visible, is nothing else than the innermost portion of the thickened wall of the original cell. In the

Fig. 193.-Cement and dentine of the root of an old tooth : $a$, pulp cavity; $b$, dentine; $c$, cement, with lacunæ; $e$, Haversian canals. From Man. 
last-mentioned lacunx, in fact, it is easy to demonstrate, by the aid of acetic acid, a special wall, which is at first thick, but subsequently becomes thinner; and occasionally such lacunæ, with walls which give off a few processes externally, may be isolated. These lacunæ are frequently empty, but in other cases they contain a substance which at first also resists hydrochloric acid, wherein, however, I could discover no nucleus.*

$\S 141$. The soft parts of the teeth are: the periosteum of the alveolus, the dental pulp, and the gum. The periosteum of the alveolus is very intimately connected with the fangs of the tooth, and has the same structure as any other periosteum, except that it is softer, contains no elastic element, and possesses an abundant nervous network, containing many thick fibres.

The dental pulp - the remains of the fotal dental papilla - arises from the periosteum at the bottom of the alveolus, enters the fangs, and, as a continuous, soft, reddish, very vascular and nervous substance, fills their canals and the pulp cavity in such a manner as to be everywhere in close adherence to the inner surface of the dentine. The pulp consists of an indistinctly fibrous connective tissue, totally destitute of the elastic element, but containing many dispersed, round and elongated nuclei; and except that it occasionally contains narrow bundles, almost like imperfect fotal connective tissue. A fluid nay be expressed from it which, like mucus, is coagulated by acetic acid and is not dissolved in an excess; and, similarly, the entire pulp is rendered whitish by acetic acid, never becoming transparent like perfect connective tissue. This substance constitutes the principal mass of the pulp; so far as the vessels and nerves extend; but upon its surface we find, immediately beneath a delicate structureless membrane, a layer of $0.02-0.04$ of a line thick, composed of many series of cells, 0.012 of a line long, 0.002-0.003 of a line broad, cylindrical or pointed at one end, with long narrow nucleolated nuclei of 0.005 of a line, arranged perpendicularly to the surface of the pulp like a cylinder epithelium. More internally these regular series are no longer recognizable, but the cells, without losing their close radial arrangement, are more intermixed, and pass, finally,

* [The structure of the cement on the fang of the still uncut molar of the calf, is very peculiar and instructive. It is here a white, friable substance, about $\frac{1}{4 \sigma}$ of an inch thick, bounded externally by a delicate Nasmyth's membrane. Its outer three-fourths are composed of straight parallel fibres, resembling those of the enamel, but $\frac{1}{60}$ of an inch long. The inner fourth consists of similar fibres inextricably interlaced, cemented into a mass by a calcareous deposit, and containing here and there, spaces or lacunæ, $\frac{16}{16} 60$ of an inch in length, as it were left among the fibres. This structure appears to become obliterated with age, as the cement on the fang of the molar immediately in front of this, which had cut the gum, had the ordinary appearance. (Huxley, l. c.)-Trs.] 
by shorter and rounder cells, without any sharp lines of demarcation, into the vascular tissue of the pulp. These cells corespond with the formative cells of the dentine, to be described presently, and they afford the materials for the deposits of dentine, upon the walls of the pulp cavity, which takes place even in the adult.

The vessels of the pulp are excessively numerous, whence its red color. 3-10 small arteries enter each pulp of a simple tooth, and ultimately form, as well in its interior as upon its surface, a loose plexus of capillaries, $0.004-0.006$ of a line in diameter, which also exhibits here and there upon the surface distinct loops from which the veins arise. The pulp appears to contain no lymphatics, but its nerves* are extremely abundant. Arising from the well-known nervi dentales, there passes into every fang a large trunk of $0.03-0.04$, and besides, as many as six or more, fine branches of $0.01-0.02$ of a line, which contain fibres of $0.0016-0.003$ of a line. They ascend at first without any considerable anastomoses and only giving off a few filarnents; but in the thicker part of the pulp they form a rich plexus, with elongated meshes and divisions of the nerve tubules, and so gradually break up into fine primitive fibres of $0.001-0.0016$ of a line. I am inclined to think their final termination is in loops, but I grant that so long as the primitive fibrils in those loops which unquestionably do occur, have not been traced from trunk to trunk, which no one has yet done, the subject is open to doubt.

The gum, gingiva, that portion of the oral mucous membrane which unites the alveolar margins of the jaw and the necks of the teeth, is a pale red vascular tissue, which is tolerably soft, but feels firm on account of the subjacent hard parts: it, attains, where it lies upon the teeth, a thickness of $\frac{1}{2}-1 \frac{1}{2}$ lines, and possesses papillæ of a considerable size (of $0.15-0.3$ of a line; in old people they even reach 0.7 of a line in length, and like the papilloe filiformes are covered with secondary papillæ), and a pavement epithelium, which, between the papillæ, has a thickness of $0.23-0.4$ of a line. I could find no glands upon the gum, but care must be taken not to confound with them certain rounded depressions of the epithelium of $0 \cdot 8-0.15$ of a line in diameter, with more cornified cells, which occur not unfrequently upon its upper portions.

\$142. Development of the Teeth.-The development of the twenty milk teeth commences in the sixth week of foetal life, by the formation of a groove in the upper and lower margin of the jaws, in which, up till the tenth week, twenty dental papillæ gradually make their ap-

* [The nerves of the alveolar periosteum and of the pulp, are particularly described by Czermák (1. c. pp. 27, 28).-Trs.] 
pearance. Partitions are now developed, so that each papilla soon lies in a special cavity. In the course of the fourth month, these cavities gradually contract, the papillæe at the same time assuming the forms of the future teeth, and finally they close up completely; this takes place, however, in such a manner that a little cavity is developed above each "tooth-sac," and thus "reserve sacs," in which the pulp begins to be developed in the course of the fifth month, are formed for the twenty anterior permanent teeth.

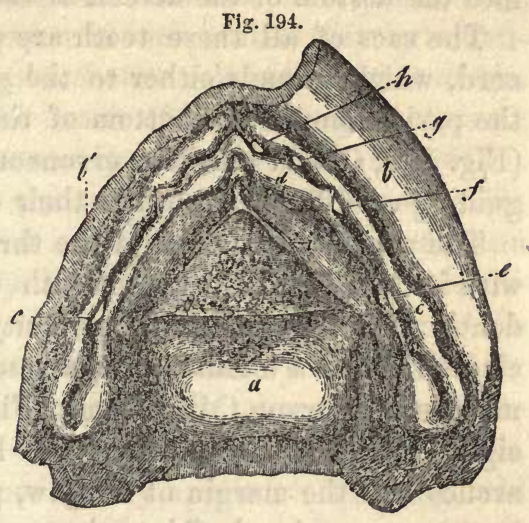

Fig. 195.
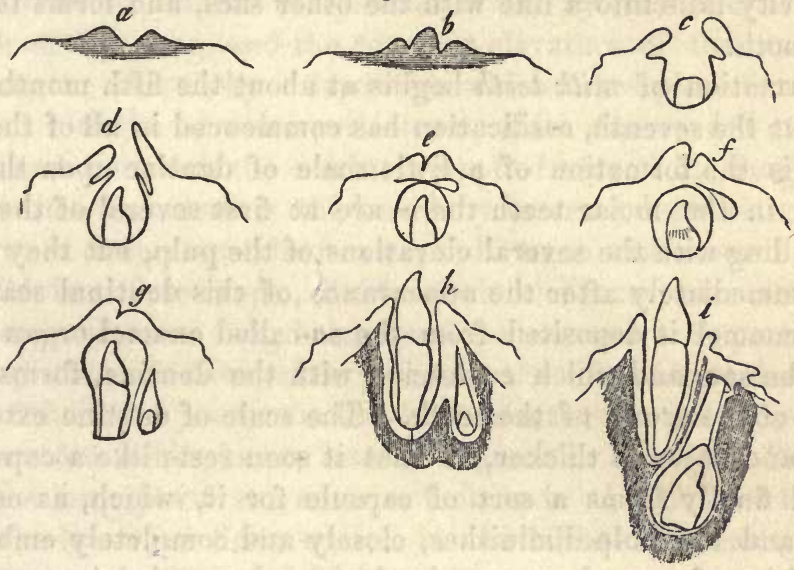

These "reserve sacs" at first lie above the milk sacs, but by degrecs they retreat backwards, and are received into hollows of the bony alveoli as they are formed round the milk teeth (Fig. 195, $g, h$ ). Those of the incisors and canines eventually become completely separated from the

FIG. 194.-Lower jaw of a human fœtus, nine weeks old, magnified 9 diameters: $a$, the tongue thrown back; $b$, right half of the lip depressed; $b^{\prime}$, left half of the lip cut off; $c$, outer alveolar wall; $d$, inner alveolar wall; e, papilla of the first molar; $f$, papilla of the canine; $g$, of the second; $h$, of the first incisor; $i$, folds where the ductus Riviniani subsequently open.

FiG. 195.-Diagram of the development of a milk-tooth, and of its corresponding permanent tooth, after Goodsir: $a$, dental furrow ; $b$, the same, with its papilla; $c$, the same beginning to close, with the rudiment of the reserve cavity; $d$, the same, still more closed ; $e$, tooth, sac completed, with a "reserve cavity ;" $f$, the reserve cavity moving backwards; $g$, the same become quite posterior, with a pulp; $h$, the alveoli of both sacs are forming, the milk-tooth has emerged; $i$, the permanent tooth forming, its deeper seated sac has a gubernaculum. 
alveoli of the corresponding milk teeth, but those of the premolars* open into the bottom of the alveoli of the deciduous molars.

The sacs of all these teeth are produced at their apices into a solid cord, which extends either to the gum, or, as in the two premolars, to the periosteum in the bottom of the alveoli of the two deciduous molars (Fig. 195, i), and has been erroneously described as a gubernaculum, or guiding cord for the teeth in their eruption.

The sac of the anterior of the three permanent molars, arises, together with its papilla, in the sixteenth or seventeenth week, quite independently, from the posterior extremity of the primitive dental groove, and closes in such a manner that a reserve sac remains between it and the mucous membrane (Mikr. Anat. Fig. 206). It is not till the seventh or eighth month after birth that the latter elongates behind the first sac, arches into the margin of the jaw, produces a papilla from its base, and becomes constricted off into the sac of the second molar. The remainder of the cavity falls into a line with the other sacs, and forms that of the wisdom tooth.

The formation of milk teeth begins at about the fifth month of foetal life, and at the seventh, ossification has commenced in all of them. The first step is the formation of a little scale of dentine upon the apex of the pulp; in the molar teeth there are at first several of these scales, corresponding with the several elevations of the pulp, but they soon coalesce. Immediately after the appearance of this dentinal scale, a thin layer of enamel is deposited from the so-called enamel organ upon the roof of the sac, and which coalescing with the dentine, forms the first rudiment of the crown of the teeth. The scale of dentine extends over the pulp and becomes thicker, so that it soon rests like a cap upon the pulp, and finally forms a sort of capsule for it, which, as ossification proceeds and the pulp diminishes, closely and completely embraces it; the deposition of enamel goes on simultaneously, so that it soon proceeds from the entire surface of the enamel organ, and becomes more and more

* [Instead of the loose phraseology "small" and "large" molars, \&c., we have adopted the philosophical nomenclature of the teeth, introduced by Professor Owen (see his Article on the Teeth, in Todd's Cyclopæedia), and thus explained by him: "Those teeth which are implanted in the premaxillary bones, and in the corresponding part of the lower jaw, are called 'incisors,' whatever be their shape or size; the tooth in the maxillary bone, which is situated at or near to the suture with the premaxillary, is the 'canine,' as is also that tooth in the lower jaw, which in opposing it, passes in front of its crown when the mouth is closed. The other teeth of the first set, are the 'deciduous molars;' the teeth which displaces them vertically, are the 'premolars :' the more posterior teeth which are not displaced by vertical successors, are the molars properly so called."

It results from this, that the so-called bicuspid and "first molar" of the permanent set in man (Professor Kölliker's "small molars") are the premolars; being, in fact, the third and fourth of the typical dentition; the first and second premolars and the third incisor of the typical dentition, not being developed in man. The nomenclature of the teeth, from being merely technical and arbitrary, has thus, by Professor Owen's recourse to development become scientific.-Trs.] 
considerable. In this manner, the whole enamel is eventually deposited around the dentinal layer of the crown, while the enamel organ and the pulp gradually diminish, until the former is represented only by a delicate membrane; and the latter presents similar relations to that of the perfect tooth. As yet there exists no trace of either fang or cement; they are not formed till the crown is nearly complete, and the tooth is about to emerge. About this time the pulp undergoes a considerable longitudinal growth, while the enamel organ becomes atrophied; and upon the newly formed portion only dentine, that of the fang, is developed. The tooth; thus forced upwards, begins to press against the upper wall of its sac, and the firm gum which is closely united with it; in which an independent process of absorption also takes place, and the tooth finally makes its appearance. The gum now contracts around it, and the rest of the dental sac becomes closely applied to the fang, and constitutes the alveolar periosteum.

The milk-tooth attains completeness: 1 , by the addition of the remainder of the fang, and the constant elevation of the crown to its normal length; and 2 , by a deposition which takes place from the sac, now united with the alveolar periosteum, which commenced even before eruption, and by which the cement is applied around the fang, while at the same time the tooth is thickened by internal deposition, the pulp diminishing to a corresponding extent. In teeth with several fangs, the pulp, which is at first simple, divides as it elongates, near its point of attachment, a separate fang being developed around each portion. The eruption of the milk teeth takes place in the following order: central incisors of the lower jaw in the 6th-8th month; central incisors of the upper jaw a few weeks later; lateral incisors in the 7 th-9th month, those of the lower jaw first; anterior molars in the 12th-14th month, those of the lower jaw first; canine in the 16th-20th month; second molars between the 20 th and 30 th months.

The permanent teeth are developed in precisely the same way as the milk-teeth. Their ossification begins, somewhat antecedent to birth, in the first molar, extends, in the first, second, and third years, to the incisors, canines, and premolars, and finally reaches the second molar; so that in the 6 th -7 th year there are 48 teeth co-existing in the two jaws, $i$. e., twenty milk teeth, and all the permanent set with the exception of the wisdom teeth (third molar). When the shedding of the teeth takes place, the bony partitions which separate the alveoli of the permanent from those of the milk teeth are absorbed, and at the same time the fangs of the latter gradually disappear from below in a manner which is not yet understood. The permanent teeth, whose fangs in the meanwhile have elongated, thus become placed immediately under the loosened crowns of the milk teeth, which finally, as the others protrude, fall out and make way for them. The permanent teeth emerge in the 
following order: first molar in the seventh year, inner incisor in the eighth year, lateral incisor in the ninth year, first premolar in the tenth year, second premolar in the eleventh year, canine in the twelfth year, second molar in the thirteenth year, third molar (wisdom tooth), between the seventeenth and nineteenth years.

The gum in the fotus, and especially in newly born infants before the eruption of the milk teeth, is whitish and very firm, almost cartilaginous, whence perhaps it has also been called gum-cartilage, although it has not the slightest resemblance to cartilage in structure, but consists of the ordinary elements of mucous membrane, but with a considerable admixture of a more tendinous tissue. The bodies of the size of milletseed, contained in it, described by Serres, the so-called glandulce
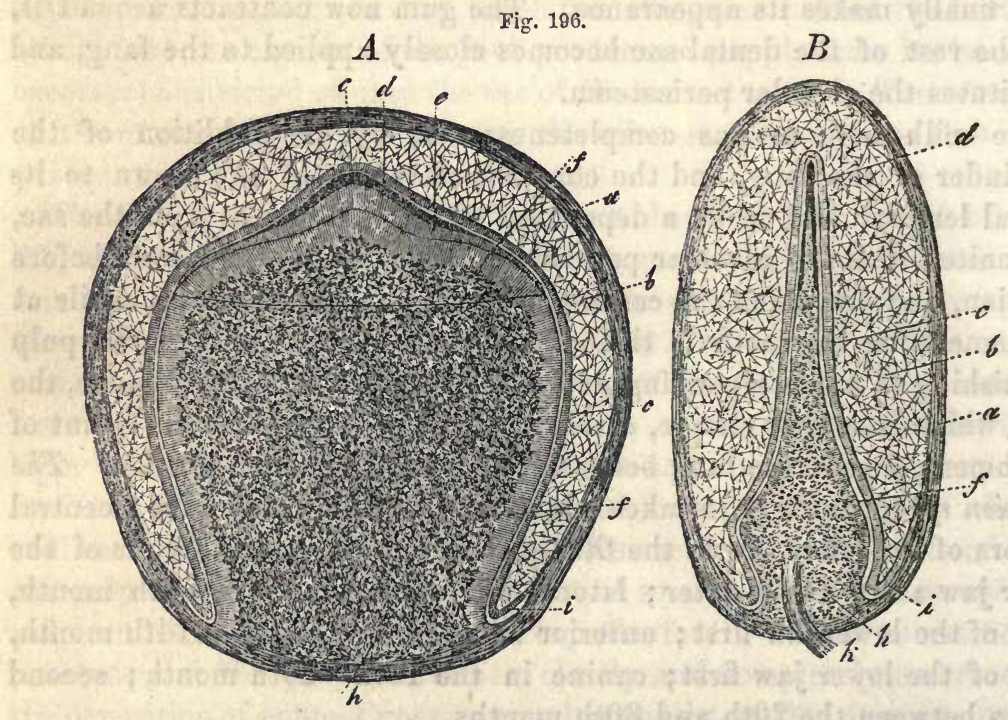

tartaricce, which are supposed to secrete the "tartar" of the teeth, are aggregations of epithelium, and are probably pathological* (see Mikr. Anat. II. 2, p. 92).

The dental sacs consist of connective tissue, in which ressels and nerves are distributed; from their base proceeds the dental pulp, which, in

FrG. 196.- $A$, tooth-sac of the second incisor of an eight-months' fuetus, from the broad surface, magnified 7 diameters : $a$, dental sac; $b$, enamel pulp; $c$, enamel membrane; $d$, enamel $; e$, dentine ; $f$, dentinal cells; $g$, limits of the cap of the dentine; $h$, dental pulp; $i$, free edge of the enamel organ. $B$, first incisor of the same embryo from the narrow surface: letters as before; $a$, dentinal cap in toto; $k$, nerves and vessels of the pulp.

* [They have a diameter of from $0.24-0.36$ of a line, and are composed throughout of numerous concentric layers of ordinary epithelial plates, or of softer scales, with cholesterine crystals and granules. Besides these, microscopic bodies of $0.02-0.12$ of a line, soft, and with only indications of lamination, are found in the gum (Kolliker, l. c.). The true rature of these glands was pointed out by Purkinje and Raschkow.-Trs.] 
form, resembles the tooth to which it belongs, and consists of an internal portion rich in vessels, and eventually in nerves also, and of a nonvascular external portion. The latter is bounded by a delicate structureless membrane, the membrana proformativa (Raschkow), which has no further relation to the development of the tooth. Beneath this, lie cells of $0.016-0.024$ of a line in length, and $0.002-0.0045$ of a line in breadth, with very beautiful, vesicular nuclei, and distinct, single or multiple nucleoli; they are arranged close together over the whole surface of the pulp (Fig. 197), like an epithelium, though not so sharply defined internally as it would be, but gradually passing, at least apparently, by smaller cells, into the parenchyma. In vascular pulps (Fig. 197), an additional boundary line may be traced, inasmuch as the capillary loops in which the vessels terminate, do not penetrate between the cylindrical cells, but end close to one another upon their inner surface; so that, considering that the dentine is produced by the cells in question, we might be justified in terming them the dentinal membrane, or membrana eboris. The internal portions of the pulp consist Fig. 197.

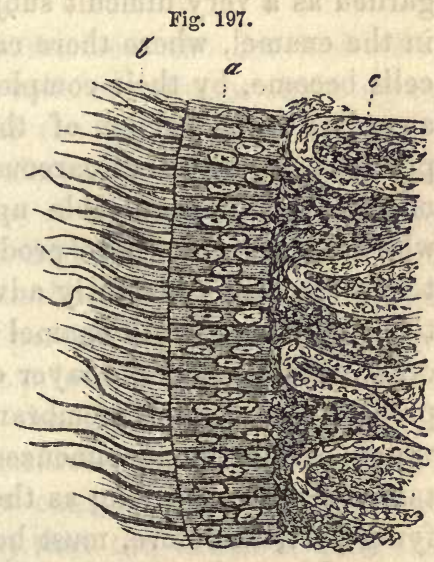
throughout of an originally granular or homogeneous, afterwards more fibrous matrix, containing many rounded or elongated nuclei, which must be regarded as a sort of connective tissue. Vessels are developed in great numbers in the pulp at the period when ossification commences; the most numerous perpendicular loops of capillaries of about 0.006 of a line existing in contiguity with the ossifying surface. The nerves accompany the vessels, but are developed later; their number is very considerable and their distribution resembles that in the pulp of the perfect tooth.

The enamel organ (organ adamantinoe), is applied to the pulp like a cap by its internal, concave surface, and is connected externally with the dental sac, in such a manner, however, that at the base of the pulp it presents a very small free edge. Its structure is very peculiar. The principal mass consists of anastomosing stellate cells (Fig. 196, b), or reticulated connective tissue, containing in its meshes a great quantity of fluid, rich in albumen and mucus. This gelatinous connective tissue is most abundant immediately before the commencement of ossification, and in its earliest stages. Thus, in the fifth and sixth months it measures $\frac{4}{10}-\frac{2}{3}$ of a line; in the new-born infant, on the other hand, only

FIG. 197.-Surface of the dentinal pulp of a newly-born infant: $a$, dentinal cells; $b$, their appendages; $c$, vascular part of the pulp; magnified 300 diameters. 
$0.16-0.2$ of a line. At this period it contains vessels in its outer third and its network is metamorphosed into true connective tissue (Mikr. Anat. ii. Fig. 211). On the inner side of the spongy tissue of the enamel organ, lies the so-called enamel membrane, membrana adamantince (Raschkow), a true cylinder epithelium, of which it need only be said that its cells measure 0.012 of a line in length, and 0.002 of a line in breadth, are finely granular and delicate, and possess nuclei frequently situated at the ends of the cells.

The development of the dental tissues has, hitherto, always been regarded as a very difficult subject. The simplest relations are presented in the enamel, where there can be no doubt whatever that the enamelcells become, by their complete ossification, the enamel-fibres. As soon as only a small portion of the cells has become ossified (without the previous deposit of calcareous matter in a granular form), a little scale of enamel is recognizable upon the somewhat larger cap of dentine, which has also just been produced. The deposition of calcareous matter in the cells constantly advances outwards, until at last they are entirely converted into enamel fibres, and extends at the same time to other cells, so that the layer of enamel increases in width. During this process, the enamel membrane does not disappear in the locality in which ossification commenced, but retains, there and elsewhere, the same thickness, so long as the deposition of enamel continues: its ossifying part, therefore, must be replaced by the incessant development of new substance, which takes place apparently, not by the apposition of new cells, but by the continual growth of the old ones. The enamel organ has assuredly some very important relation to the development of the enamel; probably serving by the abundance of albumen and of mucus in its meshes, as a storehouse, out of which the enamel membrane, distant as it is from bloodvessels, is enabled to draw the materials for its increase. In fact, the spongy tissue is seen to decrease more and more during the development of the enamel, and finally, when the enamel is complete, to disappear.

In the development of the dentine, as in that of the enamel, it is not the whole pulp which shares in the process, but only its most external, epithelium-like layer of cells, which appears to maintain a constant thickness by the elongation of the original cells, accompanied by a continual multiplication of their nuclei (Mikr. Anat. ii. 2, p. 103 et seq.) I by no means intend to assert that one and the same cell suffices for the whole duration of the development of the dentine, although this is not at all inconceivable; indeed, I consider it possible that the dental cells are from time to time replaced by others, which are formed upon their inner surface; but what I deny is, that the whole pulp is simply changed progressively, from without inwards, into dentinal cells and ossified, and I am of opinion that, like the spongy tissue of the enamel organ, the 
only import of the pulp in the development of the dentine is to support the vessels which are necessary to enable the dentinal cells to grow at all.

The diminution of the pulp, therefore, is very readily intelligible without supposing it to be ossified from without inwards; it takes place, like the diminution of the contents of the wide Haversian canals of foetal bones when the lamellæ are deposited upon their walls, by a gradual resorption of its tissue, which, as in the latter case, is soft and full of juices; and it is by no means necessary to suppose any extensive retrogressive metamorphosis of its vessels.

With regard to the formation of the dentine from the dentinal cells, it is certain that no other tissue than these cells contributes anything to its development and that they, like those of the enamel membrane, become dentine by the gradual reception of calcareous salts. The dentinal tubules are either the remains of the cavities of the dentinal cells, whose walls, in the course of ossification, thicken and harden into them, but do not quite close, or they are developed from the elongated and coalesced nuclei of the dentinal cells whose cavity persists; or finally, they are the result of a process of resorption in the primarily homogeneous dentinal tissue, analogous to the formation of the Haversian canals, or of those in the cement. Of these three hypotheses, the second would, at first sight, appear the most probable, if we consider that the dentinal tubules may be isolated, with distinct walls, that the dentinal cells are abundantly provided with nuclei, and that certain filiform prolongations of the dentinal cells which I have noticed (Figs. 9 and 197, and Mikr. Anat., II., 2, p. 105), might be regarded as elongated nuclei; but there is one very remarkable fact, that no trace of any elongation of the nuclei can be discovered by the most careful investigation. The third hypothesis is indeed conceivable, but in opposition to it, we find that pores and canals exist even in the youngest and softest dentine, when the development of the tooth is at all advanced, and therefore, that they can hardly be regarded as secondary formations. In favor of the first supposition, on the other hand, it may be said, that it would, if true, indicate a close agreement between dentine and osseous tissue, structures which are in every case nearly allied, inasmuch as the dentinal tubules would be homologous with long and narrow, simple or possibly coalesced, osseous lacunce. Certain objections may be urged, which are not, perhaps, so important as they at first appear. These are, in the first place, that the dentinal canals have special walls and may be isolated as tubes, which might be regarded as demonstrative evidence that they are developed out of peculiar vesicular structures, either nuclei or cells; and secondly, that upon this supposition, the filamentous appendages to the dentinal cells are not so readily interpreted. But as regards the former, we have recently learnt that the osseous lacunoe and canaliculi may also 
be isolated, with special walls which are not those of the original cells, and the same is true of the Haversian canals, whence it would be conceivable that the walls of the dentinal canals also, although originally and genetically not special structures, might eventually become so. Since, again, the processes of the dentinal cells may be nothing else than the still soft part of the cells in which ossification is commencing, this first hypothesis may be regarded as having a certain claim to consideration, the more so as the osseous lacunce in the teeth frequently assume forms resembling those of the dentinal canals, often communicate with them, and, at least in animals, are interposed among them.

To sum up, it may be said, that in any case, the matrix of the dentine proceeds from the cylindrical cells investing the pulp of the tooth, which undergo a greater or less elongation, coalesce and ossify. The dentinal canals either arise from the nuclei of these cells, or are, and this at present appears to me to be more probable, the remains of the cavities of the cells, whose boundaries have undergone a greater consolidation, and therefore correspond with osseous lacunce. The divisions of the canals are explained, if we conceive, either that the dentinal cells divide longitudinally from time to time, as I believe I have actually observed, or that a cell coalesces with two of its predecessors. As to the more delicate ramifications, we can only suppose that they are formed by a secondary process of resorption in already formed dentine, like that which must be assumed to occur in the osseous lacunoe, to account for the anastomoses of their canaliculi, and their communication with Haversian canals; at least, I see no possibility, whatever view we take, of explaining their formation in any other way, without coming into opposition with well-ascertained facts. No such process as the thickening and ossification of dentinal cells accompanied by the formation of pore-canals can be observed, so that the fine lateral branches appear to be entirely of secondary origin.

In the course of the ossification of the dentine, at least in man, we find that the deposition of calcareous salts in the recently-formed, structurally characterized, though only slightly hardened dentine, takes place in such a manner that the whole appears to consist of isolated globules. These globules, which are visible not only at later periods, but in the earliest cap of dentine, and are best seen at the edge of the root of a large tooth viewed externally, eventually disappear if development proceed normally, calcareous matter being deposited between them, so that the dentine becomes quite homogeneous and clear; in the opposite case, they persist in greater or less number, and the spaces between them, which are nothing but the interglobular spaces above described, contain unossified dentine.

According to my observations, the development of the cement takes place from that portion of the dental sac which lies between the pulp 
and the enamel organ, and commences, even before the eruption of the teeth, contemporaneously with the formation of their fangs. About this time the dental sac elongates inferiorly, applies itself to the growing fang, yields, from its abundant vascular network, a soft blastema, in which nucleated cells are developed, and then ossification takes place. The cement, therefore, is not formed by the ossification of the sac itself. I met with the first traces of it in newly-born infants, in the form of isolated, elongated, or rounded scales, which were firmly attached to the dentine of the, as yet, very short fang, and looked exactly like the developing osseous substance in the cranial bones; the smallest exhibited distinct osseous lacunce and a faint yellow tinge, but were quite soft and transparent, passing at their edges into a clear cellular blastema; in the larger ones, the margins were similar, but the centre was darker and firmer, and in this way every stage of transition to actual bone was presented, without any granular deposit of calcareous matter. With the elongation of the fang, new osseous scales of this kind were formed and gradually coalesced from above downwards into a single layer, to which continual additions were made from without, until the whole thickness of the cement was produced.

I am unacquainted with the Inanner in which the Nasmyth's membrane is produced. No structureless layer exists upon the enamel organ, by the ossification of which it might be supposed to be formed, and therefore I should be inclined to regard it as a calcified, amorphous exudation secreted from the enamel organ immediately after the ossification of the last enamel cells, which glues together and protects the ends of the prisms of the enamel.

If we now, in conclusion, take a general view of the different structures in the teeth and their mutual relations, we perceive that although they agree in certain respects, yet they cannot be brought under one class. Dentine and cement are much more closely allied to one another, than to enamel, and should it prove to be correct that the dentinal canals are formed by the coalescence of the cavities of thickened, elongated cells, the dentine will correspond with an osseous tissue, whose matrix is constituted only by the thickened walls of the original cells, and whose lacunce are all directly connected. Cement, or bone and dentine, often have a very close external resemblance to one another, particularly, on the one hand, when the latter is traversed by numerous Haversian canals, and, as Retzius believes he has observed, contains osseous lacunæ; and, on the other hand, when, in bone, the lacunæ are either greatly elongated, with numerous canaliculi, vascular canals also existing; or when with few lacunoe, the canaliculi are numerous and parallel, like dentinal canals. This much is certain, that the two substances never become exactly alike, and it is probable that their development is always to a certain extent different. 
The enamel may be best compared with a dentine whose cells are ossified throughout, and which, therefore presents no canals, like that in the outermost layers of fishes' teeth; at least the two substances agree in this, that they are entirely composed of elongated cells without any connecting matrix. When canals occur in the enamel, it acquires a very great similarity to dentine; but these canals probably have a totally different import to those in the dentine, viz.- - that of cavities which proceed from absorption. With the cement, the enamel has, in general, no analogy, though there is a kind of homogeneous cement with an indistinct transverse striation which, at least externally, looks somewhat like enamel, but has hardly, like the latter, arisen from elongated cells. If we consider the nature of the parts from which the various substances are developed, the dentine, formed from the vascular part of the mucous membrane of the mouth, is a true product of the homologue of the derma (schleimhaut-production), the enamel an epithelial structure, and the cement an investing substance, afforded by the mucous membrane.

$\S$ 143. The substance of the perfect tooth, though hard, is by no means incapable of molecular change, as its various diseases best show. The functions of the lacunce and their canaliculi in the bones are here performed by the dentinal canals with their ramifications, the lacunce and canaliculi in the cement, and the fissures between the prisms of the enamel. All these cavities, during life, contain a fluid, derived on the one side, from the vessel of the pulp, on the other, from those of the alveolar periosteum, and permit of changes in the substance, though they may be slow. Nothing definite, however, is known about the latter, but from the circumstance that perfect dentine is not colored when an animal is fed with madder (Hunter, Flourens, and others; compare Henle, p. 878), it may be concluded, that they are far less active than in the bones, and perhaps take place in such a manner that the calcareous matters are not at all or only very slowly renewed. The dentine is undoubtedly best provided with fluid supplies, from its being penetrated by very numerous and frequently anastomosing canals. We can as little suppose any regular circulation in it as in the bones; but it may be assumed that a certain movement takes place, proportionate to the :amount of the exudative and absorptive processes in the pulp, of the waste in the tooth itself, and of the supply afforded to the enamel and cement and probably given off from the latter tissues externally. Though the enamel is not impermeable, it permits of the passage of fluids with difficulty, as is best shown by the circumstance that the nerves of the dental pulp are not affected by acids, so long as the coating of enamel is entire, but readily enough, when, as in the incisors, the dentine is exposed. The enamel, again, is the hardest dental tissue, possesses scarcely any organic matrix and no constant systems of canals. Nasmyth's 
membrane, which is attacked with so much difficulty by chemical reagents, is, very probably, still more impenetrable than the enamel itself, and hence these two substances serve admirably to protect the teeth. The sensibility of the teeth arises from the nerves of their pulp; they are affected by contact, heat, cold, and chemical agents. Slight mechanical influence can only act by the vibrations which they may communicate to the substance of the tooth and thence to the pulp; it is therefore the more remarkable that the teeth have a certain sense of locality, so that it is possible to distinguish whether they are touched internally or externally, above or below, on the right or on the left side. The sensibility of the teeth is indeed tolerably delicate, especially on the masticating surface, where the smallest foreign bodies, as hairs, grains of sand, \&c., are perceived when these surfaces are rubbed against one another; and as regards its acuteness, it is, in disease at least, excessive, which is sufficiently explained by the considerable number of nerves in the pulp and the readiness with which they may be compressed within their hard receptacle.

With age the teeth become denser; the pulp cavity is filled with a kind of irregular dentine and may be totally obliterated, which is, perhaps, the normal cause of their falling out. In certain cases observed by Tomes, the fangs in old age were quite transparent, like horn.

The following remarks may be made upon the pathology of the teeth. Permanent teeth which have fallen out are sometimes replaced by a third dentition; but it must not be forgotten that the milk teeth occasionally remain beyond their time, and care must be taken not to confound a second tooth, late in its eruption, with a third. Teeth which have been extracted may be replaced (in fifteen months a canine tooth which had been extracted from the upper jaw was perfectly firm again). An abnormal development of the teeth takes place particularly in the ovarium, but also elsewhere. Fractures of the teeth may be reunited when they occur within the alveoli, by imperfect dentine or cement. Regeneration of the worn down parts takes place only in animals (Rodents, e.g.) in which the teeth constantly grow. Hypertrophy of the cement (the so-called exostosis), deposits of dentine in the walls of the pulp cavity and ossification of the pulp itself, are exceedingly common, and result from chronic inflammation of the periosteum and pulp.* A partial dis-

* [Wedl in his recent work (Grundzüge der path. Histol.) has added some very interesting observations to our knowledge of the structural changes occurring in the different parts of teeth. In hypertrophy of the cement he observed the canaliculi dilated, so as to form Haversian canals, and agrees with Kolliker (vid. \$140 supra) as to the frequency of the hypertrophy of the entire cement in old teeth. In partial hypertrophies of the cement he detected numerous dentinal globules, bounded by irregular fissures, and on their external border many bone-corpuscles. These latter were separated from each other by a yellowish intercorpuscular substance, and in many instances by peculiar sinuous cavities, which traversed the lamellæe of the osseous substance. 
appearance of the fang is not uncommon. Necrosis of the teeth takes place when the periosteum has been stripped off, or the pulp has died. The teeth become rough and dark, even black, and finally fall out. The nature and causes of dental caries are doubtful. It attacks living and false teeth (Tomes), and always begins on the exterior, from Nasmyth's membrane (Ficinus), whence the fluids of the mouth have been supposed to have considerable influence upon it; it does not follow, however, that one living tooth may not be more disposed to it than another, being rendered less capable of resistance either by its chemical composition, or by the mode of its nutrition. Caries, however, is assuredly not a simple solution of the salts by the oral fluids, but a solution accompanied by a putrefactive decomposition of the organic elements of the tooth, which becomes covered with infusoria and fungi; in fact, according to Ficinus's observations, the latter growths would appear to play the more important part, inasmuch as the decay of the teeth usually commences in those localities in which undisturbed opportunity is given to these organisms to develop, as in the cracks and pits of the enamel, in the depressions of the molar teeth, in the clefts between the teeth, but not where the dentine is otherwise exposed, as on the masticating surface, in filed places, \&c. The usual course of caries is, that the discolored spots of the cuticle of the enamel, covered with living and growing organisms (infusorial animalcules, similar to a Vibrio, which Ficinus calls Denticola, mucedinous fungi (Erdl, Klenke, Tomes), similar to those which are found upon the tongue, and which Ficinus wrongly refers to the Denticolce) first lose their calcareous salts, and then break up into angular, cellular pieces, as if they had been treated with hydrochloric acid. The decay then penetrates through the enamel to the dentine, always first softening it, so that it yields not more than 10 per cent. of ash (Ficinus), and then decomposing it. The dentine is more affected by this process than the enamel, its canal first becoming filled with the fluids proceeding from its decomposition, which may reach the pulp and give rise to pain, unless, as Tomes found, the dentinal canals in the neighboring healthy

The deposits of dentine in the walls of the pulp-cavity, the "osteo-dentine" of Owen, he regards as mainly originating from the dentinal globules, which to him are protein-bodies. This osteo-dentine partakes in some instances more of the nature of bone, than of dentine, but consists generally of a central substance and of tubules radiating from it; it frequently appears to be formed of concentric layers. The central substance Wedl describes as consisting either of hyaline dentinal globules, of a grayish, amorphous mass, or of distinct bone-corpuscles of varying shapes, separated by spaces resembling Haversian canals. The newly-formed tubules run from this central mass towards the dentine of the tooth, with the canals of which they communicate. Sometimes they are intersected in their course by the presence of many dentinal globules, or by irregular lacunæ. In all instances of these formations, that he has examined, the dentinal globules existed in great abundance. The dark color of the globules, wherever met with, Wedl is disposed to attribute to their retrogressive metamorphosis, whilst he regards the dark color of the interglobular spaces as dependent on a deposit of brown pigment in their interior.-DaC.] 
portions become obliterated by deposits, or the pulp is protected by new masses of dentine developed in the cavity* (Ficinus, Tomes). Eventually a brownish deposit takes place in the tubules and then the intermediate substance becomes completely broken up. In this manner the process of decomposition extends further and further, until at last the crown collapses, the root also becoming dissolved and finally falling out.

In jaundice, the teeth not uncommonly assume a yellow color, which is occasionally almost as intense as in the skin, and in asphyxiated persons they are said frequently to be red; both facts being explicable only by the supposition that the coloring matter of the bile and of the blood transudes into the dentinal tubuli. In rachitis the teeth remain unaffected. In the mucus upon the teeth, an abundant growth of the mucedinous fungi which have been mentioned, is always to be met with in a finely granular matrix, surrounding mucus- or epithelium-corpuscles; besides which we find the infusoria of carious teeth and the earthy deposits of the oral fluids. If this mucus accumulates, it hardens and forms the tartar of the teeth, which consists, according to Berzelius, of earthy phosphates $79 \cdot 0$, mucus $12 \cdot 5$, ptyalin $1 \cdot 0$, organic matter, soluble in hydrochloric acid, $7 \cdot 5$.

The best mode of examination of the teeth is by making fine sections and preparations softened in hydrochloric acid. To obtain good specimens of the former it is necessary to employ only young and fresh teeth, as the enamel otherwise readily breaks off. A longitudinal or transverse slice should be first taken off with a fine saw, and may then be rubbed down, first upon a coarser and then upon a finer stone, as thin as possible; the section should then be cleaned and polished between two glass plates, until its surface is as smooth and shining as it can be made, and finally washed with ether in order to remove any impurities it may have contracted. When well polished and dried, all the dentinal canals and lacunce will be filled with air, and the section may be preserved without further addition under a glass plate, cemented by some thick and quickly solidifying varnish. Such polished sections are preferable to any others, which, on account of their irregular surface, require to be covered with different fluids, as Canada balsam, oil of turpentine, \&c., in order to be examined by high magnifying powers. It almost always happens, in fact, that some portion of these fluids enters the dentinal tubules, which then become quite clear and indistinct and invisible in their finer ramifications. A very viscid varnish alone is of any service. In preparing these sections of the teeth, the slices may

* ["It is worthy of mention, also, that in the teeth of the hare, the sow, and the stag, especially in the molars, stony masses are constantly found. They are semi-transparent, for the most part oval and rounded bodies, situated in the axis of the dental pulp, towards its apex, in irregular rows, never extending the whole length of the dental pulp, but only to a greater or less distance from the coronal extremity." Raschkow, Meletemata, \&c., cited and translated in Nasmyth's "Researches" (1839), p. 139.-Trs.] 
also first be affixed to pieces of glass with Canada balsam, and then be rubbed down with a file and polished, on one side first, and then by warming the balsam and turning the section round, upon the other. When such a section has been washed with ether and dried, it is as good as one prepared with water only. Two sections made perpendicularly to one another through the middle of the crown and fang of a tooth, from before backwards, and from right to left, are sufficient to exhibit the most important features of the teeth; but sections ought also to be prepared, showing the surface of the pulp cavity and that of the enamel ; and also different oblique and transverse sections through the commencement of the dentinal canals of the fangs, to exhibit the anastomoses of their branches. The dental cartilage is easily demonstrable by maceration in hydrochloric acid, a process which requires a longer or shorter time according to the concentration of the acid and its more or less frequent renewal, taking 3-4 days in strong acid and in dilute, from 5-8. If it be desired to soften the tooth so much that the tubules may be isolated, it must be left for about eight days in concentrated hydrochloric acid; in thin sections of dental cartilage 12-24 hours' treatment with sulphuric and hydrochloric acid, and a few hours with dilute solutions of caustic potassa and soda, are sufficient for this purpose. It is very instructive also to macerate thin sections of teeth in acid and to examine them upon glass plates at intervals, until they entirely break up. The enamel prisms are readily isolated in developing enamel; the transverse lines are seen best when the object is moistened with hydrochloric acid, and the transverse sections of the prisms are seen exceedingly well in longitudinal sections, in some layers. The early development may be studied in embryos of two, three or four months with the simple microscope and in transverse sections of parts hardened in spirit; the structure of the dental sac, and the development of the dental tissues in foetuses of four, five, and six months, and in new-born infants, both in fresh subjects and, if it be desired to recognise the relations of the enamel organ, in spirit-preparations also, in which its structure is very well retained. The pulp of mature teeth is obtained by breaking them in a vice, and their nerves are best seen on the addition of dilute solution of caustic soda.

Literature of the Teeth.-L. Fränkel, "De penitiori dentium humanorum structurâ observationes," Vratislav, 1835 ; and Retzius, "Bemerkungen über den innern Bau der Zähnen," in Müll. "Arch.," 1837; J. Tomes, "A Course of Lectures on Dental Physiology and Surgery," London, 1848; R. Owen, "Odontography," London, 1840-45, 1 vol., with atlas of 150 plates; and article "Teeth," in "Cyclopædia of Anatomy," IV. p. 864 ; Krukenberg, "Zur Lehre vom Röhrensysteme der Zähne und Knochen," in Müll. "Archiv," 1849, p. 403 ; J. Czermak, "Beiträge zur mikroskopischen Anatomie der menschlichen Zähne, 
in Zeitschr. für. wiss. Zool." 1850, bd. II. p. 295 ; Arnold, in " der Salzbürger med. Zeitung," 1831, p. 236 ; Raschkow, "Meletematacirca dentium mammalium evolutionem," Vratisl. 1835; Goodsir, in " Edinb. Med. and Surg. Journal," 1838, No. XXXI. 1; and Froriep's "Neue Notizen," Nos. 199, 200, 202, 203; Marcusen, "Ueber die Entwicklung der Zahne der Säugethiere," aus dem "Bulletin Phys. Math." VIII., No 20, Petersburgh, 1850. On Dental Caries consult Erdl. in "Allg. Zeitung für Chirurgie von Rohatzsch," 1843, No. 19; Ficinus, in "Journal für Chirurgie von Walther and Ammon," 1846, p. 1; Klenke, "Die Verderbniss der Zähne," Leipsig, 1850. The Comparative Anatomy of the teeth is treated of microscopically in the works of Owen and Retzius above cited; also by Erdl, in the "Abhandlungen der Math. Phys. Klasse, der Königl. Bayer. Akad." bd. III. Abth. 2; Tomes, in the "Phil. Trans." 1849-50 (Marsupialia and Rodentia); Agassiz, in the "Poissons fossiles;" Henle and J. Müller, "Syst. Beschreibung der Plagiostomen," 1838.

[To these should be added: Blake, "Essay," \&c., 1801; Hunter, "Treatise on the Natural History and Diseases of the Human Teeth," edited by Thomas Bell (Works by Palmer, 1835, vol. ii.); Tomes, "On the Structure of the Teeth, the Vascularity of those Organs, and their relation to Bone," Proceedings of the Royal Society, June, 1838; Owen, "On the Structure of the Teeth, and the resemblance of Irory to Bone," British Association Reports, 1838; Nasmyth, "Medico-Chirurgical Transactions," 1839; "Proceedings of the British Association," 1839; "Researches on the Development, Structure, and Diseases of the Teeth," 1849 ; Huxley, "On the Development of the Teeth," "Quarterly Journal of Micr. Science," 1853; Salter, "On certain appearances occurring in Dentine," ibid. 1853.-Trs.]

\section{OF THE ORGANS OF DEGLUTITION.}

\section{THE PHARYNX.}

$\S 144$. The alimentary canal assumes a greater independence in the pharynx, acquiring a special investment of transversely striated muscles, the constrictores and levatores, which, however, do not entirely surround it and arise for the most part from bones. The thickness of the walls of the pharynx is about 2 lines on an average, depending principally upon this muscular layer, external to which there is a tense fibrous membrane, composed of connective tissue and elastic fibres, while internally it is separated by a layer of submucous connective tissue from the mucous membrane. The latter is paler than that of the oral cavity and its structure in the upper half of the pharynx differs considerably from that in the lower half. In the latter locality, that is, below the pharyngo-palatine arches, or in the region through which the food passes, it possesses a tessellated epithelium similar in structure and thickness to 
that of the oral cavity; above them, on the other hand, that is, on the posterior surface of the soft palate from its free edge, upon the upper surface of the uvula, in the region of the choance and Eustachian tubes, and upon the vault of the pharynx, there is a ciliated epithelium like that in the nasal cavity and larynx, to the description of which, below, the reader may be referred. In this upper or respiratory section, the mucous membrane is also redder, thicker, and more glandular than in the lower division, otherwise, however, its structure is pretty much the same, with the exception that it presents no papilla, which, however, in some parts of the lower division are very little developed and rare, and would even appear to be entirely wanting. Compared with that of the oral cavity I find the mucous membrane of the pharynx to possess much more and much stronger elastic tissue, which, in the deeper layers, forms connected, very dense, elastic membranes.

The pharynx contains two sorts of glands ; 1 , racemose mucous glands (vide supra, § 134), and, 2, follicular glands. The former $\frac{1}{3}-1$ line in diameter, have distinct apertures and abound more particularly in the upper portions of the pharynx, where they form a perfectly continuous layer on the posterior wall, in the neighborhood of the pharyngeal opening of the Eustachian tubes, and upon the posterior surface of the velum, diminishing in number lower down. Follicular glands, simple as well as compound, analogous to the tonsils, are met with in the vault of the pharynx, where the mucous membrane is closely attached to the base of the skull. Here a glandular mass, stretching from one Eustachian opening to the other, and from one to four lines thick, may constantly be met with; it is, upon the whole, smaller, but otherwise its structure resembles, in all essential respects, that of the tonsils ( $\$ 135)$. Besides this mass, whose largest sacculations are situated in the middle of the roof of the pharynx, and in the recesses behind the Eustachian apertures, and which, in aged persons, frequently present enlarged cavities, filled with puriform masses, there occur round the apertures of the tubes, and upon them, towards the choance, on the posterior surface of the velum palati, and on the lateral walls of the pharynx, as far as the level of the epiglottis, more or less numerous, smaller and larger follicles, whose size is too great for apertures of the mucous glands, and which have in all probability the same structure as the simple follicles of the root of the tongue, and receive the excretory ducts of the mucous glands.

The mucous membrane of the pharynx is rich in bloodvessels and lymphatics. The former constitute superficially a network with elongated meshes, sending short loops into the rudimentary papillæ. The nerves are very numerous, form superficial and deep plexuses, the former with fine fibres of $0.001-.0015$ of a line, which occasionally divide, and whose ultimate terminations escape the eye. 
II. THE CESOPHAGUS.

$\S 145$. The wall of the osophagus, $1 \frac{1}{2}-1 \frac{3}{4}$ lines thick, consists, externally, of a fibrous membrane composed of connective tissue, with exceedingly beautiful elastic fibres. To this succeeds a muscular membrane 3-1 line thick, composed of an external, longitudinally fibrous layer, having a thickness of 0.5 of a line, and of an internal, circularly fibrous layer of $0.24-0.3$ of a line, which are in close apposition. From the pharynx, where the longitudinal fibres arise in two bundles from the constrictor infimus, united with a third from the cricoid cartilage, they extend as far as the stomach, with whose muscles they are partly continuous. In the upper third of the oesophagus, as far as its entrance into the thorax, transverscly striated muscles alone are found, arranged in bundles of $0.04-0.24$ of a line, which sometimes distinctly anastomose. Further downwards smooth muscles of the same structure as those in the intestine (infra) make their appearance, in the first place in the circular layer, and subsequently, among the longitudinal fibres; the pro-

Fig. 198.

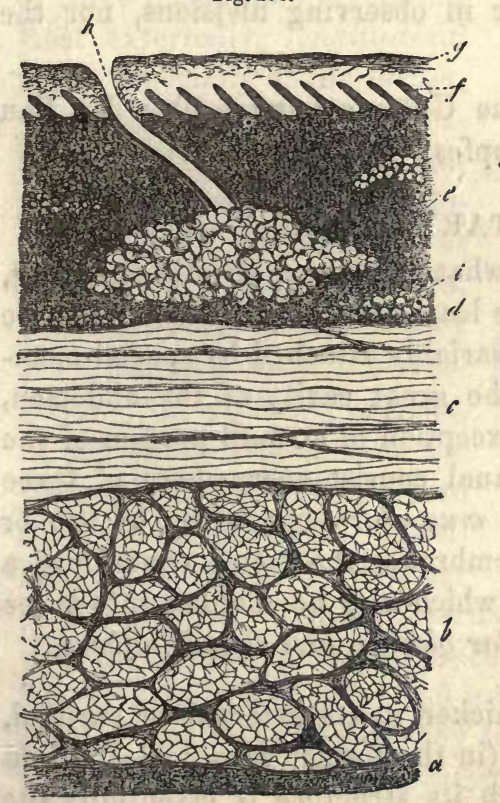

Fig. 199.

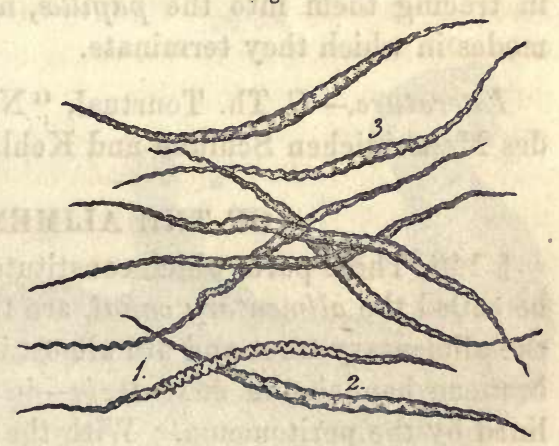

portion of these gradually increases, until at last, in the lower fourth, smooth muscle altogether predominates. Isolated, transversely-striated muscles, however, are, according to Ficinus, to be met with as far as the cardia. Most internally we find, separated from the muscular coat by a white, soft layer of sub-

mucous connective tissue (tunica nervea of the ancients), the pale-red mucous membrane, which below takes on a whitish tint. Its total thick-

Fig. 198.-Transverse section from the middle of the œsophagus (Man), magnified 1-2 diameters : $a$, fibrous investment ; $b$, longitudinal muscles; $c$, transverse muscles; $d$, tunica nervea $; e$, longitudinal muscles of the mucous membrane; $f$, papillæ; $g$, epithelium; $h$, aperture of a mucous gland; $i$, mass of fat.

Fic. 199.-Muscular-fibre cells from the csophageal mucous membrane of the Pig, after being treated with nitric acid of 20 per cent.; magnified 15 diameters. 
ness of $0 \cdot 36-0 \cdot 45$ of a line is due, to the extent of about $0 \cdot 1-0 \cdot 12$ of a line, to its laminated, tessellated epithelium, which presents the same structure as in the oral cavity, with the exception, however, that the actual epithelial plates constitute about a moiety of the whole, and, after a short maceration, or, as frequently happens in the dead subject, spontaneously, may be readily stripped off in large white sheets, either alone, or accompanied by adherent portions of the deeper layers. The proper mucous membrane, measuring on the average 0.3 of a line, possesses numerous conical papilloe of $0.04-0.05$ of a line in length, and consists of ordinary connective tissue, with fine elastic fibres, among which, however, as Brücke and I have ascertained, a great quantity of longitudinal bundles of smooth muscles, and in addition, more isolated groups of ordinary fat cells and small racemose mucous glands, may be observed.

The oesophagus is moderately provided with lymphatics and bloodvessels; the latter send loops into the papilloe and form at their bases, a not very wide network, like that in the pharynx. Nerves may also be met with in considerable numbers in the mucous membrane, containing fine fibres of $0.0012-0.0015$ of a line, but I have not yet succeeded in tracing them into the papilloe, nor in observing divisions, nor the modes in which they terminate.

Literature.-C. Th. Tourtual, "Neue Untersuchungen über den Bau des Menschlichen Schlund-und Kehlkopfes," Leipzig, 1846.

\section{OF THE ALIMENTARY CANAL.}

$\S 146$. Those parts which constitute what may, more strictly speaking, be called the alimentary canal, are the least fixed of all which compose the alimentary tract and are almost invariably attached by special membranous bands - the mesenteria -in the great cavity of the abdomen, lined by the peritonæum. With the exception of a small portion of the rectum, the walls of the alimentary canal consist everywhere of three tunics: a serous-the peritonoeum; a muscular-consisting of two or even three layers; and a mucous membrane, the latter containing a great number of glandular structures, which may be divided into three groups, racemose mucous glands, tubular glands, and closed follicles.

$\S 147$. The peritonoeum is much thicker in its external or parietal, than in its internal or visceral layer (in the former case $0.04-0.06$, in the latter $0.02-0.03$ of a line), though its structure is essentially the same in each locality. It consists principally of connective tissue with distinct, variously interwoven bundles, and abundant reticulated elastic fibres, which are coarser in the parietal lamina. A loose subserous connective tissue, containing more or less fat, unites the peritonceum with the other organs, or, as in the mesenteric folds, connects its layers together; under the visceral lamina, however, it is very little developed except in certain localities (colon, appendices epiploicoe), and in certain 
folds of the peritonceum, it does not exist at all. The free surface of both lamellæ of the peritonoeum is lined by a simple tessellated epithelium, whose slightly flattened, polygonal, nucleated cells measure, on the average, 0.01 of a line, they are so closely united and so constantly kept moist, that the free serous surface appears perfectly smooth and shining.

The peritonceum is, in general, but scantily supplied with vessels; they are most abundant in the omenta, in the visceral layer and in the subserous tissue, in which last alone, lymphatics have as yet been found. The nerves are also but few, and are especially to be met with in the omentum, the mesenteria, and hepatic ligaments, where they accompany the arteries.

$\S 148$. Muscular tunic of the alimentary canal.-The whole alimentary tract, from the stomach to the rectum, possesses a special muscular coat, which, however, does not everywhere present the same conditions.

In the stomach the muscular tunic varies in thickness; at the fundus it is thin ( $\frac{1}{4}-\frac{1}{3}$ of a line); in the middle, it has a thickness of about $\frac{1}{2}$ a line; in the pyloric region, finally, about $\frac{3}{4}$ or even 1 line. It consists of three incomplete layers: 1 , most externally, longitudinal fibres, especially at the cardia, where they arise from the expansion of a part of the longitudinal fibres of the cesophagus; and also at the pylorus and in the pars pylorica, whence, tensely stretched, they are continued upon the duodenum; 2, circular muscles, in the middle region, from the fundus to the pylorus; where they are accumulated, constituting the so-called sphincter Fig. 200.

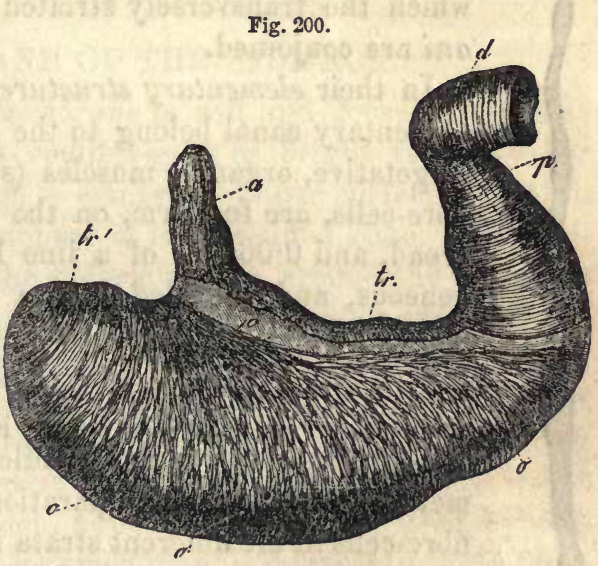
of the pylorus; 3 , most internally, oblique fibres, which, in connection with the circular fibres, embrace the fundus as in a sling, and run obliquely upon the anterior and posterior walls of the stomach, towards its greater curvature, where they terminate upon the outer surface of the mucous membrane or unite together.

In the small intestine, the muscular coat is somewhat thicker in the duodenum and the upper portions, than in the lower; it has, in general, a thickness of $\frac{1}{4}-\frac{1}{6}$ of a line, and is composed only of longitudinal and transverse fibres. The former are always less developed and do not

* Fig. 200.-Stomach of Man, reduced: $a$, œsophagus, with the longitudinal fibres ; $t r$, transverse fibres (second layer), for the most part dissected off; $t r^{\prime}$, transverse fibres of the fundus; o, fibra obliqua ; $p$, pylorus ; $d$, duodenum. 
form a continuous layer, since they are very few or entirely absent along the attachment of the mesentery; they are usually most distinct upon the free border, though even here they may be readily torn away with the serous membrane, so as, at once, to leave the second layer exposed. The latter is complete and continuous, consisting of circular bundles, which not uncommonly anastomose at very acute angles.

In the large intestine, the longitudinal fibres are reduced to the three ligamenta coli, muscular bands of $4-6$, or even 8 lines broad, and $\frac{1}{3}-\frac{1}{2}$ line thick, which commencing upon the ececum are united upon the sigmoid flexure, into a single longitudinally fibrous layer, which is continued upon the rectum. Beneath these bands there lies a continuous, circularly fibrous layer, thinner than in the small intestines and more especially developed in the duplicatures, which are known under the name of the plicoe sigmoidece.

Fig. 201. The rectum possesses a muscular layer of 1 line and more thick, in which the more abundant longitudinal fibres lie external to the circular. The ultimate, somewhat thickened extremity of the circular fibres is the sphincter ani internus, with which the transversely striated sphincter externus and levator ani are conjoined.

In their elementary structure, all the muscles of the proper alimentary canal belong to the so-called smooth or non-striated (vegetative, organic) muscles (see § 26). Their elements, the fibre-cells, are fusiform, on the average $0.002-0.003$ of a line broad, and $0.06-0.1$ of a line long, pale, flattened, and homogeneous, and provided with a nucleus $0.006-0.012$ of a line long, and $0.001-0.0028$ of a line broad.

Many of the fibres present knot-like enlargements and frequently zigzag flexures, which produce the transversely striated appearance of the entire bundles of such muscles so frequently met with in spirit preparations. The arrangement of the fibre-cells in the different strata is simply this ; mutually applied in their length and breadth and coherent, they are united into thin muscular bands, which then, invested with a coating of connective tissue and, frequently, also united into secondary bundles, constitute the thinner or thicker muscular tunics of the different regions; which, again, are surrounded and separated from the contiguous parts, by considerable layers of connective tissue.

Bloodvessels are very abundant in the smooth muscles; and their capillaries, of $0.003-0.004$ of a line, constitute a characteristic* network with rectangular meshes.

FIG. 201.-Muscular fibre cell from the small intestine (human).

* [Hardly characteristic; the vessels are arranged in precisely the same way in the fascial aponeuroses; e. g., the fascia lata of the thigh.-Trs.] 
Nothing is known about the lymphatics; nor are the relations of the nerves yet ascertained, except that Ecker has observed the division of

Fig. 202.

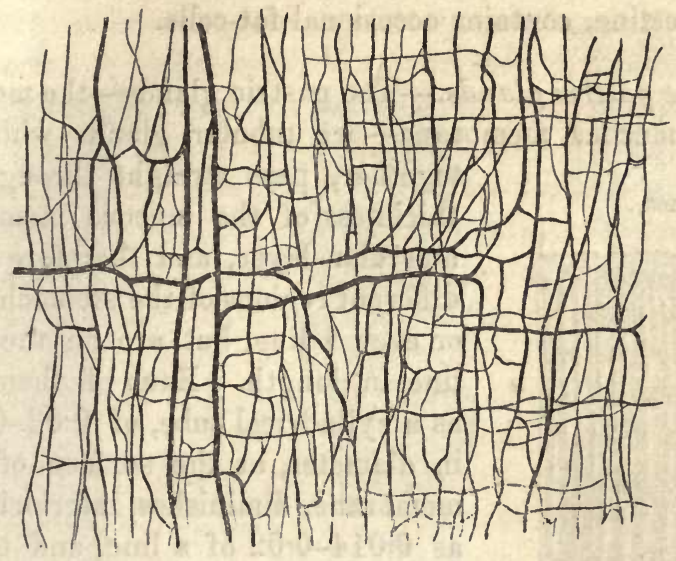

fine nerrous tubules in the muscular tunics of the stomach of the Frog and Rabbit.

\section{MUCOUS MEMBRANE OF THE STOMACH.}

$\S 149$. The gastric mucous membrane is soft and loose in texture; and its color, during digestion, is reddish-gray or bright red, at other times grayish. When the stomach is empty, the inner surface is thrown into longitudinal folds, which disappear in its distended state. Furthermore, it presents, especially in the pyloric region, around the apertures of the tubular or gastric glands, little reticulated folds or even isolated villi* (plicoe villosce, Krause) of $0.024-0.048$ or even 0.1 of a line, $\left(\frac{1}{30}-\frac{1}{20}\right.$ Krause). Not unfrequently, also, the mucous membrane is marked out especially upon the right side, by little shallow depressions, into slightly raised polygonal areæ of $\frac{1}{2}-2$ lines, the so-called "état mamelonne" of pathologists, which, however, is also exhibited by perfectly healthy stomachs. The mucous membrane is thinnest $\left(\frac{1}{6}-\frac{1}{4}\right.$ of a line), at the cardia, in the middle it becomes thickened to $\frac{1}{2}$ a line, and in the

Fia. 202.-Bloodvessels of the smooth muscles of the intestine, magnified 45 diameters.

* [These gastric villi have been recently accurately described and depicted by Dr. Neill, (vid. Amer. Journ. of Med. Sc., Jan. 1851.) By the aid of minute injections, Dr. Neill found that the ridges between the orifices of the tubuli, formed of convoluted capillaries, become larger and more elevated in the antrum pylori, and that as the pyloric orifice is approached, distinct, conical villi make their appearance, which he thinks, have not been previously described as constant by any of the writers on the subject. These villi are not as large as those of the small intestines, but, otherwise, similar in appearance. They are mainly composed of capillaries closely united by a basement membrane, and are covered with a cylinder-epithelium. In the antrum pylori, alveoli of different shapes exist in the interstices and at their bases. Whether they contain lacteals or not is doubtful.

The uses of these gastric villi have not as yet been ascertained. 1)r. Neill (1.c.) suggests, that they may be connected with the absorption of solutions of albuminous compounds. - DaC.] 
pyloric region to $\frac{3}{4}$ or 1 line, which depends entirely upon the glandular layer, since the epithelium and muscular layer everywhere possess the same thickness. The submucous tissue is abundant and, as throughout the whole intestine, contains occasional fat-cells.

$\S 150$. The gastric glands. - The gastric glands-the most important part of the mucous membrane-are tubular glands which, set close

Fig. 203.

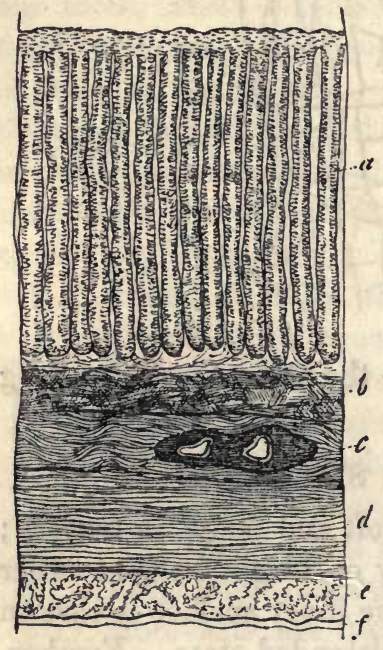
together, pass straight through the entire thickness of the mucous membrane to its muscular layer, and therefore vary, in the different regions of the stomach, from $\frac{1}{5}$ to $\frac{3}{4}$, or even 1 line, but are on the average $\frac{1}{2}$ a line in length. Each of them commences as a cylindrical tube, of $0.03-0.04$ of a line in diameter, at the surface of the mucous membrane, diminishes inferiorly to as little as 0.014-0.02 of a line, and terminates by a clavate or flask-shaped enlargement of $0.02-0.026-0.036$ of a line. The lower third of the glands is usually undulated or even twisted into a corkscrew shape, especially at the pylorus; occasionally it gives off a shorter or longer cæcal branch before its termination. Every gastric gland is surrounded by a delicate membrana propria and possesses in its upper third, a cylindrical epithelium continuous with that of the surface of the stomach; for the inferior three-fourths of its extent, on the other hand, it presents pale, finely granular, polygonal nucleated cells of $0.006-0.01$ of a line, which probably never constitute a distinct epithelium, but appear completely to fill the tubes.*

FIG. 203.-Perpendicular section through the tunics of the Pig's stomach, from the pylorus, magnified 30 diameters : $a$, glands; $b$, muscular layer of the mucous membrane; $c$, submucous tissue (tunica nervea), with divided vessels; $d$, transverse layer of muscles; $e$, longitudinal layer of muscles; $f$, serous membrane.

* [Professor Kölliker ("Verhandlungen der. Pliys. Med. Gesellsch. zu Würzb." vol. IV. 1, p. 52), has recently had the opportunity, in a case of suicide by drowning, of examining the human gastric mucous membrane in a fresh and normal state. Ho directed particular attention to the glandular apparatus, and found that the gastric glands are not as uniform in structure as above-described, but present three distinct types. 1. Simple tubular glands with peptic cells, ${ }^{1}$ (Lab-zellen). 2. Compound tubular glands with peptic cells. 3. Compound tubular glands with cylinder epithelium. The first are the most common; they occur in the middle zone of the stomach, and are generally simple tubular glands, some of which at their termination, give off short creal branches. The compound tubular glands with peptic cells occur in the narrow cardiac zone. They commence by a tube of 0.04 to $0.08^{\mathrm{mm}}$ long, 0.03 to $004^{\mathrm{mm}}$ broad, lined with cylinders. This divides first into two or three, and then into four or seven equally long cylindrical tubules, which 
In animals, the gastric glands are more complicated than in man, frequently presenting dichotomous divisions and subdivisions of their

Fig. 204.

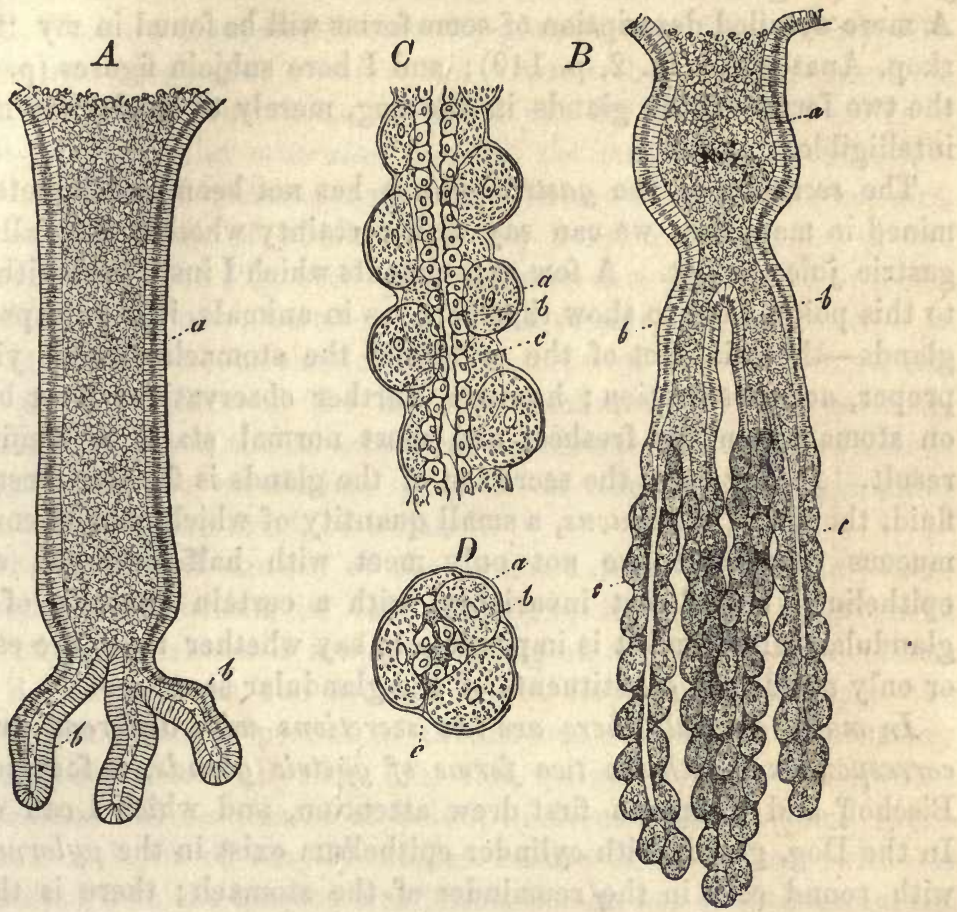

Frg. 204.- $A$, mucous gastric gland of a Dog, from the pylorus, with cylinder epithelium: $a$, wide cavity of the gland; $b$, its cæcal appendages. $B$, peptic gastric gland from the middle of the stomach : $a$, common trunk of the gland; $b$, its chief branches; $c$, terminal cæca. Magnified 60 diameters. $C$, a portion of the cæca, magnified 350 diameters, and viewed longitndinally. $D$, the same viewed in transverse section: $a$, membrana propria $; b$, large cells close to it; $c$, small epithelium round the cavity.

are lined by rounded or oval cells (peptic-cells), and which run side by side to the base of the mucous membrane. In these cells minute oil-globules are frequently observed. The terminal tubules have a peculiar twisted appearance, which is dependent on numerous lateral dilatations. In this part of the stomach no racemose glands exist, althongh they are found in the lower part of the œsophagus.

Between both these forms of "peptic gastric glands," (Magensaftdrüsen) Professor Kölliker observed, fasciculi of contractile fibre-cells, but he denies the existence of the spiral fibre-cells investing the glands, as described by Ecker.

The compound tubular glands with cylinder-epithelium occur in the pyloric zone, and resemble the last-described variety, with the exception, that the tubules are larger and devoid of the rounded finely granulated peptic cells. Neither simple glands, nor racemose glands, as stated by Ecker, are here observable.

These investigations of Professor Kölliker confirm the opinion of Donders, as to the existence of two varieties of glands in the human stomach, viz.: the proper gastric glands (called by Kölliker from their secretion "peptic" gastric glands) and the simple mucous glands. These two distinct forms have been previously described in the stomach of Mammalia by Kolliker (Vid. \$150, infra and "Mikroscopische Anatomie" II. 2, p. 240), but in Man their existence has hitherto not been satisfactorily ascertained.-DaC.] 
free ends; in many genera, they are of two very distinct kinds, the mucous gastric glands, with a cylinder epithelium and the peptic gastric glands (Magensaftdrüsen) with cells similar to those which exist in man. A more detailed description of some forms will be found in my "Mikroskop. Anatomie" (II. 2, p. 140); and I here subjoin figures (p. 507) of the two forms of the glands in the dog, merely to render my meaning intelligible.

The secretion of the gastric glands has not been so completely examined in man, that we can say with certainty whether they all secrete gastric juice or not. A few experiments which I instituted with regard to this point, tend to show that here, as in animals, it is only particular glands - those in fact of the middle of the stomach-which yield the proper, active secretion; however, further observations must be made on stomachs in the freshest and most normal state, to confirm this result. In any case, the secretion of the glands is for the most part a fluid, though in the mucus, a small quantity of which usually covers the mucous membrane, we not only meet with half-destroyed cylinder epithelium, but almost invariably, with a certain quantity of proper glandular cells; and it is impossible to say whether these are essential, or only accidental constituents of the glandular secretion.

In many animals there are two secretions with different properties corresponding with the two forms of gastric glands, a fact to which Bischoff and Wasmann first drew attention, and which I can confirm. In the Dog, glands with cylinder epithelium exist in the pylorus; those with round cells in the remainder of the stomach; there is the same arrangement in Ruminants and in the Rabbit; whilst in the Pig, it is only the middle of the stomach and especially the great curvature, which presents the latter glands. A series of experiments on artificial digestion, which were carried out by Dr. Goll, of Zurich, and myself, principally with the pig's stomach, afforded the distinct result, that, so far as their solvent powers are concerned, the glands present very different relations; those with round cells act upon protein compounds which have been coagulated by acids in a very short time, while those with cylinder epithelium, either have no action at all or take a long time to produce a slight effect. Furthermore a well-marked acid reaction is presented by that region of the stomach only in which the former glands are situated. The active organic substance, pepsin, is not contained in the gastric mucus, which consisting of detached epithelium cylinders, often form a thick covering over the mucous membrane, but in the finely granulated, rounded cells of the peptic gastric glands, to which therefore the term peptic cells (Labzellen, Frerichs)* may well be applied. According to my observations, however, these peptic cells do not necessarily become thrown off, nor take any direct share in digestion, but * [Literally, "rennet-cells."-Trs.] 
frequently exert their action simply by pouring the juice which they prepare into the glands.

$\S 151$. We have seen, that beside the glands, only a very scanty tissue enters into the mucous membrane. Around their extremities alone, do we find a dense, continuous, reddish layer $0.022-0.044$ of a line in thickness (Brïcke), the muscular layer of the mucous membrane, consisting of bundles of common connective tissue and of smooth muscles, interwoven, the latter of which cross one another principally in two directions, and, in the Pig, even pass between the glands and into the plicce villosce. In man, we meet only with vessels, and an amorphous connective tissue, without elastic fibrils, interposed between the glands, forming at the surface of the mucous membrane, a clear, perfectly homogeneous stratum, the structureless membrane of authors, which is continuous with the membrance proprice of the separate glandular tubes, but cannot, like them, be isolated.

The whole internal surface of the stomach from the cardia (where the tessellated epithelium of the cesophagus terminates by a sharp notched edge), possesses a simple covering of cylindrical cells, about 0.01 of a line long on the average, which lie immediately upon the outermost homogeneous portion of the mucous membrane, without any interposed substance. During life, this cylinder epithelium-whose other relations will be treated of below, in describing the small intestine, where a layer of exactly similar nature is to be met with-is closely united with the mucous membrane, though not so intimately, but that its elements are, at times, detached to a larger or smaller amount by the mechanical violence to which it is necessarily occasionally subjected in the stomach. After death this takes place so readily, that the cells can be seen in situ, in man, only under very favorable circumstances. Perhaps, also, detachment of the epithelium to a certain extent may take place normally, in one way or another, during digestion; at least, in animals the quantity of loose epithelial cells is often very great and they frequently almost entirely constitute the mucous coating which covers the surface of the stomach.

Besides the tubular glands, the stomach also contains, though they are inconstant and vary very much in number, closed follicles-the socalled lenticular glands, which are identical in structure with the solitary follicles of the small intestine, and therefore need not be further described in this place.*

* ["Although it may be that the lenticular glands of the stomach are always present in children, they are certainly inconstant in adults, since in many cases no trace whatever can be discovered of them. In other instances they are exceedingly numerous, covering the whole surface of the stomach, but in this case, the invariably diseased state of the alimentary tract, suggests the idea, that they stand in some connection with it. In many mammalia no trace of such structure is to be found, while, according to Bischoff (Mull. Arch. 1838), 
The bloodvessels of the gastric mucous membrane are very numerous, and their distribution is quite characteristic (compare Fig. 205, repre-

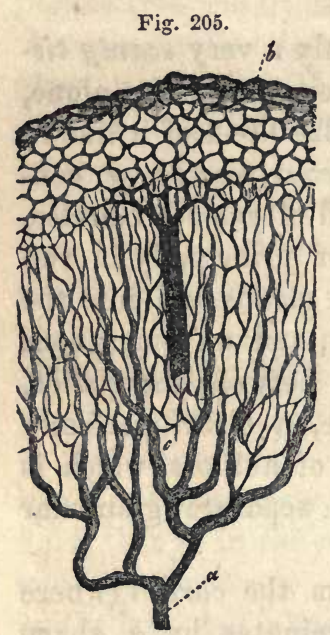
senting the vessels of the large intestine, whose arrangement is almost the same). The arteries begin to divide in the submucous connective tissue, in such manner, that only their finer branches reach the mucous membrane, in which, gradually breaking up into capillaries, they ascend in great numbers, perpendicularly, between the glands and form a network of fine capillaries of $0.002-0.003$ of a line, around the tubes, which extends as far as to the apertures of the glands. Here this network, which we may regard as continuous through the whole stomach, passes into a superficial reticulation of somewhat larger capillaries, of $0.004-0.008$ of a line, whose meshes, in man, are polygonal, $0.02-0.04$ of a line in diameter, and encircle the apertures of the glands; it is more complicated or more simple, according to the breadth of the interspaces of the glands and the occurrence of elevations upon them, but seems never to consist of simple vascular rings. From this network the veins, which are relatively wide, arise by many radicles; they then, further apart from one another than the arteries, and receiving no more blood, penetrate the glandular layer, and upon the outer surface of the mucous membrane, enter, often at right angles, a wide venous network with partly horizontal vessels, in the submucous tissue.

The lymphatics of the stomach form a superficial fine network and a deep, coarse, one; and can only be demonstrated by injection. The numerous small branches which pass from the mucous membrane, their aggregation into larger trunks, and final penetration of the muscular tunic, are readily seen in large Mammalia, killed during digestion. The

Fig. 205.-Vossels of the large intestine of a $\mathrm{Dog}$, the mucous membrane being cut through perpendicularly; $a$, artery; $b$, capillary network of the surface, with glandular apertures; $c$, vein; $d$, capillary network round the glandular tubules in the thickness of the mucous membrane.

they occasionally exist in the dog, invariably in the pig, and so far as the latter animal is concerned, I can, with Wasmann, confirm this statement. They are here, as Bischoff supposes and as is evident from Wasmann's description, not isolated, but aggregated glands, true

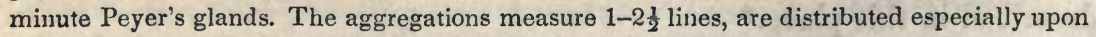
the cardia and small curvature, and are readily seen upon stripping off the muscular and submucous tissue. At first sight, they appear to lie entirely in the last-named layer, but if the attempt be made to detach them, it is found that this cannot be done without tearing the mucous membrane, to which they closely adhere. On the internal surface, small depressions are seen where these patches occur, and the gastric glands are here either absent or undeveloped." Kölliker, Mikr. Anat. II. 2, p. 151.-Trs.] 
nerves of the stomach, derived from the vagus and sympathetic, are readily traced into the submucous tissue and they may also be observed entering the muscular layer of the mucous membrane; but it becomes impossible to follow them further, principally because, in the interior of the mucous membrane itself, they present no more dark-edged tubules, but probably only the pale embryonic fibres.*

\section{MUCOUS MEMBRANE OF THE SMALL INTESTINE.}

$\S 152$. The mucous membrane of the small intestine is thinner than that of the stomach, but more complex in its structure, inasmuch as, besides the tubular or Lieberkuhnian glands, it presents a great number of permanent folds and villi; also, imbedded in its substance, peculiar closed follicles, the so-called solitary and Peyer's glands and, in the submucous tissue of the duodenum, Brunner's glands.

The mucous membrane consists of connective tissue, which is internally homogeneous or indistinctly fibrillated; except where certain glands exist, there is but little submucous tissue, so that it is pretty closely connected with the muscular tunic. Upon the inner surface of the mucous membrane, there rests a cylinder epithelium, to which further reference will be made under the head of the villi; whilst externally, towards the submucous tissue, it is bounded by a layer of smooth muscles, discovered by Brücke, which measures, at most, 0.0177 of a line; they are disposed longitudinally and transversely, but in man their slight development renders it often very difficult to discover them.

$\S 153$. The villi of the small intestine are small, whitish elevations of the innermost portion of the mucous membrane, readily distinguishable with the naked eye and which, distributed upon and between the valvulce conniventes (Kerkringian valves) through the whole extent of the small intestine, from the pylorus to the sharp edge of the ileo-creal valve (valvula Bauhini), are set so close together as to give the mucous membrane its well-known velvety appearance. They are most numerous (50-90 upon a square line) in the duodenum and jejunum, less so in the ileum (40-70 upon a square line). In the duodenum they are broader and less elevated, resembling folds and laminæ 1-10-1-4 of a line in height, 1-6-1-2 or even 3-4 of a line in breadth. In the jejunum, they

* [ In his "Mikroskopische Anatomie," B. II. 2, pp. 149, 153, 164, Professor Kölliker shows that the muscular layer of the mucous membrane of the stomach and intestine was discovered by Middeldorpf (De Glandulis Brunnianis, Vratisl. 1846, c. tab.) but remained unnoticed until it was rediscovered by Brücke and himself. In the small intestine there are, when this muscular stratum is well developed, two layers, though they are not always complete; the external layer is composed of longitudinal, the internal of transverse fibres.

In the villi, the smooth muscular fibres have been found not only in many mammalia, but also in birds. The contraction of the villi which they effect appears to have been noticed by Lacauchie, Gruby and Delafond, so long ago as 1842.-Trs.] 
appear for the most part to be conical and flattened; frequently, they are even foliated or cylindrical, clavate or filiform, the three latter forms

Fig. 206.

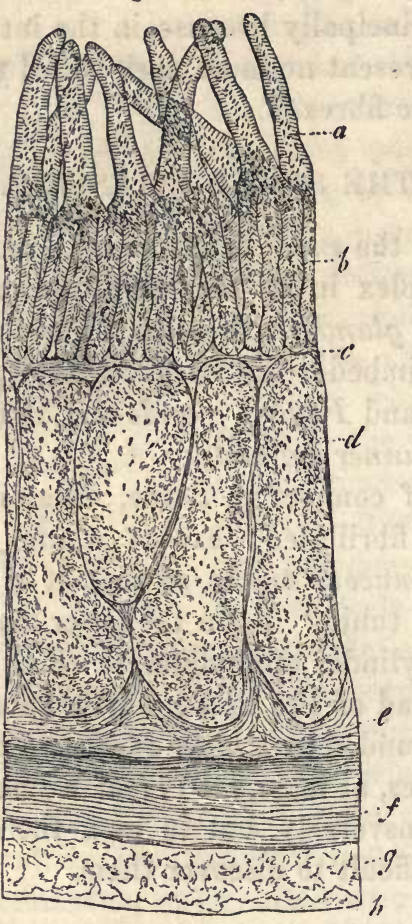

Fig. 207.

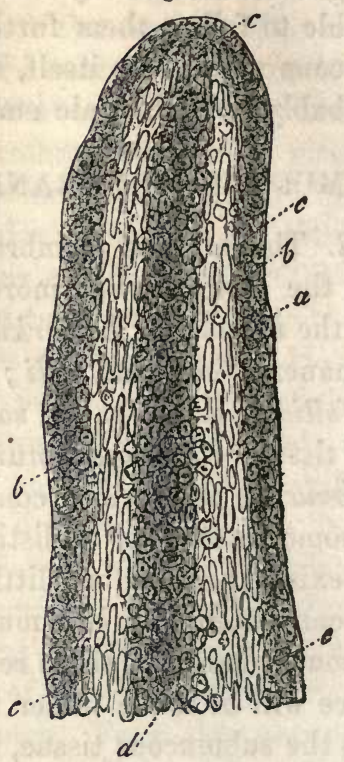

predominating in the jejunum. The length of the villi is from 1-5-1-2 of a line; the breadth from 1-6-1-10, even $1-25$ of a line; the thickness in the flattened forms $1-20$ of a line.

The villi are composed of two portions, a decper, belonging to the mucous mernbrane and an epithelial, superficial coat. The contour of the former or villus proper, is similar to that of the entire villus; it is simply a solid process of the mucous membrane, containing bloodvessels, lymphatics and smooth muscles, whose matrix, through which a variable number of roundish nuclei are scattered, in general exhibits no morphological character more decided than that of the mucous membrane itself, yet must most undoubtedly be regarded as a metamorposed connective tissue, without any intermixture of elastic tissue. The bloodvessels of the

Fig. 206.-Section through the walls of the lowest portion of a Calf's ileum, magnified 60 diameters: $a$, villi ; $b$, Lieberkuhn's glands; $c$, muscular layer of the mucous membrane; $d$, follicles of a Peyer's patch; e, remainder of the submucous tissue under them; $f$, circular muscles; $g$, longitudinal muscles.

Fra. 207.-Intestinal villus of a young Kitten without its epithelium, to which acetic acid has been added; $a$, boundary of the villus; $b$, subjacent nuclei; $c$, nuclei of the smooth muscles; $d$, round nuclei in the centre of the villus. 
villi (Fig. 208) are so numerous, that when well injected, those whose epithelium has been detached become colored throughout; and, in living animals, or those which have just been killed, each villus, if viewed from above, appears as a red dot surrounded by a clear ring. In man, every villus contains a close network of capillaries of $0.003-0.005$ of a line, with rounded or elongated nuclei, which lies immediately beneath the homogeneous external layer of the matrix* and is supplied by one, two, or three small arteries of

Fig. 208.

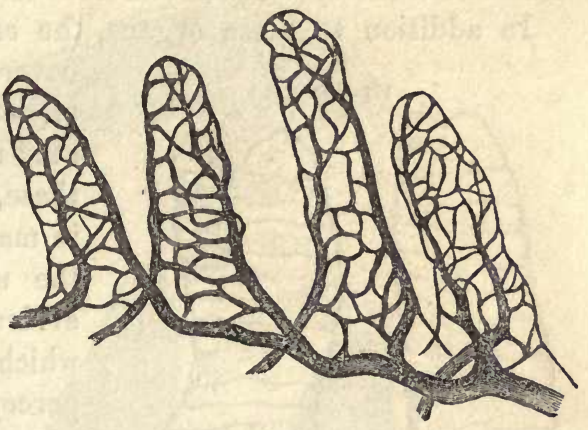
$0.01-0.016$ of a line. The blood is usually carried back directly into the larger trunks of the submucous tissue, by a vein of 0.022 of a line, which does not arise, as in animals, by the arching round of the artery, but proceeds from the gradual confluence of the finest capillaries.

The relations of the lacteals in the villi of man, have not hitherto been perfectly made out; for although the majority of investigators are inclined, like the older observers, to suppose that they commence by one or two crcal branches, yet recently, more and more voices appear to be raised for the view that they originate in a plexiform manner. As to my own opinion, I can affirm nothing with respect to the human subject, since I have never succeeded in meeting with villi distended with chyle, and in empty ones, I have been unable to obtain any decisive evidence; on the other hand, in animals, I feel certain that in many cases only a single lacteal, which has a cæcal and frequently enlarged end, and whose diameter is much greater than that of the capillaries, traverses the axis of the villus (Fig. 209.)

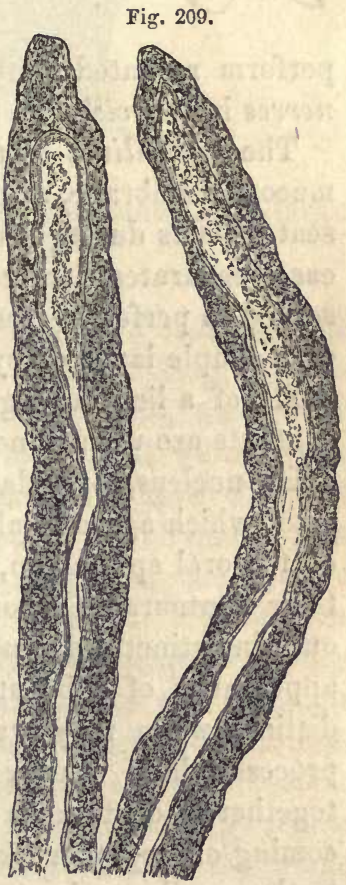

FIG. 208.-Vessels of a few villi of the Mouse, after one of Gerlach's injections; magni45 diameters.

Fra. 209.-Two villi without epithelium and with the lacteal in their interior (from the Calf), magnified 350 diameters, and treated with a dilute solution of caustic soda.

* [The " basement membrane" of Todd and Bowman.-ED.] 
For my own part in fact, I believe that all the narrow cylindrical and filiform villi will be found to present this condition, but that, on the other hand, the number and mode of origin of the lacteals may possibly be different in the broad and foliaceous forms (see Mikr. Anat., ii. 2, p. 160).

In addition to these organs, the villi also contain, as Brücke dis-

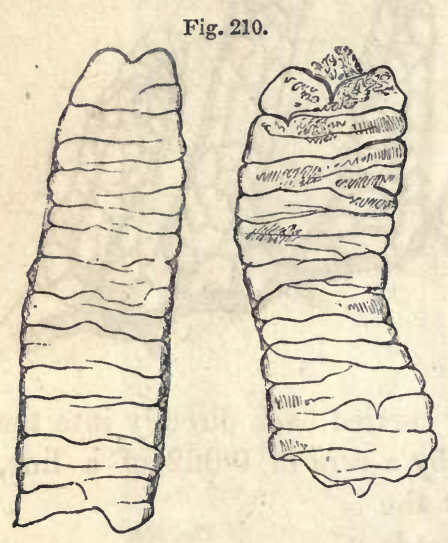
covered a short time ago, a thin layer of longitudinal smooth muscles situated more centrally round the lacteals; these, however, are not always distinct in man. They produce contractions of the villi (Fig. 210), which are very evident immediately after death, and which, according to Brücke, are also perceptible in the living animal. They have, in all probability, an important influence over the propulsion of the chyle and of the venous blood in the villi-always supposing that there is no objection to the assumption that they perform repeated contractions during life. Nothing is known of the nerves in the villi.

The epithelium of the villi and of the rest of the surface of the mucous membrane, although it is very intimately united with the deeperseated parts during life, only becoming detached accidentally or by disease, separates very readily in the dead subject, and can only be observed in perfectly fresh portions of intestine. It consists everywhere of a simple layer of cylindrical cells slightly narrowed below, of 0.01 0.012 of a line in length, and $0.003-0.004$ of a line in breadth, whose contents are usually nothing but fine granules and an oval, clear, vesicular nucleus, provided with one or two nucleoli. During life, these cells, which agree in all their chemical characters with the deeper cells of the oral epithelium, are so intimately united, that even after death their contours, in a longitudinal view, are at first either not at all, or only indistinctly distinguishable, though on the surface they have the appearance of a beautiful mosaic. The cylinders only become quite distinct when they are either spontaneously or artificially detached, a process which usually takes place in such a manner that they hang together in continuous portions, all the cells covering a villus sometimes coming off together like the calyptra of a moss. The addition of water to these cells produces a separation of the cell contents from the broad end, giving rise, in separate cells, to the appearance of a membrane thickened upon one side and in series of cells or entire villi, to that of

FIG. 210.-Two intestinal villi of the Cat, contracted, and magnified 60 diameters. 
a peculiar structureless coat, like the cuticle of plants; by its longer action, however, or by that of the intestinal fluids, the bursting of the

Fig. 211.
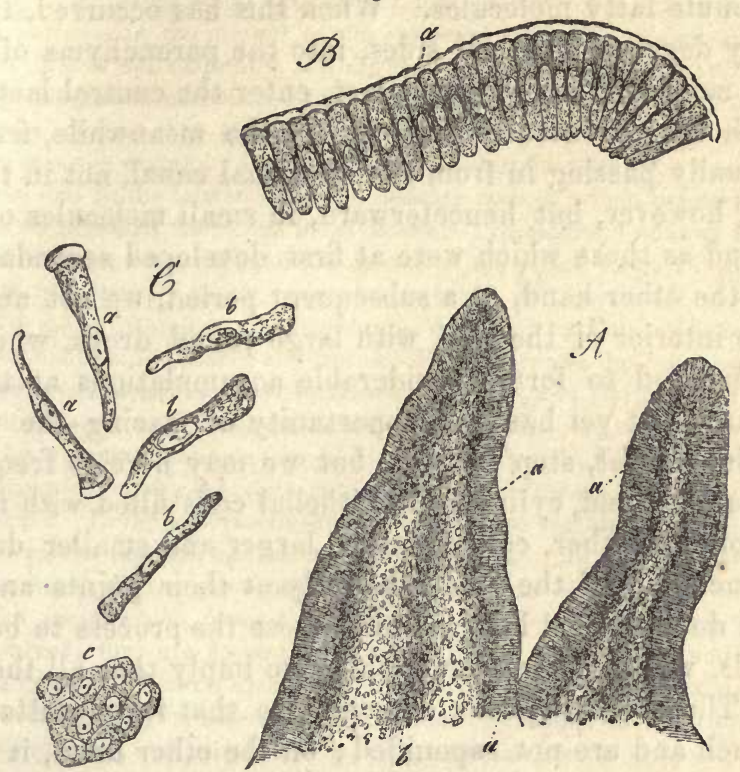

cells produces apertures in them, or they become distended into large pyriform clear vesicles.

We may here refer to the changes which the epithelial cells and the villi in general undergo during digestion. The most striking circumstance is the occurrence of fat in different parts of the villi, which may always be observed during the formation of a fatty, milk-white chyle. The succession of the morphological steps, at least as I have observed them in animals, is as follows: The fat contained in the chyme at first enters only isolated epithelial cells in different regions of the villi, so that in each we soon observe a large ovate shining drop. The number of these fat-cells rapidly increases, and then the villi acquire a very peculiar appearance, often as if beset with pearls, from the irregular alternation of cells filled with fat and consequently bright and shining, with those which are empty and pale. In the end, all the cells become filled with these drops and the epithelium appears quite dark by transmitted, but whitish by reflected light, giving its aspect to the whole villus, With the repletion of the entire epithelial covering of the villus

Fia. 211. $-\mathcal{A}$, two villi, with their epithelium, from the Rabbit; magnified 73 diameters: $a$, epithelium; $b$, parenchyma of the villus. $B$, a detached sheet of epithelium, magnified 300 diameters : $a$, membrane raised up by the action of water. $C$, single epithelial cells, magnified 350 diameters : $a$, with, $b$, without, a raised-up membrane; $c$, a few cells from the surface. 
absorption commences, but up to this time nothing has entered the lacteals. This, however, soon takes place, and the first indication we observe, is the breaking up of the large drops of fat in the cells into many tolerably minute fatty molecules. When this has occurred, these drops penetrate by degrees, from all sides, into the parenchyma of the villus itself, fill it more and more and, at last, enter the central lacteal, whose whole length they eventually occupy. In the meanwhile, fresh fat has been continually passing in from the intestinal canal, not in the form of large drops, however, but henceforward, in small molecules or drops of the same kind as those which were at first developed secondarily in the cells. On the other hand, at a subsequent period, we not uncommonly meet, in the interior of the villi, with large round drops, which appear especially inclined to form considerable accumulations at their apex. In man, I have not yet had the opportunity of tracing the process of the absorption of fat, step by step, but we may here so frequently observe, on the one hand, cylindrical epithelial cells filled with fatty molecules, and on the other, collections of larger and smaller drops of fat in the parenchyma of the villi, especially at their points and in their axes, that I do not at all hesitate to suppose the process to be the same as in animals, without, however, wishing to imply that all the steps are identical. These observations demonstrate that fatty matters are absorbed as such and are not saponified; on the other hand, it cannot at present be certainly stated how it is possible that they penetrate the membrane of the epithelial cells, the parenchyma of the villi, and the walls of the lacteals. I should be most inclined to compare the whole process to the imbibition of an emulsive fluid, such as milk, by a porous body; and I believe that the fatty molecules of the chyme are absorbed simply in consequence of their being carried along with its fluid part.*

* [Professor Brücke, in a series of important papers on the "Lacteals and on the Absorption of Chyle" (Ueber die Chylusgefasse und die Resorption des Chylus), published in the Transactions of the Vienna Academy for 1854, bas thrown much light on the difficult question of the anatomy and physiology of the parts concerned in digestive absorption.

As the result of his observations on Man and on the Mammalia, Brücke states, that the cylindrical epithelial cells covering the villi, are open at one extremity, and are not, as is generally supposed, closed by a membrane, but merely by a thin layer of a mucilaginous substance. This, he asserts, can be proved by observations on animals recently killed; for if a portion of their intestinal mucous membrane be brought under the field of the microscope, the formation of transparent vesicles, corresponding to each epithelial cell may be easily seen. These vesicles form along the margin of the villus, and are the cell-contents which have escaped from the open extremity of the cylindrical cell, enclosed by the mucilaginous substance. They have hitherto been regarded as the eell-wall rendered prominent by an expansion of the cell-contents (vid. supra, Fig 211, $C$ a); but that this view is incorrect, is evident from the circumstance, that they lie perfectly isolated by the side of the cells, without any change in the membrane of the latter being observable.

As another evidence of these epithelial cells not being closed cavities, Professor Brücke adduces the fact, that even by the aid of the highest magnifying powers, no cell membrane with pores can be clistinguished. Yet the oil globules, which are always of a distinctly recognizable size, are known to enter the cells, and it is difficult to understand how they can traverse a homogeneous membrane. 


\section{While digestion is going on, we frequently find the whole parenchyma of the villi densely filled with small nuclei, here and there surrounded}

This difficulty is increased when we consider, that a trustworthy observer (Österlein) has detected solid particles in the interior of the epithelial cells. By assuming, however, that these latter are open at one extremity, and are isolated from the intestinal tube merely by a thin layer of mucilaginous substance, which is easily traversed, Professor Brücke thinks the difficulty may be solved. The chyle, he states, passes from within the cell through an opening in the opposite extremity into the villus. Within the villus, the molecules of chyle lie in the lacteal and in the interstices of the delicate stroma, which connects the walls of the villus with its muscular fibres and bloodvessels. The lacteal, Professor Brücke regards not as a vessel, but as a mere cavity in the centre of the villus, without distinct walls. In cylindrical villi he generally found one cavity, in broad villi, two, three, or even four distinct canals. Of the structureless, thin membrane, described by Kolliker as surrounding the lacteals, he was not able to observe any traces. He also denies the commencement of the lacteal by a distinct network, as stated by Hewson, Krause, and Hyrtl, and thinks that these observers have mistaken capillaries for lacteals. The lacteal he regards, on the contrary, as originating by irregular channels formed by the interstices in the tissues of the villus.

The commencement of the complete chyliferous vessels occurs in the deeper part of the mucous membrane. In the submucous areolar tissue large vessels with distinct walls, and furnisbed with valves and an epithelium, are observable. In the smaller ramifications these cannot be detected; nor can a separate tunica propria of the walls be distinguished from the areolar tissue which forms the adventitia. In the muscular layer the chyliferous vessels have valves; they are accompanied on each side by an artery and vein. In the mucous and submucous tissue no such arrangement of the bloodvessels exists.

The chyle, Professor Brücke asserts, does not enter the vessels merely from the villi, but also from between them, and principally from between the glands of Lieberkuhn, where it may be observed lying free in the interstices of the tissue. This commencement of the chyliferous vessels by open extremities in the interstices of the strona of tissues, he regards as sufficient to account for the circumstance, that absorption may take place in parts of the inteștinal canal not possessed of villi, as in the large intestine. The lymphatics, as well as the chyliferous vessels, he considers to commence by channels in the tissues, and thus the curious fact noticed by him (vid. "Denkschriften der Wien Acad.," vol. ii. p. 21), that a colored solution may penetrate through the capsules of the Peyerian glands and reach the thoracic duct, is readily explained.

With regard to the anatomy of the chyliferous vessels after they leave the intestinal walls, Bracke confirms the recent observations of Ludwig and Noll (vid. \$219, infra). The best method to examine the commencements of the chyliferous vessels, is to allow the chyle time to coagulate in the vessels, and then to render the walls of the intestine transparent. This is most readily effected by a solution of albumen in caustic potassa, to which enough hydrochloric aeid has been added to neutralize the alkali.

In the animals he examined, Professor Brubcke found the arrangement of the chyliferous vessels to be in the main similar to that in Man. In the Weasel there is no difference. In the Rablit the chyle is not contained in separate channels, but is situated, throughout the whole thickness of the intestinal wall, in the interstices between the sheaths of the bloodvessels. In this animal, therefore, the chyle is separated from the blood merely by the thickness of the membranes of the bloodvessels, whilst in Man this is the case only in the mucous layer of the intestine. In the Pig, the walls of the chyliferous vessels exhibit a distinct layer of muscular fibres. In the Motse, the deposits of chyle in the interstices between the glands of Lieberkühn are readily seen.

The contractions of the intestinal canal Brücke considers to be the principal means by which the chyle is forced into the villi, these latter being kept erect and distended by the pressure of the blbod in their bloodvessels. As the chyle in the villi surrounds the bloodvessels, a mutual interchange of some of the elements takes place; the blood yields up fibrine to the chyle, and the chyle a portion of its soluble parts to the blood.

When the villi are filled, the contraction of their muscular fibres presses the chyle into 
by cell membranes, elements which are perhaps never entirely absent in a villus, but are at other times far fewer, and particularly are not to be distinguished in its interior.*

\section{$\S 154$. Glands of the small intestine.-The small intestine contains only two kinds of true glands; viz., 1, tubular glands, which are dis-}

the channels between the mucous and submucous membrane. If the villi be much distended, a portion of the chyle passes back again through the epithelial cells into the cavity of the intestinal tube, and this is especially apt to occur when the chyle contains any particles too coarse to circulate. The chyle is propelled further by the muscular contractions of the intestines into the vessels of the mesentery, whence it is pumped, as it were, into the thoracic duct, by the alternating pressure on the intestinal walls during the movements of respiration. This action is also aided to a slight extent by the contractions of the muscular tissue of the chyliferous vessels.

The results arrived at by Professor Brücke, will, if corroborated by future research, tend greatly to elucidate much that has hitherto been obscure in the physiology of the digestive as well as of interstitial absorption.-DaC.]

* [One of the most important contributions to our knowledge of the anatomy of the villi and the general physiology of digestive absorption, which has appeared for a long time, is Professor Brüch's "Beiträge zur Anatomie und Physiologie der Danndarm-Schleimhaut," in Siebold and Kolliker's "Zeitschrift," for April, 1853. We subjoin the principal results at which the Professor has arrived.

The epithelium is not cast off during normal digestion and in freshly killed animals it is somewhat difficult to detach it from the mucous membrane. The cells of the epithelium do not, as Weber stated, undergo any change of form during digestion and chylification, but they become filled with fat, which gradually passes on into the villi, \&c., so that in the fasting state they are again free from any foreign contents.

The villi ordinarily contain two, but sometimes many, capillary trunks, which ramify principally at their apices, and superficially. Ramifications and anastomoses in the body of the villi are less common. In dogs, many parallel vessels often run undivided for a considerable distance, and have doubtless been confounded with a central lacteal.

In all the animals Brüch examined, and in man, he found that the villi had a striking uniformity of structure. A single lacteal ran, without dividing, through the villus, and terminated shortly before reaching its apex, in a cacal commonly enlarged end (Liberkühn's ampulla). The lacteal had no wall, appearing to be a mere excavation in the villus. In cleft villi, the lacteal was cleft, each end terminating in a cæcum. In very rare cases, there were in broad villi two lacteals, a shorter and a longer, terminating in distinct ampulla, side by side. In the mucous membrane itself, the lacteals form a wide superficial network.

Brach accounts for the supposed lacteal network of the villi, by showing that the bloodvessels are as capable of absorbing fat as the lacteals, and when filled, of course acquire the appearance of a lacteal network. In some cases he found the superficial capillary network of a villus half red and half white, and it was frequently possible, when the fatty contents of the capillary network were hidden by the preponderating blood, to render them obvious by the action of water, which dissolved out the coloring matter and thus apparently converted a capillary, into a lacteal network.

Professor Brüch considers that the absorption of fat is a purely mechanical process, "just as quicksilver is pressed through leather," and he doubts altogether that the lacteals have any special absorbent function, or differ from ordinary lymphatics. However, we think that the mechanical nature of the process is open to very great question, and we should rather compare the manner in which fat enters a villus, to that in which the ingesta enter an Actinophrys; one can as readily comprehend the existence of a selective power in the former as in the latter case. That some such faculty exists would seem to be indicated by the fact stated by Bruch, that the Lieberkuhnian and Peyerian glands take no share in fatty absorption; though, on the other hand, it must be remembered that Kolliker found the eggs of Entozoa in the villi of rabbits ("Mikr. Anat." B. II. 2, 173).-Trs.] 
posed over the whole mucous membrane; and 2, racemose glands, in the submucous tissue of the duodenum.

The racemose glands, or as they are more commonly named, after their discoverer, Brunner's glands, form, at the commencement of the duodenum, upon the outer side of the mucous membrane, a continuous layer, which is best developed and thickest, close to the pylorus, where it constitutes a considerable glandular ring and extends about as far as the aperture of the biliary ducts. If the two layers of the muscular tissue be dissected off a stretched or distended duodenum, the glands may readily be recognized as yellowish, flattened bodies of $\frac{1}{10}-1 \frac{1}{2}$ lines (on the average $\frac{1}{4}-\frac{1}{2}$ a line,) with their angles rounded off, which, enclosed within a little connective tissue, lie close to the mucous membrane and send short excretory ducts into it. In their minute structure, Brunner's glands, the terminal vesicles of which measure $0.03-0.06$, even 0.08 of a line, agree perfectly with the racemose glands of the oral cavity and oesophagus. Their secretion is an alkaline mucus, in which no formed elements are contained, having no digestive action upon coagulated protein compounds and probably merely subservient to mechanical ends.

The tubular, or Lieberkühnian glands (cryptoe mucosce), are distributed over the whole small intestine, including the duodenum, as innumerable, straight, narrow cæca, which occupy the entire thickness of the mucous membrane and are frequently slightly enlarged at their extremities, though hardly ever dichotomously divided. The best idea of their number is obtained by viewing the mucous membrane, either from above or in vertical section, under a low power. In the latter case, we see the crca standing close together, almost like palisades (Fig. 206); in the former, we observe that the glands do not occupy the whole surface, but only the interspaces between the villi; here, however, they exist in such numbers, as to leave no intervals of any width, the mucous surface between the villi appearing pierced like a sieve. Even on Peyer's patches and over the solitary follicles, these glands are to be met with; but in man, they leave those portions of the mucous membrane which lie immediately over the centre of the follicles free, and therefore are arranged like rings around the follicles. The length of the Lieberkühnian glands equals the

Fig. 212.

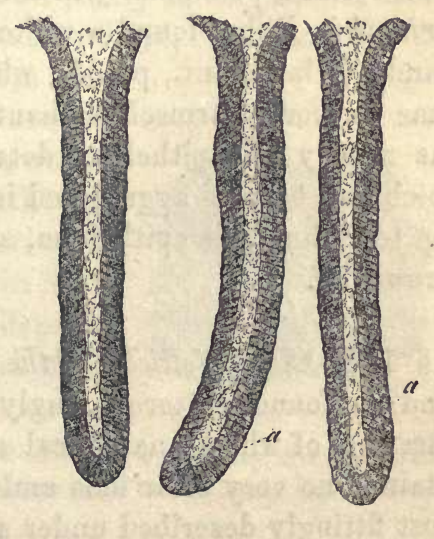

FIG. 212.-Lieberkuhnian glands of the Pig, magnified 60 diameters : a, membrana propria and epithelium; $b$, cavity. 
varies from ${ }_{5}^{1}-\frac{1}{7}$ of a line; their breadth is $0.028-0.036$ of a line; that of their aperture, $0.02-0.03$ of a line. They are composed of a delicate homogeneous membrana propiria and of a cylindrical epithelium, which, even during chylification, never, like that of the intestine, contains fat; their cavity is filled, during life, by a clear, fluid secretion, the so-called intestinal juice, which, however, becomes rapidly changed after death, or on the addition of water, so that the glands appear to be filled with cells, or with a granular mass.

The vessels of Brunner's glands have the same arrangement as those of the salivary, whilst around Lieberkühn's caeca they follow exactly the type of those of the stomach. A fine network of capillaries of 0.003 of a line, passes up round the caeca and, upon the surface of the mucous membrane, enters an elegant polygonal reticulation of somewhat wider $(0.01$ of a line) vessels, which communicates on one side with the vessels of the villi, on the other is directly continuous with veins, which, after communicating with those of the villi, run directly out of the mucous membrane. Hence, in this case also, the veins are connected only with the superficial network round the glandular apertures and with that in the villi, but not with that which surrounds the glands, so that, as in the stomach, the vessels which supply the secretion immediately succeed the arteries, and precede those to which the absorbent function is more especially assigned (comp. Frei, cited below).

Whence the small round cells, with a single nucleus, which are to be met with in the intestinal mucus, proceed, is doubtful. I have not found them in the glands and I can only refer them to the epithelium, whence I am inclined to suppose that these cells, which are usually few, arise upon the surface of the mucous membrane, like the mucous corpuscles of the oral cavity.

In various, particularly intestinal, disorders, in inflammations, typhus, peritonitis, Böhm found a white viscid secretion in many Lieberkühnian glands (Gland. int., p. 34), which, as subsequent observations of the same author (Darmschleimhaut in der Cholera, p. 63) would indicate, was merely an epithelium detached from the walls of the cavity, and which had become aggregated into a compact plug. In cholera, according to Böhm, this epithelium, as well as that of the whole intestine, is thrown off.

$\S 155$. Closed follicles of the small intestines.-Vesicles of a peculiar kind are found scattered, singly or in groups, over the walls of the small intestine, of whose anatomical and physiological import we have, as yet, attained no very clear idea and which may therefore, for the present, be most fittingly described under a general denomination.

The most important of these are Peyer's patches (glandulce agminatce). They are rounded, flattened organs, invariably situated along that sur- 
face of the intestine which is opposite to the mesentery; they are most distinct upon the inner surface, where they appear as rather depressed, smooth spots, without any very sharp definition, but they are also recognizable from the exterior, by the slight elevation to which they give rise; by transmitted light they look like more opaque portions of the membrane. These patches are usually most abundant in the ileum, but they are not uncommonly to be met with in the lower part of the jejunum:occasionally they exist in its upper portion close to the duodenum and even in the inferior horizontal portion of the duodenum itself. Ordinarily there are 20-30 of them; when they are found higher up there may be as many as $50-60$; but they are always most closely set in the lowest portion of the ileum. The dimensions of the separate patches are in general the larger, the closer they are to the $c e$ cum; their length is usually $5-1 \frac{1}{2}$.lines, but may diminish to 3 and increase to $3-5$ lines, or even 1 inch; their breadth varies from 3 to 5 or 9 lines. Where the patches lie, the valvulce conniventes are usually interrupted; in the jejunum, however, these folds are also to be met with; upon the Peyer's patches and in the ileum, rows of closely set villi often take their place.

More minutely examined, every Peyer's patch is seen to be an aggregation of closed follicles, of 1-6-1-2-1 line in Fig. 213.

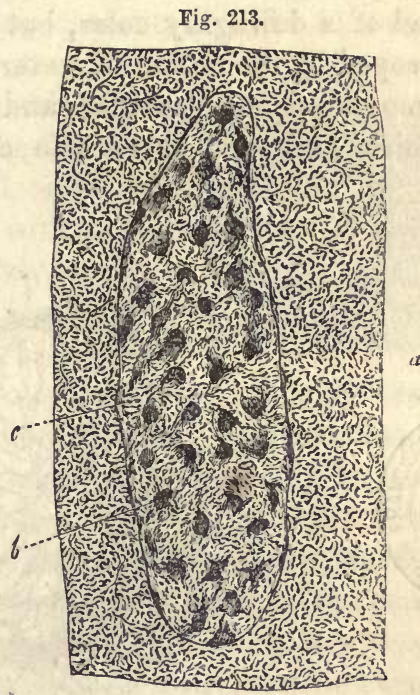
diameter, either rounded or slightly conical towards the intestinal cavity, which lie partly in the mucous membrane itself, partly in the submucous tissue, and are, on the one side not more than $0.02-0.03$ of a line distant from the mucous surface, while on the other, they are in immediate contact with the muscular tunic, which is here somewhat more closely united with the mucous membrane. Viewed from the interior of the intestine, their most striking feature in Man is the presence of many small rounded depressions $\frac{1}{3}-\frac{1}{2}-1$ line apart, which correspond with the separate follicles, and whose floor is, indeed,

Fig. 214.

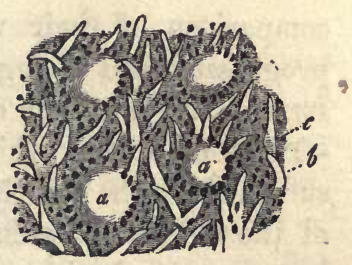

Fra. 213.-A Peyer's patch (Man), magnified 4 diameters: $a$, ordinary mucous surface, with villi; $b$, depressions upon the patches corresponding with the follicles; $c$, intermediate substance, with small villi.

FIG. 214.-Portion of a Peyer's patch of an old Man, after Flouch : $a$, follicle, surrounded by the apertures of the Lieberkühnian glands; $b$, villi; $c$, more isolated Lieberkühnian follicles. 
rendered slightly convex by the latter, but which present no villi whatsoever. The remainder of the patch is occupied by common villi, or by reticulated folds and by the apertures of the Lieberkuihnian glands; the latter are disposed around the slight elevations produced by the follicles, in circlets of 6-10 and more apertures, the coronce tubulorum of authors.

Each follicle of a patch possesses a perfectly closed, thick and tolerably strong coat of indistinctly fibrillated connective tissue, with interspersed nuclei; within this are the contents, which are soft and grayish (never milk-white). They become slowly diffused through water and consist of a little fluid, with innumerable nuclei and round cells of $0.004-0.008$ of a line, which, when recent, appear quite homogeneous and of a dull gray color, but are first cleared up and ultimately destroyed by the action of water and of acetic acid, the nuclei at the same time becoming granular and very distinct. Among these elements, which here and there also contain fatty granules, and which, as the

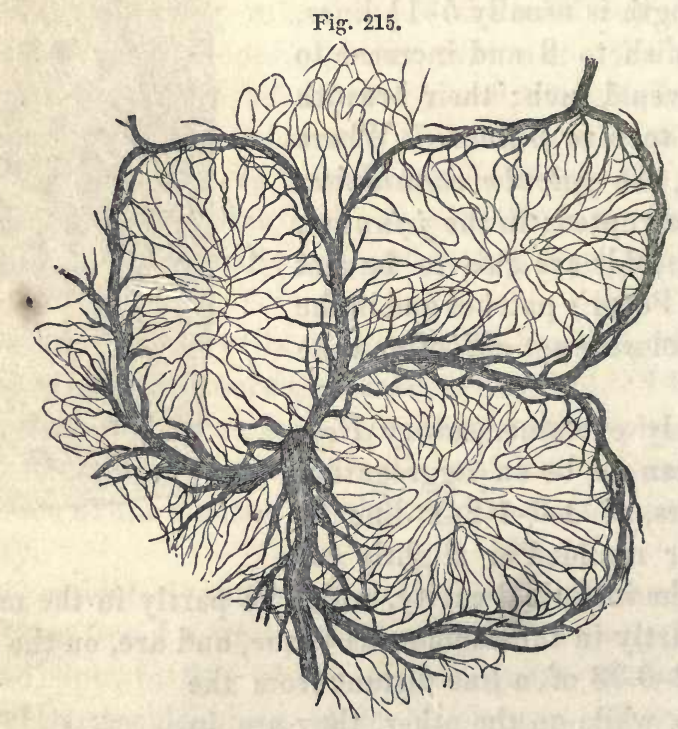

comparison of their various forms shows, are constantly undergoing progressive and retrogressive development, Frei and Ernst have demonstrated the existence of numerous, but very fine bloodvessels of 0.0015 -0.004 of a line, which are connected with a rich vascular network surrounding the follicle, and may be readily recognized in the contents of the follicles of animals (Pig, e. g.), if they be quite fresh, and have been extracted with care.

Little is known of the lymphatics of Peyer's patches. This much is certain, however, that the number of lacteals which may be traced du-

Fig. 215.-Horizontal section from the middle of three Peyer's follicles of the Rabbit, in order to show their internal vessels. After an injection by Frei. 
ring digestion from the Peyerian patches, is greater than that in other parts of the intestine, although their villi are fewer and less developed; on the other hand, we know nothing of the internal relations of these vessels. They would seem to form networks around the separate follicles, at least we see that they compass them externally; but they do not become inserted into, nor enter them, at any rate upon this surface, as their milk-white color would render their detection easy. Although, then, Brücke has recently affirmed the direct communication of the follicles with lymphatics, I must, for these and other reasons (see Mikr. Anat. II. 2, p. 188), at present doubt the fact.

The solitary follicles (glandula solitarioe) resemble the separate elements of Peyer's patches so closely in size, contents (I have also seen the internal vessels in them), and general structure, that there is no reason for considering them as distinct, particularly since the number of the follicles is subject to all possible varieties; and since, in animals at least, we find Peyer's patches with 2-35 follicles. In man, as all writers justly agree, their number is exceedingly inconstant; sometimes not one can be found, whilst in other cases, the whole

Fig. 216.

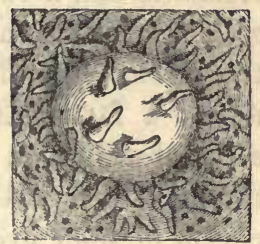
intestine, as far as the margins of the ileo-cæcal valve, is thickly beset with them, or lastly, they may occur in the ileum and jejunum, but in no very great number. Their entire absence must probably be considered abnormal, since they are constant in newly-born children, being more abundant in the jejunum than in the ileum.. The miliary vesicles, however, which are often met with in immense quantities in the small intestine and stomach, in catarrhal affections of the alimentary tract, may very probably be entirely or partially pathological, since the occurrence of such follicles has been demonstrated in other organs also (in the liver, according to Virchow). The solitary follicles have the same position as the elements of the patches, only they occur also in the mesenteric border and support villi upon their intestinal surface, which is usually somewhat convex.

I consider it as quite certain, that the follicles of Peyer's patches have no apertures, but I may here adduce the following facts. 1. In animals examined while fresh, the capsules are invariably closed, as may be very readily seen in the well-developed patches of the Pig, Sheep, Cat, Dog, \&c., which I particularly recommend for the examination of these organs, because the patches in the human subject have so frequently undergone alteration. 2. The appearance of an aperture may proceed from the depression of the mucous membrane over the single follicles, especially when the projecting portion of the wall of the folli-

Fra. 216.-A solitary follicle, covered with villi, from the small intestine. After Böhm. 
cles is not very tense. 3. In man, the closed follicles of the intestine are subject to very many morbid changes; they are frequently ruptured and so altered, that in place of the patches nothing remains but a reticulated, indistinctly pitted surface. As Virchow was the first to show (Med. Reform. 1848, No. 10, p. 64), they may also burst after death, if they are allowed to stand in water or in a warm place; whence, perhaps, many of those apertures which are met with in the dead subject should be regarded as the result of putrefactive change.

It is easy to understand, that little can be said concerning the physiology of Peyer's follicles so long as their relations to the lymphatics are not understood. They, and the follicles of the intestine in general, appear to me to be closed glandular organs, analogous to the splenic follicles, the tonsils, and the lymphatic glands, which contain peculiar elements and a vascular network. In these a constant development of cells takes place and at the same time, substances are elaborated from the plasma, supplied by the bloodvessels and perhaps also from matters not of a fatty nature, absorbed from the intestine, a part of which, in all probability, is at once taken up by the internal bloodvessels, while the larger proportion is exereted and absorbed by the lymphatics. The period of their greatest activity (when they become distended) coincides with that of the intestinal absorption, either because they absorb from the intestine or because they simply participate in the greater activity of the intestine at this time; and perhaps the more albuminous matters which they yield may be connected with the development of cells in the chyle. This hypothesis will hold good in its principal outlines, even if in future the direct connection of the lymphatics with the follicles, or the occurrence of lacteals within them, should be demonstrated; at any rate, it will not be blamed for being too wide of the facts.

$\S 156$. Mucous membrane of the large intestine.-The structure of the mucous membrane in the large intestine agrees so closely in essentials with that of the small intestine, that it may suffice here to draw attention to a few points only.

The mucous membrane of the large intestine, if we except the rectum, has no proper folds, for the transversely fibrous muscular layer also enters into the plicae sigmoidece. The villi also are absent, from the edge of the ileo-creal valve, into which the muscular tunic likewise enters, onwards; and the mucous surface, apart from some occasional, hardly perceptible, small, wart-like elevations, is even and smooth. It is difficult to detect the muscular layer of the mucous membrane in the human colon, though it is unquestionably present; it is more distinct in the rectum. In animals I find it well developed. According to Brücke, in the colon (of animals?) its longitudinally and transversely fibrous layers, which also exist here, are only 0.013 of a line thick, the diminution having taken place at the expense of the external longitudinal 
fibres, which are reduced to a threefold or even only a twofold stratum; in the rectum, the layers are again of equal thickness, and, taken together, measure about 0.022 , at the anus even 0.088 of a line and more.

The glandular organs of the large intestine are Lieberkühn's glands and solitary follicles; the former, also termed glands of the large intestine, are distributed over its whole surface from the ileo-cæcal valve to the anus and in the processus vermicularis. They are closely set, and have exactly the same structure as those of the small intestine, only, in accordance with the greater thickness of the mucous membrane, they are longer and broader $\left(\frac{1}{4}-\frac{1}{5}\right.$ of a line long, $\frac{1}{12}-\frac{1}{20}$ of a line broad). Here also $I$ have found, in man and in animals, in the fresh state, no formed contents besides a beautiful cylinder epithelium; so that we have no reason to suppose that the secretion is at all different from that in the glands of the small intestine, especially as the mucous membrane has, like that of the latter, an alkaline reaction, and, so far as my own experiments go, is equally devoid of digestive action.

The solitary follicles are arranged close together in the processus vermicularis, are very frequent in the cacum and rectum, and are also usually more abundant in the colon than in the small intestine. They are distinguished from those of the latter locality by their larger size $\left(\frac{3}{4}-1-1 \frac{1}{2}\right.$ lines $)$ and by the circumstance that upon each of the little prominences of the mucous membrane to which the follicles give rise, there is a small pit-like, elongated or rounded aperture, of 1-9-1-12 of a line, which leads to a little depression of the mucous membrane above the follicles. These pits, which are Fig. 217.

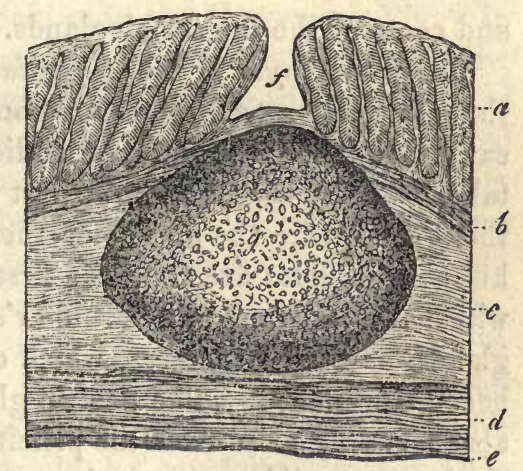
totally absent in the normal follicles of the small intestine, led Böhm formerly to regard the follicles as crcal glands provided with apertures : this, however, is incorrect, for at the bottom of this depression lies, as Brücke has also remarked, a closed, somewhat flattened follicle of exactly the same structure, even to the internal vessels, as those of the small intestine.

The bloodvessels of the glands and follicles of the large intestine present the same relations as in the small. Every Lieberkühnian aperture is encircled by a ring of vessels of $0.006-0.01$ of a line, which is some-

FIG. 217.-Solitary follicle from the colon of a Child, magnified 45 diameters: $a$, Lieberkuhn's glands; $b$, muscular layer of the mucous membrane; $c$, submucous tissue; $d$, transverse muscles; e, serous membrane; $f$, depression of the mucous membrane above the follicle, $a$. 
times single, sometimes, especially in the neighborhood of the solitary follicles, multiple.

From these vessels wider venous trunks arise and penetrate deeply between the glands, which are themselves surrounded by a dense network of fine capillaries derived immediately from the arteries (Fig. 205). Nothing is known of either the lymphatics or the nerves in the mucous membrane. The epithelium is precisely similar to that of the small intestine, and, at the anus, is separated by a pretty sharp line of demarcation, from the external epidermis.

$\S 157$. Development of the intestinal canal. The entire wall of the intestine, various as its different structures may afterwards become, proceeds from two points of development: viz. in the first place from the inferior lamina of the germinal membrane (mucous layer of Pander and Baer; mucous tunic, Reichert; glandular layer or intestinal glandular layer, of Remak), which is not the foundation of the whole mucous membrane, but only of the intestinal epithelium and of the intestinal glands; and 2, from the middle layer of the germinal membrane (vascular lamina, Pander, membrana intermedia, Reichert), which gives rise, in addition to many other parts (muscles, bones, nerves, heart), to the vascular and nervous fibrous coats of the intestine, as well as to the vessels, nerves, and coats of the intestinal glands.

The inner layer or the epithelial tube consists from first to last of nothing but cells and becomes metamorphosed by their continual multiplication, superficially and perpendicularly, which, according to Remak, takes place by division, in the first place, into the future epithelia; and in the second, into the glands of the intestine. Of the latter, the Lieberkühnian follicles are from the first, hollow diverticula of the epithelium, whilst the salivary and Brunner's glands arise, like the sudoriparous glands, as solid processes, which only subsequently acquire cavities and become branched. The gastric glands and those of the large intestine also certainly arise from the primitive epithelial tube-whether as diverticula or as solid processes is not yet made out-and form at the commencement a layer completely separated from the fibrous lamina of the intestine; whence, also, the epithelium in their neighborhood appears much thicker than it subsequently is. At a later period, delicate vascular processes grow from the fibrous layer between the glands, until at length both layers, intimately united, constitute the proper mucous membrane. Similar and more considerable processes of the fibrous layer form the villi, whilst the muscular and serous tissues are developed from its external portion.

The examination of the intestinal mucous membrane presents greater difficulties than that of other parts. The epithelium is usually in a good 
state of preservation only in quite fresh subjects, and generally breaks up into its elements with extreme ease. The villi are best seen in thin perpendicular sections, made with fine scissors, viewed with a low power, and illuminated from above. During absorption, they are usually found full of fat and nuclei, so that their separate portions are not perceived, with the exception of the lacteals, which become distinct by the use of acetic acid and still better by that of dilute caustic soda. At other times, the muscles of the villi are easily recognized by their nuclei, on the addition of acetic acid. Injections are required for the bloodvessels : the best are made by injecting from both arteries and veins at the same time, and should be preserved in fluid. The same holds good of the other parts of the intestine, for which perpendicular sections are especially instructive. For the glands, recent pieces of intestine are particularly required, although it is often, as in the stomach, exceedingly difficult to prepare them. Mucous membrane, hardened in alcohol, pyroligneous acid, or chromic acid, or boiled in acetic acid of 80 per cent. and dried, according to the method of Purkinje and Middeldorpf, or saturated with gum and dried, thin transverse and longitudinal sections being made with a sharp knife, according to Wasmann's method, may be used, being first rendered clear by a little soda. The analysis of the gastric mucous membrane into its elements, presents the greatest difficulties, especially when it is as thick as in the Horse and Pig. In the Dog, Cat, Rabbit, and in the Ruminants, the process is easier, since, frequently, by merely scraping the mucous membrane with the back of a knife, the epithelium of the glands may be drawn out in a connected state and affords all the information required as to their form and lining. Simple teasing out is also frequently sufficient to reduce the mucous membrane of the animals in question into its elements.

Brunner's glands offer no difficulties except in their excretory ducts, which may, however, be clearly seen in perpendicular sections and in animals, by teasing out the mucous membrane. The Lieberkühnian glands, also, may generally be very readily isolated in their entire length; while the closed follicles should be carefully exposed from without, isolated and opened; 'or they may be studied in perpendicular sections. The muscular tissue of the mucous membrane must be exposed by removing the tunica nervea on its exterior and then separating it in small segments from the glandular layer; its elements may be very well seen by macerating it in nitric acid of 20 per cent.

Literature of the intestinal canal.-Th. L. W. Bischoff, "Ueber den Bau der Magenschleimhaut," Mull. Arch. 1838, p. 503, with figures; Wasmann, "De digestione nonnulla," Berol., 1839, cum tab.; L. Böhm, "De glandularum intestinalium structurâ penitiori," Berol., 1835, 8 c. tab.; and "Die kranke Darmschleimhaut in der Asiatischen Cholera," Berl., 1838; J. Henle, "Symbolre ad anatomiam villorum 
intestinalium impr. eorum epithelii et vasorum lacteorum," c. tab. Berol., 1837, 4to.; J. Flouch, "Recherches sur la membrane muqueuse intestinale, in Mém. de la société d'histoire natur. de Strasbourg," III. 3, Strasb., 1845; A. Th. Middeldorpf, "De glandulis Brunnianis," Vratisl. 1846, c. tab. ; E. H. Weber, in "Müller's Archiv," 1847, p. 400 ; and in "Berichte der Königlichen Sachsischen Gesellschaft der Wissenschaften," Heft VII. 18 May, 1847, p. 245; Frerichs (and Frei), Article, "Verdauung, in Wagner's Handw. d. Physiologie," Bd. III. p. 738-755; R. O. Ziegler, "Ueber die solitären und Peyer'schen Follikel," Würzburg, 1850, Diss.; E. Brücke, 1. "Ueber den Bau und die physiologische Bedeutung der Peyer'schen Drüsen, in Denkschriften der Wiener Akademie," bd. II. 1850, p. 21, with 1 plate; 2. "Das Muskelsystem der Schleimhaut des Magens ;" and 3. "Ueber ein in der Darmschleimhaut aufgefundenes Muskelsystem," in the "Berichten der Akademie," 1851; Kölliker, "Ueber das Vorkommen von glatten Muskelfasern in Schleimhäuten," in "Zeitschrift für wiss. Zoologie," III. 1851, p. 106, und Nachtrag dazu. Heft II.; F. Ernst, "Ueber die Anordnung der Blutgef ässe in den Darmhäuten," Zürich, 1851, Diss. c. tab.

[Brüch, "Beiträge zur Anatomie und Physiologie des DünndarmSchleimhaut," Siebold and Kölliker's "Zeitschrift," 1853; also three most important papers by Brücke, "Ueber die Aufsaugung des Chylus," Sitzungsberichte d. Wiener Akad. Dec. 1852; "Ueber den Ursprung und den Verlauf der Chylusgefässe," ibid. January, 1853, and "Ueber die Chylusgefässe und die Fortbewegung des Chylus," ibid., March, 1853, which have come into our hands too late for further reference.*-Trs.]

\section{OF THE LIVER.}

$\S 158$. The liver, a large gland, is at once distinguished from those compound glands, such as the salivary, which have hitherto been described, by the intimate connection of its larger subdivisions and by the very peculiar structure of its secreting parenchyma, which elaborates the bile. The component parts are, the secreting parenchyma, consisting of the lobules or islets of the liver and of the networks of hepatic cells; the biliary passages which are formed in this, and the efferent biliary ducts; very numerous bloodvessels; a considerable number of lymphatics and nerves; and finally, a peritoneal investment.

$\S 159$. Secreting parenchyma, hepatic lobes and hepatic substance.If the surface or a section of the liver be regarded, it generally exhibits a mottled appearance, which is usually of such a kind, that small,

* [See note, p. 516.-DaC.] 
stellate, reddish or brown spots are enclosed within a more yellowish-red substance-medullary and cortical substance (Ferrein). This variegation proceeds from the usually unequal distribution of the blood in the smallest trunks and in the capillaries, and in healthy persons it is replaced by a uniform reddish-brown color. The mottling of the surface of the liver is frequently so regular as to have given rise to the supposition that it consists of lobes, especially as in an animal which is a frequent subject of investigation,-the Pig,-they are very obvious; but, as E. H. Weber was the first to demonstrate, in 1842 , in the human liver nothing of the kind exists; here, in fact, not only the secreting elements, but the most important parts of the vascular system, $i$. e. the capillary network between the portal and hepatic veins are intimately connected together throughout the whole organ. Nevertheless, it would be very erroneous to suppose that the secreting parenchyma of the liver is everywhere homogeneous. Ultimate segments may be observed in it, which have a certain independence, although they are in nowise isolated. These hepatic lobules, as they may be called, if the term be used in its most general signification, or hepatic islets, are thus produced: 1 . The smallest branches of the afferent and efferent bloodvessels, the vence inter-and intra-lobulares (Kiernan) are distributed at pretty equal intervals through the whole liver, so that a portion of hepatic substance of $\frac{1}{3}-\frac{1}{2}-1$ line in diameter, is always found to give origin in its interior, to a small twig of the hepatic vein receiving externally a certain number of the minutest branches of the portal vein and of the hepatic artery; and, 2, The hepatic ducts do not commence irregularly in the parenchyma, but are so disposed that they invariably arise at a distance of $\frac{1}{7}-\frac{1}{2}$ a line from the origins of the hepatic veins and take the same course as the finest ramifications of the portal vein. In this manner little masses, containing only secreting parenchyma, capillaries and the origins of hepatic veins, are marked out in the liver; whilst in their interspaces, together with parenchyma and capillaries, lie the ultimate branches of the portal vein and hepatic artery and the origins of the hepatic ducts, which, as they do not approach the masses from one, but always from many sides, and are also supported and partially united by connective tissue, form, if not complete, at all events partial zones around them.

The livers of those animals which present lobes (Polar Bear, J. Müller, Pig), are of the greatest value in comprehending the structure of the organ, and I therefore here subjoin an account of the structure of the Pig's liver. If we examine this organ in sections or otherwise, it is always seen to be divided into numerous small, rounded, polygonal, not very regular areoe of tolerably uniform size $\left(\frac{1}{2}-1 \frac{1}{4}\right.$ lines), which consist of the proper parenchyma of the liver and are bordered by whitish parti- 
tions, readily visible to the naked eye. If a cut surface be scraped with the handle of a scalpel, angular masses of liver, equal in size to these arece, are detached, the capsules which surrounded them remaining behind as empty compartments, like a honeycomb. These become still more distinct if a thin section of the liver is gently kneaded with the fingers in water, and then washed and examined on a black ground, in which case many compartments remain almost completely closed, and still more resemble closed capsules. It must not be supposed, however, that there are any complete special investments around each hepatic lobule. On the other hand, the membranes by which they are formed, always appertain to many lobules in common, so that the whole constitutes a cellulated substance continuous throughout, whose partitions

Fig. 218.

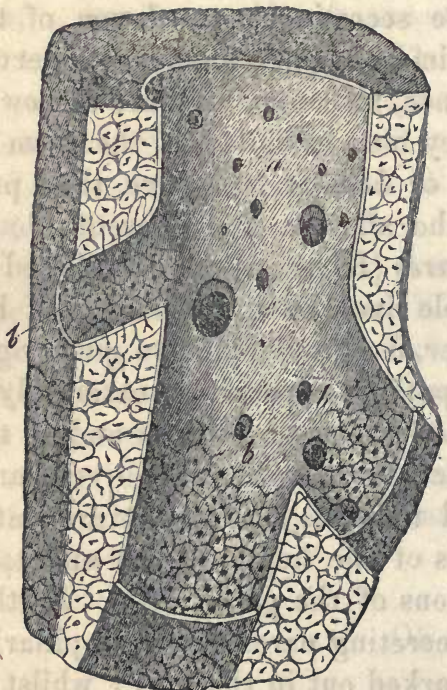

Fig. 219.

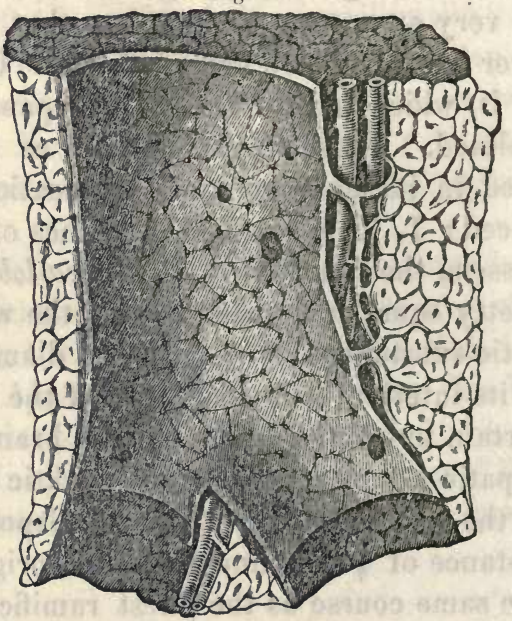

are all simple and cannot be divided into a number of lamellæ. If we trace out the capsules, or as they might better be termed, the partitions of the lobes, we find that they are, for the most part, expansions of the connective tissue, which accompanies the vena portoe, frc., or of the socalled capsule of Glisson, but are also connected with the serous investment of the liver and accompany the larger hepatic veins. Kiernan was the first to take a just view of the relation of the lobules to the hepatic vessels, when he said, that they are seated upon the branches of the hepatic vein, like leaves upon their stalk. In fact we find, if a small branch of the hepatic vein be slit up (Fig. 218, $b \quad b \quad b$ ) that it is

- Fia. 218.-Segment of a Pig's liver, with an hepatic vein laid open, somewhat magnified: $a$, large vein, into which as yet no intralobular veins open; $b$, its branches, with intralobular veins, and the bases of the lobes shining through. After Kiernan.

Fig. 219.-Portal vessel of the Pig, cut open, with its accompanying branches of the hepatic artery and duct. After Kiernan. 
surrounded on all sides by the hepatic lobules and receives a single vein from each, so that they actually appear to be attached to it by short pedicles. Now since this takes place in exactly the same manner from the veins of moderate size up to the intralobular veins, the hepatic veins and lobules may, not without reason, be compared to a tree whose branches are so numerous and so closely beset with polygonal leaves that the foliation, so to speak, constitutes one mass. Imagine now, that another ramified system, composed of vessels, is introduced into the expanded head of this tree, in such a manner that the larger vessels lie in the clefts between its principal masses, the smaller and smallest in those between the subordinate masses, ultimately penetrating into the lobules themselves, so that every lobule is connected with many of the finest twigs, receiving a coat from the connective tissue which accompanies them, and we shall have as distinct an idea as possible of the relations of the vena portce. The hepatic duct and artery merely accompany the vena portce, and, therefore, require no special notice.

In form, the lobes of the Pig's liver are angular, usually presenting irregular four, five and six-sided figures in longitudinal and transverse sections.

In the human liver, but very little connective tissue accompanies the vena portoe between the hepatic islets, and the latter can neither be said to possess eoats nor to be in any complete manner enclosed by the vessels. In cirrhosis of the liver, on the other hand, an enormous increase takes place in the amount of connective tissue contained in the parenchyma of the liver, and the individual secreting segments may become prominent or even form isolated lobules. The reddish-brown hepatic substance is softer, because more macerated, and sinks in more than the rest, upon the surface and in sections; it may also be more easily scraped away and sometimes readily falls out in fine segments. The cortical layer, which forms a reticulation around the reddish-brown spots, presents narrower places, fissurce interlobulares, Kiernan, and wider, spatia interlobularia, in which not uncommonly a bloody point may be seen, arising from a portal vessel, but not so regularly as in the brown spots, where it arises from the vena intralobularis and often appears stellate.

By the more complete filling up of the capillary network, it may happen, and, according to Theile, this is in fact the usual case in the majority of human livers, that the fissurce interlobulares disappear, the brown substance representing a network, and the yellow, occurring in isolated spots. I find, as I have stated above, that perfectly fresh livers are, for the most part, uniformly colored throughout. Kiernan describes, in children, even a reversal of the coloring, which he considers to be dependent upon congestion, more particularly of the vena porto, the external portions of the hepatic lobes being thus more injected. Neither Theile nor I have hitherto noticed this form. 
\$ 160. Hepatic cells and cell-networks.-Every hepatic islet contains two elements; 1 , a network of capillaries, which, on the one hand, is continuous with the finest portal branches, and on the other, unites into the intralobular vein, one of the roots of the hepatic vein; and 2, an interlaced tissue of delicate columns, composed of nothing but cells, the so-called hepatic cells, in close and immediate apposition. These two networks are so interwoven that the interstices of the one are completely filled by the solid portions of the other and leave no interspaces, at least when the vessels contain blood or are injected. Not a trace of biliary ducts is to be observed in this network: they are first met with at the periphery of the hepatic islets, where also the finest portal branches occur, without its having been possible, hitherto, to make out, directly, their connection with the hepatic-cell-network, which is indubitably the secreting portion of the liver.

The hepatic cells, which may be isolated with the greatest ease, ${ }^{*}$ have a diameter of $0.008-0.012$ of a line on the average, $0.006-0.016$ of a line in extreme cases, and resemble tessellated epithelium-cells in form, except that they are more irregular. Their membrane is delicate, and

Fig. 220.

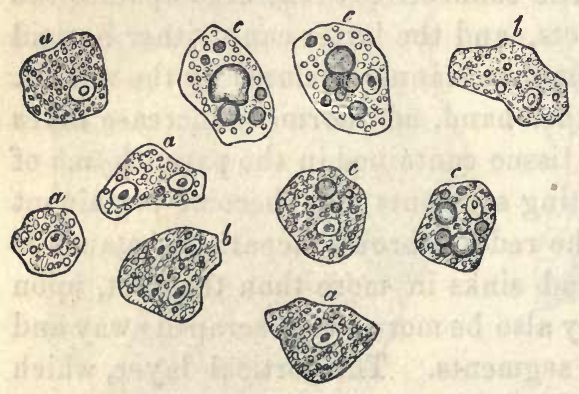
perfectly closed, and their contents, in perfectly normal livers, such as are rarely met with in man, but readily enough in animals, are a granular, yellowish, semifluid substance, which, as microscopic investigation shows, probably contains the essential element of the bile. In this lies a round vesicular nucleolated $n u$ cleus, of 0.003-0.004 of a line; and in many cells there are two.

Besides these, fat-drops and pigment granules are frequently to be met with. The former (Fig. $220 \mathrm{e}$ ) occur in all the cells, when the liver has undergone fatty degeneration, in such quantities that they become very similar to certain forms of fat-cells; and generally, as a few large or many small drops, entirely fill the cell, so that the nucleus becomes invi-

FIG. 220.-Hepatic cells of Man, magnified 400 diameters : $a$, more normal cells; $b$, with pigment; $c$, with fat.

* [This is true of the hepatic "cells" of Man and many Mammalia, but not of all. In the Rat, we could find no demarcation of the hepatic tissue into cells, the tissue resembling very nearly that of the spleen. In Fishes, Dr. Handfield Jones has pointed out the existence of all varietes in this respect, from free "nuclei," with oily and granular matter scattered through a matrix, up to perfectly formed "cells." In the Pigeon, the same author finds no fully formed cells, nor in the Duck; and he therefore draws the general conclusion, "that the secreting process by no means requires the formation of perfect cells in order to effect its peculiar changes; these may certainly occur in blastematous matter, if the nucleus only be present." "Philosoph. Transactions," 1849, p. 132.-Trs.] 
sible. Every transition may be traced from these well-marked forms to ordinary cells with a few minute drops or a single somewhat larger one; and, in fact, these less fatty cells occur to a certain extent in almost every body ordinarily subjected to examination, so that if their absence in animals were not kept in' mind, their occurrence, at least to a small amount, might be regarded as normal. The same may be said with respect to the pigment molecules (Fig. 220 b). When these are very abundant they are certainly pathological; but when few, they can only be regarded as a slight deviation from the normal state. They are small, hardly exceeding 0.001 of a line, of a yellow, or brownish yellow.color; their chemical reactions are identical with those of the coloring matter of the bile as it occurs in the intestinal canal, inasmuch as they are not altered in color by nitric acid, nor dissolved by caustic alkalies.

The hepatic cells are so arranged in the islets as to form a network by the simple apposition of their flat surfaces, without the assistance of any foreign connecting intermediate substance or investing coat. The simple or branched columns of hepatic cells, which are almost always to be found among scraped off particles of the liver, and to which Henle drew attention (Allg. Anat., p. 903), are nothing but fragments of the hepatic cell-network, whose elements do not cohere very firmly. Taken altogether, the network of every hepatic islet presents more rounded meshes at the periphery, while in the centre they are constantly disposed radially, whence, in a transverse section through the interlobular vein, long ramified columns of hepatic cells are seen stretching from the latter on all sides and uniting by short lateral anastomoses, so that the intermediate meshes appear like narrow elongated clefts. The hepatic columns consist of 1-3, more rarely of $4-5$ rows of cells, have a diameter of $0.01-0.015$ of a line on the average, 0.006 0.02 of a line in extreme cases, and are generally cylindrical or prismatic, but not at all regularly

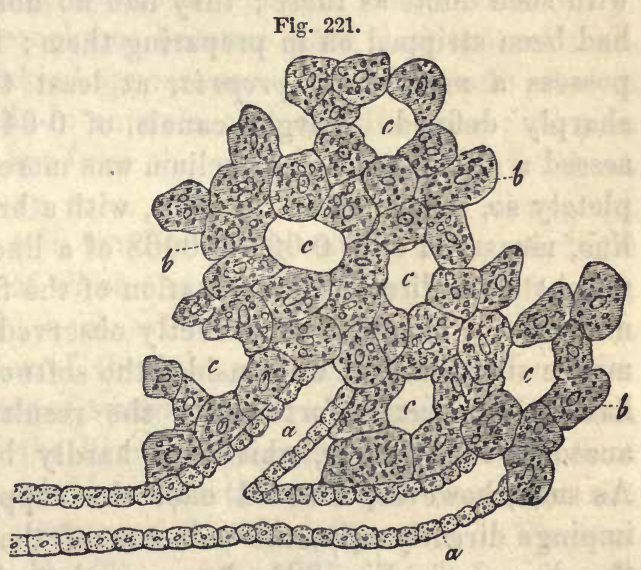
so; their surfaces are arched, plane, or, in some localities, depressed, and have rounded or sharp angles. The meshes of the hepatic cellnetwork correspond with the diameters of the capillaries and of the larger

Fia. 221.-Hepatic cell-network, $b$, and finest ductus interlobulares, $a$, of Man, after nature, the union of the two, diagrammatic; magnified 350 diameters : $c$, vascular spaces. 
vessels which border upon the hepatic islet, by which they are perfectly filled during life, and will be more thoroughly considered below.

If, as results from the above analysis, the secreting parenchyma of the liver consists of a solid network of hepatic cells, one cannot but be struck with its great difference from all the other glands of the body; and the important question arises, how, with this arrangement, the secretion is conveyed from the interior of the cells, in which we suppose it to be formed, and finally carried away. Anatomy here gives no sufficient reply; for although the ramifications of the hepatic ducts have been traced accompanying the vena portce as far as the hepatic islets, yet, as respects the connection of their finest twigs with the hepatic network of cells, no definite answer has been afforded; and indeed, up to the present time, no satisfactory account even of the structure of the former has been given. Without entering further, in this place, into the distribution of the hepatic ducts, I will only observe, that in carefully made microscopic preparations, we not unusually find fragments of the finer and finest ducts, the ductus interlobulares of Kiernan, between the hepatic islets, and readily obtain evidence that they are constructed according to the ordinary type of excretory ducts. The minutest of these canals, which I have met with, measured ${ }_{10}^{1} \frac{1}{0}$ of a line in diameter, possessed a cavity of 0.0033 of a line, and were composed of a simple layer of common tessellated epithelium-cells, which were distinguished from the hepatic cells by their small size $(0.004-0.005$ of a line), their pale contents and the minuteness of their nuclei. I hare frequently met with such ducts as these; they had no fibrous coat, perhaps because it had been stripped off in preparing them; but occasionally they seem to possess a membrana propria, at least their external contours were sharply defined. Larger canals, of $0.04-0.05$ of a line, always possessed a coat, and the epithelium was more cylindrical, though not completely so, inasmuch as the cells, with a breadth of $0.0048-0.0056$ of a line, measured only $0.006-0.0068$ of a line in length. Often as I have sought for a direct communication of the finest canals with the hepatic networks, I have not yet directly observed it; which is, indeed, by no means surprising, if we consider the softness of the parts with which we have to do; but unfortunately the result is a hiatus in the minute anatomy of the parts, which can hardly be made good by hypotheses. As such, however, I would offer the supposition, that the finest ducts impinge directly upon the columns of the network of hepatic cells, as the diagram in Fig. 221 shows, so that their cavity is terminated by hepatic cells : and, judging from the scantiness of the finest branches of the hepatic duct, I believe that such communications exist, in no very great numbers, at the circumference of the hepatic islets.

Whatever view we may take of the connection of the hepatic cell-networks with the efferent biliary canals, it is undeniable that any such 
connection only takes place upon the surface of the hepatic islets and not in their interior, and that, therefore, the bile which is formed here, must be transmitted outwards from cell to cell. Such a process of transmission through closed cells, involves, as vegetable physiology teaches us, no impossibilities; but it can hardly take place so rapidly as in those localities where actual canals subserve this purpose. Since the bile, as late investigations tend to show with increasing clearness, is not only excreted from the blood, but absolutely formed in the liver, and is at the same time by far the most complicated of all the secretions, it may be presumed that the peculiar arrangement of the secreting parenchyma in the liver stands in relation with these peculiarities. In fact, the plasma of the blood, in passing through many cells and being subjected to the metabolic influence of each before it reaches the efferent duct, must undergo very different changes from those which it suffers when it is separated from the glandular canals only by a single layer of cells and one or two structureless membranes. The resulting necessary slowness of the secretion is compensated by the size of the organ and the elaboration of the product.

If nitric acid be added to the hepatic cells, they assume a greenishyellow color, as was originally stated by Backer. Sugar and sulphuric acid turn them red; water produces an abundant precipitate of dark granules in the cells, which are usually, readily and completely soluble in acetic acid, so that they become more or less pale, often to a considerable extent; the same thing occurs if the acid be added directly. If the liver be boiled, its parenchyma becomes hard and the cells acquire a concentrated and wrinkled appearance. Dilute caustic alkalies rapidly attack the hepatic cells in animals, and dissolve them; in man they resist somewhat longer, but from the very first swell up to about twice their size, become very pale, and eventually also disappear. Ether and alcohol render the cells smaller and granular, as do sulphuric and nitric acids. The result of these and the above-mentioned facts is, that the hepatic cells contain a considerable quantity of nitrogenous substances, fat, coloring matter of the bile, and perhaps also its acids. The nitrogenous compounds are of several kinds: albumen, which is met with in the watery extract of the liver, and a substance which is precipitated by water, is readily soluble in acetic acid and resembles the casein-like matter found in the serum of the blood (Pannum in Virchow and Reinhard's Archiv, b. IV. 1). The existence of the coloring matter of the bile in the hepatic cells is not so much manifested by their reaction with nitric acid, which is also exhibited by many other cells, as by their general tint, and the frequent occurrence in them of precipated coloring matter of the bile. The existence of the fatty acids of the bile in the hepatic cells is not directly demonstrable, since both albumen and fat become red by the action of sulphuric acid and sugar (Schultze), but 
is probable. Fat, however, certainly exists in them, even when it is not microscopically demonstrable, as the collective analyses of the liver show: Probably, also, the sugar which recent researches have demonstrated in the liver will be found to exist in the parenchyma; in the cells, therefore, and not in the blood only.

As the principal mass of the liver is formed by the hepatic cells, I here add the results of one of the many analyses of the liver by Von Bibra ("Chem. Fragmente über die Leber und Galle," Braunschweig, 1849). He found, in 100 parts of the substance of the liver of a young man who died suddenly-

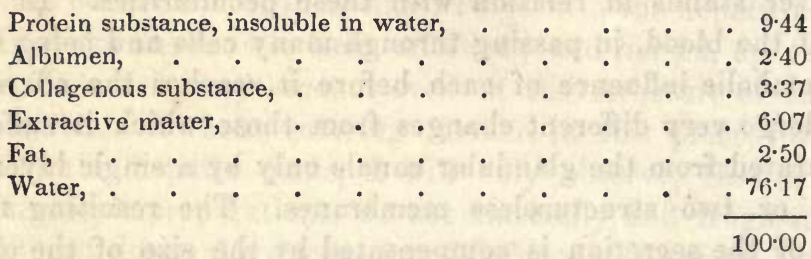

One hundred parts of the water yielded 3.99 of ash, containing especially phosphate of potassa, then phosphate of lime, some silica and iron, and chloride of sodium. The protein substance, insoluble in water, proceeds from the nuclei and membranes of the hepatic cells and from the contents to which we have referred. The albumen partly proceeds from the blood, but certainly from the cells also. Von Bibra found neither kreatin nor kreatinin in the extractive matters; the coloring matter which they contained did not present the same reaction as that of the bile, whence Von Bibra draws the conclusion that this ingredient does not exist as such in the cells. Finally, I may advert to the acid reaction of the parenchyma of fresh liver, which I discovered (Art. "Spleen," in Todd's Cyclopædia), and which, in this case, is even more remarkable than in the spleen. Von Bibra also found the watery extract of the $O x$ 's liver to have an acid reaction (l. c., p. 33), and has demonstrated the existence of lactic acid in it.

There is no subject of minute anatomy upon which opinions are so various, at the present time, as upon the structure of the secreting parenchyma of the liver; and yet, with the views which have been expressed in the preceding section, the only question that can arise is, whether the finest biliary ducts are intercellular spaces, canalicular spaces between the hepatic cells, as Henle and Gerlach consider, or whether they consist of the columns of hepatic cells surrounded by membrance proprioe. I have endeavored to show, in my "Mikr. Anat." II. p. 221, that these notions are untenable, and that nothing remains but to accept the view which has been offered, however paradoxical, as the only one which at all corresponds with nature.*

* [In animals the structure of the secreting parenchyma of the liver differs in many respects 
\$ 161. Excretory ducts of the liver.-The biliary duct and its branches accompany the vena portoe and hepatic artery, so that on one side of a portal branch there is always a much smaller biliary duct and artery, which are included with it in a sheath of connective tissue, the so-called capsule of Glisson. The hepatic ducts ramify, in Man, with the vena portce and may be dissected out for a long distance; and with the microscope they may, in fresh and injected livers, be traced as far as the lobules. Before reaching the latter, they either do not anastomose at all or very sparingly; the ductus interlobulares, however, as they have been termed, appear to be continuous with each other and thus to invest the hepatic islets. From these ducts of 1-90-1-120 of a line branches of $1-100-1-120$ of a line proceed, in no very great numbers, to the hepatic islets and become continuous with the hepatic network in the mode above described. Perhaps the very fine biliary ducts, which I have, as stated above, observed microscopically, with a diameter of 0.01 and a cavity of 0.0033 of a line, are identical with a part, at all events,

from that of Man. In the lowest forms of animal life, where no distinct digestive apparatus exists no trace, according to Prof. Leidy, of biliary structure is observable. When we rise a little in the scale, as in the Polypi, certain cells forming part of the digestive cavity, possess the power of secreting a fluid analogous to bile. In many of the Annelida, we find appended to the sides of the alimentary canals cæca lined by small cells, wlich probably secrete a biliary fluid. A similar substance is formed in the Myriapoda by the cells of the long and delicate tubes, which empty into the intestine.

In insects the liver is formed of distinct, filiforn, tortuous tubes, opening near the pyloric extremity of the stomach by separate orifices into the sides of the alimentary canal. These tubes consist of a transparent, amorphous, basement membrane, the inner surface of which is covered by secreting cells. These cells are generally rouncl or oval, and contain molecular matter, numerous fine oil-globules, a central granular nucleus and a transparent nucleolus. The tubes are filled with fine granules and a great amount of oil ; extremely minute respiratory tracheæ are distributed to them.

In the Crustacea, as in the Cray-fish, the liver is formed of two large lobes, one on each side of the intestine, united by an isthmus. Each lobe consists of conical cæca, composed of a sac of basement membrane lined with numerous secreting cells, of a more or less polygonal form. These cells have an average diameter of 0.2 of a line; they increase in size from the bottom of the cæcum upwards, and obtain a gradual addition of oil-globules. The central cavity of the cæca is filled with fat-globules and a finely granular mass.

- In the Molluscs, as in the Snail and Slug, the liver is formed of several lobes, subdivided into lobules, which are composed of numerous bulbiform cæca of a polygonal shape. These crea do not differ in structure from the cæca in the liver of the Crustacea. They are lined with cells of an average diameter of 0.2 to 0.4 of a line, filled with oil-globules, and containing a granular nucleus with a hard transparent nucleolus. Around the cæca there is a network of bloodvessels, to the parietes of which, Prof. Leidy states, minute oil-globules and nucleated fat-cells are frequently attached.

In the Vertebrata, the facts observed by Prof. Leidy confirm the researches of Kiernan and other recent observers. He considers, however, the biliary tubes to commence within the lobules, and not merely, as stated by Kölliker, as a layer of cells, but as a network of distinct tubules lined with a basement membrane and an epithelium. Their diameter in different animals is generally two and a half times the size of the secreting cells. (Vid. Leidy, "Researches into the Comparative Structure of the Liver," in American Journal of Medical Sciences, January 1848.)-DaC.] 
of those which have been injected as the origins of the hypothetical lobular biliary ducts.

All the biliary ducts, down to those which have a diameter of 0.1 of a line, possess a thick fibrous membrane, composed of dense connective tissue with many nuclei and elastic fibres, and a cylinder epithelium 0.01 of a line thick, which, in ducts under $0.04-0.05$ of a line, becomes gradually changed into a tessellated epithelium. The ductus communis choledochus and the cystic duct are similarly constituted, only their walls are thinner, and they may be readily separated into a fibrous and a mucous layer, the latter of which contains also a few muscular fibre-cells, but on the whole so sparingly, that these ducts cannot be said to possess any special muscular coat.

The gall-bladder has, between its peritoneal covering and the abundant subserous connective tissue, a delicate layer of muscles, whose fibre-cells, 0.03-0.04 of a line long, take more particularly a longitudinal and a transverse direction and present only indistinct nuclei. The mucous membrane is distinguished by many reticulated, more or less prominent folds, which contain a capillary network, exactly like that of the foliaceous intestinal villi, and it is also provided with a cylinder epithelium, whose cells are often, like the membranes of the gall-bladder, thoroughly stained with bile; their nuclei are not always distinct.

The walls of the biliary ducts contain a multitude of small, racemose, yellowish mucous glands, the glands of the biliary ducts, whose vesicles of 0.016-0.024 of a line, differ in no essential respect from those of other racemose glands. In the ductus hepaticus, choledochus, and in the lower portion of the systicus, the glands in the fibrous tunic and parts external to it, are very numerous, $\frac{1}{4}-1$ line in diameter, opening singly or many together by foramina of $0 \cdot 1-0.14$ of a line, visible to the naked eye, which give the mucous membrane of those canals a reticulated appearance. At the commencement of the cystic duct the glands are few, and in the gall-bladder itself, in which they are said to have been met with, their occurrence is certainly not constant. On the other hand, in the branches of the hepatic duct, down to $\frac{1}{3} d$ of a line in diameter, such glands are again met with, many of them opening by a double series of fine apertures which exist in these canals.

We may refer here to certain peculiar ramifications of the biliary duct, vasa aberrantia (E. H. Weber). They exist: 1 , in the ligamentum triangulare sinistrum, as 6-10 and more canals, $0.006-0.0027$ of a line in diametcr, consisting of a fibrous membrane and small cells. Ferrein and Kiernan traced them as far as the diaphragm, though for the most part they only extend to the middle of the ligament, or not so far, branching out, forming networks, or anastomosing in loops. According to Theile, tolerably large biliary ducts frequently proceed as far as the 
edge of the left lobe of the liver without entering the triangular ligament.6 2. Anastomosing biliary ducts are also to be met with in the membranous bridge which unites the Spigelian and right lobes behind the inferior vena cava, also in the membranous band which frequently covers the umbilical vein and at the edge of the cystic fossa. 3. In the transverse fissure of the liver, according to $\mathrm{E}$. $\mathrm{H}$. Weber, the right and left branches of the ductus hepaticus and their smaller twigs give off numerous fine ramuscules, which are distributed through the connective tissue of the capsule of Glisson covering the fossa and form a network, which unites the right and left hepatic ducts. Many small branches of these ducts terminate by enlarged ends of 1-25-1-18 of a line, and upon their walls a multitude of rounded elevations are met with, which, like the walls of the smallest bronchice, appear to be formed by flattened cells, which have coalesced with the canals and retain wide communications with their cavities. What Weber thus describes as vasa aberrantia, were subsequently described by Theile as glands of the biliary ducts. He says, that the elongated glands are not merely curved in various directions, but divide, the resulting branches reuniting with one another, and with the surrounding glands, as may be observed in the glands of the coarser and middle-sized biliary ducts-especially, also, in the connective tissue of the transverse fissure, where the glandular networks are connected with both branches of the biliary ducts. In opposition to these views, Weber, in his latest work, adheres to his former interpretation, and shows, against Theile, that mucous glands and their ducts form networks and connect the ducts of other glands nowhere else; furthermore, that in the new-born infant, although the network of the biliary ducts exist in the transverse fissure, those branches which terminate in enlarged extremities are almost entirely absent.

The relations of the finest ramifications of hepatic ducts, or of the ductus interlobulares of Kiernan, have not yet been perfectly made out, a subject which gave rise to many remarks in the previous section. I will only add here, that some, as more especially Guillot, suppose, not only that the ductus interlobulares anastomose, but also that their branches are inter-connected in many ways, whilst others, as Theile, describe their communications as scanty. For myself, though I have observed anastomoses of the interlobular ducts, I have as yet met with no communications between their branches, which, though they do not enter the hepatic islets, may be called lobular branches. If they occur, they are certainly few in number, for such branches may be isolated for a considerable distance without any other trunks being seen either to be given off from or to join them. Upon the whole, the interlobular branches are anything but abundantly distributed, and, therefore, the slowness of the biliary secretion is determined, not only by the pecu- 
liar structure of the hepatic parenchyma, but also by the small number of the excreting canals.

The bile is normally quite fluid, bcing only accidentally mixed with cylindrical epithelium-cells, derived from the coarser biliary ducts. I have never met with hepatic cells in them, and the statements of those who have affirmed their existence have arisen, either from a mistake, or from confounding with them the polygonal cells of the epithelium of the ductus interlobulares. Constitutents which, though abnormal, are very frequent, are-fat-drops, coloring matter of the bile in granules or granular masses, which, as in the hepatic cells, so also in the bile itself, are occasionally abundantly excreted; more rarely there are crystals of cholesterine, and especially the reddish needles of bilifulvin, lately observed by Virchow ("Mittheil d. Würzburg, Phys. Med. Ges." I. p. 311).*

* [The view which Professor Koblliker takes of the mode of termination of the hepatic ducts, can hardly be said to present any essential difference from that which Dr. Handfield Jones has ably advocated in several successive papers in the "Philosophical Transactions" (for 1846,1849, and 1853), and which we here subjoin in his own words :-

"The liver, in all vertebrate animals, may be regarded as consisting of a secretory parenchyma and of excretory ducts.

"The size of the excretory apparatus bears only a small proportion to that of the secretory.

"These two portions of the liver are not continuous with one another, but are disposed simply in a relation of juxtaposition.

"The action of the liver seems to consist in the transmission of bile, as it is formed, from cell to cell, till it arrives in the neighborhood of the excretory ducts; by which it is absorbed. This action is probably slow and very liable to be interfered with, contrasting remarkably with that of the kidney, where a particular apparatus is added to insure completeness and rapidity of action.

"The secretion of the hepatic cells is very liable to be retained within the gland, either in the cells or in a free state.

"This circumstance, as well as its structural relations, seem to point out the liver as approximating to the class of ductless glands.

"For the same reason it is highly probable that a part of the secretion of the cells is directly absorbed into the blood which traverses the lobules" ("Pliil. Trans.," 1849, p. 132).

From an extensive series of researches in all classes of the Vertebrata, Dr. H. Jones comes to the conclusion that the excretory system of the liver always terminates in closed tubes. The ducts of the Sheep's liver, which in all essential particulars agrees with that of Man and of the Pig, are thus described:-

"In the minutest branches (of the biliary ducts) which seem to be approaching their termination, and which can sometimes be examined and isolated in the most satisfactory manner, the epithelial particles are remarkably modified; they can scarcely be said to exist as separate individuals, but rather their nuclei, which are often large and distinct, are set close together in a subgranular or homogeneous basis substance. In ducts where this condition of epithelium exists, there is seldom any distinct trace of basement membrane; the margin, though sufficiently even, yet exhibiting the bulging outlines of the component nuclei; still less is there any proper fibrous coat, though the ducts may be more or less involved in the filamentary expansions of the capsule of Glisson. Ducts of this character have usually a diameter of about $\frac{1}{1000}$ th of an incli; they can sometimes be followed for a considerable distance without being seen to give off any branches, or to diminish much in calibre. Their mode of termination is various-several have been distinctly seen to terminate by rounded 


\section{$\S 162$. Vessels and nerves of the liver. - The arrangement of the blood-} vessels in the liver distinguishes it from all other organs, inasmuch as, besides an artery and an efferent vein, it possesses an additional, afferent vein, the vena portoe. While the latter is appropriated to the supply of the secreting parenchyma, being directly continuous through the capillary network with the hepatic vein, the artery is more especially distributed to the walls of the biliary ducts and of the portal vein, to Glisson's capsule, and to the serous investment of the liver, taking only a subordinate share in supplying the capillary network of the hepatic islets. The ramifications of the portal vein, and of a few small veins of the stomach and gall bladder (see Weber, Ann. Acad. 1845), which enter the liver independently, take place for the most part dichotomously; but both larger and smaller branches, besides the main trunk, into which they divide, give off a number of small vessels at right angles. The latter, often after a very short course, at once enter the hepatic islets contiguous to the largest vascular canals, while the larger portal branches, ramifying continually, and becoming finer and finer, have, according to their diameter, to take a longer or a shorter course through the hepatic parenchyma in the canals lined by the capsule of Glisson, before they enter the hepatic islets or lobules. Each of these receives,

and closed extremities, which have nearly the same diameter as the duct itself; others seem to lose their tubular character, their nuclei becomes less closely set together, and the uniting substance more faintly granular and indefinite; the duct, in short, gradually ceases, losing all determinate structure. In some, of rather minute size, $\frac{1}{3000}-\frac{1}{200}$ th of an inch in diameter, the exterior form remains distinct, but the canal is almost obliterated by the close approximation of the nuclei of the opposite walls. These structures now described, I believe to be truly the terminal branches of the hepatic duct, from which they certainly originate. They seem gradually to lay aside the several component tissues of the larger ducts, the fibrous coat blending with the ramifications of Glisson's capsule, the basement membrane imperceptibly ceasing, and the epithelium becoming resolved at last into its simple fundamental nuclei" (1. c., p. 125).

It is important to remark, that in a Dog, Dr. H. Jones found biliary matter in the interlobular fissures.

From the fact that in the contents of the hepatic ducts of Man and the Sheep, extracted by means of a forceps and without injuring the organ, hepatic cells may be detected, Mr. Wharton Jones ("Phil. Trans.," 1848) draws the conclusion that the hepatic cells are endogenous cells, answering to the epithelium of other glands-which was Henle's view. It is impossible to doubt a fact stated by so careful an observer; but, however these cells may have got into the large biliary ducts, it is quite clear, from a comparison of diameters, that they cannot enter the minutest ones-the total diameter of the latter being the same as that of the cells, viz. I $^{1} 0^{\text {th }}$ of an inch.

We are strongly inclined to believe that the view taken by Dr. H. Jones is in the main correct-that the liver is essentially of the same order as the "ductless" glands, and should be placed in the same category as the Peyerian follicles, spleen, \&c. In fact, startling as this view may at first appear, a very clear transition between the Peyerian follicles, \&c., and the liver, is afforded by the tonsils; which, on the one hand, are identical with Peyer's follicles, in so far as they are solid vascular networks, whose meshes are filled by a morphologically indifferent tissue; while, on the other hand, without differing from the liver in this respect they resemble it in having these elements arranged around diverticula of the intestinal mucous membrane.-Trs.] 
from one or other set of vessels, at least 3 , usually 4-5, smaller vessels of 1-120-1-60 of a line, which Kiernan calls vence interlobulares. Such a vein, however, is never distributed to only one hepatic islet, but always to two, or even three. Their ultimate branches, the rami lobulares of Kiernan, 10-20 in number, enter the neighboring hepatic islets, usually at a right angle, and divide immediately into the capillary network, without becoming, in man, directly united with one another. In fact, the branches of the portal vein nowhere anastomose, but are connected merely by the finest vascular network of the organ.

Fig. 222.

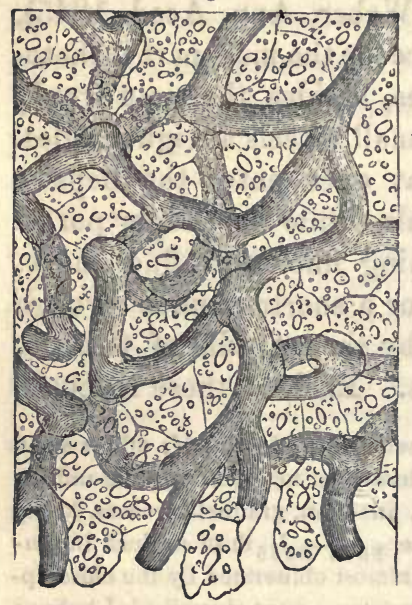

Fig. 223.

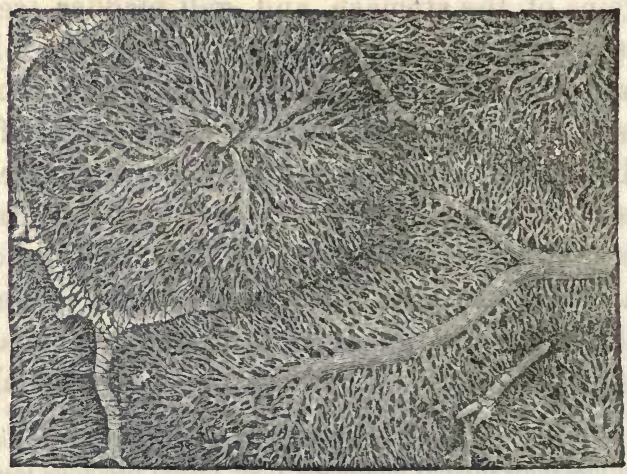

The capillary network of the islets (Fig. 222) completely fills the interspaces of the hepatic cell-network, so that the secreting parenchyma consists actually of only two elements, the hepatic cells and the capillaries. Exactly as the hepatic cell-network is continuous through the entire liver, though being interrupted by the biliary ducts which pass off and the vessels which enter, at regular intervals, it is divided into separate, very minute areace-so the capillary network of the bloodvessels passes from one hepatic islet to another, but is nevertheless discontinuous in certain spots. The diameter of the capillaries is, in general, somewhat less than that of the hepatic cell-network, though relatively considerable; in man it is, on the average, $0.001-0.0055,0.002-0.01$ of a line, in extreme instances; the wide vessels being more especially found in the neighborhood of the afferent and efferent veins, the narrowest in

Fig. 222.-Hepatic cell-network and its capillaries, magnified 350 diameters; from the Pig. Spaces are purposely left here and there between the cells and capillaries, which do not exist in nature.

Fia. 223.-Segment of a very successful injection of the hepatic veins of the Rabbit, magnified 35 diameters. One vena intralobularis is visible in its entire course, but only the radicles of the other. The capillaries of the lobules partly coalesce, and, in one place, two venous radicles do so. 
the interval between them. The meshes of the network correspond, of course, in form, with the hepatic cell-network, and are thence more elongated in the interior of the hepatic islets, more rounded externally, whilst their breadth corresponds with that of the columns of the hepatic cells, being about $0.006-0.02$ of a line.

The hepatic veins essentially resemble the portal vein, in so far as they possess no valves, branch out at acute angles and do not anastomose; their larger branches also receive numerous minute vessels, but these lie isolated in special canals in the hepatic substance to which they are firmly attached, whence they do not collapse when cut across, and, at least in their finer ramifications, possess no external investment of connective tissue, which is indeed but very rudimentary, even in the largest trunks. The relations of the ultimate branches of the hepatic vein, termed by Kiernan intra-lobular veins, and by Krukenberg, venoe centrales lobulorum, are, however, totally different from those of the portal ramuscules. These veins, which in Man are $0.012-0.03$ of a line in diameter, are best studied in some animal whose liver breaks up into isolated lobules, as the Pig; after which Kiernan has given his somewhat diagrammatic figure. If we here open a small branch of the hepatic vein, polygonal areæ are descried through the walls of the vessel-the outlines of those surfaces of the lobules which are turned towards the vein (Fig. 218).

A minute vein which, in the centre of each of these surfaces, termed by Kiernan "bases of the lobules," opens directly in to the larger vessel leads, if we trace it in the opposite direction, into the interior of a lobule, where it arises from the capillary network; but under no circumstances is it continued into a second or third lobule. In this way only a single vein, which may thence be called vena intralobularis, arises in each lobule. The vessels into which these veins directly open are called by Kiernan sublobulares, because they run along the basal surfaces of the lobules. They are sometimes large, attaining as much as 1-2 lines in the Pig, and then lie in canals which are surrounded by the basal surfaces of a certain number of lobules; at other times they are smaller, down to 1-30 of a line, in which case they only pass between the lobules. The sublobular veins unite into larger veins, which continue to receive, directly, but few or no other intralobular veins, and thence, are only partly or not at all bounded by the basal surfaces of the lobules, but only by their lateral or apical surfaces ("capsular surfaces," Kiernan). Such veins, when they are smaller, still receive sublobular veins from the groups of lobules which immediately surround them; or, lastly, only larger veins, which have the same relations as themselves.

The intralobular veins are very simply arranged. Each of them penetrates directly into the axis of an hepatic islet, or lobule, dividing in the middle into two or three principal branches, which frequently 
again subdivide. The capillaries open, not merely into the terminations of these veins, but also into their trunks throughout their course; in- deed, according to Theile, the origins of the sublobular veins also receive capillaries. In all those hepatic lobules or islets whose apices are turned either towards the surface of the liver, or to a large vascular trunk, the interlobular veins extend nearly to their extremities; whilst in others they stop more nearly in the middle, so that they are always about half the diameter of the lobules distant from the nearest interlobular veins of the vena portoe.

The hepatic artery, for the most part, accompanies the portal vein and the biliary canals, is enclosed with the latter in Glisson's capsule, and, in its principal ramifications, presents precisely the same relations as the portal vein. It is finally distributed upon the vessels and biliary ducts, in Glisson's capsule, in the fibrous and serous coats of the liver, and in the hepatic islets, whence its branches are denominated raini vasculares, capsulares, and lobulares.

1. Rami vasculares. - As it divides, in company with the vena portoe, the hepatic artery gives off numerous small branches, almost at right angles, which form a plexus in Glisson's capsule, from which some lobular branches for the parietes of the portal canal arise, on the side opposite to the arterial trunks; while many twigs proceed to the walls of the portal vein, the larger branches of the artery itself, the hepatic veins, Glisson's capsule and the biliary ducts. The distribution of the vessels is particularly remarkable in the latter canals, so that, in a good injection, they appear almost as red as the arteries thernselves.

A moderately close capillary network exists around all these parts, even the glands of the biliary ducts, whence the vence vasculares arise and open, as Ferrein discovered and as all the moderns since Kiernan have agreed, not into the hepatic vein, but into small portal twigs, as these are leaving the larger branches in Glisson's capsule, and are therefore to be regarded as internal, or hepatic radicles of the portal vein. From this cause the portal vein may be partially injected from the hepatic artery and conversely. Again, in injecting the hepatic artery and the portal vein, the vascular network in question may be filled from both sides; while it is not possible directly to force injection from the hepatic vein into them.

2. Rami capsulares. - Independently of a few branches given off by the artery, before its entrance into the liver, to the fossa ductus venosi, to the ligamentum teres and suspensorium, all the arterial twigs of the coats of the liver are the terminal prolongations of certain arteries which penetrate the liver and appear in different parts of its surface between the hepatic islets. At the points of exit, and even before reaching them, these vessels, which have a diameter in the adult of 1-30-1-20 of a line, in children as much as 1.5 of a line, break up into $3-5$ radiated subordi- 
nate branches, take for the most part a remarkable, corkscrew-like, coiled course, repeatedly anastomosing, and thus spread over the whole surface of the organ, as far as the great venous trunks (vence hepaticce, vena portce, cava inferior), the fossce of the liver and its edges, as an elegant arterial network. In the end, these arteries everywhere form a capillary plexus with wide meshes, from whence, in many partswhether universally or not I do not know-veins arise, which run back

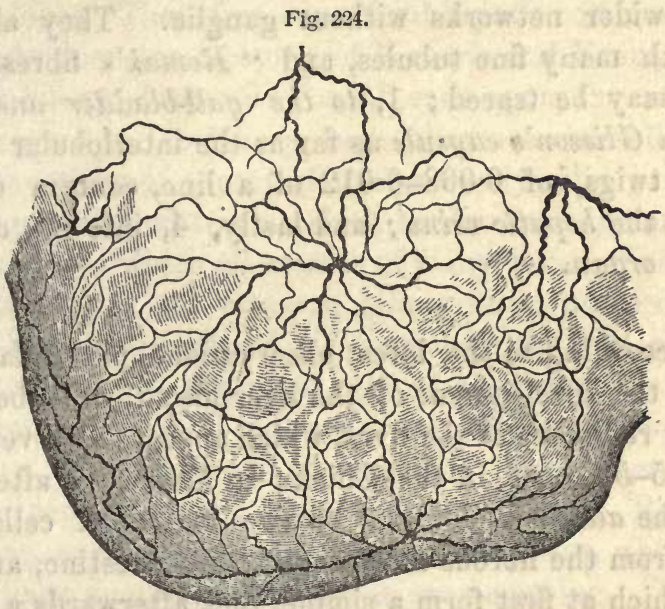

parallel with the arteries, enter the liver and open into the portal branches. Portal radicles, or vence advehentes capsulares, must be derived, therefore, from this region also. The arteries and veins of the hepatic coats are in their terminal expansion connected, on the one hand, with prolongations of the internal mammary, phrenic, cystic and even the right suprarenal and renal vessels (Theile), and anastomose, on the other side, in the hepatic fossoe, with those of Glisson's capsule, with the vena cava and hepatic vein.

3. Rami lobulares.-With every interlobular vein there runs a branch of the hepatic artery, of at most $\tau_{1}^{\frac{1}{3} \sigma}$ of a line in diameter (Theile), which, in the Pig, divides between the hepatic islets, in the capsules of the lobules, into fine anastomosing twigs, and is directly connected with the peripheral part of the capillary network of the hepatic islets or lobules, formed, as stated above, by the portal vein. Arterial blood, therefore, takes a part, although, perhaps, a minor one, in the preparation of the bile, and the hepatic artery is thus distinguished from the bronchial arteries, whose blood is carried away by special veins.

The lymphatics of the liver are very numerous, and may be divided into superficial networks, under the peritoneum; and deep vessels,

FrG. 224.-Arterial network upon the convex surface of a child:s liver. Natural size. 
which accompany the portal vein, and, in animals, at least, the hepatic veins also. Both kinds of vessels are connected and proceed partly through the diaphragm into the thorax, partly to small lymphatic glands in the porta hepatis, and to the intestinal plexus. The lymphatics of the gall-bladder are also exceedingly numerous.

The nerves of the liver are relatively very abundant. They arise from the sympathetic, and, in a smaller proportion, from the vagus, and are chiefly distributed with the hepatic artery, around which they form closer and wider networks without ganglia. They always contain, together with many fine tubules, and "Remak's fibres," a few thick fibres, and may be traced; 1, to the gall-bladder and large biliary ducts ; 2, in Glisson's capsule as far as the interlobular arteries, where their finest twigs, of $0.008-0.012$ of a line, contain only nucleated fibres; 3 , to the hepatic veins; and lastly, 4 , into the coats and ligaments of the organ.

$\S 163$. According to the latest observations, particularly of Bischoff and Remak, the development of the liver may thus be best understood. The primary rudiment of the liver, which appears at a very early period (about the 55-58 hour, in the Chick; in Mammals, after the Wolffian bodies and the allantois) consists of two masses of cells, an external, proceeding from the fibrous membrane of the intestine, and an internal, epithelial, which at first form a simple, and afterwards a dichotomously divided sac. Solid processes, the hepatic cylinders of Remak, are now developed from the epithelial lamina, which, as in the intestine, consists at first of round cells, probably in many layers, by the multiplication of its cells, and extend into the outer lamina, branching out and anastomosing, whilst, at the same time, the cells of the outer lamina included in the meshes of this network, multiply and become successively changed into vessels, nerves, connective tissue, \&c. The difficulty is to say how this peculiar, reticulated parenchyma of cells and rudimentary vessels becomes ultimately arranged as we know it to be. In the first place, as regards the hepatic-cell-network and the islets or lobules of the complete liver. They evidently proceed from the further growth of the original hepatic-cell-network, to which by a continual new development of cells, fresh processes are added, which unite into new networks, so that the hepatic-cell-network of the adult liver is the direct progeny of the original reticulation. More detailed information concerning the separate steps of the formation of the hepatic-cell-network, is at present wanting; yet from what is known it would appear to take place in somewhat different modes. Sometimes, in the subsequent stages, free cylindrical processes of the hepatic-cell-network do not exist to any extent, but it would appear to increase by the continual addition of new meshes at its edges, perhaps also by the constant elongation of the existing columns of hepatic cells and the development of fresh anastomoses between them; 
this is, if I have observed rightly, the case in man, where even in the seventh week I did not succeed in clearly distinguishing free hepatic columns. At other times, free terminations of the hepatic columns are apparently developed for a considerable period, perhaps until the whole organ has nearly arrived at perfection, their formation appearing to precede by some time that of new anastomoses, as is the case in the Chick and other Birds, and according to J. Müller, in a few Mammals ; in the latter of which, according to Müller's figures, the hepatic columns are grouped in lobes. These free, superficial hepatic columns may perhaps throw some light upon the meaning of Weber's and Krause's statements respecting the biliary. ducts with cœcal ends upon the surface of the liver. With regard to the biliary ducts, they are assuredly nothing but secondary excavations of a part of the primarily solid hepatic columns and of the larger internal tracts, which border upon the original epithelial diverticulum and which all consist of many series of cells. The excavation commences in the common biliary duct, proceeds towards its branches, and must be considered to take place exactly as in other glands, $i$. $e$. either by solution of the inner cells of the rudimentary structures, or by the excretion of a fluid between them and the consequent production of a cavity. In this mode of regarding the matter, there is only one point for consideration; viz., that according to Remak, all the hepatic columns, even the largest, form anastomoses, whilst, as is well known, the biliary ducts ramify without anastomosing. The only solution of this difficulty, consists in assuming that the anastomoses of the primary, largest hepatic columns do not continue in the course of the further development, but that they are re-absorbed, a process which has its analogue in many phenomena of foetal growth. In Man alone might we find-an exception, for it seems to me that the anastomoses of the right and left hepatic duct, in the fossa hepatis, described by $\mathbf{E}$. $\mathbf{H}$. Weber, are perfectly well explained by Remak's observations, and are simply the embryonic anastomoses of the rudiments of these canals, which have attained to some, though no very great development. The mode of origin of the fibrous membranes of the biliary ducts becomes readily comprehensible, if we reflect how the networks of hepatic columns and the fibrous layers of the liver interdigitate; so that layers of connective tissue, \&c., might be readily formed around the hepatic cylinders from those elements of the fibrous layer which are nearest to them. The further development of the vessels, nerves, \&c., presents no difficulties, taking place in the same way as in other organs. The gall-bladder in the Chick, according to Remak, is a process, at first solid, of one hepatic duct, which subsequently becomes hollow and rapidly increases in size. I saw the folds of its mucous membrane, as early as in the fifth month, in a human fotus.*

* [In his last memoir (Philosophical Transactions, 1853), Dr. Handfield Jones maintains that, in Fishes, Amphibia, and Birds, the liver is developed independently of the intestine, 
The investigation of the liver is best undertaken in the Pig, in which animal the distinct demarcation of the lobules greatly facilitates the comprehension of the relations of the secreting parenchyma, to the vessels and hepatic ducts. The hepatic cells may be isolated with the greatest ease in all animals, either singly, in series, or in reticulated fragments; but to comprehend rightly their collective arrangeinent no better means exist than the making of fine sections in a fresh liver with the double knife, for which, sections made off-hand with a razor, even in a liver previously hardened in alcohol, pyroligneous acid, chromic acid, \&c., are by no means sufficient substitutes. We do not mean to say that the hepatic-cell-network cannot be seen, at all in this manner, for it is visible even in opaque sections of liver by reflected light, but merely that no complete view can thus be obtained. The finest hepatic ducts are not readily found, though a careful search in nearly all sections which include many lobules, will almost certainly detect scattered fragments of them, readily recognizable by their small polygonal cells, at the edges of the lobules, and long examination may perhaps eventually discover such a fragment in connection with the hepatic-cell-network, which, however, I have not yet succeeded in doing. The coarser biliary ducts present no difficulties. Their glands are seen readily, partly with the raked eye, partly by the use of dilute caustic soda. Weber's anastomoses of the two hepatic ducts in the fossa transversa, are visible in good injections. The vasa aberrantia, in the left triangular ligament and in other localities, are readily perceived even without injection, on the addition of acetic acid or of caustic soda. The nerves and lymphatics of the liver are, except their finest portions, easily seen in Man. The bloodressels require good injections, for which purpose, in the human subject, I especially recommend children's livers, in which the distribution of the arteria hepatica in the serous coat, on' the vessels, \&c., is beautifully distinct. The capillary network of the lobes may readily be filled with fine injection, and a series of excellent preparations of this kind, by various masters of the art, are everywhere to be met with.

Literature of the Liver.-F. Kiernan, "The Anatomy and Physiology of the Liver," in "Phil. Trans.," 1833; E. H. Weber, "Annotat. Anat. and Physiol.," Prol. VI. VII. and VIII. Lips. 1841 and 1842, and "Programmata collecta Fasc.," II. Lips., 1851; "Ueber den

and, that the first rudiment of the excretory apparatus is the gall bladder, whence ducts extend on the one hand into the intestine, and on the other into the liver. He has not traced the development of the liver in Mammalia.

Vogt, however (Embryogenie des Saumons, p. 175), states that in Coregonus palea, the liver is at first a rounded, solid mass of cells, in contact with a diverticulum of the intestine-the future ductus choledochus; and that, in the course of development, the diverticulum grows into and ramifies in the mass-its ultimate branches terminating in creca. The gallbladder is not formed till very late.-TRs.] 
feineren Bau der menschlichen Leber," in "Müller's Archiv," 1843, p. 318; "Zusätze zu seinen Untersuchungen über den Bau der Leber," in "Berichte d. K. Sächs. Ges. d. Wissensch. zu Leipzig," 1850, p. 151; A. Krukenberg, "Untersuchungen über den feineren Bau der menschlichen Leber," in "Mull. Arch.," 1843 ; Joh. Müller, in his great Work on the Glands, in his "Physiology," and "Müller's Archiv," 1843, p. 338; Theile, article "Leber," in R. Wagner's "Handw. der Phys." II., p. 308, 1844; C. L. J. Backer, "De Structurâ subtiliori Hepatis sani et morbosi. Diss. Inaug. Trajecti ad Rhenum," 1845; Natalis Guillot, "Sur la Structure du Foie des Animaux vertébrés," Ann. d. Sc. Nat., 1848, p. 129 ; R. Retzius, "Ueber den Bau der Leber," in "Müll. Arch.," 1849, II. p. 141; C. Wedl, "Ueber die traubenförmigen Gallengangdrïsen," in "Sitzungsbericht der Wiener Akad.," 1850 , Dec., p. 480, c. Tab.; N. Weja, "Beiträge zur feineren Anatomie der Leber," in "Müll. Arch." 1851, p. 79 ; E. Von Bibra, "Chemische Fragmente iiber die Leber und die Galle," Braunschweig, 1849. The more minute comparative anatomy of the Liver is treated of by $\mathrm{H}$. Karsten, "Disq. microsc. et chem. hepatis et bilis Crustaceorum et Molluscorum," in "Nova Acta Acad. Cur.," vol. XXI. 1, p. 295; T. F. G. Schlemm, "De hepate et bile crustaceorum et molluscorum quorundam," Diss. Berol., 1844; Williams, in "Guy's Hosp. Rep." 1846; H. Meckel, "Mikrographie einiger Drüsenapparate der niederen Thiere," in "Mull. Arch.," 1849, p. 1 ; Fr. Will. "Ueber die Absonderung d. Galle," Erlangen, 1849; H. Jones, "Phil. Trans.," 1846, 1849; [J. Leidy, "Researches into the Comparative Structure of the Liver," in Amer. Jour. of Med. Sciences, 1848; Wharton Jones, "Phil. Trans.," 1848; Handfield Jones, "Phil. Trans.," 1853.-DaC.]

\section{OF THE PANCREAS.}

$\S 164$. The pancreas is a compound racemose gland, which so closely resembles the salivary glands, that a short exposition of its peculiarities will suffice. As in all such glands, larger, smaller, and smallest lobes, may be very distinctly made out, the last being composed of microscopic glandular vesicles, which are here characterized by their moderate size $0.02-0.04$ of a line, and their usually rounded form. They possess a membrana propria and a tessellated epithelium, whose cells are very frequently remarkable from the great number of fat-granules, so that the glandular vesicles appear quite opaque and as if entirely filled with secretion. The excretory ducts, which, as elsewhere, are connected with the glandular vesicles, uniting into larger canals and, eventually, into the duct of Wirsung, or pancreatic duct, are whitish and somewhat thin walled. They are composed of connective tissue and of elastic fibrils, and all possess an epithelium with small cylindrical cells, scarcely exceeding $0.006-0.008$ of a line in length, and 0.002 of a line in breadth. In the walls of the pancreatic duct, and its larger branches, small 
racemose glands of $0.06-0.08$ of a line with vesicles of $0.016-0.02$ of a line, and a less fatty epithelium, are situated in considerable numbers; whether they are mucous glands analogous to those of the biliary ducts, or parts of the pancreas itself, I cannot say. The pancreas possesses the ordinary investing tissue of the glands, with more or less abundant fat-cells, in which the ressels and nerves of the gland are distributed.

Fig. 225.

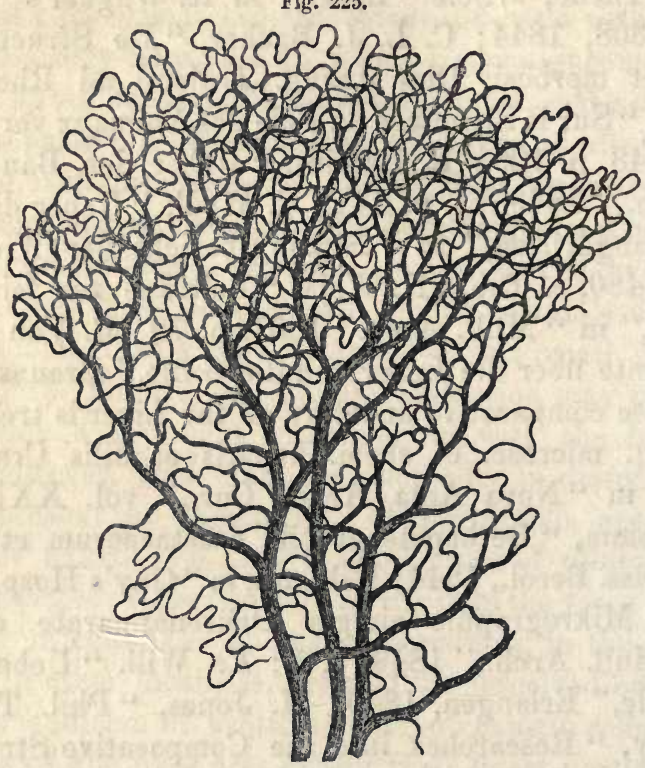

The former present exactly the same relations as in the parotid, except that the lymphatics appear to be more numerous; the latter would seem only to accompany the vessels and arise from the sympathetic, possessing fine, and a few moderately thick tubules. The secretion of the pancreas is normally perfectly fluid, only accidentally containing formed constituents, as detached epithelium of the glandular vesicles and of the ducts.

The development of the pancreas commences by the formation of a diverticulum of the posterior wall of the duodenum and in its further progress, exactly resembles the salivary glands, except that the rudiment of the gland forms, from the first, a more compact mass and thence is not so readily made out in detail.

The examination of the pancreas presents no difficulties, except that, in Man, the fat in the epithelial cells of the glandular vesicles offers some impediment and therefore the pancreas of Mammalia (Rabbit, Mouse), which usually contains less fat, should be made use of. The glandules in the ducts are best rendered visible by acetic acid.

[Literature.-J. G. Wirsung, "Figura ductus cujusdam cum multiplicibus suis ramulis noviter in pancreate observati," Padov., 1643; J.

Fra. 225. Vessels of the pancreas of the Rabbit, magnified 45 diameters. 
Müller, "De glandul. sec. structura penitiori," Wharton Jones in Philosophical Transactions, 1848, II. p. 227; Verneuil, "Anatomie du Panereas," in Gazette Medicale, 1851.-DaC.]

\section{OF THE SPLEEN.}

$\S 165$. The spleen, is a so-called blood-vascular gland, which is in some way concerned in the renewal of the blood, and probably with the secretion of the bile also. It consists of a fibrous and serous coat and of a soft parenchyma, the latter being principally composed of reticularly interwoven solid bands, the splenic trabeculoe, enclosing a red substance, the splenic pulp. In the latter we find, in addition, many peculiar white corpuscles, the splenic or Malpighian corpuscles, while abundant vessels and a certain number of nerves are distributed through its whole interior.

$\S 166$. Coats and Trabecular Tissue.-The peritoneal investment covers the whole surface of the spleen, with the exception of the hilus, where, forming a sheath around the vessels and nerves, it passes on to the fundus of the stomach as the ligamentum gastro-lienale, and of the upper extremity, from which it becomes detached, as the lig. phrenicolienale; it adheres so closely to the fibrous coat in Man (though not in Ruminants), that it can only be dissected from the organ in fragments.

The fibrous coat (tunica albuginea seu propria) completely surrounds the surface of the spleen, as a moderately thin and semi-transparent but very strong membrane, and at the hilus, passes into its interior, like Glisson's capsule, accompanying the vessels in the form of peculiar sheaths, the vagince vasorum. In man, it is composed of common connective tissue, with abundant networks of elastic fibres, whilst in some animals, the Dog, Pig, Ass, Cat (not in the Rabbit, Horse, Ox, Hedgehog, Guiena-pig, and Bat), I find it to contain smooth muscles in considerable numbers.*

The trabeculoe of the spleen are white, shining, flattened or cylindrical fibres, having on an average, a diameter of $\frac{1}{10}-\frac{1}{3}$ of a line, which are attached in great numbers to the inner surface of the fibrous coat, and less frequently to the outer surface of the sheaths of the vessels, and unite with similar trabeculce in the interior, into a network which extends through the whole organ. The interstices included in

Fig. 226.

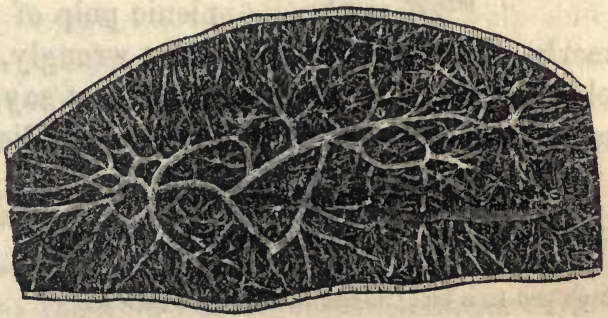

Fra. 226.- Transverse section through the middle of an Ox-spleen, washed out, to show the trabeculæ and their arrangement. Natural size.

* [The existence of these muscles in the ox's spleen was first pointed out by Dr. Sharpey. See Quain and Sharpey's Anatomy, p. 1086 (Vol. II., p. 498. Am. Ed.)-Trs.] 
it all communicate, contain the red pulp of the spleen and the Malpighian corpuscles, and although no one exactly resembles another, yet all as regards form and size, present a certain similarity.

The older anatomists considered them to be regular cavities lined by a membrane, like those of the corpora cavernosa penis, to which, indeed, they are very similar in the arrangement of the limitary trabeculoe, but there is nothing of this kind, as may be best demonstrated in sections of the spleen, in which pulp has been removed by washing. Such a preparation is best fitted for the study of the relations and connections of the trabeculce; and it is readily seen, that although very various in size, they do not ramify after the fashion of vessels, but unite quite irregularly. Where 4,5 , or more of these unequally thick trabeculoe unite, a flattened cylindrical enlargement, like a nerrous ganglion, usually exists; these are more frequent towards the external surface of the organ than in its internal portions and at the hilus, where the large vessels already afford a sufficient support to the parenchyma and an intimate union of the trabeculce is less necessary.

The structure of the trabeculoe of the human spleen perfectly re-

Fig. 227.

A
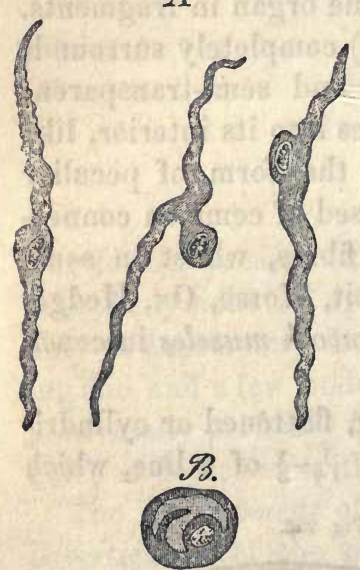
sembles that of the fibrous coat; they consist of longitudinally fibrous connective tissue, with intermingled fine elastic fibres. In animals, on the other hand, smooth muscles exist, as I showed in the year 1846, sometimes in all the trabeculae (Pig, Dog, Cat), sometimes (Ox), only in the smaller ones, with respect to whose distribution further particulars will be found in my "Mikroskopische Anatomie," II. 2, p. 256. In the trabeculoe also, we find peculiar spindle-shaped fibres, of $0.02-0.03$ of a line in length, and 0.002 of a line in breadth, with undulated ends and prominent enlargements, in which rounded nuclei are situated. They are to be met with in great numbers in the splenic pulp of Man (Fig. $227 \mathrm{~A}$ ), and I formerly, though as I now believe wrongly, took them to be smooth muscles. What their nature is I cannot say, and I can only add that they are also found coiled up in cell-like bodies* (Fig. $227 \cdot B$ ).

§ 167. Malpighian corpuscles, the splenic corpuscles, Malpighian corpu-

Fig. 227.-Peculiar fibres from the pulp of the human spleen: $A$, the saine free; $B$, one inclosed in a cell; magnified 350 diameters.

* [According to Mr. Wharton Jones ("British and Foreign Med. Chir. Review," Jan., 1853), these cells, containing "peculiar fibres," are nothing but the ordinary nucleated fibres of the pulp " circularly coiled, the coil being maintained by a tenacious intercellular substance filling up the middle space."-TRs ] 
scles or vesicles, are white roundish bodies, which are imbedded in the red substance of the spleen and are connected with the smallest arteries. They are constant only in quite fresh and healthy subjects; but not at all, or rarely, in those who die of disease, or after long fasting. It hence becomes comprehensible that Von Hessling found the corpuscles only 116 times in 960 examinations. In subjects whose age was between the first and second year, they were present in every second individual; from the second to the tenth year, in every third; from the tenth to the fourteenth, in every sixteenth; and from the fourteenth onwards, only in every thirty-second. In the bodies

Fig. 228.

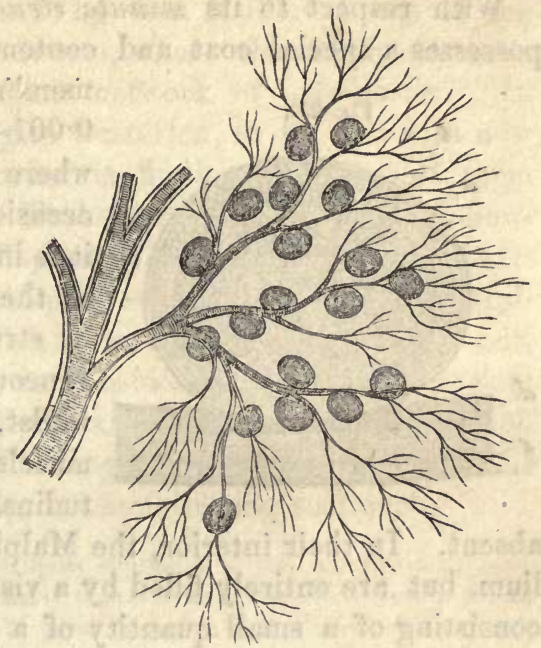
of those who die suddenly, as in consequence of accidents, suicide, or judicial sentence (of the latter of whom I have examined three cases), they are probably never absent; and it is the same with the majority of children. In these cases they are as numerous and as distinct as in Mammalia. The size of the splenic corpuscles is liable to certain variations in Man and in animals, and has hitherto, for the most part, been over-estimated, in consequence of their having been incompletely isolated. Their diameter is from 1-10-1-3, on the average 1-6 of a line; and very probably depends upon the varying condition of the chylopoietic organs, so that the corpuscles are larger after food has been taken than at other times; though, in confirmation of Ecker's statement, I can affirm that they are to be met with, beautifully developed, in fasting animals also. We have no data of any kind with regard to this point in Man.

The Malpighian corpuscles, though imbedded in the red pulp and hardly separable from it, are nevertheless always attached to a branch of an artery, in such a manner that they either rest laterally immediately upon a vessel, or are situated in its angle of division, or finally appear stalked; in which latter case, however, the stalk itself, again, is usually a small artery. Their number is very considerable, arterial twigs of $0.02-0.04$ of a line carrying 5-10 corpuscles, so that extracted with them from the pulp, they present the figure of an elegent raceme (Fig. $2: 28$ ). It appears to me that it would be rather under, than over

Frg. 228.-A portion of a small artery, with a branch covered with Malpighian corpuscles (Dog), magnified 10 diameters. 
estimating the number of the Malpighian corpuscles to assume that every $1-1 \frac{1}{2}$ cubic line of the pulp contains a corpuscle.

With respect to its minute structure, every Malpighian corpuscle possesses a special coat and contents, and is therefore a vesicle. The

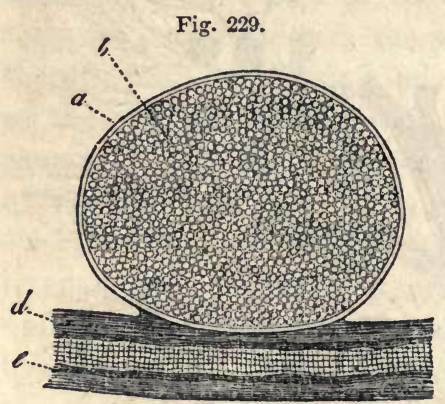
membrane is colorless and transparent, $0.001-0.002$ of a line thick, and everywhere exhibits a double contour, with occasional intermediate concentric lines; it is intimately connected with the sheath of the vessel, with which it also agrees in structure, so far as it contains homogeneous connective tissue and elastic fibrils ; whilst, on the other hand, the smooth muscles which are also present as longitudinal fibres in these sheaths, are entirely absent. In their interior, the Malpighian corpuscles contain no epithelium, but are entirely filled by a viscid, grayish, continuous substance, consisting of a small quantity of a clear, neutral fluid, coagulable by heat, and therefore albuminous; of many, rounded, larger and smaller (from $0.003-0.006$ of a line), pale cells, which usually possess a single

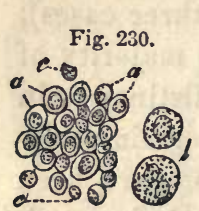
nucleus and become granulated by the action of water and of a varying number of free nuclei. Besides these cells, which frequently contain single fat-granules and offer the most distinct evidence that in the Malpighian corpuscles a constant development of cells is going on, we occasionally meet with blood.corpuscles, either free or in cells and, as I am inclined to believe from a single observation in the spleen of a Cat, with fine bloodvessels, as in Peycr's follicles (see § 155).

The Malpighian corpuscles are completely closed, and are not connected with the lymphatics, although this has been assserted by different authors-among the moderns by Huschke, Gerlach, Pölmann and Schaffner. Anatomically, they are perfectly similar to the follicles of Peyer's patches, and of the solitary glands, described above, and very closely agree with those of the tonsils and lymphatics, whence they may for the present be denominated gland-like follicles.*

Fia. 229.-A Malpighian corpuscle from the spleen of an Ox, magnified 150 diameters: $a$, wall of the corpuscle; $b$, contents; $d$, sheath; and $e$, wall of the artery to which it is attached.

FIG. 230.-Contents of a Malpighian corpuscle from the $\mathrm{Ox}: a$, small ; $b$, large, cells; $c$, free nuclei.

* [In a case of suicide, Prof. Kölliker (Würzb. Verhand. IV. 1) has lately detected distinct capillaries within the Malpighian corpuscles. Capillaries within the corpuscles he had hitherto only seen in the spleen of a Cat; they have, however, from independent observations, already been described by Gerlach and Huxley as occurring in Man.

The Malpighian corpuscles undergo, in some diseases, a peculiar degeneration. Thus, in the disease termed "waxy," or "colloid" spleen, they present the appearance of gelatinous 
Malpighian corpuscles have been discovered in all the Mammalia which have hitherto been examined, and also occur in Birds. Among the scaly Amphibia they were found by Joh. Müller in one of the Chelonia, and by myself in the Blindworm, where the corpuscles were surrounded by an exceedingly, elegant network of capillaries. In Frogs and Toads they are, according to Oesterlen, to be met with now and then; but I have not yet succeeded in finding any trace of them in the naked Amphibia, nor in the fresh-water fishes. Leydig, however, has observed them in the Plagiostomata (Beiträge zur Anat. der Rochen und Haie). Joh. Müller's supposition that the Malpighian corpuscles exist in all Vertebrata, is, therefore, not borne out, a fact which is not without weight in considering their physiological import. In a few Mammals the Malpighian corpuscles contain, though not constantly, the same forms of retrograding blood corpuscles as will be described to occur in the pulp, in the following section.*

granules, and are filled with a homogeneous mass, consisting of soft concentrically laminated bodies. These bodies, which were formerly regarded as colloid, have recently been found by Virchow (Archiv, f. Path. Anat. VI. 2) to be the result of a cellulose metamorphosis, for a watery solution of iodine, and the subsequent addition of sulphuric acid caused them to assume the peculiar violet color which is known to belong to vegetable cellulose.- $\mathrm{DaC}$.]

* [The Malpighian follicles of the spleen present three points of importance to the investigator. 1. Whether they have a capsule, and what is its nature? 2. The arrangement of their vessels. 3. The structure of the substance which they contain.

1. The capsule and its nature.-We have been quite unable to convince ourselves of the existence of any such capsule as that described by Professor Kolliker, in the Malpighian follicles of Man, the Sheep, the Pig, the Cat (Kitten), or the Rat; in all of which we have made very careful investigations with regard to this point. In Man, in the Pig, and in the Cat, we are unable to distinguish any boundary at all between the follicles and the surrounding red pulp - the substance of the one appearing to pass into the other. At the line of transition, however, the indifferent tissue of the follicle underwent a partial metamorphosis and broke up, when teased out, into spindle-shaped bodies, containing "nuclei," or short delicate fibres with " nuclei," exactly resembling, in Man, the structures described by Professor Kölliker as peculiar fibres, and represented in Fig. 227.

In the Rat, this border zone of metamorphosed tissue was somewhat broader and firmer, and when the follicle was compressed, appeared, particularly under a low power, like an indistinctly fibrous coat, such as Professor Kölliker describes; but when closely examined, it was readily seen to be no distinct closed capsule, but to pass gradually, on the one hand, into the pulp, and, on the other, into the contents of the follicle. The same is true of the Malpighian follicles of the Sheep, where the appearance of a capsule, under a low power, is often very distinct; and where imperfect elastic fibres may be met with in it.

In fact, our own observations are perfectly in agreement with those of Mr. Wharton Jones (British and Foreign Med. Review, Jan., 1853), and have led us completely to the opinion of Remak (Ueber runde Blut-gerinnsel und über Pigment Kugelhaltige Zellen, Müller's "Archiv," 1852), that the capsules of the follicles are by no means their essential element, and that we must consider the spleen to be formed by two principal constituents; the first being the parenchyma, and the second a superadded fabric of bloodvessels, nerves, lymphatics, elastic and contractile elements. The manner in which the latter are arranged in and about the parencluyma is, in a manner, accidental and very variable. It may be, as Remak says, either intercapillary, as in the pulp; or vaginal, as in the sheaths of the arte. ries; or encysted, as at the angles of division of the arteries, in the Malpighian follicles of the Sheep.

To insist, therefore, upon the follicular arrangement of the spleen, or indeed of any other 


\section{$\S 168$. The red substance of the spleen, the pulp, or parenchyma.of the spleen, is a soft reddish substance, which fills up all the interspaces}

of the vascular glands, as Sanders (On the Structure of the Spleen, "Annals of Anatomy and Physiology," 1850) and Kolliker do, seems to us to mistake accidental for essential characters.

The results of comparative anatomical examination are strikingly in favor of this view of the matter.

The Malpighian follicles of Birds and Amphibia have no capsules (Remak, Leydig). In Bombinator igneus, according to Leydig, a white substance, the representative of the Malpighian follicles, lies in the middle of the spleen, surrounded by a red cortical pulp, into which it directly passes.

On the other liand, Coluber natrix, a Reptile, presents the very opposite characters. Here the red pulp is absent, and the spleen has, as nearly as possible, the structure of an ordinary lymphatic gland, consisting of a fibrous stroma, containing cavities full of indifferent tissue, through which a capillary network, arising from the vessels of the stroma, is distributed (Leydig "Anatomisch-Histologische Untersuchungen äber Fische und Reptilien," 1853).

In Fishes the same variation occurs. In Hexanchus there are thick-walled Malpighian corpuscles (Leydig). In other Plagiostomes and many osseous Fish there are no distinct follicles, but the indifferent follicular tissue follows the sheaths of the arteries.

2. The arrangement of the vessels of the Malpighian follicles.-Johannes Maller, who gave the first good account of the Malpighian follicles of vegetable feeders (Müller's "Archiv," 1834), not only states that the follicles are, what all recent researches have shown them to be-the representatives of portions of the sheaths of the arteries, but also that the arterial twigs which supply them "sometimes run beside the Malpighian bodies without giving any branches to them, sometimes pass straight through the corpuscles" (p. 88). However, he appears to be inclined to the opinion that the arterial twigs pass "not so much through the middle of the corpuscles" as in the thickness of their walls.

All subsequent writers have affirmed that the arterial twigs pass over the surface and not through the substance of the Malpighian follicles, with the exception of Günsburg and of Dr. Sanders-who states, not in the paper we have cited, but in a subsequent communication to the Edinburgh Physiological Society (Jan. 31st, 1851), that by a peculiar method of preparation, he had observed arterial twigs passing diametrically throngh the substance of the follicles, "stains of blood also, often in linear arrangement, indicating capillaries, were seen in the interior of the sacculi." With regard to the latter point, it will be observed that, in the text, Professor Kölliker-also records a single observation of minute bloodvessels in the Malpighian follicles of the Cat.

In all the Mammalian spleens we have examined (Man, Sheep, Pig, Cat, Rat), we have observed the passage of arteries through the Malpighian follicles and the existence of a capillary network in them, with the utmost ease; we are, indeed, at a loss to comprehend how it is that previous observers have so generally overlooked facts so patent. The method we have pursued has been merely to make a tolerably fine section, containing a Malpighian follicle, with a sharp knife-to spread it out with needles, adding nothing but a little wealc syrup, and then, placing a thin glass plate over it, we have examined it with both the simple and the compound microscope. The use of the former is especially to be recommended, because by manipulating the covering plate, the whole follicle may be readily rolled about under the eye, and the clearest evidence thus obtained that the arterioles pass through and not over the surface of, the follicles. Acetic acid should not be used, as it renders the contents of the follicle opaque; but the syrup is of great service, as it keeps the coloring matter of the blood in the capillaries and renders them extremely obvious. The walls of the capillaries are exceedingly delicate and often indistinguishable as distinct structures. Both the longitudinal inner coat and the transverse muscular coat of the arterioles are very well developed in Man.

3. Structure of the "contents" of the Malpighian follicles.-We have been unable to find either cavity or fluid in the contents of the Malpighian follicles. So far as we have seen, they are solid bodies-their outer portion or "wall" being.constituted as above described; 
between the larger trabecula and the coarser vessels, and is so soft as to be readily removed from a fragment of the organ. It consists of three elements; viz., of the most delicate bloodvessels of the spleen, of microscopic fibres and trabeculoe, and of peculiar cells of the parenchyma. In Man and in Animals the occurrence of extravasated blood, in manifold stages of metamorphosis, is so frequent, that it may be almost regarded as a normal constituent. According to its amount and to the distension of the bloodvessels, the pulp appears sometimes of a brighter, sometimes of a darker blood-red; besides which, however, it must be noted that the pulp has also its own proper red coloring matter.

The fibres of the pulp are of two kinds. Firstly, there are microscopic trabeculoe, answering completely to those visible with the naked eye and possessing the same structure, except that in many Mammals they contain more smooth muscles, or are even entirely composed of them. As a general rule, their diameter varies between 0.005 and 0.01 of a line; in quantity they differ in different animals and in different parts of the spleen. In Man I find them to be more rare and broader than in other

while the inner is, to use the accurate phraseologyof Mr. Wharton Jones, composed, of " nucleated granular corpuscles and nucleated cells, similar to those of the red substance, cohering together in a mass by means of a diffluent infercellular substance, and, interspersed among these, a few somewhat larger nucleated cells" (1. c., p. 35).

The idea that the Malpighian corpuscles were hollow bodies originated with Malpighi himself; but Müller, in opposition to him and to Rudolphi, asserts very justly that in the Pig, Sheep, and $\mathrm{Ox}$, they are firm and resistant.

From all that has been said, it results very clearly that the only difference between the "pulp" and the "Malpighian follicles" of the spleen is one of degree, consisting in the greater or less development of the vascular network and the greater or less amount of metamorphosis, which the elements of the parenchyma have undergone. It is, furthermore, suffciently obvious that the anatomical differences between a solitary follicle of the intestine, a Peyer's patch, a lymphatic gland, and a spleen, are also questions of degree. It is inpossible to distinguish, under the mieroscope, a minute lymphatic gland-such as may be met with in the mesentery of the Rat, for example-from one of the follicles of the Peyer's patches of the same animal. But as the intestinal follicles are aggregated to form the Peyer's patches, so the lymplatic follicles are aggregated to form the large lymphatic glands; increase the vascularity of the stroma of a lymphatic gland and we have a spleet.

On the other hand, we can state decidedly that the follicles of the tonsils, both in Man and in the Sheep, are traversed abundantly by capillaries, so that they come under the same category; differing, lowever, from the lymphatic glands and spleen, in that the follicles are arranged, not in solid masses, but aronnd diverticula of the intestinal mucous membrane, which, in the tonsils (both in Man and in the Sheep), take the form of more or less irregularly ramified duets. Starting, therefore, from the simple intestinal follicle, we have two series of vascular glands-the one, the solid series, reaching its utmost complexity in the spleen (and perhaps the supra-renal bodies and thymus); the other, the diverticular series. Does the latter, however, reach its highest complication in the tonsils? or rather do not these lead in the plainest way to the liver, which is, like them, essentially a solid meslwork of capillaries, filled by indifferent tissue and arranged around a complex diverticulum of the intestine? It appears to us that the structure of the tonsils affords, in this way, an analogical basis for the views of Dr. Handfield Jones; and tends greatly to support the doctrine, that the liver is essentially one of the vascular glands. While adding the liver, however, we should exclude the thyroid; its structure being totally different from that of the rest of the class.-Trs.] 
Mammals, and perfectly identical in their structure with the larger trabeculoe. Other fibres which occur in the pulp are plainly the terminations of the vascular sheaths. They are very numerous, and usually have the appearance of delicate, indistinct, fibrous membranes, without any elastic element, which appear to connect the capillaries, and are, perhaps, continuous with the finest trabeculoe.

The cells of the pulp, or parenchyma-cells of the spleen, round cells of 0.003-0.005 of a line, with single nuclei, are for the most part so similar to those in the Malpighian corpuscles, that it is unnecessary to enter upon a more minute description of them; intermingled with them, and, indeed, in larger quantities than in the Malpighian corpuscles, we find free nuclei. Besides these, a few other elements may be met with: 1 , pale round bodies, with a homogeneous aspect, somewhat larger than the corpuscles of the blood, which appear either as free nuclei, or as homogeneous nuclei, closely surrounded by a delicate investment; 2 , larger cells, up to 0.01 of a line in diameter-both of the completely pale kind, with one or two nuclei, and also what I have called colorless granule-cells-that is, cells with more or fewer colorless, dark fat-granules. Each of these elements exists in the splenic corpuscles also, but never to so great an amount. The quantity of the different kinds of parenchyma cells and of free nuclei in the pulp, is so considerable, that, together with a small amount of a reddish-yellow fluid which unites them, they constitute probably one-half of the bulk of the spleen. They are not collected in large masses, but in small irregular aggregations of different sizes, which occupy the interspaces between all the trabeculce and vessels of every description, and surround the Malpighian corpuscles. The clearest conception of the arrangement is obtained, if we consider that every segment of the red substance included within the larger trabeculce has the same composition; on the small scale, as the whole spleen has on the large. In fact, the microscopic trabeculoe, the terminations of the vascular sheaths and the finest vessels, present the same relations as the large trabeculce and vessels, while the small masses of parenchyma cells correspond with the apparently homogeneous masses of pulp, visible to the naked eye. There are no special investments around the parenchyma-cells, but they lie everywhere in direct contact with the sheaths of the vessels, the trabeculoe, and the sheaths of the Malpighian corpuscles.

The red pulp of the spleen of Man and of Animals has a different color at different times; or rather the blood-corpuscles which it contains and which, without the participation of any other element, give rise to its color, present different conditions. In some animals, for instance, its color is sometimes paler, more grayish-red, sometimes brown, or even blackish-red. In the latter case, a quantity of changed blood-corpuscles will be met with (to which we shall return subsequently); in the former, on the other hand, it may be microscopically demonstrated that the red 
color proceeds from unchanged blood-corpuscles, which may also be readily expressed from the substance of the spleen and, on the addition of water, in a short time lose all their coloring matter. In other animals the spleen, although it always has about the same, usually dark color, yet contains in addition, sometimes only unchanged blood-corpuscles, sometimes multitudes of them in every stage of metamorphosis. These are very striking, and in all animals consist essentially in thisthat, 1, the blood-corpuscles, becoming smaller and darker and, in the lower Vertebrata, losing their elliptical form and taking a circular shape, agglomerate together into rounded masses, which either persist in this condition, or, combined with a certain amount of the plasma of the blood, develop an envelope externally and a nucleus in their interior, thus passing into round blood-corpuscleholding cells, of $0.005-0.015$ of a line, containing 1-20 blood-corpuscles; and 2 , these masses and cells, their contained blood-corpuscles gradually diminishing in size and assuming a golden yellow, brownish-red, or black color, either in their entire state or after breaking up into pigment granules, change into pig-

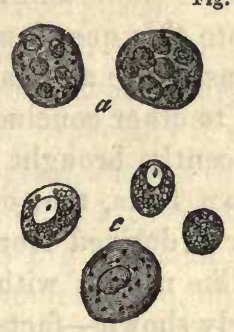
Fig. 231.

ment masses and pigmented granule cells; and, finally, the latter, their granules gradually becoming pale, pass into perfectly colorless cells. In many cases the blood-corpuscles form no masses or cells, but pass through the above-described stages of coloration and disintegration, like the others.*

Fig. 231.-Blood-corpuscle-holding cells and their metamorphoses, from the spleen of the Rabbit, magnified 350 diameters : $a$, two nucleated cells, with blood-corpuscles; $b$, similar cells metamorphosed into brown pigment cells; $c$, cells from which the color has disappeared again; $d$, pigment granules which have arisen from metamorphosed free blood-corpuscles.

* [In his "Microscopical Anatomy," Prof. Kolliker states, that he has observed bloodcorpuscle-loolding cells, and their breaking up into pigment granules, in the spleen of almost all animals. In Mammalia the cells were generally seen with difficulty. In the Cat, Horse, Ass, and Bat, no distinct cells could be detected, although the pigment masses and the pigmented granule-cells were easily observable. In Birds he met with cells containing blood-corpuscles only in the Blackbird although in many (Falco albicillus, Cuculus canorus) he was able to detect yellow pigmented granule-cells changing into black pigment. In the naked Amphibia and in Fishes, he frequently found cells enclosing 5 to 20 bloodcorpuscles, in which the metamorphosis into pigment could easily be studied.

The "blood-corpuscle-holding cells" and the supposed result of their metamorphosespigment granules, are met with in great abundance in some of the diseases of the spleen. Thus in atrophy of this organ the trabeculæ and the parenchymatous structure are filled with masses of a dark molecular pigment. In the enlarged spleen of typhoid and intermittent fever, Heschl detected many pigmented granule cells and dark pigment granules. It is probable that cells enclosing blood-corpuscles exist, not only in the spleen, but wherever many blood-corpuscles and large masses of pigment are observed. I have seen cells resembling "blood-corpuscle-holding cells" in melanosis of the eye, in congested lungs, and as part of the peculiar dark granular matter, observed under the microscope, in the black vomit of yellow fever. If in this last-named instance the cells were possessed of distinct membranes or not, I was unable to determine.-DaC.] 
The changes undergone by the blood in the spleen, a subject which is more fully treated of in my "Mikr. Anat.," II., 2, pp. 268-270, and which were observed and interpreted by Ecker, at the same time and in the same manner as by myself, have lately become the subject of much discussion. Gerlach, Schaffner, and lately O. Funke also (l. c.), who are completely at one with Ecker and myself as to the facts, differ altogether in their interpretation of them; and believe that, instead of their being the result of a dissolution of the blood-corpuscles, they proceed from a process of development of new corpuscles, and that therefore the spleen-as, indeed, was Hewson's opinion-is a formative organ for blood-corpuscles.

I have already, in another place (Zeitsch. für wiss. Zool., p. 115), confuted Gerlach's views, and I therefore consider it to be unnecessary to enter, again, into this question, the less, as Ecker, after repeated, careful investigations, quite agrees with me; indeed no unprejudiced observation can tend to other conclusions than those at which we have arrived. Remak has recently brought forward an entirely new view (l. $i . c$. From the known facts, that other pigment cells exist than those whose coloring matter is derived from blood-corpuscles, and that effused blood may form cell-like masses with blood-corpuscles, as Hasse and I have more particularly shown-facts which he has strengthened by new cases - Remak has allowed himself to be misled into asserting, not only that no cells with enclosed blood-corpuscles exist at all, but also, that in the spleen no blood-corpuscles are destroyed, $i$. e. change into pigment granules. This is so strong an assertion, that I see no necessity for my contradicting it; one might at last be required to demonstrate that there are such things as cells and blood-corpuscles. It will interest Remak to learn that Virchow, as he tells me, has satisfied himself of the existence of the cells in question, though he explains their origin in another mode, believing that the blood-corpuscles pass from without into already existing cells-a supposition with which, at present, I cannot exactly agree.

An important question arises, as to the import of the changes in the blood-corpuscles, whether they are physiological or pathological? On the one hand, very weighty reasons present themselves for considering the phenomena to be normal, particularly their constant occurrence, as it may be said, in so many living animals, especially in those living under natural conditions, as Amphibia and Fishes; secondly, the apparent continuance of perfect health, notwithstanding the enormous quantity of disintegrating blood-corpuscles; thirdly, the occurrence of bloodcorpuscle-holding cells in bloodvessels which are not cut off from the general circulation, as may be demonstrated in the Amphibia; fourthly, the absence of similar, constant changes in the blood, repeated at short intervals, in other organs in the higher Vertebrata; and much more might be added. To these facts, however, careful observation opposes 
many others, which almost involuntarily lead to the idea, that perhaps all the changes of the blood-corpuscles in the spleen are abnormal, a view to which my observations in Fishes also tend. Here, 1, the rnetamorphoses of the blood-corpuscles in the spleen do not go on in the interior of bloodvessels, but in extravasations which resemble pathological aneurismata spuria (see "Mikr. Anat.," II. 2 ; and Todd's "Cyclopædia of Anatomy and Physiology," Art. Spleen, fig. 533; Ecker, "Icon. Phys.," tab. vi. figs. 15, 16); 2, extravasations and metamorphoses of their blood-corpuscles occur, not only in the spleen, but in other organs, especially in the kidneys, where they are constant, and frequently also in the liver and peritoneum.

If to these facts we also add, that in certain animals, $e . g$. the Cat, the Sheep, \&c., the changes of the blood-corpuscles in the spleen are very rarely met with-furthermore, that their progress is not always coincident with the stages of digestion-it becomes very difficult not to believe that the phenomena are abnormal, especially if we consider that similar phenomena certainly not physiological, such as the small effusions of blood into the lungs, bronchial glands and thyroid in Man, in the lymphatic glands of the mesentery of the Pig and Rabbit, \&c., are also, on the one hand, almost as constant phenomena, and, on the other hand, are accompanied by perfectly similar metamorphoses of the blood-corpuscles. However, in the latter cases, the quantity of the metamorphosed blood-corpuscles is not to be compared to the immense number of those which are constantly undergoing disintegration in the spleen; and in the second place, it is also possible that effusion of blood may occur as a physiological phenomenon, as into the Graafian follicles, and during menstruation and the detachment of the placenta. And although all animals do not present a microscopically demonstrable disintegration of the blood-corpuscles in their spleen, yet it does not follow that the process may not occur and that when it can actually be demonstrated, it is pathological. This much is at least certain, that congestions of blood in the spleen occur in all animals, without exception; and it is almost certain, that these congestions are, in Mammals, attended by extravasation. In these stagnations of blood, the blood-corpuscles may be disintegrated, in some cases rapidly, in others slowly, which would constitute an important difference for the observer; it is also conceivable that they and their consequences are physiological and have some great influence upon life, since it is a fact, that in many animals they are constant, and occur upon a very large scale.

For the present, therefore, so long as the pathological character of the phenomena in question is not conclusively demonstrated, I must maintain their physiological nature and regard the disintegration of blood-corpuscles in the spleen as a normal occurrence.*

* [With respect to the "blood-corpuscle-holding cells," the reader will do well to consult 


\section{§169. Vessels and nerves.-As they enter the organ, the relatively} very large splenic artery and the still larger splenic vein, are accompanied by those processes of the fibrous membrane, which have been referred to as the vascular sheaths. In Man these processes form complete investments around the vessels and nerves, somewhat after the fashion of the capsule of Glisson, so that the arteries and nerves especially, can be readily isolated, while the veins, which on the side opposite to the artery are more intimately connected with the sheath, are less easily separable. At first the sheaths are as thick as the fibrous coat itself and they retain this thickness so long as they surround the principal branches of the ressels. The finer ramifications of the latter and even those small branches which are given off from the large ones, have finer and finer sheaths, until at last, when the vessels are quite minute, they become lost as thin membranes in the pulp. The thickness of any sheath is always less than that of the wall of the artery to which it belongs and greater than that of the vein, but after division the sheaths become relatively stronger. It was remarked above, that a number of the trabeculoe are inserted into the vascular sheaths and they therefore take a share, together with the vessels which they inclose, in the formation of the dense network in the interior of the spleen. In Mammalia, as in the Horse, Ass, Ox, Pig, Sheep, \&c., the sheaths present different relations, inasmuch as the smaller veins have none at all, and the larger possess them only on the side on which the arteries and nerves lie. Only the two principal venous trunks near the hilus have perfect sheaths,

Remak's very valuable and elaborate paper, "Ueber runde Blutgerinnsel und über Pigment kugel haltige Zellen," in Muller's "Archiv," for 1852, and Mr. Wharton Jones's article on the same subject in the "British and Foreign Med. Chir. Review," for 1853, to which we liave already referred. Having carefully studied them, he will, we think, arrive very much at our own conclusion, that as the question now stands, the very existence of "blood-corpuscle-holding cells" must be considered as highly problematical.

Mr. Wharton Jones found the blood of the splenic vein to contain nucleated corpuscles and fibres identical with those of the pulp, together with free nuclei similar to those of the nucleated corpuscles; on the occurrence of which, he considers the statements as to the abundance of colorless corpuscles in the blood of the splenic vein are founded. Some of these elements were traced as far as the vena porta, but in the hepatic veins they had mostly, though not entirely, disappeared. He appears to be inclined to draw the conclusion that some of the venous radicles of the spleen are connected with the pulp in the same way as the hepatic ducts with the parenchyma of the liver, and that the materials thus derived by the blood from the spleen may concur in fitting it for the secretion of bile. Moleschott ("Ueber die Entwickelung der Blutkörperchen," Müller's "Archiv," 1853), gives some curious results obtained by extirpating the liver and spleen of Frogs. Normally, the cardiac blood of Frogs contains about 8 red corpuscles to 1 colorless; after extirpation of the liver, the proportion is 2-3 red corpuscles to 1 colorless. The blood of the spleen of Frogs contains, normally, six times fewer red corpuscles in proportion to 1 white, than that of the heart. After extirpation of the liver, there are 1-6 colorless corpuscles to 1 red corpuscle in the splenic blood. When the spleen alone lias been extirpated, the proportion of red corpuscles is slightly increased. Moleschott concludes that the liver favors the metamorphosis of colorless into red corpuscles. However, we must confess that the results of the individual experiments, from the average of which his conclusions are drawn, vary so widely as to throw some doubt on the latter.-TRs.] 
whilst the arteries, from the main trunks to the finest ramifications, all possess them. The structure of the sheaths is precisely that of the trabecula, but muscles are not always found in the former when they are contained in the latter-e. $g$. in the $0 \mathrm{x}$-while in the Pig they are also very distinct in the sheaths.

The splenic artery, immediately it enters the organ, and all its principal branches, divide and spread out into a great number of ramifications, the larger of which proceed towards the anterior margin of the organ, the smaller towards the posterior, forming no anastomoses with those of other principal branches. When they have diminished to the diameter of $1-5-1-10$ of a line, they separate from the veins, which till then had run in the same sheath with them, and become connected by

Fig. 232.

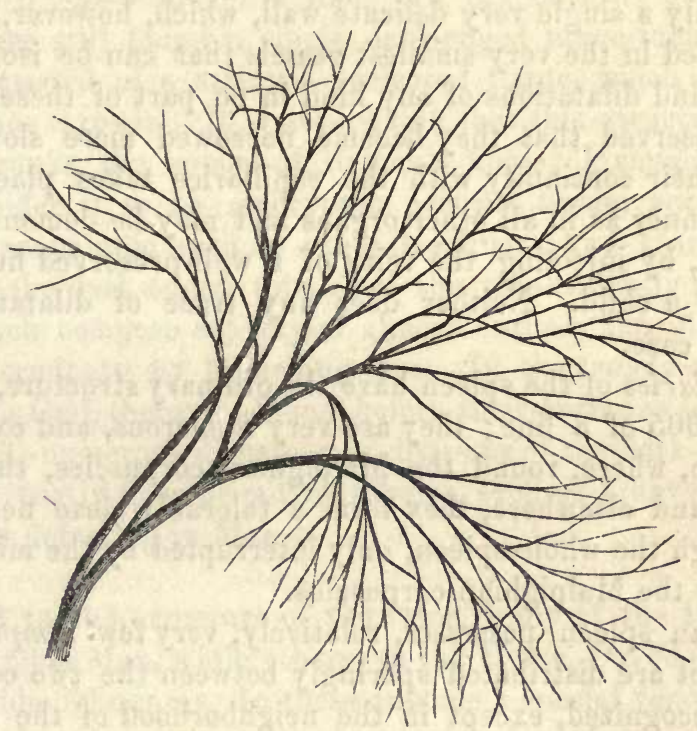

branches of $0.01-0.02$ of a line, with the Malpighian corpuscles in the manner which has been described above; perhaps, also, sending fine branches into their interior (see $\S 167$ ). Then, often closely applied to the surface of the corpuscles, but, so far as I can observe, not passing through them, as Joh. Müller formerly supposed, they enter the red pulp and immediately break up into elegant bundles of minute arteries, the so-called penicilli (Fig. 232), which finally subdivide into true capillaries of $0.003-0.005$ of a line, which throughout the pulp, round the Malpighian corpuscles, as well as elsewhere, unite into a somewhat wider network.

With respect to the reins, I must especially express myself against

FIG. 232.-An artery with its penicillate ends, from the spleen of the Pig, magnified 25 diameters. 
the existence of the venous tissues or spaces of ancient and modern anatomists, in the human spleen. The larger veins which still accompany arteries, present no peculiarities, except in their width : all possess a membrane, which is, at least upon the side of the artery, easy of demonstration, and, like the vascular sheath, gradually becomes thinner. Apertures of more minute veins, the so-called stigmata Malpighii, exist only in inconsiderable numbers in the largest of these veins, while in the smaller, they are more frequent. From the point of divarication of the arteries and veins, the relations of the latter become somewhat different. In the first place, they give off upon all sides a vast number of small veins, usually at right angles, whence their walls appear in places almost cribriform; and secondly, their membranes become completely coalescent with the sheaths of the vessels, so that ultimately the two constitute only a single very delicate wall, which, however, may still be easily detected in the very smallest vessels that can be isolated by dissection. I find dilatations of any kind in no part of these veins, only, it is to be observed that they become narrowed more slowly than the arteries. Their continuity with the capillaries takes place in exactly the same manner as in all other organs and may, be demonstrated without difficulty, by injecting the veins of a well-preserved human spleen, especially of a child. Neither does any trace of dilatations present itself in this case.

The capillaries of the spleen have the ordinary structure, and a width of $0.003-0.005$ of a line; they are very numerous, and exist throughout the pulp, where, round the Malpighian corpuscles, though not in their coats, and elsewhere, they form a tolerably close network continuous through the whole spleen, only interrupted by the minutest trabecula and by the Malpighian corpuscles.

The human spleen possesses, relatively, very few lymphatics. The superficial set are distributed sparingly between the two coats, but can hardly be recognized, except in the neighborhood of the hilus and in perfectly healthy spleens. The deep set may be discovered in the hilus, whence also, few in number and small in diameter, they accompany the arteries, but cannot be traced by any means so far as these. In the hilus both sets of lymphatics join, traverse a few small glands, which exist in this locality, and finally unite into a trunk which opens into the thoracic duct opposite the 11 th or 12 th dorsal vertebra. In diseased spleens no trace of the superficial lymphatics can ordinarily be detected.

The nerves of the spleen, consisting of many fine and a few thick tubules, with a moderate proportion of Remak's fibres, are derived from the splenic plexus, formed by two or three trunks which surround the splenic artery, and are continued on the arteries into the interior of the organ, each dividing into one or two branches, anastomosing here and there. In the Sheep and Ox, these splenic nerves are truly colossal, so 
that, taken together, they equal the empty and collapsed splenic artery, this size, however, being attributable principally to the unusual quantity of Remak's fibres.

In animals, these nerves, which never possess ganglia, may be followed into the spleen further than in Man; and, by the aid of the microscope, I have frequently met with them even upon the arteries which support the splenic corpuscles. As to their terminations, I can only state that they pass into the pulp, and are still to be met with upon the arterial penicilli. Here they ultimately become as delicate as the finest capillaries, no longer possess dark-edged tubules, and, according to Ecker's observations (1. c., p. 149, Fig. 10), probably end in free dichotomous divisions. In the Calf, the nerves upon arteries of 1 line measure $0.024-0.028$, on the penicilli arteriarum $0.0048-0.0056$, in the midst of the pulp $0.003-0.004$ of a line. In branches of $0.012-0.028$ of a line, I here still found a single dark-edged nerve-tubule, whilst all the rest consisted of a striated nucleated tissue, which alone constituted the fincr threads. It is improbable that this structure has here, as in the trunks, the import of Remak's fibres; I should rather with Ecker, consider it to be embryonic nervous tissue, such as we are sufficiently acquainted with in other localities; and I'am inclined to believe that the dark-edged tubules of the trunks finally pass into pale fibres, as such compose entirely or almost entirely the ultimate twigs, and then terminate by branching out. In the trunks of the splenic nerves of the Calf, there are found, before their entrance into the spleen and within it, numerous dichotomous divisions of the dark-edged, partly. coarser and partly finer primitive tubules, such as I have not hitherto succeeded in detecting in Man.

In regard to the structure of the veins, many of the Mammalia appear to resemble Man, while others, as the Horse, Ox, Sheep, Pig, present very wide differences. In these animals a special venous membrane and a vascular sheath are found only at the origins of the largest venaus trunks, while further in, they are visible only upon the side of the arteries. In all the smaller veins, which run independently (without arteries), no further trace of two coats is to be met with, in fact, these veins appear to be mere excavations in the substance of the spleen, especially as a number of anastomosing trabeculoe with red pulp often forming projecting knobs between them, are apparent upon their walls. However, they always have a perfectly smooth and shining surface, arising from spindle-shaped, tessellated epithelium-cells of $0.005-0.01$ of a line, which are only microscopically demonstrable. This epithelium corresponds perfectly with that of the larger veins, only that here it no longer lies upon a special wall, but immediately upon the substance of the spleen, $i$. e. upon the trabeculce and upon a delicate membranous 
substance which bounds the pulp between them. Under these circumstances we may, with perfect justice, speak of venous sinuses; the more, if we consider that these, so to say, wall-less veins have a colossal width, and are pierced by innumerable veins which open into them. These smaller veins, again, may be traced for a considerable distance with the scissors, but I have as yet never been able to succeed in demonstrating in what manner they are continuous with the capillary network, proceeding from the penicilli arteriarum, which here also is very distinct. I can hardly believe that it will ever be possible to make out this continuity completely, for the finest veins, which are often bounded by but a few trabeculoe, and indeed for the most part by the red pulp alone, are such delicate canals, that the slightest mechanical force, as in inflating or injecting them, destroys them; and even by the microscope they are not discoverable. It may always be observed, however, that they eventually become very minute, so small that it is impossible to consider their origins as enlargements. For my own part, I believe, that here also, the connection with the capillaries takes place quite in the ordinary manner, with this distinction, however, that the veins in arising from them possess only one membrane, an epithelium, and are therefore perhaps connected in some other manner with their structureless coat. Smaller series of more rounded epithelial cells, which are not unfrequently found on teasing out the pulp, probably belong to the smallest veins.

In mammalia the lymphatics are stated by all authors to be extremedy numerous, and this is perfectly true for the superficial vessels, which in the Calf, for example, are exceedingly abundant and present numerous anastomoses in the subserous cellular tissue. On the other hand, I find that the deeper lymphatics are scanty. In the hilus of a calf's spleen, for instance, I found but four lymphatic trunks with a collective diameter of 0.17 of a line. The superficial and deep lymphatics would appear to be, to a certain extent, connected; inasmuch as a few scattered lymphatics, which are probably connected with those which proceed from the hilus, accompany the arteries which pass from the interior of the spleen, to be distributed in its coats, and open into the superficial trunks. The latter may readily, in the $0 x$, be traced for a certain distance into the interior, so far that it can be seen that they not only at first, but subsequently, always accompany the arteries. Their origin is unknown, and I can only say, that in the Malpighian corpuscles and in the penicilli, as microscopic investigation shows, the arteries are no longer accompanied by lymphatics. They probably, as in the liver, belong only to the vascular sheaths. In structure, the splenic lymphatics present no peculiarities, and they have valves.

The arteries in the human spleen are exceedingly muscular, which sufficiently explains the dilatation and subsequent contraction of the 
organ observed 5 or 6 hours after the ingestion of food, noticed by many observers. In animals, besides these contractile elements, the muscles of the coats and trabeculoe which I have discovered, may take some part in this process, and their presence further accounts for the circumstance that the spleens of Animals contract by galvanism, while that of man does not (vid. Mikr. Anat. II. 2, p. 265).

$\S$ 170: Physiological remarks.-The spleen is developed at the end of the second month, in the foetal mesogastrium, at the fundus of the stomach, from a blastema which, derived from the middle layer of the germ, independently of the stomach, the liver, or the pancreas, collects in this situation. It is, at first, a whitish, often slightly lobed body $(0.72$ of a line in length, 0.4 of a line in breadth, in the ninth to the tenth week), which gradually becomes red and is very soon as rich in blood and in vessels as in the adult. The roundish small cells, of which the spleen is at first entirely constituted, become, in the third month, partly developed into vessels and fibres, whilst another portion remains as parenchyma cells. The Malpighian corpuscles are not formed till subsequently, but may always be found at the end of the foetal period, although considerably smaller than afterwards. I do not know how they are formed, but I presume that they proceed from simple masses of cells, whose external elements become metamorphosed into the coat of connective tissue, whilst the internal ones, partly persisting in their original condition, partly becoming metamorphosed into vessels, form the contents.

This is not the place to discuss at length the functions of the spleen; I must refer to my "Mikroskopische Anatomie," II. 2, p. 282, and content myself here with stating, that I consider the spleen to be an organ into whose parenchyma constituents of the blood enter bodily and at times in increased quantity, and, with the co-operation of cellular elements, which are in a state of continual formation and solution, undergo, more especially a retrogressive, but partly also a progressive metamorphosis, in the end to be taken up again by the blood and lymphatics, in order to be excreted from the body or further applied to the purposes of the organism.

Up to a certain point the investigation of the spleen presents no difficulties; the pulp, the trabeculoe, and the Malpighian corpuscles, are at once obvious. The latter are most readily examined in the Pig and $\mathrm{Ox}$, where the coat and the contents may easily be isolated, and the connection with the vessels is also apparent. To see blood-corpuscleholding cells, the addition of water must be avoided. The muscular fibres are beautifully seen in the finer trabeculce of the $0 x$, and in the trabeculoe of the Pig and Dog; and here maceration in nitric acid, of 
20 per cent., is of service. The arteries and capillaries are easily injected; the veins with great difficulty; most readily in Man. The nerves are found with ease on the arteries; the lymphatics may be best studied in the $\mathrm{Ox}$.

Literature of the Spleen.-M. Malpighi, "De liene," in "Exercit. de visc. struct.," Lond., 1669; J. Müller, "Ueber die Structur der eigenthümlichen Körperchen in der Milz einiger pflanzenfressenden Thiere," Müller's "Archiv," 1834 (the first good anatomical work since Malpighi); T. C. H. Giesker, "Splenologie, I. anatomisch. physiologische Untersuchungen über die Milz," Zürich, 1835 (a very elaborate treatise); Schwager-Bardeleben, "Observationes micros. de gland. ductu excretorio carentium structurâ," Berol., 1841; Th. von Hessling, "Untersuchungen über die weissen Körperchen der menschlichen Milz," Regensburg, 1842; A. Kölliker, "Ueber den Bau und die Verrichtungen der Milz," in Mittheil. "Der Zürch. nat. Gesellschaft," 1847, p. 120 ; "Ueber Blutkörperchen haltige Zellen," in "Zeitsch. für wiss. Zool.," Bd. I. p. 261, and Bd. II. p. 115; art. "Spleen," in Todd's "Cyclopædia of Anatomy," June, 1849; A. Ecker, "Ueber die Veränderungen, welche die Blutkörperchen in der Milz erleiden," in "Zeitsch. für Rat. Medicin," VI. 1847, and art. "Blutgefässdrüsen," in R. Wagner's "Handw. der Phys.," IV. 1, 1849; J. Landis, "Beiträge zur Lehre über die Verrichtungen der Milz," Zürich, 1847; Gerlach, "Ueber die Blutkörperchen haltenden Zellen der Milz," in "Zeitschrift für Rat. Medicin," VII. 1848; "Gewebelehre," p. 218; R. Sanders, "On the Structure of the Spleen," in Goodsir's "Annals of Anat.," I. 1850; O. Funke, "De sanguine venæ lienalis," Lips., 1851.

[Gray, "On the Development of the Ductless Glands in the Chick," "Phil. Trans.," 1852.-Ths.]

\section{OF THE RESPIRATORY ORGANS.}

$\S 171$. Under the head of respiratory organs are usually enumerated only the larynx, trachea, and lungs; but I consider it as most suitable here to describe two organs connected genetically with those respiratory organs of the embryo, which remain undeveloped, that is to say, the branchial arches; and which, physiologically, perhaps, belong to the lungs-the thyroid gland and the thymus.

\section{OF THE LUNGS.}

$\S 172$. The structure of the lungs corresponds, in all respects, with that of a compound racemose gland, presenting, in the lobes, lobules, and air-cells, the proper glandular parenchyma; whilst the bronchioe, 
trachea, and larynx, constitute the excretory apparatus. They differ from common glands in this, that since in the lungs a double processan excretion and an absorption of matters-is carried on, which affects the whole mass of blood, the eavities are proportionately more capacious, and also, on account of the special nature of their contents, of a peculiarly compact, and, at the same time, elastic structure.

$\S 173$. The larynx is the most complex portion of what are termed the air-passages, and consists, in the first place, of a firm framework, the cartilages of the larynx, together with their ligaments; secondly, of numerous small muscles attached to them; and lastly, of a mucous membrane, abounding in glands, with which they are lined.

The cartilages of the larynx are not all alike in their structure, some being composed of common cartilaginous tissue, others of fibro-cartilage, whilst others, again, are constituted of the so-termed reticular or yellow cartilage. To the former belong the thyroid-, cricoid-, and arytenoidcartilages; all of which present a more homogeneous, hyaline matrix, with scattered cartilage cells imbedded in it (Fig. 20), and approach nearest to the costal among the other true cartilages. Most externally they contain flattened cells, to which succeeds a whitish layer, with numerous large parent-cells and a more fibrous fundamental substance; and, lastly, in the interior, a larger proportion of matrix and minute radiating cavities. The membranes of the cells are thickened, and, in their interior, a large oil-drop is most usually found. Incrustations of the cartilage-cells and of the matrix, with minute calcareous granules, are very frequent in the laryngeal cartilages; but besides these, true ossifications occur, which are always attended with the formation of larger cavities, filled with a well-marked, gelatiniform, vascular medulla. The epiglottis and the cartilages of Santorini and of Wrisberg, consist of yellow or reticular cartilage (vide $\$ 22$, Fig. 21), presenting opaque, very closely interlaced fibres, which, in animals (e.g. the $0 x)$, are much thicker than in Man, and clear cells, $0.01-0.02$ of a line in size, in which Henle, in one instance, noticed a concentric disposition of such a kind that the remaining cavity of the cell resembled a simple bone-lacuna with a few prolongations (Allg. Anat., Tab. V., Fig. 8). The cartilago triticea consists of connective tissue with scattered cartilage-cells, and is, consequently, common fibro-cartilage.

Of the ligaments of the larynx, the ligg. crico-thyreoideum medium and thyreo-arytcenoidea inferiora contain a preponderance of elastic tissue and are of a yellow color; whilst others, such as the thyreo-arytoenoidea superiora, hyo- and thyreo-epiglottica, and the membr. hyo-thyreoidea, are characterized, at all events, by the great abundancerof that element which they present. The elastic fibres of the laryngeal ligaments are of the finer sort, scarcely exceeding 0.001 of a line, and are united in 
the usual manner into a very close elastic network; which, however, even where it is apparently most unmixed, contains some connective tissue. The muscles of the larynx are all transversely striated with fibres of $0.016-0.024$ of a line, and present the same structure as those of the trunk. They arise from the cartilages, and are inserted into them, and also into their elastic ligaments; the latter being the case with the thyreo-arytoenoideus, which is for the most part lost on the concave side of the vocal ligaments.

The mucous membrane of the larynx, the continuation of that of the throat and mouth, is smooth, whitish red, and connected with the subjacent parts by the ordinary, in some places abundant submucous connective tissue. Except in the glottis, the mucous membrane, covered only by a ciliated epithelium, and presenting no papilloe, abounds in finer elastic fibrous networks, particularly in its deeper portions; whilst the innermost layer, $0.03-0.04$ of a line thick, consists principally of connective tissue, and ceases in an inseparable, homogeneous border of 0.004 of a line. The ciliated epithelium, in the adult, commences at the base of the epiglottis and, above the upper vocal ligaments, is composed of several laminæ (vide $\S 21$ ), on the whole $0.024-0.04$ of a line thick; with the exception of the vocal liganents, which, as was discovered by H. Rheiner and I can confirm, have a squamose epithelium, it lines the rest of the larynx throughout. The proper ciliated cylinders $0.015-$ 0.02 of a line long, and $0.0025-0.004$ of a line broad in the mean, with elongated round nuclei of $0.003-0.0045$ of a line, and occasionally with a few fat-granules, are mostly much acuminated, frequently even prolonged into slender filaments, which may attain such a length that the entire cell may equal $0.024-0.027$ of a line. The cilia are fine, transparent, soft processes of the cell-membrane, 0.0016-0.0022 of a line long, which arise from it with a rather broader basis, and terminate in a pointed extremity. Most usually they are placed close together, over the whole of the terminal surface of the cells, according to Valentin, on the average, to the number of 10 to 22 , which appears to be rather under the mark; more rarely they occur in smaller number, or even, as it is said, singly upon a cell. But in this case, care must be taken not to regard cohering cilia as single ones, as might happen, particularly in the embryo. In their chemical relations the cells of the ciliated epithelium correspond precisely with those of the cylinder-epithelium, and especially, the separation of the cell membrane on the addition of water, may also be remarked in them. The cilia are of much more delicate consistence than the cell membrane and are very readily detached upon any maceration of the epithelium; more or less altered by almost all reagents, they are, by many, at once destroyed; in chromic acid, however, they may be preserved pretty well. In man, the ciliary motion is directed, in the trachea, from below upwards and may often be perceived 
fifty-two or even fifty-six and seventy-eight hours after death (Biermer, Gosselin).* There is nothing to show the occurrence, normally, of a desquamation of the ciliated epithelium of the larynx and air-passages.

Fig. 233.
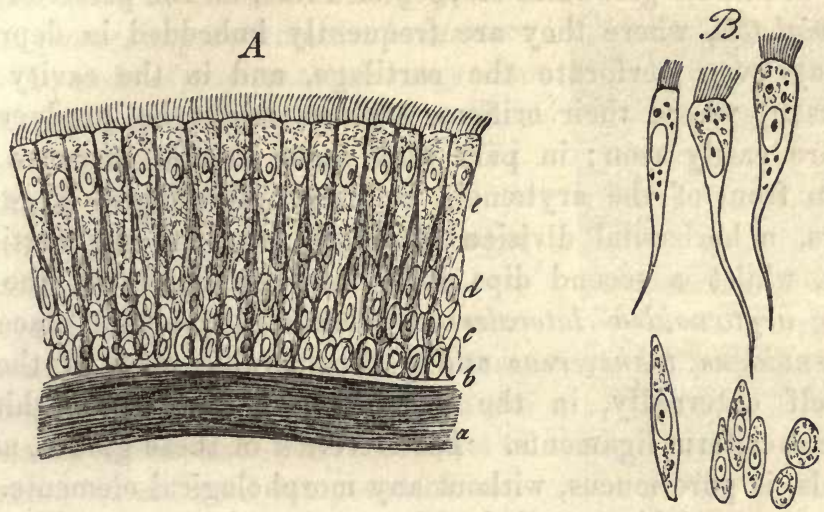

Occasionally, it is true, isolated, ciliated cylinders are thrown off and expelled with the mucus of the trachea, but of an extensive detachment of the ciliated cells there is no indication. Even in diseases of the respiratory passages, the detachment of the ciliated cells is by no means so common a phenomenon as is believed by many, and the epithelium may be frequently found uninjured under puriform mucus, or even beneath croupose exudations. The mode in which the ciliated cylinders that have been thrown off are replaced, is probably simply this: that the deep cells multiply, perhaps by division (vide $\S 12$ ), and succeed them, the outermost again producing cilia.

The laryngeal mucous nembrane contains a considerable number of

Fra. 233.-Ciliated epithelium from the human trachea, magnified 350 diam. A, epithelium in situ: $a$, most external portion of the elastic longitudinal fibres; $b$, homogeneous, most external layer of the mucous membrane; $c$, deepest, rounded cells; $d$, middle, elongated cells; $e$, most superficial cells, supporting cilia. $B$, isolated cells from the various layers.

* [The motion of the cilia is destroyed by many chemical and mechanical agents, but hitherto, notwithstanding the careful researches of Purkinje and Valentin (De phenomeno. gen. et fundam. motus vibratorii continui, Vratisl. $1835, \mathrm{pp} .74-76)$ none have been known which possessed the power of re-exciting it. Virchow (Archiv f. path. Anat. VI. 1) has, however, quite lately, whilst examining the epithelium of a human trachea, discovered that by the application of a solution of potassa the ciliary motion may be recalled. Under the action of the potassa, he states, isolated cilia begin at first to exhibit irregular, jerking movements. These gradually acquire more regularity and force, until at last the rapid, rhythmical, sweeping movement of a whole series of cells is restored. A solution of soda acts in the same manner as one of potassa; a solution of ammonia produces, on the other hand, at once a chemical decomposition. These experiments, Virchow thinks, prove con. clusively, that the substance of the vibratile cilia approximates the contractile substance of muscle (Syntonin of Lehmann).-DaC.] 
glandules, all of which belong to the category of minute racemose glands ; and, like those of the oral cavity, pharynx, \&c., present rounded glandvesicles of $0.003-0.04$ of a line with a tessellated epithelium, and excretory ducts lined with a cylinder-epithelium. They are situated, in part scattered as minute glandules of $\frac{1}{10}-\frac{1}{2}$ of a line, on the posterior surface of the epiglottis, where they are frequently imbedded in depressions, which may even perforate the cartilage, and in the cavity of the larynx itself, where their orifices, such as might be produced by a needle, are easily seen; in part they occur at the entrance of the larynx, in front of the arytænoid cartilages, forming a large aggregate mass, a horizontal division of which envelops the cartilage of Wrisberg, whilst a second dips down into the cavity of the larynx (glandulce arytcenoidece laterales). Glandules are also placed upon the arytoenoideus transversus and a considerable mass of them presents itself externally, in the ventricles of Morgagni, behind and above the sacciform ligaments. The secretion of these glands, as of the oral glands, is pure mucus, without any morphological elements.

The larynx is richly supplied with vessels and nerves. The former, in the mucous membrane, present the same conditions as in the pharynx and ultimately breaking up into capillaries, 0.003-0.004 of a line, form a superficial plexus. The lymphatics are numerous and are received by the deep cervical glands. Of the nerves, we learn from Bidder-Volckmann, that the more sensitive laryngeus superior contains a preponderance of fine fibres, whilst the inferior, whose properties are more of a motor nature, has more thick fibres. They terminate in the muscles, the perichondrium and, especially, in the mucous membrane, in which they are disposed as in the pharynx; the branches going to the epiglottis are also furnished with microscopic ganglia.

The glands of the larynx and of the air-passages are frequently altered in catarrh, so that their vesicles measure as much as 0.08 , or even 0.15 of a line, and are filled with minute, rounded cells, which may probably be compared with the mucous corpuscles formed on the surfaces of mucous membranes.

$\S 174$. The trachea and its branches are united to the contiguous parts by a connective tissue abounding in well-defined elastic fibres; they are then surrounded by a tough, elastic, fibrous tissue, which covers the cartilaginous rings, like a perichondrium, connects them together, and, in a somewhat thinner layer, invests the posterior membranous wall of the canal in question. To this layer succeed the cartilages, in front and on the sides, while posteriorly there is a layer of smooth muscles. The former, $\frac{1}{3}-\frac{1}{2}$ a line thick, are constituted exactly like the larger cartilages of the larynx, but exhibit no tendency to become ossified. The muscles, on the contrary, from the trachea onwards, cease to be of the striped kind and constitute, on the pos- 
terior wall of the tube, only an incomplete layer, 0.3 of a line thick, composed of transverse fibres; on the outer aspect of which are isolated longitudinal bundles, whose elements, 0.03 of a line long and $0.002-0.004$ of a line wide, are united into small fasciculi, which arise by delicate minute tendons of elastic tissue, in part from the inner surface of the ends of the tracheal rings; in part, particularly the longitudinal bundles, from the external fibrous membrane. ( $V i d$. Mikr. Anat. II. 2, Fig. 277.)

On the inner aspect of the cartilages and muscles, which, to a certain extent, are to be regarded as one layer, we find a stratum about 0.12 of a line thick, of more common, close, connective tissue, and then the true mucous membrane. This consists of two layers; an external composed of connective tissue, 0.12 of a line in thickness; and an internal, yellow, of $0.09-0.1$ of a line, almost wholly elastic, the plexiform fibres of which, 0.0015 of a line in diameter, run longitudinally, and in places, especially on the posterior wall, often constitute flattened fasciculi joined at acute angles. The innermost portion of the elastic layer, 0.024-0.03 of a line thick, is, particularly in the posterior wall, as in the larynx, frequently composed more of connective tissue with fine elastic fibrils; it may also be raised

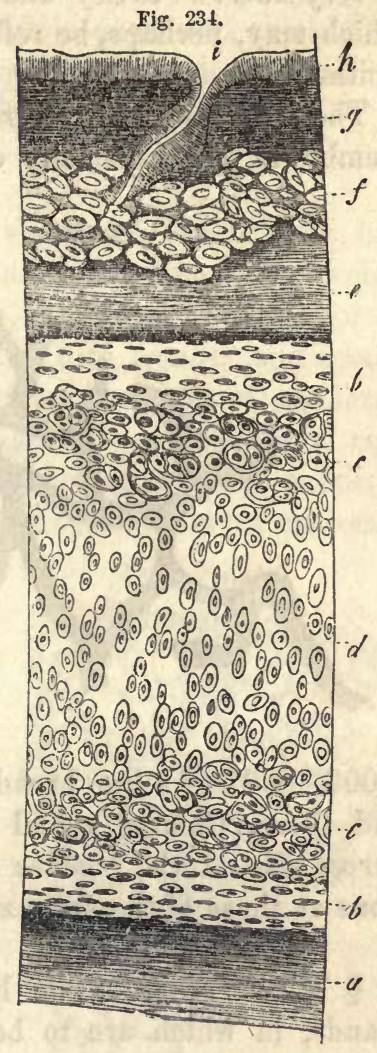
as a thin pellicle from the thicker elastic layer, and internally always presents a more homogeneous layer, 0.005 of a line thick. Upon this lies the ciliated epithelium, which is laminated and differs in no respect from that of the larynx. Numerous glands exist in the mucous membrane; these are: 1 , smaller ones of 1-10-1-4 of a line, found especially on the anterior wall, within the mucous membrane and immediately exterior to the elastic layer; and 2, larger, of $\frac{1}{4}-1$ line, which occur more in the posterior wall, externally to the muscles and the whole mucous membrane, or between the cartilages. These glands differ from those of the larynx only inasmuch as the larger of them alone have the usual tessellated epithelium in their vesicles;

Fra. 234.-Vertical section through the anterior wall of the human trachea, magnified 45 diam.: $a$, fibrous coat; $b, c, d$, cartilage; $b$, external layer, with flattened cells; $d$, internal layer, with elongated elements; $e$, submucous connective tissue; $f$, portion of a mucous gland; $g$, elastic longitudinal fibrous layer; $h$, epithelium, on which the cilia are not visible; $i$, glandular orifice. 
whilst the smaller, situated in the mucous membrane itself, and some of which are in the strictest sense simple, or only bifurcated cæcal follicles, present in their oval gland-vesicles, $0.02-0.03$ of a line in size, a very narrow cavity and walls of $0.006-0.01$ of a line, a thickness which may, perhaps, be referred altogether to the well-marked cylinderepithelium.

The bloodvessels of the trachea are very numerous, and, in the mucous membrane, are especially characterized by the circumstance, that the

Fig. 235.

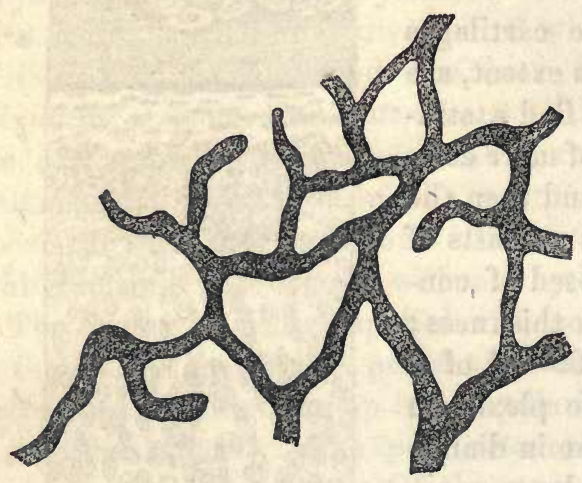
larger branches run chiefly in a longitudinal direction, whilst the superficial capillary plexus, which is frequently met with above the elastic elements, close beneath the homogeneous layer, more commonly forms rounded-angular meshes. The trachea is abundantly furnished with lymphatics; and in one case I found their commencement in the mucous membrane, in the form of wide-meshed plexuses $0.003-0.001$ of a line broad, of thin-walled vessels, from which, here and there, isolated, crecal processes were given off (Fig. 235). The nerves also of the trachea are numerous, and present the same conditions as those of the larynx.

$\S$ 175. Lungs.-The lungs are two large, compound, racemose glands, in which are to be distinguished: 1 , a special serous coatthe pleura; 2, the secreting parenchyma, consisting of the ramifications of the two bronchi, with their terminations, the air-cells, and numerous vessels and nerves; and 3 , an interstitial tissue interposed between these parts and uniting them into larger and smaller lobules.

The pleurce, in their structure, entirely correspond with the peritoneum, as in which, the parietal layer is the thicker, and consist of connective tissue abounding in finer or coarser elastic elements, with a tessellated epithelium, to which constituents, on the walls of the thorax, as on the exterior of the pericardium, a more purely fibrous lamella is superadded. Vessels are seen most abundantly in the pleura pulmonalis, where, arising from the bronchial and pulmonary arteries, they ramify in the subserous tissue; whilst the parietal lamella is more scantily supplied by the intercostal and mammary arteries. Luschka found nerves

FIG. 235.-Commencement of the lymphatics in the tracheal mucous membrane of Man, magnified 350 diameters. 
with finer and coarser fibres and traced them, in the outer portions of the membrane, to the phrenic and to the thoracic divisions of the sympathetic. In Man, I have myself also seen, in the pleura pulmonalis, nerves, as much as 0.036 of a line in diameter, accompanying the branches of the bronchial arteries, with middle-sized and thick fibres and occasionally large scattered ganglion-globules, which were derived from the plexus pulmonalis, and were probably afforded chiefly by the vagus.

$\S 176$. Air-vessels and cells.-When the right and left bronchi have reached the root of the lungs, they begin to branch, like the excretory ducts of one of the larger glands, such as the liver, dividing for the most part dichotomously and at acute angles, into smaller and smaller branches; but giving off, at the same time, from the sides of the larger and middle-sized branches, numerous minute air-vessels, at a right angle, which like the terminations of the main ramifications, subdivide in an arborescent manner. Thus is ultimately constituted an extremely rich tree of air-vessels, whose finest terminations, never anastomosing, extend through the entire lung and are to be found in every part, on the surface as well as in the interior. With them, also, are connected the ultimatc elements of the airpassages - the air-cells or pulmonary vesicles (vesiculce s. celluloe aêrece s. Malpighiance, alveoli pulmonum, Rossignol), not, as was formerly believed, by each finest bronchial twig terminating in a single vesicle, but always by their communicating with a whole group of air-cells. These groups of vesicles correspond to the smallest lobules of racemose glands, and conseFig. 236.

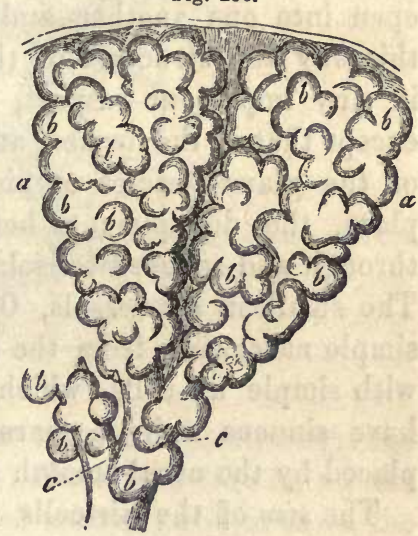
quently there is no occasion whatever to designate them under any other name, as was done by Rossignol, who calls them infundibula, although it must be allowed that their structure, in many respects, is peculiar. For, whilst in other glands the vesicles, if not quite so isolated from each other as has hitherta been supposed, still enjoy a certain degree of independence, the pulmonary elements corresponding to them, - the air-cells, - are, to a considerable extent, confluent with each other, so that all the vesicles belonging to one lobule open, not into ramifications of the finest bronchial twig going to it, but into a common space, from which the air-vessel is afterwards developed. That this is

FIG. 236.-Two small pulmonary lobules, $a a$, with the air-cells, $b b$, and the finest bronchial twigs, $c c$, upon which air-cells are also placed. From a new-born child; magnified 25 diameters. Half-diagrammatic. 
the true condition of these parts is most readily shown when sections, in various directions, of an inflated and dried lung are prepared, or a preparation injected with a colored resinous material is corroded by hydrochloric acid. In preparations of this kind, vesicles either terminal or otherwise pedunculated, or opening independently, are never met with; on the contrary, they always open in such a way, one into the other, and coalesce to such an extent, as, in the aggregate, to form, most usually, a pyriform sacculus, with sinuous walls. These sacculi, which are also identical with the finest lobules, or the infundibula of Rossignol, must not, however, be regarded as sacs, furnished on their walls with closely placed simple cells or alveoli, the latter, on the contrary, being always grouped in such a way that many of them do not open directly in the larger space, but first into other alveoli, and through them into the common carity. An idea of the whole relations of these parts may be best arrived at if each pulmonary lobule be viewed as an amphibian lung in miniature, or if it be conceived that the outer surface of the dilated extremities of the bronchial tubes is thickly beset with numerous racemose groups of vesicles, the constituents of which all open into one another and into the common cavity. Understood in this way the structure of the lungs, then, does not differ in the least, in any important respect, from that of the other racemose glands, except that in the former, at all events in the adult, a partial confluence of the gland-vesicles or air-cells of a lobule, appears to have taken place, the dissepiments between them being here and there broken through and reduced to isolated trabeculoe, as Adriani correctly observes. The smallest air-vessels, $0 \cdot 1-0 \cdot 16$ of a line in diameter, arising by simple narrowing from the most minute lobules, are at first still beset with simple air-cells, which may be termed parietal, and at first also, have sinuous walls, a character, howerer, which is soon lost to be replaced by the usual smooth appearance which is afterwards retained.

The size of the air-cells varies very considerably even in a healthy lung, amounting after death, and when they are wholly undistended with air, to 1-6-1-10-1-18 of a line. But owing to its elasticity, every air-cell may be dilated to twice or three times its natural size without rupture, and is capable afterwards of returning to its pristine condition. It will not be wrong to assume, that, in life, when the lungs are filled with the average quantity of air, the air-cells are at least one-third larger than we find them after death; and that, on the deepest possible inspiration, the expansion reaches, perhaps to twice that dimension. In emphysema, dilatations to this and even to a much more considerable extent, are permanent, and ultimately lead to the rupture of the walls of the alveoli belonging to a lobule, or even to the confluence of the lobules themselves. The form of the alveoli, in a recent collapsed lung, is most usually rounded or oval and, in one that has been inflated or 
injected, in consequence of their mutual pressure, rounded-angular; the air-cells of the surface of the lung are invariably polygonal and their external sides are almost always nearly plane.

The lobulated structure of the lung is not nearly so distinct in the human adult as in younger individuals and in animals. It is therefore advisable, in the first instance, to seek for these conditions in the lungs of a child. In this case the separate lobules are still all distinctly parted from each other by connective tissue and admit of being isolated, so that the tolerably regular pyramidal form of the superficial, and the more irregular one of the interior lobules can be satisfactorily perceived. In the adult, also, these smallest lobules, in size $\frac{1}{4}-\frac{1}{2}-1$ line, still exist, but are so intimately united, that, even on the surface of the lung, their outlines are only perceived with difficulty and imperfectly; and, in the

Fig. 237.

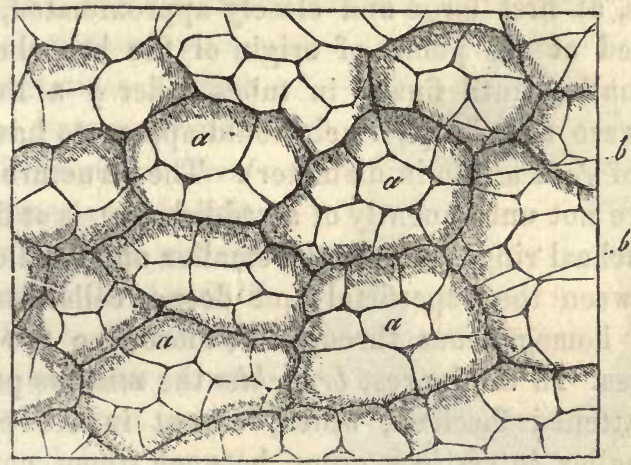

interior of the organ, a more homogeneous structure, something like that of the liver, is apparently presented. Secondary lobules, on the other hand, of $\frac{1}{4}-\frac{1}{2}-1$ inch in size (lobules of authors) are, even in the adult, most usually evident and the more so, because their boundaries are indicated by streaks of pigmentary matter, which, in course of time, is deposited in the continuous interlobular, connective tissue. These lobules are ultimately united together by a more abundant interstitial tissue, so as to form the large well-known lobes.

Thus the lung consists entirely of larger and smaller groups of aircells and smallest bronchial tubes, and accordingly the larger air-vessels also fall into certain definite groups, each of which stands in relation with only one of the former.

FIG. 237.-External surface of the lung of a Cow, with the air-cells injected with wax, magnified 30 diameters; after Harting : $a a$, air-cells; $b b$, borders of the smallest lobules or infundibula (Rossignol). 
$\S 177$. The intimate structure of the bronchioe and air-cells is as follows. The bronchice are in general constituted in the same way as the air-tubes and their branches, although from the very commencement some differences are presented, which become greater and greater in their further course. It is most proper to distinguish in them two membranes, a fibrous, still in part containing cartilages, and a mucous, with a smooth muscular layer. The former, constituted of connective tissue and elastic fibrils, is at first thick, as in the bronchi, but gradually becomes thinner and thinner; in bronchice less than $\frac{1}{2}$ a line in diameter, it is scarcely demonstrable with the scalpel; and ultimately, in their terminations, it coalesces with the mucous membrane, and the more lax connective tissue uniting the bronchice with the parenchyma of the lung. In this membrane are lodged the cartilages of the bronchice, which, instead of being half-rings, are irregular angular plates, distributed around the entire circumference of the tubes. These cartilaginous plates, at first large and closely approximated, are soon more widely separated at the points of origin of the branches, and become smaller and smaller, until finally in tubes under $\frac{1}{2}$ a line in diameter they usually cease to exist (Gerlach would appear to have noticed some even in tubes of $\frac{1}{10}$ of a line in diameter). The structure of these cartilages, which are not unfrequently of a reddish hue, is at first exactly like that of the tracheal rings, but in the smaller and smallest of them the differences between the superficial and deeper cells disappear and the tissue becomes homogeneous throughout, more like the interior of the larger cartilages. In the largest bronchice the muscles present the form of circular flattened fasciculi, which, except in old people, in whom larger and smaller interstices occur between them, constitute a completely continuous layer, and as they are still seen in twigs of 1-10-1-12 of a line in diameter, probably exist even in the pulmonary lobules. The mucous membrane is intimately united with the muscles, and at first is of the same thickness as in the trachea, but this is gradually reduced, so that tubes of less than $\frac{1}{2}$ a line have only an extremely thin wall. This everywhere consists, externally, of elastic, longitudinal fibres, the bundles formed by which give the characteristic longitudinally striped aspect to the inner surface of the bronchioe, and also produce a less distinct longitudinal plication of the mucous membrane; secondly, of a homogeneous layer $0.002-0.003$ of a line thick; and thirdly and lastly, of a ciliated epithelium, which, in the larger bronchial tubes and down to those of 1 line in diameter, is distinctly composed of several laminæ, but is gradually reduced to a single layer of ciliated cells 0.006 of a line in length. The bronchix are at first also furnished with racemose glands, even in considerable number, which, however, are wanting in tubes of $1-1 \frac{1}{2}$ lines. 
In the air-cells, I cannot admit the existence of more than two layers -a fibrous membrane and an epithelium. The former is manifestly the much attenuated mucous membrane and fibrous tunic of the bronchice, entirely deprived of the smooth muscles, and consisting of a homogeneous matrix of connective tissue, together with elastic fibres and numerous vessels. The elastic fibres, $0.0005-0.002$ of a line in size, present the form chiefly of separate trabeculx and filaments, running especially at the angles of the air-cells, which have been flattened in the distended condition, as well as around their openings; they anastomose with each other on every side and thus constitute a firm frame, on which the softer, vascular parts of the air-alveolæ, composed of connective tissue, are stretched. The structure of these elastic trabeculoe, which, at the points where the air-cells abut upon each other, mutually coalesce, so that the boundaries of the separate cells cannot for the most part be recognized, is almost everywhere one of the most close elastic networks possible, the interstices of which appear only as extremely narrow fissures, although occasionally the fibres are more loosely united, so that they are plainly recognizable as elastic elements of the usual kind. From the trabeculoe also, but everywhere sparingly, elastic fibres, in part very fine, proceed into the remainder of the walls of the air-cells, in which, by their union, they constitute a wide network. The connective tissue of the air-cells, which appears to be altogether homogeneous, is quite a subordinate element

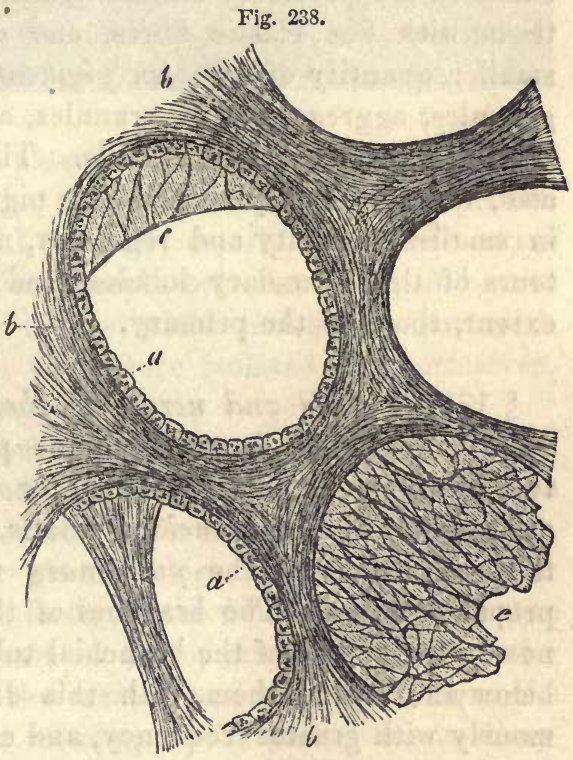
in their composition, compared with the elastic elements and vessels, presenting itself, as it may be said, only in the walls of the alveoloe, between the elastic trabeculce, as a connective medium between the numerous capillaries.

The epithelium of the air-cells is of the common tessellated kind, without cilia, and composed of polygonal, pale, granular cells, in mor-

FIa. 238.-Human air-cell, with the surrounding tissues, magnified 350 diameters: $a$, epithelinm; $b$, elastic trabecula; $c$, more delicate wall between the latter, with finer elastic fibres. 
bid states, containing fat, $0.005-0.007$ of a line in diameter, and $0.003-0.004$ of a line in thickness, resting immediately upon the fibrous membrane of the air-cells. A regular detachment of this epithelium is not to be supposed, any more than with that of the trachea and bronchioe, whilst it is indubitable, that by chance, or in diseases of the air-passages, its isolated elements may become mixed with the bronchial mucus. In Man, these cells are detached with remarkable readiness, and then lie free in the air-cells and finest ramifications of the bronchice, although in almost every lung, at all events in some of the alveolce, they may still be seen in situ; and in animals recently killed, the observation of their disposition presents no difficulty whatever.

The interlobular connective tissue of the lungs, which is contained sparingly, even between the secondary lobules, and between the primary, exists in inappreciably minute quantity, consists of cornmon connective tissue with fine elastic fibres, and contains, in the adult, a larger or smaller quantity of blackish pigment, in the form of irregular, minute granules, aggregations of granules, and also crystals, which, it may be said, are never enclosed in cells. The walls of the alveolce themselves, also, very frequently contain this pigment, which, when it is deposited in smaller quantity and regularly, marks out very distinctly the contours of the secondary lobules, and not unfrequently, also, to some extent, those of the primary.

\$178. Vessets and nerves of the lungs.-As regards their bloodvessels, the lungs occupy a unique position, inasmuch as they possess two complete vascular systems, for the most part distinct from each other-that of the bronchial vessels, for the nutrition of certain portions, and that of the pulmonary vessels, for the fulfilment of their proper function. The branches of the pulmonary artery follow pretty nearly the course of the bronchial tubes, which are most usually placed below and behind them, with this difference, that they divide dichotomously with greater frequency, and consequently diminish more rapidly in diameter. Ultimately, a twig goes to each secondary lobule, which then subdivides into still finer ramuscules, in general corresponding in number with the smallest lobules, and supplying the individual air-cells. The course of these finest lobular arteries, as they may be termed, is very easily traced in injected, inflated, and dried preparations; and it is apparent, that whilst traversing the uniting tissue between the lobules (infundibula), they supply not one lobule alone, but always two or even three of them with finer twigs. These penetrate from without, upon and between the air-cells, divide repeatedly while running in the larger elastic trabeculo, anastomosing also occasionally, though not 
regularly, with each other, or with branches of other lobular arteries, and, finally, terminate in the capillary plexus of the air-cells. This plexus, which is one of the closest existing in Man, as estimated in moist preparations, presents rounded or oval meshes $0.002-0.008$ of a line wide, and ressels of $0.003-0.005$ of a line in diameter. It lies in the wall of the air-cells, at a distance of about 0.001 of a line from the epithelium, in the middle of the fibrous tissue, and is continuous, not only over all the alveolce of one of the smallest lobules, but, also, at all events, in the adult, is

Fig. 239.

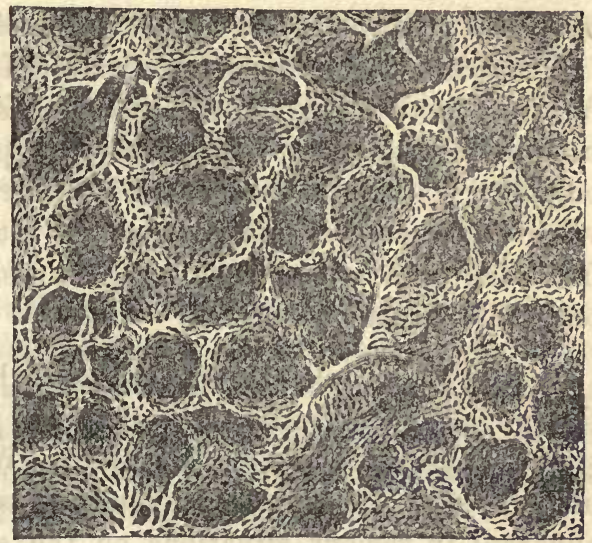
partially in connection with the plexuses of the contiguous lobules. The pulmonary veins arise from the above-described capillary plexus, with roots which lie more superficial than the arteries, and more externally on the smallest lobules, then run deeply between them, and unite with other lobular veins into larger trunks, which proceed in part with the arteries and bronchial tubes, in part more isolated by themselves, through the pulmonary parenchyma.

The bronchial arteries are distributed, firstly, to the greater bronchice, whose vessels present the same conditions as those of the trachea, then to the pulmonary veins and arteries, the latter of which, in particular, possess an extremely rich, vascular plexus, which may be traced as far as branches of $\frac{1}{3}$ of a line, and less; lastly, to the pleura pulmonalis, the branches destined for which are, sorne of them, given off even at the hilus and in the fissures between the main lobes, some also from the vessels accompanying the bronchice, coming out between the secondary lobules. Small vessels, moreover, which are not derived from the bronchial arteries, pass on the pulmonary ligaments to the pleura.

The lymphatics of the lungs are very numerous. The superficial lymphatic vessels run in the subserous connective tissue and in the inter. spaces between the larger and smaller lobules, forming a superficial, finer, and a deep, coarser, angular network, which covers the entire surface of the lungs, and on the one hand empties itself into special, superficial trunks accompanying the bloodvessels of the pleura towards the root of the lung, and, on the other, opens into the deeper vessels by numerous trunks which penetrate between the lobules. These arise

FIG. 239.-Capillary plexus of the human air-cell, magnified 60 diameters. 
from the walls of the bronchice and bloodvessels, particularly those of the pulmonary arteries, running with those canals through the substance of the lung, and through some minute lymphatic glands (glandulce pulmonales), towards the root of the lung, in order, ultimately, to communicate with the larger bronchial glands.

The nerves of the lungs are derived from the vagus and sympathetic, form the rnore scanty plexus pulmonalis anterior, and the richer plex. p. posterior, and are distributed principally with the bronchice and the pulmonary artery, occasionally, however, accompanying the pulmonary veins and vasa bronchialia. In the interior of the lung they are also furnished with microscopic ganglia, and may be traced nearly to the termination of the bronchice.

It is very remarkable, that besides the air-cells, some other parts of the lungs are also supplied by the vasa pulmonalia, such as the surface of the lungs and the finer bronchice. With respect to the former, even in uninjected lungs, minute ramuscules of the pulmonary artery are apparent in various situations on the surface of the lungs, ramifying under the pleura. Reisseisen (p. 17) describes these vessels, and figures them very beautifully (Table IV., V.); recently, Adriani has traced them in injected lungs and states that they penetrate into the interior, much convoluted and frequently anastomosing; they are considerably thicker, however, and form wider plexuses than those of the alveoli. The blood from these plexuses is conveyed away, on the one hand, by the superficial roots of the pulmonary veins, and, on the other, through anastomoses with the ramifications of the vasa bronchialia in the pleura pulmonalis. That the pulmonary artery also supplies the bronchice to some extent was stated by Arnold (Anat. II., p. 171); and to Adriani we are indebted for more precise information on this interesting subject. According to them, the pulmonary artery and veins chiefly participate in the formation of the capillary plexus on the surface of the bronchice, which is characterized by the elongated form of its meshes, and is constituted by vessels almost as fine as those of the air-cells (in Man, of $0.004-0.006$ of a line), whilst the bronchial vessels are specially destined for the supply of the muscular and fibrous coats of those canals. It is comprehensible, also, that in this situation the two vascular systems are, to a certain extent, connected; and consequently the older anatomists, such as Haller, Sömmering, and Reisseisen, who speak of a connection between the two vascular systems of the lungs, were quite right. According to Adriani and Rossignol, the bronchial arteries and veins may be injected from the pulmonary veins, and inversely, the pulmonary veins from the bronchial arteries; but the bronchial vessels cannot be filled from the pulmonary arteries.

In accordance with these facts, a participation in the interchange of gases may be ascribed also to the finest bronchice, but, on account of the 
already somewhat increased thickness of the epithelium in them, and the rather wider capillary plexus, to a less extent than in the air-cells. Here, also, we might recall to mind the dilatation of the bronchial arteries and extension of their zone of distribution, in cases where the circulation through the pulmonary arteries is interrupted (vide Virchow, in his "Archiv." III., 3, p. 456), in which cases the bronchial arteries frequently wholly replace branches of the pulmonary artery, and become respiratory vessels; conditions which, from the occurrence of numerous normal anastomoses between the two vascular systems, it is not difficult to explain.

\$179. Development of the lungs.-In the Mammalia, the lungs appear a little after the liver, in the form of two hollow protrusions of the anterior wall of the pharynx, which are in close apposition, and soon become furnished with a common peduncle-the rudiment of the larynx and trachea-and in the composition of which the epithelial tube and the fibrous membrane of the intestine take an equal share.

In the further course of development, there springs from the extremities of the original protrusions, a continually increasing number of arborescent processes, which differ entirely from what may be observed in most other glands. From their first formation they are always hollow, and in the sixth month the air-cells are developed from their invariably clavate, dilated extremities. During this growth of the glandular elements, the interior epithelium extends itself by spontaneous multiplication of its cylindrical cells (probably by division), whilst, at the same time, the fibrous layer surrounding them also grows, and finally constitutes the fibrous membrane of the bronclioe and air-cells, together with the ressels and nerves. In the second month, in the human embryo, the large pulmonary lobes are already formed, and besides them, smaller divisions also, 0.16 of a line in size, may be recognized, originating in the dilated extremities of the bronchioe, which, even at this time, are considerably ramified. As development proceeds and the ramifications of the bronchice are multiplied, these gland-granules, as I have termed them, become more and more numerous, and ultimately, in the fifth month, are aggregated so as to form smaller lobules, of $0.24-0.48$ of a line in size, each of which, in all probability, is produced from a single gland-granule, or bronchial termination of the second month. Each of the gland-granules of these lobules, which correspond with the secondary lobules of the future lung, by continued budding, finally constitutes a primary lobule, which, with air-cells of $0.025-0.3$ of a line in size, first becomes distinctly visible in the sixth month, although, up to the time of birth, new alveoli are constantly superadded (vide "Mikr. Anat." II. 2, p. 323). In the new-born child the secondary lobules measure 2-3-4 lines; the alveoli, before they are filled with air, 0.03 , and after 
the first inspiration, $0.03-0.04-0.06$ of a line ; the latter, at this time, zppear to exist in the same number as in the adult, the further increase of the lungs proceeding only from the expansion of all its parts.

[The pathological changes occurring in the tissue of the lung are numerous. An atrophy of the parenchyma takes place in old age and in emphysema. In the former, a thinning of the walls of the air-cells, and a partial obliteration of the capillaries exists. In emphysema the alveoli of the cells are enlarged, but the walls much attenuated; sometimes their rupture ensues and a communication between the separate cells is established. Hypertrophy of the air-cells, with enlargement of the capillaries, is observed in the hypertrophy of the lung which occurs from increased functional action. Obliteration of the aircells is mostly caused by exudation or deposit into the air-vesicles or the interstitial tissue. In pneumonia this exudation occurs mainly into the alveoli, sometimes, however, into the walls of the cell, the interstitial, or even into the interlobular tissue (interlobular pneumonia). The granular appearance of red hepatization is owing to a complete expansion of the air-cells by the plastic exudation. In gray hepatization the walls of the cells and the interstitial tissue become softened, and undergo a fatty metamorphosis.

Deposits of pigment occur as a consequence of hepatization, or from a simple congestion of the lung. The pigment is found in the interstitial tissue, and sometimes exists in sufficient quantity to compress the air-vesicles and produce their atrophy. It is formed either from the coloring matter of the blood, or else by the blood corpuscles breaking up into distinct pigment-cells and granules (Virchow). Tubercle is most generally deposited in the air-cells; cancer more frequently in the interstitial tissue.

Effusions of serum, or of blood, occur either into the interstitial tissue, or into the air-vesicles. In oedema the effused serum has its seat in the air-cells, whilst in apoplexy of the lung, the blood is effused in the interstitial tissue. A fatty degeneration of the epithelial cells is observed in portions of lung, compressed by a pleuritic effusion, in atelectasis (Reinhardt), and in splenization. Accidental formations (bone, cartilage) and cysts have their seats generally in the interstitial tissue. Destruction of the parenchyma of the lung is the result of inflammation, gangrene, tubercle, or cancer. A microscopical examination of the destroyed parts shows them to be infiltrated with fine fatty molecules; the elastic elements of the air-cells are generally found well preserved.-DaC.]

The investigation of the lungs presents no real difficulty, except in one point; that is, with respect to the relation of the pulmonary cells to the terminations of the bronchice, but here the difficulties are very 
considerable. In recent preparations it is obvious that the air-cells communicate in many ways, and in any case, that they are not merely terminal on the extremities of the bronchioe. If it be desired to investigate the whole subject, inflated and dried lungs (it is better in an inflated lung to tie off an end and dry it by itself), or corroded preparations, or lungs injected with uncolored substances (wax and resin) are most suitable, and with such, a definite result will be obtained, after a series of observations.

Before the injection of the bronchice is proceeded with, the air must be exhausted in the air-pump, for which purpose, also, though less conveniently, a well-fitted syringe may be employed. The injection of the bloodvessels is readily effected and the preparations should be kept wet; sometimes, when injected with opaque material, sometimes, following the processes of Schröder and Harting, with transparent substances (Prussian blue, \&c.), dried preparations are to be preferred. The aircells and bronchioe, the larynx and trachea, are readily examined. The epithelium of the air-cells is obtained in large quantity in every section through the lung, as well as ciliated cells. If it be wished to study the alveoli, the air must previously be carefully removed. These are best displayed in Man, in whom, also, all the other parts, such as cartilage, elastic elements, muscles, and glands, are easily obtainable.

Literature.-M. Malpighi, "De pulmonibus epistolæ II ad Borellum." Bonon. 1661; F. D. Reisseisen, "Ueber den Bau der Lungen, eine gekrönte Preisschrift," Berlin, 1822; [Magendie, "Mém sur la structure du poumon, in 'Journ. de Phys. exper.;" Rainey, "On the minute structure of the lungs," Philosoph. Transact. 1845;] J. Moleschott, "De Malpighianis pulmonum vesiculis," Heidl. 1845, Diss. and, "Ueber die letzten Endigungen der feinsten Bronchien," in the Holländischen Beiträgen, I. p. 7 ; Rossignol, "Recherches sur la structure intime du Poumon, Brux.," 1846; A. Adriani, "De subtiliori pulmonum structurâ." Trajecti ad Rhen. 1847, Diss.; Köstlin, "Zur normalem und patholog. Anatomie der Lungen," in Gries. "Archiv," 1848, Heft. IV. p. 282, and 1849, Heft II. p. 167 ; E. Schultz, "Disquisitiones de structurâa et texturâ canalium aëriferorum," cum tab., DorpatiLiv. 1850. Dissert.

\section{OF THE THYROID GLAND.}

$\S 180$. The thyroid gland, or body (glandula thyroidea), is a so-termed "ductless gland," which in its external aspect much resembles the racemose glands, seeing that its round, closed gland-vesicles, $\frac{1}{50}-\frac{1}{20}$ of a line in size, are surrounded by a fibrous stroma and subdivided into rounded or elongated, often slightly polygonal lobules, $\frac{1}{4}-\frac{1}{2}$ of a line in size,the gland-granules of authors; - and these again are associated into larger, though not completely separated, lobes, out of which the main 
divisions of the organ are then constituted; these have special, and indeed thicker couts, with which, lastly, a fibrous membrane investing the whole organ is conjoined.

$\S 181$. With respect to its intimate structure, there is not much to be said about the fibrous tissue or stroma of the thyroid gland, inasmuch as it consists of common interlaced bundles of connective tissue, intermixed with fine elastic fibres and also, on the surface, contains a certain quantity of fat-cells. The gland vesicles themselves, in Man, present such varied conditions of structure, that it is not easy to say what is their normal state. According to what $I$ have observed in Man and also in animals, I must, with regard to this point, declare that, analogous to the true gland vesicles, for instance, of the mucous glands, they consist of a membrana propria, an epithelium, and fluid contents. The membrane is quite homogeneous, transparent, and delicate, 0.0008 of a line thick; and, like all membranes of the sort, is rendered more distinct by caustic alkalies, in which it swells up. On its inner surface lies a single layer of epithelium, composed of polygonal, finely granular, transparent cells of $0.004-0.006$ of a line, with simple nuclei; whilst the space surrounded by these cells is occupied with a clear, somewhat viscous fluid, with a tinge of yellow in its color, the behavior of which towards alcohol and nitric acid and when the gland is boiled, clearly manifests the presence of much albumen. This is the character of the contents in the healthy thyroid gland of Man, particularly in children; if the organ, however, is but very little altered, conditions, in many respects different, are presented. Very frequently, instead of a regular epithelium, nothing is met with

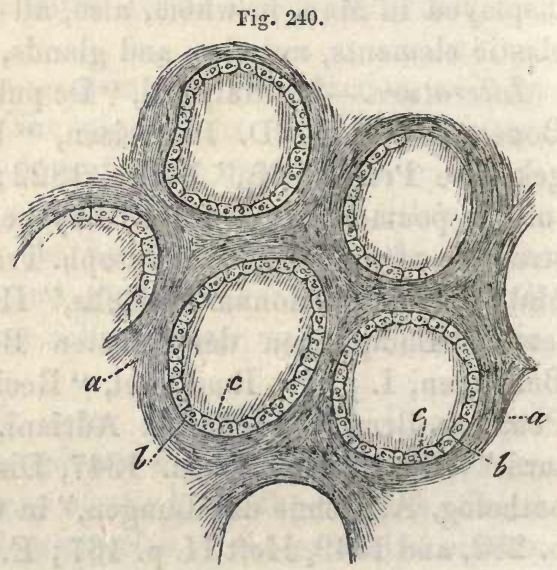
but a fluid mixed with minute, clearer or darker granules and free nuclei; although I am ignorant whether this condition of the contents does not take place till after death, or whether it is to be regarded as abnormal. For we so frequently observe, in the granular fluid, a greater or less number of the same cells, which at other times exist as epithelium, often pale and as if half dissolved, that it is impossible to avoid the conclusion, that in these cells we have an instance of that post-mortem decomposition, so frequently observed in the human subject. On the other hand, the pathological nature of the change in the thyroid

Fig. 240.- Some gland-vesicles from the thyroid gland of a Child, magnified 250 diam.: $a$, connective tissue between thern; $b$, membrane of the gland-vesicles; $c$, their epithelium. 
body and its vesicles, termed colloid, cannot be doubted, although this morbid condition is so frequent, in certain minor degrees, that many authors enumerate it under the physiological occurrences. In this degeneration, there is deposited in the gland vesicles, which increase in size at the same time, the colloid substance, which also occurs in other situations, in the form of transparent, amorphous, light yellowish, soft, solid, masses, by which they are more or less filled. In the lesser degrees of this change, the vesicles are but little enlarged, to as much as 0.05 of a line in a transverse section, presenting the appearahce of transparent yellowish-white spots or granules, which have

Fig. 241.

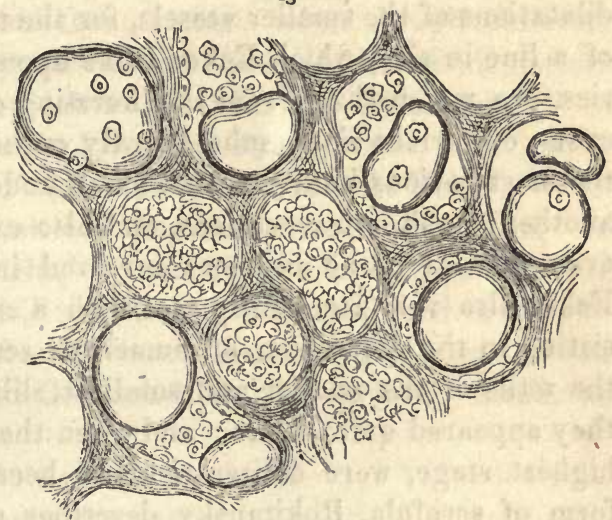
been very aptly compared, by Ecker, with boiled sago, and otherwise retaining the usual structure. In a higher degree, the vesicles containing colloid are transformed into larger cysts, of ${ }_{10}^{1}-\frac{1}{2}$ of a line, in which the epithelium is rarely any longer distinct, but, together with the abnormal contents, rounded, pale cells, filled with colloid matter or granules, and nuclei, may occur; these cysts compress the stroma and ultimately, orving to a partial absorption of the walls, coalesce into still larger sinuous cavities, the contents of which are afterwards frequently altered in various ways, by extravasations and their metamorphoses. In Mammalia and Birds, the thyroid body also occasionally contains gland-resicles slightly distended with colloid matter.

The bloodvessels of the thyroid gland are, as is well known, disproportionately numerous, but in their coarser ramifications present nothing worthy of remark. Each gland-vesicle is provided with some smaller arteries, the subordinate branches of which are distributed and subdivided in the stroma between the vesicles, finally constituting around each of them a delicate capillary plexus, with rounded-angular and elongated meshes of $0.008-0.016$ of a line, and vessels of $0.003-0.005$ of a line, resembling that of the air-cells, except that the interstices are wider. From this plexus the veins arise, which, in their further course, only partially accompany the arteries, which they much exceed in number. Lymphatics also occur in considerable number in the thyroid gland; the relations of which, however, in the interior, are unknown. The few nerves, lastly, are only vascular nerves, and are derived from the cervical portions of the sympathetic.

Fig. 241.-Gland-vesicles of the thyroid gland, filled with colloid matter, magnified 50 diameters. 
Ecker distinguishes the struma, which is by far the most frequent degeneration of the thyroid, into a vascular and a glandular. In the latter, the above-described changes of the gland-vesicles take place, whilst in the vascular struma, which is not regarded by Rokitansky as a special form, besides a hyperæmiated condition, numerous aneurismal dilatations of the smaller vessels, for the most part of those $0.030-0.040$ of a line in size, which Ecker looks upon as arteries and larger capillaries, are met with. From the bursting of these dilatations, apoplectic cysts, of various sizes, subsequently ensue, which may exhibit phases of the most various kinds, as the blood undergoes changes of one kind or another; fresh extravasations, and also exudations, are superadded, and even normal tissue becomes involved in them. In vascular struma, Ecker also very frequently met with a cretification of the vessels, consisting in the deposition of numerous scattered calcareous particles in the walls of the smaller and smallest, dilated or normal vessels, so that they appeared quite white; and when the affection had advanced to the highest stage, were obliterated and became concretions. In a certain form of scrofula, Rokitansky describes an hypertrophy of the thyroid gland, due to a multiplication of the normal glandular elements, taking place by the production of new gland-vesicles, sometimes independent, sometimes occurring in enlarged gland-vesicles, in growths inwards of their walls.

According to Remak, the thyroid body is developed by the constriction of a portion of the anterior wall of the pharynx, and the division of this into two halves. In a human embryo, at the third month, I found that the thyroid body was already composed of isolated vesicles $0.016-0.05$ of a line in size, consisting of a homogeneous envelop and rounded-angular cells in the interior, and I think I perceived that this follicle was multiplied by the formation of rounded buds and their separation by constriction. If this really be the case, the entire development, probably, of the thyroid gland would have to be regarded as a continued growth and division of the glandular follicles, of which the fission of the primary vesicular rudiment, observed by Remak, would be merely the first phase. In this respect, also, a certain resemblance with the thymus would be established, only that in the latter, both the buds of the primary rudiment, as well as the later ones, are not detached, but all remain in connection. The follicles of the thyroid gland, therefore, would not be any kind of enlarged cells, and still less metamorphosed nuclei (Rokitansky), but be equivalent to true glandfollicles.

The investigation of the vesicles of the thyroid gland has mainly been pursued in animals, especially in Birds, Amphibia, and in children; and sections obtained by means of the double-bladed knife, or glands that have been hardened, are most suitable for the purpose of studying 
the vesicles in detail, and in their mutual relations; but the same object may also be attained by minute dissection, and the teasing out of the structure. Injections are very easily made and run very finely in children; they best exhibit the plexuses around the vesicles, in sections taken from the surface.

Literature. - Schwager-Bardeleben, "Obs. micr. de glandularum ductu excret. carentium structurâ," Berol. 1841, Diss.; Panagiotides and K. Wagener, "Einige Beobachtungen über die Schilddrüse," in Froriep's "N. Notiz." Bd. XL., p. 193; and Panagiotides, "De gland. thyreoideæ structurâ penitiori," Diss. Berol., 1847 ; A. Ecker, "Versuch einer Anatomie der primitiven Formen des Kropfes, \&c.," in "Henle and Pfeuffer's Zeitschrift," f. rat. Med. VI., Bd., p. 123, and Article "Blood-vascular glands," in Wagner's "IIandw. d. Physiol." III. ; Rokitansky, in "Zeitsch. d. Wiener Aerzte," 1847, and "Zur Anatomie des Kropfes," in "Denkschriften der kaiserl. Akad. zu Wien," Bd. I., Wien, 1849.

\section{OF THE THYMUS.}

$\S 182$. The internal thoracic gland or thymus, also one of the sotermed blood-vascular glands, is a bilobate, elongated, flattened organ, broad inferiorly, invested and united to the surrounding parts by a lax connective tissue. Larger lobules, measuring on the average 2-5 lines, and of a rounded, oval, or pyriform shape, though for the most part flattened figure, are very distinctly apparent, even on superficial inspection; these, although pretty closely approximated, are still united merely by a yielding connective tissue, and may be separated without difficulty. If these lobules be traced from without inwards, it is easily perceived that they have no further mutual connection, althqugh they are invariably attached by a more slender portion, to a canal, which traverses the interior of the gland, and is, in general, spirally convoluted, though not quite regularly so. When this canal, normally $\frac{1}{2}-1 \frac{1}{2}$ lines in diameter, is opened, there are found on its inner surface a great number of oval fissures or apertures, each of which leads to a lobule and constitutes the outlet of a cavity contained in it. The resemblance of this canal of the thymus and of the closely approximated lobules opening into it, to the excretory duct and the lobules of a true gland, is still further heightened by the circumstance, that the lobules are composed of smaller, also hollow subdivisions and the latter of rounded corpuscles, 1-5-1-3 of a line in size, like gland-vesicles, the gland-granules (acini of authors), which may be recognized even on the exterior, and, from their polygonal shape, give the surface of the lobules a delicate mosaic aspect, not unlike that of the lungs. These gland-granules, however, are not vesicles at all, such as the air-cells, which, among the 
elements of the true glands, approach nearest to them in size, but solid bodies, which, towards the cavity of the lobules or its accessory cavities, are intimately coherent, whilst on the outer side they are separated

Fig. 242.

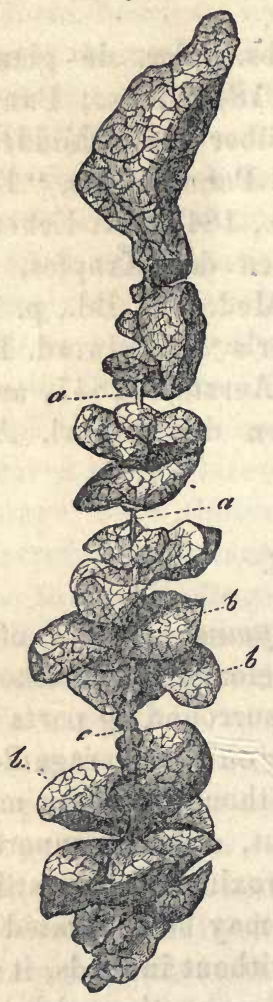

Fig. 243.

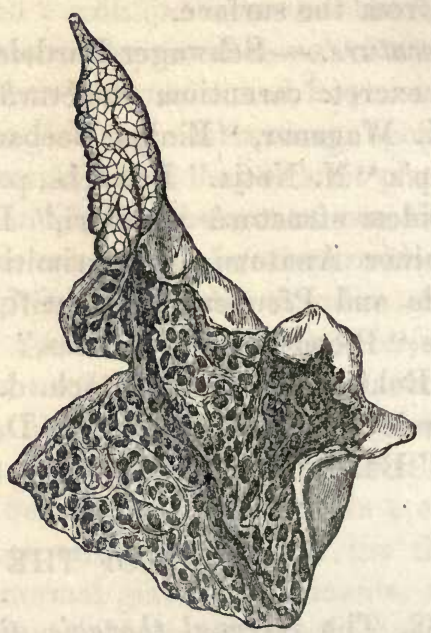

from each other. Each lobule may also be regarded as a thick-walled vesicle, with protrusions, whose inner surface is even and continuous, whilst the outer is subdivided into the above-mentioned gland-granules, by more or less deep fissures.

In many eases, a condition different from that just described is met with, inasmuch as, instead of a contracted canal, into which the cavities of the gland-lobules open, each thymus contains a larger though contracted cavity,$\frac{1}{2}-1$ inch wide, with which the gland-lobules communicate by larger fissure-like openings. Many anatomists, and among the more modern, particularly Sir A. Cooper, consider the existence of this cavity as normal; whilst others, at the head of whom is Simon, are disposed to regard it as produced by the methods of investigation employed

Frg. 242.-Portion of the thymus of a Calf, unfolcled: $a$, main canal ; $b$, glandular lobules ; $c$, isolated gland-granules, seated upon the main canal. Natural size.

Fia. 243.-Half of the human thymus, with a large cavity in the inferior wide portion and numerous orifices leading into the lobules. 
(injections, inflation). I believe that Simon is correct, when he asserts that in such a delicate structure as the thymus, injection or inflation, unless effected with the greatest care, necessarily leads to error, and I am also satisfied, that many of the observed "reservoirs" in the thymus are only artificially produced; but, nevertheless, I am of opinion, that there really are thymus glands, containing, in life, a central cavity, because I have seen such cavities extending through the entire thymus or only through separate segments of it, and this in cases where no preparation of any kind or injection had been used. I look upon the occurrence of a narrow central canal, as the original and usual condition, but believe, that in certain cases it may be expanded by a more abundant formation of the secretion, and ultimately be converted into a large cavity.

$\S$ 183. Intimate structure of the thymus. - When a lobule is stripped of its investing coat, consisting of common connective tissue, with fine elastic fibres of the finer sort and frequently with scattered fat-cells, its external surface, fissured in correspondence with the separate gland-granules, comes into view. Under strong mag. nifying powers, there is now presented a very thin $(0.0005-0.001$ of a line), indistinctly fibrous, or almost homogeneous membrane, quite correctly described by Simon, which is continuous over an entire lobule or even the whole gland, and must be placed in the same category with the wall of the follicles in Peyer's patches, the tonsils, \&c. Within this envelop, between it and the cavity of the lobule, lies a grayishwhite, soft, easily lacerable substance, $\frac{1}{6}-\frac{1}{3}$ of a line thick, which, when examined microscopically, appears to consist of nothing but free nuclei and minute cells, and on this account has, by agreement of all observers, hitherto been regarded as the secretion of the supposed glandvesicles. But this substance cannot be washed away, which would have been the case had it lain loosely in the space enclosed by the delicate membrane; on the contrary, it exhibits a considerable degree of toughness and resistance. When examined more closely, it is, by degrees,

FIG. 244.-Transverse section through the summit of an injected lobule of the thymus in a Child, magnified 30 diam.: $a$, membrane of the lobule; $b$, membrane of the gland-granules; $c$, cavity of the lobule from which the larger vessels branch out into the granules, on the surface of which they terminate, occasionally forming loops. 
apparent that other elements, to some extent of quite an unexpected kind, enter into its composition; as for instance, bloodvessels, and also a smaller quantity of a fibrous substance of the nature of connective tissue, so that a structure not unlike that of the contents of the Peyerian follicles is presented.

Of the elements of the walls of the thymus-lobules, the vesicular, together with a small quantity of a connecting fluid, constitute the main bulk. Among these, free nuclei $0.002-0.005$ of a line in size, of a round slightly flattened shape, with homogeneous, clear contents, which become troubled and granular in soda and acetic acid and with or without a nucleolus, are always present in very great numbers. Secondly, as I find, agreeing with Ecker but in opposition to Simon, cells also are never wanting, though existing of very various sizes, from 0.004 to 0.01 of a line, and, though varying in number as well, still much less numerous than the nuclei. Their nuclei are for the most part simple and distinct, and the contents pale or with scattered fat-granules, or, and this Ecker says that he has noticed after the complete development of the organ, they are without nuclei and entirely filled with fat. In the midst of these elements run numerous bloodvessels of larger and smaller size. The main trunks running on the outer aspect and close upon the central cavity in the longitudinal direction of the organ, give off a large number of branches to the central cavity, which, penetrating its walls, reach its internal surface, and there ramify minutely in a delicate pellicle, composed of connective tissue, with which it is lined, anastomosing and also forming tolerably close capillary plexuses. From these arterial plexuses, at every point where the lobules open, numerous vessels arise and enter them, taking their course in the innermost portion of the thick boundary wall, and then ramify towards the exterior in the separate gland-granules, so as to constitute a capillary plexus entirely filling them, composed of vessels $0.003-0.005$ of a line in diameter, and with meshes of 0.01-0.02 of a line (Fig. 244). The distribution of these vessels, in Man, is so limited to the interior of the gland-granules, that, even when these have been most completely displayed, not a single vessel is found on the outer aspect of their structureless investing membrane; on the contrary they are all seen terminating in loops close upon it. Besides these bloodvessels a small quantity of connective tissue also appears to enter into the formation of the thick walls of the glandular lobes; at all events, in their innermost portions, where the larger vessels are situated, we find, often with tolerable distinctness, a membrane supporting them, analogous to that lining the central cavity. In other cases, hewever, and especially in animals, an internal limitary membrane of this kind cannot be demonstrated, and the cavities of the lobules are bounded immediately by the granular substance connecting the ressels, between which, only some delicate indications of fibres are 
apparent. In no case does there exist any epithelium lining the cavities and consequently, the comparison of the innermost part of their wall with a mucous membrane is untenable.

The common cavity or central canal of the thymus, presents the same structure as the lobules, except that externally there is a thicker fibrous layer, and internally a less thick granular stratum, with rather larger vessels. In a fully developed thymus it, as well as all the secondary cavities, contains a grayish-white or milky, faintly acid fuid, often in large quantity, in which, together with a clear fluid abounding in albumen, numerous nuclei, isolated cells and, under certain circumstances, also concentrical corpuscles (vide infra) are contained. The lymphatics of the thymus are numerous and nerves may be readily demonstrated upon the arteries, although they cannot be traced as far as their terminations.

Besides the above-described normal elements, there occur, especially at the period of involution of the organ, other peculiar spherical structures, which, with Ecker, I would term the concentric corpuscles of the thymus. They present very various forms, which, however, it seems to me may conveniently be reduced to two; viz. 1 , simple, $0.006-0.01$ of a line in size, with a thick concentrically striated membrane and a granular substance in the interior, sometimes appearing like a nucleus, sometimes as a cell; and 2, compound, as much as 0.04 or even 0.08 of a line in size, and consisting of several simple corpuscles, surrounded by a common laminated envelop. These bodies, which were first noticed by Hassall and Virchow, and were further investigated by Ecker and Bruch, it appears to me arise not from a direct metamorphosis of the nucle $i$ and cells in the wall of the glandular lobule, but from the successive deposition of an amorphous material around them; and consequently that they are analogous in their mode of formation to the corpuscula amylacea of the brain, the prostatic concretions, \&c. The laminated portion consists of a substance certainly not of a fatty nature which offers considerable resistance to alkalies, approaching the colloid substance and the substance of the prostatic concretions and corpuscula amylacea, being probably formed from a change in the albumen in the glandular walls. The situation of these concentric corpuscles is external to the secretion of the thymus, and principally in the innermost part of the glandular parietes, where the larger vessels occur.*

$\S 184$. Development of the Thymus.-According to Remak, the thymus of the Chicken originates in the separation, by constriction, of the borders of the last two (third and fourth) branchial fissures, which are lined

* [Whatever may be the true nature of the Hassallian corpuscles, they do not seem to be an essential element of the thymus or its secretion, as they are not found in that body in Fishes (Skate, Ray, Sturgeon, Zeus faber); although present in the Reptilia. Leydig, " Anat. histol. Untersuchung. u. Fische und Reptilien," pp. 21 and 66.-Trs.] 
by the intestinal epithelium; and at the period when the last three aortic arches become detached from the walls of the pharynx, these follow them, and eventually lie as two elongated sacculi, on each side, between them.

In the earliest state observed in Mammalia, e. g., in the foetal Calf, 1 line long, according to Bischoff the gland represents two delicate tracts of blastema, which descend from the larynx as far as the thorax and appear to be connected above with the thyroid body. Simon gives a similar description of the thymus in the foetal Calf and Swine, $\frac{3}{4}-1 \frac{1}{2}$ inches long, except that he makes no mention of any connection with the thyroid body, figuring the tract as a tube bounded by a delicate structureless membrane and filled with nuclei and a granular substance, which is further developed by becoming thicker and longer, whilst at the same time it pushes out, at first, simple and afterwards, more and more widely ramifying buds. Thus in fotal calves, $2 \frac{1}{2}-3$ inches long, wart-like and spherical, in part even shortly pedunculated offsets already existed, which subsequently increased in number, becoming produced, at first into two and four and afterwards, successively, into still more globular bodies, until ultimately the lobules were completed. In this way the primitive tube would be converted into the central cavity of the thymus and each offset of it, in course of time, into an entire lobule of the organ. In the human subject, as early as in the seventh week, I have seen the thymus lobate at the lower end and single above. In an embryo ten weeks old, the upper extremity was a delicate walled tube, $0.04-0.06$ of a line in diameter, filled with polygonal cells; the lower portion, $0 \cdot 16$ of a line thick, presented several rounded outgrowths, in part isolated, in part grouped, to the number of from two to five together. The thicker, inferior portion of the gland, was entirely covered with further developed lobules $0.08-0.1$ of a line in size, of which, again, more simple glandgranules, each with a structureless membrane and contained cells, were visible. In the twelfth week I found the thymus not much larger, but the horns broader, and, like the rest of the organ, covered with lobules $0 \cdot 12-0.24$ of a line in size. From this, although the first stages have not yet been observed in Man, there can be no doubt that the mode of development is the same as that observed by Simon in other Mammalia.

The later development of the thymus affords other interesting conditions. In the embryo it continues to grow slowly from the third month; in the sixth it extends as far as to the thyroid gland; and from and after the seventh month begins to contain a whitish secretion. After birth it is not stationary, as was formerly believed, but usually continues to grow until the second year, and, indeed, at first very considerably. After that period its growth ceases, though it still usually remains for some time longer unchanged, until ultimately it becomes atrophied, and finally disappears. The period at which these changes 
take place varies very much. Simon places the commencement of the atrophy between the eighth to the twelfth years, a statement which, from my own observations, and in accordance with those of Ecker, I cannot consider as universally correct; because, up to the twentieth year, the thymus is frequently met with in a good state of nutrition, distended with fluid, without any fatty metamorphosis, and presenting the same structure as in children. It is still more difficult to assign the time of its complete disappearance, for which no determinate age can be indicated, although it is true that the thymus is usually not to be found after the fortieth year. The disappearance takes place in consequence of a gradual absorption, with a simultaneous development of fat in the gland-granules, and of fat-cells in the interlobular connective tissue. At the same time, also, the concentric corpuscles multiply more and more, and ultimately, according to Ecker, even connective tissue is developed in the lobules, the glandular structure being completely lost.

The investigation of the thymus is not easy. I recommend, in the first place, boiled preparations, which of themselves are very well adapted for the investigation of the connection of the lobes with the central canal, and the cavities in the lobules, and when hardened in spirit are convenient for the making of fine sections. Besides this, the hardening of recent preparations in alcohol, pyroligneous and chromic acid, and the boiling of them in acetic acid, are advisable. The thymus, also, of small Mammalia, which is membranous at the edges, is well adapted to afford a general knowledge. But, moreover, and above all, are injections of the human thymus indispensably requisite, without which no satisfactory conclusions can be arrived at.

Literature.-S. C. Lucae, "Anat. Untersuchung. d. Thymus im Menschen und in Thieren," Frankfurt am M. 1811 u. 12, 4to, und "Anat. Bemerk. über die Divertikel am Darm. u. die Höhlen des Thymus," Nurnb. 1813, 4to ; F. C. Haugsted, "Thymi in hom. et per ser. animal descrip.," Hafn., 1832, 8vo; A. Cooper, "Anatomy of the Thymus Gland," Lond., 1832, 4to ; J. Simon, "A Physiological Essay on the Thymus Gland," Lond., 1845, 4to ; Ecker, Art. "Blutgefässdrüsen," in Wagner's "Handw. der Phys." III.

\section{OF THE URINARY ORGANS.}

$\S 185$. The urinary organs consist of the two kidneys-true glands, having a tubular structure, which secrete the urine-and of the secretory urinary passages, the ureters, bladder, and urethra.

$\S 186$. In the kidneys, are to be distinguished the coats and the secre- 
ting parenchyma. To the former belong the adipose capsule, as it is termed (capsula s. tunica adiposa), constituted of lax connective tissue abounding in fat cells, scarcely deserving the name of a special membrane, and the fibrous tunic (tunica propria s. albuginea), a thin but strong coat, of a whitish color, composed of common connective tissue and numerous fine elastic networks, which closely invests the kidney, and, at the hilus, is in apposition with the pelvis of the gland and the vessels, but does not penetrate into the interior of the organ.

The secreting parenchyma, which is abruptly defined from the fibrous membrane, consists, as seen by the naked eye, of two portions, the medullary and the cortical substance, the former of which constitutes 8-15, isolated, conical, masses converging towards the hilus, the pyramids of Malpighi; whilst the latter forms the entire cortical part of the organ, and moreover, sends processes between the separate pyramids which extend as far as the hilus, - the columnoe Bertini,-and is apparently continuous throughout the gland. Examined microscopically, however, the cortical substance is found to be divided into as many segments as there are pyramids, and the kidneys, therefore, may be regarded as made up of a certain number of large, though closely connected lobes.

§ 187. Composition of the Renal Substance.-Both portions of the kidneys consist, essentially, of the uriniferous canals (tubuli uriniferi), cylindrical tubules, measuring, on the average, $0.016-0.025$ of a line. They commence in each renal lobe or segment, in those portions of the pyramids which are surrounded by the calices renales, or on the renal papillæ, by, on the average, from 200 to 500 orifices, $0.024-0.1$ of a line wide, scattered over the surface of those processes; they traverse the pyramids in nearly a straight line and in close contiguity, whence they are termed in that situation, tubuli recti (or Belliniani) (Fig. $245 \mathrm{k}$ ). In this course, each of the straight canals divides repeatedly, most usually under very acute angles and at first with a considerable diminution of size, either into two (Fig. 245 l), or more rarely into three or four, so that, ultimately, a complete bundle of finer tubules is produced from them; and in this way the continued increase in breadth of the pyramids towards the exterior is accounted for. At the same time, towards the base of the pyramids, the connection of the ducts of Bellini is rendered less close, by the interpolation between them, at regular distances, of large vascular bundles (arterioloe and venuloe rectoe), and they become separated on all sides from each other, so that, in perpendicular sections, the pyramids (the papillce of course excepted) in the entire circumference appear to spread out into numerous small bundles or pencils - the pyramids of Ferrein of authors-but which, as sections across them show, are only to be regarded as separate, sharply-defined fasciculi. The tubuli uriniferi, even here, assume a slightly undulating 
course, but this becomes much more manifest in the cortical substance, where they constitute the convoluted uriniferous tubules (tubuli contorti s. corticales), which at first sight, appear to be inextricably and irregularly interwoven, each terminating, ultimately, as Bowman discovered in the year 1842 , in a vesicular, dilated extremity, $0.06-0.01$ of a line in diameter, containing a vascular plexus of a peculiar kind-the so-called Malpighian body. Upon more minute observation, however, it is easy to perceive the convoluted tubuli are arranged in columnar masses, $\frac{1}{3}-\frac{1}{6}$ of a line wide, extending through the entire thickness of the cortical substance and in close apposition, which, notwithstanding their incomplete limitation from each other, may nevertheless be designated fasciculi corticales, or lobuli renum (or the "pyramids of Ferrein" of the older anatomists). In these columns (Fig. 245), the tubuli uriniferi are disposed, in miniature, in the same way as in a renal lobe, so that in their interior, more especially at the periphery, convoluted canals may be distinguished. When the arrangement of these parts is accurately investigated, it is seen that the ducts of Bellini, entering a cortical lobule in a fascicular manner, at first run in perfectly straight lines (Fig. 245 o). Soon, however, some, and further on, more and more, of the canals are curved laterally (Fig. $245 \mathrm{~m}$ ), in order to reach, in a ser-

Fig. 245.

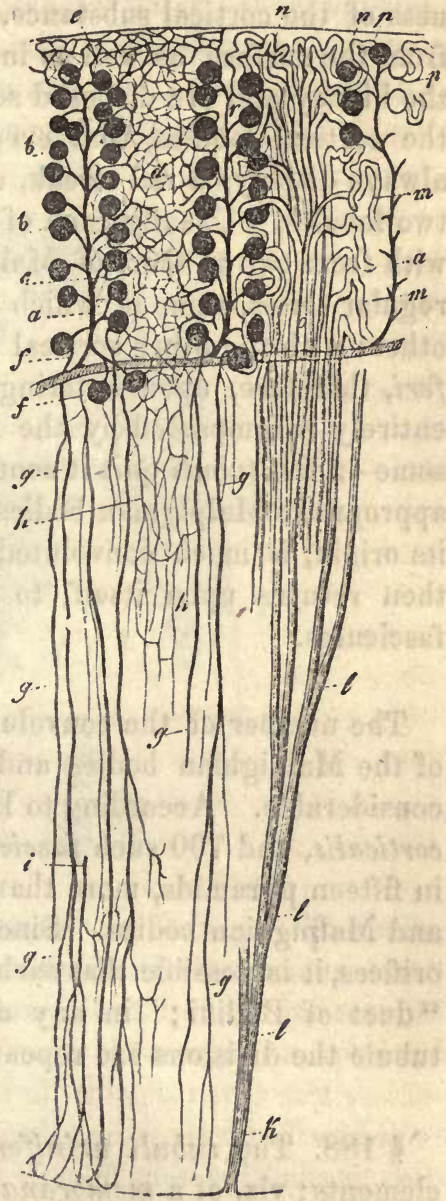

Fig. 245.- Vertical section through a portion of a pyranid and the cortical substance belonging to it, of an injected Rabbit's kidney. The figure is half-diagrammatic ; magnified 30 diam. The vessels are rspresented on the left side, and on the right the course of the tubuli uriniferi: $a$, arterice interlobulares, with the glomeruli Malpighiani, $b$, and their vasa efferentia : c, vasa efferentia ; $d$, cortical capillaries; e, vasa efferentia of the outermost corpuscles, proceeding to the superficial capillaries; $f$, vasa efferentia of the innermost glomeruli, continuous with the arteriola recta, $g \mathrm{~g} g ; h$, capillaries of the pyramids which are formed out of the latter; $i$, a venula recta, commencing at the papilla; $l$, commencement of a straight canal at the papilla; $l$, divisions of the same; $m$, convoluted tubules in the cortex, their whole course not shown; $n$, the same at the surface of the gland; 0 , their continuation in the straight tubules of the cortex; $p$, their connection with the Malpighian capsules. 
pentine course, the arterial twigs surrounding the cortical lobules; until, at last, the entire bundle of tubules is broken up, at some distance from the surface of the kidney (or of the centre of the columnce Bertini), into convoluted canaliculi. The Malpighian bodies (Fig. 245 b), from which the tubuli uriniferi arise, are found throughout the entire thickness of the cortical substance, from the pyramids to within $\frac{1}{5}$ of a line from the surface, as well as in the septa Bertini, down to the sinus of the kidney and are disposed so regularly and in such numbers, around the cortical lobules, that every transverse section through the cortex always displays a red streak, caused by these corpuscles, between each two lobules. Usually each of these streaks consists of a minute artery, with from two to four, of Malpighian bodies supported by it, but not in regular series, some of which stand more in relation with the one, and others with the other cortical bundle. Each fasciculus of tubuti uriniferi, therefore, upon entering the cortical substance, is, from the first entirely encompassed by the Malpighian bodies, and it is obvious that some of the tubuli quit it soon and others later, in order to reach their appropriate Malpighian bodies. For the rest, each cortical tubule, after its origin, is much convoluted, runs at first somewhat outwardly, and then returns upon itself, to join the straight tubules of the cortical fasciculus.

The number of the convoluted tubuli uriniferi corresponds with that of the Malpighian bodies and is consequently, in every instance, very considerable. According to Huschke, 200 tubuli exist in each fasciculus corticalis, and 700 such fasciculi in a pyramid; which calculation gives, in fifteen pyramids, more than two millions of commencements of tubuli and Malpighian bodies. Since each papilla has about 500 or even more orifices, it is possible that each cortical fasciculus proceeds from a single "duct of Bellini ;" in any case, it is evident that in every straight tubule the divisions are repeated at least ten times.*

'§ 188. The tubuli uriniferi, are everywhere composed of the same elements; viz. of a membrana propria, and a tessellated epithelium. The former is a perfectly structureless, transparent, thin $(0.0004-0.0008$ of a line), but proportionately strong and elastic coat, which, particularly in the straight tubules, may be very easily isolated for a considerable extent, when it is very prone to fall into folds, which often present the striated aspect of connective tissue. On the inner surface of this

* [It would scarcely be deduced, from what is said in the text, that the tubuli uriniferi, in the cortical part of the kidney, anastomose very freely and frequently, although the fact of their doing so has been long well known and often described. These anastomoses, however, and the general disposition of the tubules, are more particularly adverted to and well depicted by Toynbee (" Med. Chir. Transact.," 2d ser., vol. XI. p. 308, pl. 7).-Trs.] 
coat, which in its chemical characters is very closely allied to the sarcolemma (vid. §58), lies a single layer of polygonal, moderately thick cells, surrounding the cavity of the tubulus, which, from the readiness with which they alter, have given rise to many erroneous representations respecting the structure of the urinary ducts and their contents. For instance, in the usual mode of examination in water, they expand, owing to its absorption and become vesicularly distended, so that their polygonal form and regular arrangement are lost; the renal ducts, within the structureless membrane, appearing to be entirely filled with

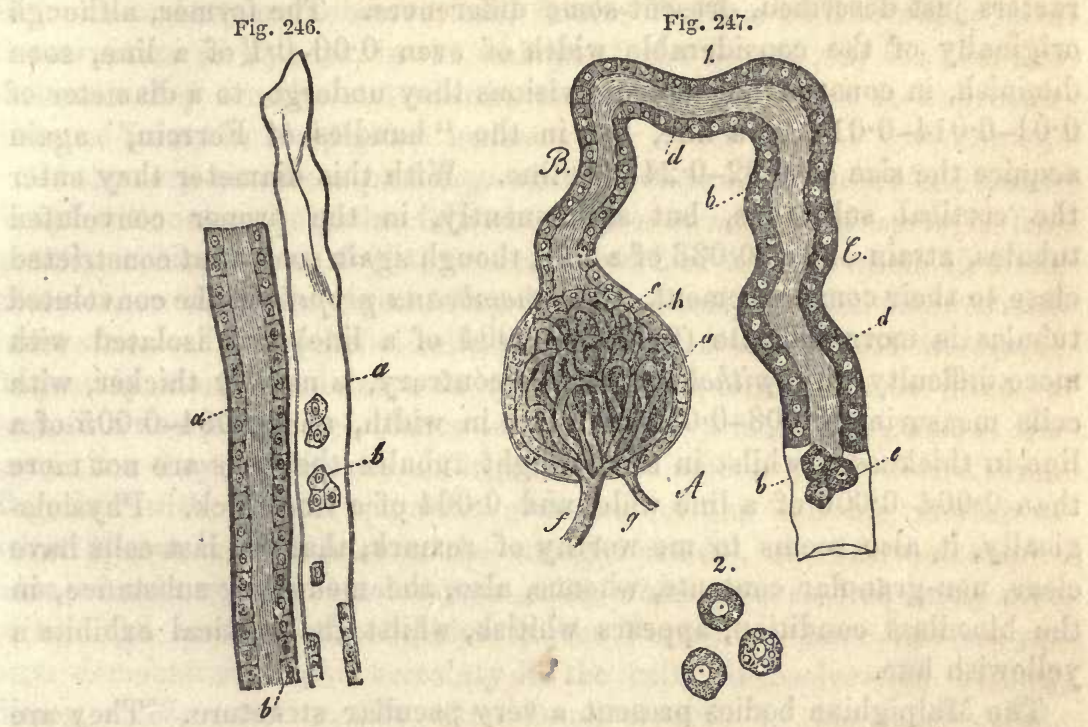

rounded larger cells and no longer to possess any cavity. The cells, also, frequently burst, in which case the tubuli contain nothing but a fine granular substance, with nuclei and clear albuminous drops escaped from the cells. In kidneys not quite fresh, these changes proceed spontaneously; and, therefore, it is, above all things, necessary to examine the organ as soon as possible after death and to avoid all additions capable of producing change. The contents of the epithelium cells are, besides round nuclei of the usual kind, a most usually, very finelygranular substance, which, on the addition of water, affords clear, lightyellowish drops, probably of albumen, but is not otherwise changed;

Fig. 246.-Two straight tubuli uriniferi of Man, one with perfect epithelium, the other half empty : $a$, membrana propria ; $b$, epithelium.

Fig. 247.-1, a Malpighian corpuscle, $\mathcal{A}$, with the tubulus uriniferus springing from it, $B, C$; Human; magnified 300 diam.; figure half-diagrammatic: $a$, membrane of the Malpighian body, continuous at $b$, with the membrana propria of the convoluted tubule; $c$, epithelium of the Malpighian body; $d$, that of the tubule; $e$, detached epithelinm; $f$, vas afferens; $g$, vas efferens; $h$, glomerulus Malpighianus. 2, three epithelial cells from the convoluted tubule, magnified 350 diam.; one with oil drops. 
under acetic acid the contents, together with the cell-membrane, are rendered pale, and soon dissolve; whilst the nuclei at the same time soon become pale, and finally, on the application of caustic alkalies, disappear in the same manner as the membrare. Besides these granules, which I do not hesitate to declare are a protein substance, and the albumen in solution in the contents, the cells very commonly contain some dark oil-drops, and more rarely one or another, exhibits granules of yellow pigment.

The straight and convoluted canals, together with the general characters just described, present some differences. The former, although originally of the considerable width of even $0.06-0.1$ of a line, soon diminish, in consequence of the divisions they undergo, to a diameter of $0.01-0.014-0.018$ of a line, but in the "bundles of Ferrein," again acquire the size of $0.02-0.24$ of a line. With this diameter they enter the cortical substance, but subsequently, in the proper convoluted tubules, attain one of 0.033 of a line, though again somewhat constricted close to their commencement. The membrana propria in the convoluted tubules is more delicate $(0.0003-0.0004$ of a line) and isolated with more difficulty, the epithelium, on the contrary, is usually thicker, with cells measuring $0.008-0.012$ of a line in width, and $0.004-0.005$ of a line in thickness; whilst in the straight tubules the cells are not more than 0.004-0.006 of a line wide, and 0.004 of a line thick. Physiologically, it also seems to me worthy of remark, that the last cells have clear, non-granular contents, whence, also, the medullary substance, in the bloodless condition, appears whitish, whilst the cortical exhibits a yellowish hue.

The Malpighian bodies present a very peculiar structure. They are to be regarded as appendages of the convoluted tubuli uriniferi, and contain imbedded in their epithelium, and, as it may be said, entirely filling their cavity, a compact, rounded vascular plexus-the glomerulus Malpighianus, the structure of which will be afterwards described. The same membrana propria, which surrounds the tubuli uriniferi, also somewhat thickened $(0.0005-0.0008$ of a line) invests these bodies (Fig. $247 \mathrm{a})$; and the epithelium is likewise continued into the capsules thus formed, only that its cells are smaller and less distinct, and invest the vascular coil on the side directed towards the canal of the emergent tubulus uriniferus. This latter, generally somewhat constricted (Fig. $247, B$ ), is inserted into the Malpighian capsule, most usually on the opposite side to the afferent and efferent vessels; but in accordance with what has been said, its cavity penetrates into the capsule, only to an inconsiderable extent; inasmuch as the latter is almost entirely occupied by the vessels and the epithelium surrounding them.*

* [With respect to the question of the cæcal termination, or commencement, as it might more properly be termed, of the tubuli uriniferi in the Malpighian capsules, there is an 
The ciliary motion discovered by Bowman in the neck of the Malpighian bodies of the Frog, and in the commencement of the tubuli uriniferi, with the direction of the stream towards the ureter, is readily confirmed, when the addition of water is avoided. It is absent, however, in Birds (Gerlach thinks he has seen it on one occasion in the Fowl) and other Mammalia, and was not noticed by me in the cases of two executed criminals, examined especially with respect to this point, whilst it is found in Serpents, in the Salamander, Triton, Bombinator, Bufo, and is very well marked in Fishes, and also, according to Remak's and my own observations, in the Wolffian bodies, which have the structure of kidneys, in the embryo of the Lizard; in the last two instances they are also met with in the uriniferous ducts at a greater distance from the Malpighian bodies.

Of the very numerous pathological degenerations of the tubuli uriniferi, I will notice only the following: The membrana propria is frequently thickened to 0.001 , or even 0.002 of a line, when it often presents, on the inner aspect, very delicate, closely approximated transverse strix. The epithelial cells, particularly in the cortical substance, frequently contain oil-drops in considerable quantity, so as often to present a deceptive resemblance to the cells of a fatty liver, and at the same time they are usually enlarged to a diameter of 0.02 of a line. Together with the oil, pigment granules (of the coloring matter of urine?) occur in them (also in the straight canals), whereas the concretions of uric acid and calcareous salts, which are so frequently met with in the canals of the tubules in the Vertebrata have not as yet been demonstrated with certainty in the cells themselves (in Fishes, Simon, "Thymus," p. 69, often found crystals in the renal cells). Colloid-like, bright yellow masses are frequent in the epithelial cells, which then most usually increase in size, dilate into slender cysts as

apparent discrepancy of opinion among anatomists. Bowman states that each tube commences in a Malpighian capsule; whilst Gerlach says that the capsules do not form the cxtremities of the uriniferous tubes, but are merely diverticula, which commnnicate by a small neck with the angle formed by the uriniferous tubes, winding throngh the cortical part of the kidneys; or, as the same thing is described by Leydig (1. c., p. 32), with respect to the kidney of the Sturgeon, two closely contignous, uriniferons tubules, are connected with the (Malpighian) capsule and continuous with it; in other words, the tubules join in a loop, at the apex of which is a globose diverticulum, in which the glomerulus is lodged; and according to him, the same arrangement obtains in the Reptilia. Now it seerns that this discrepancy admits of an easy explanation, for if we suppose the constricted neck of the diverticulum to be lengthened into a tube, we have at once the disposition described by Bowman, viz.: a tube commencing in a dilatation containing the glomerulus and afterwards anastomosing with another or with other tubules; and if the neck of the so-called diverticulum be very short, or, in other words, if the Malpighian capsule be sessile, we have the arrangement described by Gerlach, \&c. Mr. Toynbee's notion that the tubulus uriniferus inerely passes through the Malpighian capsule, forming a coil within it, appears to us to be wholly inadinissible; but many of the appearances depicted in his very carefully-executed figures (1.c.) would serve to support the opinion that the Malpighian body is more often sessile than it would seem to be from Bowman's account.-Trs.] 
much as $0.05-0.072$ of a line long, and finally, by bursting, empty themselves of the colloid substance, whence the latter is found free in the uriniferous ducts, and also in the urine. A development of the epithelial cells into other cysts, as is stated to take place by J. Simon, and also by Gildemeester (Tijdschr. d. Nederl. Maatsch. 1850) has not yet occurred to my observation, whilst I have noticed, as did Johnson, very distinctly, in an atrophied kidney, a partition of the convoluted tubules into closed cysts, to all appearance by a connective tissue developed between and constricting them. These cysts had the same structure as the tubules, and were either of the same diameter, or distended into vesicles, 0.1 of a line in width. The Malpighian bodies also may expand into cysts, in which, together with a clear fluid, the atrophied glomerulus is often visible on the wall. As abnormal contents, the tubuli uriniferi present: 1 , blood, most frequently in the commencement of the convoluted tubules, especially the superficial, often in such quantity as to produce bloody points as big as a pin's head, and visible to the naked eye, which were formerly erroneously regarded as distended Malpighian bodies; 2, fibrine, in cylindrical masses, corresponding to the cavity of the tubules; 3 , the abovementioned colloid-like substance; 4, concretions in the ducts of Bellini, consisting, in the adult, chiefly of carbonate and phosphate of lime ("Kalkinfarct"); in new-born infants, of uric acid-salts ("Harnsäureinfarct," Virchow), which giye the pyramids a brilliant gold-yellow color, and, if not exclusively, still usually occur only in children who have already respired (between the third and twentieth day after birth). In the later stages of "Bright's disease," many tubuli, which have lost their epithelium in consequence of the exudations poured out in them, become atrophied, and ultimately disappear altogether, whilst groups of others are seen, filled with a fatty, broken-up exudation, and dilated into minute nodosities (granulations, Christison).

$\S 189$. Vessels and Nerves.-The large renal artery divides, in the pelvis of the kidney, into a certain number of branches, which, after supplying the parts lying in the hilus, enter, above and below the renal veins, the cortical substance interposed between the pyramids (the columnoe Bertini). From this point they are continued, repeatedly dividing, close upon the boundary of the two renal substances, so that around each pyramid a delicate ramification, but without any anastomoses, usually afforded only by two large arteries, is formed. From this ramification, on the side looking towards the cortical substance, there arise, with great regularity, and for the most part at right angles, smaller arteries, which after a few or more repeated divisions give off fine twigs, $0.06-0.1$ of a line in diameter, which run outwardly in a straight course between the cortical fasciculi or lobules, and are most 
fitly termed arterice interlobulares (Fig. $245 a$ ). It is these twigs which support the Malpighian bodies, and, with the exception of some branches to the coats of the organ, they terminate exclusively in the formation of their vascular coils. In fact, each interlobular artery gives off, in its whole length, on two, three, or four sides, a great number of fine twigs possessing the structure of arteries and $0.008-0.02$ of a line in diameter,

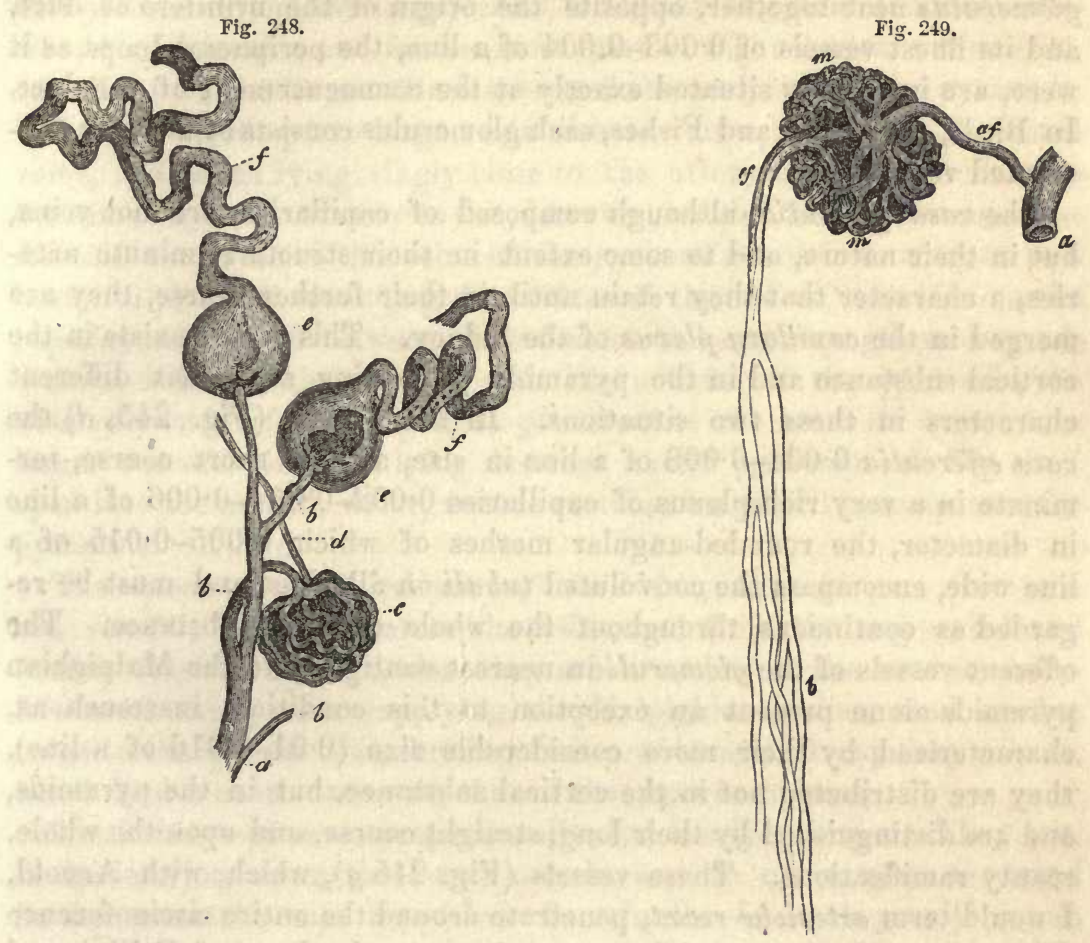

which, after running a short distance, either directly or after dividing once, penetrate the tunic of the Malpighian body, becoming the vasa afferentia of its vascular coil. Each of these (Fig. 247, 248) consists of a close convolution of fine vessels $0.004-0.008$ of a line in diameter, having the usual structure of capillaries (structureless coat and nuclei) and possesses, besides the afferent artery, an efferent vessel, the vas efferens. The mode in which these two vessels are connected is not that which usually obtains in arteries and veins, but corresponds with

Fia. 248.-From the Human kidney, after Bowman: $a$, extremity of an interlobular artery ; $b$, afferent arteries; $c$, naked glomerulus; $d$, efferent vessel; $e$, glomeruli, surrounded by the Malpighian capsules; $f$, tubuli uriniferi springing from them.-Magnified 45 diameters.

Fia. 249.-Glomerulus from the innermost part of the cortex of the kidney of the Horse, after Bowman: a, arter. interlobularis; af, vas afferens; $m$, glomerulus; ef, vas efferens $s$. arteriola recta; $b$, divisions of the same in the medullary substance.-Magnified 70 diameters. 
the arrangement presented in the bipolar retia mirabilia, as they are termed; the vas afferens, immediately after its entrance into the coil, dividing into 5-8 branches and each of these into a bundle of capillaries, which are much convoluted and interlaced, without anastomosing, and ultimately, in the same way as that in which they were formed, reunite into a single trunk. Usually the two main vessels enter and quit the glomerulus near together, opposite the origin of the urinifero us duct, and its finest vessels of $0.003-0.004$ of a line, the peripheral loops as it were, are invariably situated exactly at the commencement of the duct. In Birds, Amphibia, and Fishes, each glomerulus consists of a single convoluted vessel.

The vasa efferentia, although composed of capillaries, are not reins, but in their nature, and to some extent in their structure, minute arteries, a character that they retain until, in their further course, they are merged in the capillary plexus of the kidney. This plexus exists in the cortical substance and in the pyramids, presenting somewhat different characters in these two situations. In the former (Fig. 245, d) the vasa efferentia $0.004-0.008$ of a line in size, after a short course, terminate in a very rich plexus of capillaries $0.002-0.004-0.006$ of a line in diameter, the rounded-angular meshes of which, 0.005-0.015 of a line wide, encompass the convoluted tubuti on all sides and must be regarded as continuous throughout the whole cortical substance. The efferent vessels of the glomeruli in nearest contiguity to the Malpighian pyramids alone present an exception to this condition, inasmuch as, characterized by their more considerable size $(0.01-0.016$ of a line), they are distributed not in the cortical substance, but in the pyramids, and are distinguished by their long, straight course, and upon the whole, scanty ramifications. These ressels (Fig. $215 \mathrm{~g}$ ), which, with Arnold, I would term arterioloe reccet, penetrate around the entire circumference of the pyramids, in a straight course, between the ducts of Bellini, and descend, repeatedly dividing at acute angles, and gradually attenuated to the diameter of $0.004-0.01$ of a line, towards the papilloe, in which, as in the interior of the medullary substance (in the latter situation, either by their extremities or by twigs given off at right angles), they are continuous with the capillaries in those regions, measuring from 0.002 to 0.004 of a line. These capillaries are very importantly distinguished from those of the cortical substance by their less number and the elongated figure of the meshes of the plexus which they form, although at the boundary of the pyramids the two sets are continuous with each other. The renal veins commence in two situations, viz., at the surface of the organ and at the apices of the papillæ. In that situation, minute venous radicles collect together from the outermost portions of the capillary plexus of the cortical substance; which either regularly surround each cortical lobule, and between them unite in a stellate 
manner (stellulce Verheynii) into somewhat larger roots, or, extending over several or numerous lobules, collect into larger trunks. These two sets of veins, forming the vence interlobulares, then penetrate more deeply in company with the arteries of the same name, between the cortical fasciculi, where they are enlarged by the accession of numerous other venous radicles from the interior of the cortical substance, and proceed to join the larger veins, either directly or united into somewhat larger trunks, and for the most part at right angles. These lie, together with the larger arteries, around the periphery of the pyramids, ultimately opening into large veins without valves, as are all the renal veins, and which lying singly close to the arteries, quit the kidney in the same way. Previously to this, however, besides those of the columnce Bertini, they receive the veins of the pyramids, which commence in a beautiful plexus surrounding the orifices of the tubuli uriniferi on the papilloe, and ascend between the tubuli recti, being strengthened by additional radicles; these united with the arteries of the pyramids, the vasa efferentia of the innermost glomeruli or the arterioloe rectoe, into larger vascular bundles, lying between the "pyramids of Ferrein," open into the arched, wider, venous ramification which encompasses the pyramids.

The vessels of the membranes of the kidney, arise in part from the art. renalis, before it enters the hilus, and from the suprarenal and lumbar arteries, in part, also, they are branches of the arterice interlobulares, which, after supplying the Malpighian bodies, send on fine twigs to the fibrous coat, forming in it a wide-meshed capillary plexus, which is also continuous with that of the capsula adiposa.

The kidneys present, proportionally, but few lymphatics. They run, in the interior, along the larger vessels, and do not appear to extend further than the vasa interlobularia. In the hilus, they unite into a few small trunks, which also receive lymphatic vessels from the pelvis of the kidney and then open into the lumbar glands. Superficial lymphatics, which have been described by the older anatomists (Nuck, Cruikshank, Mascagni, \&c.), I have as yet not seen, except in the capsula adiposa, but I am unwilling positively to deny their existence.

The renal nerves, from the cæliac plexus of the sympathetic are tolerably numerous, form a plexus around the arteries, continue to present a few ganglia in the hilus, and may be traced, in company with the vessels, as far as the interlobular arteries. Where and how they terminate is unknown.*

* [With respect to the termination of the nerves in the kidney, Mr. Toynbee (1.c., p. 805) makes the remarkable and very important observation, that "the filaments end by becoming continuous with the parenchyma of the organ, precisely in the same way," be goes on to say, "as he has observed those in the tail of a Tadpole to become directly continuous with the radiating fibres of stellated corpuscles, and the filaments from the corpuscles to communicate with each other."-Trs.] 
All these vessels and nerves are supported by a connective tissue, which, at the same time, serves as a stroma for the secreting elements, and is much more developed in the medullary substance than in the cortical. At the surface of the gland, it is condensed into a membrane, often very distinct, $0.01-0.02$ of a line thick, which is but loosely connected with the fibrous tunic, partly supports the superficial capillary plexus, and is continuous with the internal stroma by numerous delicate processes.

Fig. 250.

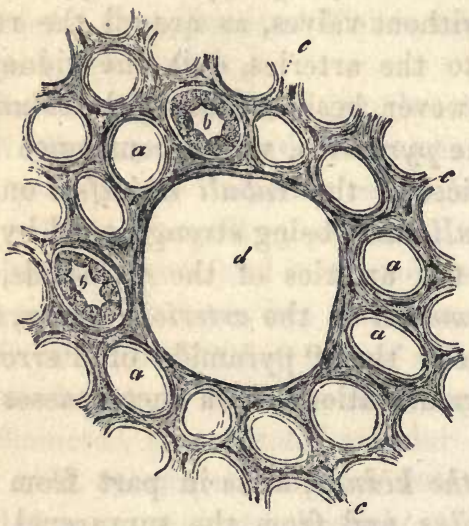

Fig. 251.
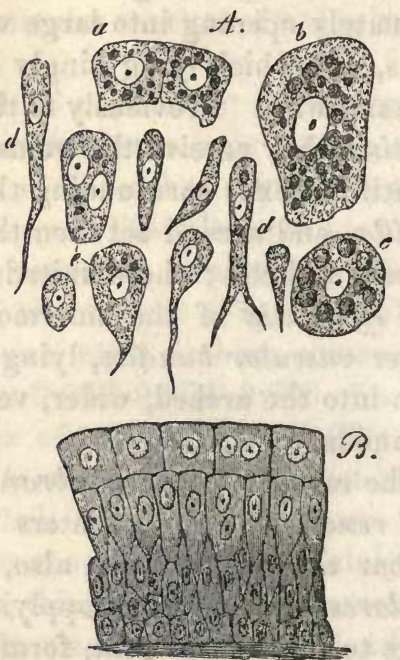

In inflammations and exudations, the stroma is frequently so much condensed as to be apparent on the most superficial inspection, or even, more or less to compress the tubuli uriniferi. The additional elements consist chiefly of a fibrinous exudation, presenting various stages of transition into connective tissue, partly also of such forms as are peculiar to immature normal connective tissue, as fusiform cells, \&c. In the case of the Malpighian bodies, these new formations present the form of concentric, often very thick deposits, which constrict the afferent and efferent vessels, thus inducing atrophy of the glomerulus, and very essentially and prejudicially affecting the secretion of urine. In other cases the increase of the stroma is only apparent, and depends upon the atrophy of the secreting elements.

FIG. 250.-Transverse section through some straight cortical tubules, magnified 350 diameters; from Man : a, transverse section of tubuli urinifcri, the membrana propria only of which rernains; $b$, similar tubules, with the epithelium still remaining; $c$, stroma of connective tissue, with elongated nuclei; $d$, cavity in which a Malpighian corpuscle was contained.

FIG. 251.-Epithelium of the pelvis of the Human kidney, magnified 350 diameters: $A$, isolated cells; $B$, the same in situ: $a$, small; $b$, large tessellated cells; $c$, the same with nucleated corpuscles in the interior; $d$, cylindrical and conical cells from the deeper layers; $e$, transitional forms. 
\$190. Excretory urinary passages.-The ureters, the pelvis, and calices of the kidney, are all composed of an external fibrous membrane, a smooth muscular layer, and a mucous membrane. The first, formed of common connective tissue and elastic fibres, chiefly of the finer kind, is continuous, at the point where the calices surround the papilloe, with the fibrous tunic of the kidney. The muscular layer in the ureters is very distinct, with external, longitudinal, and internal transverse fibres, to which, towards the bladder, internal longitudinal fibres also are superadded. In the pelvis of the kidney the two muscular layers are quite as thick as in the ureter, whilst in the calices they become thinner and thinner, ceasing where the latter are inserted into the papilla. The mucous membrane of all these parts is thin, tolerably vascular, without glands or papilloe, and becoming very much thinner $(0.005-0.01$ of a line, without epithelium), is also continued upon the renal papillæ, being likewise connected with their interior stroma. Its epithelium, $0.02-0.04$ of a line thick, is laminated and characterized by the variety of form and size of its elements, of which the most deeply-seated cells are rounded and small, those in the middle cylindrical or conical, $0.01-$ 0.02 of a line in length, and the superficial, rounded-polygonal scales $0.006-0.04$ of a line in size, or more flattened, and reaching a diameter of 0.02 of a line. The frequent occurrence of two nucle $i$ in these cells is a striking fact, as well as of clear, darkish-colored round granules $0.001-0.002$ of a line in size, which often almost assume the aspect of nuclei.

The urinary bladder, besides its peritoneal investment, possesses the same membrane as the ureters. The muscular coat presents, externally, the well-known longitudinal fibrous layer (detrusor urinoe), with parallel bundles, from which isolated fibres are continued upon the urachus; beneath these is a plexiform arrangement of oblique and transverse, stronger and slender fasciculi, interwoven into a true plexus, which do not completely cover the entire mucous membrane, and, at the neck of the bladder, constitute a strong continuous circular fibrous layer (sphincter vesicce). The corpus trigonum, in the fundus of the bladder, is a thick layer of whitish-yellow fibres, lying immediately beneath the mucous membrane, continuous with the longitudinal muscular fibres of the ureters passing through the muscular coat of the bladder, and contains chiefly longitudinal, but also some transverse fine elastic elements, connective tissue, and smooth muscular fibres. The mucous membrane, pale, smooth, and tolerably thick, except where the corpus trigonum is situated, presents an abundant submucous layer, and consequently, when the bladder contracts, is thrown into numerous folds. It presents no villi, is tolerably rich in vessels, especially at the fundus and neck, less so in nerves, which, however, especially in those two situations where they are more abundant, may be recognized as dark-bordered, fine and 
medium-sized fibres, and is covered with a laminated epithelium $0.03-$ 0.05 of a line thick, whose deeper elements are usually fusiform, conical or cylindrical, the more superficial, rounded-polygonal, or flattened. They exhibit the same want of uniformity in size as those of the pelvis of the kidney, to which irregularity the numerous depressions of various depths on the under surface of the uppermost cells, for the reception of the ends of the deeper, elongated cells, much contribute; peculiar stellate and dentate forms being thence produced. In the neck of the bladder and towards the fundus, there occur minute glands, in the form of simple pyriform follicles, or small aggregations of these (simple racemose glands). These glands, $0.04-0.24$ of a line in size, have orifices of $0.02-0.05$ of a line, a cylindrical epithelium, and contain a clear mucus. In pathological conditions, as Virchow informs me, they are occasionally enlarged and filled with whitish mucous plugs.

The male urethra will be described with the sexual organs. That of the female presents a reddish mucous membrane with numerous vessels, especially in the form of much-developed venous plexuses in the submucous tissue (which Kobelt, without any reason, has described as a corpus spongiosum), and a squamous epithelium, the deeper-seated cells of which are elongated, as in the bladder. There is an external muscular tunic united with the mucous membrane, consisting of a thin layer of longitudinal and transverse smooth muscles, intermixed with much connective tissue and elastic fibres, and of the thick substance of the musculus urethralis, the direction of the fibres in which is chiefly transverse. A certain number of larger and smaller racemose mucous glandules ("glands of Littre"), resembling in their structure those of the bladder, except that they are usually somewhat larger and more closely placed, pour their secretion into the urethra. Occasionally these glands occur of larger size (as much as 2 lines), and prominent, containing a colloid-like material in the distended vesicles.

\$ 191. Physiological remarks. Development of the urinary organs. -According to Remak, the kidneys in the Chicken are formed as two protrusions of the intestine, in the constitution of which the epithelial and the fibrous layers both take part, and, like the lungs, grow by the ramification of their epithelial tube, and the augmentation in bulk of the fibrous layer (Unters. z. Entw. d. Wirbelth. Tab. II., fig. 83-85). In the Mammalia, the earliest stage of the development of the kidneys has not yet been observed; but what we do know from the labors of Rathke, J. Müller, Valentin, and Bischoff, with respect to its subsequent conditions, perfectly accords with the statements of Remak, except that, in this case, the glandular canals appear to be developed upon the type of the salivary glands, and are not hollow from the commencement. The rudimentary kidneys in the Mammalia, at first, 
present nothing but the pelvis and a certain number of clavate hollow protrusions continuous with it-the calices. From each of the latter is subsequently formed, by continued budding, a bundle of tubuli uriniferi, each of which bundles ultimately constitutes a Malpighian pyramid and the cortical substance appertaining to it; whilst, at the same time, the kidney expands into a corresponding number of lobes. The tubuli uriniferi are at first solid, composed solely of cells, and without any membrana propria. In the course of development the latter arises, probably from a plasma afforded by the cells, and the cavity of the canal is formed, in consequence, it may be supposed, of the connection of a fluid between the cells; simultaneously with which the tubules begin to grow rapidly in length, and to become convoluted. The Malpighian bodies are originally nothing more than solid, clavate, thickened extremities of the rudimentary tubuli uriniferi. The interior cells of these pyriform or rounded bodies, subsequently become capillaries, which are continuous at two points, with the vessels outside, whilst the most exterior form the epithelium, which joins that of the tubulus uriniferus, and, like it, is invested with a membrana propria, which is, of course, deficient where the afferent and efferent vessels enter and emerge, at which point it may be said to be perforated. In the newborn child, according to Harting, the renal canals are three times more slender than in the adult, whence, as the kidney of the latter is only twice the size of that of the child, it is obvious that, at any rate after birth, no tubules are formed.

With respect to the physiological relations, I would merely offer the following remarks. There can be no question that the peculiar vascular conditions in the kidney, in accordance with which the blood circulates in special coils projecting into the commencement of the tubuli uriniferi, before it passes into the proper capillary plexus of the organ, are most intimately connected with the secretion of a large proportion of water in the urine. The hindrance to the flow of the blood gives rise to a considerable lateral pressure in the glomeruli, and a large quantity of blood-plasma is forced through the thin opposing membrane (the walls of the capillaries and the epithelium). Since all the elements of the plasma are not found in the urine, and those that are, present in it totally different proportions to those in which they exist in the blood, it is obvious that the membrane in question does not act simply as a filter, but also, for reasons at present unknown, retains certain substances (protein-compounds, fat), whilst it allows others (urea, \&c.). to pass through with peculiar facility. In this way there is formed in the commencements of the tubuli uriniferi, probably a very diluted urine, which afterwards, as it flows towards the pelvis of the kidney, reciprocally acts and is acted upon by the blood circulating around the tubuli uriniferi, receiving additional substances from it (perhaps more urea), but 
also yielding up certain of its own constituents (water and salts), and thus, at last, becomes true urine.

As regards the chemical composition of the kidney, we know very little. Frerichs (1. c. p. 42) found, in a healthy kidney, 16.30-18: solid matter, $72-73.70$ water. Of the former, the fat amounted to $0 \cdot 63-0 \cdot 1 \frac{\mathrm{n}}{0}$, although, according to $O$ wen Rees, it may amount to 1.86 ; the greater portion, however, is probably albumen, with regard to which, Ludwig especially has shown, that it exists in large quantity in the kidney, a fact that, from the micro-chemical characters of the epithelial cells of the tubuli uriniferi, cannot be wondered at.

In the higher animals, the secretion of the urine takes place without any formation or dissolution of cells, and consequently the normal urine just evacuated contains no morphological elements. It is only occasionally that epithelial cells from the urinary passages, especially from the bladder and urethra, occur in it ; but we almost always find mucus from the same localities, forming clouds or a light sediment, occasionally with mucous corpuscles; and lastly, spermatic filaments after emissions. In inflammations, hemorrhages, exudations, fatty kidney, we find pus corpuscles, oil drops, blood globules, blood, and fibrinous coagula, moulded in the tubuli uriniferi, in the form of cylindrical casts, and epithelium from the tubuiti, isolated or in continuous strings or tubes. Sedimentary deposits of the salts of the urine are readily formed as the products of decomposition. All normal urine without sediment, at a mean temperature, undergoes an acid fermentation, under the influence of the mucus contained in it; and whilst fermentation and filamentary fungi are developed, forms, from the decomposition of the urinary coloring matter, lactic or acetic acid, in consequence of which uric acid is set free from its compounds and deposited, in the form of rhombic or prismatic crystals, colored yellow or reddish by the coloring matter of the urine. Sooner or later the acid disappears, and from the decomposition of the urea, perhaps also of the coloring matter, the urine becomes ammoniacal and alkaline, with large colorless pyramidal prisms, or needles grouped in a stellate fashion and soluble in acetic acid, of the triple phosphate of magnesia and ammonia, which, intermixed with numerous infusoria (vibriones and monades) form a superficial pellicle, and with granules of urate of ammonia, and also, it may be, of carbonate of lime, a white sediment. Under conditions not as yet known, and rarely, hexahedral prisms of cystin appear in the urine; more frequently, after the use of drinks containing carbonic acid, and also in pregnant women, we find the octohedrons of oxalate of lime insoluble in acetic acid. If the quantity of uric acid be augmented, as after the inordinate use of nitrogenous food with deficient exercise, in impaired digestion, fevers, \&c. a more or less abundant yellowish precipitate of urate of soda, in the form of 
isolated or aggregated granules, is formed as soon as the urine cools, and becomes redissolved when it is again warmed. If, under these circumstances, the acid fermentation is set up, very considerable sediments of colored crystals of uric acid (brickdust sediment) are often thrown down. In injuries of the bladder, the urine frequently becomes alkalescent with great rapidity, when the above-mentioned crystals of triple phosphate at once make their appearance; they are also very frequent in pregnancy; and when assuming the pellicular form above described, were at one time regarded as a peculiar substance (Kiesteine).

The occurrence of albumen, fibrin, and fat, within the tubuli uriniferi, is, in my opinion, easily explained, upon the supposition, that in such cases the circulation is obstructed, and an increased secretion of the elements of the blood takes place in the Malpighian bodies and tubuli uriniferi, in consequence of which the epithelium of those parts which, as is well known, is found in these cases in considerable quantity in the urine, is washed away, whence, of course, any further hindrance to the continued passage of the above substances is removed. A permeation of fibrin through the epithelium is also conceivable, just as much, for instance, as upon the mucous membrane of the respiratory organs, although I do not believe that an increased pressure of such a kind as to induce a transudation of fibrin could fail to affect the delicate epithelium. When the epithelium is once removed, it becomes an important question whether it is quickly restored; and it appears to me, that the frequent occurrence of small quantities of albuminous matter in the urine often depends simply upon local deficiencies of the epithelium caused in one way or another.

Investigation of the kidney. - The tubuli uriniferi are readily isolated when the tissue of the organ is teased out; the epithelium, membrana propria, and canal being distinctly recognizable if blood-serum, or a solution of albumen be employed to moisten it. Together with entire tubules, there will be found in every preparation numerous epithelial cells, separate, or in groups, or even, as especially in the pyramids, in the form of long continuous tubules. The latter often present a peculiar aspect, for the most part collapsing, exhibiting somewhat flattened cells, and resembling vessels. Equally frequent are longer or shorter tubes of the membrana propria, which, when much plicated, cannot always at once be recognized. In the examination of the pyramids, the extremely numerous vessels must not be confounded with the "ducts of Bellini," or the epithelial casts that have escaped from them. The connection of the tubuli uriniferi with the Malpighian bodies is easily discerned in the kidney of the Frog and of Fishes, upon careful teasing out of the structure; but in Mammalia, also, it will rarely be 
missed in fine, bardened sections, and especially in injected preparations. The glomeruli themselves may frequently be recognized when naturally injected, and still better when artificially filled, which we may very readily succeed in doing with any fine material thrown in by the arteries. Similar injections, also, when successful, fill the whole capillary plexus of the cortical substance and of the pyramids; when this portion of the circulatory apparatus, especially in perpendicular sections, may be very satisfactorily made out. For the same purpose, kidneys injected from the veins are employed, in which not only the capillary plexus, but the glomeruli are filled ;* and for the study of the vasa efferentia, preparations not completely filled from the arteries are suitable. The course of the tubuli uriniferi should be studied in fine sections of kidneys hardened by immersion in alcohol, by boiling in dilute nitric acid and drying (Wittich), or by chromic acid, and rendered transparent by acetic acid; or in sections, made with the doublebladed knife, of recent, as well as of injected kidneys, in which the most important conditions, even the divisions of the "ducts of Bellini," may be observed. Nevertheless, it is always expedient to inject the tubuli uriniferi; and for this purpose, among the Mammalia, the Horse appears to be best adapted. This filling may happen, in consequence of accidental extravasation into the Malpighian capsules; in the injection of the arteries by this means, however, rarely more than the convoluted tubuli are filled; or it may be effected by injection from the ureter with the aid of the air-pump (Huschke, Isis, 1826), or the pelvis of the kidney being kept filled, if an endeavor be made by the kneading of it with the hand, to force the material into the "ducts of Bellini," and beyond them (Cayla). By means of a very fine canula, the individual tubuli may also be injected directly from the papillce.

Literature.-M. Malpighi, "De Renibus, in Exercit. de visc. struct.;" Al. Schumlansky, "Diss. de structurâ renum," c. tab. Argentor. 1782, 4 to; W. Bowman, "On the structure and use of the Malpighian Bodies of the Kidney," in "Phil. Trans.," 1842. I. p. 57; C. Ludwig, "Beiträge zur Lehre vom Mechanismus der Harnsecretion," Marburg, 1843; and Art. "Niere," in Wagner's "Handw. d. Physiolog.," II. p. 628; J. Gerlach, in "Müller's Archiv.," 1845 and 1848; Kölliker, in "Müll. Archiv.," 1845; Remak, in "Froriep's N. Notiz.," No. 786, 1845, p. 308 ; F. Bidder, in "Müll. Archiv.," 1845, and "Untersuch. über die Geschlechts- und Harnwerkzenge der Amphibien," Dorpat, 1846; J. Hyrtl, in "Zeits. d. Wien. Aerzte," 1846 ; [Jos. Toynbee, "On the Intimate Structure of the Human Kidney," "Med. Chirur. Transact.," $2 d$ Ser. vol. II., p. 303, 1846;] C. v. Patruban, "Beiträge zur Anatomie

* [I have found that the Malpighian corpuscles are filled with much more difficulty, and always imperfectly by injections thrown in by the veins. - DaC.] 
der menschlichen Niere," in "Prag. Viertelj." 1847, III. ; G. Johnson, Art. "Ren," in "Cyclop. of Anat.," May, 1848; V. Carus, in "Zeitsch. f. wiss. Zool.," II., p. 61; v. Wittich, in "Arch. für path. Anat." III., 1, 1849 ; v. Hessling, in "Froriep's N. Notiz.," 1849, p. 264, and "Histologische Beiträge zur Lehre von der Harnsecretion," Jena, 1851. Besides which should be consulted the usual Manuals of Anatomy, especially those of Henle, Valentin, J. Müller, and myself; the "Memoirs on Development," particularly of Valentin, Rathke, "Abhand zur Entw." II., p. 97; J. Müller, "De Gland. sec. structurâ;" and lastly, the "Annual Reports of Reichert," 1846 and 1849.

\section{OF THE SUPRARENAL GLANDS.}

$\S 192$. The suprarenal glands or capsules, glandulce suprarenales, are a pair of organs, in their structure approaching nearest to the blood-vascular glands, but whose function is as yet altogether unknown. Each consists of a moderately firm but thin coat composed of connective tissue, which closely invests the entire organ and is connected by numerous processes with the proper parenchyma, composed of a cortical and a medullary substance. The former, substantia corticalis, is more compact, $\frac{1}{3}-\frac{1}{2}$ a line thick, tearing readily in the direction of its thickness, and, when torn, presenting a fibrous aspect. Its color is for the most part whitish-yellow, or yellow, in the innermost third, however, usually passing into brownish yellow or brown, so that in a transverse section, two layers may be distinguished, an external, bright-colored layer and an internal, narrow, dark border. The medullary substance is, normally, of a brighter color than the cortical, being of a grayish-white with a tinge of red, although when its numerous veins are full of blood, it may assume a darker and more venous hue. Its consistence is softer than that of the cortical substance, though not so much so as is usually believed, and with respect to its thickness, it is very inconsiderable $\left(\frac{1}{6}-\frac{1}{3}\right.$ of a line) at the thin borders and at the upper and outer extremity of the organ, whilst in the middle, and in the lower and inner half, it amounts to as much as 1 or even $1 \frac{1}{2}$ lines. In the dead human subject, the cortical substance very readily becomes detached from the medullary, when the suprarenal gland presents a cavity, frequently occupying the entire organ, and containing a dirty, pultaceous substance derived from the half disintegrated brown layer of the cortex, mixed with blood, together with the less altered medullary substance; which, however, in more rare instances, also becomes broken up.

$\S 193$. Intimate structure.-The cortical substance presents as a foundation, a delicate meshwork of connective tissue, which, in connection with the tunic and the thin, mutually connected lamince proceeding from 
it, pervades the entire layer, and forms the boundaries of very numerous, closely-approximated compartments, $0.016-0.02$, or even 0.03 of a line in diameter, which extend in a vertical direction from without to within, through the entire thickness of the cortex. In these compartments is lodged a granular substance, subdivided by delicate, oblique or transverse dissepiments, into larger and smaller aggregations, which Ecker describes as gland-follicles, and as containing, within a structureless membrane, a granular material mixed with nuclei or even cells. But in these "cortical cylinders," as I would term them, I have, in most instances, noticed nothing but rounded-angular cells, $0.006-0.012$ of a line in size, and believe that Ecker, from the more rare occurrence of true follicles, has been induced to regard the compact aggregations

Fig. 252.

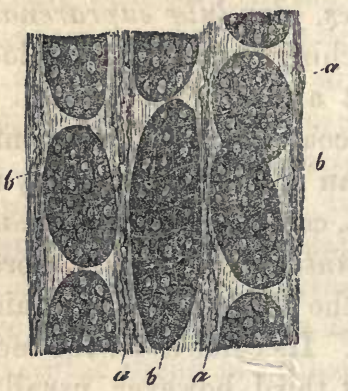

Fig. 253.

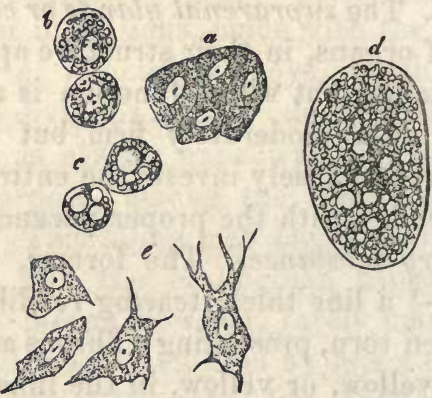

of so-termed cells which occur in the interior of the cortex, and which are $0.024-0.048-0.06$ of a line long, as special follicles. In fact, the cortical cells, which, on the external and internal portions of the cortex, are to be met with more isolated in the compartments, are in its interior, closely united into oval or cylindrical masses, in which the outlines of the cells have frequently coalesced into a single, general contour-line. I have never been able to detect any other membrane surrounding the cells besides the connective tissue of the corresponding compartment, and I have almost always succeeded, by pressure, or the addition of alkalies, in isolating the cells, without bringing into view any special sac. True follicles, I have hitherto seen only in the inner portions of the cortex, in the form of round or oval vesicles $0.02-0.3$ of a line in diameter, within which no cells like those of the cortical cylinders are formed, but only a collection of oil-drops could be recognized, and which

Fia. 252.-Portion of a vertical section through the cortex of the suprarenal body in Man: $a$, septa of connective tissue; $b$, cortical cylinder, whose composition out of cells is more or less distinctly manifest.-Magnified 300 diameters.

Fig. 253.-From the suprarenal body of Man : $a$, five cells filled with pale contents, from the summit of a cortical cylinder; $b$, pigment-cells from the innermost layer of the cortex; $c$, fat-containing cells, from the yellow cortical layer; $d$, a larger cyst filled with fat, from a cortex of that kind (gland-follicle, Ecker); e, cells from the medullary substance, some with processes.-Magnified 300 diameters. 
I have been inclined to regard as enlarged cells. The contents of the cortical cells normally consist of fine granules of a nitrogenous substance; but to these are almost always added solitary fat-granules, which in many cases (in the yellow cortical substance), exist in such quantity, as entirely to fill the cells, which then assume a deceptive resemblance to those of a fatty liver. In the brown layer of the cortex, the cells are entirely filled with brown pigment granules.

The medullary substance also has a stroma of connective tissue, which, prolonged from the cortical lamellæ, pervades the whole interior, for the most part, in more delicate fasciculi, constituting a network with rather narrow, rounded meshes. In this network lies a pale, fine-granular substance, in which, in Man, by careful manipulation, and in recent preparations, I have almost always noticed pale cells of $0.008-0.016$ of a line, which, in their fine-granular contents, occasionally presenting a few fat, or pigment granules, their frequently very distinct nucleus with large nucleoli, their angular form, and occasionally single or multiple, or even branched processes, resemble the nerve-cells of the central organs, although they cannot definitively be declared to be such.

$\S 194$. Vessels and nerves.-The bloodvessels of the suprarenal glands are numerous, lie in the stroma of connective tissue, and form two kinds of capillary plexuses; one in the cortex with elongated meshes, and one in the medullary substance with more rounded interstices. The arteries arise as numerous (amounting to twenty) small vessels from the neighboring larger trunks (phrenic, cæliac, aortic, renal), and either penetrate directly into the medullary substance or ramify in the cortical. The latter, which are the more numerous, cover the outer surface of the organ with their multiplied ramifications, and form a wide capillary plexus even in the outer tunic. They then subdivide into numerous fine twigs, and dip down into the dissepiment of the cortex, in which, becoming more and more attenuated, they run straight towards the medulla, being mutually connected in their course by pretty numerous transverse anastomoses, so that the cortical cylinders are surrounded by blood on all sides. The extremities of these vessels extend to the interior of the medulla, where, in common with the arteries which penetrate directly to the same point (of which, however, according to Nagel, in the Sheep, some proceed from the medulla entirely to the cortex) they form a rich capillary plexus of rather large vessels. The veins arise chiefly from this latter plexus, and, within the medullary substance, join the principal vein of the organ,-the $v$. suprarenalis -which comes out on the anterior surface, at the so-termed hilus, emptying itself, on the right side, into the vena cava and on the left, into the renal vein. Besides these, a good many smaller veins arise from the cortex, which either accompany the arteries in pairs, or pro- 
ceed independently and open into the renal and phrenic veins, and into the inferior vena cava. Of lymphatics, I have as yet noticed only a few stnall trunks on the surface of the organ, but none in the interior or coming out from it. The nerves of the suprarenal glands are, as was correctly stated by Bergmann, extremely numerous, arising from the semilunar ganglion and the renal plexus; according to Bergmann also, to a small extent, from the vagus and phrenic nerves. In Man, in the right suprarenal gland, I have counted thirty-three trunks, 8 of

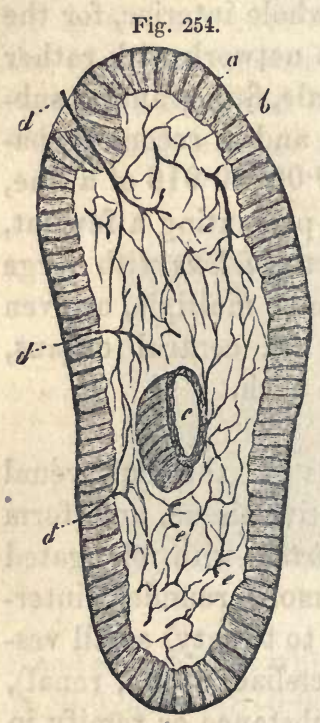
$1-5-1-10,5$ of $1-14-1-20,7$ of $1-25-1-33$, and 13 of 1-45-1-50 of a line, and found that, without exception, or at all events in a very preponderating proportion, they were constituted of dark-bordered, finer and medium-sized, or even thick fibres; were whitish or white and furnished with isolated, larger or smaller ganglia. They are especially apparent on the inferior half and inner border of the organ, and appear to be all destined for the medullary substance, in which, at least in the Mammalia, an extremely rich plexus of dark-bordered, finer fibres occurs, inclosed in the trabeculoe and connective tissue, their terminations, however, being nowhere perceptible. In man, the medullary substance is, in most instances, so altered, that the nerves cannot be traced farther than to their entrance into it, it being impossible to follow their farther distribution.

§ 195. Physiological remarks.-The suprarenal glands are developed simultaneously with the kidneys and independently of them, from a blastema derived from the middle germinal lamella (Remak), the first appearance and growth of which is unknown, and are originally larger than the kidneys. In the third month the two organs are of equal size; in the embryo at six months, the weight of the suprarenal capsule is, to that of the kidney, as 2 to 5 , in the mature embryo as 1 to 3 , in the child at birth as 1 to 8 (Meckel). In other Mammalia the suprarenal glands are, from the first, smaller than the kidneys, and increase in the same proportion with them. Little is known with respect to the histological development of the organ. I have, hitherto, investigated this only in an embryo of three months, in which, like Ecker, I found the cortex whitish, the medulla whitish-red, and both constituted of cells and fibres. The cells measured $0.012-0.02$ of a line, had well marked in part colossal nuclei, with distinct nucleoli, and in the cortical part also fatty

Fig. 254.-Transverse section of the suprarenal body of the Calf, magnified about 15 diameters: treated with soda: $a$, cortex; $b$, medulla; $c$, central vein surrounded with some cortical substance; $d$, three entering nerves; $e$, nerves, and their distribution in the interior. 
molecules. Of the nerves at that period I saw nothing. In a newly-born Rabbit, Ecker observed no appearance of follicles, whilst in a foetal Calf, 1 foot 6 inches long, he found them very distinct, but small $\left(0.05-0 \cdot 15^{\mathrm{mm}}\right)$. As regards the functions of the suprarenal glands, in the absence of all physiological indications, and so long as the course of the nerves in them is not much more accurately known, only very general observations can at present be offered. I consider the cortical and medullary substances as physiologically distinct. The former may, provisionally, be placed with the so-termed "blood-vascular glands," and a relation to secretion assigned to it; whilst the latter, on account of its extremely abundant supply of nerves, must be regarded as an apparatus appertaining to the nervous system, in which the cellular elements and-the nervous plexus either exert the same reciprocal action as they do in the gray nerve-substance, or stand in a relation as yet wholly unascertained, towards each other. (For a more detailed account vid. Mikros. Anat. II. 2, zw. Hälfte.)*

* [The former and more recent researches of Leydig ("Anatomisch-histologische Unters. üb. Fische $u$. Reptilien," 1853) appear satisfactorily to show the identity of the suprarenal glands of the Mammalia, with the yellow, vascular bodies seated, either on the kidney itself and its ernulgent veins, or at a greater distance from those glands, on the veins near the epididymis or ovaries, or upon the sympathetic nerve. The study of these more simple forms has also thrown very considerable light upon the structure and true import of the more complicated organ in the Mammalia. In the Salamander, the ganglia of the sympathetic have a very remarkable structure, and contain cellular elements of two very distinct kinds. Each ganglion has a tunic of connective tissue which sends septa into. the interior, which is thus divided into distinct and occasionally, perfectly separate compartments. The cells of both kinds are enclosed in these compartments, and are never intermixed in one and the same. In some of the compartments, or as they might almost be termed, vesicles, the cells are large, clear, and finely granular, with a vesicular nucleus and very transparent nucleolus. From some of these cells, nerve-fibres are distinctly seen to proceed, and they would appear to be of the bipolar kind of ganglion- or nerve-cells. The other kind of cells, contained in perfectly distinct compartments, are much smaller and their contents of a peculiar dirty yellow color, owing to which the perfectly transparent nucleus is rendered very distinct.

These two kinds of cells occur in very variable proportions in different ganglia. Now, if the dirty yellow-colored ganglion-globules of one of these sympathetic ganglia be compared, side by side, with the contents of one of the yellow bodies on the veins, or of the yellow granular masses on the kidney, it will be at once apparent, that by a gradual transformation, they directly pass into the fatty, granular cells of the so-termed suprarenes. And the same transformation may take place even in the sympathetic ganglia.

According to Leydig, the cortical substance of the suprarenal capsules of the Mammalia corresponds to the yellow, granular and striped suprarenal bodies of Fishes and Amphibia; whilst the medullary substance of the Mammalian organ, which is abundantly supplied with nerves and cells, very like the ganglion-globules, represents the other divisions of the sympathetic ganglia: whence he concludes that Bergmann's view, according to which the suprarenal capsules are closely related to the nervous system, is undoubtedly correct, and that those organs bear the same relation to the ganglia of the sympathetic nerves, as the pituitary body bears towards the brain. Besides this relation to the nervous system, however, they have an intimate one with the vascular; and are, therefore, always pervaded by a very close capillary plexus. But in any case, he says, the suprarenal bodies must be removed from the category of the so-termed blood-vascular glands, which wonld then include only the thyroid and thymus, or should probably be abolished altogether, as an unmeaning term.-TRs.] 
In the investigation of the suprarenal glands, the larger Mammalia ought, in the first place, to be chosen, and not till afterwards should they be studied in Man. The cortical portion is readily examined when its elements contain but little fat, and, above all, perpendicular sections of fresh specimens, or of such as have been hardened in alcohol and in chromic acid, which are afterwards to be rendered transparent by soda, are to be recommended. The medullary substance, even in animals, is very easily disintegrated, so that its elements are either not to be seen at all in the normal state, or only partially, although they are occasionally very beautifully displayed without any addition, as well as in chromic-acid preparations. In animals, the nerves are very easily discerned in fine sections, after the addition of soda; and when their external visible points of entrance are exactly hit upon in the making of the section, their course through the cortical substance is readily brought into view. For the vessels, injections should be employed, which are best effected in the Sheep or sucking Pig, and which readily succeed when made, as well from the arteries as from the non-valvular veins.

Literature.-Nagel. "Diss. sistens ren. succent. mammal. descript.," Berol., 1838, and Müll. "Arch.," 1836; C. Bergmann, "Diss. de glandulis suprarenal," c. tab. Gött., 1839; A. Ecker, "Der feinere Bau der Nebennieren beim Menschen," u. den 4 "Wirbelthierclassen," Braunschweig, 1846, und Art. "Blutgefässdrüsen," in Wagner's "Handw. d. Physiologie," Bd. IV. 1849; H. Frey, Art. "Suprarenal Capsules," in Todd's "Cyclop. of Anat.," Oct., 1849.

[H. Gray, "On the development of the ductless Glands in the Chick," "Phil. Trans." 1852.-T'Trs.]

\section{OF THE SEXUAL ORGANS.}

\section{A. MALE SEXUAL ORGANS.}

$\S 196$. The male sexual organs consist, 1 , of two glands for the secretion of the semen, the testes, which, together with special tunics, the tunicce vaginales, are contained in the scrotum; 2 , of their efferent canals, the vasa deferentia and ejaculatory ducts with their appendages, the vesiculoe seminales; 3 , of the copulatory organs, the penis and its muscles; 4, and lastly, of special accessory glands, the prostate and Cowper's ylands.

$\S 197$. The testicles, testes, are a couple of true glands, containing within a special tunic, the tunica albuginea s. fibrosa, the secreting elements, in the form of complexly convoluted tubules, the spermatic tubes or tubuli seminiferi. The tunic is a white, dense and thick membrane, 
corresponding in structure, in all respects, with other fibrous membranes (the dura mater especially), and everywhere surrounding the parenchyma of the testis as a closed capsule. Its external surface, except where the epididymis is attached to the testis, is rendered smooth and glistening by a special covering, the tunica adnata, whilst the internal is united with the substance of the gland by a thin layer of loose connective tissue, and moreover, sends a considerable number of processes into the interior; of which, the most important is the corpus Highmori

Fig. 255.

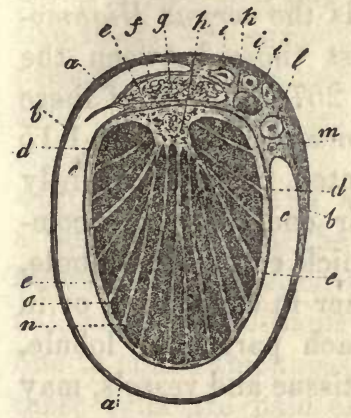

Fig. 256.

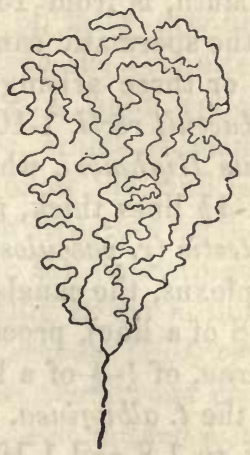

Fig. 257.

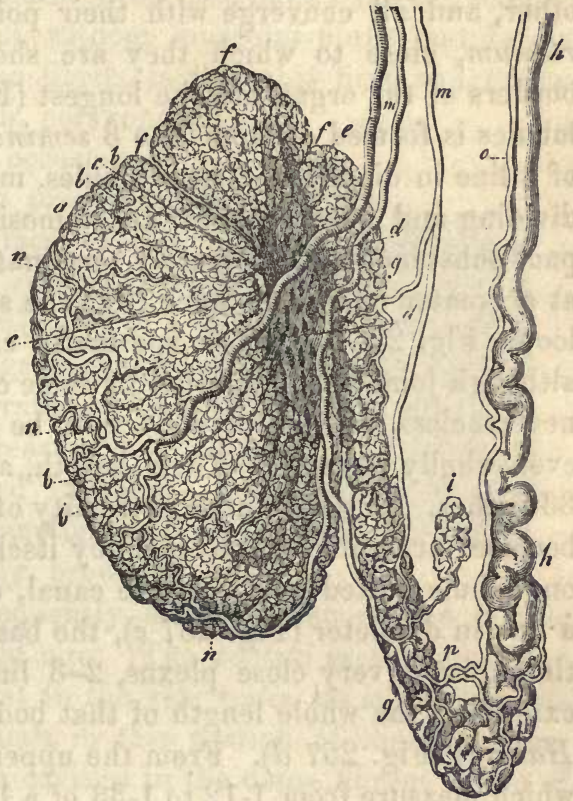

s. mediastinum testis, a vertical lamella of dense connective tissue $\frac{3}{4}-1$ inch long, and thick at its commencement, which extends from the posterior border of the testis to a depth of 3-4 lines into the interior (Fig.

Fia. 255.-Transverse section through the right testis and its tunics, in Man: a, t. vaginalis communis; $b, t$. vaginalis propria, outer lamella; $c$, cavity of the $t$. $v$. propria, which does not exist in life; $d$, inner lamella of the t. v. propria (adnata), coalescent with $e$, the $t$. albuginea; $f$, continuation of the $t$. propria on the epididymis, $g ; h$, corpus Highmorianum; $i i i$, branches of the spermatic artery; $k$, vena spermatica interna; $l$, vas deferens; $m$, artery of the vas deferens; $n$, lobuli testis; s, septula.

Fia. 256.-Diagram of the course of a spermatic tubule.

FIG. 257.-Human testis and epididymis, after Arnold: $a$, testis; $b$, lobes; $c$, ductuli recti; $d$, relé vasculosum; $e$, vascula efferentia ; $f$, coni vasculosi; $g$, epididymis; $h$, vas deferens; $i$, vas aberrans; $m$, branches of the spermatica interna of the testis and epididymis; $n$, ramification on the testis; 0 , art. deferentialis; $p$, anastomosis with a branch of the spermatic. 
$255 \mathrm{~h}$ ); but besides this, there are numerous lamellar processes (septula testis) (Fig. $255 \mathrm{~s}$ ) arising from the internal surface of the tunica albuginea, composed of more lax connective tissue, which, separating the divisions of the glandular structure from each other, and conveying the vessels belonging to them, converge from all sides towards the corpus Highmori, and are attached by their acuminate terminations to its surfaces.

The glandular substance of the testis is not absolutely homogeneous, but consists of a certain number (100-250) of pyriform lobules, lobuli testis, which are not, however, everywhere wholly separated from each other, and all converge with their points towards the corpus Highmorianum, close to which they are shortest, whilst those between the borders of the organ are the longest (Fig. 255 n, 257 b). Each of these lobules is formed of from 1 to 3 seminal tubules or canaliculi, 1-8-1-15 of a line in diameter. These tubules, much convoluted, pretty frequently dividing and also, perhaps, anastomosing, in their course, form a compact substance and ultimately terminate at the thick end of the lobule, at a greater or less distance from the surface, either in crcal ends or in loops (Fig. $256 \mathrm{~g}$ ). The spermatic canals of each particular lobule, although joined to each other by some connective tissue and vessels, may nevertheless, by careful dissection, be separated to a great extent, or even wholly isolated. Their length, according to Lauth, is from 13 to 33 inches. At the pointed extremity of each lobule the spermatic canals become straighter, when each by itself, or the two or three arising in one lobule united into a simple canal, enter as the ductuli recti, 1-10 of a line in diameter (Fig. $257 \mathrm{c}$ ), the base of the corpus Highmori, where they form a very close plexus, $2-3$ lines broad and $1 \frac{1}{2}$ lines thick, and extending the whole length of that body, - the rete testis ( $r$. vasculosum Halleri) (Fig. $257 d$ ). From the upper end of this plexus, the canals in which measure from $1-12$ to $1-33$ of a line $(0.03-0.08$ of a line), proceed 7-15 efferent canals, vasa efferentia testis $s$. Graafiana, of $\frac{1}{5}-\frac{1}{6}$ of a line $(0.16-0.18$ of a line) (Fig. 257 e), which, traversing the $t$. albuginea, are continued into the epididymis. Here they contract to 1-8 and 1-10 of a line, are convoluted in exactly the same way as the spermatic canals in the lobuli testis, but without presenting divisions or anastomoses, and thus produce a certain number of conical bodies, the apices of which are directed towards the testis, - the spermatic cones (coni vasculosi s. corpora pyramidalia) (Fig. $257 \mathrm{f}$ ). These cones, united together by connective tissue, constitute the head (globus major) of the epididymis, at the upper and posterior border of which their canals gradually coalesce; and thus is formed the simple thick duct of the epididymis, $0 \cdot 16-0 \cdot 2$ of a line in diameter (Fig. 257, g). This duct, convoluted in a well-known manner, forms the body and tail (globus minor) of the epididymis, usually giving off at its inferior extremity a cæcal prolongation, vas aberrans Halleri (Fig. 257, i) and is ultimately continuous with the 
spermatic duct or vas deferens, which at first $\frac{1}{4}-\frac{1}{3}$ of a line in diameter and convoluted, soon increases to a size of $\frac{3}{4}-1$ line, and becomes straight (Fig. 257, h). The epididymis, moreover, has an extremely delicate fibrous tunic ( $\frac{1}{6}$ of a line) of a grayish-white color.

$\S$ 198. Structure of the seminiferous tubes.-The seminal tubes of the testis are, in proportion to their diameter, rather more firmly constructed than other glandular canals and consist of a fibrous membrane and an epithelium. The former, from $0.0024-0.005$, or in the mean $0.003-0.004$ of a line thick, composed of an indistinctly fibrous connective tissue with longitudinal nuclei, without muscular fibres and rarely presenting an indication of elastic fibrils, is tolerably firm and extensible. A simple layer of roundish, polygonal cells, of $0.005-0.008$ of a line, occasionally with an indication of a membrana propria as a substratum, on the inner surface of this fibrous membrane, completes the vascular canal, which thus obtains a wall, $0.007-0.01$ of a line in entire thickness. In younger subjects, these cells are pale and finely granular, but as age advances, a continually increasing number of fatty granules is collected in them, whence the seminiferous tubes sometimes acquire a light yellowish, partially brownish

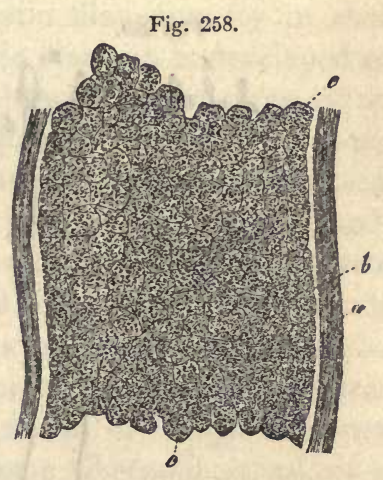
color, which is manifest very frequently in men even at the middle period of life, and invariably in old age. The ductuli recti present the same structure as the tubes; whilst in the rete testis, no special fibrous tunic can be distinguished, the canals in this portion of the gland appearing to be nothing more than cavities in the dense fibrous tissue of the corpus Highmorianum, lined by an epithelium. In the coni vasculosi the fibrous coat again makes its appearance, and to it there is now, also, added a layer of smooth muscles, which, in the form of transverse and longitudinal fibres, are recognizable even in canals of $\frac{1}{5}$ to $\frac{1}{6}$ of a line in diameter. The thicker portions of the canal of the epididymis are constructed in the same way as the vasa deferentia (vid. infra), with a cylindrical epithelium, which, moreover, commences even in the head of the epididymis.

The contents of the seminiferous tubes vary according to age. In Boys and young Animals the slender tubuli contain nothing but minute, clear cells, the most external of which might be regarded as epithelial cells, although not always clearly distinguishable from the others. At the age of puberty, together with the increased size of the tubuli semini-

Fia. 258.-Portion of a spermatic tube in Man, magnified 350 diameters : a, fibrous coat with longitudinal nuclei; $b$, clear border, probably a membrana propria ; c, epithelium. 
feri, the elements contained in them also increase in circumference; and when the formation of semen has actually commenced, they appear as clear, round cells and cysts of 0.005-0.03 of a line, which, according to their size, enclose a variable number of, from 1 to 10 , or even 20 , clear nuclei of $0.0025-0.0035$ of a line with nucleoli. At this time, in many cases, an epithelium is not manifest, the seminal tubes rather appearing to be occupied entirely and solely by the cells in question; at other times, and particularly in advancer years, an epithelium is presented containing fat, or pigment-cells, and surrounding the other elements. The latter, whether they occur in the one way or the other, are the precursors of the semen, which, in the mature condition, entirely consists of an extremely small quantity of a viscid fluid, and of innu-

Fig. 259.

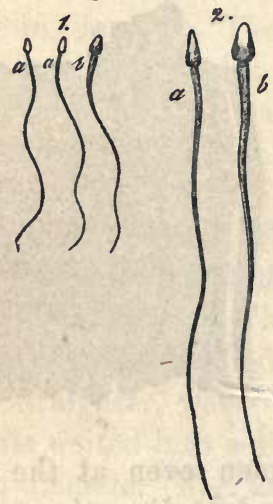

Fig. 260.
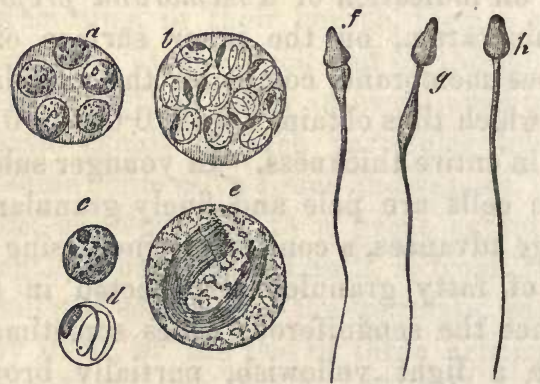

merable minute, linear corpuscles having a peculiar movement, the spermatic filaments or animalcules, fila spermatica, spermatozoa (also spermatozoida). These spermatic filaments are perfectly homogeneous, soft corpuscles, in which are distinguishable a thicker portion - the body or head,-and a filamentary appendage, - the filament or tail. The former is flattened and, viewed on the side, pyriform, with the acute end in front; on the surface oval, or even rounded anteriorly, and also, though more towards the anterior part, slightly cupped; so that it sometimes appears clear in the middle, sometimes opaque. It is $0.0016-$ 0.0024 of a line long, $0.008-0.0015$ of a line wide, $0.0005-0.0008$ of a line thick, and according as it lies on the surface or edge, does it appear clearer or more opaque, always presenting a peculiar fat-like, glistening

FIG. 259.-Spermatic filaments (human); 1, magnified 350 diameters; 2, magnified 800 diameters; $a$, viewed on the edge; $b$, on the flat surface.

FIG. 200.-Development of the spermatic filaments in the Rabbit: $a$, parent.cell (cyst) with five nuclei: $b$, one with ten nuclei, each of which contuins a convoluted spermatic filament; $c$, an isolated nucleus with nucleoli; $d$, one with the spermatic filament; $e$, a cyst with a bundle of spermatic filaments; $f f, g \mathrm{~g}$, immature spermatic filaments, with enlargements in the filamentary portion; $h$, a perfect spermatic filament. $a, b, c$, magnified 350 diarneters; $d-h$, magnified 500 diameters. 
aspect, and, particularly in a lateral view, dark borders. The pale filamentary portion has an average length of 0.02 of a line, and at its anterior end, where it joins the broader extremity of the body, with a slight constriction, it is wider $(0.0003-0.0005$ of a line, and also flattened, being gradually prolonged into an extremely fine point, scarcely visible even under the highest magnifying powers. In vigorous men the semen, throughout the vas deferens and in the globus minor of the epididymis is composed of these corpuscles, occasionally intermixed with isolated granules, nuclei, and cells, whilst in the upper part of the epididymis and in the body of the testis other elements, such as the abovedescribed cells and cysts, preponderate more and more, and finally constitute the entire secretion. These spermatic cells and cysts, as I term them, have a definite relation to the spermatic filaments, for in each $n u c l e u s$, as was first shown by me, a spermatic filament is developed on the inner wall, in the form of a spiral corpuscle with two or three turns. How this really arises is unknown; very probably as a sort of deposit from the contents of the nucleus, which at the same time become clear, in the same way as the spiral filaments of the vegetable cell are formed; but it may at all events be asserted as a positive fact, that the testis itself is the proper site of this development, so that, under normal conditions, developed, spermatic filaments may always be found in the nuclei, in the internal portions of the gland, and frequently in every seminiferous tube without exception. But in the normal course of things the spermatic filaments in the testis itself do not become liberated at all, or in very small proportion, and the tubuli seminiferi, consequently, are by no means the situation in which spermatic filaments are to be sought for, although even here, on the addition of water, which causes the substance by which they are enclosed to burst, they will always be found. They do not occur in the free state before reaching the rete testis and coni vasculosi. First, the nuclei burst, and the filaments remain in the spermatic cells, in which, when numerous (10-20), they are very regularly disposed in close apposition, with the heads and tails together, in a curved bundle, or when in less number, confusedly aggregated. Ultimately these cells and cysts also burst, the filaments are liberated, and, forming a dense entangled crowd, entirely fill the epididymis, still in part associated in bundles, which, however, also are soon broken up, in part isolated. In the lower portion of the epididymis, the entire process of development is usually concluded, though it happens, not unfrequently, that isolated transitional forms are conveyed still farther, and are not completely developed before reaching the vas deferens.

The semen, regarded as a whole, as it is found in the vas deferens, is a whitish, viscid, inodorous material, consisting almost entirely of spermatic filaments, and containing between those bodies an extremely minute quantity of a connective fluid. The chemical composition of 
this unmixed semen has not yet been investigated in Man; but we know through Frerichs, with regard to the semen of the Carp, that the spermatic fluid contains no albumen, some little mucus, and, of salts, chloride of sodium, and a small quantity of alkaline sulphates and phosphates; whilst the spermatozoa consist of a protein compound (according to Frerichs, binoxide of protein), and contain besides $4.05 \%$ of a yellowish, butyraceous fat, and $5 \cdot 21_{0}^{\circ}$ of phosphate of lime. The semen, as ejaculated, is a mixture of pure semen, and of the secretions of the vesiculoe seminales, the prostate and Cowper's glands. It is, in this condition, colorless, opalescent, with an alkaline reaction and peculiar odor; when emitted, it is viscid and glutinous, like albumen, but on cooling becomes gelatinous, and after some time again thinner and fluid. When examined microscopically it presents, besides the spermatozoa, a moderate quantity of a clear fluid, which, on the addition of water, presents irregular-sized whitish flocculi and fragments, and is undoubtedly derived principally from the vesiculce seminales. This gelatinizing substance, which Henle described as fibrin, and Lehmann regards as an albuminate of soda, has been described by Vauquelin, who analyzed human emitted semen, together with the substance of the spermatic filaments, as spermatin, of which he found $6_{0}^{0}$, whilst besides it there were present $90_{0}^{\circ}$ water, 30 earthy phosphates, and $1_{0}^{0}$ soda. When semen is dried, innumerable crystals of the triple phosphate of magnesia and ammonia are formed among the undestroyed spermatozoa, which generally, owing probably to the considerable quantity of carbonate of lime contained in them, are not easily destructible. They may be demonstrated in seminal spots even after a long time, when they are moistened; resist putrefaction for a lengthened period in water and animal fluids (Donné observed them even after three months in putrid urine), and retain their form unchanged even after incineration (Valentin). Acetic acid has but little effect upon the spermatic filaments. Caustic potassa and soda render them pale, and dissolve - them, after from 15 to 30 minutes. Nitric acid $\left(20_{0}^{\circ}\right)$ at first produces scarcely any change, afterwards dissolving them. In sulphuric acid their outline becomes extremely faint, and they swell up, but are not entirely dissolved, as are, for instance, the epithelial cells of the seminal tubes. They are not colored yellow by nitric acid and potassa, nor red by sugar and sulphuric acid. Nitrate of potassa in a solution containing 6 per cent. does not dissolve them. In the pure semen the filaments exhibit no movements, or scarcely any, when it is too much concentrated. Their movements are first visible in the contents of the vesiculoe seminales, and in ejaculated semen, or when pure semen is diluted. The movement of these bodies is effected solely by the alternate flexure and extension, or serpentine motion of the filamentary portion, in which way are produced such lively and various undulating, 
rotatory, quivering changes of place, during which the head or body always precedes, that these elements of the semen were formerly regarded as animals. The duration of the movements depends upon various circumstances. In the dead body they are not unfrequently perceptible, even 12-24 hours after death (on one occasion Valentin noticed faint motion at the end of even 84 hours), and in the female genital organs in the Mammalia, they exhibit motion even after seven or eight days. Water at first renders the motions more lively, but they soon cease, and the filaments are not unfrequently curved in a looplike form. Blood, milk, mucus, pus, syrup, and a diluted saline solution usually have no injurious effect; but it is otherwise with urine and bile, the former particularly, when it is strongly acid or much diluted. All chemical reagents, acids, metallic salts, caustic alkalies, \&c., cause the motion to cease, as do narcotics when they act chemically upon the filaments, or are too much diluted.*

The formation of the spermatic filaments and of the semen, it is true, usually ceases in old age, although they are not unfrequently found in men of 60,70 , or even 80 years of age, and even accompanied-though this, it must be confessed, is an unusual phenomenon-with the procreative faculty. After diseases, the spermatic filaments are as often found to be present as absent; and, with respect to the cause of their deficiency, only this much can be stated, that it appears to depend principally upon impaired nutrition. $f$

* [Chloroform exerts the same influence upon the spermatozoa as it does upon all motile tissues in animals and plants. The spermatozoa of the Frog, when exposed to the dilute vapor of chloroform, gradually cease to move, regaining their motile property upon exposure of the fluid to the air (at any rate many of them), and the filaments thus revivified, appear to retain all their impregnating power.-TRs.]

$\dagger[$ The origin and development of the spermatic filaments have lately been accurately investigated by Dr. Burnett (vid. Mem. of Americ. Acad. of Arts and Sciences, v. i., 1853). From an extensive series of researches in the vertebrate animals, Dr. Burnett concludes that the morphological changes in the sperm-cell, preceding the formation of the spermatic filaments, are identical in their character with the changes in the ovum, which are antecedent to the formation of the new being. When the generative function begins to be developed, the character of the epithelial cells, lining the tubules, is modified. The cells pass to a higher degree in function, but do not undergo any change in structure, except a slight increase in size. In this condition, they divide and subdivide, by a process similar to the segmentation of the yelk, urtil they are entirely converted into a mulberry mass. A liquefaction of the segmented contents into a minute granular blastema then ensues, and from this the spermatic filaments are developed. In the Plagiostomes, Dr. Burnett was able to observe the disappearance of the mulberry mass, and its replacement by a fasciculus of spermatic filaments, although the exact metamorphosis by which the granular cellular mass formed the bodies of the spermatozoids could not be detected. The spermatic filaments, Dr. Burnett thinks, are not formed, as stated by Kolliker, by a deposit from the contents of the sperm.cell or nucleus, but by an elongation of the cell or nucleus itself. The body of the spermatozoid is developed from the cell, whilst the tail is probably subsequently formed by an accumulation of minute granules.

The absence of these spermatic particles does not always, as above stated, depend upon impaired nutrition. M. Gosselin (Archives Générales de Médicine, Sept., 1853) has noticed 
$\S 199$. Membranes, vessels, and nerves of the testis.-The testes, together with their fibrous tunic, and a portion of the epididymis, are immediately invested by the tunica vaginalis propria (Fig. 255, b, d, f), a delicate serous meinbrane, which, at one time, was a part of the peritoneum, and corresponds with it in structure. Its epithelium, constituted of a layer 0.005 of a line thick, of clear, polygonal cells, 0.005-0.008 of a line in size, with distinct nuclei, and occasionally with isolated, yellowish pigment-granules rests on the testis, immediately upon the fibrous membrane, or, at all events, in this situation, is inseparably united with the fibrous coat constituting the tunica adnata testis, or the visceral lamella of the $t$. $v$. propria; whilst, on the epididymis, the serous coat may be distinctly separated, and, like its parietal portion, consists of dense connective tissue containing elongated nucle $i$. The tunica vaginalis communis, is a dense, tolerably thick membrane, consisting, on the testis, of firm connective tissue, and higher up, of a more lax reticulated tissue with elastic fibres; it closely surrounds the tunica vaginalis propria, and also invests the spermatic cord, and the lower end of the epididymis. Between it, the tunica propria, and the epididymis, and firmly connected with both tissues, is placed a layer of smooth muscles, usually corresponding to the two lower thirds of the testis, - the internal muscular tunic of the testis; whilst on its outer side is inserted the cremaster, composed of transversely striated fibres. The scrotum, lastly, is formed by the external muscular tunic of the testis, which is more laxly connected with the $t$. $v$. communis, - the tunica dartos; with respect to which, vid. $\$ 34$; and by the external integument, which is characterized by its thinness, the absence of fat, the color of its epidermis, and its, mostly, large sebaceous and sweat-glands.

The bloodvessels of the testis and epididymis are derived from the long and slender a. spermatica interna, which, running in the spermatic cord, proceeds from its posterior aspect to the testis, and sometimes entering at once the corpus Highmorianum, sometimes divided into nurnerous branches, ramifies in the fibrous tunic of the testis, and on its inner surface, proceeding towards the anterior border of the gland. The coarser ramification in the parenchyma of the testis proceeds partly from the corpus Highmorianum, partly from the points of origin of the septula testis, from the tunica albuginea, into the septula, from which, again, numerous more minute vessels penetrate into the interior of the lobules, constituting a rather wide-meshed plexus of capillaries, $0.003-0.008$ of a line in diameter, around the tubuli seminiferi. In the epididymis

that in the semen of patients who had suffered attacks of double epididymitis, the spermatozoids remained absent for months, even for years, after a complete restoration of the general health. In some diseases, the spermatozoids themselves are changed. Thus, Lallemand (Annales des Sciences Nat., tom. xv., p. 30), states, that in patients broken down by seminal losses, they appear imperfectly formed, the tails being rough, irregular, and indistinct.DaC.] 
there is a similar though less abundant plexus, in the formation of which the artery of the vas deferens also participates (Fig. 257), whilst the scrotum and the $t$. vaginalis are richly supplied with vessels from the $a \alpha$. scrotales and spermatica externa. The veins accompany the arteries, and with respect to the lymphatics, not only are those of the scrotum and vaginal tunics extremely numerous, but, according to the beautiful researches of Panizza ("Osservazioni," Tab. VIII.), confirmed by Arnold, those of the testis are also very much developed. They proceed partly from the interior, partly from the surface of the testis and epididymis, form beneath the tunica adnata beautiful plexuses, ultimately connected by several trunks in the spermatic cord, and, together with those of the vaginal tunics, communicate with the lumbar glands.

The few nerves of the testis are derived from the internal spermatic plexus, and accompany the arteries to the gland. I have in vain endeavored to follow their course in the interior, where it but rarely happens that dark-bordered nerves are seen, even accompanying tho larger arteries of the parenchyma.

\$200. Vasa deferentia, vesiculoe seminales, accessory glands.-The spermatic ducts, or vasa deferentia, are cylindrical canals, having a mean width of $1-1 \frac{1}{2}$ lines, the walls being $\frac{1}{2}-\frac{2}{3}$ of a line thick, and the cavity $\frac{1}{4}-\frac{1}{3}$ of a line in diameter, and composed, most externally, of a thin fibrous membrane, then of a strong, smooth-muscular layer, and most, internally, of a mucous membrane. The muscular tunic, which is 0.38 -0.6 of a line thick, consists of an external, strong layer of longitudinal fibres, a middle one, also strong, of transverse and oblique fibres, and an internal thinner longitudinal layer, constituting not more than $\frac{1}{4}$ th of the whole muscular tunic. The tissue is constituted of stiff and pale fibre-cells, as much as 0.1 of a line in length, and having an average width of $0.004-0.006$ of a line, intermixed with some connective tissue, and a very few pale elastic fibrils. The mucous membrane, 0.12 of a line thick, is white, longitudinally plicated, and in the last, broadest and widest portions of the duct, presents numerous larger and smaller fossce, disposed in a reticular manner. Its external two-thirds are whiter, and contain one of the closest felted tissues of elastic fibrils with which I am acquainted; whilst towards the interior is found a more transparent, thinner layer, composed of an indistinctly fibrous connective tissue, with nuclei, upon which rests a single layer of tessellated epithelium, constituted of cells $0.005-0.008$ of a line in size, and which almost invariably contain a certain number of brownish pigment-granules, from which the internal surface of the mucous membrane acquires a yellowish hue. The vessels of the vas deferens are very distinct in the external fibrous tunic, but also penetrate into the muscular and mucous coats, constituting in each, loose plexuses of capillaries $0.003-0.005$ of a line in diameter. 
According to Swan ("Nerves of the Human Body," Pl. V., 82; VI., 81 ), the vas deferens within the pelvis is surrounded by numerous, but fine nerves, which are in connection with those of the lateral and median, vesical and rectal nerves, as well as with those of the hypogastric plexuses. I have myself also seen these nerves, which contain fine fibres, and "fibres of Remak," but have been unable to trace them in the interior.

The structure of the ejaculatory ducts and vesiculoe seminales appears to be the same as that of the vasa deferentia; the seminal vesicles, as is well known, being nothing more than appendages of the latter, furnished with wart-like, saccular, or even branched processes. The ejaculatory ducts, in their upper portions, present the same muscular structure as the spermatic canal, only that their walls are more delicate. As they approach the prostate, their membranes become still thinner, but nevertheless, even at the ultimate extremity, exhibit muscular fibres, mixed with a considerable quantity of connective tissue and elastic fibrils. The walls of the vesiculoe seminales are much thinner than those of the vas deferens, although they possess the same structure, except that the manifestly vascular mucous membrane is furnished throughout with reticular fossce. Externally, the vesiculoe seminales are invested with a membrane, in part composed solely of connective tissue, in part, as on the posterior surface, distinctly muscular, which is continued between the separate convolutions of its canal, connecting them together; and, at the inferior end, passes from one vesicula seminalis to the other, in the form of a broad muscular band. The contents of the vesiculo seminales are, normally, a clear, rather viscid fluid, which after death acquires a soft gelatinous consistence, though subsequently it becomes perfectly fluid; it contains a protein-compound very readily soluble in acetic acid and which is obviously identical with that contained in the ejaculated semen. With many other observers, I have so frequently seen spermatic filaments in the vesiculoe seminales, that I should describe their occurrence there as normal, and assign a double function to the seminal vesicles; together with its principal one, of affording a special secretion, that of also performing the part of seminal receptacles. The nerves of the vesiculce seminales are derived from the sympathetic, and spinal cord, immediately from the rich plexus seminalis, the filaments of which, in part, penetrate the membranes of the vesicles, though they cannot be traced further, in part proceed to the prostate, whose plexus-plexus prostaticus-receives additions also from the vesical and inferior pelvic plexuses.

The prostate, according to my observations, is a very muscular organ, so much so, that the glandular substance does not constitute more than one third or a half of the whole mass. Proceeding from within to without, there is presented in intimate connection with the thin mucous membrane, the epithelium of which is always in two layers, though possess- 
ing a superficial lamella composed of cylindrical cells, a yellowish longitudinally fibrous layer, which extends from the trigonum vesicae to the caput gallinaginis, is, in fact, unconnected with the muscles of the bladder, and is composed, in equal proportions, of connective tissue with elastic fibres, and of smooth muscles. To this succeeds a strong layer of circular fibres of a similar structure, continuous with the sphincter vesicoe, extending as far as the caput gallinaginis, and which I term the sphincter prostatce. Beyond these different muscular layers, we come at last to the proper glandular tissue of the prostate, which consequently occupies, principally, the more external portions of the organ, although it is true that isolated lobules encroach upon the circular fibres; its numerous excretory ducts penetrate the longitudinal and transverse fibres, and open on both sides of the caput gallinaginis. The latter consists of a grayish-red, tolerably dense substance, which may be very readily split into fibres in the direction of the transverse diameter of the organ, or more accurately described, radiates on all sides of the upper surface of the organ, and is composed, in the first place, of variously-sized bundles of evidently smooth muscle, and secondly of the glands of the prostate. The latter are 30-50 compound, racemose glands, whose aggregate form is conical or pyriform, and which are distinguished from the usual kind of racemose glands by their more lax structure, their evident composition of numerous, pedunculate, glandular vesicles, and the slight development of the extremely minute glandular lobules, a condition which is partly connected with the abundant fibrous tissue interposed between the glandular elements. The glandular vesicles are pyriform or roundish, $0 \cdot 05-0 \cdot 1$ of a line in size, and lined with polygonal or short, cylindrical epithelial cells, 0.004-0.005 of a line long, with brown pigment granules; whilst in the excretory ducts, the same cylinders are met with as exist in the prostatic portion of the urethra. The secretion of the prostate appears to be similar to that of the vesiculce seminales; at all events, according to Virchow, the prostatic concretions, or stones, as they are termed-round, concentric concretions, $0 \cdot 03-0 \cdot 1$ of a line, and more, in size, which are formed in the glandular vesicles-consist of the same protein-compound, soluble in acetic acid, which is found in the vesiculoe seminales. The prostate possesses a fibrous coat, abounding in bundles of smooth muscles, and closely investing the glandular tissue, and tolerably numerous vessels, among which numerous capillaries surrounding the glandular elements, and a rich venous plexus under the mucous membrane of the urethra, are deserving of notice. The course of the nerves above described, in the interior of the prostate, is unknown.

The uterus masculinus, or vesicula prostatica, situated in the caput gallinaginis, in the middle, between the ductus ejaculatorii, presents in its whitish-yellow walls, lined with a cylinder-epithelium, principally 
connective tissue and elastic fibrils, with which, in the neck of the bladder, a few, and, at its base, pretty numerous flat, smooth muscles are intermixed.

The glands of Cowper are compact, compound, racemose glands, whose ultimate vesicles, $0.02-0.05$ of a line in size, are lined with a tessellated epithelium, whilst a cylindrical epithelium is found in the excretory canals. The delicate membrane with which the whole gland is invested, as well as the fibrous stroma in its interior, is tolerably rich in smooth muscles, which have lately been also found by me in the excretory canals, $\frac{1}{4}$ of a line wide, as a delicate longitudinal layer. The secretion of these glands, which may be readily obtained from the excretory ducts, is common mucus.

$\S 201$. The organ of copulation, in Man, consists of the penis, an organ which is composed of three erectile, highly vascular bodies-the corpora spongiosa s. cavernosa; it is attached to the pelvis, and traversed by the urethra, invested by special fascix and by the external integument, and furnished with three proper muscles.

The corpora cavernosa penis are two cylindrical bodies separated posteriorly, united anteriorly, and parted only by a single incomplete septum, in which are to be distinguished a special fibrous membrane (tunica albuginea s. fibrosa), and the internal spongy tissue. The former, which is a white, glistening, very strong membrane, $\frac{1}{2}$ a line in thickness, constitutes both the external tunic of the spongy bodies, and also, in its anterior half-as a thin lamella, partially broken up into separate fibres and laminæ- the septum between them; it consists of common fibrous tissue, like that of tendons and ligaments, with numerous, well-developed, fine, elastic fibres. Within it lies the reddish spongy substance, consisting of innumerable fibres, bars, and laminæ, united into a fine meshwork-the trabeculce corp. cavernosorum; and with its minute, rounded-angular cavities, which anastomose on all sides, and, in life, are filled with venous blood, the venous sinuses of the spongy body, bears the most deceptive resemblance to a sponge. All the trabeculoe, without exception, present precisely the same structure. Externally they are covered by a simple layer of intimately connected tessellated epithelium, the cells of which frequently do not admit of being separated,-the epithelium of the venous spaces; to this succeeds the proper fibrous tissue, which is composed on the one hand of almost equal proportions of connective tissue and fine elastic fibres, and on the other of smooth muscles, and in many, but by no means in all, the trabeculoe, encloses larger or smaller arteries and nerves. The elements of the trabecular muscles are not only at once distinctly recognizable from their wholly characteristic nuclei, on the addition of acetic acid, but may also be isolated in great numbers, and especially after treatment 
with nitric acid of $20 \frac{\mathrm{o}}{0}$, appearing as fibre-cells $0.02-0.03$ of a-line long, $0.002-0.0025$ of a line broad.

The corpus cavernosum urethree (corpus spongiosum) is constructed essentially in the same way as that of the penis, except that, 1 , the fibrous membrane, which in the bulb also forms the rudiment of a septum, is much thinner, less white, and more abundantly supplied with elastic elements ; 2 , the intertrabecular spaces are smaller, being smallest in the glans; 3 , and lastly, the trabeculae are more delicate, and, beneath the epithelium, richer in elastic fibrils; having, however, in other respects, the same structure as elsewhere.

This is the place also to speak of the male urethra, which at the isthmus is an independent canal, whilst at its commencement and termination it consists merely of a canal of mucous membrane, supported by the prostate and corpus cavernosum urethrce. The proper mucous membrane, beneath a longitudinal layer of connective tissue abounding in elastic fibres, presents not only, as already mentioned, in the prostatic portion, but also in the membranous parts, although less developed, smooth muscles mixed with the usual fibrous tissue, disposed longitudinally and transversely; to which again succeed the animal fibres of the musculus urethralis. In the pars cavernosa, also, the submucous tissue still presents, here and there, muscles of the same kind, and at a certain depth longitudinal fibres are always found, with a greater or less intermixture of such, which, however, cannot be referred to the corpus cavernosum, seeing that there are no venous spaces betwreen them; but which rather form a continuous membrane, bounding the true corpora cavernosa,* on the side towards the mucous membrane of the urethra. The epithelium of the urethra is formed of pale cylinders, 0.012 of a line in size; beneath which, however, are found one, or perhaps two, layers of round or oval cells. In the anterior half of the fossa of Malpighi exist papillce 0.03 of a line long, and a tessellated epithelium, 0.04 of a line thick. In the isthmus and pars cavernosa urethrce are found, in considerable number, the so-termed "glands of Littre," $\frac{1}{3}-\frac{1}{2}$ a line in size, which, speaking generally, rank with the racemose glands, although distinguished from them by their' tubular form, and the frequently much convoluted course of the glandular vesi-

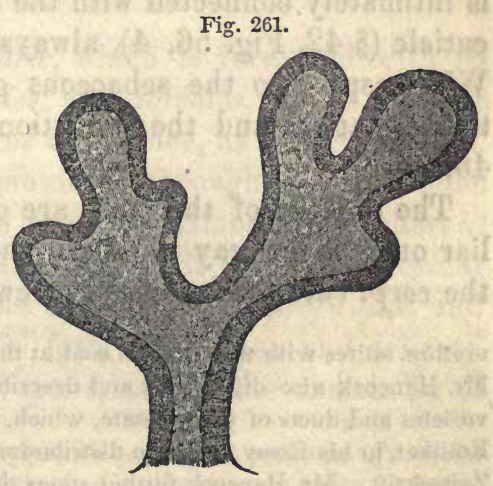

FIG. 261.- "Gland of Littre," from the fossa Morgagni, in Man; magnified 500 diameters.

* [Mr. Hancock published, in 1851, an account of the distribution of the organic muscular fibres of the urethra, essentially agreeing with the above. He states that this internal muscular coat of the corpus spongiosum, or, as Mr. Hancock prefers to call it, muscular coat of the 
cles, which are $0.04-0.08$ of a line wide. More simple forms of glands of this kind (Fig. 161) are occasionally met with intermixed with the others; and in the pars prostatica, instead of them, we find minute mucous follicles, similar to those which have been before described as occurring in the cervix vesicce. The epithelium, both in the vesicles of the "glands of Littre" and in the excretory ducts, 1-2 lines long, which are directed forwards, penetrating the mucous membrane obliquely, is cylindrical ; in the former situation, however, more or less approaching the tessellated form (Fig. 261); the secretion is common mucus, which in dilatations of the glandular follicles is, not unfrequently, collected in some quantity. Some minute, inconstant fossa of the mucous membrane have been termed lacunce Morgagni, in which $I$ have been unable to detect anything of a glandular nature. The fascia penis, a tissue abounding in finer elastic fibres, surrounds the penis, from the root to the glans, being, in the former situation, in connection with the perineal fascia, and that of the inguinal region, and also contributes to the formation of the suspensory ligament of the penis, a structure very rich in true elastic tissue, which extends from the symphysis to the dorsum penis. Externally it is continuous, without any line of demarcation, with the skin of the penis, which, up to the free border of the prepuce, a simple duplicature of it, possesses the nature of the common integument; though certainly characterized by its delicacy, and the presence of a layer of smooth muscles in the abundant, fatless, subcutaneous tissue, a continuation of the tunica dartos (vid. § 34), which extends as far as into the prepuce. At the border of the prepuce the integument of the penis assumes more of the nature of a mucous membrane, and is no longer furnished with hairs and sudoriparous glands, although it has well-developed papillo; it is still thinner than before, and on the glans is intimately connected with the spongy body, and covered with a softer cuticle ( $\$ 42$, Fig. 56, 4), always, however $0.035-0.056$ of a line thick. With respect to the sebaceous glands ( $g l$. Tysoniance), which exist in this situation, and the formation of the preputial smegma, consult $\S \S$ 46,74 .

The arteries of the penis are derived from the pudic, and are peculiar only in the way in which they supply the corpora spongiosa. In the corp. cav. penis, except a few small twigs from the a. dorsalis, the

urethra, unites with the external coat, at the lips of the urethra, so as to form a sort of sphincter. Mr. Hancock also discovered and described the abundant organic muscles surrounding the 'vesicles and ducts of the prostate, which, though admitted in the text, were denied by Prof. Kölliker, in his Essay upon the distribution of the organic muscles in Siebold and Kolliker, Zeitschrift. Mr. Hancock further states that the muscular fibres of the meinbranous portion of the urethra are continued over the inner and outer surfaces of the prostate, into the muscular coat of the bladder, so that, according to his view, there is one continuous muscular coat, from the bladder to the end of the penis, which twice separates into two layers; posteriorly, to enclose the prostate, anteriorly, to envelop the spongy tissue of the corpus spongiosum.-See Hancock on the "Anatomy and Physiology of the Urethra," 1852.-Trs.] 
a. profundoe penis alone run near the septum, surrounded by a sheath of connective tissue continuous with the trabecular network, in fact directly forwards, but giving off a small twig to the bulbs of the crura. In this course they afford numerous, occasionally anastomosing branches to the spongy substance, which, running in a convoluted manner (except at the time of erection) in the axis of the trabecula, ramify in them, and ultimately, without the formation of capillary plexuses open into the venous spaces, by capillaries of $0.006-0.01$ of a line in diameter. In the posterior part of the penis there are numerous minute arterial trunks, measuring $0.04-0.08$ of a line in diameter, and for the most part lying from 3-10 together, as was first observed by J. Müller; they are contorted and convoluted in a peculiar, tendril-like manner (arterice helicinoe), and do not terminate in cæcal ends, but, as I have ascertained, give off from their clavate extremities minute vessels of $0.006-0.01$ of a line, which, like the other arterial prolongations, are continued further, and terminate in the venous spaces. The arterial ramification is precisely

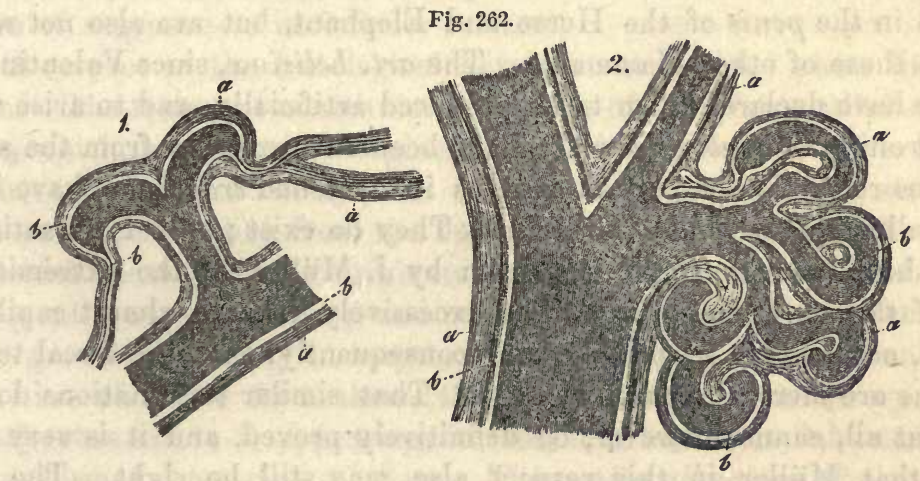

similar, in the corpus cavernosum urethroe, which is furnished with its supply from art. bulbosoe, bulbo-urethrales, and dorsales, and in the bulb there also exist art. helicinoe. The veins commence, as it may be said, in the venous spaces which intercommunicate throughout; and from which, though not universally from the same situations, short efferent canals, or emissaria, receive the blood and convey it into the external veins, furnished with special walls (vena dorsalis, v. v. profundx, and bulbosce in particular). The lymphatics form very close and delicate plexuses in the skin of the glans, the prepuce, and in the remainder of

Fia. 262.-Arteries from the corpora cavernosa penis of Man, injected ; magnified 30 diameters. 1 , one of the smaller arteries, with a lateral branch, dividing into two helicine arteries, from the extremities of which two very small vessels proceed, and are continued into the delicate trabecula ; 2 , five helicine arteries placed on a short stem of a larger arterial division; from two of these, fine vessels are seen to be given off, the others appear to terminate in cæcal ends : $a$, trabecular tissue, here presenting the form of sheaths to the arterial trunks and helicine arteries; $b$, wall of the arteries. 
the integument, and communicate with the superficial inguinal glands through trunks accompanying the dorsal vessels. According to Mascagni, Fohmann, and Panizza, there are also numerous lymphatics in the glans surrounding the urethra, which run backwards on that canal, and proceed to the pelvic glands.

The nerves of the penis are derived from the pudendal and the plexus cavernosus of the sympathetic, the former of which are distributed principally to the skin and the mucous membrane of the urethra, and, in a small proportion only, to the corpora cavernosa, to which alone the latter set of nerves is destined. The terminations of the former nerves present the same conditions as those of the integuments; numerous divisions, in particular, and faint indications of axile corpuscles, occur in the glans penis; those of the latter nerves are not as yet known, although, in the trabeculoe of the corpora cavernosa, nerves with fine fibres, and "fibres of Remak," are readily demonstrated.

The smooth muscles of the corpora cavernosa are very distinctly shown in the penis of the Horse and Elephant, but are also not wanting in those of other Mammalia. The art. helicino, since Valentin and Henle have declared them to be produced artificially, and to arise from the inrolling of trabeculce which have been cut across, or from the spontaneous retraction of certain arteries in stretched trabeculce, have been generally rejected, "but incorrectly. They do exist; only I am satisfied that the circumstance, noticed even by J. Müller, of the extremity of one of these arteries, giving off an excessively delicate, almost capillary vessel, occurs very frequently, and consequently, that the cæcal terminations are merely apparently such. That similar terminations do not exist at all, cannot however, be definitively proved, and it is very possible that Müller, in this respect also, may still be right. The art. helicince, therefore, are not simple vascular loops, as which they are described by Arnold, although, in one instance, I have noticed such an arrangement in place of them.

§ 202. Physiological remarks. - The development of the testes, commencing in the second month, takes place, according to all that we know, from a blastema which appears independently on the inner side of the Wolffian body; and, at first, the form of the male sexual glands entirely resembles that of the ovaries. At a subsequent period, when the Wolffian body begins to waste, a portion of its fine canals, the Malpighian corpuscles of which disappear, become connected with the testes, and are formed into the epididymis, whilst at the same time the excretory duct of the Wolffian body constitutes the vas deferens.* Then,

* [It is a curious fact connected with this alleged origin of the epididymis distinctly from the rest of the gland, that, in cystic disease of the testicle, either of the innocent or malig- 
by a process not as yet accurately explained, the testes, with its peritoneal investment, descends into the scrotum under the agency of the gubernaculum, a process composed of transversely striated and smooth muscles; and by the growing together of the peritoneal protrusion contained in the gubernaculum - the processus vaginalis peritonci,with its own proper serous coat, acquires its tunica vaginalis propria. The vesicula prostatica, the analogue of the uterus, and probably of the vagina, is the remainder of the "Müllerian ducts," two canals, descending on the external border of the Wolffian body, which, in the female, form the oviduct and by the coalescence of their extremities, the uterus and vagina; but in the male disappear, except the commencement, which becomes the "hydatids of Morgagni," and the last portion. The vesiculae seminales are protrusions of the $v$. deferentia; and the prostate, Cowper's, and the smaller glands, are most probably formed, in analogy with other similar glands, from the epithelium of the urethra. The penis is developed from the pelvic bones outwards, and does not, till subsequently, include the urethra which is formed by the closure of a groove on its inferior surface.

With respect to the histological development of these parts little is known. The testes are constituted originally of a uniform cellular substance, which, however, soon begins to divide into transverse rows, forming the rudiments of the seminal tubes. These are, at first, straight crcal canals, extending from the outer border of the testis to the interior, which most probably originate as solid tracts of cells, and only subsequently acquire a cavity and membrana propria. From the continued growth, especially in length, of these primary channels, and the production of offsets from them, the later, convoluted and very long seminal tubes arise; it appearing, in fact, that an entire lobule of the testis is formed from each of them. The tunica albuginea of the testis and its internal prolongations arise from the primary blastema of the gland, and make their appearance at the same time with the seminal tubes.

With regard to the physiological relations of the male sexual organs, in the adult, I would here notice the following points. The secretion of semen, in animals, does not go on continuously, like that of the urine, but is intermittent, taking place only at the time of rutting or heat. In Man, the capability of producing semen, assuredly, always exists, although it does not appear to follow from this, that semen is being continually formed, and that what is not emitted undergoes absorption; and consequently it seems justifiable to suppose that the seminal tubes secrete semen only when the secretion has been partially evacuated externally, either in consequence of sexual congress, or of seminal emissions, and nant type, the affection is "the result of morbid changes in the ducts of the rete testis; this part of the gland being the sole seat of the disease." (Curling, "Med. Chir. Transact.," XXXVI. p. 456, 1853).-Trs.] 
an excitement of the nervous system has caused an increased flow of blood to the testis. There are no certain facts in favor of an absorption of the semen when formed, which could only take place in the vasa deferentia and vesiculoe seminales; for what is observed in animals, after the rutting season is over, has no reference to this point; and the very circumstance, that in the situations above-mentioned, no traces of disintegration of the semen are ever found, appears to be very much opposed to such a supposition. At the same time, however, it is perhaps unquestionable, that without seminal evacuations, a formation of semen may be possible; for it is sufficiently established, that a rich, heating diet, and an unsatisfied sexual excitement, often produce a turgescence of these organs, attended with painful sensations, and most probably with a formation of semen. The subsequent removal of this fulness does not, however, appear to me incontestably to prove any absorption, because a difference in the quantity of blood in the testis, and the passing of the semen into the $v$. deferentia are sufficient to account for the restoration of the usual condition. The fluid constituting a seminal emission is not pure semen, but in great part the secretion of the vesiculoe seminales and prostate, and affords no criterion by which to estimate the energy of the secretion of the testes. The formation of the semen itself certainly does not proceed rapidly and copiously, as might be concluded from the relatively small quantity of blood contained in the testes, and from its slow motion in them, necessarily consequent upon the anatomical conditions; and as is also evident from the fact, that after a few previous emissions, even in the most vigorous organisms, a certain time is requisite for the preparation of a fresh secretion. The secretions of the accessory glands are perhaps simply intended for the dilution of the semen.

That the seminal filaments are not animalcules, but elementary parts of the male organism, it is useless at the present time to attempt to demonstrate; although it is still as much as ever unknown, and will not easily soon be ascertained, what is effected by their curious movements, which are obviously intended to convey them to the ovum, from the uterus, which they probably reach in fruitful congress. Nor, from the experiments of Prévost, Dumas, Schwann, and Leuckart, and the later researches of Newport (Phil. Trans. 1851, 1), can the least doubt be entertained that they are the true impregnating agent, and for the purpose of impregnation must necessarily come in contact with the ovum. The circumstance that motile spermatic filaments alone possess the fertilizing property, and, according to Newport, that the effect upon the ovum takes place immediately upon the contact, although a short duration of the contact of the spermatic filament with the ovum is necessary to render it efficient, also shows, as it appears to me, that they do not act by affording any material substance to the egg, but in consequence of their exciting actions in it, as bodies in a state of peculiar activity. In my first 
work upon the spermatic fluid, in which I expressed this view, in order to indicate a ground for discussion, I compared its influence upon the ovum to that of a nerve-fibre upon a nerve-cell, or of a magnet upon iron; and these comparisons, to which might be added the influence which a part of an organism exerts upon a self-organizing exudation, or an entire organism upon a part in a state of self-regeneration, still appear to me the most suitable, if impregnation is in any way to be assimilated with other processes; but I have no objection to offer, should the chemical side of the question be advocated in preference, as by Bischoff, and the functions of the semen be referred to the category of catalytic phenomena.*

In the act of copulation, various motile phenomena are presented, of which we need discuss only those conducive to ejaculation and erection. In the former the vasa deferentia, provided as they are with a colossal muscular apparatus, are chiefly operative; these organs, as Virchow and I found in an executed criminal, shorten and contract with remarkable energy when excited by galvanism; as also do the vesiculce seminales, the highly muscular prostate, and, of course, the transversely striated muscular tissue of the urethra and penis. Erection is caused, as I have shown ("Würzb. Verh." Bd. II.), by a relaxation of the muscular elements in the trabeculoe of the cavernous and spongy bodies, and of the tunica media of the arteries of those parts; in consequence of which the tissue, like a sponge which has been compressed, expands and becomes filled with blood. The rigidity ensues so soon as the muscles are completely relaxed, and the sinuses filled to the utmost, without there being any necessity that the return of the blood should be impeded, and the circulation stopped. It ceases when the muscles again contract, the venous spaces become narrowed, and the blood is expressed from them. In the act of ejaculation, the ischio-cavernosi,

* [The later, most important researches of Dr. Neilson, respecting the impregnation of the ovum in Ascaris mystax ("Phil. Transact.," 1852), and of Mr. Newport (op. cit.), with regard to that of the Frog, in which he has been compelled to abandon his former opinion, that the spermatozoa did not penetrate through the vitelline membrane, and has shown that, in that case, as in the one so ably described by Dr. Neilson, those bodies penetrated into the substance of the vitellus in large numbers, where they underwent changes, and finally disappeared-render much of the above speculation on the subject of their influence in impregnation futile. Whatever may be the nature of the influence conveyed to the vitellus by the spermatic filaments, it must now perhaps be regarded as an established fact, that it cannot be communicated except by an immediate contact between the motile filaments and the substance of the vitellus, which thereupon undergoes segmentation, and the series of changes is commenced, to terminate in the evolution of the embryo. Additional confirmation of the same fact would be afforded by Dr. Keber's researches on the entrance of the spermatozoon into the ovum of Unio ("De introitu Spermatozoorum," \&c., 1852), could full reliance be placed upon his results; but this, from some investigations of our own on the same subject -both in Unio and in Pholas-we consider extremely doubtful. The appearances he describes, much more resemble those noticed by Von Wittich and Carus in the ovum of Spiders.-Trs ] 
and the bulbo-cavernosus muscles, which are formed of transversely striated fibres, increase the rigidity of the anterior parts by the compression of the root of the penis and dorsal veins; but under no circumstances can they, of themselves alone, contribute to the bringing about of the erection. I am not aware that any more important function can be assigned to the helicine arteries; this much being certain, that the erection does not depend upon them, because they do not occur in every part of the human penis, and are wanting in many animals.

The investigation of the male sexual organs presents, generally speaking, no great difficulties. The tubuli seminiferi are very readily isolated, and when they are carefully unfolded some divisions are always met with. In order to trace their entire course, they must be injected according to the directions of Lauth or Cooper, which may be found quoted in all Manuals of anatomy. Lauth places the testicle for two or three hours in lukewarm water, then expresses the semen as completely as possible from the epididymis, and immerses the gland for 3-4 hours in a solution of carbonate of ammonia, or for 8-12 hours in a saturated solution of carbonate of potassa, or a weak solution of caustic potassa, which reagents partly dissolve the spermatic cells and epthelia; the testis is then again compressed, laid in alkaline water, and injected with quicksilver, at first under a weak and afterwards under a strong pressure, a process requiring from $1 \frac{1}{2}$ to 2 hours. So soon as the quicksilver has penetrated into the vas deferens, the column must be lowered to 5 inches, for otherwise the tubuli seminiferi, the filling of which demands some hours more, burst. Cooper injected from the vasa efferentia, into which he introduced a fine canula. For microscopical investigation, Gerlach recommends a solution of gelatine, with carmine or chromate of lead. The vas deferens is best studied in transverse sections, after it has been hardened or dried, as are also the prostatic glands; whilst the muscles of the prostate and the corpora cavernosa can be distinctly made out only in the recent state, or after the application of nitric acid. The helicine arteries may be seen, even in fresh preparations, close to the larger arterial trunks, but still better after injection with fine materials.

Literature.-A. Cooper, "Obs. on the structure and diseases of the testis," London, 1830, with twenty-four plates; E. A. Lauth, "Mém. sur le testicule humain," in "Mém. de la société d'histoire naturelle de Strasbourg," tom. I, 1833 ; C. Krause, "Verm. Beobachtungen" (various observations), in Müll. "Arch." 1837, p. 20 ; E. H. Weber, "De arteriâ spermatica deferente, de vesica prostatica et vesiculis seminalibus," Progr., 1836, editum in Progr. collecta, II. 1851, p. 178; "Zusätze zur Lehre vom Bau und den Verrichtungen der Geschlechtsorgane" (Contributions to the knowledge of the structure and functions of 
the sexual organs), Leipsic, 1846; C. J. Lampferhoff, "De vesicularum seminalium naturâ et usu," Berol., 1835; Kölliker, "Ueber die glatten Muskeln der Harn- und Geschlechtsorgane," in "Beiträge zur Kenntniss der glatten Muskeln" (On the smooth muscles of the urinary and genital Organs, in Contributions to the knowledge of the smooth muscles), in “Zeitsch. f. wiss. Zoologie," I. ; Fr. Leydig, "Zur Anatomie der männlichen Geschlechtsorgane und Analdrüsen der Säugethiere" (On the Anatomy of the male sexual Organs and anal glands of the Mammalia), in "Zeits. f. wiss. Zoologie," II.; A. v. Leeuwenhoek, "Arcana Naturæ," p. 59; Prévost and Dumas, "Ann. d. Sc. Nat.," III. 1824, and "Mém. de la Soc. d'Hist. Nat. de Genève," tom. I. p. 180; R. Wagner, "Die Genesis der Samenthierchen" (The genesis of the Spermatozoa), in Müll. "Archiv," 1836, and "Fragmente zur Physiologie der Zeugung" (Fragments on the physiology of Generation), Munich, 1836 ; A. Donné, "Nouv. Expér. sur les animalcules spermatiques," Paris, 1837, and "Cours de Microscopie," Paris, 1844; A. Kölliker, "Beiträge zur Kenntniss der Geschlechtsverhältnisse und der Samenfliussigkeit wirbelloser Thiere" (Contributions to the knowledge of the sexual relations and the seminal fluid of the Invertebrata), Berlin, 1841, and "Die Bildung der Samenfäden in Bläschen als allgemeines Entwicklungsgesetz" (The formation of the spermatic filaments in cells, as a general law of Development), in "Denkschr. d. schweiz. naturf. Gesellsch.," Bd. VIII. 1846; Krämer, "Obs. microsc. et experimenta de motu spermatozoorum," Gott., 1842 ; Fr. Will, "Ueber die Secretion des thierischen Samens" (On the secretion of the semen of animals), Erlangen, 1849 ; R. Wagner and Leuckart, art. "Semen," in Todd's "Cycl. of Anat." Jan. 1849; Newport, "On the impregnation of the ovum of the Amphibia," in "Phil. Trans." 1851, I.; B. Panizza, "Osservazioni anthropo-zootomico-fisiologische," Pavia, 1836; J. Müller, "Entdeckung der bei der Erection wirksamen Arterien" (Discovery of the Arteries which effect Erection), "Arch." 1835, p. 202; G. Valentin, "Ueber den Verlauf der Blutgefässe in dem Penis des Menschen" (On the course of the bloodvessels in the human penis), Müll. "Arch." 1838; Kobelt, "Die Männlichen und Weiblichen Wollustorgane" (The male and female sexual organs), Freiburg, 1844; Herberg, "De erectione penis," Lips. 1844 ; Kölliker, "Ueber das Anat. und Phys. Verhalten der Cavernösen K.örper der Männlichen Sexualorgane" (On the anatomical and physiological relations of the corpora cavernosa of the male sexual organs), in "Verhandl. der Würzb. Med. Phys." Ges. 1851. [H. Hancock, "On the Anatomy and Physiology of the Male Urethra, 1852 ;" Fr. Leydig, "Zur Anat. d. männl, Geschlechtsorgane u. Analdrüsen der Saügethiere," in "Zeitschrift für wiss. Zool." Bd. II.; Waldo J. Burnett, "Researches upon the origin, mode of development, and nature of the Spermatic Particles among the four classes of Vertebrated 
Animals, in Mem. of Americ. Acad. of Arts and Sciences," V. part 1, 1853; H. Luschka, "Die Append ienlargebilde des Hoden," in "Virchow's Archiv," VI. 3, 1854.-DaC.]

\section{B. FEMALE SEXUAL ORGANS.}

$\S 203$. The female sexual organs consist: 1, of two follicular glands, in which the ova are formed - the ovaries-with the parovaria, and the two excretory ducts, which, however, are not directly connected with them,-the oviducts, or Fallopian tubes; 2, of the uterus, for the reception and nourishment of the foetus; and 3, of the parts subservient to the expulsion of the foetus, as well as to copulation-the vagina and external genitals.

$\S 204$. Ovary, parovarium.-The ovaries, ovaria, are constituted of special tunics, and of a stroma containing the ova, or the parenchyma. The former consist of a peritoneal coat, which covers all but the inferior border, and of a firm, white, fibrous coat, the tunica albuginea s. propria, $\frac{1}{4}$ of a line thick, which closely invests the whole parenchyma, and is intimately connected with it without any abrupt line of demarcation; but does not send any processes into the interior, like the corresponding coat of the testes, with which, otherwise, it precisely corresponds in structure. The stroma is a grayish-red substance, of tolerably firm

Fig. 263.

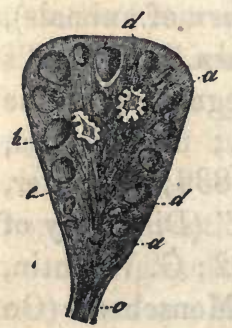
consistence, composed of a nucleated, tough, fibrous, though not distinctly fibrillar, connective tissue, in which are lodged the ovisacs and the vessels of the organ. From the inferior border of the ovary, where the vessels enter, and ovisacs are never situated, the stroma extends, in the form of a compact lamella, into the interior of the ovary, from which it then radiates, in larger and more slender bundles, towards both surfaces and the free border of the organ, so that, in a transverse section, a penicillar arrangement is presented by them. The ovisacs or follicles, usually termed Graafian vesicles, folliculi ovarii s. Grafiani, s. ovi-sacci, entirely closed, round follicles, from $\frac{1}{4}$ to 3 lines in mean size (Fig. $263 a, b$ ), are imbedded in the more peripheral portions of the stroma, so that, in a section, at all events, of well-developed and normal ovaries, the parenchyma separates, as it were, into a medullary and cortical substance, the latter of which only as it may be said, contains the follicles. Ovaries in that condition, also, should alone be made use of, for the obtaining of a correct notion

Fig. 263.-Transverse section through the ovary of a woman dead in the fifth month of pregnancy: $a$, Graafian follicle of inferior, and $b$, of the superior surface, $c$, peritoneal lamella of the lig. latum, continued upon the ovary, and coalescing with $d$, the $t$. albuginea; in the interior, two corpora albicantia (old corpora lutea) are visible; e, stroma of the ovary. 
of the size, position, and number of the Graafian follicles. The latter amounts to 30-50-100 in each ovary, and in many cases may reach 200 ; whilst in arrested or degenerated ovaries, such as are frequent especially in old women, not more than 2 to 10 , or even none at all, are often met with.

Each follicle, in the fully formed condition, consists of a membrane and contents. The former may be most aptly compared with a mucous membrane and presents: 1 , a highly vascular fibrous layer, theca folliculi v. Baer, s. tunica fibrosa, of a proportionately not inconsiderable thickness, which is united to the stroma of the ovary by a rather loose connective tissue, and consequently can be readily stripped off in its totality. Its external, somewhat firmer, reddish-white layer (Fig. 264 a), has been distinguished by v. Baer from the internal, thicker, softer, and redder portion (Fig. 264 b) ; but at the same time it should be remarked, that the inner layer also may be again divided, and that both lamince are composed of the same undeveloped, nucleated connective tissue, intermixed with numerous, mostly fusiform, formative cells. In young follicles a delicate, structureless, membrana propria, bounds the fibrous coat on the inner aspect and may, by the use of alkalies, even at a later

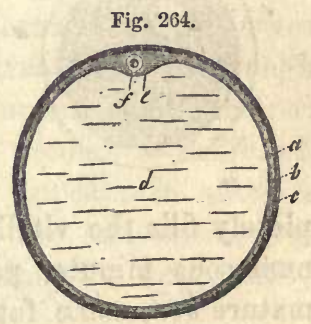
period, frequently be demonstrated as a distinct membrane. 2, an epithelium (granular layer, membrana granulosa of authors), (Fig. 264 $b, c)$. This membrane, $0.008-0.012$ of a line or more, in thickness, lines the entire follicle, and on the side looking towards the surface of the ovary, where the ovum is situated, presents a wart-like thickening, projecting towards the interior and enveloping the ovum-the germinal eminence, cumulus proligerus, $\frac{1}{3}$ of a line broad (Fig. 264 e). Its roundish, polygonal cells, $0-003-0.004$ of a line in size, with proportionately large nuclei, and, frequently, some yellowish fatty granules. disposed in several layers, are extremely delicate, and after death soon become indistinct, in consequence of which the whole epithelium presents the appearance of a fine granular membrane with numerous nuclei. The interior of the follicles is occupied by a clear, light-yeIlowish fluid-liquor folliculi - of the density of the serum of the blood, containing, almost always, isolated granules, nuclei, and cells, which are scarcely anything more than detached portions of the membrana granulosa, and do not originate in the fluid.

In the germinal eminence, close upon the fibrous membrane of the follicle, and therefore in the most prominent part of it, is placed the

Fic. 264.-Graafian follicle of the Sow, magnified about 10 diameters: $a$, external; $b$, internal, layer of the fibrous membrane of the follicle: c, membrana granulosa; $d$, liquor folliculi; $e$, germinal eminence, a projection of the membrana granulosa; $f$, ovum with a zona pellucida, vitellus, and gerıninal vesicle. 
egg (ovulum), imbedded in the cells of which the eminence is composed, and retained in its position by them. When the follicle bursts, or is ruptured, the ovulum escapes, surrounded by the cells of the cumulus, and the contiguous part of the epithelium, which encompass it, as a sort of ring or disc (discus proligerus, germinal disc, v. Baer), enclosing it, however, entirely, and by no means only attached to it in its greatest breadth. The ovulum itself is a spherical vesicle, measuring when mature 1-8-1-10 of a line, which, though in certain respects peculiar, nevertheless possesses the nature and constitution of a simple cell. The cell-membrane, or vitelline membrane, membrana vitellina, has the unusual thickness of $0.004-0.005$ of a line, and, in microscopical figures,

Fig. 265.

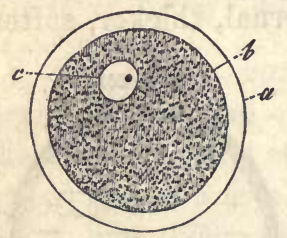
surrounds the contents, or yelk (vitellus), as a clear, transparent ring, whence it has received the name of zona pellucida. It is structureless, very elastic, and firm, so as to support a considerable degree of extension without, being torn; and in chemical characters corresponds, in every particular, with the membrance proprice, § 16. The light-yellow yelk, which in recent ovula completely fills the vitelline membrane, is composed of viscid fluid, having numerous minute, pale granules dispersed in it, with which, in the mature ova, some fatty granules are also associated, and, in the fullyformed ovum, contains, excentrically, a well-marked vesicular nucleus, 0.02 of a line, with clear contents, and a homogeneous, round, parietal nucleolus, 0.003 of a line in size, the germinal vesicle, vesicula germinativa (the "vesicle of Purkinje"), and the germinal spot, macula germinativa (or "spot of Wagner") as they are here termed.

The parovarium (Nebeneierstock)- a rudiment of the Wolffian body of the embryo, consists of a certain number of canals, $0.15-0.2$ of a line in diameter, diverging from the hilus ovarii into the "bat's wing," which in Man neither open into the ovarium nor are connected with any other parts, and contain nothing but a little clear fluid. The tubes are formed of a fibrous membrane, $0.020-0.024$ of a line thick, and of a single layer of pale, cylindrical, probably ciliated cells, and are of interest only as the remains of an embryonic structure.

The arteries of the ovary, derived from the aa. spermatica and uterina, and forming numerous minute trunks between the lamella of the broad ligaments, enter from the inferior border of the ovary, run in a serpentine course in the internal portion of the stroma, and terminate partly in the stroma itself and in the $t$. albuginea, but chiefly in the walls of the Graafian follicles, where they form an exterior more coarse, and an

FIG. 265.-Human ovulum, from a follicle of the average size, magnified 250 diam.: $a$, vitelline membrane (zona pellucida); $b$, external boundary of the yelk, and also internal boundary of the yelk-membrane; $c$, germinal vesicle, with the germinal spot. 
inner finer plexus, which extends as far as the membrana granulosa. The veins arise in the same situation, are in Man, for the most part, very beautifully displayed in the walls of the larger follicles, and terminate in the uterine and internal spermatic veins. A few lymphatic vessels come out from the hilus ovarii, and proceed, in company with the bloodvessels, to the lumbar, and pelvic glands. And with respect to the nerves, they arise from the spermatic plexus, enter as minute trunks with fine fibres, and "fibres of Remak," together with the arteries, into the ovary; but as respects their ultimate condition, they have not yet been investigated.

§ 205. Detachment and re-formation of the ova,-corpora lutea. From the commencement of puberty up to the period of involution, the ovaries are the seat of a continual detachment of the ova by dehiscence of the Graafian vesicles, which, independently of sexual congress, takes place in women and virgins, above all at the menstrual period, although it may and does frequently occur at other times, under conditions not yet accurately determined. In animals the same process is exhibited at the time of "heat," although sexual congress appears to afford a necessary impetus to its completion; and in them the anatomical processes may be more completely traced, whilst, in Man, the opportunity for such observations is much more rarely afforded.

When the Graafian follicles approach the time of bursting, they gradually enlarge to a circumference of 4 to 6 lines and more, and are continually brought more and more near to the surface, until they project beyond it, as wartlike or hemispherical elevations, covered only by a thin pellicle of the much attenuated $t$. albuginea, with its peritoneal lamella. At the same time their vessels are remarkably multiplied, and by the continual exudation from them, the liquor folliculi is rendered more and more abundant, whilst the fibrous coat of the follicle, at the bottom and sides, but not where the ovulum is situated, becomes thickened towards the interior; the membrana granulosa also swells a little, and contains larger cells (up to 0.01 of a line.) When these processes have advanced to a certain point, the thin, opposing coats can no longer withstand the continued and ever-increasing pressure from the interior of the follicle; they give way at the most elevated, and most thinned point, exactly where the ovulum is situated, and this body, surrounded by the cells of the germinal eminence, if the oviduct has applied itself exactly over the follicle, escapes into it. But the vital course of the Graafian follicle is not hereupon concluded, for now a series of partly new formations is presented in it, in consequence of which it at first - becomes a corpus luteum, as it is termed, and ultimately disappears altogether.

These corpora lutea are displayed in the most complete state, when 
conception and pregnancy ensue upon the detachment of the ovum, and, when in perfection, appear as roundish or oval, firm bodies, mostly rather larger than the former follicles, and are usually visible even on the exterior as projections, exhibiting on the summit a stellate cicatrix, arising from the rupture of the Graafian follicle. Exteriorly, these bodies are bounded, towards the stroma of the ovary, by a thin whitish fibrous membrane (Fig. 266, $2 f$ ), succeeded by a yellowish vascular lamella, which is much plicated, and consequently appears thicker (Fig. $266 \mathrm{c}$ ); and in the interior is a larger or smaller cavity filled, either with coagulated blood (blood-clot), or with a somewhat gelatinous fluid tinged with blood (Fig. $266 d e$ ). With respect to the origin of these bodies, it is easily perceptible, that their "nucleus" or contents consist of the blood poured out upon the rupture of the follicle, frequently mixed with some remains of the liquor folliculi, and that the outer fibrous membrane is the external layer of the original fibrous coat of the follicle; and as regards the yellow plicated cortical layer, this is referable for

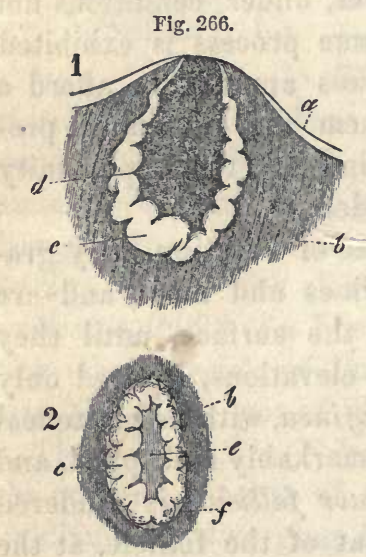
the most part to the internal layer of the fibrous membrane of the original follicle, which, even before the expulsion of the ovum, becomes loosened in texture, and afterwards rapidly expanded to the thickness of $\frac{1}{4}-\frac{1}{2}$ a line and more. The remains of the epithelium, or membrana granulosa, which were not expelled with the ovum from the follicle, also seem to contribute to this thickening, though in a subordinate degree, and by no means in the same proportion as the layer in question; the increased thickness of which is accompanied by the development of a vast number of smaller and larger cells, which are, in part, transformed into immature connective tissue and vessels, in part remain in the condition of cells, characterized by their size, which reaches as much as $0.01-0.2$ of a line, their well-marked, vesicular nucle $i$ with nucleoli, and a greater or less number of yellow oil-drops in the interior. The corpus luteum, thus constituted, retains its original size for some time, up to the second or third month of pregnancy; because, whilst the contents (whether these are a blood-clot, or a reddish gelatinous substance with a small cavity in the interior) gradually diminish and lose their color, the yellow cortical layer continues to increase in thickness, and, at the

FIG. 266.-Two corpora lutea, of the natural size, in a transverse section. 1, quite recent, eight days after conception; 2 , at the fifth month of pregnancy: $a, t$, albuginea; $b$, stroma ovarii ; $c$, thickened and plicated fibrous membrane of the follicle (inner layer); $d$, blood-clot within it; $e$, discolored blood-clot; $f$, fibrous coat bounding the corpus luteum. 
same time, its tissue to become more organized and more compact; this change taking place by the transformation, on the one side, of the internal substance into fibrous tissue, and on the other by the more intimate fusion of the yellow cortex with it, and the more abundant development of immature connective tissue in the former. In the fourth and fifth months the atrophy of the corpus luteum commences, and is slowly continued to the end of pregnancy; so that in persons dead in childbed, it still measures 4 lines on the average, but afterwards more rapidly, until ultimately, after some months, the metamorphosed Graafian follicle has entirely disappeared, or become reduced to a diminutive, variously colored corpuscle, which undoubtedly may still exist for a long time, and, perhaps, is not removed altogether for some years. Such arrested corpora lutea (corpora albicantia and nigra, of authors) at first retain a distinct limitation, a dentate nucleus, containing a minute cavity of a grayish white, or red-brown, even black color depending upon altered hematin, and a cortical substance presenting various tints of yellow or yellowish-white, or even quite white, and often still distinctly plicated, but subsequently they become mere amorphous spots, coalescent with the stroma of the ovary. Their elements are fibres, more of an embryonic character, such as also form the ovarian stroma, together with various pigmentary molecules and colored crystals (hæmatoidin), and a whitish-yellow fat, which latter at first occurs in the cortical substance still contained in larger, round, elongated, or fusiform cells, but is ultimately liberated by their rupture, and at last subjected to a more or less complete absorption.

In the corpora lutea, which are not formed at the time of a pregnancy, the same processes, in general, take place as in the others, but with much greater rapidity; so that these bodies have usually entirely disappeared in the space of one or two months, or left only the merest trace, whence they never possess the peculiar conformation of the others, which have been termed the true corpora lutea.*

The place of the numerous follicles which disappear from the ovaries during the whole of the vigorous periods of life, is supplied by the constant production, even in the adult, of new ovisacs, which are developed into Graafian follicles. In animals, these new formations which take place at the time of heat, and were first noticed by Barry, Bischoff, and Steinlin, are very abundant, and very easily observed,

* [Besides the difference in size and the rapidity of their metamorphoses, the true corpora lutea are distinguishable from the corpora lutea formed in the non-pregnant state by the thickness of the wall, and by their different color. The convoluted wall of the true corpus luteum is almost always twice as thick, whilst its color and that of the central coagulum, is never of so decided a yellow, but has generally more of a dusky and indefinite hue; a difference which is probably owing to a less number of yellow oil-globules. (Vide Dalton's Prize Essay, "On the Corpus Luteum of Menstruation and Pregnancy," Transactions of the American Medical Association for 1851.)-DaC.] 
whilst in Man no opportunity has as yet been afforded of noticing them, and it is only from the circumstance, that in this case also in normal ovaries, follicles of the most various sizes are always met with, that a continual formation of them may be concluded to take place. In Man, it is also probable, that the times of conception and of menstruation are those in which, especially, these productions take place, which in animals, as respects their histology, originate in exactly the same way, as will be afterwards described when we speak of the first follicles of the embryo. [See Appendix, § corpora lutea.-Trs.]

\$ 206. Oviducts and uterus. - Of the three coats of the oviduct, the most external, which belongs to the peritoneum, presents nothing worthy of remark. The middle, or smooth muscular coat, is of tolerable thickness, especially in the internal half of the duct; and consists of external, longitudinal, and internal transverse fibres, the elements of which, even at the time of pregnancy, can be isolated with some difficulty, and are intermixed with much more undeveloped connective tissue of the same form as in the stroma of the ovary. The innermost coat is the mucous membrane, a thin, whitish-red, soft layer, which is connected with the muscular tunic by a small quantity of submucous connective tissue, presents no glands or villi, though it has a few longitudinal folds, and consists of undeveloped connective tissue, with many fusiform formative cells. On its inner surface, from the uterus to the free border of the fimbrice, lies a single layer of conical or filiform, ciliated cells, of $0.006-0.01$ of a line, whose distinct cilia effect a current running from the ostium abdominale to the ost. uterinum, which probably assists in the locomotion of the ovula, but not of the spermatic fluid.

The uterus is constituted in the same way as the oviducts, except that the muscular coat and mucous membrane are much stronger, and, in some respects, differently constructed. In the pale red muscular coat, three layers may, most conveniently, be distinguished, which, however, cannot, as elsewhere (in the intestine for instance), be sharply defined from each other. The external layer is composed of longitudinal and transverse fibres, the former of which, forming a continuous, thin stratum, intimately united to the serous coat, extend over the fundus and the anterior and posterior surfaces, as far as the cervix, whilst the stronger transverse fibres surround the organ, and are also, to some extent, continued beyond the uterus, into the ligg. rotunda, ovarii, and lata, and upon the oviducts. The middle layer is the strongest, presents transverse, longitudinal, and oblique, flat bundles, - which are interlaced in a complex manner, and contains larger vessels, chiefly veins, whence, especially in the pregnant uterus, it presents a spongy appearance. The innermost layer, lastly, is again thinner, and 
formed of a network of more slender, longitudinal, and of stronger, transverse, and oblique fibres, which, at the openings of the oviducts, frequently form very distinct rings. In the fundus, where the uterus is thickest, the middle layer is strongest, and often appears to be composed of several lamina; whilst, at the thinner cervix, transverse fibres especially, intermixed with isolated longitudinal ones, are met with. In the neighborhood of the external os uteri, and in that part itself, highly developed transverse fibres lie immediately beneath the mucous membrane, and may be described as an occlusor of it-sphincter uteri. As to the elements, all these layers consist of short $(0.02-0.03$ of a line) fusiform fibre-cells, with elongated oval nuclei, which, on account of the great quantity of the nucleated embryonic connective tissue, of the same constitution as in the stroma ovarii, by which the layers are pervaded, can only with difficulty be isolated, and even with the aid of nitric acid, of $20 \%$, are not brought into view so distinctly as elsewhere.

The mucous membrane of the uterus is of a white or whitish-red color; it is closely united with the muscular coat, from which it cannot be raised; in transverse sections, however, it is distinguishable from it by its, mostly, brighter color, though rarely presenting any marked line of demarcation. Except in its fundamental substance, consisting of the connective tissue which exists everywhere in the female genital organs, containing undeveloped nuclei and fibre-cells without elastic elements, and the epithelium, which throughout appears as a ciliated epithelium with pale cells, as much as 0.016 of a line in size, and delicate cilia which vibrate from without to within, the mucous membrane is differently constituted in the body and fundus, and in the canal of the cervix. In the former situations it is more delicate, redder, and thinner (from $\frac{1}{2}-1$ line), smooth on the inner aspect, and without papilloe, but occasionally presenting a few large folds. In it are found very numerous minute glands, the utricular glands of the uterus, or uterine glands (glandulce utriculares s. uterinoe), which bear the closest resemblance to the Lieberkühnian glands of the intestine; they exhibit the form of thickly placed follicles, either simple or bifurcated, and not unfrequently spirally contorted at the end, and in length correspond with the thickness of the mucous membrane, being $0.02-0.03$ of a line broad. They consist of a very delicate structureless membrane, and a uniform cylinder-epithelium, and open either singly or two or three together, with orifices 1-30th of a line wide. Normally, these glands contain no morphological elements at all, but it is probable that their epithelium is very easily detached, and may appear as a grayish-white secretion filling them.

In the cervix the mucous membrane is whiter, denser, and thicker (1-1 $\frac{1}{2}$ lines), particularly on the anterior and posterior walls, where the well-known plicce palmatoe are situated; between which are found larger 
and smaller, sinuous fossæ lined with cylinder-epithelium, and as much as 1 line and more in depth, and differing very essentially from common mucous follicles, although, as the secreting organs of the viscid, crystalline mucus of the cervix uteri, they may be designated the mucous follicles of the uterus. In this region also occur, in great abundance, closed vesicles, $\frac{1}{3}-1-2$ lines and more in size, filled with the same secretion, and composed of a layer of connective tissue and short cylindercells, the so-termed ovula Nabothi, which, like the Graafian follicles, might perhaps be regarded as closed glandular vesicles, bursting periodically, but which probably are nothing more than dilated and closed mucous follicles, and in part also pathological new formations; they are likewise occasionally found in the mucous membrane of the body of the uterus. The inferior third or half of the cervical canal contains verrucose or filiform papillæ, $0 \cdot 1-0 \cdot 3$ of a line long, clothed with ciliated cylinders, containing a single or several vascular loops, with very numerous minute nuclei, and also, perhaps, pale oil-drops in their interior.

The distribution of the vessels in the unimpregnated uterus, does not present much of a special nature. The larger arterial branches run in the muscular substance, and ramify thence on both sides in the muscular and mucous coats. The latter, as everywhere, has larger vessels in the deeper, and finer in the superficial portion, and these, after they have surrounded the glands with smaller capillaries, form an extremely rich and delicate plexus of larger vessels $(0.006-0.01$ of a line) on the surface, from which arise the wide, thin-walled veins, unfurnished with valves, which follow the course of the arteries towards the exterior. The lymphatics, probably commencing in the mucous membrane, are remarkably numerous, form coarser or finer networks under the peritoneal investment and proceed, in numerous, considerable trunks, accompanying the blood-vessels, in part to the pelvic glands, in part, with the vasa spermatica, to the lumbar plexus. The nerves of the uterus, containing numerous fine, and some thick nerve-fibres coming from the hypogastric and pudendal plexuses, and united in a plexiform manner, reach the uterus through the broad ligaments, and ramify, following principally the course of the vessels in the muscular substance, from the fundus to the cervix, in which latter situation they are the most abundant. They are white and, in the uterus, are not furnished with any ganglia; their condition in the mucous membrane, and their terminations elsewhere, are unknown.

Of the ligaments of the uterus, the ligg. lata, anteriora, and posteriora, are duplicatures of the peritoneum, which contain, together with the vessels and nerves passing to and from the uterus, a considerable number of smooth muscular fibres continued into them from the uterus. The same tissue also arising from the uterus, occurs more sparingly in the ligg. ovarii, and in very considerable number in the ligg. rotunda, 
in the form of longitudinal bundles surrounded by connective tissue, with which at the internal abdominal ring a good many transversely striated muscular fibres, often extending nearly to the uterus, are associated.

\$207. Changes in the uterus at the menstrual period and in pregnancy.-At the menstrual period, the whole uterus enlarges, and its texture expands, which is perhaps to be attributed, chiefly, to the distension of the vessels, and the considerable infiltration of the entire organ with blood-plasma; at all events, beyond a greater facility in the demonstration of its elements, I have been unable to perceive any further alteration in the muscular coat. The mucous membrane, on the other hand, in many cases really increases, being thickened to 1-2, even 3 , or, in its projecting folds, even to 5-6 lines; it becomes softer and presents, in its tissue, well-marked, easily isolated, utricular glands, 1-3 lines long, and 0.036-0.04 of a line broad, and numerous immature, round and fusiform cells. The bloodvessels of the mucous membrane, which chiefly afford the menstrual flux, are throughout the uterus, and particularly in its body and fundus, extremely numerous and much distended, and this is especially the case with the superficial capillary plexus; whence also, the mucous membrane presents a bright red color. With the escape of the blood from the superficial, ruptured capillaries, the epithelium of the mucous membrane is, in great measure, thrown off, except that of the cervix, and may always be found in large quantity in the mucus mixed with blood, which fills the cavity of the uterus ; it is not, however, to be regarded as normal, if, after the menstrual period, or during it, the whole uterine mucous membrane or portions of it are detached. After the menstrual period, the parts rapidly regain their pristine condition, and the epithelium is restored.

Changes of a totally different kind are induced in the uterus by pregnancy, among which, however, in a Fig. 267.

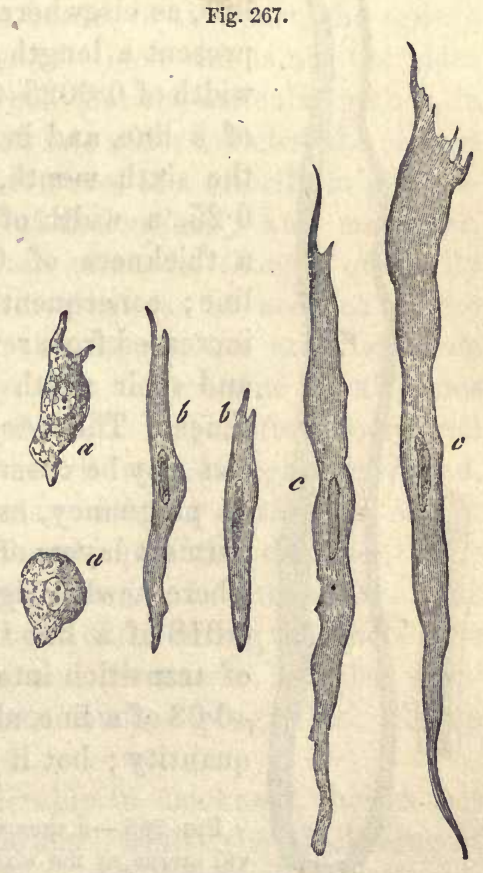
microscopical point of view, the in-

Fra. 267.-Muscular elements from the uterus, in the fifth month of pregnancy; $a$, formative cells of the muscular fibres; $b$, younger; $c$, developed, fibre-cells.-Magnified 350 diameters. 
creased bulk of the organ only, is of interest. This enlargement, as

Fig. 268.

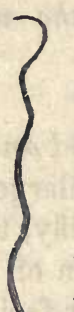
is well known, depends upon the great augmentation of the circumference and of the cavity; at first with increased, and afterwards (usually from the fifth month onwards) with a diminished thickness of the walls, and an increase in bulk, amounting on the average to twentyfour times the original size (J.F. Meckel, "Anat.," IV. 691). The mode in which this change is brought about was, as regards the histological conditions, until a recent period, it may be said, entirely unknown; but, in the main point, is now sufficiently made out. The principal changes occur in the muscular coat, to which the increased volume of the uterus is chiefly to be assigned, and there are two processes which participate in common in its production: in the first place, an enlargement of the pre-existing elements ; and secondly, a new formation of them. The former is so considerable, that the contractile fibre-cells, instead of a length of $0.002-0.003$ of a line, and width of 0.002 of a line, as elsewhere, in the fifth month present a length of $0 \cdot 06-0 \cdot 12$, and width of $0.0025-0.006$, or even 0.01 of a line, and in the second half of the sixth month, a length of $0 \cdot 1-$ 0.25 , a width of $0.004-0.006$, and a thickness of $0.002-0.0028$ of a line; consequently, their length is increased from seven to eleven times, and their width from twice to five times. The new formation of muscles may be observed in the first half of pregnancy, especially in the innermost layers of the muscular coat, where newly originating cells, $0 \cdot 01$ 0.018 of a line in size, in all stages of transition into fibre-cells of 0.02 -0.03 of a line, always occur in great quantity; but it takes place also in

Fig. 268.-a, muscular fibre-cell from a gravid uterus, at the sixth month; $b$, its middle portion, after treatment with acetic acid, and

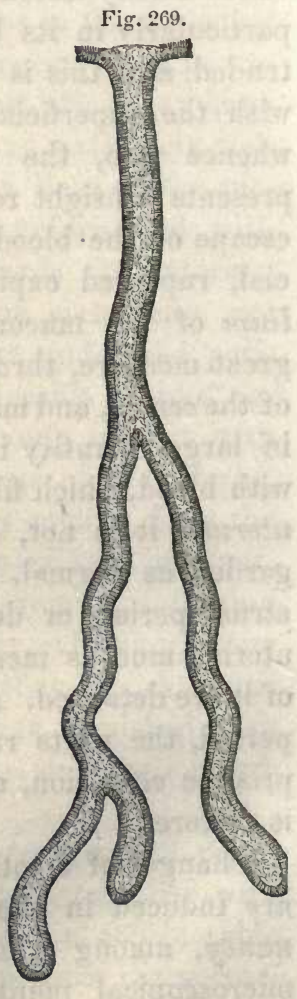
exhibiting the appearance of a membrane; $c$, nucleus of the fibre-cell.-Magnified 350 diameters.

FIG.269.-A uterine gland from a woman in the first pregnancy, eight days after conception. 
the outer layers. After the sixth month, this origination of muscles seems to cease; at least in the twenty-sixth week, in the whole uterus I have found nothing but the above-mentioned colossal fibre-cells, and no longer any trace of their earlier forms. Like the muscles, the fibrous tissue which unites them also increases, and towards the end of pregnancy, occasionally exhibits distinct fibrils. Whilst the muscular coat grows in this way, the mucous membrane has also undergone manifold changes. It is in it, especially, that the metamorphoses of the gravid uterus commence, seeing that as early as the second week, it becomes thickened to 2-3 lines, is softer, more lax, and redder, acquires more prominent plicce, and is more distinctly defined from the muscular coat; peculiarities which, as time goes on, become more and more marked. Examined microscopically, it is apparent, that not only are the vessels more distended, but also that an abundant new formation of connective tissue has taken place in its parenchyma, and a considerable enlargement of the utricular glands, which latter are, at this time, 2-3 lines long, and $0.04-0.11$, or, on the average, 0.08 of a line broad. As these changes proceed, the greater part of the hypertrophied mucous membrane is transformed into the well-known decidua vera, whilst another portion, at the point of attachment of the ovum, is converted into the placenta uterina, and by a growth from the border of this part, the reflexa is produced around the ovum; processes of which this is not the place to speak farther. It can only be remarked that the utricular glands, in the decidua vera, are gradually converted into wider follicles, the orifices of which give rise to the appearance, as it were, of a cribriform perforation in that membrane and the border of the reflexa; and moreover, that the deciduce, from the second month onwards, though gradually diminishing, it is true, in thickness, nevertheless, on account of the enlargement of the internal surface of the uterus, are still far from ceasing to increase in bulk; and lastly, that their tissue at all times consists of larger and smaller, round cells, with large, often compound nuclei, in part of colossal fibre-cells with well-formed large nuclei, and, particularly in the decidua vera, of vessels; whilst in epithelium, except in the first month, is no longer to be found upon the deciduce. The mucous membrane of the cervix takes no part in the formation of the deciduce, and retains its epithelium (without cilia) during the whole time of pregnancy. It also, however, swells, and its mucous follicles, especially, enlarge and secrete the well-known mucous plug which fills up the entire canal of the cervix.

The serous coat also increases considerably in thickness, though not to the same extent as the mucous membrane; whilst the thickening of the uterine ligaments, particularly of the round ligaments, is very evident, and also depends upon changes in their smooth muscles similar to those described in the muscles of the uterus, and probably also, upon an increase of the transversely striated bundles. The growth of the 
bloodvessels and lymphatics, in length and calibre, is also very evident, and is, in great part, to be referred to the enlargement and new formation of muscular elements, which, in the veins, are also demonstrable in the $t$. adventitia and intima. With respect to the nerves, they also become thickened, although it is doubtful whether new nerve-fibres are really produced in them. On the other hand, it is certain that the preexisting elements increase in width and length, retain their dark borders for a greater distance, and may be traced further into the interior than at other times.

The lessening of the uterus after parturition, and its restoration to a condition, not, indeed, similar to the previous state, though closely approximated to it, does not take place in its various portions exactly in the same way. In the muscular coat, an atrophy of the contractile fibrous elements manifestly plays a principal part, since, as early as three weeks after parturition, these fibres are again as

Fig. 270.

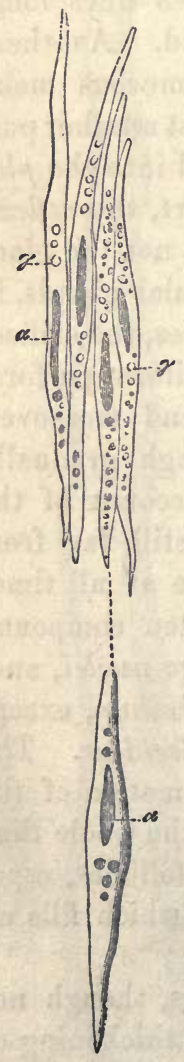
short ( 0.03 of a line) as in the virgin uterus, fat, at the same time, being developed in their interior; but a complete absorption of certain muscular fibres is also probably superadded to this. A different process takes place in the mucous membrane, which, in the form of the deciduce and placenta uterina, is completely thrown off after parturition, and consequently has to be entirely formed anew. The intimate nature of the processes accompanying this unique kind of regeneration has not yet been traced, though it is more than probable that it is completed as early as within the first two or three months after parturition. It is evident that, besides this, the serous coat, the vessels and nerves of the uterus, return to their former condition, but the precise nature of this change in them has not yet been investigated.

It has been generally assumed, since Tiedemann, that the nerves of the gravid uterus are thicker than in the virgin state; but quite recently this has been altogether disputed by Dr. Snow-Beck, and is only partially admitted by Jobert de Lamballe ("Compt. rend.," 1841, Mai), inasmuch as that he states that it is the investing connective tissue, but not the nerves themselves, that is thickened. It is evident that very accurate microscopic investigations are alone competent to decide this question; but such investigations are wanting. No

Fig. 270.-Muscular fibre-cell of the uterus, three weeks after parturition, four of them treated with acetic acid, and pale: $a$, nuclei; $g$, fat-granules.-Magnified 350 diameters. 
conclusion, in the first place, can be drawn from Remak's statement (l. c.), that the nerves, at the time of pregnancy, enlarge and acquire a gray color, a change depending upon an increase of nucleated fibres, because no grounds exist upon which it can be decided whether these nucleated fibres are embryonic nerve-fibres, or a form of connective tissue. On the other hand, we are indebted to Kilian for careful researches in animals, which prove with certainty that the uterine nerves, at the time of pregnancy, may be traced further into the substance of the uterus, in the form of dark-bordered fibres; whilst at an earlier period, in part even before they enter the uterus, in part when they have scarcely reached it, they possess the nature of embryonic non-medullated fibres. For this reason, Kilian succeeded also in tracing the nerves in the gravid uterus much further in to the parenchyma than at other times. He perceived no evidence of a formation of new nerve-fibres in the trunks, and regards such an occurrence as improbable; for otherwise a new formation of ganglionic substance must also be assumed, which is unlikely. Something of the kind appears to me by no means impossible, because the multiplication of the ganglion-cells and of the fibres would only take place once-in the first pregnancy; it is also conceivable that newly formed nerve-fibres are added to the others simply as branches, and consequently it will be more prudent to wait and see upon which side Remak's statements, with respect to the human subject, incline. Upon this, however, I would also remark, that a thickening of the nerves may undoubtedly be produced by an increased size of the already existing fibres and an augmentation of the neurilemma, and that the nerves, by a multiplication of their ultimate divisions, are fully enabled to ramify over larger spaces than at other times.

The increased size of the vessels, both of the arteries, and, above all, of the veins, at the time of pregnancy, is very considerable; owing to which, at this period, the middle layer of the muscular substance, containing the larger vessels, is much more distinct from the other two. The alteration which takes place in the vessels of the mucous membrane, at the point where the placenta is formed, cannot here be entered upon; and I will only remark that I agree with those who believe that large vascular trunks exist in the human uterine placenta, at the border and on the convex surface, whilst in the interior there are only lacunce without walls, between the villi of the chorion (vide Kiwisch, "Geburtskunde," I. p. 151, et seq.; C. Wild, "Zur Physiologie d. Placenta," Wurzb., 1849; Virchow, "Archiv," III. p. 449; Schröder v. d. Kolk, in the "Verh. d. Nied. Instituts," 1851). In the rest of the decidua, the capillaries are frequently excessively enlarged; according to Virchow ("Archiv. f. path. Anat.," III. p. 436), its superficial capillaries, in the sixth week of pregnancy, reach the size of $0.027-0.045$ of a line, and become extremely thin-walled, as are, probably, also those in the part 
where the placenta is situated, before their walls disappear and their cavity is thrown into that of the lacunce. In the venous trunks of the gravid uterus, besides the circular muscular layer, with much enlarged fibre-cells, which exist, also in other situations, I have found an external and internal longitudinal muscular layer with similar colossal elements; so that here the increase of the walls may be directly demonstrated ("Zeitsch. f. wiss. Zool." I. 84).

§ 208. Vagina and external sexual parts. The walls of the vagina, 1 line thick, consist of an external fibrous coat, a middle muscular layer, and a mucous membrane. The thin white fibrous coat presents externally a more lax, towards the interior a more dense connective tissue, with numerous elastic fibres and venous plexuses, and passes, without any line of demarcation, into the second, redder layer, which, together with connective tissue and numerous veins, contains, particularly during pregnancy, a good many, developed, smooth muscular fibres, which, with their transverse and longitudinal bundles of fibre-cells, $0.04,0.08$ of a line long, constitute a true muscular membrane. The mucous membrane is of a pale red color, with numerous larger and smaller folds and elevations-the columnoe rugosce; it is composed of a dense connective tissue, without glands, and containing elastic elements in extreme abundance, to which its great firmness and extensibility are due. Its inner surface presents numerous conical or filiform papillæ, from 0.06 to 0.08 of a line in length, and 0.025 to 0.03 of a line in breadth, which are entirely imbedded in a tessellated epithelium, $0.07-0.09$ of a line thick, of the same kind as that in the œsophagus, the uppermost scales of which, having a diameter of $0.01-0.015$ of a line, contain nuclei of 0.003 of a line. The hymen is a duplicature of the mucous membrane of the vagina, and contains the same elements.

From the vayina the mucous membrane is also continued upon the external genitals, invests the glans clitoridis and the vestibule, with the orifice of the urethra; folds of it constituting the preputium clitoridis and labia minora. On the labia majora, it is continuous uninterruptedly with the external integument, which, on their inner side, and at the commissura labiorum, still bears a close resemblance to a mucous membrane; whilst on their border and outer surface, and on the mons veneris, it resembles the cutis in all respects. The matrix of the mucous membrane of the external genital organs, is a spongy, highly vascular, fatless connective tissue, containing, however, a good many elastic fibres, and which, in its condensed external layer, $\frac{1}{4}-\frac{1}{5}$ of a line thick, corresponding to the corium, is everywhere furnished with much-developed papilloe, in the labia minora 1-10-1-20, and on the clitoris 1-24-1-33 of a line in length; and with a squamose epithelium of $0.04-0.12$ of a line, the most superficial cells of which are $0.01-0.02$ of a line in size (Fig. 
$56,4)$. The labia majora, in the structure of their coverings, correspond partly with the mucous membrane, in part approach the cutis, and contain, in the interior, common adipose tissue.

The external genital organs are furnished with various smaller and larger glands. Sebaceous glands, mostly of a rosette-form and considerable size ( $\frac{1}{4}-1$ line), occur in the labia majora, on the external and internal aspects, in connection with larger and smaller hair-follicles; moreover, in larger quantity in the labia minora, for the most part without hairs and rather smaller (from $\frac{1}{10}$ to $\frac{1}{2}$ a line); occasionally, also, around the orifice of the urethra, and laterally at the entrance of the vagina. Common racemose mucous glands, $\frac{1}{3}-1 \frac{1}{2}$ lines in size, with scarcely visible or tolerably wide openings, and having excretory ducts, either short, or as much as 6 lines long, exist in very various number around the orifice of the urethra, in the vestibule, and in the lateral portions of the entrance of the vagina. Lastly, the two "glands of Bartholini," corresponding to Cowper's glands in the male, are situated at the inferior extremity of the bulbi vestibuli; they are common racemose mucous glands, 6 lines in size, with pyriform gland-vesicles lined with a tessellated epithelium, $0.02-0.05$ of a line in diameter, and lodged in a compact nucleated connective tissue without muscular fibres. The excretory ducts of these glands, 7-8 lines long, and $\frac{1}{2}$ a line wide, have, external to their mucous membrane, invested with a cylinder epithelium 0.01 of a line thick, a delicate longitudinal layer of smooth muscles, and always contain a viscous, amorphous, clear, yellowish mucus.

The clitoris, with its two corpora cavernosa and glans attached to the bulbi vestibuli, the divided corpus cavernosum urethrce of the female, present, on a small scale, precisely the same conditions as the corresponding parts and corpora cavernosa of the male; and in them the muscular elements are even more readily isolated than in man.

The bloodvessels of the vagina and of the external genital organs, present, upon the whole, nothing much worthy of remark. In the papilloe of the various situations where they occur, we find, for the most part, simple vascular loops; it is only when the papillæ are larger or compound, such as abound around the orifice of the urethra, that more complex loops occur. The corpora cavernosa have the same structure as in man; and, according to Valentin, helicine arteries also appear to exist in the clitoris. The venous plexuses in the walls of the vagina, above the bulbi vestibuli, are extremely rich; but by no means, as Kobelt assumes, represent true corpora cavernosa. The lymphatics of the external genital organs, and of the vagina, are numerous, and communicate partly with the inguinal glands, partly with the pelvic plexus. The nerves, lastly, are derived in part from the sympathetic, in part from the pudendal plexus, and are extremely numerous, especially in the clitoris, but are also found without difficulty in the mucous 
membrane of the vagina. In the latter situations they present divisions, and their terminations have as yet been but little investigated. I have never found nerves in papillæe containing vessels, whilst, in the clitoris, I have sometimes met with them in non-vascular, minute verrucosities, which also contained rudimentary axile corpuscles; and I think I have noticed here, as well as on the surface of the mucous membrane itself, finer and coarser looplike terminations of nerves lying buried in the bodies resembling axile corpuscles, which are also occasionally met with in these situations. In the clitoris of the Sow, Dr. Nylander, of Helsingfors, found Pacinian bodies, which I have also seen; and looped terminations of the nerves in the papilloe.

§ 209. Physiological remarks.-In their development, the internal female genital organs, as was noticed above in $\$ 202$, entirely correspond, originally, with those of the male; and it is not till after some time that a difference in the histological development of the sexual glands is manifested, consisting in this, that in the female, the Wolffian body, except that it forms the parovarium, stands in no farther relation to the genital apparatus, whilst the "ducts of Müller" are formed into the oviducts, uterus, and vagina. As regards the histological conditions, the ovaries alone seem to present any great interest. These bodies are composed at first of common formative cells, $0.005-0.009$ of a line in

Fig. 271.
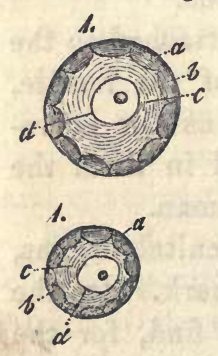
size, which afterwards pass, in part, into fibres and vessels, in part persist as cells, multiply spontaneously, probably by division, and serve for the formation of the Graafian follicles. These, according to Barry, at first appear as spherical agglomerations, 0.01 of a line in size, of some few cells, which contain, in the interior, a clear vesicle-the germinal vesicle; but, by the formation of a delicate, structureless membrane on the exterior, around the cells, which then represent an epithelium, soon assume the nature of follicles. Very young Graafian follicles of this kind (ovisacs, Barry) occur by thousands in the ovaries of nearly mature embryos, and of new-born children, in which the further development is very easily traced. Whilst the follicle increases by the multiplication of the cells of its epithelium (the membrana granulosa), and at the same time acquires an external vascular, fibrous coat; a clear substance, in Man containing but few granules, is collected in the interior, detaching the germinal vesicle,

FIa. 271.-Three Graafian follicles from the ovary of a newly-born female child, magnified 350 diameters. 1, without; 2, with, acetic acid: $a$, structureless membrane of the follicle; $b$, epithelium (membrana granulosa); $c$, yelk; $d$, germinal vesicle with spot; $e$, nucleus of the epithelial cells; $f$, vitelline membrane, very delicate. 
- $0.0065-0.008$ of a line in size, with a germinal spot of $0.001-0.0015$ of a line, from the epithelium, to which at first it was closely applied, and forcing it into the centre of the follicle. When this has attained the size of 0.02 of a line, a membrane in close apposition with the membrana granulosa, and surrounding the germinal vesicle and the whole contents of the follicle-the vitelline membrane-becomes evident; which is regarded by all authors as a secondary formation, although it probably exists, even in the very earliest rudiment of the follicle, as an extremely delicate membrane, closely surrounding the germinal vesicle. At first excessively delicate, and scarcely perceptible, the vitelline membrane, when the follicle has increased in size and contains more fluid, becomes more distinct, owing to its removal from the wall of the follicle, and rapidly thickens. In follicles of 0.04-0.05 of a line, the ova are perfectly distinct and disproportionately large, with a delicate zona pellucida, and still lying very close to the walls of the follicle. The further development is apparent of itself; and I will only remark that, in the new-born child, follicles visible to the naked eye will be more rarely found; whilst such make their appearance even before puberty, although they undergo no considerable development before that period.

According to what has been said, the mode of origin of the Graafian follicle ranks in every respect with that of the tubular glands. The former is an agglomeration of cells, at first, perhaps, without cavity or contents, to which the structureless membrane is added, not by the coalescence of the outermost cells, but probably as an excretion from them, and thus is formed the follicle, which therefore exactly corresponds with a closed gland-vesicle, or a section of a tubular gland canal. How the germinal vesicle, and the vitelline membrane arise, is doubtful ; the former is either a nucleus of new formation, originating in the minute cavity of the follicle, about which a certain amount of vitellus is subsequently collected, the cell, or vitelline membrane not being formed until after this, from a sort of cell formation "around portions of contents;" or the whole ovum, with the germinal vesicle, is nothing else than the central cell of the primordial rudiment of the Graafian follicle, and consequently co-existent with it. In any case it corresponds to a cell, and the germinal vesicle is nothing but the cell-nucleus.

With respect to the physiological conditions of the mature, female sexual organs, much has already been remarked in the preceding pages; and it will, therefore, here be sufficient to say something about their movements and secretions. In the ovaries, whose stroma frequently presents a deceptive appearance of muscularity, I have in vain sought for muscles, with nitric acid of $20_{0}^{\circ}$, although in recent preparations microscopical appearances are occasionally obtained, which one is inclined to explain as belonging to that tissue. That the oviducts are capable of 
very active movements cannot be doubted, from the results of vivisections in animals, and microscopical researches in Man; and in opposition to V. Kiwisch ("Geburtskunde," p. 96) I do not understand why their application to the ovaries should not be brought about by movements in them, together with a kind of erection dependent upon increased fulness of the vessels, as has also been established by the experiments of Gendrin and Raciborski (1. c. p. 412-417), in two women dead during menstruation, and of Laahr ("De mutat. gen. mul. brevi post concept." Halis, 1843) in the case of one who was killed shortly after coitus. As regards the movements of the uterus, they are, at all events during parturition, very energetic, but take place even at other times. The muscular tissue is so disposed, that at first a universal contraction of the uterine cavity, but afterwards local, more or less extensive contractions, also may be performed with great ease. Thus, in the act of parturition, the cervix and the os uteri are at rest, whilst the fundus and body contract, contractions of the former parts and of the vagina not ensuing till subsequently. In convulsions, the whole uterus contracts closely round the child; in retention of the placenta, the contraction is entirely local and confined to the fundus. It is probable that movements take place at the time of menstruation and in the act of congress, but the fact has not been ascertained. In the latter case, an opening of the os uteri, and a dilatation of the canal of the cervix, are commonly supposed to take place. If this is to be regarded as a spontaneous action of the cervix, it would be justifiable, with Kiwisch (1. c. p. 103), to refuse assent to the supposition, for the radiating fibres described by Kasper, which alone could effect anything of the kind, do not exist; the fact, nevertheless, is conceivable, if we assume a relaxation of the muscular element in the cervix and os, together with a contraction, especially of the longitudinal fibres in the fundus and body. In comparing the uterus, as respects the disposition of its muscular element and its movements, with other organs, none affords so apt a comparison as the bladder, in which the muscular tissue is arranged essentially in the same way, and a physiological antagonism exists between the superior and inferior portions. The sensibility of the uterus, and of the internal parts of the female genital organs in general, is very slight; careful sounding of the uterine cavity causes no sensation; in like manner, contact with the vaginal portion is, frequently, scarcely felt, whilst these parts give pain upon more powerful pressure or traction, and when in a state of inflammation. The sensibility of the vagina increases towards the inferior portion; and as regards the external organs, the clitoris is rendered especially susceptible of sensation, by its abundant supply of nerves, as is also the entrance to the vagina, particularly at the orifices of the glands of Bartholini or Duverney.

The secretions of the female genital organs, except those of the ova- 
rium, are: 1, a whitish mucus in the uterus and vagina, which, in the former situation, is derived chiefly from the uterine glands, and probably differs in some respects from the other; 2 , a transparent viscous mucus in the cervix uteri (vide supra); 3 , the clear viscid secretion of the Bartholinian glands, which is poured out in large quantity in copulation; and upon excitation, as was noticed by Huguier and Scanzoni, it even frequently escapes in jets, which may be ascribed to the muscles of the excretory ducts; 4 , the secretions of the minute subaceous and mucous follicles of the external organs.

Investigation of the female organs. - The Graafian follicles should be examined as fresh as possible, when the membrana granulosa and ova will be seen in their natural relations. In ovisacs that have been longer kept, the former floats about in flocculi in the liquor folliculi, and the "germinal eminence" is also for the most part destroyed. In order to make sure of obtaining the ovulum, the position of which is readily perceived even in the still closed follicle, in certain animals, as in the Bitch, for example, a large carefully extracted follicle is opened under water, and the larger flocculi which escape are examined with a low magnifying power; it is also readily found when the contents of a follicle are carefully transferred to an object-bearer. In rough sections, or when the structure of the ovaries is teased out, ova are also always readily found, although this is not exactly the mode of seeking for them to be recommended. The muscular elements of the oviducts, uterus, vagina, \&c., are investigated by means of careful dissection, as also in fine sections of parts that have been hardened. Kasper especially recommends that the uterus should be boiled for 10 minutes in water, and then placed for 24 hours in the most concentrated solution of carbonate of potassa, or that it should be treated with pyroligneous acid, and the sections moistened with dilute acetic acid; whilst Schwartz, according to Reichert, dries the uterus hardened in alcohol, and renders the muscular fibres distinct by acting upon them for a short time with nitric acid of $20 \frac{0}{0}$. The method, also, employed by Wittich (p. 486), should be used, according to Gerlach. The contractile fibre-cells are nowhere more beautifully displayed than in the gravid uterus. The uterine glands are best shown at the menstrual period and in the first months after conception. The ciliated epithelium is only to be seen in perfectly fresh subjects, and best in the Fallopian tubes; the non-ciliated cells, on the other hand, are readily seen. The preparation of the external parts presents no difficulty, and the directions already given are applicable to the glands, nerves, papilloe, and epithelium.

Literature.-C. E. v. Baer, "De ovi mammalium et hominis genesi, epist.," Lips. 1827, and "Commentarius," in German, in Heusinger's "Zeitsch.," II. ; Coste, "Recherches sur la génération des mammi- 
fères," 1834 ; "Embryogénie comparée," Paris, 1837 ; " Etudes ovologiques," in "Annal. Franc. et Etrang. d'Anat. et de Phys.," II. 224, 1838; "Histoire générale et part. du developpement," Paris, 1847; A. Bernhardt, "Symbolæ ad ovi. mam. hist. ante prægnat.," Vrat., 1834, Diss. ; R. Wagner, "Ueber das Keimbläschen," in Müller's "Archiv," 1835, p. 373 ; "Prodromus hist. generationis," Lips., 1836; "Beiträge zur Zeugung u. Entwicklung," in "Denkschrift der bayr. Akad.," Bd. II. 1837, p. 511 ; M. Barry, " Researches in embryology," Series I. II. III. in "Philos. Trans.," 1838, 1840; Bischoff, "Beweis der von d. Begattung unabhängigen Reifung u. Loslösung d. Eier d. Säugethiere und des Menschen," Giessen, 1824 ; "Ann. d. Sc. Nat.," 3, Ser. II. 1844, 304; Pouchet, "Théorie positive de l'ovulation spontanée," Paris, 1847 ; Zwicky, "De corpor. luteorum origine," Turici, 1844 ; Kobelt, "Der Nebeneierstock des Weibes," Heidelberg, 1847; W. Steinlin, "Ueber die Entwicklung der Graafschen Follikel und Eier der Säugethiere," in " Mittheil der Zürcher naturf. Gesellschaft," 1847, p. 156 ; Fr. Tiedemann, "Tabulæ nervorum uteri," Heidelb., 1822; G. Kasper, "De structurâ fibrosa uteri non gravidi," Vratisl., 1840; E. H. Weber, "Zusatze zur Lehre vom Bau der Geschlectsorgane," Leipzig, 1846 ; A. Kölliker, "Ueber die glatten Muskeln der weiblichen Genitalien," in "Zeitschr. für wissensch. Zool.," I.; Fr. Kilian, "Die Structur des Uterus bei Thieren," I. II. Art. in "Zeitschr. f. ration. Med.," Bd. VIII. IX. 1849 u. 1850 ; "Die Nerven des Uterus," ibid., 1850, Bd. X. st. 41 ; R. Lee, "Memoirs on the ganglia and nerves of the uterus," London, 1849; Th. Snow-Beck, "On the nerves of the uterus," in "Philos. Trans.," II. 1846; Rainey, "On the structure and use of the lig. rotundum uteri," in "Phil. Trans.," II. 1850; V. Schwartz, "Observ. microsc. de decursu muscul. uteri et vaginæ hominis," Dorpat., 1850, Diss ; Robin, "Mém. pour servir à l'hist. anat. et pathol. de la membrane muqueuse uterine," in "Arch. génér. de Méd." 1848, tom. XVII. pp. 258 à 405, tom. XVIII. p. 257; Kobelt, "Die männlichen und weiblichen Wollustorgane," Freib., 1844; Tiedemann, "Von den Duverney'schen Drüsen des Weibes," Heidelberg, 1840; C. Mandt, "Zur Anatomie der weiblichen Scheide," in "Zeitschr. für rat. Med.," VII. p. 1; Huguier, "Sur les appareils secrets des organes génit. de la femme," in "Annal. d. Sc. Nat.," 1850, p. 239. [Dalton "On the Corpus Luteum of Menstruation and Pregnancy," Philadelphia, 1851 ; Bischoff, "Die Physiologie der Menstruation und Conception," in "Zeitschr. für. rat. Med." Bd. IV. 1. 1853.-DaC.]

\section{OF THE LACTEAL GLANDS.}

$\S 210$. The lacteal glands (glandula lactiferce) are a pair of compound racemose glands, which, in the male, are only rudimentary, but in the female fully developed, and, after parturition, secrete the milk. 
With respect to their structure, the lacteal glands, in all essential particulars, completely correspond with the larger racemose glands, for instance the parotid and the pancreas. Each gland consists of 15-24 or more, irregular, flattened lobes, $\frac{1}{2}$ to 1 inch wide, with a rounded, angular outline, which, although their cavities are quite distinct from each other, cannot externally always be definitely separated. Each is composed of a certain number of smaller and smallest lobules, and these, lastly, of gland-vesicles. The latter are rounded, or pyriform, 0.05-0.07 in size, with a distinct constriction between them; and the smallest excretory ducts, as for instance, in the small mucous glands, and as everywhere else, are formed of a structureless membrane and tessellated epithelium, which, at the time of lactation, undergoes peculiar metamorphoses. All these glandular elements are surrounded by dense, white connective tissue, particularly abundant between the glandvesicles and smaller lobules, and are united into a compact, large glandulur mass, which is ultimately covered by a quantity of adipose tissue, and in part by the skin. The lacteal glands are, properly speaking, not simple glands, but like the lachrymal, aggregations of these. From each glandular lobe, by the coalescence of the excretory ducts of the smaller and larger lobules, there ultimately proceeds a shorter or longer duct, 1-2 lines in diameter, the lacteal duct or canal (ductus lactiferus s. galactophorus), which running towards the nipple, dilates beneath the areola, into an elon-

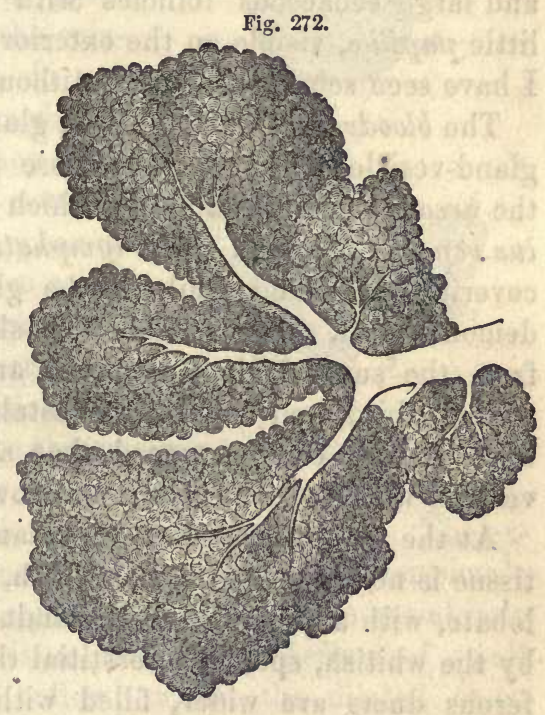
gated sacculus, 2-4 lines wide, the lacteal sac or receptacle (sacculus s. sinus lactiferus); afterwards contracting to 1 or $\frac{1}{2}$ a line, it bends round into the nipple, and ultimately opens, at its apex, in an independent orifice, not more than 1-3-1-5 of a line in diameter, between the papillce which exist in that situation. All these excretory ducts, besides an epithelium, which in the largest of them presents cylindrical cells, $0.006-0.01$ of a line long, and in the finer ramifications, rounded polygonal smaller cells, and a homogeneous layer beneath them, alsô possess a white dense fibrous membrane, longitudinally plicated in the larger canals, in which I have hitherto been unable to detect any indubitable

Fra. 272.-A few of the smallest lobules of the lacteal gland of a puerperal female, with their ducts, magnified 70 diameters. After Langer. 
muscular fibres, and nothing but a nucleated, longitudinally fibrous, connective tissue with fine elastic fibres. Henle, however, more recently, thinks that he has noticed longitudinal muscles in the lacteal ducts, not those of the nipple, but more deeply within the gland.

The nipple (mamilla) and the areola, present numerous smooth muscles, to which the contractility of those parts is owing (vid. § 34); a delicate cuticle, the horny layer of which, in the female, is not more than 0.006 of a line thick, whilst the Malpighian layer has a thickness of 0.04 of a line and is colored in the deeper portion; and compound papilloe 1-10-1-33 of a line long. On the breast itself, the papilloe are small (1-60-1-80 of a line) and simple, and the epidermis still finer $(0.032-$ 0.04 of a line), although with a thicker horny layer of $0.02-0.024$ of a line. In the areola, especially at its borders, but not on the nipple itself, there are large sudoriparous glands, often with peculiar contents, and large sebaceous follicles with fine hairs, which frequently form little papilloe, visible on the exterior (vid. $\S \S 68$ and 73). In the male, I have seen sebaceous glands without hairs, also on the nipple.

The bloodvessels of the lacteal glands are numerous, and surround the gland-vesicles with a rather close plexus of capillaries. The veins in the areola constitute a circle, which is not always quite complete (circulus venosus Halleri). The lymphatics are equally abundant in the skin covering the gland, whilst in the gland itself they have not yet been demonstrated. The nerves of the skin covering the mamma are derived from the supraclavicular nerves, and the cutaneous branches of the second, third, and fourth intercostals. In the interior of the gland, no other nerves can be traced than a few fine twigs accompanying the vessels, whose termination is unknown.

At the time of lactation the gland enlarges very considerably. Its tissue is no longer uniform, whitish, and firm, but softer, granular, and lobate, with a yellowish-red, glandular parenchyma, distinctly bounded by the whitish, spongy interstitial tissue. The gland-vesicles and lactiferous ducts are wider, filled with milk, and the vessels excessively multiplied. In the external parts, the enlargement of the areola and of the nipple is especially worthy of remark; the cause of which appears to depend upon a growth of these parts, with all their elements, including the muscular fibres and minute glands; and not in a simple extension of the color over a larger surface. In the male, the lacteal gland is quite rudimentary $\frac{1}{3}-2$ inches broad, and 1-3 lines thick, not lobed, and firm. The lacteal ducts have no sinuses, and are never so far developed as in the female, inasmuch as they either correspond in form with those met with in the new-born child, or, in larger glands, are more branched, and furnished with a certain number of terminal vesicles, which, on account of their considerable size (they are three times as large as the gland-vesicles in the female), are not to be regarded as 
true gland-vesicles. In rare, but well-established instances, the glands, even in the male, have become so much developed as to be capable of secreting milk.

§ 211. Physiological remarks. - The lacteal gland, in its development, follows the same course as the other cutaneous glands, and is, as I find ("Mittheil. d. Zürcher nat. Gesells.," 1850, No. 41) in accordance with Langer (l. c.), originally (in the fourth to the fifth month) nothing but a solid papillary projection of the mucous layer of the epidermis, which is invested by a layer of denser dermal tissue (Fig. $273,{ }^{1}$ ). In the sixth to the seventh month, it throws out a certain number of buds, and in this way arise the first rudiments of the subsequent lobes (Fig. 273, ${ }^{2}$ ). These are, at first, nothing but minute pyriform- or flask-shaped processes of the common rudiment of the gland, which do not separate from each other until towards the end of fotal life, at which time they open externally; whilst, at the same time, rounded or elongated solid buds begin to appear at their ends, which at.this time are also solid. At the period of birth, the gland measures from $1 \frac{1}{2}-4$ lines, and already distinctly exhibits a certain number (12-15) divisions, of

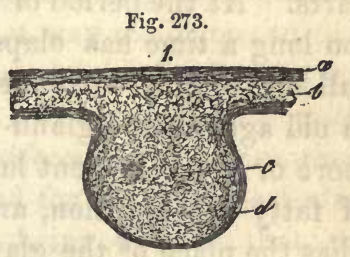

which the internal still approximate the rudimentary papilloe, in fact have either simple flask-like ends, or terminate in two or three sinuosities; whilst the others are in connection with a greater number. The excretory duct of each of these rudimentary lobules, which is either simple or possesses two or three branches, is composed of a fibrous membrane of immature, nucleated connective tissue, and an epithelium of small cylindrical cells, and is manifestly hollow; whilst the dilated ends, which cannot in this case, any more than in other glands in the process of development, at this time be termed terminal vesicles, are still solid; being wholly composed, besides the fibrous tunic continued upon them from the ducts, of minute nucleated cells. From this very simple form, the latter one is thus developed: by the long-continued gemmation of the primary and subsequently formed, clavate ends, and their simultaneous excavation, a much-branched duct, beset in its offsets with whole groups of hollow gland-vesicles, is at last formed. These processes,

Fig. 273.-Development of the lacteal gland. 1, rudiment of the gland in a male embryo, at five months; $a$, horny layer; $b$, mucous layer of the epidermis; $c$, process of the latter or rudiment of the gland; $d$, fibrous membrane around the same. 2 , lacteal gland of a female fotus, at seven months, seen from above: $a$, central substance of the gland, with larger $(b)$ and smaller $(c)$ solid outgrowths, the rudiments of the large gland-lobes. 
however, go on more slowly in the lacteal gland than in any other secretory organs. According to Langer, to whom we are indebted for careful researches upon this subject, true terminal vesicles are never met with in childhood, before menstruation is established, but, universally, only undeveloped ducts with clavate ends. On the occurrence of puberty, true gland-vesicles are formed, but at first only at the borders of the gland, until, ultimately, in the first pregnancy, the entire gland is fully developed. After the forst lactation, it is true, the gland again diminishes in size, but all its constituent parts remain, and again enlarge in the succeeding occasions of conception, without the addition of any new parts. At the period of involution-probably also, if after a pregnancy, too long a time has elapsed without the functions of the gland being called into play-it undergoes a retrograde metamorphosis, until finally, in old age, all the gland-vesicles have disappeared, and nothing but the more or less persistent lactiferous ducts, with their epithelium in a state of fatty degeneration, are to be found in the adipose cushion which supplies the place of the glandular tissue.

The milk, the secretion of the mammary glands, consists of a fluid, the milk-plasma, and innumerable spherical, opaque corpuscles, with the brilliant aspect of fat-drops, suspended in it. These corpuscles-the milk-globules - vary in size, from immeasurable minuteness up to $0.001-$ 0.002 of a line and more, and most probably do not consist of the fatty part of the milk alone, but have also a delicate investment of casein,

Fig. 274.

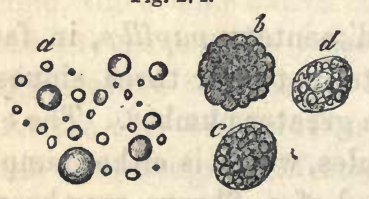
and it is to them that the whiteness of the milk is owing. With respect to the formation of the milk, it is to be remarked that, except at the periods of lactation and pregnancy, the glands contain nothing but a small quantity of a yellowish viscid mucus, with a certain number of epithelial cells, and are lined up to their extremities by an epithelium, which in that situation is tessellated, but externally is more cylindrical. With conception, this state of things is altered. The cells of the gland-vesicles begin to develop, at first a little, and subsequently more and more fatty matter within them, and to enlarge, so as entirely to fill the terminal vesicles. To this is added, before the end of pregnancy, a new formation of fat-containing cells in them, by which the older cells are forced into the lactiferous ducts, which they gradually fill. Thus it happens, that although a true secretion is not at that time set up, still in the latter half of pregnancy a few drops of fluid may be expressed from the gland, which, as is shown by its yellow color, is not milk, but nevertheless contains a certain number of fat-globules from the more or

Fia. 274.-Elementary forms in milk, magnified 350 diameters : $a$, milk-globules; $b$, colostrum corpuscles; $c, d$, cells with fat-globules from the colostrum, one (d) with a nucleus. 
less disintegrated fatty cells, exactly resembling the subsequent milkglobules, and also contains such cells either with or without a tunicthe so-termed colostrum corpuscles. On the commencement of lactation after parturition, the cell-formation in the gland-vesicles proceeds with excessive energy, in consequence of which the secretion collected in the lactiferous ducts and gland-vesicles is evacuated, as the colostrum or immature milk, the true milk taking its place.

The latter, in the extremities of the gland, consists only of some fluid and cells entirely filled with fat-globules, which sometimes occupy the gland-vesicles alone, sometimes associated with pale epithelial cells, which, however, always contain more or less fat, and originate either in a free cell-formation or from epithelial cells, in a way analogous to that in which the cutaneous sebaceous matter is formed (vide $\S 73$ ), by their continued multiplication. These cells, which I would designate milkcells, break up, so soon as they reach the lactiferous ducts into their elements, the milk-globules; the membrane, and for the most part also, the nucleus, disappearing, without a vestige being left, so that the milk, when secreted, usually presents no indication of its mode of origin. At most, there occur in it a very few larger or smaller aggregations of milk-globules, which, from their similarity to those met with in the colostrum, may likewise be termed colostrum-corpuscles. The secretion of the milk, therefore, depends essentially upon a formation of fluid and fat-containing cells in the gland-vesicles, and consequently falls into the category of those secretions into the composition of which morphological elements enter; above all to the fatty secretions, such as the cutaneous sebaceous matter, in which cells of precisely similar kind occur to those met with in the gland-vesicles of the lacteal glands, and in the colostrum.

In the new-born child, the mammary gland very frequently contains a small quantity of a fluid presenting the external and microscopical characters of milk, the origin of which is probably related to the formation of the glandular ducts.

With respect to the colostrum-corpuscles and fat-globules of the colostrum, Reinhardt was the first to prove, -that the supposition broached by Nasse and Henle, that these bodies are related to a formation of fat-containing cells in the mammary glands, and that the former in their more usual form are nothing but membraneless cells, and the latter oil-drops liberated from cells, is in every respect well founded, although he is inclined to distinguish the formation of the colostrum from the secretion of milk, and to regard the former as a pathological process, as a fatty metamorphosis, by which the old epithelial cells of the gland, previously to the formation of true milk, are evacuated externally, and particularly because, in the true milk-formation, he 
was unable to perceive any fat-containing cells. But since V. Bueren, especially, has found such cells, and consequently the formation of the milk and of the colostrum, seem to be morphologically quite identical, such a separation of the two processes can no longer be defended; and, in cases of repeated parturition, the formation of colostrum can scarcely be viewed in any other light than as the introduction to that of the milk. On the other hand, I am quite of opinion, that the production of the first colostrum is connected with the excessive development of the lacteal gland coincident with the first pregnancy; and that it is in part derived from the internal cells of the originally solid rudiments which are removed, during the formation of the ultimate terminations of the gland. I explain, in a similar way, the formation of milk in the new-born child; in which case, surely, no true secretion can be thought of.

Donne, the discoverer of the colostrum-corpuscles, states, that in inflammations and tumefactions of the breast of nursing women, the milk acquires the nature of colostrum; which is, however, denied by D'Outrepont and Münz ("Neue Zeitschrift für Geburtskunde," Bd. $10)$; in the same way, according to Lehmann ("Phys. Chemie," II., 327, [transl. II., p. 334]) it would appear, that, in acute diseases generally, and also in menstruation (Donne, d'Outrepont), the milk exhibits colostrum-corpuscles, which, when they exist in larger quantity, are regarded by Donné as indicative of bad milk. In hoof-murrain" ("Klauenseuche"), Herberger and Donné found the milk to contain a good deal of colostrum. In sour milk the casein coagulates into granules, and the milk-globules gradually run together into larger drops. Blue and yellow milk, according to Fuchs (vide Scherer, art. "Milk," in "Handw. d. Phys.," II., p. 470) contains colorless infusoria, which he terms vibrio cyanogenus and xanthogenus, which, when transferred to healthy milk, also color it; a fact which, as regards blue milk, is confirmed by Lehman (l. c., p. 335, Eng. transl.); according to Bailleul ("Comptes rend.," t. 17, p. 1138), however, and Lehmann [once only], a filamentary fungus is also found in that sort of milk. Red milk has also been noticed by C. Nägeli, and vegetable protococcuslike growths found in it.

For the investigation of the mammary glands, those of pregnant or nursing women, or of women who have borne children, should be preferably selected, because it is only in such that the gland-vesicles are well developed. When the smallest lobules are teased out, their elements come readily into view; but if it be desired to examine into their arrangement, fine sections of glands boiled in acetic acid and dried are above all to be recommended, as well as injected preparations, which it is not difficult to make from the lacteal sinuses. For the study of the development of the gland, besides recent specimens, preparations made 
with acetic acid are necessary. The smooth muscles of the areola are found by mere dissection, although not always very easily, as, except during pregnancy, they are frequently very delicate.

Literature.-Rudolphi "Bemerk. über den Bau der Brüste," in the "Abh. der Berliner Akademie," 1831, p. 337; Astley Cooper, "The Anatomy of the Breast," London, 1839, 4to ; C. Langer, "Ueber den Bau und die Entwicklung der Milchdrüsen, mit 3 Taf.," from the "Denkschr. d. Wiener Akad.," Bd. III., Wien, 1851; A. Donné, "Du lait et en particulier du lait des nourrices," Paris, 1837; "Ueber die mikroskopischen Körperehen im Colostrum," Müller's "Arch.," 1839, p. 182; "Cours de Microscopie," Paris, 1844; Fr. Simon, "Die Frauenmilch, nach ihrem chemischen u. physiol. Verhalten dargestellt," Berlin, 1838 ; "Ueber die" Corps granuleux "von Donné," in Müller's "Arch.," 1839, pp. 10 and 187; J. Henle, "Ueber die mikr. Bestandtheile der Milch," in Fror. "Notizen," 1839, No. 223; H. Nasse, "Ueber die mikr. Bestandt. d. Milch," Müller's "Archiv," 1840, p. 259 ; Reinhardt, in "Arch. f. path. Anat.," Bd. I., pp. 52-64; Lammerts van Bueren, "Onderzoekingen over de Melkbolletjes," in the "Nederl. Lancet," 2d ser., 4, Jaarg., p. 722, or "Observat. microscop. de lacte," Traject. ad Rhenum, 1849, Diss.; "De Ontwikkeling van de Vormbestanddeelen der Melk," in the "Nederl. Lancet," $2 d$ ser., 5, Jaarg, p. 1; Fr. Will, "Ueber die Milchabsonderung," Erlangen, 1850, Programm. Besides which should be consulted the "General Anatomy" of Henle, J. Müller's work on the Glands, and the Atlases of Berres, Donne, and Mandl. [Vid. also, "Observations on the muscular tissue of the Skin," by Joseph Lister, M.B., "Quart. Journ. Micros. Sc.," vol. I., p. 262, 1853.-Trs.]

\section{OF THE VASCULAR SYSTEM.}

§ 212. The vascular system, consisting of the heart, with the blood and lymphatic vessels, contains in its interior the blood and the lymph (chylus), with innumerable morphological particles. The lymphatic vascular system presents special organs-the lymphatic glands.

\section{1.-OF THE HEART.}

$\S 213$. The heart is a thick, hollow, muscular organ, divided into four compartments, invested externally by a serous membrane-the pericardium, - and lined internally by the endocardium, a continuation of the walls of the great vessels, particularly of the tunica intima.

The pericardium does not differ in structure from other serous membranes, as, for instance, the peritoneum. The outer lamella is consi- 
derably the thicker; it is more fibrous towards the exterior, presenting, towards the interior, numerous fine, elastic networks, which are immediately covered with one or two layers of tessellated epithelium. Very numerous elastic networks of the same kind are found also in the inner thin lamella, which is, partly, very intimately united with the muscular substance, and partly, especially in the sulci, separated from it by common adipose tissue, which, moreover, not unfrequently forms a subserous fatty layer, extending almost over the entire heart. The vessels present the same conditions as elsewhere; and with respect to the nerves, twigs from the phrenic and recurrent branch of the right vagus have been demonstrated in the outer lamella of the pericardium (Luschka).

The muscular fibres of the heart are red and transversely striated, but differ in many respects from those of the voluntary muscles. The individual fibres themselves are, on the average, about $\frac{1}{3} d$ more slender $(0.004-0.01$ of a line), frequently more distinctly striated in the longitudinal than in the transverse direction, and pretty readily divisible into fibrils and minute particles ("sarcous elements," Bowman); their sarcolemma is very delicate, or even wholly inappreciable; and in the fibres, there almost uniformly occur minute fatty granules, which, with the nucleus, are frequently disposed in a series in the axis of the fibre, and, where the muscular tissue is degenerated, appear most usually to be excessively multiplied, and also colored. Much more, however, than by these characters, is the muscular tissue of the heart distinguished by the intimate union of its elements, which, except on the internal surface of the organ, not only never form manifestly distinct bundles, being everywhere in close apposition with each other, and separated only by a scanty connective tissue, but, as was discovered by Leeuwenhoek, *

Fig. 275 . and I also have found (vid. p. 108), are directly united

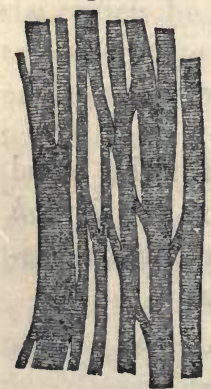
together in their elements. These anastomoses of the muscular fibres, which are a universal attribute of the cardiac muscular tissue, are effected in the human and mammalian heart generally, chiefly, by short, oblique, or transverse, usually small fasciculi, and are extremely numerous, so that, in many places in the ventricles and auricles (whether universally I know not), numerous instances of them are presented in every minute portion. Besides these, there also exist true divisions or fibres, by which the thickness of separate portions of muscle may be rendered more considerable than it was originally.

The course of the muscular fibres of the heart is extremely complex, and a general outline only of it can here be given. The muscular structures of the ventricles and of the auricles are completely distinct from

FIa. 275.-Anastomosing primitive fasciculus from the human heart.

* [See note, p. 108.-Trs.] 
each other; both, however, have as their chief point of origin the ostia venosa of the ventricles, where tough, tendinous tracts - the so-called annuli fibro-cartilaginei-are situated, two anterior, on the right and left of the aortal opening, and one posterior, which runs backwards also from the aorta to the border of the auriculo-ventricular septum, where it splits into two slender crura. In the auricles are found: 1, fibres, which are common to both, in the form of transverse, flattened bundles, which proceed chiefly anteriorly, but afterwards also superiorly and posteriorly, from one auricle to the other, and are continued in them as transverse fibres. 2, special fibres. These constitute, in the first place, complete rings at the origins of the great veins, and at the points of the appendices; and, in the second place, a longitudinal layer of some thickness beneath the endocardium, which springs from the auriculo-ventricular openings, and is especially developed in the right auricle (musculi pectinati). Besides these, there exist between the latter muscles, and also in the auricles, numerous other small fasciculi, which, on account of their irregularity, cannot be more particularly described. The septum is to some extent common to both auricles. Its muscles arise from the most anterior part of the upper border of the septum of the ventricles, immediately behind the aorta, from the posterior fibrocartilage and arch to the right, around the fossa ovalis, in which only slender fibres exist, in a superior and posterior direction, in order to terminate, partly at the vena cava inferior, partly by forming a complete ring; whilst on the left, they surround the fossa ovalis in the opposite direction.

The muscular structure of the ventricles is disposed so that on the external and internal surfaces the fibres everywhere decussate; and in the intermediate portion, every stage of transition from the one direction to the other is presented. The muscular fibres arise at the ostia venosa and at the arterial openings, in part immediately, and partly with the intervention of short tendons, run more or less obliquely (and some longitudinally or even transversely), and after they have surrounded a portion of the ventricle in the longitudinal or transverse direction, curve back again, and then terminate, some in the musculi papillares, and chordce tendinece, whilst others are again inserted in the points of origin above indicated, so that without being interrupted by tendons, they describe large involved loops; or figure-of- 8 turns of large size, and running in very numerous and diverse directions.

The endocardium is a whitish membrane, investing all the elevations and depressions of the internal surface of the heart, as well as the papillary muscles and their tendons, and the valves. It is most developed in the left auricle (as much as $\frac{1}{4}$ of a line), and thinnest in the ventricles, so that the muscular substance there presents its natural color. As regards its structure, it consists of three layers; an epithelium, an 
elastic layer, upon which the varying thickness of the endocardium in different situations depends, and a thin layer of connective tissue. The first is a single, or, according to Luschka, perhaps a double layer of polygonal, usually elongated, clear, flattened, nucleated cells, 0.007 to 0.012 of a line long, resting immediately upon the most superficial layer of the elastic membrane, which may be said to consist of nothing but very fine, longitudinal, elastic fibres. The remainder of this middle layer is constituted of a matrix of common connective tissue with scattered nuclei, through which the very abundant finer and coarser elastic networks penetrate. This elastic element is so abundant, in fact, in the auricles, and even mixed with true fenestrated membranes (vid. § 23) that the endocardium of those cavities is rendered almost entirely a yellow, elastic membrane, consisting of several laminæ. Most externally, there succeeds to this elastic layer, a stratum of connective tissue, of great tenuity indeed, but which, nevertheless, both in the ventricles and in the auricles, may easily be raised as an entire membrane. In the portion bordering upon the elastic tissue this layer contains fine elastic elements. It represents, in fact, a somewhat loose layer, like a subserous connective tissue, uniting the muscles and the true endocardium.

The auriculo-ventricular valves are lamince springing from the fibrous rings of the ostia venosa, in the thicker parts of which, a middle layer of connective tissue with numerous elastic networks, and two lamelloe of the endocardium united with it, may be clearly distinguished. Towards the free border, these three layers are conjoined so as to form a single one, composed of connective tissue and fine elastic networks, over which again the epithelium is continued. The semilunar valves present the same conditions as the free border of the others, and with respect to the chordce tendinece, they are constituted of common tendinous tissue, covered by a very thin layer of endocardium-consisting, indeed, merely of epithelium and a fine elastic lamella.

The bloodvessels of the muscular substance of the heart are very numerous, but differ in no respect from those of transversely striped muscle ( $\$ 77)$, except that the capillaries, owing to the slenderness of the muscular fibre, often encompass several of them in common. The endocardium is tolerably well supplied with vessels in its layer of connective tissue, whilst they are more scanty in the proper endocardium. In the auriculo-ventricular valves a few vessels are readily seen, not only in animals but also in Man (vid. Luschka, l. c., p. 182, Fig. 1), some of which enter them from the papillary muscles, but chiefly from the basis of the valves, and are also distributed in part, though sparingly, in their proper endocardial investment. The semilunar valves possess no vessels. Only a few lymphatics are found in the external lamella of the pericardium, whilst they occur in greater abundance on the inner lamella on the muscular substance, and may there be demonstrated 
readily enough, if the heart be placed for a few days in water, as Cruikshank correctly observes. Their trunks collect in the sulci, accompanying the bloodvessels, and terminate in the glands, behind and below the arch of the aorta, on the bifurcation of the trachea, to which the pulmonary lymphatics also proceed. Whether the substance of the heart and the endocardium are also furnished with lymphatics, as is asserted by some, is not yet determined. The nerves of the heart are numerous and proceed principally from the cardiac plexus formed by the vagus and sympathetic, beneath and behind the arch of the aorta. These nerves, forming the more scanty plexus coronarius dexter, and the richer $p . c$. sinister, accompany the vessels on the right and left ventricles and auricles, run, in part with the vessels, in part crossing them in various directions, toward the apex of the heart, and, whilst entering into numerous anastomoses with each other, usually at acute angles, enter the muscular substance at various points, some even in the coronary sulcus, in order to terminate, partly in the muscular substance, and partly to reach the layer of connective tissue of the endocardium. The cardiac nerves, in Man, are gray, and, except the largest, contain only fine and very pale fibres; the latter, however, in the greater number, and intermixed with not very numerous nucleated fibres. Although the nerves, even in the endocardium, retain their dark borders and are tolerably numerous, it has not hitherto been possible to discover their terminations in that situation, any more than in the muscular substance, Ganglia exist, not only in the cardiac plexus in various situations, but, as Remak discovered, in the Calf, also in the muscular substance of the auricles and ventricles, which is likewise true of man and other animals. These ganglia are best known in the Frog, in which they are situated, especially in the septum, and at the junction of the auricles with the ventricle, and contain apolar and unipolar cells (Ludwig, Bidder, R. Wagner, myself). The minute fusiform enlargements on the external nervous branches, especially noticed by Lee, are not ganglia, being merely thickenings of the neurilemma.

With respect to the particular direction of the muscular fibres of the ventricles, the following remarks may be offered. On the external surface of the ventricles there is a layer, $\frac{1}{2}-1$ line thick, which, on the left ventricle, runs obliquely downwards from the pulmonary artery, the anterior longitudinal sulcus and the left transverse sulcus, and in the middle of the wall of the ventricle, descends very abruptly, almost vertically. On the right ventricle, these fibres are oblique only on the conus arteriosus, whilst on the sides and posteriorly they are almost or quite transverse. At the longitudinal sulci, the superficial fibres are continued from one ventricle upon the other, so that a small portion of those of the left ventricle arise from the anterior side of the ostium venosum dextrum 
and the greater part of those belonging to the right ventricle from the posterior portion of the left ostium venosum. If the fibres of the left ventricle are traced, it will be found, with the exception of those which at the posterior longitudinal sulcus pass upon the right ventricle, that they (Fig. $276 a, a^{\prime}, a^{\prime \prime}$ ) run towards the apex of the heart, and there form the well-known vortex, and then curve inwards, forming loops; and are continued as the innermost, for the most part longitudinal, fibres of the cavity of the ventricle, and either ascend as high as the venous openings, or terminate in the posterior papillary muscle. Upon removal of this set of fibres, a thick layer comes into view, interposed between its external and internal portions, the fasciculi in which, at first sight, appear to surround the cavity of the ventricle in an oblique and transverse direction, although they seem to arise, without exception, from the ostia venosa, and again to terminate in the same situation, and to describe a figure-of- 8 turn, still more distinctly than the external muscular layer, as has been clearly shown by Ludwig. I find that the bundles of this layer (Fig. $276 c, c^{\prime} c^{\prime \prime} c^{\prime \prime \prime}$ ), after their origin from the left border

Fig. 276.

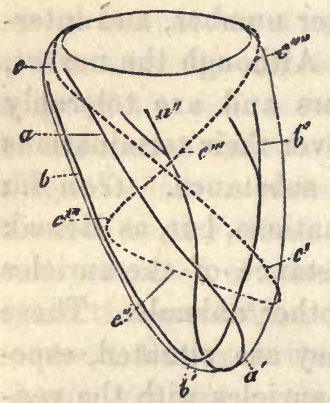

of the aorta and the anterior half of the ostium venosum sinistrum, extend obliquely downwards, and to the left $(c)$, and then, before reaching the apex of the heart, curve towards the posterior wall of the ventricle $\left(c^{\prime}\right)$, whence they again ascend on the septum $\left(c^{\prime \prime}\right)$ and the anterior wall $\left(c^{\prime \prime \prime}\right)$, and are finally inserted in the whole extent of the venous opening, and also in the upper border of the septum $\left(c^{\prime \prime \prime \prime}\right)$. It is from these fibres in the free wall of the ventricle that are derived the deep layers which decussate with the superficial, and on the septum afford the fibres which, on the left side, run obliquely from below and behind, upwards and forwards.

On the right ventricle, there are much fewer independent fibres than on the left. Most of the superficial fasciculi are continued upon the left ventricle; both the anterior, which are continued over the anterior longitudinal sulcus and lost in the vortex, and also many of those which, at the posterior longitudinal sulcus, stretch from the left to the right ventricle. These latter fibres, consequently, completely encircle the

FIG. 276.-Diagram of the left ventricle, with the septum, in order to show the course of the muscular fibres. $a a^{\prime} a^{\prime \prime}$, superficial fibres: $a$, on the anterior wall; $a^{\prime}$, the turning inwards of them at the vortex; $a^{\prime \prime}$, their passage into the posterior papillary muscle. $b, b^{\prime}, b^{\prime \prime}$, septal fibres of the right side: $b$, their course, downwards and forwards; $b^{\prime}$, their passage into the vortex and internal muscular layer of the left ventricle, as well as their termination in the anterior papillary muscle, $b^{\prime \prime} . c-c^{\prime \prime \prime \prime}$, middle muscular layer: $c$, commencement at the right side of the ostium venosum, and course on the anterior wall obliquely downwards and backwards ; $c^{\prime}$, curvature on the septum, and course on it, $c^{\prime \prime} ; c^{\prime \prime \prime}$, curvature on the anterior wall, and deep course in it, to the end of the ostium venosum, $c^{\prime \prime \prime \prime}$. 
right ventricle, part of them also entering the vortex, and part, in the anterior longitudinal sulcus, joining the middle muscular layers of the left ventricle. Independent, superficial fibres occur only: 1 , at the conus arteriosus, arising from the ostium venosum dextrum, between the right auricle and the aorta, surrounding the conus arteriosus, and returning thence, from the left, back to their point of origin; 2, at the apex of the right ventricle, where not unfrequently a distinct, second yortex exists, in which case some of the superficial fibres arising from the left ostium venosum also curve inwards in that vortex, as they do in that of the left ventricle, and are continued into the superficial fibres of the right ventricle, but, on account of their intricate interlacement, cannot be traced further. Besides these, other deeper fibres exist in the right ventricle, which are disposed as follows: 1. From the upper border of the septum, and the left posterior side of the pulmonary opening, flattened bundles commence and run in the septum, downwards and forwards, towards the apex of the heart and the anterior longitudinal sulcus, where they join the superficial fibres, and are continued with them into the vortex, whence they may be traced as far as the anterior papillary muscle of the left rentricle (Fig. $276 b, b^{\prime}, b^{\prime \prime}$ ). 2. With these fibres are associated others, running obliquely downwards and backwards, from the right side of the pulmonary opening and the right portion of the ostium venosum dextrum, beneath the superficial layer of fibres on the free wall of the ventricle, as far as the posterior longitudinal sulcus, where they curve abruptly towards the septum, in which they accompany the fibres described under (1), though more on the inferior half of the septum, to the apex of the heart, and terminate in a similar way. 3. With these fibres are also conjoined, to some extent, the elements of the great papillary muscle of the right ventricle, whilst those of the two smaller are continued into the fibres of the septum described under (1). Besides this, all these muscles furnish direct fibres, some of which descend from the ostium venosum and return upon themselves, and some proceed from the network of the trabeculce carnece and cannot be traced further.

It would appear therefore, that the auricles, as regards their muscular structure, are almost distinct; whilst in the ventricles, the entire superficial, tolerably thick muscular layer is continuous all round and is disposed as if the heart had only a single cavity. Properly speaking, the left ventricle alone in this respect, is independent, in which there is not only beneath the superficial layer a very thick muscular mass arising and ending in it, to which, also, the greater part of the septum belongs, but which also receives nearly all the deeper muscular layers arising on the right side, in the free wall of the ventricle, and inserted into the right portion of the septum. The heart, consequently, might be described as composed of two muscular sacs; the thinner of which is com- 
mon to the whole; the other and thicker belonging only to the left division, and to soine extent being interposed between the layers of the former. To the latter would belong the entire septum, and the middle muscular substance of the left ventricle ; to the former, the superficial layers with their continuations in the innermost muscular strata, and especially the whole of the free portion of the right ventricle.

\section{2.-OF THE BLOODVESSELS.}

$\S 214$. As regards their structure, the bloodvessels are divided into arteries, capillaries, and veins; but these three divisions are by no means separated by definite limits, inasmuch as the capillaries are continuous with the veins on the one hand, as imperceptibly as they commence from the arteries on the other. At the same time it is true that both kinds of larger vessels, although in their rudiments presenting a general conformity of structure, are still sharply and definitely distinguished in many respects.

Concerning the tissues which enter into the composition of the vessels, and the mode of their arrangement, the following general remarks may be made. Whilst the true capillaries possess only a single perfectly structureless coat, in the larger vessels, with few exceptions, the number of tunics is increased to three, which may most suitably be described as tunica intima, $t$. media, and $t$. externa $s$. adventitia. In these tunics there are found, of the fibrous tissues of the body, in the first place, the elastic and smooth muscular tissues, but the connective tissue, and even the transversely striped muscular tissue are also represented in them; besides which, there exists epithelia, peculiar homogeneous membranes, vessels, and even nerves; so that we have presented in them a complexity of structure which renders a general description almost impossible and which can be made clear only by an accurate examination of each particular element; and the rather so, because the more extensively distributed tissues assume very different forms. With respect to the arrangement and subdivision of these tissues, they may be said to exhibit a very strong tendency to lamination and, in the different layers, to the assumption of a constant direction in the course of their constituent elements. The former of these dispositions, however, rarely extends to the actual isolation of the individual layers; and to the latter, though more rarely, there are also exceptions. The tunica intima is the thinnest of the membranes of the vessels, and always consists of a cellular layer, the epithelium; most usually also of an elastic membrane in which a longitudinal direction of the fibres predominates; to which again may be superadded other layers of one kind or another, which also almost invariably retain the longitudinal direction. The $t$. media is for the most part a thick layer, and is especially the seat of the trans- 
verse elements and of the muscles, although in the veins it also contains numerous longitudinal fibres, and in all the larger vessels presents, in addition, elastic elements, and connective tissue in greater or less quantity. The $t$. adventitia, lastly, again exhibits a preponderating longitudinal fibrillation, is as thick as or thicker than the $t$. media, and consists, for the most part, only of connective tissue and elastic networks.

If the separate tissues of the vascular tunics are investigated somewhat more closely, it will be seen that the connective tissue appears almost universally fully developed, with fine and coarse bundles and distinct fibrils. It is only in the smallest arteries and veins that it is
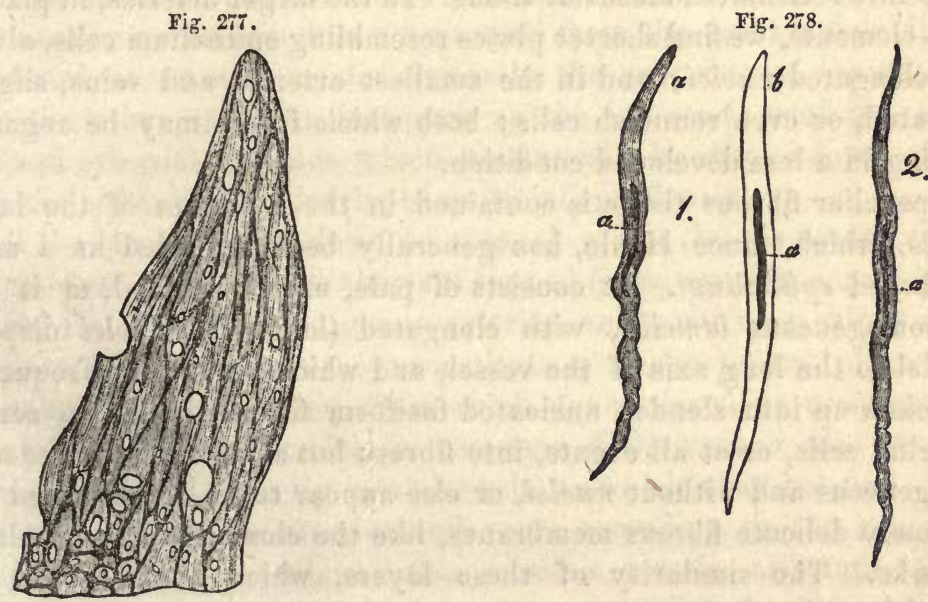

replaced by a nucleated, indistinctly fibrous tissue, and ultimately passes into a perfectly homogeneous, still occasionally nucleated, delicate membrane. The elastic tissue nowhere presents such manifold forms as it does in the vessels. From wide-meshed, lax networks of the finest, middle-sized, and thickest fibres (Fig. 22, p. 81), up to the narrowest, closest, membraniform interlacements of these fibres, all transitionary forms are here met with ; and besides this, we may notice every degree of transformation of the latter, or of the elastic reticulated membranes into true elastic membranes, which either betray their derivation, in an elastic, more or less indistinct, fbrous network pervading them, and distant openings, or are transformed, either partially or entirely, into perfectly homogeneous plates, exhibiting more or fewer openings (Fig. 25, p. 82).

Fra. 277.-Elastic membrane from the tunica media of the popliteal artery in Man, with an indication of fibrous networks; magnified 350 diameters.

Fia. 278.-Muscular fibre-cells from the human arteries, magnified 350 diameters. 1, from the popliteal artery; $a$, without, $b$, with acetic acid. 2 , from a twig, $\frac{1}{2}$ a line in diameter, of the anterior tibial artery: $a$, nuclei of the cells. 
In the smallest vessels, instead of the elastic elements, there occasionally occur, especially in the $t$. adventitia, fusiform cells, which can only be regarded as undeveloped formative cells of elastic tissue. Transversely striped muscle is found only at the openings of the largest veins into the heart; whilst, on the contrary, smooth muscles are extensively distributed, especially in vessels of a medium size, and also to some extent in the larger vessels. The elements of these muscles, or the contractile fibre-cells, in the majority of the vessels, present nothing peculiar, except that their length never exceeds 0.04 of a line, and that they are united, either directly, or with the intervention of connective tissue and elastic fibrils, into flattened bundles and muscular membranes, more rarely into reticulated muscular tissue. In the larger arteries, in place of these elements, we find shorter plates resembling epithelium cells, always with elongated nuclei; and in the smallest arteries and veins, slightly elongated, or even roundish cells; both which forms may be regarded as being in a less developed condition.

A peculiar fibrous tissue is contained in the $t$. intima of the larger vessels, which, since Henle, has generally been regarded as a metamorphosed epithelium. It consists of pale, usually striped, or it may be, homogeneous lamello, with elongated (long-oval) nuclei disposed parallel to the long axis of the vessel, and which may not unfrequently be broken up into slender nucleated fusiform fibres, similar to certain epithelial cells, or at all events, into fibres; but at other times are more homogeneous and without nuclei, or else appear to be transformed into extremely delicate fibrous membranes, like the closest and finest elastic networks. The similarity of these layers, which I shall term the striped lamelloe of the $t$. intima, or rather of the fibrous cells of which they are fundamentally constituted, to the epithelium of the vessels, is nevertheless insufficient to justify the supposition of their being derived from the latter, since there are no facts to show that the true epithelial cells and the striped lamelloe stand in any genetic connection of such a kind, as that the latter were at one time a true epithelium and the innermost layer in the vessel, being afterwards successively pushed back, and their elements made to coalesce; on the contrary, it seems to be allowable to regard the epithelial cells and the formative cells of these layers as originally equivalent, and that in the course of development, the one set are transformed in one direction, and the other in another, and, in this way, ultimately become tissues of more or less different kinds.

The epithelium of the vessels (Fig. 14, p. 76) presents two forms: firstly, especially in the great reins, it appears under that of a tessellated epithelium, with polygonal, mostly somewhat elongated cells; and secondly, as in most of the arteries, as a fusiform epithelium, with acuminated, slender cells, $0.01-0.02$ of a line long. Normally, it 
exists in all vessels, may almost always be pretty readily broken up into its elements, and like other simple epithelia, is not subject to any constant detachment and restoration. With Remak, we might describe the epithelium as the cellular membrane of the vessels, since it differs from other epithelia in this respect, that in the large vessels, it is often continuous with the striped lamellce, without any line of demarcation, so that frequently it cannot be said where the one ceases and the others commence; but I should myself rather be inclined to retain the old name, both because the innermost cellular layer of the vessels presents in all respects the relations of a simple epithelium, and is, in many situations (heart, smaller vessels) abruptly defined from the deeper tissues. Even the circumstance particularly adduced by Remak, that the vascular epithelium does not proceed from the embryonic epithelial membrane, is not with me decisive as to the propriety of separating it from the other epithelia, inasmuch as the investments of the serous sacs and synovial capsules, which no one will be disposed to separate from the epithelia, are developed quite independently.

All the larger vessels down to those $\frac{1}{2}$ a line in diameter and less, possess nutritive vessels as they are termed (vasa vasorum s. nutrientia), derived from minute contiguous arteries, and ramifying chiefly in the t. adventitia, in which they constitute a rich capillary plexus with somewhat rounded meshes, from which again the veins accompanying the arteries arise, and which, in the case of the venous vasa vasorum, pour their blood directly into the vein to which they belong. The $t$. media of the larger arteries and veins, according to the concurrent testimony of many authors, also contains vessels, although in very small number, and only in the outer layers; whilst the internal layers and the $t$. intima have always appeared to me to contain none at all; but even in these situations some observers would seem to have noticed vessels (in the $\mathrm{Ox}$, the vena cava inferior is richly provided with vessels up to the $t$. intima). Nerves derived from the sympathetic and spinal nerves may readily be seen going to many arteries, but frequently appear merely to accompany them. Where they enter the coats of a vessel they run only within the $t$. adventitia, and in favorable instances, in animals, divisions and free terminations of their fine fibres may be perceived (vide "Mikros. Anat.," II., 1, p. 532-33). Many arteries are wholly without nerves, as those of the cerebral and spinal substance, of the $t$. chorioidea, the placenta, as well as many arteries of muscles, glands, and membranes, whence it is obvious, that nerves are not as requisite for them as we have usually been inclined to believe. This may be said more decisively with respect to the veins, as it is only in the larger ones that a few fine nerves can be demonstrated. Such have been observed by Luschka in the sinuses of the dura mater, the veins of 
the vertebral canal, the vence cavce, the jugular, iliac, and crural veins, and in those of the liver by myself. They are derived both from the sympathetic and the spinal nerves, and with regard to their terminations have not yet been investigated. According to Luschka, they would appear to extend to the innermost vascular tunic; but this I have not as yet been successful in observing.

\$ 215. For more easy description, the arteries may be divided, according as the middle tunic is purely muscular, or composed of muscular and elastic fibres intermixed, or else chiefly elastic, into small, medium sized and large arteries; and the more properly so, because, concomitantly with the variations in the structure of the middle tunic, the external and internal coats present a different conformation, at all events, in many respects. A general characteristic of the arteries is presented in the circumstance, that the middle tunic is very strong, and consists of numerous, regularly disposed lamince, the elements of which observe a transverse direction. In the largest arteries the $t$. media is yellow, highly elastic, and of great thickness; towards the periphery of the body it gradually diminishes in thickness, and becomes redder and more contractile, until, just before the capillaries are reached, it appears quite thin and subsequently inapparent. The whitish $t$. intima is always much thinner, and varies in thickness within narrower limits, but this is also regulated by the size of the vessel ; whilst, on the contrary, the $t$. adventitia of the largest arteries is absolutely considerably thinner than in those of a medium calibre, in which it often equals the $t$. media in thickness, or may even exceed it. In a special exposition of these points, it is best to begin with the smallest arteries as the simplest in structure; with these the others may be afterwards readily compared.

Arteries under 4-5 or 1 line in diameter, with few exceptions, present, until close to the capillaries, the following structure (Fig. 279). The $t$. intima consists of only two lamina, an epithelium, and a peculiar, glistening, less transparent membrane, which I shall term the elastic internal tunic. The former contains well-marked, fusiform, pale cells with long-oval nuclei, which are readily separated in connection, in entire fragments, or even in the form of perfect tubes; but may also be isolated, and then present no small resemblance, on the one hand, with the fusiform cells of pathologists (also with the formative cells of the elastic fibres and of connective tissue), and on the other with contractile fibrecells; from the former, however, they are distinguished by the less attenuation of their extremities and their paleness; and from the latter by their rigidity, by the nucle $i$ never having the rod-like form, and by their chemical reactions. The elastic tunic is, on the mean 0.001 of a line 
thick, and in the living subject is smoothly stretched beneath the epithelium, whilst in empty arteries it almost always presents a greater or less number of usually strong folds, and frequently also, numerous, fine transverse rugo, which give it, although perfectly homogeneous, a peculiar longitudinally striated aspect; in addition, it appears almost always as a fenestrated membrane, as it is termed, with various sized, distinctly marked reti-

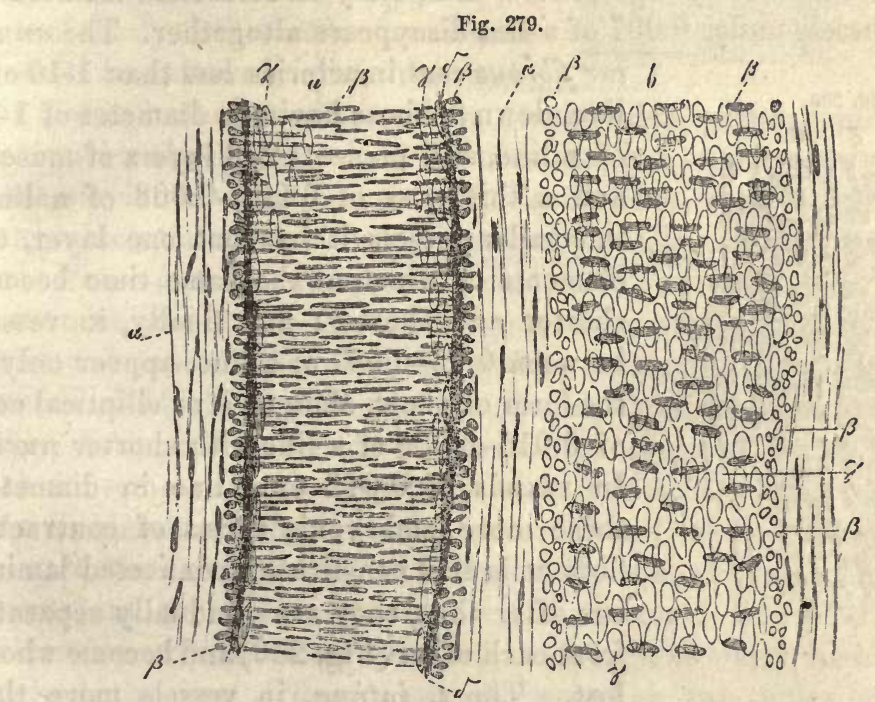

culated fibres, and usually minute elongated openings; more rarely as a true but very close network, of chiefly longitudinal elastic fibres, with narrow, elongated fissures, - and completely corresponds in aspect, in its great elasticity, and its chemical reactions, with the elastic lamelloe of the t. media of the larger arteries. The middle tunic of the small arteries is purely muscular, without the slightest admixture of connective tissue and elastic elements, and is stronger or weaker according to the size of the vessel (down to 0.03 of a line). In vessels of $1-10$ of a line in diameter, the fibre-cells, which are united into lamellce, may be pretty readily isolated by dissection, and in still smaller ones by boiling and maceration in nitric acid of 20 per cent., when they appear as delicate fibre-cells-0.02-0.03 of a line long, and 0.002-0.0025 of a line broad. The $t$. adventitia consists of connective tissue and fine elastic fibres, and is usually as thick as the $t$. media or even a little thicker.

Fra. 279.-An artery, $a, 0.062$, and vein, $b, 0.067$ of a line in diameter; from the mesentery of a child; treated with acetic acid, and magnified 350 diameters : a, tunica adventitia, with elongated nuclei; $\beta$, nuclei of the contractile fibre-cells of the $t$. media, viewed in part on the flat surface, in part in apparent transverse section; $\gamma$, nuclei of the epithelial cells; $\delta$, elastic longitudinal fibrous membrane. 
The above description of the structure holds good of arteries down to 1-8 of a line in size, but nearer the capillaries the structure changes more and more (Fig. 280). Even in arteries 1-10 of a line in diameter, the $t$. adventitia contains no elastic tissue, being composed only of connective tissue with elongated nucle $i$, which at first still retains its fibrous character, but afterwards, though always nucleated, appears more homogeneous, and ultimately represents a thin, truly structureless membrane, which, in vessels under 0.007 of a line disappears altogether. The annu-

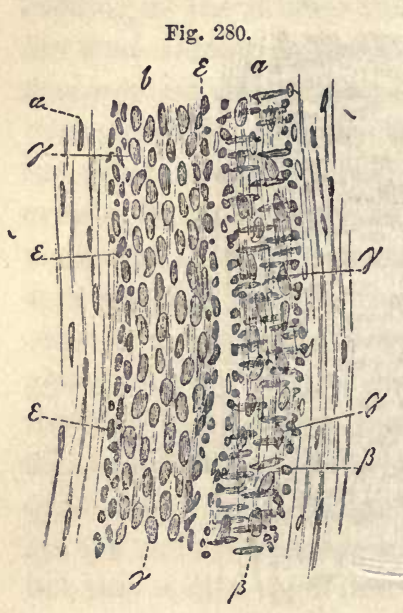
lar fibrous coat in arteries less than 1-10 of a line, down to those having a diameter of 1-25 of a line, still presents $2-3$ layers of muscles and a thickness of $0.005-0.008$ of a line; in smaller vessels it has but one layer, the elements of which at the same time become shorter and shorter; and finally, in vessels between $0.03-0.007$ of a line, appear only in the form of short, elongated or elliptical cells of $0.015-0.006$ of a line with shorter nuclei. In vessels of 0.012 of a line in diameter, these more embryonic forms of contractile fibre-cells still constitute a connected lamina, but after that, they are gradually separated from each other (Fig. 280) and become wholly lost. The t. intima, in vessels more than $0.028-0.03$ of a line in size, has an elastic inner membrane, which, however, in its earliest form, is very delicate, and does not appear to attain its full development in arteries less than $0.06-0.08$ of a line in diameter. On the other hand, the epithelium may be traced in vessels of not more than 0.01 or even of 0.07 of a line; and it may at the same time be remarked, that its cells can no longer be isolated, and its presence only be recognized from the closely placed elliptical nuclei.

Medium-sized arteries abore 4-5 or 1 line, up to those of 2 and 3 lines in diameter, at first, present no great alterations in the external and internal tunics, whilst the $t$. media is not only always increased in thickness in proportion to the size of the vessel (from $0.05-0.12$ of a line), but is also changed in structure. For now, in addition to muscular layers, the number of which constantly increases, but the elements of which are precisely the same as before, we observe fine elastic fibres, which, united into wide-meshed networks, run, at first singly and with little regularity among the muscular elements; but in larger vessels of this category

FIG. 280.-An artery, $a$, of 0.01 , and a vein, $b$, of 0.015 of a line, from the mesentery of a child, magnified 350 diameters, treated with acetic acid. The letters as in Fig. 279: $\varepsilon, t$. media of the vein, composed of nucleated connective tissue. 
are accompanied by a certain amount of connective tissue, and here and there exhibit a disposition to form special layers alternating with the muscular, though not presenting the characters of a continuous network throughout the $t$. media. Thus, although the $t$. media is deprived of its - eminently contractile structure, it must be allowed that the mus-

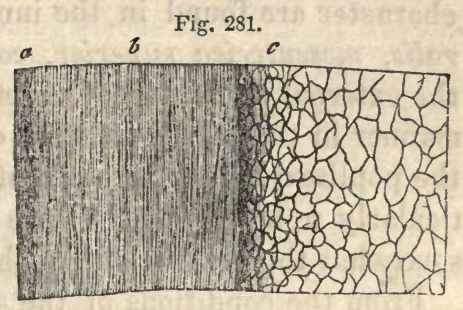
cular fibres here also still retain a considerable preponderance. The t. intima in the medium-sized arteries has, not unfrequently, between the elastic inner membrane and the epithelium, several other layers, among which the above described striped lamelloe are the most remarkable. These lamello, composed of fine elastic networks, wider towards the exterior, and lying in a homogeneous, granular or fibrillated connective substance, constitute the middle lamina of the $t$. intima, the elements of which also all run longitudinally, and are for that reason readily distinguishable from the muscular layers of the $t$. media, to which in some respects they bear a resemblance. The $t$. adventitia, lastly, in almost all these arteries, exceeds the $t$. media in thickness, attaining $0.05-0.16$ of a line. Its elastic fibres at the same time become stronger and stronger, and even in the vessels 1 line in diameter, a considerable aggregation of them, where it adjoins the $t$. media, may be perceived, the line of demarcation between the two tunics being in all these arteries extremely well defined. This elastic membrane of the $t$. Whast adventitia is extremely well marked in the largest of the ressels belonging to the class now under consideration, as in the external and internal carotids, the crural, brachial, profunda femoris, mesenteric, and cæliac, in which it measures from 0.013 to as much as 0.04 of a line, and more, and is to some extent very distinctly laminated, the structure of the lamelloe very often closely approaching that of the true elastic membranes. Besides this, the external layers of the $t$. adventitia also contain elastic networks, only that the elements of the latter are somewhat finer and constitute minute lamello, but are conjoined with less regularity. The largest of the medium-sized arteries exhibit an approach to the largest arteries, inasmuch as, in their $t$. media certain portions of the elastic networks constitute somewhat stronger elastic lamellce, which, however, are continuous through the entire thickness of the tunic, and also, more rarely, form true elastic membranes, by which they are distinguished from the elastic plates of the annular fibrous

Fig. 281.-Transverse section of the art. profunda femoris of Man, magnified 30 diameters: $a, t$. intima, with the elastic layer (the epithelium is not perceptible); $b, t$. media, without elastic lamella, but with fine elastic fibres; $c, t$. adventitia, with elastic networks and connective tissue. 
coat of the largest arteries, yet to be described. Lamellae of the former character are found in the inner layers of the $t$. media of the aa. cruralis, mesenterica superior, caliaca, iliaca externa brachialis, and in the external and internal carotids; whilst they occur in a remarkable manner in the commencement of the tibialis antica and postica, and in the popliteal artery throughout the entire middle tunic; they are particularly well developed in the last-named vessel, which has also usually somewhat thicker walls than the crural.

From the conditions of the $t$. media above stated, and in other respects also, the transition from the medium-sized to the largest arteries is rendered extremely gradual. With respect to the $t$. intima, its epithelial cells in the latter are usually no longer so much elongated as in the smaller vessels, though still retaining their fusiform figure and a length of $0.006-0.01$ of a line. The rest of this tissue does not necessarily increase in amount with the size of the vessel, although it exhibits, especially in the aorta, a great proneness to become thickened, so that it is often difficult to determine its normal thickness. As regards its

Fig. 282.

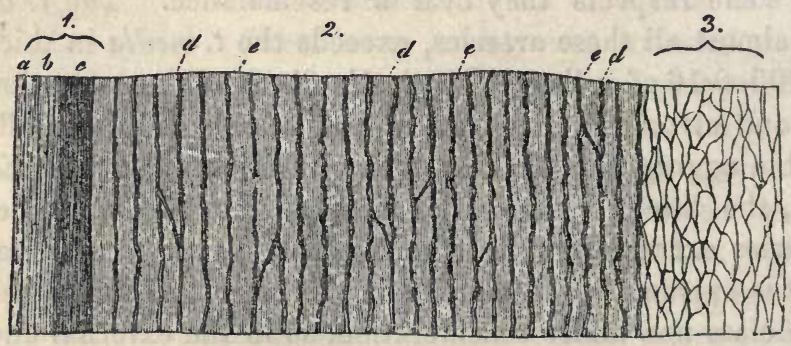

structure, it consists chiefly of lamelloe of a clear, sometimes homogeneous, sometimes striated, or even distinctly fibrillated substance, presenting, for the most part, the characters of connective tissue (Eulenberg obtained some gelatin from the $t$. intima), and is pervaded by finer and coarser longitudinal elastic networks. Usually these networks become more and more close from within to without, and their elements stronger, and on the side towards the $t$. media the inner membrane ceases, either with an elastic close network or a true fenestrated more or less fibrous membrane, obviously corresponding with the elastic inner membrane of the small arteries. Immediately beneath the epithelium the elastic networks are either very fine, or are replaced by one or several clear layers

Fig. 282.-Transverse section of the aorta below the supcrior mesenteric artery. 1, $t$. intima $; 2, t$. media $; 3, t$. adventilia : $a$, epithelium; $b$, striped lamellæ; $c$, elastic membrane of the intima; $d$, elastic lamellæ of the $t$. media; e, its muscles and connective tissue; $f$, elastic networks of the $t$. adventitia. From Man.-Magnified 30 diameters, treated with acetic acid. 
- the striped lamelloc,-which, when nucleated, often appear to consist of coalesced epithelial cells; and when homogeneous and without nuclei, to approach pale elastic membranes. In the annular fibrous layer, the largest arteries present, as a new element, peculiar elastic membranes or plates, which, except in the transverse disposition of their fibres, are constituted in all essential respects in the same way as the elastic inner membrane, particularly of the smaller arteries, and sometimes appear as very thick networks of strong elastic fibres, sometimes as true fenestrated membranes with a less evident fibrous structure. These membranes $0.001-0.0012^{\circ}$ of a line thick, and the number of which may amount to from 50 to 60 , regularly alternate, at distances of $0.003-$ 0.008 of a line, with transverse layers of smooth muscle, which are pervaded by connective tissue and networks of medium-sized elastic fibres; nevertheless, they are not to be regarded merely as tubes inserted regularly one within the other and having the interspaces occupied by muscles ; for, in the first place, they are connected with each other and with the finer elastic network pervading the muscular tissue, sometimes more frequently, sometimes more sparingly; and, in the second place, they are not frequently interrupted in spots, or replaced by coinmon elastic networks. The plates are seen most distinctly and most regularly disposed in the abdominal aorta, the a. innominata, the common carotid, and the smallest of their immediate branches, although these conditions vary considerably in different individuals, so that in the want of very Fig. 283.

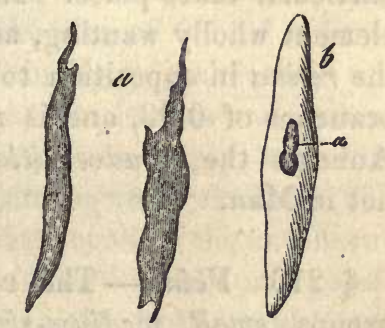
extended researches no general statement with respect to them can be propounded.

Another characteristic of the $t$. media is the slight development of its muscular element. Contractile fibre-cells, it is true, may be found also in the largest arteries throughout all the layers of the middle tunic; but, in the first place, compared with its other elements, the elastic plates, the connective tissue, and the finer elastic networks, they constitute only a very inconsiderable part of that membrane $\left(\frac{1}{3}-\frac{1}{4}\right)$; and, secondly, are so undeveloped in their elements, that it appears very doubtful whether they possess any notable contractile power. For in the aorta and the trunk of the pulmonary artery, the fibre-cells in the inner layers of the $t$. media are often not longer than 0.01 , and 0.004 0.006 of a line broad, and quite flat, so that they are not unlike certain epithelial cells; at the same time their figure is irregular, rectangular, fusiform or clavate, though they still contain the well-known, rod-like

Fra. 283.-Muscular fibre-cells from the innermost layer of the axillary artery in Man; magnified 350 diameters: $a$, without; $b$, with, acetic acid; $a$, nucleus of the fibres. 
nuclei. In the outer layers the fibre-cells are more slender and more elongated, up to 0.02 of a line, and at the same time more like the wellmarked muscular fibre-cells of other organs, though even there retaining somewhat of a rigid and peculiar aspect. In the carotids, subclavian, axillary and iliac arteries, the contractile elements have become more developed, whence also the $t$. media of those arteries does not present the pure yellow color of that of the largest vessels, but begins to assume a reddish tinge. The $t$. adventitia of the largest arteries is, relatively and absolutely, thinner than in the smaller, amounting to $0.04-0.02$ of a line. Its structure, upon the whole, is the same as in otber vessels, although the elastic inner layer is less developed, and also very indistinctly defined from the thick elastic elements of the $t$. media.

The $t$. intima of certain arteries also contains smooth muscles, as I have found in the axillary and popliteal arteries in Man, and as has been lately demonstrated, particularly in the visceral arteries of the Mammalia. In the largest arteries in Man this tunic is very frequently thickened, in which condition a vast increase of the striped lamella in particular takes place. In the $t$. media of no artery is the muscular element wholly wanting, and Henle erroneously adduces the arteries of the retina in opposition to this, for it occurs in those arteries even in branches of 0.03 , and is not absent in any above 0.02 of a line. In Animals the $t$. adventitia of the large arteries contains muscles, but not in Man.

§ 216. Veins. - The veins also admit of being divided into three groups, small, medium-sized, and large, which, however, are not so abruptly defined as is the case with the arteries. The veins, without exception, have thinner walls than the arteries, which depends just as much upon their containing a less considerable quantity of contractile, as upon a more sparing development of the elastic elements; whence also the venous walls collapse more readily, and are less contractile. The $t$. intima, in the larger veins is frequently not thicker than it is in those of medium size; it is less developed than in the arteries, but in other respects, of the same structure. The $t$. media, which is never yellow, but usually grayish-red, contains far more connective tissue, fewer elastic fibres and muscles, and, what chiefly characterizes it, always presents together with the transverse, longitudinal layers also. It is in general thin, but absolutely stronger in the medium-sized veins than in the larger, and in these also the muscular element is most vigorously developed. The $t$. adventitia, lastly, is usually the thickest of the three coats, its relative and ábsolute thickness usually increasing with the size of the vessels. In constitution it precisely resembles that 
of the arteries, except that in many veins, especially of the abdomen, longitudinal rnuscles, occasionally very well developed, appear in it, and give the entire venous wall a peculiar character.

The smallest veins (Fig. $280 \mathrm{~b}$ ), may be said to consist solely of a nucleated, indistinctly fibrous, or homogeneous connective tissue and an epithelium. The elements of the latter are elliptical or round, with oval or even rounded nuclei, whilst the former constitutes a proportionately strong $t$. adventitia, and besides that, a thinner layer, which supplies the place of the $t$. media (Fig. $280 \mathrm{\varepsilon}$ ), both having a longitudinal, fibrous arrangement. Below the size of 0.01 of a line the veins gradually lose the external connective tissue and the epithelium, the $t$. media appearing to pass into the structureless wall of the capillaries. A muscular membrane, and in general, a layer of annular fibres are first seen in veins more than 0.02 of a line in size, in the form, at first, of widely separated, transversely oval cells, with short-oval, sometimes even almost rounded, transverse nuclei. By degrees these cells become more elongated, and more numerous, and ultimately, in vessels of 0.06 0.08 of a line constitute a continuous layer (Fig. $279 \beta$ ), but which is always less developed than the corresponding arterial tissue. This continues to be the structure of veins up to the size of 0.1 of a line; when elastic networks, at first fine, begin gradually to make their appearance externally to the epithelium in the t.t. musculosa and adventitia, whilst at the same time the muscular layers multiply, and even admit connective tissue and fine elastic fibres among their elements.

$V$ eins of the medium diameter of 1-3-4 lines, such as the cutaneous veins, and deeper veins of the extremities up to the brachial and popliteal, the veins of the head and viscera, except the main trunks, are characterized (particularly those of the lower extremity) by the not inconsiderable development of their annular fibrous membrane, which, as in the arteries, is of a yellowish-red color, and striped transversely. But even where thickest, it is far from equalling that of the corresponding arterial vessels; and in thickness never exceeds 0.06 to 0.07 of a line. It consists not only of transverse but also of longitudinal layers, and in this respect likewise differs from that of the arteries. The transverse layers are composed of common, undulating connective tissue, with fine, loosely ar-

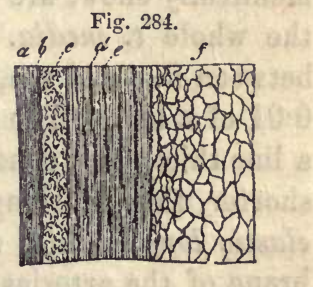
ranged, or rather isolated elastic fibres (nucleated fibres, as they are

Fra. 284.-Transverse section of the vena saphena magna, at the mallelus, magnified 30 diameters : $a$, striped lamella and epithelium of the t. intima; $b$, its elastic membrane; $c$, longitudinal, internal connective-tissue layer of the $t$. media, with elastic fibres; $d$, transverse muscles, and $e$, longitudinal elastic networks, disposed in a laminated manner; $f, t$. adventitia. 
termed), and a large quantity of smooth muscles, the fusiform elements

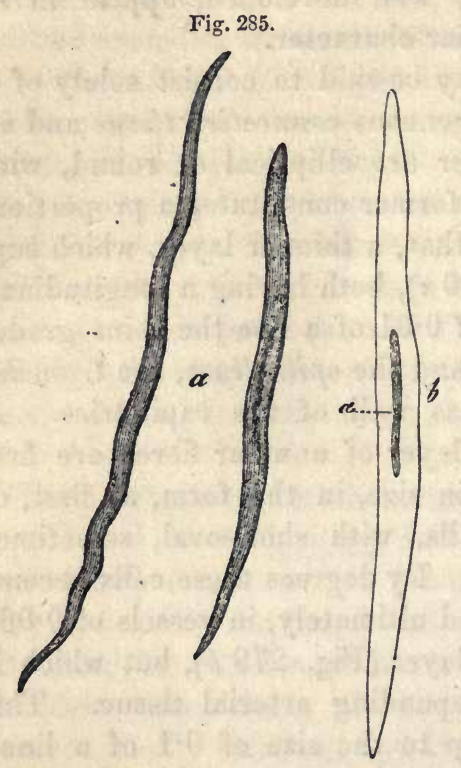
of which, 0.02-0.04 of a line long, and 0.004-0.007 broad, present the usual characters of contractile fibrecells, whilst the longitudinal laminæe consist of true, thick, and very thick, reticulated elastic fibres. With respect to the mutual relations as lamince of these tissues, it should be remarked that in certain veins (popliteal, profunda femoris, saphena major and minor), the $t$. intima is succeeded by a layer, formed solely of connective tissue and finer elastic networks, and having a longitudinal fibrillation-the longitudinal lamina of the $t$. mediawhilst in other veins the muscular elements extend also into the innermost lamina. In this case, immediately external to the $t$. intima, there is a transverse layer of muscles, with connective tissue and elastic fibrils, which three tissues, in these veins, always accompany each other; to this succeeds a regular alternation of longitudinal, elastic, reticulated membranes, always in a single layer, and transverse muscles with connective tissue, so that the $t$. media of these veins presents a laminated aspect, somewhat resembling that of the largest arteries. It should, however, be remarked, that the elastic, reticulated membranes, although frequently very closely interwoven, nevertheless never form homogeneous, elastic membranes; moreover, that they are occasionally interrupted, and, as longitudinal sections manifestly show, are invariably continuous with one another through the whole $t$. media. The number of these elastic lamelloe fluctuates between five and ten, and their interspaces vary in width from $0.004-$ 0.01 of a line. The $t$. intima of the medium-sized veins is $0.01-0.04$ of a line thick, and consists, where it is thinner, only of an epithelium with shorter, though elongated cells, a striated, nucleated lamella, and an elastic longitudinal membrane, corresponding to the elastic inner membrane of the arteries, but which scarcely ever appears as a truly homogeneous, fenestrated membrane, but most usually as an extremely close, areolated network of finer and coarser elastic fibrils. Where the $t$. intima is thicker, the striated lamellce are multiplied, and, above all, one or even several additional networks of elastic, fine fibres make their ap-

Frg. 285.-Muscular fibre-cells from the renal vein of Man; $a$, without ; $b$, with acetic acid; $a$, nucleus of the latter.-Magnified 350 diameters. 
pearance on the inner aspect of the above-described elastic membrane, which forms the limitary portion of the $t$. intima. I have also seen smooth muscles of the $t$. intima, in the veins of the gravid uterus, as well as in the saphena major and popliteal vein ; and Remak has confirmed their existence in the visceral veins of certain Mammalia. The $t$. adventitia of these veins is almost invariably thicker than the $t$.media, often twice as thick, rarely of equal strength. Usually it contains only longitudinal, much interwoven, often very well-marked elastic networks with thick fibres, and common connective tissue, but in the case of those visceral veins, the trunks of which have longitudinal muscles in the $t$. adventitia, similar muscular elements also extend for a certain distance into the branches (vid. seq.)

The largest veins are distinguished from those of the medium diameter chiefly by the sparing development of the $t$. media, and especially of its muscular elements; a deficiency, however, it is true, often counterbalanced by the presence of contractile elements in the $t$. adventitia. The thickness of the $t$. intima is usually 0.01 of a line, when it presents the same conditions as in the medium-sized veins. More rarely, as occasionally in the vena cava inferior, in the trunks of the hepatic veins, and in the vena innominata, it amounts to 0.02 and 0.03 of a line, which increase of thickness is due to striped lamelloe with nuclei, and fine, elastic, longitudinal networks, never to those composed of muscles. The $t$. media, on the average, presents a thickness of $0.02-0.04$ of a line, but may occasionally, as at the commencement of the trunk of the vena portoe, in the uppermost part of the abdominal portion of the $v$. cava inferior, and at the orifices of the hepatic veins, measure 0.05-0.12 or be wholly wanting, as in the greater part of the $v$. cava inferior in the liver, and in the further course of the largest hepatic veins. Its structure, in all essential particulars, is the same as in the previous class of vessels, except that the longitudinal elastic networks are intricately connected together, and less distinctly, or not at all, laminated; the transverse muscles, also, are scanty and indistinct, even where the $t$. media possesses the considerable thickness above stated, and are more abundantly intermixed with bundles of connective tissue. I have noticed the muscles to be most developed in the splenic vein and $v$. portoe; they appeared to me to be wholly wanting in the abdominal portion of the inferior vena cava below the liver, in certain spots, and also in the subclavian vein, and in the terminal portions of the superior and inferior vence cavce. The $t$. adventitia of the largest veins, is almost invariably nearly twice as thick as the middle tunic, or even as much as five times as thick, and exhibits, in its structure, the important difference, at least in certain veins, as Remak correctly states, that it contains a considerable quantity of longitudinal muscles. These are very distinct, as was pointed out by Bernard ("Gaz. Med. de Paris," 1849, 17, 331), in the 
hepatic portion of the inferior vena cava, where their fasciculi, 0.010.04 of a line thick, constitute a network pervading the inner half or two inner thirds of the external membrane, which, where the $t$. media is absent, rests immediately upon the $t$. intima, and may attain a thickness of as much as 0.22 of a line. Besides this, I have found, as Remak has also done, these contractile, longitudinal bundles (which never contain

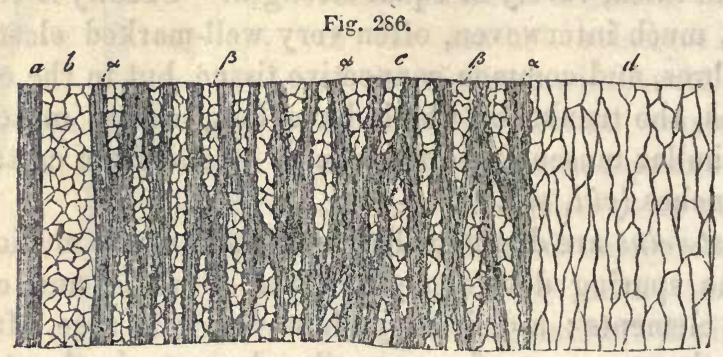

connective tissue, though probably a certain number of elastic fibres), still very well developed, in the trunks of the hepatic veins, in that of the vena porto, and in the remaining portion of the inferior vena cava, and have traced them as far as the splenic, superior mesenteric, external iliac, and renal veins. Some were also to be found in the vena azygos, whilst they were altogether absent in the superior veins. These muscles extended through the entire thickness of the $t$. adventitia, only in the renal vein and vena porto, whilst in the other veins abore enumerated, a greater or less portion of it, contained no muscular element, and consisted as usual, of longitudinal connective tissue, and elastic networks composed of strong fibres. The muscular layer of the $t$. adventitia therefore appears to be a special membrane of the vessels, and occasion would be afforded to confound it with the undeveloped, or, as has been stated, even absent $t$. media; an error, however, which might be readily avoided by tracing the conditions of the smaller veins. The muscular layer of the $t$. adventitia, besides the contractile elements, having a length of $0.02-0.04$ of a line, which present the common character, and numerous elastic longitudinal networks, invariably contains a certain amount of connective tissue, which, as it seems, is always arranged transversely, so that the transverse elements, even in these large veins, are compensated for though not exactly, by muscle. All the large veins, which open into the heart, are furnished, for a short distance, with an external annular layer of muscles similar to those of the heart itself, and which also present anastomoses of the primitive

FIG. 286.-Longitudinal section of the inferior vena cava, in the liver, magnified 30 diameters: $a, t$. intima $; b, t$.media, without muscles, containing only connective tissue and elastic fibres; $c$, inner layer of the $t$. adventitia; $a$, its longitudinal muscles; $\beta$, transverse connective tissue of the same layer; $d$, external portion of the $t$. adventitia, without muscles. 
fasciculi. According to Räuschel, these muscles, in the region of the superior vena cava, extend as far as to the subclavian vein, and may be found also in the main branches of the pulmonary veins; and even, according to Schrant, in the former case, more in the interior of the wall of the vessels, and disposed longitudinally.

The veins in which the muscular element is excessively developed demand special notice, and also those in which that element is wholly wanting. To the former class belong the veins of the gravid uterus, in which besides the $t$. media, the $t$. intima and adventitia also present muscular layers, longitudinal in the two latter, the elements of which in the 5 th and 6 th month exhibit the same colossal development as those of the uterus itself. The muscular element is wanting: 1 , in the veins of the maternal portion of the placenta, in whose walls, externally to the epithelium, large cells and fibres, which I regard as undeveloped connective tissue, occur; 2 , in most of the veins of the cerebral substance, and of the pia mater. These veins consist of a roundish epithelium in a single layer, a thin longitudinal connective layer, with solitary elongated nuclei, which supplies the place of the $t$. media, and in the smaller vessels of a more homogeneous, and in the larger, of a fibrillated and nucleated $t$. adventitia. It is but rarely that a faint indication of muscles in the $t$. media is seen in the largest of these veins, as shown in Fig. $279 ; 3$, in the sinuses of the dura mater and the veins of Breschet in the bones, which are furnished with a layer of connective tissue, occasionally containing fine elastic fibres, external to a tessellated epithelium, and which layer is continuous with that of the dura mater and of the internal periosteum. 4, in the venous sinuses of the corpora cavernosa (vid. sup.), and of the spleen of certain Mammalia (vid. § 169). 5, in the veins of the retina.

The valves of the veins consist chiefly of distinct connective tissue, which, at their free border, runs transversely, containing numerous elongated nuclei, and also isolated, undulating, usually fine, in part strong, elastic fibres. Their surface is covered either with nothing but an epithelium, with short cells, or in addition, there is beneath it, a very fine elastic network, the prevailing direction of which is longitudinal. The valves may, therefore, be regarded as continuations of the middle and internal tunics, although muscular fibres, so far as I have seen, are wanting in them (Wahlgren states that he has found such fibres in the larger valves).

§ 217. Capillaries (vasa capillaria).-With the solitary exception of the corpora cavernosa of the sexual organs, and of the uterine placenta, the arteries and veins, in Man, are universally connected by the intervention of a rich plexus of microscopically fine vessels, which, on account of their slender dimensions, have been designated under the above name. 
They are everywhere composed of a single, structureless membrane, with cell-nuclei, and are thus very essentially distinguished from the larger vessels, although the transition from the one to the other is wholly imperceptible; so that at a certain point in the course of the vessels it is quite impossible to detect the characters of either of the classes into which Histology has been accustomed to divide them. Vessels of this kind may be best described as venous and arterial transitionary vessels, according as they lie on the one side or the other, and without any further alteration of the common classification, may be referred to the capillaries.

The true capillaries, when more closely examined, exhibit the following conditions: Their structureless membrane is perfectly clear and transparent, sometimes delicate and presenting a simple contour, sometimes thicker $(0.0008-0.001$ of a line $)$, and bordered by a double line. In its microscopical reactions, it corresponds entirely with older cell membranes and the sarcolemma of the transversely striped muscles (vid. § 85), and as regards its other properties, it is perfectly smooth on both aspects, and notwithstanding its tenuity, tolerably resistant and elastic, although very probably not contractile. It invariably presents a certain number of elongated cell-nuclei, 0.003-0.004 of a line in size, which are disposed with wide interspaces, usually alternately on opposite sides of the vessel, sometimes more approximated, or in close contiguity, though rarely in actual contact; and, when the capillary tunic is thin, are situated on its inner side, when thicker, within its substance, in such a way, however, as not unfrequently to cause projections of it on the outer surface. The diameter of the capillaries, in Man, varies from 0.002 to 0.006 of a line; and, for the sake of description, they may be again subdivided into finer, of $0.002-0.003$ of a line, with few nuclei and thinner walls; and coarser, of $0.004-0.006$ of a line, with a thicker membrane and numerous nuclei; although in so doing it is not intended to draw any distinct limitation between them.

The capillaries, by their union, constitute the capillary plexuses, retia capillaria, which have already been described in detail, in speaking of the individual organs and tissues, and, consequently, here, will be referred to only in brief and general terms. The forms presented in these plexuses, which, notwithstanding considerable diversities, are constant in the different organs, and according to their similarity or diversity, more or less characteristic, depend to some extent upon the disposition of the elementary parts, and are also in some degree dependent upon the energy of the functions. With respect to the former, there are in many organs certain tissues, into which vessels never penetrate, -as the transversely striped muscular fibres, the bundles of connective tissue, nerve-fibres, cells of all kinds, gland-vesicles, and which, therefore, according to their form, 
trace out definite courses for the capillaries, so that they sometimes present elongated meshes, sometimes rounded, narrower or wider reticulations. The physiological energy is still more influential, and it is a general rule, that the greater the activity of an organ, whether as regards contractions or sensations, excretion or absorption, so much the closer is the capillary network, and so much the more abundant the supply of blood. The capillary plexuses are closest in the secernent and absorbent organs, as in the glands, above all in the lungs, liver, and kidneys; next in the integuments and mucous membranes; much wider in the organs which receive blood only for the purpose of their own nutrition, as the muscles, nerves, organs of sense, serous membranes, tendons, and bones; although among these organs differences exist, as, for instance, the muscles, and the gray nerve-substance, are more abundantly supplied than the other parts above enumerated. The diameter of the capillaries themselves is almost directly in an inverse relation, and they have the thinnest walls and are smallest $(0.002-0.003$ Fig. 287

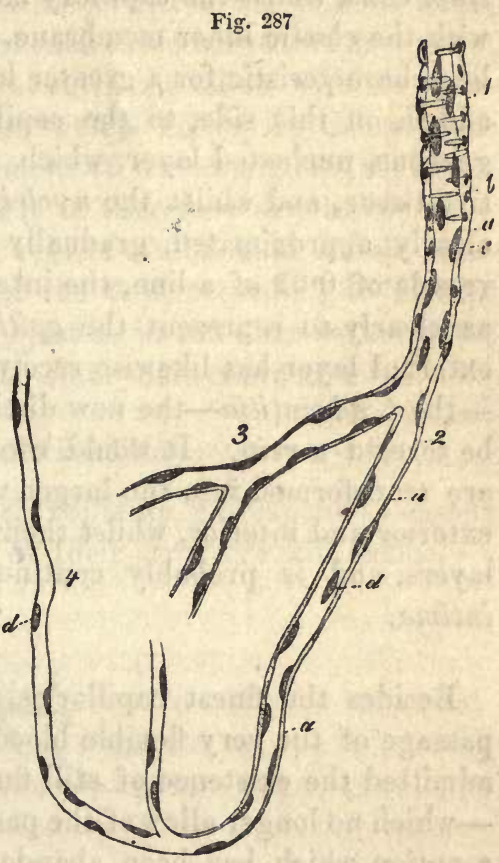
of a line) in the nerves, muscles, retina, and the Peyerian patches; in the external integument and mucous membranes they attain the size of $0.003-0.005$, in the glands and bones, lastly, one of $0.004-0.006$ of a line, and in the compact substance of the latter, although no longer having, in all respects, the structure of capillaries, even the diameter of $0.008-0.01$ of a line. Physiology is not as yet in a condition to explain these differences in all particulars, inasmuch as information is wanting with respect to the laws of diffusion in the various capillary membranes; and also because the more minute conditions of the sanguineous circulation, in the separate organs, are wholly unknown.

The mode in which the capillaries pass into the larger vessels is diffcult of investigation. On the arterial side it is found that the capil-

Fig. 287.-Finest vessels on the arterial side of the capillaries. 1, an artery of the smallest size ; 2 , transitional vessel ; 3 , coarser capillary ; 4 , finer capillary : a, structureless coat, with a few nuclei, representing the $t$. adventitia; $b$, nuclei of the muscular fibre-cells; $c$, nuclei within the minute artery, probably still belonging to an epithelium; $d$, nuclei of the capillaries and transitional vessels. From the human brain; magnified 300 diameters. 
- laries, as they become wider, present more closely placed nuclei, and are then invested externally with a structureless $t$. adventitia, and solitary muscle-cells, whence, when they have reached the diameter of 0.007 of a line, they already exhibit the aspect of the finest arteries (Fig. 287, 1). Afterwards, the nuclei seem to be replaced by epithelium cells, whilst the capillary membrane either ceases, or is continuous with the elastic inner membrane. The venous transitionary vessels are less characteristic for a greater length. The first thing that is superadded, on this side, to the capillary membrane, is an external, homogeneous, nucleated layer, which may be regarded as a sort of connective tissue, and whilst the nuclei of the capillary vessels become more closely approximated, gradually coalesces with their membranes. In vessels of 0.01 of a line, the internal nucle $i$ have become so numerous, as clearly to represent the epithelium, and as at this time also, the external layer has likewise received the addition of a nucleated lamina - the $t$. adventitia - the now distinctly laminated vessel (Fig. 280) may be termed a vein. It would consequently appear that the capillaries are transformed into the larger vessels by the addition of layers on the exterior and interior, whilst their proper membrane coalesces with these layers, and is probably continuous with the fibrous layer of the $t$. intima.

Besides the finest capillaries; which, however, always admit of the passage of the very flexible blood-corpuscles, the older anatomists have admitted the existence of still finer vessels-the so-termed vasa serosa - which no longer allow of the passage of blood, but only of its plasma,a notion which has been abandoned by most modern authors. Hyrtl alone thinks, that it is necessary to admit of the existence of vessels of this kind in the cornea, because the vessels at its border escape the sight without passing into veins, and are too small (in Man, when injected, 0.0009 of a line) to be capable of conveying blood-corpuscles. He thinks, that still further on they are continued into vasa serosa, and probably are connected with the, as yet undemonstrated, lymphatics. In opposition to this, Brücke and Gerlach remark, that the corneal vessels terminate in true loops, and that it would thence appear that Hyrtl's statements are based upon incomplete injections. I am able, however, to state that something corresponding with the vasa serosa of authors does actually exist in the cornea, having noticed that, in the Dog, fine and the finest filaments are continued still further inwards, from the terminal loops previous to the blood-corpuscles, which occur in that animal, as in all others, at the margin of the cornea; which filamentary prolongations were connected in a reticular manner, and were usually slightly dilated at the points of junction. Whether these filaments were hollow, and had any contents, and directly communicated with the canals of the true 
capillaries, could not be determined, and I should not, therefore, at present definitely declare them to be pervious parts of the vascular system; whilst I have not the least hesitation, nevertheless, in referring them to that system, for although they may have no canal, they scarcely admit of any other possible interpretation, than as being derived from the vascular plexus, which covers almost the entire cornea in the child at birth, and as being obliterated capillaries. Should these corneal elements not turn out to be vasa serosa, I am acquainted, in the adult, with no situation in which such vessels exist, whilst vessels conveying plasma are everywhere present during the development of the capillaries, as a preliminary phenomenon (vid. infra), and it is therefore intelligible that, even at a subsequent period, scattered ressels of the kind should occasionally be met with, as in the brain of the Calf, according to Henle; or may perhaps exist in great quantity, just as in the distribution of the nerves, the terminations often retain the embryonic character.

\section{3.-OF THE LYMPHATICS.}

$\S 218$. The lymphatics, except in their contents, correspond so closely with the veins, that a short exposition of their structure will suffice.

Fig. 288.

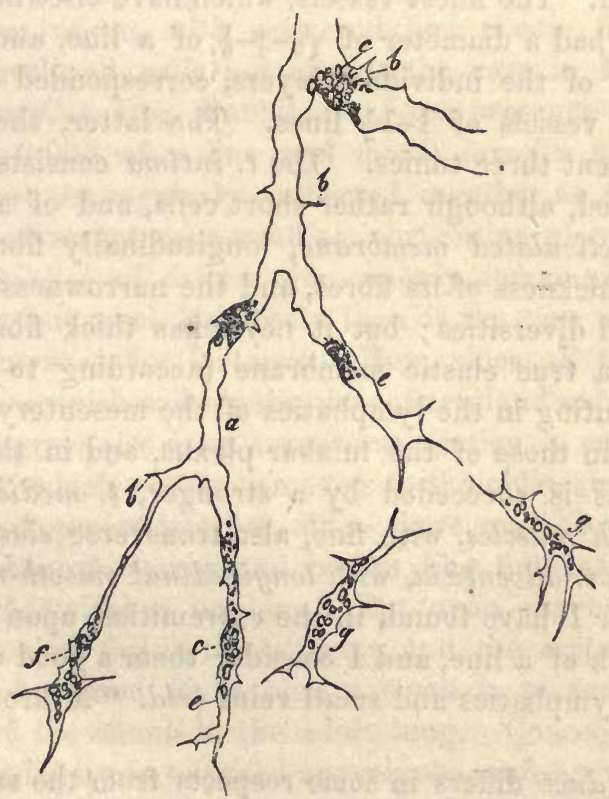

Frg. 288,-Capillary lymphatic from the tail of the Tadpole, magnified 350 diameters: $a$, membrane; $b$, processes formed by it; $c$, remains of the contents of the cells forming these vessels, in which nuclei are concealed; $e$, cæcal terminations of the vessels; $f$, one of these terminations, still pretty distinctly recognizable as a formative cell; $g$, isolated formative cells about to join with the actual vessels. 
The capillary lymphatics, which, in the three situations in which they have hitherto been seen with certainty-in the small intestine, the tail of the Tadpole, and the mucous membrane of the trachea-commence partly with free prolongations, in part in networks. I have, in a single instance in the tracheal mucous membrane in Man, had an opportunity of investigation, and they were found to consist of a delicate structureless wall, without distinct nuclei after the addition of soda, and having a diameter of $0.003-0.005-0.01$ of a line (Fig. 235). The same structure is presented in the simple lacteals of the intestinal villi in Mammalia, except that these measure $0.012-0.026$ of a line, and have a somewhat thicker wall. The lymphatics discovered by me in the tail of the Tadpole (Fig. 288), on the contrary, correspond entirely with the blood-capillaries, in the occurrence of nuclei on the inner side of the very delicate, structureless membrane, whilst they differ from them in being furnished with short, jagged processes with prolongations. The diameter of the lymphatic capillaries in this situation is $0.002-0.015$ of a line, and the two main trunks of the tail, like those of the blood-vascular system itself, have a perfect capillary structure.

In what way these capillary lymphatics are changed into the larger lymphatic canals has not been seen by any one, or at all events has not been investigated. The finest vessels, which have elsewhere come under my observation, had a diameter of $\frac{1}{10}-\frac{1}{7}-\frac{1}{6}$ of a line, and these, except in the thickness of the individual layers, corresponded in all respects with the larger vessels of $1-1 \frac{1}{2}$ lines. The latter, the medium-sized lymphatics, present three tunics. The $t$. intima consists of an epithelium, of elongated, although rather short cells, and of a single, rarely double elastic reticulated membrane, longitudinally fibrillated, which, as regards the thickness of its fibres, and the narrowness of the meshes, exhibits manifold diversities; but it never has thick fibres, nor does it ever constitute a true elastic membrane (according to Weyrich, this membrane is wanting in the lymphatics of the mesentery, whilst I have always found it in those of the lumbar plexus, and in those of the extremities). This is succeeded by a stronger, $t$. media, composed of transverse smooth muscles, with fine, also transverse elastic fibres; and lastly, there is a $t$. adventitia, with longitudinal smooth-muscular fasciculi. The latter I have found, in the extremities, upon vessels of not more than 1-10th of a line, and I consider them a good distinctive character between lymphatics and small veins (vid. "Mikrosk. Anat.," II. p. 236).

The thoracic duct differs in some respects from the smaller lymphatics. The similarly constituted epithelium is succeeded by some striped lamella, and these by an elastic reticular membrane, longitudinally fibrillated, although the entire $t$. intima scarcely measures $0.006-0.01$ of a line. The $t$. media, 0.025 of a line thick, commences with an 
extremely thin layer of longitudinal connective tissue, with fine elastic fibres, and consists, besides, of a transverse muscular layer, with fine elastic fibres. The $t$. adventitia lastly, contains longitudinal connective tissue, together with elastic fibrils, and a few reticularly connected bundles of longitudinal muscles. The valves of this duct, and of the lymphatics in general, correspond completely with those of the veins.

The bloodvessels of the lymphatics present the same Fig. 289. conditions in the thoracic duct as in the veins. No nerves have been found in them.

§ 219. Lymphatic glands. - These glands (glandulo lymphaticce) differ very considerably from the rest of the blood-vascular glands with which they are usually classed, and approach nearest to the Peyerian patches of the intestine, although they do not wholly correspond with them. Every normal lymphatic gland, within a thin but tough sheath, composed of nucleated connective tissue and fine elastic fibrils, presents a soft, whitish-red parenchyma, in which three elements, viz. a fibrous tissue, a soft, pultaceous pulp, and bloodvessels, are manifest. The fibrous tissue, formed partly of fibrous, and in part of more homogeneous connective tissue, with scattered fine elastic fibres, when the gland is well developed, as is not always the case in Man, but almost invariably in the Cat, Dog, Rabbit, Rat, \&c., presents a large number of thin (0.004-0.005 of a line and more) lamelloe arising from the sheath, which are so regularly connected together as to constitute an elegant areolated structure pervading the entire gland, all of whose roundish spaces, $\frac{1}{6}-\frac{1}{3}$ of a line wide, openly communicate, it is true, with each other, but much less freely than is the case with the cells of the corpora cavernosa, for instance. Now, since all these spaces are occupied by the grayish-white pulp, the entire gland exhibits externally, and, in some degree, also in a transverse section, a coarsely granular, vesicular aspect, which was known even to the older anatomists, almost like that of the Peyerian patches, since there may be distinguished in it a great number of clear round bodies, like follicles, surrounded by narrow, somewhat darker borders. But upon proceeding to isolate these bodies we shall fail in the attempt, and the septa by which they are parted will be found to be always common to several, something like the walls of the alveoli in the adult lung. Consequently, notwithstanding the similarity in outward appearance, and as we shall find, in contents also, there is a very essential difference between the follicles of

FIG. 289.-Transverse section of the thoracic duct in Man, magnified 30 diameters: $a$, cpithelium, striped lamclla, and elastic inner membrane; $b$, longitudinal connective tissue of the $t$. media; $c$, transverse muscles of the same tunic ; $d, t$. adventitia, with $e$, the longitudinal muscles. 
the Peyerian patches, as well as of the spleen, and of the tonsils, and the alveolar spaces in the lymphatic glands, on which account I shall describe the latter as "alveoli."

The grayish-white alkaline pulp which fills the spaces in question, agrees in nearly all respects with that contained in the Peyerian follicles, and consists of a certain proportion of fluid, and of very many morphological elements. The latter are, in part free nuclei, of $0.002-$ 0-003 of a line, usually without distinct nucleoli, with homogeneous contents, which are nevertheless rendered turbid by water; in part true, pale, uninuclear, round cells, most of which measure from $0.003-$ 0.004 of a line, with nuclei similar to those found in the free condition, a certain number of larger size $(0.005-0.007$ of a line) with larger, often distinctly vesicular nuclei and nucleoli, and occasionally a few fat granules. These morphological elements also, to some extent, correspond entirely with the cells of the lymph and chyle; a circumstance in itself of no great significance, since no specific character can be assigned to either of them. The similarity of the contents of the alveoli of the lymphatic glands, with those of the follicles of the Peyerian patches, is still further increased by the circumstance, that they are also penetrated by a fine vascular network, as I at least have observed, and have already stated in another place ("Mikr. Anat.," II. 2, p. 192). For the numerous bloodvessels of the lymphatic glands, which frequently penetrate into the interior at a depressed hilus-like spot, are not distributed merely in the septa of connective tissue, as has hitherto been generally asserted, but, as I have seen in Man, also enter the pulp filling the alveoli, where they run freely among the elements, and form a very fine capillary plexus, bearing the closest resemblance to that of the Peyerian follicles, except that in general it is rather wider, and frequently also varicose.

The most difficult part of the anatomy of these glands is the ascertaining of their connection with the lymphatic vessels. After the majority of the most recent authors had agreed in the assumption, that the vasa inferentia and efferentia were connected by numerous anastomoses of convoluted and looped vessels, the proper parenchyma of the glands being thus frequently left in the background or altogether forgotten, the number of those has latterly been much increased, who advocate the view originally propounded by Malpighi, viz. that the lymphatic glands consist of an aggregation of anastomosing cells, into which the vasa afferentia open, and from which the vasa efferentia proceed; and, in particular, Ludwig and Noll have declared themselves most decidedly in favor of this view. As for myself, it will be apparent from the preceding account, that $I$ am one of those who admit of the existence of a special glandular element in the lymphatic glands, and I consequently deny, in the most explicit terms, that they are com- 
posed merely of a plexus of lymphatic vessels. As regards the relation of the lymphatics to the glandular part or alveoli, together with their contents, I formerly felt compelled to express myself in opposition to the notion propounded by Ludwig and Noll, although without having entered deeply into the subject, principally because, whilst it seemed to me improbable, that the alveoli of the glands in question should contain bloodvessels, and at the same time communicate with the lymphatics; and, in the second place, because in cases where the vasa afferentia and efferentia were full of milk-white chyle, I was never able to perceive similarly colored contents in the alveoli. These facts, indeed, still retain all their weight with me; but they are now more than outweighed by further experience, so that it appears very doubtful whether they justify the conclusions which I formerly thought might be deduced from them. For I find, like Ludwig, in a considerable number of injections in the human subject, Dog (cervical glands), and $\mathrm{Ox}$ (lumbar glands), that it is impossible to fill lymphatic vessels in the interior of the glands, and that the injection either colors only the ramifications of the vasa inferentia upon the gland, or when it runs further, as it may be more easily made to do in animals than in Man, it enters the alveoti, which it fills according to their position in the series, and escapes through the vasa efferentia. Induced by the result of these experiments, I should now, without being desirous of giving a definitive opinion, be inclined to side with Ludwig, and to deny the existence of any direct connection between the afferent and efferent lymphatics, or rather to view the alveoli of the glands as a specially modified part of them.* In accordance with this notion, the lyinph would be poured out into the alveoli, and flow through them in fine divided streams among the elements of which their contents are composed; and, to this circumstance, it is probably owing that it never has a milk-white color. In this process it is possible that some of the cells of these contents, which so closely resemble the lymphcorpuscles, may be detached and become disintegrated, whence the chyle of the vasa efferentia abounds more in morphological elements, than the fluid conveyed by the vasa efferentia. At the same time, I am decidedly opposed to the view which would regard the morphological contents of the alveoli as directly appertaining to the lymph, as lymphcells-which are there formed, and subsequently conveyed away from the gland. I consider them rather as an independent, stationary, glandular element, standing, indeed, in the closest relation with the chyle, but not necessarily forming a part of it, or passing into the

* [In a paper since published, Prof. Kölliker states, that he is now entirely satisfied of the correctness of the views of Ludwig and Noll. He considers, however, that the lymphatics regain their coats in the medullary substance, where they form a minute plexus, from which the vasa efferentia proceed ( $V i d$. Kolliker, "Ueber den feineren Bau u. die Functionen der Lymphdrusen," in "Verhand. d. Phys. Med. Ges.," in Wurzb. IV. 2, 1854.) $-\mathrm{DaC}$. 
blood. If we ascribe to the alveoli of the lymphatic glands the function of inducing a metamorphosis and changes in the lymphatic fluid flowing through them, under the influence of the cells of its pulp, which are manifestly in a continual process of development, of such a kind probably, that its elements are rendered more capable of development, or new matters, such as fibrin, are mixed with it, the reason is at once evident, why the lymphatic fluid should form more cells after its passage through the glands than previously. The well-known cases also of "white blood," in which, together with an enormous increase in size of the lymphatic glands, a vast multiplication of the colorless bloodcells takes place (Virchow), may be explained in accordance with the above view; although, for the present, I am not disinclined to assume, that although no constant and total passage of the pulp of the lymphatic glands into the lymph takes place, which from the anatomical conditions is utterly impossible (considering the bloodvessels in the alveoli), nevertheless a sort of commixture of it from the alveoli contiguous to the vasa efferentia occurs, so that the lymphatic glands, after all, at least to some extent, appear to afford a site for the formation of lymph-corpuscles.

The lymphatics of the lymphatic glands retain all their tunics up to the gland. But as they ramify in an arborescent manner on the gland and become smaller, they lose the muscular membrane, and enter the alveoli, possessing only a layer of connective tissue with fine elastic fibres, and an epithelium. The glands, at all events the larger ones, always have some delicate nervous filaments composed of fine fibres, which enter in company with the bloodvessels, and are lost to sight in the interior. The ganglia in the lymphatic glands, mentioned by Schaffner ("Zeitsch. f. rat. Med.," VII. 177), I have not been able to find, nor is that author's description of the kind to command much confidence.

The above-described structure of the lymphatic glands does not apply to all cases. In Man, and in other animals, there are small and smallest glands, of $3-2-1$, or even $\frac{1}{2}$ a line, in which the interior does not distinctly exhibit the alveolar structure, appearing, on the contrary, to be more continuous throughout, and homogeneous, notwithstanding a good many traces of fibrous structure, which always exist in them. In the larger glands also, especially in certain animals, a similar condition of the contents is not unfrequently presented, which of course does not materially affect the above exposition of the structure of the lymphatic glands, since, in such cases, we simply see a less developed condition of the septa, and a more intimate union of the individual parts of the pulp.

O. Heyfelder has lately described (1. c.), in the lymphatic-glands of 
the Mouse; the Rat, and, to some extent, of the Rabbit, a complete muscular layer. In the Bat also, the Dog, Sheep, $\mathrm{Ox}$, Goose, and Fowl, smooth muscles are said to occur sparingly, and to be fewest in Man. Heyfelder also says that they pass into the internal septa, and that in the Rabbit he has noticed contractions of the glands upon electrical excitement, an experiment which has not yet succeeded with me.

The lymphatic glands are subject to numerous degenerations. The most frequent are extravasations of blood in the alveoli, and in consequence of these effusions, depositions of pigmentary matter, which may proceed to such an extent as to render the glands brownish-red, or even black (bronchial glands); we also find thickenings of the sheath, and of the internal septa; fatty deposits in the bloodvessels; hypertrophies, with a uniform increase of all their parts; tuberculosis and cancer.*

\section{4.-OF THE BLOOD AND THE LYMPH.}

$\S 220$. Every part of the vascular system contains in its interior a special liquid, consisting of a fluid and numerous morphological particles, and which, according to its color, its occurrence in one or other division of the system, and its other properties, is distinguishable on the one side into white and red blood, and lymph or chyle; and on the other, into blood in the more strict sense of the term. Histology is concerned only with the description of the morphological elements existing in these

* [Professor Brücke, in a valuable communication read before the Vienna Academy, March 31, 1853 ("Ueber die Chylus.gefasse und die Fortbewegung der Chylus"), confirms the account given by Ludwig and Noll of the structure of the lymphatic glands, and states that the vasa inferentia break up into the porous, glandular tissue, out of which the vasa efferentia arise anew. In the glands themselves, a distinction must be drawn between the cortical substance, composed of round or ovate bodies, like the separate glanclular bodies of Peyer's patches, and the medullary substance. The framework of the latter is formed by the large bloodvessels, with their tunica adventitic. One portion of their branches divides into capillaries in the medullary substance, the rest enter the cortical substance. The accompanying connective tissue becomes looser, the finer the branches. 'The fully developed connective fibres disappear more and more, and in their place cytoblasts appear, with closely investing cell-membranes, which run out into two or three pointed, sometimes flat, usually filiform processes, fitted together into a soft tissue, in which the capillaries of the medullary substance lie. Round cells in different stages of development follow them, resembling the lymph-corpuscles, and forming the immediate limit of the fine, irregular, frequently anastomosing canals, which render the medullary substance as porous as a sponge. The whole gland is enclosed in a membrane, which, as Heyfelder observed, is composed of connective tissue and smooth fibre-cells, and sends sheaths in towards the medullary substance, whereby imperfect compartments are formed, in which the glandular elements lie. The cliyle of the vasa inferentia traverses the glandular elements, enters the pores of the medullary substance, and passes thence on the opposite side, between the glandular elements to the vasa inferentia. "I have never," (says Brücke) "observed the fat-drops of the chyle enter into the interior of the glandular elements, which appear to be merely bathed with the fluid part of it. On the other hand, the cells which are formed in the glandular elements pass as lymph-corpuscles into the chyle."-Trs.] 
fluids, among which the blood- and lymph-corpuscles are by far the most inportant, leaving the description of their other conditions to physiology.

$\S 221$. The lymph and the chyle, like the blood, consist of a plasma, which coagulates out of the vessels; and of morphological elements, including elementary granules, nuclei, colorless cells, and red blood-corpuscles, which, however, are not found in all parts of this vascular system, nor everywhere in equal quantity. The elementary granules are immeasurably minute granules, which, as has been shown by H. Müller, consist of fat and a protein-envelop, and are contained in vast numbers in milky chyle, whose color is owing to them alone, whilst, in the more colorless lymph they are either wholly wanting, or are rare and scattered. Free nuclei, 0.001-0.002 of a line in size, and of a more homogeneous aspect, becoming vesicular and granular on the addition of water,

Fig. 290.

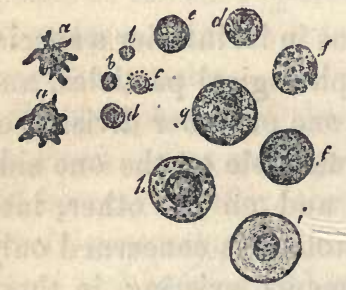

I have hitherto noticed only in the commencements of the lacteals in the mesentery, and in the vasa efferentia of the mesenteric glands, though even there scantily, and never in the thoracic duct; whilst the colorless cells, which are identical in the chyle and in the lymphthe chyle- or lymph-corpuscles of authorsare found almost everywhere in the lymphatic vascular system in considerable quantity. These are rounded, pale cells, $0.0025-0.0055$ of a line in diameter, which, when examined in their native fluid, appear homogeneous or finely granular, and contain a usually indistinctly transparent, homogeneous, slightly glistening, round nucleus; but on the addition of water, the nucleus and contents are rendered turbid by a granular deposit, and on that of acetic acid, becomes transparent and pale, exhibiting the strongly granulated contracted nuclei with extreme distinctness, bursting at the same time, and allowing the contents to escape; a change that also frequently takes place, especially in the smaller cells, on the addition of water, preceded by the appearance of clear albuminous drops. Otherwise dilute solutions, when the lymph-cells are already spherical, induce no very remarkable changes of form, whilst, in consequence of the evaporation of the fluid, and by concentrated liquids, a considerable contraction, and frequently also, a jagged outline, is caused in them (Fig. 290 a).

In size, quantity, and shape, the lymph-corpuscles present diversities, according to situation. In the commencement of the lacteals, which

FIG. 290.-Elements of the chyle: $a$, lymph-corpuscles become stellate by the escape of their contents; $b$, free nuclei; $c$, one such, surrounded by a few granules; $d, e$, minute lymphcells, some with a distinct nucleus ; $f, g$; larger cells, one with a visible nucleus; $h$, one such, after the addition of a little water; $i$, with the addition of acetic acid. 
are eminently adapted for such investigations, in the mesentery, and before it reaches the lymphatic glands, the chyle contains but few, and in the smallest mesenteric ressels which allow of being examined, frequently even no chyle-corpuscles at all. Where they do exist, which is always the case in the larger trunks, they usually appear small $(0.002-0.003$ of a line), closely investing the minute nucleus, and often even, as it were, in process of formation by the apposition of granules. As the chyle traverses the mesenteric glands, the cells become more and more numerous and larger, so that in the lacteals at the root of the mesentery (and also in the larger lymphatic trunks), together with the small cells which are still present, we find numerous larger ones, up to 0.0055 of a line in size. At the same time, at any rate in the Dog, Cat, and Rabbit, a multiplication of the lymph-corpuscles by division takes place more or less actively; in this process the larger cells elongate till they attain a length of $0.006-0.008$ of a line, and when the nucleus has divided, are separated into two by a median, circular constriction. This proceeding does not usually occur at all in the thoracic duct, and consequently the larger cell-forms, of 0.004-0.0055 of a line are here rare. In Animals, at all events, the majority of the cells in this situation are larger than the blood-corpuscles, that is to say, they are $0.0025-0.0035$ of a line, whilst in Man, at least as observed by Virchow and myself in an executed criminal, they were invariably smaller (from 0.002 of a line on the average). The nuclei of these lymph-corpuscles, which are imperceptible without the addition of acetic acid, were for the most part single and round, occasionally, also, horseshoe shaped, or constricted in the middle, very rarely truly multiple. In other Mammalia, cells having nuclei disintegrated by acetic acid, or naturally constricted and multiple ( $3-5$ fold) are very rare, omitting those in process of division; although occasionally such occur even in considerable quantity.

Red blood-corpuscles I have not as jet noticed in the human chyle, when it has been carefully taken, and under normal conditions; whilst in animals these corpuscles almost always occur in the thoracic duct in small quantity, and also frequently in the lymph of certain organs, as of the spleen. As they do not exhibit the slightest trace of a development within the lymphatics, I regard them as elements escaped from the bloodvessels, and indeed, am of opinion, so.long as direct connections between the two sets of ressels in the peripheral parts are not shown to exist, that this passage is an accidental occurrence, from the rupture of finer vessels, which, owing to the peculiar structure of certain organs, as the spleen and lymphatic glands, may be very readily conceived; and, indeed, as I have shown in the Tadpole, may be directly observed. I would, however, remark, that I have not unfrequently met with brown, round granule cells, $0.004-0.005$ of a line in 
diameter, completely corresponding with those mentioned as found in the blood, and which are probably derived from the lymphatic glands.

From the above facts, it would seem not to admit of doubt that the lymph-corpuscles are formed, like cells, by the development of membranes around free nuclei, a process which is effected, in the first place, in the commencements of the lymphatic vessels, but also, and chiefly, in the vasa efferentia of the lymphatic glands. To this is superadded the multiplication of cells by division, which does not always take place. The total quantity of the corpuscles, contained in the lymph, compared with that of the blood-corpuscles, is very inconsiderable, not only in the middle-sized and smaller trunks especially, of the lymphatics, but even in the thoracic duct itself is very far from being in an equal proportion; and even there all the elements of the fluid may readily be perceived, without any dilution. More precise enumerations have not yet been instituted, and it can only be added that considerable diversities exist, and that a milk-white chyle is not always also rich in corpuscles.*

* [Professor Kölliker appears not to be thoroughly acquainted with the very accurate and extensive researches of Mr. Wharton Jones, embodied in his memoir on "The Blood-corpuscle, considered in its different phases of development in the Animal Series," "Philosophical Transactions," 1846 ; the publication of which will, we believe, be considered hereafter to constitute an epoch in our knowledge of the blood. It is shown in this Memoir that "the lymph-corpuscle of the Vertebrata is identical with the corpuscle of their blood. In the oviparous Vertebrata it occurs, like the corpuscle of their blood, in the two phases of granule-cell and nucleated cell; whilst in man and Mammifera it occurs like the corpuscle of their blood in the three phases, of granule-cell, nucleated cell, and free cellæform nucleus.

"The only difference that exists between the corpuscle of the lymph and the corpuscle in the blood is, as regards the oviparous Vertebrata, the little degree of coloration which the colored stage of the nucleated cell as yet presents, and, as regards the Mammifera, the small degree of coloration which the colored stage of the free cellæform nucleus has yet attained" (p. 82).

Mr. Wharton Jones first pointed out in this Memoir the true nature of the process which is described and figured in the text as the "bursting," \&c., of the lymph-corpuscles. These changes of form, in fact, are not in general produced by any such cause, but they arise from the amaba-like motions of the corpuscles, observed by Mr. Jones in the Skate, Frog, and many Invertebrata, and which may be readily enough seen on a smaller scale in the colorless corpuscle of the human blood.

The subjoined description of the phenomena presented by the colorless corpuscle (granulecell, Jones) of the Skate will serve for all:-

" My attention was first attracted to the phenomenon, by observing a granule-cell with the granules apparently escaping from it, as if burst (Fig. 3). But the cell soon appearing again with all the granules collected together, I was led to watch, and soon perceived that the appearance of granules escaping as if from a burst cell, was owing to this: The transparent and colorless cell-wall bulged out on one side, leaving the granules still agglomerated and holding together, but this only for a short time; for soon, single granules were seen to separate and burst out from the rest, and to enter the hitherto empty compartment produced by the bulging out of the cell-wall. The regular manner in which this sometimes took place was remarkable. I have actually seen the granules enter the compartment by one side, and circulate along the bulging cell-wall to the other side, until the whole compartment became filled with granules. This having occurred, the bulging began to subside, but was succeeded by the bulging of another part of the cell-wall, into which again a flow of granules took place, and so on all round the cell." 
§222. Of the Blood.-The blood, so long as it is circulating in the vessels, is a slightly glutinous fluid, in which only two elements, the blood-corpuscles, -globules, -cells (corpuscula s. globuli s. cellulce sangui$n i s)$, the majority of which are of a red color while some are white, and the colorless blood-plasma (liquor s. plasma sanguinis), are tó be distinguished; when excluded from the circulation, it usually coagulates entirely, by the solidification of the fibrin in solution in the plasma, and afterwards by the contraction of the coagulated constituent, divides into the "crassamentum" and "serum." The former is of a deep red color containing, together with the fibrin, almost all the colored, and most of the colorless blood-corpuscles, with a portion of the still dissolved parts of the plasma, whilst the remainder of the latter, together with some of the colorless blood-corpuscles, constitutes the serum. In certain cases in Man, especially in disease, before the coagulation of the blood has taken place, the colored corpuscles subside more or less deeply below the surface of the fluid, when the crassamentum presents a superficial colorless, or whitish stratum (inflammatory crust), consisting only of the coagulated fibrin and colorless blood-cells, together with the fluid with which they are imbued.

The colored or red blood-globules, or simply blood-globules, in which alone the coloring matter of the blood resides, are minute non-nucleated cells of a flattened, lenticular form, which are contained in the blood in such vast quantity, that unless it be diluted with serum, they do not readily admit of exact investigation, ${ }^{*}$ appearing of themselves to constitute the blood. However important it would be to know accurately the proportion of the blood globules to the plasma, Fig. 291.

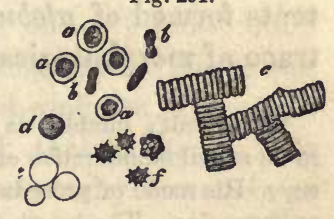
their number and their volume, all researches hitherto have failed, owing to the difficulty of the subject; and even the very recent statements of Schmidt, according to which 47-54 parts of moist blood-globules exist in 100 parts of human blood, can only be described as approximative. One method only can be successful, consisting in the direct enumeration

Fic. 291.-Blood-globules of Man: $a$, viewed on the flat surface; $b$, on the edge; $c$, united into rouleaux; $d$, rendered spherical by the addition of water; $e$, rendered colorless by the same agent; $f$, blood-globules shrunken by the drying up of the fluid.

We have already pointed out the occurrence of similar amaba-like movements in the young cells of mucous membranes detached during slight inflammation, and in the cells of the gelatinous tissue of medusæ; and we think that, very probably, it will eventually be found to be a property of all young periplastic substance.

MM. Guérin-Méneville (1849-51), Davaine (1850), and Robin (1853), appear also to have observed these amaba-like movements; without being acquainted with Mr. W. Jones's essay. (See the "Histoire nat. des Vegetaux parasites," by M. Robin, p. 567).-Trs.]

* [We must caution the reader against being guided by this statement. Nothing is easier than the examination of the blood as it is, - nothing more likely to mislead than the practice of diluting it with any fluid whatsoever.-Trs.] 
of the globules in accurately determined quantities of blood, and as precise a determination as possible of the volume of the individual corpuscles (Vierordt); but this method, if applied in such a way as to insure correct results, demands so much time and trouble, that it cannot be expected to obtain general application, and we must be contented with an accurate investigation of the total quantity of blood-globules in a single or in some few instances, an undertaking in which Vierordt is now engaged.*

The red blood-globules, more minutely examined, present the following characters: Their form is usually that of a biconcave or plane, orbicular disk, with rounded borders, and consequently they present a different aspect to the observer, according as the surfaces or borders are turned towards him. In the former case they are pale yellow, orbicular corpuscles, in which, according to the focussing of the microscope, the slight central depression which almost always exists, is indicated, sometimes by a clear, sometimes by an opaque spot in the centre, the latter appearance admitting of being confounded with a nucleus. Viewed on the edge, on the other hand, they present the form of an elongated, narrow ellipse, or of an ellipse constricted in the middle. The bloodcorpuscle is constituted of a very delicate, but nevertheless tolerably firm, and at the same time, elastic, colorless cell-membrane, composed of a protein substance closely allied to fibrin, and of colored viscid contents formed of globulin and hocmatin, which in the adult present no trace of morphological particles, of granules, or of a cell-nucleus; they

* [Vierordt's method, as lately published by him ("Schmidt's Jahrb." 4, 1852) consists in an actual enumeration of the blood-corpuscles, when mixed with fluids of a certain density. His mode of procedure is to take up a small quantity of blood by a capillary tube of uniform size. The length of the blood-column in the tube is measured under a low magnifying power, and multiplied by the diameter of the tube; and thus the total volume of the blood-column is obtained. The blood is then allowed to run from the tube on to a glass slide, is there spread out in narrow streaks, and intimately mixed with a solution of gum, or albumen. The blood-streaks are successively brought under the divisions of an ocular micrometer, and the number of corpuscles in each accurately counted. This process will enable us to determine, with tolerable accuracy, the number of corpuscles in a certain quantity of blood. It is, however, extremely laborious, since Vierordt himself states, that in order to complete a single enumeration, nearly one week is required.

A mode of procedure by which the number of the blood-corpuscles may be more readily ascertained, is the one proposed by Welcker (Archiv. des Vereins. fur. Gem. Arb. B. 1, H. 2, 1853). This observer employs a stage micrometer, divided into a known number of parallelograms. On this, the blood, mixed with a solution of albumen, is spread, and the corpuscles in each division counted, with the aid of an ocular micrometer. For practical purposes, Welcker states that the number of the blood-corpuscles may be ascertained by a yet simpler method. A measured quantity of blood is diluted with a certain quantity of fluid, the color of the diluted blood is then compared with a color scale determined upon by previous experiment, and by the degree of color, the number of the blood-corpuscles may be approximately estimated. By employing both these methods, Welcker was able to ascertain that healthy blood (his own) contained about $4,000,000$ corpuscles in a cubic millimetre, whilst in a case of hysteria he found but $3,800,000$, and in a patient in the last stage of consumption, but $2,400,000$ corpuscles. $-\mathrm{DaC}$.] 
are consequently vesicles, whence the name "blood-cells" is to be preferred. The elasticity, softness, and flexibility of the membrane is so considerable, that the corpuscles are rendered capable of passing through vessels of less diameter than themselves, and when by pressure under the microscope they have become elongated, flattened, or otherwise altered in shape, of reassuming their original form. The blood-globules are the better adapted for the former process, since their surface is perfectly smooth and slippery, so that they easily glide over the walls even of the smallest capillaries, which present the same conditions.

The size of the blood-globules may differ in different individuals; but these diversities, owing to the minuteness of the bodies concerned, are not altogether inconsiderable. As a general mean size, the most recent inquiries of Harting ("Rech. micrometr.") from measurements of recent blood-corpuscles, give a width of $0.0033(1.300)$ and a thickness of 0.00062 of a line; and of Schmidt, from the estimation of dried bloodglobules, a width of 0.0035 of a line, whilst, according to the former, the mean width in various individuals amounts to from 0.0028 to 0.0036 , and according to Schmidt to $0.0032-0.0035$ of a line, with which numbers those given by other good observers' essentially correspond. The differences in the same individual, found by Harting to exist between the two extremes, amount, as regards the width, to 0.0010 0.0017 , and for the thickness to $0.00009-0.0005$ of a line; the extremes observed in general were $0.0020-0.0040$ and $0.0005-0.0009$ of a line; and Schmidt states, that in 100 blood-corpuscles $95-98$ are of equal size. With respect to the size of the blood-globules in one and the same individual, it may be stated in general, that it necessarily differs at different times, and especially that it necessarily increases or diminishes according to the varying degrees of concentration of the blood-plasma; but on this subject we have scarcely any accurate researches. Harting only says, that the blood-corpuscles of the same individual, measured after an interval of three years, presented the same mean size, whilst in the same individual after a copious meal, somewhat less average dimensions were exhibited (by about 0.00013 of a line), and more considerable extremes. It has been remarked that determinate data are wholly wanting as to the number of the blood-globules, and we must await the results of Vierordt's researches. But, at all events, from what has been ascertained with respect to the amount of solid constituents in the blood-globules, this much in general may be concluded, that they are more numerous in the male than in the female sex; moreover, that after repeated venesection, during pregnancy and after prolonged deprivation of food, they diminish in number; and in certain diseases, as chlorosis and ancemia, are also found to be much more scanty than usual. At the same time, however, it is certain, that all the possible variations are not as yet by any means exhausted, and it can scarely be 
doubted, that in every individual the number of blood-cells is subject to numerous, even daily changes, according to the conditions of supply and waste, with which we have still to be made accurately acquainted. The volume of the blood-cells is estimated by Harting, regarding them as short cylinders, as that of a cell of 0.0763 cubic millimeters; and the weight, taking their specific gravity as equal to that of water, and abstracting their central depression, at 1-13,114,000 milligramme. If, with Schmidt, the blood is taken to contain 50 per cent. of corpuscles, and the whole quantity of blood be estimated at 10 kilogrammes, we have a total of 65 billions 570,000 millions. According to Schmidt, the specific gravity of the blood-corpuscles in men, is $1 \cdot 0885-1.0889$, and in women 1.0880-1.0886-numbers which must stand and fall with his statements respecting the quantity of blood-corpuscles. Compared with the other constituents of the blood, the corpuscles are heavier than the serum and the plasma. In the former, and in defibrinated blood, they form, upon standing, a red sediment, whilst in the plasma, owing to its rapid coagulation, they do not usually subside below the level of the fluid. This subsidence of the blood-cells, which, according to their own density and that of the fluid in which they are suspended, may be slow or rapid, is favored by their mutual cohesion, which is observed especially in inflammatory blood, in which, from the rapid subsidence of the bloodcells, part of the blood coagulates into a colorless mass; but it also takes place in perfectly healthy blood, and, in fact, constantly in little drops obtained by trifling injuries of the skin, and frequently also in the blood taken by venesection. The blood-globules in these instances apply themselves to each other by their flat surfaces, and form, as it were, columns or rouleaux, to the sides of which others may be again applied, so that very complicated branched figures, and even networks are in this way produced, covering the entire field of view (Fig. 291, c).

Besides the colored, the blood also contains a certain number of colorless elements, of two kinds-elementary granules of a fatty nature, and true cells. The former, which correspond entirely with those of the chyle (vide $\$ 221$ ), occur in very varying number, sometimes very scantily or not at all, sometimes in greater or even in vast numbers, so as to give the serum a whitish or even milk-white color. From all that we know, these must exist when fat is introduced into the blood through the chyle; thus also in common alimentation, 3-6 hours and longer after the taking of food, although in many cases they seem to disappear in the course of the pulmonary circulation; at all events Nasse (vide Nasse, "Wagn. Handw. d. Phys.," I., p. 126) and others have never found them in the systemic blood of healthy persons, a fact which I can confirm as regards my own blood. In herbivorous Animals, on the contrary, in the Goose, and in sucking Animals, the occurrence of these molecules appears to be constant; in pregnant women, also, and after the free use of milk or alcoholic drinks, and also in famishing 
persons (in consequence of an absorption of the fat of the body), it seems to be, at all events, very frequent. The colorless cells of the white blood-corpuscles are derived from the chyle; and may, consequently, be termed the chyle- or lymph-corpuscles of the blood. Some of them are uninuclear, and correspond in all respects with the small cellular elements of the chyle (vide \$221); some multinuclear, having an average size of 0.005 of a line, in which case they so closely resemble pus-corpuscles, that it is quite impossible to distinguish the one from the other. The larger corpuscles are rarely as granular as the smaller, usually tolerably homogeneous, often with clear contents, so that their two or three, rounded, minute nuclei are at once

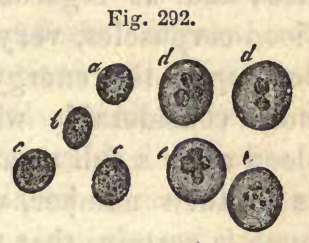
apparent.* Should this not be the case, acetic acid or water brings the nuclei distinctly into view by rendering the contents clear, of which occasionally a drop escapes from the ruptured cell; at the same time,

Fia. 292.-Colorless blood-corpuscles, or lympl-corpuscles of the blood: $a, b$, smaller cells, such as are found in the thoracic duct, viewed on the side $(a)$, and on the edge $(b)$; $c, c$, the same with visible nucleus; $d, d$, larger cells with nuclei multiple $a b$ origine; $e, e, e$, the same after having been acted upon by acetic acid, the nuclei disintegrated or becoming so.

* [With regard to the appearances presented by the "nucleus" of the colorless corpuscle, Mr. Wharton Jones (1. c. p. 67 for the Frog, p. 72 for Man, the Horse, and the Elephant) shows very clearly that the singleness or multiplicity of this body depends entirely upon the strength of the acetic acid used to bring it out, and we can fully confirm his statement so far as our own observations have gone. If the blood of Man, in fact, be diluted first with water, and then only very dilute acetic acid be added, the "nuclei" of the colorless corpuscles will all, or almost all, be circular, with even edges. If, on the other hand, strong acetic acid be added at once, every variety of form, from mere notching, to apparent division into two, three, or four smaller ones, will be found. "I am satisfied, however, that this is, in both cases (granule-cell and nucleated cell) merely an appearance produced by the shrivelling together of the walls of the single cellæform nucleus, in consequence of the action of the acetic acid.

"It is proper to observe, that I have come to this conclusion, only after having particularly tested the point by repeated, careful, prolonged, and varied observations. Nor is the determination of the point of small moment, as on the appearance of a multiple nucleus, which $I$ have thus shown to be artificially produced, and on a similar, but I believe equally artificially produced appearance of a multiple nucleus in the pus-corpuscle, a particular view has been founded as to the first formation of the nucleus, and, indeed, as to celldevelopmert generally." (Wharton Jones, 1. c., pp. 67-S.)

It will be observed that in the text Professor Kolliker admits that the multiple "nuclei" may be still further broken up by the action of reagents.

The existence of any difference of specific gravity between the colorless and the red corpuscles of the blood may be doubted; their relative positions in masses of blood, to which reference is made above, being fully accounted for by the aggregation of the red corpuscles into rolls.

With regard to the blood-corpuscles of the Invertebrata, Mr. Wharton Jones's Memoir, above cited, should be consulted, as it contains the only complete account of them extant. The statement in the text, that Amphioxus has no blood-corpuscles, is incorrect. It is, however, altogether exceptional among the Vertebrata, as its corpuscles are entirely of the colorless kind.-Trs.] 
at all events under the former reagent, the nuclei not unfrequently are farther disintegrated, and fall into irregular, jagged, and constricted corpuscles, or are even resolved into a greater number, 4, 5, 6, and more, of smaller granules, assuming, at the same time, a yellow color, whilst the cell-mernbranes gradually disappear. The other reactions of these colorless blood-corpuscles are those of the common indifferent cells, and as regards their number, it is, compared with that of the blood-corpuscles, very small, though not always the same, being dependent upon the energy with which nutrition is going on, and therefore more considerable when a large quantity of chyle has entered the blood after a full meal. It is impossible to give any definite statement as to their number, without perfectly accurate enumeration; but this much is certain, that the usual statement, that there is one colorless to every ten colored blood-corpuscles, is quite incorrect. I find, with Henle and Donders, that they are much less numerous than this, and am of opinion, that when the latter, with Moleschott, reckons $5 \cdot 1$ colorless to 2000 colored corpuscles, he is not far wrong. After meals, these authors found the number of the latter augmented to $6 \cdot 2$, whilst in fasting animals, as was also noticed by Heumann in Pigeons, they diminished in number; and after long fasting, at least in Frogs, they saw them disappear altogether.* Their increase after venesections, not only relatively but even absolutely, is a very remarkable circumstance; and this, as in the Horse, after very copious abstraction of blood (as much as $50 \mathrm{lbs}$.) may proceed to such an extent, that the colored and colorless corpuscles appear to exist in equal numbers. The white corpuscles are lighter than the colored, and they are consequently more numerous in the upper strata of the crassamentum. When the latter presents a buffy coat, it always contains a great number of these corpuscles, and especially if their number has been augmented by previous venesections, so as in such cases to constitute even half of the buffy coat (Remak, Donders). Their less tendency to subside is, moreover, increased by the circumstance, that although they have an uneven sur-

* [Moleschott has recently continued his researches on the relative number of the white and the red corpuscles, and has arrived at some interesting results. In boys from $2 \frac{1}{2}$ to 12 years of age, he found as the mean in seven enumerations of the corpuscles, 4.5 colorless to 1000 colored; in adults from 21 to 49 years, 2.9 colorless to 1000 colored; in old men from 62 to 78 years, 2.6 colorless to 1000 colored. In women from 14 to 38 years the average was only 2.6 colorless to 1000 colored corpuscles, whilst in the blood of the same women while menstruating, he counted 4 colorless to 1000 colored. An increase of the proportion of the colorless corpuscles was also observed in pregnant women $(3.6$ colorless to 1000 colored), and in the blood of persons after meals which were rich in albuminous substances. After having partaken freely of the latter, he noticed in his own case 3.5 colorless to 1000 colored corpuscles, whilst a few hours after a breakfast on non-albuminous substances, he counted only $2 \cdot 1$ colorless to 1000 colored corpuscles. These observations of Moleschott are important, and nust be borne in mind in determining the number of the colorless corpuscles in suspected cases of leucocythemia.-DaC.] 
face, and a disposition to cohere, they usually do not form any large aggregations, and never constitute rouleaux.

Condition of the blood-corpuscles in various kinds of blood.-However sensitive the blood-cells, out of the body, are towards various reagents, they appear, within it, to be so constant, at all events as regards their shape, that not only within the bounds of the physiological condition, are no notable and uniform differences to be observed in them, in the arterial and venous blood and the blood of the different organs, but, even in the most various diseases, no visible alterations are presented. And yet it cannot be doubted that, as the color and chemical composition of the blood-cells vary, so also are their forms subject to certain diversities and changes, according as the blood is more concentrated or diluted, and abounds more or less with one saline constituent or another, or with other substances; but these changes of form are so inconsiderable, that it cannot be wondered at, that we have not yet been in a condition to recognize them with certainty. At all events, with Henle, I must most expressly declare, that all these forms-the jagged blood-corpuscle on the one side, and the diminutive, spherical, colored or pale-are never met with in the circulating blood. Slight degrees of flattening and distension may probably be noticed; but in such researches it should never be forgotten how quickly the blood-corpuscles change their form, and care must be taken not to view a condition set up out of the organism, as a natural one. The relations of the bloodcells, as to their number, appear to vary more than their forms. As respects the colored, they are more numerous in the venous than in the arterial blood. In speaking of the venous blood, that of the hepatic veins stands pre-eminent, containing, according to Lehmann, far more blood-cells than that of the portal vein, and even exceeding in that respect the somewhat rich blood of the jugular vein. The colorless blood-cells, as I and Funke have found, exist in very great number in the splenic blood, and indeed sometimes more in the form of uninuclear cells, sometimes as multinuclear; and also in the blood of the hepatic veins, according to Lehmann, in which they are characterized by their very various size (vid. $\S 223$ ); I have noticed this, in many cases, though by no means invariably, but am unable to regard it as an exclusive character of the blood in the hepatic veins, because $I$ have also found the same multitude of colorless cells in perfectly healthy animals, in the portal blood, as Lehmann has done in one case, as well as in the blood of the pulmonary veins. Otherwise, however, the colorless cells are more abundant in the venous, than in the arterial blood (Remak). In the vena cava superior, and iliac vein of the Dog, Zimmermann noticed uninuclear cells, and, in the $v$. cava inferior, multinuclear ones.

Many experiments have already been made as to the influence of various reagents on the blood-globules, although the results obtained 
have in some measure been of very trifling importance; and $I$, therefore, here adduce, chiefly from my own researches on the subject of the human blood-corpuscles, only what may serve to illustrate their anatomical and physiological relations. Water at first renders the bloodglobules spherical, and, owing to the diminution of the broad diameter, consequent upon the increased thickness, smaller (from 0.002-0.0024 of a line), which may be best seen in corpuscles arranged in columns. The size then usually remains without further change, and the coloring matter and remainder of the contents slowly (sometimes suddenly, and by fits and starts) escape, so that the fluid becomes dark-red, the corpuscles at the same time losing their color, and acquiring the aspect of colorless vesicles or rings, so faint, that it is often difficult to perceive them. But by the addition of tincture of iodine, which colors them yellow, or of salts (common salt, nitre, \&c.), of gallic or chromic acid, which cause them to shrink, and to present a more defined outline, they may be readily brought into view; and it is thus satisfactorily shown that water, by no means dissolves, or destroys them. Some bloodglobules always withstand the influence of the water for a longer time, and are still colored when all the rest have lost their coloring matter; but it is not yet ascertained, whether these are to be regarded as of younger formation, as is commonly supposed, or of older. The latter notion seems to be favored by the circumstance that older cells usually have firmer membranes than younger ones, and also that blood-corpuscles, left to their fate out of the circulation-for instance in extravasated blood-always, in time, become more resistant; but it must be allowed that, at present, no decisive opinion can be given either way. Many other substances act in the same manner as water, only more powerfully and even destructively, particularly acids and alkalies; although not all with equal energy. Gallic and pyroligneous acids, aqueous solutions of chlorine and iodine, sulphuric ether and chloroform, act very much in the same way as water. In the first three the blood-globules remain as distinct, pale rings, whilst in sulphuric ether they are instantaneously transformed into the most delicate and excessively faint rings, $\frac{1}{3}-\frac{1}{4}$ the original size, and which it is very difficult to perceive in the finely granular coagulum that is formed at the same time, although they are rendered more distinct by the addition of salts (nitre for instance). I have seen no evidence of an actual solution of the cells. Chloroform acts in the same manner, only more slowly, and the corpuscles first become considerably smaller, and of a glistening yellow color. Acetic acid, of $10_{0}^{\circ}$, at once renders the corpuscles extremely faint, so as to be scarcely perceptible, but they are by no means dissolved, being visible, even at the end of several hours, in the form of delicate rings. A solution, containing 20 per cent. of acid, acts more energetically, and in glacial acetic acid, the cells are completely dissolved in the space of two hours, in the slimy brown blood. Concentrated 
sulphuric acid renders the blood black-brown. The corpuscles become pale, and although still retaining some color, are scarcely recognizable, their contours running mutually together. On the addition of nitre or water, which latter throws down a white precipitate, they again become distinct as minute, dull-yellow, round corpuscles. After some hours' action of the acid all is dissolved. Concentrated hydrochloric acid, which colors the blood brown, and produces a white precipitate, contracts most of the cells, which are gradually dissolved, and renders many of them granular internally, also producing rents in some of them, so that the contents escape, in the form of a pale streak, appearing like a stalk to the corpuscle; subsequently they become so faint in color as to be scarcely perceptible, without the aid of some saline solution. After some hours many are dissolved, though a few offer a longer resistance. Nitric acid, when concentrated, renders the blood olive-brown, and the corpuscles greenish. The latter are corrugated, but are not rendered smaller, and are partly cnclosed in the coagulum, which is formed at the same time, in part free, and lying above it. After several hours there is still no indication of a solution going on, but this takes place at the end of a day. Of the alkalies, potassa acts the most powerfully. A solution containing 10 per cent. makes the blood black, and at once dissolves all the blood-cells, first rendering them spherical and smaller. The same thing takes place with a solution containing 20 per cent. of the alkali, except that some of the cells remain for a time as pale rings, whilst a concentrated solution of two parts potassa and one part water does not attack the corpuscles, beyond making them very small ; at the same time they remain spherical, or jagged and folded. The whole blood is coagulated by this solution, and acquires, at first, a brickred, and afterwards, a bright brown color. On the subsequent addition of water, the blood-corpuscles enlarge, as in no other reagent, to a size of 0.006 of a line, remaining for the most part flat, and are then dissolved as in a dilute solution. Caustic soda, and caustic ammonia, in solutions containing about 10 per cent., act like the corresponding potassa-solution, only that the action is a little weaker, whilst concentrated caustic soda ( $1 \frac{1}{2}$ part to 1 part water) acts precisely like caustic potassa. The same phenomenon of a diminution of the blood-cells, as that caused by some of the reagents above noticed, is manifested also in many other instances, and may be referred to the abstraction of materials, chiefly water, from the cells, as it is always concentrated solutions which so act. In these cases, also, since the blood-globules reflect the light from more numerous points, the color of the blood becomes brighter, usually of a brick-red. Even the mere concentration of the blood-plasma, by evaporation, causes the cells to shrink more or less, in consequence of which they become either round, dark, brilliant globules, $0.001-0.002$ of a line in size, or jagged, stellate bodies, or, lastly; 
diversely bent and plicated discs. All concentrated solutions of metallic and other salts, act in the same way, unless, like nitrate of silver, they exert an immediately destructive influence. Donders and Moleschott have carefully investigated the reactions, especially of such soluble salts as exist in the blood, and have found that a concentrated solution (1 part salt, 7 parts water) added to an equal volume of blood, diminishes all the cells, and reddens the blood. The cells are least affected by the hydrochlorates of soda and potassa, much more by the phosphate and carbonate of soda, and nitrate of potassa, most of all by the sulphates of soda and potassa. When diluted ( 1 part salt, 17 parts water), all these salts color the blood a dark wine-red, and produce a distension of the cells, rendering them pale, and completely dissolving them at the end of four or five hours; in this regard the soda-compounds, except common salt, which exerts no destructive action, prove more energetic than those of potassa. I find changes similar to those caused by the salts, to take place on the addition of alcohol, tincture of iodine, chromic acid, and creasote, the first two of which merely render the blood-globules smaller and corrugated, the latter also causing them to become granular internally. In this respect the action of creasote is the most remarkable, which transforms the blood-corpuscles partly into perfectly opaque, even fat-like glistening granular and homogeneous granules and globules, and partly into very beautiful clear vesicles, which may even be rendered polygonal by their mutual pressure. Lastly, it is very important to notice the influence of oxygen and of carbonic acid on the blood, which by their reception into the interior of the cells, both in the body (in the pulmonary and systemic capillaries) as well as externally to it, as proved by experiment, produce sometimes a brighter, sometimes a darker color in it. This takes place without any change of form in the blood-corpuscles (J. Müller, and Todd and Bowman, in opposition to Nasse and Harless), and the experiment may be alternately made several times in succession with the same blood without any alteration of the corpuscles (Magnus, Bischoff, Del'Espinasse, and Renemann, in opposition to Harless). Those gases also act upon the coloring matter of the blood, when isolated, in the same way as upon the corpuscles (Magnus, Marchand), and it is probable that the change of color is not connected with any chemical change in the homatin, but is a physical action of a peculiar kind, analogous to similar changes of color in other fluids caused by the absorption of gases.

Blood-corpuscles of other animals.-The non-nucleated blood-corpuscles of the Mammalia do not differ in form from those of Man, except that in the Camel and Llama (Auchenia Paco, A. Glama, A. Vicugna) they are oval, and 0.0038 of a line long; they are mostly smaller than in Man, as in the Dog, 0.0031, Rabbit, Rat, 0.0028, Swine, 0.0027, Horse and $0 x, 0.0025$, Cat, 0.0024 , Sheep, 0.0022 of a line, the small- 
lest $(0.00094)$ in the Musk Deer; seldom larger ( 0.005 of a line) as in the Elephant. All the lower Vertebrata have, almost without exception, oval, nucleated blood-corpuscles, of the shape of a melon seed. Those of Birds are from 0.004 to 0.008 of a line long, and contain roundish nuclei; those of the Amphibia measure between 0.0080.025 of a line in length, have round and oval nucle $i$, and are largest in the naked Amphibia (Frog, $0.011-0.013$ of a line long, $0.007-0.008$ of a line broad; Proteus, 0.025 of a line long, 0.016 of a line broad, Salamander, 0.02 of a line long); those of Fishes, lastly, are mostly 0.005-0.007 of a line long, except that in the Plagiostomes they measure $0.01-0.015$ of a line; in the Lepidosiren they are 0.020 of a line long, and 0.012 of a line broad. In Myxine and Petromyzon they are 0.005 of a line in diameter, round and slightly biconcave. In Amphioxus the blood-corpuscles are absent.* The bloodcorpuscles of the Invertebrata resemble the colorless cells of the blood in the higher animals, and are almost always uncolored.

The following should here be also noticed as extraordinary constituents of the blood: 1, cells enclosing blood-corpuscles, noticed by Ecker and myself in the

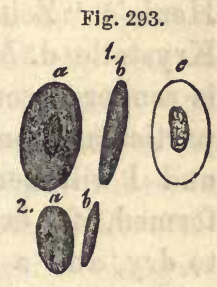
blood of the spleen and hepatic vessels, and elsewhere also in the blood (vid. Mikroskop. Anat., II. 3, p. 369, et seq.) 2, pigmented and colorless granule-cells, observed by myself, Ecker, Meckel, Virchow, and Funke, particularly in cases of intermittent fever and diseases of the spleen (1. c.) 3, pale, fine-granular, roundish aggregations, in the blood of the splenic vein (Funke). 4, peculiar concentric bodies, three to four times larger than the white blood-cells, similar to those of the thymus (vid. Henle, "Zeitsch. f. rat. Pathol.," Bd. VII. p. 44), found by Hassall in fibrinous clots in the heart. 5, cells resembling pus-corpuscles, in tumors of the spleen and leukæmia (Virchow); these bodies are found in vast quantities, but, in their form, cannot in any way be distinguished from the colorless blood-corpuscles. 6, caudate, pale or pigmented cells (Virchow, "Arch.," II.) Besides these, should be noticed, the morphological elements which are formed in the blood without the body or in cases where the circulation has been obstructed-the fibrinous coagula and crystals. The former are seen in coagulated blood, usually in the form of fine, extremely closely interwoven fibrils, disposed irregularly; occasionally as stronger, straiter fibres, having a uniform width of $0.001-0.003$ of a line; not unfrequently also in the shape of plates resembling epidermis scales (fibrinous flakes, Nasse). I noticed crystals of a red color in normal blood in the year 1849

FIa. 293.-1, blood cells of the Frog: $a$, viewed on the side; $b$, on the edge; $c$, rendered colorless by water. 2 , Blood-cells of the Pigeon: $a$, viewed on the side; $b$, on the edge.

* [rid. Note, p. 707.-Trs. ] 
("Zeitsch. für wissen. Zool.," I. p. 266, Todd's "Cyclop. of Anat." Art. "Spleen," p. 792, and Mikros. Anat., II. p. 280), in the blood of the Dog, of Fishes, and of a Python; sometimes within the bloodglobules, sometimes free in the blood, particularly of the liver and spleen. Their occurrence in the former situation especially, appeared to me to prove that they exist in the blood during life, and consist of a substance allied to hematin and hematoidin (Virchow); but I also showed that they were soluble in acetic and nitric acids, and in caustic alkalies, and consequently that they are not identical with hematoidin. Quite recently, Funke, without being acquainted with my observations, has independently noticed these crystals in the blood of the Horse, Dog, Man, and Fishes, and instituted very careful researches with respect to them ("De sanguine venæe lienalis," Lips., 1851; also in Henle's "Zeitsch. N. Folge," Bd. I. p. 172, and "Neue Beob. üb. d. Krystalle d. Milzvenen- u. Fischblutes," ibid., II. p. 199), by which it is rendered certain that these crystals arise out of the body. For more particulars concerning them reference may be made to the works cited,* and I will here only further notice that the crystals are most readily formed, if a drop of blood covered with a piece of glass be allowed nearly to dry, and a small quantity of water be added; moreover, that the crystals are formed not only in the blood of the splenic vein, as it at first seemed, but in other kinds of blood also, in Man (I can obtain crystals in my own venous blood) and other animals. They assume the form of red or pale needles, columns, and plates, probably belonging to the rhombic system (Funke), and also tetrahedral (in the Guinea Pig, Lehmann, Funke), and are characterized by their little permanency, since they perish in the air, are very soluble in water, and also in acetic acid, alkalies, and nitric acid. Those found by me in the blood of the Dog resisted the action of water, but I do not think that they were of a different nature, and I should be inclined to refer this difference to the greater resistance of the blood-globules themselves. Funke believes that they consist of the albuminous contents of the blood-cells in combination with hematin, relying, for support to this opinion, especially upon their numbers, their occurrence in blood-cells, their formation, as observed by him, from aggregations of blood-cells, and their absence from the serum of the blood; but I cannot regard this hypothesis as at

*[Also to Funke in "Schmidt's Jahrbücher" No. IX. p. 1, and in his Atlas of Pathological Chemistry, Leipsic, 1853; Robin and Verdeil "Traité de Chemie Anatomique," tom. III.p. 430 ; Kunde "Ueber Krystall-bilding in Blute" in "Henle u. Pfeufer 's Zeitschrift," II. p. 271, 1852, and in Mémoires de la Societé de Biologie," IV. 1852; Parker "Med. Times and Gazette," 1852 ; Lehmạnn "Bericht d. Königl. sach. Gesellschaft der Wiss," Leipzig, 1853, and in $2 \mathrm{~d} \mathrm{Ed}$. of his "Physiologische Chemie," Bd. II. p. 151 ; L. Teichmann, "Ueber die Krystallization der organischen Bestandtheile des Bluts," in Zeitschrift für. rat. Med. Bd. III. H. 3. This last-named writer describes crystals procured from dried blood, differing in form from the ordinary blood-crystals, and for which he proposes the name of "hamin crystals." - DaC.] 
present fully established, and it appears to me that further proof is required before we should admit the existence of crystals from the substances in question, and the more so, because colorless crystals are also formed in the blood, quite independently of the blood-cells. Robin and Verdeil, moreover, assert that they have also obtained crystals from the serum of the blood; and the quantity of the crystals is by no means opposed to the notion that they are derived from a salt of the blood merely tinged with homatin, since, in the case of fibrin we see that not much of a substance is required to occupy a large space.

\section{§223. Physiological remarks.-The development of the bloodvessets} takes place, essentially on the same type, in the heart, arteries, and veins. The rudiments of all these vessels, and even of the heart, are solid tracts of cells of greater or less thickness, which, by the liquefaction of their interior substance, and the metamorphosis of the central cells into blood-globules, become cavities, which shortly coalesce, and constitute a continuous passage for the blood. The heart and vessels having remained for some time in this condition of cellular tubes, in which state the former, moreover, exhibits contractions, the cells composing the walls, with the exception of the innermost, begin to elongate into fibres, and to represent the divers fibrous tissues and tunics. At the same time the vessels become thicker and increase in circumference, which at first is still to be referred to an increase in the number of the cells, but subsequently, is brought about chiefly, or even solely, by the growth of their elements in length and thickness. In the fifth month of foetal life, all the larger and medium-sized vessels are formed, with their tunics and tissues, and it is impossible to perceive any vestige of formative cells. The tissues, however, appear to be still far from completion, the muscular fibres being short and delicate, and instead of the strong elastic fibrous networks, we perceive only finer and the finest

* [Reichert was the first to draw attention to the occurrence and nature of crystallized albuminous matters colored with hæmatin, in his "Beobachtungen über eine eiweissartige, Substanz in Krystallform," Müller's "Archiv," 1849. These were tetrahedrons of as much in some cases as $1-15$ of a line long, and occurred upon the placenta and fetal membranes in a Guinea Pig. Their albuminous nature was confirmed by Schmidt and Buchheim; subsequently, "Bericht," Müller's "Archiv," 1852, Reichert states that he has again found the crystals in the same locality, and that he has convinced himself, by further experiments, that the difference observed between his crystals and those described by Kunde and Lehmanin, arose entirely from the action of the spirit in which his first specimens had been preserved. Reichert observed the development of crystals of the same kind, though smaller, in fresh blood taken from the heart of the Guinea Pig.

In connection with this subject, Dr. Ayres has recorded a very interesting observation with regard to the occurrence of prismatic, more or less red crystals, in a band of olivegreen, almost black matter, having the appearance of coagulated blood at the margin of the placenta of the Bitch ("Quarterly Journal of Mic. Science," vol. i. p. 299, with figures). See also the papers of Dr. Parkes ("Med. Times and Gazette," 1852), and of Dr. Sieveking ("Brit. and For. Med. Chir. Rev.," 1853) on this subject.-Tras.] 
fibrils, and in the place of the elastic membranes themselves, only layers of more or less coalescent, fusiform cells. The internal longitudinal fibrous membrane alone, in many vessels, is at this time demonstrable as a homogeneous elastic tunic immediately under the epithelium, but in the smaller vessels this is wanting, and is replaced by a layer of elongated cells, out of which it appears to be developed. It is thought that similar cells are occasionally to be seen also in the adult, in which the elastic inner membrane is likewise merged. The muscular fibres of the heart arise, as in other situations, from the union of cells, but I have not yet seen how their anastomoses are formed, whether from a branching of certain formative cells, or by the lateral apposition of small rows of cells-probably in both ways.

The mode in which the development of the capillaries is effected, differs in toto from that observed in the larger vessels. The former, as Schwann and I have shown, proceed from the coalescence of single cells. At the primary origin of these vessels, tubules of some size are formed, at first by the successive apposition in a straight line and the coalescence of rounded-angular cells, and the subsequent absorption of the septa-and of the contents, but not of the nuclei, which remain attached to the former cell-membrane, now become the eapillary tunic. Delicate pointed processes then project from the walls of these little vessels, which rapidly elongate, and meeting similar pointed processes of stellate cells dispersed in the surrounding tissue, coalesce with them. At the same time, the other processes of these cells join, so that there is soon produced a network of stellate cells, continuous with the already formed capillary tube or tubes. This net, however, is never spread, for the prolongations, given off from already formed and pervious capillaries, and the neighboring cells connected with them are constantly and rapidly transformed into fresh capillaries, by the continual increase in size of the coalescing processes from their point of origin onwards, and their becoming hollow. In this way are produced vessels which are at first extremely fine, and admit only blood-plasma-true vasa plasmatica s. serosa; but they rapidly enlarge, until at last the blood-globules are transmitted through them, and the capillaries are perfected. Owing to the circumstances, that while these processes of the stellate formative cells thus enlarge, the bodies of the cells do not expand in a corresponding manner, but appear as simple nodular points in the vessels, all vestige of the original cellular network is gradually lost, and subsequently the situation of the bodies of the cells can only be determined by the position of the persistent nuclei. When finer tubules have once been formed from the previous larger capillaries, the facilities for the passage of the blood are continually undergoing augmentation, inasmuch as new stellate cells are constantly enlarging into vessels, whilst, at the same time, fresh vascular material is as constantly furnished by the apposition of 
new cells. New connections are also frequently formed between capillaries which are already pervious, partly by the direct meeting of prolongations from them, partly also by the mutual connection of formative cells lodged in their interstices, whence, of course, the original net is rendered closer. This mode of development, so far as I have seen, obtains in all animals, without exception, in which capillaries exist, and the various objections offered to the exposition given by Schwann and myself, have chiefly arisen in the notion, that every network connecting the arteries and veins in embryos, is a capillary plexus. This, however, is by no means the case, and consequently the circumstance, that the wrongly termed capillaries of the germinal area arise after the type of the larger vessels, is not an objection of the least weight in opposition to us.

The capillaries of the lymphatic system, which may be readily traced in the tail of batrachian larvoe (Fig. 228), exhibit, essentially, precisely the same mode of development as those of the blood-vascular system (Fig. 294), except that anastomoses are rare in them, and its course is more limited to the mutual apposition of fusiform cells, or of cells furnished with three principal processes. Observations are wanting with respect to the larger trunks of this system, although it cannot be doubted, that they follow exactly the same course as the bloodvessels. Engel (l. c.) has lately treated of the lymphatic glands, and states, that they proceed from lymphatic vessels which throw out buds and are much convoluted.

The development of the blood-corpuscles is pretty accurately known, in the embryo, as concerns its principal stages. The first blood-corpuscles, in the Mammalia and other Vertebrata in general, are nucleated, colorless cells, with granular contents; they are perfectly identical with the for-

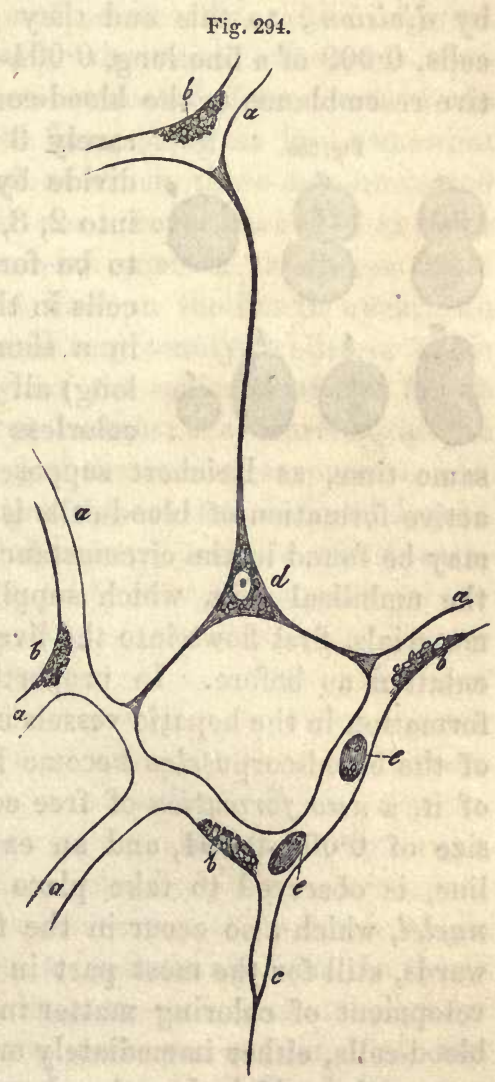

FIG. 294.-Capillaries from the tail of a Tadpole: $a$, completed capillaries; $b$, cell-nuclei, and remains of the contents of the original formative cells; $c$, creal process of a vessel; $d$, stellate formative cell, connected by three prolongations, with three processes of pervious capillaries; e, blood-globules, still retaining some granular contents.-Magnified 350 diameters. 
mative cells of every part of the young embryo, and arise in the originally solid rudiments of the heart and great vessels, in some situations very early, in others somewhat later, by the separation of the central cells contained in the rudiments, in consequence of the development of a fluid (the first blood-plasma) between them. The first perfect bloodcorpuscles arise from these colorless cells, which lose their granules, and, except the nucleus, become filled with hematin. These colorless, nucleated, primary blood-cells are spherical, of a deeper color than the blood-corpuscles of the adult, and larger (in a fotal Lamb, $3 \frac{1}{2}$ lines long, most of them were $0.005-0.0065$, the minority $0.0025-0.0035$ of a line; in a human embryo, 4 lines long, according to Paget, $0.004-0.007$ of a line), but in all other respects present the same conditions, and, with their colorless formative cells, at first constitute the sole elements of the blood. But many of them soon begin to multiply by division; to this end they grow into elliptical, or even flattened cells, 0.009 of a line long, $0.004-0.006$ of a line broad, bearing a deceptive resemblance to the blood-corpuscles of the Amphibia; produce 2,

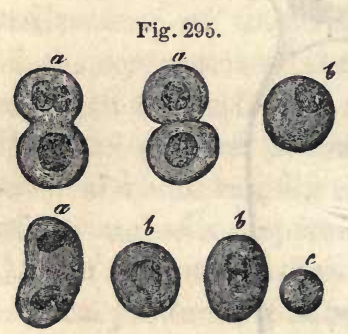
rarely 3 or 4 , rounded nuclei, and afterwards divide by one or several annular constrictions, into 2,3 , or 4 new cells. When the liver begins to be formed, this multiplication of the bloodcells in the entire mass of the blood ceases, and in a short time (in the fotal Lamb, 11 lines long) all trace even of their development out of colorless formative cells is lost; whilst at the same time, as Reichert supposed and I have directly proved, a very active formation of blood-cells is set up in the liver, a reason for which may be found in the circumstance that, at this time, all the blood from the umbilical vein, which supplies the embryo with new organizable materials, first flows into the liver, instead of entering the general circulation as before. In proportion to the extent in which this cellformation in the hepatic vessels is carried on, does the self-multiplication of the blood-corpuscles become less and less considerable; and instead of it, a new formation of free colorless nucleated cells, having a mean size of $0.003-0.004$, and an extreme diameter of $0.0015-0.006$ of a line, is observed to take place in the blood and immediately around nuclei, which also occur in the free condition; and which cells afterwards, still for the most part in the liver, are transformed, by the development of coloring matter in their contents, into colored, nucleated blood-cells, either immediately or after they have multiplied in a similar way to that which the colored corpuscles had previously followed. This

Fig. 295.-Blood-corpuscles of a fotal Lamb, $3 \frac{1}{2}$ lines long: $a$, bi- and tri-nucleated, large, colored blood-globules, in various stages of division; $b$, larger spherical, colored blood-cells, one with a nucleus undergoing spontaneous division; $c$, a smaller one of the same kind.Magnified 300 diameters. 
new formation of blood-corpuscles in the liver, with which the considerable size of, and the abundant supply of blood to, the embryonic liver, is perfectly in accord, continues probably throughout the foetal life; at all events, $I$ have found it in quite old embryos in Mammalia, and also in newly-born children, although it probably diminishes, pari passu, in connection with the appearance of the ductus venosus (which, according to Rathke, is a secondary formation) and its enlargement, because, through it, a considerable portion of the blood from the umbilical vein enters the circulation directly, and is diverted from the liver.

The further development of the nucleated, spherical blood-cells of the embryo, which have originated in either way, takes place in this manner: the cells gradually, and either directly, or after they have multiplied in the mode above described, become more and more flattened, and even present slight excavations, whilst their nuclei manifestly diminish, ard on the application of acetic acid, exhibit a great tendency to disintegration. Ultimately, the nuclei disappear altogether, and the blood-cells become non-nucleated, like those of the adult, of which they all soon assume the form, being at first somewhat irregular. With respect to the period at which these non-nucleated colored cells make their appearance, it must be remarked that in a fotal Lamb, $3 \frac{1}{2}$ lines long, I could perceive none of them, nor could Paget in a human embryo, measuring 4 lines in the fourth week. In fotal Lambs, 9 lines long, they were still very scanty, whilst in those 13 lines in length, they constituted by far the majority of the bloodcells; and in a human embryo, at three months, they formed, in the hepatic blood $\frac{1}{4}$, and elsewhere about $\frac{1}{6}-\frac{1}{8}$ of the colored corpuscles. In still older embryos, they preponderate greatly, so that in foetal Lambs of 5-13 lines in length, the nucleated colored cells in the hepatic blood constituted not more than $\frac{1}{4}$ or $\frac{2}{5}$ of the blood-cells, and in the rest of the blood, in the larger embryos, did not occur more abundantly than the lymph-globules in the blood of the adult animal. At what time, in the human embryo, the nucleated colored cells become more rare and disappear, is not yet ascertained, although Paget saw them still tolerably numerous in one instance in an embryo of five months. The blood of the larger Mammalian embryos contains, not only in the liver, but also elsewhere, besides the colored blood-globules, other colorless cells in great number, and often as numerous as the colored, which. cells, there can perhaps be no doubt, are derived mainly from the liver, in which, even in foetal Lambs 13 lines long, the colorless and slightly colored, nucleated blood-cells constitute, perhaps, one-third of the whole blood-corpuscles; and in the latter periods of foetal life, are probably also derived from the lymph. Whether these cells are metamorphosed into colored ones, is by no means determined, this much only having been ascertained, that the transitionary forms between the 
two, so numerous in the hepatic blood, are wholly wanting in the rest of that fluid.

The origination of the blood-globules after birth and in the adult, notwithstanding the great pains specially devoted to this point, still remains one of the most obscure parts in the history of the blood-cells; in my opinion, however, the notion which assumes that the red bloodcells proceed from the smaller chyle-corpuscles, which lose their nuclei, become flattened, and have hematin produced in them, is the one most deserving of credit. These cells are about of the same size as the blood-globules, or even rather smaller, have the same kind of membrane as the latter, are flattened, and not unfrequently of a faint yellow color, and consequently may, as we see in the colorless blood-cells of the embryo, pass without any considerable change into colored cells. Where and how this takes place, no one has seen; and notwithstanding all the trouble and care that I have devoted to the subject, I have never noticed a nucleated colored blood-cell in the adult. The only thing of the sort that I have met with, has been this, that in the pulmonary veins, and occasionally also in other blood, the smaller lymphcorpuscles were in many instances pretty distinctly colored, much more so than in the thoracic duct, so that, except from their faintly granular aspect, they were scarcely distinguishable from the true blood-cells lying on their flat side; and the more so, because they contained somewhat smaller nuclei than elsewhere; but even this circumstance is insufficient to decide the question. The following points, however, may be adduced as presenting very important analogies: 1 , that in all the lower Vertebrata, very distinctly, for instance, in the Amphibia, even in adult animals, the origination of nucleated blood-cells from the lymphcorpuscles may be observed; and, 2, that, in the human embryo, also, the formation of the colored blood-globules from colorless cells, very closely resembling the lymph-corpuscles, has been demonstrated by me in the most decisive way. If to this it be added, that there is not the slightest evidence of an independent, or other kind of origination of blood-cells, it may perhaps be considered quite justifiable if I maintain their origination from the lymph-corpuscles; and, in order to explain the reason why the transition itself has not yet been observed, if I broach the supposition that it may take place too rapidly to be in any way obvious with our means of observation.

Although in what has been said, I express myself in favor of the formation of the red blood-cells from the elements of the lymph and of the chyle, I would by no means assert that all the elements of those fluids become blood-cells at every period of post-embryonic life. The microscopical investigation of the blood would rather show, that they invariably contain a certain number of larger pale cells with several nuclei, or a single nucleus disintegrated by acetic acid, of which, although they are certainly derived from the chyle, or are metamorphosed elements of 
it, it is perhaps impossible to suppose that they ever become blood-cells. This being established, it is a question whether the change of the bloodcells, their formation and their dissolution, is not perhaps much slower than is commonly assumed, and whether they are not elementary parts of a more stable nature than is supposed. I am unable to throw any decided light upon this point, and will merely remark that in any case, so long as the growth of the body goes on, and the quantity of blood is augmented, an energetic formation of blood-cells must take place; in opposition to which it is quite unascertained whether, in this period of life, blood-cells are dissolved; on which account, also, it cannot be stated how many of the elements of the chyle undergo the metamorphosis into blood-corpuscles. In the adult, only this much should be regarded as certain, that when from any cause the quantity of blood has become diminished, it may be replaced together with the red bloodcells; whilst it is altogether unascertained whether, under the usual conditions, anything like an energetic solution and re-development of those cells takes place. As their formation cannot be definitely observed, nothing remains by which the question can be decided but observations respecting the dissolution of the blood-globules; these observations, however, have by no means tended to demonstrate the occurrence of a constant change of the elements of the blood, taking place at short intervals; for although in the spleen of many animals a vast quantity of blood-globules undergoing disintegration is met with, the frequent and regular recurrence of a dissolution of those bodies in that organ has not yet been proved. Taking everything into consideration, I am therefore of opinion, that the question as to when, and to what extent, blood-corpuscles perish and are again formed in the adult, cannot be definitely decided from the facts at present in our possession, although I am inclined to think that the elements of the blood are not altogether such perishable structures as is commonly believed.

I have still to mention, that, quite recently, the view that the bloodglobules are formed independently in the blood, out of colorless cells, is advocated by various authorities. Lehmann and Funke rely, the former on the large amount of colorless cells in the blood in the hepatic veins, the latter on the similar condition of the blood in the splenic vein, and they both consider it probable, that a new formation of red bloodcells takes place within the bloodvessels of the liver and spleen. It appears to me, that this question must be approached with very great care, so long as the transition of the colorless cells into blood-corpuscles has not been directly observed, which in this case has by no means been done. At present we are far too little acquainted with the vital relations of the colorless cells in the blood to conclude, merely from their existence, upon a formation of red blood-cells, and especially when we remember the facts stated; since, as I have elsewhere shown ("Mikros. 
Anat.," II. 2, p. 292), it is very possible that the colorless cells in question, in the splenic and hepatic veins, are derived from the parenchyma of the spleen, are only accidental constituents of the blood, and as their frequently multiple nucle $i$ seem to indicate, undergo no further developments, but are in a state of gradual removal.

The view propounded by Gerlach and others, that the cells containing blood-corpuscles, which are met with frequently in the spleen and occasionally in the blood, have a relation to the formation of blood-cells, must decidedly be rejected, since the blood-corpuscles of all these cells are in a state of dissolution.*

* [It is somewhat surprising that Professor Kölliker should not have thought it necessary to consider the doctrine advocated by Wharton Jones (l. c.), the truth of which the latter writer may, we think, be almost said to have demonstrated, viz. that the colored corpuscle of the blood of Mammalia is the homologue of the "nucleus" of the colorless corpuscle of the same blood, and of the "nucleus" of the corpuscle of the blood of oviparous Vertebrata and of Invertebrata.

If we consider that it is admitted on all sides: 1, that the colorless corpuscle of Mammalian blood and the lymph-corpuscle are identical. 2, that these are identical with the colorless and lymph-corpuscles of other Vertebrata. 3, that in the latter, the colored blood-corpuscle proceeds from the colorless corpuscle:-only three hypotheses can well remain with regard to the relation of the blood-and colorless corpuscles of Mammalia-viz.: that in the text; that which supposes that they have an independent origin; and that advocated by Wharton Jones. The two former of these hypotheses are deficient in all positive basis, and the first appears to us extremely improbable. On the other hand, the third theory appears to be in harmony with all the known facts; and opposed to none. It is, shortly, that in Vertebrate animals, the blood-corpuscle is found in three successive phases of development: that of a cell with granular contents - the granules being either fine or coarse; that of a cell without any contents except the "nucleus"-the cell being either colorless or colored; and, finally, in that of a free cellæform "nucleus," which is either colorless or colored. We have thus three phases, each of which has two stages. The phases of granule-cell and nucleated-cell are met with in all Vertebrata; Amphioxus alone going no further than the colorless stage of the second phase. In the oviparous Vertebrata the blood-corpuscle presents the first two phases in both their stages. In the Mammalian cell, the phases exist in all their stages; but two of the latter, that of the colored nucleated cell and that of the colorless free ccllæform nucleus, occur but rarely and scantily. That the red corpuscle of Mammals is the cellæform "nucleus" of the nucleated-cell stage, set free by the bursting of this cell itself, and become filled and red by the secretion of globulin and coloring matter into its interior, is strongly evidenced by the correspondence in size between the "nucleus" and the red corpuscle, as the latter varies in different animals. In the Elephant the red corpuscle is very large, and in the Goat it is very small; the "nucleus" of the colorless corpuscle varies correspondingly. There is a similar correspondence in form; and it is remarkable that in the Paco, whose red corpuscles are, when fully formed, elliptical, while the nuclei of the colorless corpuscles are for the most part circular, younger less-colored red corpuscles are met with, which are circular and correspond in all respects with the "nuclei" of the colorless corpuscles. In dealing with objections which might be raised from the chemical and phýsical differences between the red corpuscles and the "nuclei," Wharton Jones shows that these almost disappear if we select the youngest state of the red corpuscle as one term of the comparison.

It is rare to meet with the transition stage, between the phase of nucleated cell and that of "free cellæform nucleus" in the blood of Man. "We have, however, recently recorded an observation of the kind in unaltered blood ("Quarterly Journal of Micr. Science," vol. i. p. 145), where a well-marked red corpuscle, was observed within what would otherwise have been regarded as a colorless corpuscle, and occupying the place of its " nucleus;" and 
The investigation of the heart, as regards the muscular fibres themselves, is easy, and their anastomoses will be found without difficulty in every carefully made preparation. But great difficulties attend the tracing of the course of the fibres in that organ. Hearts that have been macerated in weak spirit are best adapted to this purpose; the boiling in water, also, of the recent heart, or of hearts that have been previously in salt for some weeks, has been long recommended, a method, instead of which Purkinjé and Palicki advise the boiling in a solution of common salt, or still better of sulphide of lime; whilst Ludwig, after removing the pericardium, lays the heart in water, and repeats this maceration each time after the removal of a layer of the muscular substance, using at the same time slight pressure. For the bloodvessels, the tearing of them into lamellæ with the forceps and scalpel, which alone was formerly employed, is not sufficient; the exarnination of transverse and longitudinal sections of the entire wall, being, in addition, indispensably requisite. The best mode of proceeding is to dry portions of the vessel stretched out upon paper, in which condition sections may be made even of very small vessels, which are to be again moistened with water, and if it be wished to study the muscular structure, treated with acetic or nitric acid of 20 per cent. (Weyrich), or else with caustic soda, by which reagents the elastic tissue is also very beautifully displayed. For the speedy, isolated demonstration of the epithelium, the elastic inner membrane, and the muscular tunic; the larger vessels at the base of the brain have appeared to me to be the best adapted; the elastic membranes of the $t$. media are readily isolated by maceration in strong acetic acid. Its muscular fibres are always to be seen upon simple teasing out; or else readily, upon the addition of nitric acid. For the study of the capillaries, the brain, the retina, the Tadpole and embryos are above all to be recommended; for their development, the Tadpole, the allantois ' of embryos, and the vascular capsule of the lens. The blood should be examined, when it is possible, in the serum itself, afterwards with the various reagents above noticed; and regard must be paid to the great tendency to change possessed by its elements. I inject lymphatic glands with carmine and size, or with sealing-wax and resin dissolved in alcohol; I also recommend sections of preparations hardened in alcohol.

Literature.-J. C. Fr. Wolff, in the "Memoirs of the Petersburg Academy," for the years 1780-92; J. Reid, Art. "Heart," and B. Searle, "Fibres of the Heart," in "Cyclop. of Anat.," II. ; Par-

we may add that the same subject has recently afforded, in blood taken from the finger one or two hours after breakfast, a very considerable proportion of such corpuscles with red "nuclei," affording every transitional stage between the ordinary colorless corpuscle and the free red cellæform nucleus. For these observations, however, water and very dilute acetic acid were added to the blood.-Trs.] 
chappe, "Du Cœur, de sa structure et de ses mouvements," Paris, 1844; C. Ludwig, "Bau der Herzventrikel," in "Zeits. für rat. Med.," Bd. VII., p. 189, and "Ueber die Herznerven der Frösche," in Müller's "Archiv," 1848, p. 139; Luschka, "Das Endocardium und die Endocarditis," in Virchow's "Archiv," IV., p. 171; Remak, "Ueber die Ganglien des Herzens," in Müller's "Archiv," 1844, p. 463, and "Ueber den Bau des Herzens," ibid., 1850, p. 76; R. Lee, "Memoir on the ganglia and nerves of the heart," London, 1851 [and "On the Nerves which supply the Muscular Structure of the Heart," "Proceed. Roy. Soc.," Nov. 17, 1853, vol. vi., No. 99, p. 337]; Bidder "Ueber die Nervencentra im Froschherzen," in Müller's "Archiv," 1852, p. 163; R. Wagner, "Symp. Ganglien des Herzens," in "Handw. d. Phys.," part XIII., p. 360 ; F. Räuschel, "De arteriar. et venar. struct.," Vratisl., 1836, Diss. ; Kölliker, "Ueber die Muskulatur der Gefässe," in "Mitth. d. Zürch. naturf.," Ges., 1847, and "Zeits. f. wiss. Zool.," I.; "Sur le developpement des vaisseaux capillaires sanguins et lymphatiques," in "Ann d. Sc. Nat.," 1846; C. Donders and H. Jansen, "Unters. über die krankh. Veränder. d. Arterienwände," in "Archiv. f. phys.," Heilk., VII., p. 361, also in "Nederl. Lancet," I., p. 473; Jaesche, "De telis epithelialibus in gener. et de iis vasorum in specie," Dorp., 1847; J. Engel, "Beiträge zur Anatomie der Gefässe," in "Zeits. der Wiener," Aerzte, 1847, pp. 152, 315, 428, 1849, p. 121 ; R. Remak, "Histologische Bemerkungen über die Blutgefässwände," in Müll. "Arch.," 1850 ; J. M. Schrant, "Ontleedkundige Studien over de aderlijke bloedvaten," in "Tijdschr. d. Maatsch. tot bevord. d. geneesk.," 1850, p. 2 ; M. Schultze, "De arteriarum structuiâ," Gryph., 1850; H. Weyrich, "De texturâ et structurâ vas lymphatic.," Dorpat, 1851; Fr. Wahlgren, "Vensystemets allmänna Anatomi," Lund., 1851; F. Noll (and Ludwig), "Ueber den Lymphstrom u. die Anatomie der Lymphdrüsen," in Henle's "Zeits.," IX., p. 52; Remak, "Ueber blutleere Gefässe im Schwanze d. Froschlarven," in Mull. "Arch.," 1850, pp. 79, 183; J. Engel, "Bau u. Entwicklung der Lymphdrüsen," in "Prag. Vierteljahrschrift," 1850 , p. 111 ; O. Heyfelder, "Ueber den Bau der Lymphdrüsen," Bresl., 1851; H. Nasse, Art. "Chylus, Lymphe, u. Blut," in Wagner's "Handw. d. Physiol.," Bd. I. ; H. Müller, "Beiträge z. Morphologie des Chylus u. Eiters," in "Zeits. f. rat. Med.," 1845; R. Wagner, "Beiträge z. vergl. Physiologie d. Blutes," Leipzig, 1833, and "Nachträge zur vergl. Physiol.," I., ibid., 1838; J. C. Fahrner, "De globulorum sanguinis origine," Turici, 1845; A. Kölliker, "Ueber die Blutkörperchen eines menschl. Embryo und die Entwickl. d. Blutk. b. Säugethieren," in "Zeitsch. f. rat. Med.," Bd. IV., 1846, p. 42; C. Donders u. J. Moleschott, "Untersuch. ii. d. Blutkörperchen," in the "Holländ. Beiträgen," III., 360; Donders, in "Ned. Lancet," 1846; 
Wharton Jones, "The blood-corpuscle considered in its diff. phases of development," in the "Phil. Trans.," 1846, II., p. 82. Besides which should be consulted the hand-books of E. H. Weber and Henle, and the recent embryological works of Vogt, Remak, Prévost, Lebert, and Courty. [The more recent publications on the vascular system are: Von Hessling "Histologische Mittheilungen," in Siebold and Kölliker's Zeitschrift, v., p. 189; A. Kölliker, "Ueber den feinern Bau u. die Functionen der Lymphdrüsen," in "Verh d. phys. med. Ges. in Wurzb.," iv. 2, 1854; Rokitansky, "Ueber einige der wichtigsten Krankheiten der Arterien," Wien, 1852; C. Wedl, "Ueber Blut. u. Blutgef äss Neubildung," in "Schmidt's Jahrbücher," No. 12, 1853; J. Moleschott, "Ueber das Verhältniss der farblosen Blutzellen zu den farbigen in verschiedenen Zuständen des Menschen," in "Wiener Mediz. Wochenbl." No. 8, 1854; T. Williams, "On the Blood proper and the Chylaqueous Fluid of Invertebrate Animals," in "Phil. Trans.," part ii., 1853, and "The Blood, its Chemistry, Physiology, and Pathology," in British and Foreign Med. Chirurg. Review, Oct. 1853, and Jan. 1854.-DaC.]

\section{OF THE HIGHER ORGANS OF SENSE.}

\section{OF THE ORGAN OF VISION.}

$\S 224$. The visual organ consists of the eyeball, or the proper sensitive apparatus, and the accessory parts, some for its protection, some for its movement: viz. the eyelids, the ocular muscles, and the lachrymal organs. The eyeball itself is a very complex organ, into the constitution of which nearly all the tissues of the body enter. It is composed essentially of three tunics : a fibrous-the sclerotica and cornea, a vascular-the chorioidea and iris, and a nervous; and of two internal refractive media-the vitreous humor and the crystalline lens.

\section{A. OF THE EYEBALL.}

\$225. Fibrous tunic of the Eye.-The external envelop of the eyeball is formed by a tough, fibrous membrane, composed chiefly of connective tissuc, which, to outward appearance, is divisible into a smaller, anterior, transparent portion-the cornea; and a larger, opaque, posterior part-the sclerotic; but, as shown by its development and more intimate structure, is to be regarded as a membrane continuous throughout.

The sclerotica, also termed the tunica albuginea, is a white, very tough and strong, fibrous membrane, which gradually diminishes in thickness as it advances forwards from the posterior part of the eye, 
where it is directly connected with the sheath of the optic nerve, although it is again strengthened, in front, by the expanded tendons of the recti muscles, with which it is blended, afterwards becoming continuous with the cornea. When boiled, it affords common gelatin, and it consists of true connective tissue, the fibrils of which are very distinctly manifest when the structure is teased out,' or transverse sections

Fig. 296.

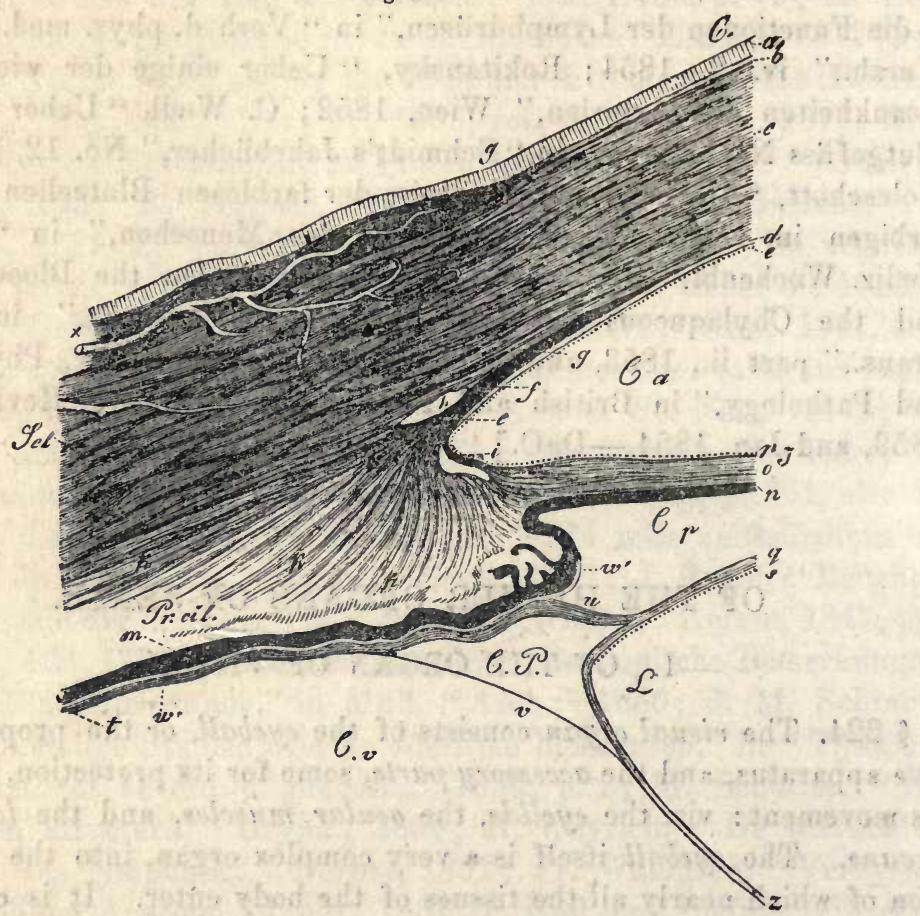

are treated with acetic acid. The bundles themselves are straighter, in other respects as in the tendons, being intimately united and conjoined into larger, thinner or thicker, flattened bands, which are disposed in

FIG. 296.-Transverse section through the tunics of the eye, in the region of the ciliary processes, magnified 12 diameters: Scl, sclerotica; C, cornea ; Pr. cil., processus ciliaris ; C.a, anterior chamber; C.p, posterior chamber; C.v. corpus vitreum; C.P. canalis Petiti; $L$, lens ; I, iris; a, conjunctiva cornec, epithelium; $b$, homogeneous lamella beneath, continuous with the conjunctiva sclerotica, $x$; $c$, fibrous layer of the cornea; $d$, membr. Demoursii ; $e$, indication of its epithelium; $f$, termination of the membrana Demoursii, and its transition into peculiar fibres, $g$, which are continued at $i$, upon the iris constituting the lig. pectinatum iridis; $h$, canalis Schlemmii ; $k$, musculus ciliaris s. tensor chorioidea, springing from its inner wall, $l ; m$, pigment layer of the ciliary processes; $n$, the iris ; 0 , fibrous layer of the iris ; $p$, indication of its epithelium; $q$, anterior wall of the capsule of the lens; $z$, posterior wall; $s$, indication of the epithelium of the capsule; $t$, zonula Zinnï, or anterior thickened portion of the hyaloid membrane; $u$, its free anterior lamina (proper zonula), inserted into the border of the lens; $v$, its posterior lamina blended with the posterior wall of the lenticular capsule; $w$; colorless epithelium of the ciliary processes; $w^{\prime}$, anterior termination of this epithelium. In part after Bowman. 
the transverse and longitudinal directions, alternating pretty regularly through the entire thickness of the tunic, and consequently in vertical sections, producing a lamellated structure. Truly independent lamelloe, however, nowhere exist, the various longitudinal layers having numerous points of connection, as have also the transverse lamince. However, on the external, but more particularly on the internal surface of the sclerotic the longitudinal fibres are collected into somewhat thicker plates, and thus acquire a greater independence.

Numerous fine elastic elements pervade the connective tissue of the sclerotic, of the same form as those in the tendons and ligaments ( $\$ 80)$, viz.: as a network of the finer or finest fibres, in which the sites of the original formative cells are indicated by enlargements with nuclear rudiments, so that the whole often very closely resembles anastomosing, fusiform, and stellate cells. During life, the elements of this network occasionally appear still to possess cavities and fluid contents; at any rate in portions of a dried sclerotic, air is always to be seen in the bodies of all the cells (these are the cretaceous corpuscles of Huschke), and consequently, in this situation the opinion propounded by Virchow, that channels of this kind are a sort of nutritive canals, would appear to be completely justified, and the more so, because the vessels of this tunic are at all events very scanty. They are derived chiefly from the ciliary arteries and from those of the muscles of the eyeball, and constitute, as I and Brücke have found, a tolerably wide-meshed network of capillaries of the last order. Bochdalek has recently described nerves (and also Rahm, in the Rabbit) in the sclerotic, but with Arnold and Huschke, I have hitherto been unable to satisfy myself that these are anything more than branches, on its inner side, running to the ciliary ligament.

The cornea (Fig. 296 C) is perfectly transparent, still more compact and tough than the sclerotic, and is composed of three special layers, viz.: 1 , of the conjunctival membrane (conjunctiva cornece); 2 , of the proper cornea; and 3, of the membrane of Descemet; the first and last of which are formed of an epithelium and a subjacent structureless membrane, and the middle one of a fibrous tissue of a peculiar kind.

The proper cornea, or the fibrous layer (Fig. 296 c), by far the most important part of the whole tunic, consists of a fibrous substance closely allied to connective tissue, but which, according to J. Müller, affords when boiled, not gelatin, but chondrin. Its elements, pale bundles, $0.002-0.004$ of a line in diameter, in which, at least when teased out, finer fibrils are usually perceptible, sometimes more and sometimes less distinctly, are united into flat bundles. These bundles, which have their flat sides always parallel with the surface of the cornea, decussate in various directions, and exhibit, if not complete lamelloe, yet a distinctly laminated structure, owing to which the cornea is very readily torn and penetrated in the direction of its surfaces, and with great difficulty in 
that of its thickness. The correspondence of the corneal elements with connective tissue is also shown by the following circuinstances: 1 , that it is continuous at the border, by its elements, which in that situation follow principally a radiating direction, directly and without interruption with the similarly disposed fibres of the sclerotic, so that there cannot be the least question as to the non-existence of any natural demarcation between the two tunics; and 2, as Virchow was the first to show, that a great number of anastomosing, fusiform and stellate, nucleated cells lie among its bundles, just as they do in undeveloped elastic tissue (connective tissue-corpuscles of Virchow), which also exist in the sclerotic, though more branched. It can perhaps scarcely be doubted, that the nutritive fluid, with which the cornea is constantly imbued in considerable quantity, and which, in the large eyes of animals, may be directly demonstrated by expression, is in great measure conveyed and distributed in the interior, by the cells in question; a view which is only strengthened by the knowledge that these cells, in morbid conditions of the cornea, very frequently contain oil-drops, and occasionally, according to Donders, even pigment, in their interior. The "corneal tubes" injected by Bowman in the eye of the $\mathrm{Ox}$ and in that of Man must not be confounded with this cellular network, and are probably to be explained as artificial dilatations of the minute interstices which normally exist between the structural elements of the cornea, and which it is thought may occasionally be perceived on microscopical examination.

The conjunctival membrane of the cornea (Fig. $296 a, b$ ) is composed principally of a soft, laminated epithelium, $0.023-0.050$ of a line thick, in which the deeper layers of cells are elongated and placed vertically upon the cornea, whilst the middle ones are more of a rounded form, and as they approach the surface, pass into a layer, $0.008-0.01$ of a line thick, corresponding to the horny layer of the epidermis, composed of plates $0.01-0.14$ of a line in size, though still nucleated and soft. Many of these latter cells, as I have shown ("Zeitsch. f. wiss. Botanik," II., p. 80), in consequence of their mutual pressure, present larger or smaller pits, like certain cells in the urinary bladder, so as when viewed on the side often to exhibit a stellate figure, which induced Valentin, who first noticed this form, to regard them as cells with processes. Beneath the epithelium, which, after death, is very soon rendered opaque by both water and acetic acid, is a structureless lamella, first described by Bowman (anterior elastic lamella, of Bowman), 0.003-0.004 of a line thick, which is especially evident in vertical sections and in folds of thin superficial sections, upon the addition of alkalies, although it is by no means so sharply defined from the true cornea as the membrane of Descemet, nor does it seem to be of the same import as that membrane, but is perhaps no more than the remainder of the vascular layer of the corneal conjunctiva, which exists at an earlier period. Arched fibres, like rigid 
bundles of connective tissue or elastic fibres, are occasionally visible, given off from it, and penetrating the cornea to a certain distance, where they are lost.

The membrane of Descemet or Demours, also termed the membrane of the aqueous humor (memb. Descemeti, s. Demoursii s. humoris aquei) (Fig. $296 \mathrm{~d}$ ), consists of an elastic membrane rather laxly attached to the corneal tissue-the proper membrane of Descemet [posterior elastic lamina of the cornea, Bowman], and of an epithelium on its inner surface. The former is as clear as glass, brilliant, quite structureless, easily lacerable, though tolerably firm, and so elastic, that when it is raised from the cornea by the scalpel and forceps, by boiling in water, or by maceration in alkalies, under which treatment, as under reagents in general, it does not lose its transparency, it always rolls up strongly and towards the front. Towards the border of the cornea, the membrane of Descemet, which is $0.006-0.008$ of a line thick, and in chemical properties approaches the homogeneous membranes ( $\$ 16)$, passes into a peculiar system of fibres, first accurately described by Bowman. This set of fibres commences at a short distance from the margin of the cornea on the anterior surface of the membrane of Descemet (Fig. $296 \mathrm{~g}$ ), as an elongated network of fine fibrils, like the finer elastic fibrils, afterwards gradually increasing in thickness, until at the very margin of the cornea, the whole thickness of the membrane of Descemet is broken up into a network of thicker fibres and trabeculoe, which turn over upon the border of the iris (Fig. $296 i$ ), and are blended with its anterior surface. Consequently, the membrane of Descemet does not cease, as is usually stated, with a free border, but, on the contrary, is continued (Fig. $296 \mathrm{f}$ ) all round the anterior chamber, by numerous free processes passing across it, upon the iris. The elements of this ligamentum iridis pectinatum, as it is termed by Huek [pillars of the iris, Bowman], and which, according to Luschka, is much more distinct in the eyes of certain animals (Dog, for instance) than in Man, were formerly ("Zeitsch. f. wiss. Zool.," I., p. 54) referred by me to reticular connective tissue, at a time when I was acquainted with their form but not with their reactions; now, however, I should rather be inclined to describe them as an intermediate form between the connective and elastic tissues. The bundles in question correspond with those of connective tissue, in their width $(0.004-0.012$ of a line) and paleness, and also in the circumstance that still finer fibrils are usually to be distinguished in them, whilst in their rigidity and chemical reactions they approach the elastic tissue and the membrane of Descemet itself, of which latter, though probably differing from it genetically, they are, in the adult at any rate, an integral constituent.

The epithelium of the "membrane of Demours" (Fig. 296 e), which, in Man, frequently does not retain the perfect condition, is a single layer, $0.002-0.003$ of a line thick, of well-formed, polygonal cells, 
$0.006-0.01$ of a line in size, with extremely fine and pale granular contents, and round nuclei of 0.003-0.005 of a line. Towards the border of the cornea the cells of which the epithelium is constituted become smaller, and then ceases as a connected layer, whilst isolated indications, usually of elongated, or even fusiform epithelial cells, are continued, over the fibrous network of the lig. pectinatum, and surrounding its elements, upon the border of the iris, where a perfect epithelium is again met with.*

The cornea in the adult is nearly altogether non-vascular, whilst, as J. Müller and Henle first observed ("De membr. pupill.," p. 44), in the human embryo and fotal Lamb a rich capillary plexus exists in the conjunctiva cornece, but which does not appear to extend as far as the centre. Towards the end of fotal life and after birth, this plexus diminishes in breadth, in animals to a less extent than in Man, so that in the latter we find bloodressels at the margin of the cornea, only in a zone of $\frac{1}{2}$ or at most of 1 line in width. These vessels are for the most part the fine and finest capillaries of 0.002-0.004 of a line, forming one or several rows of arches, and thus terminating; they are lodged in the substance of the conjunctiva, which here extends, in the form of a distinct layer, for a short distance upon the cornea, ceasing in its anterior structureless lamella. In animals these superficial or conjunctival vessels also exist, but are usually much better displayed, and extend further towards the centre; frequently over half the radius, or even beyond it. Besides these, deeper capillaries derived from the sclerotic also occur in the substance of the cornea, usually accompanying the nervous trunks, in which they either form a single or a few very much elongated loops, or extend a little beyond' them; they all terminate in loops, the finest vessels constituting which, like the superficial capillaries, measure scarcely more than 0.002 of a line. I have also noticed, in Man, these peculiar corneal vessels accompanying the nervous trunks, although not constantly and never so much developed.

Nothing certain is known of the lymphatics of the cornea (vid. also, Arnold, "Anat." II. p. 988), though I have recently observed vessels in the cornea of a young Cat (Fig. 297), which I can scarcely regard as anything else than lymphatics. In this instance, at the margin of the cornea, together with the very distinct capillary loops containing blood-corpuscles, there were numerous wider vessels (of $0.01-0.02$, or even 0.03 of a line), which either extended singly into the cornea to the

* [This statement is directly opposed to Mr. Bowman's observations (op. c., p. 22), who says, "that it would appear from what has been said concerning the conversion of the posterior elastic lamina at its border into fibrous tissue, which in part passes through the aqueous humor to the iris, that this epithelium must cease with the elastic lamina, since there is no longer any stratum upon which it can rest." He has been unable "to discover the smallest appearance of it upon the pillars of the iris, and conceives, therefore, that it is limited to the cornea." And according to the same accurate observer, the front of the iris has no true epithelial investment.-TRs.] 
same distance as the bloodvessels, and terminated in dilated clavate ends, or in acuminate points, or two, three, or more together, formed simple loops, from which in like manner other cæcal processes were given off. Notwithstanding their capacity these vessels presented a delicate, structureless coat, with scattered, appressed nuclei, and contained a clear fluid, in which frequently a few, and occasionally even a good many clear spherical cells, exactly like lymph-corpuscles, were visible. If I had found these vessels in other animals as well, I should at once have declared them to be the commencements of the lymphatics of the conjunctiva, but it appears to me, at present, more prudent to regard this explanation perhaps as probable, but not as certain. For

Fig. 297.

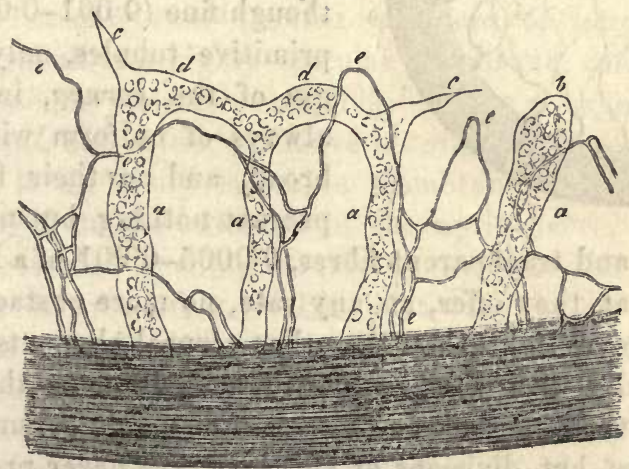

although, in this one instance of the Cat, the ressels in question were very manifest in both cornece, so that I was able to point them out to many of my colleagues, particularly to R. Virchow and H. Müller, I have since been unable to perceive any decided indication of pale vessels of the same kind, either in the adult Cat or in the newly born Kitten, or in the Dog, Ox, Sheep, Pig, and Rabbit. But it is now well known that the commencements of lymphatics, for once when they are distinct (in the intestinal villi for instance), escape the sight perhaps twenty or thirty times. Nevertheless, in this case there seems to be every reason for caution. Should the vessels in question not be lymphatics, they might be regarded as pathological excavations, or as transformations of earlier embryonic corneal vessels; but the manifest limitary membrane of the canals is opposed to the former supposition, and the latter is upset by the circumstance that they occurred in the sarne plane with true vessels, and did not enter into the least anastomosis with them.

The nerves of the cornea discovered by Schlemm, are derived from the nervuli ciliares, penetrate the sclerotic at its anterior border (in the

Fig. 297.-Capillaries and lympliatics (?) at the border of the cornea of a Kitten: $a$, trunks of the colorless vessels; $b$, creal clavate extremity of one of these vessels; $c$, pointed prolongation; $d$, loops; e, blood-capillaries.-Magnified 250 diameters. 
Rabbit, according to Rahm, in the posterior half of the globe), and thence enter the fibrous layer of the cornea. In Man, they are readily found at the margin of that tunic, in the form of 24-36 finer and thicker twigs, but scarcely exceeding 0.02 of a line in size. What

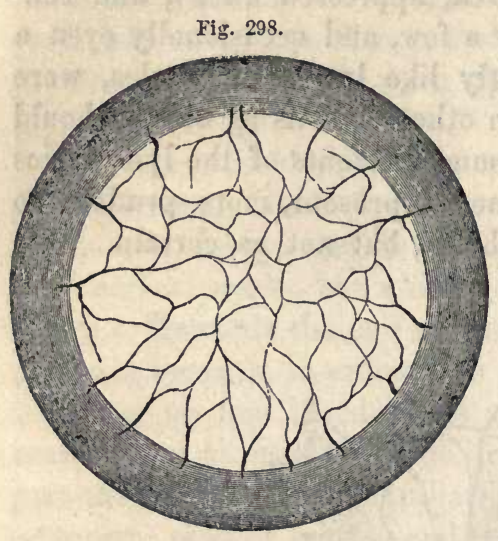
especially characterizes these nerves, is not so much their mode of distribution, which takes place with numerous bifurcations and anastomoses, so as to produce a wide nervous plexus extending throughout the cornea, as the circumstances that they still contain dark-bordered though fine $(0.001-0.002$ of a line), primitive tubules, only at the margin of the cornea, in a zone not always of uniform width, $\frac{1}{2}-1$ line broad, and in their further course present nothing but non-medullated perfectly clear and transparent fibres, $0.0005-0.001$ of a line at most in diameter, so that they offer, at any rate, no more obstacle to the passage of the rays of light than the other corneal elements, as is evident from the difficulty with which they are traced under the microscope. The trunks of these nerves exhibit, though rarely, bifurcations of the primitive tubules, but divisions of this kind are never presented in the plexus formed by them, the conditions in which, however, on account of their paleness, scarcely admit of being quite certainly traced. This plexus is situated in the proper cornea, although nearer to its anterior surface, and from the absence of any indication of free terminations to the nerve-fibres, appears to consist altogether of anastomosing branches of the finest sort, so that, though not in the form of loops, a mutual connection of the nerve-tubes may be assumed to exist.

The bloodvessels of the conjunctiva cornece, in the healthy condition of the organ, are very scanty, and the figures given by Römer (Ammon's "Zeitsch." V. 21, Tab. I. Figs. 9, 11), and Arnold ("Icon. org. sens," II. Fig. 6), I regard as exceptional instances; but, as is well known, they may become so much developed, in inflammations, as almost to cover the entire cornea. The proper corneal vessels also, in such cases, appear to be developed more deeply in the interior. With regard to the vasa serosa of the cornea, vid. \$217. The statements that have been made respecting the bloodvessels of the membrana $D e-$ moursii, in inflammation of the eye (vid. Henle, "De memb. pupill.," p.

FIg. 298.-Coarser ramifications of the nerves of the cornea of the Rabbit. Where the trunks are represented as dark, they contain dark-bordered primitive fibres. 
53), appear still to demand confirmation; and Arnold's "serous vessels," in the normal condition of the membrane of Descemet ("Anat." I. Tab. II. Fig. 5, II. p. 1015), are nothing more than the anastomosing fibres of the ligamentum pectinatum of the iris. The cornea, although vascular only at its margin, is nevertheless not unfavorably circumstanced as regards its nutritive conditions. Wounds in it rapidly unite, portions of the epithelium or even of the fibrous layer when removed, are restored, and ulcers are filled up from the bottom with new corneal substance. Fatty deposits in its tissue, particularly in its cellular elements (especially above and below, or even all round), produce a yellow zone-the so-termed arcus senilis (gerontoxon).*

$\S 226$. The vascular tunic (tunica vasculosa) or uvea.-The second tunic of the eyeball is a highly vascular membrane, containing a great amount of pigmentary matter, and divisible into a larger posterior portion-the choroid, and a less extensive anterior segment-the iris.

The choroid is an easily lacerable membrane, 1-15-1-30 of a line thick, extending from the entrance of the optic nerve, where it is perforated by a rounded opening, nearly to the anterior border of the sclerotic, where it presents a thicker part-the corpus ciliare, and is then continuous with the $i$ ris. Its external surface is attached not only by larger vessels and nerves, but also otherwise, tolerably intimately, to the sclerotic, so that in exposing the choroid a portion of the membrane always remains more or less adherent to the sclerotic, in the form of a brown tissue. This is the so-termed lamina fusca of authors, which there is no ground for separating from the vascular tunic and regarding as a distinct membrane, although in many instances scattered pigment-cells, such as exist in it, are found to extend even into the connective tissue of the sclerotic. The inner surface of the choroid is smooth and, at the ora serrata, very closely connected with the retina, elsewhere more loosely; whilst anteriorly to the ora serrata, and particularly in the processus ciliares, it is very intimately united with the hyaloid membrane (zonula Zinnii), so that the two are never completely separable.

The choroid consists essentially of two portions, a vascular, external thicker layer-the proper choroid, and an inner distinctly colored lamina - the pigmentun nigrum; the former, however, may be again subdivided into three, but by no means sharply defined layers, viz.: 1 , an external, brown, soft lamella, supporting the ciliary nerves and long ciliary vessels, and, in front, containing the ciliary muscle-the outer pigment-layer; 2 , the less deeply colored, proper vascular layer, with the larger arteries and veins; and 3, a colorless, delicate, internal layer,

* [As shown by Mr. Edwin Canton" in his "Observations on the Arcus senilis, or fatty degeneration of the Cornea."- "Lancet," vol. i. 1850, p. 560.-TRs.] 
containing an extremely abundant capillary plexus-the membrana choriocapillaris, which, however, does not extend further in front than the ora serrata. The tissue of which the proper choroid is constituted, except the vessels and nerves, which indeed make up a considerable part of it, and the ciliary muscle, is of a peculiar kind, and cannot conveniently be described under any particular head, but like the fibres of the lig. pectinatum of the iris, though in somewhat different respects, is intermediate between the connective and elastic tissues. In the outer portions of the tunic, this stroma is formed of fusiform or stellate, very irregular, and extremely pale, or more or less brown nucleated cells, 0.008-0.02 of a line long, which anastomose frequently with each other by shorter or longer, usually very delicate $(0.0005$ of a line), but rather rigid processes, and from their great number represent a lax membra-

Fig. 299.

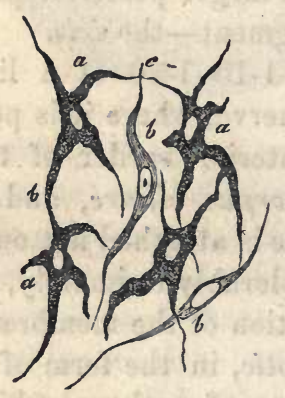
nous tissue. There would be nothing very peculiar in this, and these cellular networks might properly be classed with other similar anastomosing pigment-cells, as for instance in the batrachian larva (most characteristic in Alytes); but in the inner layers of the choroid, and especially in the membrana choriocapillaris, they gradually pass into homogeneous, nucleated tissue, at first containing a little pigment, but afterwards none at all; and which, although in appearance very similar to homogeneous connective tissue, is distinguished from it by its resistance to acids and alkalies, and approximates the elastic tissue, from which, however, it likewise differs in its trifling elasticity and paleness; whence it is better, at present, to regard it as sui generis.

The ciliary ligament of anatomists, or the musculus ciliaris s. tensor chorioidece (Fig. $296 k$ ), the really muscular nature of which was recognized almost simultaneously by Brücke and Bowman, is a tolerably thick layer of radiating smooth muscular bundles, passing from the most anterior border of the sclerotic upon the ciliary body, and ceasing in its anterior half, opposite the part where the ciliary processes are placed, internally. More precisely described, the ciliary muscle arises where the sclerotic is grooved for the formation of the venous sinus of Schlemm, and, in fact, from a special, dense, smooth tract (Fig. 296 l), which, forming the inner wall of the canal in question, coalesces with the sclerotic, and also receives a portion of the fibrous network, into which the membrana Demoursii is prolonged, the fibres of which are completely blended with the elements of the tract in question, and resemble the others in' all respects except that they are much finer,

FIG. 299.-Cells from the stroma of the choroid: $a$, pigment-cells; $b$, uncolored fusiform cells; $c$, anastomoses of the former. Human.-Magnified 350 diameters. 
anastomose more closely, and run in a circular direction. The ciliary muscle terminates at the most adherent portion of the ciliary processes, though not in those processes themselves, and as regards the elements of which it is composed, they are rather shorter $(0.02$ of a line) and broader (0.003-0.004 of a line) than the common fibre-cells; being at the same time finely granular, soft, and so perishable, as, in Man, not readily to admit of being isolated.

The pigmentum nigrum (Fig. $296 \mathrm{~m}$ ) is a continuous, purely cellular layer, completely investing the inner surface of the choroid and consisting, as far as to the ora serrata, of a single layer of well-formed, almost regularly hexahedral, contiguous cells, $0.006-0.008$ of a line in diameter, 0.004 of a line thick, disposed in an elegant mosaic manner, in which the large quantity of brownish-black pigment usually prevents the nucleus being apparent as more than a clear spot in the interior. On the side towards the retina, however, a narrow clear border is frequently left free of color, showing that the cells must, at one time, have possessed contents or have had a thickened membrane. From the ora serrata onwards, the pigment-cells are disposed in several, at least two, layers, become rounded, smaller, and entirely filled with pigment, so that the nuclei even are scarcely visible. All the pigmentcells have extremely delicate walls, and are very easily ruptured under pressure; the pig-

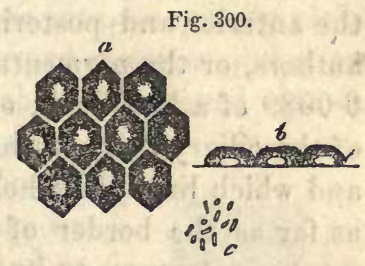
ment is composed of minute, flattened, oval corpuscles, at most 0.0007 of a line long, and presenting, sometimes even while contained in the cell, but still better when liberated, the phenomenon of molecular motion in the most marked manner. The pigment of the choroid is wanting in the eyes of albinoes, as well as, at any rate partially, in the region of the tapetum in animals; but the cells, which would elsewhere contain it, exist in both these instances, only perfectly colorless.

The iris differs from the choroid in containing true connective tissue, the delicate, lax fasciculi of which, partly radiating, in part circular, especially at the ciliary border, and much interlaced, constitute the principal bulk of the stroma of this tunic, and, towards its surface, form a more homogeneous layer. It contains a large number of elongated nucle $i$, which, at any rate in part, are situated in fusiform cells, similar to those of the choroid, only smaller; and also a few rigid, pale fibres, which, as prolongations of the ligamentum pectinatum of the iris, or of the "membrane of Demours," are continued over a part of the anterior surface; lastly; the smooth muscular fibres of the iris, presenting exactly the same characters as those of the choroid [ciliary muscle]. In Man, these fibres constitute a very distinct occlusor muscle of the pupil

Fig. 300.-Cells of the pigmentum nigrum of Man: $a$, viewed on the surface; $b$, on the side; $c$, pigment-granules. 
(sphincter pupillce) in the form of a smooth ring, $\frac{1}{4}$ of a line wide, close to the pupillary margin of the iris, and somewhat nearer to the posterior surface, which, in a blue iris, may be readily recognized after the removal of the posterior pigment, with and without the application of acetic acid, and may also be torn up into its elements, $0.02-0.03$ of a line long. Besides this larger muscular ring, I find, close to the annulus iridis minor, another very narrow ring, nearer the anterior surface of the iris, not more than 1-40 of a line in breadth. Brücke traces the dilator pupilloe as far as the ligamentum pectinatum and the border of the vitreous lamella of the cornea, but I am unable to do so; and it rather appears to me to commence in the substance of the iris at the ciliary margin. From what the difficulty of the investigation has allowed me to see of this muscle, it consists of numerous slender fasciculi, which, far from constituting a continuous membrane, run inwards, each separately between the vessels, and are inserted at the border of the sphincter.*

The iris differs from the choroid also, in possessing a cellular layer on the anterior and posterior surfaces. The latter, the so-termed uvea of authors, or the pigmentum nigrum of the iris (Fig. $295 n$ ) is a stratum, 0.0089 of a line thick, of minute, closely filled pigment-cells, like those of the ciliary body, with which they are also uninterruptedly connected, and which lines the whole of the posterior surface of the iris, extending as far as the border of the pupil. When the iris is folded, the pigmentary stratum, or its free surface, appears to be bounded by a delicate, but sharply defined line, which has been described by several authors as a special membrane (membrana pigmenti, Krause, $m$. limitans, Pacini [?], Brücke, M. Jacobi, Arnold), and, in fact, in eyes that have been kept for some time, and on the addition of alkalies, may be raised in places from the pigment. But since, in such instances, the pigmentary layer is always without any defined outline, and its granules are exposed and dispersed, it appears to me that this membrane is nothing more than the conjoined outer cell-walls of the pigment-cells, which, as is known to be the case elsewhere (intestinal villi for instance) are raised in their totality, and apparently as a special membrane. The cellular layer of the anterior surface of the iris is a simple epithelium of rounded and much flattened cells, which, when viewed in a fold of the iris, are seen to constitute, not a continuous, clear border of uniform breadth throughout, but on the contrary, only distinct, slight elevations. This layer is better seen after the removal of the posterior pigment, in a horizontal view, and also by scraping or shaving off the anterior surface of the iris.t The color of the iris, in blue eyes, depends simply upon the posterior pigment seen through its substance; whilst in

* [Compare Mr. Lister's "Observations on the contractile tissue of the Iris," "Quarterly Journal of Micr. Science," vol. i. p. 8, October, 1852.-Trs.]

† [Vid. note, p. 730 . -Trs. $]$ 
brownish-yellow, brown, and black eyes, it is owing to a special irispigment, which is very unequally distributed, and thus produces the peculiar markings of the anterior surface. This pigment is seated, in the first place, in the stroma itself, and in fact, chiefly in its fusiform cells, but also, as it appears to me, occurs free among the fibres and vessels, and in the fibre-cells of the sphincter pupillce; lastly, in the anterior epithelial layer, it consists of larger and smaller cells, goldyellow or brownish irregular-sized granules, aggregations of granules and streaks, never of the regular pigment-granules of the true ocular pigment.

The vessels of the tunica vasculosa are extremely numerous, and are variously disposed in its different parts. The choroid receives its blood from the short posterior ciliary arteries, about twenty small ressels, penetrating the sclerotic, in the posterior part of the eyeball, at a greater or less distance from the optic nerve, and which, dividing in a dichotomous manner in the middle or vascular layer of the choroid, run anteriorly, and subdivide into three sets of branches: 1 , external, which, having attained a certain fineness by continued division, pass directly into the vence vorticosce; 2 , internal, which pass into a capillary plexus immediately beneath the pigment, in the so-termed membrana choriocapillaris, or Ruyschiana; and 3, anterior, which are continued into the ciliary body and iris. The above-mentioned capillary plexus of the innermost layer of the choroid - which in animals having a tapetum lies upon its internal aspect, and may easily be demonstrated as a special membrane, as may also occasionally be done in Man-is one of the most elegant and closest that exists, inasmuch as its meshes, formed by vessels of 0.004 of a line, do not measure more than $0.002-0.005$ of a line, the capillaries arising from the larger vessels, as it were in a stelliform manner. It extends, as has been already said, only as far as the ora serrata, where it gives place to somewhat coarser convolutions of vessels, 0.004 of a line in diameter, which, proceeding from the anterior branches of the short posterior ciliary arteries, constitute the ciliary processes, and are so closely approximated that, besides the ressels and a homogeneous sheath supporting the processes, the latter seem to contain no other tissue. From thesc various points, and from the ciliary muscle, which

Fig. 301.

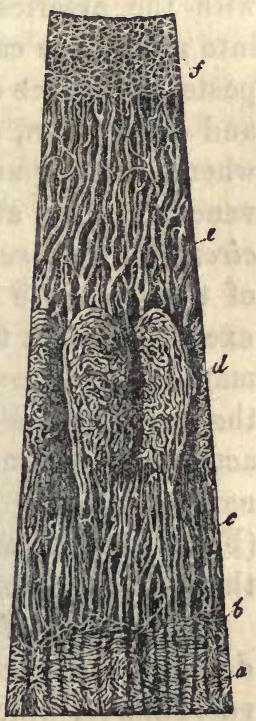

Fra. 301.-Vessels of the choroid and iris of a Child, after Arnold; viewed from within; magnified 10 diameters : $a$, capillary plexus of the posterior segment of the choroid, terminating at the ora serrata, $b ; c$, arteries of the corona ciliaris, supplying the ciliary processes, $d$, and in part passing upon the iris, $e ; f$, capillary plexus on the inner surface of the pupillary margin of the iris. 
likewise obtains some twigs from the same arteries, the blood is returned principally through the vence vorticosce, which, lying upon the arteries, constitute elegant vascular stars or vortices, two above and two below (or it may be five or six); and also at the back of the eyeball, through some minute vence ciliares posticce breves, all of which veins penetrate the sclerotic in the same way as the arteries.

The iris receives its blood in the first place from the arteries of the choroid, and secondly, from the long posterior and the anterior ciliary arteries. The former, with their anterior branches, in part immediately enter the iris, between the ciliary processes, and in part, after supplying the ciliary processes, form small trunks at their border and anterior extremity, which are also continued upon the iris. The long ciliary arteries, two in number, perforate the sclerotic on the right and left a little anterior to the short ciliary vessels, run in the external pigment layer of the choroid, as far as the tensor chorioidece, where, each dividing into two branches and joining the ciliares anticae, which to the number of five or six penetrate the sclerotic in front, they constitute a superficial irregular arterial circle in that muscle-the circulus art. iridis major. From this circle, together with small vessels passing either from it, or from the vessels forming it to the tensor muscle, very many convoluted branches, continued to the iris, are given off; which, with the arteries already mentioned from the choroid, divide, partly into a few true capillaries, a layer of which is found particularly at the posterior surface of the pupillary margin, beneath the pigment (Arnold), and partly run, continually dividing, as far as the pupillary margin, where, forming arched loops, they pass into fine, but still not capillary, venous trunks, after they have constituted a second, usually irregular circulus arteriosus minor close to the annulus iridis minor. The veins of the iris arise from the arteries and capillaries just mentioned, run, except frequent transverse anastomosing branches, also in a radiating manner, and open: 1, more from the posterior surface of the iris into the vasa vorticosa; 2, into the vence ciliares posticce longoe; and 3, according to Arnold and Retzius, also into the "canal of Schlemm," a narrow annular channel situated between the choroid and sclerotic (Fig. 295 h), from which the venulce ciliares anticce, passing through the sclerotic, afterwards convey the blood outwardly.

The nerves of the tunica vasculosa are also very numerous, but destined solely for the ciliary muscle and the iris. They are the nervuli ciliares, which perforate the sclerotic posteriorly, then run forwards in the outer lamella of the choroid, partly in grooves in the sclerotic, and, before entering the ciliary muscle, divide with repeated bifurcations. Within the muscle they break up into a rich and close plexus, numerous filaments from which proceed to the muscle and to the cornea, while others constitute the proper nerves of the iris. The latter ac- 
company the vessels, dividing repeatedly, and with frequent anastomoses, especially in the annulus minor, to the pupillary margin, where their mode of termination is at present unknown. The elements of all these nerves are, in the trunks, of the medium and fine kinds, from $0.002-0.004$, and in the iris of not more than 0.001-0.002 of a line. I have never noticed ganglion-cells in them, nor in the ciliary muscle, where Bochdalek describes them as existing.

Some authors, and among them, recently, Bochdalek, believe that they have seen scattered nerves in the choroid-a circumstance that I am unable to confirm from my own observations. Quite recently, Rainey ("Philos. Magaz.," May, 1851, p. 420) describes a transversely striped choroideal muscle, occupying the posterior part of the choroid and extending through its entire thickness in the form of variously decussating layers, which he says may be most easily demonstrated in the eye of the Sheep. I agree with Henle in thinking that these statements rest upon deceptive grounds; at all events, in the situation in question, either in animals or in Man, I can discover nothing like muscular fibres.

§ 227. Nervous membrane (retina).-The retina is the innermost of the five tunics of the eyeball, and is in close apposition with the choroid, though not coextensive with it, ending at the ora serrata in an undulated margin (margo undulato-dentatus s. ora serrata retince), which is very intimately connected, on the one side with the choroid, and on the other with the hyaloid membrane. A continuation of the retina on the ciliary portion of the hyaloid membrane, which is described by many anatomists, does not exist.

The retina is a delicate membrane; when recent, almost perfectly transparent and clear, and after death whitish and opaque. It commences at the point of entrance of the optic nerve, with which it is, in part, continuous. Its thickness at first is 0.1 of a line, but as it extends anteriorly it soon diminishes to 0.06 , until ultimately, close to the anterior border of the

Fig. 302.

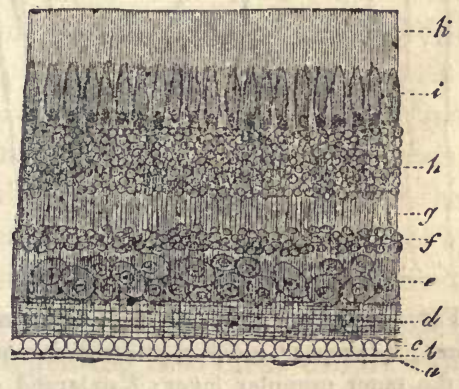

FIG. 302.-Vertical transverse section of the retina of Man, from the posterior portion of the membrane, magnified 250 diameters: a, hyaloid membrane with decumbent nuclei: $b$, membrana limitans; $c$, clear globules (epithelium?); $d$, expansion of the optic nerve; $e$, layer of gray nerve-substance; $f$, internal granular layer; $g$, fine-granular layer, in which the radiating fibres are more distinct than elsewhere; $h$, external granular layer; $i$, internal division of the bacillar layer, with the "cones;" $k$, external division, with the prolongations of the "cones" and the true "rods." 
retina, it is not more than 0.04 of a line in thickness, and finally terminates quite abruptly. Notwithstanding this various thickness, the following layers from without to within may be evidently distinguished in all parts of it; 1 , the layer of rods and cones [bacillar layer]; 2, the granular layer; 3 , the layer of gray nerve-substance; 4 , the expansion of the optic nerve; and 5, the limitary membrane. These layers, with the exception of the innermost, which is of uniform thickness throughout, in general become thinner towards the front, in correspondence with the diminished thickness of the whole retina.

1. The bacillar layer, stratum bacillorum s. memb. Jacobi (Fig. 302 $i, k)$, presents a very remarkable structure, being composed of innumerable rod-like and conical corpuscles, disposed with the utmost regularity and reflecting the light very strongly. With the exception of $\mathrm{H}$. Müller (vid. infra), this structure, in animals, has been understood quite erroneously; and even in Man it has been but very superficially known. It consists of two elements-the rods, bacilli $(k)$, and the cones, coni $(i)$, which together constitute a single layer, 0.036 of a line thick at the bottom of the eye, more anteriorly 0.024 , and quite in front not more than 0.015 of a line in thickness. In general these bodies are so

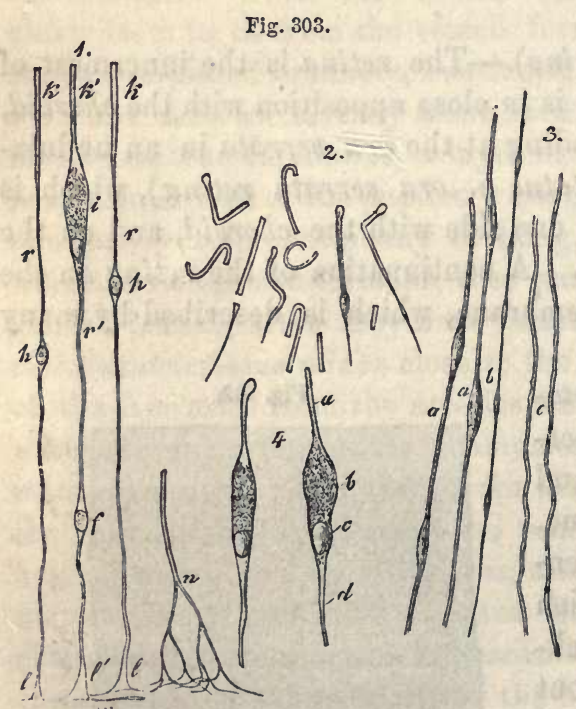
arranged that the more numerous rods have their largest end directed outwards, whilst the cones are disposed in the reverse direction, whence the latter, when imperfectly examined, appear to constitute an inner, distinct, thinner layer, lying between the inner extremities of the "rods."

In Man, the rods (Fig. 303, $1, k, 2)$ are cylindrical, slender, elongated corpuscles, in which a larger external end, the proper rod, is to be distinguished from a more slender internal portion, the prolongation or

Fra. 303.- Retinal elements of Man, magnified 350 diameters, 1, "rods" and radiating fibres: $k$, proper "rod ;" $r$, prolongation of its pointed inner extremity; $h$, "granule" (cell) of the outer granular layer; $l$, enlarged extremity of the radiating fibres proceeding from them to the surface of the optic layer; $k^{\prime}$, "rod" seated on a "cone," $i$; $r$, fibre proceeding from the latter, connected with the "granule," $f$, of the inner granular layer, and the terminal enlargement, $l$, on the inner surface of the retina; $n$, one of the fibrous bundles in which the radiating fibres frequently terminate at their innermost extremity. 2, "rods" torn off from their fibres, in various states of curvature, \&c. 3 , fibres of the optic nerve: $a, b$, straight, coarser and finer fibres, with varicosities; $c$, without varicosities. 4, two "cones," $b$, torn off from their processes, $d$, with somewhat altered "rods," $a$, at their outer ends; $c$, nucleus of the "cones." 
filament. The former portion of the rods, which alone almost has hitherto been known to anatomists, is a cylinder, $0.0075-0.012-0.015$ of a line long, 0.0008 of a line broad, and truncated at the outer end, whilst the inner is produced into a short point, $0.002-0.003$ of a line in length, which is often separated from the rest of the "rod" by a faint transverse line, and might even perhaps be assigned to the "filament." The latter is an extremely delicate process, not more than $0.0002-0.0003$ of a line in thickness, of uniform width throughout, prolonged immediately from the point of the "rod,"and, extending through the inner half of the bacillar layer; it is connected with the other elements of the retina in a manner to be afterwards described. The filament is so delicate that it is usually torn off near its origin on the slightest mechanical impression affecting the bacillar layer; on which account also it has happened that observers hitherto have been acquainted only with the "proper rods," and, though they had often seen the somewhat longer filaments attached to them, the latter were regarded merely as artificial products. Since Hannover, also, the points of these organs have been misplaced outwardly by all writers, which is wholly incorrect. The substance of the "rods" is clear, homogeneous with a faint glistening fatty aspect, very soft and flexible, and at the same time extremely fragile. Their delicacy is so great that they undergo the most manifold changes even in water, often even to their being rendered unrecognizable, bending, as it were, into a hook of various forms, curling and rolling up in all ways, or breaking up into two or more pieces, and allowing clear drops to escape, which are often met with on the external surface of the retina in vast quantity, derived partly from the "rods," partly from the ruptured pigment-cells of the choroid. One of the most usual changes consists in this, that the point, if it be not detached, which is very frequently the case, becomes distended in a varicose manner, and assumes a lancet-shape, or is even transformed into a sphere, on which the "filament" of various lengths is placed, in consequence of which, the obtuse end of the "rod" often presents a hook-like curve or a slight enlargement. The "rods" are almost invariably very much altered by reagents ; and, above all, the proper "rods," which, notwithstanding their greater bulk; yet offer less resistance than the "filaments." Ether and alcohol cause them to contract and shrivel up, often rendering them unrecognizable, but do not dissolve them. In acetic acid of 10 per cent. they are immediately shortened very considerably, swelling out in several places, and disintegrating into clear drops, which at first offer some resistance, but afterwards disappear. Concentrated acetic acid dissolves them in a short time, as do alkalies and mineral acids; whilst diluted chromic acid, although it causes them to shrink a little, is the best preservative of them.

The "cones" (Fig. $303^{4}$ ) are "rods" which instead of a filament are furnished at their inner extremity with a conical or pyriform body, the 
length of which equals half the thickness of the bacillar layer (from $0.007-0.015$ of a line), and whose breadth is from 0.0025 to 0.0045 of a line. Each of these "cones" consists of an external, thicker and longer, finely granular extremity, often more or less ventricose, which, gradually diminishing in size, passes into a common "rod" without a point, and of a shorter inner portion, somewhat constricted from the other by a slight incurvation, in which an elongated or pyriform, more opaque and brilliant body, 0.002-0.003 of a line in length, is enclosed. On the internal aspect, these "cones," in which I can see nothing but a cell with a nucleus, are, like the "rods," continued into the succeeding layers of the retina, where we shall again have to consider them, by fine filaments, $0.0004-0.0006$ of a line in size, similar to those of the "rods."

The "rods" and "cones" are arranged vertically upon the retina, like palisades, in close apposition, and consequently, one of their ends is directed towards the choroid and the other towards the granular layer. Close to the macula lutea the "cones" form an almost continuous stratum (Fig. $304{ }^{2}$ ), so that the "rods" are placed only in single series between them, but more anteriorly they are wider apart, the distance between them at first being about $0.002-0.003$ of a line, and in the anterior portions of the retina even $0.0004-0.005$ of a line (Fig. $304{ }^{3}$ ), thus affording room for more "rods" in the interspaces. Viewed from

Fig. 304 .

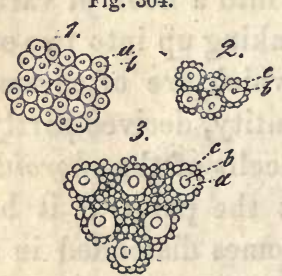
without, the bacillar layer, when its outermost surface is brought into focus, exhibits rounded spaces, placed at a greater or less depth, filled with a clear substance, which also occupies elsewhere the interstices between the elements of this layer. These clear spaces, corresponding to the "cones," present an internal, dark, smaller circle, the terminal surface or apparent transverse section of the "rod" which is seated upon the "cone," and are surrounded by the closely "crowded terminal surfaces of the "proper rods," disposed in a sort of mosaic manner, their outlines being indicated by the single, double, or multiple series of reticulations (Fig. 304).

2. The granular layer, stratum granulosum (Fig. $303 \mathrm{~h}, \mathrm{f}$ ), is composed of opaque, granular corpuscles, reflecting the light tolerably strongly, of a round or oval figure, and 0.002-0.004 of a line in size, sometimes looking like free nuclei, sometimes like minute cells almost entirely filled by large nuclei, although, according to my observations, they should all be referred to the latter category. For I find, especially in preparations made with chromic acid, that from both sides of every granule very fine filaments, $0.0002-0.0003$ of a line thick, are regularly

Fia. 304.-Bacillar layer from without. 1, at the "yellow spot" (only "cones"); 2, at the border of the same; 3 , from the middle of the retina: $a$, "cones," or vacuities corresponding with them; $b$, "rods" of the "cones," whose terminal surface is often placed rather more deeply than that of the proper "rods," c.-Magnified 350 diameters. 
given off, which in many cases may be distinctly seen to proceed from a pale border surrounding the granule, so that the whole is very like a bipolar ganglion-cell in miniature. In Man, the granules, in the greater part of the retina, are disposed in two layers-an outer, thicker of $0.013-0.016(h)$, and an inner, thinner $(f)$, of $0.006-0.008$ of a line -which are parted from each other by a clear, fine-granular, and, to some extent, vertically striated layer $(g), 0.006-0.008$ of a line thick, whilst, towards the ora serrata, the two constitute a single stratum of not more than 0.015 of a line in thickness. The granules of the inner layer are a trifle larger than those of the outer, and when they are oval, as is most usually the case, I find that they are placed with the long axis in the direction of the thickness of the retina, so that their processes, like those of the external layer, run directly outwards and inwards.

3. The layer of cineritious cerebral substance (Fig. $302 e$ ) is pretty sharply defined on the side of the granular layer, and less so towards that of the fibres of the optic nerve, between the elements of which it penetrates more or less. It is composed of a finely granular matrix, corresponding exactly with that of the gray substance on the surface of the cerebrum and cerebellum, and of numerous nerve-cells scattered in it. Of the latter, some, particularly in the outer half of this layer, which is $0.008-0.012-0.02$ of a line thick, are small (0.003-0.006 of a line), inconspicuous, and in fresh preparations recognizable only by their beautiful vesicular nuclei; whilst another portion, forming an almost continuous layer on the inner side, are of a larger size (0.006-0.016 of a line). These cells

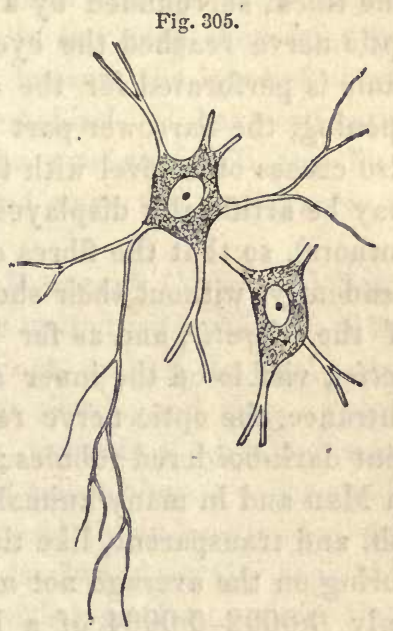
are usually pyriform or rounded, or occasionally prolonged into 3-5 angles; and most of them, perhaps all, are furnished with pale processes like those of the central nerve-cells-which were first noticed by Bowman ("Lectures," \&c., pp. 84 and 125), and afterwards also described by Hassall, Corti, and myself.* The processes occur either single or in numbers varying from two to six and more; are at first as much as 0.002 of a line wide, but in their further course continually

FrG. 305.-Nerve-cells with processes from the retina of the Ox, magnified 350 diameters.

* [Pacini appears to have been the first to perceive the existence and true nature of the caudate cells in this layer of the retina. ("Sulla tessitura int. dell. Retina," 1844, p. 32.) - Trs.] 
diminish in size, under repeated divisions, till they are reduced to fine filaments of scarcely 0.0004 of a line in diameter, which, in isolated cells, terminate in torn ends. In every case in which I have noticed these nerve-cells distinctly in situ, their processes were given off towards the exterior, and afterwards in their further course, without entering the granular layer, appeared to be curved, in order to ramify in the gray nervous layer itself. The nuclei of these nerve-cells, which behave towards reagents like those of the cerebrum, measure $0.003-0.005$ of a line, and usually present a very distinct nucleolus.

4. On the inner aspect of the layer in question we find the expansion of the optic nerve (d). This nerve, after quitting the chiasma (concerning which vid. p. 390) and till it reaches the eye, presents the same conditions as a common nerve; its dark-bordered fibres, $0.0005--0.002$ of a line in diameter, much disposed to become varicose, and between which, according to Hassall, nerve-cells would also seem to occur, but which I have not yet noticed, form polygonal bundles, $0.048-0.064$ of a line thick, surrounded by a nurilemma of the usual kind. When the optic nerve reached the eye, its sheath is lost in the sclerotic, which tunic is perforated for the entrance of the nerve by a funnel-shaped opening, the narrower part being inward; and the internal neurilemma also ceases on a level with the inner surface of the same tunic, where it may be artificially displayed, as a cribriform lamella (lamina cribrosa of authors), so that the fibres of the optic nerve enter the eye, each independently, without their sheaths of connective tissue. Within the canal of the sclerotic, and as far as the slight eminence, the colliculus nervi optici, visible on the inner surface of the retina opposite its point of entrance, the optic nerve retains its white color, and continues to present dark-bordered tubules; but, from that point onwards, its elements, in Man and in many animals, become perfectly clear, yellowish or grayish, and transparent, like the finest tubules in the central organs, measuring on the average not more than $0.0006-0.0008$ and a good many only $0.0002-0.0004$ of a line, whilst some, it is true, have a size of $0.001-0.0015$ or even of 0.002 of a line. What chiefly distinguishes these from other pale nerve-terminations, is the absence of nuclei in their course, a somewhat greater refractive power, and the frequent occurrence of varicosities, which two latter particulars would seem to indicate, if not exactly a nerve-medulla as in the common nerves, still the existence of partially semi-fluid and perhaps fatty contents, and assimilate the nerve-fibres of the retina to the most delicate elements of the cerebrum. I have not yet been able to demonstrate axis-fibres and sheaths in the fibres of the retina, although I would not from that circumstance at present conclude that they do not exist.* At any rate

* [Here again, Professor Kölliker is at variance with Mr. Bowman, who gives as one of the peculiar characteristics of the fibres of the optic nerve in the retina, that they have lost 
the retinal fibres are not composed solely of nerve-medulla, for, if they are treated never so thoroughly with ether, they always remain, smaller indeed, but more distinct and more opaque than previously. Fibres which have been thus treated enlarge again in cold acetic acid, and dissolve in alkalies, and consequently consist, perhaps without doubt, chicfly of a nitrogenous substance.

As respects the course of the nerve-fibres in the retina, this much is certain, that they radiate on all sides from the colliculus nervi optici and constitute a continuous membranous expansion, which extends as far as the ora serrata retino, and presents any considerable interruption only in the situation of the macula lutea. In this true nervous membrane the fibres are associated into larger and smaller compressed bundles, usually $0.01-0.012$ of a line wide, which either mutually anastomose at very acute angles, or run for considerable distances parallel with each other. Notwithstanding all that has been stated by various authors, it may be boldly asserted that the terminations of these nerves are as yet wholly unknown; and, as more will be said upon this subject afterwards, I shall here merely remark that, in any case, they exist not only in front, but in every part of the retina, because the layer of nervefibres becomes visibly thicker from before to behind. I have estimated its thickness, in Man, at the bottom of the eye at 0.036 , two lines beyond the yellow spot at $0.006-0.008$, and near the ora serrata at 0.002 of a line.

5. The limitary membrane, membrana limitans* (b), is a delicate membrane, 0.0005 of a line thick, intimately united with the rest of the retina, which, when that structure is teazed out, and on the application of reagents, is frequently detached in large shreds, and then appears perfectly structureless. On its inner aspect, towards the hyaloid membrane $(a)$, when the retina is folded, flattened cell-nuclei are occasionally perceptible, which certainly cannot be referred to an epithelium, and scarcely to the vitreous body, as the latter is always readily separable from the retina. It seems to be different with regard to a clear, light yellowish border, $0.002-0.003$ of a line wide, situated on the outer side of the membrana limitans, which, in folds of a perfectly fresh retina, appears, as it were, to be completely blended with the limitary membrane, but occasionally exhibits, more or less distinctly, the contours of excessively clear and transparent spherical bodies (b), 0.002-0.003 of a line in size. At a longer interval after death, as well as on the addition of water, a large number of transparent globules, like drops of albumen, are afforded by the clear border of the retina, which then disappears altogether, except the membrana limitans, frequently also,

"the tendency to fall into the varicose or beaded state; in a word, that the fibres of the nerve, in expanding into the retina, lose their white substance, but retain the axis. or central fibre."-Trs.]

* [So named by Pacini (1. c., p. 22).-Trs.] 
together with it. Todd and Bowman describe the clear bodies above noticed as cells, and also figure a minute nuclear corpuscle in them; and I will not directly contradict this notion, although I have not as yet been in any way able to satisfy myself of the existence of nucle $i$ and true cells in this layer.

The condition of the retinal elements at the "yellow spot" is in many respects peculiar. In the first place, any continuous layer of optic fibres is there wholly wanting, and the stratum of nerve-cells, which are in close mutual apposition, lies immediately upon the membrana limitans. Between these cells, however, nerve-fibres run equally from the sides and the internal end of the spot into it, either isolated or in very minute bundles, and terminate in a way that cannot be accurately determined. In the centre of the macula lutea there is a thin uncolored spot in which the granular layer is wanting, from $0.08-0.1$ of a line in diameter, through which the pigment of the choroid is visible-the so-termed foramen centrale. The plica centralis never exists during life; but this is not the case, probably, with the yellow color, which depends upon diffused pigment pervading all the parts of the retina, except the "rods." The latter, in this situation, assume the form of "cones" exclusively, inasmuch as the "proper rods," as Henle ("Zeitsch. f. rat. Path.," 1852, II. p. 307) correctly states, are wholly wanting in the "yellow spot" and its immediate neighborhood. Instead of them, the "cones" form a perfectly continuous layer, are more slender than elsewhere (not more than $0.002-0.0024$ of a line in breadth), and support at their outer end, here as elsewhere, not short points, as Henle states, but the usual "rods," which in this situation are not more than 0.0006 0.0007 of a line broad (Fig. 304).

After this description of the elements of the various retinal layers, it will be as well to cast a glance upon their mutual connection. I have ascertained, in the human eye, that the fibres proceeding from the "rods" and "cones" inwards, and from the "granules," on both sides, are connected, and simply constitute parts of a fibrous system of the retina, as yet not recognized as a connected whole, except by $\mathrm{H}$. Müller. This system, the greater number of whose elements are vertical, penetrates the entire thickness of the tunic, and might be termed the radiating fibre-system (radial fibres, $\mathrm{H}$. Müller), in contradistinction to the horizontal, referable to the expansion of the optic nerve. Proceeding from the bacillar layer, it is obvious, that the fine filaments arising from the "rods" and "cones" are directly continuous with the similar processes given off from the external side of the "granules," in such a way that the filaments of the "rods" (Fig. $303^{1}, r r$ ) are connected. with the "granules" of the outer granular layer, and those of the "cones" (Fig. $303^{1}, r$ ) with the "granules" of the inner layer; in fact, each "cone" or "rod" is in connection with a granule, the latter also per- 
haps with several. The filaments which run inwards from both kinds of "granules," which may be pretty readily traced in vertical sections, are continued in a straight line, or slightly curved, through the layer of gray nerve-substance without any connection with its elements, and enter the expansion of the optic nerve, where, as especially in the posterior part of the eye, in which situation the layer of nerve-fibres is thick, it is easy to perceive that they run in the narrow interstices between the nervous bundles, in a fascicular manner towards the membrana limitans. I have taken much pains in the investigation of their relations at the surface of the retina, and have arrived at the following results. If the inner surface of the retina be examined under a strong magnifying power, in its posterior half, where the fibrous bundles of the optic nerve are still very distinct, a peculiar marking will be observed between them, consisting of series of points, of minute stelliform figures, or of little streaks, which often (also in animals, as the Ox for instance) regularly converge towards each other from the bundles of fibres like the rays of a feather. If these structures are traced in vertical sections, it is easily seen that they are nothing but the extremities of the radiating fibres dipping down between the nervous bundles, and presenting a somewhat peculiar aspect. For, whilst in the deeper part of the retina they are simple pale fibres, of at most 0.0008 of a line in size, they are here so modified that some of them simply expand, and terminate in a triangular pale corpuscle, 0.0015-0.003 of a line in length and breadth (Fig. $303^{1} \mathrm{l}$ ), from the internal angles of which, one or two horizontal fibres are again given off; whilst the others, without expanding, end in a complete bundle of 5-9 or more fine fibres (Fig. $303{ }^{1} n$ ), which also turn to the sides and continue in the plane of the nervous expansion. What further becomes of these latter, innermost processes of the radiating fibre-system, I have not yet been fortunate. enough to observe, however zealously I have investigated the matter, and regret that the decision of this very important point in the anatomy of the retina must still be left in abeyance. The radiating fibres either actually terminate in the filaments observed by me on the surface of the expansion of the optic nerve, or they are continuous with the true fibres of that nerve, or at any rate are in connection with them. In a physiological point of view the latter supposition would, in any case, be the most plausible; and in support of it, it may be stated, that in the true fibrous bundles of the retina, together with the varicose nervetubules (Fig. $303^{3} a, b$ ), there are fibres of another sort (Fig. $303^{3} c$ ), which, although of equal size, agree in all respects with the radiating fibres in the absence of varicosities, and in their less straight or more serpentine and irregular course. It may be that these fibres are the direct continuations of the horizontal terminal processes of the radiating fibres, which subsequently, in their further progress towards' the 
optic nerve, acquire more and more of the character of common nervetubules, and follow a more direct course. However interesting this notion may be, according to which the "rods" would be the terminations of the optic fibres, the considerable difficulties attending it must not be concealed; among which not the least is the circumstance, that, although the "rods" and "cones" are certainly fifty times more numerous than the fibres of the optic nerve, yet the radiating fibres arising from the former, on their passage into the optic fibres, subdivide, and, as it must probably be assumed, are continuous with several of them-a difficulty, which might indeed be removed on the hypothesis, that a single optic fibre receives or gives off numerous radiating fibres, but is nevertheless of such a kind that I do not consider it advisable to proceed any further upon a basis unsupported by facts.

In spite of the obscurity which, from what precedes, still hangs over a very important point in the anatomy of the retina, physiology may nevertheless even at present draw some useful conclusions from the facts in our possession. In the first place, since the demonstration by $\mathrm{H}$. Müller and myself of its connection with the radiating fibre-system and the "granules," the bacillar layer appears in quite a different light from that in which it was previously held, and it is now obviously impossible to regard it, with Brücke, as a catoptric, reflecting apparatus. I look upon the "rods" and "cones," which may also be said to correspond in all chemical characters with the nerve-fibres of the retina, and the whole of the radiating fibre-system of the retina, as true nervous elements; and venture at the same time to broach the bold supposition, founded upon a less established basis, which has been already thrown out, that the "rods" and "cones" are the true percipients of light, and that they communicate their condition to the fibres of the optic nerve, by means of the direct or indirect connection of their fibrous processes with the former, through which again the impressions are conveyed to the sensorium. That the optic-fibres in the nervous expansion of the retina, do not perceive light, appears to me to be proved by the circumstance: 1 , that the point of the retina, where those fibres alone, and no other elements of the retina, are found, viz. at the entrance of the optic nerve, is not sensitive to light; 2 , that the optic fibres are superimposed upon each other in such numbers, in almost every part of the retina, and above all in the neighborhood of the macula lutea, that it is impossible they should perceive light, inasmuch as each luminous impression, owing to the transparency of the fibres, must in any case always affect many of them, and consequently would of necessity give rise to confused sensations; and 3 , because the part of the retina in which there is no continuous layer of nerve-fibres on the inner surface, that is to say, the "yellow spot," is the most sensitive to luminous impressions. Under this notion, the import of the "rods," and their remarkable arrange- 
ment, would be intelligible, and the almost inexplicable correspondence in the size of the images of the smallest distinguishable interspaces between two objects, with the diameter of the "rods" and "cones," be placed in its true light. I consider it impossible to say anything with respect to the import of the other elements of the retina, for although the "granules" may be compared to minute bipolar ganglion-corpuscles, and their continuity with the radiating fibres is known, this affords as little ground for discussion as to their function, as is given by the knowledge of the fact that the large nerve-cells of the inner layers have numerous processes and probably free terminations.

The vessels of the retina are derived from the art. centralis retino, which enters the eye enclosed in the optic nerve, and begins to ramify from the centre of the colliculus nervi optici in 4 or 5 main branches. Lodged at first only beneath the membrana limitans, these vessels penetrate through the layer of nerve-fibres into that of gray nerve-substance, ramify in an elegant arborescent manner as far as the ora serrata, and pass by their terminal prolongations on all sides into a rather widemeshed network of very fine capillaries $(0.002-0.003$ of a line), which is lodged chiefly in the gray layer, but partly also in the expansion of the optic nerve. In animals, the veins commence with a complete circle, circulus venosus retince, at the ora serrata, accompany the arteries in single trunks, and converge to the vena centralis, which quits the eye together with the artery. No large vessels exist in the "yellow spot," where there are only numerous capillaries. I have never yet met with nerves on the retinal ressels, whilst on the outside of the larger vessels I have occasionally noticed traces of an accompanying fibrous tissue, approaching nearest to the embryonic, reticular connective tissue.

The radiating fibre-system of the retina, though known in its separate parts to older and more recent observers, had not been comprehended in its connection, and we are very greatly indebted to $H$. Müller for his accurate investigations into this important structure in animals of every class. I have repeated Müller's observations in the human eye, which was not investigated by him, and have been enabled to confirm in it all that he has stated, and in some respects to carry the observations further. The reason why the radiating fibre-system and the relation of the "rods" to it has hitherto remained unknown, is because no one except Bowman, so far as I am aware, has engaged in the indispensable study of vertical sections of the retina, and moreover, because no one had thought of applying chromic acid to that tunic, which has elsewhere in the nervous tissues been of such important service, and although I had particularly shown that the multipolar retinal cells were well preserved in it. If parts still fresh be taken for examination, in transverse sections and chromic acid preparations, very satisfactory views of the 
structures above described will be obtained, and it will excite surprise that such numerous fibres pervading the entire retina should have been hitherto overlooked. A wholly new investigation of the retina has commenced with what has been made known by Müller and myself, but it will still demand much time and pains before it can be conducted to any certain results. Future inquirers should take up particularly the relations of the radiating and optic fibres in the eye, also the point whether the latter subdivide in the retina, as is asserted by Hassall and Corti, and lastly, whether the nerve-cells are directly connected with the nerve-fibres (Corti) or not.*

$\S 228$. The crystalline lens is a perfectly transparent body, in relation by its posterior surface with the vitreous humor, and laterally with the termination of the hyaloid membrane, the zonula Zinnii; and in which are to be distinguished the lens, properly so termed, and its capsule.

The capsule of the lens consists of two elements-the proper capsule and the epithelium. The former is a perfectly structureless and transparent, highly elastic membrane, enclosing the lens on all sides, as if moulded to it, and parting it from the neighboring structures. If the lens with its capsule be placed in water, the latter becomes considerably distended by imbibition, whence it is apparent, that membranes of that kind, notwithstanding their homogeneous structure, are yet very permeable, so that the nutrition of the non-vascular lens is provided for without difficulty, by means of materials penetrating from without. The lenticular capsule, measuring in its anterior wall, $0.005-0.008$ of a line, and posteriorly to the attachment of the zonula Zinnii, where it is abruptly thinned, not more than $0.002-0.003$ of a line, may be readily torn, punctured, or incised, whilst it offers considerable resistance to a blunt instrument. If an uninjured capsule be punctured, it contracts

* [Profs. Kölliker and Müller (vid. Comptes. rend. tom. xxxvii., Sept. 1853) have since been able by further researches, to confirm their views on the structure of the retina. They - state also, that they have succeeded in tracing in the human retina the direct connection of the nerve-fibres with the nerve-cells-a fact first noticed by Corti in the Ruminants, and to which they attach extreme physiological importance: The radial fibres proceeding from the "rods," Kölliker observed frequently to unite into fasciculi, which was not the case in those derived from the "cones." At the yellow spot, he found the nerve-cells to constitute a thick layer, formed of from 9 to 12 rows of cells; but both he and Müller deny most strongly the presence of nerve-fibres in this situation, although their existence has been quite lately again positively affirmed by Hannover (Zeitschrift für wiss. Zool. B. V. H. 1, 1853).

The physiological conclusions which Kölliker and Müller deduce from these more recent anatomical observations are, in the main, the same as those above expressed. They seem, however, less inclined to view the "rods" and "cones" as the only percipients of light, but regard them more as conductors of the luminous impressions to the nerve-cells of the retina. These they consider to form a true ganglion, capable of perceiving the light, the expansion of the fibres of the optic nerve merely serving as a means of communication with the sen. sorium.-DaC.] 
to such an extent, owing to its elasticity, that the lens not unfrequently escapes spontaneously. In its micro-chemical reactions the capsule of the lens behaves exactly like other transparent membranes, except that, according to Strahl ("Archiv f. phys.," Heilk., 1852) it would appear to be dissolved by boiling in water. The epithelium of the capsule is placed, not on the outer surface, as Brücke states, but on the inner, towards the lens, lining the anterior half of the capsule with a single layer of beautifully clear, polygonal cells, of $0.006-0.01$ of a line, with round nuclei. After death its elements are readily separated, expand into transparent spherical vesicles, many of which burst, and together with a few drops of aqueous humor which have penetrated into the interior, constitute the so-termed aqua Morgagni, which during life, when the epithelium is accurately applied to the surface of the lens, does not exist at all.

The lens itself consists entirely of elongated, flat, hexahedral elements, $0.0025-0.005$ of a line broad, and 0.009-0.0014 of a line thick, of a perfectly transparent aspect, very flexible and soft, and having a considerable degree of toughness, which have usually been described as the fibres of the lens, although they are nothing more than thin-walled tubes with clear, viscous, albuminous contents, which, when the tubes are torn, escape from them in the form of large irregular drops, and consequently might suitably be described as the tubes of the lens. As concerns the microscopic characters of these bodies, they are distinguished by the circumstance of their becoming opaque and more distinct in all reagents by which albumen is coagulated; consequently, reagents of that kind, particularly nitric acid, alcohol,

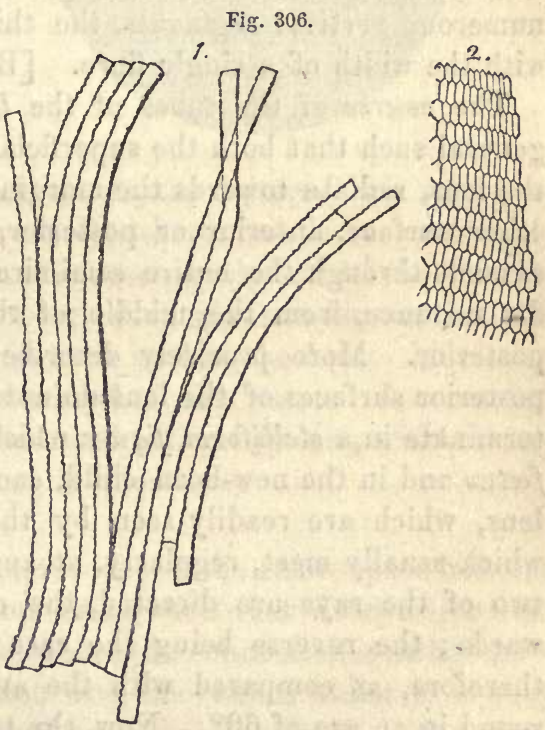
creasote, and chromic acid, are especially suitable for the investigation of the lens; but in caustic alkalies they are quickly dissolved, and they are also speedily attacked by acetic acid. The union of the tubes which are more solid, slender, and opaque in the more compact inner layers of the lens-the so-termed nucleus-than in the softer external portions, is brought about simply by their apposition. They invariably

FIG. 306.-Fibres or tubes of the lens. 1, from the Ox, with slightly toothed borders; 2 , transverse scction of the lenticular tubes of Man.-Magnified 350 diameters. 
lie with their sides parallel to the surface of the lens, regularly interdigitating with each other by their acute borders, so that, as is shown in Fig. $306^{2}$, in the interior of the lens, each tube is surrounded by six others, and their transverse section presents the aspect of a wall built up of hexagonal bricks. As the edges and corresponding surfaces of the tubes are usually somewhat uneven, or even toothed (in animals, particularly Fishes, beautifully so) their lateral union is rendered more intimate than it is between their border surfaces, and on this account also the structure is more readily torn into lamellæ in the direction of the surfaces, than into vertical plates in that of its thickness. For the same reason, also, a lamellar structure may be assigned to the lens, as is commonly done, seeing that it is constituted of concentric laminæ like an onion, only it must not be forgotten that these laminæ are not regularly defined layers, and never consist of a single stratum of tubes, and, moreover, what may prove of great physiological importance, that the elements of the lens are, properly, still more regularly disposed in the direction of its thickness, so that, throughout the lens, they cover each other, and the datter might be regarded as consisting of very numerous vertical segments, the thickness of which would correspond with the width of a single fibre. [Bowman, l. c., p. 69.]

The course of the tubes of the lens in the separate lamellæ is in general such that both the superficial and the deeper, in the centre of the lens, radiate towards the margins, and then curve round upon the other surface, anterior or posterior, but in such a way that no fibre extends through the entire semi-circumference of the lens, or reaches, for instance, from the middle of the anterior surface to that of the posterior. More precisely described, the tubes on the anterior and posterior surfaces of the lens do not proceed exactly to the middle, but terminate in a stelliform figure which exists in that situation. In the foetus and in the new-born child, each of these stelliform figures of the lens, which are readily seen by the naked eye, presents three rays, which usually meet, regularly, at angles of $120^{\circ}$; in the anterior star, two of the rays are directed, the one upwards and the other downwards; the reverse being the case with the posterior "star," which, therefore, as compared with the anterior, appears as it were turned round in an arc of $60^{\circ}$. Now, the tubes arising from the middle of the anterior "star" extend, on the posterior aspect, only as far as the extremities of the three rays; and, on the other hand, those commencing from the posterior pole do not reach the middle of the anterior. Similar conditions obtain in all the tubes situated between these two points, so that none of them reach entirely round; and all the tubes in a layer are of equal length. Now precisely the same thing also exists in the "nucleus" of the lens in the adult, whilst in the superficial lamello, and on the surface itself, we observe a more complex "star," 
with from nine to sixteen rays of various lengths, and rarely quite uniform, among which, however, three principal rays may be distinguished. The course of the fibres is consequently rendered more complex, and the rather so, because fibres attached to the sides of the rays converge in an arched manner, so that the latter appear, as it were, feathered, or form whirls (vortices lentis); but, notwithstanding this, the course of the fibres remains essentially the same in all respects as that above described, inasmuch as in this case also the anterior and posterior "stars" do not correspond with each other, and no fibre

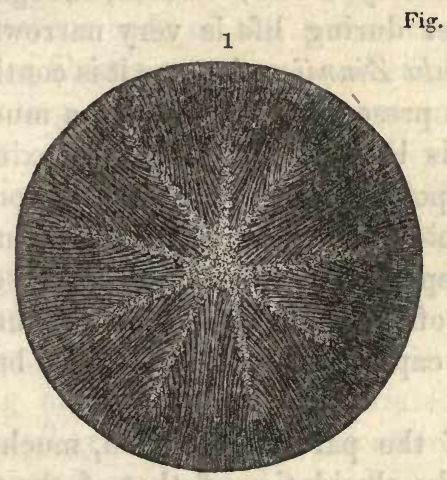

Fig. 307.

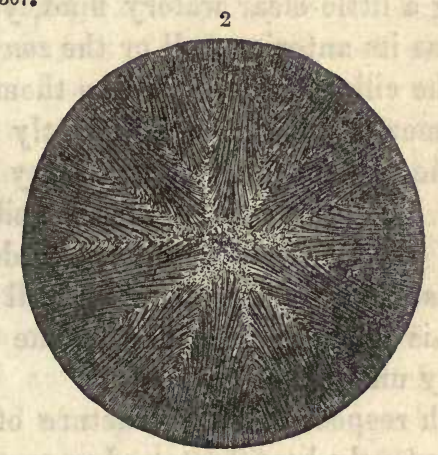

extends from the one pole to the other. In the "stars," the substance of the lens is not formed of tubes as elsewhere, but is in part finely granular, in part homogeneous; and, consequently, since the "stars" involve all the layers, three or more, vertical, non-fibrous lamellæe ("central planes," Bowman) exist in each half of the lens. Moreover, the tubes themselves in the neighborhood of the "stars" become less distinct, are gradually fused together, and ultimately lost, without any line of demarcation, in the substance in question.

$\S 229$. The vitreous body or humor, occupies the entire space between the lens and the retina; its relations being such that excepting at the point of entrance of the optic nerve, where the connection is rather more intimate, it is only in loose apposition with the retina, whilst it is very closely united with the corona ciliaris and the lens itself. The membrane enclosing the vitreous body, or the hyaloid membrane, which behind the ora serrata constitutes an extremely fine and delicate, perfectly transparent membrane scarcely perceptible under the microscope, in front of that part becomes rather firmer (Fig. $296 t$ ), and is continued to the border of the lens as the pars ciliaris hyaloidece s. zonula Zinnii ("suspensory ligament of the lens," Bowman [and Retzius]), where it

Fra. 307.-Lens of the adult, after Arnold, to show the "star." 1, anterior aspect; 2, posterior. 
becomes blended with the capsule of that body. In doing this it splits into two lamellae, a posterior $(v)$, which is blended with the capsule of the lens a little behind its border, and cannot be traced further, so that beyond that point the posterior wall of the lenticular capsule and the vitreous body are directly in contact; and an anterior $(u)$ connected with the ciliary processes,- the zonula in the more restricted sense,which is attached to the capsule of the lens a little in front of its margin. Between the two lamelloe and the border of the lens, there is left a space surrounding the latter in an annular manner, and, in a transverse section, of a triangular form-the canal of Petit; - which, though containing a little clear watery fluid, yet during life is very narrow, inasmuch as its anterior wall or the zonula Zinnii so long as it is continuous with the ciliary processes, like them presents the aspect of a much plicated membrane, and consequently is brought into close approximation with the posterior wall, at as many points as there are ciliary processes. These folds, however, are still visible where the zonula, quitting the ciliary processes, is continued independently upon the border of the lens, as a part of the posterior wall of the posterior chamber of the eye ; and it is therefore attached to the capsule, not in a straight but in a slightly undulating line.

With respect to the structure of the parts in question, much pains have recently been bestowed upon the elucidation of that of the proper vitreous body; although it cannot be asserted that at present the truth has been arrived at. Brücke's view, according to which the vitreous body, like an onion, consists of concentric lamella parted by a gelatinous fluid, was contradicted by Bowman, who has shown, that the concentrated solution of acetate of lead used by Brïcke for the exposition of these lamellce, produces the appearance of lamination not only on the superficial surface but also on that of any section whatever, but without rendering true lamellæ manifest. Hannover's opinion, according to which, after treatment of the vitreous body with chromic acid, numerous dissepiments are found in it, running from the surface towards the axis, so that in a vertical section a number of "rays" are perceptible proceeding from the central point, and the whole resembles an orange laid open, appears to have more in its favor, inasmuch as, at any rate, the vitreous body of the new-born child, according to Bowman (Lectures, $p$. 100, and Fig. 5, p. 97), when treated with chromic acid, very distinctly exhibits an areolated aspect of the kind described, but it should be remarked that from the same author's observations, the conditions are widely different in the eye of the adult, for, in this instance, in chromic acid preparations, a few concentric lamelloe are found externally, to which succeed very irregular radiating septa, and lastly an irregular central cavity. If to this it be added that these lamelloe formed by chromic acid also cannot be demonstrated as true membranes, and that 
in the fresh vitreous body no trace of them is perceptible, the appearances produced by this second reagent can likewise not to be considered as proving much.

A more correct notion of the constitution of the vitreous body would appear to be derivable from the study of its development. It has been long known that the vitreous body in the foetus has vessels on its surface and in the interior; it might thence have been concluded that some tissue for the support of these vessels must also exist-but no one has, till recently, sought farther information with the aid of the microscope. Bowman was the first (1. c., p. 100 and p. 97, Fig. 7) to remark, that the vitreous body of the new-born child exhibited a very distinct and peculiar fibrous structure, consisting in fact of a close network of fibres, presenting at nodular enlargements [where the fibres join] "minute nuclear granules resembling oil-particles, but not soluble in ether;" the whole exhibiting "a peculiar fibrous texture not at all unlike that of the enamel pulp" in the foetal tooth-sac, that is to say, to its gelatiniform connective tissue. This agrees pretty nearly with what Virchow has recently found. According to the latter author, the vitreous body of a foetal Pig, 4 lines long, consists of a homogeneous substance, containing mucus, and faintly striated at distant spots, in which round nucleated granular cells lie, scattered at regular distances apart. This substance is surrounded by a delicate membrane, with very elegant vascular networks and a fine fibrous areolated mesh-work containing nucle $i$ at the nodular intersections, and also enclosing in its meshes a gelatinous mucus with rounded cells. Consequently, and also because he found mucus in the vitreous body in the adult, Virchow believes that the tissue of the foetal corpus vitreum should be ranked with what he terms "mucous tissue," corresponding with my gelatinous connective tissue ( $(24)$; and that it might be assumed that, in the course of development, the structure may so change that the cells disappear and the intercellular substance alone remains. As regards my own views, I can only partially agree with these authors. In the vitreous body of the human fotus and of that of animals, I can perceive nothing but a homogeneous matrix containing mucus and numerous round or elongated granular, nucleated cells, $0.004-0.01$ of a line in size, dispersed in it at pretty regular distances of $0.01-0.02$, or even 0.03 of a line apart. It is true I have also noticed stelliform anastomosing cells also, but only on the outer side of the hyaloid membrane, and which when the vessels once began to convey blood, could be easily shown to communicate with them, and to be, in fact, capillaries in process of development. Of membranes, such as Hannover describes, I have never seen any certain indication with the microscope; and yet such membranes, did they exist, would incontestably be as easily recognized, where they are folded, as the excessively delicate hyaloid membrane itself. In the 
vitreous body of the adult, of the previous conditions there was usually nothing left but the homogeneous matrix, the cells having disappeared; I noticed the latter, however, in many instances, though rare and indistinct, particularly in those parts of the organ bordering upon the lens and the hyaloid membrane in general. From these observations I conclude that the vitreous body, at an early period probably presents a sort of structure most nearly approximated to embryonic cellular tissue, but that subsequently all trace of such a structure is normally lost, and it consists merely of a niore or less consistent mucus.

Zonula Zinnii.-At the ora serrata, the hyaloid membrane comes into intimate contact with the retina, and the latter again with the choroid, so that it is extremely difficult to display the relations of the above-noticed zonula Zinnii. If this part be exposed from the outside, some of the pigmentum nigrum of the ciliary processes is almost always left adherent at certain spots and often over a considerable extent. If the places where this is not the case are examined, it is evident that the outermost lamina of the zonula is a grayish layer, extending exactly so far as the processus ciliares are in connection with the zonula, and ceasing anteriorly in a slightly toothed, irregular border. Under the microscope there are always visible in this layer, even when the zone appears quite clear, and particularly towards the front, a good many rows of pale pigment-cells belonging to the choroid, which are situated principally in the folds in which the processus ciliares were contained, and give the entire zone a striped aspect. On the inner side of this lies a single layer of clear, frequently very pale, polygonal, nucleated cells, of $0.006-0.012$ of a line in size, but which is never entire, being always partially removed, together with the ciliary processes, on which Henle and others have noticed it. This layer of cells does not belong to the retina, as most authors assume, and still less to the hyaloid membrane, but to the choroid, and is nothing more than a stratum of cells not containing pigment, lying immediately upon the corona ciliaris, internally to the pigment (Fig. $296 w, w^{\prime}$ ); it does not, however, in any way appear to be a distinct epithelial lafer, but only the uncolored part of the pigmentary stratum, to which it would stand in the same relation as the colorless epidermis-cells to the colored, in dark skins. This colorless epithelium of the corona ciliaris, as I shall term these cells, is most distinctly shown on the ciliary processes, as a clear border sharply defined on the inner side, and often $0.006-0.008$ of a line broad, whose large, frequently shortly cylindrical cells, may usually be recognized without any trouble, and are always rendered distinct by acetic acid; whence it is evident that the boundary is composed only of them, and is not a special membrane. Posteriorly, this cellular stratum reaches as far as the ora serrata, extending anteriorly to the termination of the ciliary processes (Fig. $296 w^{\prime}$ ), and on either side it is con- 
tinuous, without any line of demarcation, with the pigmentary layer, the clear cells being gradually replaced by cells containing pigment.

Excepting these cells, the zonula is a thin, transparent but tolerably firm membrane, stretching from the ora serrata retince as far as the border of the lens, and appearing to be a continuation of the hyaloid membrane. It consists of peculiar, pale fibres, already very well characterized by Henle, resembling certain forms of reticular connective tissue, except that they are more rigid, usually present no distinct fibrils, and are less swollen in acetic acid. They commence, very fine, a little behind the ora serrata retince on the outer side of the hyaloid membrane, although most intimately connected with it, in part like fibrils of connective tissue, then run forwards, forming a layer at first more lax, and becoming more and more dense, and increasing in thickness (up to 0.004 or even 0.01 of a line and more), with numerous divisions and anastomoses, and for the most part parallel with each other, until they constitute, at the free portion of the zonula, a perfect, continuous layer-though still containing a few isolated bundles, and are ultimately blended with the capsule of the lens. From the ora serrata to the commencement of the "canal of Petit," no hyaloid membrane besides the fibres of the zonula, can be any longer distinguished, whilst at the canal itself, where the substance of the vitreous body is separated from the fibrous layer, it is again furnished with a limitary membrane, only thinner than before, which constitutes the posterior wall of the "canal of Petit," and extends no farther than to the border of the lens, where it ceases as a special membrane, the vitreous body being most intimately united with the posterior lamina of the capsule of the lens.

M. Langenbeck, some years ago described, in the free portion of the zonula Zinnii, what he believes to be a muscular ring, and terms the musculus compressor lentis s. accommodatorius ("Klinisch. Beiträge zur Ophthalmol.," 1849, p. 66), of which I have been unable to perceive any indication. He has probably confounded the zonular fibres with such a structure.

\section{B. ACCESSORY ORGANS.}

$\$ 230$. The eyelids are supported by the so-termed tarsal cartilages (tarsi), thin, semilunar, flexible, but tolerably elastic plates, attached internally and externally by the fibrous, tarsal ligaments,-and belonging, as far as their structure is concerned, to the solid, fascicular, connective tissue, though occasionally containing a certain number of minute cartilage cells. These plates, $0.3-0.4$ of a line thick, the fibres in which chiefly run parallel with the borders, are covered externally by the orbicularis palpebrarum and the integuments, and internally by the con- 
junctiva. The skin is here very thin (1-5-1-8 of a line), with scanty, subcutaneous connective tissue containing no fat, a delicate cuticle, $0.055-0.058$ of a line thick, and short papilloe (of $0.060-0.066$ of a line); but it is furnished throughout with minute sudoriparous glands (of 1-10-. 1-12 of a line) and almost invariably, with numerous minute hairs (frequently, with contiguous sebaceous glands, but whether always so provided I do not know). At the edges of the palpebrce these hairs are more considerably developed and constitute the eyelashes, which are also furnished with sebaceous follicles. Agreeing in all respects, in structure and secretion, with the sebaceous glands, the Meibomian glands, nevertheless, differ somewhat in form. They are imbedded in the tarsal cartilages, to the number of from twenty to forty, in the form of elongated, white, delicate, parallel, racemose follicles, disposed in such a direction that the long axes of the glands, cut those of the tarsal cartilages at a right angle. Each of these glands which are visible at once upon inverting the eyelid, and do not occupy the entire width of the tarsi, consists of a straight excretory duct, $0.04-0.05$ of a line wide, which at its orifice on the inner edge of the free palpebral border, is lined with common epidermis, including the horny and the mucous layers, and more internally presents the usual structure observed in the sebaceous glands. This canal, throughout its length, is beset with round or pyriform, shortly pedunculated, gland-vesicles, $0.04-0.07-0.1$ of a line in diameter, either isolated or aggregated several together, in which, in a mode similar to that already described in speaking of the sebaceous glands ( $\$ 74)$, a constant production of spherical, adipose cells, $0.005-0.01$ of a line in size, takes place; the cells differing from the sebaceous cells, only in the circumstance that the oil-drops contained in them do not usually run together into a single large drop, but remain separate. As these cells advance towards the excretory duct they gradually break up into a whitish pultaceous substance composed of oil-drops, and form the so-termed lema s. sebum palpebrale. The orbicularis palpebrarum, constituted of transversely striped, though rather slender and pale muscular fibres, lies immediately beneath the skin, its stratum internum being separated from the tarsi by a layer of lax, and to some extent adipose connective tissue, so that it may be readily raised into a fold together with the integuments. It is only towards the free margin that this muscle is more closely attached to the tarsi, and there presents a bundle of fibres situated at the very verge of the eyelid, which is parted from the rest of the muscle by the follicles of the cilia-the so-termed ciliar muscle (musculus ciliaris, Riolan).

The conjunctiva (a mucous membrane), commences at the free palpebral margin, as an immediate continuation of the external integument, lines the posterior surface of the eyelids, and is then reflected upon the eyeball, investing the anterior part of the sclerotic and the entire cornea. 
The palpebral conjunctiva is a reddish membrane, $0 \cdot 12-0.16$ of a line thick, very intimately connected with the posterior surface of the tarsi, and consisting of a dense layer of connective tissue corresponding to the cutis, $0.08-0.11$ of a line thick, and of a squamose epithelium, 0.04 of a line in thickness, containing deeper cells of an elongated form, and more superficially, polygonal, slightly flattened, nucleated, and (so far as I have seen in Man) non-ciliated cells. Papilloe also, similar to those of the cutis, are met with in the palpebral conjunctiva, some of which are smaller and more cylindrical, whilst others, particularly towards the point of reflection of the membrane where it is generally thicker, are larger (as much as 1-10 of a line long), more verrucose and fungiform. At the line of reflection itself, Krause describes minute racemose mucous glands, 1-5-1-26 of a line in size, but which do not always exist. The conjunctiva sclerotico, is white, less dense and thinner than that of the lids, tolerably rich in fine elastic fibres, and loosely and movably attached to the sclerotic by an abundant submucous connective tissue, containing more or fewer fat-cells. Papilloe are wholly wanting in this portion, except at the line of reflection, as well as glands, whilst the epithelium is well developed, as on the conjunctiva corneos, and beneath it there is not unfrequently an outermost layer of the proper mucous membrane, in the form of a very distinct structureless, narrow seam. At the margin of the cornea, particularly in elderly persons, the conjunctiva scleroticce forms a slight annular elevation $\frac{1}{2}-1$ line broad, - the annulus conjunctivce, which encroaches a little upon the cornea at the lower, and especially at the upper border. The corneal conjunctiva has been already described, and it only remains to notice the plica semilunaris, or the third palpebra at the inner canthus of the eye. This is a simple duplicature of the sclerotic conjunctiva, which rises in front into a conical elevation - the caruncula lachrymalis, - in which are seated about a dozen fine hairs, surrounded by an equal number of rosette-like sebaceous follicles 1-5-1-4 of a line in size, encompassed by numerous fat-cells.

The lachrymal apparatus consists, in the first place, of the lachrymal glands-a certain number of larger and smaller compound raeemose glands, disposed in two groups-termed the superior and inferior lachrymal glands, and in the structure of the larger and smaller lobules, as well as in the rounded gland-vesicles, $0.02-0.04$ of a line in diameter, precisely resembling the salivary and mucous glands $(\$ \S 134,135)$. The excretory ducts of these glands, 6-12 in number, perforate the conjunctiva in the fold between the outer part of the eyelid and the globe of the eye; they are excessively fine canaliculi, composed of connective tissue, with a few nuclei and elastic fibrils, and of a cylindrical epithelium. It is extremely difficult to display these canals in man, whilst in animals (the $\mathrm{Ox}$ for instance) they are easy of demonstration. The passages by which the tears are conveyed away from the eye are con- 
structed in the same simple manner as the excretory ducts of the lachrymal glands, consisting merely of a dense connective tissue with numerous networks of fine elastic fibres, particularly abundant in the lachrymal canals, which appears to be a continuation of the mucous membrane of the nose and of the conjunctiva, and of an epithelium which, in the lachrymal canals, is of the squamous kind, and in the lachrymal sac and nasal duct is furnished with vibratile cilia, as in the cavity of the nares. The muscles of the globe of the eye and of the eyelids, as well as the musculus Horneri, are all composed of transversely striped muscular fibres, and together with their tendons, present no differences from those of the trunk and extremities. The fascia bulbi oculi s. Tenoni is a true fibrous membrane, and the trochlea is formed principally of dense, connective tissue, in which only a few cartilage cells can be seen.

The vessels of the organs described in this section present little worthy of remark. Excepting those of the muscles and skin, they are most abundant in the palpebral conjunctiva, in which they chiefly enter the papilloe, and in the next place in the lachrymal glands and the caruncula lachrymalis. The sclerotic conjunctiva also contains numerous vessels, and the Meibomian glands within the tarsi are also surrounded by a few. Except in the skin of the eyelids, lymphatics have only been demonstrated by Arnold in the conjunctiva scleroticoe, where they form, at the border of the cornea, a closer, and more externally a looser plexus, passing outwards in several small trunks. The palpebrce and conjunstiva are everywhere well supplied with nerves, but their relations have been minutely examined only in the conjunctiva. In this membrane, in Man, I have found terminal plexuses as in the external integument, with numerous divisions of fibres, $0.001-0.006$ of a line thick, extending up to the margin of the cornea, together with pretty clear indications of loops and free terminations. Besides which, in one instance, there were presented, towards the palpebral conjunctiva, peculiar "nerve-coils," $0.02-0.028$ of a line in size, into which a single nerve-fibre usually entered, whilst 2-4 were given off from it (vid. "Mik. Anat." II. 1, p. 31, Fig. 13, $A, 3$ ). The relations of the nerves of the lachrymal apparatus are entirely unknown.

§ 231. Physiological remarks. - The eyeball is not developed from a single point as a whole, but arises from the conjunction of formations, proceeding on one side from the central nervous system, on another from the skin, and, thirdly, from the parts lying between the tro. In the Chick, the primitive ocular vesicles arise before the commencement of the second day, from the primitive cerebral vesicle or the anterior cerebrum, in the form of two protrusions, at first sessile, but afterwards having a hollow peduncle-the rudiment of the optic nerve. At the 
beginning of the third day, the formation of the lens commences, from the skin of the face covering these vesicles, by the thickening on the inner aspect, and inversion of the epidermis, in consequence of which the anterior wall of the primitive ocular vesicle is also inverted, and becomes applied to the posterior wall, so that the cavity of the vesicle is wholly obliterated. Now, at first, this secondary ocular vesicle encompasses the lens, which in the mean time has been separated by constriction from the epidermis, and comes into exact apposition with it beneath, like a cup; subsequently, however, the vitreous body is developed between the two, in a special new cavity. How the latter is formed has not yet been ascertained, although, as Schöler observes, it is most probable that it also grows in from the skin,-in fact, from the region below and behind the lens, - and participates with the latter in the inversion of the primitive ocular vesicle. According to Remak, the retina is formed from the inner, thicker wall of the inverted or secondary ocular vesicle, and from the outer and thinner, the choroid, from the anterior border of which the iris is not produced till afterwards. The sclerotic and cornea are applied from without upon the eyeball thus constituted, the former being to some extent a production of the skin.

An interesting phenomenon is presented in the vessels existing in the fotal eye, even in the transparent media. The vitreous body, on its outer surface, between the hyaloid membrane and the retina, presents a tolerably wide-meshed vascular plexus, which is supplied by branches of the arteria centralis retince, given off from it at its entrance into the eye, and anteriorly, at the border of the lens on the zonula Zinnii, forms a vascular circle, the circulus arteriosus Mascagnii, from which again vessels are given off to the membrana capsulo-pupillaris, presently to be described. Besides this, a special arteria hyaloidea, also derived from the central artery of the retina, runs in the so-termed canalis hyaloideus, in a straight line through the vitreous body, to the lens, and ramifies in the most elegant arborescent manner, at very acute angles, in a membrane closely applied to the posterior wall of the lenticular capsule. This is nothing else than a portion of an external vascular capsule, which at first very closely surrounds the lens, and in its anterior wall is supplied by the continuations of the hyaloid artery, coming round the border of the lens towards the front, with which branches of the circulus arteriosus Mascagnii and of the anterior border of the uvea are connected. Afterwards, when the lens retreats from the cornea, with which it is, at first, in close apposition, and the iris buds out from the border of the uvea, the anterior wall of the vascular lenticular capsule is divided into two portions: one central and anterior, which, arising from the border of the iris, and connected with that membrane by vessels, closes the pupil-the membrana pupillaris ; 
and another, external and posterior, extending backwards from the same points upon the border of the lens-the membrana capsulo-pupilaris. The latter becomes more and more distinct as the lens and aqueous chambers are developed and the lens retreats, until at last it represents a delicate membrane stretching across the posterior chamber. The venous blood from all these parts is returned through the veins of the iris, and from the outer surface of the vitreous body, also through those of the retina, and perhaps through a vena hyaloidea, said to take the same course as the artery, but of the existence of which many authors doubt, and which I have never myself seen. With respect to the genetic import of the vascular capsule, nothing has as yet been ascertained. I find it to be composed of a homogeneous tissue, with a few scattered cells, and regard it as a structure corresponding to the cutis, which, in the formation of the lens, is detached from the skin, together with a portion of the epidermis, and remains in the eye. The vitreous body, then, may be understood as modified subcutaneous connective tissue, - a supposition not at all incongruous with the observations above adduced, and the more so, because, as I have shown (\$24), all the subcutaneous connective tissue of the foetus is at one time perfectly gelatinous, and, like the enamel organ which also belongs to the same tissue in specie, strikingly resembles the vitreous body in aspect and consistence.

Concerning the histological development of the eyes, the following only need be remarked. At an early period they consist in all their parts of formative cells of uniform size, which, in process of time, are metamorphosed into the various tissues. In the fibrous coat, in the second and third month, the cells are developed, in the mode already described ( $\$ 24)$, into connective tissue, and at the same time the distinction is set up between the cornea and sclerotic, which are at first, externally, exactly alike, and constitute only a single membrane. In the uvea the cells are for the most part employed in the formation of vessels; another portion goes to the formation of the inner and outer pigment-layers, pigment-granules being deposited in them at the commencement of the third month, whilst another is transformed into muscles, nerves, the epithelia and connective tissue of these membranes. The development of the nerve-cells and of the so-termed "granules" from embryonic cells, may be readily traced. I have observed the same thing also with respect to the "cones;" and I think that, in the Frog, it may be assumed with respect to the "rods" likewise, that they are nothing but elongated cells; whilst in the Mammalia, the formation of the "rods," and of the nerve-fibres themselves, has not yet been traced. The lens, lastly, is originally composed entirely of cells, which, in course of time, are transformed into the tubes. The precise nature of the processes attending these changes has not yet been 
investigated, although I agree with $\mathrm{H}$. Meyer in the conclusion, that since the tubes, both in the foetus and child, present only a single nucleus, each of them is developed out of a single cell. These nuclei taken as a whole, constitute a thin layer, extending from the borders of the lens, through the middle of its anterior half, and slightly convex in front ("nuclear zone," Meyer); the nuclei being smaller in the interior portions, and as it were in progress of solution, whence it may certainly be concluded that the lens increases by the apposition of thin layers from without. The formative cells of the tubes of the lens are those which exist on the anterior half of the capsule and the starting point of the formation of the lenticular elements, according to my observation, is the entire anterior surface and the border of the organ. Nuclei are visible in the tubes even in the lens of the adult, as was known to Harting, though only at its margin.

With respect to the vessels of the foetal eye, Dr. Thiersch has quite recently communicated to me a mass of interesting details, accompanied by beautiful injections, to which I shall refer in the concluding part of my "Microscopical Anatomy."

Investigation of the visual organ.-The fibrous tunic of the eye should be examined in the recent condition, and in moistened sections of dried preparations, which latter, especially of the cornea and at its point of transition into the sclerotica, afford very useful information. If, after the removal of the vitreous body and lens, the iris and choroid are dried, their connection with each other and with the fibrous tissue may be studied. In order to view the nerves and vessels of the cornea, the latter is removed by a circular section in the recent eye, together with the margin of the sclerotic, the whole is divided into three or four segments, which, in order that they may lie the better, have little incisions made into them around the edge, are moistened with the aqueous humor, and covered with a thin plate. The nervous trunks, which are here usually opaque, are then sought for at the border of the cornea, first with a low power, and afterwards traced under a higher. The nerves are beautifully displayed in the eye of the Rabbit, where I can perceive their trunks with the naked eye, though they may usually be readily found in other eyes also, but are always traced with difficulty towards the centre. If the epithelium is cloudy, it must be removed by caustic soda, which at first does not affect the nerves. The vessels under these circumstances are usually full of blood, and consequently present no difficulties. The corneal epithelium is visible on the surface in sections of dried preparations, and is very well shown when the surface is scraped. The "membrane of Demours" is very distinct in sections, and frequently its epithelium also; otherwise the latter is well seen on the surface, and in detached shreds of the membrane. The passage of this membrane into the ligamentum pectinatum of the iris, may be seen 
in sections, and by careful dissection. In the latter case, the inner wall of the "canal of Schlemm" should be carefully removed, together with the iris and choroid, and an attempt made to raise from it portions of the membrana Demoursiana, which is not unfrequently successful. The uvea offers no difficulty. The pigment-cells of the stroma, with their processes, and the inner pigment are readily seen; the latter at the margin of folds, and in carefully detached portions. For the investigation of the ciliary muscle, a fresh eye is requisite, as its elements very soon become unrecognizable. The muscles of the iris should be studied in a blue eye, and best in that of a child, after removal of the posterior pigment; and also in the eye of a white Rabbit, in which the sphincter pupillo may be readily seen without farther trouble on the application of acetic acid. The same preparation should be employed in order to examine the nerves of the iris, but a perfectly fresh eye and a dilute solution of soda are indispensable. The retina should be examined in the recent state, on the surface, in vertical sections, and at the edges of folds, moistened with aqueous humor, and without any covering glass; and also with the aid of slight compression, and by the teasing out of the tissue. Chromic acid preparations are very important in the study of this structure. This reagent, it is true, affects the "rods" to some extent, but preserves the other parts so much the better, and without its aid Müller and I should never have arrived at the results above stated, although Hannover, on account of its influence upon the "rods," erroneously considered it an unfit agent to employ. The most advantageous mode of applying it, is to treat a fresh retina at once with chromic acid, and to trace all the stages of its effect step by step. If the solution be much diluted, the elements are very little changed, and in particular may be easily isolated; and if more concentrated, sections through the retina may be prepared, without which no complete view of the structure of that tunic can be arrived at. I apply it by extending a portion of the retina upon an object-bearer, with a little chromic acid, in such a way that it should lie flat and not float. Extremely fine slices may then be taken by a sharp convex scalpel or razor from any sectional surface, by pressing downwards, which, with a little pains, may be done easily enough. It is as well, however, to guide the cutting scalpel by the handle of another held in the other hand, until the edge of the former is brought immediately over the border of the retina. When the nervous layers, which are very well defined from each other, have been studied in sections of this kind, which should be taken especially from the neighborhood of the macula lutea, as well as from other situations in the transverse and longitudinal directions, and which when useful necessarily exhibit only a few layers of the elements, they may be carefully teased out or rendered more transparent by soda, which last, however, is not generally of much use, since it makes the elements pale. The hyaloid membrane 
is posteriorly always very readily detached from the retina, together with the vitreous body, and may be recognized in every eye, in sections from the surface of that body, examined under the microscope, and, in folds, occasionally by the naked eye. The zonula Zinnii, on the other hand, in the recent eye, is always so covered by detached pigment and the colorless epithelium of the ciliary processes, and at its posterior border by the retina, that it cannot well be recognized in that situation, and almost only in its free, most anterior portion. In such preparations, also, after the removal to the greatest possible extent of the adherent parts by means of a hair pencil, pretty good views of it may be obtained, particularly if, in addition to the viewing of the external and internal surface of segments of the zonula detached from the vitreous body, and of preparations made by the teasing out of the structures, the borders of folds, especially of the inner surface, are also examined, which, with some care, may be obtained to the whole extent of the zonula, and of its points of connection with the retina. The zonula, in connection with the hyaloid membrane, is very beautifully and distinctly isolated from the retina and the cells of the ciliary processes, in half-putrid eyes, and in macerated preparations of the vitreous body; and preparations of this kind are especially adapted to show that the zonula is a part of the hyaloid membrane, as well as the mode of origin and course of its fibres. For the study of the zonular fibres I can also particularly recommend chromic acid preparations, in which they become quite opaque and glistening, almost like elastic fibres. The capsule of the lens and its epithelium present no difficulties. The tubes of the lens, when fresh, are very transparent, but in dilute chromic acid they are rendered quite distinct. Sections of the lens may be easily procured from preparations made in alcohol, or chromic acid, or from dried and hardened preparations, which may be rendered transparent again by means of acetic acid. The accessory organs of the eyes require no particular remark, unless, with respect to the Meibomian glands, it may be stated, that they are best seen in tarsi which have been cleanly dissected and treated with acetic acid and alkalies, and in longitudinal and transverse sections of similar preparations, dried.

Literature.-THE "EyE" AS A WHOLE: Valentin, in his "Repert.," 1836 and 1837, and "Handw. d. Physiol.," I.p. 748 ; S. Pappenheim, "Gewebelehre d. Auges," Berlin, 1842 ; E. Brücke, "Anat. Beschreib, d. menschlichen Augapfels," Berlin, 1847; W. Bowman, "Lectures on the parts concerned in the operations on the eye and on the structure of the Retina and Vitreous Humor," London, 1849 ; A. Hannover, "Bidrag til Ojets Anatomie, Physiologie og Pathologie," Copenhagen, 1850. ScleRoTrCA: M. Erdl, "Disquisit. anat. de oculo," I. "De m. scleroticâ," 
Monach., 1839; Bochdalek, "Ueber die Nerven der Sclerotica," in "Prag. Viertelj.," 1849, IV., 119. ConNEA: Kölliker, "Ueber die Nerven der Hornhaut," in "Mitth. d. naturf." Ges., in Zürich, 1848, No. 19; Rahn, "Ueber die Nerven der Hornhaut," in "Mitth. d. naturf.," Ges., in Zü̈rich, 1850, No. 45; Luschka, "Die Nerven der durchsichtigen Augenhaut," in "Zeitsch. f. rat. Med.," X., p. 20, and "Die Structur der serösen Häute des Auges," in "Str. d. serösen Häute," Tubingen, 1851 ; Strube, "Der normale Bau der Cornea," Diss., Wurzburg, 1851. Choroid AND IRIS : C. Krause, "Ueber die Pigmenthaut," in Müller's "Arch.," 1837, p. 33 ; E. Brïcke, "Ueber den Musc. cramptonianus u. d. Spannmuskel der Chorioidea," in Müll. "Archiv.," 1846; Bochdalek, "Beiträge zur Anatomie des Auges," "Prag. Viertelj.," 1850, I. Retina: G. Treviranus, "Ueber den Bau der Netzhaut," in his "Beiträgen," Bremen, 1835 and 1837 ; C. M. Gottsche, "Ueber d. Nervenausbreitung d. Retina," in Pfaff's "Mittheil. a. d. Geb. d. Med.," 1846 [and "Ueber die Retina im Auge der Grätenfische," in Müll. "Archiv," 1835, p. 457]; A. Michaelis, in Müller's "Archiv," 1837, p. 12, and "N. Acta," T. XIX., 1842; B. Langenbeck, "De retinâ observat.," Gott., 1836 ; R. Remak, "Zur mikrosk. Anatomie der Retina," in Müll. "Archiv," 1839; B. Lersch, "De retinæ struct. microsc.," Berolini, 1840; A. Burow, "Ueber den Bau der macula lutea," in Müll. "Archiv," 1840; F. Bidder, "Zur Anatomie der Retina," in Müller's "Archiv," 1839 and 1841; R. Hannover, "Ueber die Netzhaut," in Müller's "Archiv," 1840 and 1843, and "Recherches microsc. sur le syst. nerveux," Copenh., 1844; E. Brücke, "Ueber die physiologische Bedeutung der stabförmigen Körper," in Müller's "Archiv," 1844, p. 444, and "Anat. Untersuchung über die sog. leuchtenden Augen," ibid., 1845, p. 337 ; F. Pacini, "Sulla tessitura intima della retina," in "Nuovi Annali delle scienze naturali di Bologna," 1845; H. Müller, "Zur Histologie des Netzhaut," in "Zeitsch. für wissenschaft. Zool.," 1851, p. 234; Corti, "Beitrag zur Anatomie der Retina," in Müller's "Archiv," 1850, p. 274. [A. Kölliker, "Zur Anatomie und Physiologie der Retina," in "Verhandl, d. Würzb. phys. med., Ges." Bd. III., p. 216; H. Müller, "Bemerkungen über den Bau n. die Funktion der Retina," ib., Bd. III., p. 336, and Bd. IV., p. 96; also conjointly with Kölliker, in "Verhandl. d. Würzb. phys. med. Ges.," p. 3, Bands. III. and IV., and in "Comptes rendus de l'Academie des Sciences," tom. XXXVII., No. 13, Sept. 1853; A. Hannover, "Zur. Anatomie und Physiologie der Retina," in "Zeitsch. f. wiss. Zool.," Bd. X., p. 17, 1853.-Ev.] VITREOUS BODY : E. Brïcke, "Ueber den innern Bau des Glaskörpers," in Müll. "Archiv," 1843, p. 345, and 1845, p. 130 ; Hannover, " Entdeckung des Baues des Glaskörpers," in Müller's "Archiv," 1845, p. 467 ; W. Bowman (op. cit.), and in "Dublin Quarterly Journal," Aug. 
1845, p. 102 ; Virchow, "Notiz über den Glaskörper," in "Arch. f. path. Anatomie," IV., p. 468 [and V. 2, p. 298], and in "Verh. d. Würzb. phys. med." Gesellsch., II., p. 317. Lens : W. Werneck, "Mikr. Betracht. der Wasserhaut u. des Linsensystems," in Ammon's "Zeitsch.," Bd. IV. and V.; R. Hannover, "Beobachtungen über den Bau der Linse," in Müll. "Archiv," 1845, p. 478; Harting, "Histolog. Anteekennigen," 1846, pp. 1-7, and "Recherch. micrométriques." Development of the Ere: H. Schöler, "De oculi evolutione," Mitav., 1849, Diss.; Remak, in his large work, "Ueber Entwicklungsgeschichte," 1850-51; Gray, "On the development of the retina and the optic nerve," in "Phil. Trans.," I., 1850; Henle, "De membr. pupilJari," Bonn., 1832; Reich, "De membr. pupillari," Berolini, 1833; J. Muller, also Arnold, and Henle, on the "M. capsulo-pupill.," in Ammon's "Zeitsch.", II., p. 391, III., p. 37, IV., pp. 23 and 28. Besides which, see Arnold, "Org. sensuum."

\section{II.-OF THE ORGAN OF HEARING.}

$\S 232$. The auditory organ consists of the proper sentient parts with the expansion of the acoustic nerve, which are contained in the osseous substance of the labyrinth; and of special accessory apparatus, the external and middle ear, intended chiefly for the reception and conduction of the undulations of sound.

§ 233. External and middle ear.-The auricle (pinna) and the cartilaginous external auditory canal, are supported by the cartilage of the ear (cartilago auris), $\frac{1}{8}-1$ line thick, and while retaining the thick perichondrium very flexible, but otherwise extremely brittle, and the form of which is well known. This cartilage, in its more intimate structure, approaches the yellow or reticular cartilages, though it is distinguished by a considerable preponderance of cartilage-cells, 0.01 of a line in diameter, in the striated matrix. It is covered by the external integument, which, except in the lobule, contains no fat and on the concave side of the auricle is closely adherent to the cartilage, where it is characterized by a great abundance of glands. These are, in the first place, common sebaceous follicles, which are most developed in the concha and fossa scaphoidea, where they attain the diameter of $\frac{1}{4}-1$ line; secondly, minute sudoriparous glands of $1-16$ of a line on the convex side of the pinna; and, lastly, the ceruminous glands, already described $(\S 71,72)$, in the cartilaginous, external auditory canal itself. In the latter, the cutis measures 1-5-1-8 of a line in thickness, without the epidermis, which is 1-75-1-50 of a line thick; and presents, besides the glandulce ceruminosce, hairs and sebaceous follicles in a dense subcutaneous tissue, whilst in the osseous part of the passage it is very thin, 
contains no organs of any kind, and is blended very intimately with the periosteum of the canal.

The middle ear, in all its cavities, together with the ossicles, tendons, and nerves contained in it, is lined with a delicate mucous membrane, which, in the mastoid cells and on the ossicula auditûs, where it also forms the memb. obturatoria stapedis, and on the membrana tympani, is still more delicate than in the accessory sinuses of the nose, being thickest in the Eustachian tube. In the latter situation, its epithelium is of the squamose, ciliated kind, 0.024 of a line thick, whilst in the tympanic cavity it is changed into a thin, tessellated epithelium, composed of one or two layers, extending as far as the accessory cavities. The membrana tympani, which, according to Todd and Bowman, is furnished with a ciliated epithelium, consists of a middle fibrous plate, which, at the sulcus tympanicus, in connection with the periosteum of the cavity of the tympanum and of the osseous meatus and with the cutis lining the latter, commences in a dense tract of chiefly annular fibres-the so-termed annulus cartilagineus, - and further inwards is composed principally of slender radiating fasciculi converging towards the centre, where the handle of the malleus is inserted into this membrane, and in part reticulated, with undeveloped fine elastic fibres ("connective tissue corpuscles," Virchow). Externally, this membrane is covered by a delicate continuation of the epidermis of the external meatus, and internally is lined by a fine investment of the mucous membrane of the tympanum.

The ossicula auditûs are composed principally of spongy osseous substance, with a thin compact cortex; and their articulations and ligaments resemble, in miniature, those of other similar organs in all respects, even down to the cartilaginous layer, consisting of scarcely more than a single stratum. Their muscles, like those of the external ear, are transversely striped. The Eustachian tube has in part, as a foundation, cartilage which in structure approaches the true cartilages; usually, however, presenting a pale, fibrous matrix; and containing, in the cartilaginous portion, especially towards the aperture, numerous racemose mucous glands, of precisely the same conformation as those of the pharynx, in the mucous membrane of which organ that of the Eustachian tube is imperceptibly lost. The external ear is supplied with vessels and nerves, in the same manner as the external integuments. In the middle ear, the mucous membrane, especially of the walls of the tympanum, is highly vascular, as is also the Eustachian tube and the membrana tympani, in which latter the largest arteries and veins run along the manubrium of the malleus in the middle coat, constituting arterial and venous circles around the periphery of the membrane, and also ramifying abundantly in the mucous membrane. The nerves are derived principally from the ninth and fifth pairs, and upon the whole 
are scantily distributed in the mucous membrane and membrana tympani. With their terminations we are unacquainted, whilst it is known that the tympanic nerve contains numerous large ganglion-cells, either isolated or aggregated into small ganglia.

\$234. The inner surface of the vestibule and of the osseous semicircular canals is lined by an extremely thin periosteum, consisting of a rigid, finely-fibrous connective tissue, without elastic fibres, but with numerous nucle $i$ and in many respects resembling the forms of fibre met with in the inner wall of the "canal of Schlemm" in the eye. On the surface of this periosteum rests a tessellated epithelium in a single layer, with delicate, polygonal, nucleated cells of $0.007-0.009$ of a line, which, as well as its certainly not very numerous vessels, stands in relation to the peritymphas. aqua Cotunni, filling the osseous labyrinth. By the conjunction of the periosteum of the labyrinth and the lining of the tympanum, are produced the membrance tympani secundarioe, which, like the proper membr.tympani, are composed of a middle fibrous layer with vessels and scattered nervous filaments, and two epithelial layers.

The two sacculi and canals, contained in the interior of the vestibule and of the osseous semicircular canals, all essentially present the same structure. Their firm, transparent, and elastic walls, which are tolerably thick in proportion to the minuteness of the parts $(0.012-0.015$ of a line in the canals, and 0.016 in the sacculi), present, most externally, a membrane composed of reticulated, fine fibres, approaching very nearly to the outer colored layer of the choroid or the lamina fusca, like which it also occasionally contains irregular brownish pigment-cells. This is succeeded, by a transparent, glassy membrane, sharply defined, especially on the inner aspect, $0.004-$ 0.008 of a line thick, presenting in part a distinct, delicate, longitudinal striation, and on the addition of acetic acid always exhibiting a multitude of elongated nuclei, and which consequently cannot well be placed in the same class with the membrance proprice, the capsule of the lens, \&c., although it very nearly approaches them in its chemical reactions. The innermost layer, lastly, is a simple, readily disintegrated, tessellated epithelium, 0.003 of a line, with sornetimes larger, sometimes smaller $(0.004-0.008$ of a line) polygonal cells, lining all the spaces in question, and enclosing the so-termed endolymph. s. aquula vitrea auditiva, in which, in Fishes, Barruel has ascertained the presence of mucus.

The vessels of the membranous labyrinth are tolerably numerous, and are distributed in minute arteries and veins and abundant capillary net-

FrG. 308.-Transverse section of a semicircular canal, magnified 250 diameters : $a$, fibrous membrane with nucle ; $b$, homogeneous membrane; $c$, epithelium. From the Calf. 
works, on the fibrous membrane and vitreous tunic of these parts, being most abundant near the terminations of the nerves. Of these, we are acquainted only with that of the acoustic nerve, which, with the nervus vestibuli, supplies the three membranous canals and the elliptic sacculus, and, with a branch of the cochlear nerve, the round sacculus. In the semicircular canals, the nerves are distributed only on the ampulla, and, indeed, enter each, as Steifensand has shown, in an inversion or duplicature of the wall situated on the concave side of the canal, which appears, viewed on the inside, as a transverse projection extending through about one-third of the circumference. Within these folds, the nerves divide at first into two principal branches, which, diverging towards both sides, penetrate into the vitreous membrane of the ampulloe, where each of them breaks up into a rich bundle of smaller, frequently anastomosing ramuscules, which ultimately appear to terminate free, in fine twigs, composed of from two to ten primitive fibres, 0.001-0.0015 of a line thick. In the sacculi, the distribution of the nerves is the same, except that it occupies a larger space, and does not occur in a projection of the wall. In this situation, also, I think I have noticed free prolongations of the attenuated nerves, although it may be very possible, as Todd and Bowman point out, that they are continuous with very pale fibres, in which they ultimately terminate. In the situation of the nervous expansion, we find, in each of the sacculi, a sharply defined spot, as white as chalk, and readily seen by the naked eye, which is attached to the inner wall of the sacculus by a perfectly clear membrane, 0.01 of a line thick, and probably epithelial. These are the so-termed otoconia of Breschet, or otolithes, which are constituted of innumerable corpuscles, 0.0004-0.005 of a line long, and (in the largest) 0.001-0.002 of a line broad, of a rounded or elongated form, or distinctly pointed at each end, probably hexahedral prisms, suspended in a homogeneous substance. They are composed of carbonate of lime, and are said to leave a residuum of some organic matter; but this I have not succeeded in observing.

$\S 235$. Cochlea.-The canal of the cochlea, filled by the fluid of the labyrinth, is lined in both its scalce by a periosteum, here and there presenting a small quantity of pigment, and which is constituted precisely like that of the vestibule, and also partially invests the lamina spiralis ossea. An epithelium, 0.0005 of a line thick, with delicate, flattened polygonal cells, $0.007-0.009$ of a line in size, covers this ligamentous membrane, and is also continued upon the lamina spiralis membranacea, where its nature is, to some extent, altered. The most important part of the cochlea is the lamina spiralis, which, in its osseous zone, contains narrow-meshed anastomosing canals for the reception of the cochlear nerves, which canals, towards the free border of the lamina, coalesce so as to form a fissure-like cavity and consequently, in this situation, the osseous spiral lamina actually consists of two plates. 
The membranous zone, having a constant width of 0.2 of a line, is again

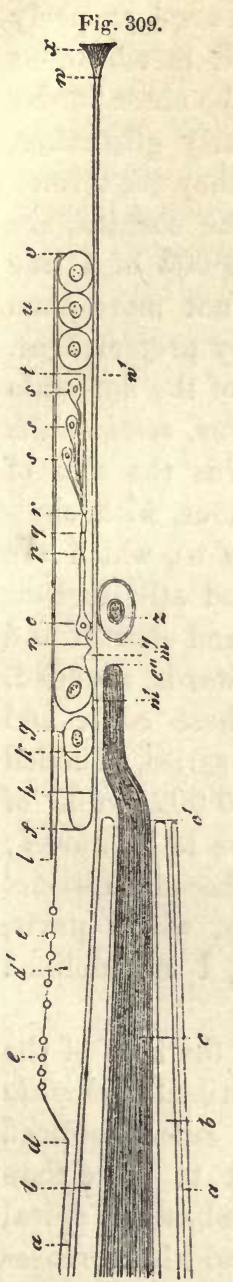
subdivided into a zona denticulata and a zona pectinata, the former of which constitutes about the inner two-thirds, and the latter the outer third of the breadth of the membranous lamina, and are both characterized by a great complexity of structure, the importance of which was first, in more recent times, pointed out by Corti (l. c.) (vid. Figs. 309, 310).

1. The zona denticulata $(d-v)$ may be again subdivided in to two portions, an internal, the habenula interna s. sulcata $(d-g)$, and an external, the habenula externa s. denticulata $(h-t)$. The former is developed at $d$, as an immediate continuation of the periosteum of the lamina spiralis ossea, and, in fact, only from that portion of it which looks towards the scala vestibuli, increasing in width and thickness from the commencement to the termination of the cochlear canal. In the first and second turns of the cochlea, its under surface supplies the place of the periosteum upon the most external portion of the osseous

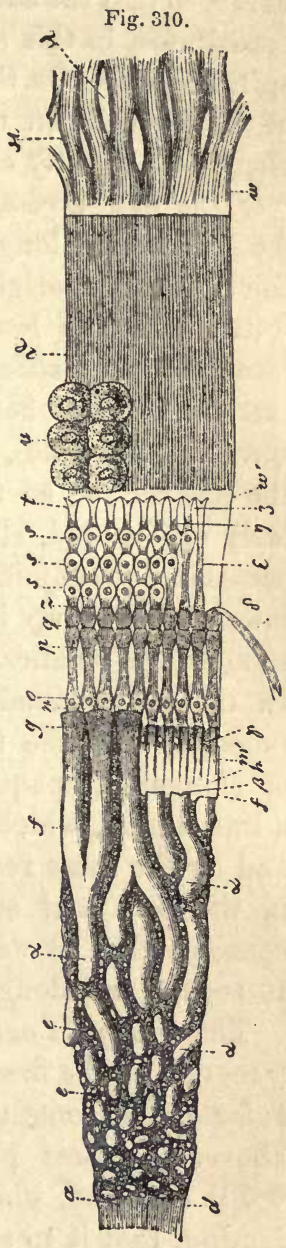

Fra. 309.-Vertical section of the lamina spiralis, at 6 lines from its commencement, magnified about 225 diameters (Cat or Dog); the epithelial layer investing its upper and lower surfaces is removed: $a$, periosteum of the osseous zone; $b$, the two lamellæo of the osseous zone near its free border; $c, c^{\prime}, c^{\prime \prime}$, termination of the auditory nerves; $d$ - $w$, lamina spiralis membranacea; $d-w$, zona denticulata $d d \cdot d^{\prime}-f$, habenula sulcata; $d$, place where the periosteum is thickened; $e$, granules in the groove of the habenula sulcata ; $f$-g, "teeth of the first series ;" $g-f-h$, sulcus s. semicanalis spiralis; $h$, its inferior wall: $k$, epithelial cells at the entrance of the sulcus spiralis; $h-w^{\prime}$, habenula denticulata; $h-m$, apparent teeth; $n-t$, "teeth of the second series;" $n-p$, their posterior joint; $o$, nucleated enlargement upon them; $p-q$ and $q-r$, articular portions ; $r-t$, anterior joint of the second row; $s s s$, three cylinder-cells, placed upon them; $l \cdot v$, membrane covering the habenula denticulata; $u$, one of the epithelial cells underneath; $w \cdot w$, zona pectinata ; $x$, periosteum, attaching the lamina spiralis (musc. cochlearis, Todd and Bowman); $y$, vas spirale internum; $z$, its inner coat. Aftcr Corti.

Fia. 310.-Vestibular surface of the lamina spiralis membranacea, magnified 225 diameters. The letters are in part the same as in Fig. 309: $a$ a, cylindrical elevations of the habenula 
zone, whilst in the last half turn it is bounded only by the expansion of the nerve, so that the habenula sulcata, in the strict sense of the word, properly constitutes, in this situation, only a part of the so-termed membranous spiral lamina. On the upper surface of this layer, and on the outer border of it, there is an uninterrupted series of clear, peculiarly glistening, elongated, slightly clavate processes ( $g$ ), the "teeth," as they are termed "of the first series," which, in the first spiral turn of the cochlea, are 0.02 of a line long; $0.004-0.005$ of a line wide, and 0.003 of a line thick, at their origin, whilst in the last turn they are not more than 0.015 of a line long and 0.003 of a line broad. They project, free, into the scala vestibuli, and arch over the commencement of the habenula externa, so that between the two a tolerably deep sulcus, semicanalis spiralis (Huschke), opening externally, is left. Towards the axis of the cochlea the so termed "teeth" are directly continuous, with similarly constituted, elongated ridges or costce (Fig. $309 a$ a), which are occasionally conjoined in pairs, or divided into two, and still further inwards break up into divisions, which become shorter and shorter and smaller and smaller, being at first elongated and afterwards rounded. In the longitudinal and transverse grooves between these costce and tubercles and the teeth, there usually exists, in single series, rounded or elongated, opaque glistening corpuscles (e), 0.0015-0.002-0.003 of a line in size, which, on the addition of acetic acid, prove to be nuclei; and by the same reagent, nucleiform strice occasionally become distinct in the pale and somewhat swollen "teeth" and costoe, which parts, consequently, as well as those presently to be described, I am inclined to regard as belonging to the connective-tissue group.

The habenula externa s. denticulata $(h-t)$ arises under the base of the "teeth" of the first series, directly from the just-described habenula sulcata, and constitutes at first the bottom of the spiral sulcus noticed above. In most places its thickness does not amount to more than 0.001 of a line, which is also that of the rest of the membranous spiral lamina, that is to say, of the zona pectinata, and its width increases towards the cupola in proportion to the decreasing breadth of the habenula sulcata, so that it measures at first, not more than 0.05 , and ultimately, $0 \cdot 1$ of a line. With respect to its structure, it presents, on the side of the scala vestibuli, a considerable number of elevations, whilst towards the scala tympani it is perfectly smooth and even. These elevations, proceeding from within to without, are as follow:-first, come the so-termed "apparent teeth" ("dents apparents," Corti), a crowded

sulcata: $\beta$, spot where a "tooth of the first series" originates; $\gamma$, spaces between the "apparent teeth ;" $\delta$, anterior portion of a "tooth of the second series" thrown back; $\varepsilon$, the same in its natural position, without its epithelial cells; $\zeta$, the same with only the lowermost epithelial cells; $n$, the same with two of the lowermost cells; $\vartheta$, streaks or slight elevations of the zona pectinata: $x$, periosteum, by which the lamina spiralis is attached, with spaces, $\lambda$, between the bundles. After Corti. 
series of elongated projections, 0.08 of a line long, 0.002 of a line broad, which, separated from each other by shallow grooves, are slightly raised at the outer end, and then suddenly depressed. Externally to these processes which, in the first turn of the cochlea, are still situated on the zona ossea, beneath the "teeth of the first series," and in that situation present minute elongated hollows between their outer ends (Fig. 309, \%), but in the second and third turns are placed more externally than the "teeth of the first series," being bounded on the under surface only by the nerves, succeed in equal number the "teeth of the second series," (Corti) $(n-t)$, very remarkable structures, of which the adjoining figure will afford a better exposition than any description. Each of them represents a little rod, somewhat compressed from above to below, and lies free and movable on the membranous spiral lamina, to which it is affixed only by the inner extremity, as a continuation of which, therefore, these teeth must be regarded. Viewed more closely, each of them presents three joints. The innermost $(n-p)$, an attached joint, resembles a cell of cylinder epithelium, and contains in its somewhat dilated internal extremity $(o)$ a round nucleus, 0.005 of a line in size; to this succeed the middle joints $(p, q, r)$ two equal, elongated, quadrangular segments, 0.0044 of a line long, of the same homogeneous and glistening substance as that of which all these "teeth" in general are composed (the coni articulares of Corti), which are connected with each other and with the internal and external joints, so as to allow the latter a certain extent of motion up and down. The last joint, finally $(r-t)$, is at first attenuated, but towards the extremity again becomes wider and bifurcate, and supports three nucleated segments attached to its inner extremity, resembling pedunculated cells $(s, s, s)$, one above the other, the undermost being the longest, which may be termed "teeth of the third series" ("cylinder epithelium-cells," Corti). The habenula denticulata, as far as to the "teeth of the second series," is covered by round or oval epithelium cells $(h)$ which also occupy the spiral sulcus below the "teeth of the first series," though lying free and separate in immediate contiguity, and forming a continuous layer only on the $h a$ mulus membranaceus. Upon these cells and over the entire habenula denticulata, we then find a peculiar, thin, finely striated membrane ( $(-v)$, which externally projects a little over the commencement of the zona pectinata, though separated from it by some large epithelial cells $(u)$, and passing internally upon the habenula sulcata, where it is gradually thinned and ultimately lost. This membrane, covered by the epithelium of the cochlear canal, can hardly be viewed as anything but a continuation of the habenula sulcata, and may be most suitably compared with the zona pectinata.

2. The zona pectinata (Todd and Bowman) $\left(w^{\prime}-w\right)$, is the outer portion of the membranous spiral lamina, smooth on both the upper and 
under surfaces, and affixed at the outer side, on a projection of the external wall of the cochlear canal. It is a perfectly homogeneous lamella, but, except at the borders, appears to be very closely ribbed, and thence assumes a fibrous aspect. At its outer edge this lamina, which seems to be opened out in a narrow border, receives a peculiar fibrous substance $(x)$ arising from the wall of the cochlea, which there presents a minute osseous ridge (the lamina spiralis accessoria), Huschke; which Todd and Bowman describe as a cochlear muscle, but in which I can perceive nothing but a form of nucleated connective tissue, whence I shall term it the ligamentum spirale.

The nerves of the cochlea enter the cavities of the osseous zone from the canal of the modiolus, and there form a close plexus of darkbordered fibres, 0.0015 of a line in diameter, throughout its whole length, and which, as discovered by Corti, contains, at a very definite spot not far from the border of the zone, an aggregation, at first, $0 \cdot 1$ of a line in width, of bipolar, oval, minute $(0.011-0.016$ of a line long, $0.0066-0.0097$ of a line broad), and pale ganglion-cells, which it is very probable intercept all the fibres of the cochlear nerves in their course.

Fig. 311. The dark-bordered fibres proceeding from the external side of

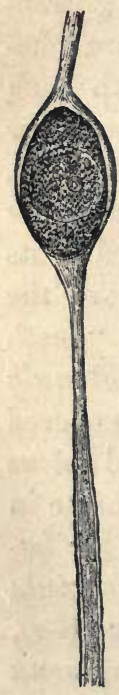
these cells, are again disposed in anastomosing, and afterwards in simply parallel, flattened bundles, which become less and less close as they approach the hamulus, so that, upon that process, the fibres may be perceived forming a single layer, and even separated by interstices. The end of these nerves, in all the parallel bundles and fibres, is always in the same line, but in the first spiral turn will be found nearer to the outer wall of the cochlea than it is higher up. Besides this, there are terminations, also situated between the two plates of the osseous zone, although exactly at its border; in the second turn, in an extent of $0.02-0.03$ of a line, and even outside it, on the under surface of the commencement of the habenula denticulata, consequently within the scala tympani; in the third half turn, lastly, they appear in the form of a nervous border, 0.08-0.09 of a line broad, also on the under surface of the habenula sulcata. The actual termination of the nerve-fibres which are reduced to 0.001 of a line in diameter, appears to me, as well as to Corti, to take place by their first becoming pale, and still finer, and afterwards ceasing-and I must here express myself as decidedly opposed to the notion of the existence of loops.*

Fig. 311.-Bipolar ganglion-globules from the zonula osseo of the lamina spiralis of the Pig, magnified 350 diameters. After Corti.

* [More recent rescarches have induced Prof. Kolliker to alter materially his views on the termination of the cochlear nerve, and indeed of the structure of the cochlea itself. As the result of careful investigations in the Ox, Cat, Pig, Rabbit, and in Man, he found that 
The vessels of the cochlea, though fine, are yet very numerous; they are distributed, in the first place, to the parietal periosteum of the cochlear canal, and secondly, to the lamina spiralis. In the former situation, besides the capillary plexus, wnich is found throughout, they

the cochlear nerves do not terminate in the scala tympani, but that the fibres penetrate through openings in the membranous lamina spiralis into the scala vestibuli, where they become attached to the "teeth of the second series." These latter as well as the pedunculated cells they support, he now regards as a nervous apparatus constituting the true terminations of the cochlear nerves, and, he addnces in proof of the correctness of this opinion the following histological observations.

The membranous lamina spiralis, is not merely a continuation of the periosteum of the vestibular surface of the osseous zone, but also of that of its tympanal surface. The firstmentioned periosteal lamella forms the habenula sulcata with its teeth and the commencement of the habenula denticulata, and then unites externally to the "apparent teeth," and the "teeth of the second series" with the other periosteal layer. This besides lining the bone, extends beyond the edge of the osseous zone as a covering to the nerve-fibres, which are thus, even after leaving the bone, enclosed between two layers of periosteum, and hence do not, as stated by Corti, lie free in the scala tympani. Now in vertical sections of the lamina spiralis, treated with chromic acid, the pale nerve-fibres may be traced running towards the scala vestibufi, and perforating the habenula denticulata near the external extremities of the " apparent teeth.' Exactly over the perforated portion of the habenula denticulata (habenula perforata) are seated the teeth of the second series, which may be seen to be connected with the dark-bordered nerve-fibres, by means of delicate pale processes.

The nervous nature of the "teeth of the second series" Kolliker states, becomes further apparent by a micro-chemical analysis. Corti affirms them to be chemically similar to the membranous lamina spiralis, but this Kolliker proves to be incorrect. $\mathrm{He}$ found them on the contrary to correspond most closely to the dark-bordered nerve-fibres, and the processes of the ganglion-cells. Caustic soda and potassa destroyed them almost immediately. The addition of water produced in them two or three varicose enlargements, containing a filament precisely similar to an axis-cylinder. The nucleated swelling at their commencement described by Corti, he regards as a bipolar ganglion globule, one process of which is connected with the dark-bordered nerve-fibres, whilst the other supports three unipolar nervecells ("three epithelial cells" of Corti).

Based upon these histological data, Kölliker advances some interesting and novel views with regard to the physiology of the cochlea. The existence of the peculiar nervous apparatus at the extremity of the cochlear nerves, renders it, he thinks, highly probable that this is the only portion of the nerve which possesses the power of receiving sonorous undulations. These undulations reach the cochlea through the fenestra ovalis, and are not communicated, as most physiologists suppose, by the osseous lamina to the cochlear nerve, since the latter lies free in the labyrinth and is unconnected with the osseous zone. The isolated position of the cochlear nerve, its movability and great softness, renders it, Kolliker further surmises, specially applicable to receive the undulations transmitted by the fluid of the labyrinth. Regarding the special function of the cochlea, he is inclined to attribute to it a greater physiological importance, than to any of the other parts of the labyrinth. The free position of the expanded portion of the nerve, and the extent of surface over which its numerous terminal fibres are spread, constitute it, he thinks, an organ of great delicacy of hearing, enabling us to distinguish several sounds at once, and also to estimate accurately their exact pitch. The ganglionic termination of the cochlear nerves, Kolliker concludes, establishes a surprising analogy between the auditory, and visual apparatus, since the sensory nerves of botli are possessed at their peripheral expansion of a ganglion, which receives the impressions, the nerve-fibres merely serving to conduct them to the brain. (Vid. Kölliker, "Ueber die Endigimgen des Nervus Cochleæ und die Functions der Schnecke," Würzburg, 1854.) $-\mathrm{DaC}$.] 
constitute a peculiar vascular tract in the scala vestibuli, immediately under the ligamentum spirale, - the stria vascularis of Corti, and which though continuous with the vessels of the periosteum, still lies above it, imbedded, as it were, in the partially colored epithelium. In the zona spiralis, we find in the osseous portion, but also in the nervous expansion itself, a rich capillary plexus, continuous with a vas spirale running on the under or vestibular surface of the zona membranacea, through the whole extent of the cochlea. This vessel, which is probably renous, lies immediately under the habenula denticulata, sometimes more towards the interior, sometimes more extcrnally, becoming, in the last lalf turn of the cochlea, a capillary vessel of not more than 0.004 of a line in diameter; but towards the base, it gradually enlarges to 0.013 of a line, and is distinctly composed of two coats. In rare instances, there are two capillary vasa spirala in the situation above indicated, and on two occasions, in Man and in the Sheep, Corti also noticed an external vas spirale, near the ligamentum spirale on the zona pectinata, which, however, did not communicate with the internal vessels, so that, speaking generally, the zona pectinata is non-vascular.

Lastly, we have to consider the acoustic nerve. The fibres of its trunk, in Man, measure $0.002-0.005$ 'of a line, are very readily destroyed, and have only a delicate neurilemma. Among these, in the trunk itself and in the vestibular and cochlear nerves, there occur numerous bipolar, apolar, and unipolar, pale and colored ganglion-cells, measuring, in the Mammalia and in Man, 0.02-0.07 of a line, of which the two latter forms are, as Stannius correctly observes, probably only truncated bipolar cells, inasmuch as, particularly in Fishes, the acoustic nerve contains cells of this kind only or nearly so. Similar cells, but smaller, are also met with, as already mentioned, in the cochlea, as well as in the nervous twigs in the vestibule (Pappenheim, Corti). Divisions of the fibres of the auditory nerve were noticed by Czermák in the ultimate ramifications in the ampulloe and sacculus of the Sturgeon, by myself and Harless in the Frog, and by Leydig in the Chimcera.

Of the development of the auditory organ, it need here merely be mentioned, that according to Huschke's discovery, confirmed by Reissner and Remak, the membranous portions of the labyrinth are formed from the external integument, simply by its inversion, and consequently in their origin may be compared with the lens and vitreous body. To this inversion, in which the cellular layers corresponding to the epidermis, principally, but not alone, as Remak believes, take part, the auditory nerves are afterwards continued from the brain; and from the middle germinal layer are afforded the hard tissues and the rest of the soft parts in order to complete the sentient organ. With respect to the 
histological development of the soft parts of the labyrinth, nothing of consequence is known.

In the description of the cochlea, I have altogether followed Corti, having formerly satisfied myself of the correctness of his statements, when that zealous and intelligent observer pursued his investigations in Wurzburg; and having also, recently, confirmed the greater part of them by the examination of the human cochlea. At the same time the parts of the habenula denticulata, which are so difficult of investigation, have always appeared to me to demand further consideration, and particularly with respect to the ascertaining of the kind and mode of the movements of its processes, the relations of the epithelium, and of the investing, structureless membrane. So long as these points are not ascertained, all suppositions respecting the function of these delicate structures are without any secure basis, and the physiology of the cochlea, otherwise so difficult, is rendered only still more perplexed (vid. Harless, l. c.). As regards the nature of the so-termed "teeth," they are certainly, as Corti assumes, nothing more than developments of the periosteum of the cochlear canal, and may, in my opinion, although in chemical respects very similar to the vitreous membranes, yet be regarded as belonging to the connective-tissue group. Whether the three so-termed cylinder epithelium-cells (Corti) on the habenula denticulata are really epithelial, as Corti supposes, still demands investigation. These bodies, notwithstanding their great delicacy, appear to me rather to be referable to the category of the other tissues of the habenula denticulata; and on that account I have termed them "teeth of the third series."*

For the investigation of this organ, which, however, presents any very considerable difficulties only in the labyrinth, perfectly fresh objects are indispensable, and are best taken from animals just killed. For the moistening of them, serum or syrup should be employed, when it is desirable to view the parts in a perfectly normal condition. Successful results will be obtained, especially if a certain amount of practice, in the exposing and dissection of the delicate tissues with which we have to do, be combined with great patience, seeing that it is often a matter of chance whether any given relations are brought into view. In order to see the nervous plexus of the osseous zone of the cochlea, the latter must be deprived of its calcareous constituents by dilute muriatic acid; whilst in the case of the ganglion-cells in that situation, the only means of attaining our object consists in a careful breaking down of the osseous zone in a neutral medium. 
Literature.-E. Huschke, in "Fror. Not.," 1832, No. 707; "Iris," 1833, No. 18, 34; K. Steifensand, "Untersuchungen über die Ampullen des Gehörorgans," in Müller's "Archiv," 1835; S. Pappenheim, "Die specielle Gewebelehre des Gehörorgans," Breslau, 1840, and Froriep's "Not.," 1838, Nos. 141, 194, and 195; G. Breschet, "Recherches sur l'organe de l'ouie dans l'homme et les animaux vertébrés," $2 \mathrm{~d}$ ed. Paris, 1840 ; E. Krieger, "De otolithis," Berol. 1840 ; Wharton Jones, "The Organ of Hearing," in Todd's " Cyclopædia," Vol. II. p. 529; J. Hyrtl, "Ueber das innere Gehörorgan des Menschen und der Säugethiere," Prag., 1845; A. Corti, "Recherches sur l'organe de l'ouie des mammifères," in Zeitsch., f. wiss. Zool. III., p. 109 ; Reissner, "De auris internæ formatione," Dorpat, 1851; E. Harless, Art. "Hören," in Wagner's "Handw. der Physiol." IV., p. 311, and "Münchn. Gel. Anzeiger," 1851, No. 31 and 37; Stannius, "Ueber die gangliöse Natur des Nervus acusticus," in Gött. Nachr., 1850, No. 16 ; Ib., 1851, No. 17 ; [A. Kölliker, "Ueber die letzten Endigungen des Nervus Cochleæ und die Function der Schnecke," Würzburg, 1854.-DaC.] Besides which should be consulted the general works of Krause, Huschke, Arnold, Todd and Bowman, Remak (Entwicklungsgeschichte), and the "Icones org. sensuum," of Arnold.

\section{III.-OF THE OLFACTORY ORGAN.}

$\S 236$. The olfactory organ consists of the two nasal cavities, or fossce, supported by bones and cartilages, and lined by a mucous membrane, and of a certain number of accessory cavities, viz., the frontal, sphenoidal, and ethmoidal sinuses and the antrum Highmori. Of all these cavities, however, only the uppermost portions of the nasal fossæ, where the olfactory nerve is distributed, are subservient to the sense of smell itself, the others being either simple conducting canals, and participating as well in the action of respiration, or at any rate having no direct relation to the olfactory sense.

The hard structures, just named, present nothing much worthy of remark; and of the bones it need merely be mentioned, that the thinnest parts of the ethmoid consist only of a fundamental substance and bone-fibres, without any Haversian canals. The nasal cartilages are true cartilages, and most nearly approach those of the larynx, except that the contents of the cartilage-cells are usually very pale, and without fat, the cell-walls little thickened, and the matrix finely granular. Beneath the perichondrium there is, also in this situation, a layer of flattened cells, which, on the septum attains a thickness of 0.024 of a line, whilst in the interior the cells are more rounded, larger, and disposed in rows, in the direction of the thickness of the septum.

With respect to the coverings of these parts, the integument of the nose may be first noticed: it is characterized by a thin epidermis 
$0.024-0.032$ of a line thick, a dense cutis of 1.4 with minute undeveloped papilloe of 1-40-1-66 of a line, and fine hairs, as well as by a close adipose tissue 1 line thick, intimately united with the cartilage, containing large sebaceous follicles, extending into the latter, and minute sudoriparous glands 1-10-1-12 of a line in diameter. This external integument, with its sebaceous follicles, and with stronger hairs (vibrissee) also extends a short distance into the nasal fossoe, but not quite so far as the point where the cartilaginous portion of the nose terminates, passing imperceptibly into the mucous membrane of the olfactory organ, by which all the remaining cavities are lined, though it does not everywhere present the same conditions. As was discovered by Todd and Bowman, and $I$ can fully confirm, this mucous membrane is subdivided into a ciliated and a non-ciliated portion, the latter being limited to the uppermost parts of the nasal fosse, where the olfactory nerve is distributed, and consequently should perhaps be termed the olfactory mucous membrane in the stricter sense, whilst the other might retain the old name of "Schneiderian membrane."

Upon first viewing the latter, it will be found that, although the epithelium vibrates throughout, still that its structure is not everywhere the same, and that we may conveniently distinguish in it the thicker glandular mucous membrane of the proper nasal fossce from the thinner membrane of the accessory sinuses, and of the interior of the spongy bones. The epithelium, in both situations, is squamose and ciliated, like that of the larynx (Fig. $311^{2}$ ), measuring, in some places, 0.018-0.020 of a line in thickness, and in others as much as 0.042 of a line. In Man it presents pale, fine-granular cells, of which the outermost ciliated ones attain a length of 0.03 of a line, and in animals produce a current running from before to behind. To this succeeds, a true $m$. mucosa, wholly without elastic elements, or at all events very scantily supplied with them, and composed chiefly of common connective tissue. In the proper nasal fossa there are imbedded in this membrane very numerous larger and smaller, racemose mucous glands of the usual kind, with gland vesicles of $0.02-0.04$ of a line, so that, in places, especially at the borders of the septal cartilage, and on the inferior spongy bones, it presents a thickness of 1-2 lines. The thickness of the mucous membrane of these regions, however, does not depend upon the glands alone, but also, especially at the border and posterior extremity of the inferior spongy bone, upon abundant, almost cavernous venous plexuses in its interior. In the accessory cavities, the glands are almost wholly wanting, and $I$ have as yet only occasionally found them in the antrum Highmori, where their excretory ducts and gland-vesicles are sometimes dilated into cysts containing mucus, $\frac{1}{2}$ a line in diameter. Except in these places, the mucous membrane of the accessory cavities is extremely delicate and inseparable, as a distinct membrane, from the periosteum lining them; 
and the same may be said of it in the nasal fossæ themselves, particularly in the glandular parts, notwithstanding the intimate connection of the two. A remarkable appearance presented itself to me in the body of a youth aged 15 (who, as I was informed by Virchow, also exhibited ossifications in the lungs), consisting in the deposition-in the mucous membrane, in all these accessory cavities, as well as in the similarly constituted mucous membrane on the concave side of the spongy bones, immediately beneath the epithelium - of calcareous salts, to such an extent, that its uppermost layer was transformed into a peculiar ossified though still flexible membrane, in which there existed, in places, larger and smaller, often very regularly disposed openings, but no evidence of a special structure. Under this layer, which, where well developed, appeared perfectly white, like a membrane filled with air-vesicles, as which I at first regarded it, there always occurred a looser connective tissue with vessels, of which latter, however, some were also incrusted; and in the deeper parts of the epithelial layer itself, there were scattered, smaller, simple or aggregated concretions, like "brain-sand" in miniature.

The proper olfactory mucous membrane of all the divisions of the organ, occupies only the uppermost parts of the septum and of the

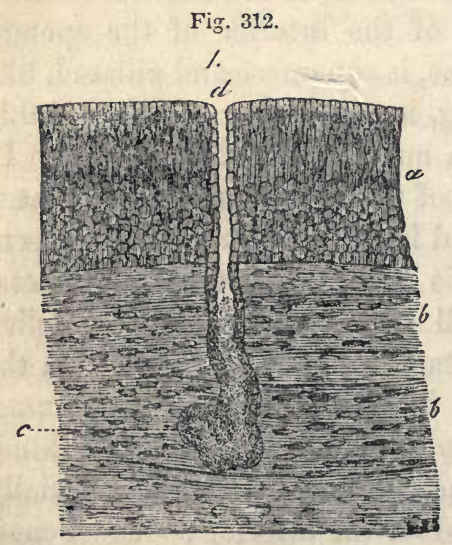

2.

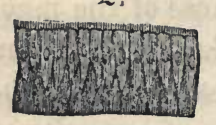
walls of the proper nasal fosse, where the superior spongy bones are situated, to a distance of about $\frac{3}{4}-1$ inch downwards from the lamina cribrosa. It is distinguishable, even by the naked eye, from the contiguous ciliated membrane, by its greater thickness and its color, which is sometimes yellowish, as in Man, the Sheep, and Calf; sometimes yellowish-brown or brown, as in the Rabbit and Dog; and, when examined microscopically, it is seen to be bounded by a tolerably well-defined, toothed or undulated border. The differences of the structure depend upon the condition of the epithelium, the occurrence of numerous peculiarly constructed glands, which I shall term "Bowman's glands," and upon the relations of the nerves. The

Fig. 312.-From the nasal mucous membrane of the Sheep; magnified 150 diameters. 1 , from the regio olfactoria, transverse section of the mucous membrane : $a$, epithelium without cilia; $b$, olfactory nerves, with a dividing, pale, nucleated fasciculus; $c$, one of "Bowman's glands ;" $d$, its orifice. 2 , ciliated epithelium of the Schneiderian membrane. 
epithelium is not ciliated, of which I have most fully satisfied myself, not indeed in Man, in whom I have never yet met with the epithelium of the true olfactory region in a perfect condition, although the ciliated epithelium occurred frequently enough still in a state of vibration, but in the animals just named. It is also much thicker, so that in the Sheep, in which the ciliated epithelium is 0.03 of a line, it measures 0.05 , and in the Rabbit, the one is 0.04 , and the other 0.07 of a line thick. Notwithstanding this thickness, which is considerable for a mucous membrane, it is remarkably delicate and soft, and is so much affected by almost all reagents as to allow of its being studied only with considerable trouble. According to my observations, it should be described as a squamose cylinder-epithelium; at all events, in opposition to Todd and Bowman, I always find as its outermost layer, one or two strata of slender, vertical cells, $0.005-0.007$ of a line long, whilst it must be allowed that, more deeply, spherical elements, of 0.003 0.004 of a line, alone appear to exist. All these cells have minute, round nuclei, usually pale, finely granular contents of a brownish hue in the deepest layer only in the Rabbit and Dog, and such delicate membranes, that in water they instantly burst. Although the ciliated cells of the nasal fossoe are much more readily affected by water than those of other situations, this is true to a much greater extent as regards the cells of the olfactory region; and the destructive effect of the filling of the nasal cavities with water (E. H. Weber) and other fluids is thus easily accounted for, as well as, on the other hand, is the ready transition of volatile substances through the epithelium rendered intelligible. For the moistening and protection of this epithelium throughout the region in which it exists, it is furnished with a great number of the "glands of Bowman," which is the more remarkable, because the immediately contiguous, ciliated mucous membrane is but scantily supplied with glands, or is wholly without them. These glands are simple cylinders, either straight or slightly convoluted at the lower end, and $0.08-0.1$ of a line in length, or elongated pyriform follicles, situated principally between the larger branches of the olfactory nerves, in crowded rows, in part, however, more isolated, as at the lower boundaries of the olfactory region. They most nearly approach certain forms of the Lieberkühnian glands, and the sudoriparous glands of the foetus. I have never yet noticed divisions of the follicles, although it is very possible that I may have overlooked them, since these organs are extremely delicate, and easily altered. Their canals, 0.014-0.025 of a line wide, are lined by a beautiful, simple epithelium, composed of rounded polygonal cells, $0.006-0.008$ of a line in size, containing more or fewer yellowish or brownish pigment-granules, to which is due the varying color of the olfactory mucous membrane. Their excretory 
ducts are rather more contracted $(0.008-0.012$ of a line) than the glandular canals, and ascend, always lined by rounded larger cells, straight through the epithelium, in order to terminate on the surface with rounded orifices, 0.01 of a line in diameter, surrounded by a few large cells. The tissue beyond these glands is, as in other regions, soft connective tissue, without elastic elements.

The mucous membrane, in the proper nasal cavities, is very richly supplied with vessels, and less so in the accessory sinuses. The terminal branches of these vessels form loose plexuses around the glands, and in the trunks and branches of the olfactory nerves; while on the surface of the mucous membrane itself, they constitute a more close network with numerous horizontal loops, at first sight leading to the supposition of the presence of papillo, which, however, do not exist. The branches, also, of the arteries and veins enter into numerous anastomoses, and constitute (the latter especially) on the inferior

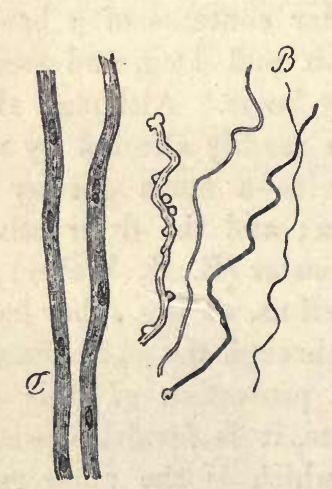

Fig. 313.

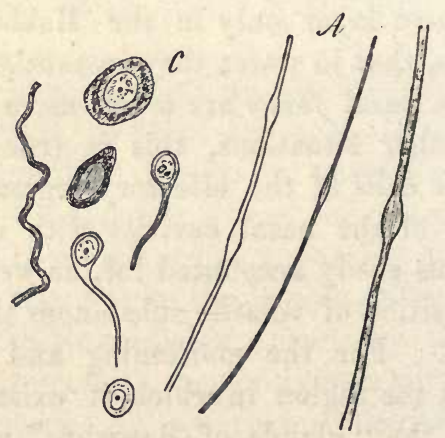

spongy bones, the abundant spongiform plexus already noticed. Nothing is known of the lymphatics of the nasal mucous membrane. The nerves are, in the first place, branches of the fifth pair (ethmoidal, posterior nasal, and a branch of the greater anterior dental nerve), which supply especially the ciliated region of the organ, presenting there the same conditions as the nerves in other sentient mucous membranes (of the pharynx, for example), but also extend to the proper olfactory region; and, as I noticed in one instance in the Calf, send scattered dark-bordered primitive fibres in the course of the branches of the olfactory nerves. The olfactory nerve, in its tract and bulb, contains dark-bordered fibres and nerve-cells, of which we have already spoken.

Fig. 313.-From the olfactory nerve of Man; magnified 350 diameters: $\mathcal{A}$, nerve-tubes from the tractus, in water; $B$, in syrup, appearing contracted; $C$, nerve-cells, from the bulb; $D$, nerve-fibres, from the branches in the olfactory organ. 
The nervi olfactorii, on the other hand, in Man and in other Mammalia, even in the main trunks given off from the olfactory bulb, present no white medullated fibres at all, but are wholly constituted of pale, slightly granular flattened fibres, $0.002-0.003$ of a line wide, with elongated nuclei, which are closely adherent and retained in connection by common sheaths of connective tissue, which are somewhat thicker and therefore whiter in the rami ad septum. With respect to the origin of these fibres, which very closely resemble the embryonic nerveelements, whether they are derived from the olfactory bulb, or from the cerebrum itself, has by no means been as yet ascertained in Man, or in other Mammalia, although, from the observations of Leydig in the "Plagiostomata," ("Beiträge," p. 34, Tab. I., fig. 6), it is probable that the former is the case. The termination of these nerves is yet more doubtful. This much is readily seen, that the nervi olfactorii, in their course in the mucous membrane of the olfactory region, are gradually attenuated as they descend, in consequence of numerous divisions at acute angles, and form a plexus which may also, in Mammalia, be traced almost throughout the olfactory region; but shortly before reaching its border, these plexuses are always lost to sight, nor is any other indication of terminal branches presented; so that, as regards the main fact, I remain quite in the dark. At present it appears to me most probable that the terminal distribution takes place throughout the non-ciliated region, and above all at its border; at any rate $I$ have never yet been able to detect the filaments of the olfactory nerves in the ciliated epithelium, although they can be traced down to twigs of the size of $0.005-0.01$ of a line. I have not seen the ganglion-globules on the inner surface of the finer plexus mentioned by Valentin ("Nervenlehre," p. 303), and the rather strange-looking "glands of Bowman," might have been mistaken for such bodies.

In the investigation of the olfactory organ, the chief difficulty arises from the softness of the epithelium, and on this account a solution of albumen, or humor vitreus, only should be employed to moisten it. Vertical sections in detached portions of the mucous membrane, are best made with the scissors; and the edges of folds not unfrequently afford good sectional views. The mucous glands, are found in sections; those of Bowman on teasing out the structure. Chromic acid cannot be recommended for the olfactory nerves: teasing out the membrane is the most suitable mode of proceeding; as well as the compression of fresh preparations or of preparations moistened with soda or acetic acid; lastly, the examination of mucous membrane macerated in water, in which the nerves are preserved for a long time. 
Literature.-Todd and Bowman (op. cit., II.) [Horn, "Ueber die Endschlingen des Geruchsnerven," Müll. "Archiv," 1850. Dr. Horn professes to have discovered looped terminations of the olfactory nerve in the Frog (Trs.); O. Kohlrausch, "Ueber das Schwellgewebe an den Muscheln der Nasenschleimhaut in 'Müller's Archiv,'" 1853; A. Kölliker, "Ueber den Bau der grauen Nervenfasern der Geruchsnerven in 'Verhand. d. phys. med. Ges.' zu Wurzburg," IV. 1, 1853.-DaC.] 


\section{APPENDIX.}

\section{BY THE TRANSLATORS.}

$\S 1$. The first section of the Appendix should, according to promise, have been constituted of an exposition of the views which we have taken with regard to the Cell-theory. Finding, however, that it would be inexpedient to extend this already somewhat voluminous work by the amount of space and number of illustrations which would be necessary for a more complete discussion of the subject than has already appeared in the pages of the "British and Foreign Medico-Chirurgical Review" (October, 1853), we must refer the reader to the article in question.

We hope, however, in the course of a short time, to treat of the whole subject at length in another place.

Corpuscula tactûs and Pacinian bodies.-In an essay on this subject, contained in the "Quarterly Journal of Microscopical Science," for October, 1853, we have endeavored to show that papillæ which contain "corpuscula" may also possess a vascular loop, and that there is, at any rate, no inverse relation between nerves and ressels in the papillæ, since in the Frog, the terminations of the nerves in the fungiform papillæ of the tongue, first described by Dr. Waller ("Phil. Trans.," 1848), take place in obviously vascular papillæ. We regard the "corpusculum" as no peculiar body, but simply as embryonic connective tissue, differing from that of the rest of the papilla only in the regular arrangement of its elastic element; it is, in fact, the dilated termination of the neurilemma of the nerve of the papilla.

With regard to the mode of termination of the nerves, while not venturing to deny the existence of loops, we doubt it; on the other hand, repeated instances of the so-called free termination of dark-contoured nerve-tubules on the surface of the corpuscula are described and figured (1. c., p. 3, Fig. 4). The termination is not really "free," inasmuch as the tubules become continuous, both here and in the Frog's tongue, with the imperfect, reticulated, elastic fibrils of the papillæ.

As respects the Pacinian bodies, we stated, at that time in opposition to all authorities, that their central portion is solid and not hollow, and that in Birds and in the human hand, the fluid supposed to exist be- 
tween the concentric laminæ was equally hypothetical. In structure, the Pacinian body, in fact, is identical with the corpusculum tactûsbeing a solid mass of connective tissue, whose apparent lamination depends on the regular disposition of its elastic elements. We stated further, that the central nerve-tubule gradually terminates, passing into the central solid axis of the Pacinian body. In reality, the Pacinian bodies are also nothing more than thickened processes of the neurilemma of the nerve to which they are attached, and differ from the tactile corpuscles only in the circumstance that in the latter the thickening takes place on one side of the nerve-fibril, while in the Pacinian body it takes place on both sides.

In the meanwhile, contemporaneous observations on this subject were made by Leydig (" Ueber die Vater-Pacinischen Körperchen der Taube") and by Kölliker ("Einige Bemerkungen über die Pacinischen Körperchen,") and were published in Siebold and Kölliker's "Zeitschrift," B. V., H. 1.

Leydig and Kölliker's results are, in the main, in accordance with our own, especially as respects the solidity of the Pacinian body. The "central cavity" is given up by both, but Kölliker still maintains the existence of a fluid between the outer layers, at least in the Cat. Leydig regards the central solid axis of the Pacinian body in the Bird as the expanded termination of the nerve itself.

Wagner had already drawn attention to the resemblance between the corpuscula tactus and the Pacinian bodies; Leydig further shows that the latter form one series with the Savian bodies and the so-called muciparous canals of osseous and cartilaginous Fishes. We ventured, in the paper in question, to add the "tactile hairs" or "vibrissce" of Mammalia to this series of cutaneous organs, by showing that they are but a further development of the muciparous canals-pointing out, at the same time, that even the highest organs of sense, the Eye and the Ear, are constructed upon the same principle.

§ 2. Malpighian bodies of the spleen.-Having recently carefully investigated the structure of these organs, we have arrived at the following conclusions (vid. "Quarterly Journal of Micr. Science," Jan. 1854).

1. In the various animals examined (Man, Sheep, Pig, Rat, Kitten), we find, as Dr. Sanders had already demonstrated in the Pig, that the minute arterial twigs supplying the Malpighian bodies are not only distributed over, but enter and ramify in them, breaking up into their fine penicillate branches as they pass out.

Furthermore, connecting these arterioles, there is a network of fine capillaries, whose walls are hardly distinguishable, but which are readily detected by using syrup, which retains the coloring matter in their contained blood-corpuscles. 
The pulp of the Malpighian body stands, as Remak pointed out, in the relation of tunica adventitia to the arterioles; it is composed of indifferent tissue, consisting of endoplasts imbedded in a homogeneous periplast, and which may or may not become surrounded by cell-walls.

The Malpighian body has no wall, but passes insensibly, as Wharton Jones had already shown, into the fusiform fibres of the red pulp.

We adduce evidence from Remak and Leydig that the Malpighian bodies of other Vertebrata present similar relations, and that the spleen, lymphatics, Peyer's patches and the glanduloe solitarioe, the supra-renal capsules, thymus, and pituitary body, belong to the category of the socalled "vascular" glands, all consisting essentially of masses of indifferent tissue contained in a vascular plexus.

We proceed to show that the follicles of the tonsil are not closed, but traversed by capillaries; and that it is a gland of the same class, distinguished from a Peyer's patch only in the fact of its elements being aggregated, not on a plane, but round a diverticulum of mucous nembrane.

Finally, we suggest that the liver itself is but a huge tonsil-a vascular gland, with what might be termed a false duct; and we indicate the agreement of this doctrine with the view taken by Dr. H. Jones with regard to this organ, and we may add, with the recent beautiful researches of Bernard.

§3. Corporalutea.-Prof. Kölliker does not appear to be acquainted with the exact description of the structure of the corpora lutea, given by Mr. Wharton Jones so long ago as 1843-4, in his "Report on the Ova of Man and the Mammifera before and after Fecundation," "Brit. and For. Med. Review," 1843, and in a paper entitled "Microscopical Examination of an early Corpus Luteum," in the "London Med. Gazette" for 1844. From the latter we extract the following passages, premising that to the naked eye a section of this corpus luteum presented the appearance of a dark clot with a central membranous shred from which processes radiated.

"The body in question (early corpus luteum) is of a lenticular form, about 6-10 inch in diameter, and about 4-10 inch thick, and projects on the surface of the ovary by somewhat more than half its diameter. The prominent part being covered merely by the indusium of the ovary, the dark brown red color of the body shines through. Examined microscopically the central membranous shred was found to present the following structure :

"1. On its free surface a fine film of tessellated epithelium. 2. Invested by this epithelium was a stratum of finely interwoven transparent fibres, with dark contours, somewhat like elastic tissue. 3. Outside all was a layer identical in structure with the stroma of the ovary, 
the same structure as that composing the principal thickness of the walls of Graafian follicles.

"The membranous processes possessed a similar structure, and were found to be continuous with the stroma of the ovary. That part of the body next the substance of the ovary, had, by its pressure, so condensed the stroma at the place, that the latter looked somewhat like an external capsule sending processes inwards, which met and interwove with those sent outward by the central membranous striæ. But that this appearance of external capsule was the result simply of the matting of the stroma of the ovary by pressure, is shown by the circumstance that it was absent at the peripheral part of the clot-like body, there being there, as already said, merely the indusium.

"As to the microscopical characters of the clot-like matter itself, this was found to consist of granulous corpuscles somewhat like socalled compound inflammation globules closely aggregated, and red blood-corpuscles interspersed amongst them. The latter had lost some of their coloring matter, but the granulous corpuscles were tinged red, as if they had imbibed it.

"The conclusion which is to be drawn as to the nature of the body from this investigation is, that it is a true corpus luteum in an early stage; that the central membranous shred is the wall of the Graafian follicle, from which the ovum had escaped; and that the clot-like mass (which would by and by have acquired the characteristic yellow appearance of the corpus luteum, traces of which could indeed in some deep places be detected), together with the membranous processes extending through it from the central shred to the stroma of the ovary, is the stroma surrounding the Graafian follicle infiltrated with bloody-looking matter."

With regard to the important question of the nature and reason of the differences between true and false corpora lutea, Mr. Wharton Jones observes :

"If in the human female, Graafian follicles burst periodically, independently of coitus, why is there no corpus luteum left? The explanation which might be given of this is :

"By a slow, natural, and regular process, a Graafian follicle maturates, points, bursts, and is evacuated like a small healthy abscess or pimple, and like this the part quickly cicatrizes. A Graafian follicle, at the time of coitus, not happening to be in this fully matured state, its bursting is hastened by the process of congestion, exudation, and extravasation above-described; but when burst, the lymph and blood in an altered state remain for a while in the form of a corpus luteum, which may thus be compared to the hard base of an abscess evacuated prematurely. Though true corpora lutea, there is reason to believe, are formed only after coitus, impregnation may take place without the for- 
mation of a corpus luteum. How is this? The explanation which might be offered of such rare cases is this: Coitus may have chanced to take place at the very time when a Graafian follicle, having become mature, had spontaneously given way and expelled the ovum. No congestion, exudation, and extravasation, would in this case take place, but the part would quickly close and cicatrize. 4. Lastly, what explanation can be given of the origin of false corpora lutea? To this it might be answered, that from some circumstance or other, effusion of blood takes place into the interior of a Graafian follicle, perhaps on the point of bursting spontaneously. This blood coagulates and remains filling and distending the Graafian follicle, even although its walls may have subsequently given way. But besides this effusion of blood, an exudation of lymph takes place on the inner surface of the walls of the Graafian follicle, in consequence of the irritation produced, which, becoming organized, presents the same yellow appearance as the substance of the true corpus luteum formed outside the walls of the Graafian follicle.

"In conclusion, I would remark that, though physiologically one may be permitted to speculate, as I have done, on the relation between the occurrence of corpora lutea in the ovaries and preceding coitus, it would be rash and unwarrantable in any one to pronounce positively from the occurrence of a corpus luteum in the ovaries that coitus had taken place. The discovery of an ovum in the uterus, in process of development, could alone, in the present state of knowledge, warrant such an affirmation in a court of law. But, on the other hand, the absence of a corpus luteum could not warrant the affirmation that coitus had not taken place."

$\S 4$. Development of the Teeth.-In an Essay on this subject in the "Quarterly Journal of Microscopical Science," for April, 1853, we have taken a very different view from that advocated by Professor Kölliker, and which amounts to this, that all the tunics of the teeth are the result of calcareous deposition from the pulp, the so-called "enamel organ" taking no direct share whatever in the process. This view was based upon observations made upon the teeth of all the principal orders of the Vertebrata, i.e., the Mackerel, the Skate, the Frog, the Calf, and Man; and subsequent observations on these and on other animals, have only confirmed our belief in the substantial truth of the matters of fact there stated.

The keystone of the theory of dental development there enunciated is the fact, that, in all the orders of the Vertebrata, a membrane homologous with the so-called "persistent capsule," discovered, in 1839 , by Mr. Nasmyth, and which we have therefore denominated "Nasmyth's membrane," can be demonstrated covering the enamel of the teeth and extending over the dentine, to be continuous with the membrana preformativa and basement membrane of the sac, in an incompletely formed 
tooth; or with the surface of the cement, in a fully developed one. Nasmyth's membrane, in fact, is at first the membrana preformativa more or less altered.

We hold this statement to be incontrovertible, nor less so the correlated doctrine, that the enamel and cement, as well as the dentine, are developed beneath Nasmyth's membrane, between it and the pulp; that the enamel is, consequently, during the whole course of its formation, separated from the enamel-organ by Nasmyth's membrane; and that, therefore, the direct conversion of the long cylinders of the epithelium into the fibres of the enamel, strongly as their mutual resemblance may suggest the notion, is, to say the least, highly improbable.

Thirdly, we have met with no facts in opposition to what we have stated with regard to the mode of development of dentine and the relation of the latter to the pre-existing elements of the pulp, and we believe that there is every reason to regard what we have there called the "Deposition theory," as an established position. According to this theory, the dentine is not the result of the conversion of the elements of the pulp; the endoplasts of the latter never becoming engaged in the calcareous deposit, as they are in bone; but the young dentine is formed by a deposit of transparent calcareous granules in a thin layer between the pulp and the membrana preformativa. It must be understood, however, that the latter two structures are continuous, and that when the dentine is said to be deposited between them it is not meant that any real interval exists, but only that the outer portion of the periplast of the pulp, of which the membrana preformativa constitutes a part, increases and receives a calcareous deposit without any corresponding implication of the endoplasts of the pulp.

The thinnest and youngest layer of the dentine appears to be structureless, which may, however, arise from the small quantity of calcareous matter which it contains: subsequently, minute cavities, irregular in form, and 1-5000th of an inch apart, appear in it; and these corresponding with one another in successive layers of the dentine, become the dentinal tubuli. The appearance of walls, \&c., to these tubuli, we consider to be the result of a subsequent differentiation in the dentine.

A careful study of the mode in which the dentine-like tegumentary organs of many of the lower animals (Fishes, Articulata, Mollusca) are formed, has afforded the fullest confirmation of this theory of the development of dentine, and we would recommend those who have any doubt upon the subject to study the development of the spines of the Skate, or that of the shell of the Crab or Lobster.

The mode of development of the enamel appears to us to be a very difficult subject, and requires to be most carefully studied. Taking into consideration the facts that a distinction of a superficial and a deep layer of calcified tissue is very general in the tegumentary organs- 
that in a Molluscan shell, for instance (e. g. Trigonia), we may have a superficial membranous layer corresponding with Nasmyth's membrane, a deeper prismatic layer, whose prisms precisely resemble those of enamel on a large scale, and an internal laminated tubular layer, corresponding with dentine; and knowing, further, that these varieties of structure thus arranged are (whatever view we take of shell-structure) nothing but the result of the different modes in which calcified deposit has successively taken place in the same organ, it is sufficiently obvious that there are abundant analogical grounds for considering the enamel and the dentine as modifications of one and the same tissue.

Nor does the structure of the enamel in the Fish or the Batrachian present any difficulty in the way of this view. It is, at most, indistinctly fibrous and contains so large a quantity of calcareous matter in proportion to the dentine, that the differences between the two may well be supposed to arise-as we believe they do-from this circumstance alone.

In the higher Vertebrata, however, when the enamel in its young state consists of definite fibres composed of organic substance, which are added to the surface of the tooth only after the formation of a subjacent scale of dentine, it becomes more difficult to comprehend the development of the former. There appears to be three possibilities.

1. What we call the primary scale of dentine is not, on the crown of the tooth, dentine at all, but young enamel, becoming converted into the latter structure, and not into the former, as development proceeds. This appears, as first sight, a startling hypothesis enough; but there are, so far as we know, no means of disproving it. Young dentine can only be known to be such by its relations; in structure it is neither like perfect dentine nor like perfect enamel; but might readily be supposed to be converted into either by variation in the quantity and mode of deposition of its calcareous element. If this deposit be comparatively small, leaving much of the organic basis, and not encroaching upon the existing cavities, we have dentine ; increase the quantity of calcareous salts, and break up the organic basis at the same time into fibres, and enamel would be produced.

2. The enamel is the indirect product of the prismatic cells of the enamel organ, whose inner extremities pass into successive layers of membrane, which are applied upon and indistinguishably unite with the membrana preformativa over the whole surface of the developing enamel. The laminated membrane thus formed receives a calcareous deposit, and breaks up into the prisms of the enamel.

This hypothesis likewise, at first sight, appears somewhat improbable, but it may be strictly parallel with what occurs in the formation of prismatic shell substance, where a laminated membranous substance is 
produced from the cellular epidermis of the mantle, and subsequently breaks up into the characteristic, large, transversely striated prisms.

3. The enamel is neither the result of the modification of the primary "dentine," nor superimposed on this from the enamel organ, but a tertium quid, the product of the growth and metamorphosis of that excessively thin layer of organic matter which lies between the dentine and the enamel.

In support of this view, also, a very close analogy may be found in the mode of development of the shaft of the hair-a structure which exhibits the closest correspondence with the teeth. The fibrous cortex of the hair is, in fact, homologous with dentine; it is a horny dentine, containing rudimentary canals. External to this substance we find two layers; the inner composed of parallel horny, structureless plates, closely united and set obliquely on the shaft, in fact, a rudimentary horny enamel; the outer consisting of a tough areolated membrane, outer layer of the cuticle, whose resemblance to Nasmyth's membrane cannot be overlooked. Now, if we trace the development of these layers in the long hairs of the head, we find that they pass on the bulb into a structureless limitary membrane, beneath which lie the endoplasts of the pulp; this is, in fact, a membrana preformativa of the hair pulp. Passing from the base towards the apex of the hair, the deep endoplasts become surrounded by the horny matter and the pigment-granules of the cortex, while the superficial layer remains free from the latter, but gradually becomes horny, and loses its endoplasts. Its outer portion then becomes the areolated outer (Nasmyth's membrane) cuticular layer, while its inner portion breaks up into the parallel plates of the inner (enamel) cuticular layer. So far as we have been able to observe in the long hair, however, the disappearance of the endoplasts takes place before the areolation and lamination of the periplast which corresponded to them, so that, as we have already stated (note, page 181), the cuticle does here pass into an apparently structureless layer. This, however, is not, as it seemed, a real discrepancy from Prof. Kölliker's views, for in the short thick hairs, such as those of the nostril, the endoplasts persist longer, and we see that, as he states, the areolations of the outer cuticle are the representatives of the cell-cavities of the outer layer of the pulp; while the laminæ of the inner layer are the result of the lamination of the next layer of the pulp, whose endoplasts may be seen gradually disappearing, whilst its periplast breaks up into plates.

Now in the long hairs we have a relation of the outer cuticle to the cortex very similar to that of Nasmyth's membrane to the dentine before the development of the enamel, and the conclusion is obvious, that as the development of the inner layer of the cuticle takes place by the differentiation of the intermediate substance between the cuticle and cortex, 
so that of the enamel may take place in the same way in relation to Nasmyth's membrane and the dentine.

These would appear to be the alternatives concerning the development of the enamel. At present facts would seem to be wanting to determine definitely which should be accepted.

Finally comes the question of interpretation of the phenomena of development of the dental tissues, and the determination of the homologies of the latter with the pre-existing elements of the mucous membrane. Professor Kölliker's views are stated in the text. He considers the dentine and the cement to be the calcified corium of the mucous membrane, while the enamel is the calcified epithelium.

The view we have ourselves taken is that cement, dentine, and enamel, are calcifications in the same constituent of the mucous membrane, and, in fact, that they entirely belong to its corium or dermal element. Taking for granted that the membrana preformativa was a basement membrane, and furthermore, the received doctrine that a basement membrane marks the boundary between the dermal and epidermal elements of integument or mucous membrane, it was, in fact, impossible to come to any other conclusion. An extensive study of the integumentary organs, however, has led us to reflect more closely upon this matter, and to inquire what is a basement membrane, and what is the real distinction between the epidermic and the dermic elements of a membrane? We cannot here enter into the grounds for our conclusions (which will be stated in full in a forthcoming article on the "Tegumentary System," in Todd's "Cyclopædia of Anatomy and Physiology"), but must be content merely to state our conclusions that the existence of a basement membrane, $i$. e., of a structureless membrane, internal or external to it, proves nothing with regard to the dermic or epidermic nature of an organ, but that we must be guided entirely by the direction of its growth and metamorphosis. Every integument and every mucous membrane may, in fact, be distinguished into three portions; a central plane of indifferent tissue, from which growth and metamorphosis take place externally, to constitute the representative of epidermis or epithelium, to which we propose to give the name of ecderon; while internally, growth and metamorphosis take place from the central plane, so as to constitute the representative of the derm or "mucosa,". which we have termed the enderon.

Now the dental pulp is a process of the whole integument, and its outer surface, although bounded by a "basement membrane," truly represents the deepest layer of the ecderon of ordinary integument, while its inner substance belongs to the enderon. Although, therefore, the dentine is not a calcified cellular epidermis, it is a calcified ecderon, and grows in the same manner as an ecderon would do. The cement 
follows the dentine, and whatever the view we take of the development of the enamel, it also belongs to the ecderon. Although, therefore, the teeth are not, in the ordinary sense, epidermic structures, they are homologous with the ecderon, and not with the true derma or enderon of the mucous membrane. 


\section{IN D EX.}

A.

Air-cells of lungs, structure of, $575-575,579$.

Alimentary canal, muscular tunic of, 503 ; literature of, 527. See Stomach, Intestine.

Arachnoid membrane, structure of, in spinal cord, 393 ; of brain, 395 ; vessels of, 398 ; nerves of, $i b$.

Areolar tissue, 94; subcutaneous areolar tissue, 119 ; fat cells in, 120, 125.

Arteries, structure of tunics of, $678-684$; literature of, 724 .

Articular capsules, 295.

Articular cartilage, structure of, 292, 293, note; condition of bone beneath, 293 ; vascularity of, 294.

A scherson, vesicles of, 41 .

Axile-corpuscles, nature of, 130 ; terminations of nerves in, 134.

Axillary glands, 202.

Axmann, Dr., on ganglion-fibres, 406 , note; on axis-cylinder, 409 , note.

A yres, Dr., on prismatic crystals in coagulated blood of the Bitch, 715, note.

B.

Barry, Dr., on structure of muscular fibre, 227.

Bernard, M., on secretion of submaxillary, 465 ; on muscular fibres in inferior vena cava, 687 .

Berthold, Dr., on growth of hair, 196.

Berzelius, Prof, on medulla of bone, 282.

Bibra, Von, his analysis of muscle, 250 ; on chemical composition of dentine, 471 ; of enamel, 477; of the liver, 536.

Bichat, Prof., his influence on Histology, 33; on vessels in bone, 301 .

Bidder, Dr., on nerve-cells in the glossopharyngeal nerve, 417.

Bile, constituents of, 540 .

Bischoff, Prof., on lenticular glands of sto. mach, 509 , note.

Bladder. See Urinary Bladder.

Blood, changes of, in spleen, 560 ; elements of, 703 ; elementary granules of, 706 ; extraordinary constituents of, 713 : occurrence of crystals in, 714 .

Blood-corpuscles, red, form and character of, 703,704 ; nucleus of, 704, 722, note; me. thod of enumerating, 704 , note; size of, 705 ; diminution of number in disease, $i b$.; presence of, in chyle, 701 ; conditions of, iu various kinds of blood, 709 ; influence of reagents upon, 710 ; forms in blood of ani. mals, 712, 722, note; development of, 717 , 722.

Blood-corpuscles, colorless, form and origin of, 707 ; relative number of, compared with red, 708, 709 ; development of, into red, 718-723 ; literature of, 724 .

Blood-corpuscle-holding cells, occurrence of in spleen, 558-562; in congested lungs, 559 , note; in black vomit, ib.; import of, 560.

Blood-crystals, 714 .

Blood-vascular glands, tissue of, 116-118.

Blondvessels, varieties of, 674 ; tissues enter. ing into their composition. 674-678; development of, 715 ; literature of, 724. See Arteries, Veins, and Capillaries.

Bühm, Dr., on detachment of epithelium in cholera, 520.

Bone, intimate structure of, 266-281; Haversian canals of, 267-270; lamellæ of, 270275 ; lacunæ and cells of, 275,279 ; canaliculi of, 276 ; contents of lacunæ of, 280 ; peri. osteuın, 281 ; marrow of, 282 ; cells of medulla of, 283,284 , note; connections of, 284-291 ; physical and chemical properties of, 299 ; vessels of, 301 ; lymphatics of, 302 ; nerves of, 303 ; development and formation of, 306,330 ; vital phenomena of, 336 ; pathological changes of, 340 ; investigation of, 342 ; literature of, 102,344 .

Bownan, Mr., on structure of muscular fibre, 225,228 , note; on rupture of muscular fibrils in tetanus, 258 ; on ciliary motion in Malpighian corpuscles, 601 ; on structure of cornea, 728,729 ; on nerve-cells of retina, 743 ; on constitution of vitreous humor, 754,755 ; on glands in olfactory mucous membrane, 780. See 'Todd and Bowman.

Brain, ganglia of, 382. See Cerebrum and Cerebellum.

Brain-sand, 402, note, 403.

Bright's disease, tubuli uriniferi in, 602 .

Bronchiæ, intimate structure of, 578 ; arteries of, 581 .

Brüch, Prof., on the anatomy of the intestinal villi, 518 , note.

Brücke, Prof., on cadaveric rigidity, 253 ; on muscular fibre in villi, 514 ; on structure of lacteals and absorption of chyle, 516 , note; on lymphatic glands, 699 ; on function of bacillar layer of retina, $\mathbf{7 4 8}$; on structure of vitreous body, 754 .

Brullé and Huguény, MM., experiments upon bones of growing animals, 329, 338 .

Brunner's glands, strucıure of, 519 ; vessels of, 520 ; method of examining, $52 \%$.

Burnett, Dr., on development of spermatic filaments, 625, note.

Bursæ mucosæ, structure of, $123,239$.

C.

Capillaries, structure of, $689-693$; develop. ment of, 716. 
Carpenter, Prof., on structure of muscular fibrils, 229 , note; on basement membrane in corium, 141.

Cartilage, its composition, 79; varieties of, 80 ; in pathological structure, 322 ; literature of, 81 ; costal cartilage, 290; of articulations, 291 ; structure of, when loose in joints, 298; chemical composition of, 300 ; nerves of, 305 ; development of, 307 , 309 , note; changes during ossification of, 313,316 ; vessels of, 315 .

Cells, general anatomy of, 43-70; furms and contents of, 44 ; size of, 44,45 ; membranes of, 45,47 ; chemical composition of, 45 ; nuclei of, $44,45,46$; nucleoli of, 46 ; comparison with primordial utricle in plants, 47 ; development of, 48,54 ; multiplication by division, 54 ; vital phenomena of, 59,60 ; processes in the interior of, 61 ; changes and chemical composition of contenis, 165 ; elasticity of cell-membranes, 67 ; excretive processes, ib. ; contractility of, 68 ; mctamorphoses of, 69 , 70 .

Cellular tissue. See Areolar tissue.

Cement, chemical composition of, $4 \mathrm{SO}$; struc. ture of, 481; development of, 492.

Cerebellum, elementary tissues of, 379 ; gray layer of, 380 ; composition of its crura, 381 .

Cerebral nerves, structure of, 416 .

Cerebro-spinal fluid, analysis of, 401.

Cerebrum, ganglia of, 382 ; structure of hypophysis, 385 : corpora striata, 382 ; corpora quadrigemina, 384 ; optic thalami, 384 ; hemispheres of, 385 ; intimate structure of convolutions, 386 ; origin of nerve.fibres in 390 ; membranes of, 392-399; ependyma of ventricles of, 397 ; vessels of, 400 .

Ceruminous glands, structure of, 209 ; secre. tion of, 210-212; literature of, 212.

Choroid coat of eye, layers of, 733 ; pigmentum nigrum, 735; vessels and nerves of, $73 \%-739$.

Chyle, elements of, 700 .

Cilia, structure of, in cells lining larynx, 570:

Ciliary ligament, 734 .

Ciliary motion, agents for re-exciting it, 571 , note; in neck of Malpighian border of kidney, 601.

Clark, Prof., on vegetable growths on the tongue, 452 , note.

Clitoris, 655 .

Cochlea, parts of, $770-774$; zona pectinata, 773 ; nerves of, $774-775$, vessels of, 775 ; physiology of, 775 , note; investigation of, $77 \%$; literature of, 778 .

Colostrum, 665, 666 .

Conjunctiva, structure of palpebral, 759; of corneal, 728; vessels of, 732, 760.

Connective tissue, elements of, 89,94 ; chemical reactions of, 90 ; development of, 90,95 , 97 , note; forms of, 91-94; corpuscles of, 85 .

Connective-tissue-corpuscles, 85.

Contractile tissue, dependent on nuscular fibre-cells, 104.

Colloid, occurrence of, in thyroid gland, 587 ; in tubuli uriniferi of kidney, 601 .

Cooper, Sir Astley, on cavity of thymus gland, 590.

Copulation, motile phenomena in act of, $63 \%$.

Corium, structure of, 123 ; development of, 134.

Cornea, elements and structure of, $727-733$; vesselsand ly mphatics of, 730 ; nerves of, 731 .
Corpora Intea, formation of, 643 ; structure of, $645,78 \%$.

Corpuscula amylacea, of nervous system, 41 ; in ventricles of the brain, 401, 402, note.

Corpus striatum, nerve-fibres of, 382 .

Corti, Marquis, on connection of the nerve. cells of the retina with the nerve-fibres, 750 ; on structure of cochlea, 772, 775, note, 777 .

Costal cartilage, structure of, 290 .

Cowper, glands of, 630,635 .

Cruveilhier, Prof., on pathological changes in articular cartilage, 295 ; on lymphatics of articular cartilage, 303.

Czermak, Prof., on structure of dentine, 475, note.

\section{D.}

Dalton, Dr., on corpus luteum of nienstruation and pregnancy, 645 , note.

Deciduæ, 651, 653.

Deglutition, organs of, 499-502. See Pharynx, CEsophagus.

Demours, membrane of, 729 .

Dentine, structure of, 469 ; canals of, 470 ; chemical composition of, 471 ; formation of, 491.

Digestion, changes which the epithelial cells and the villi undergo during, 515 ; organs of, 436-568. See Intestinal Canal, Liver, and Pancreas.

Donders, Prof., on development of connective tissue, 85 ; on nature of fibres of intervertebral ligaments, 287 ; on composition of carilage, 300 .

Donné, M., on spermatozoa, 624 ; on colostrum in human milk, 666 .

Ductless glands, tissue of, 116. See Spleen, 'Thymus, 'Thyroid, and Supra-renal bodies.

Duhamel, M., his experiments upon growing animals, 328.

Dura mater, structure of, 392,394 ; vessels of, 397 ; nerves of, 393 .

\section{E.}

Ear. structure of external canal, 767 ; ossicula of, 768 : membrana tymparii, 768 ; vestibule and osseous semicircular canals, 769 cochlea, 770-778; development of, 776 ; literature of, 778 .

Ecker, Prof., on degenerations of thyroid, 588 ; on structure and development of thy. mus, 592,595 .

Elastic ligaments, 284.

Elastic Tissue, elements of, 81 ; chemical reactions of, 82 ; development of, 83 ; literature of, 88 ; presence of, in ligamentum nuchæ, 284.

Elementary parts, simple, 39-70 ; higher, 70118 ; literature of, 71.

Enamel, structure of, 476,478 ; chemical composition of, 477 ; Nasmyth's membrane, 480 ; formation of, 489 .

Endocardium, structure of, 669 ; nerves of, 671.

Ependyma, of ventricles, 396, 401 ; of spine, 397 , note.

Epidermic Tissue, character of, 74 ; forms of, 75 ; prevalence of, in animal kingdom; in 
pathological formations, 78 ; literature of, 78.

Epidermis, 139-158; Malpighian layer, 140 ; horny layer, 142; color of, 144 ; pliysical and vital properties of, 146 : action of reagents on, $14 \%, 148$; on epidermis of Negro, 149 , note; grow th and regeneration, 151 ; development of, 154

Epididymis, 620, 626 .

Epiglottis, structure of, 569; glandules of, 572.

Epithelium, forms of, 76 ; of stomach, 509 ; of villi, 514 ; changes of, during digestion, 515,516 , note; ciliated, of larynx, 570; of air-cells, 579 ; of bronchiæ, 578 ; of uterus, 647 ; varieties of lining vessels, 676 ; of membrane of Descemet, 729 ; of olfactory organ, 779, 781.

Eulenberg, Dr., his case of black fur of the tongue, 453.

Eye, tunics of, 725 ; sclerotic coat, 725 ; vascular tunic, 733-739; retina, 739-750 ; lens, $750-753$; vitreous humor, $753-777$; accessory organs of, $75 \tau-760$; physiological remarks on, 760 ; vessels of fœtus, 761 ; histological development of, 762 ; mode of investigating, 763 ; literature of, 765 .

Eyelids, $75 \%$.

\section{F.}

Fibro-cartilage, 240 ; structure of interarticular, 297; chemical composition of, 300.

Fibrous tissues, 81, 89. See Connective and Elastic Tissue.

Filum terminale, 367 ; corpuscula amylacea in, 401 .

Follicles, solitary, of small intestine, 523 ; of large intestine, 525 .

Fremy, M., on chemical composition of brain, 359.

Frerichs, Prof., on synovia, 298; on chemical composition of kidney, 610 ; on semen of the carp, 624.

Fuchs, Prof., on infusoria in milk, 666 .

Funke, Dr., on crystals in the blood, 214.

\section{G.}

Gall-bladder, structure of, 538 .

Ganglia, fibres of, 405 ; cells of, 109, 406 ; upon glosso-pharyngeal nerve, 454 .

Ganglion-globules. See Nerve-cells.

Ganglionic nerves, 418. See Sympathetic nerve.

Gastric glands, structure of, 506, 507, note; 509 , note; secretions of, 508 .

Gelatiniform connective tissue, 96 .

Genital organs, external, of male, 630 ; of female, 655 .

Gerber, Prof., his method of examining the skin, 158.

Gerlach, Prof., on Malpighian bodies, 601, note; on formation of blood-globules, 722 .

Glands, structure of, in oral cavity, 456 ; follicular, 459; salivary, 463 ; of stomach, 506 ; of intestine, 518-525; of larynx, 572; of trachea, 573; of Cowper, 630; of Littre, 631; of uterus, 647 ; of external genital organs in female, 655 ; Meibomian, of eye. lid, 758; "of Bowman," 781.
Glundular tissue, varieties of, 113-115; lite. rature of, 116.

Glisson, capsule of, $532,544,546$.

Gosselin, M., on composition of semen after epidid ymitis, 625 , note.

Graafian vesicles, 640, 643, 645 .

Granules, general consideration of, 41 ; tremulous motion of, 64 ; formation of, in nuclei, 64 .

Gums, composition of, 484 ; in fœtus, 488.

\section{H.}

Hair, disposition and size of, 171 ; chemical composition of, 172; intimate structure of, 173-182; properties of, 182 ; papilla, 183; root sheath of, 184 ; development of, 186 ; shedding of, 191 ; physiological observa tions on, 193 ; abnormal conditions of, 197 ; method of investigating, $i b$.; literature, 198.

Hancock, Mr., on organic muscular fibres of the urethra, 631 , note.

Hannover, Dr., on nerve-fibre in the yellow spot of the eye, 750 , note; on membranes in fœtal vitreous humor, 755 .

Harting, Mr., his measurements of blood-corpuscles, 705.

Hassall, Mr., on corpuscles in Thymus, 593; on peculiar concentric bodies in fibrinous clots in the heart, 713.

Hasse, Prof., on medulla-cells in cylindrical bones, 283 .

Haversian canals, origin of, 318,327 .

Haversian glands, $296^{\circ}$.

Heart, muscular structure of, $668,671-674$; endocardium of, 669 ; bloodvessels of, $6 \% 0$; valves of, $i b$.; nerves of, 671 ; development of, 715 ; mode of examining its structure, 723 ; literature of, 724 .

Henle, Prot., on smooth muscles in skin, 121; on hair-bulb, 195, note; on cells of reticular cartilage, 569 ; on composition of semen, 624 ; on absence of the "rods" in the yellow spot of the eye, 746 .

Hepatic artery, branches of, 544-546.

Hepatic cclls, 532-536.

Hepatic ducts, $537-539,540$, note; vasa aberrantia of, 538 .

Heyfelder, Dr., on muscular layer in lymphatic glands, 698.

Horner, Prof., on axillary glands in Negro, 202 , note.

Hyaloid membrane, 753, 755.

Hymen, tissues of, 654 .

Hypophysis cerebri, structure of, 385 .

I.

Interlobular connective tissue of lungs, 580 .

Interosseous membranes, structure of, 238 .

Intervertebral ligaments, structure of, 286 .

Intestiral canal, elementary composition of, 436 ; development of, 526 ; literature of, 527. See Oral Cavity, Stomach, and Intestine.

Intestine, small, mucous membrane of, 511 ; villi of, 511-518; lacteals of, 513,516 , note; glands of, 518-520; closed follicles of, 520524 ; mode of examination of, 527 ; literature of, $52 \%$. 
Intestine, large, mucous membrane of, 524 ; glands of, 5i25; literature of, 527.

Iris, elements of, 735- 738 ; cause of color of, 736 ; vessels and nerves of, 738, 739 .

\section{J.}

Jacob, membrane of, 782 .

Jaundice, teeth in, 497.

Jobert de Lamballe, M., on nerves of gravid uterus, 652 .

Jones, Dr. Hanfield, his views on termination of hepatic duct, 540, note; on the development of the liver, 547 , note.

Jones, Mr. Wharton, on Malpighian follicles of spleen, 555, note; on blood of splenic vein, 562, note; on the blood-corpuscles in animals, 702, note; on nucleus of colorless blood-corpuscles, $70 \%$, note; 722 , note; on corpora lutea, 787 .

\section{K.}

Keber, Dr., on entrance of spermatozoon into the ovum of Unio, 637, note.

Kidney, general composition of, 596 ; urinife. rous tubules of, 597-602; Malpighian corpuscles, 598, 600; pathological degenerations of, $601,606,611$; vessels and nerves of, 602,606 ; struma of, 606 ; calices and pelvis, 607 ; chemical composition of, 610 ; mode of investigation of, 611 ; literature, 612.

Kiernan, Mr. on structure of liver, 539, 543.

Kilian, Prof.. on nerves of gravid uterus, 653.

Krause, Prof., on investigation of sudoriparous glands, 208; on gastric villi, 505, note.

L.

Lacteal glands, structure of, 661 ; enlargement of, during lactation, 662; physiological remarks on, 663 ; secretion of. 664 ; method of examining, 666 ; literature of, 667 .

Langenbeck, Prof., on muscular ring in zone of Zinn, 757.

Larynx, cartilages of, 569 ; ligaments of, ib. ; mucous membrane of, 570 ; glands of, 572 ; vessels and nerves of, $i h$.

Lassaigne, M., his analysis of brain, 359 .

Lee, Dr., on ganglia in the heart, 671 .

Leeuwenhoek, on anastonıosis of primitive fasciculi of muscle, 108, 668 .

Lehmann, Prof., on fluid of vesiculæ seminales, 624 ; on presence of colostrum-corpuscles in acute diseases, 666 .

Leidy, Prof., on elements of articular cartilage, 293; on structure of liver, 537 , note.

Lens, crystalline, elements of, $750-754$; capsule of. 750 .

Leydig, Dr., on nerve-fibres in the skin of Fishes, 415; on Malpighian corpuscles in Fishes and Reptiles, 555, 556, note; on structure of supra-renal capsules of mammalia, 617, note; on origin of fibres of the olfactory nerves in the Plagiostomata, 783 .

Lieberkühnian glands, structure of, 519,525 ; viscid secretion of, 520 .

Ligamenta flava, structure of, 284.
Ligaments, connecting bone, 284 ; between vertebræ, 285 ; nerves of, 305.

Ligament un nuchæ, structure of, 284 ; nerves of, 305 .

Liston, Mr., on vessels of articular cartilage, 294.

Liver, component parts of, 528: arrangement of, in animals, 529,536, note; in Man, 531; secreting cells of, 532-536; capsule of, 532 , 546 ; chemical analysis of, 536 ; excretory ducts of, $53 \tau-540$; secretion of, 540 ; arrangement of vessels in, 541-545; lymphatics in, 545 ; nerves of, 546 ; development of, 546,547 ; mode of investigating, 548 ; literature of, 548 .

Loose cartilages, in joints, structure of. 298.

Ludwig, Prot., on albumen in healthy kid. neys, 610 ; on muscular tibres of left ventricle, 672 .

Ludwig and Nolla, MM., on structure of lymphatic glands, 696,699 .

Lungs, intricate structure of, 574-581; vessels and nerves of, $581-583$; development of, 583 ; pathological changes in parenchyma, 584 ; mode of investigation, $i b$.; literature of, 585 .

Luschka, Prof., on nerves of bone, 304 ; on cellulose corpuscles in the ganglion of Gasser, 403; on ligament of the iris in animals, 729 .

Lyinph, elements of, 700 ; formation of corpuscles of, 702 .

Lymphatics, structure of, 693.

Lymphatic glands, structure of, 695,698 ; connection of, with lymphatic vessels, 696 ; degenerations of, 699 .

\section{M.}

Malpighi, Prof., on structure of lymphatic glands, 696.

Malpighian corpuscles, of spleen, 552-556, 557 , note, 786; of kidney, 59\%, 600 .

Mammary Glands. See Lacteal glands.

Matrix, of tissues in general, 40 ; of bone, 270 . Marrow. See Medulla.

Measurements, those generally employed, 43 ; table of, $i b$.

Medulla, of bone, 282 ; of cartilage, 291; composition of, in fœtus, 322 .

Medulla oblongata, structure of, 372 ; gray substance of, 374 ; origin of nerves from, $376,3 \pi \%$.

Meibomian glands, 758 .

Membrane of Descemet, 729,734 .

Microscopes, varieties of, 38 .

Milk, constituents of, 664 ; varieties of, 666 .

Mohl, Prof. Von, observations on cell-division in plants, 56 , note.

Moleschott, Dr., on development of bloodcorpuscles, 562 , note; on relative number of red and white corpuscles, 708 .

Mouth. See Oral Cavity.

Mucous bursæ, 239.

Mucous corpuscles, 465 .

Mucous membranes, general structure of, 436 ; of mouth, $i b$.; of tongue, 446 ; intimate structure of glands of, 456 ; of pharynx. 500; of intestine, 511, 524; of larynx, 570; of bronchiæ, 5\%8; olfactory, 780.

Mucous sheaths, 239. 
Müller, Dr. H, on glands in ichthyosis congenita, 221; on development of bone, 396 ; on radiating fibre-system in retina, $\mathbf{7 4 6}$, 749,750 , note.

Muiller, Prof. J., on nerve-fibres in orbital muscle of the Pike, 249; on interarticular cartilnge, 300 ; on Malpighian corpuscles of spleen, 555; on helicine arteries, 633 , 634 ; on composition of cornea, 727.

Mulder, Prof.. on chemical examination of the nails, 165 ; on organic basis of cartilage, 200.

Muscle, striatcd, elements of, 223 ; constitution of fibres of, $225-230$; nature of primitive fibrils, 227; connection of, with other parts, 231; vessels in, 243; lymphatics in, 245 ; nerves of, 245-250; chemical and physical relations of, 250 ; development of, 2.53-257 ; parhological conditions of, 257259 ; physiological remarks on, 259-264; method of investigating, 264-266 ; literature, $109,266$.

Muscular fibre. See Muscle.

Muscular fibre-cells. Se€ Snooth muscular fibres.

Muscular system, constituents of, 222,266 .

Muscular tissue, smooth, structure of, 102 ; physiological importance of, 103 ; distribu. tion of, 103; in contractile tissues, 104 ; in vertebrata, 105 ; literature of, 106 ; action of reagents on, 105 ; presence of, in walls of œsophagus, 501 ; in intestine, 511 ; in villi, 514 ; in corpora cavernosa of penis, 630 ; in coats of vessels, 676 ; in arteries, 683 ; in veins, 686,689 .

Muscular tissue, striated, elements of, 106, 225 ; chemical composition of, 106 ; distribution of, 107, 108; literature of, 108.

\section{N.}

Nails, layers of, 159 ; structure of, 161 ; action of reagents on, 163 ; growth of, 166 ; pathological conditions of, 168; development of, 169; method of investigating, 1\%0; literature, 170.

Nasmyth, Mr., on structure of enamel, 476 , note.

Neill, Dr., on gastric villi, 505 , note.

Neilson, Dr., on impregnation of ovum in Ascaris mystax, 637, note.

Nerve-cells, structure of, 357-359; in me. dulla oblongata, 376 ; in cerebellum, 380 ; in ganglia, 406 ; pale processes of, 408 ; connection of, with nerve-fibre, 409 ; in the sympathetic, 421 ; development of, 427 .

Nerve-fitre, structure of, 345-356; medulla of, 346,350 ; axis-cylinder of, 347,351 , $353-356$; sheath of, 349,350 ; in the skin of animals, 414 ; terminations of, 415 ; by loops, 415, 418; development of, in Tad. pole, 423.

Nervous system, elements of, $345-359$; divisions of, 345 ; central nervous system, 359 ; peripheral nervous system, 404 ; de. velopment of elements of, $427-431$; functions of, 431 : literature of, 112, 434. See Cerebrum, Spiral Cord, Cerebellum, Medulla oblongata, Nerve-fibre, Nerve-cells, and Nervous Tissue.

Nervous tissue, elements of, 109,345 ; varie. ties of, 110; physiological importance of,
111 ; distribution of, 112 ; literature of, 112 , 434 ; chemical composition of, 111,359 ; pathological changes of, 431 ; method to be employed in investigations of, 434 .

Newport, Mr., on impregnation of ovum of Frog, 636, 63\%, note.

Nipple, structure of, 662 .

Nose. See Olfactory Organ.

Nuclei, occurring free, 42 ; multiplication of by division, 50 ; origin and development of, 58 ; importance of, in cell development, $i b$.; occurrence of, in blood-corpuscles, 722, note. Nucleus-fibres, $81,84,85$.

\section{O.}

Esophagus, structure of walls of, 501 ; literature of, 502 .

Esterlein, Prof., on epithelial cells of intestine, 517, note.

Olfactory nerve, fibres of, 390,783 .

Olfactory organ, structure of, $778-783$; mucous membrane of, $780-783$; mode of investigating, 783 ; literature of, 784 .

Optic nerve, origin of fibres of, 390 ; course of fibres in the eye, 744,745 .

Oral cavity, mucous membrane of, 436 ; minute structure of mucous membrane of, 437 ; vessels and lymphatics of, 438 ; epithelium of, 439-441; mucous glands of, 455; their intimate structure, 456-459; mode of examining its mucous membrane, 466 ; literature of, $46 \%$. See Teeth, Tongue. Organs, classification of, 73,74 .

Osseous system, 266; nerves of, 303-306. (See Bone.)

Osseous tissue, elements of, 99, 267; distribution of, in vertebrata, 101 ; literature of, 102, 344 ; forms of, 266. See Bone.

Otolithes, composition of, 770 .

Ovary, structure of, 640-642 ; mode of examining, 659 ; literature, $i b$.

Oviducts, 646 .

Ovum, composition of, 642 ; detachment and reformation of, 643 .

Owen, Prof., on "contour lines" of dentine, 472; on dentine containing Haversian canals, 474 ; on structure of dentine, 475 , note.

\section{P.}

Pacchionian granulations, 403.

Pacini, Prof., on termination of nerves in corpuscles, 412.

Pacinian bodies, structure of, 413,785 .

Pancreas, structure of, 549 ; literature of, 550 .

Panum, Dr., experiments on formation of membranes, 43 .

Parotid gland, 463.

Parovarium, 642.

Penis, structure of, 630,634 ; arteries of, 633 ; literature of, 639 .

Pericardium, 667.

Periosteum, structure of, 281 ; lymphatics of, 303 ; layers of bone formed on inner aspect of, 324, 328; lining osseous semicircular canals, 769.

Peritoneum, structure of, 502.

Peyer's glands, composition of, 521, 523 : lymphatics of, 523,524 . 
Pharynx, mucous membrane of, 500; glands of, $i b$.

Pia mater, of spinal cord, 394 ; of brain, 395 ; bloodvessels and lymphatics of, 398 ; nerves of, 399; Pacchionian granulations of, 403.

Pigment cells, 144; in skin of Negro, 144, note.

Pineal gland, structure of, 385 .

Placenta, 653.

Pleuræ, structure of, 574 .

Pons Varolii, 372, 376.

Prepuce, 632.

Primordial cartilaginous skeleton, 306 ; metamorphoses of, 310 .

Prostate gland, structure of, 628-630; concretions in, 629.

Purkinje, Prof., observations on endocardium of Ruminants, 108 ; on nerves in the pia mater of the $\mathrm{Ox}, 399$.

\section{Q.}

Quekett, Prof., on elastic fibres in Giraffe, 81.

R.

Reichert, Dr., on crystalline form of histogenetic substances, 57 ; on fibrous tissue, 94 ; on cortical substance of hair, 176, note; on epithelium upon articular cartilages, 291; on cartilage corpuscles, 311 , note; on crys. tallized albuminous matter, 715 .

Reid, Dr. John, observations on paralyzed muscles, 258.

Reiney, Mr., on existence of a choroideal muscle, 739 .

Reinhardt, Dr., on colostrum, 665.

Remak, Prof., observations on cell-division, 55 ; on cartilage-corpuscles, 311 , note; on "fibres of Remak," 423,426 ; on ganglia upon glosso-pharyngeal nerve, 454 ; on development of thyroid, 588 ; on development of kidneys, 608 .

Respiration, organs of, 568. See Larynx, Trachea, and Lungs.

Retina, structure of layers of, 739-746 ; condition of elements of, at yellow spot, 746 ; connection of its various layers, 746-751; physiological importance of the separate layers, 748 ; especially of the radiating fibre-system, $748,749,750$, note; mode of investigating, 764; literature, 766 .

Retzius, Prof., on cells of dentine, 475, note; on Nasmyth's membrane, 477 , note.

Robin, M., on structure of cartilage, 311 , note; on formation of bone, 322 ; on vegetable growth on the tongue, 452 , note; on crystals obtained from blood-serum, 715.

Rokitansky, Prof., on new formation of mus. cular fibre, 257 ; on hypertrophy of thyroid, 588.

S.

Saliva, 465

Salivary glands, structure of, 464 ; secretion of, 465 .

Salter, Mr., on structure of dentine, 475, note.

Schwann, Prof., importance of his researches on Histology, 34 ; theory of cell develop. ment, 56,57 ; on development of connec- tive issue, 88,95 ; on development of cartilage, 310 ; on nerves of vas deferens, 628 .

Sclerotic coat of eye, structure of, $725-727$.

Sebaceous glands, occurrence of, 212-215 ; structure of, 216 ; development of, 218 220 ; abnormal conditions of, 221 ; method of investigating, 221 ; literature, 222.

Semen, spermatozoids of, $622-624$; chemical composition of, 624 ; formation of, 635,636 . Seminal tubes, 621 .

Septum narium, nerves in, 305 ; structure of cartilage of, 310 , note.

Sesamoid bones, 240.

Sexual organs, male, 618 ; physiological remarks on, 634; literature of, 638. See T'estes, Penis, Prostate Gland.

Sexual organs, female, 640 ; physiological remarks on, 656 ; mode of investigating, 659 ; literature of, $i b$.

Sharpey, Dr., on formation of certain bones of the skull, 335, note.

Simon, Dr., on increase of sudoriparous glands in elephantiasis, 207.

Simon, Mr., on structure and development of thymus gland, 590,595 .

Skin, laycrs of, 119-138; subcutaneous cellular tissue, 120 ; corium, 121-125; fat cells, 125 ; vessels, 127 ; papillæ, 121, 130 ; nerves of, 129, 132 ; physiological remarks on, 135 ; epidermis, 139-158; literature, 158; method of investigating. 157; glands of, 199-222.

Sinooth muscular fibres, 102. See Muscular Tissue, smooth.

Spermatozoa, composition of, 622 ; move. ments of, 624 ; action of reagents on, ib.; formation of, 623,625 ; absence and changes of, in disease, 625, note; as impregnating agents, 636 .

Spinal cord, division of, 360 ; intimate structure of white substance, 361 ; structure of gray substance, 362-365; posterior roots of nerves in, 366 ; filum ter minale, 367 ; agents for investigating the course of the fibres, 368 ; course of fibres in, 369 ; membranes of, 392-399; vessel of, 400 ; structure of ganglia of, 405.

Spinal nerves, origin of, 404; structure of ganglia on, 405, 406, note; course and termination of, 411 ; termination of, in Pacinian bodies, 412 ; neurilemma of, 414 ; ves. sels of, $i b$.

Spleen, coats and trabecular tissue of, 551 ; Malpighian corpuscles, 552-556; pulp or parenchyma, 556-559; changes undergone by blood-corpuscles in, 559-562; vessels and nerves of, 562-565; lymphatics of, 566 ; physiological remarks on, 567 ; inves. tigation of, $i b$.; literature of, 563 .

Stilling, Dr., on structure of spinal cord, 377.

Stomach, muscular tunic of, 503 ; mucous membrane, 505 ; villi of, 505 , note; glands of, 506-509; epithelium, 509 ; bloodvessels of, 510 ; lymphatics of, $i b$.; mode of examining, 527 ; literature, 527.

Sudoriparous glands, structure of, 200 ; secre. tion of, 202 ; ducts of, 204 ; development of, 205 ; pathological conditions of, 207; me. thod of investigation of, 208 ; literature of, $i b$.

Sublingual gland, 463.

Submaxillary gland, 463.

Supra renal capsules, intimate structure of, $613-615,617$, note; vessels and nerves of, 
615 ; physiological remarks on, 616 ; investigation of, 618 ; literature of, $i b$.

Sympathetic nerve, fibres of, 418,422 ; rami communicantes of, 419,421 ; cells of ganglia of. 421,424 ; peripheral distribution of, 423 ; fibres of Remak, 423,425 ; ultimate course of branches of, 425 .

Synapta digitata, development of cell in, 52 .

Symphysis pubis, cartilaginous layer of, 288; formation of bone corpuscles in, $i b$.

Synovia, microscopical appearance of, 298 ; chemical composition of, 298 .

Synovial membrane, 239; intimate structure of, 296.

Sweat-glands. See Sudoriparous Glands.

Synovial sacs, 242 ; structure of, in muscular system, 242; occurrence of cartilage cells in, 242 .

\section{T.}

Tactile corpuscles. See Axile Corpuscles.

Tarsal cartilages, 757 .

T'eeth, parts of, 468 ; dentine, 468 ; enamel, 476 ; cement, 480 ; pulp of, 483 ; development of, 484-494, 789; pathology of, 495; mode of examining, 497 ; literature, 498.

Tendons, structure of, 232-235 ; ligaments of, 239 ; vessels of, 244 ; nerves of, 247 ; chemical composition, 252 ; development of, 255.

Testes, glandular substance of, 620 ; seminiferous tubes of, 621-624; secretion of, 624 ; membranes, vessels, and nerves of, 626 ; structure of vasa deferentia and ejaculatory duct, 627,628 ; development of, 634 ; method of examining, 638 ; literature of, $i b$.

Thoracic duct, 694 .

Thyroid gland, structure of, 586 ; bloodvessels of, 587 ; development of, 588 ; pathological changes in, $i b$.; literature of, 589.

Thynus, structure of, 589-593; concentric corpuscles of, 593 ; development of, 594 ; investigation of, 595 ; literature of, $i b$.

Tissues, general anatomy of, 39-118; classification of, 72 .

'Todd and Bowman, Profs., on ligaments of the lower jaw, 285 ; on vessels of bone, 301 ; on epirhelium of membrana tympani, 768 ; on epithelium of olfactory nucous membrane, 779,780 .

Tomes, Mr., on composition of teeth, 471 ; on enamel prisms, 478, note.

Tomes and De Morgan, MM., on structure and development of bone, 2\%0, note; 2 $\mathbf{i}$, note; 280 , note; 323 , note.

Tongue, muscles of, 441-446; division of striated fibres in, 446 ; mucous membrane of, 447 ; papilla of, 447-451; mucedinous fungi on, 451 ; fur of, in diseases, 451,452 , note; physiology of papillæ of, 453 ; nerves of, 454 ; follicular glands of, 459 ; mode of examining papillæ, 466 ; literature, 467.

Tonsils, structure of, 461 ; vessels and nerves of, $i b$.; secretion of, 462 .

Toynbee, Mr., on termination of nerves in kidney, 605 , note.

Trachea, tissues of, 572 ; glands of, 573 ; ciliated epithelium of, $i b$.; bloodvessels of, 574 .

Tracheæ, termination of, 71 .

Tunica media, of arteries, 679, 682 .
U.

Ureters, structure of, 607.

Urethra, female, structure of, 608.

Urerhra, male, structure of, 631.

Urinary bladder, structure of, 607.

Urinary organs, enumeration of, 595 ; development of, 608; literature, 612. See Kidneys, Ureters, Bladder, and Urethra.

Urine, secretion of, 610 ; sediments in, 610 ; presence of albumen, fibrin, and fat in, 610 , 611.

Uterus, structure of, 646-649 ; distribution of vessels in, 648 ; ligaments of, $i b$.; changes in, at menstrual period and in pregnancy, 649-654; nerves of, 652 ; mode of invest $i$ gating, 659; literature of, 660 .

\section{V.}

Vagina, 654 .

Valentin, Prof., on termination of nerve-fibres in muscle, 248; on development of bone, 335 ; on movements of spermatozoa, 625 .

Vascular system, 667-725; literature of, 723 . See Heart, Bloodvessels, and Blood.

Vas deferens, 627.

Vasa vasorum, 677 .

Vascular tunic of eye, 733. See Choroid and Iris.

Vauquelin, M., his analysis of the brain, 359 ; of human semen, 624 .

Veins, structure of coats of, 684-689; valves of, 689.

Ventricles of heart, fibres of, 672 .

Vesicles, of Ascherson, 41; in milk, 41 ; in the yelk-substance, 42 .

Vesiculæ seminales; structure of, 628 .

Vierordt, Dr., on enumeration of blood-corpuscles, 704, note.

Villi, intestinal, 511; structure of, 512-514; epithelium of, 514,516 , note; changes they undergo during digestion, 515,516 , note: lacteals of, 513,516 , note.

Vitreous humor of eye, constitution of, 754757 ; investing membrane of, 754 ; develop. ment of, 755 .

Virchow, Prof., on connective-tissue corpuscles, 85 ; on gelatinous tissue of Wharton, 95 ; on mucoús tissue, 96 ; on contents of bursæ mucosæ, 239; on interarticular fibro-cartilage, 297 , note; on cartilage. corpuscles, 311, note; on microscopical conditions of osseous growths, 341 ; on spinal ependyma, 397 , note; on cellulose nature of the corpora amylacea, 402 , note; on colloid spleen, 554, note; on agents for restoring ciliary motion, $5 \% 1$, note; on prostatic concretions, 629 ; on cells resembling pus-corpuscles in the blood, 713 ; on the composition of the vitreous body in the fœetus, 755.

Volkmann, Prof., on origin of the fibres in the spinal cord, 370 ; on structure of glosso. pharyngeal nerve, 417 .

\section{W.}

Wagner, Prof., on axile-corpuscles, 130 ; on 
termination of nerves in muscle, 248,249 ; on ganglion-globules in the Ray, 391, 392 ; on bipolar cells in Fishes, 410.

Weber, Prof., his experiments on touch, 137 ; on smegma præputii, 215 ; on elasticity of muscles, 262 ; on branches of hepatic duct, 539.

Wedl, Dr., on hypertrophy of muscular fibre, 257, note; on structure of discased teeth, 495, note.

Welcker, Dr., his method of counting bloodcorpuscles, 704, note.

Wrisberg, cartilage of, $569,572$.
X.

Xanthogenus, presence of, in milk, 666 .

Y.

Yelk, cleavage of, 51 .

Yellow fibrous tissue. See Elastic Tissue.

Z.

Zinn, zone of, $753,754,756$.

Zona denticulata, 771 . 

RETURN TO the circulation desk of any University of California Library or to the

NORTHERN REGIONAL LIBRARY FACILITY Bldg. 400, Richmond Field Station University of California Richmond, CA 94804-4698

ALL BOOKS MAY BE RECALLED AFTER 7 DAYS

- 2-month loans may be renewed by calling (510) 642-6753

- 1-year loans may be recharged by bringing books to NRLF

- Renewals and recharges may be made 4 days prior to due date.

DUE AS STAMPED BELOW

\section{AUG 162001}

\section{AUG 212001}




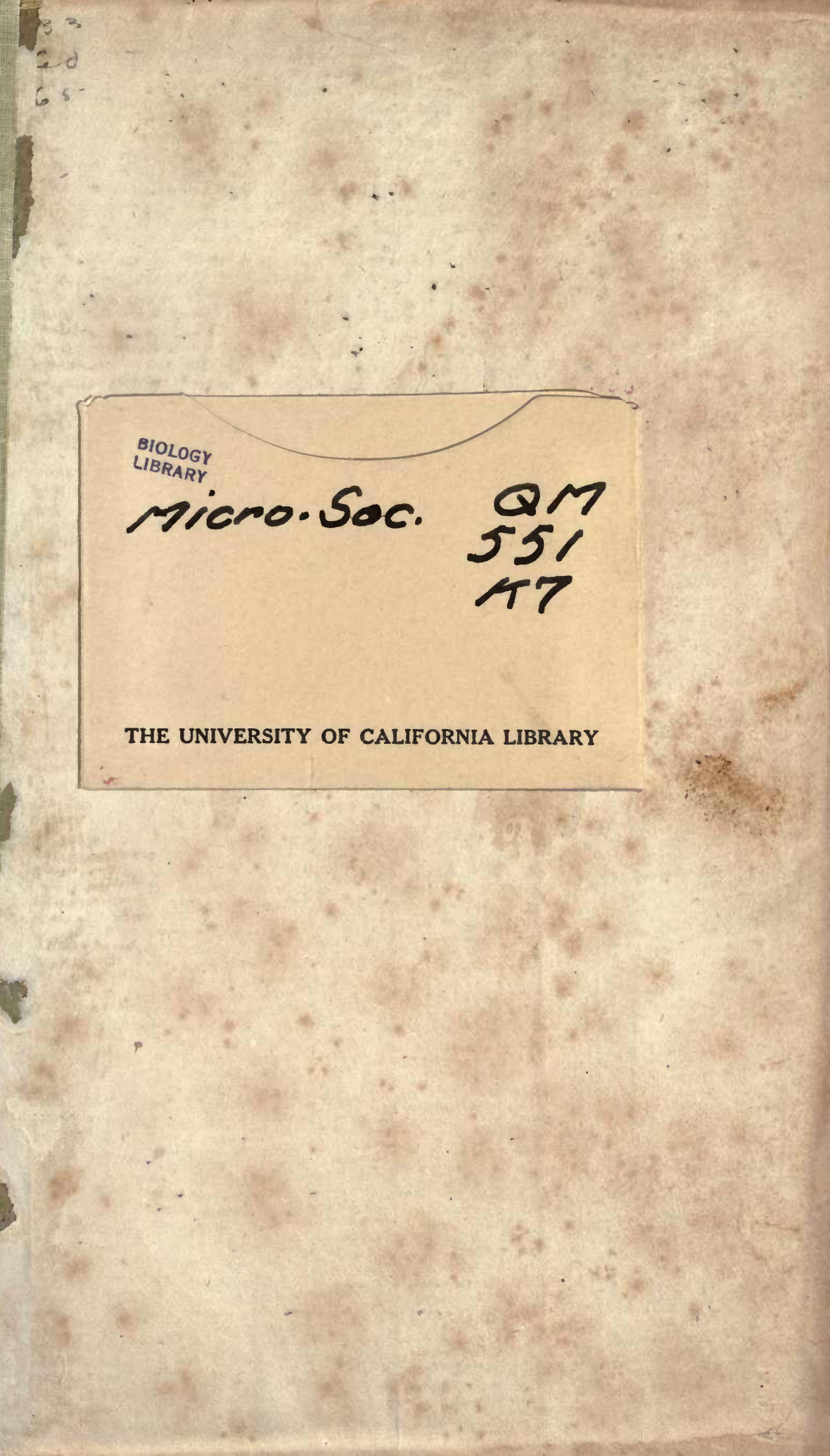


